\title{
Accident Fault Trees for Defense Waste Processing Facility
}

by

A. G. Sarrack

Westinghouse Savannah River Company

Savannah River Site

Aiken, South Carolina 29808

E. Nomm

L. P. Stringfield

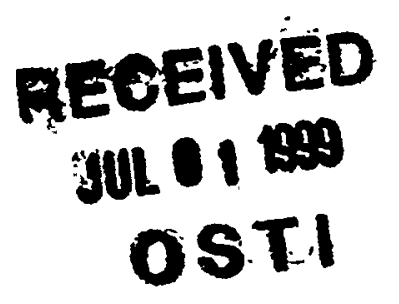

RECEIVED

JUL 01 WDO

$f$

This paper was prepared in connection with work done under the above contract number with the U.S.

Department of Energy. By acceptance of this paper, the publisher and/or recipient acknowledges the U.S. Government's right to retain a nonexclusive, royalty-free license in and to any copyright covering this paper, along with the right to reproduce and to authorize others to reproduce all or part of the copyrighted paper. 
X-ESR-S-00001

REVISION 0

\section{ACCIDENT FAULT TREES FOR DEFENSE WASTE PROCESSING FACILITY (U)}
A. G. Sarrack
E. Nomm
L. P. Stringfield

\section{Classification}
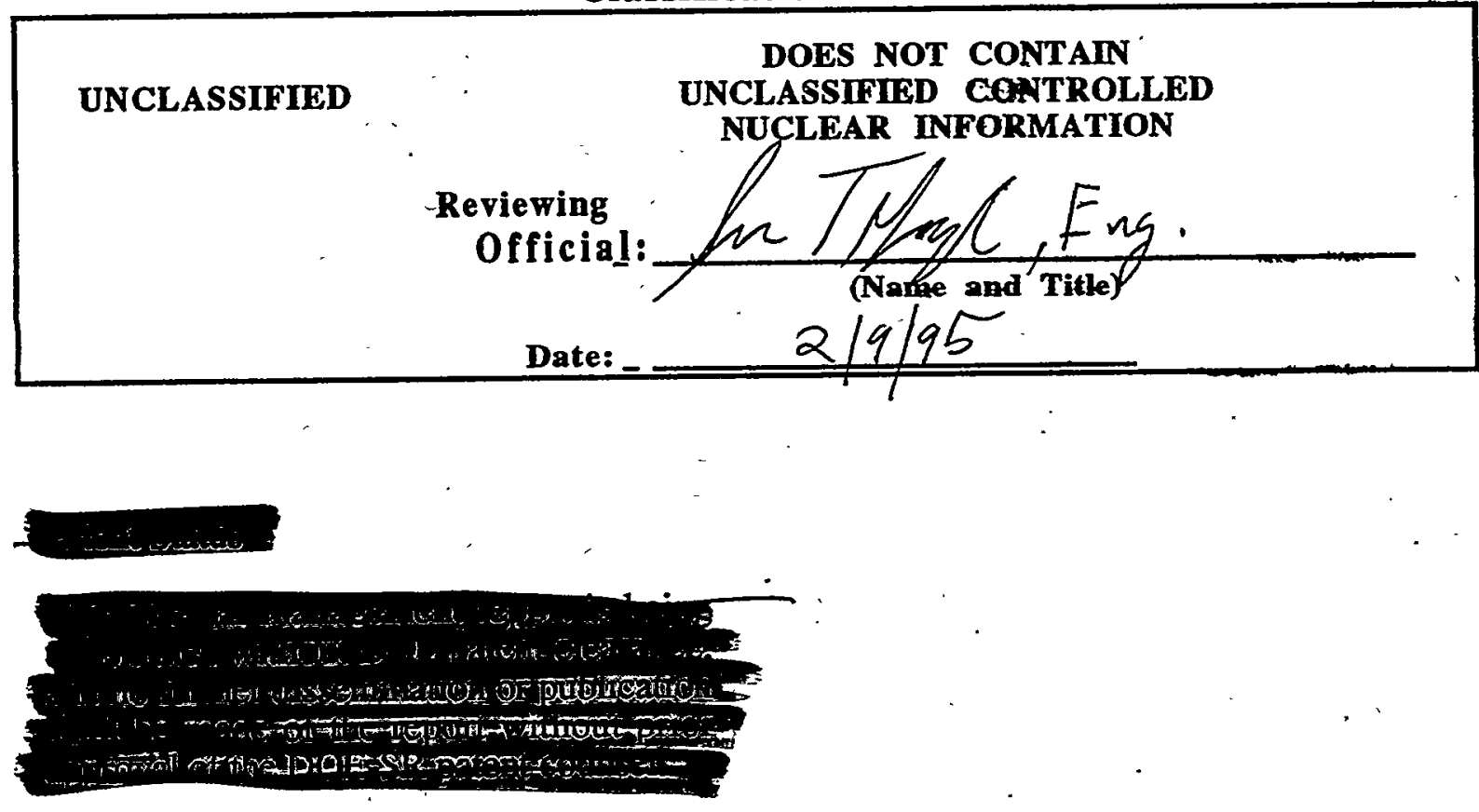

Westinghouse Savannah River Company Engineoring \& Conetruction Services Aiken, SC 29808

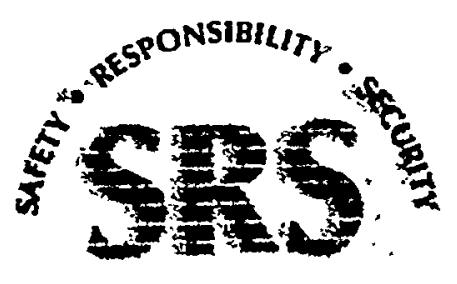

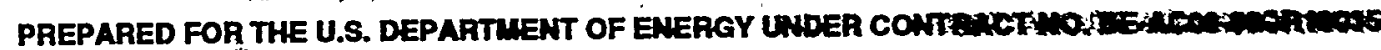




\section{DISCLAIMER}

This report was prepared by the Westinghouse Savannah River Corporation (Westinghouse) for the United States Department of Energy under Contract DE-AC0989 SR18035 and in an account of work performed under that contract. Neither the United States or the United States Department of Energy, nor Westinghouse, nor any of their employees, makes any warranty, expressed or implied, or assumes any legal liability or responsibility for the accuracy, completeness, or usefulness of any information apparatus, product, or process disclosed herein, or represents that its use will not infringe privately owned rights. Reference herein to any specific commercial product, process or service by trade name, mark, manufacturer, or otherwise does not necessarily constitute or imply endorsement, recommendation, or favoring of same by Westinghouse or by the United States Government or any agency thereof. The views and opinions of authors expressed herein do not necessarily state or reflect those of the United States Government or any agency thereof. 
$S A E S$

SAFETY ANALYSIS ENGINEERING SECTION
KEY WORDS:

DWPF

FAULT TREES

INTERNAL

ACCIDENTS

SEISMIC

RETENTION: PERMANENT

\section{ACCIDENT FAULT TREES FOR DEFENSE WASTE PROCESSING FACILITY (U)}
A. G. Sarrack
E. Nomm
L. P. Stringfield

Publication Date: January 1995

\section{Classification}

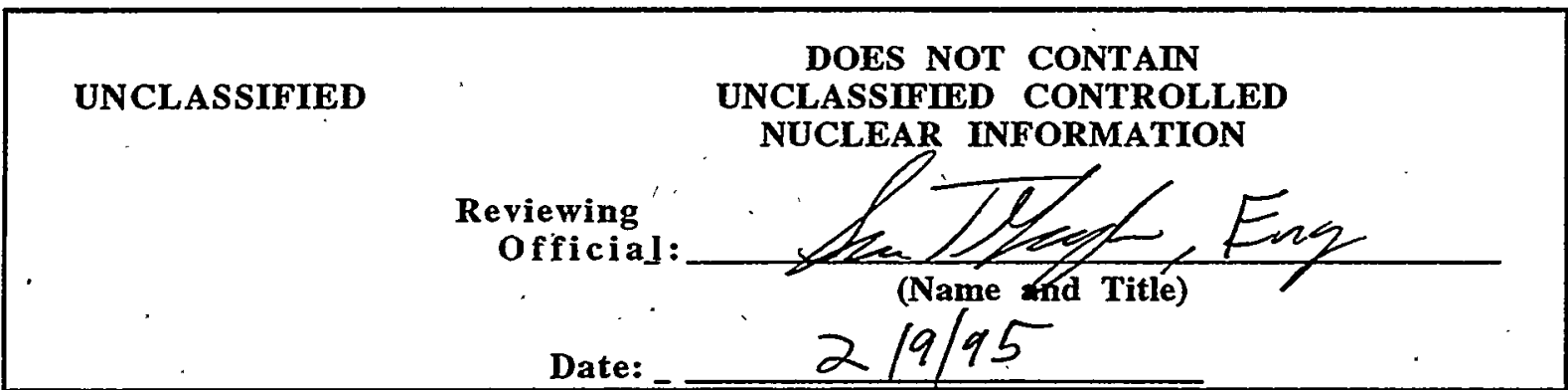

Westinghouse Savannah River Company

Engineering \& Construction Services Aiken, SC 29808

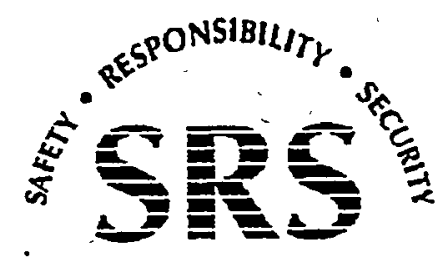

PREPARED FOR THE U.S. DEPARTMENT OF ENERGY UNDER CONTRACT NO. DE-AC09-89SR18035 
(This page is left blank intentionally.) 
PROJECT: N/A

DOCUMENT: $\quad$ X-ESR-S-00001, REV. 0

TITLE:

ACCIDENT FAULT TREES FOR DEFENSE WASTE PROCESSING FACILITY (U)

QA TASK NUMBER: N/A

REVISION NUMBER: $\quad 0$

APPROVALS:

Signature

assanese

AG Sarrack, Authbr, RAG

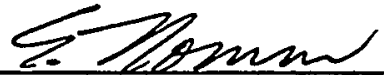

E Nomm, Author, RAG

2 - 2 flumen.

LeStringfield, Author, SAIC

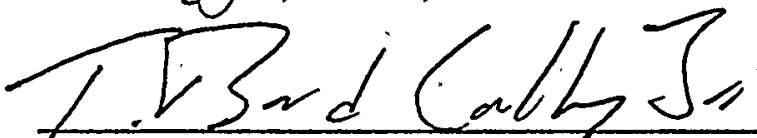

TB Calloway, Technical Reviewer, DWPF
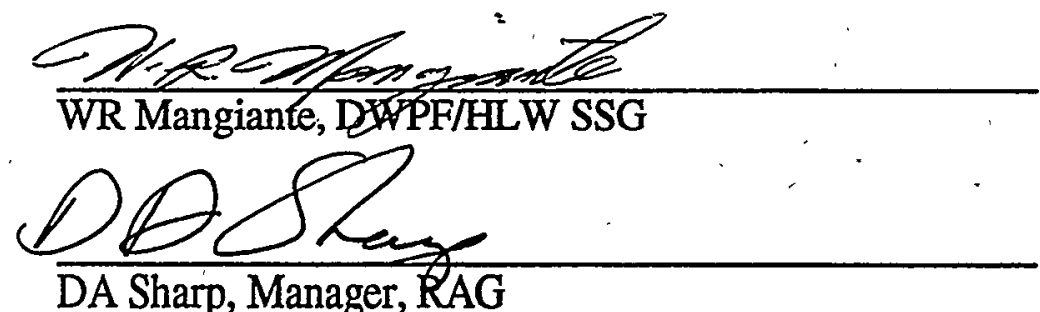

DA Sharp, Manager, RAG

IyJ fiitchler, Manager, SAES

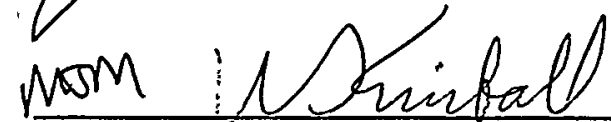

WD Kimbăll, Manager Engineering, DWPF $\underline{\text { Date }}$

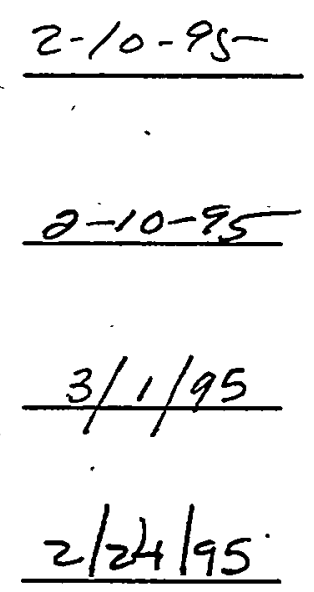

$3 / 2125$
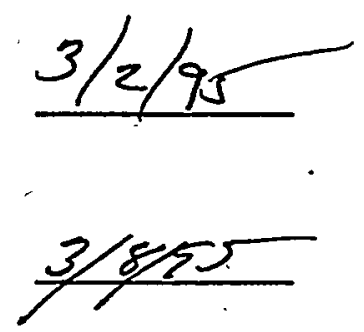

$3 / 10 / 95$ 

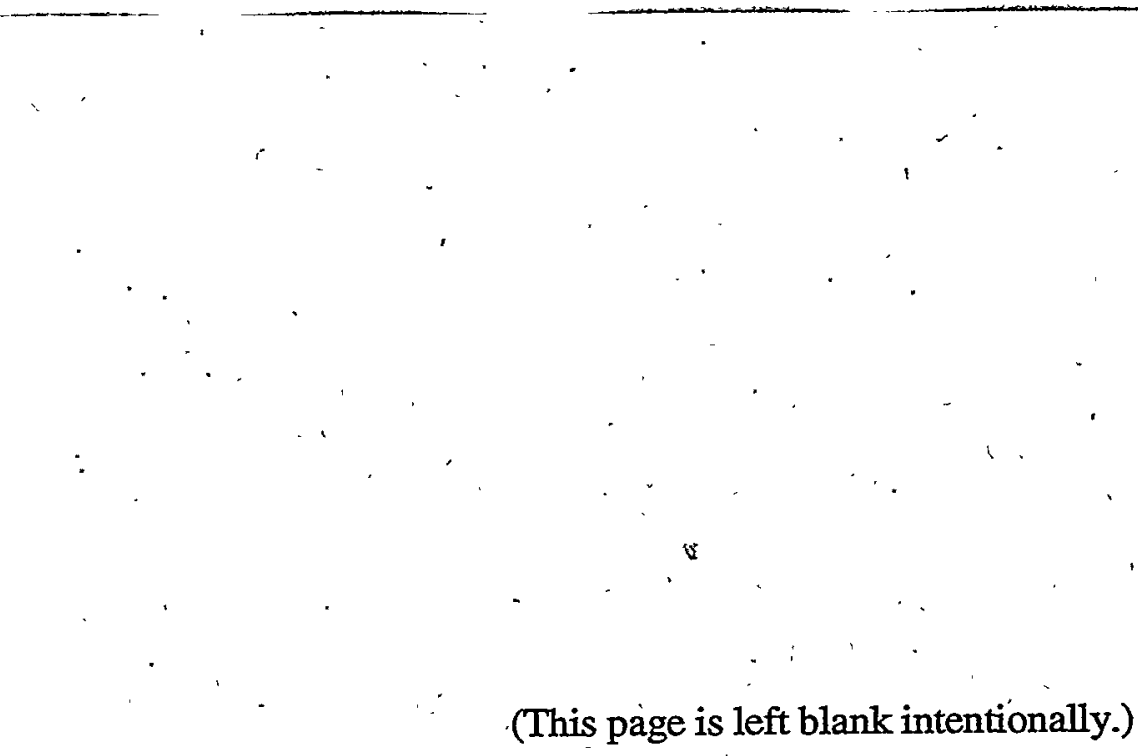

(This page is left blank intentionally.) 


\begin{abstract}
The purpose of this report is to document fault tree analyses which have been completed for the Defense Waste Processing Facility (DWPF) safety analysis. Logic models for equipment failures and human error combinations that could lead to flammable gas explosions in various process tanks, or failure of critical support systems were developed for internal initiating events and for earthquakes. These fault trees provide frequency estimates for support systems failures and accidents that could lead to radioactive and hazardous chemical releases both on-site and off-site. Top event frequency results from these fault trees will be used in further APET analyses to calculate accident risk associated 'with DWPF facility operations. This report lists and explains important underlying assumptions, provides references for failure data sources, and briefly describes the fault tree method used. Specific commitments from DWPF to provide new procedural / administrative controls or system design changes are listed in the "Facility Commitments" section. The purpose of the "Assumptions" section is to clarify the basis for fault tree modeling, and is not necessarily a list of items required to be protected by Technical Safety Requirements (TSRs).
\end{abstract}


(This page is left blank intentionally.)

\section{)}




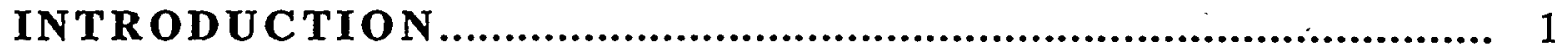

METHOD OR APPROACH...................................................... 1

RES UL TS ......................................................................... 3

INPUTS AND ASSUMPTIONS ................................................ 5

Assumptions Related to Accident Fault Trees ................................... 5

Process Vessel Vent Header (PVVH) Explosion.......................... 5

Sludge Receipt and Adjustment Tank (SRAT) Explosion................. 6

Slurry Mix Evaporator (SME) Explosion...................................... 7

Precipitate Reactor Bottoms Tank (PRBT) Explosion...................... 8

Melter Feed Tank (MFT) Explosion........................................... 9

Precipitate Reactor (PR) Explosion.............................................. 10

Organic Evaporator (OE) Explosion.............................................. 10

Organic Evaporator Condensate Tank (OECT) Explosion.................. . 11

Precipitate Reactor Feed Tank (PRFT) Explosion.......................... 12

Steam Explosion in Melter (SMELT)....................................... 12

Melter Off-gas (OFFGAS) Explosion............................................. 13

Sludge Pump Tank (SPT) Explosion........................................ 15

Precipitate Pump Tank (PPT) Explosion...................................... 16

Organic Waste Storage Tank (OWST) Explosion............................. 17

Salt Processing Cell (SPC) Explosion....................................... 18

Assumptions Related to Support Systems Fault Trees.......................... 18

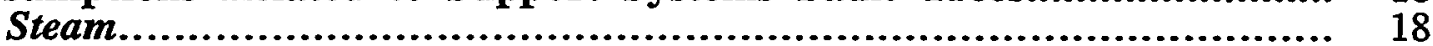

Process Chilled Water........................................................... 19

Process Cooling Water................................................................. 19

Cooling Tower Water............................................................. 20

Bulk Nitrogen System............................................................... 20

Bulk Nitrogen Tank.......................................................... 21

CPC Backup Purge Nitrogen Tank............................................. 21

CPC Backup Purge................................................................ 21

CPC Primary Purge............................................................... 22

Backup Electric Power.............................................................. 22

Primary (Normal) Electric Power............................................... 23

Zone 1 Ventilation............................................................. 24

SPC Primary Purge .................................................................... 25

SPC Backup Purge ............................................................. 25

SPC Low Pressure Nitrogen Supply.......................................... 26

LPPP Nitrogen System........................................................... 26

Instrument Air...................................................................... 26

General Assumptions.................................................................. 27

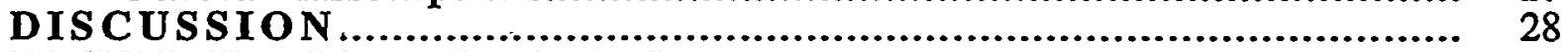

FACILITY COMMITMENTS ....................................................... 29

REEERENCES ................................................................... 32 
Table 1. Accident Fault Tree Results (Internal Initiators)................................. 3

Table 2. Support System Fault Tree Results (Internal Initiators̈)............................... 4 4 


\section{LIST OF ATTACHMENTS}

Attachment 1. List of Drawings (P\&ID's) Used ....................................................................33

Attachment 2. Basic Event Nomenciature...................................................................................37

Attachment 3. Basic Event (BE) File .................................................................................42

Attachment 4. Type Code (TC) File..............................................................................................96

Attachment 5. Accident Fault Tree Plots, Cutsets, and BE Importance .....................................109

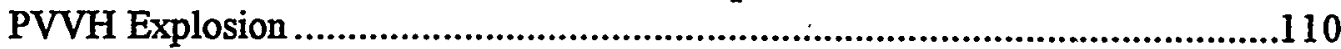

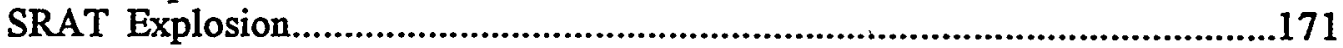

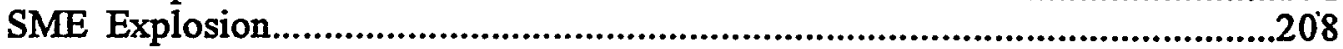

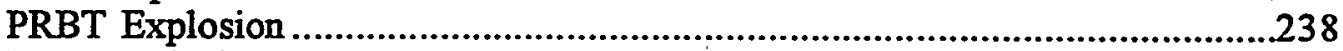

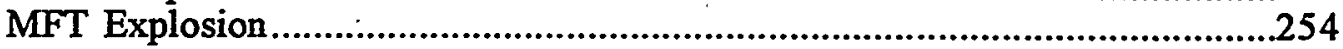

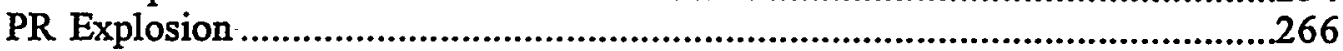

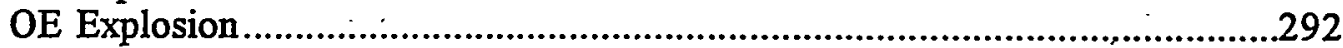

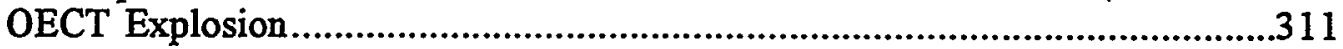

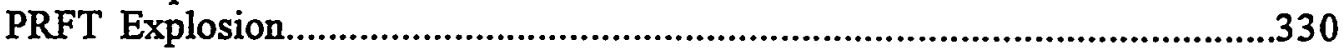

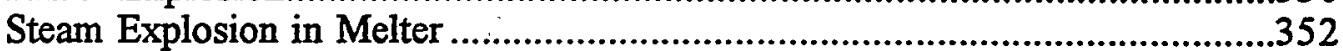

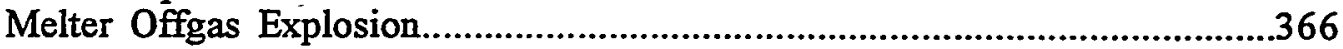

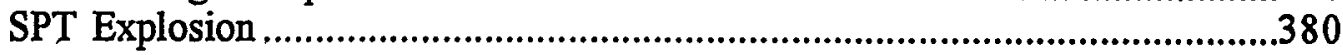

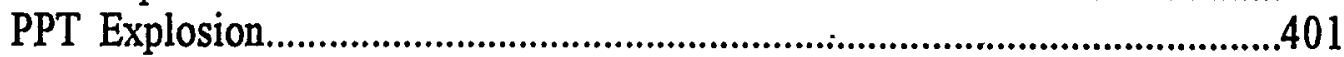

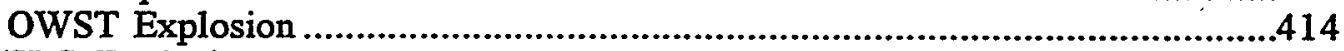

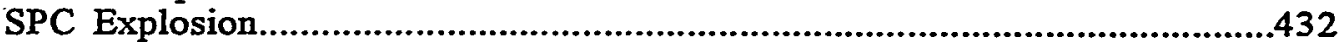

Attachment 6. Support System Fault Tree Plots, Cutsets, and BE Importance ..............................401

Steam Process steam failure .......................................................................446

SSW Process chilled water failure ...............................................................452

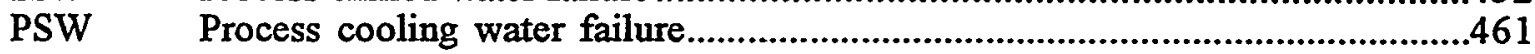

CTWCC Cooling tower water (common cause) failure.....................................................470

DEEP Cooling tower water (deep well) failure …….............................................474

CTWP . Cooling tower water (pumping) failure ........................................................479

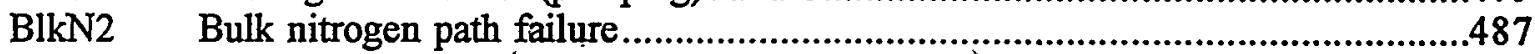

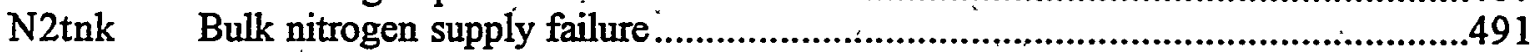

CPCtnk CPC nitrogen tank supply unavailable........................................................495

CPCBP CPC backup purge path unavailable...........................................................499

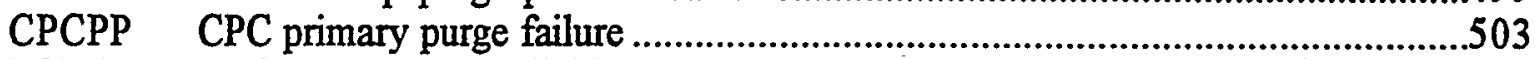

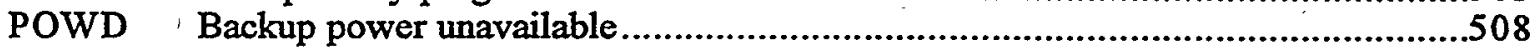

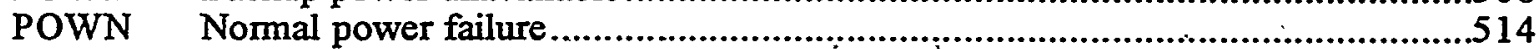

W Vitrification building ventilation failure (POWN and Inair available) ...............524

VV-E Vitrification building ventilation failure (POWN failed, Inair available) ............532

VV-EI Vitrification building ventilation failure (POWN and Inair failed) ......................538

SPCPP SPC primary purge failure ................................................................546

SPCBP $\quad$ SPC backup purge unavailable ..............................................................554

SPCN2 SPC nitrogen blanket path unavailable ....................................................565

LPPPN2 LPPP nitrogen supply path failure...................................................................572

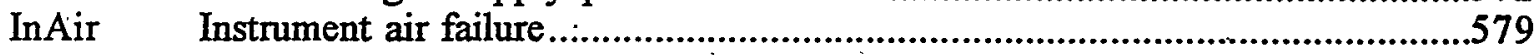

Attachment 7. Seismic Fault Trees .........................................................................................597

Atachment 8. Basic Event Importance Factor Explanation ......................................................644 


\section{ABBREVIATIONS/ACRONYMS}

\begin{tabular}{|c|c|c|c|c|}
\hline APET & & & & Accident Progression Event Tree . \\
\hline $\mathrm{BE}$ & & & & Basic Event \\
\hline CAFTA & & & & Computer Aided Fault Tree Analysis \\
\hline $\mathrm{CPC}$ & & & & Chemical Processing Cell \\
\hline CSRAM & & & & Cut-Set Reliability, Availability, and Maintainability \\
\hline DCS & & & & Distributed Control System \\
\hline DWPF & & & & Defense Waste Processing Facility \\
\hline FCV & & & & Flow Control Valve \\
\hline HEPA. & & & & High Efficiency Particulate Air (filter) \\
\hline HLW & & & & High Level Waste \\
\hline LEL & & & & Lower Explosive Limit \\
\hline$\overline{\text { LFL }}$ & & . & & Lower Flammability Limit \\
\hline LPPP & & & & Low Point Pump Pit \\
\hline $\mathrm{MCC}$ & & & & Motor Control Center \\
\hline MFT & & & & Melter Feed Tank \\
\hline $\mathrm{MOC}$ & & & & Minimum Oxygen for Combustion \\
\hline $\mathrm{OE}$ & & & & Organic Evaporator \\
\hline OECT & & & & Organic Evaporator Condensate Tank \\
\hline OGCT & & & & Off-gas Condensate Tank \\
\hline OWST & & & & Organic Waste Storage Tank \\
\hline P\&ID & & & & Piping \& Instrument Drawing \\
\hline $\mathrm{PCV}$ & & & & Pressure Control Valve \\
\hline PHA & 、 & . & & Precipitate Hydrolysis Aqueous \\
\hline PHR & & & & Process Hazard Review \\
\hline PPT & & & & Precipitate Pump Tank (in LPPP) \\
\hline PR & ' & & . & Precipitate Reactor \\
\hline PRBT & & & & Precipitate Reactor Bottoms Tank \\
\hline PRC/D & & & & Precipitate Reactor Condenser/Decanter \\
\hline PRFT & & & & Precipitate Reactor Feed Tank \\
\hline PSV & & & & Pressure Safety Valve \\
\hline PSW & & & & Primàry Service Water (process cooling water) \\
\hline PVVH & & & & Process Vessel Vent Header \\
\hline $\mathrm{RAG}$ & . & & & Risk Analysis Group \\
\hline SAE & . & & & Safety Analysis Engineering \\
\hline SAR & & & & Safety Analysis Report \\
\hline SME & & & & Slurry Mix Evaporator \\
\hline SPC & & & & Salt Processing Cell \\
\hline SPT & & & . & Sludge Pump Tank (in LPPP) \\
\hline SRAT & & ' & & Sludge Receipt and Adjustment Tank \\
\hline SRS & & & & Savannah River Site \\
\hline SSW & & & & Secondary Service Water (process chilled water) \\
\hline $\mathrm{TC}$ & & & & Type Code \\
\hline WSRC & & & & Westinghouise Savannah River Company \\
\hline
\end{tabular}




\section{INTRODUCTION}

A systematic, safety analysis is being conducted for the Defense Waste Processing Facility (DWPF) at the Savannah River Site to meet the requirements of DOE Order 5480.23, "Nuclear Safety Analysis Reports." Fault tree analyses for internal initiator scenarios and seismic events have been completed which provide estimates of failure rates for support systems and accident frequencies for possible explosions in process tanks and piping systems that could lead to radioactivity and hazardous chemical releases and potential consequences to people on-site and off-site. The process systems and their support systems are sufficiently complex that use of probabilistic risk assessment methods is warranted. The frequency results from these fault trees will be used in further Accident Progression Event Tree (APET) analyses to evaluate a broad spectrum of potential accident scenarios, to characterize the risk of the DWPF when waste processing operations begin.

An important input to the frequency determinations are all the underlying assumptions and data for basic component failures and human errors that can initiate and enable the sequence of events leading to an accidental release. These assumptions and data are documented in this report along with the fault tree logic used in the analyses. Information included here serves as the technical basis for assignment of each accident scenario to a given likelihood-of-occurrence range (e.g., Anticipated: $1 / \mathrm{yr}$ to $10^{-2} / \mathrm{yr}$, Unlikely: $10^{-2} / \mathrm{yr}$ to $10^{-4} / \mathrm{yr}$, Extremely Unlikely: $10^{-4} / \mathrm{yr}$ to $10^{-6} / \mathrm{yr}$, and beyond extremely unlikely: $<10^{-6} / \mathrm{yr}$ ) as defined in DOE Standard3009, "Preparation Guide for U. S. Department of Energy Nonreactor Nuclear Facility Safety Analysis Reports."

\section{METHOD OR APPROACH}

Of the accident scenarios identified in the hazard analysis for DWPF, fifteen explosion accidents (Table 1) were selected for additional analysis to determine frequencies of occurrence in various DWPF process vessels. These scenarios were for hydrogen, benzene, or steam explosions caused by internal initiator events. The fault tree method was selected as most suitable to perform the frequency analyses, with a separate tree provided for each scenario.

Components and systems which serve to prevent, detect, or mitigate potential consequences were identified in the hazard analysis and were modeled in the fault trees. Detailed design P\&W's (listed in Attachment 1) were used to define process systems and their support systems. Operating procedures were reviewed as needed to define required operator actions which could potentially prevent or mitigate the progression of an accident.

CAFTA (Computer Aided Fault Tree Analysis) software, Version 2.2e (Reference 1) was used to construct fault trees and quantitatively solve them. A revised cutset editor, CSRAM, Version 2.1e, was substituted for the CUTSET module in the code. CAFTA generated the fault tree plot, determined minimal cutsets and basic event importance, and computed the overall failure rate for each fault tree. 
In addition to the logic structure of gates and events with their names and descriptions, CAFTA input included information needed to determine event probabilities or frequencies of occurrence. Depending on the information supplied, CAFTA calculated appropriate probabilities and frequencies and combined them according to specified logical relationships to calculate the frequency of the top event. Attachment 2 describes the naming convention for basic events. Attachment 3 lists the Basic Event (BE) file, which shows for each event the probability/frequency data and the reference source for each item. of data. Along with the information in the BE file, the Type Code (TC) file listed in Attachment 4 has additional information used by CAFTA to evaluate probabilities for basic events. The details of how these calculations were done are described in Reference 1, which gives numerous examples.

Support system fault trees (Table 2) were developed to model possible failures of support systems. Some of these contributed as both initiator and enabler events in the accident fault trees, while some were only enabler events. An initiator is an event that must be included in the set of postulated failures and errors which combine in the analysis to produce the accident of concern. In a way they can be thought of as events that could start the sequence of events ending in an accident, although fault tree analysis per se does not deal with time sequences. Enabler events are those which only contribute to the possible ways that the accident could come about. An event may be either or both an initiator or an enabler. This distinction determines the types of input failure data and the data computations made in the analysis (Reference 1).

Support system failures were modeled within the accident fault trees as basic events. Also, a particular support system failure event could factor into more than one accident fault tree. Since these events were modeled as if they were independent events, it was necessary to make support system fault trees independent of each other. For example, SPC primary purge fails if normal power is lost. The purge fault tree does not include power within it, and the top event is called, "SPC Primary Purge Fails (excluding normal power)." The dependency of purge on normal power is handled by the APET, which considers the purge system in a failed state if normal power is in a failed state.

General seismic fault trees were developed for accidents initiated by earthquakes. These trees were not used to develop specific frequency numbers as for internal initiators, but provide the logic which APET combines with fragility data for various magnitude earthquakes. Each of the accident trees has a corresponding seismic tree. Many of the support system trees also have seismic counterparts; however, some support systems which did not have any seismic qualifications were assumed to fail in an earthquake (i.e., a probability of 1.0 of failure). 


\section{RESULTS}

Table 1 summarize accident frequencies due to internal event initiators during normal operations, excluding maintenance activities.

Table 1. Accident Fault Tree Results (Internal Initiators)

\begin{tabular}{|c|c|c|c|}
\hline Likelihood Range & System & Description & $\begin{array}{l}\text { Frequency } \\
\text { (per year) }\end{array}$ \\
\hline $\begin{array}{l}\text { Unlikely } \\
\text { Unlikely }\end{array}$ & $\begin{array}{l}\text { SRAT } \\
\text { SPC }\end{array}$ & $\begin{array}{l}\text { Sludge Receipt-and Adjustment Tank explosion } \\
\text { Salt Processing Cell explosion }\end{array}$ & $\begin{array}{l}9.7 \mathrm{E}-4 \\
1.1 \mathrm{E}-4\end{array}$ \\
\hline Extremely Unlikely & SME & Slurry Mix Evaporator explosion & $8.8 \mathrm{E}-5$ \\
\hline Extremely Unlikely & MFT & Melter Feed Tank explosion & $4.6 \mathrm{E}-5$ \\
\hline Extremely Unlikely & PR & Precipitate Reactor explosion & $4.5 \mathrm{E}-5$ \\
\hline Extremely Unlikely & SMELT & Steam Explosion in Melter & $1.8 \mathrm{E}-5$ \\
\hline Extremely Unlikely & $\sigma E$ & Organic Evaporator explosion & $1.2 \mathrm{E}-5$ \\
\hline Extremely Unlikely & PRFT & Precipitate Reactor Feed Tank explosion & $8.2 \mathrm{E}-6$ \\
\hline Extremely Unlikely & OECT & Organic Evaporator Condensate Tank explosion & $6.7 \mathrm{E}-6$ \\
\hline Extremely Unlikely & PRBT & Precipitate Reactor Bottoms Tank explosion & $5.8 \mathrm{E}-6$ \\
\hline Extremely Unlikely & PVVH & Process Vessel Vent Header explosion & $3.1 \mathrm{E}-6$ \\
\hline Extremely Unlikely & PPT & Precipitate Pump Tank (in LPPP) explosion & $1.8 \mathrm{E}-6$ \\
\hline Extremely Unlikely & $\begin{array}{l}\text { OWSTT } \\
\mathrm{K}\end{array}$ & Organic Waste Storage Tank explosion & $1.3 \mathrm{E}-6$ \\
\hline $\begin{array}{l}\text { Beyond Ext. Unlikely } \\
\text { Beyond Ext. Unlikely }\end{array}$ & $\begin{array}{l}\text { SPT } \\
\text { OFFGAS }\end{array}$ & $\begin{array}{l}\text { Sludge Pump Tank (in LPPP) explosion } \\
\text { Melttr Off-oas explosion }\end{array}$ & $\begin{array}{ll}3.2 \mathrm{E}-7 \\
15 \mathrm{E}-11\end{array}$ \\
\hline
\end{tabular}

None of the accidents are in the "anticipated" frequency range. Two of the explosion scenarios (SRAT and SPC) are at the low end of the "unlikely" frequency range. The remainder are distributed across the "extremely unlikely" and "beyond extremely unlikely" ranges. Fault tree plots, the most significant cutsets, and basic event importance for all the listed accidents are included in Attachment 5.

Each fault tree and cutset report is followed by two basic event importance listings. The first listing ranks each basic event by the Risk Achievement Worth importance factor, and the second listing ranks events by Risk Reduction Worth importance factor. From the Risk Achievement Worth one can tell how much more likely the top event is if a given basic event always happens. The risk achievement worth for a given basic event is equal to the top event frequency if that basic event is assumed to fail divided by the top event frequency if that basic event failure occurs at its predicted failure rate.

The Risk Reduction Worth provides a measure of how much less likely the top event is, if you assume a given basic event never occurs. The Risk Reduction Worth importance factor for a given basic event is equal to the normal top event frequency divided by the top event frequency, given that event never occurs. See Attachment 8 for further details. 
Table 2 shows failure frequencies, unavailability data, and repair times for support system failures modeled by fault trees included in Attachment 6, which includes fault tree plots, significant cutsets, and basic event importance: The support system fault trees were modeled as basic event inputs in the various accident fault trees as appropriate. The failure frequency for a support system was not dependent on the type of internally initiated accident. This independence would not be true for externally caused, natural phenomena accidents, such as earthquakes.

Systems listed in Table 2 that show only unavailability data are pure enabler events. They are backup systems whose isolated failures cannot cause an accident, and their failures are expressed as probabilities with no associated frequencies. The systems which have frequencies associated with them are both initiators and enablers. Failures of these systems could be causes of accidents, or they could enable other sets of failure or human error events to progress to an accident or to more severe consequences. For example, loss of CPC primary purge can eventually lead to a hydrogen gas explosion.

The seismic fault trees are grouped together in Attachment 7.

Table 2. Support Sýstem Fault Tree Results (Internal Initiators)

\begin{tabular}{|c|c|c|c|c|}
\hline Title & Description & $\begin{array}{l}\text { Frequency } \\
\text { (per hour) }\end{array}$ & Unaviailability & $\begin{array}{c}\text { Repair } \\
\text { Time (hrs) }\end{array}$ \\
\hline STEAM & Process Steam & $2.1 E-4$ & $1.2 \mathrm{E}-2$ & 55 \\
\hline SSW & Process chilled water & $2.3 \mathrm{E}-5$ & $3.3 \mathrm{E}-4$ & 14 \\
\hline PSW & Process cooling water & - & 2.3E-3 & - \\
\hline CTWCC & Cooling tower water - common & $1.1 \mathrm{E}-5$ & $5.8 \mathrm{E}-5$ & 5.5 \\
\hline DEEP & Cooling tower water - deep well & - & $5.4 \mathrm{E}-3$ & - \\
\hline CTWP & Cooling tower water - pumping & $6.3 \mathrm{E}-6$ & $5.6 \mathrm{E}-4$ & 90 \\
\hline BLKN2 & Bulk nitrogen path & $5.5 \mathrm{E}-6$ & $1.1 \mathrm{E}-4$ & 20 \\
\hline N2TNK & Bulk nitrogen supply & $7.7 \mathrm{E}-6$ & $1.3 \mathrm{E}-4$ & 17 \\
\hline CPCTNK & CPC nitrogen tank supply & - & $3.7 \mathrm{E}-5$ & - \\
\hline CPCBP & CPC backup purge path & - & $3.1 \mathrm{E}-3$ & - \\
\hline СРCPP & CPC primary purge & $1.9 \mathrm{E}-5$ & $9.5 \mathrm{E}-4$ & 51 \\
\hline POWD & Backup power & ـ & $1.1 \mathrm{E}-2$ & $=$ \\
\hline POWN & Normal power & $3.5 \mathrm{E}-5$ & $2.9 \mathrm{E}-4$ & 8.5 \\
\hline & Vitrification Bldg. Ventilation & - & $3.3 \mathrm{E}-5$ & - \\
\hline VV-E & $\begin{array}{l}\text { Vit. Bldg. Ventilation (normal } \\
\text { electric power lost) }\end{array}$ & - & $1.1 \mathrm{E}-3$ & - \\
\hline VV-EI & $\begin{array}{l}\text { Vit. Bldg. Ventilation (normal } \\
\text { electric power and inst. air lost) }\end{array}$ & - & $7.6 \mathrm{E}-3$ & - \\
\hline SPCPP & SPC primary purge & $6.7 \mathrm{E}-5$ & $5.9 \mathrm{E}-4$ & 8.8 \\
\hline $\mathrm{BP}$ & SPC backup purge & - & $.6 .7 \mathrm{E}-3$ & - \\
\hline SPCN2 & SPC nitrogen blanket path & & $4.1 \mathrm{E}-4$ & - \\
\hline LPPPN2 & LPPP nitrogen supply path & - & $5.3 \mathrm{E}-4$ & - \\
\hline INAIR & Instrument air & 1:1E-5 & $2.9 \mathrm{E}-4$ & 26 \\
\hline
\end{tabular}




\section{INPUTS AND ASSUMPTIONS}

\section{Assumptions Related to Accident Fault Trees}

\section{Process Vessel Vent Header (PVVH) Explosion}

The Process Vessel Vent Header (PVVH) may carry flammable concentrations of hydrogen gas and benzene vapor. Potentially explosive levels of hydrogen can only be reached in the PVVH if base load air flow fails, and hydrogen levels are high from the SRAT or SME. Hydrogen generation rates in PRBT and MFT are insufficient to produce explosive concentrations in PVVH. Explosive benzene levels may be reached in the PVVH if benzene generation in PR or OE is so high that dilution with $\mathrm{CPC}$ and baseload air flow is inadequate. Such a condition can occur if the condenser for those vessels fails or if the SPC is not isolated from PVVH when the base load air flow fails. Interlocks are provided to protect against these occurrences. Normally, PVVH base load air flow is designed to reduce SPC benzene off-gas and the CPC hydrogen/benzene off-gas to concentrations less than LFL. The benzene concentration is flammable in a short section of PVVH piping, but is diluted to less than LEL in the remainder of the system downstream.

The PVVH is overwhelmed by benzene if PR steam supply valves fail open and either SCVC or PRC/D is not operating. If both condensers are working, enough benzene is removed that the PVVH flow reduces it to less than LFL. OEC/D or SCVC operation is adequate to prevent benzene from OE overwhelming PVVH.

The following statements pertain to the PVVH seismic fault tree:

1. Purge failure to CPC vessels results in LFL being reached in those vessels and propogating to the PVVH, but the time it takes to reach LFL in the PVVH is sufficiently long to justify use of delayed (internal) ignition source probability.

2. Excess benzene is received by the PVVH from the SPC if they are not isolated from each other by the seismic trigger interlock.

PVVH system boundaries include ventilation piping from the Formic Acid Vent Condenser (FAVC) and the Salt Cell Vent Condenser (SCVC) up to the Zone 1 ventilation connection, heater, filters, and fans. Support systems include: nitrogen supply tank, CPC primary and backup purge systems, process cooling water, cooling tower water, chilled water, process steam, instrument air, normal electric power, and backup electric power.

A temperature monitor detects high temperature in the SCVC and gives an alarm to alert operators in addition to automatic interlock actions. Loss of base load air flow is detected by a flow monitor; low flow gives an alarm. A benzene analyzer and detector on the PVVH actuates automatic interlocks when a high concentration is detected to stop PR transfers and turn off the $\mathrm{OE}$ steam supply. 


\section{Sludge Receipt and Adjustment Tank (SRAT) Explosion}

The Sludge Receipt and Adjustment Tank (SRAT) in CPC is purged by the primary air purge system for CPC. The nitrogen purge system for CPC vessels serves as a backup to the primary system. The purge flow prevents buildup of high concentrations of flammable hydrogen gas or benzene vapors. Excessive hydrogen levels may be reached if a failure of interlock actions occurs when purge flow fails. Improper additions of PHA, formic acid, or nitric acid into SRAT could also cause high generation of hydrogen gas that could exceed the removal capacity of the purge system. Benzene levels in SRAT could exceed LFL if transfer or operator errors result in high benzene content of material coming from the SPC.

SRAT system boundaries include CPC air purge and nitrogen purge piping, a flow control valve, a needle valve, two hydrogen gas detectors, and interlock circuitry to shut down processing operations that could contribute to high generation of hydrogen gas. Essential support systems include: normal and backup electric power, nitrogen supply system, process cooling water; cooling tower water, and instrument air.

Flow monitors on the CPC purge system detect loss of purge flow and alarm to initiate operator actions. Hydrögen gas detectors (GC's) actuate interlock circuits that shut down the SRAT steam supply, start cooling water circulation, and stop all transfers of materials to SRAT.

Three additional assumptions were made in the fault tree analysis for the SRAT. These were:

1. The fault tree analysis assumes that an out of calibration steam flow element can not cause steam to fail to the SRAT during transfer and allow steam to flow thereafter.

2. Successful interlock actions in the SRAT or SME prevent explosive mixtures from accumulating. The cause of interlock actions is repaired before radiolytic decomposition causes hydrogen concentration to reach LFL.

3. Interlock actions for SME and SRAT are unsuccessful if any of the actions are unsuccessful (stop steam, start cooling, and stop transfers).

The following statements pertain to the SRAT seismic fault tree:

1. Purge failure results in LFL being reached, but the time it takes to reach LFL is sufficiently long to justify use of delayed (internal) ignition source probability.

2. Nitric acid or PHA over-addition to the SRAT are likely enough to be considered in the seismic fault tree.

3. The frequency of inadequate sludge characterization by SRTC coupled with an earthquake is low enough to be left out of the seismic fault tree. 
4. Low boil up during transfer to the SRAT is not included because steam must be restarted for significant benzene to be released from the SRAT, and seismic response procedures prevent starting process steam before ensuring it will not cause such a problem.

\section{Slurry Mix Evaporator (SME) Explosion}

The hazard in the Slurry Mix Evaporator (SME) is possible hydrogen gas accumulation in the vessel. The Chemical Processing Cell (CPC) primary purge air system purges the SME. There is a backup nitrogen purge system for CPC vessels. Excessive hydrogen gas concentrations may be reached in the SME if the primary and backup purge systems fail or the rate of hydrogen gas generation exceeds purge design. High rates of hydrogen gas generation are reduced to safe levels by an interlock system that monitors hydrogen concentration and automatically takes actions to shut down the SME process. These interlock actions cause the SME steam supply to be turned off, cooling water flow to be started, and transfers from feed and chemical addition tanks to be stopped.

The SME system boundary includes purge system piping, control valve, needle valve, hydrogen detectors, and interlock instrumentation. In addition to proper operation of the purge system, chemical additions to SME must be carefully controlled. Essential support systems include: electric power, process cooling water system, cooling tower water system, and instrument air system.

Instruments monitor purge air flow and give an alarm upon loss of flow. Gas chromatographs monitor hydrogen concentration. If a high level of hydrogen occurs, interlock circuits shut the process down and start cooling water flow to reduce the hydrogen generation rate to radiolytic decomposition.

Two additional assumptions were made in the fault tree analysis for the SME. These are:

1. Successful interlock actions in the SRAT or SME will prevent explosive mixtures of hydrogen gas from accumulating. If loss of purge air flow is the initiator of interlock action, it is assumed that purge flow will be restored before hydrogen gas concentration reaches LFL.

2. Interlock actions for SME and SRAT are unsuccessful if any single action is unsuccessful (stop steam, start cooling, and stop transfers).

The following statements pertain to the SME seismic fault tree:

1. Purge failure results in LFL being reached, but the time it takes to reach LFL is sufficiently long to justify use of delayed (internal) ignition source probability.

2. The frequency of inadequate sludge characterization by SRTC or over addition of formic acid, coupled with an earthquake is low enough to be left out of the seismic fault tree. 


\section{Precipitate Reactor Bottoms Tank (PRBT) Explosion}

The Chemical Processing Cell (CPC) primary purge air system draws air through the Precipitate Reactor Bottoms Tank (PRBT) to prevent buildup of flammable hydrogen gas or benzene vapor. $\mathrm{CPC}$ also has a backup nitrogen blanket gas system should the primary purge system fail. In normal operration benzene vapor generation is maintained below the removal capability of the purge system by limiting the amount of benzene discharged to the PRBT from the PR in the Salt Processing Cell (SPC). Normally the precipitate reactor feed material from PRFT does not have enough benzene to cause high benzene concentrations in the PRBT with the purge air system on, even if an immediate transfer takes place from the PR before the processing is completed in the PR. It would take an operator error involving draining benzene from the PRC/D into the PR and erroneous transfer of this high benzene material to the PRBT to result in a vapor concentration greater than LFL in the PRBT.

Hydrogen gas evolves continually from the waste solution in PRBT. An extended loss of purge air flow and backup nitrogen purge would allow hydrogen to build up. If PRBT purge air stops and an alarm sounds, this analysis assumed that one of several independent nitrogen purge systems could be started by the operator before the hydrogen LFL is reached.

The following statements pertain to the PRBT seismic explosion fault tree:

1. Purge failure results in LFL being reached, but the time it takes to reach LFL is sufficiently long to justify use of delayed (internal) ignition source probability.

2. The probability of PRBT receiving a high benzene content transfer just prior to an earthquake is sufficiently low that it is negligible, when coupled with the seismic event frequency.

3. The frequency of inadequate sludge characterization by SRTC coupled with an earthquake is low enough to be left out of the seismic fault tree.

System boundaries for the PRBT fault tree include purge system piping, flow control valves, manual block valves, and purge flow monitoring instruments. The electric power system is an essential support system for CPC air purge system. The nitrogen supply tank and its associated piping are required by the backup nitrogen purge system.

Flow monitors can detect purge system failure and sound an alarm for operator actions. Conditions which cause benzene vapor generation to exceed the removal capacity of the purge system would go undetected if the equipment failures or operator errors were not recognized: 
Errors in Precipitate Reactor (PR) operations are possible causes for high benzene vapor concentrations in PRBT. The PRBT is a holding tank for SRAT feed from PR. Because material transferred from PR to PRBT is going from a system that controls the gas or vapor space to minimum oxygen for combustion (MOC) to one that controls the benzene vapor concentration to its lower flammability limit (LFL), there is a potential for high benzene transferred from one system to the other. One scenario for high benzene present in the transferred fluid involves errors in PRC/D cleaning. The PRC/D is a decanter on the PR that holds some benzene from PR operations. If it is cleaned and benzene is emptied into the PR before the contents of PR are transferred to PRBT, then PRBT could receive a high benzene solution.

\section{Melter Feed Tank (MFT) Explosion}

The Chemical Processing Cell (CPC) primary purge air system draws air through the Melter Feed Tank (MFT) to prevent buildup of flammable hydrogen gas, which slowly evolves from the melter feed solution.

The following statements pertain to the MFT seismic fault tree:

1. Purge failure results in LFL being reached, but the time it takes to reach LFL is sufficiently long to justify use of delayed (internal) ignition source probability.

2. The frequency of inadequate sludge characterization by SRTC coupled with an earthquake is low enough to be left out of the seismic fault tree.

3. MFT purge can not be overwhelmed by hydrogen generation with nominal inventory, regardless of termperature.

4. MFT purge can not be overwhelmed by hydrogen generation with nominal operating temperature, regardless of conceivable chemical addition errors.

5. The case of MFT purge being overwhelmed by hydrogen generation due to chemistry errors coupled with high termerature is negligible, because the two events are each low probability and independent of each other.

System boundaries for MFT fault tree include purge system piping, flow control valve, manual block valves, and purge flow monitoring instruments with alarms. The electric power system is an essential support system for CPC air purge system. The bulk nitrogen or CPC backup purge supply tanks and associated piping are required by the backup nitrogen purge system.

Flow monitors detect loss of purge air flow. Temperature monitoring instruments detect high temperature in the MFT. Both instrument systems alarm to initiate operator actions. 


\section{Precipitate Reactor (PR) Explosion}

The primary carbon dioxide purge system in the Salt Processing Cell (SPC) purges the gas space in the Precipitate Reactor (PR) vessel. The primary system generates carbon dioxide gas by vaporizing liquid $\mathrm{CO}_{2}$ with applied heat from an electric heater. There is also a backup supply of carbon dioxide from a bank of high pressure gäs cylinders. 'The SPC nitrogen purge system may also be used as a backup. A high benzene vapor concentration is unavoidable in the PR; consequently, the approach used to prevent possible explosions is to exclude oxygen gas by maintaining either a carbon dioxide or nitrogen atmosphere in the vessel. The precipitate reactor operation is a batch operation. The $\mathrm{CO}_{2}$ purge rate must be checked periodically and adjusted manually. If a high concentration of oxygen is detected in the PR, an automatic interlock circuit increases the gas pressure in the vessel higher than the surrounding SPC pressure to prevent air from leaking into the PR.

For SPC vessel seismic fault trees, it is modeled that if the isolation of SPC from PVVH or nitrogen addition to SPC vessels is not successful, SPC vessels explode.

Precipitate Reactor purge system boundaries included SPC carbon dioxide piping, a purge pressure control valve, a pressure safety relief valve, vacuum breaker, and various isolation valves. There are two oxygen gas detectors and associated interlock instrumentation: Support systems include normal and backup electric power and SPC primary, backup, and nitrogen purge.

$\mathrm{PR}$ purge failure is detected by flow instruments that give an alarm to alert operators. Two oxygen analyzers detect possible air leaking into the vessel. High oxygen concentration will actuate an interlock circuit that closes control valves to raise the gas pressure in the PR. The interlock also stops transfers in the SPC, turns off the steam supply to the PR, starts PR cooling water circulation, and stops the vessel agitator.

\section{Organic Evaporator (OE) Explosion}

The primary carbon dioxide purge system in the Salt Processing Cell (SPC) purges the gas space in the Organic Evaporator (OE) vessel. The primary system generates carbon dioxide gas by vaporizing liquid $\mathrm{CO}_{2}$ with applied heat from an electric heater. There is also a backup supply of carbon dioxide from a bank of high pressure gas cylinders. The SPC nitrogen purge system may also be used as a backup. A high benzene vapor concentration is unavoidable in the $\mathrm{OE}$; consequently, the approach used to prevent possible explosions is to exclude oxygen gas by maintaining either a carbon dioxide or nitrogen atmosphere in the vessel. The organic evaporator operation is a batch operation. The $\mathrm{CO}_{2}$ purge rate must be checked periodically and adjusted manually. If a high concentration of oxygen is detected in the $\mathrm{OE}$, an automatic interlock circuit increases the gas pressure in the vessel higher than the surrounding SPC pressure to prevent air from leaking into the $\mathrm{OE}$.

For SPC vessel seismic fault trees, it is modeled that if the isolation of SPC from PVVH or nitrogen addition to SPC vessels is not successful, SPC vessels explode. 
Organic Evaporator purge system boundaries included SPC carbon dioxide piping, a purge pressure control valve, a pressure safety relief valve, vacuum breaker, and various isolation valves. There are two oxygen gas detectors and associated interlock instrumentation. Support systems include normal and backup electric power and SPC primary, backup, and nitrogen purges.

$\mathrm{OE}$ purge failure is detected by flow instruments that give an alarm to alert operators. Two oxygen analyzers detect possible air leaking into the vessel. High oxygen concentration will actuate an interlock circuit that closes control valves to raise the gas pressure in the $\mathrm{OE}$. The interlock also stops transfers in the SPC, turns off the steam supply to the OE, starts OE cooling water circulation, and stops the vessel agitator.

\section{Organic Evaporator Condensate Tank (OECT) Explosion}

The primary carbon dioxide purge system in the Salt Processing Cell (SPC) purges the gas space in the Organic Evaporator Condensate Tank (OECT). The primary system generates carbon dioxide gas by vaporizing liquid $\mathrm{CO}_{2}$ with applied heat from an electric heater. There is also a backup supply of carbon dioxide from a bank of high pressure gas cylinders. The SPC nitrogen purge system may also be used as a backup. A high benzene vapor concentration is unavoidable in the OECT; consequently, the approach used to prevent possible explosions is to exclude oxygen gas by maintaining either a carbon dioxide or nitrogen atmosphere in the vessel. The precipitate reactor operation is a batch operation. The $\mathrm{CO}_{2}$ purge rate must be checked periodically and adjusted manually. If a high concentration of oxygen is detected in the OECT, an automatic interlock circuit increases the gas pressure in the vessel higher than the surrounding SPC pressure to prevent air from leaking into the OECT.

For SPC vessel seismic fault trees, it is modeled that if the isolation of SPC from PVVH or nitrogen addition to SPC vessels is not successful, SPC vessels explode.

Organic Evaporator Condensate Tank purge system boundaries included SPC carbon dioxide piping, a purge pressure control valve, a pressure safety relief valve, vacuum breaker, and various isolation valves. There are two oxygen gas detectors and associated interlock instrumentation. Support systems include normal and backup electric power and SPC primary, backup, and nitrogen purges.

OECT purge failure is detected by flow instruments that give an alarm to alert operators. Two oxygen analyzers detect air leaking into the vessel. High oxygen concentration will actuate an interlock circuit that closes control valves to raise the gas pressure in the OECT. The interlock also stops transfers in the SPC and stops the vessel agitator. 


\section{Precipitate Reactor Feed Tank (PRFT) Explosion}

The primary carbon dioxide purge system in the Salt Processing Cell (SPC) purges the gas space in the Precipitate Reactor Feed Tank (PRFT). The primary system generates carbon dioxide gas by vaporizing liquid $\mathrm{CO}_{2}$ with applied heat from an electric heater. There is also a backup supply of carbon dioxide from a bank of high pressure gas cylinders. The SPC nitrogen purge system may also be used as a backup. A high benzene vapor concentration is unavoidable in the PRFT; consequently, the approach used to prevent possible explosions is to exclude oxygen gas by maintaining either a carbon dioxide or nitrogen atmosphere in the vessel. The precipitate reactor operation is a batch operation. The $\mathrm{CO}_{2}$ purge rate must be checked periodically and adjusted manually. If a high concentration of oxygen is detected in the PRFT, an automatic interlock circuit increases the gas pressure in the vessel higher than the surrounding SPC pressure to prevent air from leaking into the PRFT.

For SPC vessel seismic fault trees, it is modeled that if the isolation of SPC from PVVH or nitrogen addition to SPC vessels is not successful, SPC vessels explode.

Precipitate Reactor Feed Tank purge system boundaries included SPC carbon dioxide piping, a purge pressure control valve, a pressure safety relief valve, vacuum breaker, and various isolation valves. There are two oxygen gas detectors and associated interlock instrumentation. Support systems include normal and backup electric power and SPC primary, backup, and nitrogen purge systems.

PRFT purge failure is detected by flow instruments that give an alarm to alert operators. Two oxygen analyzers detect air leaking into the vessel. High oxygen concentration will actuate an interlock circuit that closes control valves to raise the gas pressure in the PRFT: The interlock also stops transfers in the SPC and stops the vessel agitator.

\section{Steam Explosion in Melter (SMELT)}

Conditions that could lead to a steam explosion in the melter require the presence of both water and partially melted excess salt. Water is a normal constituent of the process. Melter feed is a slurry which is approximately $50 \%$ water. Also, procedures require flushing of the feed tube with water before and after each feed operation. Salt content is monitored by sampling and controlled by procedures in both the Liquid Radioactive Waste Handling Facilities (LRWHF) and DWPF.

There are no major process systems or support systems modeled in the SMELT fault tree. The failure events in the tree include only human errors and failures of sampling equipment at both LRWHF and DWPF. 
High salt content of feed material is detected through sampling at one location at LRWHF and at two locations at DWPF. This analysis assumed that:

1. Each macro batch to the melter (approximately one every two years) has a high salt content level... This assumption is conservatively estimated since there is no sitespecific data available to accurately estimate this occurrence.

2. At least two analyzers are available at each analytical lab location for sample analysis. This assumption is based on the content of quality control procedures which require redundancy in the sampling process.

3. If an operator/analyst/engineer conducts faulty sampling at one location, he/she will fail to properly conduct any further sampling at any other locations or time with a probability of unity.

4. An excess amount of salt within a batch that is not detected will always accumulate on the top layer of the melter cap.

5. All calculations required to compare lab results to a standard at DWPF are performed in PCCS. Additionally, the DWPF process engineer must enter all lab results into PCCS to complete the sample analysis process.

6. Failure to prepare a batch for sampling according to procedure will result in a batch sample that contains an erroneously low salt content level.

7. Water is present in the melter with a probability of unity due to the frequency of water purging in the melter operations.

8. Lab sample analysis results at the tank farm are provided as a report; these results do not require any further data processing (such as input to PCCS) before the batch is transferred.

9. MFT sampling is not performed on every micro batch.

10. Two consecutive batches containing a high salt content must accumulate in the melter for sufficient molten salt to be generated for a steam explosion. 


\section{Melter Off-gas (OFFGAS) Explosion}

The melter off-gas system treats gases that are driven off in the melter before these are released to the atmosphere. Features of this system control melter pressure and initiate glass. pouring at the appropriate time. The lid heaters provide the energy to initiate complete combustion of gases in the melter vapor space; consequently, there will be no combustible gases in the effluent from the melter. A low temperature interlock will automatically shut down the feed pumps when low temperature resulting from lid heater failure is detected in the melter vapor space. If equipment failures or operating error release combustible gases into the off-gas piping, the gas is most likely to explode down stream of the quencher near the offgas condensate tank. At this point, excess water vapor is condensed and conditions are most favorable for ignition of a mixture that is above LEL.

Following are assumptions specific to the Melter Off-gas Explosion fault tree:

1. An explosive concentration in the off-gas system cannot be attained unless excess organics accumulate within the system through either a loss of the dilution air flow or faulty sampling of organics in the sludge.

2. At least two analyzers are available at the analytical lab in DWPF. This assumption is based on the content of quality control procedures which require redundancy in the sampling process.

3. If an operator/analyst/engineer conducts faulty sampling at the SRAT and this person also conducts sampling at the SME, he/she necessarily fails to properly conduct sampling there.

4. All calculations required to compare lab results to a standard at DWPF are performed within PCCS. Additionally, the DWPF process engineer must enter all lab results into PCCS to complete the sample analysis process.

5. Failure to prepare a batch for sampling according to procedure will result in a batch sample that contains an erroneously low organic content.

6. Two out of four lid heater thermocouples must fail to cause failure of a lid heater. Similarly, two out of the four lid heaters must fail to cause sufficient heat loss in the melter plenum.

7. Thermocouple components do not require calibration.

8. Two out of three thermocouples in the melter plenum must fail in order to cause failure of the feed pump shut off interlock. Failure of one thermocouple will result in removal of this temperature input (will be considered as an outlier).

9. MFT sampling is not performed on every melter batch. 
System boundaries for the melter off-gas system include melter feed pumps, lid heaters, backup off-gas film cooler, off-gas condensate tank, and piping connecting these pieces of equipment. Interlock controls for the feed pumps and thermocouples are also included. The plant air system and instrument air system are important support systems related to the backup off-gas film cooler air and pressure control air systems. Electric power is not needed, because a loss of electric power results in a safe condition when the feed pumps stop due to the power loss.

When the air flow for the backup off-gas film cooler fails, and automatic interlock shuts down the feed pumps and opens valves to provide an alternate flow pathway from the melter cell to the plenum. Low temperature in the melter plenum gives an alarm and shuts off the feed pump through an interlock circuit.

\section{Sludge Pump Tank (SPT) Explosion}

A Process Vessel Ventilation System (PVVS) provided in the Low Point Pump Pit (LPPP) purges combustible gas from the Sludge Pump Tank (SPT) to prevent potentially explosive hydrogen gas mixtures from accumulating therein. The ventilation system is backed up by a nitrogen blanket gas addition system, which has two, manually operated addition valves. In normal operation vessel ventilation air passes first through a HEPA filter and then enters the general volume of the LPPP cells. The SPT is maintained at a lower pressure than its surrounding cell space by the ventilation exhaust fan. As a result, ventilation air is pulled into the SPT through the tank overflow line. Exhaust air, including any combustible vapors, from the SPT flows through a moisture condenser and a mist eliminator. This stream of air then combines with the larger stream of air from the LPPP cell volume to further dilute combustible gas and vapor. The combined stream of air flows through a heater and a bank of four parallel HEPA filters. There are two redundant exhaust fans in the PVVS. Their operation is automatically controlled by an instrument system that monitors differential pressure between the Precipitate Pump Tank (PPT) and the ventilation duct downstream of the mist eliminator. If the ventilation air flow fails, nitrogen blanket gas will continue to purge the SPT, which then vents through the tank overflow line.

The primary purge system boundaries are the PVVS inlet filters and the exhaust stack. Components included in the system are ventilation ducts, valves, dampers, fans, filters, condenser, preheater, mist elimínator, and controllers based on flow and temperature. The backup nitrogen purge system consists of a nitrogen gas supply, manual actuation valves, pressure control valves, and flow monitoring instrumentation. These components in either the primary purge system of backup nitrogen purge system must operate as designed to prevent the accumulation of an explosive mixture of hydrogen gas in the SPT. Important support systems include: electric power, process steam, instrument air, and chilled water.

Inadequate SPT purge air flow is detected by differential pressure across the exhaust HEPA filters. The instrument alarms at very high, high, and low differential pressure. There is also a flow monitor with a low flow alarm in the exhaust duct just before the exhaust fans. Inadequate flow due to plugged inlet HEPA filters is detected by a differential pressure monitor and alarm. The backup nitrogen purge flow is monitored and checked during routine surveillance rounds. 


\section{Precipitate Pump Tank (PPT) Explosion}

Benzene vapor explosion is the safety concern for the Precipitate Pump Tank (PPT) in the Low Point Pump Pit (LPPP). PPT is operated with a primary nitrogen gas purge to keep the oxygen concentration from reaching MOC. The primary nitrogen source is the bulk nitrogen gas system; the backup nitrogen sources are a bank of high pressure nitrogen cylinders and the local LPPP nitrogen tank supply. The backup system actuates automatically upon loss of primary purge supply. Instruments monitor oxygen concentration in the effluent stream from PPT which goes to the Process Vessel Vent. System (PVVS). If the oxygen level gets too high, an outlet control valve closes automatically isolating the tank from the PVVS, to allow nitrogen gas pressure to build up in the vessel, thereby preventing leakage of air into the PPT.

Following are assumptions specific to the PPT:

1. All maintenance performed on the nitrogen supply lines to the precipitate tank do not involve closing of manual valves in these lines (with the exception of the pressure indicators; these have manual valves located in an isolated line and do not result in a loss of flow through the line).

2. Failure of the nitrogen purge at the sources (i.e., bulk nitrogen tanks and backup cylinders) will result in a loss of the nitrogen calibration line to the oxygen analyzers. These analyzers will fail as is and will result in failure of valve HCV-8772 to close.

3. The benzene concentration in the precipitate tank is at or above the lower explosive limit (LEL).

4. Loss of the PVVS exhaust fans or inadvertent closure of valve HCV-8772 due to either a loss of power or individual component failures, results in a safe configuration of the system in the PPT due to the positive pressure supplied to the precipitate tank via the nitrogen purge system. (Air does not enter PPT.)

5. Filter plugging and pressure control valve failures in the nitrogen purge lines are immediately detected.

6. The oxygen analyzers are calibrated and tested bi-weekly.

The PPT purge system boundaries include the primary and backup nitrogen system supply tanks, piping, and valves. Components included in the system are portions of PVVS ventilation ducts, control valves and controllers based on nitrogen purge flow and oxygen gas concentration. There are no essential support systems (other than the nitrogen purge systems) for PPT. Loss of electric power and loss of instrument air fail to a safe configuration. 
Inadequate PPT nitrogen purge flow is detected by a flow monitor which alarms to initiate operator actions. There also are pressure indicators on the common nitrogen lines. Two oxygen analyzers are connected to the tank vent exhaust line. A high oxygen signal from either analyzer automatically closes an exhaust valve and shuts off the PPT agitator and pump.

\section{Organic Waste Storage Tank (OWST) Explosion}

The Organic Waste Storage Tank (OWST) is a 150,000 gallon tank for organic byproducts of the DWPF precipitate hydrolysis process. The major organic constituent is benzene. OWST will receive approximately 350 gallon batches of benzene every 43 hours. Benzene vapor explosions are prevented by keeping the oxygen concentration below the minimum oxygen for combustion (MOC) level with a slightly pressurized blanket of nitrogen gas in the tank. The primary source of nitrogen gas is from the same bulk liquid nitrogen system that provides nitrogen purge to the Precipitate Pump Tank (PPT) and Sludge Pump Tank (SPT) in the Low. Point Pump Pit (LPPP) and other tanks and sumps. There are backup sources of nitrogen to the OWST consisting of a dedicated liquid nitrogen tank and a bank of high pressure nitrogen gas bottles. Pressure control valves regulate the flow of nitrogen gas to the OWST and prevent unwanted flow reversals through the system.

The system boundary includes nitrogen system piping, control valves (PCYs), nitrogen tanks, and various associated instrumentation. All PCVs must remain operable, and the sensors which input to the control systems must be properly calibrated. The liquid nitrogen inventory must be adequate, and the piping system must remain leak tight and not plugged. .

Loss of OWST nitrogen blanket gas is detected by low pressure alarms in the OWST inner tank and in the pump well. An oxygen analyzer will also alarm if air enters the vessel.

'The OWST outer tank is protected from benzene explosion by two methods:

1. The amount of time the outer tank contains benzene following a leak from the inner tank is minimized by monitoring for benzene and correcting the problem immediately, after the leak is detected.

2. Ignition sources to set off an explosion in the outer tank are minimized. The tank is grounded, lightning protection is provided, and maintenance uses spark-resistant tools. 


\section{Salt Processing Cell (SPC) Explosion}

The Zone 1 ventilation system purges the Salt Processing Cell (SPC) which prevents the accumulation of potentially flammable benzene vapor within the cell volume. Ventilation system failure with either SPC vessel overpressurization, vessel overheating, or spill of waste solution into the SPC sump without pumping to the PR, could result in benzene vapor concentrations reaching LFL within the SPC. Pressurization of process vessels in SPC is a safety response to detected high oxygen levels within any of these vessels. However, this increases the risk of benzene vapor accumulation in the cell volume outside the vessels. While explosion prevention by inerting is the main concern within SPC vessels, the concern in the Process Vessel Vent Header (PVVH) is LFL (dilution) control. The off-gas ventilation from SPC combines with the flow from CPC, thus diluting the benzene concentration in PVVH. If the PVVH flow gets too low, an automatic interlock closes two valves and isolates SPC from PVVH. This interlock also turns off $\mathrm{CO}_{2}$ supply pressure to SPC vessels. SPC vessels are protected from reaching MOC by a nitrogen purge. Pressure interlocks stop the supply of steam to $\mathrm{PR}$ and $\mathrm{OE}$ and starts cooling water flow to these vessels.

Since the seismic trigger interlock is designed to isolate the SPC from the PVVH and supply a constant flow of nitrogen to SPC vessels, the SPC Explosion seismic fault tree models benzene to always enter the cell following an earthquake. The fault tree concludes that if Zone 1 ventilation fails, a cell explosion occurs.

SPC system boundaries include support systems, such as: Zonè 1 -ventilation system components, instrument air system, cooling tower water system, normal and backup electric power systems, and the control instrumentation associated with the PVVH low flow interlock.

\section{Assumptions Related to Support Systems Fault Trees}

\section{Steam}

The process steam system is a closed loop system that provides heat to the following process vessels: $P R, O E, S M E$, and SRAT. The process steam generator is a heat exchanger connected to the area steam supply through a 260 pound pressure control valve. Process steam piping includes an individual flow control valve (FCV) to each of the process vessel steam coils. The pressure in each steam coil is regulated by a PCV located on the discharge side of the coil. All steam effluent pipes from the vessels connect to a common header, which goes to the steam system condenser (i.e., a heat exchanger cooled by the cooling tower water, CTW, system). Condensate collects in the condensate.tank and from there is returned to the steam generator by a set of redundant pumps. If condensate return flow stops;because of pump failure, the closed loop process steam system will stop functioning.

Process steam system boundaries include piping, FCV's, PCV's, and temperature sensing instruments. Essential support systems include: the 260 pound area steam system, the cooling tower water (CTW) system, normal electric power, and instrument air system. 
Process steam system failure is detected by low temperature within the system and also by low temperatures in the various process vessels requiring steam heat.

\section{Process Chilled Water}

The chilled water system, which is shown as the secondary service water (SSW) system on P\&ID's, is a closed loop cooling system. It serves to remove heat from several DWPF condensers and condensate tanks. These include the primary off-gas condenser and its condensate tank, the backup off-gas condenser and its condensate tank, the facility air ventilation condenser, and the salt cell ventilation condenser. Heat from the SSW is transferred through a heat exchanger to the cooling tower water (CTW) system.

System boundaries for the chilled water (SSW) system include heat exchangers pumps, valves, and instruments in the SSW piping system. Two valves on the CTW system controlled by condenser pressure are also included. CTW is an essential support system for SSW. Other support systems include normal and backup electric power, and instrument air (needed by CTW).

Failures of the SSW are detected by a low flow alarm circuit, a high temperature alarm circuit, trouble alarms; or high temperature indications within the individual systems cooled by SSW.

The fault tree for SSW assumes that the system would fail to provide needed cooling if the closed loop flow were to stop and the bypass line did not open. Failure of the chilled water system due to insufficient inventory was not modeled because a large volume of water is available in the system, an automatic makeup system is capable of handling small leaks, and large pipe leaks have a very low frequency of occurrence.

\section{Process Cooling Water}

The process cooling water system, which is shown as the primary service water (PSW) system on P\&ID's, is a closed loop cooling system. It serves to remove heat from the melter sleeve, the secondary backup off-gas condenser, and other miscellaneous vessels and condensers. Heat from the PSW is transferred through a heat exchanger to the cooling tower water (CTW) system. There is a second PSW/CTW heat exchanger on standby.

System boundaries for the process cooling water (PSW) system include redundant heat exchangers, redundant pumps, pressure control and flow control valves, and instruments in the PSW piping system. A valve on the CTW system controlled by PSW temperature is also included. CTW is an essential support system for PSW. Other support systems include normal and backup electric power, and instrument air (needed by CTW).

Failures of the PSW are detected by a low flow alarm circuit, a low pressure alarm circuit, a high temperature alarm circuit, a low differential pressure alarm circuit, and temperature indications within the individual systems cooled by PSW. 
The fault tree for PSW assumes that the system would fail to provide needed cooling if the closed loop flow were to stop and the bypass line did not open. Failure of the process cooling water system due to insufficient inventory was not modeled because a large volume of water is available in the system, an automatic makeup system is capable of handling small leaks, and large pipe leaks have a very low frequency of occurrence.

\section{Cooling Tower Water}

The cooling tower water (CTW) system transfers process heat from the process cooling water (PSW) system and the chilled water (SSW) system to the atmosphere. Heat exchangers provide the interfaces between the closed loop cooling systems and CTW. There are four pumps in the CTW system. Two are rated as $50 \%$ pumps and two as $25 \%$ pumps. Normally, one large and one small pump are on-line, while the other two are in standby. One $50 \%$ primary pump or two $25 \%$ primary pumps provide adequate pumping. . A deep well water system can provide backup water to CTW for up tó four hours.

A temperature control valve at the heat exchanger outlet regulates CTW flow through the PSW heat exchanger. The temperature is measured at the PSW discharge side of the heat exchanger. A pressure control valve regulate's flow through the SSW chillers. The pressure sensor is in the condenser. A bypass line returns excess flow from the pump discharge line to the CTW basin. If the bypass valve fails open, the CTW system will be inoperable.

The CTW system boundaries include piping, CTW pumps, deep well pumps, heat exchangers, control valves, and the forced convection fans on the cooling tower. Fans (forced convection) are required 6 months per year, and two of the three fans must operate to provide adequate air flow. Electric power and instrument air systems are essential support systems for CTW. If normal power fails, the primary pumps fail (requiring deep well system to function). If instrument air fails, the cooling tower water system fails, due to improper valve positions (Recirculation valve opens and main flow valves close).

Low flow and pressure instruments detect possible failure of CTW pumps. Abnormal conditions produce alarms for operator actions.

The fault tree analysis for CTW assumes that the pump pit inlet screens will not plug. This assumption was made because there is no identifiable debris, the basin is visually checked regularly, and the debris would have to be of varying density so that it covered the screens at varying depths.

\section{Bulk Nitrogen System}

The bulk.nitrogen system vaporizes liquid nitrogen from the bulk nitrogen tank in an ambient vaporizer. The system has a second vaporizer that is kept in standby. The bulk nitrogen system supplies primary purge gas to the PPT and backup purge to SPT in the low point pump pit, primary blanket gas to the OWST, isolation blanket gas to SPC vessels, and nitrogen blanket gas to miscellaneous cold chemical storage tanks. 
System boundaries for the bulk nitrogen system include two ambient vaporizers, piping, a pressure control valve, several pressure safety relief valves, and pressure monitoring instruments with alarms. No support systems other than the bulk nitrogen supply tank are required for the bulk nitrogen system to perform its intended function.

Pressure monitors detect bulk nitrogen system failures. Also if the bulk nitrogen tank becomes depleted, the bulk nitrogen system will be unavailable. Loss of bulk nitrogen tank supply is detected by low level sensors in the tank. This instrument gives an alarm to alert operators.

\section{Bulk Nitrogen Tank}

The bulk nitrogen tank supply consists of a tank of liquid nitrogen that supplies nitrogen to the bulk nitrogen system and the CPC backup purge system.

System boundaries for the bulk nitrogen tank supply include the tank, pressure safety relief valves, and monitoring instruments to track inventory and warn of low level or loss of inventory. No support systems are required for the bulk nitrogen tank supply to perform its intended function. Level sensors in the bulk nitrogen tank detect and alarm low levels in the tank.

\section{CPC Backup Purge Nitrogen Tank}

The CPC purge nitrogen tank supply provides liquid nitrogen to the CPC purge backup system. A minimum of qa 4 day supply of liquid nitrogen is stored in a tank that is protected against overpressurization. An alternate liquid nitrogen supply can be established from the bulk nitrogen supply tank, and it is possible to provide gaseous or liquid nitrogen from a nitrogen delivery truck through piping that is separate from the normally used tanks and vaporizers.

The boundaries for the CPC purge nitrogen tank supply include the liquid nitrogen tank, piping, pressure control valves, pressure safety relief valves, and tank low level instruments and alarm. No support systems are needed for the CPC nitrogen tank supply to perform its intended function. A loss of CPC nitrogen tank supply is detected by a low level sensor in the tank. This instrument provides a low level alarm to initiate operator actions.

\section{CPC Backup Purge}

The CPC backup purge system is a nitrogen gas purge system which vaporizes liquid nitrogen in ambient pressure vaporizers. Both ambient vaporizers are required to supply proper nitrogen flow. Interlocks which monitor for loss of primary purge flow automatically isolate the primary purge system piping and initiate backup nitrogen purge flow. The isolation feature prevents loss of backup purge gas through the primary purge supply lines. 
CPC backup purge system boundaries include the ambient vaporizers, piping, pressure control valves, check valves, and pressure safety relief valves. The CPC liquid nitrogen tank supply is an essential support system for backup purge. If the CPC backup purge nitrogen tank and the bulk nitrogen tank supplies fail or run out, CPC backup purge will be unavailable.

There are no specific alarms provided to detect backup purge failure. The low flow alarms associated with individual $\mathrm{CPC}$ vessels would indicate purge system failure. If a pressure relief safety valve fails open, it would be detected by low level in the liquid nitrogen supply tank.

\section{CPC Primary Purge}

The primary purge air system in the chemical processing cell (CPC) provides air ventilation for CPC process vessels to continuously remove flammable gases and vapors so that these do not accumulate to concentrations above LFL.

CPC system boundaries include redundant air compressors, an air receiver tank, piping, pressure.relief valves, and pressure control.valves that not only regulate air pressure but prevent reverse flow from the process vessels. Normal electric power is an essential support system for CPC primary purge.

Air compressor failures are detected by trouble alarms and a low receiver pressure alarm. In addition, purge air flow monitors on the individual CPC vessels actuate interlocks upon failure of primary purge.

\section{Backup Electric Power}

Backup electric power for all essential services in DWPF comes from two, $2000 \mathrm{~kW}$. each, diesel generator sets located in the fan house. Upon demand they can supply all the essential loads through four main load centers, which in turn, supply twenty motor control centers.

Following are assumptions specific to the Loss of Normal Power fault tree:

1. Maintenance is performed on the diesels annually.

2. All latent human errors are incorporated into the diesel generator fail to run and fail to start data. Human errors associated with failing to restore a generator following maintenance are modeled separately within the fault tree.

3. One operator is responsible for placing both diesels in standby following maintenance. Additionally, the operator will always fail to place the second diesel in standby when he fails to place the first diesel in standby. 
System boundaries for backup electric power include two diesel driven generators, power cables or buses, normal power breakers, and supply breakers up to the four main load centers. Support systems include instrument air, a dedicated compressed air system for starting the diesels, 125 volts DC control system power supply from a bank of batteries, fuel oil supply, lubrication oil supply, and a closed loop jacket cooling water system with a radiator and fan.

A loss of normal electric power will first be detected as an under voltage on the load centers. This will open normal power system breakers and send a start signal to the diesel generators. When the generators are up to speed and the frequency and voltages are correct, the generator supply breakers will close. If one of the generators does not start within ten seconds of the other, the tie breaker closes and the one operating diesel generator will power all of the essential loads.

\section{Primary (Normal) Electric Power}

Normal electric power is supplied to S-Area by two overhead $13.8 \mathrm{kv}$ feeders from H-Area. These are connected to separate $13.8 \mathrm{kv}$ feeders. These are normally connected together through a tie breaker, which allows either feeder to supply both buses. Secondary substations step down the voltage from $13.8 \mathrm{kv}$ to $480 \mathrm{v}$ to supply the various loads in SArea. Each secondary substation is connected to the two $13.8 \mathrm{kv}$ primary buses through a redundant distribution system that allows selection, through a duplex interrupter switch, of one or the other of the $13.8 \mathrm{kv}$ buses.

Following are assumptions specific to the Loss of Normal Power fault tree:

1. Human errors associated with maintenance activities are immediately detected and corrected. Maintenance performed on the normal power system requires lockout procedures; removal of the lockout with subsequent restoration of the power to the component is immediately detected.

2. Maintenance will not be performed on a redundant power train until power has been restored to the alternate train.

System boundaries for normal electric power include all electrical buses, feeders, and breakers necessary to supply power to the primary buses of DWPF. The $13.8 \mathrm{kv}$ feeders connect to 1he $115 \mathrm{kv}$ electric power grid through substation transformers. The $115 \mathrm{kv}$ grid derives its electric power from the southeastern commercial electric power grid and the power station in D-Area. Other than the ultimate power sources, no support systems were considered in the analysis of the normal electric power system.

Normal electric power failure is self indicating through multiple equipment failure alarms and indications. The frequency that power is lost to equipment fed from a normal power bus was equated to the frequency that normal power is lost. The frequency of individual circuit breaker failures (i.e., failed open) was judged to be insignificant compared to loss of DWPF normal power. 


\section{Zone 1 Ventilation}

Zone 1 ventilation system exhausts air from the vitrification building zone 1 through a sand filter before release to the atmosphere. In addition to the normal mode of operation, the fault tree analysis considered several abnormal modes of operation given electric power or instrument air system failures.

Following are assumptions specific to the Zone 1 ventilation fault tree:

1. Failure of either a discharge or suction damper to remain open on an operating fan line does not result in a complete blockage of flow in that line. Additionally, failure of a discharge damper to open or failure of the operator to open' a suction damper following maintenance on the standby fan line results in failure of the fan to start.

2. Complete closure of the fan inlet vanes reduces the inlet orifice to 4 " which is sufficient to maintain the fan operation.

3. Fan vibration monitors no longer have the capability to shut off a fan through DCS.

4. Fan timing relays will not cause a fan to fail to run if the relay spuriously opens following successful start-up of the fan.

5. Under a loss of normal power condition, the timing relay will automatically start up the second fan on an emergency bus when the first fan has failed to start (i.e., within a short period of time). No credit is given for automatic start-up of the second fan when the first fan fails after successfully starting.

6. It is not possible to plug the sand filter during normal operating conditions (i.e., conditions other than a seismic event).

7. Under a loss of normal power condition, the fans will not re-start if UPS power is unavailable at the time of the power loss.

8. Under loss of normal power and instrument air conditions, the time it takes for the instrument air pressure to drop below $85 \mathrm{psi}$ in the fan house (i.e., the minimum pressure required for start of the fan house compressor) is greater than the time period in which the fans are re-started by the timing relay.

9. Successful operation of zone 1 ventilation is defined as one ventilation fan running.

The system includes ventilation exhaust ducts, sand filter exhaust plenum, four exhaust fans, dampers, DCS control circuits, electric power circuits, UPS power system, and monitoring instruments with alarms. Essential support systems include normal and backup electric power systems, instrument air, and the 125 volts DC battery power supply to operate mechanical circuit breakers upon loss of normal power. 
Instruments monitor differential pressure between building zones and give alarms for abnormal zone pressure differences. There are high pressure alarms for continuously occupied areas and areas containing radioactive materials. Two pressure transmitters are located immediately upstream of zone 1 sand filter exhaust plenum. Air flow through each exhaust fan is also monitored.

Under normal operating conditions, when normal electric power is available, three fans are on and one is in ready standby. A high pressure indication from either transmitter in the sand filter exhaust plenum will start the fourth fan automatically. A low flow condition can also lead to automatic start of the standby fan; however, this analysis does not take credit for the low flow initiated starting feature.

If normal electric power is lost, all operating fans will stop until the backup electric power from the diesel generator comes on line. An automatic timing relay starts two fans from two separate electric power buses. If one of the fans connected to the emergency bus fails to start, the timing circuit will start the other fan connected to that same bus automatically. Operators may also restart fans manually.

\section{SPC Primary Purge}

The salt processing cell (SPC) primary purge system uses carbon dioxide gàs. Liquid carbon dioxide from a large storage tank is converted to gas in an electrically heated vaporizer. Gas pressure is regulated to provide purge flow through the various SPC vessels.

System boundaries for the SPC primary purge system include the carbon dioxide storage tank, electric vaporizer, piping, supply shutoff valve, pressure control valves, pressure safety relief valves, heater control circuit, and low tank level and low pressure alarm circuits. Normal and backup electric power systems are the only support systems necessary for SPC primary purge to operate. If electric power fails, the vaporizer will not generate enough carbon dioxide gas for adequate purge flow to SPC vessels.

A failure of SPC primary purge (e.g., if the pressure control valve fails closed) is indirectly . detected by a high flow alarm on the SPC backup purge system, which automatically supplies purge flow when the pressure in the primary system gets too low. Loss of liquid carbon dioxide inventory is detected by low tank level and low tank pressure alarms.

\section{SPC Backup Purge}

Salt processing cell backup purge is a carbon dioxide gas system supplied from a bank of compressed gas cylinders. Gas flow from the backup purge system is regulated by pressure in the primary purge piping system and comes on only if the primary system pressure gets too low. The backup system has enough capacity for short outages of the primary purge system. 
System boundaries for SPC backup purge include four high pressure carbon dioxide cylinders, piping, isolation valves, pressure regulators, two pressure control valves, pressure safety relief valves, and alarms for low gas supply. No support systems are required for the SPC backup purge system to perform its intended function. The carbon dioxide supply for SPC backup purge is monitored by cylinder weight and pressure.

\section{SPC Nitrogen Supply}

The salt processing cell low pressure nitrogen supply system provides nitrogen purge to SPC vessels whenever SPC is isolated from the PVVH. The purpose of the low pressure blanket gas is to keep oxygen from entering the process vessels and to minimize the amount of benzene vapor released from the vessels into the surrounding cell space. Nitrogen gas is produced in ambient pressure vaporizers supplied from the bulk liquid nitrogen tank.

System boundaries for the low pressure nitrogen system include two vaporizers, piping, isolation valves, pressure control valves, and pressure safety relief valves. No support systems are required for the SPC low pressure nitrogen system to perform its intended function. Failures of the low pressure nitrogen system do not produce alarms but may be detected through DCS indications or field surveillance.

\section{LPPP Nitrogen System}

The low point pump pit (LPPP) nitrogen system supplies backup nitrogen purge gas to the precipitate pump tank (PPT) and the sludge pump tank (SPT). This system has both a liquid nitrogen supply tank and compressed nitrogen gas cylinders. The backup system automatically continues the purge flow if primary purge systems should fail.

System boundaries for the LPPP nitrogen system include the liquid nitrogen tank, a vaporizer, high pressure nitrogen gas cylinders, piping, check valves, pressure control valves, pressure safety relief valves, and nitrogen supply tank low level alarm. No support systems are required for the LPPP nitrogen system to perform its intended function.

A low level alarm instrument detects failure or depletion of the liquid nitrogen supply. Periodic surveillance would detect failures of the compressed gas supply. Also, low purge flow alarms on the SPT or PPT would indicate possible problems with the nitrogen supply system.

\section{Instrument Air}

The instrument air system supplies high pressure air to instruments and pneumatic equipment throughout the DWPF. Two compressors are on line and a third is normally in standby. The system has redundant dryers and filters so that units can be repaired or replaced while the air system remains operable. 
System boundaries for instrument air include, three compressors, air receiver tank, filters, redundant dryers, supply solenoid valves, pressure control valves, pressure safety relief valves, cooling water supply piping and valves, and differential pressure monitors and alarms. Normal and backup electric power systems, and the cooling tower water system are essential support systems for instrument air.

Loss of instrument air is detected by low pressure instruments with alarms and would also be . evident by numerous failures of pneumatic equipment.

\section{General Assumptions}

The following general assumptions were factored into the fault tree analysis:

1. Assumptions pertaining to individual basic events are printed in the fault trees, identified by "a:" before the assumption. For example, "a: calibrated twice/year" means that it is assumed that the piece of equipment is calibrated at that frequency.

2. Most equipment or component failures are assumed to be corrected within $\mathbf{8}$ hours or the systems are configured in such a way that those failures do not continue to put the facility at risk. Exceptions to this recovery assumption are indicated in the fault trees.

3. Possible sabotage is not included in the analysis.

4. Ammonium nitrate explosions cannot occur in any vessel or ventilation system in DWPF.

5. If a pressure safety relief valve (PSV) fails open on a pressurized gas system, it is assumed the valve will not close again and must be isolated from the system by operator action.

6. If a PSV can be isolated from the piping system by a three-way valve that connects to another PSV, system recovery time is short (e.g., 1 hour). But if a PSV must be replaced; the repair time is assumed to be 24 hours.

7. Manual valves will not fail closed if normally open, and will not fail open, if normally closed. An incorrect valve position may result from operator error.

8. Failure rates for components such as electrical breakers or switches related to individual electric motors were determined to be insignificant compared to the failure rate of the overall DWPF power system.

9. If a temperature element controlling a temperature control valve fails or a pressure element controlling a pressure control valve fails, the valve moves to the position which adversely affects system reliability. 
10. If a device is out of calibration, it affects system performance adversely:

11. Temperature elements are functionally checked twice per year, unless otherwise specified.

12. If purge systems fail to the SRAT or SME, and interlock actions are unsuccessful, the flammable gases in the vessiel are assumed to reach LFL. If interlock actions are successful, the purge is restored before LFL is reached.

13. If purge systems fail to the MFT or PRBT, and alarm response actions are not successfully completed, the flammable gases in the vessel are assumed to reach LFL. If alarm response actions are successfully completed, the purge is assumed to be restored before LFL is reached.

14. Each DWPF macro-batch from the tank farm will be chemically characterized by SRTC.

15. Instrument calibration errors are pure enabler events. They can cause protective equipment to not detect abnormal' conditions, but they cannot initiate a failure sequence.

16. An ignition source is necessarily present for seismic scenarios that lead to an explosive mixture quickly (within a few hours) following the earthquake. For scenarios where explosive mixtures take longer, ignition probability is equivalent to the internal ignition probability.

17. CPC backup nitrogen supply, LPPP local liquid nitrogen supply, and OWST local liquid nitrogen supply all have adequate capacity that they will continue to provide nitrogen for a minimum of 96 hours following an earthquake, taking into account the possibility of additional leakage in piping due to the earthquake.

\section{DISCUSSION}

The hazard analysis for the Defense Waste Processing Facility (DWPF) identified process vessels in which flammable mixtures of hydrogen gas or benzene vapor could accumulate if certain vessel ventilation or purge systems were to fail. Once the concentration of these substances builds up above LFL, then only a spark source is required to set off a deflagration or in the extreme case a detonation that could disperse radioactive waste material into the environment. Operability of purge systems or ventilation systems is a crucial requirement in preventing explosion accidents. These important systems could fail from a number of causes such as random failures of system components (i.e., internal initiators) or from external natural disasters that damage process facilities and equipment (i.e., external initiators). This fault tree analysis included, only those accidents identified in the hazards analysis which are based on internal initiators and seismic initiators. Other external initiators such as high wind storms or tornadoes and external events. related to man's activities (e.g., airplane crash), are covered elsewhere in the accident analysis. 
An additional limitation on the scope of this study is that only normal operations were analyzed. Unusual or major maintenance activities and special testing were not included. Routine equipment checks and instrument testing and component replacements (e.g., filters, bulbs, fuses, etc.) if covered under operating procedures, were considered to be part of normal operation and were taken-into account. Similarly, unusual occurrence recovery actions specified in procedures were also included.

\section{FACILITY COMMITMENTS}

Developiment of explosion accident fault trees for DWPF was an iterative process with design activities. Preliminary fault trees were developed and submitted to DWPF for review. In those cases where unacceptably high accident frequencies were calculated, commitments were made by DWPF to change procedures, add administrative controls, or modify system design to reduce the likelihood of explosion accidents (Reference 5). The analyses were changed to reflect the commitments listed below in this section. Fulfillment of these commitments must be verified in order for facility design to match the accident analysis in the SAR.

1. The PRC/D drain valve operations procedure will be revised to reduce the probability of opening the drain valve after PR sampling. Changes include adding statements at the step where the valve is opened to stress the importance of not opening the valve at the wrong time, and to add a review and sign off for the technical group representative. \{Impacts PRBT fault trees\}

2. A supervisor permissive switch for opening the PRC/D drain valve will be added. This permissive switch will time out and must be approved again if not completed in the allotted time. \{Impacts PRBT fault trees\}

3. An interlock will be provided that prevents PR steam supply if PRC/D drain valve is open. \{Impacts PRBT fault trees\}

4. Sampling procedures for the precipitate reactor (PR) will be submitted for review to ensure that nominal error probabilities are justified: These procedures will be revised as necessary, based on comments generated during these reviews. \{Impacts PRBT fault trees\}

5. Training manuals, lesson plans, and objectives will be revised as necessary to emphasize the hazards of SPC (fuel rich, oxygen below MOC) contents being transferred to CPC vessels (diluted below LFL, oxygen present). \{Impacts PRBT fault trees\}

6. The SRAT double batch procedure will be revised to incorporate technical review and approval. \{Impacts SRAT fault trees\}

7. Emergency Response Procedure developed for seismic event will include warnings of restoring steam, power, or instrument air, following a seismic event. \{Impacts all seismic fault trees\} 
8. SPC purge system will be modified, adding a nitrogen purge, activated by seismic triggers, loss of normal power, and low PVVH flow, as described in Reference 8. \{Impacts PR, PRFT, OE, OECT, and PVVH fault trees\}

9. SPC primary purge will have two normally open, fail closed, isolation valves in series, closed by seismic trigger activation or low PVVH flow. (See Reference 8.) \{Impacts $P R, P R F T, O E, O E C T$, and PVVH fault trees\}

10. The SPC backup purge $\left(\mathrm{CO}_{2}\right.$ cylinders $)$ will be modified so that it ties into the primary line upstream of the isolation valves referred to in commitment \#9. (See Reference 8.) \{Impacts SPCN2 fault trees\}

11. Valves will be added to isolate the $\mathrm{SPC}$ nitrogen addition lines from the $\mathrm{CO}_{2}$ supplies upon nitrogen system activation. (See Reference 8.) \{Impacts SPCN2 fault trees\}

12. Nitrogen supply to the CPC backup purge system will be increased to include a four day supply (using CPC and SPC demand rates) in a seismically qualified tank separate from the bulk nitrogen tank. (See Reference 8.) \{Impacts CPCTNK fault trees\}

13. A pressure control valve will be added in parallel to PCV-0967 on the CPC backup purge line. (See Reference 8.) \{Impacts CPCBP fault trees\}

14. A manual valve will be added in parallel to each of the CPC purge supply flow control valves. (See Reference 8.) \{Impacts SRAT, SME, MFT, and PRBT fault trees\}

15. SCVC/PVVH isolation valves HCV-1041 and HCV-9278 will each be supplied power by separate UPSs, with control logic provided that closes them on low PVVH flow, loss of control power, or high seismic activity. \{Impacts PVVH, PR, OE, PRFT, and OECT fault trees\}

16: A seismically qualified liquid nitrogen tank with a four day supply for the OWST blanket gas system will be provided. It will feed the OWST blanket supply lines downstream of the compressed nitrogen gas supply from high pressure cylinders. Dual check valves in series isolate this dedicated supply from the high pressure cylinders and the bulk nitrogen supply. (See Reference 8.) \{Impacts OWSTTK fault trees\}

17. The OWST nitrogen supply will be provided through parallel pressure control valves. (See Reference 8.) \{Impacts OWSTTK fault trees\}

18. The Low Point Pump Pit will be provided with nitrogen through parallel pressure control valves from a tank with adequate capacity to supply both the precipitate pump tank and the sludge pump tank for four days. (See Reference 8.) \{Impacts LPPPN2 foult trees\}

19. The SPT and PPT in the LPPP will be supplied by nitrogen purge through manual valves. (See Reference 8.) \{Impacts SPT and PPT fault trees\} 
20. The PPT off-gas will be modified to have a second fail closed valve in series with HCV 8772. HCV 8772 and the new HCV will be provided an interlock to close upon low LPPP PVVH flow. The manual valve on the bypass line (with SV 8773) will be maintained normally closed. (See Reference 8.) \{Impacts PPT fault trees\}

21. The SPC fire damper will be removed, (reducing the frequency of cell air flow loss). \{Impacts SPC fault trees\}

22. A seismic switch will be installed to isolate the PPT from the PVVH (reducing the probability of reaching MOC in the tank). \{Impacts PPT fault trees\}

23. The SRAT nitric acid addition system will be modified so that the display reading will properly indicate the amount added. (FIC-0718 will read directly, not with a 5:8 factor difference from actual flow.) \{Impacts SRAT fault trees\}

24. MFT purge failure response procedure, AOP-S-8009, will add the procedure connector symbol between pages 2 and 3 . \{Impacts MFT fault trees\}

25. MFT purge flow low, alarm response procedure, S-FAL0683, will be rewritten so that steps 3 and 4 are placed in reverse order (i.e., the purge must be re-established before any work order is performed to repair faulty low elements). \{Impacts MFT fault trees\}

26. The commitments listed in Reference 6 and 7 regarding fragility values for systems and equipment will be met. \{Impacts all seismic trees\}.

27. A temperature interlock for the melter vapor space plenum will be installed to automatically shut off the operating feed pump if a low temperature signal is detected. \{Impacts Melter Offgas fault trees\} 


\section{REFERENCES}

1. J. Koren, et al, "CAFTA/CAFTA 386 version 2.2c," Science Applications International Corporation, May 29, 1992 (Proprietary Software).

2. C. H. Blanton and S. A. Eide, "Savannah River Site Generic Data Base Deevelopment (U)," WSRC-TR-93-262, Rev. 0, June 30, 1993.

3. H. C. Benhardt, et al, "Savannah River Site Error Data Base Development for Nonreactor Nuclear Facilities (U)," WSRC-TR-93-581, Rev. 0, February 28, 1994,

4. E. V: Brown and A. G. Sarrack, "DWPF Accident Analysis Fault Trees - Explosions in the SPC and CPC (U)," WSRC-RAM-93-90018, August 2, 1993.

5. T. B. Calloway, "Fragility Data for DWPF Mode C Analysis (U)," OPS-DTL-940152, October 27, 1994.

6. G. B. Rawls, "Additional Seismic Fragilities for DWPF Safety Basis (U)," ECS-EAT-940088, October 27, 1994.

7. G. B. Rawls, "Seismic Fragilities for DWPF Safety Basis (U)," ECS-EAT-94-0084, October 18, 1994.

8. J. P. Schwenker, "Resolution of DWPF's Safety Basis (U)," WSRC-TR-94-0395, Revision 1, December, 1994. 


\section{Attachment 1 List of P\&IDs used}

\begin{tabular}{|c|c|}
\hline Drawing & $\frac{\text { (page } 1 \text { of } 4 \text { ) }}{\text { Description }}$ \\
\hline 750040 & PPT vessel \\
\hline 750041 & LPPP PPT vent and N2 blanket \\
\hline 750042 & PPT cell \\
\hline 750104 & MFT (Process Cooling Water) \\
\hline 750108 & Melter (Process Cooling Water) \\
\hline 750109 & Melter feed piping \\
\hline 750110 & Melter feed piping \\
\hline 750115 & Melter pressure relief \\
\hline 750116 & SME Condenser \\
\hline 750122 & RCT (Cooling Coils and Chilled water) \\
\hline 750123 & PVVH Blowers and dampers \\
\hline 750124 & Base Air Load to PVVH and HEPAs \\
\hline 750147 & DWTT (Process Steam and Cooling Coils) \\
\hline 750152 & LPPP tank ventilation prefilters \\
\hline 750162 & SPC Sump (CO2 purge) \\
\hline 750172. & FAVC \\
\hline 750179 & CPC Vent Header \\
\hline 750181 & SCVC and OG Exhauster gas sampling \\
\hline 750182 & PVVH gas sampling \\
\hline 750183 & PVVH gas sampling (after filter) \\
\hline 750184 & PVVH gas sampling \\
\hline 750185 & SRAT (Process Steam and Cooling Coils) \\
\hline 750186 & SRAT (Air/N2 purge) \\
\hline 750187 & SME (Process Steam and Cooling Coils) \\
\hline 750195 & PVVH \\
\hline 7.50196 & SRAT Condenser \\
\hline 750197 & PVVH Heater and filter \\
\hline 750199 & DWTT Condenser \\
\hline 750203 & Melter quencher \\
\hline 750204 & Melter shell cooling \\
\hline 750205 & Melter vessel cooling \\
\hline 750208 & PVVH gas sampling (before filter) \\
\hline 750222 & Off-Gas Condensate Tan \\
\hline
\end{tabular}


X-ESR-S-00001, REV. 0

\section{Attachment 1 List of P\&IDs used}

\begin{tabular}{l} 
(page 2 of 4) \\
\begin{tabular}{|c|l|}
\hline Drawing & \multicolumn{1}{|c|}{ Description } \\
\hline 750224 & BOGCT (Chilled water) \\
\hline 750225 & Off-Gas Condenser (Chilled Water) \\
\hline 750227 & BOGC (Chilled Water and Process Cooling Water) \\
\hline 750235 & MFT (Cooling Coils) \\
\hline 750234 & Melter electrodes \\
\hline 750235 & MFT (Vent) \\
\hline 750237 & Melter dome heaters \\
\hline 750240 & Cold Feed Organic Acid Vent Cond. (Chilled water) \\
\hline 750251 & SPT Cell \\
\hline 750252 & RPT Cell \\
\hline 750253 & LPPP Sludge tank (Vent) \\
\hline 750254 & LPPP Recycle tank (Vent) \\
\hline 750294 & LPPP Cell Vent. supply and tank vent blowers \\
\hline 750297 & Melter dome heaters \\
\hline 750303 & Melter dome heaters \\
\hline 750304 & Melter dome heaters \\
\hline 750308 & SME gas chromatographs \\
\hline 750312 & SME (Air/N2 Purge) \\
\hline 750313 & MFT (Air/N2 Purge) \\
\hline 750451 & PRBT and MFT vent header \\
\hline 750476 & SRAT Vent header \\
\hline 750477 & CPC Vent Header to FAVC \\
\hline 750478 & SRAT Ammonia Scrubber \\
\hline 750479 & SME Ammonia Scrubber \\
\hline 750481 & RCT/MFT Ammonia Scrubber \\
\hline 750483 & SRAT gas chromatographs \\
\hline 750485 & \\
\hline 750494 & LPPP tank ventilation filters \\
\hline 751008 & Liquid N2 Supply tank and steam vaporizer \\
\hline 751058 & N2 ambient vaporizers for CPC \\
\hline 751539 & CPC Purge compressors and receiver \\
\hline 751540 & DeepWell System \\
\hline 751548 & CPC Purge Supply Header \\
\hline 751580 & CTW Tower \\
\hline
\end{tabular} \\
\hline
\end{tabular}




\section{Attachment 1 \\ List of P\&IDs used}

\begin{tabular}{l} 
(page 3 of 4) \\
\begin{tabular}{|c|l|}
\hline Drawing & \multicolumn{1}{|c|}{ Description } \\
\hline 751581 & CTW Pumps \\
\hline 751588 & PCW/CTW Heat Exchangers \\
\hline 751589 & Process Cooling Water head tank and recirc. pumps \\
\hline 751593 & Melter Cooling Water Pumps \\
\hline 751594 & Melter / CTW Heat Exchangers \\
\hline 751595 & Chilled water head tank and recirc. pumps \\
\hline 751596 & Chillers \\
\hline 751598 & Process Steam Heat Exchanger \\
\hline 751602 & Process Steam Cond. Tank and pumps \\
\hline 751603 & Process Steam Generator \\
\hline 751610 & Plant/instrument air supply (2 of 2) \\
\hline 751611 & Instrument supply header \\
\hline 751612 & Plant/instrument air supply (1 of 2) \\
\hline 751650 & N2 header to PR, LPPP, and OWST \\
\hline 751654 & CO2 main supply header and backup cylinders \\
\hline 751655 & Liquid CO2 supply and vaporizer \\
\hline 751664 & Process Steam supply header \\
\hline 751665 & Process Steam return header \\
\hline 751669 & Melter cooling distribution headers \\
\hline 751670 & Process Cooling Water supply and return headers \\
\hline 751671 & Chilled water supply and return headers \\
\hline 751672 & CTW supply header \\
\hline 751673 & CTW return header \\
\hline 751685 & Instrument air piping \\
\hline 751745 & N2 supply header (to LPPP precipitate tank) \\
\hline 751832 & Zone 1 Ventilation (fan house air accumulator) \\
\hline 754001 & PR (Process Steam) \\
\hline 754003 & PR (Vent) \\
\hline 754004 & PR (CO2 purge) \\
\hline 754006 & PR (Cooling Coils) \\
\hline 754007 & PRFT (Cooling Coils, CO2 purge) \\
\hline 754010 & OE (Process Steam and Cooling Coils) \\
\hline 754011 & OE (CO2 purge) \\
\hline 754013 & OECT (CO2 purge) \\
\hline 754016 & PRC/D \\
\hline
\end{tabular} \\
\hline
\end{tabular}


X-ESR-S-00001, REV. 0

\section{Attachment 1 List of P\&IDs used}

\begin{tabular}{|l|l|}
\hline Drawing & \multicolumn{1}{|c|}{ (page 4 of 4) } \\
\hline 754018 & Description \\
\hline 754020 & SCVC \\
\hline 754021 & PRBT (Cooling Coils and Air/N2 purge) \\
\hline 754037 & PVVH gas sampling \\
\hline 754061 & OWST \\
\hline 754063 & OWST HEPA \\
\hline 754064 & N2 Supply to OWST \\
\hline 756677 & Critical Plant air supply header \\
\hline 766583 & Cooling Tower return valve logic. (CLD) \\
\hline 766827 & Cooling Tower discharge valve logic (CLD) \\
\hline 766828 & Deep Well Water to CTW logic (CLD) \\
\hline 7.66829 & Deep Well Water to CTW logic (CLD) \\
\hline 766901 & SRAT and SME interlock logic (CLD) \\
\hline 766963 & SRAT and SME interlock logic (CLD) \\
\hline 767909 & Melter Cooling Radiation Interlock logic (CLD) \\
\hline 770311 & Electrical Distribution (sécond main substation) \\
\hline 776559 & LPPP cell vent. filters, blower and stack \\
\hline 776631 & PVVH and Cell exhaust \\
\hline 776662 & Sand filter and exhaust fans and dampers \\
\hline 776663 & Exhaust fans and dampers and stack \\
\hline
\end{tabular}




\section{Attachment 2}

\section{Naming Convention for Fault Trees}

Form Page 1 of 5

aaabbbbbcccdefff

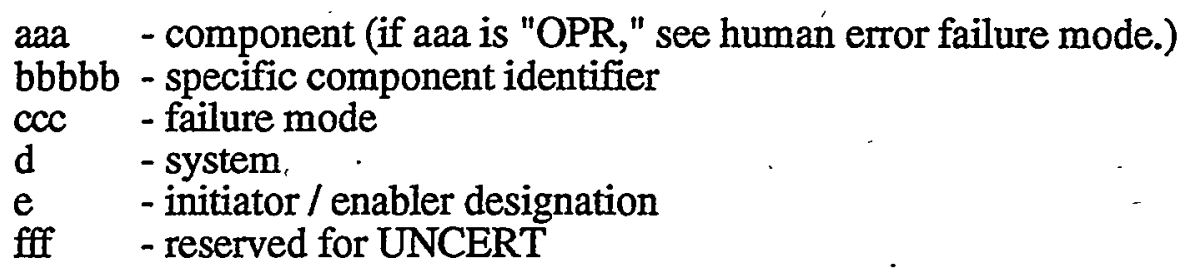

(aaa) Component Abbreviations (digits 1, 2, and 3)

\begin{tabular}{|l|l|}
\hline Abbr. & Component \\
\hline AE- & Analyzing element \\
\hline AEG & Gas Chromatograph \\
\hline AX- & Analysis calculation module \\
\hline BCH & Batch of product \\
\hline BKR & Breaker \\
\hline BNZ & Benzene (explosive amount) \\
\hline BOT & Bottle (gas cylinder) \\
\hline BUS & Electrical bus \\
\hline CC- & Common cause event \\
\hline CC2 & Common cause: 2 events \\
\hline CC3 & Common cause: 3 or more events \\
\hline CHL & Chiller \\
\hline CMP & Compressor \\
\hline CO2 & Carbon dioxide \\
\hline CP- & Common person (same operator) \\
\hline CV- & Check valve \\
\hline CYL & Gas cylinder \\
\hline DCS & Distributed control system \\
\hline DG- & Diesel generator \\
\hline DMP & Damper \\
\hline DRY & Air dryer \\
\hline EXP & Explosive gas \\
\hline FAL & Low flow alarm \\
\hline FAN & Fan \\
\hline FCV & Flow control valve \\
\hline FE- & Flow element (sensor) \\
\hline FED & Electrical feeder \\
\hline FI- & Flow indicator \\
\hline FIT & Flow indicator / transmitter \\
\hline FLG & Flange \\
\hline & \\
\hline
\end{tabular}


X-ESR-S-00001, REV. 0

\section{Attachment 2 \\ Page 2 of 5 \\ Naming Convention for Fault Trees}

(aaa) Component Abbreviations (Continued)

\begin{tabular}{|l|l|}
\hline Abbr. & Component \\
\hline FLT & Filter \\
\hline FSH & High flow switch \\
\hline FSL & Low flow switch \\
\hline FT- & Flow transmitter \\
\hline FY- & Flow relay \\
\hline HCV & Manually controlled, motor operated valve \\
\hline HPW & H-area power \\
\hline HTR & Heater \\
\hline HX- & Heat exchanger \\
\hline IA- & Instrument air \\
\hline IGN & Ignition source \\
\hline INK & Interlock \\
\hline JMP & Jumper \\
\hline LCV & Level control valve \\
\hline LE- & Level element (sensor) \\
\hline LPW & Loop power \\
\hline LSL & Low level switch \\
\hline MOV & Motor operate valve \\
\hline N2- & Nitrogen \\
\hline OFV & Overflow valve \\
\hline OPR & Operator (see human error type of failure mode) \\
\hline PC-. & Pressure control module \\
\hline PCS & Process control system \\
\hline PCV & Pressure control valve \\
\hline PDA & Differential pressure alarm \\
\hline PDE & Differential pressure element \\
\hline PDS & Differential pressure switch \\
\hline PDT & Differential pressure transmitter \\
\hline PE- & Pressure element \\
\hline PGV & Plug valve \\
\hline PIC & Pressure indicator/controller \\
\hline PIP & Pipe or line \\
\hline PMP & Pump \\
\hline POP & Process Operating Procedure (DCS) \\
\hline PR- & Precipitate reactor \\
\hline PSE & Rupture disk \\
\hline PSH & High pressure switch \\
\hline PSL & Low pressure switch \\
\hline
\end{tabular}




\begin{tabular}{l} 
Naming Convention for Fault Trees \\
\multicolumn{1}{|c|}{$\begin{array}{c}\text { Attachment } 2 \\
\text { Page } 3 \text { of } 5\end{array}$} \\
\begin{tabular}{|l|l|} 
(aaa) & Component Abbreviations (Continued) \\
\hline Abbr. & Component \\
\hline PSV & Pressure relief valve \\
\hline PT- & Presssure transmitter \\
\hline REG & Pressure regulator \\
\hline RY- & Relay \\
\hline SC- & Speed controller \\
\hline SLG & Sludge \\
\hline SLR & Excess salt \\
\hline SRA & SRAT batch \\
\hline STM & Steam \\
\hline SV- & Solenoid valve \\
\hline TC- & Temperature controller \\
\hline TCV & Temperature control valve \\
\hline TE- & Temperature element \\
\hline TNK & Tank \\
\hline TOP & Fault tree top event \\
\hline TRF & Transformer \\
\hline TRS & Debris screen (in water system) \\
\hline TSH & High temperature switch \\
\hline TX- & Transfer \\
\hline UA- & Trouble alarm \\
\hline UPS & Uninterruptable Power Supply \\
\hline VB- & Vacuum breaker \\
\hline WE- & Weight element \\
\hline WSL & Low weight alarm \\
\hline WTR & Water \\
\hline & \\
\hline
\end{tabular} \\
\hline
\end{tabular}


X-ESR-S-00001; REV. 0

\section{Attachment 2 \\ Page 4 of 5 \\ Naming Convention for Fault Tree}

(ccc) Failure Mode Abbreviàtions (digits 9, 10, and 11)

\begin{tabular}{|l|l}
\hline Abbr. & Failure mode \\
\hline
\end{tabular}

ADJ Adjusted

BFL Basic failure

BLU Built up

BYP Bypassed

CAL Calibrated

CHK Checked

DBL 1 Double

\begin{tabular}{l|l}
\hline FCL & Fail closed \\
\hline
\end{tabular}

FHI Fail high.

FLO Fail low

FOP Fail open

FOU Fouled

\begin{tabular}{l|l} 
FTA & Fail to alarm \\
\hline
\end{tabular}

FTC Fail to close

FTO $\quad$ Fail to open

FTR $\quad$ Fail to run

FTS $\quad$ Fail to start

HI- $\quad$ High condition

IDL Idle condition

、 \begin{tabular}{|l|l|}
\hline LKS & Leaks \\
\hline
\end{tabular}

OFF Not on

ON- On, running, or in use

OUT Ran out (inventory)

PLG Plugged

PRE Present

REP 1 Replaced

RUP 1 Ruptured

- T\&M Testing and Maintenance (out of service for T\&M)

UNA Unavailable due to T\&M

USD Used (demanded)

\begin{tabular}{l|l}
\hline VCR & Vehicle crash \\
\hline
\end{tabular} 


\section{Attachment 2}

\section{Page 5 of 5 \\ Naming Convention for Fault Tree}

(ccc) Failure Mode Abbreviations for Human Errors

\begin{tabular}{|l|l|}
\hline Abbr. & Failure mode \\
\hline AC- & Failure of an administrative control \\
\hline CA- & Chemical addition or elution error \\
\hline CS- & Fail to respond to a compelling signal \\
\hline CV- & Check / verification error \\
\hline DE- & Diagnosis error \\
\hline IR- & Incorrect reading or recording of data \\
\hline LA - & Laboratory analysis error \\
\hline MC- & Miscalibration \\
\hline NC- & Failure to verify parameter with calculation \\
\hline OV- & Overfilling of a tank \\
\hline RA- & Random actuation / shutdown of system \\
\hline RM- & Failure to restore to service following maintenance \\
\hline SO- & Error in selecting control outside control room \\
\hline SR- & Error in selecting control within control room \\
\hline SV- & Supervisor verification error \\
\hline VI- & Failure of visual inspection \\
\hline
\end{tabular}

The third character of each human error failure mode is either $\mathrm{H}$ (high), $N$ (nominal), or L (low).

\section{(d) System (digit 12)}

The system designation is used in cases where two components with the same failure mode have different failure probabilities, due to being in different conditions. For example, a check valve in a water system may have a different probability of failing to open than a check valve in a compressed gas system. Character " $\mathrm{d}$ " of the naming convention allows a different type code (and thus a different value) to be used.

Example of two type codes with different system designations:

CV-FTO 1 (check valve in water system fails to open) $=5 \mathrm{E}-5 /$ demand

CV-FTO 2 (check valve in compressed gas system fails to open) $=1 \mathrm{E}-4 /$ demand

(e) Event Type (digit 13)

\begin{tabular}{|l|l|}
\hline Abbr. & Event type \\
\hline$*$ & Pure intiator \\
\hline$\#$ & Pure enabler \\
\hline+ & Initiator/ enabler \\
\hline
\end{tabular}




\section{Attachment 3 Basic Event (BE) File}

Basic event (BE) file Master.BE contains all events used in the DWPF accident analysis fault trees. The BE file printout follows the following format:

\begin{tabular}{|l|l|l|l|l|l|}
\hline Event & C & Input & Calc. & Description & Source \\
\hline
\end{tabular}

Event - The name of each basic event, which follows the naming convention in Attachment 2. Each event has a unique name shared by all branches of the fault trees that model that event.

C- Calculation type, which depends on the type of failure being entered. The result van be a probability or a frequency. Details on how the calculation types are chosen are provided in References 1 and 2 of this attachment.

Input - The input column is provided to enter data on the mission time of a component, the frequency of inspection, or other information that may be necessary to calculate the probability or frequency of component failures. See References 1 and 2 of this attachment for further details.

Calc. - Calculated probability of the event. (This column is not entered, but is derived by CAFTA.

Description - The description column allows a more detailed description of the basic event entered.

Source - The source column is provided to enter information regarding the source of failure rate data or calculation type choice:

\section{References for Attachment 3}

1. CAFTA User's Manual, CAFTA 386 Version 2.2c, SAIC, May, 1992 (proprietary software)

2. CAFTA User's Manual, CSRAM 386 Version 2.1c, SAIC, May, 1992 (proprietary software) 
Basic Event Report for I: \CAFTA IDATA MMASTER.BE

$11 / 11 / 94 \quad 11: 42 \mathrm{AM}$

\begin{tabular}{|c|c|c|c|c|c|c|c|c|}
\hline Event & & C & nput & Calc. & Description & Source & & \\
\hline $\begin{array}{l}\text { AE--RAD-BFLG\# } \\
\text { AE-2898-BFLG\# } \\
\text { AE-3405-FLOK\# } \\
\text { AE-3409-FLOK\# } \\
\text { AE-6852-BFLQ\# } \\
\text { AE-68523CCAQ\# } \\
\text { AE-6853-BFLQ\# } \\
\text { AE-9233-BFLP\# } \\
\text { AE-DWPF1CCLU\# } \\
\text { AE-DWPF2CCLU\# } \\
\text { AE-DWPFOCCLU\# } \\
\text { AE-SRTC-CCLU\# } \\
\text { AEG3407-FLO5\# } \\
\text { AEG3407-LKS5\# } \\
\text { AEG3408-FLO5\# } \\
\text { AEG3408-LKS5\# } \\
\text { AEG8795-FLO5\# } \\
\text { AEG8795-LKS5\# } \\
\text { AEG8796-FLO5\# } \\
\text { AEG8796-LKS5\# } \\
\text { AEG8797-FLOD\# } \\
\text { AEG8797-LKS4\# } \\
\text { AEG8798-FLOD\# } \\
\text { AEG8798-LKS4\# }\end{array}$ & $\checkmark$ & 5 & $\begin{array}{r}8 \mathrm{H} \\
5.0 \mathrm{E}-06 \mathrm{H} \\
8 \mathrm{H} \\
5.0 \mathrm{E}-06 \mathrm{H} \\
43 \mathrm{H} \\
5.0 \mathrm{E}-06 \mathrm{H} \\
43 \mathrm{H} \\
5.0 \mathrm{E}-06 \mathrm{H} \\
8 \mathrm{H} \\
1.0 \mathrm{E}-05 \mathrm{H} \\
8 \mathrm{H} \\
1.0 \mathrm{E}-06 \mathrm{H} \\
8 \mathrm{H} \\
1.0 \mathrm{E}-05 \mathrm{H} \\
168 \mathrm{H} \\
5.0 \mathrm{E}-06 \mathrm{H} \\
86 \mathrm{H} \\
5.0 \mathrm{E}-07 \mathrm{H} \\
86 \mathrm{H} \\
5.0 \mathrm{E}-07 \mathrm{H} \\
86 \mathrm{H} \\
5.0 \mathrm{E}-07 \mathrm{H} \\
86 \mathrm{H} \\
5.0 \mathrm{E}-07 \mathrm{H} \\
86 \mathrm{H} \\
5.0 \mathrm{E}-05 \mathrm{H} \\
1 \mathrm{Q} \\
7.5 \mathrm{E}-07 \mathrm{H} \\
86 \mathrm{H} \\
5.0 \mathrm{E}-05 \mathrm{H} \\
1 \mathrm{Q} \\
7.5 \mathrm{E}-07 \mathrm{H} \\
86 \mathrm{H} \\
5.0 \mathrm{E}-05 \mathrm{H} \\
.5 \mathrm{Y} \\
7.5 \mathrm{E}-07 \mathrm{H} \\
86 \mathrm{H} \\
5.0 \mathrm{E}-05 \mathrm{H} \\
1 \mathrm{Q} \\
7.5 \mathrm{E}-07 \mathrm{H} \\
86 \mathrm{H} \\
5.0 \mathrm{E}-05 \mathrm{H} \\
.5 \mathrm{Y} \\
7.5 \mathrm{E}-07 \mathrm{H} \\
86 \mathrm{H} \\
5.0 \mathrm{E}-05 \mathrm{H} \\
.5 \mathrm{Y} \\
7.5 \mathrm{E}-07 \mathrm{H}\end{array}$ & $\begin{array}{l}4.00 \mathrm{E}-05 \\
4.00 \mathrm{E}-05 \\
1.07 \mathrm{E}-04 \\
1.07 \mathrm{E}-04 \\
4.00 \mathrm{E}-05 \\
4.00 \mathrm{E}-06 \\
4.00 \mathrm{E}-05 \\
4.20 \mathrm{E}-04 \\
2.15 \mathrm{E}-05 \\
2.15 \mathrm{E}-05 \\
2.15 \mathrm{E}-05 \\
2.15 \mathrm{E}-05 \\
2.15 \mathrm{E}-03 \\
1.63 \mathrm{E}-03 \\
2.15 \mathrm{E}-03 \\
1.63 \mathrm{E}-03 \\
2.15 \mathrm{E}-03 \\
1.64 \mathrm{E}-03 \\
2.15 \mathrm{E}-03 \\
1.63 \mathrm{E}-03 \\
2.15 \mathrm{E}-03 \\
1.64 \mathrm{E}-03 \\
2.15 \mathrm{E}-03 \\
1.64 \mathrm{E}-03\end{array}$ & $\begin{array}{l}\text { COOLING TOWER RADIATION MONITOR FAILS } \\
\text { PROCESS CONDENSATE IINE RADIATION } \\
\text { MONITOR FAIIS } \\
\text { SCVC O2 concentration analyzer } 3405 \\
\text { fails low } \\
\text { SCVC O2 concentration analyzer } 3409 \\
\text { fails low } \\
\text { OXYGEN ANALYZING ELEMENT } 6852 \text { FAILS } \\
\text { COMMON CAUSE FAILURE OF OXYGEN } \\
\text { ANALYZING ELEMENTS 6852 AND 6853 } \\
\text { OXYGEN ANALYING ELEMENT 6853 FAILS } \\
\text { O2 Sensor Fails } \\
\text { CC: two analyzers in DWPF fail low - } \\
\text { batch \#1 } \\
\text { CC: two analyzers in DWPF fail low - } \\
\text { batch \#2 } \\
\text { CC: two analyzers in DWPF fail low } \\
\text { CC: two analyzers at tank farm fail - } \\
\text { false low reading } \\
\text { PVVH benzene analyzer } 3407 \text { element } \\
\text { fails low (LEL) } \\
\text { Air inleakage in PVVH benzene analyzer } \\
3407 \\
\text { PVVH benzene analyzer } 3408 \text { element } \\
\text { fails low (LEL) } \\
\text { Air inleakage in PVVH benzene analyzer } \\
3408 \\
\text { SRAT offgas detector \#1 analyzer } \\
\text { element fails low (LEL) } \\
\text { Air inleakage in SRAT offgas detector } \\
\# 1 \text { sample line } \\
\text { SRAT offgas detector \#2 analyzer } \\
\text { element fails low (LEL) } \\
\text { Air inleakage in SRAT offgas detector } \\
\text { \#2 sample line } \\
\text { SME LFL analyzer } 8797 \text { fails low } \\
\text { Air inleakage. in SME detector } 8797 \\
\text { sample line } \\
\text { SME hydrogen detector } 2 \text { (8798) fails } \\
\text { low inleakage in SME detector } 8798 \\
\text { Air ing } \\
\text { sample line }\end{array}$ & $\begin{array}{l}\text { a: } 8 \text { hours to repair } \\
\text { a: } 8 \text { hours to repair } \\
\text { checked once/cycle } 43 \\
\text { checked once/cycle (43 } \\
\text { a: } 8 \text { hours to repair } \\
\text { a: } 8 \text { hrs to repair } \\
\text { a: } 8 \text { hours to repair } \\
\text { osR } 3 \text { 1.2, sR } 4.1 .2 .2 \\
\text { functional test } \\
\text { a: functionally tested } \\
\text { a: functionally tested } \\
\text { a: functionally tested } \\
\text { a: functionally tested } \\
\text { a: checked every batch } \\
\text { a: checked twice/year } \\
\text { a: checked every batch } \\
\text { a:checked twice/year } \\
\text { a: checked every batch } \\
\text { a: checked twice/year } \\
\text { a: checked every batch } \\
\text { a:checked twice/year } \\
\text { a: checked every batch } \\
\text { a: checked twice/year } \\
\text { a: checked every batch } \\
\text { a: checked twice/year }\end{array}$ & $\begin{array}{l}\text { hrs) } \\
\text { hrs) } \\
\text { weekly } \\
\text { each batch } \\
\text { each batch } \\
\text { each batch } \\
\text { each batch }\end{array}$ & \\
\hline
\end{tabular}


Basic Event Report for I: \CAFTA \DATA IMASTER.BE 11/11/94 11:42 AM (CONT.)

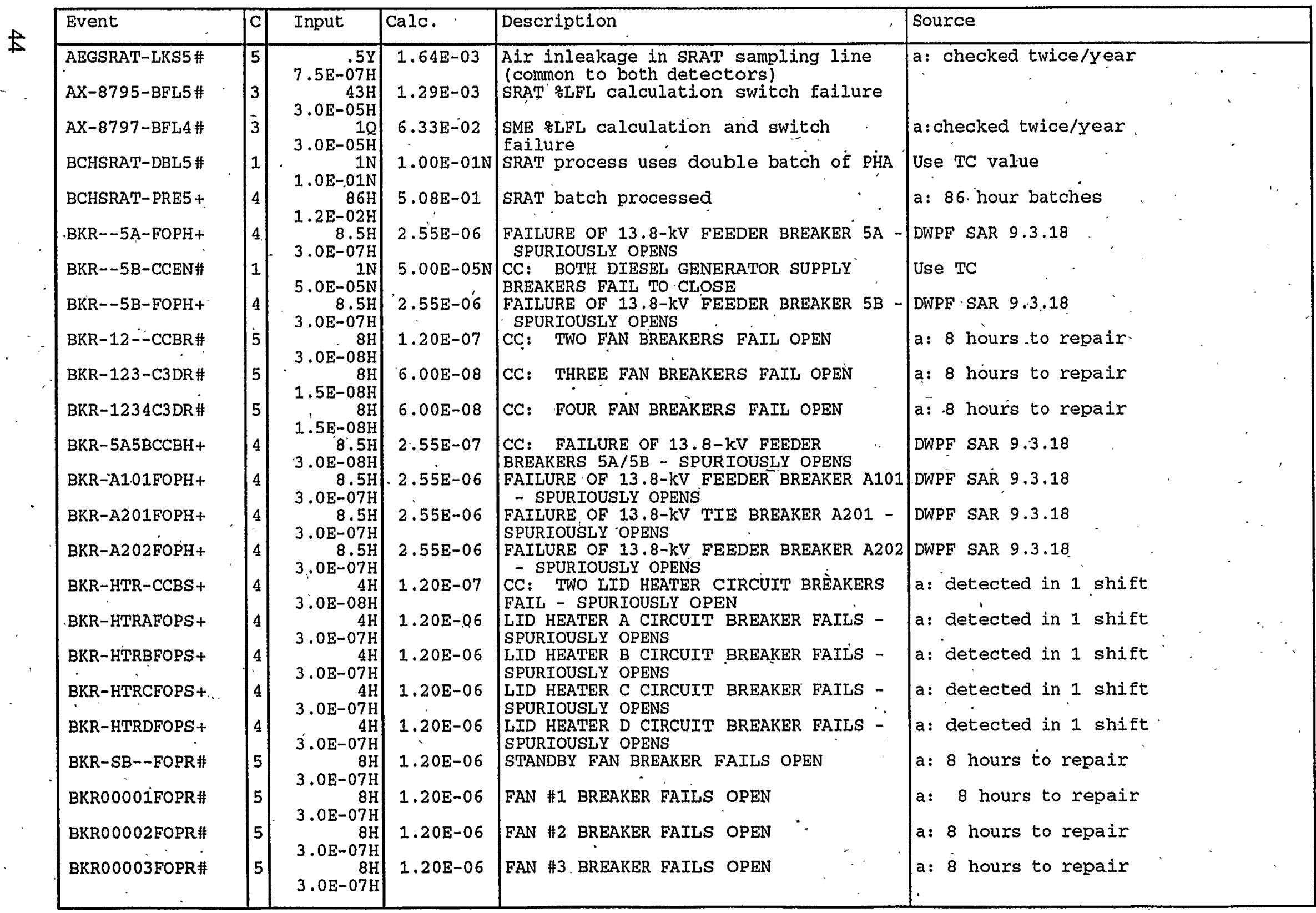


Basic Event Report for I: ICAFTAIDATAlMASTER.BE 11/11/94 11:42 AM (CONT.)

\begin{tabular}{|c|c|c|c|c|c|}
\hline Event & C & Input & Calc. & Description & Source \\
\hline $\mathrm{BKI}$ & 3 & $\begin{array}{r}8.5 \mathrm{H} \\
3.0 \mathrm{E}-08 \mathrm{H}\end{array}$ & $55 \mathrm{E}-07$ & $\begin{array}{l}\text { CC: BOTH DIESEL GENERATOR SUPPLY } \\
\text { BREAKERS TRANSFER OPEN }\end{array}$ & DWPF SAR 9.3 .18 \\
\hline $\mathrm{CBH}+$ & 4 & $\begin{array}{r}8.5 \mathrm{H} \\
3.0 \mathrm{E}-08 \mathrm{H}\end{array}$ & $2.55 E-07$ & $\begin{array}{l}\text { CC: FAILURE OF } 13.8-\mathrm{kV} \text { FEEDER } \\
\text { BREAKERS A101/A202,- SPURIOUSLY OPEN }\end{array}$ & DWPF SAR 9.3.18 \\
\hline BKRB102BFTON\# & 1 & $5.0 E-04 N$ & $5.00 \mathrm{E}-04 \mathrm{~N}$ & $\begin{array}{l}\text { NORMAL POWER BREAKER B10-2B FAILS TO } \\
\text { OPEN }\end{array}$ & Use TC \\
\hline BKRB105BFOPN\# & 3 & $\begin{array}{r}8.5 \mathrm{H} \\
3.0 \mathrm{E}-07 \mathrm{H}\end{array}$ & $2.55 \mathrm{E}-06$ & SUPPLY BREAKER B10-5B TRANSFERS OPEN & DWPF SAR 9.3 .18 \\
\hline BKRB1 & 1 & $5.0 \mathrm{E}-04 \mathrm{~N}$ & $5.00 \mathrm{E}-04 \mathrm{~N}$ & SUPPLY BREAKER B10-5B FAILS TO CLOSE & Use TC \\
\hline BKRB9-2BFTON\# & 1 & $5 . \mathrm{OE}$ & $5.00 \mathrm{E}-04 \mathrm{~N}$ & $\begin{array}{l}\text { NORMAL POWER BREAKER B9-2B FAILS TO } \\
\text { OPEN }\end{array}$ & Use TC \\
\hline BKRB9-5BFOPN\# & 3 & $\begin{array}{r}8.5 \mathrm{H} \\
\text { 3. } . \mathrm{OE}-07 \mathrm{H}\end{array}$ & $2.55 \mathrm{E}-06$ & SUPPLY BREAKER B9-5B TRANSFERS OPEN & DWPF SAR 9.3 .18 \\
\hline TCN\# & 1 & 5. $0 \mathrm{E}-0 \frac{1 \mathrm{~N}}{4 \mathrm{~N}}$ & $5.00 \mathrm{E}-04 \mathrm{~N}$ & SUPPLY BREAKER B9-5B FAILS TO CLOSE & Use TC \\
\hline BKRB9B10CCDN\# & 1 & 5. $0 \mathrm{E}-05 \mathrm{~N}$ & $5.00 \mathrm{E}-05 \mathrm{~N}$ & $\begin{array}{l}\text { CC: NORMAL POWER BREAKERS B9-2B AND } \\
\text { B10-2B FAIL TO OPEN }\end{array}$ & Use TC \\
\hline BKRSRATSFOPD\# & 3 & $\begin{array}{r}8 \mathrm{H} \\
3.0 \mathrm{E}-07 \mathrm{H}\end{array}$ & $2.40 \mathrm{E}-06$ & $\begin{array}{l}\text { SRAT offgas sample pump-circuit } \\
\text { breaker fails open }\end{array}$ & a: closed in 8 hours \\
\hline BNZ-XFR-PREP+ & 4 & $2.3 \mathrm{E}-02 \mathrm{H}$ & $2.25 \mathrm{E}-02$ & Benzene transferred to OWST & Transfer lasts one hour \\
\hline BNZOEHOTPREP\# & 3 & $2.3 \mathrm{E}-02 \mathrm{H}$ & $4.50 \mathrm{E}-02$ & Benzene being boiled off in $\mathrm{OE}$ & a: 2 hours per batch \\
\hline BNZPRHOTPREP\# & 3 & $2.3 \mathrm{E}-\mathrm{16} \mathrm{H}$ & $3.08 \mathrm{E}-01$ & Hot benzene present. in PR & a: 16 hours per batch \\
\hline BOTCO2B-REP1\# & 5 & $6.7 \mathrm{E}-0 \frac{1 \mathrm{M}}{5 \mathrm{H}}$ & $2.37 \mathrm{E}-02$ & Bottles were used since last refilling & a: Refilled in 1 month \\
\hline BOTCO2B-T\&M1\# & 3 & $\begin{array}{l}7 \dot{D} \\
1 Y\end{array}$ & & $\begin{array}{l}\text { SPC backup CO2 bottles isolated for } \\
\text { maintenance }\end{array}$ & $\begin{array}{l}\text { a: isolated annually, error corrected } \\
\text { in } 1 \text { week }\end{array}$ \\
\hline BOTCO2B1T\&M1\# & 3 & $\begin{array}{l}7 D \\
1 Y\end{array}$ & $1.90 \mathrm{E}-02$ & $\begin{array}{l}\text { SPC backup } \mathrm{CO} 2 \text { bottle } 1 \text { isolated for } \\
\text { maintenance }\end{array}$ & $\begin{array}{l}\text { a: isolated annually, error corrected } \\
\text { in } 1 \text { week }\end{array}$ \\
\hline BOTCO2B2T\&M1\# & 3 & $\begin{array}{l}7 \mathrm{D} \\
1 \mathrm{Y}\end{array}$ & $1.90 \mathrm{E}-0.2$ & $\begin{array}{l}\text { SPC backup } \mathrm{CO} 2 \text { bottle } 2 \text { isolated for } \\
\text { maintenance }\end{array}$ & $\begin{array}{l}\text { a: isolated annually; error corrected } \\
\text { in } 1 \text { week }\end{array}$ \\
\hline BOTCO2B3 T\&M1\# & 3 & $\begin{array}{l}7 D \\
1 Y\end{array}$ & $1.90 \mathrm{E}-02$ & $\begin{array}{l}\text { SPC backup } \mathrm{CO} 2 \text { bottle } 3 \text { isolated for } \\
\text { maintenance }\end{array}$ & $\begin{array}{l}\text { a: isolated annually, error corrected } \\
\text { in } 1 \text { week }\end{array}$ \\
\hline BOTCO2B $4 \mathrm{~T} \& \mathrm{M} 1 \#$ & 3 & $\begin{array}{l}7 \mathrm{D} \\
1 \mathrm{Y}\end{array}$ & $1.90 \mathrm{E}-02$ & $\begin{array}{l}\text { SPC backup } \mathrm{CO} 2 \text { bottle } 4 \text { isolated for } \\
\text { maintenance }\end{array}$ & $\begin{array}{l}\text { a: isolated annually, error corrected } \\
\text { in } 1 \text {.week. }\end{array}$ \\
\hline BUS--A1-BFLH+ & 4 & $\begin{array}{r}8.5 \mathrm{H} \\
1.0 \mathrm{E}-07 \mathrm{H}\end{array}$ & $8.50 \mathrm{E}-07$ & FAILURE OF $13.8-\mathrm{kV}$ BUS AI & DWPF SAR 9.3.18. \\
\hline BUS--A1-UNAH\# & 1 & $3.8 \mathrm{E}-02 \mathrm{~N}$ & $3.80 \mathrm{E}-02 \mathrm{~N}$ & $\begin{array}{l}\text { BUS A1 UNAVAILABLE DUE TO } \\
\text { TESTING/MAINTENANCE }\end{array}$ & Use TC \\
\hline BUS--A2-BFLH+ & 4 & $\begin{array}{r}8.5 \mathrm{H} \\
1.0 \mathrm{E}-07 \mathrm{H}\end{array}$ & $8.50 \mathrm{E}-07$ & FAILURE OF $13.8-\mathrm{kV}$ BUS A2 & DWPF SAR 9.3 .18 \\
\hline $\begin{array}{l}\text { BUSA1-A2CCAH+ } \\
\text { CC2-AIR-DRY1+ }\end{array}$ & 4 & $\begin{array}{r}8.5 \mathrm{H} \\
1.0 \mathrm{E}-08 \mathrm{H} \\
8 \mathrm{H} \\
5.0 \mathrm{E}-08 \mathrm{H}\end{array}$ & $\begin{array}{l}8.50 \mathrm{E}-08 \\
4.00 \mathrm{E}-07\end{array}$ & $\begin{array}{l}\text { CC: FAILURE OF } 13.8-\mathrm{kV} \text { BUSSES A1 AND } \\
\text { A2 } \\
\text { CC: both air dryers fàil }\end{array}$ & $\begin{array}{l}\text { DWPF SAR } 9.3 .18 \\
\text { a: } 0.1 \text { X single rate, repiared in } 8 \\
\text { hours }\end{array}$ \\
\hline
\end{tabular}


Basic Event Report for I: \CAFTA \DATA IMASTER.BE 11/11/94 11:42 AM (CONT.)

\begin{tabular}{|c|c|c|c|c|c|}
\hline Event & $\mathrm{C}$ & Input & Calc. & Description & Source \\
\hline $\begin{array}{l}\mathrm{CC} 2-\mathrm{AIR}-\mathrm{PT}-1 \# \\
\mathrm{CC} 2 \text {-CPC-CMP2+ } \\
\text { CC2-MFT-FE-7\# } \\
\text { CC2-MFT-FE-7+ } \\
\text { CC2-PSW-PMPG\# } \\
\text { CC2-SME-H2-1\# } \\
\text { CC2-SPC-PCV1\# } \\
\text { CC2-VAP-PSV1+ } \\
\text { CC2BENZ-AEG1\# } \\
\text { CC2CHILLCHL1+ } \\
\text { CC2CPCBPCV-J\# } \\
\text { CC2CPCBPPCVJ\# } \\
\text { CC2CTW--FAN1+ } \\
\text { CC2DEEP-DG-1\# } \\
\text { CC2LPPN2CV-J\# } \\
\text { CC2LPPN2PCVJ\# } \\
\text { CC2LPPV'-FAN1+ } \\
\text { CC2MAINTHCV1\# } \\
\text { CC2OWST-PCVN\# } \\
\text { CC2OWST-PT-1\# } \\
\text { CC2PRBT-FE-7\# } \\
\text { CC2PRBT-FE-7+ } \\
\text { CC2PURGESPCK\# } \\
\text { CC2PVVH-FAN1+ }\end{array}$ & $\mid \begin{array}{l}4 \\
5\end{array}$ & $\begin{array}{r}1.08 \mathrm{H} \\
1.07 \mathrm{H} \\
7 \mathrm{D} \\
5.0 \mathrm{E}-06 \mathrm{H} \\
1 \mathrm{M} \\
3.0 \mathrm{E}-07 \mathrm{H} \\
1 \mathrm{M} \\
3.0 \mathrm{E}-07 \mathrm{H} \\
8 \mathrm{H} \\
3.0 \mathrm{E}-06 \mathrm{H} \\
1 \mathrm{M} \\
5.0 \mathrm{E}-06 \mathrm{H} \\
1 \mathrm{~N} \\
3.0 \mathrm{E}-04 \mathrm{~N} \\
4 \mathrm{H} \\
1.0 \mathrm{E}-06 \mathrm{H} \\
1 \mathrm{M} \\
5.0 \mathrm{E}-06 \mathrm{H} \\
8 \mathrm{H} \\
5.0 \mathrm{E}-06 \mathrm{H} \\
1 \mathrm{~N} \\
5.0 \mathrm{E}-05 \mathrm{~N} \\
1 \mathrm{~N} \\
3.0 \mathrm{E}-04 \mathrm{~N} \\
3.0 \mathrm{E}-06 \mathrm{H} \\
1 \mathrm{~N} \\
3.0 \mathrm{E}-03 \mathrm{~N} \\
.0 \mathrm{~N} \\
5.0 \mathrm{E}-05 \mathrm{~N} \\
1 \mathrm{~N} \\
3.0 \mathrm{E}-04 \mathrm{~N} \\
1 \mathrm{H} \\
3.0 \mathrm{E}-06 \mathrm{H} \\
1 \mathrm{M} \\
5.0 \mathrm{E}-08 \mathrm{H} \\
1 \mathrm{~N} \\
3.0 \mathrm{E}-04 \mathrm{~N} \\
1.0 \mathrm{E}-07 \mathrm{H} \\
3.0 \mathrm{E}-07 \mathrm{H} \\
1 \mathrm{M} \\
3.0 \mathrm{E}-07 \mathrm{H} \\
1 \mathrm{~N} \\
1.0 \mathrm{E}-04 \mathrm{~N} \\
8 \mathrm{H} \\
3.0 \mathrm{E}-06 \mathrm{H}\end{array}$ & $\begin{array}{l}2.40 \mathrm{E}-06 \\
8.39 \mathrm{E}-04 \\
2.16 \mathrm{E}-04 \\
2.16 \mathrm{E}-04 \\
2.40 \mathrm{E}-05 \\
1.80 \mathrm{E}-03 \\
3.00 \mathrm{E}-04 \mathrm{~N} \\
4.00 \mathrm{E}-06 \\
1.80 \mathrm{E}-03 \\
4.00 \mathrm{E}-05 \\
5.00 \mathrm{E}-05 \mathrm{~N} \\
3.00 \mathrm{E}-04 \mathrm{~N} \\
5.04 \mathrm{E}-04 \\
3.00 \mathrm{E}-03 \mathrm{~N} \\
5.00 \mathrm{E}-05 \mathrm{~N} \\
3.00 \mathrm{E}-04 \mathrm{~N} \\
3.00 \mathrm{E}-0.6 \\
1.80 \mathrm{E}-05 \\
3.00 \mathrm{E}-04 \mathrm{~N} \\
4.38 \mathrm{E}-04 \\
2.16 \mathrm{E}-04 \\
2.16 \mathrm{E}-04 \\
1.00 \mathrm{E}-04 \mathrm{~N} \\
2.40 \mathrm{E}-05\end{array}$ & 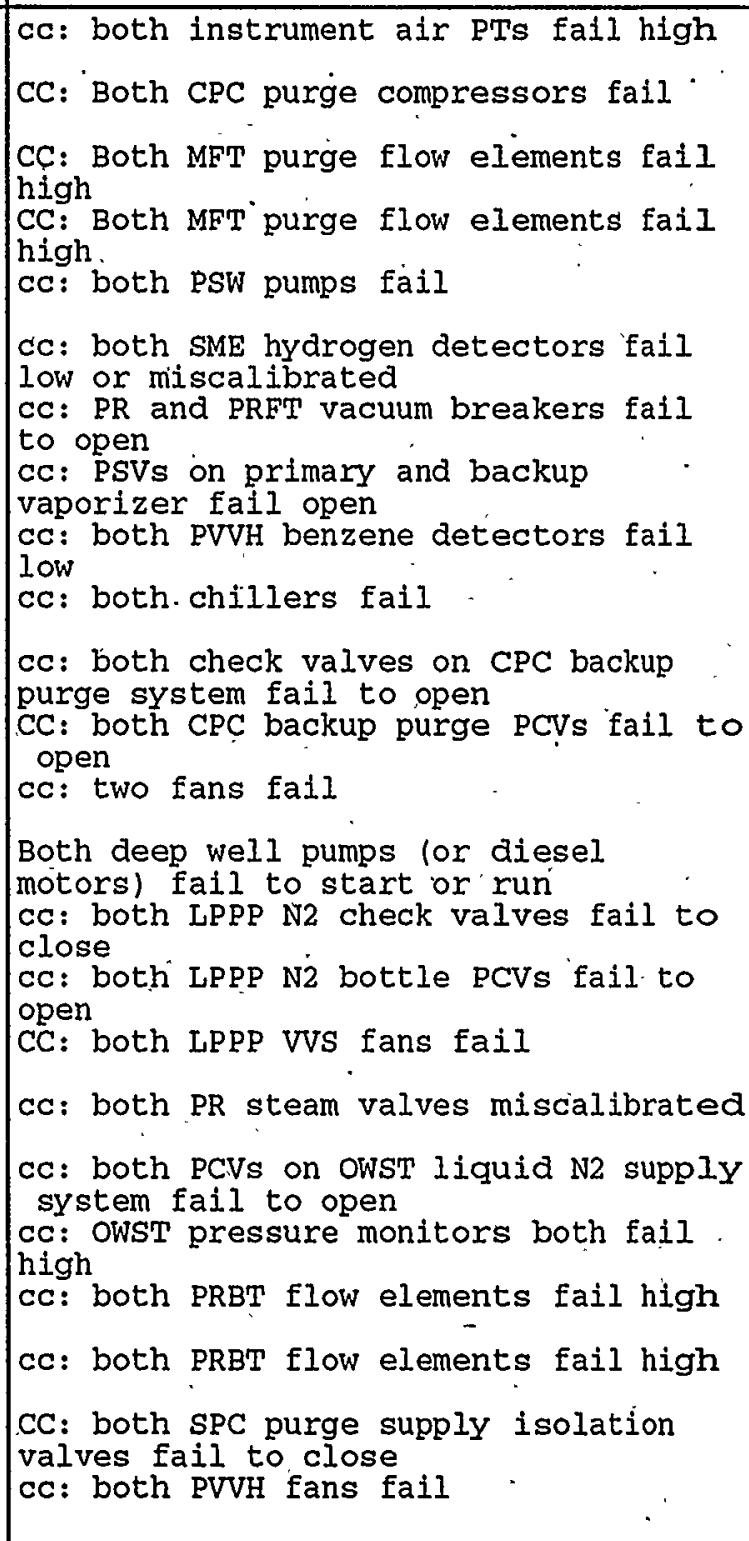 & 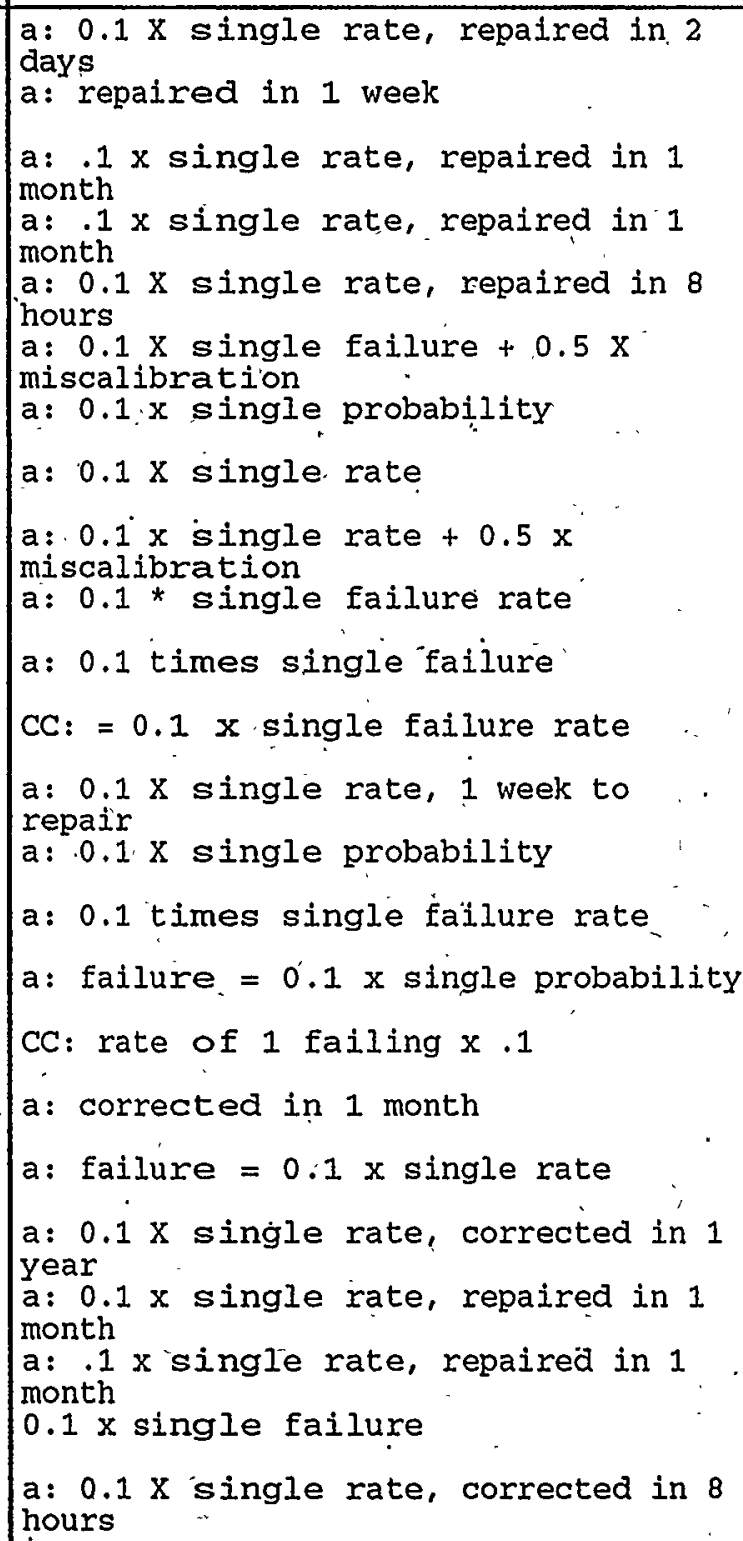 \\
\hline
\end{tabular}


Basic Event Report for I: ICAFTAIDATA IMASTER.BE 11/11/94 11:42 AM (CONT.)

\begin{tabular}{|c|c|c|c|c|c|}
\hline Event & $\mathrm{C}$ & aput & IC. & escription & Source \\
\hline $\begin{array}{l}\text { CC2SPCN2RTH1\# } \\
\text { CC2SPCN2SV-N\# } \\
\text { CC2SPCN3 PCVN\# } \\
\text { CC2SPCP-PT-K\# } \\
\text { CC2SPCPPLSL1\# } \\
\text { CC2SPCV-MOVK\# } \\
\text { CC2SPTN2LINJ\# } \\
\text { CC2SRAT-LFL5\# } \\
\text { CC2SRAT-SMP6\# } \\
\text { CC2STEAMHCV1+ } \\
\text { CC2STEAMPMPG+ } \\
\text { CC3-CTW-PMP1+ } \\
\text { CC3-SME-FSL4\# } \\
\text { CC3-SPC-FE-1\# } \\
\text { CC3CO2B-REG1\# } \\
\text { CC3CO2B-RMH1\# } \\
\text { CC3MAINTFSL1\# } \\
\text { CC3SRAT-FSLD\# } \\
\text { CHLBACK-FTRE\# }\end{array}$ & 3 & $\begin{array}{r}1 \mathrm{M} \\
3.0 \mathrm{E}-07 \mathrm{H} \\
1 \mathrm{~N} \\
3.0 \mathrm{E}-04 \mathrm{~N} \\
4.3 \mathrm{E}-02 \mathrm{~N} \\
5.0 \mathrm{E}-03 \mathrm{~N} \\
1 \mathrm{~N} \\
5.0 \mathrm{E}-04 \mathrm{~N} \\
1 \mathrm{~N} \\
3.0 \mathrm{E}-04 \mathrm{~N} \\
.12 \mathrm{~N} \\
3.0 \mathrm{E}-03 \mathrm{~N} \\
1 \mathrm{~N} \\
1.0 \mathrm{E}-04 \mathrm{~N} \\
1 \mathrm{~N} \\
3.0 \mathrm{E}-04 \mathrm{~N} \\
7 \mathrm{D} \\
1.0 \mathrm{E}-07 \mathrm{H} \\
1 \mathrm{~N} \\
3.0 \mathrm{E}-03 \mathrm{~N} \\
1 \mathrm{~N} \\
3.0 \mathrm{E}-04 \mathrm{~N} \\
1 \mathrm{~N} \\
4.1 \mathrm{E}-04 \mathrm{~N} \\
1 \mathrm{M} \\
5.0 \mathrm{E}-06 \mathrm{H} \\
1 \mathrm{~N} \\
3.0 \mathrm{E}-05 \mathrm{~N} \\
4 \mathrm{H} \\
5.0 \mathrm{E}-08 \mathrm{H} \\
8 \mathrm{H} \\
3.0 \mathrm{E}-06 \mathrm{H} \\
4 \mathrm{H} \\
1.5 \mathrm{E}-06 \mathrm{H} \\
1 \mathrm{~N} \\
1.1 \mathrm{E}-04 \mathrm{~N} \\
7 \mathrm{D} \\
1.5 \mathrm{E}-07 \mathrm{H} \\
48 \mathrm{H} \\
1.5 \mathrm{E}-07 \mathrm{H}-04 \mathrm{~N} \\
1 \mathrm{~N} \\
8 \mathrm{H} \\
2.5 \mathrm{E}-03 \mathrm{~N} \\
.2 \mathrm{~N} \\
1.5 \mathrm{E}-03 \mathrm{~N} \\
1 \mathrm{~N} \\
\end{array}$ & $\begin{array}{l}3.00 \mathrm{E}-04 \mathrm{~N} \\
2.15 \mathrm{E}-04 \mathrm{~N} \\
5.00 \mathrm{E}-04 \mathrm{~N} \\
3.00 \mathrm{E}-04 \mathrm{~N} \\
3.60 \mathrm{E}-04 \mathrm{~N} \\
1.00 \mathrm{E}-04 \mathrm{~N} \\
3.00 \mathrm{E}-04 \mathrm{~N} \\
8.40 \mathrm{E}-06 \\
3.00 \mathrm{E}-03 \mathrm{~N} \\
3.00 \mathrm{E}-04 \mathrm{~N} \\
4.10 \mathrm{E}-04 \mathrm{~N} \\
1.80 \mathrm{E}-03 \\
3.00 \mathrm{E}-05 \mathrm{~N} \\
2.00 \mathrm{E}-07 \\
2.40 \mathrm{E}-05 \\
6.00 \mathrm{E}-06 \\
1.10 \mathrm{E}-04 \mathrm{~N} \\
2.52 \mathrm{E}-05 \\
3.60 \mathrm{E}-06 \\
2.50 \mathrm{E}-03 \mathrm{~N} \\
3.00 \mathrm{E}-04 \mathrm{~N} \\
1.10 \mathrm{E}-04 \mathrm{~N} \\
4.00 \mathrm{E}-04\end{array}$ & 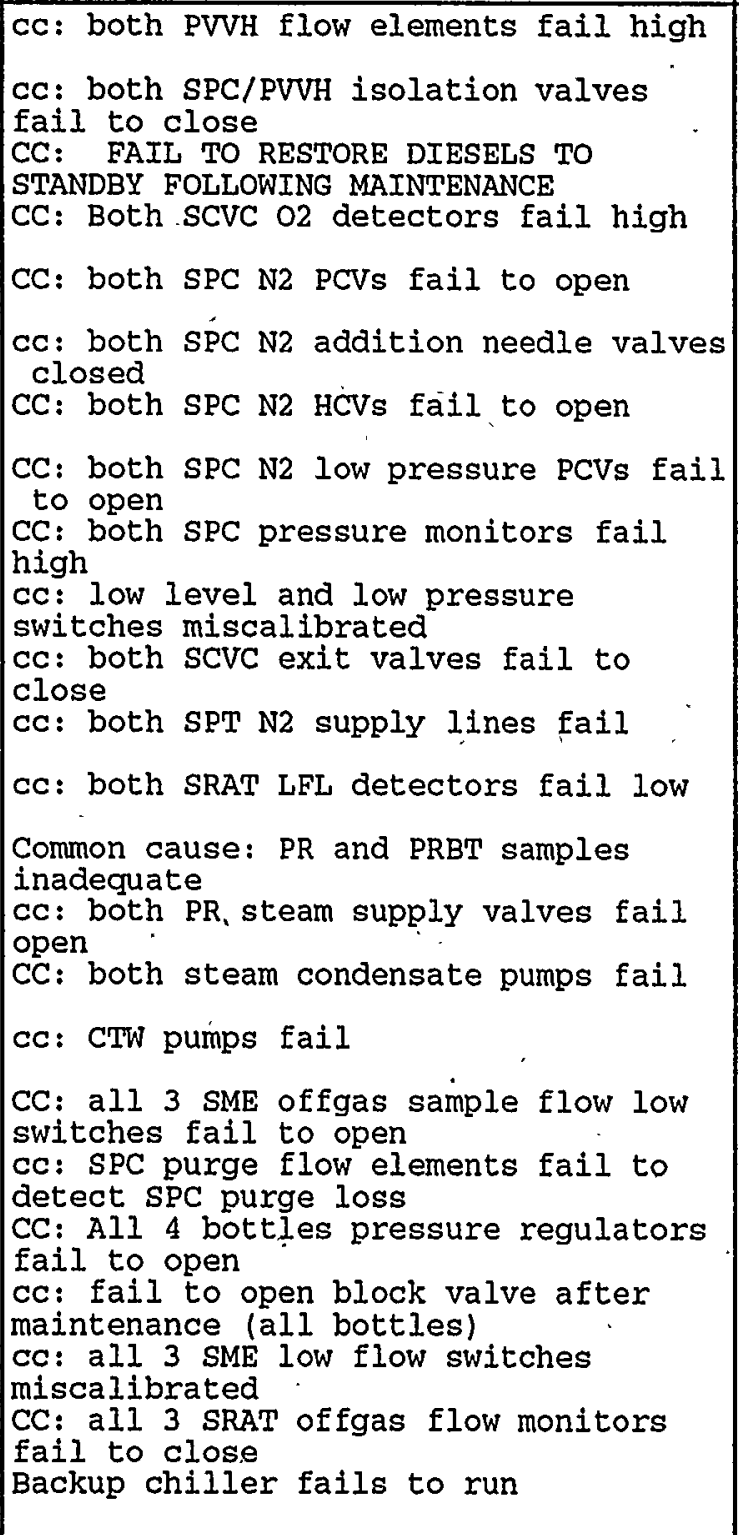 & 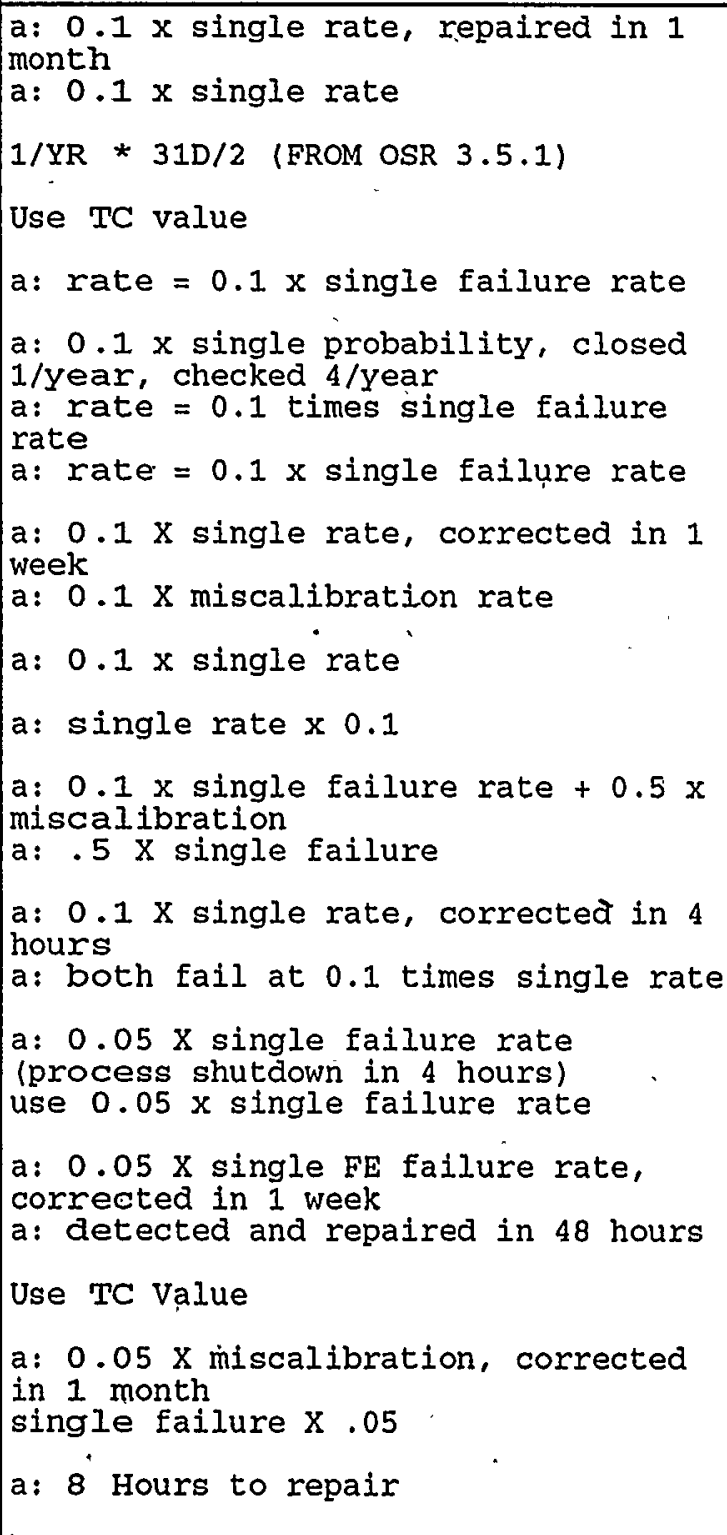 \\
\hline
\end{tabular}


Basic Event Report for I: \CAFTA IDATA IMASTER.BE 11/11/94 11:42 AM (CONT.)

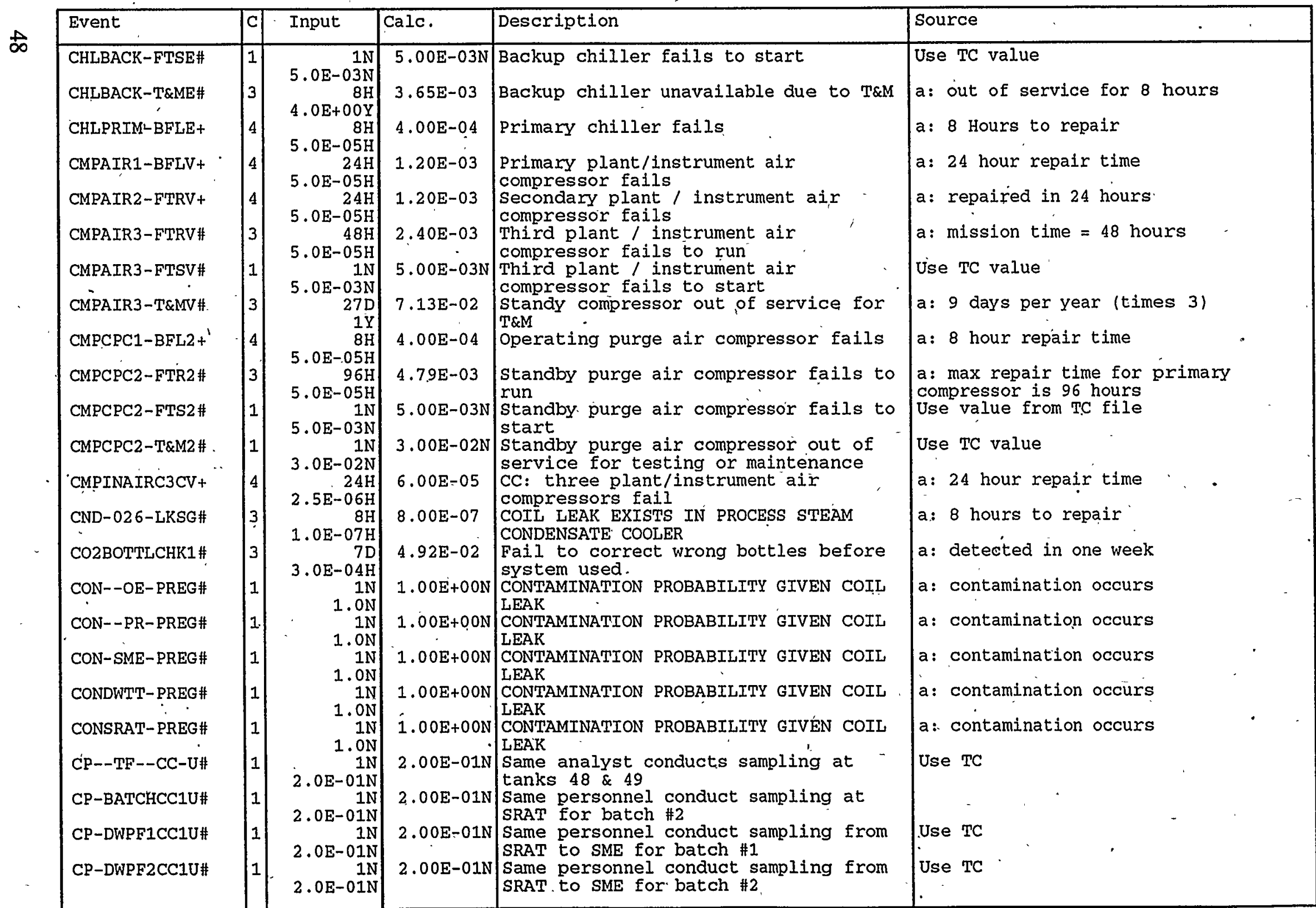




\begin{tabular}{|c|c|c|c|c|c|}
\hline Event & $\mathrm{C}$ & Input & Calc. & Description & Source \\
\hline $\begin{array}{l}\text { CP-DWPFOCC1U\# } \\
\text { CV-2362-FTOF\# } \\
\text { CV-9145-FTOO\# } \\
\text { CV-9147-FTOO\# } \\
\text { CV-CPCB1FTOJ\# } \\
\text { CV-CPCB2 FTOJ\# } \\
\text { CV-CPCB3FTCJ\# } \\
\text { CV-DXXX-FTCJ\# } \\
\text { CV-DXXY-FTCJ\# } \\
\text { CV-MAIN-FCLG+ } \\
\text { CV-OE---FTCJ\# } \\
\text { CV-OECT-FTCJ\# } \\
\text { CV-PMP1-FCLG+ } \\
\text { CV-PMP2-FTOG\# } \\
\text { CV-PR---FTOJ\# } \\
\text { CV-PR1--FTCJ\# } \\
\text { CV-PR2--FTCJ\# } \\
\text { CV-PRFT-FTCJ\# } \\
\text { CV-PRFT-FTOJ\# } \\
\text { CYLOWST-OUTP\# } \\
\text { DCS-----BFL2\# } \\
\text { DCS--EP-BELE\# } \\
\text { DG-1/200CCCN\# } \\
\text { DG-1/200CCDN\# }\end{array}$ & 1 & $\begin{array}{r}1 \mathrm{~N} \\
2.0 \mathrm{E}-01 \mathrm{~N} \\
1 \mathrm{~N} \\
5.0 \mathrm{E}-05 \mathrm{~N} \\
1 \mathrm{~N} \\
5.0 \mathrm{E}-05 \mathrm{~N} \\
1 \mathrm{~N} \\
5.0 \mathrm{E}-05 \mathrm{~N} \\
1 \mathrm{~N} \\
5.0 \mathrm{E}-04 \mathrm{~N} \\
1 \mathrm{~N} \\
5.0 \mathrm{E}-04 \mathrm{~N} \\
1 \mathrm{~N} \\
5.0 \mathrm{E}-04 \mathrm{~N} \\
1 \mathrm{~N} \\
5.0 \mathrm{E}-04 \mathrm{~N} \\
1 \mathrm{~N} \\
5.0 \mathrm{E}-04 \mathrm{~N} \\
8 \mathrm{H} \\
5.0 \mathrm{E}-07 \mathrm{H} \\
1 \mathrm{~N} \\
5.0 \mathrm{E}-04 \mathrm{~N} \\
1 \mathrm{~N} \\
5.0 \mathrm{E}-04 \mathrm{~N} \\
8 \mathrm{H} \\
5.0 \mathrm{E}-07 \mathrm{H} \\
1 \mathrm{~N} \\
5.0 \mathrm{E}-05 \mathrm{~N} \\
1 \mathrm{~N} \\
5.0 \mathrm{E}-04 \mathrm{~N} \\
1 \mathrm{~N} \\
5.0 \mathrm{E}-04 \mathrm{~N} \\
1 \mathrm{~N} \\
5.0 \mathrm{E}-04 \mathrm{~N} \\
1 \mathrm{~N} \\
5.0 \mathrm{E}-04 \mathrm{~N} \\
1 \mathrm{~N} \\
5.0 \mathrm{E}-04 \mathrm{~N} \\
1 \mathrm{~N} \\
3.6 \mathrm{E}-01 \mathrm{~N} \\
8 \mathrm{H} \\
3.0 \mathrm{E}-03 \mathrm{~N} \\
8.5 \mathrm{H} \\
14 \mathrm{D} \\
3.0 \mathrm{E}-06 \mathrm{H} \\
1 \mathrm{~N} \\
5 \mathrm{H}\end{array}$ & $\begin{array}{l}2.00 \mathrm{E}-01 \mathrm{~N} \\
5.00 \mathrm{E}-05 \mathrm{~N} \\
5.00 \mathrm{E}-05 \mathrm{~N} \\
5.00 \mathrm{E}-05 \mathrm{~N} \\
5.00 \mathrm{E}-04 \mathrm{~N} \\
5.00 \mathrm{E}-04 \mathrm{~N} \\
5.00 \mathrm{E}-04 \mathrm{~N} \\
5.00 \mathrm{E}-04 \mathrm{~N} \\
5.00 \mathrm{E}-04 \mathrm{~N} \\
4.00 \mathrm{E}-06 \\
5.00 \mathrm{E}-04 \mathrm{~N} \\
5.00 \mathrm{E}-04 \mathrm{~N} \\
4.00 \mathrm{E}-06 \\
5.00 \mathrm{E}-05 \mathrm{~N} \\
5.00 \mathrm{E}-04 \mathrm{~N} \\
5.00 \mathrm{E}-04 \mathrm{~N} \\
5.00 \mathrm{E}-04 \mathrm{~N} \\
5.00 \mathrm{E}-04 \mathrm{~N} \\
5.00 \mathrm{E}-04 \mathrm{~N} \\
3.60 \mathrm{E}-01 \mathrm{~N} \\
1.20 \mathrm{E}-05\end{array}$ & 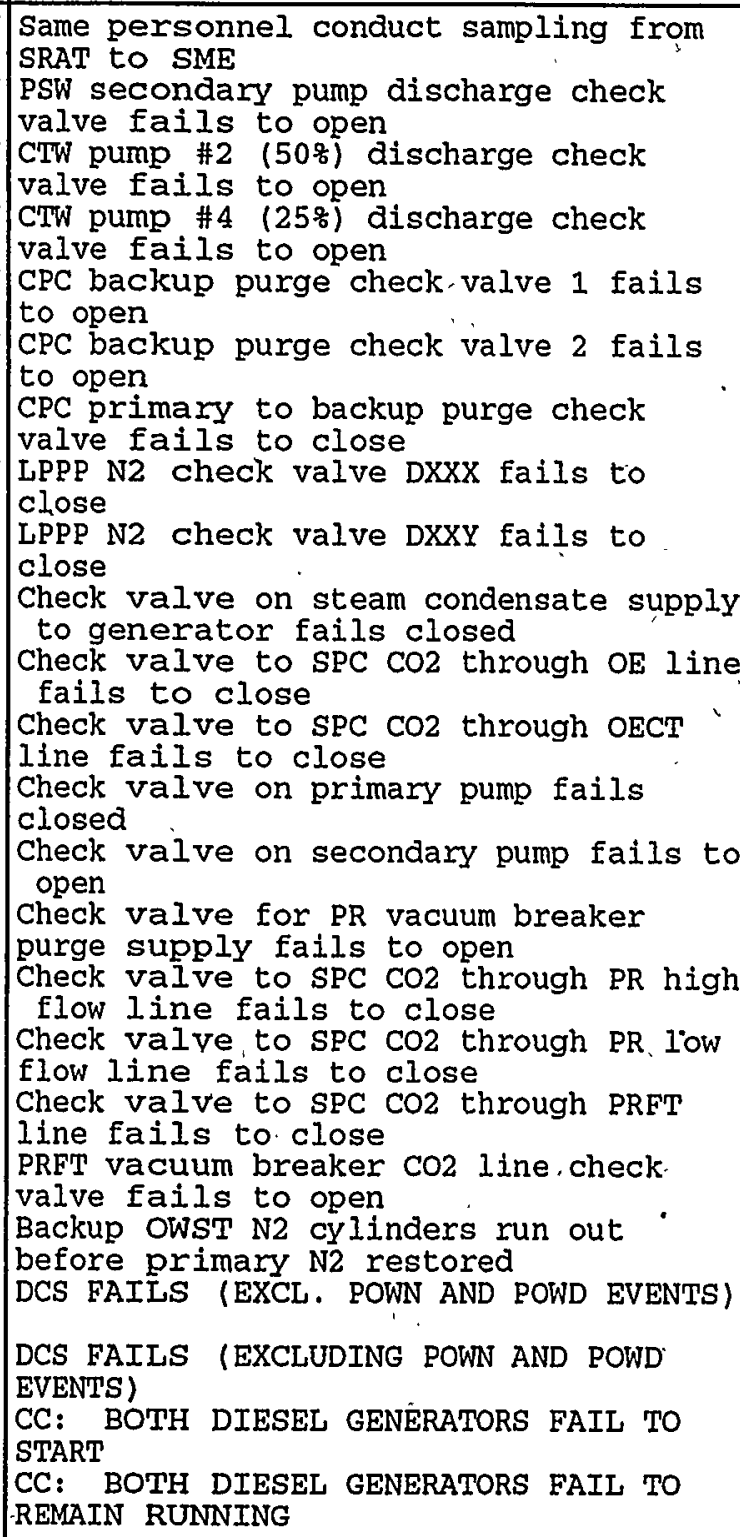 & $\begin{array}{l}\text { Use TC } \\
\text { a: } 8 \text { hour restoration time } \\
\text { Use TC value } \\
\text { Use TC value } \\
\text { Use TC value } \\
\text { Use TC value } \\
\text { Use TC value } \\
\text { Use TC value } \\
\text { Use TC value } \\
\text { a: Detected and repaired or process } \\
\text { shut down in } 8 \text { hours } \\
\text { Use TC value } \\
\text { Use TC value } \\
\text { a: Detected and repaired or process } \\
\text { shut down in } 8 \text { hours } \\
\text { Use TC value } \\
\text { Use TC value } \\
\text { Use TC value } \\
\text { Use TC value } \\
\text { Use TC value } \\
\text { Use TC value } \\
\text { a: supply capacity less than } \\
\text { restoration time } \\
\text { a: } 8 \text { hours repair time } \\
\text { a: } 14 \text { days repair time } \\
\text { Use TC } \\
\text { DwPF sAR } 9.3 .18 \\
\text { Un }\end{array}$ \\
\hline
\end{tabular}


Basic Event Report for I: \CAFTA \DATA MMASTER.BE 11/11/94 11:42 AM (CONT.)

\begin{tabular}{|c|c|c|c|c|c|}
\hline Event & C & nput & Calc. & Description & Source \\
\hline $\begin{array}{l}\text { FANO0003 FTRR\# } \\
\text { FANO0004 FTSR\# } \\
\text { FANCTW--ADJO\# } \\
\text { FANCTW1-FTRO+ } \\
\text { FANCTW2-FTRO+ } \\
\text { FANCTW3-FTRO+ } \\
\text { FANLPPV1BFLT+ } \\
\text { FANLPPV2BFLT\# } \\
\text { FANLPPV2 FTST\# } \\
\text { FANPVV1-BFLD+ } \\
\text { FANPVV2-BFLD\# } \\
\text { FANPVV2-FTSD\# } \\
\text { FANPVV2-T\&MD\# } \\
\text { FCV0683-FCL7+ } \\
\text { FCV1027-FOP9+ } \\
\text { FCV1893-ADJE+ } \\
\text { FCV1893-FTOE\# } \\
\text { FCV2044-FTC4\# } \\
\text { FCV2056-ADJI+ } \\
\text { FCV2056-FOPI+ } \\
\text { FCV2369-ADJF\# } \\
\text { FCV2369-FTOF\# }\end{array}$ & 3 & 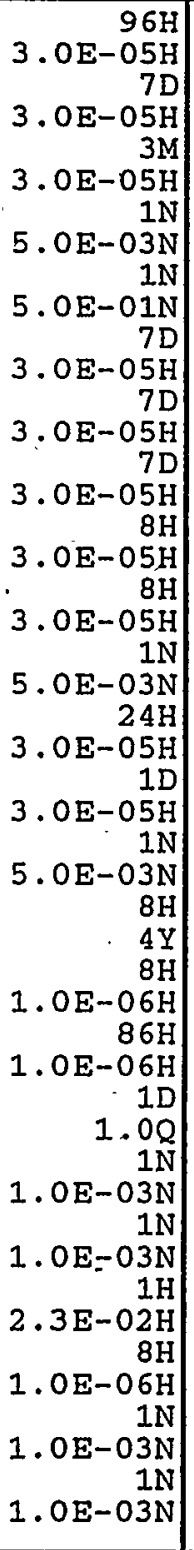 & $\begin{array}{l}2.52 \mathrm{E}-03 \\
3.17 \mathrm{E}-02 \\
5.00 \mathrm{E}-03 \mathrm{~N} \\
5.00 \mathrm{E}-01 \mathrm{~N} \\
5.01 \mathrm{E}-03 \\
5.01 \mathrm{E}-03 \\
5.01 \mathrm{E}-03 \\
2.40 \mathrm{E}-04 \\
2.40 \mathrm{E}-04 \\
5.00 \mathrm{E}-03 \mathrm{~N} \\
7.19 \mathrm{E}-04 \\
7.20 \mathrm{E}-04 \\
5.00 \mathrm{E}-03 \mathrm{~N} \\
3.65 \mathrm{E}-03 \\
8.00 \mathrm{E}-06 \\
8.60 \mathrm{E}-0.5 \\
1.09 \mathrm{E}-02 \\
1.00 \mathrm{E}-03 \mathrm{~N} \\
1.00 \mathrm{E}-03 \mathrm{~N} \\
2.25 \mathrm{E}-02 \\
8.00 \mathrm{E}-06 \\
1.00 \mathrm{E}-03 \mathrm{~N} \\
1.00 \mathrm{E}-03 \mathrm{~N} \\
.\end{array}$ & $\begin{array}{l}\text { FAN \#1 FAILS TO REMAIN RUNNING } \\
\text { FAN \#2 FAILS TO REMAIN RUNNING } \\
\text { FAN \#3 FAILS TO REMAIN RUNNING } \\
\text { STANDBY FAN FAILS TO START } \\
\text { Forced convection required in cooling } \\
\text { tower } \\
\text { CTW tower fan } 1 \text { fails } \\
\text { CTW tower fan } 2 \text { fails } \\
\text { CTW tower fan } 3 \text { fails } \\
\text { LPP VVH primary fan fails to run } \\
\text { LPP vVH secondary fan fails to run } \\
\text { LPP VVH secondary fan fails to start } \\
\text { Primary PVVH blower fails } \\
\text { PVVH backup fan fails to run } \\
\text { Standby PVVH blower fails to start } \\
\text { PVVH standby fan unavailable, due to } \\
\text { testing or maintenance fails closed } \\
\text { MFT purge supply valve fa } \\
\text { PR steam supply valve FCV-1027 fails } \\
\text { open } \\
\text { Chilled water bypass valve required to } \\
\text { open (no system flow) } \\
\text { Flow control valve for chilled water } \\
\text { bypass fails to open } \\
\text { SME formic acid flow control valve } \\
\text { fails to close } \\
\text { PR formic acid supply valve opened } \\
\text { (1/43 hours) } \\
\text { Formic acid addition valve to PR fails } \\
\text { open } \\
\text { PSW bypass valve required to be open } \\
\text { (no process flow) } \\
\text { PSW bypass valve fails to open }\end{array}$ & $\begin{array}{l}\text { a: } 96 \text { hr mission time (bounded by } \\
\text { seismic event) } \\
\text { a: } 7 \text { days to repair } \\
\text { a: } 3 \text { months to repair } \\
\text { Use TC } \\
\text { Use TC value } \\
\text { a: detected and repaired in } 1 \text { week } \\
\text { a: detected and repaired in } 1 \text { week } \\
\text { a: detected and repaired in } 1 \text { week } \\
\text { osR } 3.2 .1 \text {, sR } 4.2 .1 .1 \text {, checked } \\
\text { shiftiy } \\
\text { a: Primary fan repaired, or shutdown } \\
\text { within } 8 \text { hours } \\
\text { Use TC value } \\
\text { a: } 24 \text { hours to repair } \\
\text { a: } 24 \text { hour mission time } \\
\text { Use TC value } \\
\text { a: out } 8 \text { hours per quarter } \\
\text { a: } 8 \text { hours to repair } \\
\text { a: detected and corrected before next } \\
\text { batch } \\
\text { a: demand lasts for } 1 \text { day } \\
\text { a: checked annually } \\
\text { Use TC value } \\
\text { a: valve remains open } 1 \text { hour } \\
\text { a: } 8 \text { hours to repair } \\
\text { Use TC value } \\
\text { a: checked annually } \\
\text {. }\end{array}$ \\
\hline
\end{tabular}




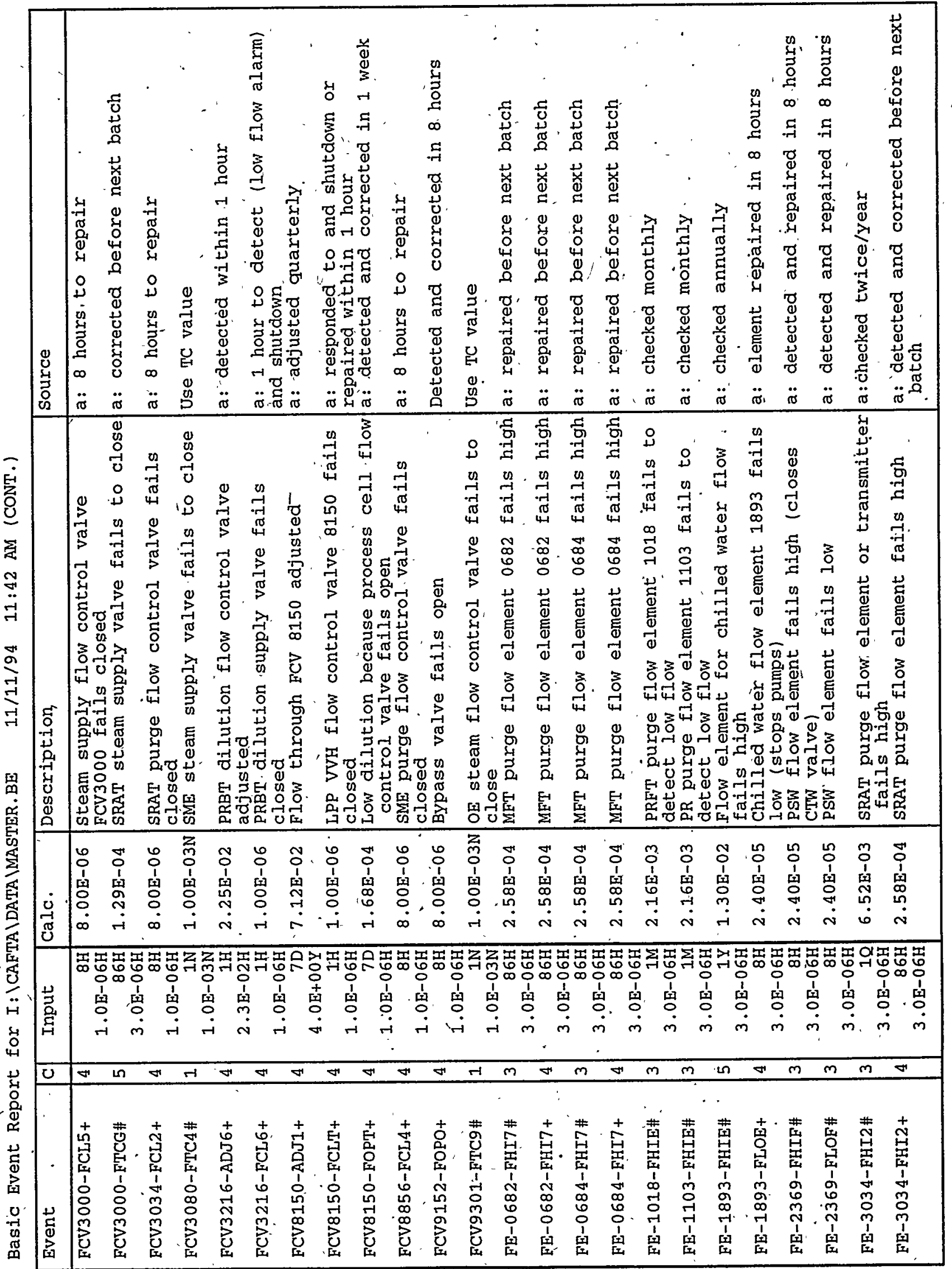


Basic Event Report for I: ICAFTA IDATA IMASTER.BE 11/11/94 11:42 AM (CONT.)

\begin{tabular}{|c|c|c|c|c|c|}
\hline Event & C & aput & Calc. & Description & Source \\
\hline $\begin{array}{l}\text { FED--2--BFLH+ } \\
\text { FED-1-2-CCAH+ } \\
\text { FED-PR--PREK\# } \\
\text { FIT8150-FHIT+ } \\
\text { FLGN2SUPLKSP+ } \\
\text { FLGOFICELKSP\# } \\
\text { FLT-062-PLGQ+ } \\
\text { FLT-BP--PLGQ+ }\end{array}$ & 4 & $\begin{array}{r}86 \mathrm{H} \\
3.0 \mathrm{E}-06 \mathrm{H} \\
86 \mathrm{H} \\
3.0 \mathrm{E}-06 \mathrm{H} \\
86 \mathrm{H} \\
3.0 \mathrm{E}-06 \mathrm{H} \\
86 \mathrm{H} \\
3.0 \mathrm{E}-06 \mathrm{H} \\
86 \mathrm{H} \\
3.0 \mathrm{E}-06 \mathrm{H} \\
8 \mathrm{H} \\
3.0 \mathrm{E}-06 \mathrm{H} \\
86 \mathrm{H} \\
3.0 \mathrm{E}-06 \mathrm{H} \\
8 \mathrm{H} \\
3.0 \mathrm{E}-06 \mathrm{H} \\
86 \mathrm{H} \\
3.0 \mathrm{E}-06 \mathrm{H} \\
86 \mathrm{H} \\
3.0 \mathrm{E}-06 \mathrm{H} \\
1 \mathrm{M} \\
3.0 \mathrm{E}-06 \mathrm{H} \\
1 \mathrm{M} \\
3.0 \mathrm{E}-06 \mathrm{H} \\
1 \mathrm{M} \\
1.0 \mathrm{E}+00 \mathrm{Y} \\
1 \mathrm{M} \\
1.0 \mathrm{E}+00 \mathrm{Y} \\
1 \mathrm{M} \\
1.0 \mathrm{E}+00 \mathrm{Y} \\
8.5 \mathrm{H} \\
1.0 \mathrm{E}-06 \mathrm{H} \\
8.5 \mathrm{H} \\
1.0 \mathrm{E}-06 \mathrm{H} \\
8.5 \mathrm{H} \\
1.0 \mathrm{E}-07 \mathrm{H} \\
2 \mathrm{H} \\
2.3 \mathrm{E}-02 \mathrm{H} \\
1 \mathrm{H} \\
3.0 \mathrm{E}-06 \mathrm{H} \\
8 \mathrm{H} \\
1.0 \mathrm{E}-06 \mathrm{H} \\
8 \mathrm{H} \\
1.0 \mathrm{E}-06 \mathrm{H} \\
4 \mathrm{H} \\
3.0 \mathrm{E}-06 \mathrm{H} \\
4 \mathrm{H} \\
\end{array}$ & $\begin{array}{l}2.58 \mathrm{E}-04 \\
2.58 \mathrm{E}-04 \\
2.58 \mathrm{E}-04 \\
.2 .58 \mathrm{E}-04 \\
1.29 \mathrm{E}-04 \\
2.40 \mathrm{E}-05 \\
1.29 \mathrm{E}-04 \\
2.40 \mathrm{E}-05 \\
2.58 \mathrm{E}-04\end{array}$ & 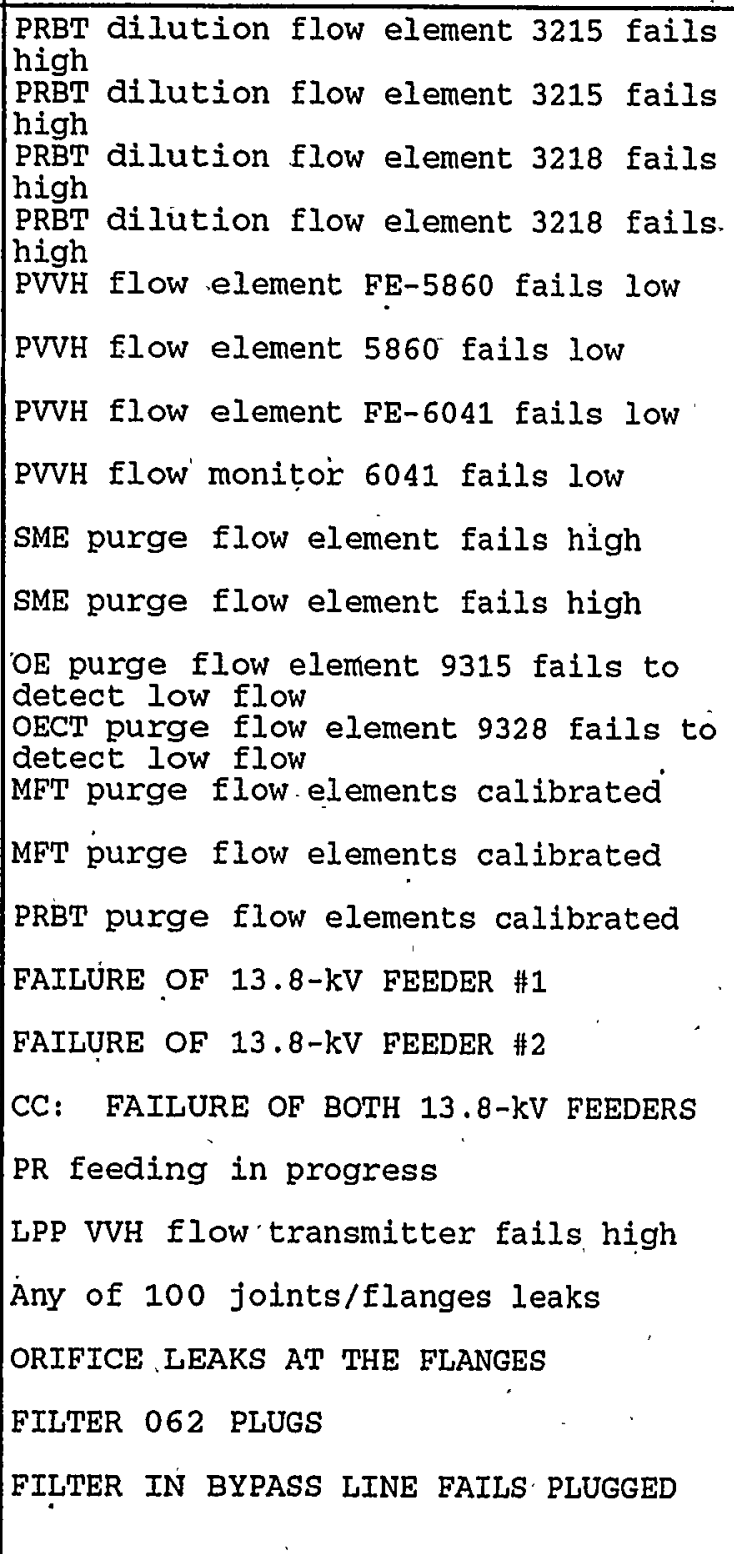 & $\begin{array}{l}\text { Repaired before next batch } \\
\text { reparired before next batch } \\
\text { repaired before next batch } \\
\text { repaired before next batch } \\
\text { a: corrected before next sRAT batch } \\
\text { a: detected and corrected in } 8 \text { hours } \\
\text { a: corrected by next sRAT batch } \\
\text { a: detected and corrected in } 8 \text { hours } \\
\text { a: repaired before next batch } \\
\text { a: repaired before next batch } \\
\text { a: checked monthly } \\
\text { a: checked monthly } \\
\text { a: calibrated annually, corrected in } \\
1 \text { month } \\
\text { a: calibrated annually, corrected in } \\
1 \text { month } \\
\text { a.: calibrated annully, corrected in } \\
1 \text { month } \\
\text { DWPF sAR } 9.3 .18 \\
\text { DwPF sAR } 9.3 .18 \\
\text { DwPF sAR } 9.3 .18 \\
2 \text { hours per pR batch } \\
\text { a: responded to and shutdown or } \\
\text { repaired within } 1 \text { hour } \\
\text { a: } 8 \text { Hr Repair } \\
\text { a: } 8 \text { hr detection } \\
\text { a: } 8 \text { hours to repair } \\
\text { a: } 8 \text { hours to repair } \\
\text {. }\end{array}$ \\
\hline
\end{tabular}


Basic Event Report for I: \CAFTA \DATA \MASTER.BE 11/11/94 11:42 AM (CONT.).

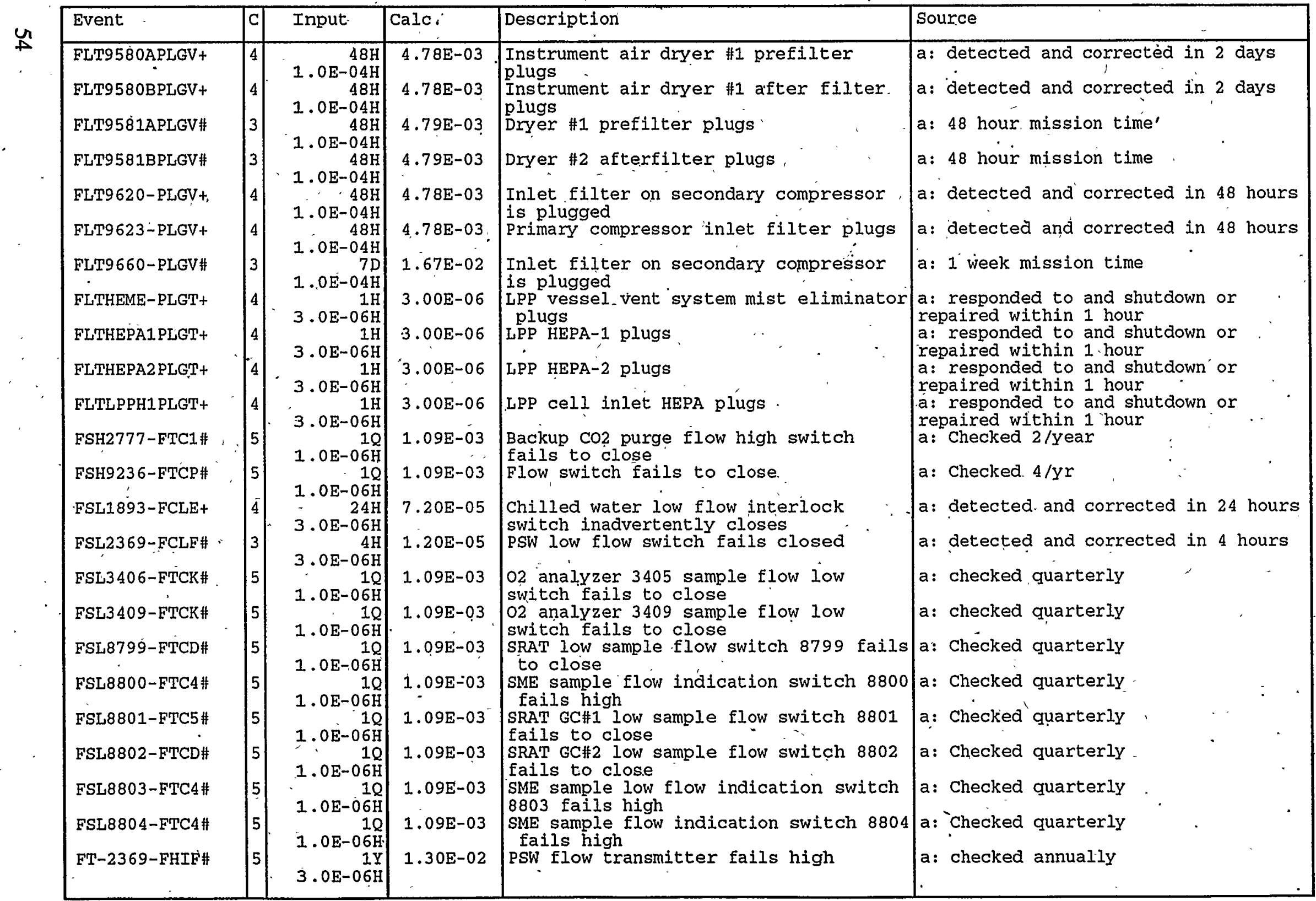


Basic Event Report for I: \CAFTA \DATA IMASTER.BE 11/11/94 11:42 AM (CONT.j)

\begin{tabular}{|c|c|c|c|c|c|}
\hline Event & C & Input & Calc. & Description & Source \\
\hline $\begin{array}{l}\text { FT-8155-FLOT\# } \\
\text { FT-9152-FLOO+ } \\
\text { FY-8150-BFLT+ } \\
\text { HCV1017-FCLB+ } \\
\text { HCV1033-FTCO\# } \\
\text { HCV1038-FOP9+ } \\
\text { HCV1102-FCL9+ } \\
\text { HCV1142-FTCO\# } \\
\text { HCV1885-FCLE+ } \\
\text { HCV1897-FTOE\# } \\
\text { HCV3010-FTCO\# } \\
\text { HCV3010-FTOF\# } \\
\text { HCV3089-FCL4 \# } \\
\text { HCV3089-FTCO\# } \\
\text { HCV3212-FTCO\# } \\
\text { HCV3252-FTCO\# } \\
\text { HCV5845-FTCO\# } \\
\text { HCV5907-FTCO\# } \\
\text { HCV8772-FOPQ\# } \\
\text { HCV8772-FTCQ\# } \\
\text { HCV9116-FCLO+ } \\
\text { HCV9116-FTCG\# } \\
\text { HCV9122-FTOG\# } \\
\text { HCV9150-FCLG\# } \\
.\end{array}$ & 5 & $\begin{array}{r}1 \mathrm{Y} \\
3.0 \mathrm{E}-06 \mathrm{H} \\
8 \mathrm{H} \\
3.0 \mathrm{E}-06 \mathrm{H} \\
1 \mathrm{H} \\
1.0 \mathrm{E}-07 \mathrm{H} \\
8 \mathrm{H} \\
5.0 \mathrm{E}-07 \mathrm{H} \\
1 \mathrm{~N} \\
1.0 \mathrm{E}-03 \mathrm{~N} \\
86 \mathrm{H} \\
5.0 \mathrm{E}-07 \mathrm{H} \\
8 \mathrm{H} \\
5.0 \mathrm{E}-07 \mathrm{H} \\
1 \mathrm{~N} \\
1.0 \mathrm{E}-03 \mathrm{~N} \\
8 \mathrm{H} \\
5.0 \mathrm{E}-07 \mathrm{H} \\
1 \mathrm{~N} \\
1.0 \mathrm{E}-03 \mathrm{~N} \\
1 \mathrm{~N} \\
1.0 \mathrm{E}-03 \mathrm{~N} \\
1 \mathrm{~N} \\
1.0 \mathrm{E}-03 \mathrm{~N} \\
8 \mathrm{H} \\
5.0 \mathrm{E}-07 \mathrm{H} \\
1 \mathrm{~N} \\
1.0 \mathrm{E}-03 \mathrm{~N} \\
1 \mathrm{~N} \\
1.0 \mathrm{E}-03 \mathrm{~N} \\
1 \mathrm{~N} \\
1.0 \mathrm{E}-03 \mathrm{~N} \\
1 \mathrm{~N} \\
1.0 \mathrm{E}-03 \mathrm{~N} \\
1 \mathrm{~N} \\
1.0 \mathrm{E}-03 \mathrm{~N} \\
8 \mathrm{H} \\
5.0 \mathrm{E}-07 \mathrm{H} \\
1 \mathrm{~N} \\
1.0 \mathrm{E}-03 \mathrm{~N} \\
4 \mathrm{H} \\
5.0 \mathrm{E}-07 \mathrm{H} \\
1 \mathrm{~N} \\
1.0 \mathrm{E}-03 \mathrm{~N} \\
1 \mathrm{~N} \\
1.0 \mathrm{E}-03 \mathrm{~N} \\
-8 \mathrm{H} \\
5.0 \mathrm{E}-07 \mathrm{H}\end{array}$ & $\begin{array}{l}1.30 \mathrm{E}-02 \\
2.40 \mathrm{E}-05 \\
1.00 \mathrm{E}-07 \\
4.00 \mathrm{E}-06 \\
1.00 \mathrm{E}-03 \mathrm{~N} \\
4.30 \mathrm{E}-05 \\
4.00 \mathrm{E}-06 \\
1.00 \mathrm{E}-03 \mathrm{~N} \\
4.00 \mathrm{E}-06 \\
1.00 \mathrm{E}-03 \mathrm{~N} \\
1.00 \mathrm{E}-03 \mathrm{~N} \\
1.00 \mathrm{E}-03 \mathrm{~N} \\
4.00 \mathrm{E}-06 \\
1.00 \mathrm{E}-03 \mathrm{~N} \\
1.00 \mathrm{E}-03 \mathrm{~N} \\
1.00 \mathrm{E}-03 \mathrm{~N} \\
1.00 \mathrm{E}-03 \mathrm{~N} \\
1.00 \mathrm{E}-03 \mathrm{~N} \\
2.00 \mathrm{E}-06 \\
1.00 \mathrm{E}-03 \mathrm{~N} \\
2.00 \mathrm{E}-06 \\
1.00 \mathrm{E}-03 \mathrm{~N} \\
1.00 \mathrm{E}-03 \mathrm{~N} \\
4.00 \mathrm{E}-06\end{array}$ & 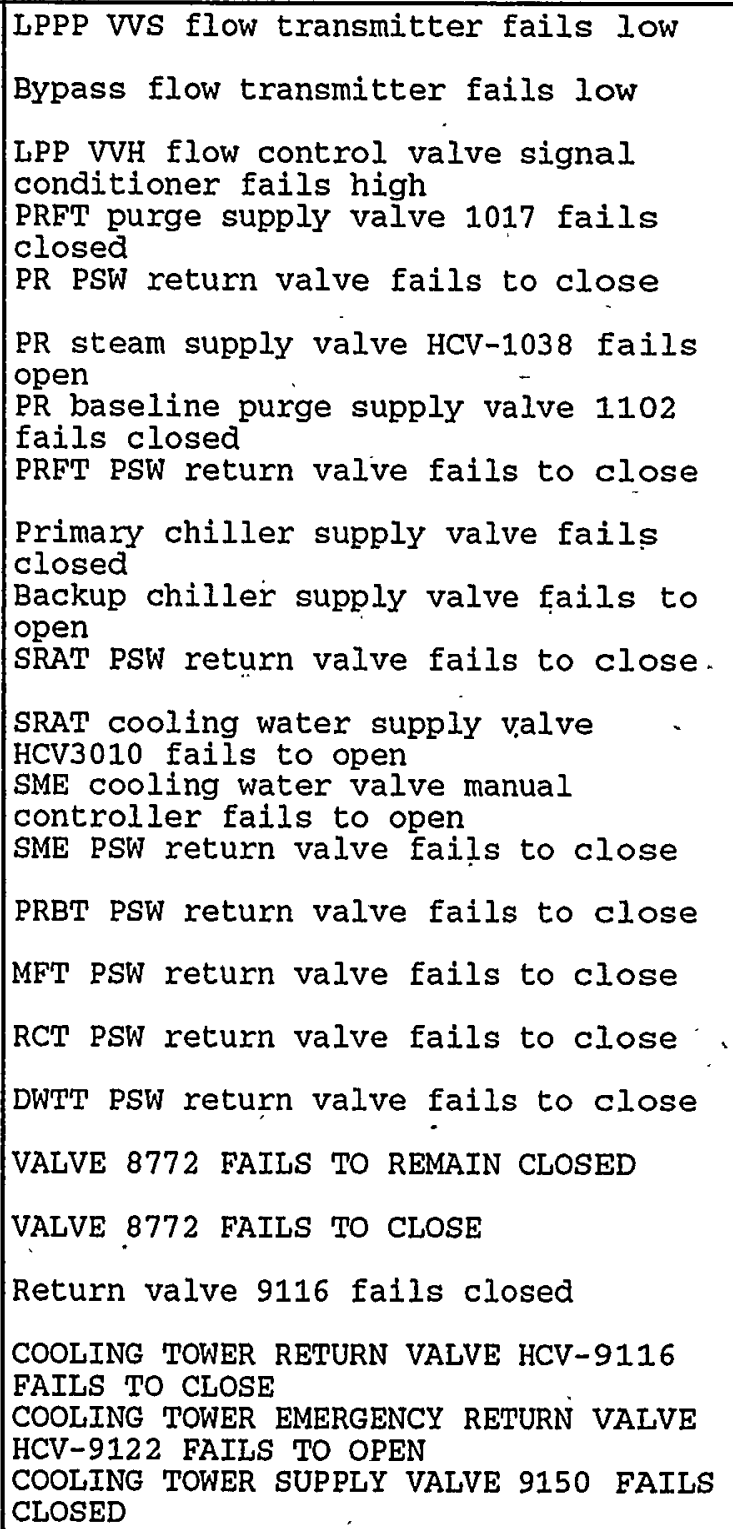 & $\begin{array}{l}\text { a: checked annualy } \\
\text { Detected and corrected in } 8 \text { hours } \\
\text { a: responded to and shutdown or } \\
\text { repaired within } 1 \text { hour } \\
\text { a: } 8 \text { hours to repair or shutdown } \\
\text { Use TC value } \\
\text { a: detected and repaired before next } \\
\text { batch } \\
\text { a: } 8 \text { hours to repair or shutdown } \\
\text { Use TC value } \\
\text { a: } 8 \text { hours to repair } \\
\text { Use TC Value. } \\
\text { Use TC value } \\
\text { Use TC value } \\
\text { A: } 8 \text { HouRs To REPAIR } \\
\text { Use TC value } \\
\text { Use TC value } \\
\text { Use TC value } \\
\text { Use TC value } \\
\text { Use TC value } \\
\text { a: } 8 \text { hours to repair } \\
\text { Use TC } \\
\text { a: detected and repaired, bypassed, } \\
\text { or shut down in } 4 \text { hours } \\
\text { Use TC } \\
\text { Use TC } \\
\text { a: } 8 \text { hours to repair } \\
\text { Un }\end{array}$ \\
\hline
\end{tabular}


Basic'Event Report for I: \CAFTA IDATA IMASTER.BE $11 / 11 / 94 \quad 11: 42$ AM (CONT.)

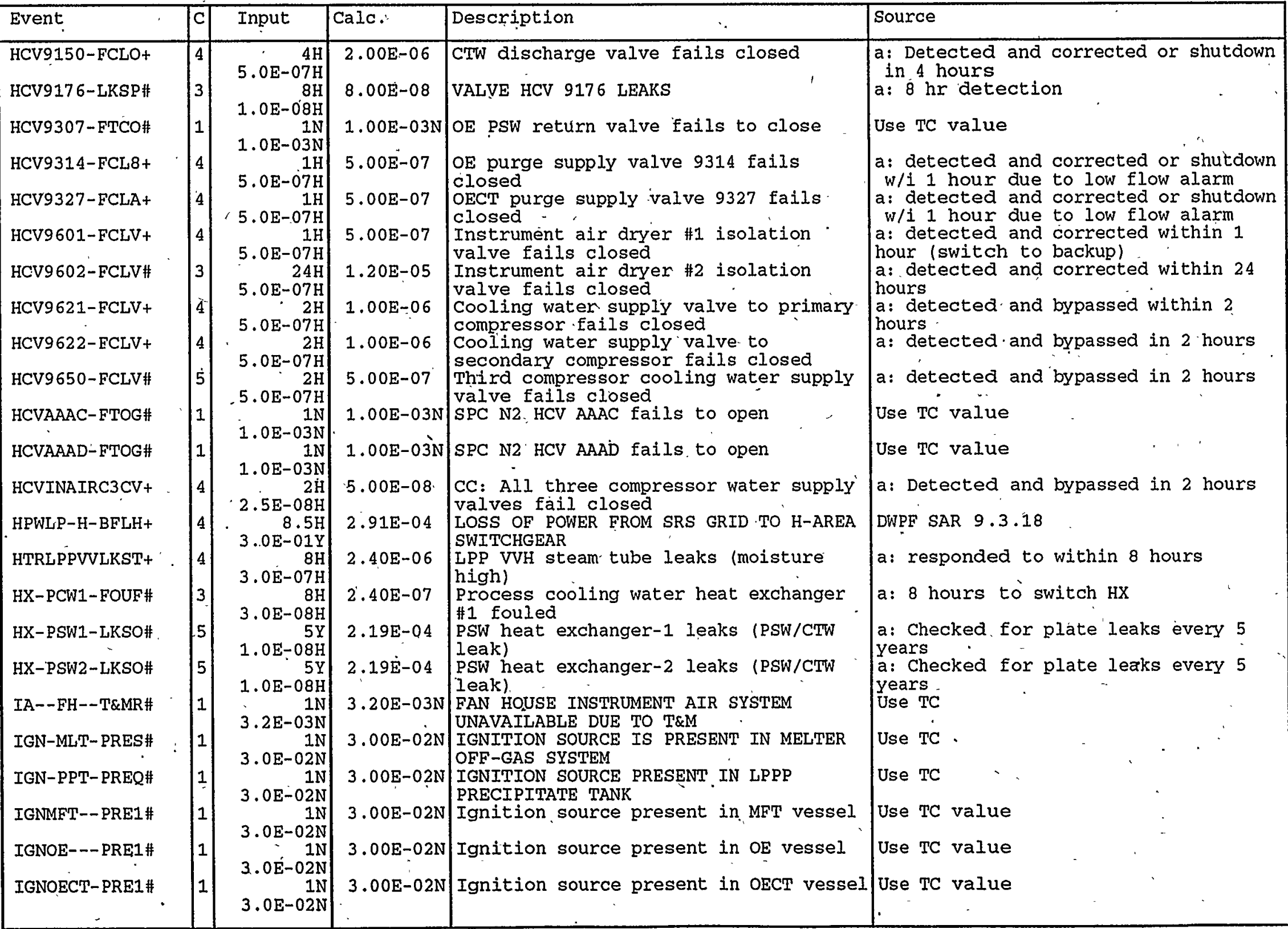


Basic Event Report for I: \CAFTA IDATA IMASTER.BE 11/11/94 11:42 AM (CONT.)

\begin{tabular}{|c|c|c|c|c|c|}
\hline Event & c & Input & Calc. & Description & Source \\
\hline $\begin{array}{l}\text { IGNPRFT-PRE1\# } \\
\text { IGNPVVH-PRE1\# } \\
\text { IGNSME--PRE1\# } \\
\text { IGNSPC--PRE1\# } \\
\text { IGNSPT--PRE1\# } \\
\text { IGNSRAT-PRE1\# } \\
\text { INKSRLFLBYP5\# } \\
\text { JMP-HTR-CCAS+ } \\
\text { JMP-HTRABFLS+ } \\
\text { JMP-HTRBBFLS+ } \\
\text { JMP-HTRCBFLS+ } \\
\text { JMP-HTRDBFLS+ } \\
\text { JMP-SPC-LKS1+ } \\
\text { LCV2220AFCLG+ } \\
\text { LCV2220BFCLG+ } \\
\text { LE-2220-CALG+ } \\
\text { LE-2220-FHIG+ } \\
\text { LE-2262-FLOG\# } \\
\text { LE-5925-FLOG\# . } \\
\text { LPWST-LPBFLH+ }\end{array}$ & 1 & $\begin{array}{r}1 \mathrm{~N} \\
3.0 \mathrm{E}-02 \mathrm{~N} \\
.1 \mathrm{H} \\
2.3 \mathrm{E}-5 \mathrm{H} \\
1 \mathrm{~N} \\
3.0 \mathrm{E}-02 \mathrm{~N} \\
1 \mathrm{~N} \\
3.0 \mathrm{E}-02 \mathrm{~N} \\
1 \mathrm{~N} \\
3.0 \mathrm{E}-02 \mathrm{~N} \\
1 \mathrm{~N} \\
3.0 \mathrm{E}-02 \mathrm{~N} \\
1 \mathrm{~N} \\
3.0 \mathrm{E}-02 \mathrm{~N} \\
1 \mathrm{~N} \\
3.0 \mathrm{E}-02 \mathrm{~N} \\
1 \mathrm{~N} \\
3.0 \mathrm{E}-02 \mathrm{~N} \\
1 \mathrm{~N} \\
3.0 \mathrm{E}-02 \mathrm{~N} \\
1 \mathrm{~N} \\
4.6 \mathrm{E}-04 \mathrm{~N} \\
4 \mathrm{H} \\
1.0 \mathrm{E}-09 \mathrm{H} \\
4 \mathrm{H} \\
1.0 \mathrm{E}-08 \mathrm{H} \\
4 \mathrm{H} \\
1.0 \mathrm{E}-08 \mathrm{H} \\
4 \mathrm{H} \\
1.0 \mathrm{E}-08 \mathrm{H} \\
4 \mathrm{H} \\
1.0 \mathrm{E}-08 \mathrm{H} \\
43 \mathrm{H} \\
2.0 \mathrm{E}-05 \mathrm{H} \\
8 \mathrm{H} \\
5.0 \mathrm{E}-07 \mathrm{H} \\
8 \mathrm{H} \\
5.0 \mathrm{E}-07 \mathrm{H} \\
10 \\
2.3 \mathrm{E}-04 \mathrm{H} \\
8 \mathrm{H} \\
5.0 \mathrm{E}-07 \mathrm{H} \\
8 \mathrm{H} \\
5.0 \mathrm{E}-07 \mathrm{H} \\
1 \mathrm{Y} \\
5.0 \mathrm{E}-07 \mathrm{H} \\
8.5 \mathrm{H} \\
8.0 \mathrm{E}-04 \mathrm{Y}\end{array}$ & $\begin{array}{l}3.00 \mathrm{E}-02 \mathrm{~N} \\
2.30 \mathrm{E}-06 \\
3.00 \mathrm{E}-02 \mathrm{~N} \\
3.00 \mathrm{E}-02 \mathrm{~N} \\
3.00 \mathrm{E}-02 \mathrm{~N} \\
3.00 \mathrm{E}-02 \mathrm{~N} \\
3.00 \mathrm{E}-02 \mathrm{~N} \\
3.00 \mathrm{E}-02 \mathrm{~N} \\
3.00 \mathrm{E}-02 \mathrm{~N} \\
3.00 \mathrm{E}-02 \mathrm{~N} \\
4.60 \mathrm{E}-04 \mathrm{~N} \\
4.00 \mathrm{E}-09 \\
4.00 \mathrm{E}-08 \\
4.00 \mathrm{E}-08 \\
4.00 \mathrm{E}-08 \\
4.00 \mathrm{E}-08 \\
8.59 \mathrm{E}-0.4 \\
4.00 \mathrm{E}-06 \\
4.00 \mathrm{E}-06 \\
3.34 \mathrm{E}-01 \\
4.00 \mathrm{E}-06 \\
4.00 \mathrm{E}-06 \\
2.19 \mathrm{E}-03 \\
7.76 \mathrm{E}-07\end{array}$ & $\begin{array}{l}\text { Ignition source present in OWST inner } \\
\text { tank } \\
\text { Ignition source present in OWST outer } \\
\text { tank } \\
\text { Ignition source present in PR vessel } \\
\text { Ignition source present in PRBT vessel } \\
\text { Ignition source present in PRFT vessel } \\
\text { Ignition source present in PVVH } \\
\text { Ignition source present in SME vessel } \\
\text { Ignition source present in SPC } \\
\text { Ignition source present in SPT vessel } \\
\text { Ignition source present in SRAT } \\
\text { SRAT high LFI interlock bypassed } \\
\text { CC: TWO FUSES FOR LID. HEATERS FAIL } \\
\text { FUSE FOR LID HEATER A FAILS } \\
\text { FUSE FOR LID HEATER B FAILS } \\
\text { FUSE FOR LID HEATER C FAILS } \\
\text { FUSE FOR LID HEATER D FAILS } \\
\text { SPC jumper leak - benzene leaks onto } \\
\text { SPC Eioor } \\
\text { Steam generator cond. level control } \\
\text { valve A fails closed } \\
\text { Steam generator cond. level control } \\
\text { valve B fails closed } \\
\text { Steam generator condensate level } \\
\text { transmitter is calibrated } \\
\text { Steam generator condensate level } \\
\text { transmitter fails high } \\
\text { LEVEL SENSOR/SWITCH FAILS (FALSE LOW } \\
\text { SIGNAL) } \\
\text { SPC sump level element fails low } \\
\text { FAILURE TO suPPLY POWER TO GRID FROM } \\
\text { ON-SITE AND OFF-SITE SOURCES }\end{array}$ & $\begin{array}{l}\text { Use TC value } \\
\text { Ignition source lasts } 0.1 \text { hour } \\
\text { Use TC value } \\
\text { Use TC value } \\
\text { Use TC value } \\
\text { Use TC value } \\
\text { Use TC value } \\
\text { Use TC value } \\
\text { Use TC value } \\
\text { Use TC value } \\
\text { Use TC value } \\
\text { a: detected in } 1 \text { shift } \\
\text { a: detected in } 1 \text { shift } \\
\text { a: detected in. } 1 \text { shift } \\
\text { a: detected in } 1 \text { shift } \\
\text { a: detected in } 1 \text { shift } \\
\text { a: corrected before next batch } \\
\text { Estimated } 8 \text { hour resotration time } \\
\text { Estimated } 8 \text { hour resotration time } \\
\text { a: calibrated twice/year } \\
\text { Estimated } 8 \text { hour resotration time } \\
\text { a: } 8 \text { hours to repair } \\
\text { D: checked annually saR } 9.3 \text {. } 18 .\end{array}$ \\
\hline
\end{tabular}


Basic Event Report for I:ICAFTA \DATA IMASTER.BE · 11/11/94 11:42 AM (CONT.)

\begin{tabular}{|c|c|c|c|c|c|}
\hline Event & C & Input & Ca:lc: & Description & Source \\
\hline LSL4033XFTC1\# & 5 & $2 \mathrm{D}$ & $2.40 \mathrm{E}-05$ & CO2 tank low level switch fails to & a: failure detected in 48 hours \\
\hline LSL7863-ETC3\# & 3 & $\begin{array}{r}1.0 \mathrm{E}-06 \mathrm{H} \\
10\end{array}$ & $2.18 \mathrm{E}-03$ & $\begin{array}{l}\text { loose } \\
\text { Low N2 tank level switch fails to }\end{array}$ & a: Checked $2 / \mathrm{yr}$ \\
\hline MOV1041-FCLK+ & & $1.0 \mathrm{E}-06 \mathrm{H}$ & 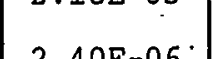 & close & d: Cnecked alys \\
\hline MOV1041-FCLK+ & $|4|$ & $3.0 \mathrm{E}-07 \mathrm{H}$ & $2.40 \mathrm{E}-06^{\circ}$ & $\begin{array}{l}\text { SpC/PVVH isolation valve } 1041 \text { fails } \\
\text { closed }\end{array}$ & a: returned to open in 8 , hours. \\
\hline MOV1041-FTCK\# & 1 & $3.0 \mathrm{E}-03 \mathrm{~N}$ & $3.00 \mathrm{E}-03 \mathrm{~N}$ & $\begin{array}{l}\text { SPC/PVVH isolation valve MOV- } 1041 \\
\text { fails to close }\end{array}$ & Use TC value : \\
\hline MOV1100-ETCK\# & 1 & $\begin{array}{r}1 \mathrm{~N} \\
3,0 \mathrm{E}-03 \mathrm{~N}\end{array}$ & $3.00 \mathrm{E}-03 \mathrm{~N}$ & $\begin{array}{l}\text { PRFT to PR transfer valve fails to } \\
\text { close }\end{array}$ & Use TC value \\
\hline MOV1131-ADJ6+ & 4 & $1.0 \mathrm{E}+000$ & $4.59 \mathrm{E}-04$ & PRCD cleaned (drain valve opened) & $\begin{array}{l}\text { a: valve remains open } 1 \text { hour, } \\
\text { quarterly }\end{array}$ \\
\hline MOV1131-FTCK\#. & 1 & $3.0 \mathrm{E}-03 \mathrm{~N}$ & $3.00 \mathrm{E}-03 \mathrm{~N}$ & PRC/D drain valve fails to close & Use TC value \\
\hline MOV1131-LKS6+ & 4 & $\begin{array}{r}24 \mathrm{H} \\
1.0 \mathrm{E}-06 \mathrm{H}\end{array}$ & $2.40 \mathrm{E}-05$ & PRCD drain valve leaks (benzene to PR) & a: detected and repaired in 24 hours \\
\hline MOV5862-FCLD+ & 4 & $3.0 \mathrm{E}-07 \mathrm{H}$ & $7.20 \mathrm{E}-06$ & $\begin{array}{l}\text { PVVH primary fan suction valve fails } \\
\text { closed }\end{array}$ & a: repaired in 24 hours \\
\hline MOV5864-FTOD\# & 1 & $3.0 \mathrm{E}-03 \mathrm{~N}$ & $3.00 \mathrm{E}-03 \mathrm{~N}$ & $\begin{array}{l}\text { PVVH standby fan suction valve fails } \\
\text { to open }\end{array}$ & Use TC value \\
\hline MOV99278-FCLK+ & 4 & $\begin{array}{rr}8 \mathrm{H} \\
3.0 \mathrm{E}-07 \mathrm{H}\end{array}$ & $2.40 \mathrm{E}-06$ & $\begin{array}{l}\text { Co open } \\
\text { SPC/PVVH isolation valve } 9278 \text { fails } \\
\text { closed }\end{array}$ & a: returned to open in 8 hours \\
\hline MOV9278-FTCK\# & 1 & $3.0 \mathrm{E}-03 \mathrm{~N}$ & $3.00 \mathrm{E}-03 \mathrm{~N}$ & $\begin{array}{l}\text { SPC/PVVH isolation valve MOV-9278 } \\
\text { fails to close }\end{array}$ & Use TC value \\
\hline N2-CPCBPT\&M1\# & 3 & $\begin{array}{r}24 \mathrm{H} \\
1 \mathrm{Y}\end{array}$ & $2.74 \mathrm{E}-03$ & $\begin{array}{l}\text { CPC backup purge system out of service } \\
\text { for T\&M }\end{array}$ & a: system shutdown in 24 hours \\
\hline N2-CYL--USD1\# & 5 & $\because \quad \begin{array}{ll}7 \mathrm{D} \\
\because \quad 1 \mathrm{Y}\end{array}$ & $9.53 \mathrm{E}-03$ & LPPP local N2 bottles empty & $\begin{array}{l}\text { a: used at primary failure rate, } \\
\text { refilled in } 1 \text { week }\end{array}$ \\
\hline N2-LPPP-T\&M1\#_ & 3 & $\begin{array}{r}14 \mathrm{D} \\
1 \mathrm{Y}\end{array}$ & $3.76 \mathrm{E}-02$ & $\begin{array}{l}\text { LPPP local N2 system out of service } \\
\text { for T\&M }\end{array}$ & a: 2 weeks/year \\
\hline N2-OWST-T\&M1\# & 3 & $\begin{array}{r}14 \mathrm{D} \\
1 \mathrm{Y}\end{array}$ & $3.76 \mathrm{E}-02$ & $\begin{array}{l}\text { OWST local liquid N2 system out of } \\
\text { service for T\&M }\end{array}$ & a: 14 days per year \\
\hline N2-TRUCKBFL1\# & 1 & $1.0 \mathrm{E}-02 \mathrm{~N}$ & $1.00 \mathrm{E}-0.2 \mathrm{~N}$ & N2 delivery fails to arrive at DWPF & estimated \\
\hline OFV-018-FOPP+ & 4 & $\begin{array}{r}24 \mathrm{H} \\
5.0 \mathrm{E}-09 \mathrm{H}\end{array}$ & $1.20 \mathrm{E}-07$ & $\begin{array}{l}\text { Overflow (inner to outer tank) fails } \\
\text { open }\end{array}$ & a: 24 hours to repair \\
\hline OPR--5B-LOHH\# & 1 & $\begin{array}{l}1.8 \mathrm{E}-04 \mathrm{~N} \\
5.0 \mathrm{E}-03 \mathrm{~N}\end{array}$ & $9.00 \mathrm{E}-07 \mathrm{~N}$ & $\begin{array}{l}\text { OPERATOR FAILS TO REMOVE LOCKOUT ON } \\
\text { FEEDER BREAKER 5B }\end{array}$ & $1 / 5$ YRS * $16 \mathrm{H} / 2$ (WMS) \\
\hline OPR--A1-LOHH\# & 1 & $\begin{array}{l}1.8 \mathrm{E}-04 \mathrm{~N} \\
5.0 \mathrm{E}-03 \mathrm{~N}\end{array}$ & $9.00 \mathrm{E}-07 \mathrm{~N}$ & $\begin{array}{l}\text { OPERATOR FAILS TO REMOVE LOCKOUT ON. } \\
\text { BUS A1 }\end{array}$ & $1 / 5$ YRS * $16 \mathrm{H} / 2$ (WMS) \\
\hline OPR- - PT-MCHR\# & 1 & $3.0 \mathrm{E}-02 \mathrm{~N}$ & $3.00 \mathrm{E}-02 \mathrm{~N}$ & $\begin{array}{l}\text { OPERATOR MISCALIBRATES PRESSURE } \\
\text { TRANSMITTERS (OUT -OF RANGE) }\end{array}$ & Use TC. \\
\hline OPR-22 62MCHG\# & 1 & $\begin{array}{r}2.5 \mathrm{E}-01 \mathrm{~N} \\
.3 .0 \mathrm{E}-02 \mathrm{~N}\end{array}$ & $7.50 \mathrm{E}-03 \mathrm{~N}$ & $\begin{array}{l}\text { OPERATOR MISCALIBRATES LEVEL } \\
\text { SENSOR/SWITCH }\end{array}$ & $1 / \mathrm{yr} * 0.5 \mathrm{y} / 2$ \\
\hline OPR-A2 01LOHH\# & 1 & $\begin{array}{l}1.8 \mathrm{E}-04 \mathrm{~N} \\
5.0 \mathrm{E}-03 \mathrm{~N}\end{array}$ & $9.00 \mathrm{E}-07 \mathrm{~N}$ & $\begin{array}{l}\text { OPERATOR FAILS TO REMOVE LOCKOUT ON } \\
\text { TIE BREAKER A201 }\end{array}$ & $1 / 5$ YRS * $16 \mathrm{H} / 2$ (WMS) \\
\hline OPR-A202LOHH\# & 1 & $\begin{array}{l}1.8 \mathrm{E}-04 \mathrm{~N} \\
5.0 \mathrm{E}-03 \mathrm{~N}\end{array}$ & $9.00 \mathrm{E}-07 \mathrm{~N}$ & $\begin{array}{l}\text { OPERATOR FAILS TO REMOVE LOCKOUT ON } \\
\text { FEEDER BREAKER A202 }\end{array}$ & $1 / 5 Y R S * 16 \mathrm{H} / 2$ (WMS) \\
\hline
\end{tabular}


Basic Event Report for I: /CAFTAIDATA.IMASTER.BE 11/11/94 11:42 AM

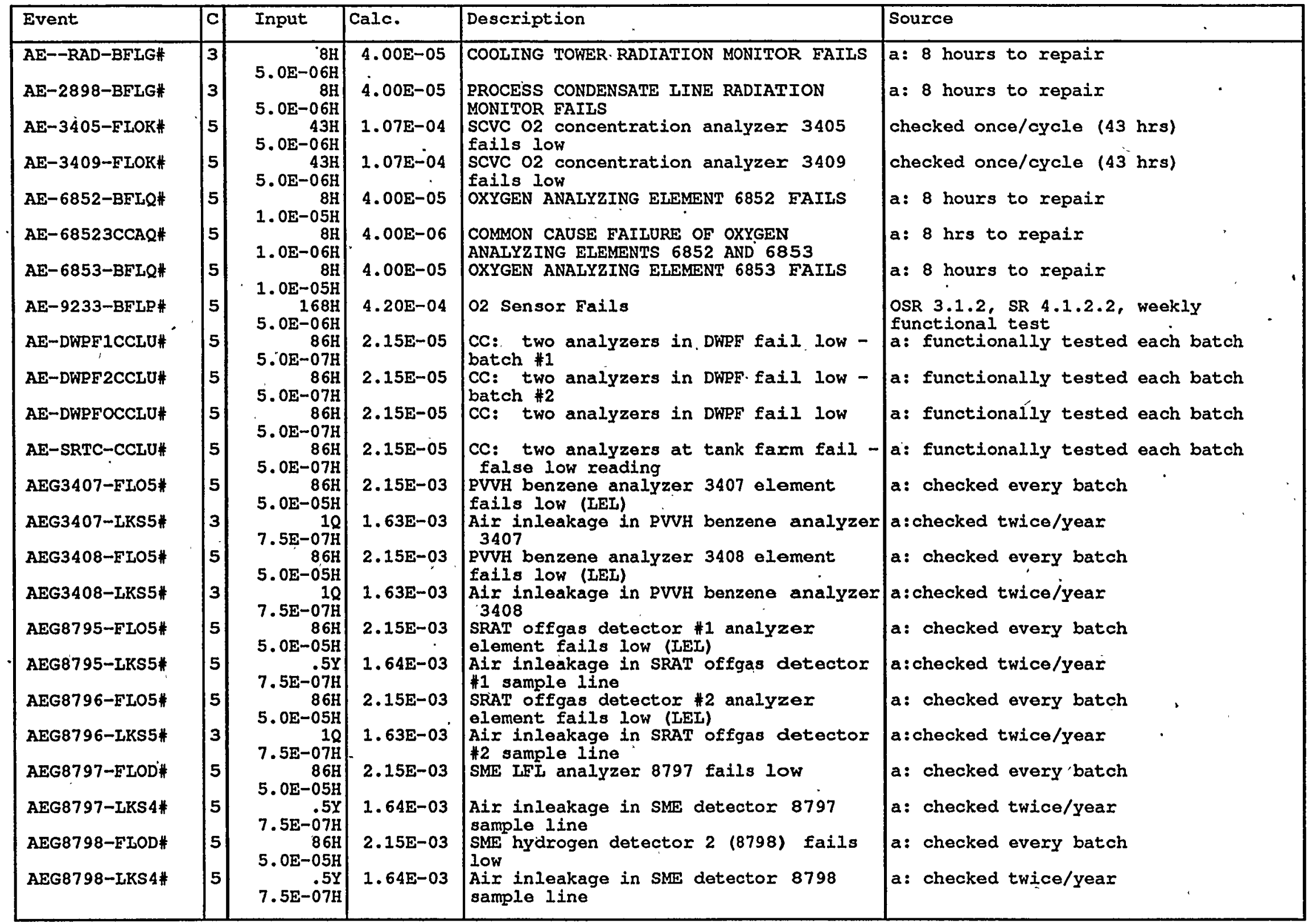


Basic Event Report for I: \CAFTA \DATA \MASTER.BE

11/11/94 11:42 AM (CONT.)

\begin{tabular}{|c|c|c|c|c|c|c|c|c|}
\hline Event & c) & iput & Calc. & Description & Sor & & & \\
\hline $\begin{array}{l}\text { AEGSRAT-LKS5\# } \\
\text { AX-8795-BEL5\# } \\
\text { AX-8797-BEL4\# } \\
\text { BCHSRAT-DBL5\# } \\
\text { BCHSRAT-PRE5+ } \\
\text { BKR--5A-FOPH+ } \\
\text { BKR--5B-CCEN\# } \\
\text { BKR--5B-FORH+ } \\
\text { BKR-12--CCBR\# } \\
\text { BKR-123-C3DR\# } \\
\text { BKR-1234C3DR\# } \\
\text { BKR-5A5BCCBH+ } \\
\text { BKR-A101FORH+ } \\
\text { BKR-A201FOPH+ } \\
\text { BKR-A202FOPH+ } \\
\text { BKR-HTR-CCBS+ } \\
\text { BKR-HTRAFORS+ } \\
\text { BKR-HTRBFOPS+ } \\
\text { BKR-HTRCFOPS+ } \\
\text { BKR-HTRDFOPS+ } \\
\text { BKR-SB--FOPR\# } \\
\text { BKR00001FOPR\# } \\
\text { BKR00002FOPR\# } \\
\text { BKR00003FOPR\# }\end{array}$ & $5 \mid$ & $\begin{array}{r}7.5 \mathrm{Y} \\
7.5 \mathrm{E}-07 \mathrm{H} \\
43 \mathrm{H} \\
3.0 \mathrm{E}-05 \mathrm{H} \\
1 \mathrm{Q} \\
3.0 \mathrm{E}-05 \mathrm{H} \\
1 \mathrm{~N} \\
1.0 \mathrm{E}-01 \mathrm{~N} \\
86 \mathrm{H} \\
1.2 \mathrm{E}-02 \mathrm{H} \\
8.5 \mathrm{H} \\
3.0 \mathrm{E}-07 \mathrm{H} \\
1 \mathrm{H} \\
5.0 \mathrm{E}-05 \mathrm{H} \\
8.5 \mathrm{H} \\
3.0 \mathrm{E}-07 \mathrm{H} \\
8 \mathrm{H} \\
3.0 \mathrm{E}-08 \mathrm{H} \\
8 \mathrm{H} \\
1.5 \mathrm{E}-08 \mathrm{H} \\
8 \mathrm{H} \\
1.5 \mathrm{E}-08 \mathrm{H} \\
8.5 \mathrm{H} \\
3.0 \mathrm{E}-08 \mathrm{H} \\
8.5 \mathrm{H} \\
3.0 \mathrm{E}-07 \mathrm{H} \\
8.5 \mathrm{H} \\
3.0 \mathrm{E}-07 \mathrm{H} \\
8.5 \mathrm{H} \\
3.0 \mathrm{E}-07 \mathrm{H} \\
4 \mathrm{H} \\
3.0 \mathrm{E}-08 \mathrm{H} \\
4 \mathrm{H} \\
3.0 \mathrm{E}-07 \mathrm{H} \\
3.0 \mathrm{E}-07 \mathrm{H} \\
4 \mathrm{H} \\
3.0 \mathrm{E}-07 \mathrm{H} \\
3.0 \mathrm{E}-07 \mathrm{H} \\
8 \mathrm{H} \\
3.0 \mathrm{E}-07 \mathrm{H} \\
8 \mathrm{H} \\
3.0 \mathrm{E}-07 \mathrm{H} \\
8 \mathrm{H} \\
3.0 \mathrm{E}-07 \mathrm{H} \\
8 \mathrm{H} \\
3.0 \mathrm{E}-07 \mathrm{H}\end{array}$ & $\begin{array}{l}1.64 \mathrm{E}-03 \\
1.29 \mathrm{E}-03 \\
6.33 \mathrm{E}-02 \\
1.00 \mathrm{E}-01 \mathrm{~N} \\
5.08 \mathrm{E}-01 \\
2.55 \mathrm{E}-06 \\
5.00 \mathrm{E}-05 \mathrm{~N} \\
2.55 \mathrm{E}-06 \\
1.20 \mathrm{E}-07 \\
6.00 \mathrm{E}-08 \\
6.00 \mathrm{E}-08 \\
2.55 \mathrm{E}-07 \\
2.55 \mathrm{E}-06 \\
2.55 \mathrm{E}-06 \\
2.55 \mathrm{E}-06 \\
1.20 \mathrm{E}-07 \\
1.20 \mathrm{E}-06 \\
1.20 \mathrm{E}-06 \\
1.20 \mathrm{E}-06 \\
1.20 \mathrm{E}-06 \\
1.20 \mathrm{E}-06 \\
1.20 \mathrm{E}-06 \\
1.20 \mathrm{E}-06 \\
1.20 \mathrm{E}-06\end{array}$ & 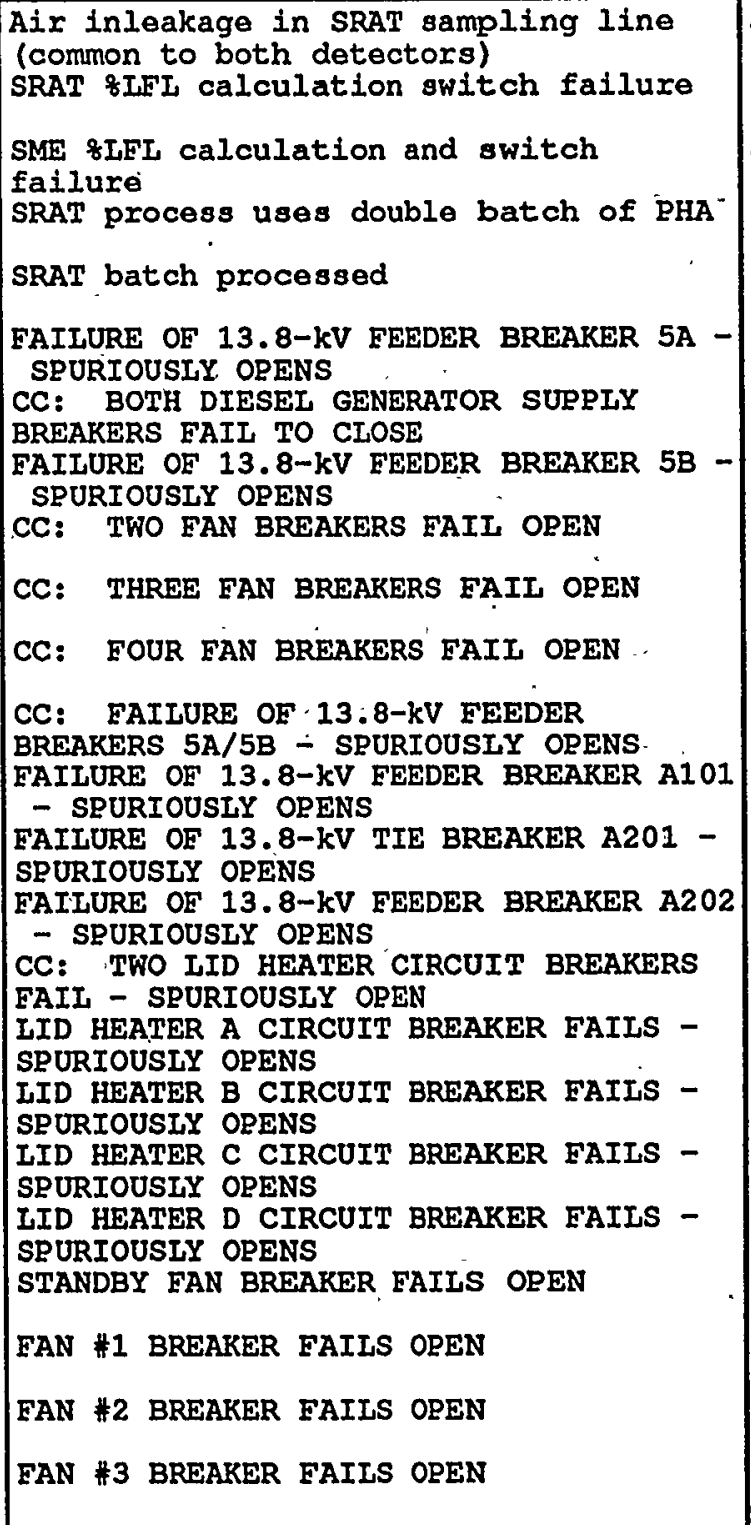 & $\begin{array}{l}\text { a:checked twice/year } \\
\text { Use TC value } \\
\text { a: } 86 \text { hour batches } \\
\text { DWPF SAR } 9.3 .18 \\
\text { Use TC } \\
\text { DWPF SAR } 9.3 .18 \\
\text { a: } 8 \text { hours to repair } \\
\text { a: } 8 \text { hours to repair } \\
\text { a: } 8 \text { hours to repair } \\
\text { DWPF SAR } 9.3 .18 \\
\text { DWPF SAR } 9.3 .18 \\
\text { DFPF SAR } 9.3 .18 \\
\text { DWPF SAR } 9.3 .18 \\
\text { a: detected in } 1 \text { shift } \\
\text { a: detected in } 1 \text { shift } \\
\text { a: detected in } 1 \text { shift } \\
\text { a: detected in } 1 \text { shift } \\
\text { a: detected in } 1 \text { shift } \\
\text { a: } 8 \text { hours to repair } \\
\text { a: } 8 \text { hours to repair } \\
\text { a: } 8 \text { hours to repair } \\
\text { a: } 8 \text { hours to repair }\end{array}$ & . & . & 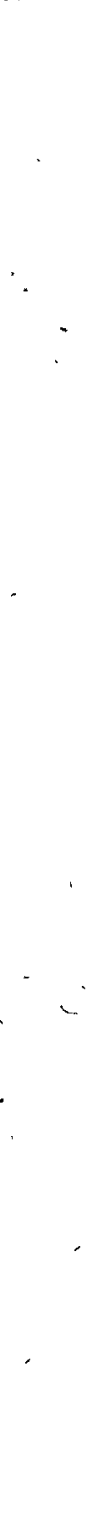 \\
\hline
\end{tabular}


Basic Event Report for I: \CAFTA \DATAIMASTER.BE 11/11/94 11:42 AM (CONT.)

\begin{tabular}{|c|c|c|c|c|c|}
\hline Event & c & input & Calc. & Description & Source \\
\hline $\begin{array}{l}\text { BKRB105BFTCN\# } \\
\text { BKRB9-2BFTON\# } \\
\text { BKRB9-5BFORN\# } \\
\text { BKRB9-5BFTCN\# } \\
\text { BKRB9B1 OCCDN\# } \\
\text { BKRSRATSFORD\# } \\
\text { BNZ-XER-PREP+ } \\
\text { BNZOEHOTPREP } \\
\text { BNZPRHOTPREP } \\
\text { BOTCO2B-REP1* } \\
\text { BOTCO2B-T\&M1\# } \\
\text { BOTCO2B1T\&M1\# } \\
\text { BOTCO2B2T\&M1\# } \\
\text { BOTCO2B3T\&M1\# } \\
\text { BOTCO2B4T\&M1\# } \\
\text { BUS--A1-BELH+ } \\
\text { BUS--A1-UNAH\# } \\
\text { BUS--A2-BELH+ } \\
\text { BUSA1-A2CCAH+ } \\
\text { CC2-AIR-DRY1+ }\end{array}$ & 3 & $\begin{array}{r}8.5 \mathrm{H} \\
3.0 \mathrm{E}-08 \mathrm{H} \\
8.5 \mathrm{H} \\
3.0 \mathrm{E}-08 \mathrm{H} \\
1 \mathrm{~N} \\
5.0 \mathrm{E}-04 \mathrm{~N} \\
8.5 \mathrm{H} \\
3.0 \mathrm{E}-07 \mathrm{H} \\
1 \mathrm{~N} \\
5.0 \mathrm{E}-04 \mathrm{~N} \\
1 \mathrm{H} \\
5.0 \mathrm{E}-04 \mathrm{~N} \\
8.5 \mathrm{H} \\
3.0 \mathrm{E}-07 \mathrm{H} \\
1 \mathrm{~N} \\
5.0 \mathrm{E}-04 \mathrm{~N} \\
1 \mathrm{~N} \\
5.0 \mathrm{E}-05 \mathrm{~N} \\
8 \mathrm{H} \\
3.0 \mathrm{E}-07 \mathrm{H} \\
1 \mathrm{H} \\
2.3 \mathrm{E}-02 \mathrm{H} \\
2 \mathrm{H} \\
2.3 \mathrm{E}-02 \mathrm{H} \\
16 \mathrm{H} \\
2.3 \mathrm{E}-02 \mathrm{H} \\
1 \mathrm{M} \\
6.7 \mathrm{E}-05 \mathrm{H} \\
7 \mathrm{D} \\
1 \mathrm{Y} \\
7.0 \mathrm{D} \\
1.0 \mathrm{E}-08 \mathrm{H} \\
8 \mathrm{H} \\
1.08 \mathrm{H}\end{array}$ & $\begin{array}{l}2.55 \mathrm{E}-07 \\
2.55 \mathrm{E}-07 \\
5.00 \mathrm{E}-04 \mathrm{~N} \\
2.55 \mathrm{E}-06 \\
5.00 \mathrm{E}-04 \mathrm{~N} \\
5.00 \mathrm{E}-04 \mathrm{~N} \\
2.55 \mathrm{E}-06 \\
5.00 \mathrm{E}-04 \mathrm{~N} \\
5.00 \mathrm{E}-05 \mathrm{~N} \\
2.40 \mathrm{E}-06 \\
2.25 \mathrm{E}-02 \\
4.50 \mathrm{E}-02 \\
3.08 \mathrm{E}-01 \\
2.37 \mathrm{E}-02 \\
1.90 \mathrm{E}-02 \\
1: 90 \mathrm{E}-02 \\
1.90 \mathrm{E}-02 \\
1.90 \mathrm{E}-02 \\
1.90 \mathrm{E}-02 \\
8.50 \mathrm{E}-07 \\
3.80 \mathrm{E}-02 \mathrm{~N} \\
8.50 \mathrm{E}-07 \\
8.50 \mathrm{E}-08 \\
4.00 \mathrm{E}-07\end{array}$ & 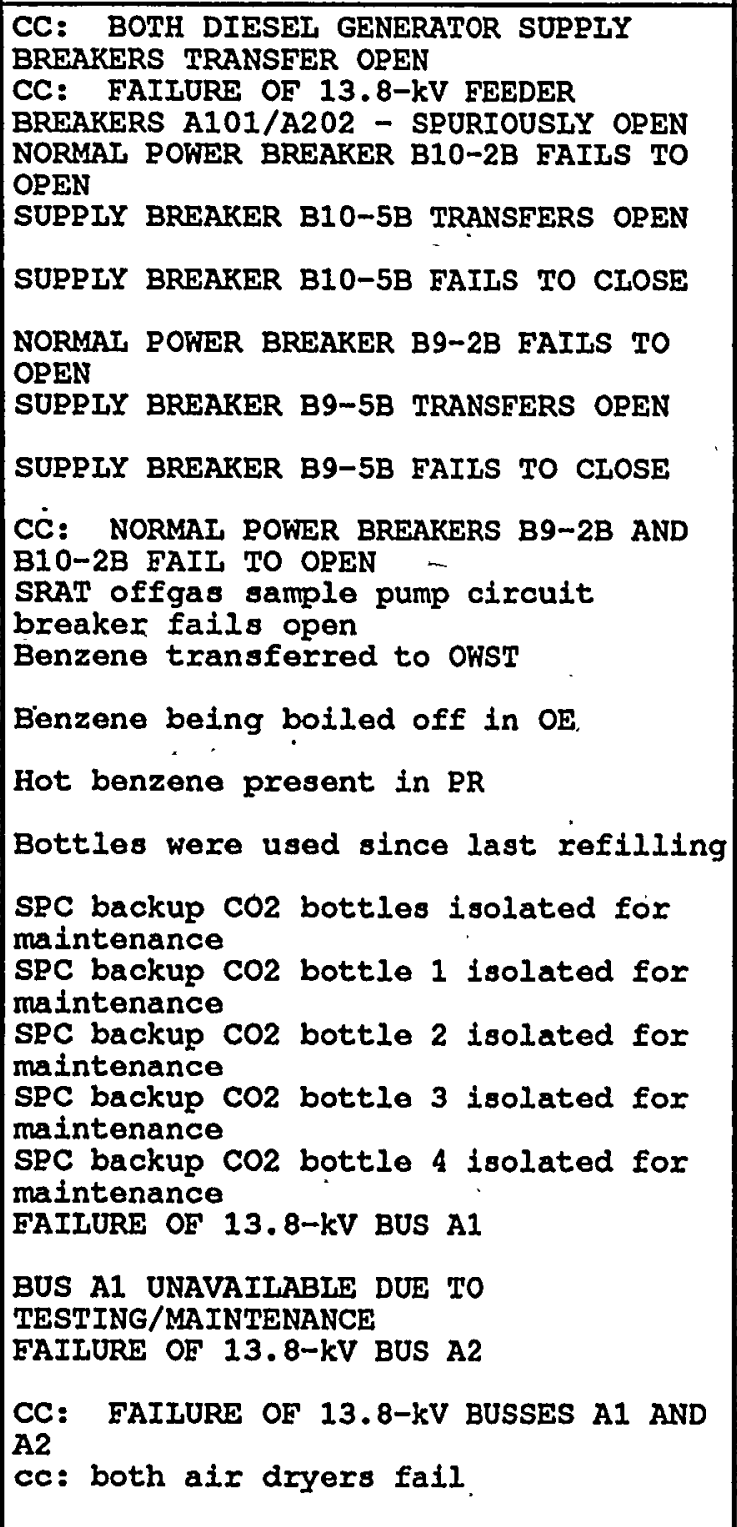 & 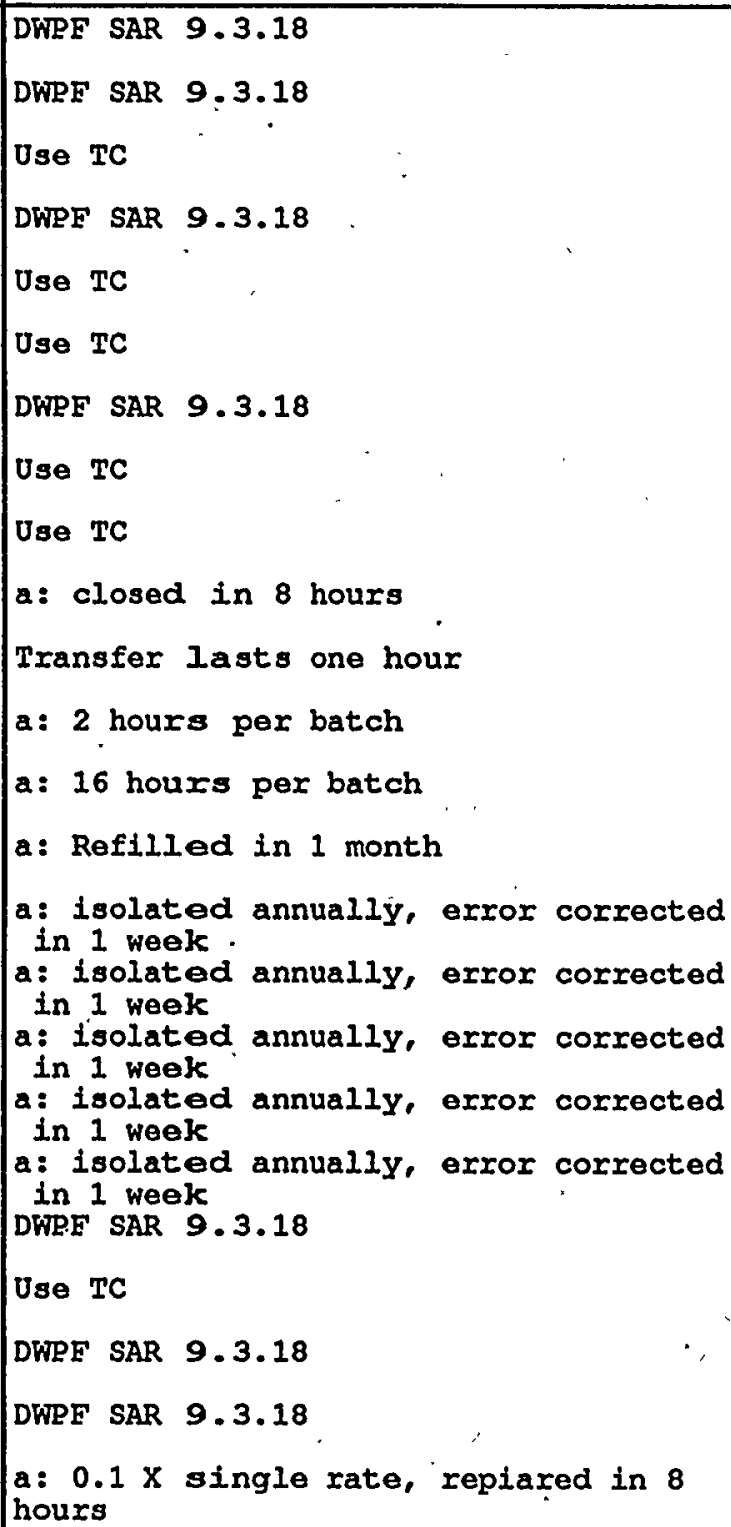 \\
\hline
\end{tabular}




\begin{tabular}{|c|c|c|c|c|c|}
\hline vent & \begin{tabular}{l|l}
$c$ \\
\end{tabular} & aput & Calc. & Description & Source \\
\hline 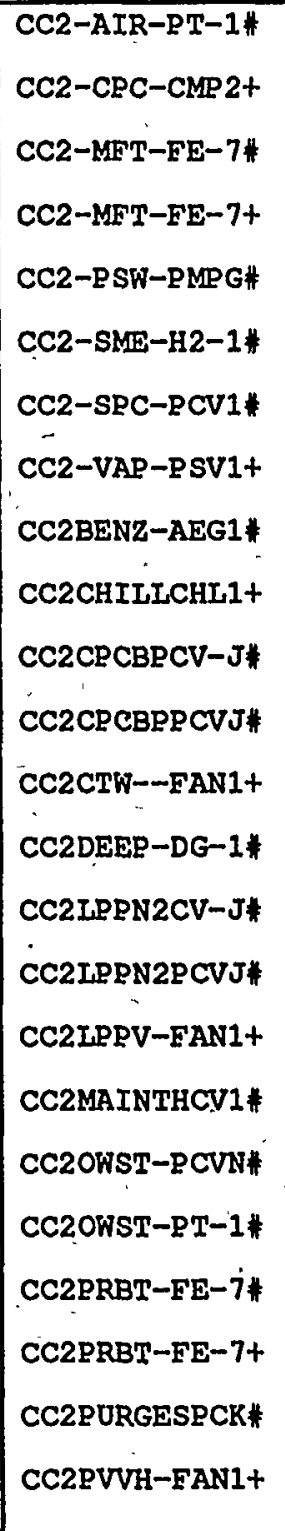 & $\begin{array}{l}1 \\
4 \\
1 \\
1 \\
1\end{array}$ & $\begin{array}{r}48 \mathrm{H} \\
1.0 \mathrm{E}-07 \mathrm{H} \\
7 \mathrm{D} \\
5.0 \mathrm{E}-06 \mathrm{H} \\
1 \mathrm{M} \\
3.0 \mathrm{E}-07 \mathrm{H} \\
1 \mathrm{M} \\
3.0 \mathrm{E}-07 \mathrm{H} \\
8 \mathrm{H} \\
3.0 \mathrm{E}-06 \mathrm{H} \\
1 \mathrm{M} \\
5.0 \mathrm{E}-06 \mathrm{H} \\
1 \mathrm{~N} \\
3.0 \mathrm{E}-04 \mathrm{~N} \\
4 \mathrm{H} \\
1.0 \mathrm{E}-06 \mathrm{H} \\
1 \mathrm{M} \\
5.0 \mathrm{E}-06 \mathrm{H} \\
8 \mathrm{H} \\
5.0 \mathrm{E}-06 \mathrm{H} \\
1 \mathrm{~N} \\
5.0 \mathrm{E}-05 \mathrm{~N} \\
1 \mathrm{~N} \\
3.0 \mathrm{E}-04 \mathrm{~N} \\
7 \mathrm{D} \\
3.0 \mathrm{E}-06 \mathrm{H} \\
1 \mathrm{~N} \\
3.0 \mathrm{E}-03 \mathrm{~N} \\
1 \mathrm{~N} \\
5.0 \mathrm{E}-05 \mathrm{~N} \\
1 \mathrm{~N} \\
3.0 \mathrm{E}-04 \mathrm{~N} \\
1 \mathrm{H} \\
3.0 \mathrm{E}-06 \mathrm{H} \\
1 \mathrm{M} \\
5.0 \mathrm{E}-08 \mathrm{H} \\
1 \mathrm{~N} \\
3.0 \mathrm{E}-04 \mathrm{~N} \\
1.0 \mathrm{Y}-07 \mathrm{H} \\
1 \mathrm{M} \\
3.0 \mathrm{E}-07 \mathrm{H} \\
1 \mathrm{M} \\
3.0 \mathrm{E}-07 \mathrm{H} \\
1 \mathrm{~N} \\
1.0 \mathrm{E}-04 \mathrm{~N} \\
8 \mathrm{H} \\
3.0 \mathrm{E}-06 \mathrm{H}\end{array}$ & \begin{tabular}{|l|}
$2.40 \mathrm{E}-06$ \\
$8.39 \mathrm{E}-04$ \\
$2.16 \mathrm{E}-04$ \\
$2.16 \mathrm{E}-04$ \\
$2.40 \mathrm{E}-05$ \\
$1.80 \mathrm{E}-03$ \\
$3.00 \mathrm{E}-04 \mathrm{~N}$ \\
$4.00 \mathrm{E}-06$ \\
$1.80 \mathrm{E}-03$ \\
$4.00 \mathrm{E}-05$ \\
$5.00 \mathrm{E}-05 \mathrm{~N}$ \\
$3.00 \mathrm{E}-04 \mathrm{~N}$ \\
$5.04 \mathrm{E}-04$ \\
$3.00 \mathrm{E}-03 \mathrm{~N}$ \\
$5.00 \mathrm{E}-05 \mathrm{~N}$ \\
$3.00 \mathrm{E}-04 \mathrm{~N}$ \\
$3.00 \mathrm{E}-06$ \\
$1.80 \mathrm{E}-05$ \\
$3.00 \mathrm{E}-04 \mathrm{~N}$ \\
$4.38 \mathrm{E}-04$ \\
$2.16 \mathrm{E}-04$ \\
$2.16 \mathrm{E}-04$ \\
$1.00 \mathrm{E}-04 \mathrm{~N}$ \\
$2.40 \mathrm{E}-05$ \\
\end{tabular} & $\begin{array}{l}\text { cc: both. instrument air PTs fail high } \\
\text { CC: Both CPC purge compressors fail } \\
\text { CC: Both MFT purge flow elements fail } \\
\text { high } \\
\text { CC: Both MFT purge flow elements fail } \\
\text { high } \\
\text { cc: both PSW pumps fail } \\
\text { cc: both SME hydrogen detectors fail } \\
\text { low or miscalibrated } \\
\text { cc: pR and pRFT vacuum breakers fail } \\
\text { to open } \\
\text { cc: PSVs on primary and backup } \\
\text { vaporizer fail open } \\
\text { cc: both PVVH benzene detectors fail } \\
\text { low both chillers fail } \\
\text { cc: both } \\
\text { cc: both check valves on cPC backup } \\
\text { purge system fail to open } \\
\text { CC: both CPC backup purge PCVs fail to } \\
\text { open } \\
\text { cc: two fans fail } \\
\text { Both deep well pumps (or diesel } \\
\text { motors) fail to start or run } \\
\text { cc: both LPPP N2 check valves fail to } \\
\text { close } \\
\text { cc: both LPPP N2 bottle PCVs fail to } \\
\text { open } \\
\text { CC: both LPPP vvs fans fail } \\
\text { cc: both PR steam valves miscalibrated } \\
\text { cc: both PCVs on owsT liquid N2 supply } \\
\text { system fail to open } \\
\text { cc: OwST pressure monitors both fail } \\
\text { high } \\
\text { cc: both PRBT flow elements fail high } \\
\text { cc: both PRBT flow elements fail high } \\
\text { cC: both SPC purge supply isolation } \\
\text { valves fail to close } \\
\text { cc: both PVvH fans fail }\end{array}$ & 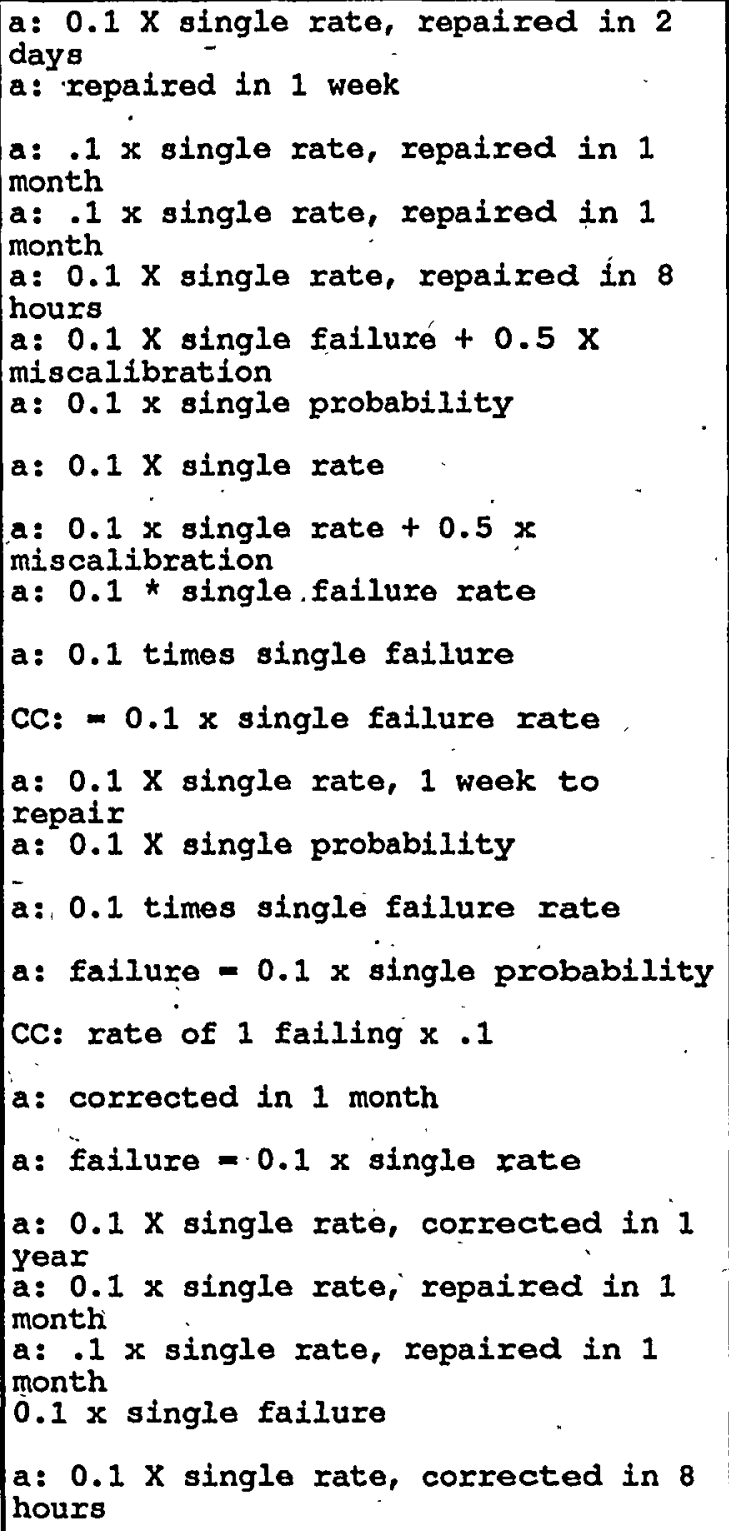 \\
\hline
\end{tabular}


Basic Event Report for I: \CAFTA IDATA MMASTER.BE 11/11/94 11:42 AM (CONT.)

\begin{tabular}{|c|c|c|c|c|c|}
\hline Event & C & nput & Calc. & Description & Source \\
\hline $\begin{array}{l}\text { CC2PVVH-FE-7\# } \\
\text { CC2PVVH-MOV1\# } \\
\text { CC2SBSWTRMNN" } \\
\text { CC2SCVC-O2-K\# } \\
\text { CC2SPCN2PCVN\# } \\
\text { CC2SPCN2RTH1\# } \\
\text { CC2SPCN2SV-N\# } \\
\text { CC2SPCN3PCVN\# } \\
\text { CC2SPCP-PT-K\# } \\
\text { CC2SPCPPLSL1\# } \\
\text { CC2SPCV-MOVK\# } \\
\text { CC2SPTN2LINJ\# } \\
\text { CC2SRAT-IFL5\# } \\
\text { CC2SRAT-SMP6\# } \\
\text { CC2STEAMHCV1+ } \\
\text { CC2STEAMPMPG+ } \\
\text { CC3-CTH-PMP1+ } \\
\text { CC3-SME-FSL4\# } \\
\text { CC3-SPC-FE-1\# } \\
\text { CC3CO2B-REG1\# } \\
\text { CC3CO2B-RMH1\# } \\
\text { CC3MAINTFSL1\# } \\
\text { CC3SRAT-FSLD\# } \\
\text { CHLBACK-FTRE\# }\end{array}$ & 3 & $\begin{array}{r}1 \mathrm{M} \\
3.0 \mathrm{E}-07 \mathrm{H} \\
1 \mathrm{~N} \\
3.0 \mathrm{E}-04 \mathrm{~N} \\
4.3 \mathrm{E}-02 \mathrm{~N} \\
5.0 \mathrm{E}-03 \mathrm{~N} \\
1 \mathrm{~N} \\
5.0 \mathrm{E}-04 \mathrm{~N} \\
1 \mathrm{~N} \\
3.0 \mathrm{E}-04 \mathrm{~N} \\
.12 \mathrm{~N} \\
3.0 \mathrm{E}-03 \mathrm{~N} \\
1 \mathrm{~N} \\
1.0 \mathrm{E}-04 \mathrm{~N} \\
1 \mathrm{~N} \\
3.0 \mathrm{E}-04 \mathrm{~N} \\
7 \mathrm{D} \\
1.0 \mathrm{E}-07 \mathrm{H} \\
1 \mathrm{~N} \\
3.0 \mathrm{E}-03 \mathrm{~N} \\
1 \mathrm{~N} \\
3.0 \mathrm{E}-04 \mathrm{~N} \\
1 \mathrm{~N} \\
4.1 \mathrm{E}-04 \mathrm{~N} \\
1 \mathrm{M} \\
5.0 \mathrm{E}-06 \mathrm{H} \\
1 \mathrm{~N} \\
3.0 \mathrm{E}-05 \mathrm{~N} \\
4 \mathrm{H} \\
5.0 \mathrm{E}-08 \mathrm{H} \\
8 \mathrm{H} \\
3.0 \mathrm{E}-06 \mathrm{H} \\
4 \mathrm{H} \\
1.5 \mathrm{E}-06 \mathrm{H} \\
1 \mathrm{~N} \\
1.1 \mathrm{E}-04 \mathrm{~N} \\
7 \mathrm{D} \\
1.5 \mathrm{E}-07 \mathrm{H} \\
48 \mathrm{H} \\
1.5 \mathrm{E}-07 \mathrm{H} \\
1 \mathrm{~N} \\
2.5 \mathrm{E}-03 \mathrm{~N} \\
.2 \mathrm{~N} \\
1.5 \mathrm{E}-03 \mathrm{~N} \\
1 \mathrm{~N} \\
1.1 \mathrm{E}-04 \mathrm{~N} \\
8 \mathrm{H} \\
5.0 \mathrm{E}-05 \mathrm{H}\end{array}$ & $\begin{array}{l}3.00 \mathrm{E}-04 \mathrm{~N} \\
2.15 \mathrm{E}-04 \mathrm{~N} \\
5.00 \mathrm{E}-04 \mathrm{~N} \\
3.00 \mathrm{E}-04 \mathrm{~N} \\
3.60 \mathrm{E}-04 \mathrm{~N} \\
1.00 \mathrm{E}-04 \mathrm{~N} \\
3.00 \mathrm{E}-04 \mathrm{~N} \\
8.40 \mathrm{E}-06 \\
3.00 \mathrm{E}-03 \mathrm{~N} \\
3.00 \mathrm{E}-04 \mathrm{~N} \\
4.10 \mathrm{E}-04 \mathrm{~N} \\
1.80 \mathrm{E}-03 \\
3.00 \mathrm{E}-05 \mathrm{~N} \\
2.00 \mathrm{E}-07 \\
2.40 \mathrm{E}-05 \\
6.00 \mathrm{E}-06 \\
1.10 \mathrm{E}-04 \mathrm{~N} \\
2.52 \mathrm{E}-05 \\
3.60 \mathrm{E}-06 \\
2.50 \mathrm{E}-03 \mathrm{~N} \\
3.00 \mathrm{E}-04 \mathrm{~N} \\
1.10 \mathrm{E}-04 \mathrm{~N} \\
4.00 \mathrm{E}-04\end{array}$ & 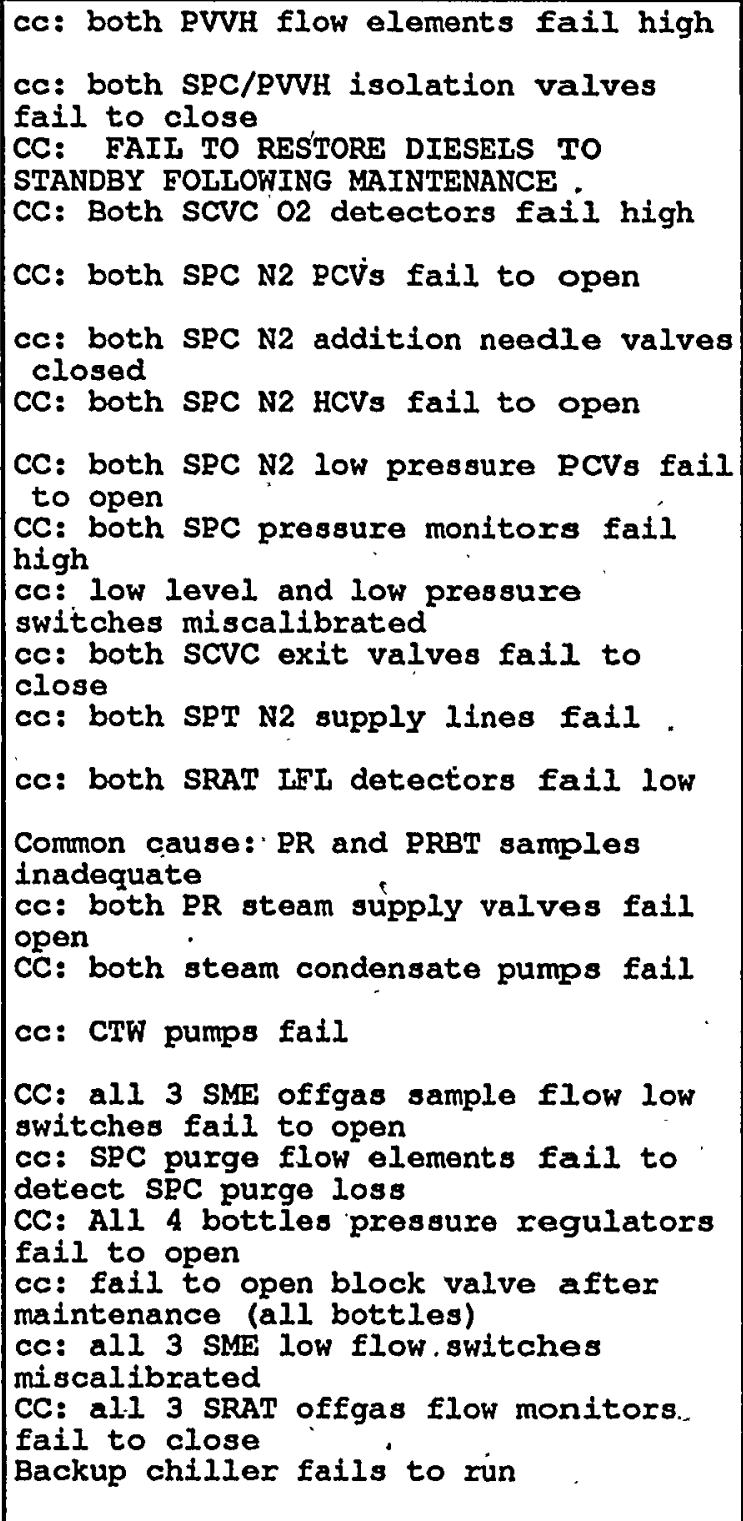 & 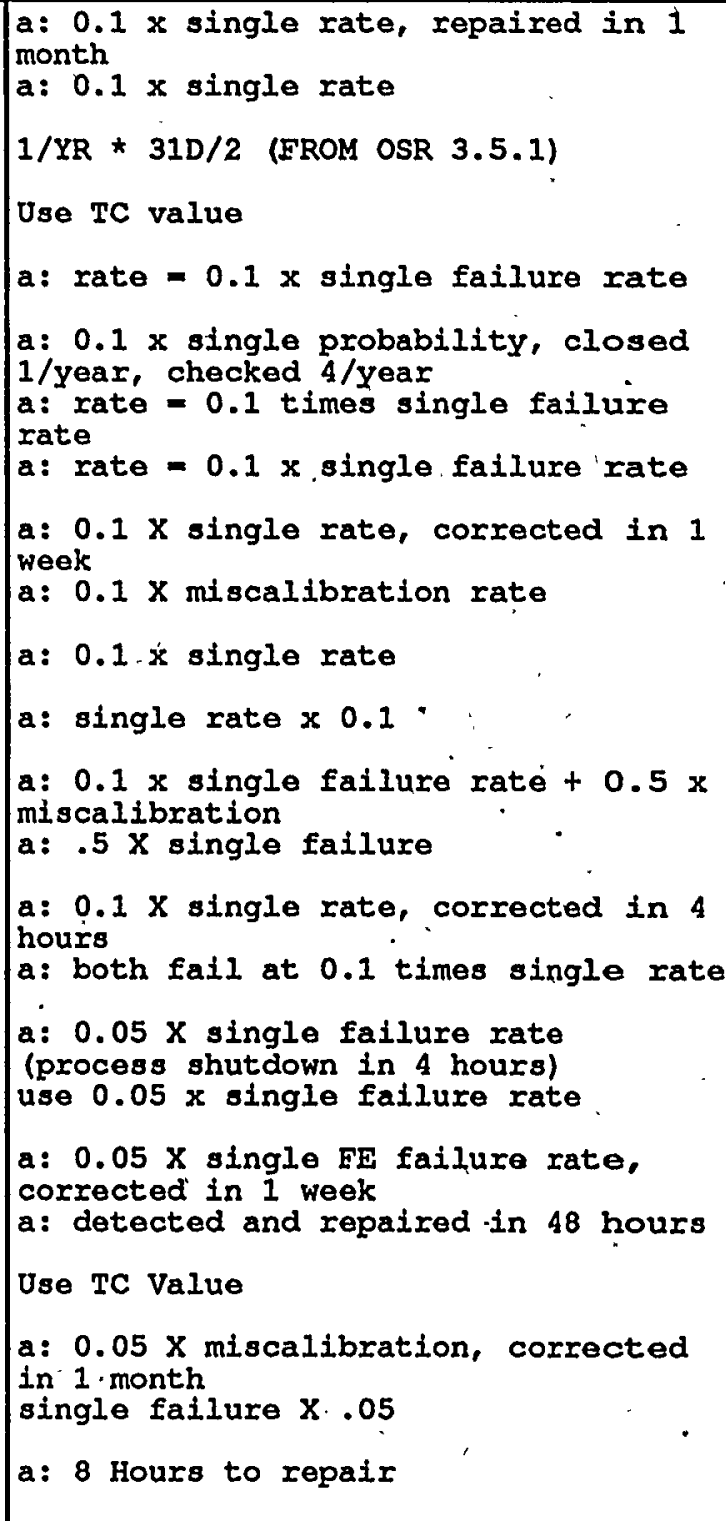 \\
\hline
\end{tabular}


Basic Event Report for I: \CAFTA DATAMIMASTER.BE 11/11/94 11:42 AM (CONT.)

\begin{tabular}{|l|}
\hline EVENT \\
\hline CHLBACK-FTSE\# \\
CHLBACK-T \&ME\# \\
CHLPRIM-BFLE+ \\
CMPAIR1-BFLV+ \\
CMPAIR2-FTRV+ \\
CMPAIR3-FTRV\# \\
CMPAIR3-FTSV\# \\
CMPAIR3-T\&MV\# \\
CMPCPC1-BEL2+ \\
CMPCPC2-FTR2\# \\
CMPCPC2-FTS2\# \\
CMPCPC2-T\&M2\# \\
CMPINAIRC3CV+ \\
CND-026-IKSG\# \\
CO2BOTTLCHK1\# \\
CON--OE-PREG\# \\
CON--PR-PREG\# \\
CON-SME-PREG\# \\
CONDWTT-PREG\# \\
CONSRAT-PREG\# \\
CP--TF--CC-U\# \\
CP-BATCHCC1U\# \\
CP-DWPF1CCIU\# \\
CP-DWPE2CC1U\# \\
\end{tabular}

Calc. \begin{tabular}{l|l} 
& Dèscription \\
\hline $5.00 \mathrm{E}-03 \mathrm{n}$ & Backup
\end{tabular} $5.0 \mathrm{E}-03 \mathrm{~N}$ $4.0 \mathrm{E}+00 \mathrm{Y}$ $5.0 \mathrm{E}-05 \mathrm{H}$ 5. $0 \mathrm{E}-05 \mathrm{H}$ 5. $0 \mathrm{E}-05 \mathrm{H}$ 5. $0 \mathrm{E}-05 \mathrm{H}$

3.65E-03 Backup chiller unavailable due to T\&M

4.00E-04 Primary chiller fails

$1.20 \mathrm{E}-03$

$1.20 \mathrm{E}-03$

Primary plant/instrument air compressor fails

Secondary plant / instrument air compressor fails.

2.40E-03 Third plant / instrument ais compressor fails to run

Third plant / instrument air.

compressor fails to start

$5.0 \mathrm{E}-03 \mathrm{~N}$

27D $7.130-02$

$7.13 \mathrm{E}-02$

$4.00 \mathrm{E}-04$

$5.0 \mathrm{E}-05 \mathrm{H}$

5. $0 \mathrm{E}-05 \mathrm{H}$

$4.79 \mathrm{E}-03$

T\&M

Operating purge air compressor fails

$1 \mathrm{~N}$

$5.00 \mathrm{E}-03 \mathrm{~N}$

Standby purge air compressor fails to

run

3.00E-02N start

$6.00 \mathrm{E}-05$

starge air compressor out of

service for testing or maintenance

CC: three plant/instrument air

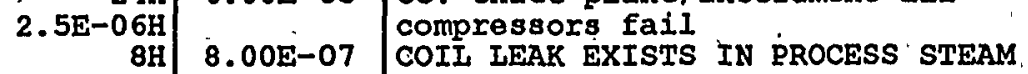

$1.0 \mathrm{E}-07 \mathrm{H}$

3. $0 \mathrm{E}-04 \mathrm{H}$

4.92E-02 Fail to correct wrong bottles before

Fail to correct

1.00E+OON CONTAMINATION PROBABILITY GIVEN COIL

$1.0 \mathrm{~N}$

$1 \mathrm{~N} 1.00 \mathrm{D}$ LEAK

$1.0 \mathrm{~N}$

$1 \mathrm{~N} 1.00 \mathrm{E}+00 \mathrm{~N}$ LEAK

1. ON

$1.0 \mathrm{~N}$

$1.0 \mathrm{~N}$

$1 \mathrm{~N}$

2. $0 \mathrm{E}-01 \mathrm{~N}$

2. $0 \mathrm{E}-01 \mathrm{~N}$

. $2.0 \mathrm{E}-02 \mathrm{~N}$

2. $0 \mathrm{E}-01 \mathrm{~N}$
IEAKR

CONTAR

$1.00 \mathrm{E}+00 \mathrm{~N}$

tanks 48 \& 49 conducts sampling at

Same personnel conduct sampling at

SRAT for batch \#2

.00E-01N Same personnel conduct sampling from SRAT to SME for batch \#1

2.00E-01N Same personnel conduct sampling from SRAT to SME for batch $\# 2$

\section{Source}

Use TC value

a: out of service for ' 8 hours

a: 8 Hours to repair

a: 24 hour repair time

a: repaired in 24 hours

a: mission time -48 hours

Use. TC value

a: 9 days per year (times 3 )

a: 8 hour repaix time

a: max repair time for primary

compressor is 96 hours.

Use value from TC file

Use TC value

a: 24 hour repair time

a: 8 hours to repaix

a: detected in one week

a: contamination occurs

a: contamination occurs

a: contamination occurs

a: contamination occurs

a: contamination occurs

Use TC

Use TC

Use TC 


\begin{tabular}{|c|c|c|c|c|c|}
\hline Event & C & aput & Calc. & Description & Source \\
\hline 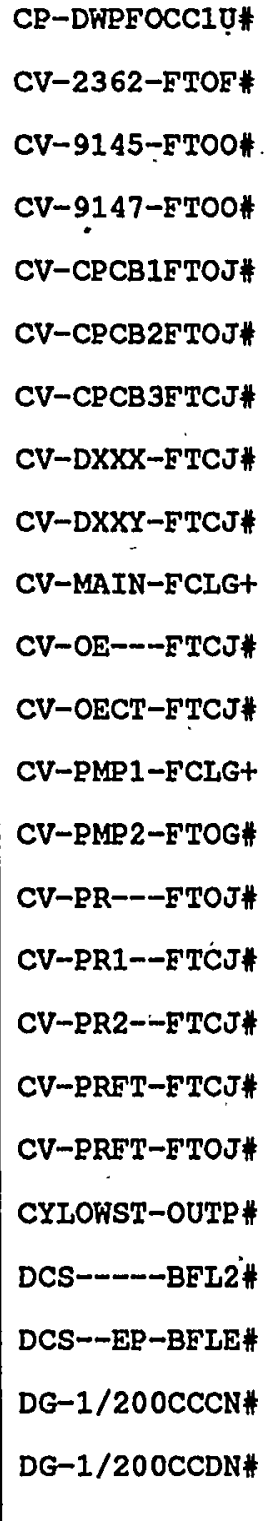 & 1 & $\begin{array}{r}1 \mathrm{~N} \\
2.0 \mathrm{E}-01 \mathrm{~N} \\
1 \mathrm{~N} \\
5.0 \mathrm{E}-05 \mathrm{~N} \\
1 \mathrm{~N} \\
5.0 \mathrm{E}-05 \mathrm{~N} \\
1 \mathrm{~N} \\
5.0 \mathrm{E}-05 \mathrm{~N} \\
1 \mathrm{~N} \\
5.0 \mathrm{E}-04 \mathrm{~N} \\
1 \mathrm{~N} \\
5.0 \mathrm{E}-04 \mathrm{~N} \\
1 \mathrm{~N} \\
5.0 \mathrm{E}-04 \mathrm{~N} \\
1 \mathrm{~N} \\
5.0 \mathrm{E}-04 \mathrm{~N} \\
1 \mathrm{~N} \\
5.0 \mathrm{E}-04 \mathrm{~N} \\
8 \mathrm{H} \\
5.0 \mathrm{E}-07 \mathrm{H} \\
1 \mathrm{~N} \\
5.0 \mathrm{E}-04 \mathrm{~N} \\
1 \mathrm{~N} \\
5.0 \mathrm{E}-04 \mathrm{~N} \\
8 \mathrm{H} \\
5.0 \mathrm{E}-07 \mathrm{H} \\
1 \mathrm{~N} \\
5.0 \mathrm{E}-05 \mathrm{~N} \\
1 \mathrm{~N} \\
5.0 \mathrm{E}-04 \mathrm{~N} \\
1 \mathrm{~N} \\
5.0 \mathrm{E}-04 \mathrm{~N} \\
1 \mathrm{~N} \\
5.0 \mathrm{E}-04 \mathrm{~N} \\
1 \mathrm{~N} \\
5.0 \mathrm{E}-04 \mathrm{~N} \\
1 \mathrm{~N} \\
5.0 \mathrm{E}-04 \mathrm{~N} \\
1 \mathrm{~N} \\
3.6 \mathrm{E}-01 \mathrm{~N} \\
8 \mathrm{H} \\
3.0 \mathrm{E}-06 \mathrm{H} \\
-14 \mathrm{D} \\
3.0 \mathrm{E}-06 \mathrm{H} \\
1 \mathrm{~N} \\
1.0 \mathrm{E}-03 \mathrm{~N} \\
8.5 \mathrm{H} \\
5.0 \mathrm{E}-04 \mathrm{H}\end{array}$ & $\begin{array}{l}2.00 \mathrm{E}-01 \mathrm{~N} \\
5.00 \mathrm{E}-05 \mathrm{~N} \\
5.00 \mathrm{E}-05 \mathrm{~N} \\
5.00 \mathrm{E}-05 \mathrm{~N} \\
5.00 \mathrm{E}-04 \mathrm{~N} \\
5.00 \mathrm{E}-04 \mathrm{~N} \\
5.00 \mathrm{E}-04 \mathrm{~N} \\
5.00 \mathrm{E}-04 \mathrm{~N} \\
5.00 \mathrm{E}-04 \mathrm{~N} \\
4.00 \mathrm{E}-06 \\
5.00 \mathrm{E}-04 \mathrm{~N} \\
5.00 \mathrm{E}-04 \mathrm{~N} \\
4.00 \mathrm{E}-06 \\
5.00 \mathrm{E}-05 \mathrm{~N} \\
5.00 \mathrm{E}-04 \mathrm{~N} \\
.5 .00 \mathrm{E}-04 \mathrm{~N} \\
5.00 \mathrm{E}-04 \mathrm{~N} \\
5.00 \mathrm{E}-04 \mathrm{~N} \\
5.00 \mathrm{E}-04 \mathrm{~N} \\
3.60 \mathrm{E}-01 \mathrm{~N} \\
1.20 \mathrm{E}-05 \\
5.04 \mathrm{E}-04 \\
1.00 \mathrm{E}-03 \mathrm{~N} \\
4.24 \mathrm{E}-03\end{array}$ & 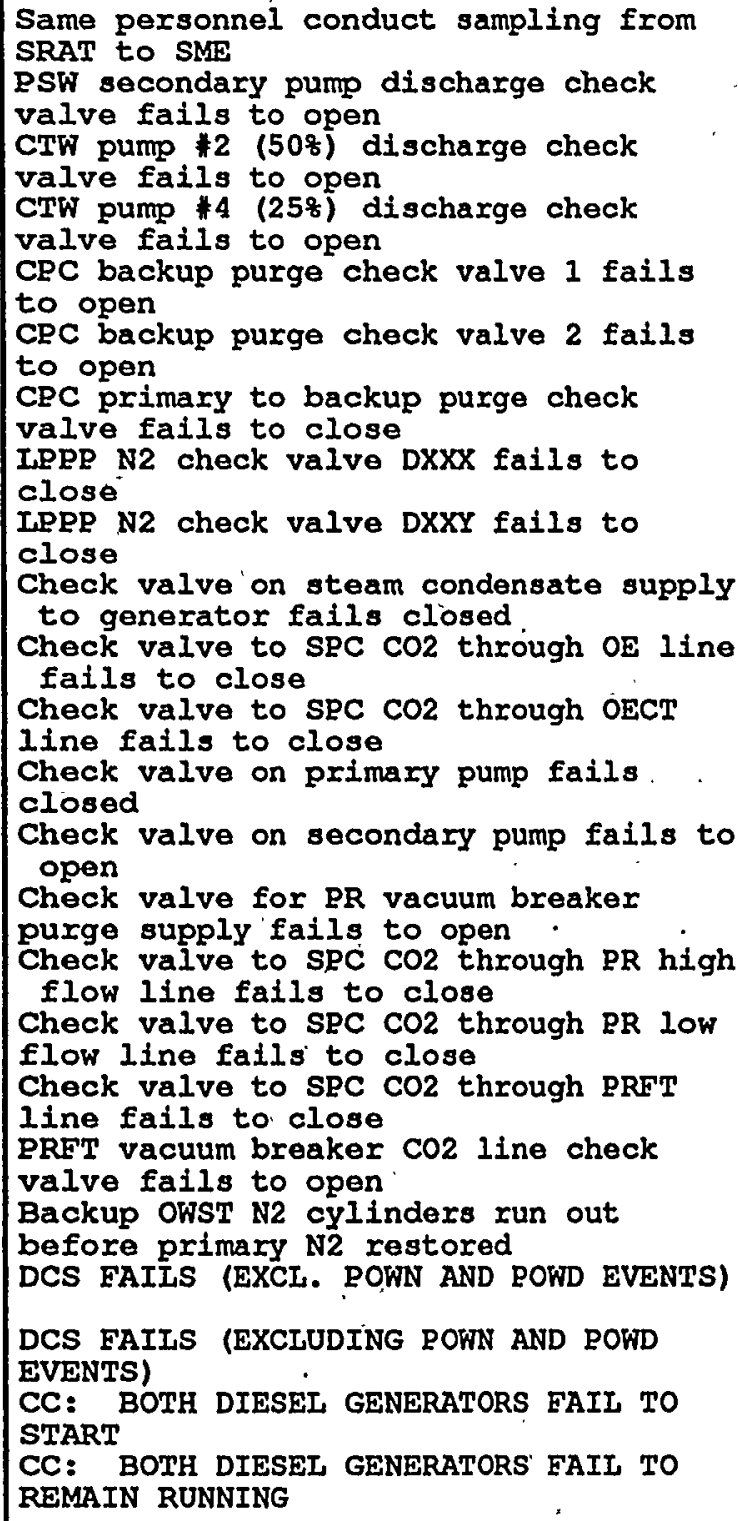 & 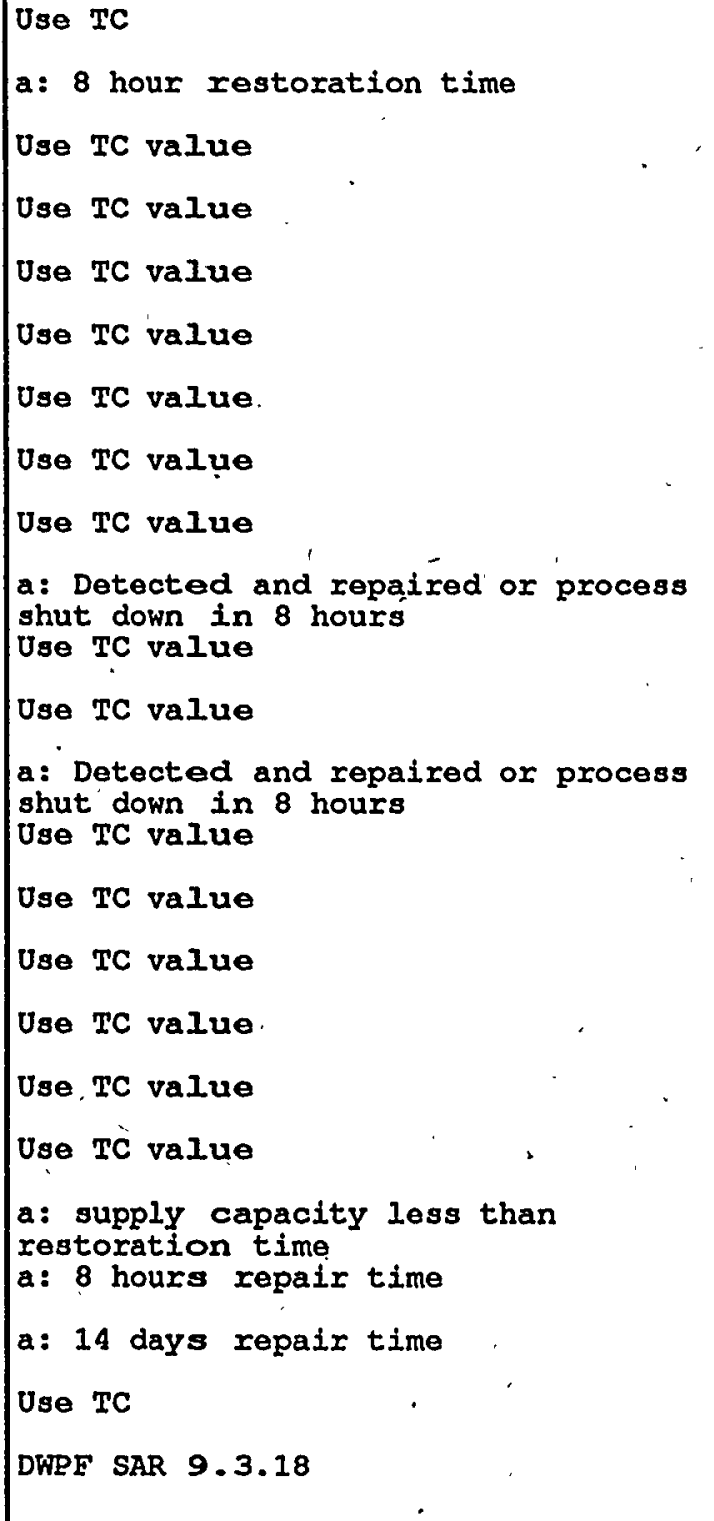 \\
\hline
\end{tabular}




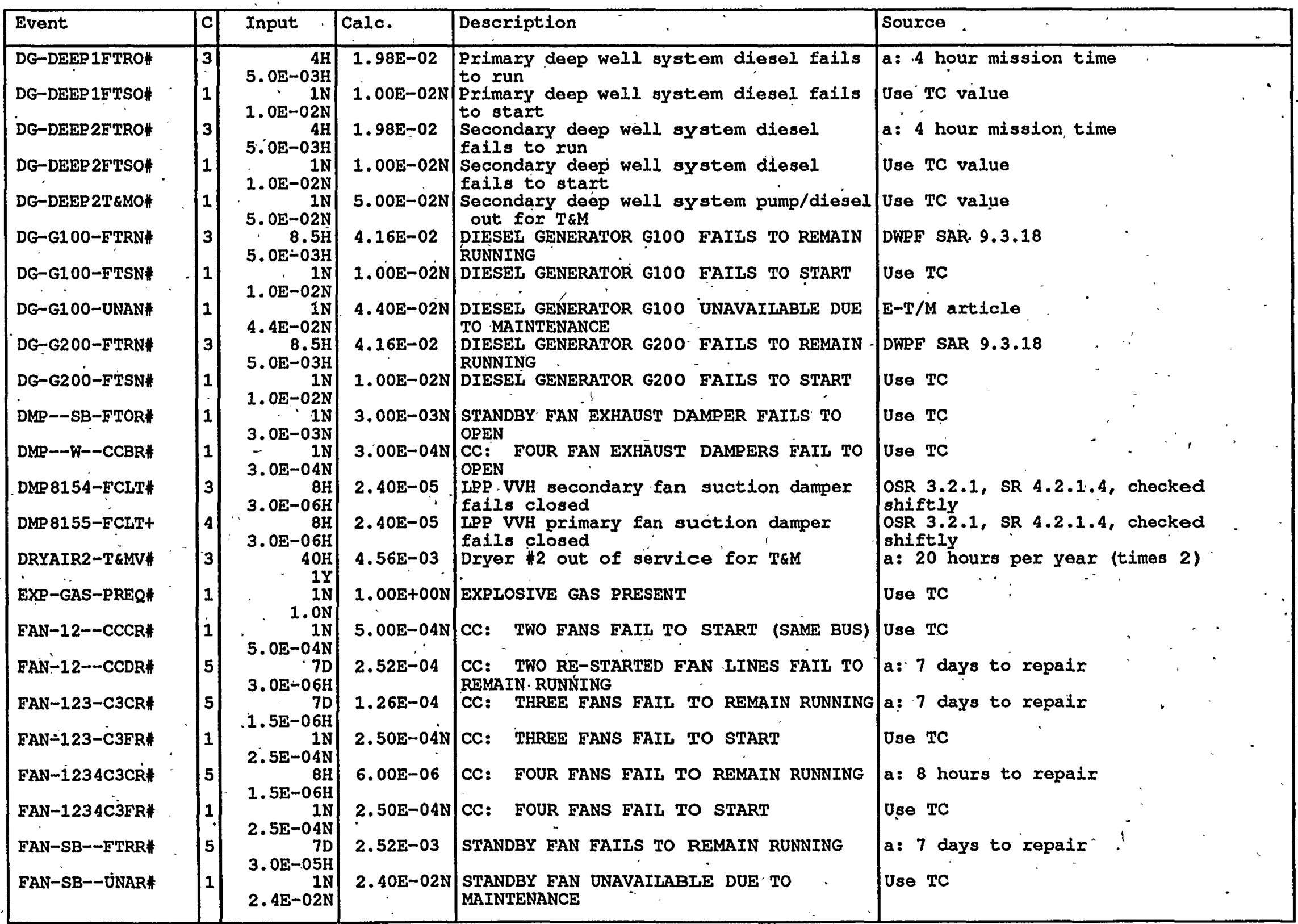


Basic Event Report for I: \CAFTA \DATA

\begin{tabular}{|c|c|c|c|c|c|}
\hline Event & c & put & Calc. & Description & Source \\
\hline 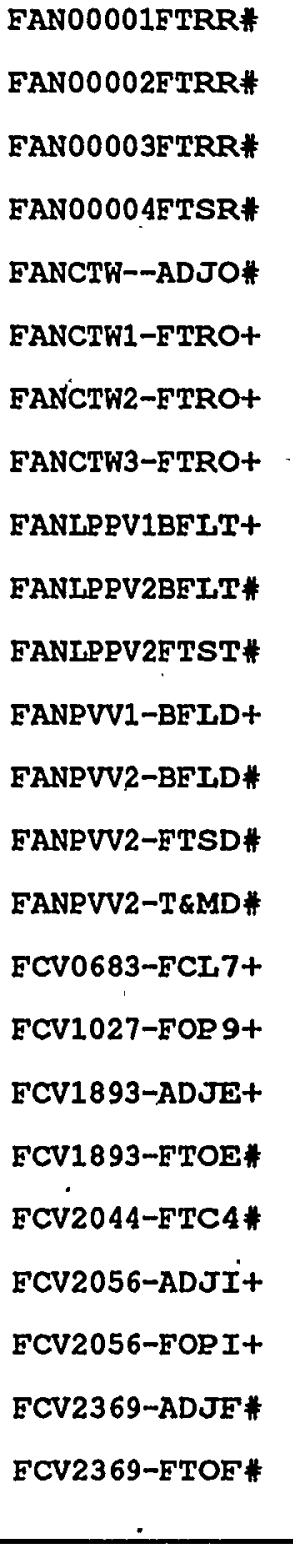 & 3 & $\begin{array}{r}96 \mathrm{H} \\
3.0 \mathrm{E}-05 \mathrm{H} \\
7 \mathrm{D} \\
3.0 \mathrm{E}-05 \mathrm{H} \\
3 \mathrm{M} \\
3.0 \mathrm{E}-05 \mathrm{H} \\
1 \mathrm{~N} \\
5.0 \mathrm{E}-03 \mathrm{~N} \\
1 \mathrm{~N} \\
5.0 \mathrm{E}-0 \mathrm{~N} \\
7 \mathrm{D} \\
3.0 \mathrm{E}-05 \mathrm{H} \\
7 \mathrm{D} \\
3.0 \mathrm{E}-05 \mathrm{H} \\
7 \mathrm{D} \\
3.0 \mathrm{E}-05 \mathrm{H} \\
8 \mathrm{H} \\
3.0 \mathrm{E}-05 \mathrm{H} \\
8 \mathrm{H} \\
3.0 \mathrm{E}-05 \mathrm{H} \\
1 \mathrm{~N} \\
5.0 \mathrm{E}-03 \mathrm{~N} \\
24 \mathrm{H} \\
3.0 \mathrm{E}-05 \mathrm{H} \\
1 \mathrm{D} \\
3.0 \mathrm{E}-05 \mathrm{H} \\
1 \mathrm{~N} \\
5.0 \mathrm{E}-03 \mathrm{~N} \\
8 \mathrm{H} \\
4 \mathrm{Y} \\
8 \mathrm{H} \\
1.0 \mathrm{E}-06 \mathrm{H} \\
86 \mathrm{H} \\
1.0 \mathrm{E}-06 \mathrm{H} \\
1 \mathrm{D} \\
1.00 \\
1 \mathrm{~N} \\
1.0 \mathrm{E}-03 \mathrm{~N} \\
1 \mathrm{~N} \\
1.0 \mathrm{E}-03 \mathrm{~N} \\
1 \mathrm{H} \\
2.3 \mathrm{E}-02 \mathrm{H} \\
8 \mathrm{H} \\
1.0 \mathrm{E}-06 \mathrm{H} \\
1 \mathrm{~N} \\
1.0 \mathrm{E}-03 \mathrm{~N} \\
1 \mathrm{~N} \\
1.03 \mathrm{~N}\end{array}$ & $\begin{array}{l}2.88 \mathrm{E}-03 \\
2.52 \mathrm{E}-03 \\
3.17 \mathrm{E}-02 \\
5.00 \mathrm{E}-03 \mathrm{~N} \\
5.00 \mathrm{E}-01 \mathrm{~N} \\
5.01 \mathrm{E}-03 \\
5.01 \mathrm{E}-03 \\
5.01 \mathrm{E}-03 \\
2.40 \mathrm{E}-04 \\
2.40 \mathrm{E}-04 \\
5.00 \mathrm{E}-03 \mathrm{~N} \\
7.19 \mathrm{E}-04 \\
7.20 \mathrm{E}-04 \\
5.00 \mathrm{E}-03 \mathrm{~N} \\
3.65 \mathrm{E}-03 \\
8.00 \mathrm{E}-06 \\
8.60 \mathrm{E}-05 \\
1.09 \mathrm{E}-02 \\
1.00 \mathrm{E}-03 \mathrm{~N} \\
1.00 \mathrm{E}-03 \mathrm{~N} \\
2.25 \mathrm{E}-02 \\
8.00 \mathrm{E}-06 \\
1.00 \mathrm{E}-03 \mathrm{~N} \\
1.00 \mathrm{E}-03 \mathrm{~N}\end{array}$ & 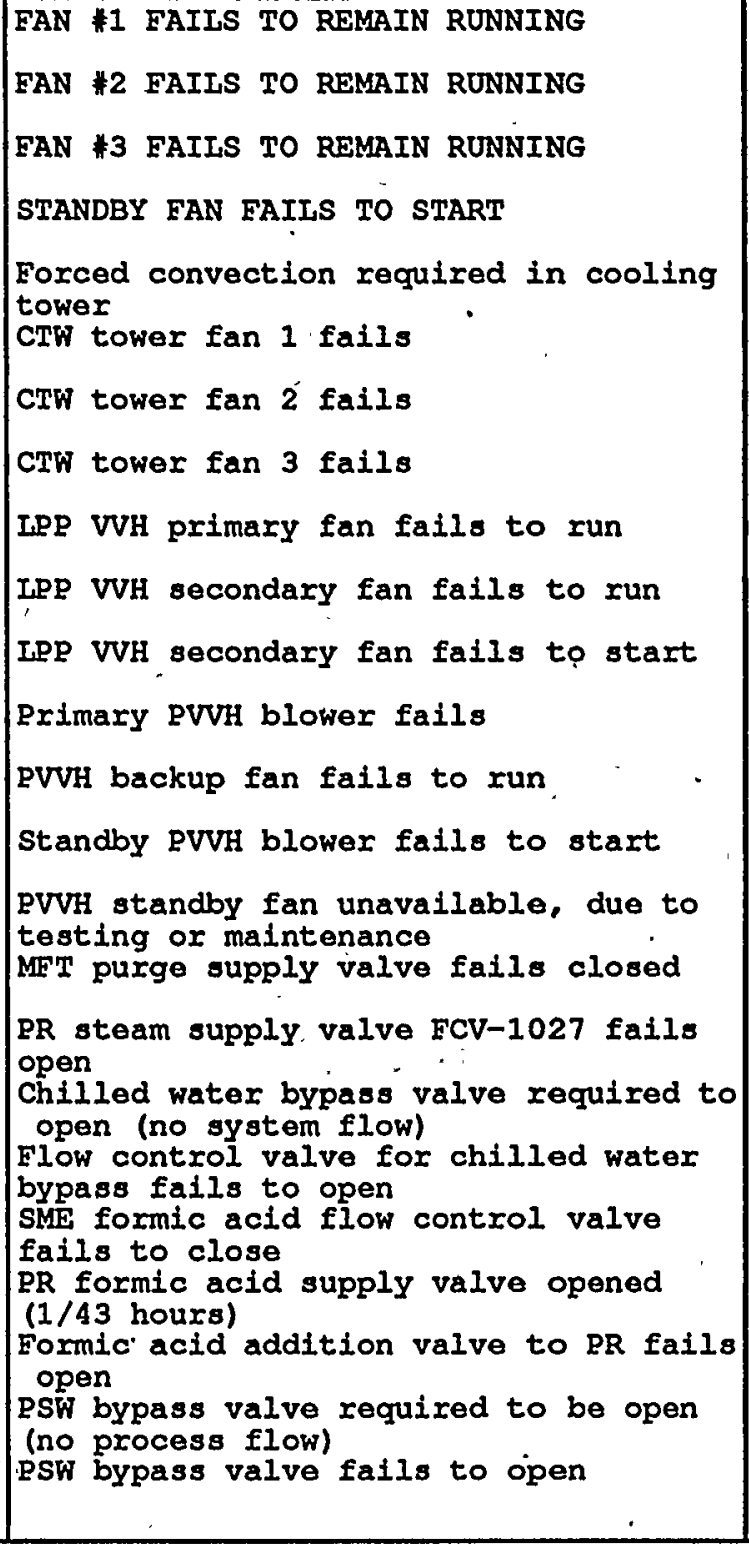 & $\begin{array}{l}\text { a: } 96 \text { hr mission time (bounded by } \\
\text { seismic event) } \\
\text { a: } 7 \text { days to repair } \\
\text { a: } 3 \text { months to repair } \\
\text { Use TC } \\
\text { Use TC value } \\
\text { a: detected and repaired in } 1 \text { week } \\
\text { a: detected and repaired in } 1 \text {. week } \\
\text { a: detected and repaired in } 1 \text { week } \\
\text { OsR } 3.2 .1 \text {, sR } 4.2 .1 .1 \text {, checked } \\
\text { shiftiy } \\
\text { a: Primary fan repaired, or shutdown } \\
\text { within } 8 \text { hours } \\
\text { Use TC value. } \\
\text { a: } 24 \text { hours to repair } \\
\text { a: } 24 \text { hour mission time. } \\
\text { Use TC value } \\
\text { a: out } 8 \text { hours per quarter } \\
\text { a: } 8 \text { hours to repair } \\
\text { a: detected and corrected before next. } \\
\text { batch } \\
\text { a: demand lasts for } 1 \text { day } \\
\text { a: checked annually } \\
\text { Use TC value . } \\
\text { a: valve remains open } 1 \text { hour } \\
\text { a: } 8 \text { hours to repair } \\
\text { Use TC value } \\
\text { a: checked annually }\end{array}$ \\
\hline
\end{tabular}


Basic Event Report for I: \CAFTA \DATA MASTER.BE 11/11/94 11:42 AM (CONT.)

\begin{tabular}{|c|c|c|c|c|c|}
\hline Eve & 0 & det & $\therefore$ & iption & $\theta$ \\
\hline $\begin{array}{l}\text { FCV3216-ADJ6+ } \\
\text { FCV3216-FCL6+ } \\
\text { FCV8150-ADJ1+ } \\
\text { ECV8150-FCLT+ } \\
\text { FCV8150-FORT+ } \\
\text { FCV8856-FCL4t } \\
\text { FCV9152-FORO+ } \\
\text { FCV9301-FTC9\# } \\
\text { FE-0682-FHI7* }\end{array}$ & 4 & $\begin{array}{r}8 \mathrm{H} \\
1.0 \mathrm{E}-06 \mathrm{H} \\
86 \mathrm{H} \\
3.0 \mathrm{E}-06 \mathrm{H} \\
8 \mathrm{H} \\
1.0 \mathrm{E}-06 \mathrm{H} \\
1 \mathrm{~N} \\
1.0 \mathrm{E}-03 \mathrm{H} \\
1 \mathrm{H} \\
2.3 \mathrm{E}-02 \mathrm{H} \\
1 \mathrm{H} \\
1.0 \mathrm{E}-06 \mathrm{H} \\
7.0 \mathrm{D} \\
4.0 \mathrm{E}+0 \mathrm{OY} \\
1 \mathrm{H} \\
1.0 \mathrm{E}-06 \mathrm{H} \\
7 \mathrm{D} \\
1.0 \mathrm{E}-06 \mathrm{H} \\
8 \mathrm{H} \\
1.0 \mathrm{E}-06 \mathrm{H} \\
8 \mathrm{H} \\
1.0 \mathrm{E}-06 \mathrm{H} \\
1 \mathrm{H} \\
1.0 \mathrm{E}-03 \mathrm{~N} \\
86 \mathrm{H} \\
3.0 \mathrm{E}-06 \mathrm{H} \\
86 \mathrm{H} \\
3.0 \mathrm{E}-06 \mathrm{H} \\
86 \mathrm{H} \\
3.0 \mathrm{E}-06 \mathrm{H} \\
86 \mathrm{H} \\
3.0 \mathrm{E}-06 \mathrm{H} \\
1 \mathrm{M} \\
3.0 \mathrm{E}-06 \mathrm{H} \\
1 \mathrm{M} \\
3.0 \mathrm{E}-06 \mathrm{H} \\
1 \mathrm{Y} \\
3.0 \mathrm{E}-06 \mathrm{H} \\
8 \mathrm{H} \\
3.0 \mathrm{E}-06 \mathrm{H} \\
8 \mathrm{H} \\
3.0 \mathrm{E}-06 \mathrm{H} \\
8 \mathrm{H} \\
3.0 \mathrm{E}-06 \mathrm{H} \\
10 \\
3.0 \mathrm{E}-06 \mathrm{H} \\
86 \mathrm{H} \\
3.0 \mathrm{E}-06 \mathrm{H}\end{array}$ & $\begin{array}{l}1.29 \mathrm{E}-04 \\
8.00 \mathrm{E}-06 \\
1.00 \mathrm{E}-03 \mathrm{~N} \\
2.25 \mathrm{E}-02 \\
1.00 \mathrm{E}-06 \\
7.12 \mathrm{E}-02 \\
1.00 \mathrm{E}-06 \\
1.68 \mathrm{E}-04 \\
8.00 \mathrm{E}-06 \\
8.00 \mathrm{E}-06 \\
1.00 \mathrm{E}-03 \mathrm{~N} \\
2.58 \mathrm{E}-04 \\
2.58 \mathrm{E}-04 \\
2.58 \mathrm{E}-04 \\
2.58 \mathrm{E}-04 \\
2.16 \mathrm{E}-03 \\
2.16 \mathrm{E}-03 \\
1.30 \mathrm{E}-02 \\
2.40 \mathrm{E}-05 \\
2.40 \mathrm{E}-05 \\
2.40 \mathrm{E}-05 \\
6.52 \mathrm{E}-03 \\
2.58 \mathrm{E}-04 \\
\end{array}$ & $\begin{array}{l}\text { SRAT steam supply valve fails to close } \\
\text { SRAT purge flow control valve fails } \\
\text { closed } \\
\text { SME steam supply valve fails to close } \\
\text { PRBT dilution flow control, valve } \\
\text { adjusted } \\
\text { PRBT dilution supply valve fails } \\
\text { closed } \\
\text { Flow through FCV } 8150 \text { adjusted } \\
\text { IPP vvH flow control valve } 8150 \text { fails } \\
\text { closed } \\
\text { LOw dilution because process cell flow } \\
\text { control valve fails open } \\
\text { SMT purge flow control valve fails } \\
\text { Closed } \\
\text { BYpass valve fails open } \\
\text { OE steam, flow control valve fails to } \\
\text { close } \\
\text { MFT purge flow element } 0682 \text { fails high } \\
\text { MFT purge flow element } 0682 \text { fails high } \\
\text { MFT purge flow element } 0684 \text { fails high } \\
\text { MFT purge flow element } 0684 \text { fails high } \\
\text { PRFT purge flow element } 1018 \text { fails to } \\
\text { detect low flow } \\
\text { PR purge flow element } 1103 \text { fails to } \\
\text { detect low flow } \\
\text { Flow element for chilled water flow } \\
\text { fails high } \\
\text { Chilled water flow element } 1893 \text { fails } \\
\text { low (stops pumps) fails high (closes } \\
\text { PSW flow element fails } \\
\text { CTw valve) } \\
\text { PSW flow element fails low } \\
\text { SRAT purge flow element or transmitter } \\
\text { fails high } \\
\text { SRAT purge flow element fails high }\end{array}$ & $\begin{array}{l}\text { a: } 8 \text { hours to repair } \\
\text { a: corrected before next batch } \\
\text { a: } 8 \text { hours to repair } \\
\text { Use TC value } \\
\text { a: detected within } 1 \text { hour } \\
\text { a: } 1 \text { hour to detect (low flow alarm) } \\
\text { and shutdown } \\
\text { a: adjusted quarterly } \\
\text { a: responded to and shutdown or } \\
\text { repaired within } 1 \text { hour } \\
\text { a: detected and corrected in } 1 \text { week } \\
\text { a: } 8 \text { hours to repair. } \\
\text { Detected and corrected in } 8 \text { hoirs } \\
\text { Use rc value } \\
\text { a: repaired before next batch } \\
\text { a: repaired before next batch } \\
\text { a: repaired before next batch } \\
\text { a: repaired before next batch } \\
\text { a: checked monthly } \\
\text { a: checked monthly } \\
\text { a: checked annually } \\
\text { a: element repaired in } 8 \text { hours } \\
\text { a: detected and repaired in } 8 \text { hours } \\
\text { a: detected and repaired in } 8 \text { hours } \\
\text { a: checked twice/year } \\
\text { batch }\end{array}$ \\
\hline
\end{tabular}


Basic Event Report for I: \CAFTA IDATA MASTER.BE 11/11/94 11:42 AM (CONT.)

\begin{tabular}{|c|c|c|c|c|c|}
\hline Event & \begin{tabular}{|l|l|}
$c$ & \\
\end{tabular} & Input & Calc. & escription & Source \\
\hline 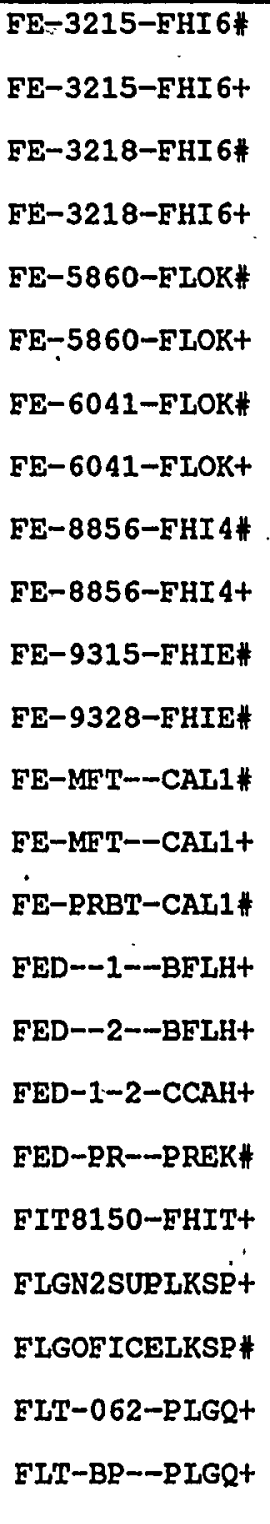 & 3 & $\begin{array}{r}8 \mathrm{8} 6 \mathrm{H} \\
3.0 \mathrm{E}-06 \mathrm{H} \\
86 \mathrm{H} \\
3.0 \mathrm{E}-06 \mathrm{H} \\
86 \mathrm{H} \\
3.0 \mathrm{E}-06 \mathrm{H} \\
86 \mathrm{H} \\
3.0 \mathrm{E}-06 \mathrm{H} \\
86 \mathrm{H} \\
3.0 \mathrm{E}-06 \mathrm{H} \\
8 \mathrm{H} \\
3.0 \mathrm{E}-06 \mathrm{H} \\
86 \mathrm{H} \\
3.0 \mathrm{E}-06 \mathrm{H} \\
8 \mathrm{H} \\
3 . \mathrm{OE}-06 \mathrm{H} \\
86 \mathrm{H} \\
3.0 \mathrm{E}-06 \mathrm{H} \\
86 \mathrm{H} \\
3.0 \mathrm{E}-06 \mathrm{H} \\
1 \mathrm{M} \\
3.0 \mathrm{E}-06 \mathrm{H} \\
1 \mathrm{M} \\
3.0 \mathrm{E}-06 \mathrm{H} \\
1 \mathrm{M} \\
1.0 \mathrm{O}+00 \mathrm{Y} \\
1 \mathrm{M} \\
1.0 \mathrm{E}+00 \mathrm{Y} \\
1 \mathrm{M} \\
1.0 \mathrm{E}+00 \mathrm{Y} \\
8.5 \mathrm{H} \\
1.0 \mathrm{E}-06 \mathrm{H} \\
8.5 \mathrm{H} \\
1.0 \mathrm{E}-06 \mathrm{H} \\
8.5 \mathrm{H} \\
1.0 \mathrm{E}-07 \mathrm{H} \\
2 \mathrm{H} \\
2.3 \mathrm{E}-02 \mathrm{H} \\
1 \mathrm{H} \\
3.0 \mathrm{E}-06 \mathrm{H} \\
8 \mathrm{H} \\
1.0 \mathrm{E}-06 \mathrm{H} \\
8 \mathrm{H} \\
1.0 \mathrm{E}-06 \mathrm{H} \\
3.0 \mathrm{E}-06 \mathrm{H} \\
4.0 \mathrm{E}-06 \mathrm{H} \\
\end{array}$ & $\begin{array}{l}2.58 \mathrm{E}-04 \\
2.58 \mathrm{E}-04 \\
2.58 \mathrm{E}-04 \\
2.58 \mathrm{E}-04 \\
1.29 \mathrm{E}-04 \\
2.40 \mathrm{E}-05 \\
1.29 \mathrm{E}-04 \\
2.40 \mathrm{E}-05 \\
2.58 \mathrm{E}-04 \\
2.58 \mathrm{E}-04 \\
2.16 \mathrm{E}-03 \\
2.16 \mathrm{E}-03 \\
7.89 \mathrm{E}-02 \\
7.59 \mathrm{E}-02 \\
7.89 \mathrm{E}-02 \\
8.50 \mathrm{E}-06 \\
8.50 \mathrm{E}-06 \\
8.50 \mathrm{E}-07 \\
4.50 \mathrm{E}-02 \\
3.00 \mathrm{E}-06 \\
8.00 \mathrm{E}-06 \\
8.00 \mathrm{E}-06 \\
1.20 \mathrm{E}-05 \\
1.20 \mathrm{E}-05\end{array}$ & 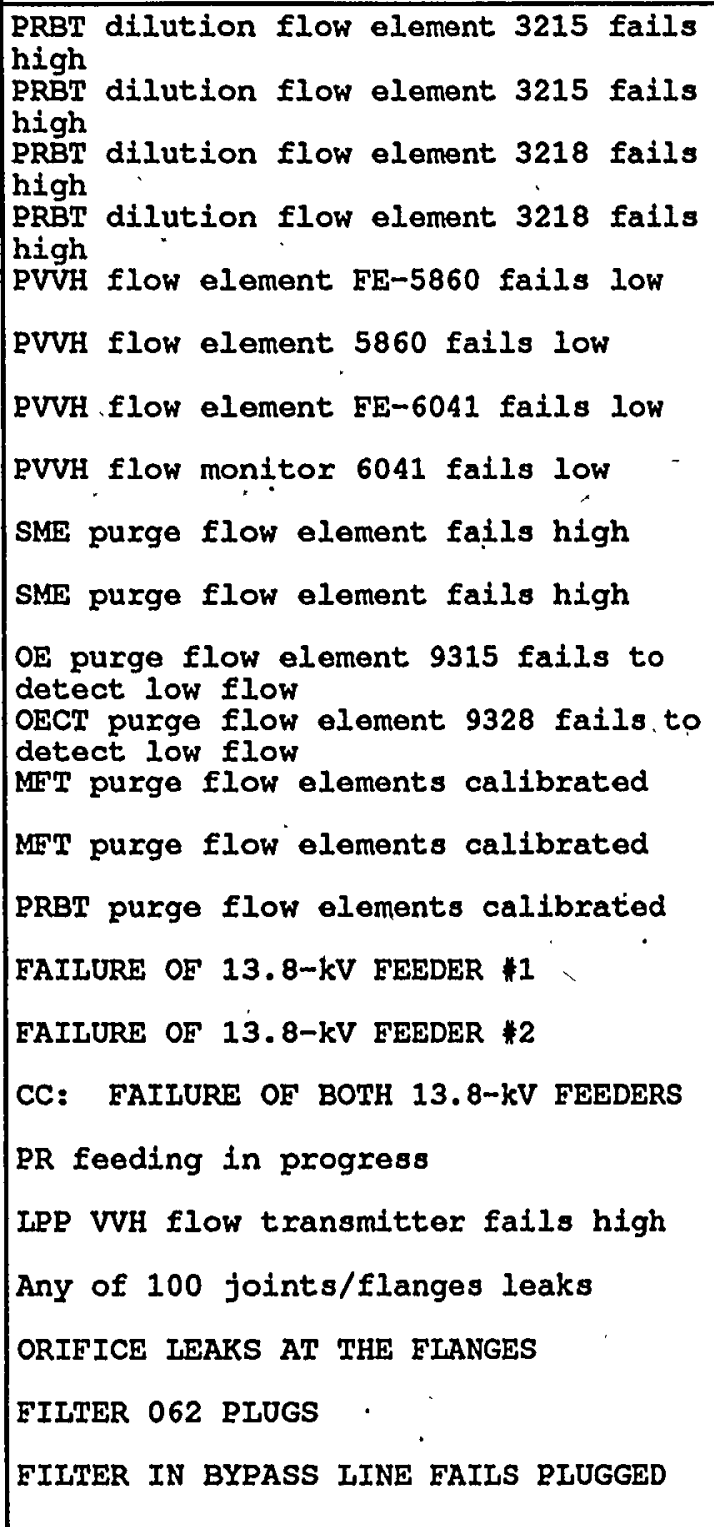 & $\begin{array}{l}\text { Repaired before next batch } \\
\text { reparired before next batch } \\
\text { repaired before next batch } \\
\text { repaired before next batch } \\
\text { a: corrected before next SRAT batch } \\
\text { a: detected and corrected in } 8 \text { hours } \\
\text { a: corrected by next sRAT batch } \\
\text { a: detected and corrected in } 8 \text { hours } \\
\text { a: repaired before next batch } \\
\text { a: repaired before next batch, } \\
\text { a: checked monthly } \\
\text { a: checked monthly } \\
\text { a: calibrated annually, corrected in } \\
1 \text { month } \\
\text { a: calibrated annually, corrected in } \\
1 \text { month } \\
\text { a: calibrated annually, corrected in } \\
\text { 1 month } \\
\text { DWPF sAR } 9.3 .18 \\
\text { DWpF sAR } 9.3 .18 \\
\text { DwPF sAR } 9.3 .18 \\
2 \text { hours per pR batch } \\
\text { a: responded to and shutdown or } \\
\text { repaired within } 1 \text { hour } \\
\text { a: } 8 \text { Hr Repair } \\
\text { a: } 8 \text { hr detection } \\
\text { a: } 8 \text { hours to repair } \\
\text { a: } 8 \text { hours to repair } \\
\text { a }\end{array}$ \\
\hline
\end{tabular}


Basic Evènt Report for I: \CAFTA \DATA\MASTER.BE '11/11/94 11:42 AM (CONT.)

\begin{tabular}{|c|c|c|c|c|c|}
\hline vent & c) & nput & Calc. & Description & Source \\
\hline 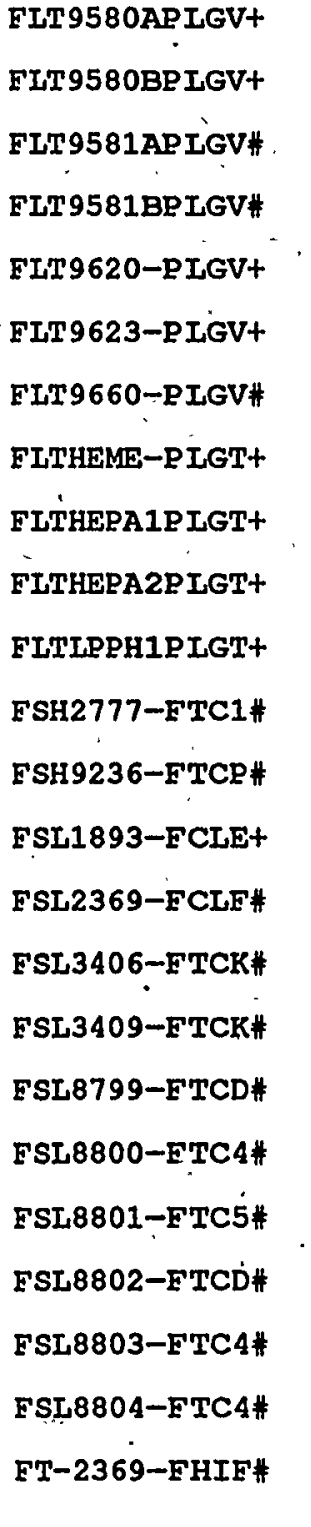 & $\begin{array}{l}5 \\
- \\
5\end{array}$ & $\begin{array}{r}48 \mathrm{H} \\
1.0 \mathrm{E}-0.4 \mathrm{H} \\
48 \mathrm{H} \\
1.0 \mathrm{E}-04 \mathrm{H} \\
48 \mathrm{H} \\
1.0 \mathrm{E}-04 \mathrm{H} \\
48 \mathrm{H} \\
1.0 \mathrm{E}-04 \mathrm{H} \\
48 \mathrm{H} \\
1.0 \mathrm{E}-04 \mathrm{H} \\
48 \mathrm{H} \\
1.0 \mathrm{E}-04 \mathrm{H} \\
7 \mathrm{D} \\
1.0 \mathrm{E}-04 \mathrm{H} \\
1 \mathrm{H} \\
3.0 \mathrm{E}-06 \mathrm{H} \\
1 \mathrm{H} \\
3.0 \mathrm{E}-06 \mathrm{H} \\
1 \mathrm{H} \\
3.0 \mathrm{E}-06 \mathrm{H} \\
1 \mathrm{H} \\
3.0 \mathrm{E}-06 \mathrm{H} \\
10 \\
1.0 \mathrm{E}-06 \mathrm{H} \\
10 \\
1.0 \mathrm{E}-06 \mathrm{H} \\
24 \mathrm{H} \\
3.0 \mathrm{E}-06 \mathrm{H} \\
4 \mathrm{H} \\
3.0 \mathrm{E}-06 \mathrm{H} \\
10 \\
1.0 \mathrm{E}-06 \mathrm{H} \\
10 \\
1.0 \mathrm{E}-06 \mathrm{H} \\
10 \\
1.0 \mathrm{E}-06 \mathrm{H} \\
10 \\
1.0 \mathrm{E}-06 \mathrm{H} \\
10 \\
1.0 \mathrm{E}-06 \mathrm{H} \\
10 \\
1.0 \mathrm{E}-06 \mathrm{H} \\
10 \\
1.0 \mathrm{E}-06 \mathrm{H} \\
10 \\
1.0 \mathrm{E}-06 \mathrm{H} \\
1 \mathrm{Y} \\
3.0 \mathrm{E}-06 \mathrm{H}\end{array}$ & $\begin{array}{l}4.78 \mathrm{E}-03 \\
4.78 \mathrm{E}-03 \\
4.79 \mathrm{E}-03 \\
4.79 \mathrm{E}-03 \\
4.78 \mathrm{E}-03 \\
4.78 \mathrm{E}-03 \\
1.67 \mathrm{E}-02 \\
3.00 \mathrm{E}-06 \\
3.00 \mathrm{E}-06 \\
3.00 \mathrm{E}-06 \\
3.00 \mathrm{E}-06 \\
1.09 \mathrm{E}-03 \\
1.09 \mathrm{E}-03 \\
7.20 \mathrm{E}-05 \\
1.20 \mathrm{E}-05 \\
1.09 \mathrm{E}-03 \\
1.09 \mathrm{E}-03 \\
1.09 \mathrm{E}-03 \\
1.09 \mathrm{E}-03 \\
1.09 \mathrm{E}-03 \\
1.09 \mathrm{E}-03 \\
1.09 \mathrm{E}-03 \\
1.09 \mathrm{E}-03 \\
1.30 \mathrm{E}-02\end{array}$ & 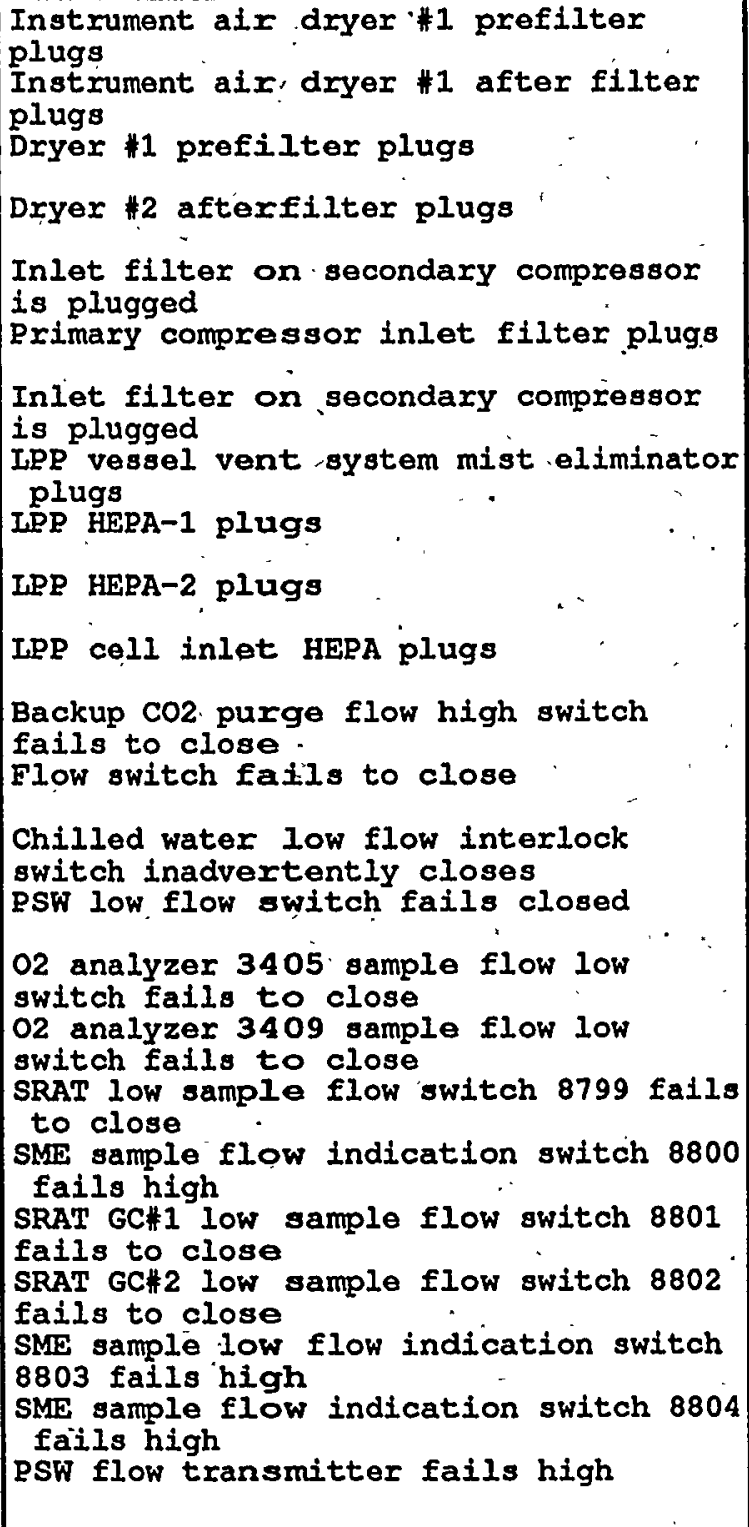 & $\begin{array}{l}\text { a: detected and corrected in } 2 \text { days } \\
\text { a: detected and corrected in } 2 \text { days } \\
\text { a: } 48 \text { hour mission time' } \\
\text { a: } 48 \text { hour mission time } \\
\text { a: detected and corrected in } 48 \text { hours } \\
\text { a: detected and corrected in } 48 \text { hours } \\
\text { a: } 1 \text { week mission time } \\
\text { a: responded to and shutdown or } \\
\text { repaired within } 1 \text { hour } \\
\text { a: responded to and shutdown or } \\
\text { repaired within } 1 \text { hour } \\
\text { a: responded to and shutdown or } \\
\text { repaired. within } 1 \text { hour } \\
\text { a: responded to and-shutdown or } \\
\text { repaired within } 1 \text { hour } \\
\text { a: Checked } 2 / y e a r . \\
\text { a: Checked } 4 / y x \text {. } \\
\text { a: detected and corrected in } 24 \text { hours } \\
\text { a: detected and corrected in } 4 \text { hours } \\
\text { a: checked quarterly } \\
\text { a: checked quarterly } \\
\text { a: Checked quarterly } \\
\text { a: Checked quarterly } \\
\text { a: Checked quarterly } \\
\text { a: Checked quarterly } \\
\text { a: Checked quarterly } \\
\text { a: Checked quarterly } \\
\text { a: checked annually }\end{array}$ \\
\hline
\end{tabular}


Basic. Event Report for I: \CAFTA IDATA \MASTER.BE 11/11/94 11:42 AM (CONT.)

\begin{tabular}{|c|c|c|c|c|c|}
\hline Event & c| & Input & Calc. & escription & Source \\
\hline $\begin{array}{l}\text { FT-8155-FLOT* } \\
\text { FT-9152-FLOO+ } \\
\text { FY-8150-BFLT+ } \\
\text { HCV1017-FCLB+ } \\
\text { HCV1033-FTCO* } \\
\text { HCV1038-FOP9+ } \\
\text { HCV1102-FCL9+ } \\
\text { HCV1142-FTCO\# } \\
\text { HCV1885-FCLE+ } \\
\text { HCV1897-FTOE* } \\
\text { HCV3010-FTCO* } \\
\text { HCV3010-FTOF* } \\
\text { HCV3089-FCL4* } \\
\text { HCV3089-FTCO* } \\
\text { HCV3212-FTCO* } \\
\text { HCV3252-FTCO* } \\
\text { HCV5845-FTCO* } \\
\text { HCV5907-FTCO* } \\
\text { HCV8772-FOPQ } \\
\text { HCV8772-FTCQ* } \\
\text { HCV9116-FCLO+ } \\
\text { HCV9116-FTCG* } \\
\text { HCV9122-FTOG } \\
\text { HCV9150-FCLG\# }\end{array}$ & $\begin{array}{l}4 \\
4 \\
1 \\
4 \\
1 \\
1 \\
1 \\
3 \\
1 \\
1 \\
1 \\
1 \\
1 \\
5 \\
1 \\
4 \\
1 \\
1 \\
3\end{array}$ & $\begin{array}{r}1 \mathrm{Y} \\
3.0 \mathrm{E}-06 \mathrm{H} \\
8 \mathrm{H} \\
3.0 \mathrm{E}-06 \mathrm{H} \\
1 \mathrm{H} \\
1.0 \mathrm{E}-07 \mathrm{H} \\
8 \mathrm{H} \\
5.0 \mathrm{E}-07 \mathrm{H} \\
1 \mathrm{~N} \\
1.0 \mathrm{E}-03 \mathrm{~N} \\
86 \mathrm{H} \\
5.0 \mathrm{E}-07 \mathrm{H} \\
8 \mathrm{H} \\
5.0 \mathrm{E}-07 \mathrm{H} \\
1.0 \mathrm{~N}-03 \mathrm{~N} \\
8.0 \mathrm{~B}-07 \mathrm{H} \\
1 \mathrm{~N} \\
1.0 \mathrm{E}-03 \mathrm{~N} \\
1.0 \mathrm{~N}-03 \mathrm{~N} \\
1.5 \mathrm{~N} \\
1.0 \mathrm{E}-03 \mathrm{~N} \\
8 \mathrm{H} \\
5.0 \mathrm{E}-07 \mathrm{H} \\
1.0 \mathrm{~N}-03 \mathrm{~N} \\
1 \mathrm{~N} \\
1.0 \mathrm{E}-03 \mathrm{~N} \\
1.0 \mathrm{~N}-03 \mathrm{~N} \\
1 \mathrm{~N} \\
1.0 \mathrm{E}-03 \mathrm{~N} \\
1.0 \mathrm{E}-03 \mathrm{~N} \\
8 \mathrm{H} \\
5.0 \mathrm{E}-07 \mathrm{H} \\
1.0 \mathrm{E}-03 \mathrm{~N} \\
4 \mathrm{H} \\
5.0 \mathrm{E}-07 \mathrm{H} \\
1.0 \mathrm{~N}-03 \mathrm{~N} \\
1 \mathrm{~N} \\
1.0 \mathrm{E}-03 \mathrm{~N} \\
5.0 \mathrm{~B}-07 \mathrm{H} \\
\end{array}$ & $\begin{array}{l}1.30 \mathrm{E}-02 \\
2.40 \mathrm{E}-05 \\
1.00 \mathrm{E}-07 \\
4.00 \mathrm{E}-06 \\
1.00 \mathrm{E}-03 \mathrm{~N} \\
4.30 \mathrm{E}-05 \\
4.00 \mathrm{E}-06 \\
1.00 \mathrm{E}-03 \mathrm{~N} \\
4.00 \mathrm{E}-06 \\
1.00 \mathrm{E}-03 \mathrm{~N} \\
1.00 \mathrm{E}-03 \mathrm{~N} \\
1.00 \mathrm{E}-03 \mathrm{~N} \\
4.00 \mathrm{E}-06 \\
1.00 \mathrm{E}-03 \mathrm{~N} \\
1.00 \mathrm{E}-03 \mathrm{~N} \\
1.00 \mathrm{E}-03 \mathrm{~N} \\
1.00 \mathrm{E}-03 \mathrm{~N} \\
1.00 \mathrm{E}-03 \mathrm{~N} \\
2.00 \mathrm{E}-06 \\
1.00 \mathrm{E}-03 \mathrm{~N} \\
2.00 \mathrm{E}-06 \\
1.00 \mathrm{E}-03 \mathrm{~N} \\
1.00 \mathrm{E}-03 \mathrm{~N} \\
4.00 \mathrm{E}-06\end{array}$ & 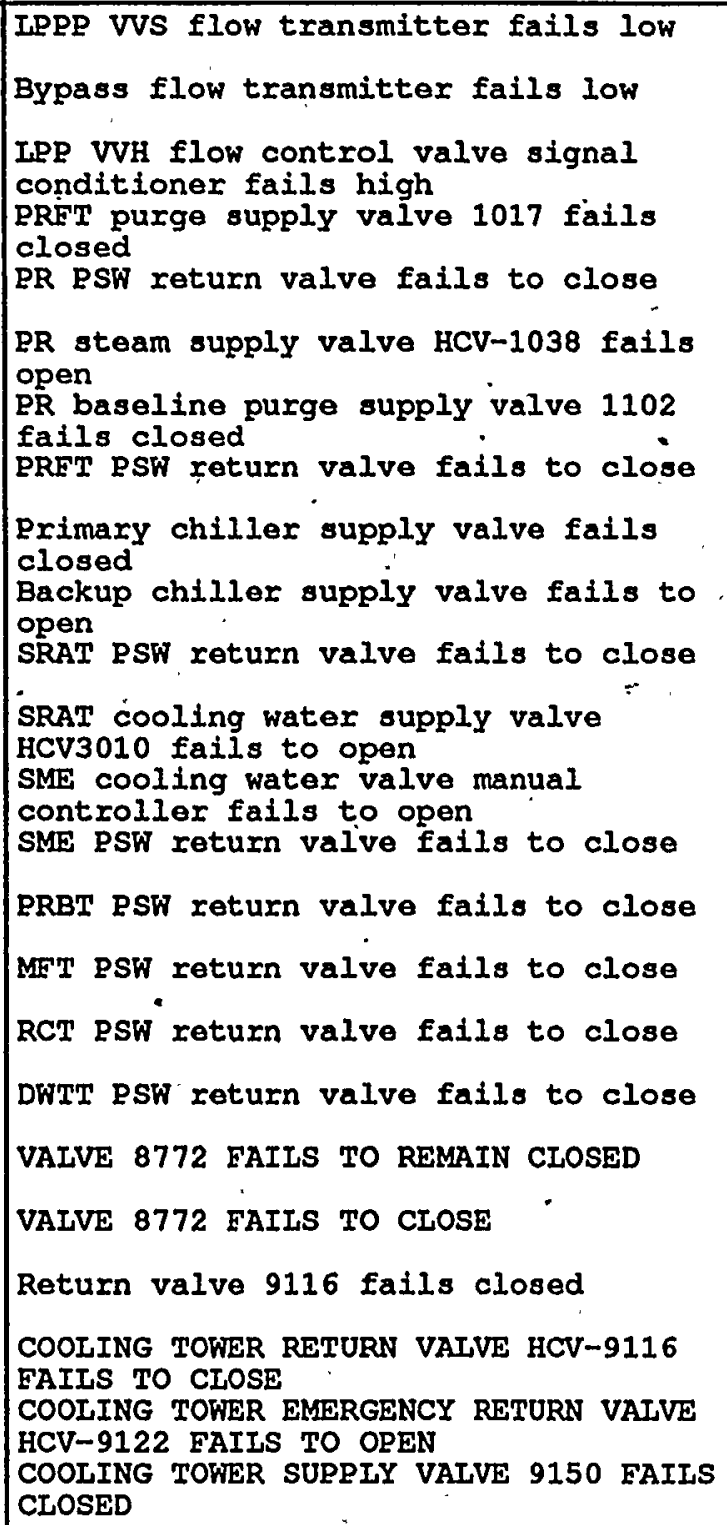 & $\begin{array}{l}\text { a: checked annualy } \\
\text { Detected and corrected in } 8 \text { hours } \\
\text { a: responded to and shutdown or } \\
\text { repaired within } 1 \text { hour } \\
\text { a: } 8 \text { hours to repair or shutdown } \\
\text { Ose TC value } \\
\text { a: detected and repaired before next } \\
\text { batch hours to repair or shutdown } \\
\text { a: } 8 \text { hours } \\
\text { Use TC value } \\
\text { a: } 8 \text { hours to repair } \\
\text { Use TC Value } \\
\text { Use TC value } \\
\text { Use TC value } \\
\text { A: } 8 \text { HOURS TO REPAIR } \\
\text { Use TC value } \\
\text { Use TC value } \\
\text { Use TC value } \\
\text { Use TC value } \\
\text { Use TC value } \\
\text { a: } 8 \text { hours to repair } \\
\text { Use TC } \\
\text { a: detected and repaired, bypassed, } \\
\text { or shut down in } 4 \text { hours } \\
\text { Use TC } \\
\text { Use TC } \\
\text { a: } 8 \text { hours to repair. }\end{array}$ \\
\hline
\end{tabular}


Basic Event Report for I: \CAFTA IDATAlMASTER.BE 11/11/94 11:42 AM (CONT.)

\begin{tabular}{|c|c|c|c|c|c|}
\hline Event & $\mathrm{C}$ & iput & Calc. & Description & ce \\
\hline 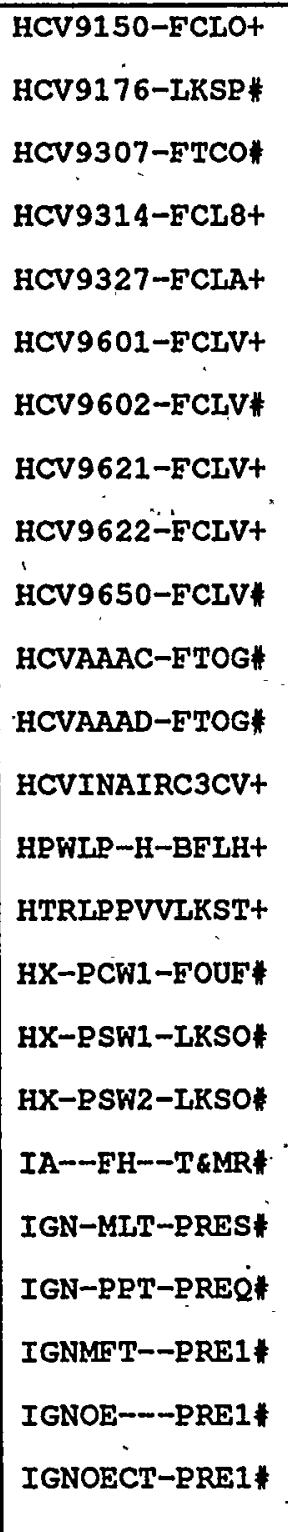 & 1 & $\begin{array}{r}4 \mathrm{H} \\
5.0 \mathrm{E}-07 \mathrm{H} \\
8 \mathrm{H} \\
1.0 \mathrm{E}-08 \mathrm{H} \\
1 \mathrm{~N} \\
1.0 \mathrm{E}-03 \mathrm{~N} \\
1 \mathrm{H} \\
5.0 \mathrm{E}-07 \mathrm{H} \\
1 \mathrm{H} \\
5.0 \mathrm{E}-07 \mathrm{H} \\
1 \mathrm{H} \\
5.0 \mathrm{E}-07 \mathrm{H} \\
24 \mathrm{H} \\
5.0 \mathrm{E}-07 \mathrm{H} \\
2 \mathrm{H} \\
5.0 \mathrm{E}-07 \mathrm{H} \\
2 \mathrm{H} \\
5.0 \mathrm{E}-07 \mathrm{H} \\
2 \mathrm{H} \\
5.0 \mathrm{E}-07 \mathrm{H} \\
1 \mathrm{~N} \\
1.0 \mathrm{E}-03 \mathrm{~N} \\
1.0 \mathrm{E}-03 \mathrm{~N} \\
2 \mathrm{H} \\
2.5 \mathrm{E}-08 \mathrm{H} \\
8.5 \mathrm{H} \\
3.0 \mathrm{E}-01 \mathrm{Y} \\
8 \mathrm{H} \\
3.0 \mathrm{E}-07 \mathrm{H} \\
8 \mathrm{H} \\
3.0 \mathrm{E}-08 \mathrm{H} \\
5 \mathrm{Y} \\
1.0 \mathrm{E}-08 \mathrm{H} \\
5 \mathrm{Y} \\
1.0 \mathrm{E}-08 \mathrm{H} \\
1 \mathrm{~N} \\
3.2 \mathrm{E}-03 \mathrm{~N} \\
1 \mathrm{~N} \\
3.0 \mathrm{E}-02 \mathrm{~N} \\
1 \mathrm{~N} \\
3.0 \mathrm{E}-02 \mathrm{~N} \\
1 \mathrm{~N} \\
3.0 \mathrm{E}-02 \mathrm{~N} \\
1 \mathrm{~N} \\
3.0 \mathrm{E}-02 \mathrm{~N} \\
1 \mathrm{~N} \\
3.0 \mathrm{E}-02 \mathrm{~N}\end{array}$ & $\begin{array}{l}2.00 \mathrm{E}-06 \\
8.00 \mathrm{E}-08 \\
1.00 \mathrm{E}-03 \mathrm{~N} \\
5.00 \mathrm{E}-07 \\
5.00 \mathrm{E}-07 \\
5.00 \mathrm{E}-07 \\
1.20 \mathrm{E}-05 \\
1.00 \mathrm{E}-06 \\
1.00 \mathrm{E}-06 \\
5.00 \mathrm{E}-07 \\
1.00 \mathrm{E}-03 \mathrm{~N} \\
1.00 \mathrm{E}-03 \mathrm{~N} \\
5.00 \mathrm{E}-08 \\
2.91 \mathrm{E}-04 \\
2.40 \mathrm{E}-06 \\
2.40 \mathrm{E}-07 \\
2.19 \mathrm{E}-04 \\
2.19 \mathrm{E}-04 \\
3.20 \mathrm{E}-03 \mathrm{~N} \\
3.00 \mathrm{E}-02 \mathrm{~N} \\
3.00 \mathrm{E}-02 \mathrm{~N} \\
3.00 \mathrm{E}-02 \mathrm{~N} \\
3.00 \mathrm{E}-02 \mathrm{~N} \\
3.00 \mathrm{E}-02 \mathrm{~N}\end{array}$ & 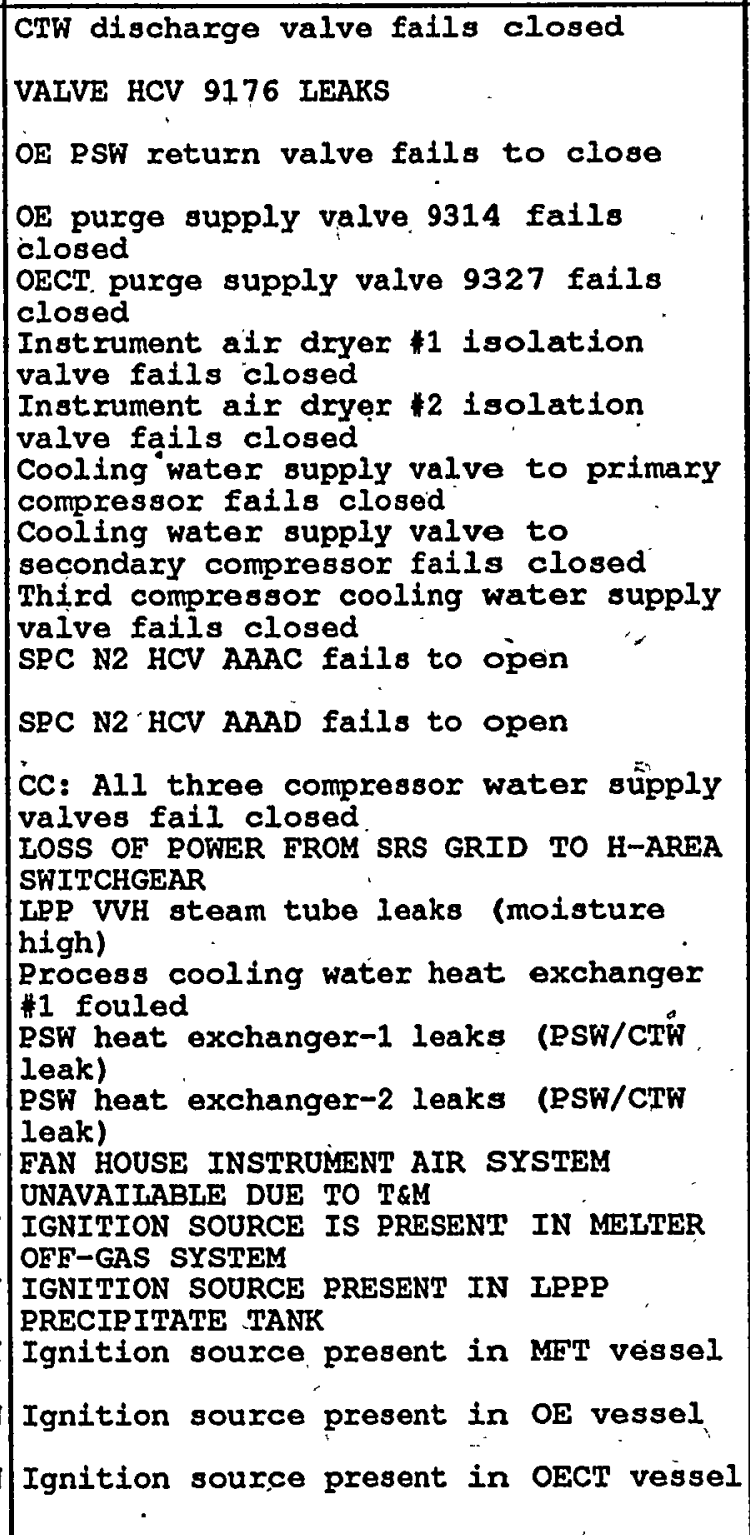 & $\begin{array}{l}\text { a: Detected and corrected or shutdown } \\
\text { in } 4 \text { hours } \\
\text { a: } 8 \text { hr detection } \\
\text { Use TC value } \\
\text { a: detected and corrected or shutdown } \\
\text { w/i } 1 \text { hour due to low flow alarm } \\
\text { a: detected and corrected or shutdown } \\
\text { w/i } 1 \text { hour due to low flow alarm } \\
\text { a: detected and corrected within } 1 \\
\text { hour (switch to backup) } \\
\text { a: detected and corrected within } 24 \\
\text { hours } \\
\text { a: detected and bypassed within } 2 \\
\text { hours } \\
\text { a: detected and bypassed in } 2 \text { hours } \\
\text { a: detected and bypassed in } 2 \text { hours } \\
\text { Use TC value } \\
\text { Use TC value } \\
\text { a: Detected and bypassed in } 2 \text { hours } \\
\text { DwPF sAR } 9.3 .18 \\
\text { a: responded to within } 8 \text { hours } \\
\text { a: } 8 \text { hours to switch HX } \\
\text { a: Checked for plate leaks every } 5 \\
\text { years } \\
\text { a: Checked for plate leaks every } 5 \\
\text { years } \\
\text { Use TC } \\
\text { Use TC } \\
\text { Use TC } \\
\text { Use TC value } \\
\text { Use TC value } \\
\text { Use TC value }\end{array}$ \\
\hline
\end{tabular}


Basic Event Report for I: \CAFTA IDATAIMASTER.BE 11/11/94 11:42 AM (CONT.)

\begin{tabular}{|c|c|c|c|c|c|}
\hline Event & c & Input & Calc. & Description & Source \\
\hline $\begin{array}{l}\text { IGNPRBT-PRE1* } \\
\text { IGNPRET-PRE1* } \\
\text { IGNPVVH-PRE1* } \\
\text { IGNSME--PREI* } \\
\text { IGNSPC--PRE1* } \\
\text { IGNSPT--PRE1* } \\
\text { IGNSRAT-PRE1* } \\
\text { INKSRLFIBYP5* } \\
\text { JMP-HTR-CCAS+ } \\
\text { JMP-HTRABFLS+ } \\
\text { JMP-HTRBBELS+ } \\
\text { JMP-HTRCBFLS+ } \\
\text { JMP-HTRDBFLS+ } \\
\text { JMP-SPC-LKS1+ } \\
\text { LCV2220AFCLG+ } \\
\text { LCV2220BECLG+ } \\
\text { LE-2220-CALG+ } \\
\text { LE-2220-EHIG+ } \\
\text { LE-22 62-ELOG\# } \\
\text { LE-5925-FLOG* } \\
\text { IPWST-IRBELH+ }\end{array}$ & 5 & $\begin{array}{r}1 \mathrm{~N} \\
3.0 \mathrm{E}-02 \mathrm{~N} \\
-1 \mathrm{H} \\
2.3 \mathrm{E}-5 \mathrm{H} \\
1 \mathrm{~N} \\
3.0 \mathrm{E}-02 \mathrm{~N} \\
1 \mathrm{~N} \\
3.0 \mathrm{E}-02 \mathrm{~N} \\
1 \mathrm{~N} \\
3.0 \mathrm{E}-02 \mathrm{~N} \\
1 \mathrm{~N} \\
3.0 \mathrm{E}-02 \mathrm{~N} \\
1 \mathrm{~N} \\
3.0 \mathrm{E}-02 \mathrm{~N} \\
1 \mathrm{~N} \\
3.0 \mathrm{E}-02 \mathrm{~N} \\
1 \mathrm{~N} \\
3.0 \mathrm{E}-02 \mathrm{~N} \\
1 \mathrm{~N} \\
3.0 \mathrm{E}-02 \mathrm{~N} \\
1 \mathrm{~N} \\
4.6 \mathrm{E}-04 \mathrm{~N} \\
4 \mathrm{H} \\
1.0 \mathrm{E}-09 \mathrm{H} \\
4 \mathrm{H} \\
1.0 \mathrm{E}-08 \mathrm{H} \\
4 \mathrm{H} \\
1.0 \mathrm{E}-08 \mathrm{H} \\
4 \mathrm{H} \\
1.0 \mathrm{E}-08 \mathrm{H} \\
4 \mathrm{H} \\
1.0 \mathrm{E}-08 \mathrm{H} \\
43 \mathrm{H} \\
2.0 \mathrm{E}-05 \mathrm{H} \\
8 \mathrm{H} \\
5.0 \mathrm{E}-07 \mathrm{H} \\
8 \mathrm{H} \\
5.0 \mathrm{E}-07 \mathrm{H} \\
1 \mathrm{Q} \\
2.3 \mathrm{E}-04 \mathrm{H} \\
8 \mathrm{H} \\
5.0 \mathrm{E}-07 \mathrm{H} \\
8 \mathrm{H} \\
5.0 \mathrm{E}-07 \mathrm{H} \\
1 \mathrm{Y} \\
5.04 \mathrm{Y}\end{array}$ & $\begin{array}{l}3.00 \mathrm{E}-02 \mathrm{~N} \\
2.30 \mathrm{E}-06 \\
3.00 \mathrm{E}-02 \mathrm{~N} \\
3.00 \mathrm{E}-02 \mathrm{~N} \\
3.00 \mathrm{E}-02 \mathrm{~N} \\
3.00 \mathrm{E}-02 \mathrm{~N} \\
3.00 \mathrm{E}-02 \mathrm{~N} \\
3.00 \mathrm{E}-02 \mathrm{~N} \\
3.00 \mathrm{E}-02 \mathrm{~N} \\
3.00 \mathrm{E}-02 \mathrm{~N} \\
4.60 \mathrm{E}-04 \mathrm{~N} \\
4.00 \mathrm{E}-09 \\
4.00 \mathrm{E}-08 \\
4.00 \mathrm{E}-08 \\
4.00 \mathrm{E}-08 \\
4.00 \mathrm{E}-08 \\
8.59 \mathrm{E}-04 \\
4.00 \mathrm{E}-06 \\
4.00 \mathrm{E}-06 \\
3.34 \mathrm{E}-01 \\
4.00 \mathrm{E}-06 \\
4.00 \mathrm{E}-06 \\
2.19 \mathrm{E}-03 \\
7.76 \mathrm{E}-07\end{array}$ & $\begin{array}{l}\text { Ignition source present in OWST inner } \\
\text { tank } \\
\text { Ignition source present in OWST outer } \\
\text { tank } \\
\text { Ignition source present in PR vessel } \\
\text { Ignition source present in PRBT vessel } \\
\text { Ignition source present in PRFT vessel } \\
\text { Ignition source present in PVVH } \\
\text { Ignition source present in SME vessel } \\
\text { Ignition source present in SPC } \\
\text { Ignition source present in SPT vessel } \\
\text { Ignition source present in SRAT } \\
\text { SRAT high IFL interlock bypassed } \\
\text { CC: TWO FUSES FOR LID HEATERS FAII } \\
\text { FUSE FOR LID HEATER A FAILS } \\
\text { FUSE FOR LID HEATER B FAILS } \\
\text { FUSE FOR LID HEATER C FAILS } \\
\text { FUSE FOR IID HEATER D FAIIS } \\
\text { SPC jumper leak - benzene leaks onto } \\
\text { SPC floor } \\
\text { Steam generator cond. level control } \\
\text { valve A fails closed } \\
\text { Steam generator cond. level control } \\
\text { valve B fails closed } \\
\text { Steam generator condensate level } \\
\text { transmitter is calibrated } \\
\text { Steam generator condensate level } \\
\text { transmitter fails high } \\
\text { IEVEI SENSOR/SWITCH FAILS (FALSE LOW } \\
\text { SIGNAI) } \\
\text { SPC sump level element, fails low } \\
\text { FAIIURE TO sURPLY powER TO GRID FROM } \\
\text { ON-SITE AND OFF-SITE SOURCES. }\end{array}$ & $\begin{array}{l}\text { Use TC value } \\
\text { Ignition source lasts } 0.1 \text { hour } \\
\text { Use TC value } \\
\text { Use TC value } \\
\text { Use TC value } \\
\text { Use TC value } \\
\text { Use TC value } \\
\text { Use TC value } \\
\text { Use TC value } \\
\text { Use TC value } \\
\text { Use TC value } \\
\text { a: detected in } 1 \text { shift } \\
\text { a: detected in } 1 \text { shift } \\
\text { a: detected in } 1 \text { shift } \\
\text { a: detected in } 1 \text { shift } \\
\text { a: detected in } 1 \text { shift } \\
\text { a: corrected before next batch } \\
\text { Estimated } 8 \text { hour resotration time } \\
\text { Estimated } 8 \text { hour resotration time } \\
\text { a: calibrated twice/year } \\
\text { Estimated } 8 \text { hour resotration time } \\
\text { a: } 8 \text { hours to repaix } \\
\text { a: checked annually } \\
\text { DwPF saR } 9.3 .18\end{array}$ \\
\hline
\end{tabular}


Basic Event Report for I: \CAFTA IDATA MASTER.BE 11/11/94 11:42 AM (CONT.)

\begin{tabular}{|c|c|c|c|c|c|}
\hline vent & c) & nput & Calc. & Description & Source \\
\hline 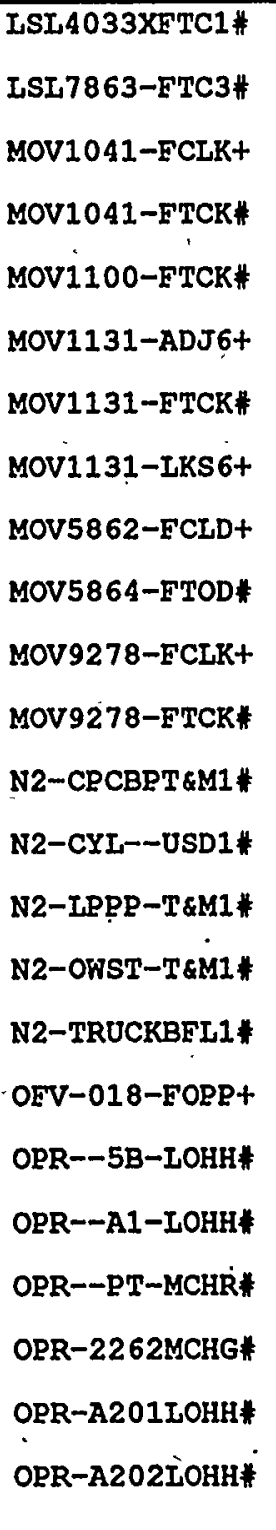 & $4 \mid$ & $\begin{array}{r}2 \mathrm{D} \\
1.0 \mathrm{E}-06 \mathrm{H} \\
1 \mathrm{O} \\
1.0 \mathrm{E}-06 \mathrm{H} \\
8 \mathrm{H} \\
3.0 \mathrm{E}-07 \mathrm{H} \\
1 \mathrm{~N} \\
3.0 \mathrm{E}-03 \mathrm{~N} \\
1 \mathrm{~N} \\
3.0 \mathrm{E}-03 \mathrm{~N} \\
1 \mathrm{H} \\
1.0 \mathrm{E}+00 \mathrm{Q} \\
1 \mathrm{~N} \\
3.0 \mathrm{E}-03 \mathrm{~N} \\
24 \mathrm{H} \\
1.0 \mathrm{E}-06 \mathrm{H} \\
1 \mathrm{D} \\
3.0 \mathrm{O}-07 \mathrm{H} \\
1 \mathrm{~N} \\
3.0 \mathrm{E}-03 \mathrm{~N} \\
8 \mathrm{H} \\
3.0 \mathrm{E}-07 \mathrm{H} \\
1 \mathrm{~N} \\
3.0 \mathrm{E}-03 \mathrm{~N} \\
24 \mathrm{H} \\
1 \mathrm{Y} \\
7 \mathrm{D} \\
1 \mathrm{Y} \\
14 \mathrm{D} \\
1 \mathrm{Y} \\
14 \mathrm{D} \\
1 \mathrm{Y} \\
1.0 \mathrm{E}-02 \mathrm{~N} \\
24 \mathrm{H} \\
5.0 \mathrm{E}-09 \mathrm{H} \\
1.8 \mathrm{E}-04 \mathrm{~N} \\
5.0 \mathrm{E}-03 \mathrm{~N} \\
1.8 \mathrm{E}-04 \mathrm{~N} \\
5.0 \mathrm{E}-03 \mathrm{~N} \\
1 \mathrm{~N} \\
3.0 \mathrm{OE}-02 \mathrm{~N} \\
2.5 \mathrm{E}-01 \mathrm{~N} \\
3.0 \mathrm{E}-02 \mathrm{~N} \\
1.8 \mathrm{E}-04 \mathrm{~N} \\
1.0 \mathrm{E}-03 \mathrm{E}-04 \mathrm{~N} \\
5.0 \mathrm{E}-03 \mathrm{~N}\end{array}$ & $\begin{array}{l}2.40 \mathrm{E}-05 \\
2.18 \mathrm{E}-03 \\
2.40 \mathrm{E}-06 \\
3.00 \mathrm{E}-03 \mathrm{~N} \\
3.00 \mathrm{E}-03 \mathrm{~N} \\
4.59 \mathrm{E}-04 \\
3.00 \mathrm{E}-03 \mathrm{~N} \\
2.40 \mathrm{E}-05 \\
7.20 \mathrm{E}-06 \\
3.00 \mathrm{E}-03 \mathrm{~N} \\
2.40 \mathrm{E}-06 \\
3.00 \mathrm{E}-03 \mathrm{~N} \\
2.74 \mathrm{E}-03 \\
9.53 \mathrm{E}-03 \\
3.76 \mathrm{E}-02 \\
3.76 \mathrm{E}-02 \\
1.00 \mathrm{E}-02 \mathrm{~N} \\
1.20 \mathrm{E}-07 \\
9.00 \mathrm{E}-07 \mathrm{~N} \\
9.00 \mathrm{E}-07 \mathrm{~N} \\
3.00 \mathrm{E}-02 \mathrm{~N} \\
7.50 \mathrm{E}-03 \mathrm{~N} \\
9.00 \mathrm{E}-07 \mathrm{~N} \\
9.00 \mathrm{E}-07 \mathrm{~N}\end{array}$ & 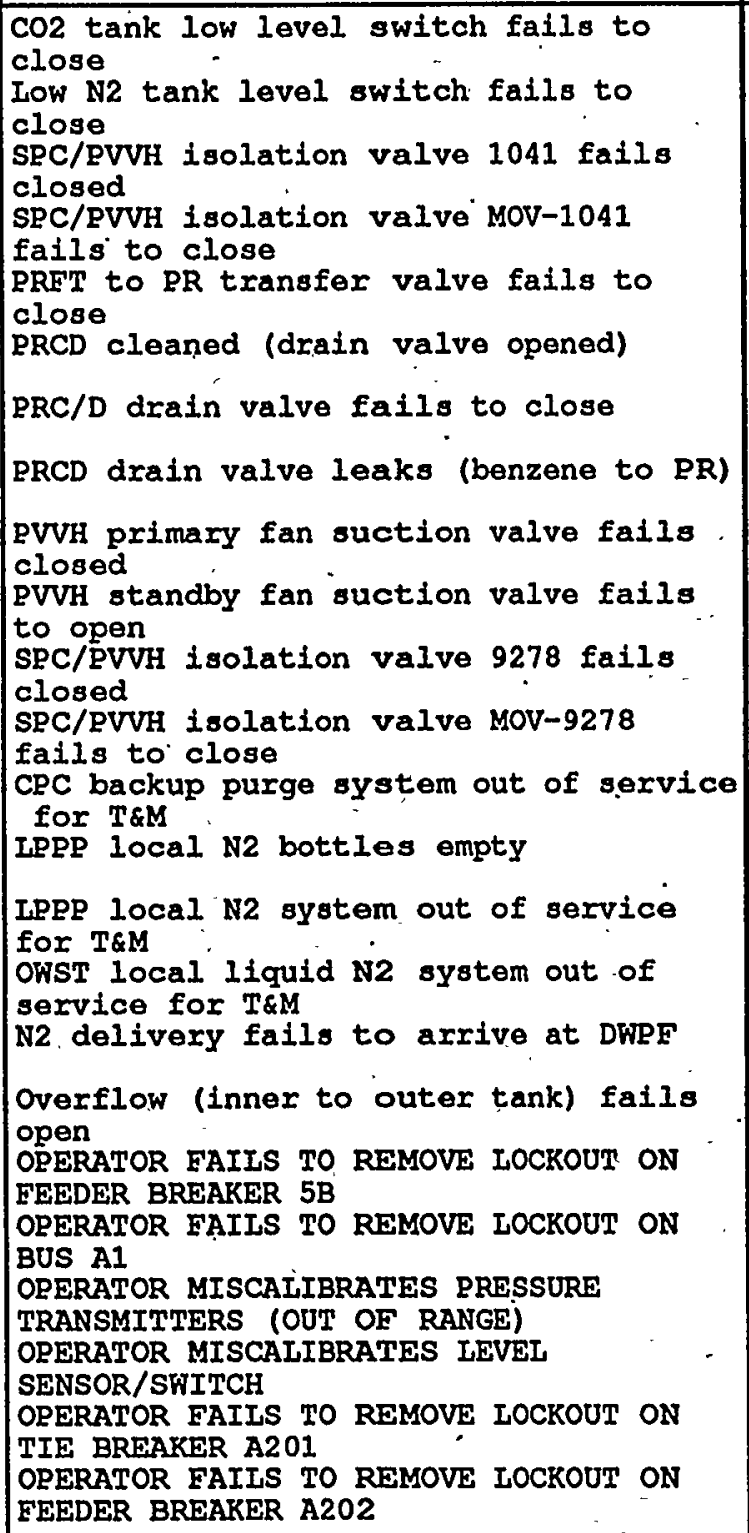 & 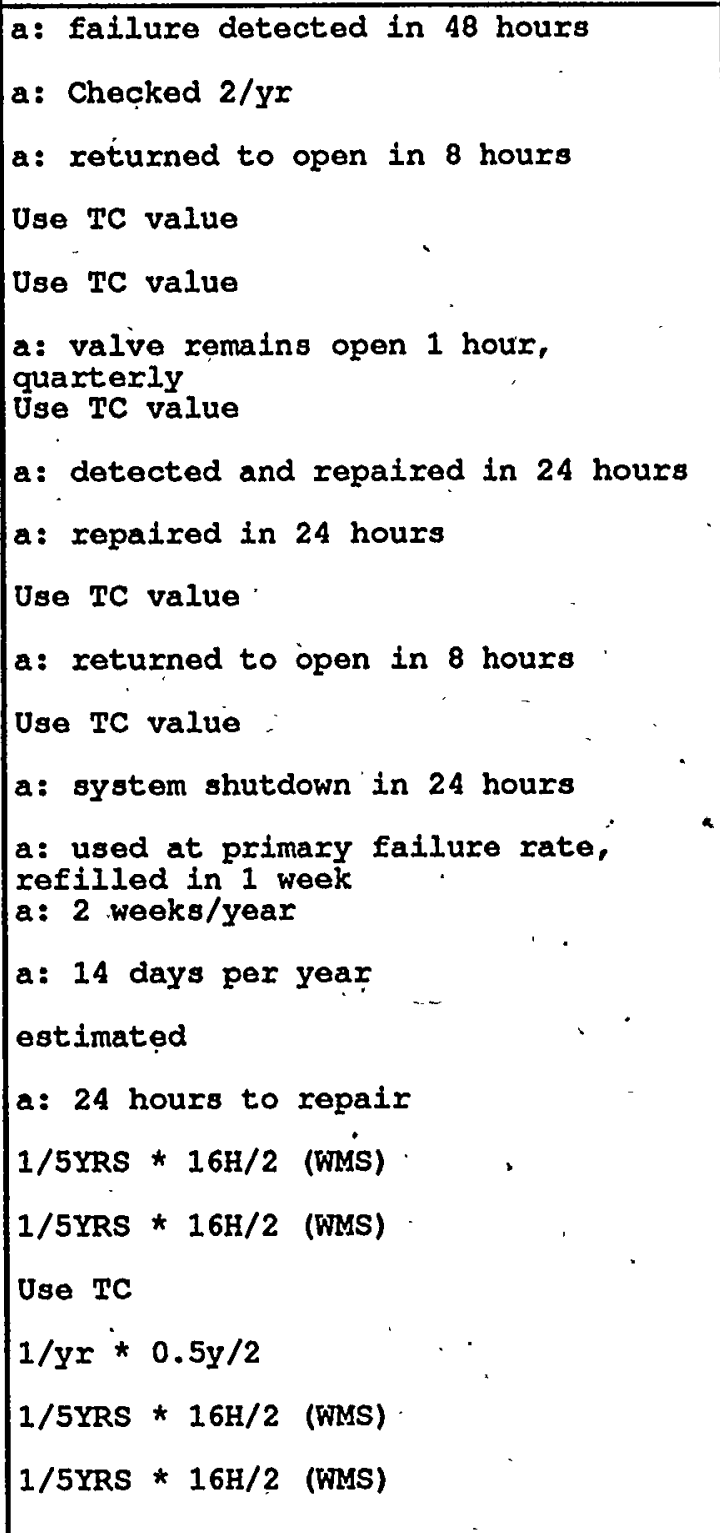 \\
\hline
\end{tabular}


Basic Event Report for I: \CAFTA \DATA MMASTER.BE 11/11/94 11:42 AM (CONT.)

\begin{tabular}{|c|c|c|c|c|c|}
\hline Event & C & put & Calc. & Description & Source \\
\hline 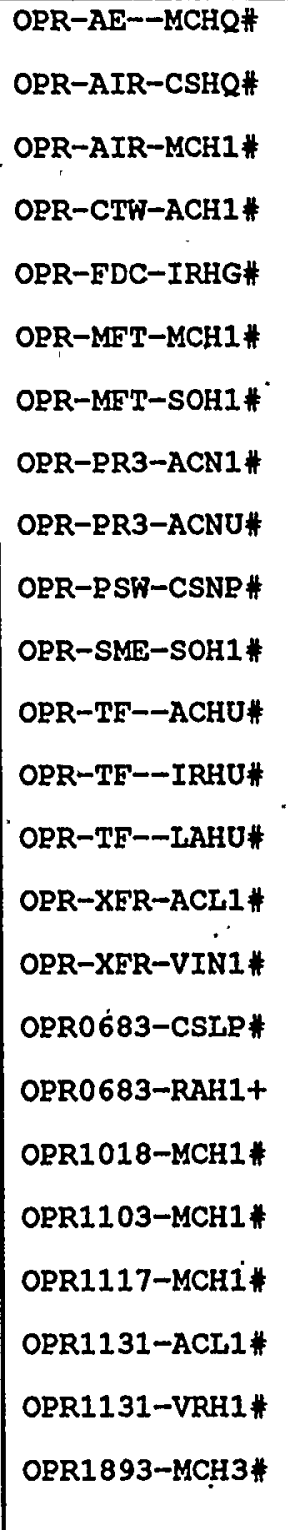 & $|1|$ & $\begin{array}{r}14 \mathrm{D} \\
1.5 \mathrm{E}-02 \mathrm{Y} \\
1 \mathrm{~N} \\
1.0 \mathrm{E}-01 \mathrm{~N} \\
1 \mathrm{~N} \\
3.0 \mathrm{E}-02 \mathrm{~N} \\
1 \mathrm{~N} \\
5.0 \mathrm{E}-02 \mathrm{~N} \\
1 \mathrm{~N} \\
5.0 \mathrm{E}-01 \mathrm{~N} \\
1 \mathrm{~N} \\
3.0 \mathrm{E}-02 \mathrm{~N} \\
1 \mathrm{~N} \\
5.0 \mathrm{E}-02 \mathrm{~N} \\
1 \mathrm{~N} \\
5.0 \mathrm{E}-03 \mathrm{~N} \\
1 \mathrm{~N} \\
5.0 \mathrm{E}-03 \mathrm{~N} \\
1 \mathrm{~N} \\
1.0 \mathrm{E}-02 \mathrm{~N} \\
1 \mathrm{~N} \\
5.0 \mathrm{E}-02 \mathrm{~N} \\
1 \mathrm{~N} \\
5.0 \mathrm{E}-02 \mathrm{~N} \\
1 \mathrm{~N} \\
5.0 \mathrm{E}-01 \mathrm{~N} \\
1 \mathrm{~N} \\
1.0 \mathrm{E}-03 \mathrm{~N} \\
1 \mathrm{~N} \\
5.0 \mathrm{E}-04 \mathrm{~N} \\
1 \mathrm{~N} \\
1.0 \mathrm{E}-01 \mathrm{~N} \\
3.0 \mathrm{E}-03 \mathrm{~N} \\
12 \mathrm{H} \\
5.0 \mathrm{E}-05 \mathrm{H} \\
4 \mathrm{E}-3 \mathrm{~N} \\
3.0 \mathrm{E}-02 \mathrm{~N} \\
4 \mathrm{E}-3 \mathrm{~N} \\
3.0 \mathrm{E}-02 \mathrm{~N} \\
4 \mathrm{E}-3 \mathrm{~N} \\
3.0 \mathrm{E}-02 \mathrm{~N} \\
1 \mathrm{~N} \\
5.0 \mathrm{E}-04 \mathrm{~N} \\
1 \mathrm{~N} \\
5.0 \mathrm{E}-02 \mathrm{~N} \\
.01 \mathrm{~N} \\
3.0 \mathrm{E}-02 \mathrm{~N}\end{array}$ & \begin{tabular}{|l|}
$2.88 \mathrm{E}-04$ \\
$1.00 \mathrm{E}-01 \mathrm{~N}$ \\
$3.00 \mathrm{E}-02 \mathrm{~N}$ \\
$5.00 \mathrm{E}-02 \mathrm{~N}$ \\
$5.00 \mathrm{E}-01 \mathrm{~N}$ \\
$-3.00 \mathrm{E}-02 \mathrm{~N}$ \\
$5.00 \mathrm{E}-02 \mathrm{~N}$ \\
$5.00 \mathrm{E}-03 \mathrm{~N}$ \\
$5.00 \mathrm{E}-03 \mathrm{~N}$ \\
$1.00 \mathrm{E}-02 \mathrm{~N}$ \\
$5.00 \mathrm{E}-02 \mathrm{~N}$ \\
$5.00 \mathrm{E}-02 \mathrm{~N}$ \\
$5.00 \mathrm{E}-01 \mathrm{~N}$ \\
$1.00 \mathrm{E}-03 \mathrm{~N}$ \\
$5.00 \mathrm{E}-04 \mathrm{~N}$ \\
$1.00 \mathrm{E}-01 \mathrm{~N}$ \\
$3.00 \mathrm{E}-03 \mathrm{~N}$ \\
$6.00 \mathrm{E}-04$ \\
$1.20 \mathrm{E}-04 \mathrm{~N}$
\end{tabular} & 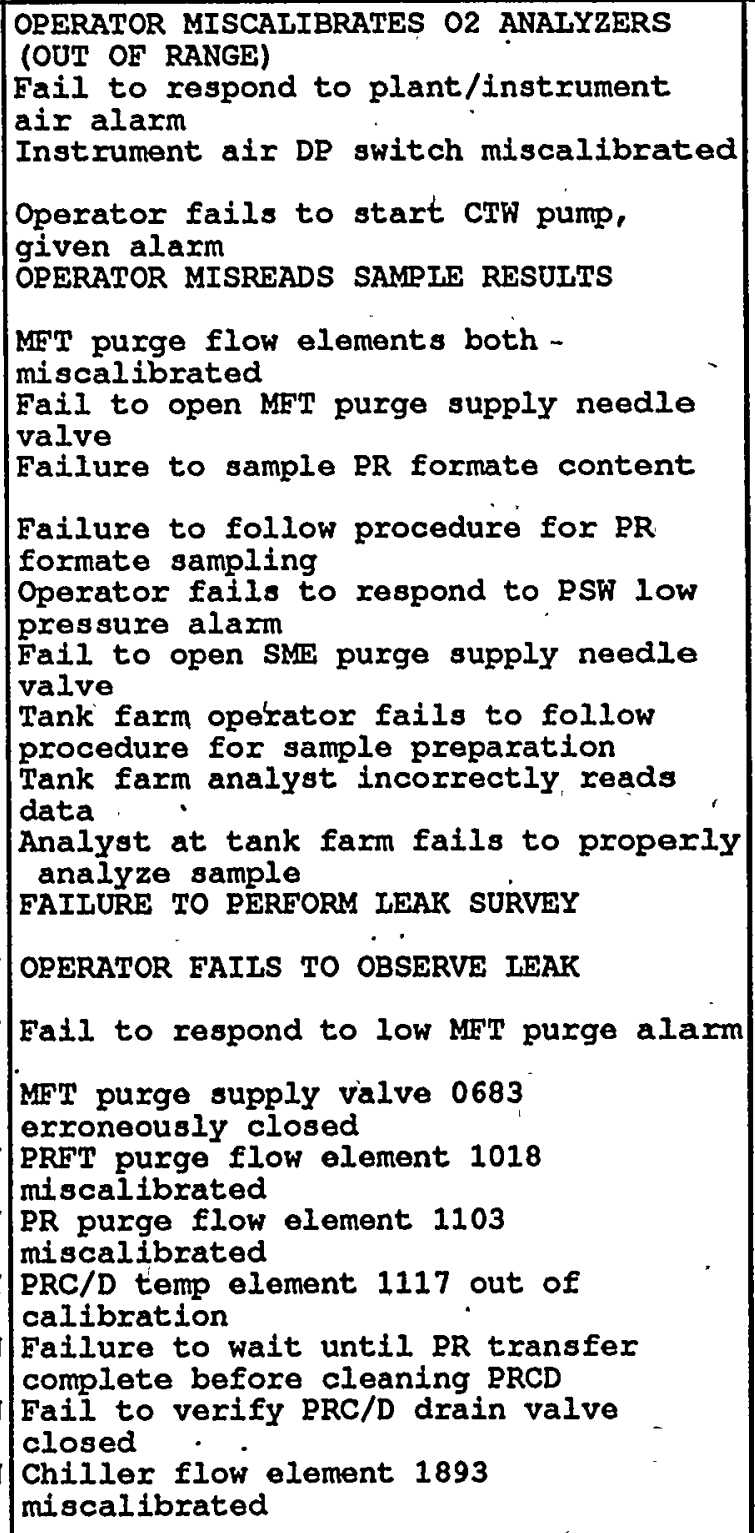 & $\begin{array}{l}\text { a: } 2 \text { weeks to detect \& repair } \\
\text { Use TC value } \\
\text { Use TC value } \\
\text { Use TC value } \\
\text { Use TC } \\
\text { a: complete dependence between FEs } \\
\text { Use TC value } \\
\text { Use TC Value } \\
\text { Use TC value } \\
\text { Use TC value } \\
\text { Use TC value } \\
\text { Use TC } \\
\text { Use TC } \\
\text { Use TC } \\
\text { use tc value } \\
\text { use tc value } \\
\text { Use TC value } \\
\text { a: corrected in } 12 \text { hours } \\
\text { a: calibrated annually, corrected by } \\
\text { next batch } \\
\text { a: calibrated annually, corrected by } \\
\text { next batch } \\
\text { a: calibrated annually, corrected by } \\
\text { next batch } \\
\text { Use TC Value } \\
\text { Use TC Value } \\
\text { a: calibrated annually, corrected in } \\
\text { I week }\end{array}$ \\
\hline
\end{tabular}


Basic Event Report for I: \CAFTA IDATA \MASTER.BE 11/11/94 11:42 AM (CONT.)

\begin{tabular}{|c|c|c|c|c|c|}
\hline rent & 0 & nput & $21 c$. & escription & Source \\
\hline 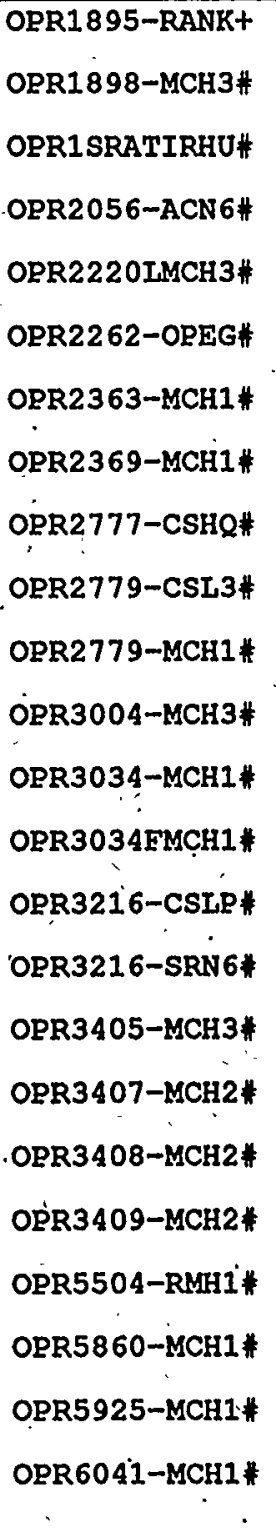 & 1 & $\begin{array}{r}1 \mathrm{H} \\
5.0 \mathrm{E}-06 \mathrm{H} \\
1 \mathrm{~N} \\
3.0 \mathrm{E}-02 \mathrm{~N} \\
1 \mathrm{~N} \\
5.0 \mathrm{E}-01 \mathrm{~N} \\
1 \mathrm{~N} \\
5.0 \mathrm{E}-03 \mathrm{~N} \\
1 \mathrm{~N} \\
3.0 \mathrm{E}-02 \mathrm{~N} \\
1 \mathrm{~N} \\
1.0 \mathrm{~N} \\
.04 \mathrm{~N} \\
3.0 \mathrm{E}-02 \mathrm{~N} \\
1 \mathrm{~N} \\
3.0 \mathrm{E}-02 \mathrm{~N} \\
1 \mathrm{~N} \\
1.0 \mathrm{E}-01 \mathrm{~N} \\
1 \mathrm{~N} \\
3.0 \mathrm{E}-03 \mathrm{~N} \\
1 \mathrm{~N} \\
3.0 \mathrm{E}-02 \mathrm{~N} \\
1 \mathrm{~N} \\
3.0 \mathrm{E}-02 \mathrm{~N} \\
.02 \mathrm{~N} \\
3.0 \mathrm{E}-02 \mathrm{~N} \\
0.01 \mathrm{~N} \\
3.0 \mathrm{E}-02 \mathrm{~N} \\
1 \mathrm{~N} \\
3.0 \mathrm{E}-03 \mathrm{~N} \\
1 \mathrm{~N} \\
1.0 \mathrm{E}-02 \mathrm{~N} \\
0.1 \mathrm{~N} \\
3.0 \mathrm{E}-02 \mathrm{~N} \\
1.2 \mathrm{E}-1 \mathrm{~N} \\
3.0 \mathrm{E}-02 \mathrm{~N} \\
1.2 \mathrm{E}-1 \mathrm{~N} \\
3.0 \mathrm{E}-02 \mathrm{~N} \\
0.1 \mathrm{~N} \\
3.0 \mathrm{E}-02 \mathrm{~N} \\
1 \mathrm{~N} \\
5.0 \mathrm{E}-02 \mathrm{~N} \\
3 \mathrm{E}-3 \mathrm{~N} \\
3.0 \mathrm{E}-02 \mathrm{~N} \\
1 \mathrm{~N} \\
3 \mathrm{E}-3 \mathrm{~N} \\
3 \mathrm{~N}-02 \mathrm{~N} \\
3.02 \mathrm{~N}\end{array}$ & $\begin{array}{l}3.00 \mathrm{E}-02 \mathrm{~N} \\
5.00 \mathrm{E}-01 \mathrm{~N} \\
5.00 \mathrm{E}-03 \mathrm{~N} \\
3.00 \mathrm{E}-02 \mathrm{~N} \\
1.00 \mathrm{E}+00 \mathrm{~N} \\
1.20 \mathrm{E}-03 \mathrm{~N} \\
3.00 \mathrm{E}-02 \mathrm{~N} \\
1.00 \mathrm{E}-01 \mathrm{~N} \\
3.00 \mathrm{E}-03 \mathrm{~N} \\
3.00 \mathrm{E}-02 \mathrm{~N} \\
3.00 \mathrm{E}-02 \mathrm{~N} \\
6.00 \mathrm{E}-04 \mathrm{~N} \\
3.00 \mathrm{E}-04 \mathrm{~N} \\
3.00 \mathrm{E}-03 \mathrm{~N} \\
1.00 \mathrm{E}-02 \mathrm{~N} \\
3.00 \mathrm{E}-03 \mathrm{~N} \\
3.60 \mathrm{E}-03 \mathrm{~N} \\
3.60 \mathrm{E}-03 \mathrm{~N} \\
3.00 \mathrm{E}-03 \mathrm{~N} \\
5.00 \mathrm{E}-02 \mathrm{~N} \\
9.00 \mathrm{E}-05 \mathrm{~N} \\
3.00 \mathrm{E}-02 \mathrm{~N} \\
9.00 \mathrm{E}-05 \mathrm{~N}\end{array}$ & 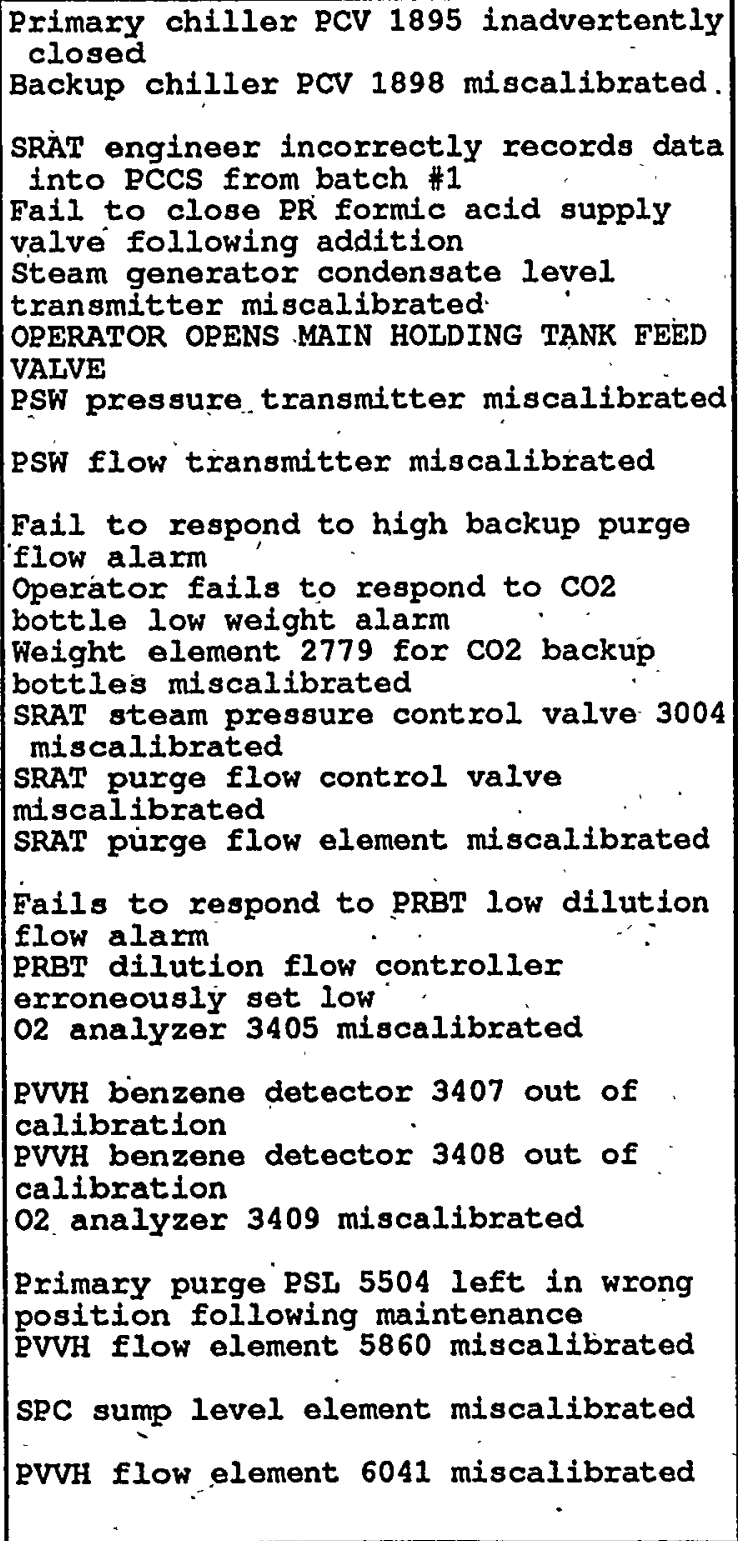 & $\begin{array}{l}\text { a: detected and corrected in } 1 \text { hour } \\
\text { Use TC value } \\
\text { Use TC } \\
\text { Use TC value } \\
\text { Use TC value } \\
\text { Use TC } \\
\text { a: calibrated annually, error } \\
\text { corrected in } 2 \text { weeks } \\
\text { Use TC value } \\
\text { Use TC value } \\
\text { Use TC value } \\
\text { Use TC value } \\
\text { Use TC value } \\
\text { a: calibrated annually, corrected in. } \\
1 \text { week } \\
\text { a: calibrated annually, corrected in } \\
\text { I week : } \\
\text { Use TC Value } \\
\text { Use TC value } \\
\text { a: calibrated } 1 / 10 \text { batches, corrected } \\
\text { by next batch } \\
\text { a: calibrated monthly, tested every } \\
\text { batch } \\
\text { a: calibrated monthly, tested every } \\
\text { batch } \\
\text { a: calibrated } 1 / 10 \text { batches, corrected } \\
\text { by next batch } \\
\text { Use TC value } \\
\text { a: calibrated annually, corrected in } \\
24 \text { hours } \\
\text { Use TC value } \\
\text { a: calibrated annually, corrected in } \\
24 \text { hours }\end{array}$ \\
\hline
\end{tabular}




\begin{tabular}{|c|c|c|c|c|c|}
\hline Event & c & put & $\therefore$ & Description & Source \\
\hline 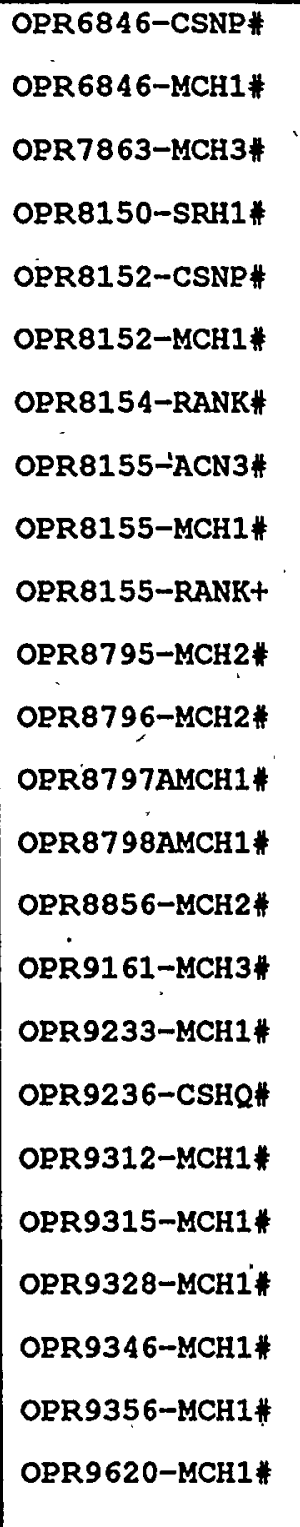 & 1 & $\begin{array}{r}1 \mathrm{~N} \\
1.0 \mathrm{E}-02 \mathrm{~N} \\
1 \mathrm{~N} \\
3.0 \mathrm{E}-02 \mathrm{~N} \\
1 \mathrm{~N} \\
3.0 \mathrm{E}-02 \mathrm{~N} \\
1 \mathrm{~N} \\
3.0 \mathrm{E}-02 \mathrm{~N} \\
1 \mathrm{~N} \\
1.0 \mathrm{E}-02 \mathrm{~N} \\
1 \mathrm{~N} \\
3.0 \mathrm{E}-02 \mathrm{~N} \\
24 \mathrm{H} \\
5.0 \mathrm{E}-06 \mathrm{H} \\
1 \mathrm{~N} \\
5.0 \mathrm{E}-03 \mathrm{~N} \\
1 \mathrm{~N} \\
3.0 \mathrm{E}-02 \mathrm{~N} \\
24 \mathrm{H} \\
5.0 \mathrm{E}-06 \mathrm{H} \\
1.2 \mathrm{E}-1 \mathrm{~N} \\
3.0 \mathrm{E}-02 \mathrm{~N} \\
1.2 \mathrm{E}-1 \mathrm{~N} \\
3.0 \mathrm{E}-02 \mathrm{~N} \\
1.2 \mathrm{E}-1 \mathrm{~N} \\
3.0 \mathrm{E}-02 \mathrm{~N} \\
1.2 \mathrm{E}-1 \mathrm{~N} \\
3.0 \mathrm{E}-02 \mathrm{~N} \\
1 \mathrm{E}-02 \mathrm{~N} \\
3.0 \mathrm{E}-02 \mathrm{~N} \\
1 \mathrm{~N} \\
3.0 \mathrm{E}-02 \mathrm{~N} \\
1 \mathrm{~N} \\
3.0 \mathrm{E}-02 \mathrm{~N} \\
1.0 \mathrm{E}-01 \mathrm{~N} \\
3 \mathrm{E}-3 \mathrm{~N} \\
3.0 \mathrm{E}-02 \mathrm{~N} \\
4 \mathrm{E}-3 \mathrm{~N} \\
3.0 \mathrm{E}-02 \mathrm{~N} \\
4 \mathrm{E}-3 \mathrm{~N} \\
3.0 \mathrm{E}-02 \mathrm{~N} \\
4 \mathrm{E}-3 \mathrm{~N} \\
3.0 \mathrm{E}-02 \mathrm{~N} \\
1 \mathrm{~N} \\
3.0 \mathrm{E}-02 \mathrm{~N} \\
3.0 \mathrm{E}-02 \mathrm{~N}\end{array}$ & $\begin{array}{l}3.00 \mathrm{E}-02 \mathrm{~N} \\
3.00 \mathrm{E}-02 \mathrm{~N} \\
3.00 \mathrm{E}-02 \mathrm{~N} \\
1.00 \mathrm{E}-02 \mathrm{~N} \\
3.00 \mathrm{E}-02 \mathrm{~N} \\
1.20 \mathrm{E}-04 \\
5.00 \mathrm{E}-03 \mathrm{~N} \\
3.00 \mathrm{E}-02 \mathrm{~N} \\
1.20 \mathrm{E}-04 \\
3.60 \mathrm{E}-03 \mathrm{~N} \\
3.60 \mathrm{E}-03 \mathrm{~N} \\
3.60 \mathrm{E}-03 \mathrm{~N} \\
3.60 \mathrm{E}-03 \mathrm{~N} \\
3.00 \mathrm{E}-04 \mathrm{~N} \\
3.00 \mathrm{E}-02 \mathrm{~N} \\
3.00 \mathrm{E}-02 \mathrm{~N} \\
1.00 \mathrm{E}-01 \mathrm{~N} \\
9.00 \mathrm{E}-05 \mathrm{~N} \\
1.20 \mathrm{E}-04 \mathrm{~N} \\
1.20 \mathrm{E}-04 \mathrm{~N} \\
1.20 \mathrm{E}-04 \mathrm{~N} \\
3.00 \mathrm{E}-02 \mathrm{~N}\end{array}$ & $\begin{array}{l}\text { Operator fails to respond to LPPP VVS } \\
\text { HEPA high DP alarm } \\
\text { HEPA filter DP switches miscalibrated } \\
\text { N2 tank low level switch out of } \\
\text { calibration } \\
\text { Misadjustment - FCV set low } \\
\text { Operator fails to respond to high } \\
\text { inlet HEPA alarm } \\
\text { High DP switch miscalibrated } \\
\text { Operator erroneously closes IPP VVH } \\
\text { fan-2 inlet damper } \\
\text { Operator fails to respond to PVV flow } \\
\text { low alarm FAL-8155 } \\
\text { PVVH flow transmitter } 8155 \\
\text { miscalibrated } \\
\text { Operator erroneously closes LPP VVH } \\
\text { fan-1 inlet damper } \\
\text { SRAT offgas detector } 1 \text { out of } \\
\text { calibration } \\
\text { SRAT offgas detector } \$ 2 \text { out of } \\
\text { calibration } \\
\text { SME hydrogen detector } 1 \text { out of } \\
\text { calibration } \\
\text { SME hydrogen detector } 2 \text { out of } \\
\text { calibration } \\
\text { Operator miscalibrates sME purge flow } \\
\text { element } \\
\text { Valve HCv-9161 miscalibrated } \\
\text { O2 analyzer miscalibrated } \\
\text { Operator fails to respond to high } \\
\text { backup N2 flow alarm } \\
\text { OE vapor space PT-9312 out of } \\
\text { calibration } \\
\text { OE purge flow element } 9315 \\
\text { miscalibrated } \\
\text { OECT purge flow element } 9328 \\
\text { miscalibrated } \\
\text { OEC/D temp element } 9346 \text { out of } \\
\text { calibration } \\
\text { SCVC chilied water supply valve } 9356 \\
\text { miscalibrated } \\
\text { Secondary compressor inlet filter DP } \\
\text { miscalibrated }\end{array}$ & $\begin{array}{l}\text { Use TC value } \\
\text { a: complete dependence } \\
\text { Use TC value } \\
\text { Use TC value } \\
\text { Use TC value } \\
\text { Use TC value } \\
\text { a: corrected in } 24 \text { hours } \\
\text { Use TC value } \\
\text { Use TC value } \\
\text { a: corrected in } 24 \text { hours } \\
\text { a: calibrated monthly, tested every } \\
\text { batch } \\
\text { a: calibrated monthly, tested every } \\
\text { batch } \\
\text { a: tested every batch, calibrated. } \\
\text { monthly } \\
\text { a: tested every batch, calibrated } \\
\text { monthly } \\
\text { a: calibrated annually, corrected by } \\
\text { next batch } \\
\text { Use TC value } \\
\text { Use TC value } \\
\text { Use TC value } \\
\text { a: calibrated annually, corrected in } \\
\text { 24 hours } \\
\text { a: calibrated annually, corrected by } \\
\text { next batch } \\
\text { a: calibrated annually, corrected by } \\
\text { next batch } \\
\text { a: calibrated annually, corrected by } \\
\text { next batch } \\
\text { Use TC value } \\
\text { Use TC value }\end{array}$ \\
\hline
\end{tabular}


Basic Event' Report for I: \CAFTA \DATA \MASTER.BE 11/11/94 11:42 AM' (CONT.)

\begin{tabular}{|c|c|c|c|c|c|c|c|c|c|}
\hline Event & c & Input & Calc. & Description & Source - & - & . & & \\
\hline 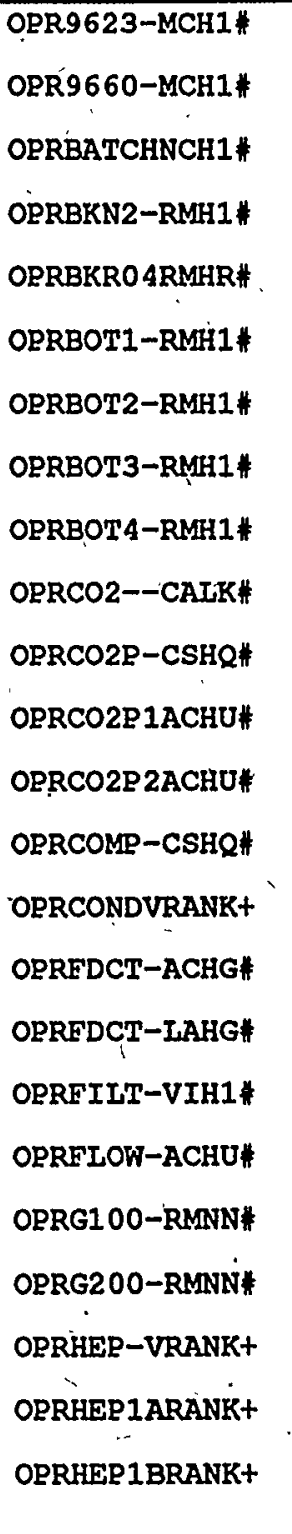 & $\begin{array}{l}1 \\
1 \\
1 \\
1 \\
1 \\
1 \\
1 \\
1 \\
1 \\
1 \\
1 \\
1 \\
1 \\
1 \\
4 \\
1 \\
1\end{array}$ & $\begin{array}{r}1 \mathrm{~N} \\
3.0 \mathrm{E}-02 \mathrm{~N} \\
1 \mathrm{~N} \\
3.0 \mathrm{E}-02 \mathrm{~N} \\
1 \mathrm{~N} \\
1.0 \mathrm{E}-01 \mathrm{~N} \\
1 \mathrm{~N} \\
5.0 \mathrm{E}-02 \mathrm{~N} \\
1 \mathrm{~N} \\
5.0 \mathrm{E}-02 \mathrm{~N} \\
1 \mathrm{~N} \\
5.0 \mathrm{E}-02 \mathrm{~N} \\
1 \mathrm{~N} \\
5.0 \mathrm{E}-02 \mathrm{~N} \\
1 \mathrm{~N} \\
5.0 \mathrm{E}-02 \mathrm{~N} \\
5.0 \mathrm{E}-02 \mathrm{~N} \\
1 \mathrm{~N} \\
3.0 \mathrm{E}-04 \mathrm{~N} \\
1 \mathrm{~N} \\
1.0 \mathrm{E}-01 \mathrm{~N} \\
1.0 \mathrm{~N} \\
5.0 \mathrm{E}-02 \mathrm{~N} \\
1 \mathrm{~N} \\
5.0 \mathrm{E}-02 \mathrm{~N} \\
1.0 \mathrm{E}-01 \mathrm{~N} \\
7 \mathrm{D} \\
5.0 \mathrm{E}-06 \mathrm{H} \\
5.0 \mathrm{~N}-02 \mathrm{~N} \\
1.0 \mathrm{E}-03 \mathrm{~N} \\
1 \mathrm{~N} \\
5.0 \mathrm{E}-01 \mathrm{~N} \\
5.0 \mathrm{E}-02 \mathrm{~N} \\
4.3 \mathrm{E}-02 \mathrm{~N} \\
5.0 \mathrm{E}-03 \mathrm{~N} \\
4.3 \mathrm{E}-02 \mathrm{~N} \\
5.0 \mathrm{E}-03 \mathrm{~N} \\
7 \mathrm{D} \\
5.0 \mathrm{E}-06 \mathrm{H} \\
7 \mathrm{D} \\
5.0 \mathrm{E}-06 \mathrm{H} \\
5.0 \mathrm{DD} \\
5.06 \mathrm{H}\end{array}$ & $\begin{array}{l}3.00 \mathrm{E}-02 \mathrm{~N} \\
3.00 \mathrm{E}-02 \mathrm{~N} \\
1.00 \mathrm{E}-01 \mathrm{~N} \\
5.00 \mathrm{E}-02 \mathrm{~N} \\
5.00 \mathrm{E}-02 \mathrm{~N} \\
5.00 \mathrm{E}-02 \mathrm{~N} \\
5.00 \mathrm{E}-02 \mathrm{~N} \\
5.00 \mathrm{E}-02 \mathrm{~N} \\
5.00 \mathrm{E}-02 \mathrm{~N} \\
3.00 \mathrm{E}-04 \mathrm{~N} \\
1.00 \mathrm{E}-01 \mathrm{~N} \\
5.00 \mathrm{E}-02 \mathrm{~N} \\
5.00 \mathrm{E}-02 \mathrm{~N} \\
1.00 \mathrm{E}-01 \mathrm{~N} \\
8.39 \mathrm{E}-04 \\
5.00 \mathrm{E}-02 \mathrm{~N} \\
1.00 \mathrm{E}-03 \mathrm{~N} \\
5.00 \mathrm{E}-01 \mathrm{~N} \\
5.00 \mathrm{E}-02 \mathrm{~N} \\
2.15 \mathrm{E}-04 \mathrm{~N} \\
2.15 \mathrm{E}-04 \mathrm{~N} \\
8.39 \mathrm{E}-04 \\
8.39 \mathrm{E}-04 \\
8.39 \mathrm{E}-04\end{array}$ & 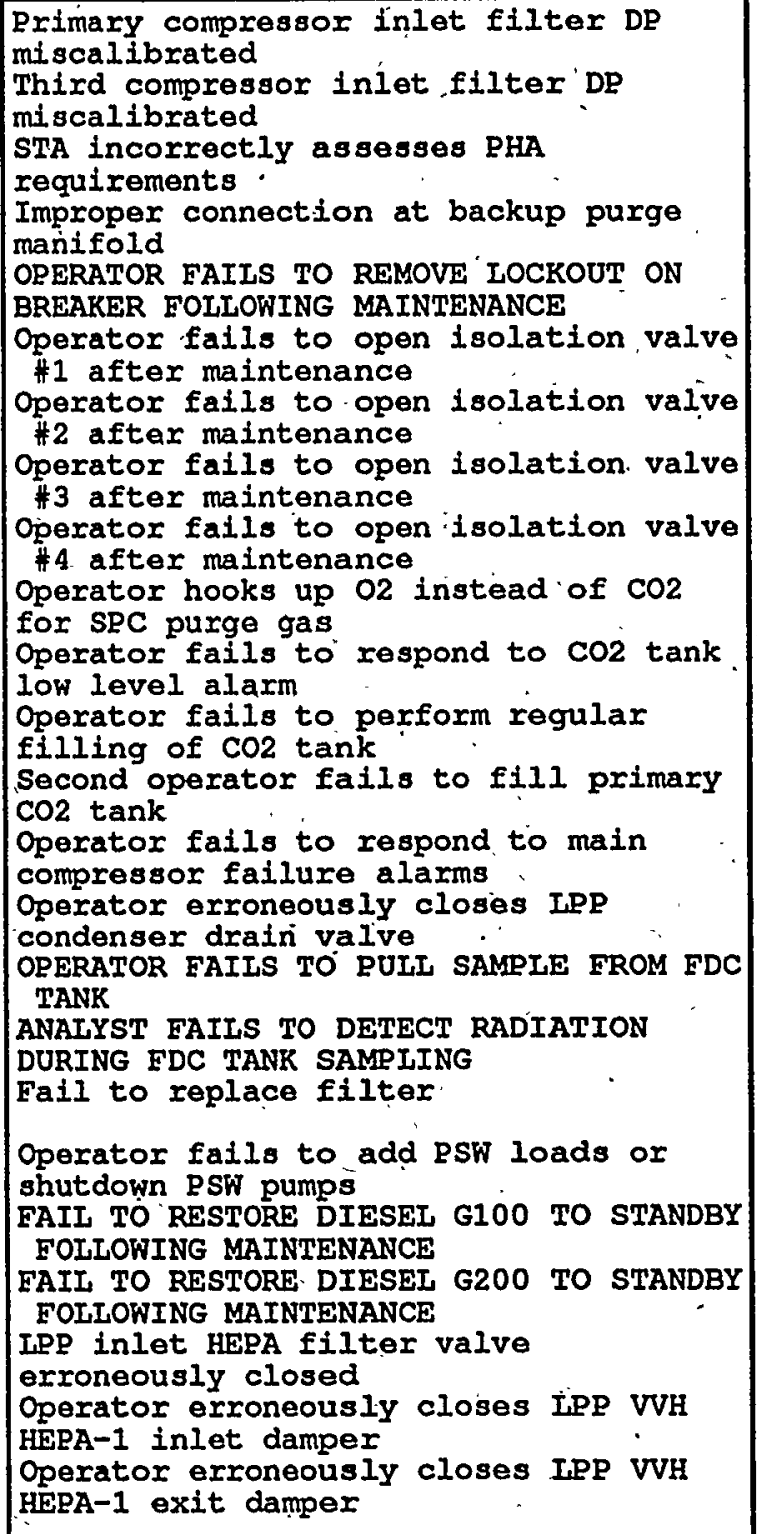 & $\begin{array}{l}\text { Use TC value } \\
\text { Use TC value } \\
\text { Use TC value } \\
\text { Use TC Value } \\
\text { Use TC } \\
\text { Use TC Value } \\
\text { Use TC Value } \\
\text { Use TC Value } \\
\text { Use TC Value } \\
\text { Use TC Value } \\
\text { Use TC value } \\
\text { Use TC value } \\
\text { Use TC value } \\
\text { Use TC value } \\
\text { a: Corrected } \\
\text { Use TC } \\
\text { Use TC } \\
\text { Use TC value } \\
\text { Use TC value } \\
1 / Y R * 31 D / 2 \\
1 / Y R * 31 D / 2 \\
\text { a: corrected } \\
\text { a: corrected } \\
\text { a: corrected }\end{array}$ & $\begin{array}{l}\text { in } 1 \text { week } \\
\text { (FROM OSR } \\
\text { (FROM OSR } \\
\text { in } 1 \text { week } \\
\text { in } 1 \text { week } \\
\text { in } 1 \text { week }\end{array}$ & $\begin{array}{c}. \\
\cdot \\
\cdot \\
3.5 .11 \\
3.5 .1) \\
k \\
k \\
k\end{array}$ & & $\therefore$ \\
\hline
\end{tabular}


Basic Event Report for I: \CAFTA \DATA MMASTER.BE 11/11/94 11:42 AM (CONT.)

\begin{tabular}{|c|c|c|c|c|c|}
\hline Event & c & nput & Calc. & Description & Source \\
\hline 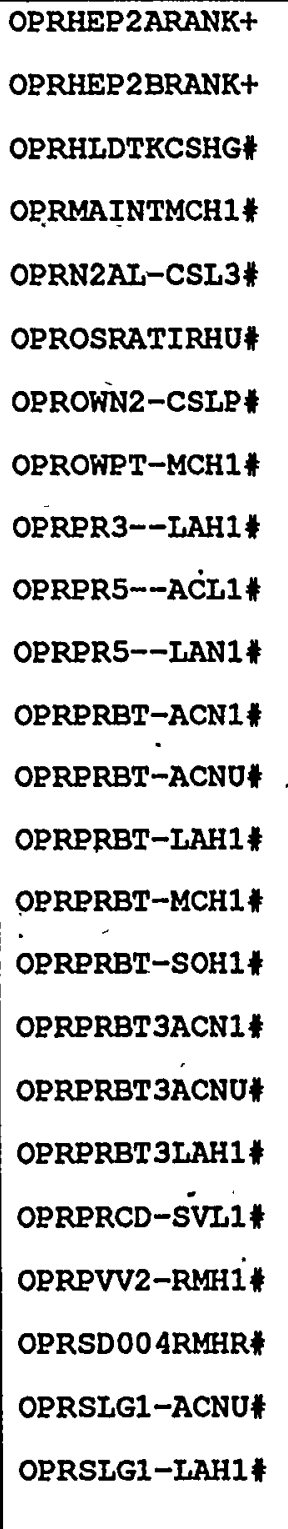 & 4 & 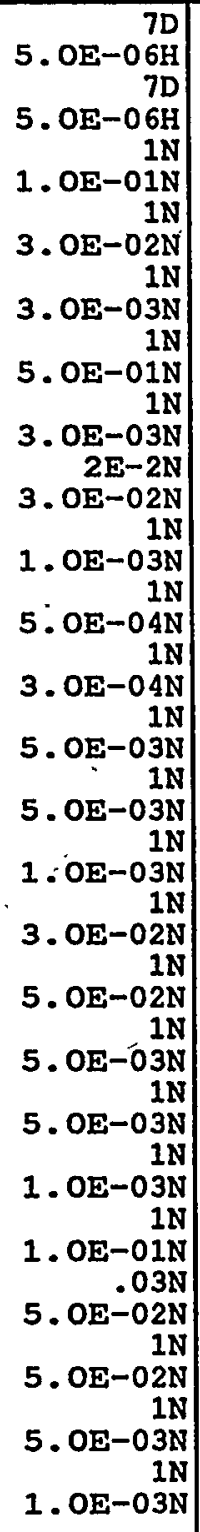 & $\begin{array}{l}8.39 \mathrm{E}-04 \\
8.39 \mathrm{E}-04 \\
1.00 \mathrm{E}-01 \mathrm{~N} \\
3.00 \mathrm{E}-02 \mathrm{~N} \\
3.00 \mathrm{E}-03 \mathrm{~N} \\
5.00 \mathrm{E}-01 \mathrm{~N} \\
3.00 \mathrm{E}-03 \mathrm{~N} \\
6.00 \mathrm{E}-04 \mathrm{~N} \\
1.00 \mathrm{E}-03 \mathrm{~N} \\
5.00 \mathrm{E}-04 \mathrm{~N} \\
3.00 \mathrm{E}-04 \mathrm{~N} \\
5.00 \mathrm{E}-03 \mathrm{~N} \\
5.00 \mathrm{E}-03 \mathrm{~N} \\
1.00 \mathrm{E}-03 \mathrm{~N} \\
3.00 \mathrm{E}-02 \mathrm{~N} \\
5.00 \mathrm{E}-02 \mathrm{~N} \\
5.00 \mathrm{E}-03 \mathrm{~N} \\
5.00 \mathrm{E}-03 \mathrm{~N} \\
1.00 \mathrm{E}-03 \mathrm{~N} \\
1.00 \mathrm{E}-01 \mathrm{~N} \\
1.50 \mathrm{E}-03 \mathrm{~N} \\
5.00 \mathrm{E}-02 \mathrm{~N} \\
5.00 \mathrm{E}-03 \mathrm{~N} \\
1.00 \mathrm{E}-03 \mathrm{~N}\end{array}$ & 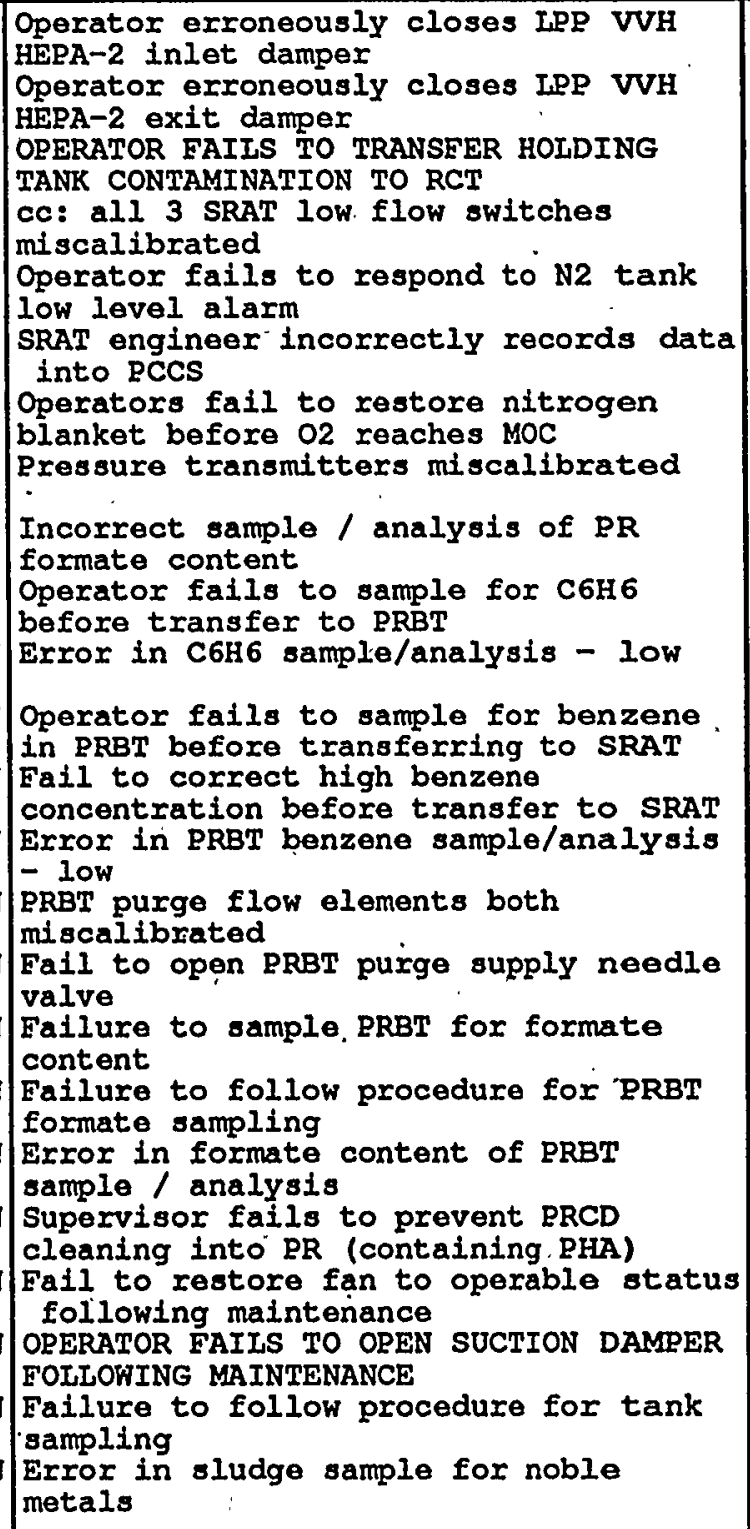 & $\begin{array}{l}\text { a: corrected in } 1 \text { week } \\
\text { a: corrected in } 1 \text { week } \\
\text { Use TC } \\
\text { a: same as single miscalibration } \\
\text { Use TC value } \\
\text { Use TC } \\
\text { a: > 4 hours to MOC } \\
\text { a: calibrated annually, corrected in } \\
\text { I week } \\
\text { Use TC value } \\
\text { Use TC Value } \\
\text { Use TC value } \\
\text { Use TC value } \\
\text { Use TC value } \\
\text { as: complete dependence between FEs } \\
\text { Use TC value } \\
\text { Use TC value } \\
\text { Use TC value } \\
\text { Use } \\
\text { Use TC value } \\
\text { Use }\end{array}$ \\
\hline
\end{tabular}




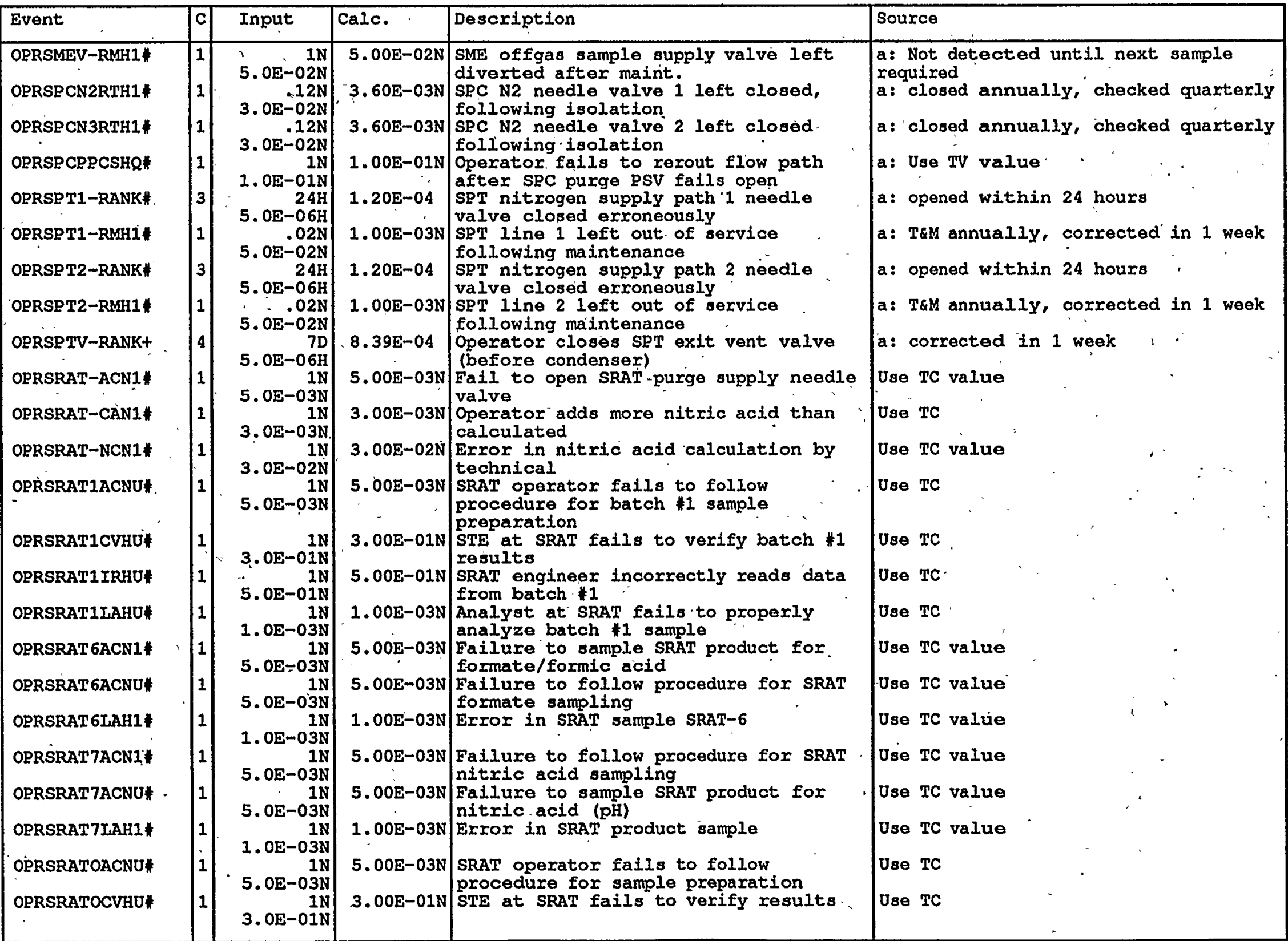


Basic Event Report for I: (CAFTA \DATAMMASTER.BE 11/11/94 11:42 AM (CONT.)

\begin{tabular}{|c|c|c|c|c|c|}
\hline Event & c & nput & Calc. & Description & Source \\
\hline $\begin{array}{l}\text { ORRSRATOIRHU\# } \\
\text { ORRSRATOLAHU\# } \\
\text { ORRSRATURMH5\# } \\
\text { ORRSUMP-ACHG\# } \\
\text { ORRSUMP-CSHQ\# } \\
\text { ORRSV74ARANK+ } \\
\text { ORRSV74BRANK+ } \\
\text { ORRSV74CRANK+ } \\
\text { OPRSV74DRANK+ } \\
\text { ORRVESI-OVH1+ } \\
\text { OPRVVE1DRANK+ } \\
\text { OPRVVE2DRANK\# } \\
\text { PC-1041-FLOK\# } \\
\text { PCSSRAT-BEL5\# } \\
\text { PCV-7172FCLQ+ } \\
\text { PCV-BP--FCLQ+ } \\
\text { PCV0963-FCL2+. } \\
\text { PCV0963-FTCJ\# } \\
\text { PCV0964-FCL2+ } \\
\text { PCV0967-FTOJ\# } \\
\text { PCV1016-FCLB+ } \\
\text { PCV1019-FTOB\# } \\
\text { PCV1101-FCL9+ } \\
\text { PCVII04-FTO9\# }\end{array}$ & 1 & $\begin{array}{r}1 \mathrm{~N} \\
5.0 \mathrm{E}-01 \mathrm{~N} \\
1 \mathrm{~N} \\
1.0 \mathrm{E}-03 \mathrm{~N} \\
1.4 \mathrm{E}-3 \mathrm{~N} \\
5.0 \mathrm{E}-02 \mathrm{~N} \\
1 \mathrm{~N} \\
5.0 \mathrm{E}-02 \mathrm{~N} \\
1 \mathrm{~N} \\
1.0 \mathrm{E}-01 \mathrm{~N} \\
24 \mathrm{H} \\
5.0 \mathrm{E}-06 \mathrm{H} \\
43 \mathrm{H} \\
5.0 \mathrm{E}-06 \mathrm{H} \\
43 \mathrm{H} \\
5.0 \mathrm{E}-06 \mathrm{H} \\
43 \mathrm{H} \\
5.0 \mathrm{E}-06 \mathrm{H} \\
8 \mathrm{H} \\
5.0 \mathrm{E}-05 \mathrm{H} \\
24 \mathrm{H} \\
5.0 \mathrm{E}-06 \mathrm{H} \\
7 \mathrm{D} \\
5.0 \mathrm{E}-06 \mathrm{H} \\
43 \mathrm{H} \\
1.0 \mathrm{E}-06 \mathrm{H} \\
1 \mathrm{~N} \\
1.0 \mathrm{E}-03 \mathrm{~N} \\
4 \mathrm{H} \\
3.0 \mathrm{E}-06 \mathrm{H} \\
4 \mathrm{H} \\
3.0 \mathrm{E}-06 \mathrm{H} \\
8 \mathrm{H} \\
3.0 \mathrm{E}-06 \mathrm{H} \\
1 \mathrm{~N} \\
3.0 \mathrm{E}-03 \mathrm{~N} \\
8 \mathrm{H} \\
3.0 \mathrm{E}-06 \mathrm{H} \\
1 \mathrm{~N} \\
3.0 \mathrm{E}-03 \mathrm{~N} \\
8 \mathrm{H} \\
3.0 \mathrm{E}-06 \mathrm{H} \\
1 \mathrm{~N} \\
3.0 \mathrm{E}-03 \mathrm{~N} \\
8 \mathrm{H} \\
3.0 \mathrm{E}-06 \mathrm{H} \\
1 \mathrm{~N} \\
3.0 \mathrm{E}-03 \mathrm{~N}\end{array}$ & \begin{tabular}{|l|}
$5.00 \mathrm{E}-01 \mathrm{~N}$ \\
$1.00 \mathrm{E}-03 \mathrm{~N}$ \\
$7.00 \mathrm{E}-05 \mathrm{~N}$ \\
$5.00 \mathrm{E}-02 \mathrm{~N}$ \\
$1.00 \mathrm{E}-01 \mathrm{~N}$ \\
$1.20 \mathrm{E}-04$ \\
$2.15 \mathrm{E}-04$ \\
$2.15 \mathrm{E}-04$ \\
$2.15 \mathrm{E}-04$ \\
$4.00 \mathrm{E}-04$ \\
$1.20 \mathrm{E}-04$ \\
$8.40 \mathrm{E}-04$ \\
$2.15 \mathrm{E}-05$ \\
$1.00 \mathrm{E}-03 \mathrm{~N}$ \\
$1.20 \mathrm{E}-05$ \\
$1.20 \mathrm{E}-05$ \\
$2.40 \mathrm{E}-05$ \\
$3.00 \mathrm{E}-03 \mathrm{~N}$ \\
$2.40 \mathrm{E}-05$ \\
$3.00 \mathrm{E}-03 \mathrm{~N}$ \\
$2.40 \mathrm{E}-05$ \\
$3.00 \mathrm{E}-03 \mathrm{~N}$ \\
$2.40 \mathrm{E}-05$ \\
$3.00 \mathrm{E}-03 \mathrm{~N}$ \\
\end{tabular} & 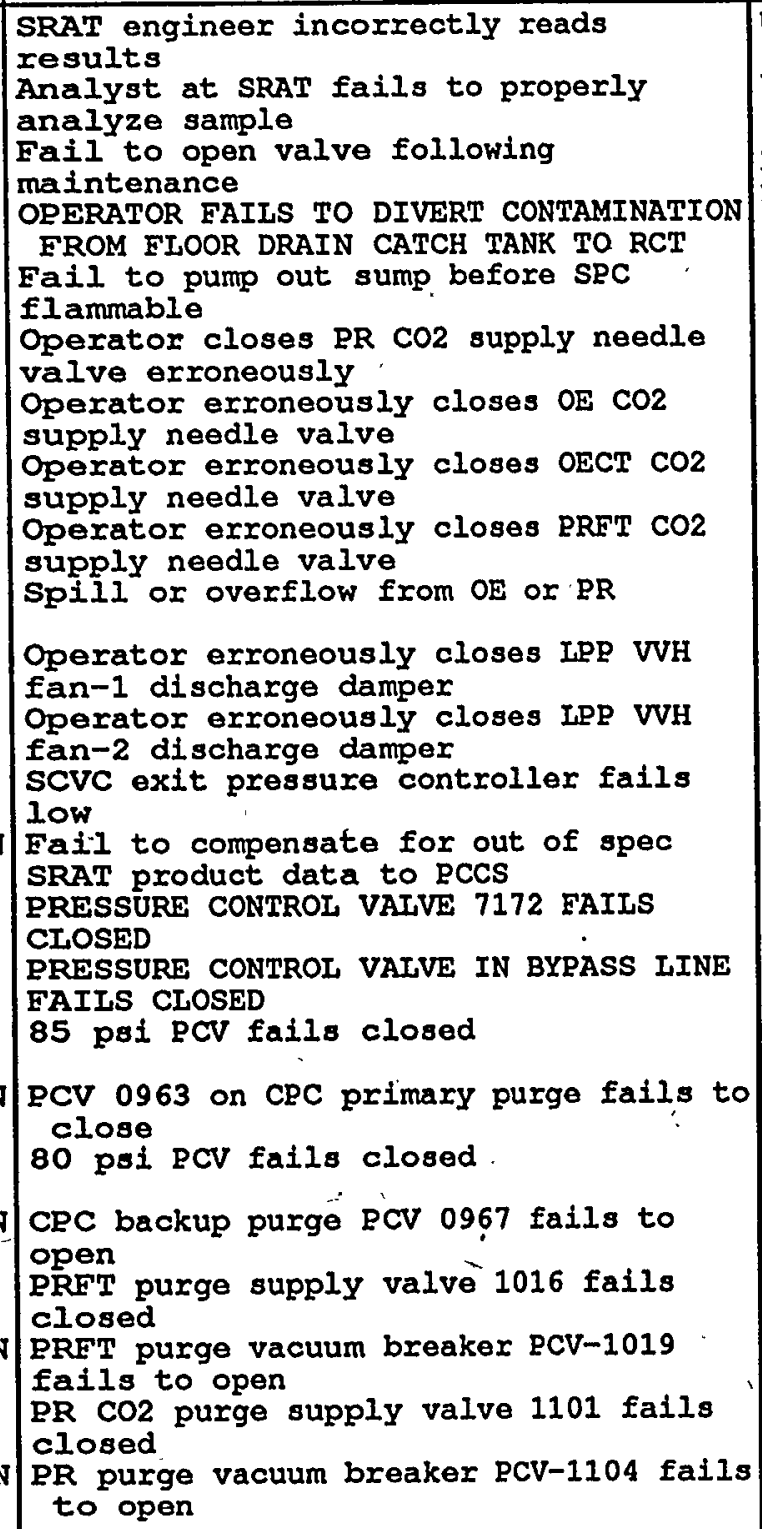 & $\begin{array}{l}\text { Use TC } \\
\text { Use TC } \\
\text { a: maintained annually, corrected } \\
\text { before next batch } \\
\text { Use TC } \\
\text { Use TC value } \\
\text { a: corrected in } 24 \text { hours } \\
\text { a: opened by next batch } \\
\text { a: opened by next batch } \\
\text { a: opened by next batch } \\
\text { a: } 2 \text { tanks, } 4 \text { hour recovery } \\
\text { a:corrected in } 24 \text { hours } \\
\text { a: corrected in } 1 \text { week } \\
\text { a: repaired before next PR batch } \\
\text { Use TC Value } \\
\text { a: } 8 \text { hours to repair } \\
\text { a: } 8 \text { hours to repair } \\
\text { a: repaired or process shutdown in } 8 \\
\text { hours } \\
\text { Use TC value } \\
\text { a: Repaired or process shutdown in } 8 \\
\text { hours } 8 \text { Hours to repair } \\
\text { Use TC value } \\
\text { a: repaired in } 8 \text { hours } \\
\text { Use TC value } \\
\text { ase } \\
\text { alue } \\
\text { a }\end{array}$ \\
\hline
\end{tabular}


Basic Event Report for I: \CAFTA DDATA MASTER.BE 11/11/94 11:42 AM (CONT.)

\begin{tabular}{|c|c|c|c|c|c|}
\hline Event & c & unt & alc. & escription & Pource \\
\hline $\begin{array}{l}\text { PCV1898-CAL1* } \\
\text { PCV1898-FTOE* } \\
\text { PCV2221-FCLG } \\
\text { PCV2775-FTO1* } \\
\text { PCV2778XFTO1* } \\
\text { PCV3004-CAL1* } \\
\text { PCV3004-FCLG+ } \\
\text { PCV7418XFTOQ\# } \\
\text { PCV7420-FTOQ\# } \\
\text { PCV7420XFTOQ\# } \\
\text { PCV7601XFCL1+ } \\
\text { PCV7603XFOR1* } \\
\text { PCV7603XT\&M1\# }\end{array}$ & 4 & $\begin{array}{r}8 \mathrm{H} \\
3 . \mathrm{OE}-06 \mathrm{H} \\
7 \mathrm{D} \\
1.0 \mathrm{E}+0 \mathrm{OY} \\
1 \mathrm{~N} \\
3.0 \mathrm{E}-03 \mathrm{~N} \\
8 \mathrm{H} \\
3 . \mathrm{OE}-06 \mathrm{H} \\
1 \mathrm{~N} \\
3.0 \mathrm{E}-03 \mathrm{~N} \\
1 \mathrm{~N} \\
3.0 \mathrm{E}-03 \mathrm{~N} \\
7 \mathrm{D} \\
1.0 \mathrm{E}+0 \mathrm{OY} \\
8 \mathrm{H} \\
3.0 \mathrm{E}-06 \mathrm{H} \\
1 \mathrm{~N} \\
3.0 \mathrm{E}-03 \mathrm{~N} \\
1 \mathrm{~N} \\
3.0 \mathrm{E}-03 \mathrm{~N} \\
1 \mathrm{~N} \\
3.0 \mathrm{E}-03 \mathrm{~N} \\
1 \mathrm{H} \\
3.0 \mathrm{E}-06 \mathrm{H} \\
1 \mathrm{M} \\
3.0 \mathrm{E}-06 \mathrm{H} \\
24 \mathrm{H} \\
3.0 \mathrm{E}-01 \mathrm{Y} \\
24 \mathrm{H} \\
3.0 \mathrm{E}-06 \mathrm{H} \\
24 \mathrm{H} \\
3.0 \mathrm{E}-06 \mathrm{H} \\
24 \mathrm{H} \\
3.0 \mathrm{E}-06 \mathrm{H} \\
7 \mathrm{D} \\
1.0 \mathrm{E}+00 \mathrm{Y} \\
1 \mathrm{~N} \\
3.0 \mathrm{E}-03 \mathrm{~N} \\
24 \mathrm{H} \\
3.0 \mathrm{E}-06 \mathrm{H} \\
8 \mathrm{H} \\
3.0 \mathrm{E}-06 \mathrm{H} \\
1 \mathrm{~N} \\
3.0 \mathrm{E}-03 \mathrm{~N} \\
24 \mathrm{H} \\
1 \mathrm{~N} \\
3 \mathrm{~N} \\
3\end{array}$ & $\begin{array}{l}9.53 \mathrm{E}-03 \\
3.00 \mathrm{E}-03 \mathrm{~N} \\
2.40 \mathrm{E}-05 \\
3.00 \mathrm{E}-03 \mathrm{~N} \\
3.00 \mathrm{E}-03 \mathrm{~N} \\
1.90 \mathrm{E}-02 \\
2.40 \mathrm{E}-05 \\
3.00 \mathrm{E}-03 \mathrm{~N} \\
3.00 \mathrm{E}-03 \mathrm{~N} \\
3.00 \mathrm{E}-03 \mathrm{~N} \\
3.00 \mathrm{E}-06 \\
1.08 \mathrm{E}-03 \\
1.10 \mathrm{E}-03 \\
7.20 \mathrm{E}-05 \\
7.20 \mathrm{E}-05 \\
7.20 \mathrm{E}-05 \\
1.88 \mathrm{E}-02 \\
1.50 \mathrm{E}-03 \mathrm{~N} \\
7.20 \mathrm{E}-05 \\
2.40 \mathrm{E}-05 \\
3.00 \mathrm{E}-03 \mathrm{~N} \\
7.20 \mathrm{E}-05 \\
3.00 \mathrm{E}-03 \mathrm{~N}\end{array}$ & 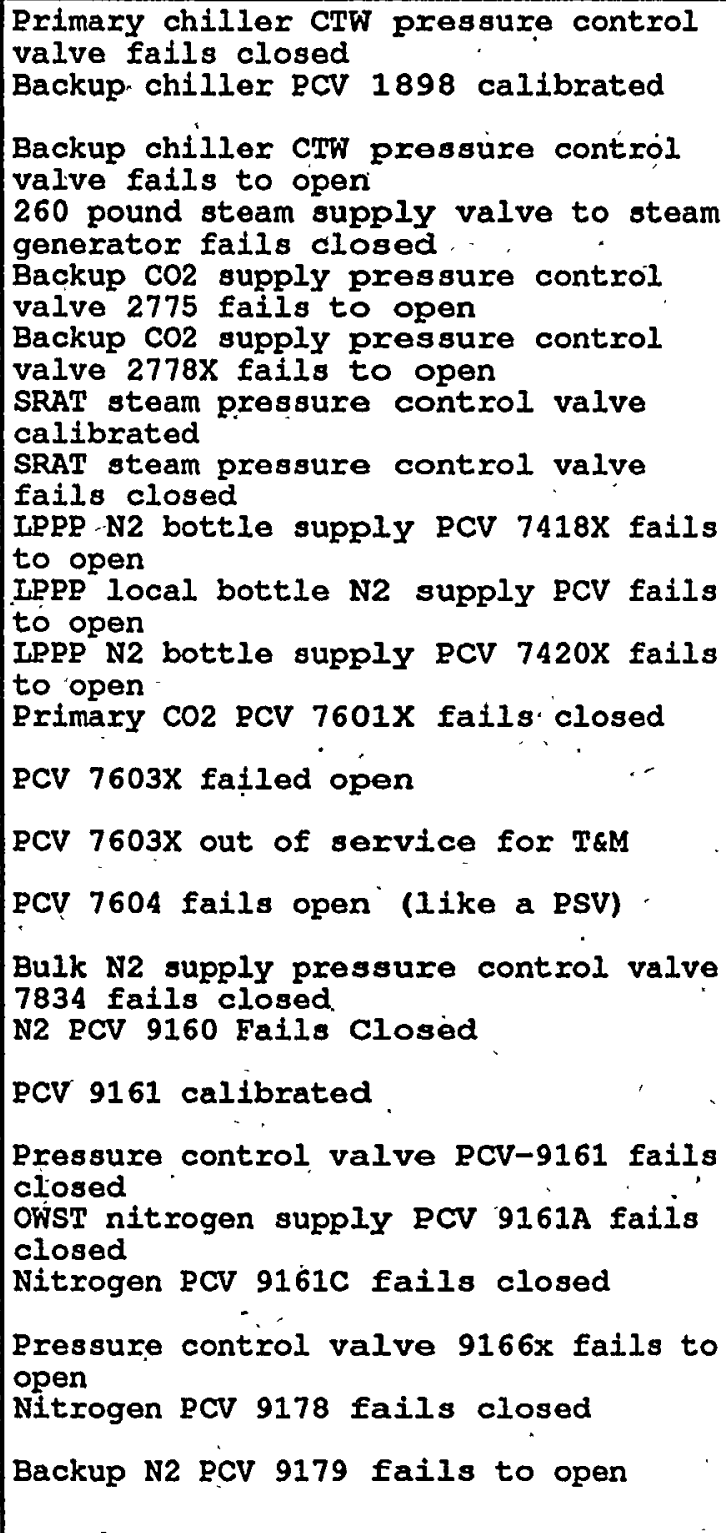 & $\begin{array}{l}\text { a: } 8 \text { hours to repair } \\
\text { a: calibrated annually, error } \\
\text { corrected in } 1 \text { week } \\
\text { Use TC value } \\
\text { a: detected and repaired or process } \\
\text { shutdown in } 8 \text { hours } \\
\text { A:Demands will be supplied by, loss of } \\
\text { primary CO2 } \\
\text { A:Demands will be supplied by loss of } \\
\text { primary cO2 } \\
\text { a: calibrated annually, error } \\
\text { corrected in } 1 \text { week } \\
\text { a: } 8 \text { hours to repair } \\
\text { Use TC value } \\
\text { Use TC value. } \\
\text { Use TC value } \\
\text { a: } 1 \text { hour to detect and rerout (high } \\
\text { backup flow alarm) } \\
\text { a: paths rerouted monthly } \\
\text { a: taken out of service for } 24 \text { hours } \\
\text { at a time } \\
\text { a: } 24 \text { hours to repair } \\
\text { a: } 24 \text { hours to repair/replace } \\
\text { a: } 24 \text { Hr Repair } \\
\text { a: calibrated annually, corrected in } \\
\text { I week } \\
\text { Use TC value } \\
\text { a: } 24 \text { Hr Repair } \\
\text { a: } 8 \text { Hr Repair } \\
\text { Use TC value } \\
\text { a: } 24 \text { hours to detect and repair } \\
\text { Use TC value }\end{array}$ \\
\hline
\end{tabular}




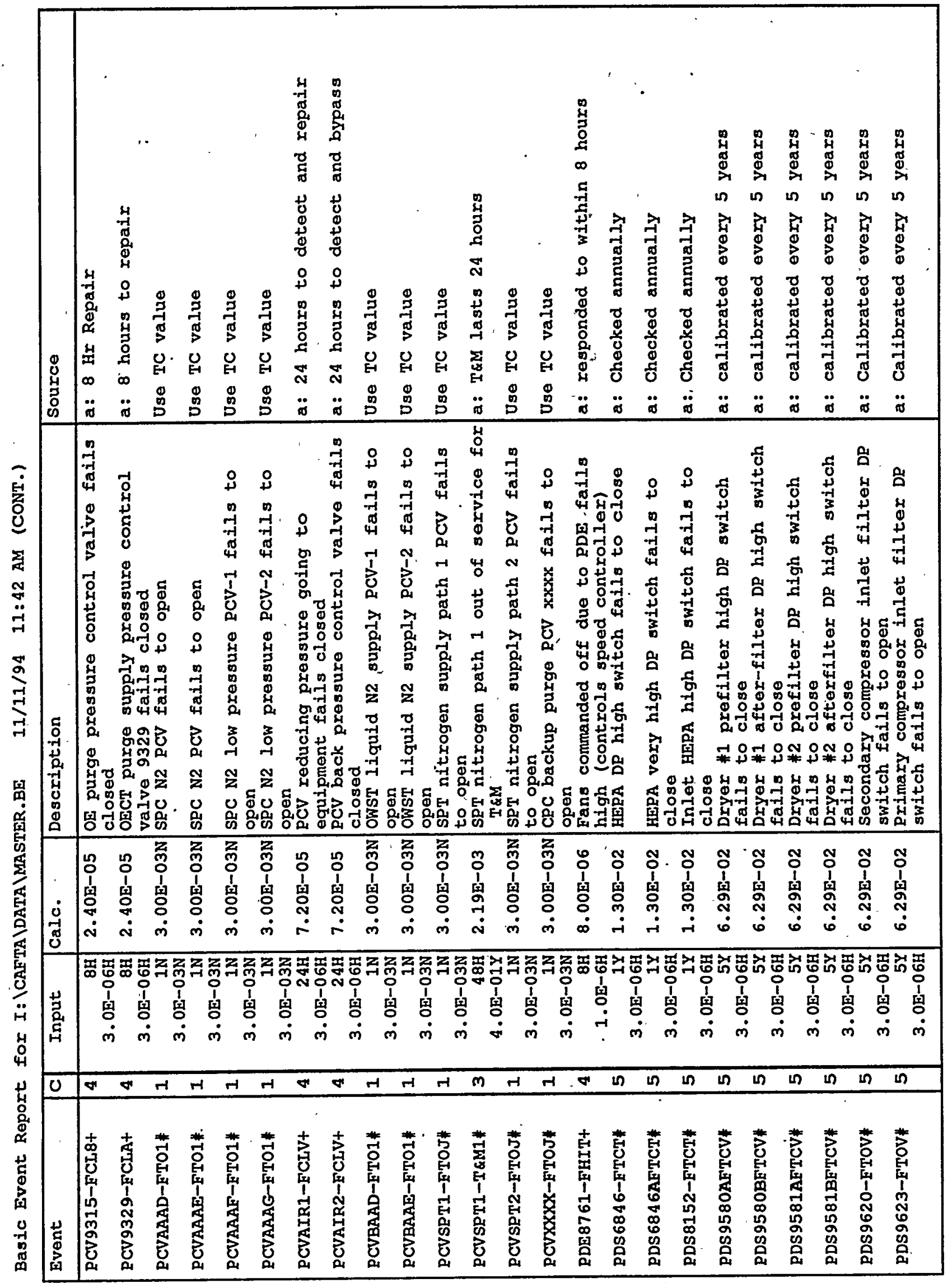


Basic Event Report for I: \CAFTA IDATA \MASTER.BE $11 / 11 / 94^{\prime} \quad 11: 42$ AM (CONT.)

\begin{tabular}{|c|c|c|c|c|c|}
\hline Event & C) & Input & alc. & Description & Source \\
\hline 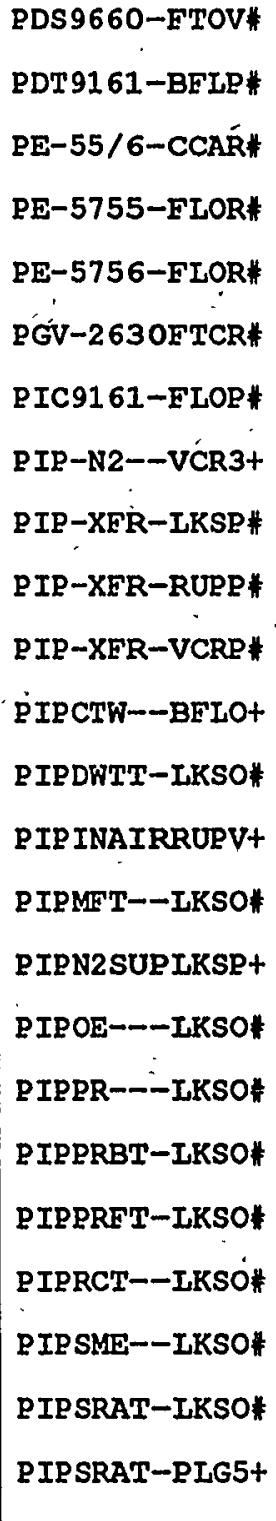 & 5 & $\begin{array}{r}5 \mathrm{Y} \\
3.0 \mathrm{E}-06 \mathrm{H} \\
1 \mathrm{Q} \\
3.0 \mathrm{E}-06 \mathrm{H} \\
14 \mathrm{D} \\
1.0 \mathrm{E}-07 \mathrm{H} \\
14 \mathrm{D} \\
1.0 \mathrm{E}-06 \mathrm{H} \\
14 \mathrm{D} \\
1.0 \mathrm{E}-06 \mathrm{H} \\
1 \mathrm{~N} \\
3.0 \mathrm{E}-03 \mathrm{~N} \\
10 \\
1.0 \mathrm{E}-05 \mathrm{H} \\
48 \mathrm{H} \\
2.55 \mathrm{E}-08 \mathrm{H} \\
8 \mathrm{H} \\
9.0 \mathrm{E}-09 \mathrm{H} \\
8 \mathrm{H} \\
2.18 \mathrm{E}-08 \mathrm{H} \\
1 \mathrm{H} \\
1.27 \mathrm{E}-09 \mathrm{H} \\
4 \mathrm{H} \\
6.0 \mathrm{E}-06 \mathrm{H} \\
5 \mathrm{Y} \\
1.5 \mathrm{E}-07 \mathrm{H} \\
8 \mathrm{H} \\
2.18 \mathrm{E}-08 \mathrm{H} \\
5 \mathrm{Y} \\
1.5 \mathrm{E}-07 \mathrm{H} \\
750 \mathrm{H} \\
9.0 \mathrm{E}-09 \mathrm{H} \\
1.5 \mathrm{E}-07 \mathrm{H} \\
5 \mathrm{Y} \\
1.5 \mathrm{E}-07 \mathrm{H} \\
5 \mathrm{Y} \\
1.5 \mathrm{E}-07 \mathrm{H} \\
5 \mathrm{Y} \\
1.5 \mathrm{E}-07 \mathrm{H} \\
5 \mathrm{Y} \\
1.5 \mathrm{E}-07 \mathrm{H} \\
5 \mathrm{Y} \\
1.5 \mathrm{E}-07 \mathrm{H} \\
1.5 \mathrm{E}-07 \mathrm{H} \\
1.0 \mathrm{E}-07 \mathrm{H}\end{array}$ & $\begin{array}{l}6.29 \mathrm{E}-02 \\
3.26 \mathrm{E}-03 \\
1.68 \mathrm{E}-05 \\
1.68 \mathrm{E}-04 \\
1.68 \mathrm{E}-04 \\
3.00 \mathrm{E}-03 \mathrm{~N} \\
1.08 \mathrm{E}-02 \\
1.22 \mathrm{E}-06 \\
7.20 \mathrm{E}-08 \\
1.74 \mathrm{E}-07 \\
1.27 \mathrm{E}-09 \\
2.40 \mathrm{E}-05 \\
3.28 \mathrm{E}-03 \\
1.74 \mathrm{E}-07 \\
3.28 \mathrm{E}-03 \\
6.75 \mathrm{E}-06 \\
3.28 \mathrm{E}-03 \\
3.28 \mathrm{E}-03 \\
3.28 \mathrm{E}-03 \\
3.28 \mathrm{E}-03 \\
3.28 \mathrm{E}-03 \\
3.28 \mathrm{E}-03 \\
3.28 \mathrm{E}-03 \\
1.00 \mathrm{E}-07\end{array}$ & 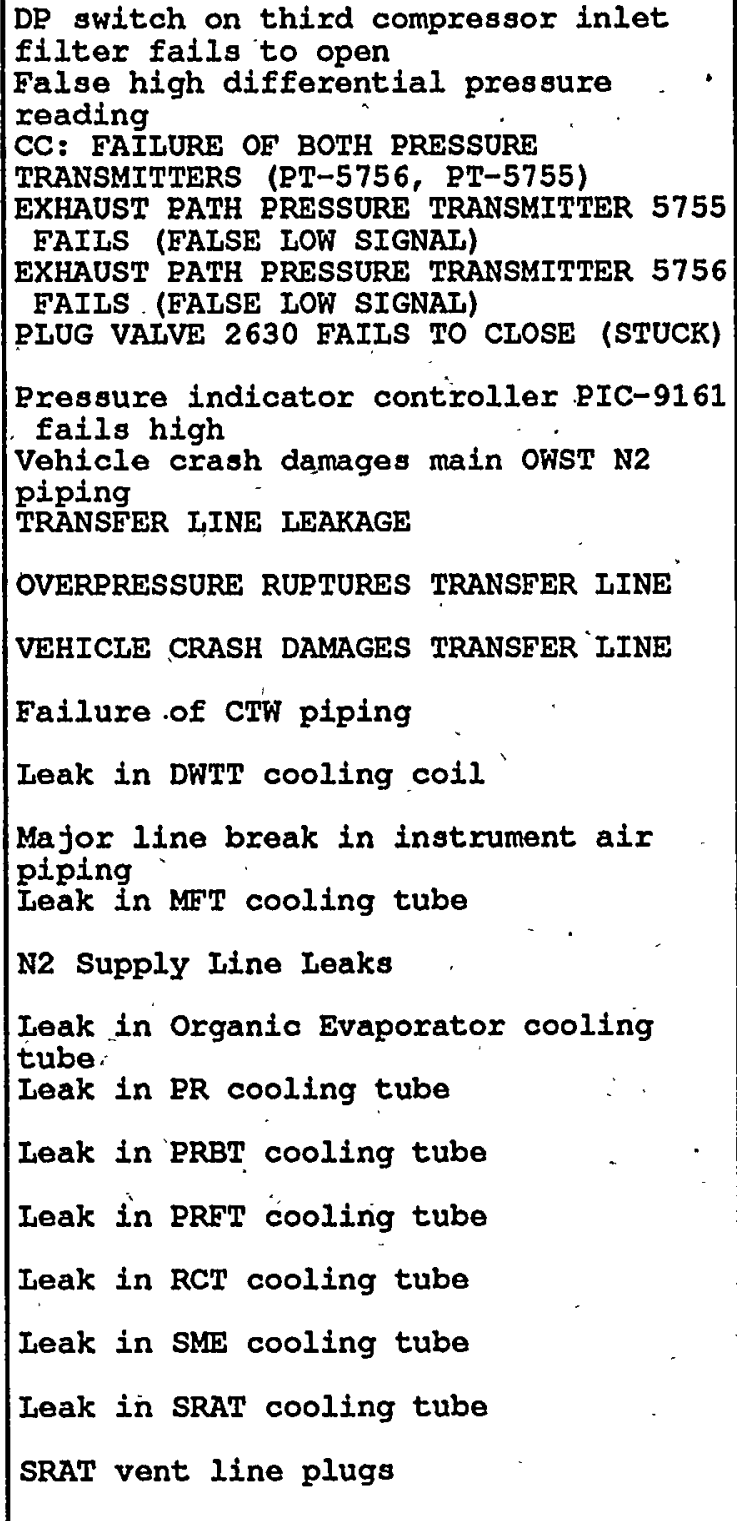 & 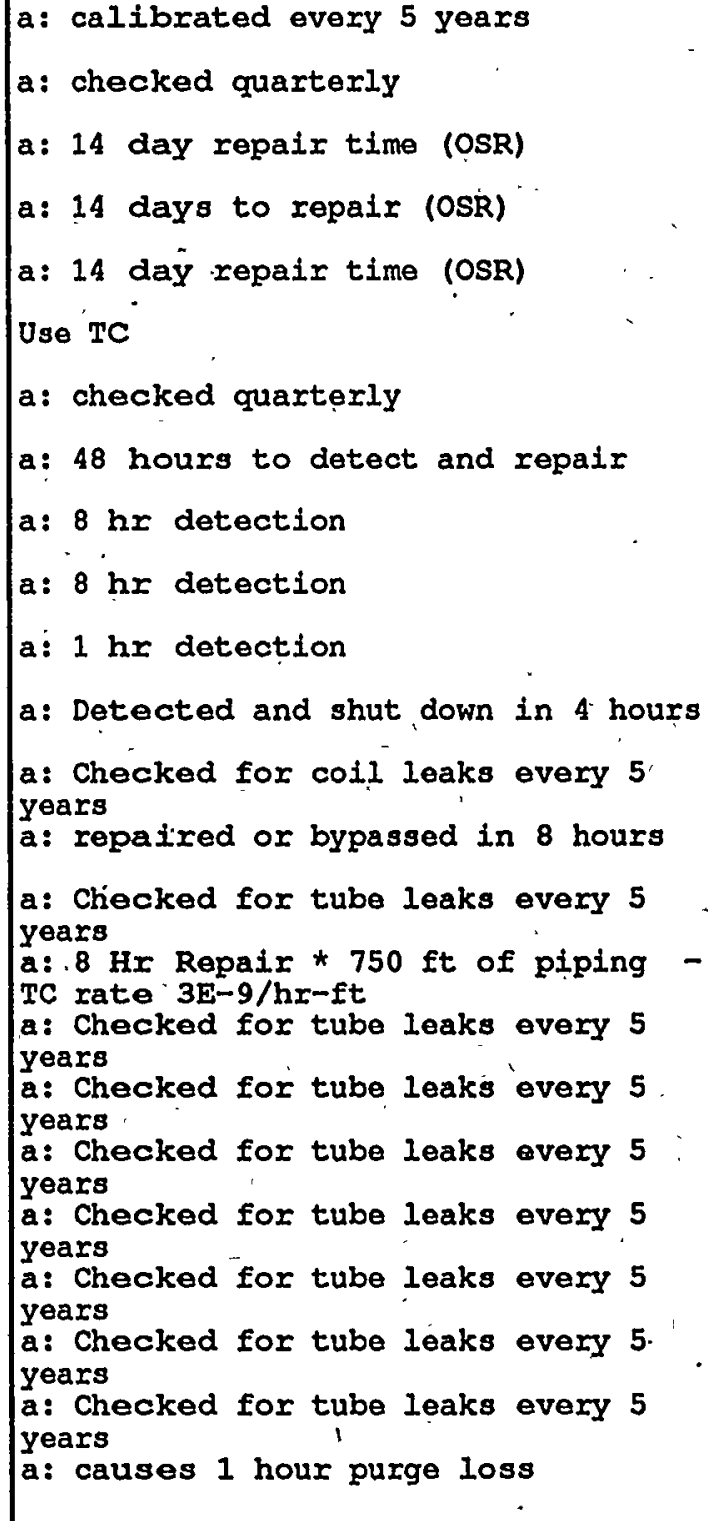 \\
\hline
\end{tabular}


Basic Event Report for I: ICAFTAIDATAIMASTER.BE 11/11/94 11:42 AM (CONT.)

\begin{tabular}{|c|c|c|c|c|c|}
\hline Event & c) & put & Calc. & Description & Source \\
\hline $\begin{array}{l}\text { PMPCHIL2OFFE* } \\
\text { PMPCOOLABFLF* } \\
\text { PMPCOOLBETRF* } \\
\text { PMPCOOLBETSF* } \\
\text { PMPCTW1-ETRO+ } \\
\text { PMPCTW2-ETRO* } \\
\text { PMPCTW2-ETSO\# } \\
\text { PMPCTW2-T\&MO* } \\
\text { PMPCTW3-ETRO+ } \\
\text { PMPCTW4-ETRO } \\
\text { PMPCTW4-ETSO* } \\
\text { PMPCTW4-T\&MO\# } \\
\text { PMPDEEP 1ETRO\# }\end{array}$ & 5 & $\begin{array}{r}168 \mathrm{H} \\
3.0 \mathrm{E}-05 \mathrm{H} \\
8 \mathrm{H} \\
3.0 \mathrm{E}-05 \mathrm{H} \\
1 \mathrm{~N} \\
3.0 \mathrm{E}-03 \mathrm{~N} \\
1 \mathrm{~N} \\
1.0 \mathrm{~N} \\
168 \mathrm{H} \\
3.0 \mathrm{E}-05 \mathrm{H} \\
8 \mathrm{H} \\
3.0 \mathrm{E}-05 \mathrm{H} \\
8 \mathrm{H} \\
1.5 \mathrm{E}-06 \mathrm{H} \\
8 \mathrm{H} \\
3.0 \mathrm{E}-05 \mathrm{H} \\
8 \mathrm{H} \\
3.0 \mathrm{E}-05 \mathrm{H} \\
1 \mathrm{~N} \\
3.0 \mathrm{E}-03 \mathrm{~N} \\
1 \mathrm{~N} \\
5.0 \mathrm{E}-02 \mathrm{~N} \\
8 \mathrm{H} \\
3.0 \mathrm{E}-05 \mathrm{H} \\
8 \mathrm{H} \\
3.0 \mathrm{E}-05 \mathrm{H} \\
1 \mathrm{~N} \\
3.0 \mathrm{E}-03 \mathrm{~N} \\
7 \mathrm{D} \\
3.0 \mathrm{E}-05 \mathrm{H} \\
4 \mathrm{H} \\
3.0 \mathrm{E}-05 \mathrm{H} \\
1 \mathrm{~N} \\
3.0 \mathrm{E}-03 \mathrm{~N} \\
1 \mathrm{~N} \\
1.0 \mathrm{E}-02 \mathrm{~N} \\
7 \mathrm{D} \\
3.0 \mathrm{E}-05 \mathrm{H} \\
3.0 \mathrm{E}-05 \mathrm{H} \\
1 \mathrm{~N} \\
3.0 \mathrm{E}-03 \mathrm{~N} \\
1 \mathrm{~N} \\
1.0 \mathrm{E}-02 \mathrm{~N} \\
3.0 \mathrm{E}-05 \mathrm{H} \\
4 \mathrm{H} \\
3.05 \mathrm{H}\end{array}$ & $\begin{array}{l}1.20 \mathrm{E}-04 \\
3.00 \mathrm{E}-03 \mathrm{~N} \\
6.32 \mathrm{E}-01 \mathrm{~N} \\
2.52 \mathrm{E}-03 \\
1.20 \mathrm{E}-04 \\
6.00 \mathrm{E}-06 \\
2.40 \mathrm{E}-04 \\
2.40 \mathrm{E}-04 \\
3.00 \mathrm{E}-03 \mathrm{~N} \\
5.00 \mathrm{E}-02 \mathrm{~N} \\
2.40 \mathrm{E}-04 \\
2.40 \mathrm{E}-04 \\
3.00 \mathrm{E}-03 \mathrm{~N} \\
5.01 \mathrm{E}-03 \\
1.20 \mathrm{E}-04 \\
3.00 \mathrm{E}-03 \mathrm{~N} \\
1.00 \mathrm{E}-02 \mathrm{~N} \\
5.01 \mathrm{E}-03 \\
1.20 \mathrm{E}-04 \\
3.00 \mathrm{E}-03 \mathrm{~N} \\
1.00 \mathrm{E}-02 \mathrm{~N} \\
1.20 \mathrm{E}-04 \\
1.20 \mathrm{E}-04\end{array}$ & 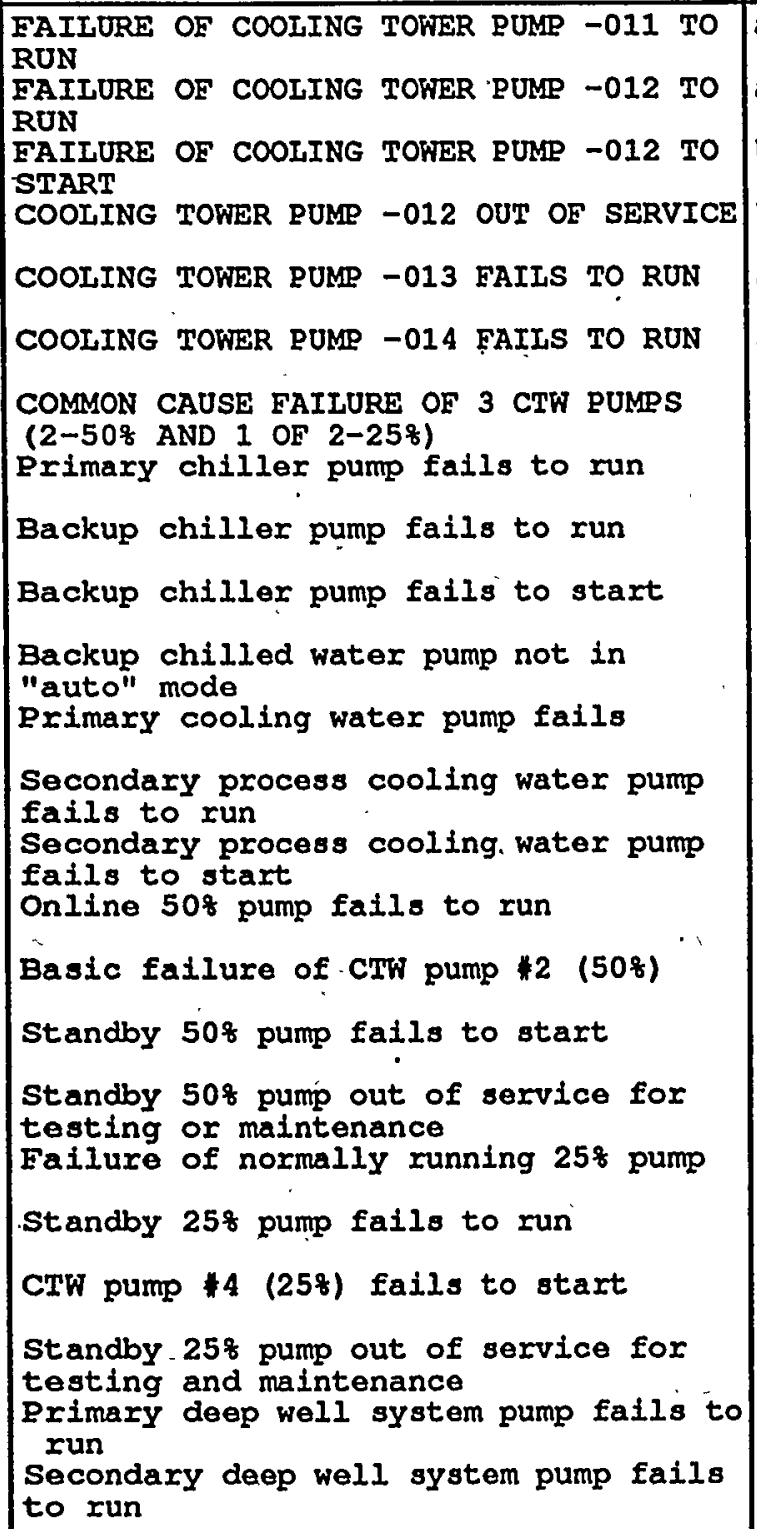 & $\begin{array}{l}\text { a: } 1 \text { week to repair } \\
\text { a: } 8 \text { hours to repair } \\
\text { Use TC } \\
\text { Use TC } \\
\text { a: } 1 \text { week to repair } \\
\text { a: } 8 \text { hours to repair } \\
\text { a: } 8 \text { hours to repair } \\
\text { a: } 8 \text { hours to repair } \\
\text { a: } 8 \text { hour mission time } \\
\text { Use TC Value, } \\
\text { estimated by system engineer } \\
\text { a: } 8 \text { hour repair time } \\
\text { a: } 8 \text { hour repair time } \\
\text { Use TC } \\
\text { a: failed pump repaired in } 1 \text { week } \\
\text { a: Detected and shut down in } 4 \text { hours } \\
\text { if standby pump fails } \\
\text { Use TC value } \\
\text { a: } 4 \text { hour mission time } \\
\text { a: } 4 \text { hour mission time } \\
\text { a: Failed pump repaired in. } 1 \text { week } \\
\text { a: detected and process shut down in } \\
\text { 4 hours if standby fails } \\
\text { a: Not repaired or noticed and shut } \\
\text { down until next batch } \\
\text { a }\end{array}$ \\
\hline
\end{tabular}


Basic Event Report for I: \CAFTA IDATA MMASTER.BE 11/11/94 11:42 AM (CONT.)

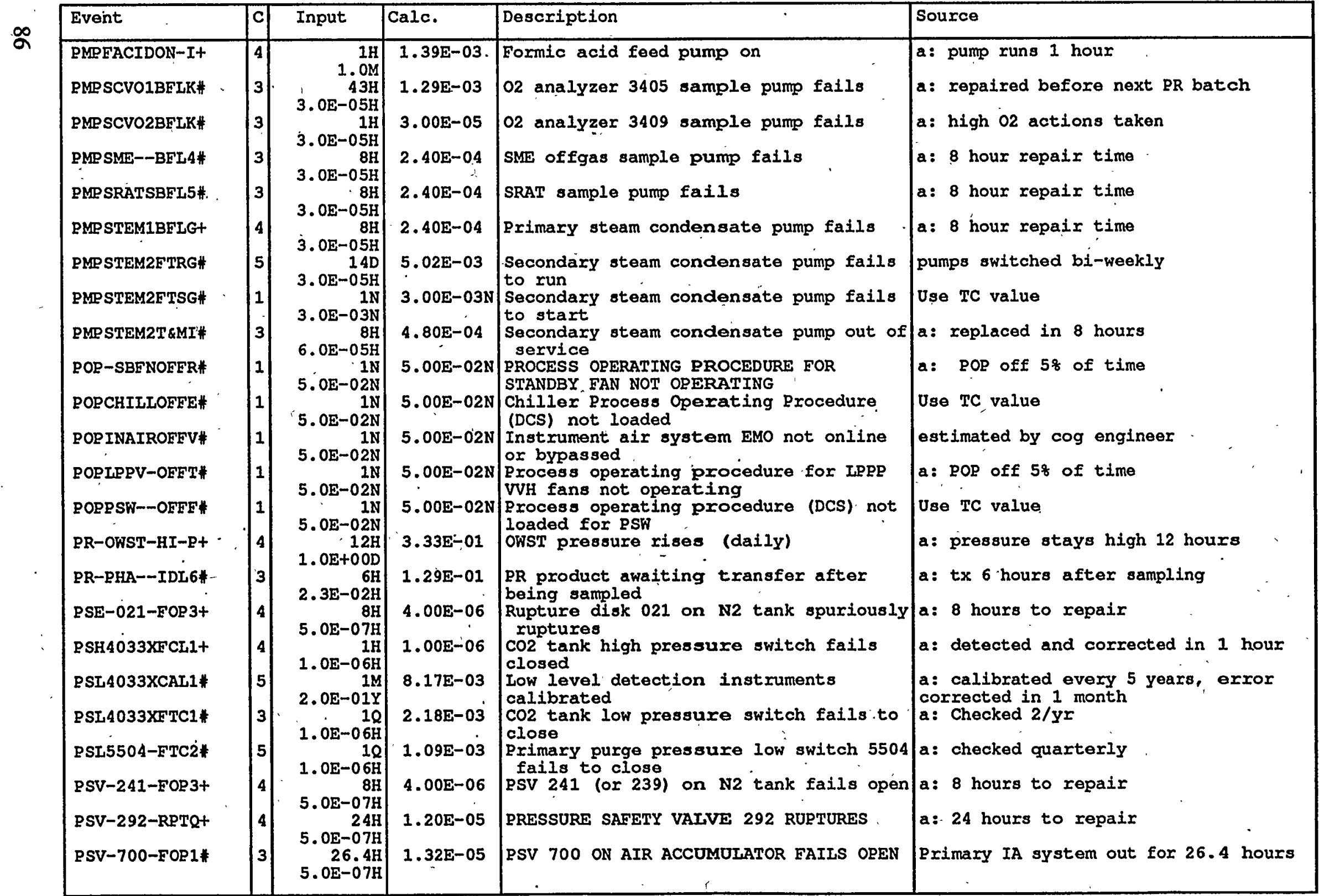


Basic Event Report for I: \CAFTA IDATA MMASTER.BE 11/11/94 11:42 AM (CONT.)

\begin{tabular}{|c|c|c|c|c|c|c|c|}
\hline $\mathrm{Ev}$ & c & put & Calc. & Description & Source & & \\
\hline 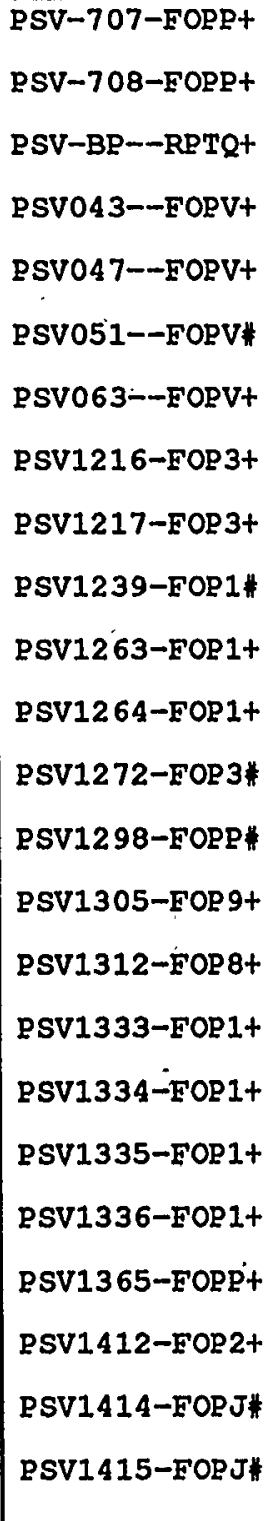 & 4 & $\begin{array}{r}24 \mathrm{H} \\
1.0 \mathrm{E}-06 \mathrm{H} \\
168 \mathrm{H} \\
1.0 \mathrm{E}-06 \mathrm{H} \\
24 \mathrm{H} \\
5.0 \mathrm{E}-07 \mathrm{H} \\
8 \mathrm{H} \\
5.0 \mathrm{E}-07 \mathrm{H} \\
8 \mathrm{H} \\
5.0 \mathrm{E}-07 \mathrm{H} \\
8 \mathrm{H} \\
5.0 \mathrm{E}-07 \mathrm{H} \\
8 \mathrm{H} \\
5.0 \mathrm{E}-07 \mathrm{H} \\
8 \mathrm{H} \\
5.0 \mathrm{E}-07 \mathrm{H} \\
8 \mathrm{H} \\
5.0 \mathrm{E}-07 \mathrm{H} \\
24 \mathrm{H} \\
5.0 \mathrm{E}-07 \mathrm{H} \\
24 \mathrm{H} \\
5.0 \mathrm{E}-07 \mathrm{H} \\
24 \mathrm{H} \\
5.0 \mathrm{E}-07 \mathrm{H} \\
24 \mathrm{H} \\
5.0 \mathrm{E}-07 \mathrm{H} \\
24 \mathrm{H} \\
1.0 \mathrm{E}-06 \mathrm{H} \\
24 \mathrm{H} \\
5.0 \mathrm{E}-07 \mathrm{H} \\
24 \mathrm{H} \\
5.0 \mathrm{E}-07 \mathrm{H} \\
8 \mathrm{H} \\
5.0 \mathrm{E}-07 \mathrm{H} \\
24 \mathrm{H} \\
5.0 \mathrm{E}-07 \mathrm{H} \\
24 \mathrm{H} \\
5.0 \mathrm{E}-07 \mathrm{H} \\
24 \mathrm{H} \\
5.0 \mathrm{E}-07 \mathrm{H} \\
8 \mathrm{H} \\
1.0 \mathrm{E}-06 \mathrm{H} \\
8 \mathrm{H} \\
5.0 \mathrm{E}-07 \mathrm{H} \\
24 \mathrm{H} \\
5.0 \mathrm{E}-07 \mathrm{H} \\
24 \mathrm{H} \\
5.0 \mathrm{E}-07 \mathrm{H} \\
5 \\
5 \\
5\end{array}$ & $\begin{array}{l}2.40 \mathrm{E}-05 \\
1.68 \mathrm{E}-04 \\
1.20 \mathrm{E}-05 \\
4.00 \mathrm{E}-06 \\
4.00 \mathrm{E}-06 \\
4.00 \mathrm{E}-06 \\
4.00 \mathrm{E}-06 \\
4.00 \mathrm{E}-06 \\
4.00 \mathrm{E}-06 \\
1.20 \mathrm{E}-05 \\
1.20 \mathrm{E}-05 \\
1.20 \mathrm{E}-05 \\
1.20 \mathrm{E}-05 \\
1.20 \mathrm{E}-05 \\
1.20 \mathrm{E}-05 \\
1.20 \mathrm{E}-05 \\
4.00 \mathrm{E}-06 \\
1.20 \mathrm{E}-05 \\
1.20 \mathrm{E}-05 \\
1.20 \mathrm{E}-05 \\
8.00 \mathrm{E}-06 \\
4.00 \mathrm{E}-06 \\
6.00 \mathrm{E}-06 \\
6.00 \mathrm{E}-06\end{array}$ & 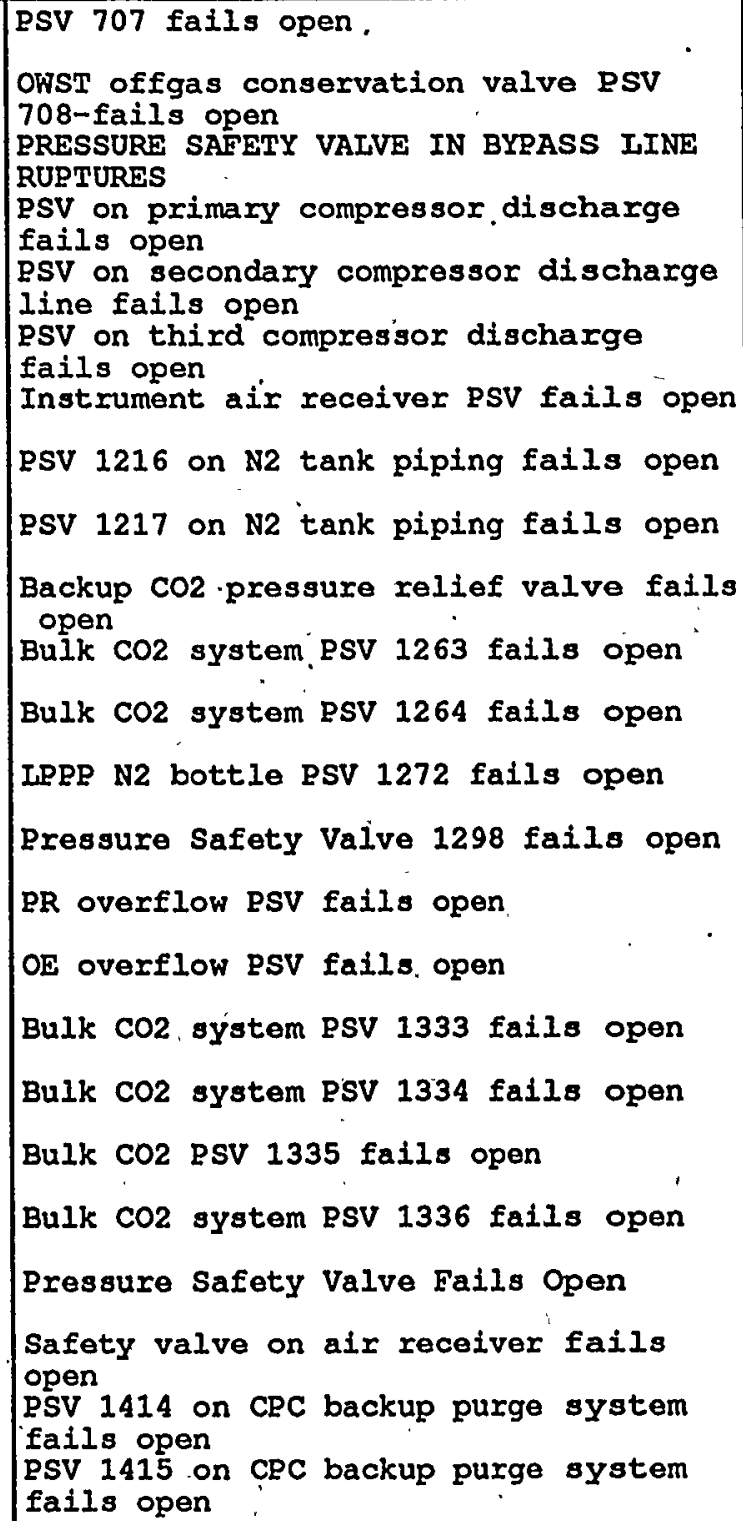 & $\begin{array}{l}\text { a: } 24 \text { hours to repair } \\
\text { a: corrected in } 1 \text { week } \\
\text { a: } 24 \text { hours to repair } \\
\text { a: } 8 \text { hours to repair } \\
\text { a: } 8 \text { hours to repair } \\
\text { a: } 8 \text { hours to repair } \\
\text { a: } 8 \text { hours to repair } \\
\text { a: } 8 \text { hours to repair } \\
\text { a: } 8 \text { hours to repair } \\
\text { a: repair time - } 24 \text { hours } \\
\text { a: } 24 \text { hours to repair } \\
\text { a: } 24 \text { hours to repair } \\
\text { a: repaired in } 24 \text { hours } \\
\text { a: } 24 \text { hours to repair } \\
\text { a: } 24 \text { hours to repair } \\
\text { a: } 24 \text { hours to repair } \\
\text { a: } 8 \text { hours to repair } \\
\text { a: } 24 \text { hours to repair } \\
\text { a: } 24 \text { hours to repair } \\
\text { a: } 24 \text { ours to repair } \\
\text { a: } 8 \text { Hr Repair } \\
\text { a: } 8 \text { hour repair time } \\
\text { a: } 24 \text { hours to repair } \\
\text { a: } 24 \text { hours to repair }\end{array}$ & 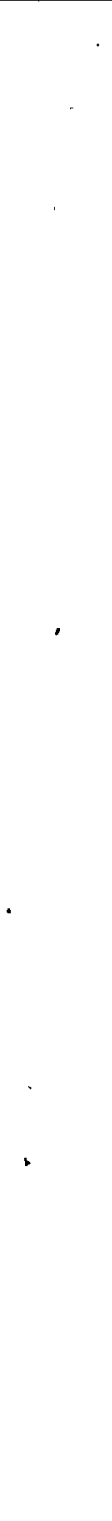 & $\begin{array}{l}\cdot \\
\cdot \\
1\end{array}$ \\
\hline
\end{tabular}


Basic Event Report fox I: \CAFTA (DATA \MASTER.BE 11/11/94 11:42 AM (CONT.)

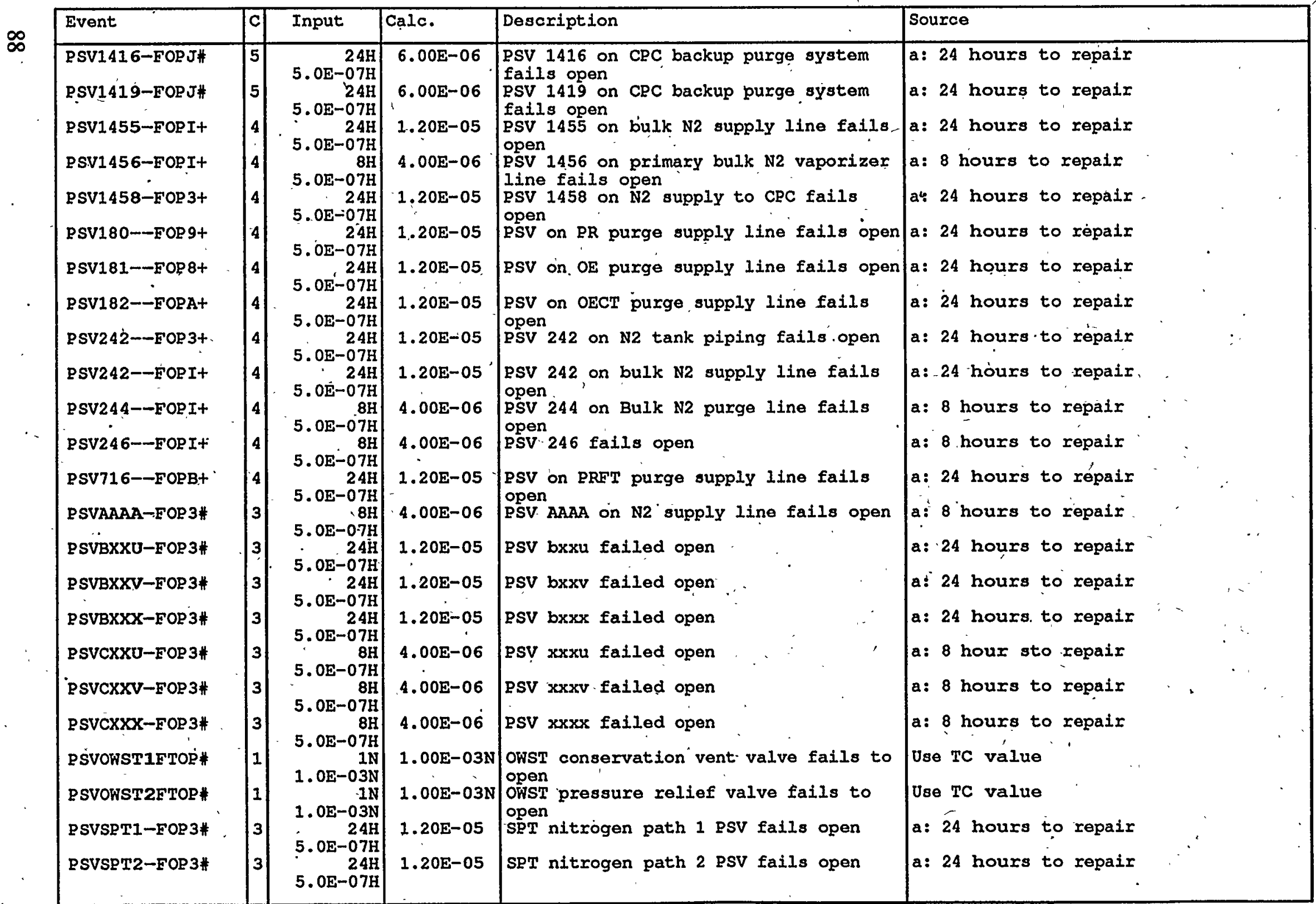




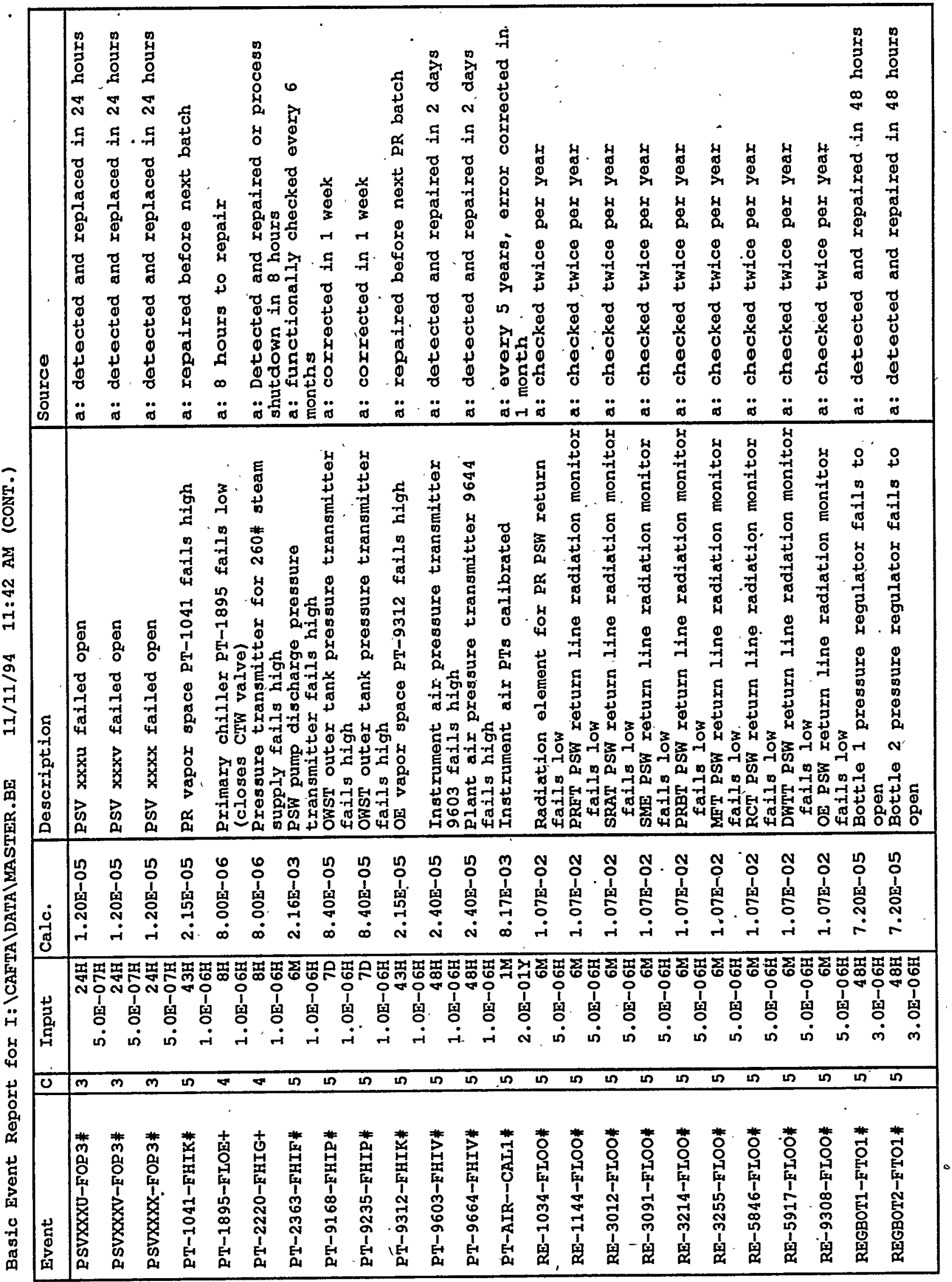


Basic Event Report for I: \CAFTA\DATA MASTER.BE 11/11/94, 11:42 AM (CONT.)

\begin{tabular}{|c|c|c|c|c|c|}
\hline Event & $|c|$ & Input & Calc. & Description & Souxce \\
\hline $\begin{array}{l}\text { REGBOT3-FTO1\# } \\
\text { REGBOT4-FTO1\# } \\
\text { RY--12--C2DR\# } \\
\text { RY--12--CCER\# } \\
\text { RY--123-C3DR\# } \\
\text { RY--123-C3GR\# } \\
\text { RY--1234C3GR\# } \\
\text { RY--FD--FTOS\# } \\
\text { RY--SB--FORR\# } \\
\text { RY--SB--FTCR\# } \\
\text { RY-00001FORR\# } \\
\text { RY-00002FORR\# } \\
\text { RY-00003FOPR\# } \\
\text { RY-1234-C3DR\# } \\
\text { RY-4041-FTO5\# } \\
\text { RY-9667AFTOV\# } \\
\text { RY-BASINFORR\# } \\
\text { RY-PFSFTETO4\# } \\
\text { RY-PRPMPETO5\# } \\
\text { RY-SRTL-FTO5\# } \\
\text { SC-8760-FLOT\# } \\
\text { SC-8762-FLOT+ } \\
\text { SLGNOBL-PRE5\# } \\
\text { SLRSALT-BLUO\# }\end{array}$ & $\left|\begin{array}{l}5 \\
1\end{array}\right|$ & $\begin{array}{r}38 \mathrm{H} \\
3.0 \mathrm{E}-06 \mathrm{H} \\
48 \mathrm{H} \\
3.0 \mathrm{E}-06 \mathrm{H} \\
8 \mathrm{H} \\
3.0 \mathrm{E}-08 \mathrm{H} \\
1.0 \mathrm{~N}-04 \mathrm{~N} \\
8 \mathrm{H} \\
1.5 \mathrm{E}-08 \mathrm{H} \\
1 \mathrm{~N} \\
5.0 \mathrm{E}-05 \mathrm{~N} \\
1 \mathrm{~N} \\
5.0 \mathrm{E}-05 \mathrm{~N} \\
1 \mathrm{~N} \\
1.0 \mathrm{E}-05 \mathrm{~N} \\
8 \mathrm{H} \\
3.0 \mathrm{E}-07 \mathrm{H} \\
1 \mathrm{~N} \\
1.0 \mathrm{E}-05 \mathrm{~N} \\
8 \mathrm{H} \\
3.0 \mathrm{E}-07 \mathrm{H} \\
8 \mathrm{H} \\
3.0 \mathrm{E}-07 \mathrm{H} \\
3.0 \mathrm{E}-07 \mathrm{H} \\
8 \mathrm{H} \\
1.5 \mathrm{E}-08 \mathrm{H} \\
1.0 \mathrm{E}-05 \mathrm{~N} \\
1.0 \mathrm{~N}-05 \mathrm{~N} \\
8 \mathrm{H} \\
3.0 \mathrm{E}-07 \mathrm{H} \\
-1 \mathrm{~N} \\
1.0 \mathrm{E}-05 \mathrm{~N} \\
1 \mathrm{~N} \\
1.0 \mathrm{E}-05 \mathrm{~N} \\
1 \mathrm{~N} \\
1.0 \mathrm{E}-05 \mathrm{~N} \\
8 \mathrm{H} \\
1.0 \mathrm{E}-06 \mathrm{H} \\
8 \mathrm{H} \\
1.0 \mathrm{E}-06 \mathrm{H} \\
1 \mathrm{~N} \\
1.0 \mathrm{E}-02 \mathrm{~N} \\
1.0 \mathrm{~N}+00 \mathrm{~N}\end{array}$ & $\begin{array}{l}7.20 \mathrm{E}-05 \\
7.20 \mathrm{E}-05 \\
1.20 \mathrm{E}-07 \\
1.00 \mathrm{E}-04 \mathrm{~N} \\
6.00 \mathrm{E}-08 \\
5.00 \mathrm{E}-05 \mathrm{~N} \\
5.00 \mathrm{E}-05 \mathrm{~N} \\
1.00 \mathrm{E}-05 \mathrm{~N} \\
1.20 \mathrm{E}-06 \\
1.00 \mathrm{E}-05 \mathrm{~N} \\
1.20 \mathrm{E}-06 \\
1.20 \mathrm{E}-06 \\
1.20 \mathrm{E}-06 \\
6.00 \mathrm{E}-08 \\
1.00 \mathrm{E}-05 \mathrm{~N} \\
1.00 \mathrm{E}-05 \mathrm{~N} \\
2.40 \mathrm{E}-06 \\
1.00 \mathrm{E}-05 \mathrm{~N} \\
1.00 \mathrm{E}-05 \mathrm{~N} \\
1.00 \mathrm{E}-05 \mathrm{~N} \\
8.00 \mathrm{E}-06 \\
8.00 \mathrm{E}-06 \\
1.00 \mathrm{E}-02 \mathrm{~N} \\
1.00 \mathrm{E}+00 \mathrm{~N}\end{array}$ & 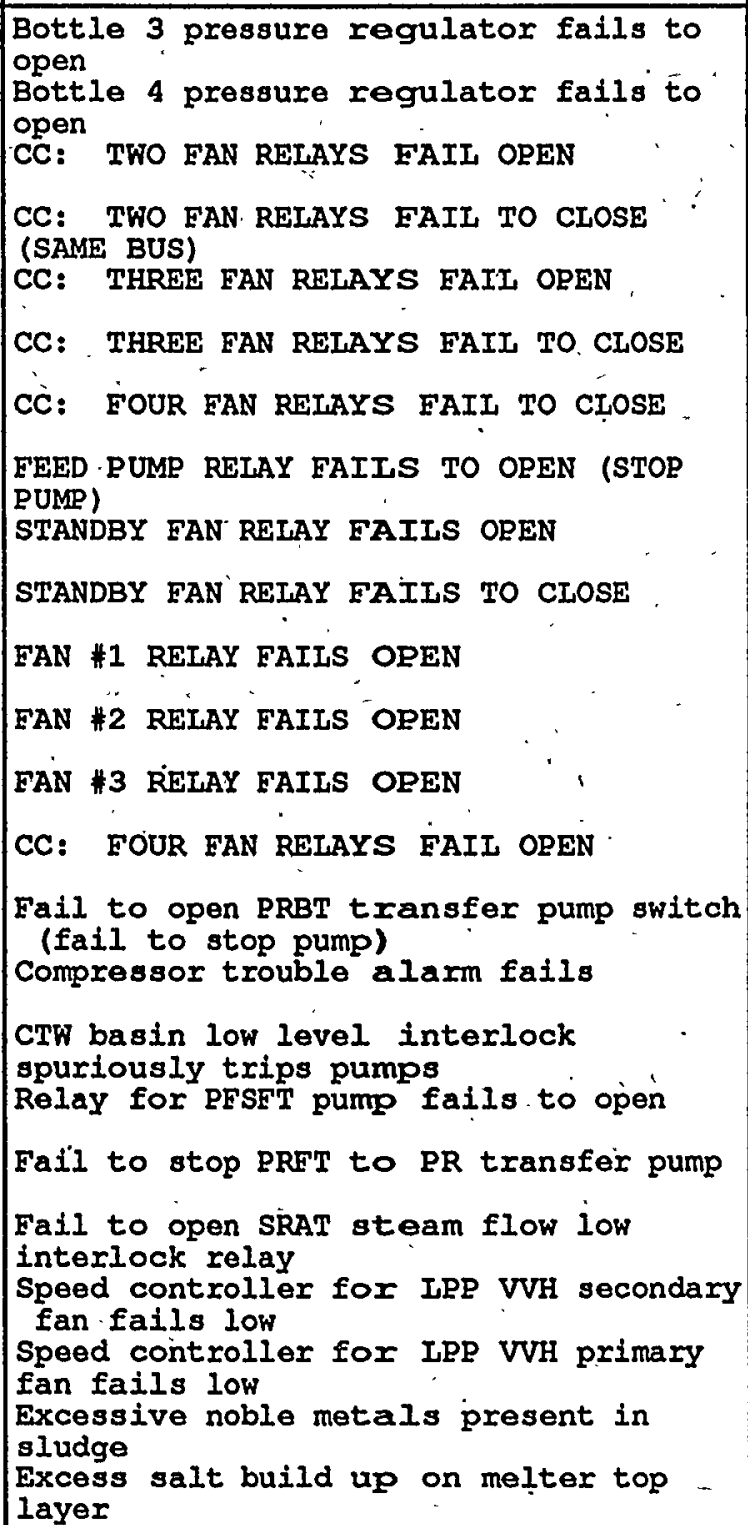 & $\begin{array}{l}\text { a: detected and repaired in } 48 \text { hours } \\
\text { a: detected and repaired in } 48 \text { hours } \\
\text { a: } 8 \text { hours to repair } \\
\text { Use TC } \\
\text { a: } 8 \text { hours to repair } \\
\text { Use TC } \\
\text { Use TC } \\
\text { Use TC : } \\
\text { a: } 8 \text { hours to repair } \\
\text { Use TC } \\
\text { a: } 8 \text { hours to repaix } \\
\text { a: } 8 \text { hours to repair } \\
\text { a: } 8 \text { hours to repaix } \\
\text { a: } 8 \text { hours to repair } \\
\text { Use TC value } \\
\text { a: relay fails to open } \\
\text { a: } 8 \text { hour repair time } \\
\text { Use TC value } \\
\text { Use TC value } \\
\text { Use TC value } \\
\text { a: Primary failure repaired or } \\
\text { responded to within } 8 \text { hours } \\
\text { a: } 8 \text { hours to repair } \\
\text { a: present during entire cycle } \\
\text { a: any salt build up results in } \\
\text { excess amount }\end{array}$ \\
\hline
\end{tabular}




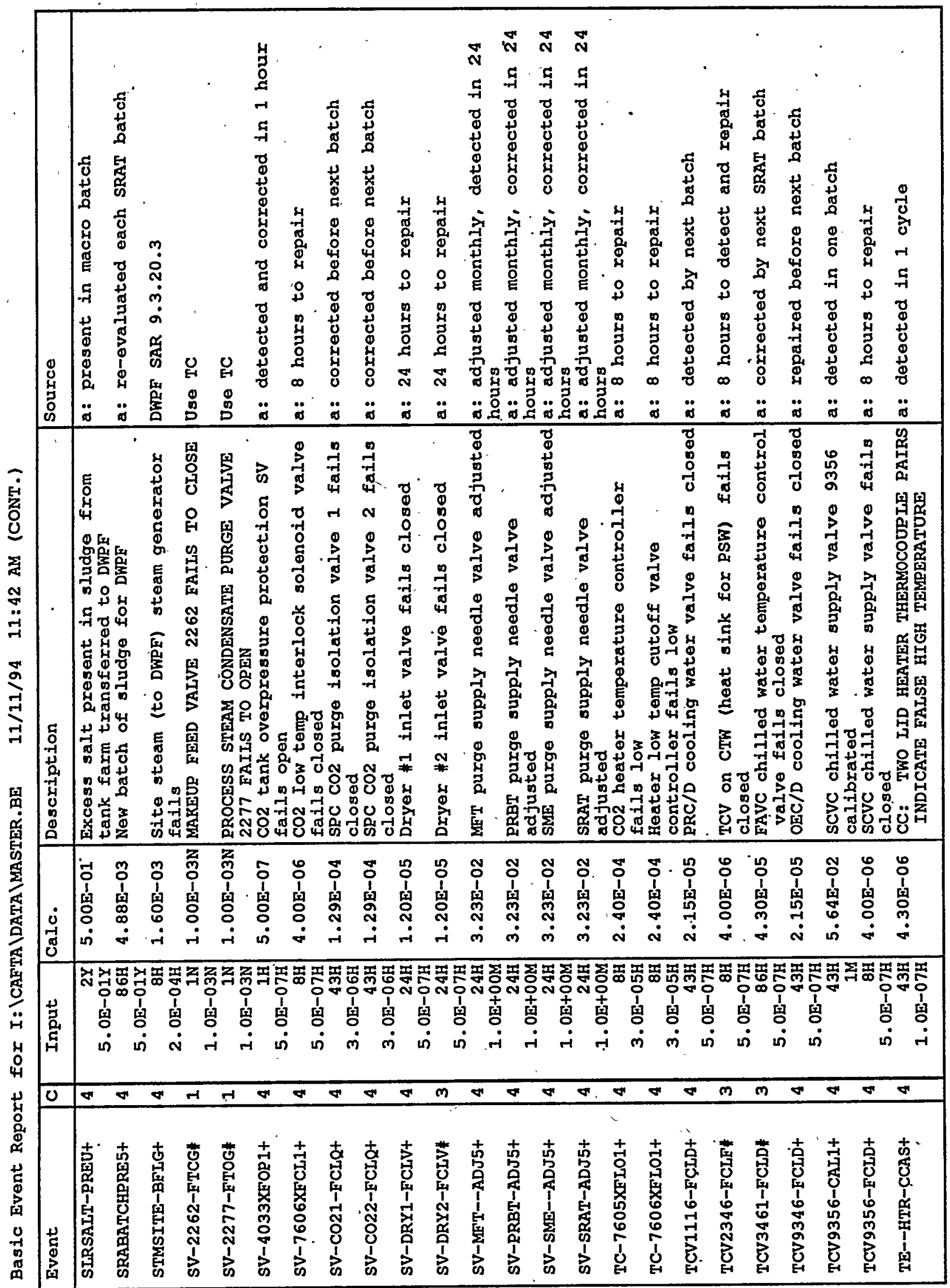


Basic Event Report for I: \CAFTA IDATA IMASTER.BE 11/11/94 11:42 AM (CONT.)

\begin{tabular}{|c|c|c|c|c|c|c|c|c|}
\hline Event & c & nput & Calc. & Description & Sor & & & \\
\hline 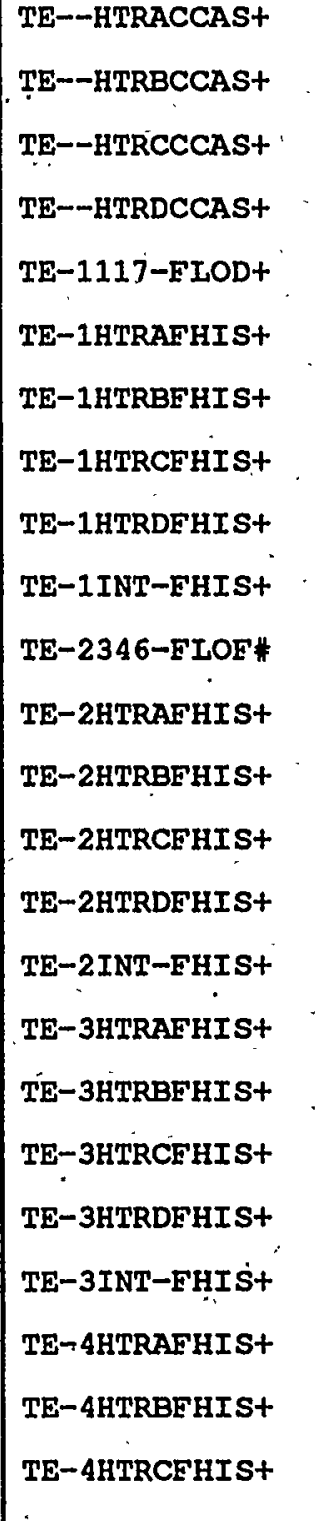 & 4 & $\begin{array}{r}43 \mathrm{H} \\
1.0 \mathrm{E}-07 \mathrm{H} \\
43 \mathrm{H} \\
1.0 \mathrm{E}-07 \mathrm{H} \\
43 \mathrm{H} \\
1.0 \mathrm{E}-07 \mathrm{H} \\
43 \mathrm{H} \\
1.0 \mathrm{E}-07 \mathrm{H} \\
43 \mathrm{H} \\
1.0 \mathrm{E}-06 \mathrm{H} \\
43 \mathrm{H} \\
1.0 \mathrm{E}-06 \mathrm{H} \\
43 \mathrm{H} \\
1.0 \mathrm{E}-06 \mathrm{H} \\
43 \mathrm{H} \\
1.0 \mathrm{E}-06 \mathrm{H} \\
43 \mathrm{H} \\
1.0 \mathrm{E}-06 \mathrm{H} \\
43 \mathrm{H} \\
1.0 \mathrm{E}-06 \mathrm{H} \\
6 \mathrm{M} \\
1.0 \mathrm{E}-06 \mathrm{H} \\
43 \mathrm{H} \\
1.0 \mathrm{E}-06 \mathrm{H} \\
43 \mathrm{H} \\
1.0 \mathrm{E}-06 \mathrm{H} \\
43 \mathrm{H} \\
1.0 \mathrm{E}-06 \mathrm{H} \\
43 \mathrm{H} \\
1.0 \mathrm{E}-0.6 \mathrm{H} \\
43 \mathrm{H} \\
1.0 \mathrm{E}-06 \mathrm{H} \\
43 \mathrm{H} \\
1.0 \mathrm{E}-06 \mathrm{H} \\
43 \mathrm{H} \\
1.0 \mathrm{E}-06 \mathrm{H} \\
43 \mathrm{H} \\
1.0 \mathrm{E}-06 \mathrm{H} \\
43 \mathrm{H} \\
1.0 \mathrm{E}-06 \mathrm{H} \\
43 \mathrm{H} \\
1.0 \mathrm{E}-06 \mathrm{H} \\
43 \mathrm{H} \\
1.0 \mathrm{E}-06 \mathrm{H} \\
43 \mathrm{H} \\
1.0 \mathrm{E}-06 \mathrm{H} \\
43 \mathrm{H} \\
1.0 \mathrm{E}-06 \mathrm{H}\end{array}$ & $\begin{array}{l}4.30 \mathrm{E}-06 \\
4.30 \mathrm{E}-06 \\
4.30 \mathrm{E}-06 \\
4.30 \mathrm{E}-06 \\
4.30 \mathrm{E}-05 \\
4.30 \mathrm{E}-05 \\
4.30 \mathrm{E}-05 \\
4.30 \mathrm{E}-05 \\
4.30 \mathrm{E}-05 \\
4.30 \mathrm{E}-05 \\
2.16 \mathrm{E}-03 \\
4.30 \mathrm{E}-05 \\
4.30 \mathrm{E}-05 \\
4.30 \mathrm{E}-05 \\
4.30 \mathrm{E}-05 \\
4.30 \mathrm{E}-05 \\
4.30 \mathrm{E}-05 \\
4.30 \mathrm{E}-05 \\
4.30 \mathrm{E}-05 \\
4.30 \mathrm{E}-05 \\
4.30 \mathrm{E}-05 \\
4.30 \mathrm{E}-05 \\
4.30 \mathrm{E}-05 \\
4.30 \mathrm{E}-05 \\
\end{array}$ & 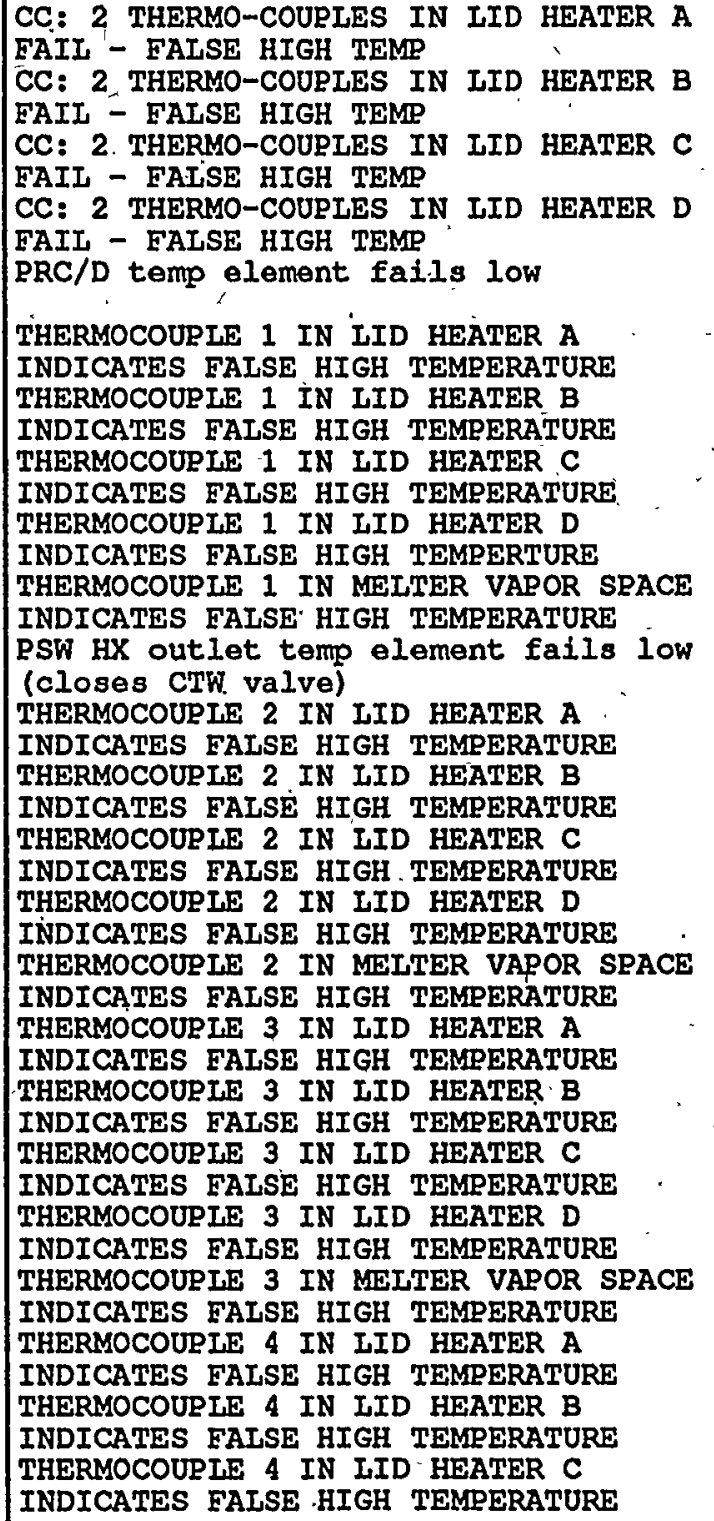 & $\begin{array}{l}\text { a: detected in } 1 \text { cycle } \\
\text { a: detected in } 1 \text { cycle } \\
\text { a: detected in } 1 \text { cycle } \\
\text { a: detected in } 1 \text { cycle } \\
\text { a: detected before next } \\
\text { a: detected in } 1 \text { cycle } \\
\text { a: detected in } 1 \text { cycle } \\
\text { a: detected in } 1 \text { cycle } \\
\text { a: detected in } 1 \text { cycle } \\
\text { a: detected in } 1 \text { cycle } \\
\text { a: Checked twice a year } \\
\text { a: detected in } 1 \text { cycle } \\
\text { a: detected in } 1 \text { cycle } \\
\text { a: detected in } 1 \text { cycle } \\
\text { a: detected in } 1 \text { cycle } \\
\text { a: detected in } 1 \text { cycle } \\
\text { a: detected in } 1 \text { cycle } \\
\text { a: detected in } 1 \text { cycle } \\
\text { a: detected in } 1 \text { cycle } \\
\text { a: detected in } 1 \text { cycle } \\
\text { a: detected in } 1 \text { cycle } \\
\text { a: detected in } 1 \text { cycle } \\
\text { a: detected in } 1 \text { cycle } \\
\text { a: detected in } 1 \text { cycle }\end{array}$ & batch & . & \\
\hline
\end{tabular}


Basic Event Report, for I: (CAFTA \DATA MASTER.BE, 11/11/94 11:42 AM (CONT.)

\begin{tabular}{|c|c|c|c|c|c|}
\hline Ev & C & & Calc. & Description & Source \\
\hline 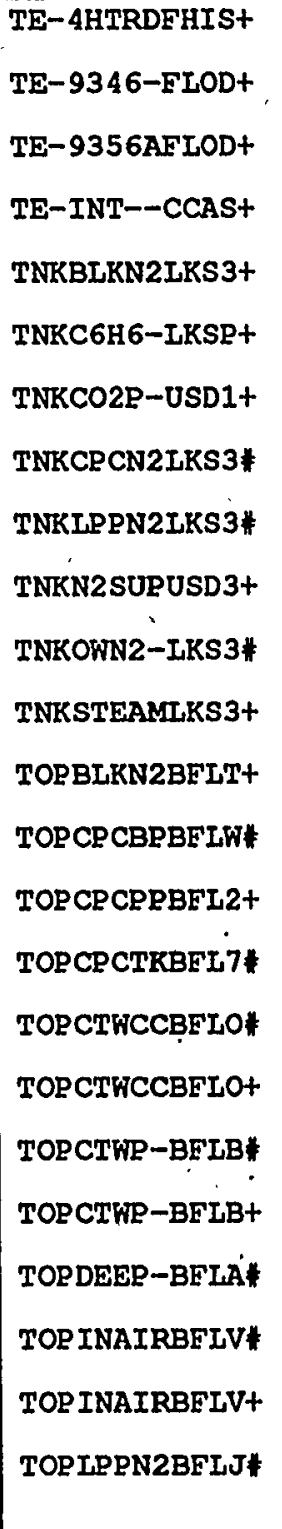 & 1 & $\begin{array}{r}43 \mathrm{H} \\
1.0 \mathrm{E}-06 \mathrm{H} \\
43 \mathrm{H} \\
1.0 \mathrm{E}-06 \mathrm{H} \\
24 \mathrm{H} \\
1.0 \mathrm{E}-06 \mathrm{H} \\
43 \mathrm{H} \\
1.0 \mathrm{E}-07 \mathrm{H} \\
48 \mathrm{H} \\
1.0 \mathrm{E}-08 \mathrm{H} \\
24 \mathrm{H} \\
1.0 \mathrm{E}-07 \mathrm{H} \\
24 \mathrm{H} \\
1 \mathrm{M} \\
48 \mathrm{H} \\
1.0 \mathrm{E}-08 \mathrm{H} \\
48 \mathrm{H} \\
1.0 \mathrm{E}-08 \mathrm{H} \\
24 \mathrm{H} \\
3.3 \mathrm{E}-01 \mathrm{D} \\
7 \mathrm{D} \\
1.0 \mathrm{E}-08 \mathrm{H} \\
8 \mathrm{H} \\
1.0 \mathrm{E}-08 \mathrm{H} \\
20 \mathrm{H} \\
5.5 \mathrm{E}-06 \mathrm{H} \\
1 \mathrm{~N} \\
3.1 \mathrm{E}-03 \mathrm{~N} \\
51 \mathrm{H} \\
1.9 \mathrm{E}-05 \mathrm{H} \\
1 \mathrm{~N} \\
3.7 \mathrm{E}-05 \mathrm{~N} \\
5.5 \mathrm{H} \\
1.1 \mathrm{E}-05 \mathrm{H} \\
5.5 \mathrm{H} \\
1.1 \mathrm{E}-05 \mathrm{H} \\
90 \mathrm{H} \\
6.3 \mathrm{E}-06 \mathrm{H} \\
90 \mathrm{H} \\
6.3 \mathrm{E}-06 \mathrm{H} \\
1 \mathrm{~N} \\
5.4 \mathrm{E}-03 \mathrm{~N} \\
26 \mathrm{H} \\
1.1 \mathrm{E}-05 \mathrm{H} \\
26 \mathrm{H} \\
1.1 \mathrm{E}-05 \mathrm{H} \\
1.3 \mathrm{E}-04 \mathrm{~N} \\
\end{array}$ & $\begin{array}{c}4.30 \mathrm{E}-05 \\
4.30 \mathrm{E}-05 \\
2.40 \mathrm{E}-05 \\
4.30 \mathrm{E}-06 \\
4.80 \mathrm{E}-07 \\
2.40 \mathrm{E}-06 \\
3.23 \mathrm{E}-02 \\
4.80 \mathrm{E}-07 \\
4.80 \mathrm{E}-07 \\
2.48 \mathrm{E}-01 \\
1.68 \mathrm{E}-06 \\
8.00 \mathrm{E}-08 \\
1.10 \mathrm{E}-04 \\
3.10 \mathrm{E}-03 \mathrm{~N} \\
9.68 \mathrm{E}-04 \\
3.70 \mathrm{E}-05 \mathrm{~N} \\
6.05 \mathrm{E}-05 \\
6.05 \mathrm{E}-05 \\
5.67 \mathrm{E}-04 \\
5.67 \mathrm{E}-04 \\
5.40 \mathrm{E}-03 \mathrm{~N} \\
2.86 \mathrm{E}-04 \\
2.86 \mathrm{E}-04\end{array}$ & 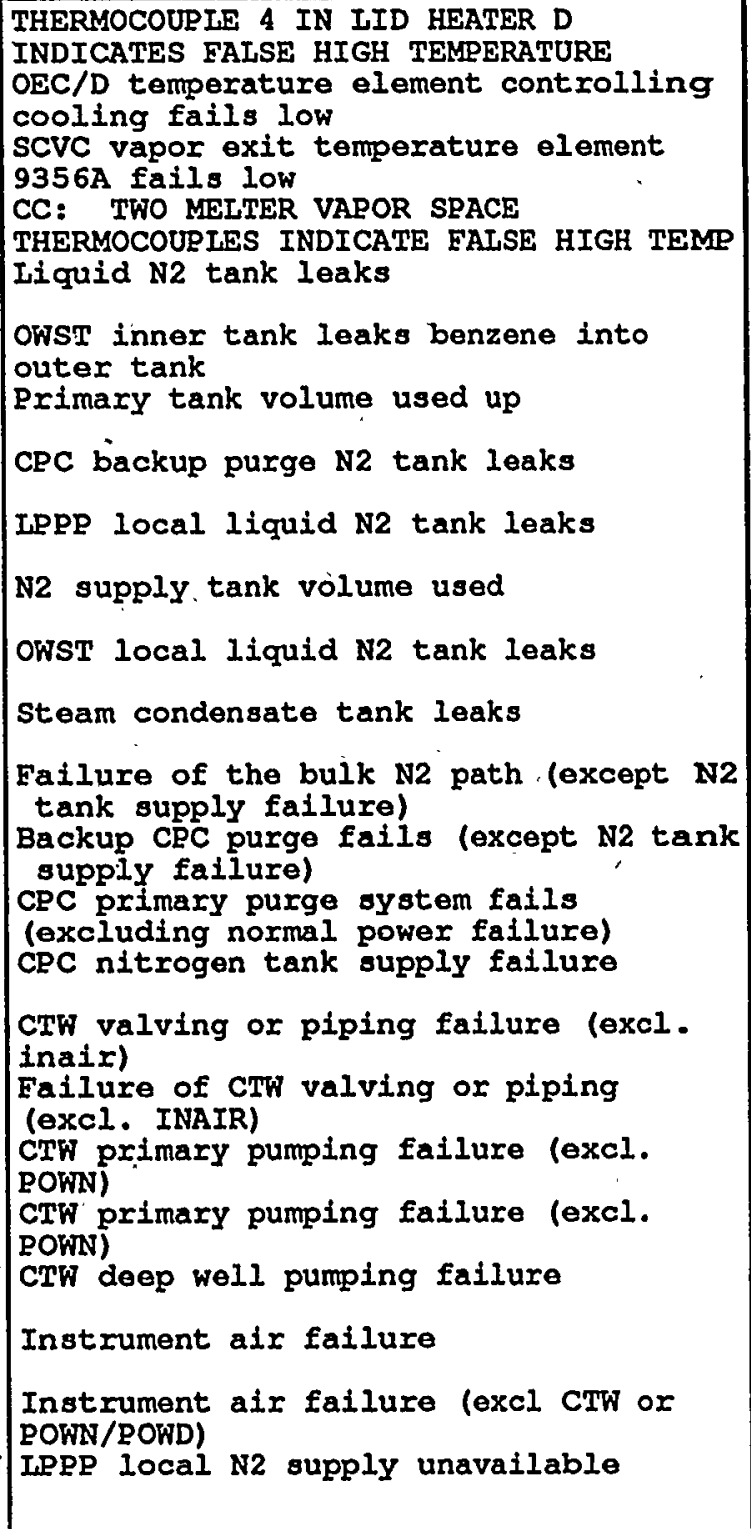 & $\begin{array}{l}\text { a: detected in } 1 \text { cycle } \\
\text { a: repaired before next batch } \\
\text { a: detected and corrected in } 24 \text { hours } \\
\text { a: detected in one cycle } \\
\text { a: detected and alternate source } \\
\text { supplied in } 48 \text { hours } \\
\text { a: } 24 \text { hours to rectify } \\
\text { a: } 1 \text { month supply } \\
\text { a: repaired and refilled in } 48 \text { hours } \\
\text { a: repaired and refilled in } 48 \text { hours } \\
\text { Use TC value } \\
\text { a: repaired and refilled in } 1 \text { week } \\
\text { a: Process shut down in } 8 \text { hours } \\
\text { From fault tree Blkn2.CAF } \\
\text { From fault tree CPCBP.CAF } \\
\text { From fault tree CPCPR.CAF } \\
\text { From fault tree CPCTNK.CAF } \\
\text { From fault tree CTWCC.CAF } \\
\text { From fault tree CTWCC.CAF, } \\
\text { From fault tree CTWP.CAF } \\
\text { From fault tree CTWP.CAF } \\
\text { From fault tree DEEP.CAF } \\
\text { From fault tree Inair.CAF } \\
\text { From fault tree Inair.CAF' } \\
\text { From fault tree IPRPN2.CAF }\end{array}$ \\
\hline
\end{tabular}


Basic Event Report for I: \CAFTA \DATA\MASTER.BE 11/11/94 11:42 AM (CONT.)

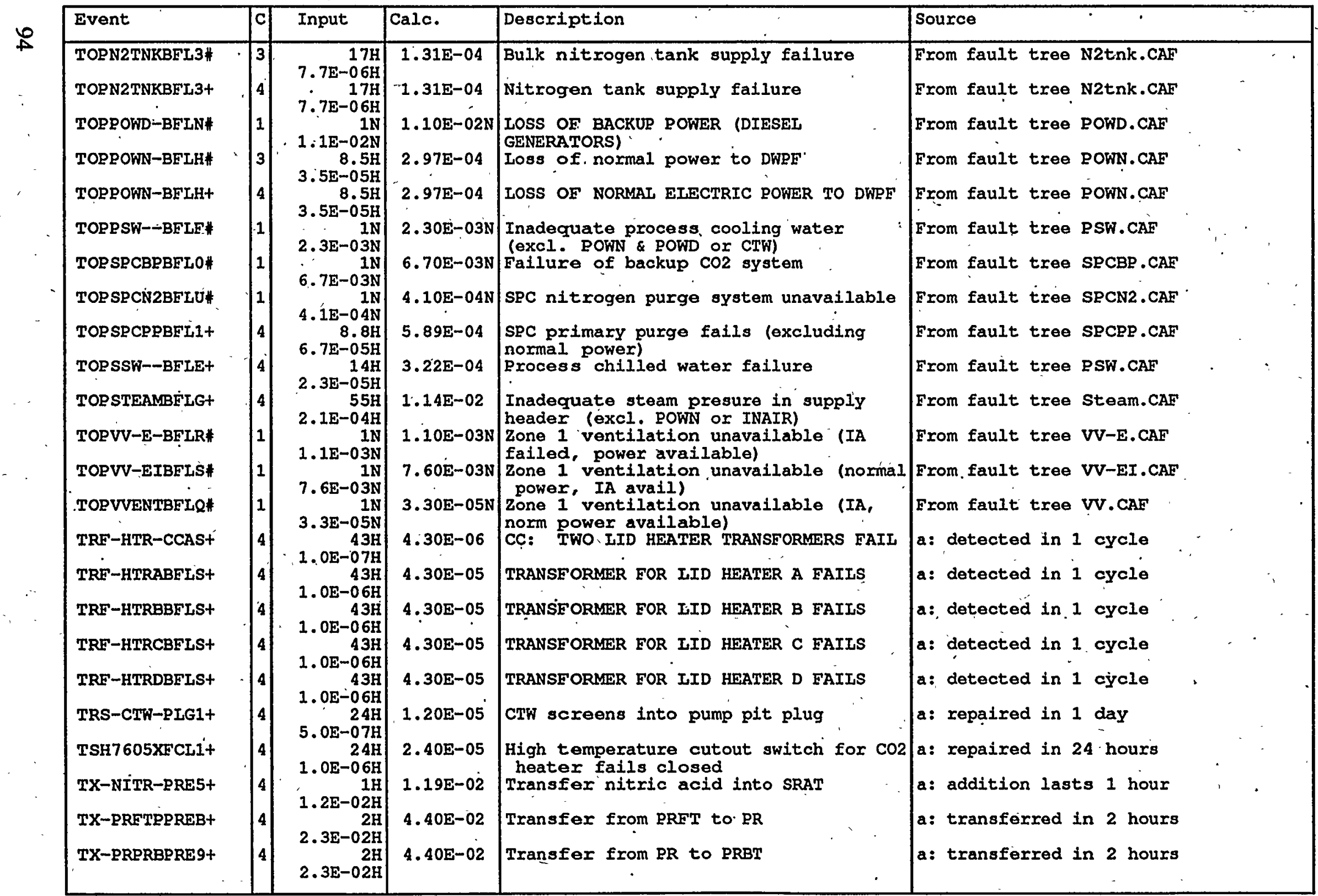


Basic Event Report for I: \CAFTA IDATAIMASTER.Bg $11 / 11 / 94 \quad 11: 42$ AM (CONT.)

\begin{tabular}{|c|c|c|c|c|c|}
\hline Event & c & Input & Calc. & Description & Source \\
\hline $\begin{array}{l}\text { TX-SRAT-PRE5+ } \\
\text { UA-1882-FTAE* } \\
\text { UA-5502-FTA2* } \\
\text { UPS-FAN-BFLR* } \\
\text { UPS1041-BELR* } \\
\text { URS9278-BELR* } \\
\text { VAP--OE-LKSG+ } \\
\text { VAP--PR-IKSG+ } \\
\text { VAP-SME-IKSG+ } \\
\text { VAPDWTT-LKSG+ } \\
\text { VAPSRAT-LKSG+ } \\
\text { VB-418--FORP+ } \\
\text { VLV9176-LKSP* } \\
\text { VLVDWTOTLKSP* } \\
\text { VLVRCIRCLKSP* } \\
\text { WE-2779-CAL1* } \\
\text { WE-2779-FHI1* } \\
\text { WSL2779BETC1* } \\
\text { WTR-MLT-PREU* }\end{array}$ & 5 & $\begin{array}{r}23 \mathrm{H} \\
1.2 \mathrm{E}-02 \mathrm{H} \\
3 \mathrm{M} \\
3.0 \mathrm{E}-06 \mathrm{H} \\
1 \mathrm{Y} \\
3.0 \mathrm{E}-06 \mathrm{H} \\
1 \mathrm{~N} \\
4.7 \mathrm{E}-04 \mathrm{~N} \\
1 \mathrm{~N} \\
4.7 \mathrm{E}-04 \mathrm{~N} \\
1 \mathrm{~N} \\
4.7 \mathrm{E}-04 \mathrm{~N} \\
8 \mathrm{H} \\
1.0 \mathrm{E}-07 \mathrm{H} \\
8 \mathrm{H} \\
1.0 \mathrm{E}-07 \mathrm{H} \\
8 \mathrm{H} \\
1.0 \mathrm{E}-07 \mathrm{H} \\
8 \mathrm{H} \\
1.0 \mathrm{E}-07 \mathrm{H} \\
8 \mathrm{H} \\
1.0 \mathrm{E}-07 \mathrm{H} \\
24 \mathrm{H} \\
5.0 \mathrm{E}-07 \mathrm{H} \\
8 \mathrm{H} \\
1.0 \mathrm{E}-08 \mathrm{H} \\
8 \mathrm{H} \\
1.0 \mathrm{E}-08 \mathrm{H} \\
8 \mathrm{H} \\
1.0 \mathrm{E}-08 \mathrm{H} \\
1 \mathrm{M} \\
2.0 \mathrm{E}-01 \mathrm{Y} \\
12 \mathrm{H} \\
3.0 \mathrm{E}-06 \mathrm{H} \\
12 \mathrm{H} \\
3.0 \mathrm{E}-06 \mathrm{H} \\
1 \mathrm{~N} \\
1.0 \mathrm{E}+00 \mathrm{~N}\end{array}$ & $\begin{array}{l}2.16 \mathrm{E}-01 \\
3.23 \mathrm{E}-03 \\
1.30 \mathrm{E}-02 \\
4.70 \mathrm{E}-04 \mathrm{~N} \\
4.70 \mathrm{E}-04 \mathrm{~N} \\
4.70 \mathrm{E}-04 \mathrm{~N} \\
8.00 \mathrm{E}-07 \\
8.00 \mathrm{E}-07 \\
8.00 \mathrm{E}-07 \\
8.00 \mathrm{E}-07 \\
8.00 \mathrm{E}-07 \\
1.20 \mathrm{E}-05 \\
8.00 \mathrm{E}-08 \\
8.00 \mathrm{E}-08 \\
8.00 \mathrm{E}-08 \\
1.63 \mathrm{E}-02 \\
1.80 \mathrm{E}-05 \\
1.80 \mathrm{E}-05 \\
1.00 \mathrm{E}+00 \mathrm{~N}\end{array}$ & 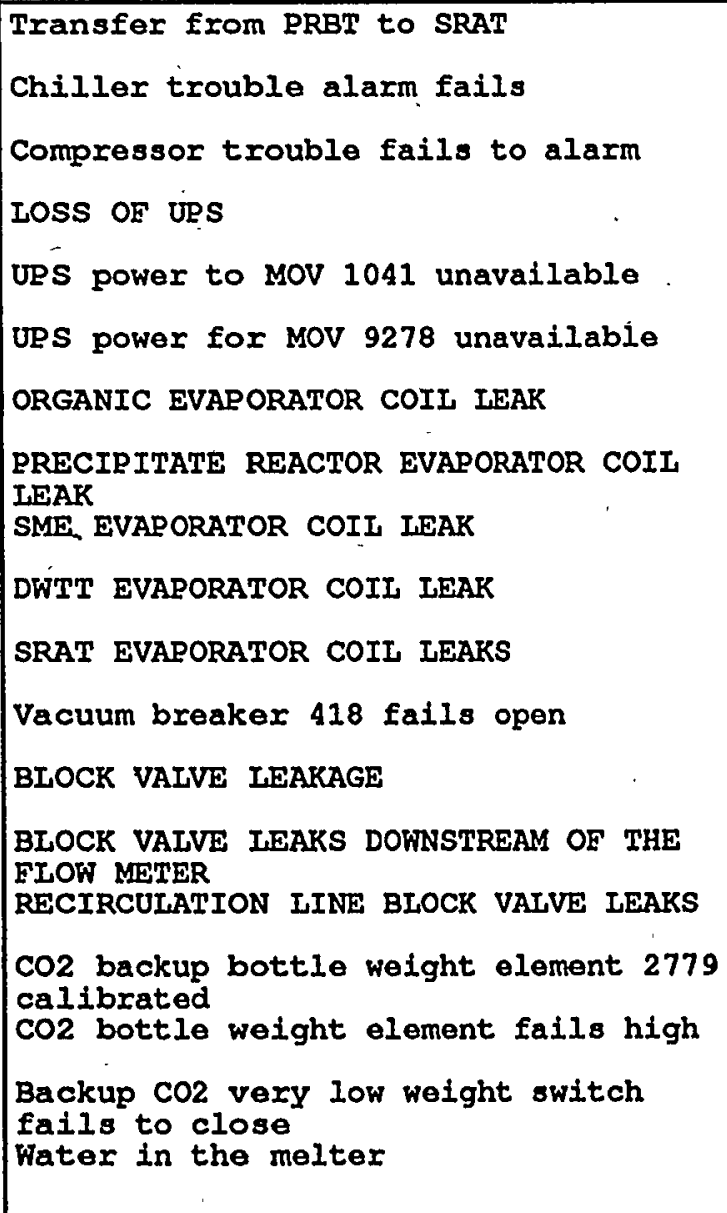 & $\begin{array}{l}\text { Transfer lasts } 23 \text { hours } \\
\text { a: checked quarterly } \\
\text { a: checked annually } \\
\text { Use TC } \\
\text { Use TC value } \\
\text { Use TC value } \\
\text { a: } 8 \text { hours to repair } \\
\text { a: } 8 \text { hours to repair } \\
\text { a: } 8 \text { hours to repair } \\
\text { a: } 8 \text { hours to repair } \\
\text { a: } 8 \text { hours to repair } \\
\text { a: } 24 \text { hours to repair } \\
\text { a: } 8 \text { hr detection } \\
\text { a: } 8 \text { hr detection } \\
\text { a: } 8 \text { hr detction } \\
\text { a: error detected in } 1 \text { month } \\
\text { a: detected and repaired in } 12 \text { hours } \\
\text { a: detected and repaired in } 12 \text { hours } \\
\text { Due to water purge }\end{array}$ \\
\hline
\end{tabular}




\section{Attachment 4 Type Code (TC) File}

The Type Code (TC) file contains all failure types used in the DWPF accident analysis fault trees. The TC file is given in the following format and van be interpreted as follows:

Type - The type code follows the naming convention in Attachment 2. Each type code has a unique name shared by all basic events that represent the same component type, failing in the same mode, in the same type of system. For example, all pumps failing to run in water systems have the same failure frequency assigned by the TC file.

Rate - The failure rate is entered in the Rate column. This number is from reliability data, as found in References 1 and 2 or supplied by DWPF cognizant engineers (where appropriate).

U - Units for the calculation

$$
\begin{array}{ll}
\mathrm{H}=\text { Hour } & \mathrm{D}=\text { Day } \quad \mathrm{M}=\text { Month } \\
\mathrm{Y}=\text { Year } & \mathrm{N}=\text { no units (probability) }
\end{array}
$$

Desc. - The description column provides room for a longer description of the type code to be entered.

Source - The source column is provided to enter information regarding the source of failure rate data.

\section{References for Attachment 4}

1. WSRC-TR-93-262, SRS Generic Data Base Development (U), June 30, 1993.

2. WSRC-TR-93-581, SRS Human Error Generic Data Base Development for Nonreactor Nuclear Facilities (U), February, 1994. 
Type codes for I: ICAFTAIDATA MASTER.TC

$11 / 11 / 94 \quad 11: 43$ AM

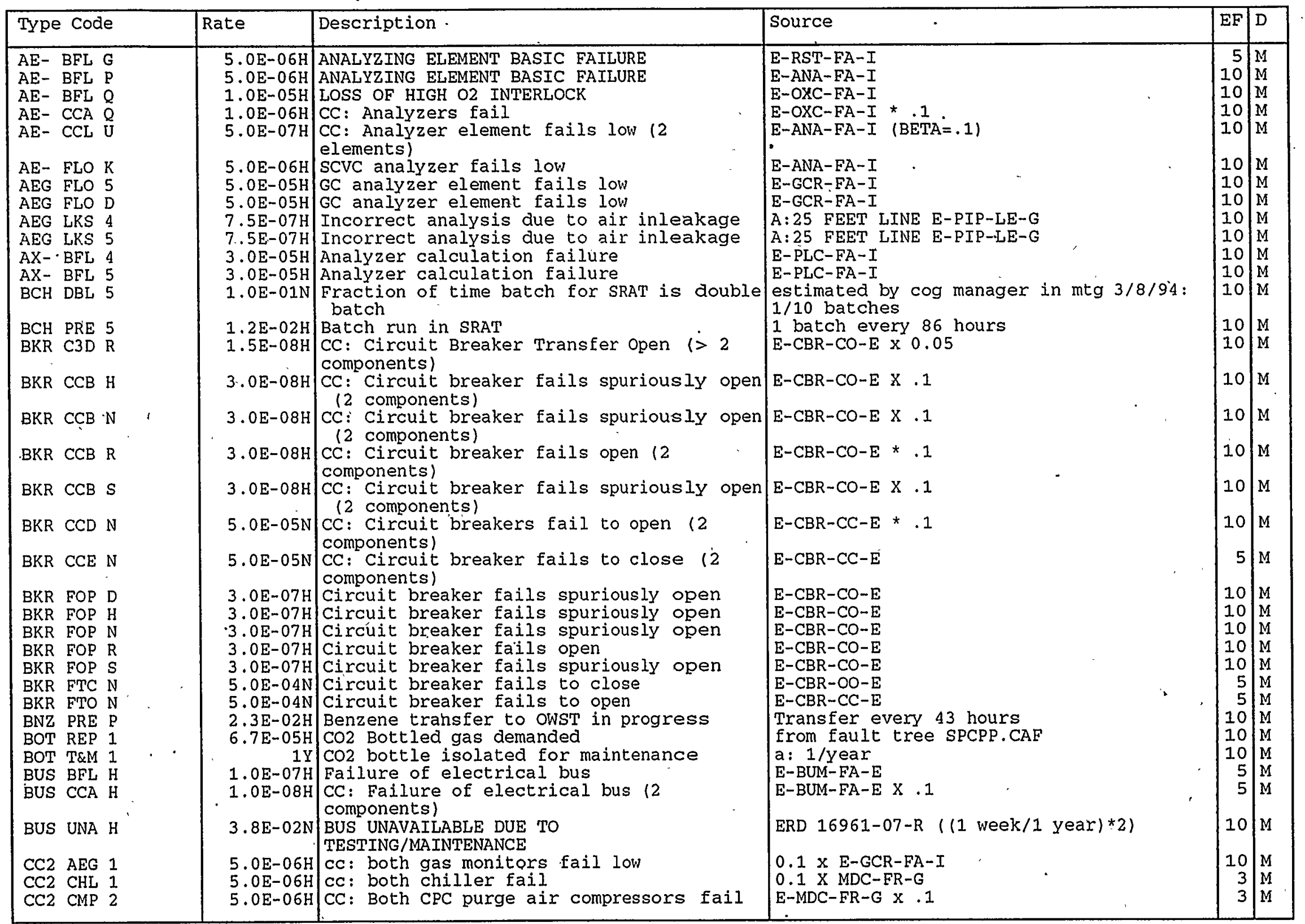


Type Codes for I: \CAFTA \DATA IMASTER.TC

11/11/94 11:43 AM (CONT.)

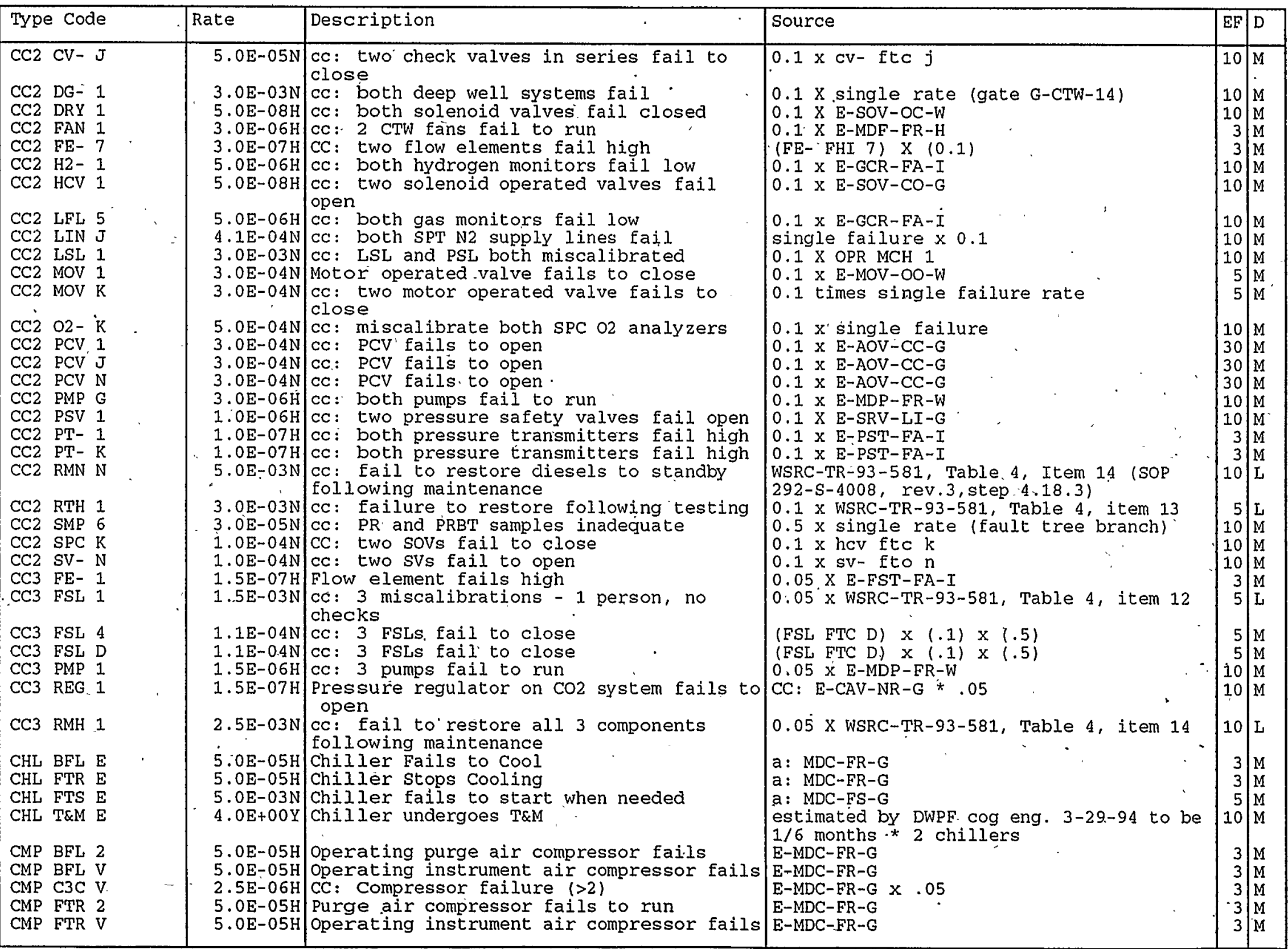


Type codes for I: \CAFTA IDATA MMASTER , TC

11/11/94 11:43 AM (CONT.)

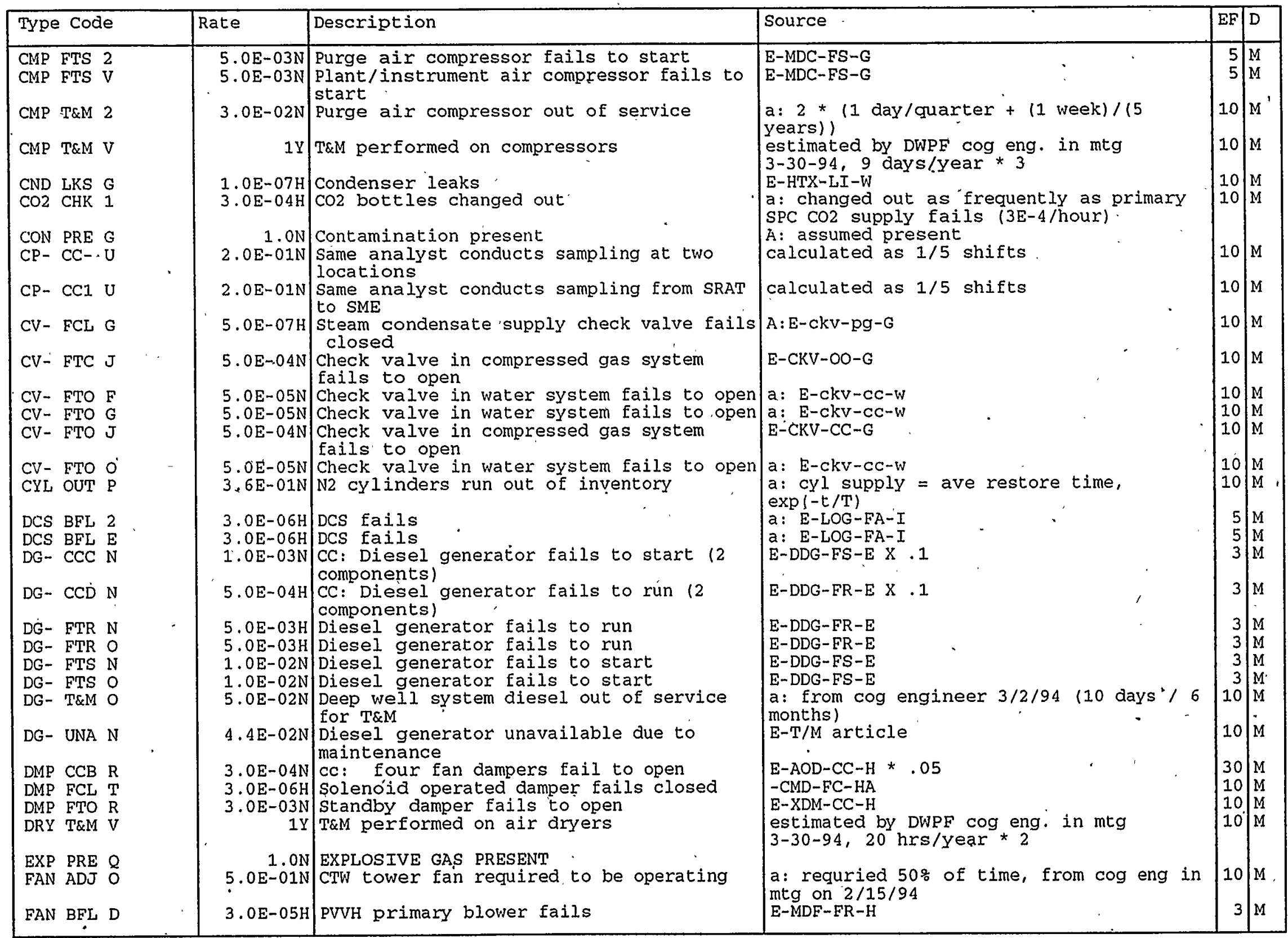


Type Codes for I: \CAFTA \DATA IMASTER.TC

11/11/94 11:43 AM (CONT.)

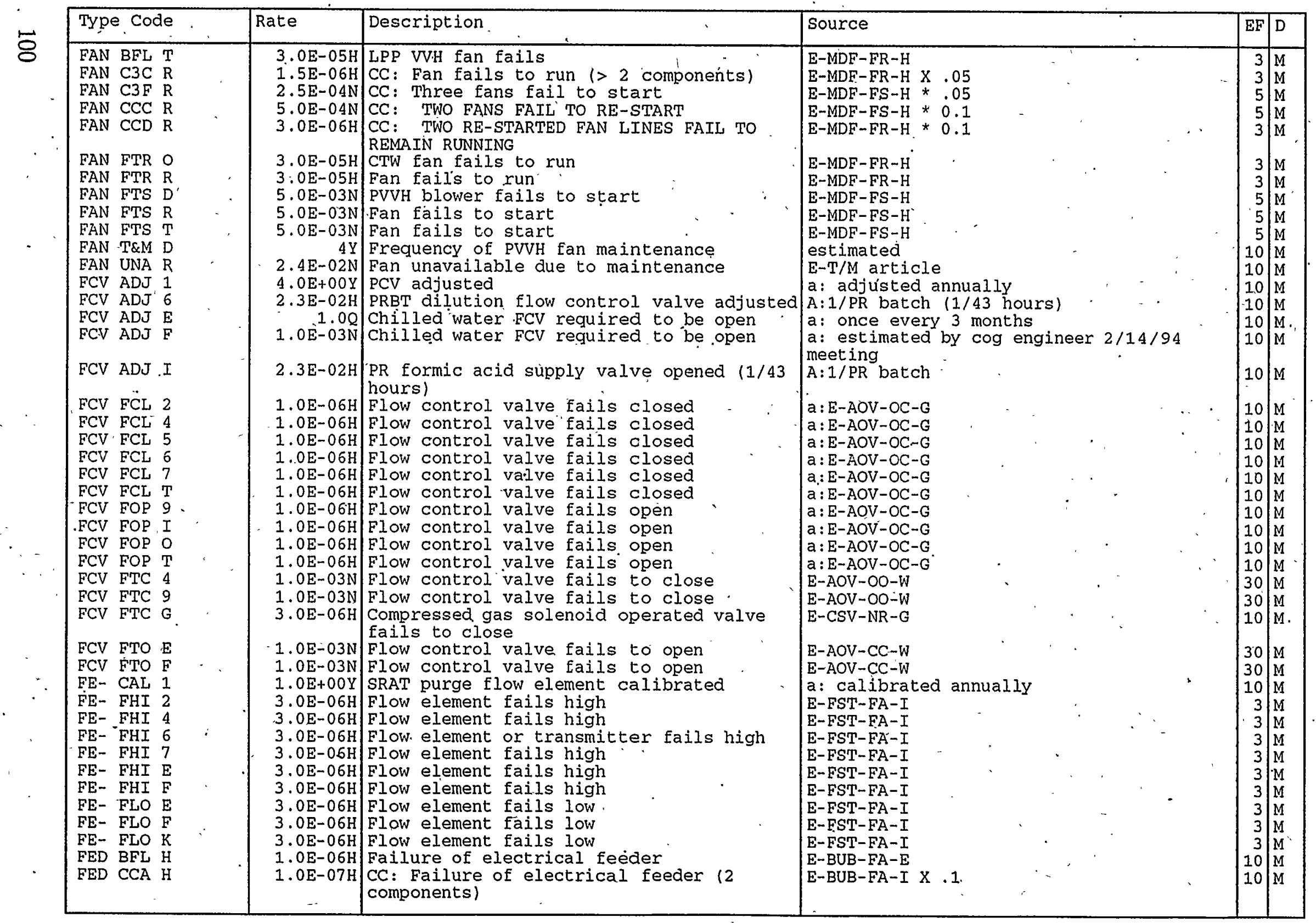


Type Codes for I: ICAFTA IDATA IMASTER.TC 11/11/94 11:43 AM (CONT.)

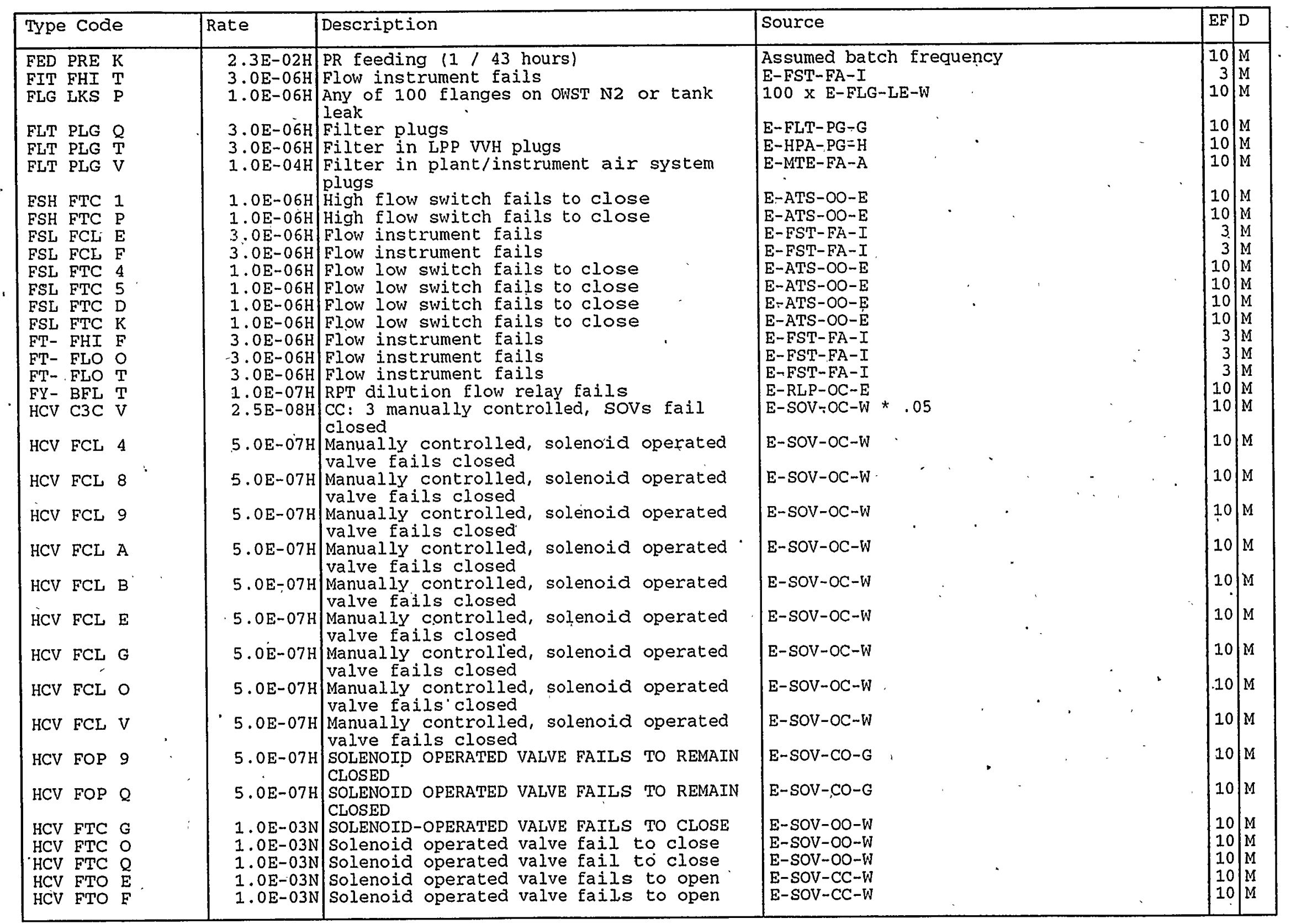


Type codes for I: \CAFTA \DATA IMASTER.TC

\begin{tabular}{|c|c|c|c|c|c|c|c|c|}
\hline & Type Code & & Rate & Description & ource & & $\mathrm{EF}$ & D \\
\hline 1 & 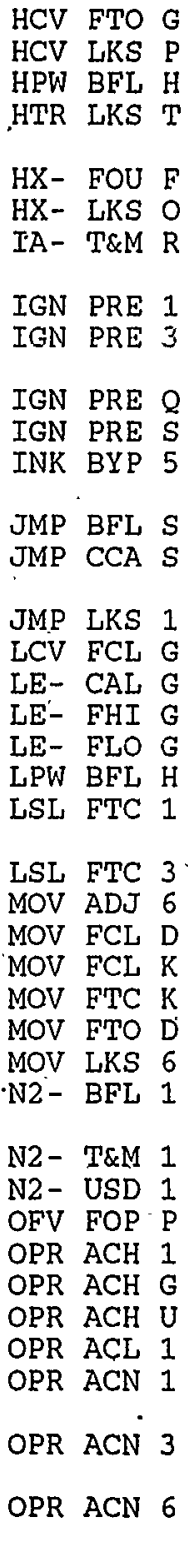 & . & $\begin{array}{r}3.0 \mathrm{E}-02 \mathrm{~N} \\
2.3 \mathrm{E}-5 \mathrm{H} \\
3.0 \mathrm{E}-02 \mathrm{~N} \\
3.0 \mathrm{E}-02 \mathrm{~N} \\
4.6 \mathrm{E}-04 \mathrm{~N} \\
1.0 \mathrm{E}-08 \mathrm{H} \\
1.0 \mathrm{E}-09 \mathrm{H} \\
2.0 \mathrm{E}-05 \mathrm{H} \\
5.0 \mathrm{E}-07 \mathrm{H} \\
2.3 \mathrm{E}-04 \mathrm{H} \\
5.0 \mathrm{E}-07 \mathrm{H} \\
5.0 \mathrm{E}-07 \mathrm{H} \\
8.0 \mathrm{E}-04 \mathrm{Y} \\
1.0 \mathrm{E}-06 \mathrm{H} \\
1.0 \mathrm{E}-06 \mathrm{H} \\
1.0 \mathrm{E}+00 \mathrm{Q} \\
3.0 \mathrm{E}-07 \mathrm{H} \\
3.0 \mathrm{E}-07 \mathrm{H} \\
3.0 \mathrm{E}-03 \mathrm{~N} \\
3.0 \mathrm{E}-03 \mathrm{~N} \\
1.0 \mathrm{E}-06 \mathrm{H} \\
1.0 \mathrm{E}-02 \mathrm{~N} \\
5 \\
5.0 \mathrm{E}-09 \mathrm{H} \\
5.0 \mathrm{E}-02 \mathrm{~N} \\
5.0 \mathrm{E}-02 \mathrm{~N} \\
5.0 \mathrm{E}-02 \mathrm{~N} \\
5.0 \mathrm{E}-04 \mathrm{~N} \\
5.0 \mathrm{E}-03 \mathrm{~N}\end{array}$ & $\begin{array}{l}\text { Solenoid operated valve fails to open } \\
\text { VALVE LEAKAGE } \\
\text { Failure of H area power } \\
\text { Heater steam tubes leak } \\
\text { Heat exchanger fouled } \\
\text { Heat exchanger leaks (tube/shell) } \\
\text { Fan house IA system unavailable due to } \\
\text { T\&M } \\
\text { Ignition source in tank probability } \\
\text { Ignition source frequency due to nearby } \\
\text { lightning strike, } \\
\text { IGNITION souRCE PREsENT } \\
\text { Ignition source present in. melter } \\
\text { SRAT temp low interlock bypassed } \\
\text { ELECTRIC JUMPERS FoR LID HEATERS FAIL } \\
\text { CC: ELECTRIC JUMPERS FoR LID HEATERS FAIL } \\
\text { (2 components) } \\
\text { SPC jumper leaks } \\
\text { Level control valve fails closed. } \\
\text { Level sensor, is calibrated } \\
\text { Level sensor fails high } \\
\text { LEVEL ELEMENT (SENSOR) FAILs Low } \\
\text { Failure to supply loop power } \\
\text { Low level switch on co2 system fails to } \\
\text { close } \\
\text { Low level switch fails to close } \\
\text { PRCD cleaned (drain valve operated) } \\
\text { Motor operated valve fails closed } \\
\text { Motor operated valve fails closed } \\
\text { Motor operated valve fails to close } \\
\text { Motor operated valve fails to open } \\
\text { Motor operated valve leaks (internal). } \\
\text { DWPF liquid nitrogen delivery fails to } \\
\text { arrive } \\
\text { Maintenance of N2 system } \\
\text { LPPP N2 bottles used } \\
\text { Vacuum breaker fails open } \\
\text { Failure of administrative control - high } \\
\text { Failure of administrative control - high } \\
\text { Failure of administrative control - high } \\
\text { Failure of administrative control - low } \\
\text { Failure of administrative control - } \\
\text { nominal } \\
\text { Failure of. administrative control - } \\
\text { nominal } \\
\text { Failure of administrative control - } \\
\text { nominal }\end{array}$ & 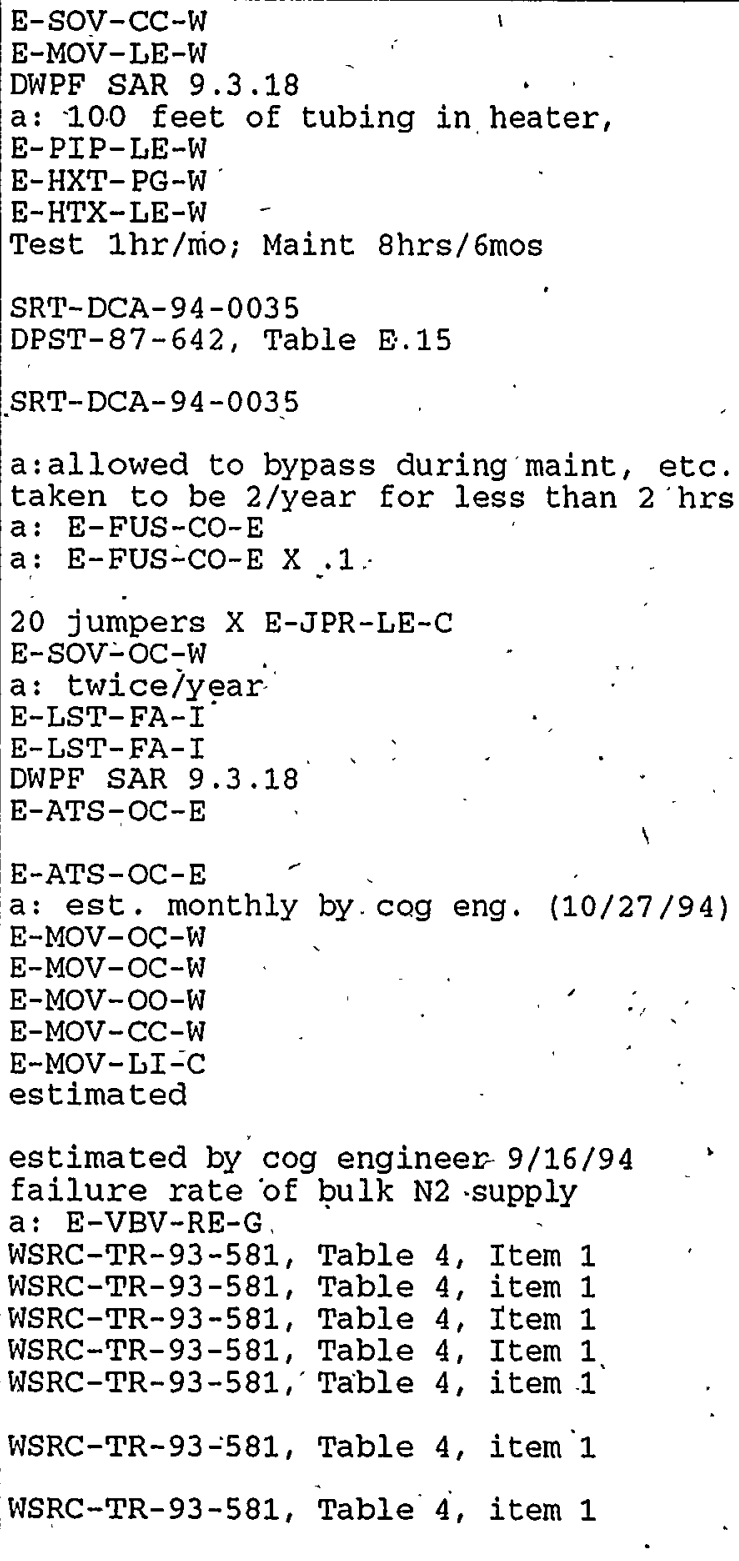 & & $\begin{array}{r}10 \\
10 \\
5 \\
5 \\
5 \\
5 \\
10 \\
10\end{array}$ & $\begin{array}{l}\mathrm{M} \\
\mathrm{M} \\
\mathrm{M} \\
\mathrm{M} \\
\mathrm{M} \\
\mathrm{M} \\
\mathrm{M} \\
\mathrm{M} \\
\mathrm{M} \\
\mathrm{M} \\
\mathrm{M}\end{array}$ \\
\hline
\end{tabular}


Type Codes for I: ICAFTA \DATA \MASTER.TC 11/11/94 11:43 AM (CONT.)

\begin{tabular}{|c|c|c|c|c|c|c|}
\hline Type Code & & Rate & Description & Source & EF & D \\
\hline OPR ACN U & & $5.0 \mathrm{E}-03 \mathrm{~N}$ & $\begin{array}{l}\text { Failure of administrative control - } \\
\text { nominal. }\end{array}$ & $\begin{array}{l}\text { WSRC-TR-93-581, Table 4, item } 1 \\
\text { (SW4-16.2, Rev. 0, Draft) }\end{array}$ & 10 & I \\
\hline OPR CAL K & & $3.0 \mathrm{E}-04 \mathrm{~N}$ & $\begin{array}{l}\text { Chemical addition error - low (simple. } \\
\text { process) }\end{array}$ & WSRC-TR-93-581, Table 4, item 16 & 10 & $\mathrm{~L}$ \\
\hline OPR CAN 1 & & $3.0 \mathrm{E}-03 \mathrm{~N}$ & $\begin{array}{l}\text { Chemical addition error - low (simple } \\
\text { process) }\end{array}$ & WSRC-TR-93-581, Table 4, item 16 & 10 & L \\
\hline OPR CSH G & & $1.0 \mathrm{E}-01 \mathrm{~N}$ & $\begin{array}{l}\text { Failure to respond to compelling signal, } \\
\text { many competing signals }\end{array}$ & WSRC-TR-93-581, table 4, item 2, high & 10 & $\mathrm{~L}$ \\
\hline OPR CSH Q & & $1.0 \mathrm{E}-01 \mathrm{~N}$ & $\begin{array}{l}\text { Failure to respond to compelling signal, } \\
\text { many competing signals }\end{array}$ & WSRC-TR-93-581, table 4, item 2, high & 10 & $\mathrm{~L}$ \\
\hline $\begin{array}{l}\text { OPR CSL } 3 \\
\text { OPR } \text { CSL } \\
\text { OPR } \\
\text { CSN }\end{array}$ & & $\begin{array}{l}3.0 \mathrm{E}-03 \mathrm{~N} \\
3.0 \mathrm{E}-03 \mathrm{~N} \\
1.0 \mathrm{E}-02 \mathrm{~N}\end{array}$ & $\begin{array}{l}\text { Failure to respond to compelling signal } \\
\text { Failure to respond to compelling signal } \\
\text { Failure to respond to compelling signal, }\end{array}$ & $\begin{array}{l}\text { WSRC-TR-93-581, table 4, item 2, low } \\
\text { WSRC-TR-93-581, table 4, item 2, low } \\
\text { WSRC-TR-93-581, table 4, item 2, nominal }\end{array}$ & $\left|\begin{array}{l}10 \\
10 \\
10\end{array}\right|$ & $\begin{array}{l}L \\
L \\
L\end{array}$ \\
\hline OPR CVH U & & $3.0 \mathrm{E}-01 \mathrm{~N}$ & $\begin{array}{l}\text { several competing signals } \\
\text { Independent checker fails to verify }\end{array}$ & WSRC-TR-93-581, Table 4, Item 8 & & DL \\
\hline OPR IRH G & & $\mathrm{LN} \mid$ & $\begin{array}{l}\text { results } \\
\text { Operator misreads results - high }\end{array}$ & WSRC-TR-93-581, Table 4, Item & & \\
\hline OPR IRH U & & & $\begin{array}{l}\text { Incorrect reading or recording of data - } \\
\text { high }\end{array}$ & WSRC-TR-93-581, Table 4, Item? & & L \\
\hline $\begin{array}{ll}\text { OPR LAH } & 1 \\
\text { OPR LAH } & \text { L }\end{array}$ & & $\begin{array}{l}.0 \mathrm{E}-03 \mathrm{~N} \\
.0 \mathrm{E}-03 \mathrm{~N}\end{array}$ & $\begin{array}{l}\text { Laboratory analysis error - high } \\
\text { Laboratory analysis error - high }\end{array}$ & $\begin{array}{l}\text { WSRC-TR-93-581, Table 4, Item } 19 \\
\text { WSRC-TR-93-581, Table 4, Item } 19\end{array}$ & $\left|\begin{array}{l}10 \\
10 \\
10\end{array}\right|$ & $\begin{array}{l}L \\
L\end{array}$ \\
\hline OPR LAH U & & $03 \mathrm{~N}$ & Laboratory analysis error - high & WSRC-TR-93-581, Table 4, Item 19 & 10 & \\
\hline OPR LAN 1 & & & Laboratory analysis error - nominal & WSRC-TR-93-581, Table 4, Item 19 & $\left|\begin{array}{c}10 \\
10\end{array}\right|$ & \\
\hline $\begin{array}{ll}\text { OFR } & \text { LOH } \\
\text { OPR } & \text { MCH } \\
1\end{array}$ & & $\begin{array}{l}03 \mathrm{~N} \\
02 \mathrm{~N}\end{array}$ & $\begin{array}{l}\text { Fail to remove lockout on breaker } \\
\text { Miscalibration - } 1 \text { person, no che }\end{array}$ & $\begin{array}{l}\text { WSRC-TR-93-581， Table 4, Item } 15 \\
\text { WSRC-TR-93-581， Table 4, item } 12\end{array}$ & $\left|\begin{array}{r}10 \\
5\end{array}\right|-200$ & \\
\hline OPR MCH 2 & & $02 \mathrm{~N}$ & Miscalibration - 1 person, no checks & WSRC-TR-93-581, Table 4, item 12 & & \\
\hline OPR MCH 3 & & 02N & Miscalibration - 1 person, no checks & WSRC-TR-93-581, Table 4, item 12 & & $\mathrm{~L}$ \\
\hline OPR MCH G & & & Miscalibration - 1 person, no checks & $\begin{array}{l}\text { WSRC-TR-9:-381, } \\
\text { years); a: calib. performed } 1 / 2 \text { years }\end{array}$ & & \\
\hline OPR MCH & & $1.5 \mathrm{E}-02 \mathrm{Y}$ & Miscalibration - 1 person, no checks & $\begin{array}{l}\text { WSRC-TR-93-581, Table } 4 \text {, item } 12 *(1 / 2 \\
\text { years); } a \text { : calib. performed } 1 / 2 \text { years }\end{array}$ & & $\mathrm{L}$ \\
\hline $\begin{array}{l}\text { OPR MCH R } \\
\text { OPR NCH } 1\end{array}$ & & $\begin{array}{l}3.0 \mathrm{E}-02 \mathrm{~N} \\
1.0 \mathrm{E}-01 \mathrm{~N}\end{array}$ & $\begin{array}{l}\text { Miscalibration - } 1 \text { person, no checks } \\
\text { Fail to verify paramenter with } \\
\text { calculation }\end{array}$ & $\begin{array}{l}\text { WSRC-TR-93-581, Table 4, item } 12 \\
\text { WSRC-TR-93-581, Table 4, item } 20\end{array}$ & & L \\
\hline OPR NCN 1 & & $3.0 \mathrm{E}-02 \mathrm{~N}$ & $\begin{array}{l}\text { Fail to verify paramenter with } \\
\text { calculation }\end{array}$ & WSRC-TR-93-58̣1, & & L \\
\hline $\begin{array}{l}\text { OPR OPE } \\
\text { OPR OVH } \\
\text { OPR RAH } \\
\text { OPR } \\
\text { OPR RAN }\end{array}$ & & $\begin{array}{l}1.0 \mathrm{~N} \\
5.0 \mathrm{E}-05 \mathrm{H} \\
5.0 \mathrm{E}-05 \mathrm{H} \\
5.0 \mathrm{E}-06 \mathrm{H}\end{array}$ & $\begin{array}{l}\text { Operator opens makeup feed valve } \\
\text { Overflow from OE, OECT, or } \mathrm{PR} \\
\text { Random actuation/shutdown of system } \\
\text { Random actuation/shutdown of system - } \\
\text { nominal }\end{array}$ & $\begin{array}{l}\text { Assumed to occur given low level signal } \\
\text { (WSRC-TR-93-581, item 18) X (3) } \\
\text { WSRC-TR-93-581, Table 4, item } 21 \\
\text { WSRC-TR-93-581, Table 4, item } 21\end{array}$ & $\left|\begin{array}{l}10 \\
10 \\
10\end{array}\right|$ & $\begin{array}{l}L \\
L \\
L\end{array}$ \\
\hline $\begin{array}{lll}\text { OPR RMH } & 1 \\
\text { OPR RMH } & \text { RM } \\
\text { OPR } & \text { RMH } & \text { R }\end{array}$ & & $\begin{array}{l}02 N \\
02 N \\
02 N\end{array}$ & $\begin{array}{l}\text { Failure to restore following maintenance } \\
\text { Failure to restore following maintenance } \\
\text { Failure to restore following maintenance }\end{array}$ & $\begin{array}{l}\text { WSRC-TR-93-581, Table 4, item } 14 \\
\text { WSRC-TR-93-581, Table 4, item } 14 \\
\text { WSRC-TR-93-581, Table 4, Item } 14\end{array}$ & & $\begin{array}{l}\mathrm{L} \\
\mathrm{L} \\
\mathrm{L}\end{array}$ \\
\hline OPR RMN N & & $5.0 \mathrm{E}-03 \mathrm{~N}$ & Failure to restore following maintenance & ble 4 , Item 14 & 10 & $\mathrm{~L}$ \\
\hline & & $3.0 \mathrm{E}-02 \mathrm{~N}$ & - nominal & $\begin{array}{l}292-S-4003 / 4008 \\
\text { WSRC-TR-93-581, Table 4, item } 13\end{array}$ & & L \\
\hline
\end{tabular}


Type codes for I: ICAFTA IDATA IMASTER.TC

11/11/94 11:43 AM (CONT.)

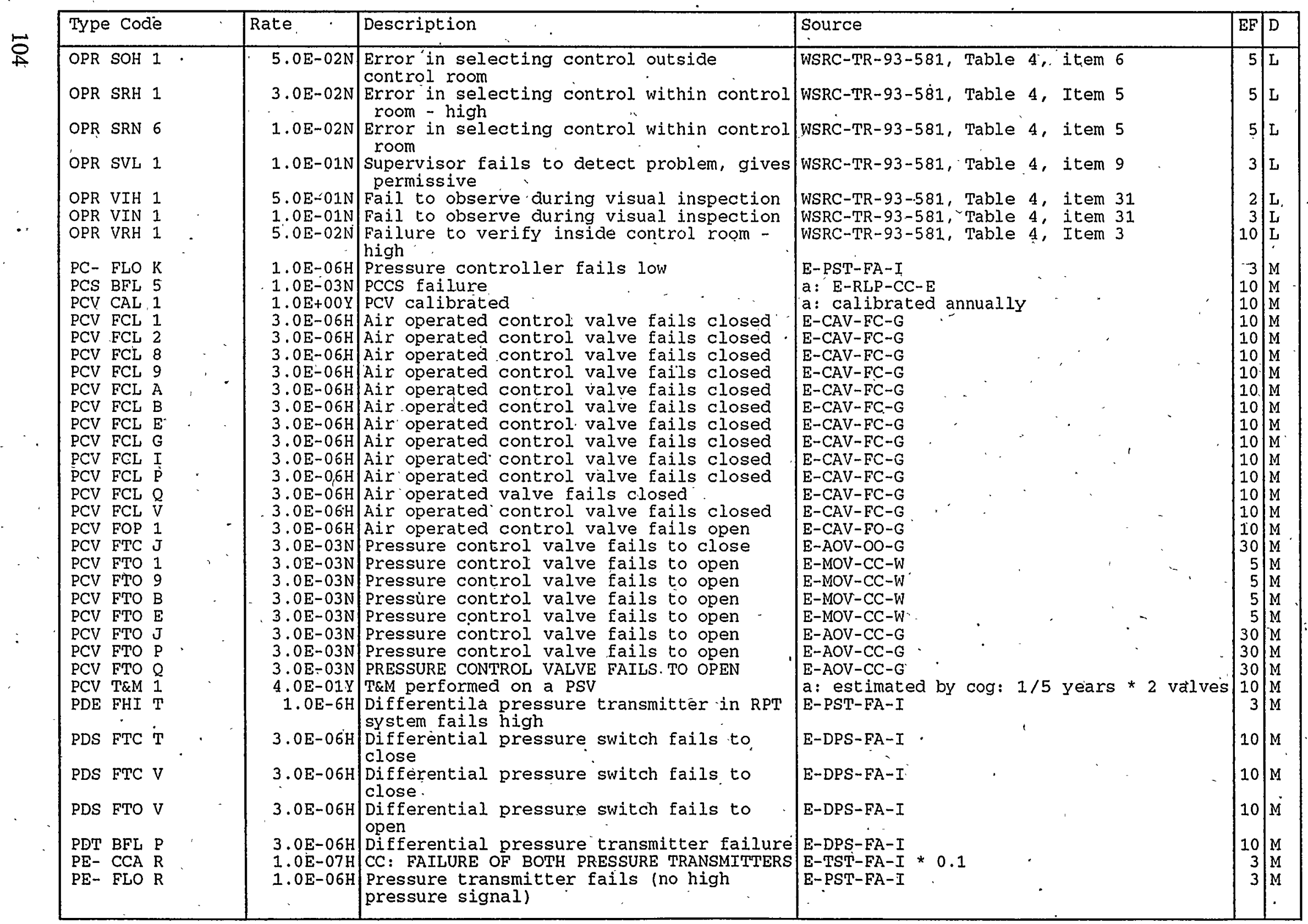


Type Codes foṛ I: ICAFTA IDATA IMASTER.TC

11/11/94 11:43 AM (CONT.)

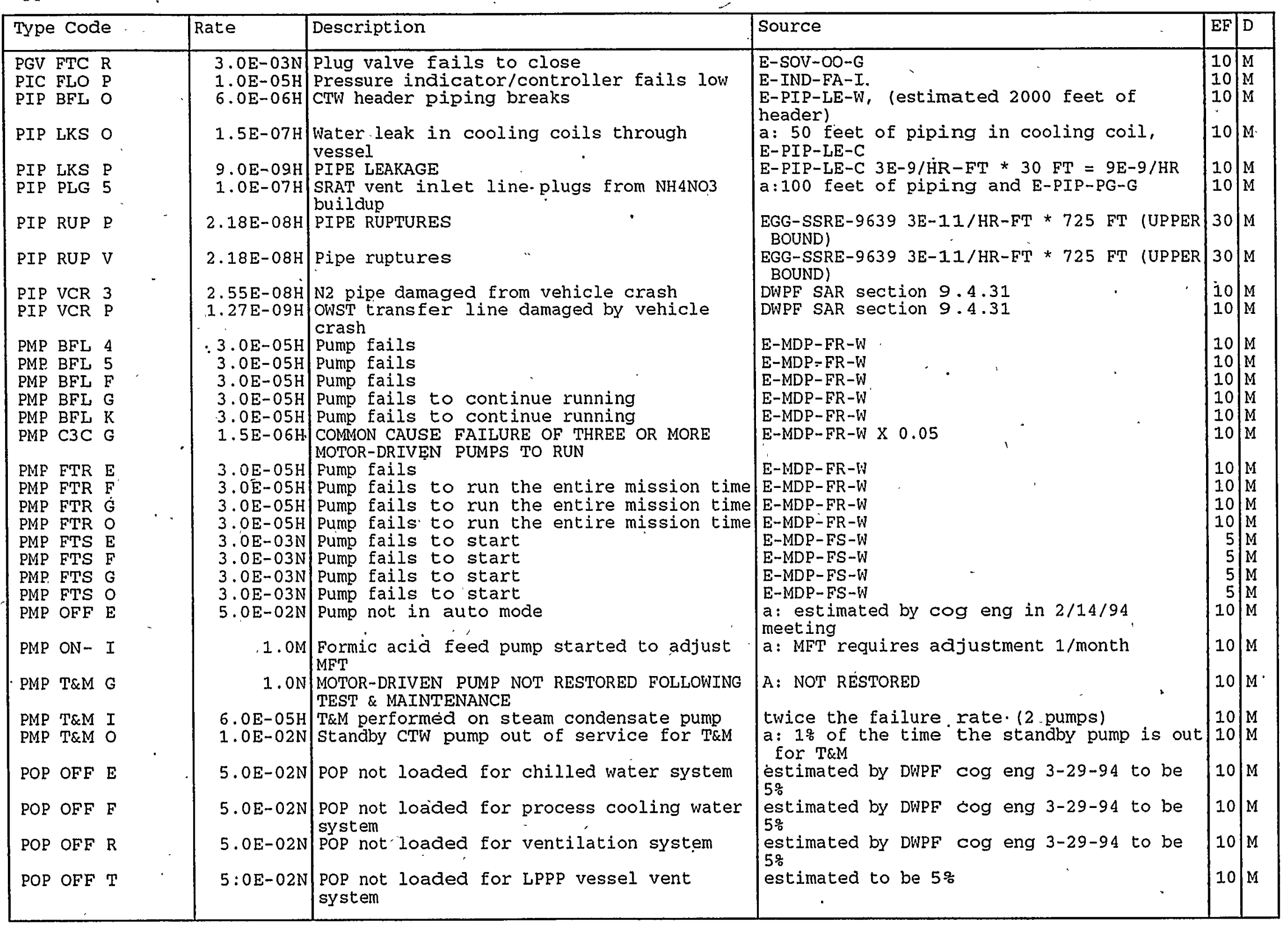


Type Codes for I: \CAFTA \DATA \MASTER.TC

11/11/94 11:43 AM (CONT.)

\begin{tabular}{|c|c|c|c|c|c|}
\hline Type Code & Rate & Description & Source & EF & D \\
\hline SLR PRE U & $5.0 \mathrm{E}-01 \mathrm{Y}$ & $\begin{array}{l}\text { Excessive salt content present in melter } \\
\text { feed solution }\end{array}$ & assumed present every batch (1/86 hours) & 10 & $M$ \\
\hline SRA PRE 5 & $5.0 \mathrm{E}-01 \mathrm{Y}$ & $\begin{array}{l}\text { Macro batch of sludge to DWPF (every } 2 \\
\text { years) }\end{array}$ & $\begin{array}{l}\text { Estimated by TNX and DWPF (in meeting } \\
\text { minutes } 3 / 8 / 94 \text { ) }\end{array}$ & $10 \mid$ & $\mathrm{M}$ \\
\hline STM BFL $G$ & $2.0 \mathrm{E}-04 \mathrm{H}$ & $\begin{array}{l}\text { Site steam (to DWPF) steam generator } \\
\text { fails }\end{array}$ & $\begin{array}{l}\text { DWPF SAR Section } 9.3 .20 .3 \text { steam Supply } \\
\text { and Distribution Systems, Frequency }\end{array}$ & 10 & $\mathrm{M}$ \\
\hline $\begin{array}{l}\text { SV- ADJ } 5 \\
\text { SV- FCL } 1\end{array}$ & $\begin{array}{l}1.0 \mathrm{E}+00 \mathrm{M} \\
5.0 \mathrm{E}-07 \mathrm{H}\end{array}$ & $\begin{array}{l}\text { Needle valve adjusted } \\
\text { Solenoid valve fails closed }\end{array}$ & $\begin{array}{l}\text { estimated by cog eng } \\
\mathrm{E}-\mathrm{SOV}-\mathrm{OC}-\mathrm{W}\end{array}$ & $\left|\begin{array}{l}10 \\
10\end{array}\right|$ & $\begin{array}{l}\mathrm{M} \\
\mathrm{M}\end{array}$ \\
\hline $\begin{array}{l}\text { SV- FCL } 0 \\
\text { SV- FCL }\end{array}$ & $3.0 \mathrm{E}-06 \mathrm{H}$ & Solenoid operated valve fails closed & E-CSV-FC-G & 10 & \\
\hline SV- FOP 1 & $\begin{array}{l}5.0 \mathrm{E}-\mathrm{U} / \mathrm{H} \\
5.0 \mathrm{E}-07 \mathrm{H}\end{array}$ & $\begin{array}{l}\text { Solenold valve talls closed } \\
\text { Solenoid valve fails open }\end{array}$ & $\begin{array}{l}E-S O V-C C-W \\
E-S O V-C O-W\end{array}$ & $\left|\begin{array}{l}100 \\
10\end{array}\right|$ & $\mathrm{M}$ \\
\hline SV- FTC G & $1.0 \mathrm{E}-03 \mathrm{~N}$ & Solenoid valve fails to close & E-SOV-OO-W & 10 & $\mathrm{M}$ \\
\hline $\begin{array}{l}\text { SV-FTO G, } \\
\text { TC- FLO } 1\end{array}$ & $\begin{array}{l}1.0 \mathrm{E}-03 \mathrm{~N} \\
3.0 \mathrm{E}-05 \mathrm{H}\end{array}$ & $\begin{array}{l}\text { SOLENOID VALVE FAILS TO OPEN } \\
\text { Temperature controller fails low }\end{array}$ & $\begin{array}{l}E-S O V-C C-W \\
E-P L C-F A-I\end{array}$ & $\left|\begin{array}{l}10 \\
10\end{array}\right|$ & \\
\hline TCV CAL 1 & $1 \mathrm{M}$ & $\begin{array}{l}\text { Maintenance performed on SCVC chilled } \\
\text { water system }\end{array}$ & estimated & 10 & $\mathrm{M}$ \\
\hline TCV FCL D & $5.0 \mathrm{E}-07 \mathrm{H}$ & $\begin{array}{l}\text { Temperature control valve (solenoid } \\
\text { operated) fails closed }\end{array}$ & E-SOV-OC-W & 10 & $\mathrm{M}$ \\
\hline TCV FCL $\mathrm{F}$, & $5.0 \mathrm{E}-07 \mathrm{H}$ & $\begin{array}{l}\text { Temperature control valve (solenoid } \\
\text { operated) fails closed }\end{array}$ & E-SOV-OC-W & 10 & $\mathrm{M}$ \\
\hline TE- CCA S & $1.0 \mathrm{E}-07 \mathrm{H}$ & $\begin{array}{l}\text { CC: Temperature elements fail } 12 \\
\text { components) }\end{array}$ & a: E-TST-FA-I X .1 & 3 & $\mathrm{M}$ \\
\hline TE- FHI $S$ & $1.0 \mathrm{E}-06 \mathrm{H}$ & Temperature element fails & E-TST-FA-I & 3 & $\mathrm{M}$ \\
\hline $\begin{array}{l}\text { TE- FLO D } \\
\text { TE- FLO F }\end{array}$ & $\begin{array}{r}1.0 \mathrm{E}-06 \mathrm{H} \\
1.0 \mathrm{E}-06 \mathrm{H}\end{array}$ & $\begin{array}{l}\text { Temperature element fails } \\
\text { Temperature element fails }\end{array}$ & $\begin{array}{l}E-T S T-F A-W \\
E-T S T-F A-T\end{array}$ & & $\mathrm{M}$ \\
\hline TNK LKS 3 & $1.0 \mathrm{E}-08 \mathrm{H}$ & Liquid N2 tank leaks & E-TKP-LE-W & 10 & $M$ \\
\hline TNK LKS $\mathrm{P}$ & $1.0 \mathrm{E}-07 \mathrm{H}$ & TANK LEAKS & E-TKP-LE-G & 10 & $\mathrm{M}$ \\
\hline TNK USD 1 & & CO2 supply tank volume used & estimated by cog eng. supply $=30$ days & 10 & $M$ \\
\hline $\begin{array}{l}\text { TNK USD } 3 \\
\text { TOP BFL } 0\end{array}$ & $\begin{array}{l}3.3 \mathrm{E}-01 \mathrm{D} \\
6.7 \mathrm{E}-03 \mathrm{~N}\end{array}$ & $\begin{array}{l}\text { N2 supply tank volume used } \\
\text { Failure of CO2 backup system }\end{array}$ & $\begin{array}{l}\text { a: Told by cog eng, supply }=7 \text { days } \\
\text { Fault tree calculation of SPCBP.CAF }\end{array}$ & $\begin{array}{r}10 \\
5\end{array}$ & $M$ \\
\hline $\begin{array}{l}\text { TOP BFL } 1 \\
\text { TOP BFL } 2\end{array}$ & $\begin{array}{l}6.7 \mathrm{E}-05 \mathrm{H} \\
1.9 \mathrm{E}-05 \mathrm{H}\end{array}$ & $\begin{array}{l}\text { SPC primary purge system fails } \\
\text { Loss of CPC primary (air) purge system }\end{array}$ & $\begin{array}{l}\text { Fault tree sPCPP.CAF }(10-6-94) \\
\text { Fault tree calculation of CPCPP. CAF } \\
(10-6-94)\end{array}$ & $\begin{array}{r}10 \\
5\end{array}$ & $\frac{M}{M}$ \\
\hline TOP BFL 3 & $7.7 \mathrm{E}-06 \mathrm{H}$ & Bulk N2 tank supply failure & Fault tree calculation of N2TNK.CAF & $\cdot 5$ & M \\
\hline TOP BFL 7 & $3.7 \mathrm{E}-05 \mathrm{~N}$ & CPC nitrogen tank supply failure & $\begin{array}{l}\text { Fault tree calculation of CPCTNK. CAF } \\
(10-6-94)\end{array}$ & 5 & M \\
\hline TOP BFL A & $5.4 \mathrm{E}-03 \mathrm{~N}$ & CTW Deep well system unavailable & $\begin{array}{l}\text { Fault tree calculation of Deep.CAF } \\
(10-6-94)\end{array}$ & 10 & M \\
\hline TOP BFL B & $6.3 \mathrm{E}-06 \mathrm{H}$ & CTW primary pumps fail & Fault tree calculation of CTWP.CAF & 10 & M \\
\hline TOP BFL E & $2.3 \mathrm{E}-05 \mathrm{H}$ & Inadequate chilled water to supply header & $\begin{array}{l}\text { Fault tree calculation of CHW.CAF } \\
(10-6-94)\end{array}$ & 10 & M \\
\hline TOP BFL $\mathrm{F}$ & $2.3 \mathrm{E}-03 \mathrm{~N}$ & $\begin{array}{l}\text { Inadequate process cooling water to } \\
\text { supply header }\end{array}$ & $\begin{array}{l}\text { Fault tree calculation of PSW.CAF } \\
(10-6-94)\end{array}$ & 10 & M \\
\hline TOP BFL G & $2.1 \mathrm{E}-04 \mathrm{H}$ & Failure to supply steam to supply header & $\begin{array}{l}\text { Fault tree calculation of STEAM.CAF } \\
(10-6-94)\end{array}$ & 10 & M \\
\hline
\end{tabular}


Type Codes for I : \CAFTA \DATA \MASTER. TC

11/11/94 11:43 AM (CONT.)

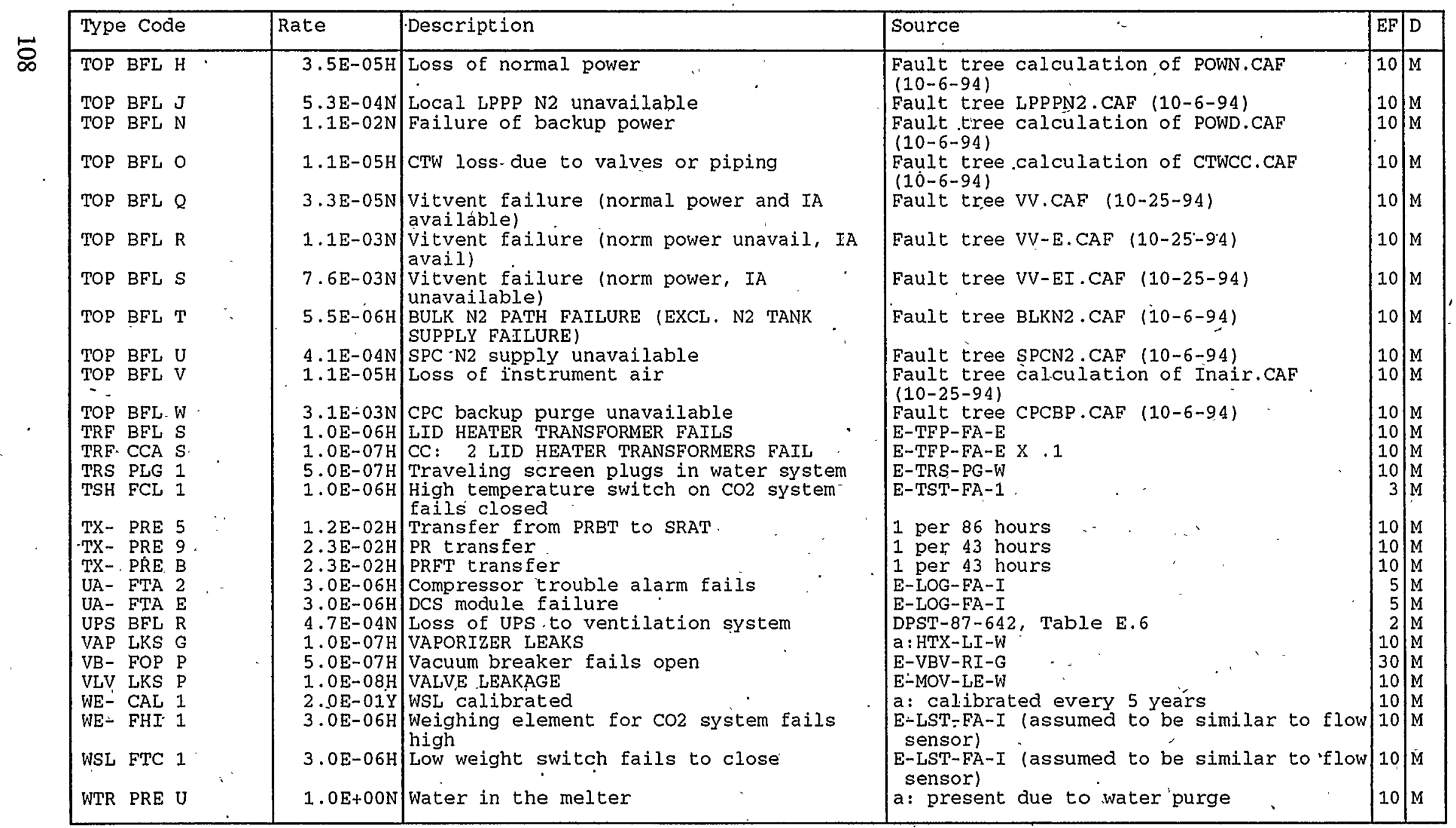




\section{Attachment 5.}

Accident Fault Tree Plots and Cutsets

PVVH Explosion

110

SRAT Explosion

171

SME Explosion

208

PRBT Explosion

238

MFT Explosion

254

PR Explosion

OE Explosion

292

OECT Explosion

311

PRFT Explosion

330

Steam Explosion in Melter

352

Melter Offgas Explosion

SPT Explosion

PPT Explosion

OWST Explosion

SPC Explosion

401

414

432 
X-ESR-S-00001, REV. 0 


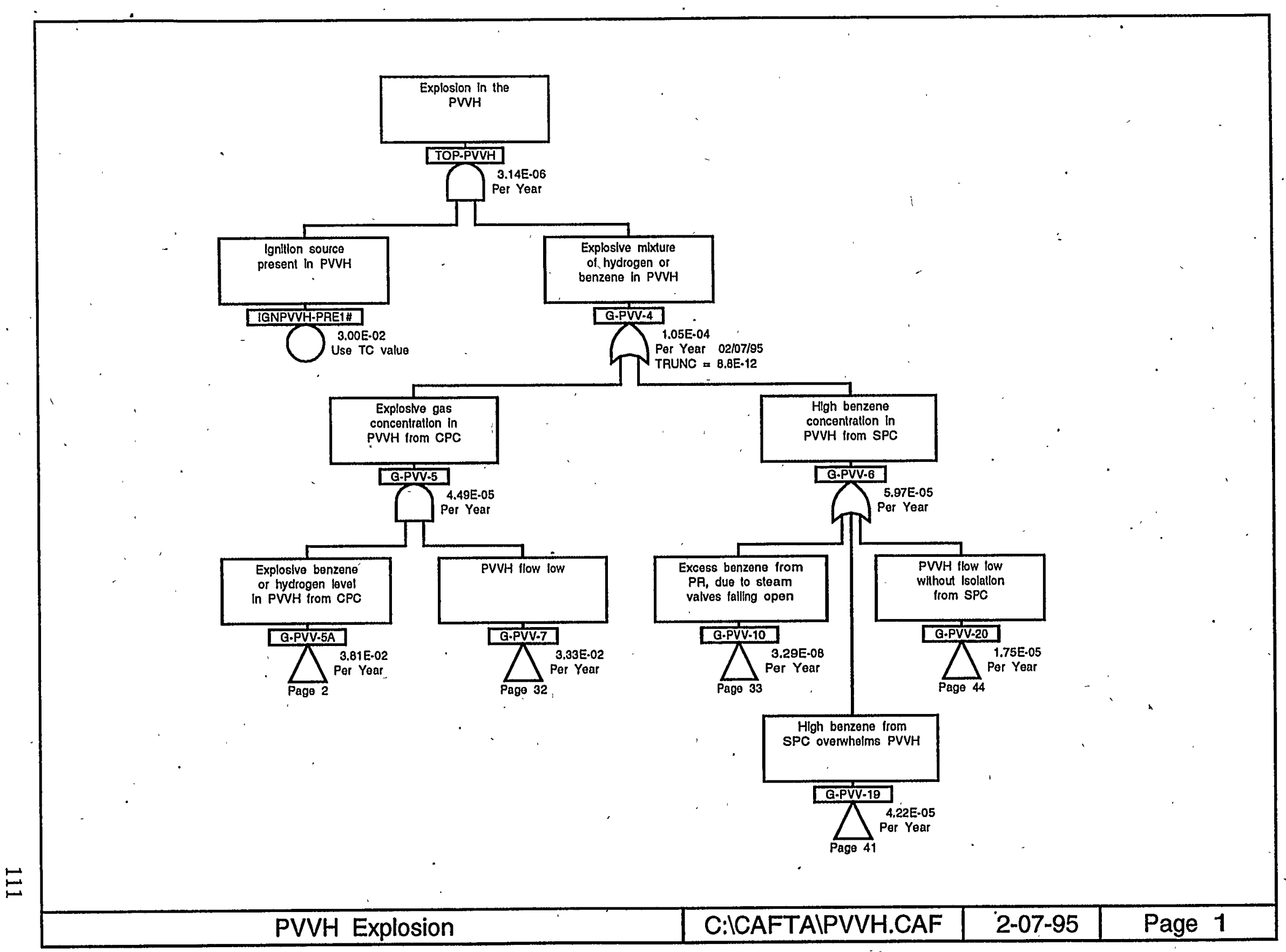




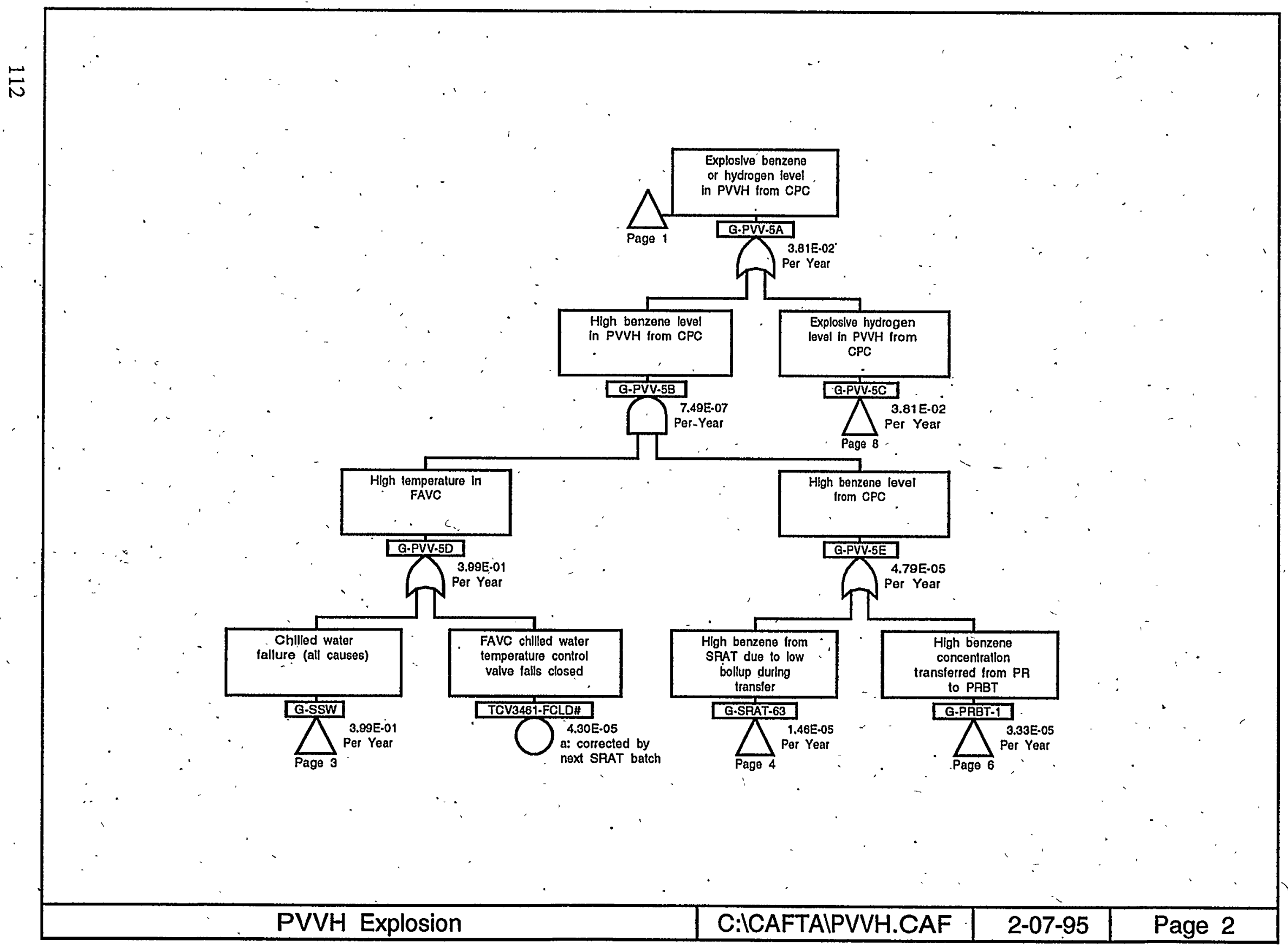




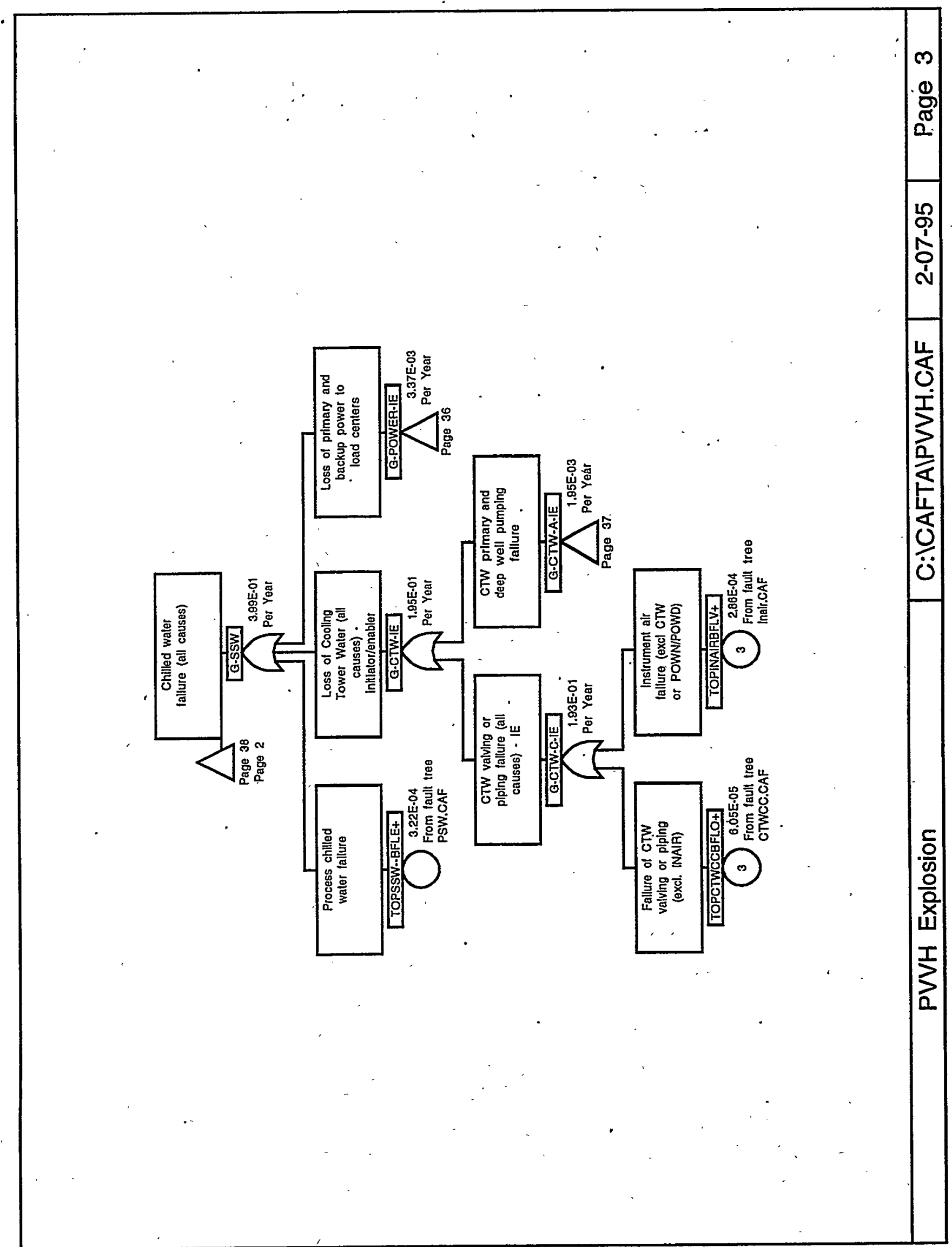




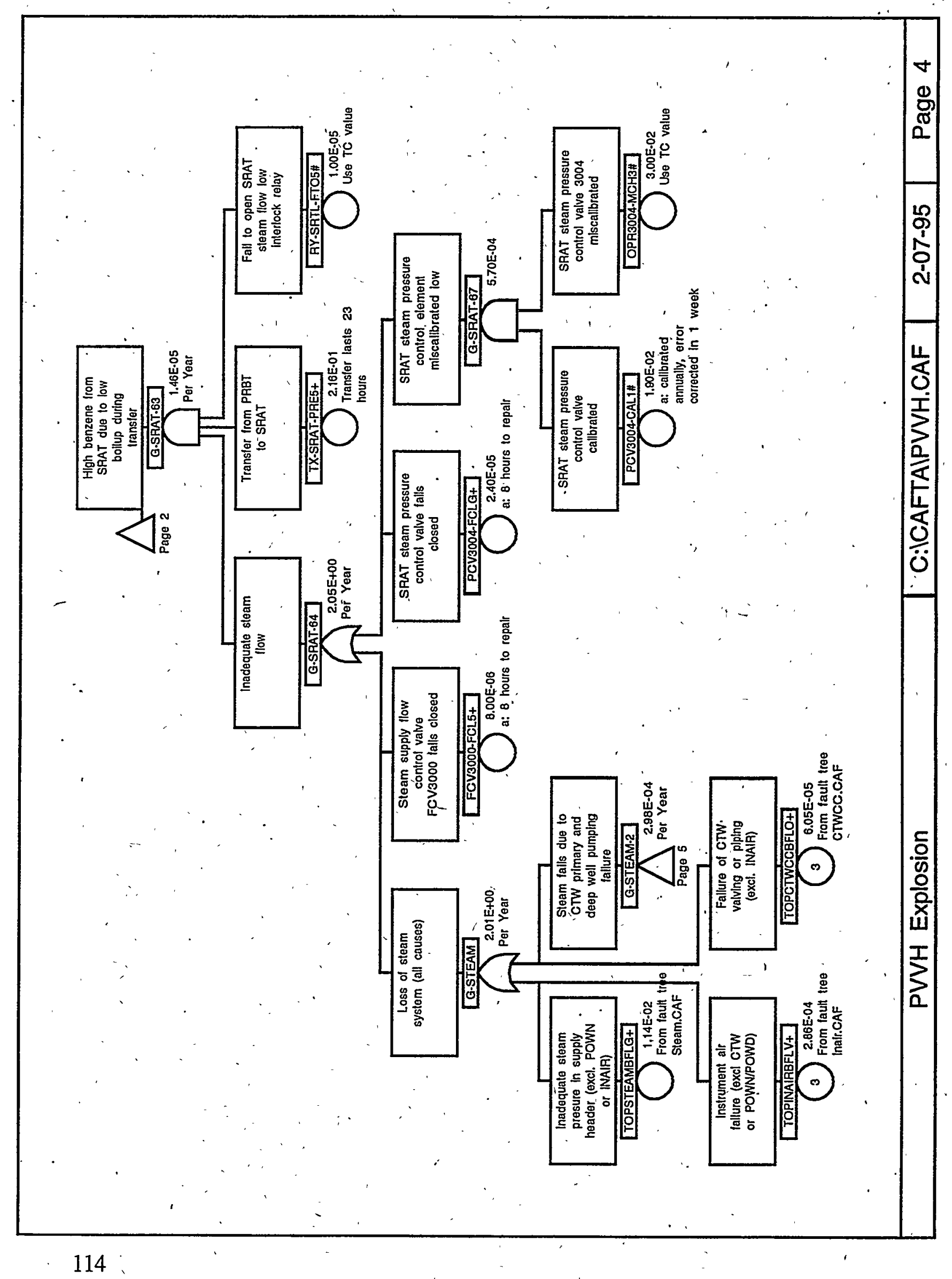




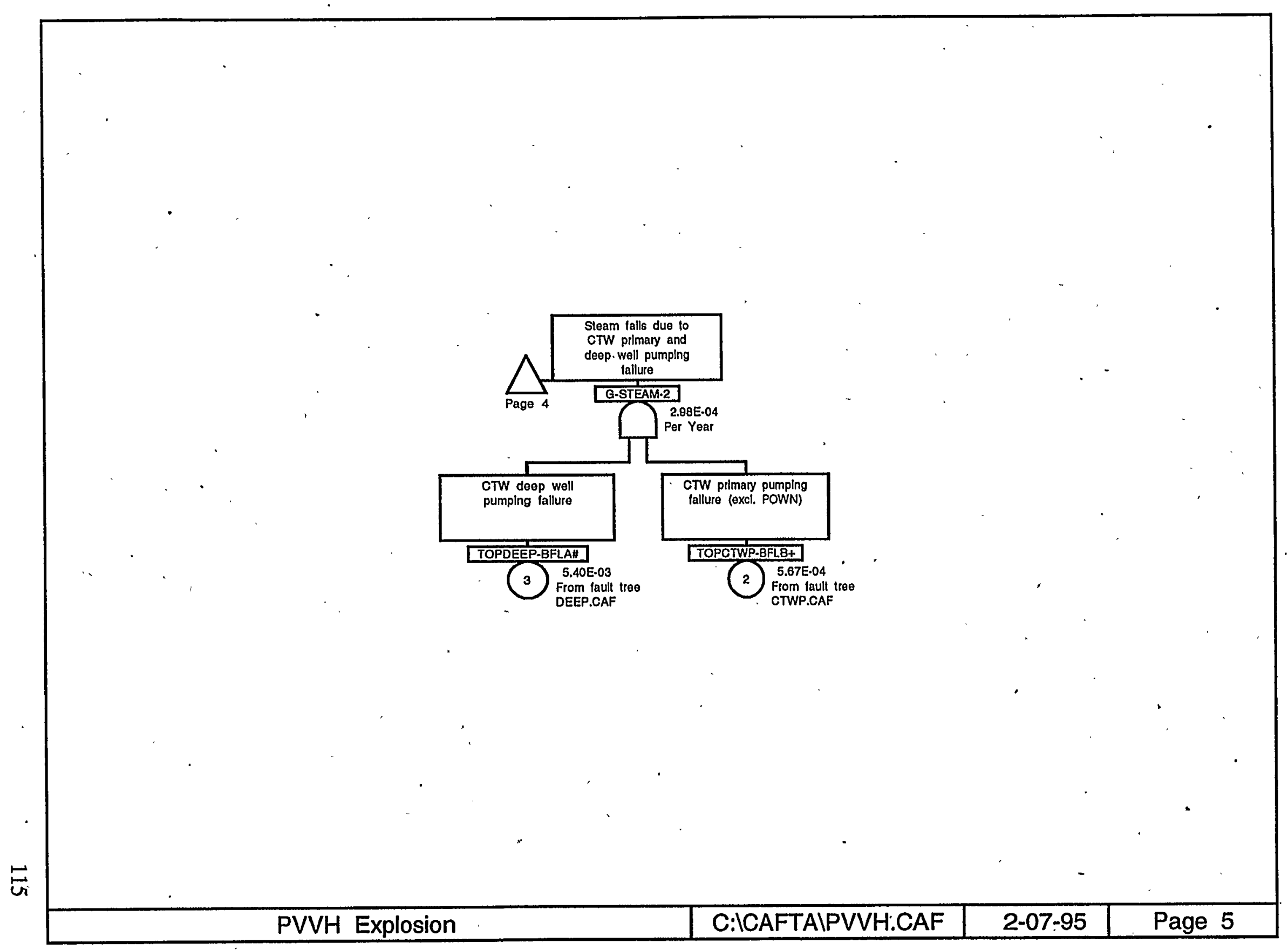




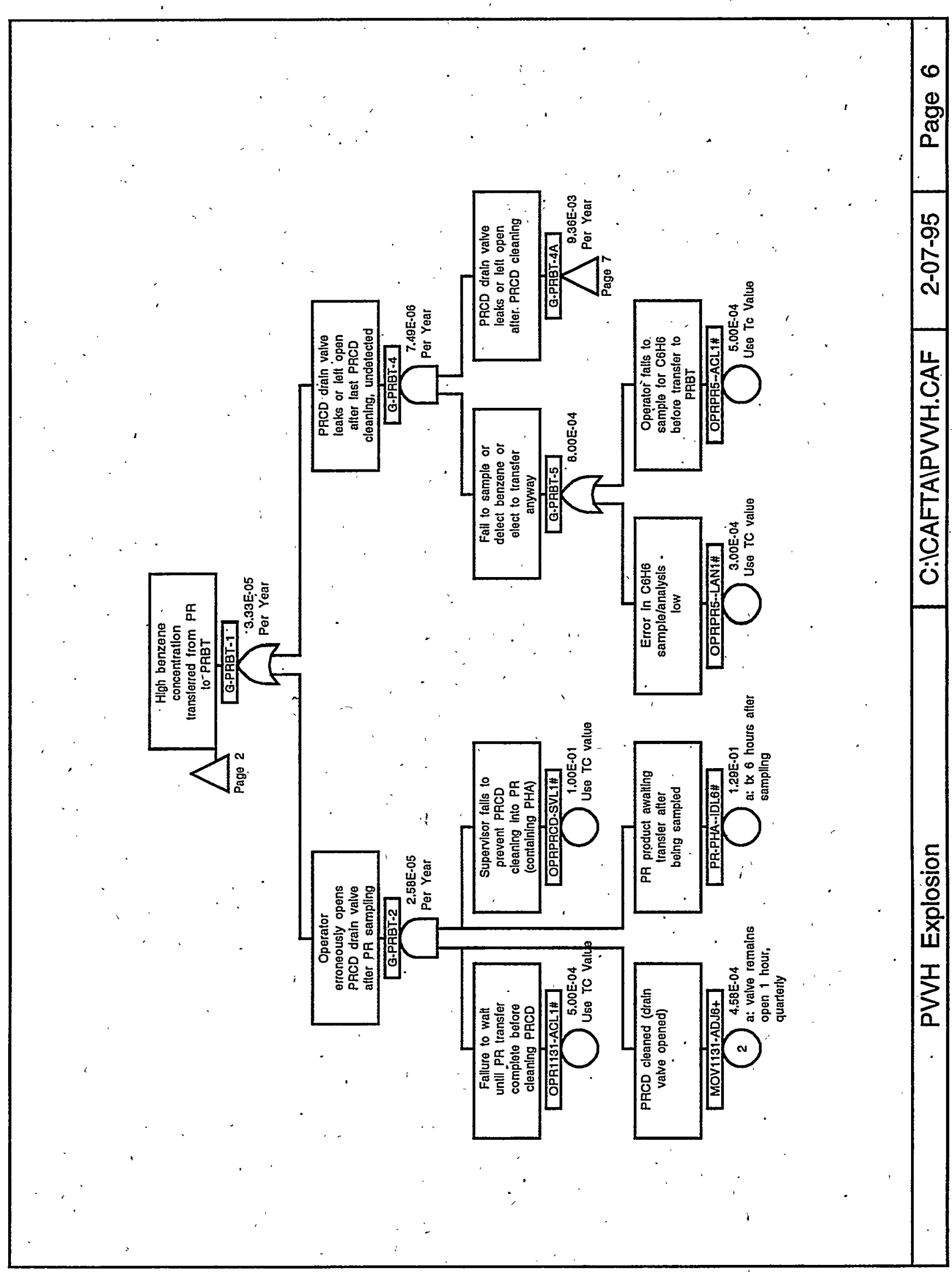




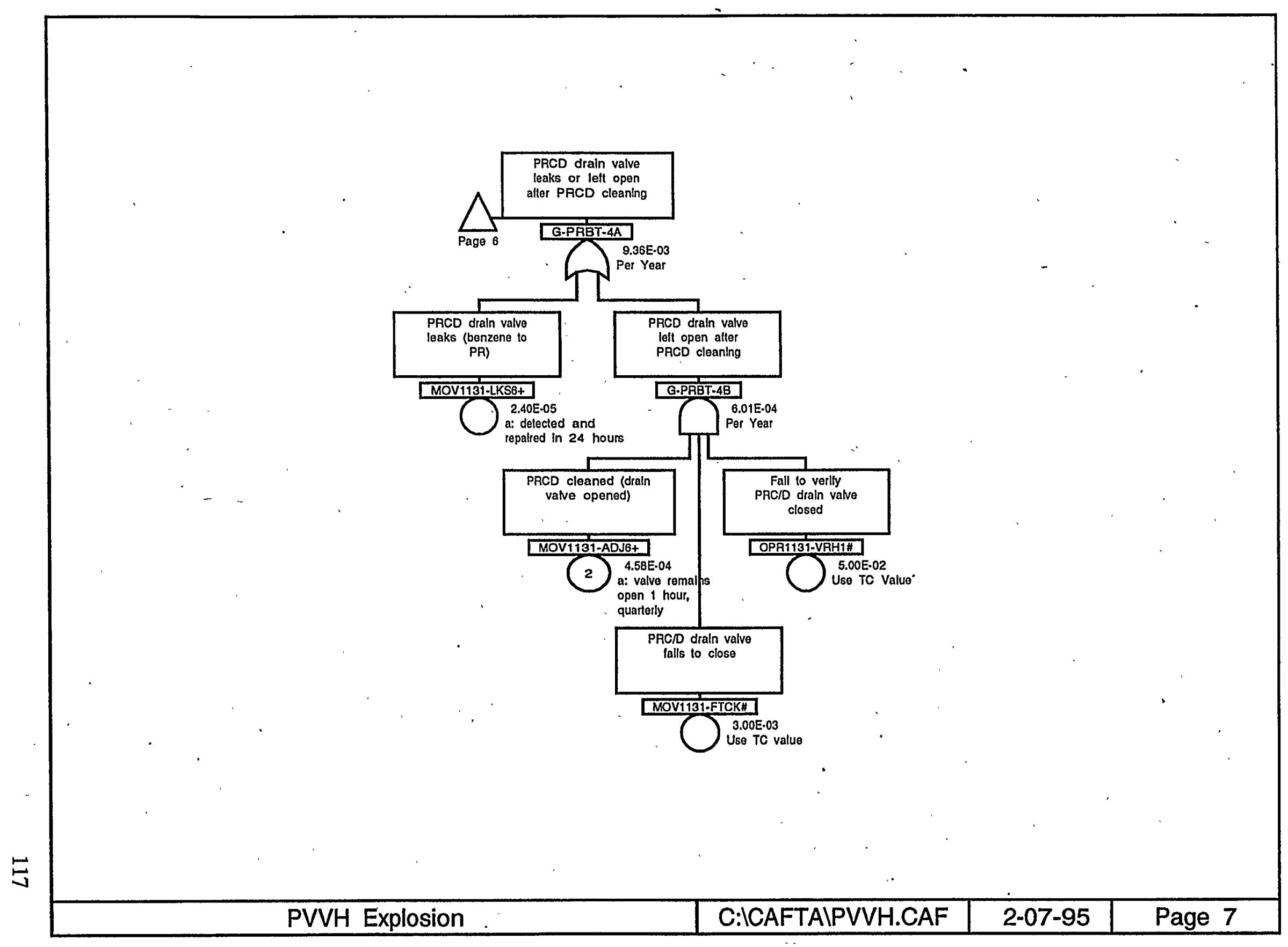




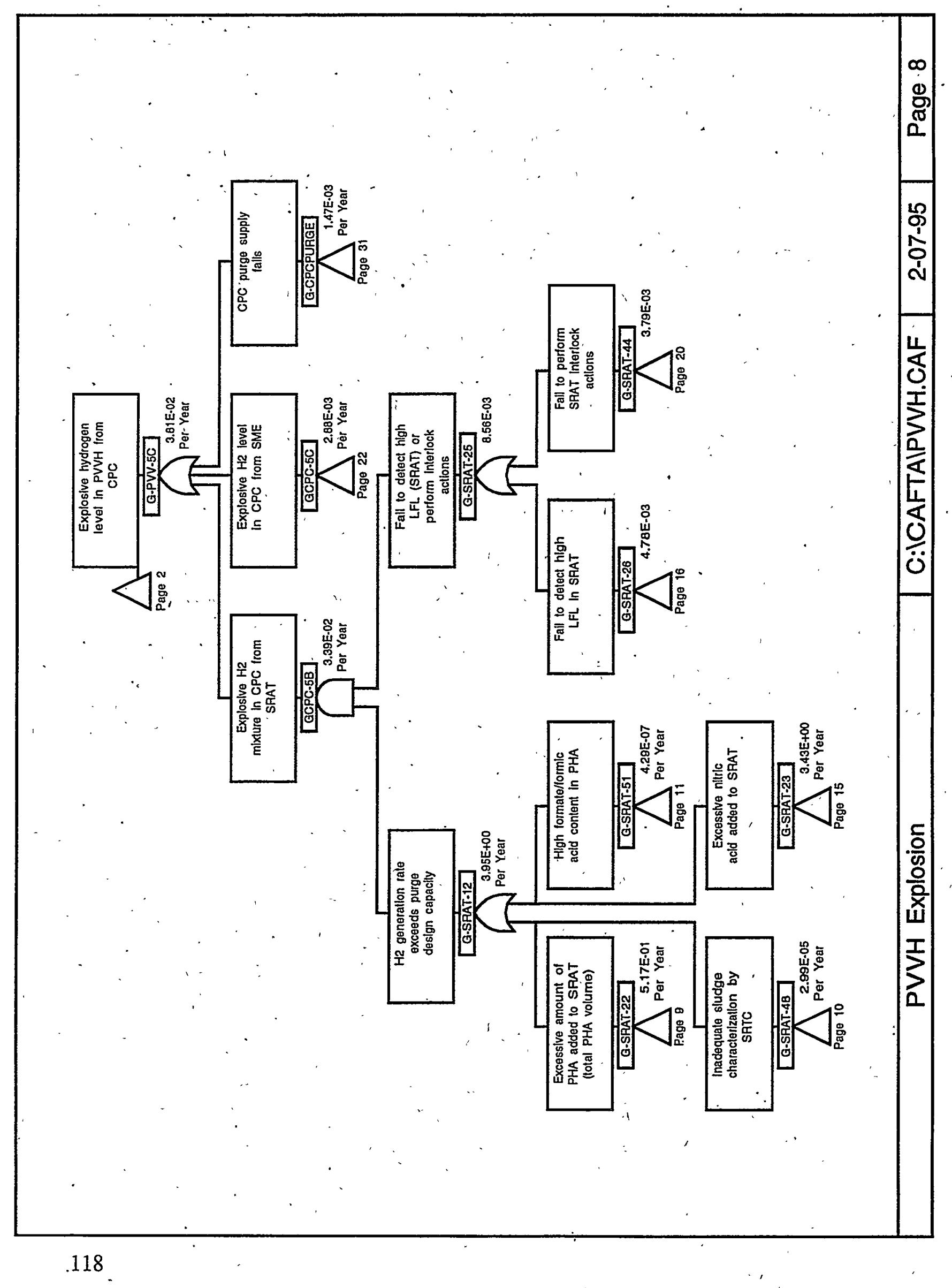




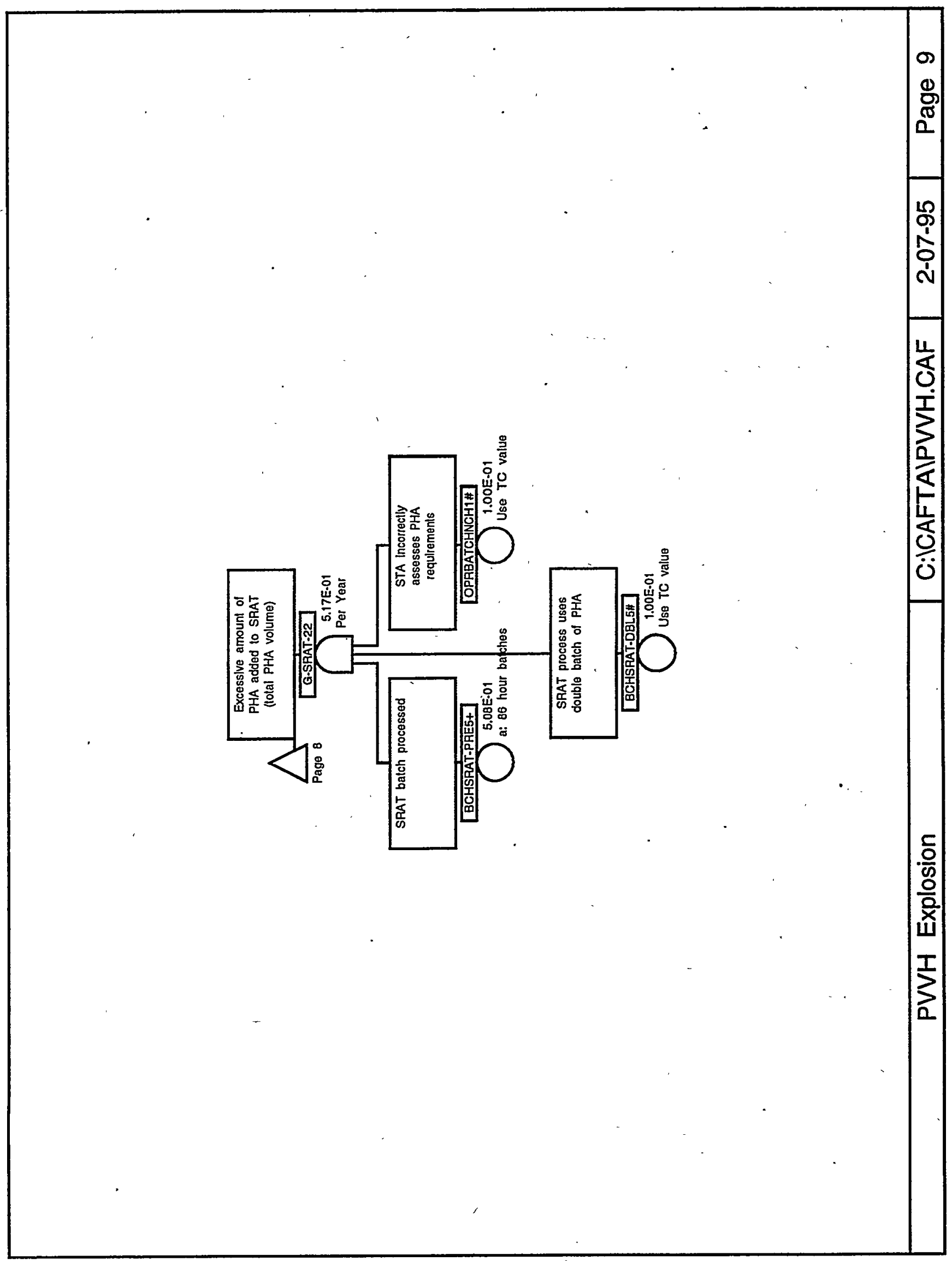


X-ESR-S-00001, REV. 0

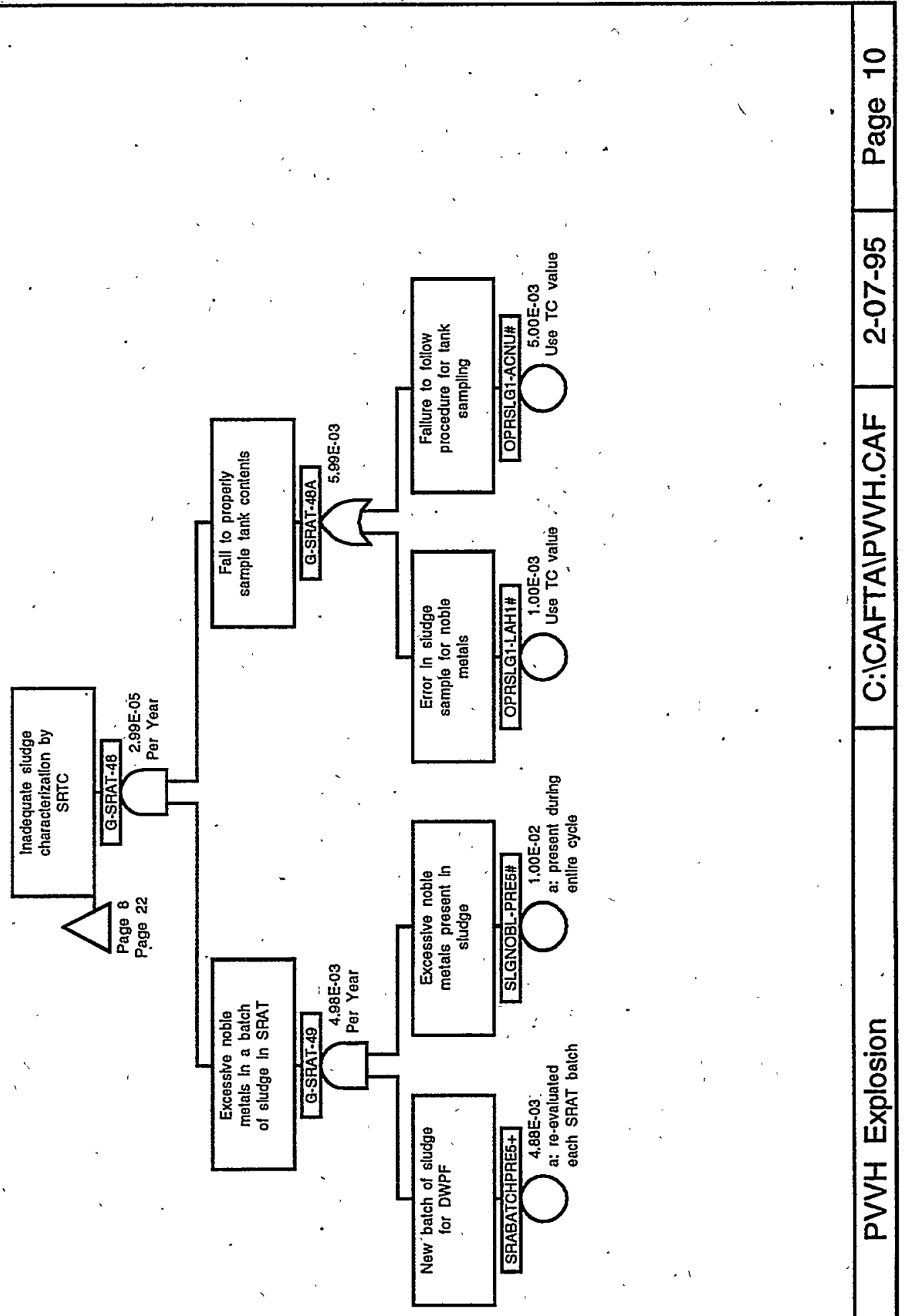




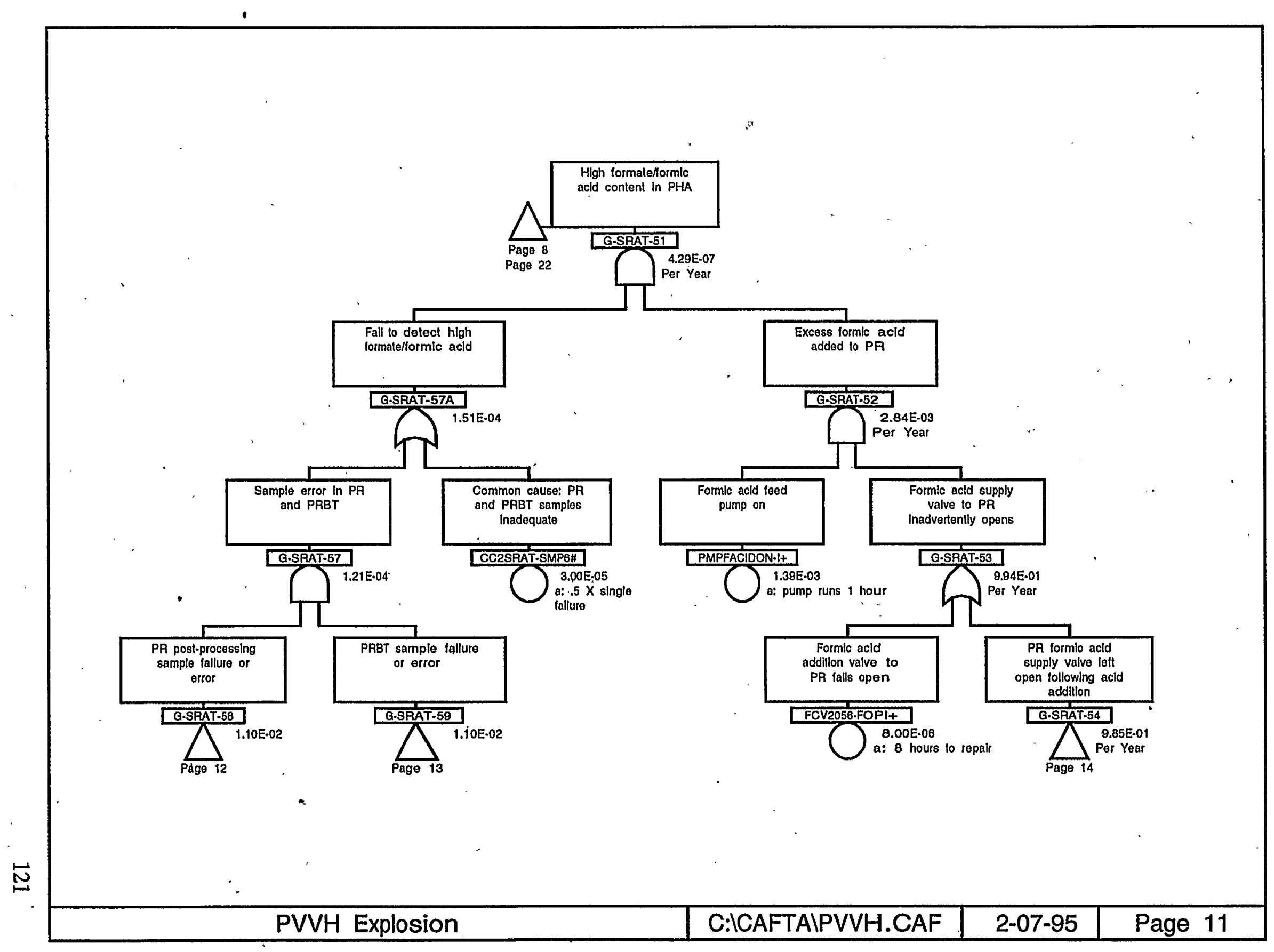




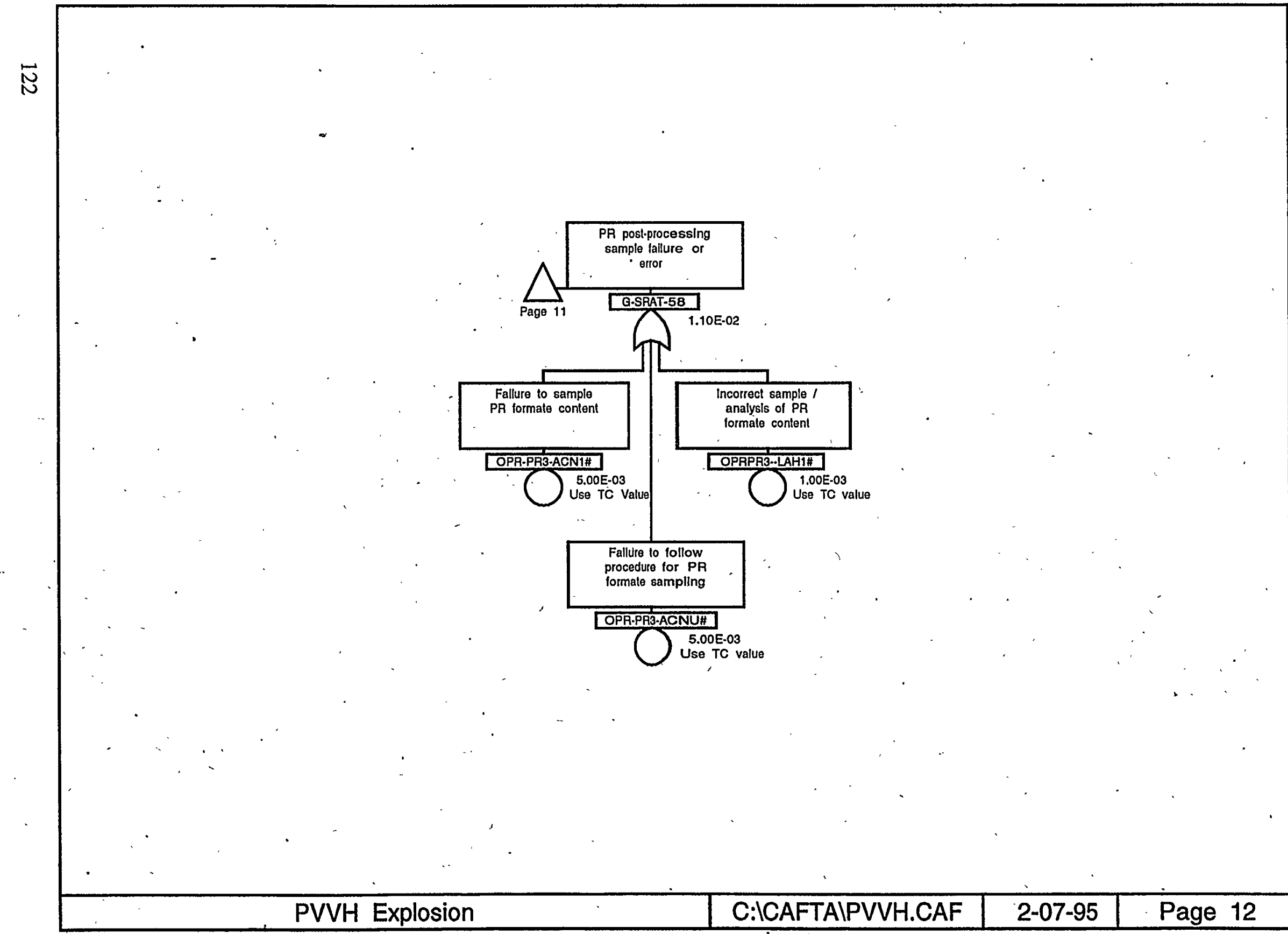




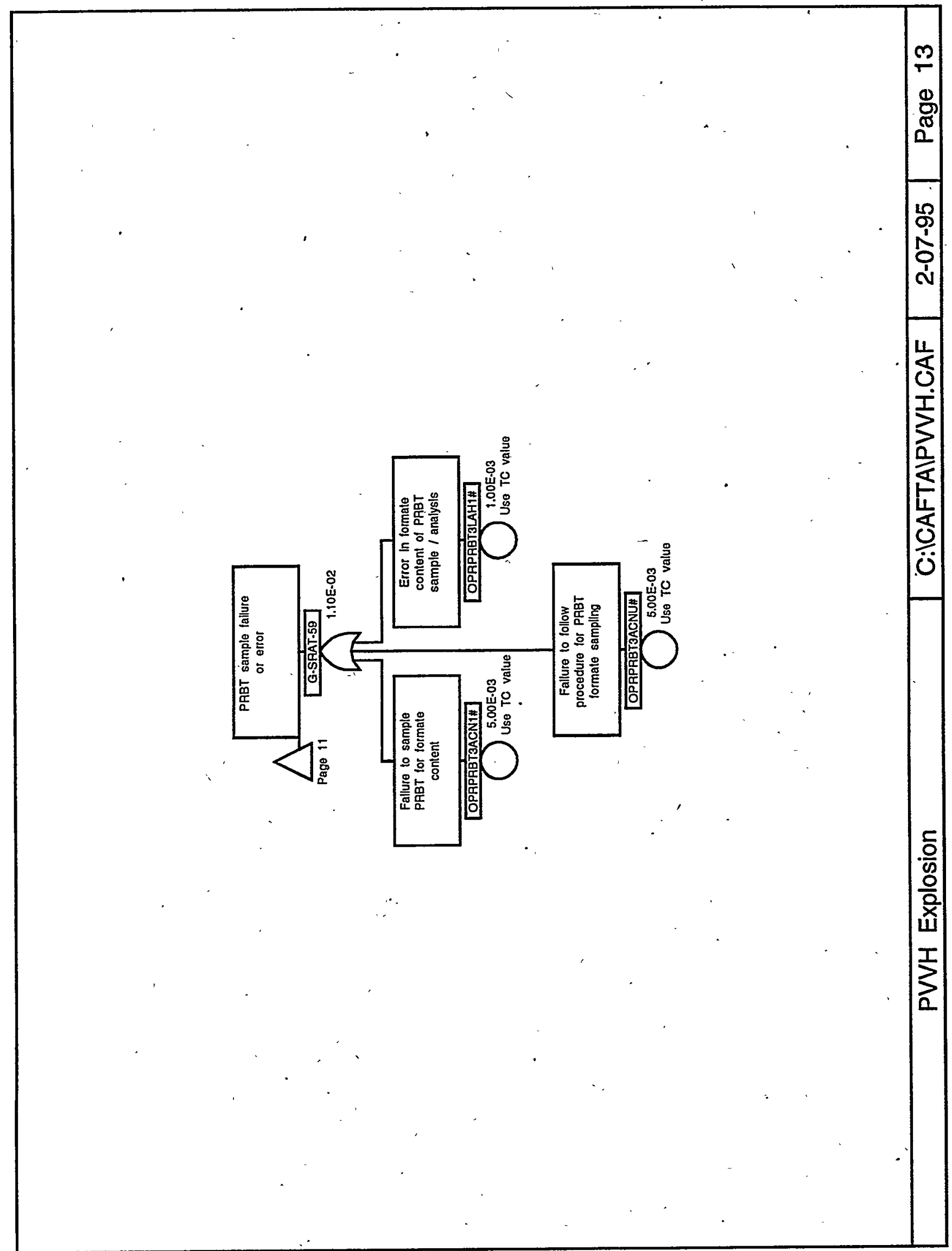


X-ESR-S-00001, REV. 0

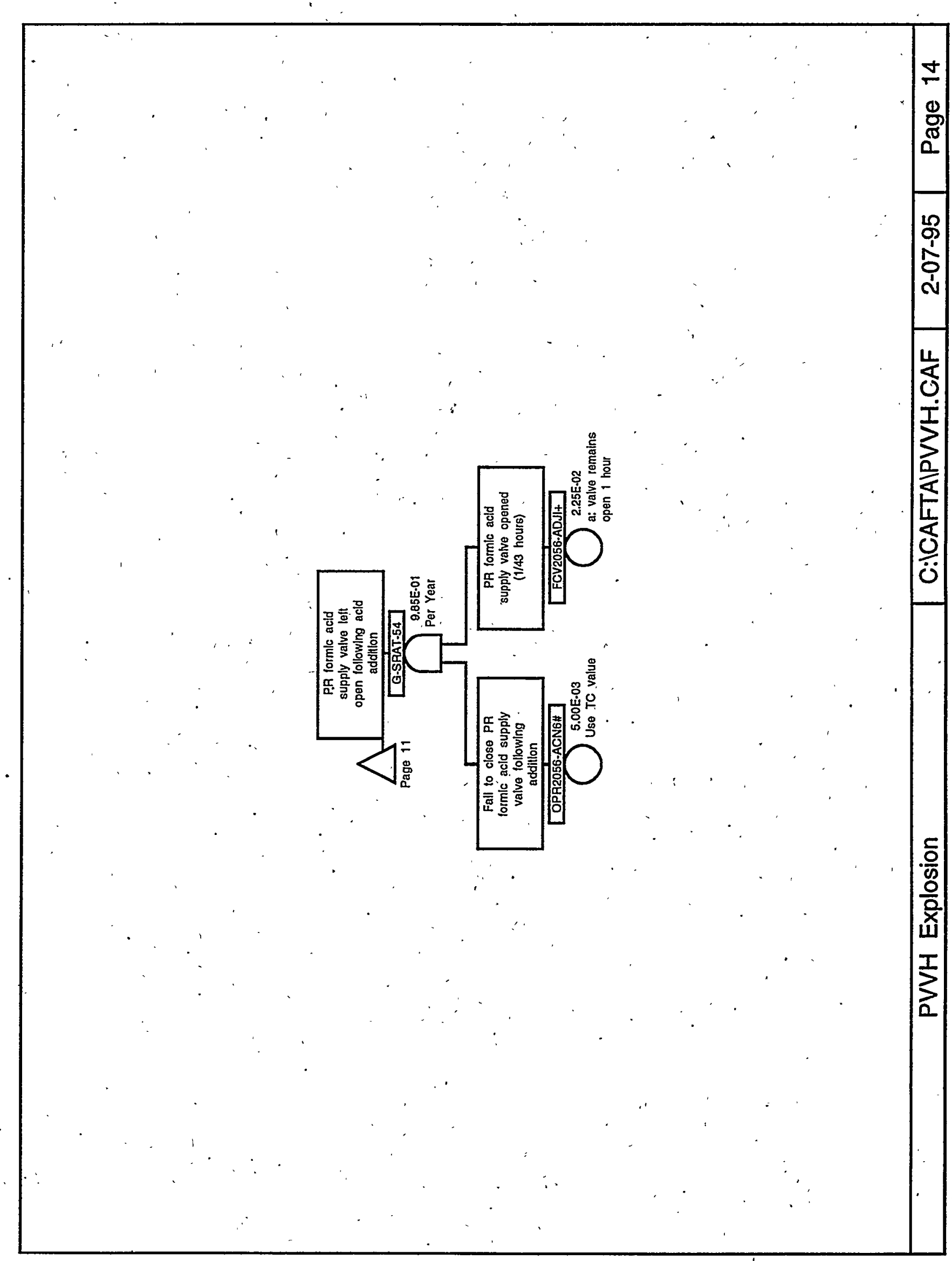




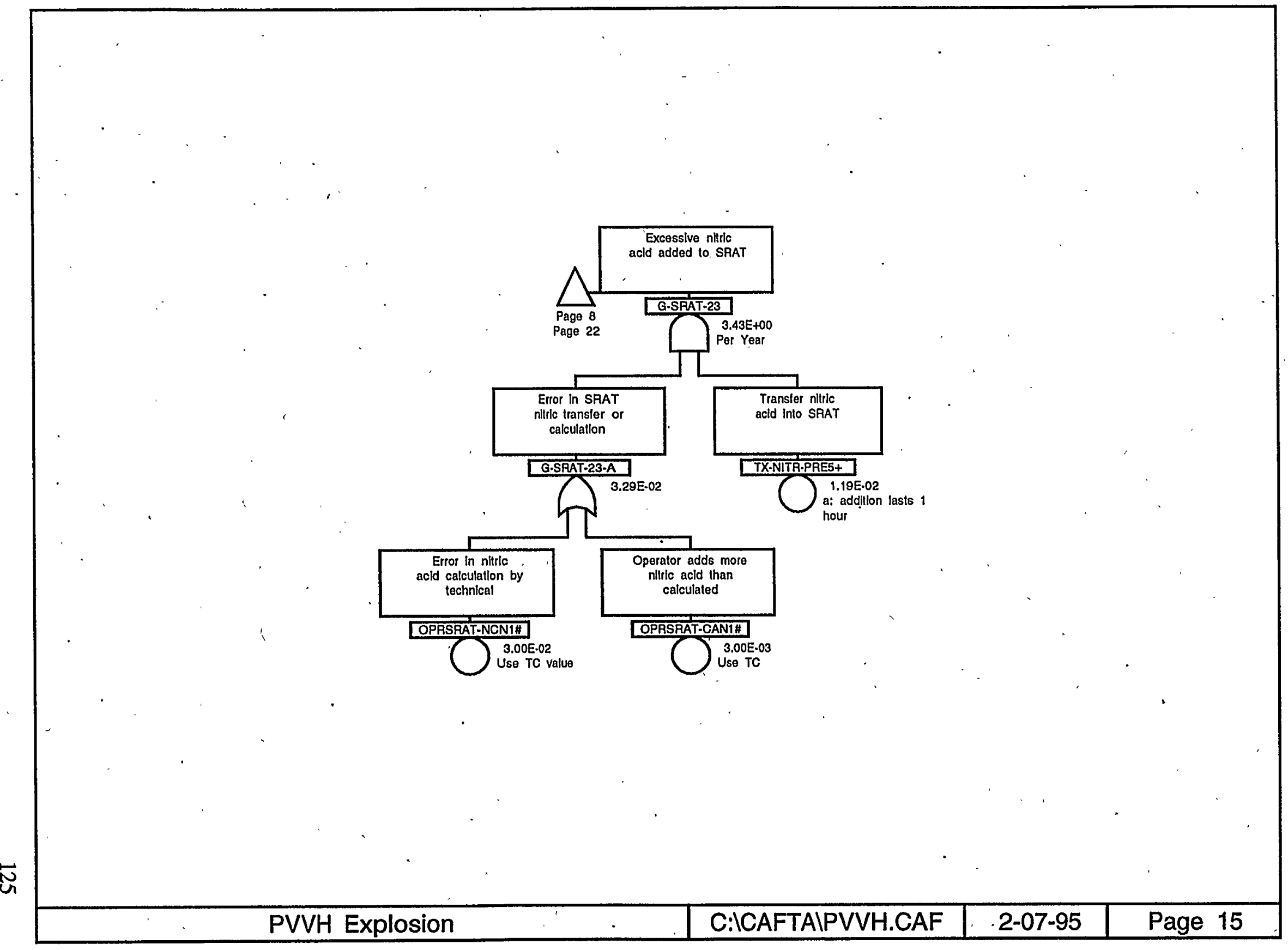




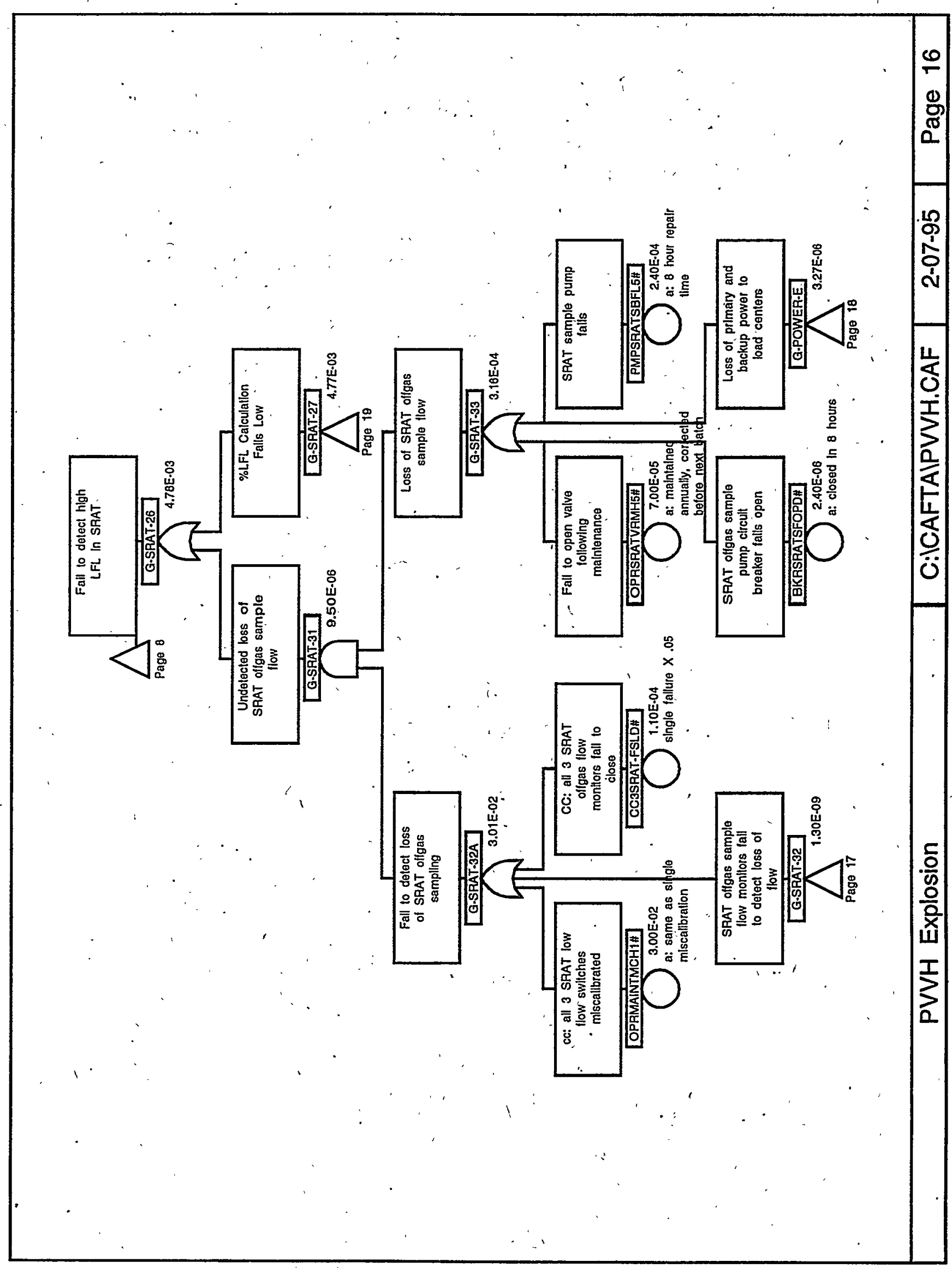




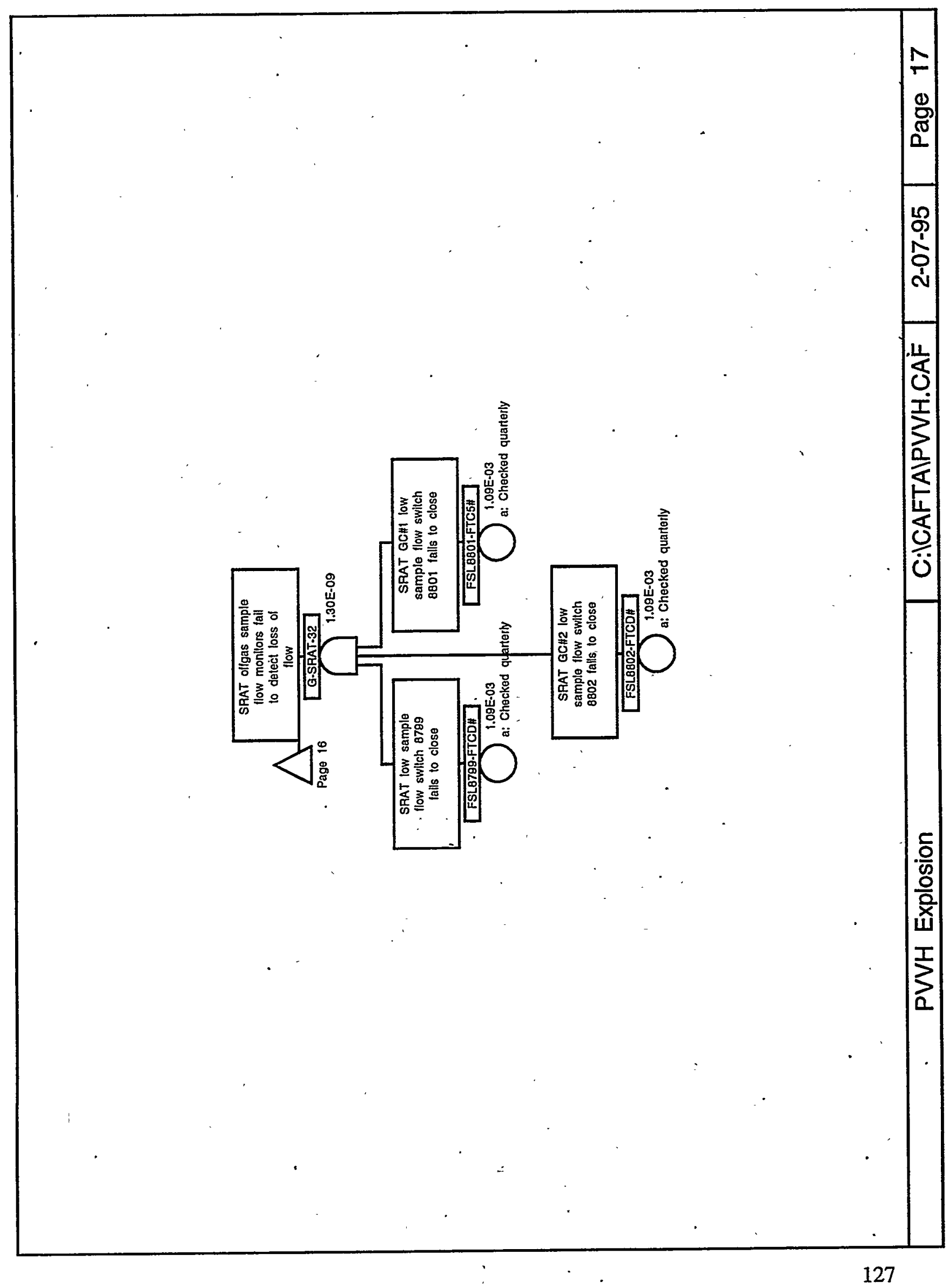


- X-ESR-S-00001, REV.'0 '

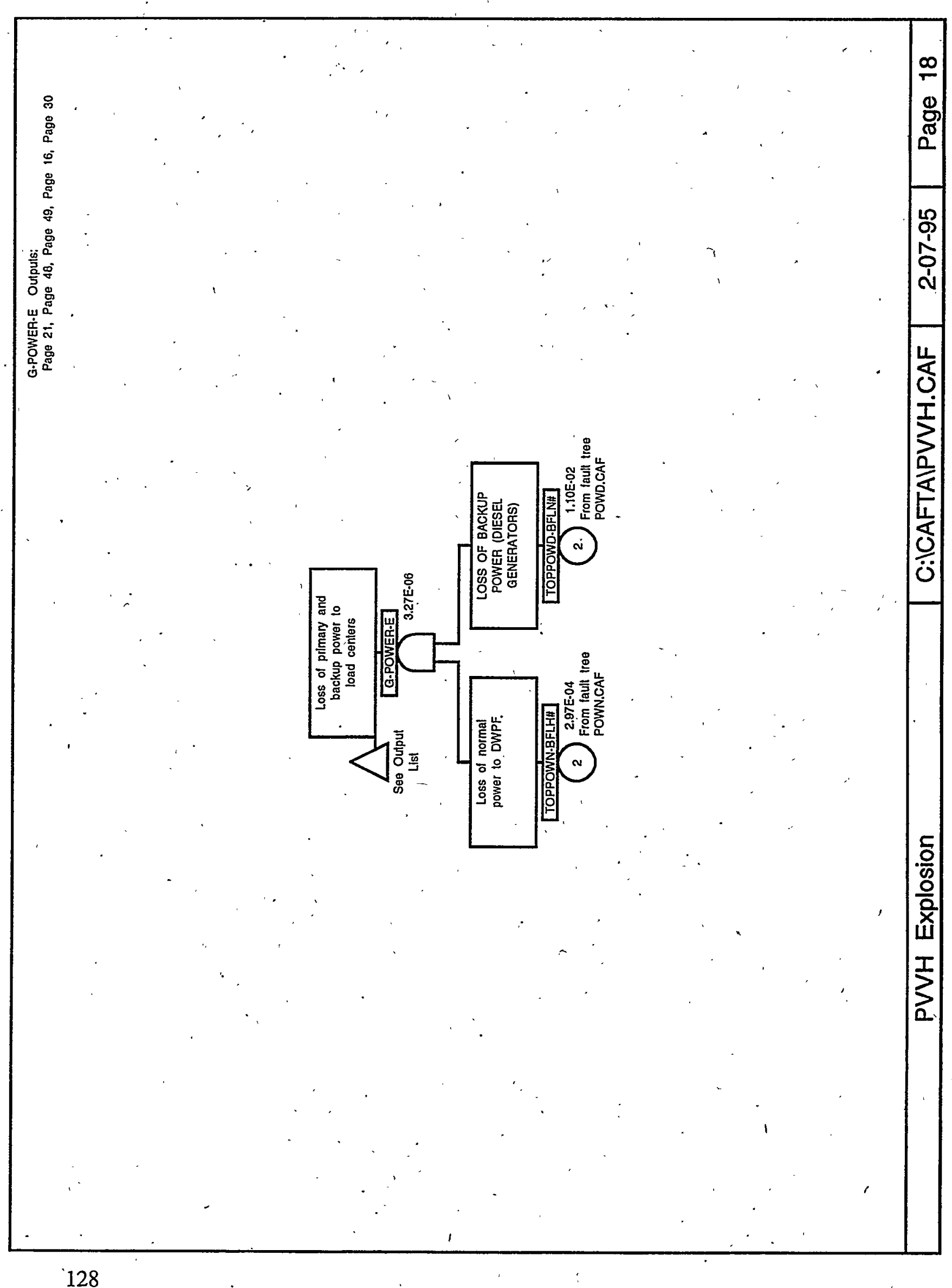




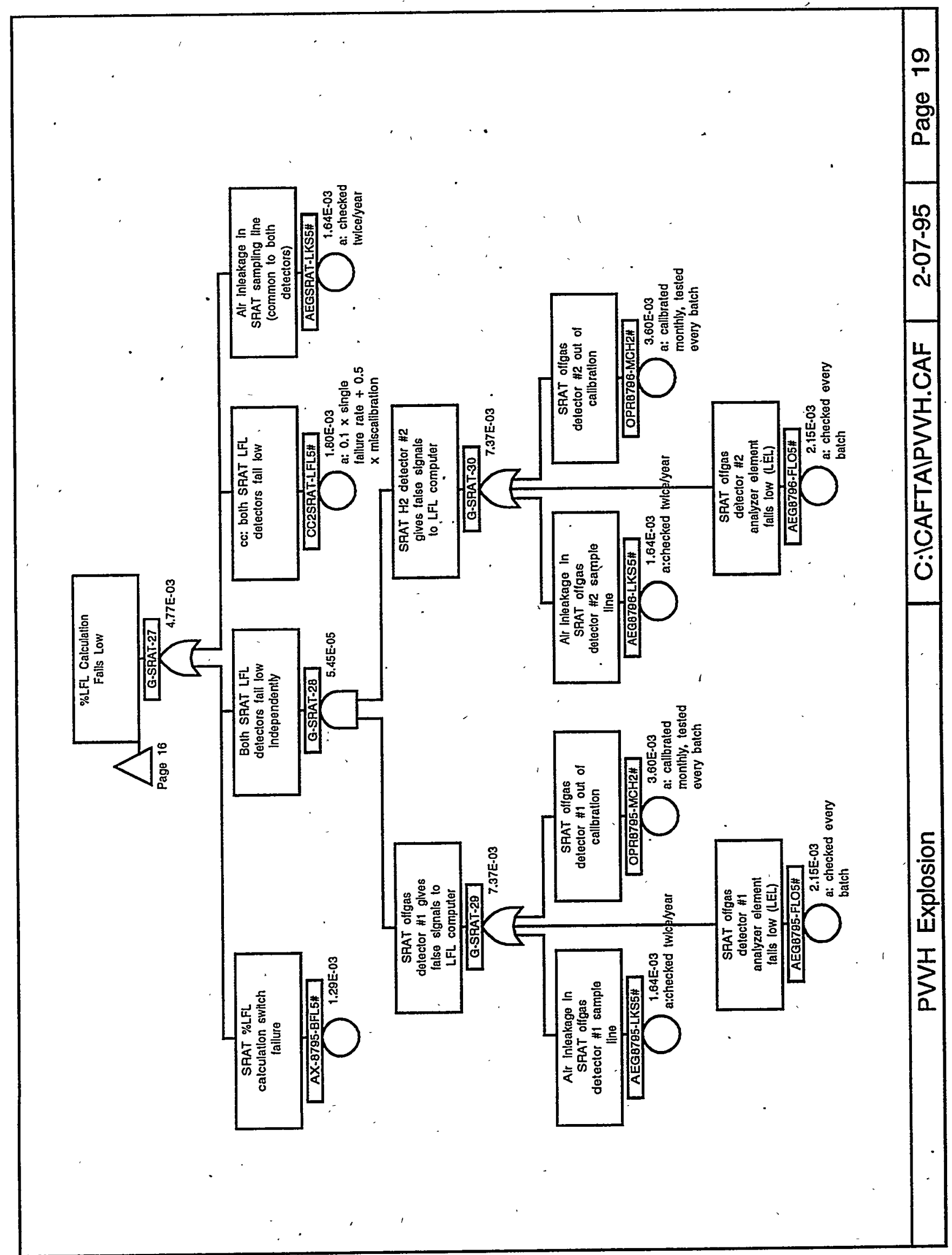


X-ESR-S-00001,REV. 0.

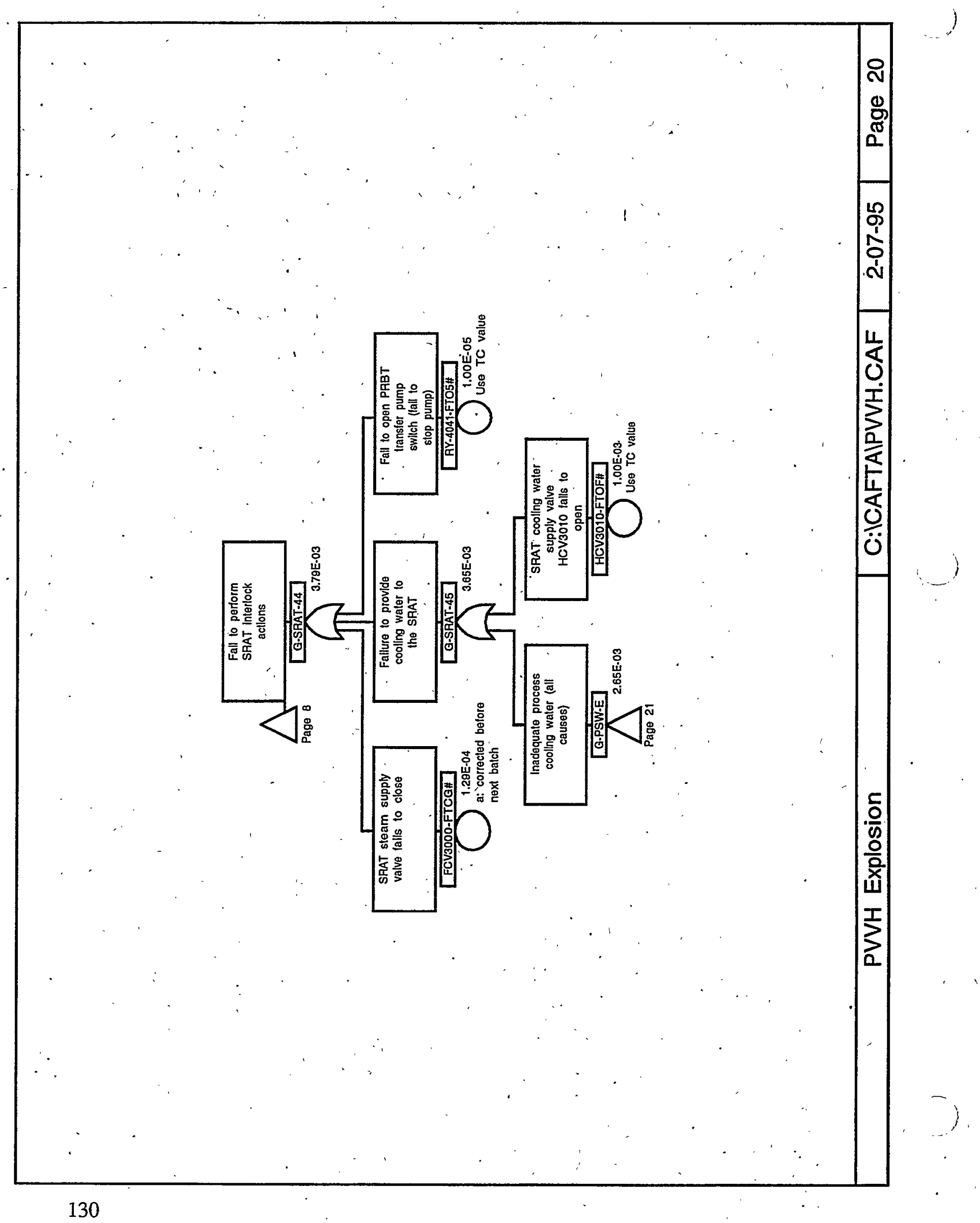




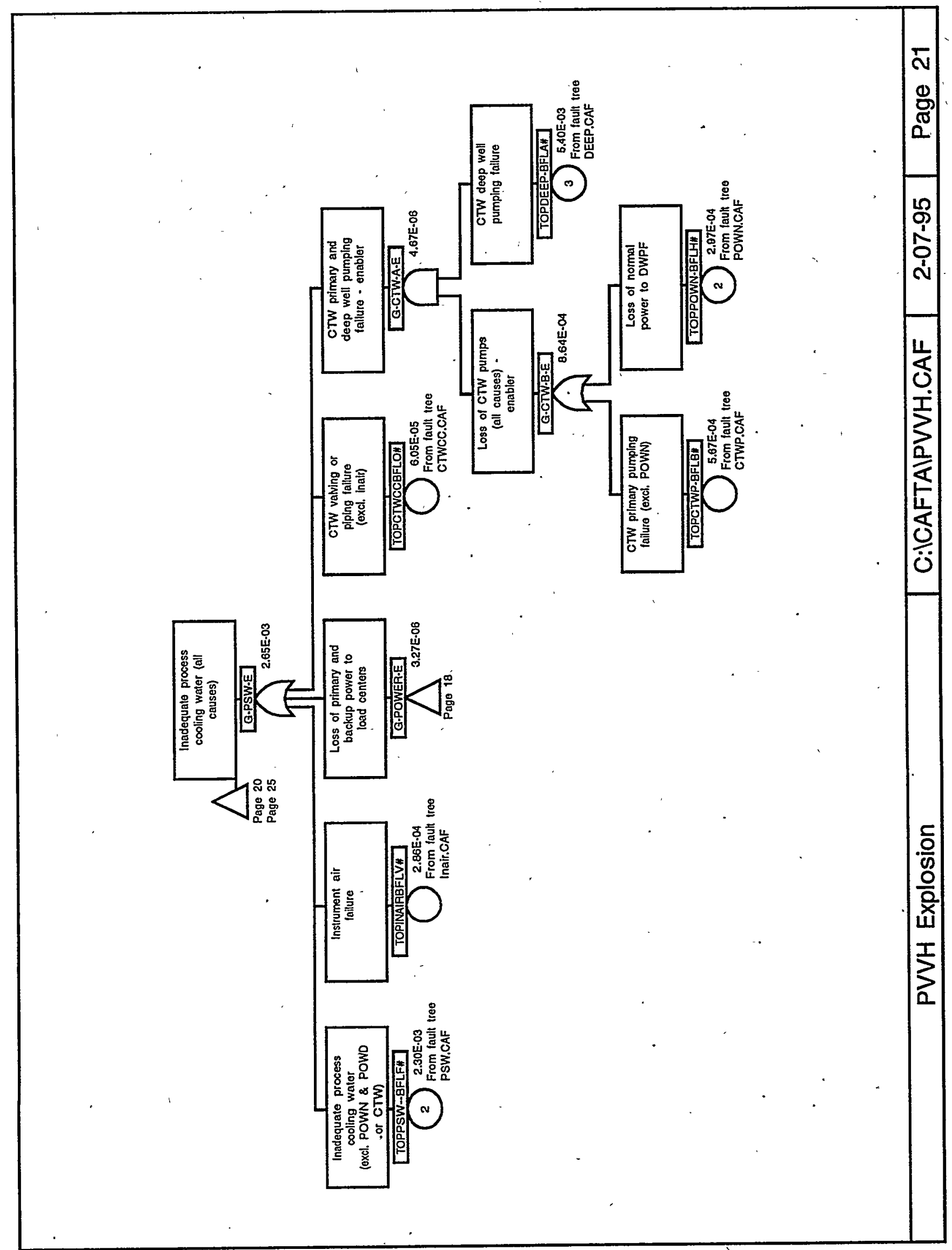


X-ESR-S-00001, REV. 0

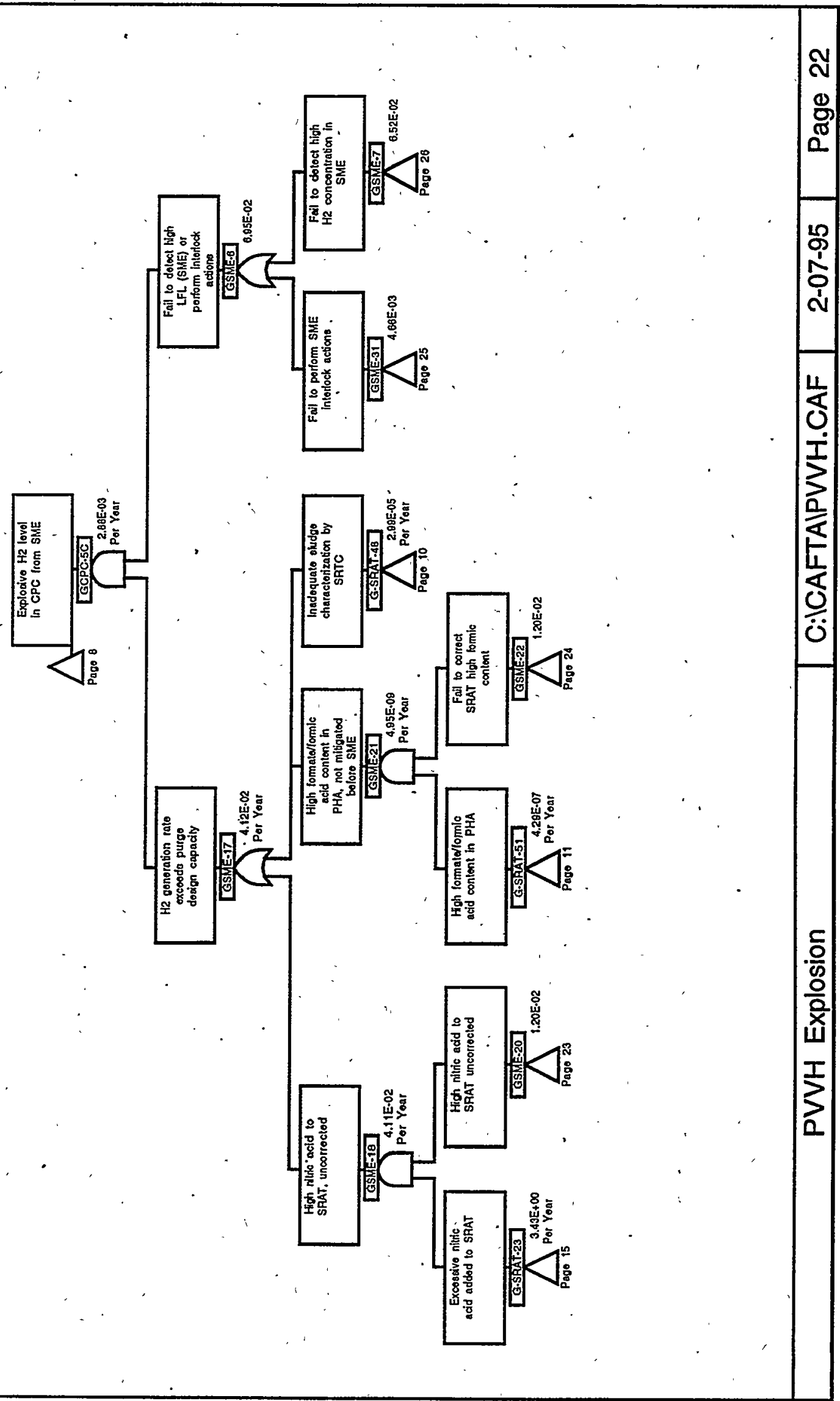




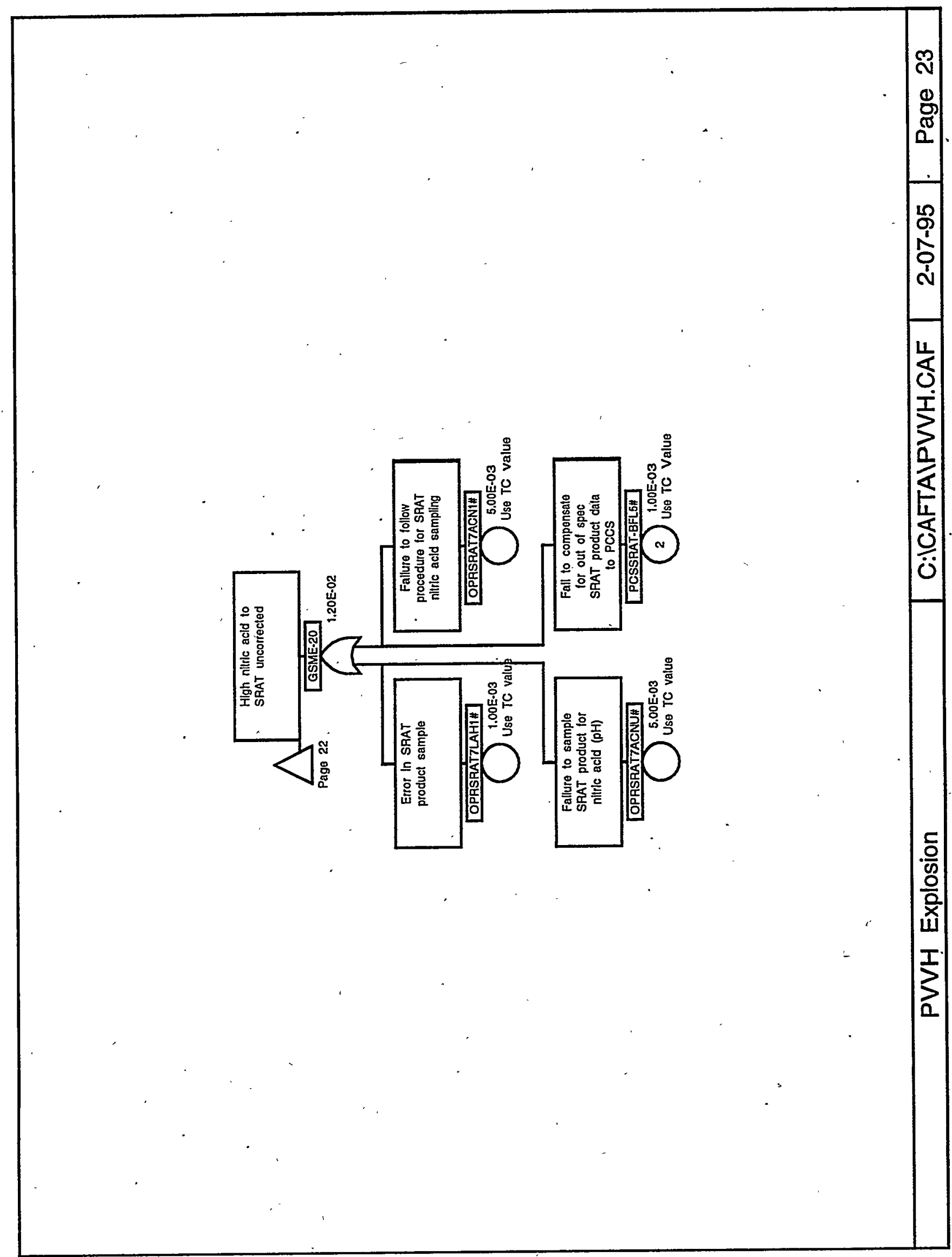


X-ESR-S-00001, REV. 0

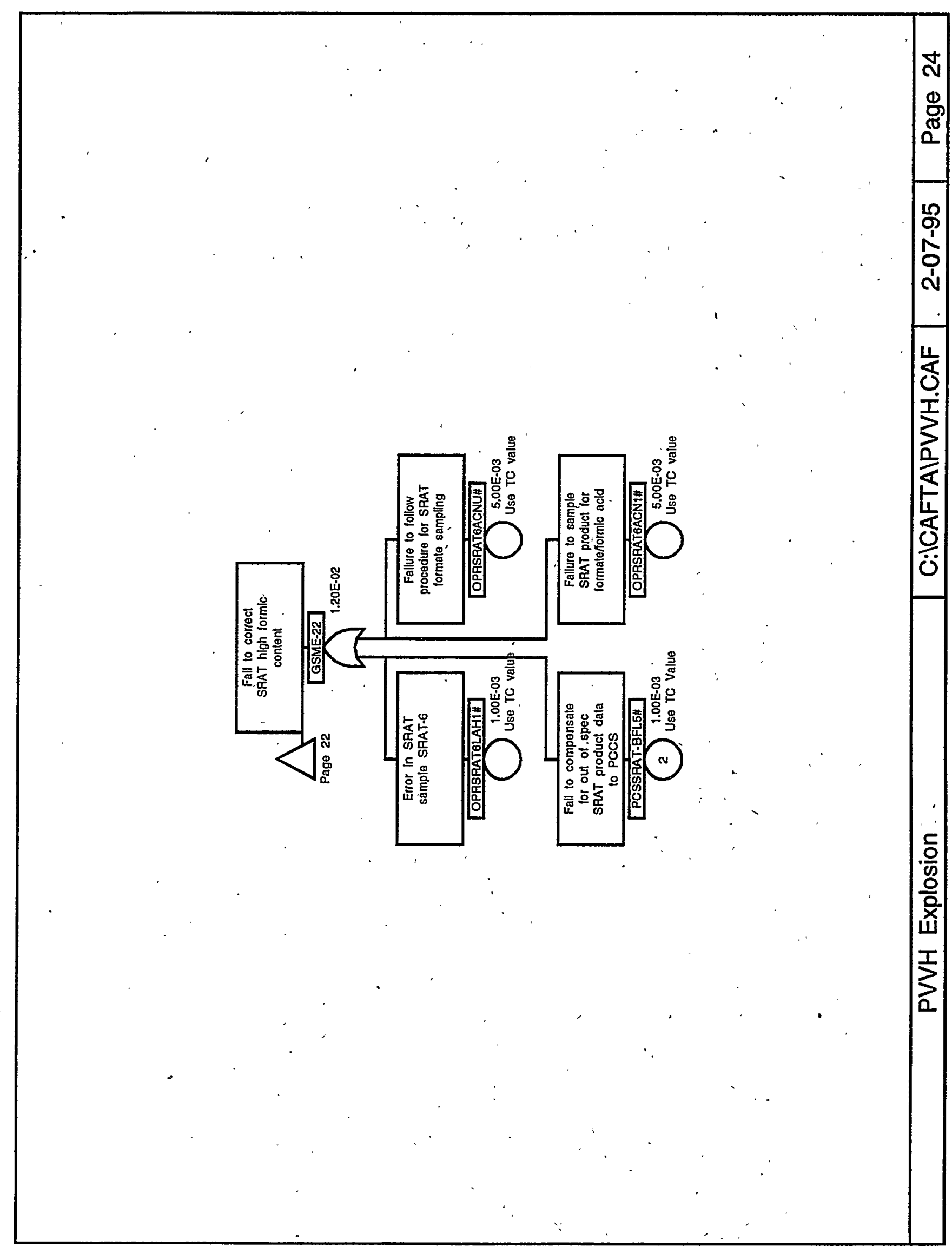




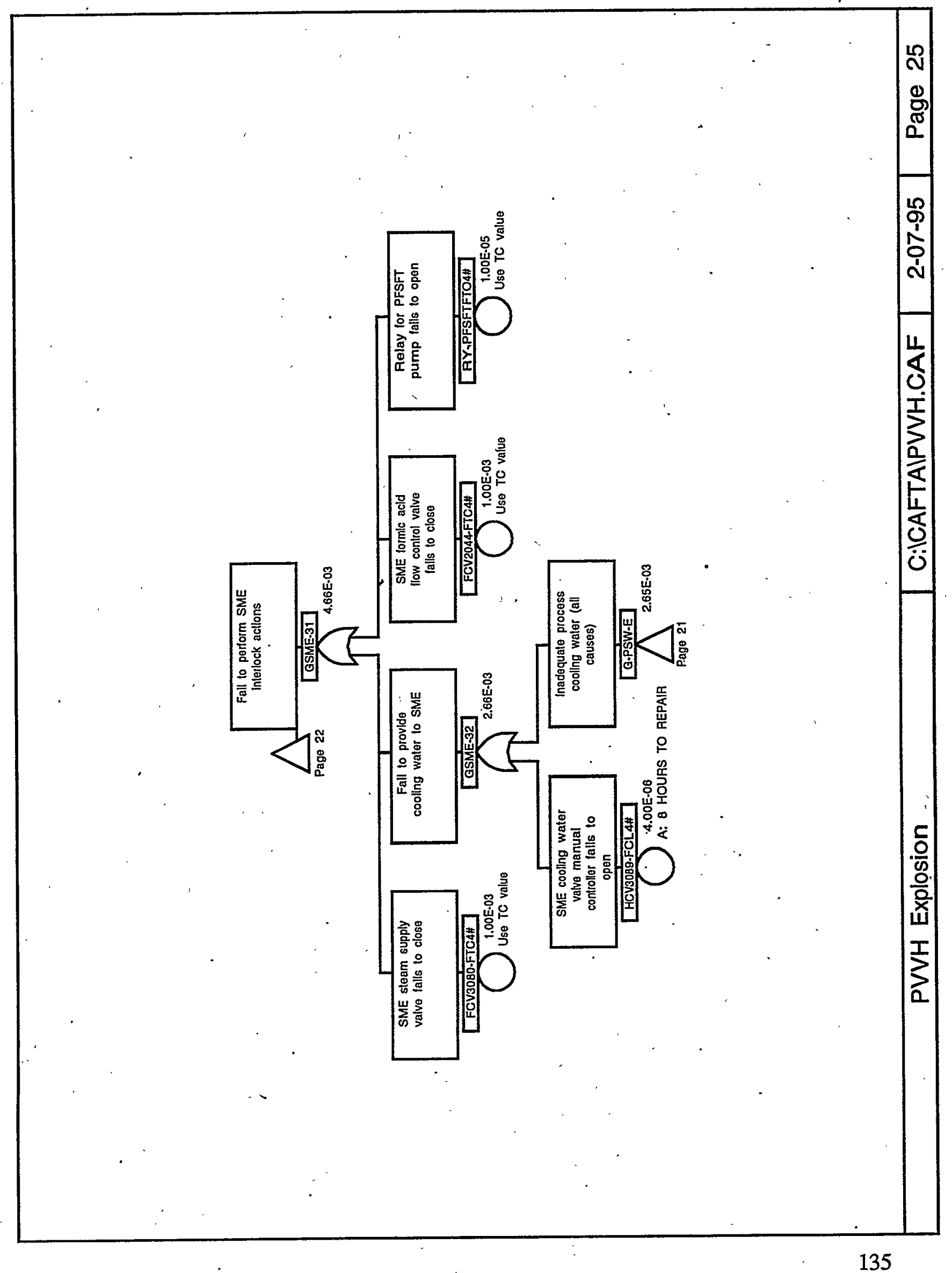




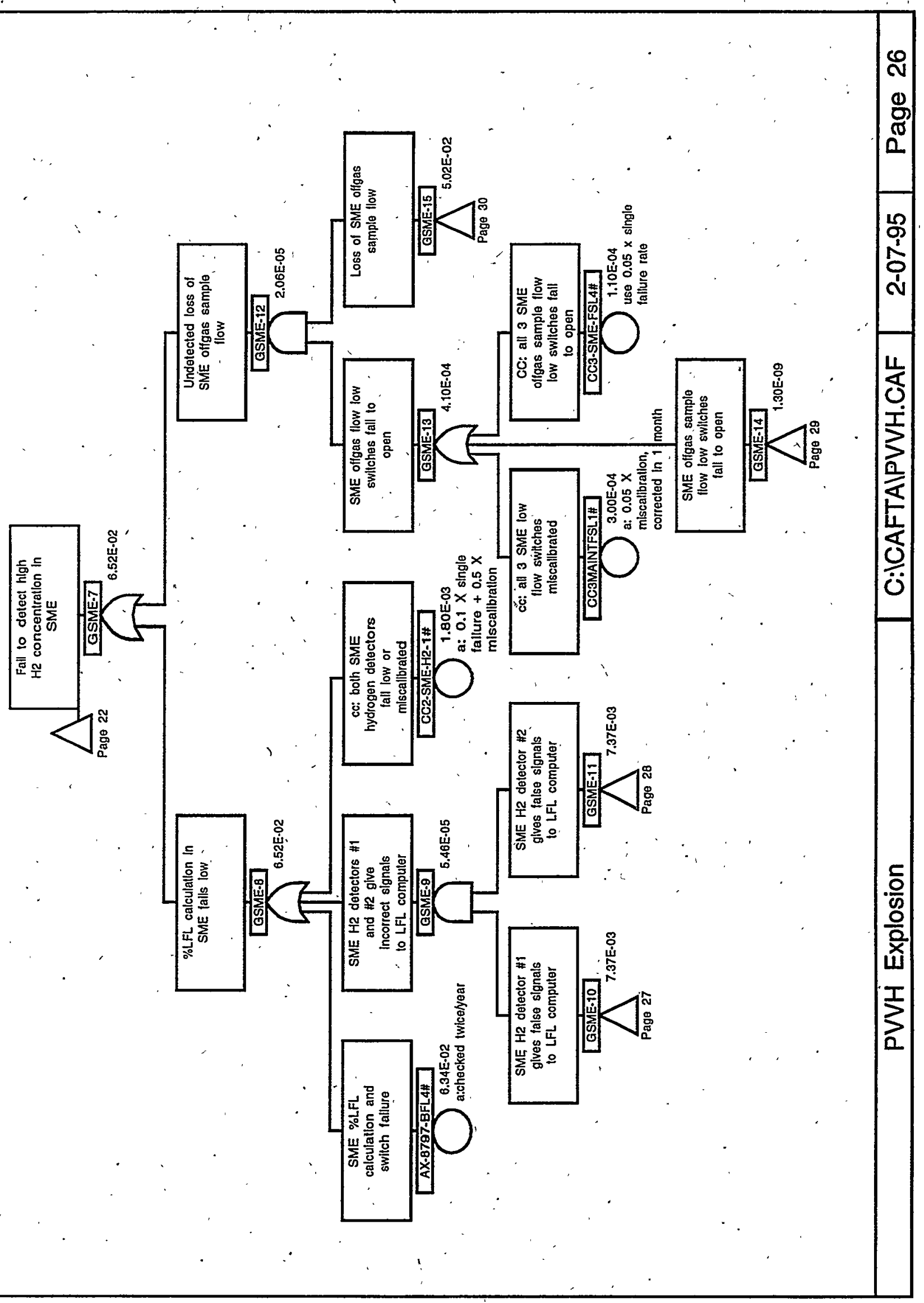




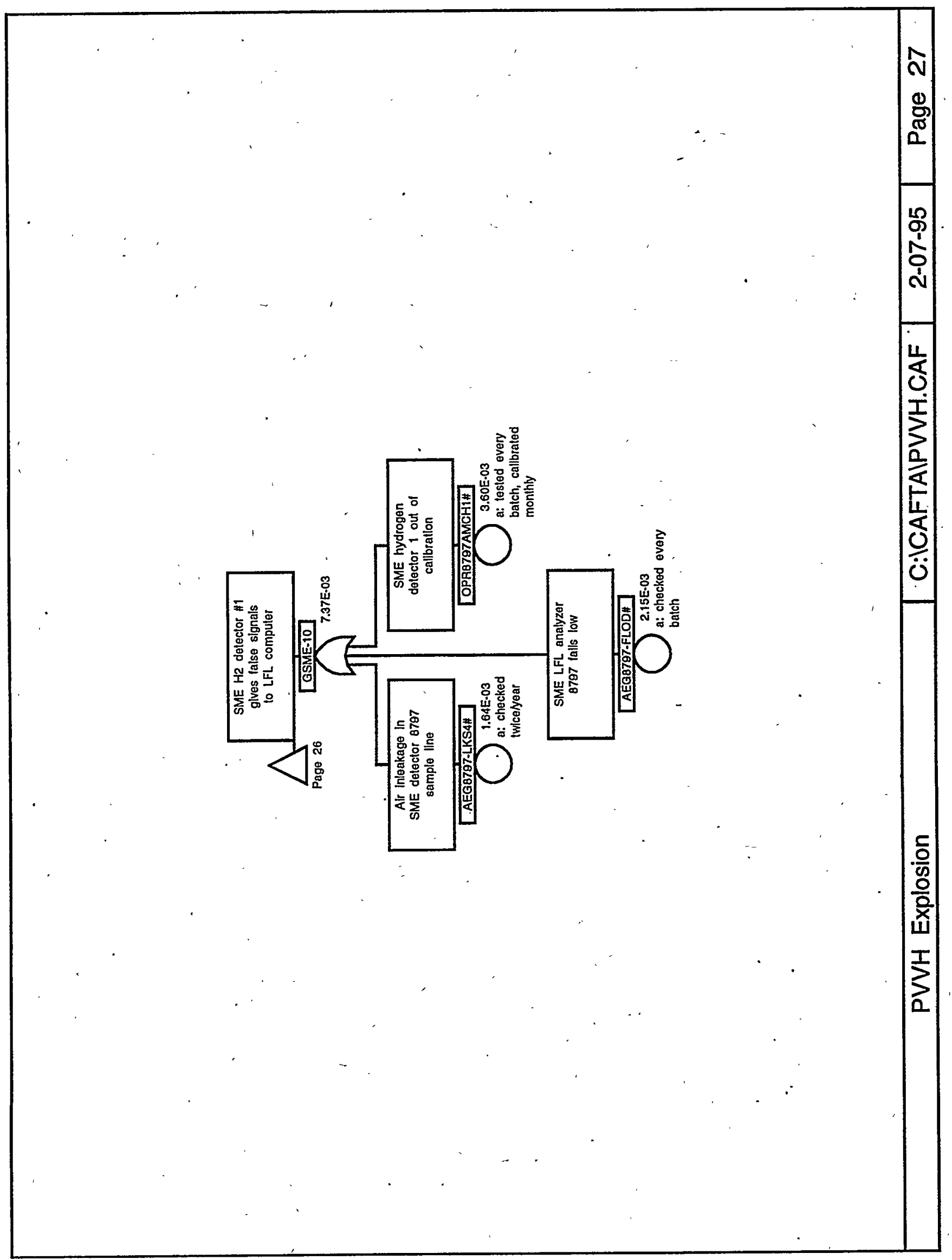




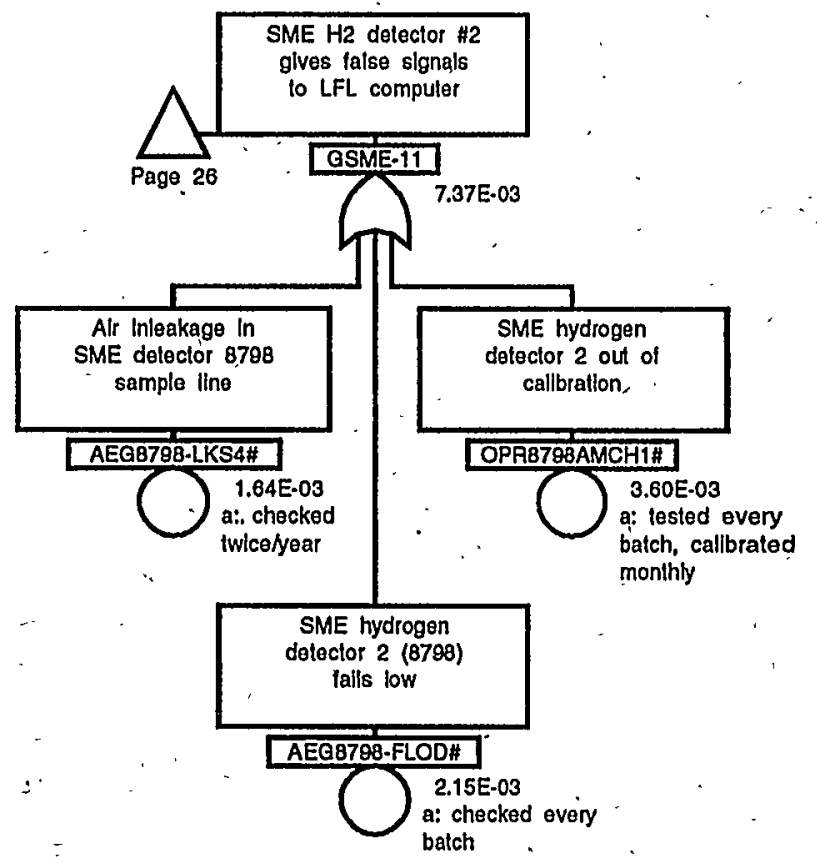




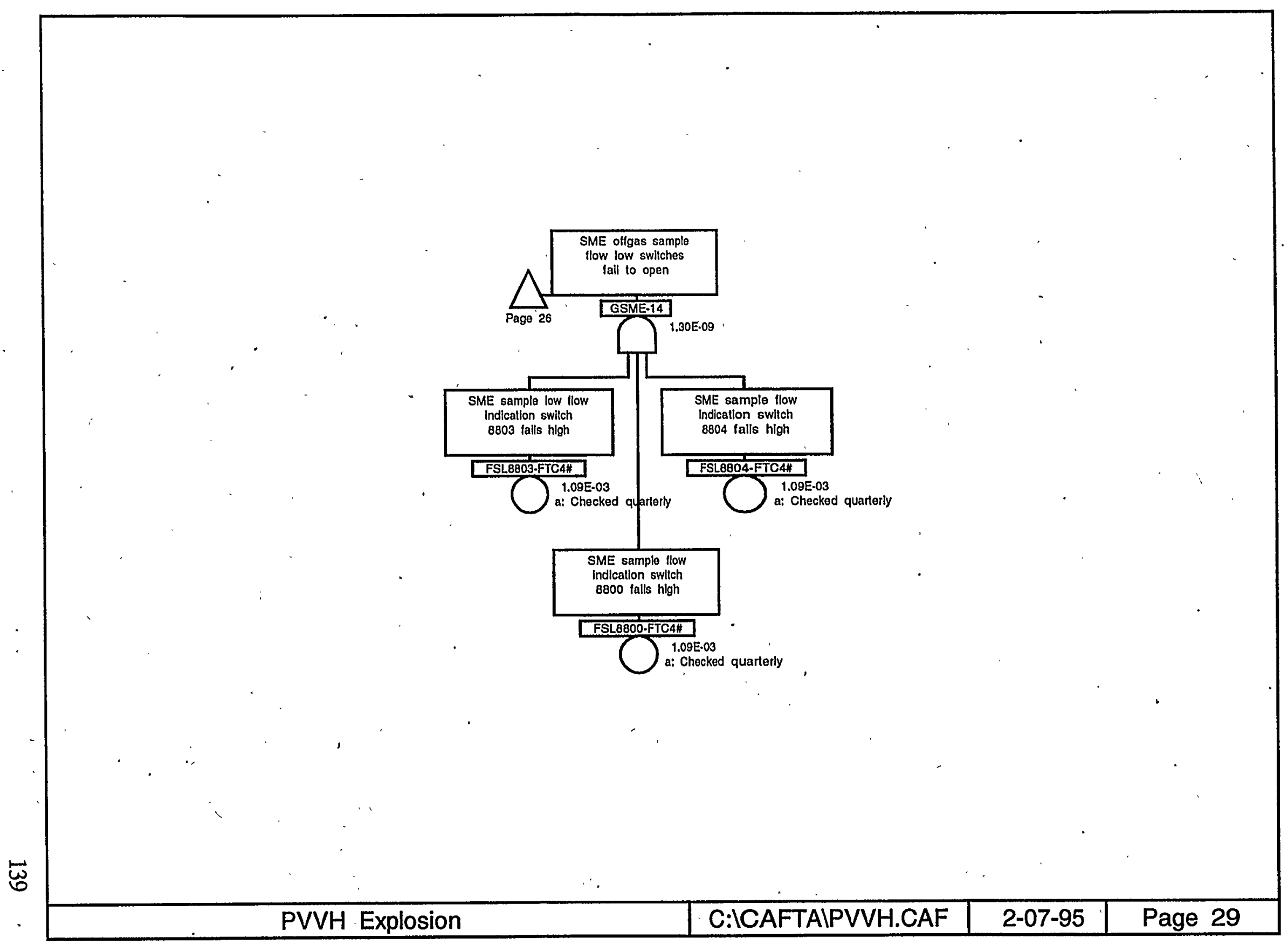


X-ESR-S-00001, REV. 0

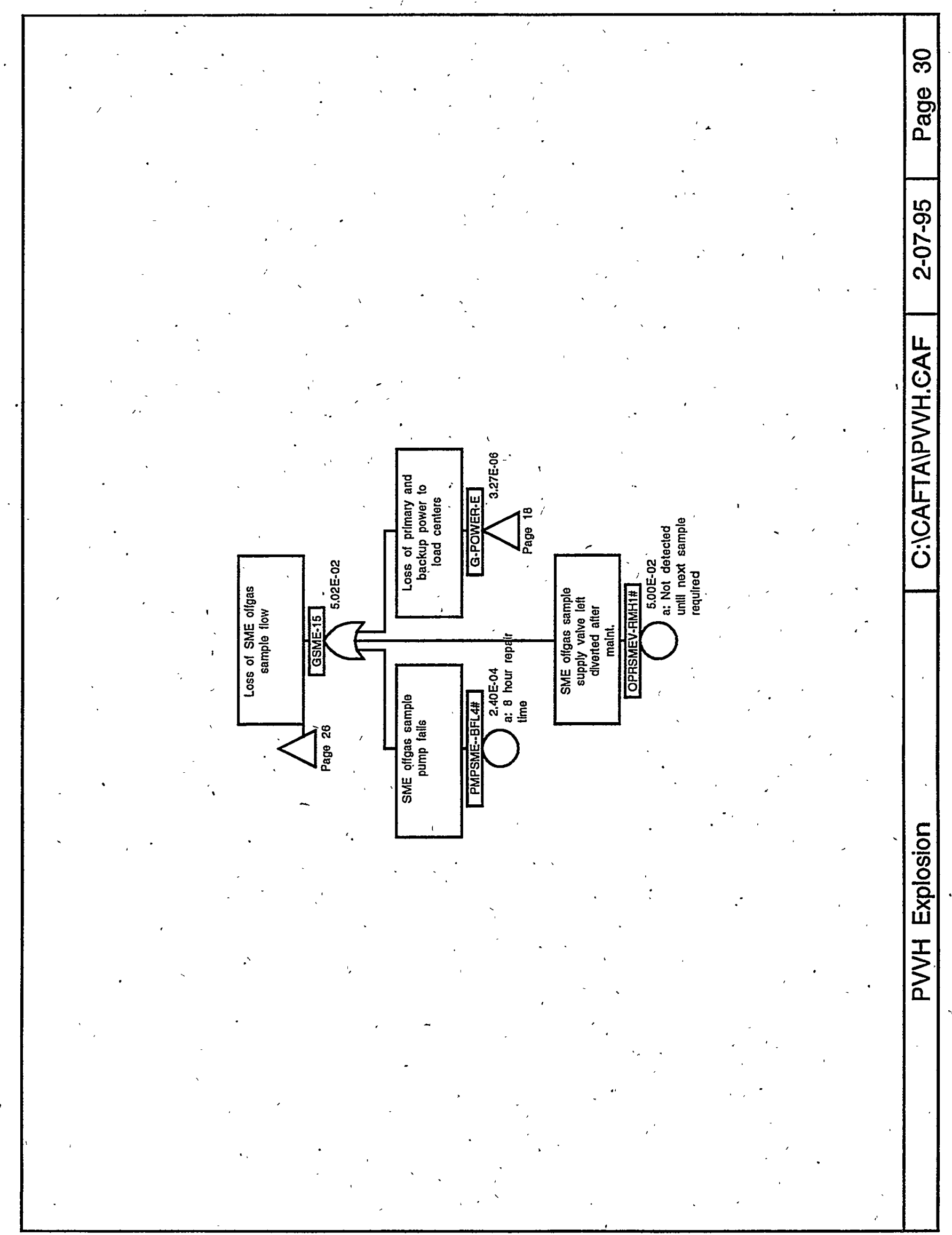




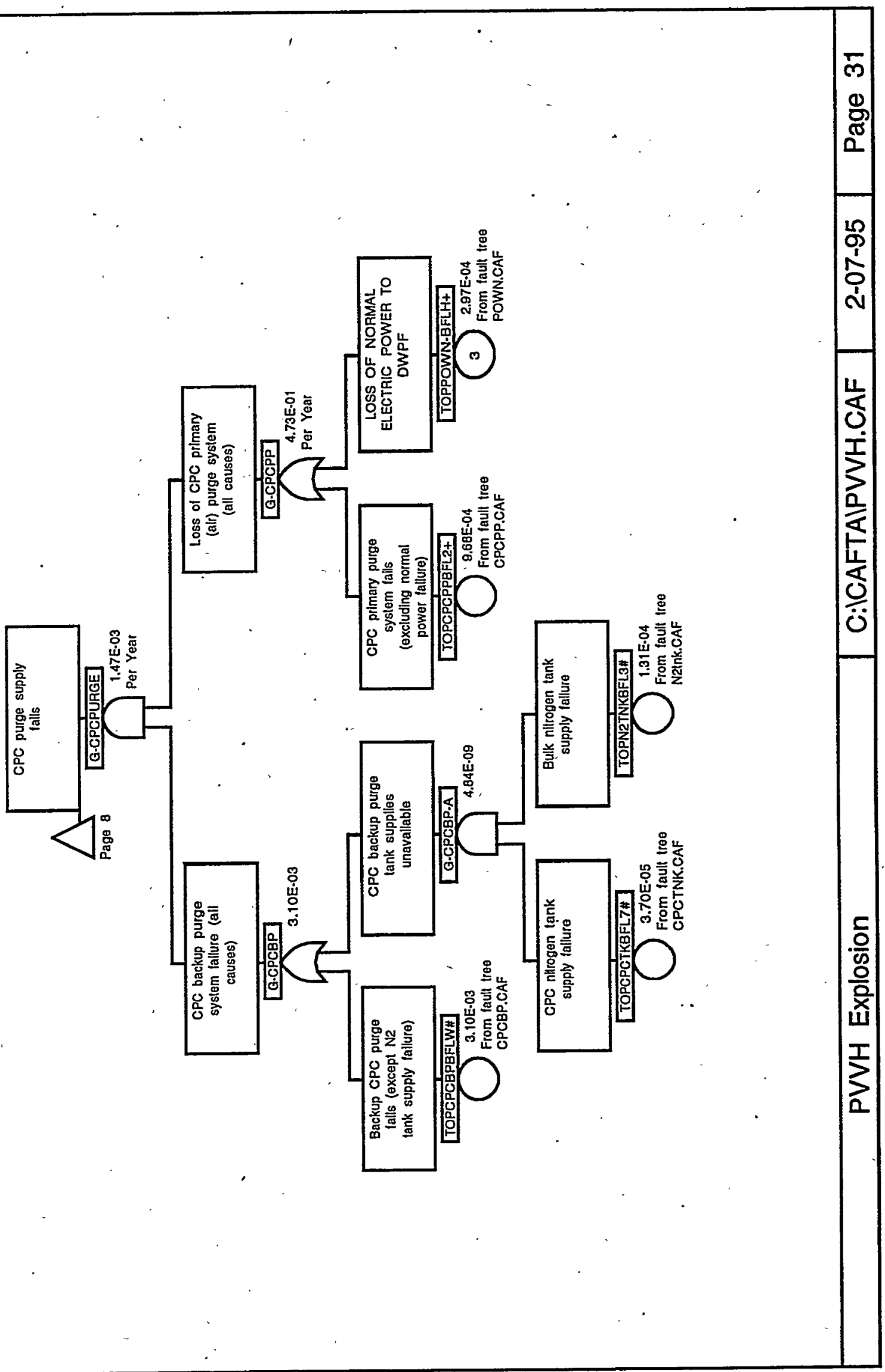




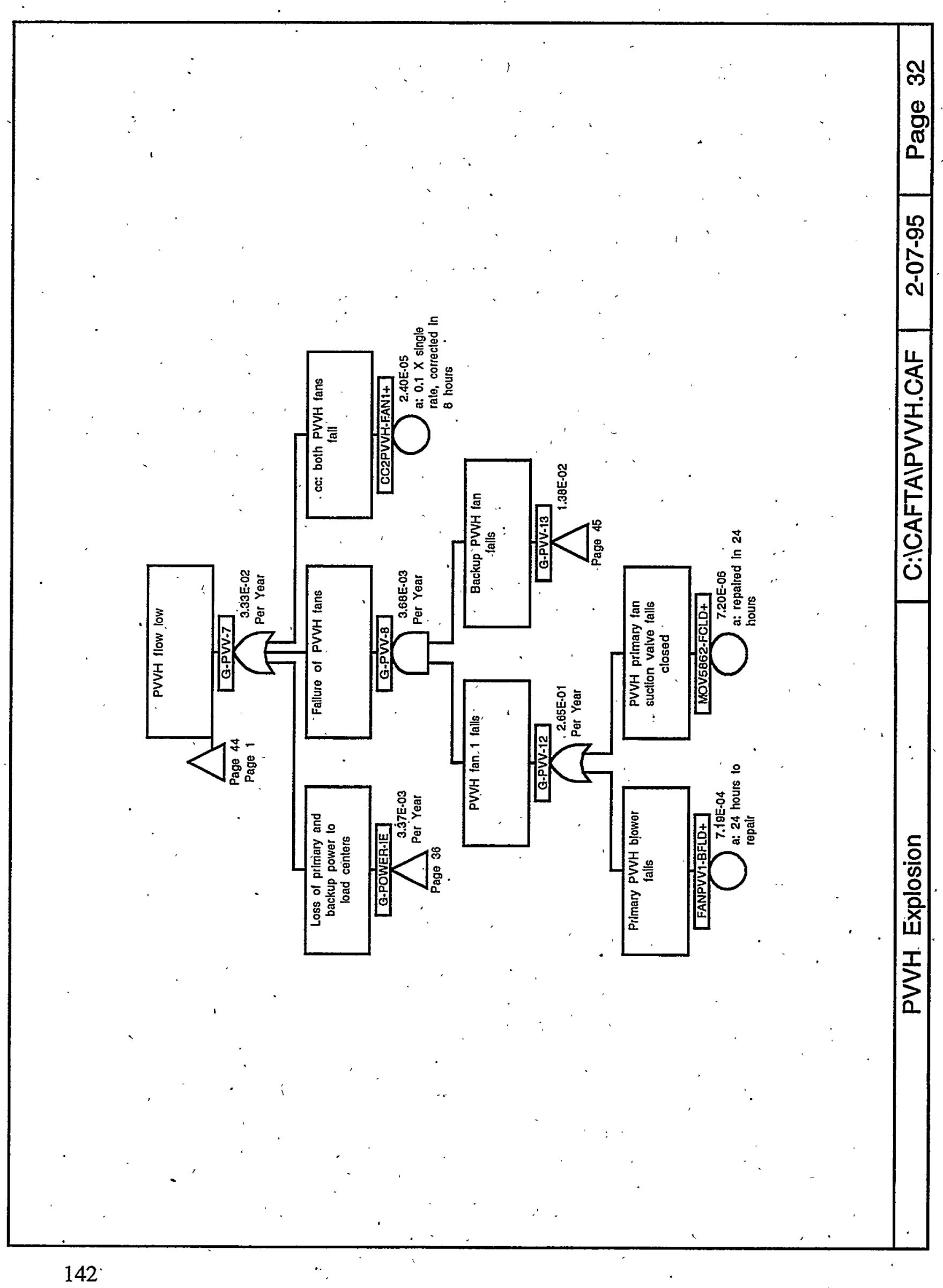




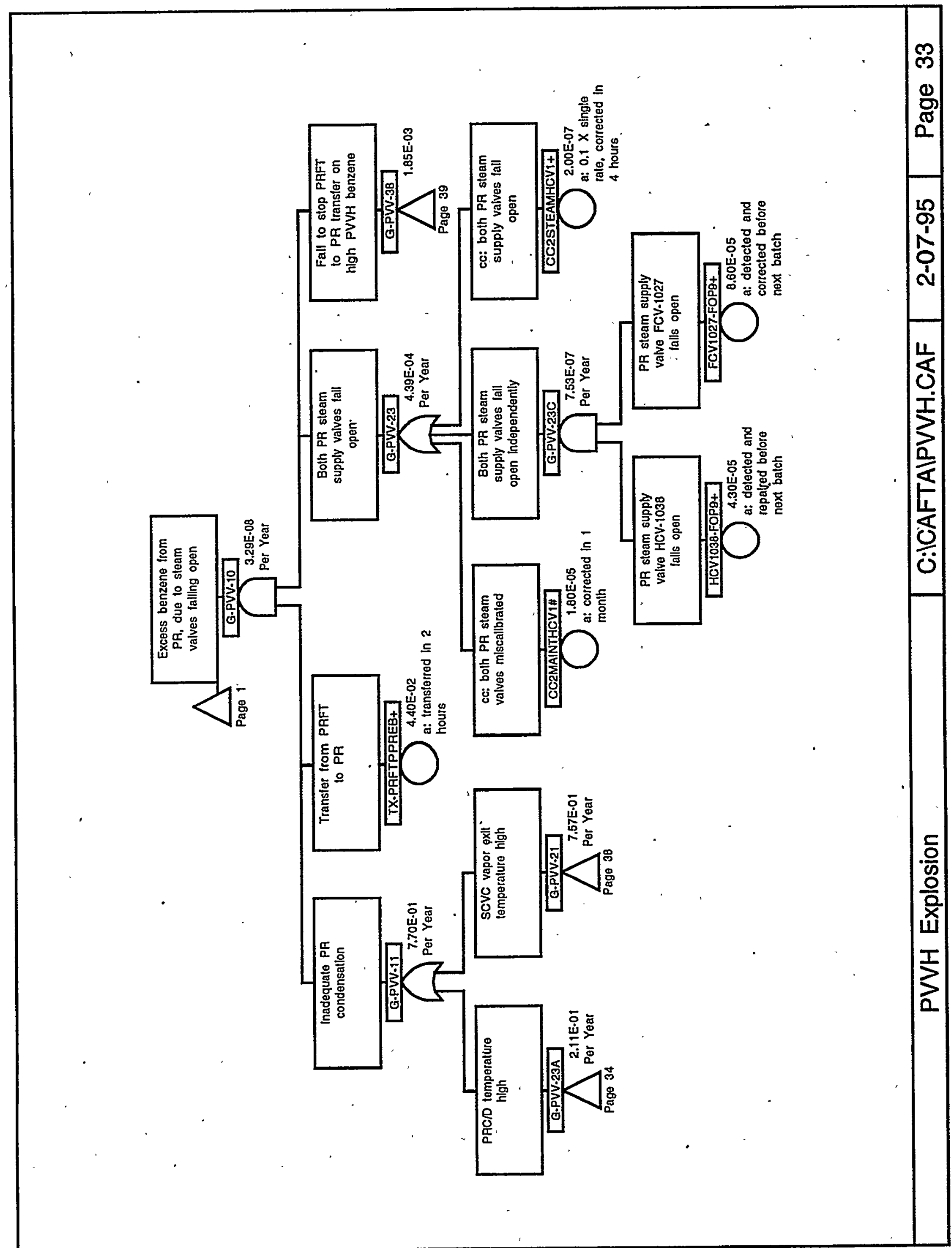


X-ESR-S-00001, REV. 0

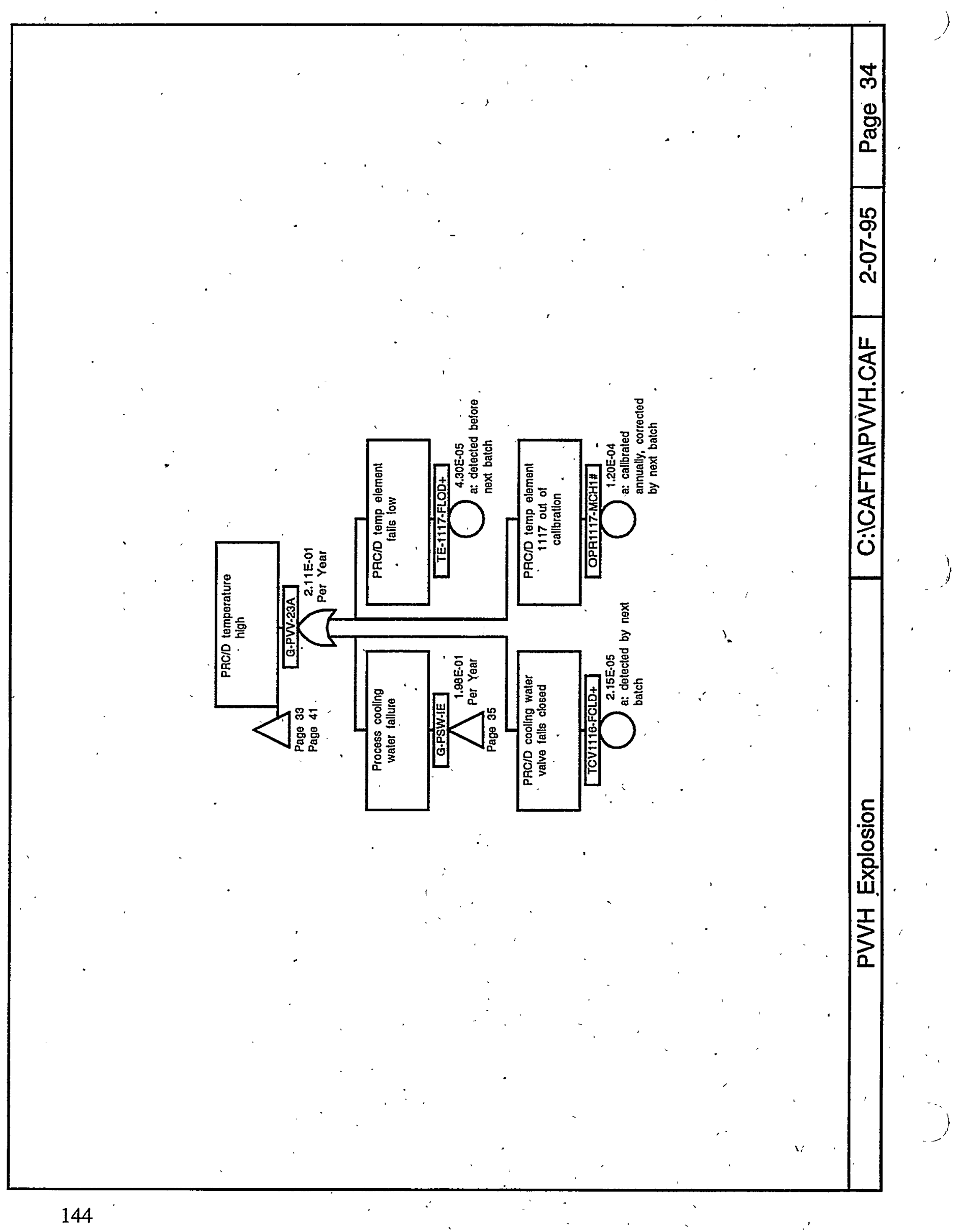




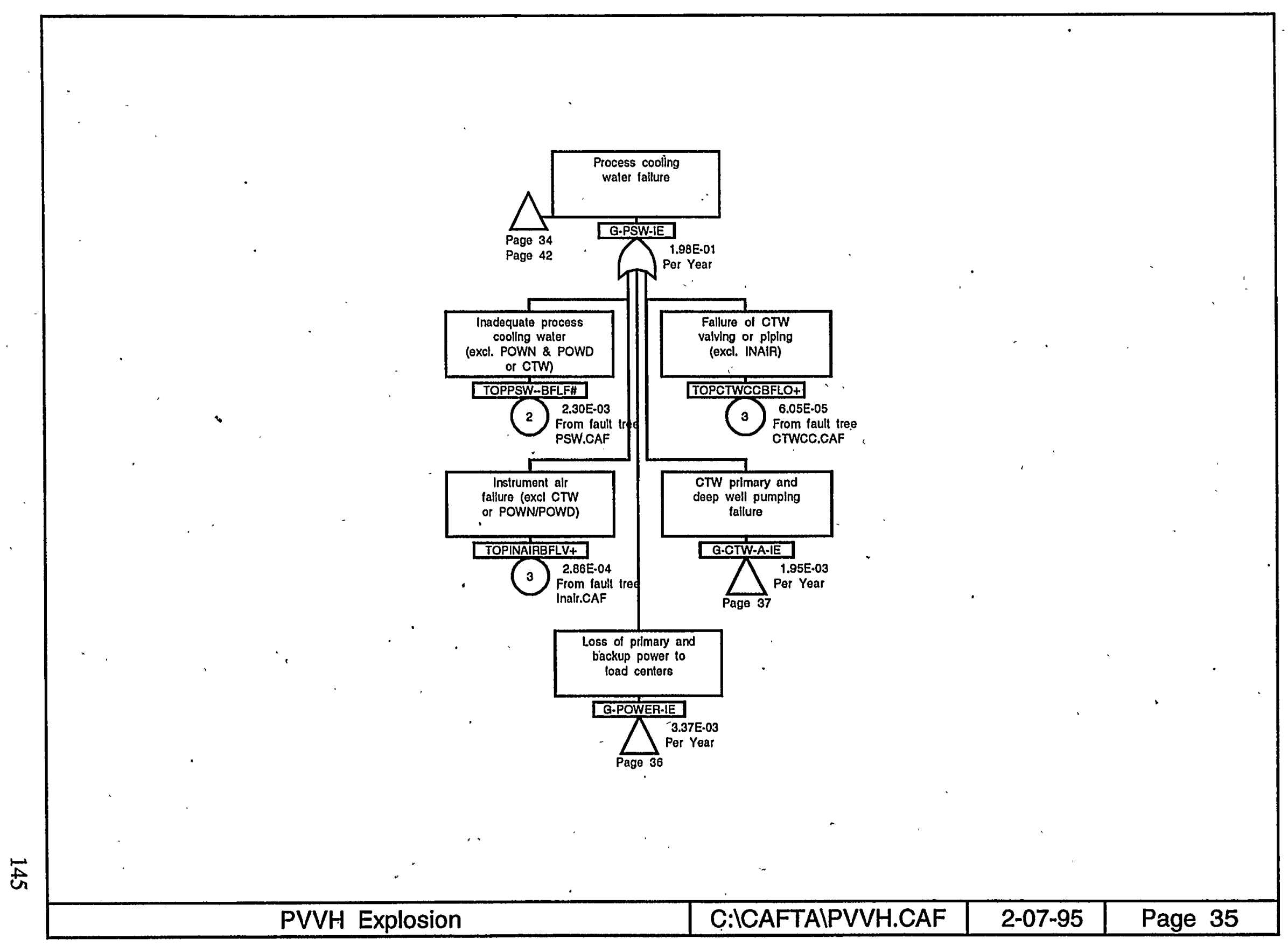


X-ESR-S-00001, REV. 0

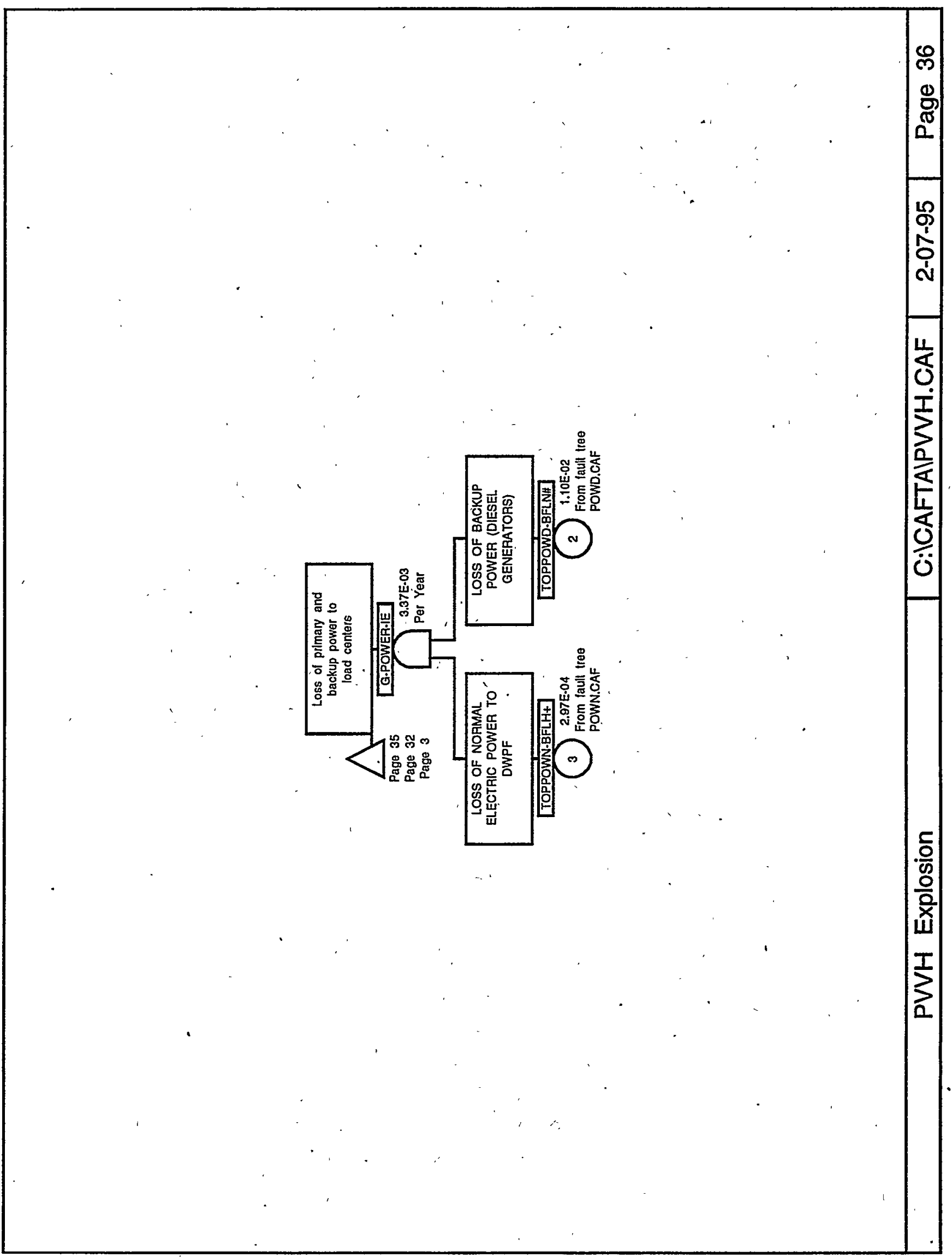




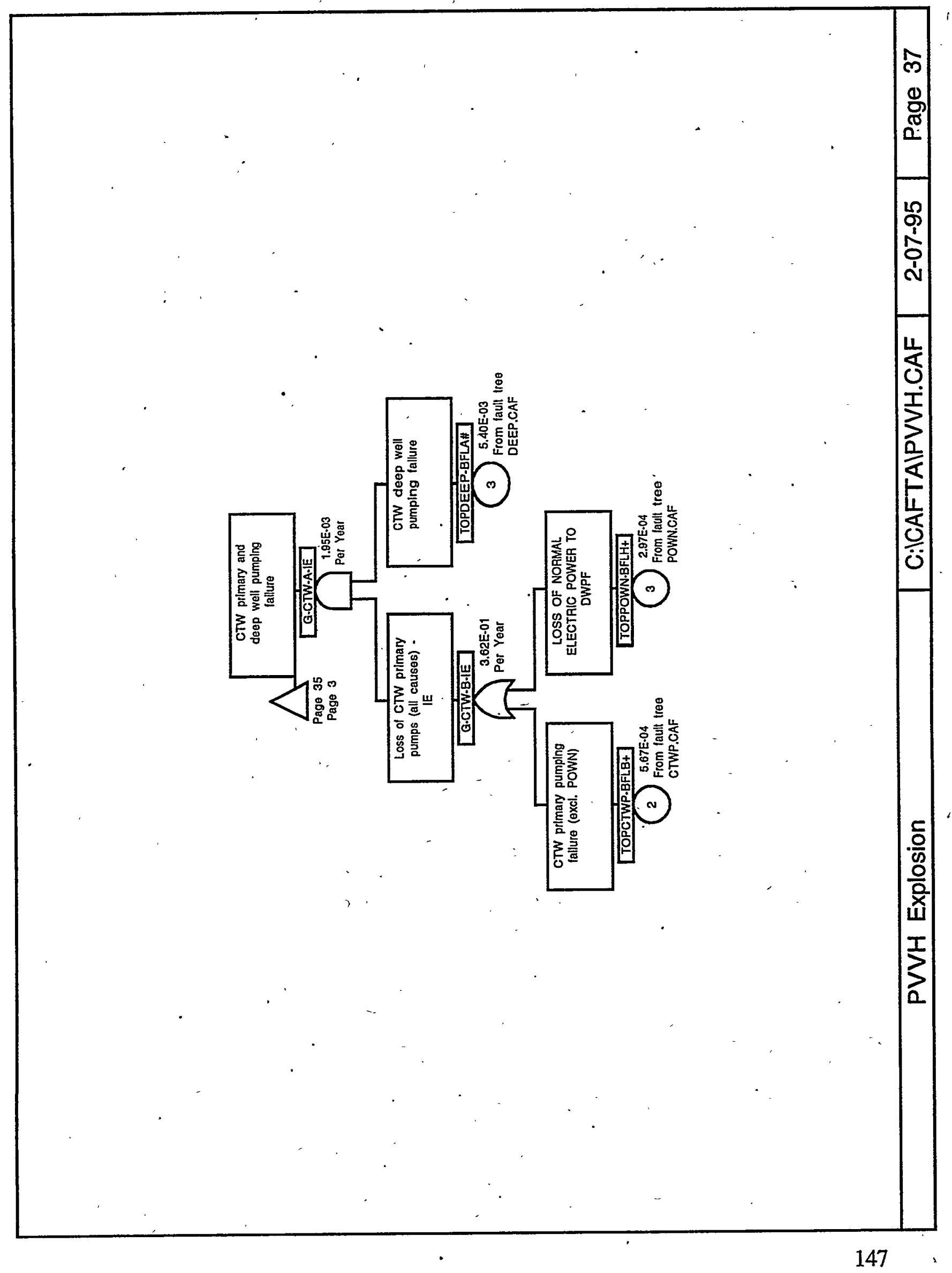




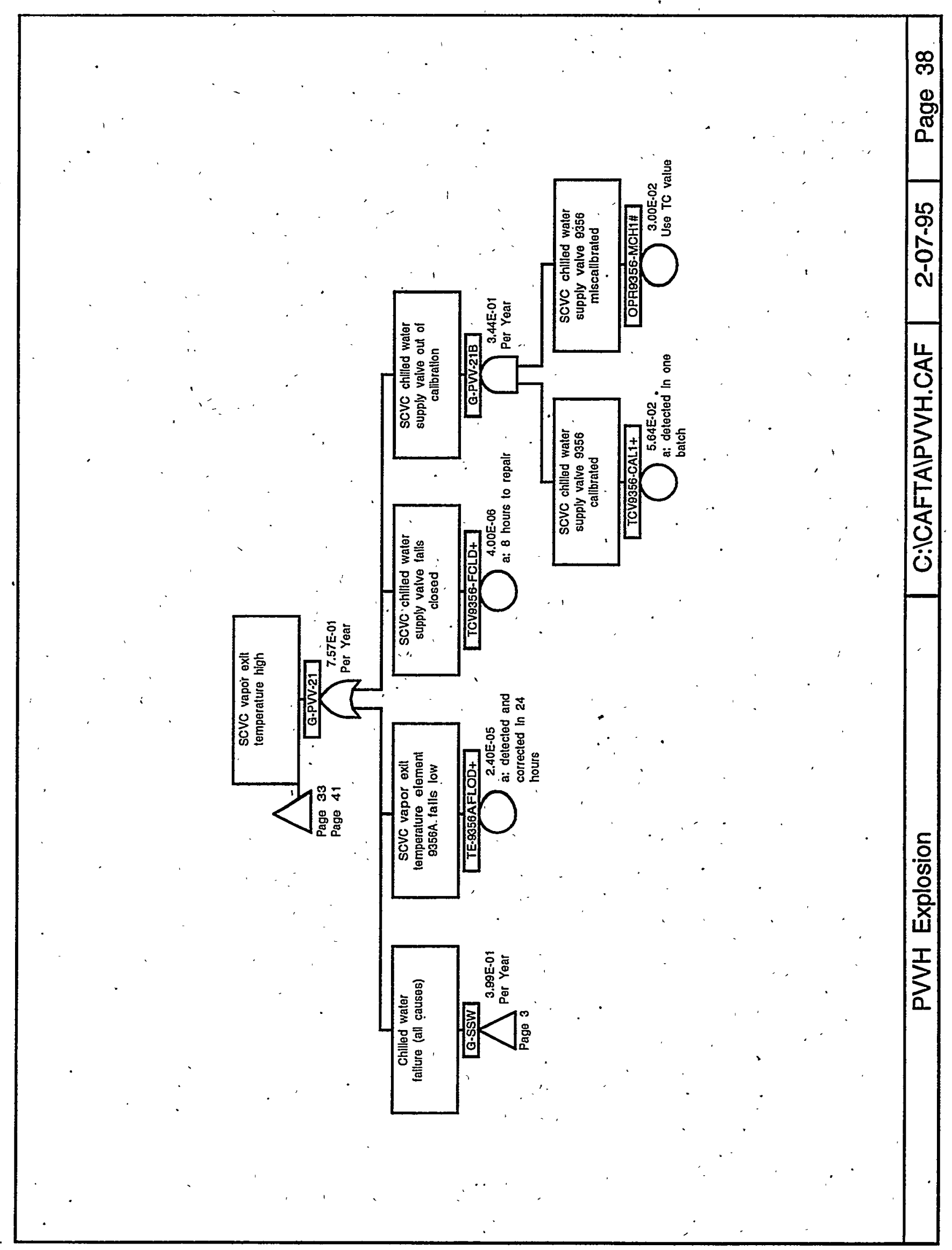




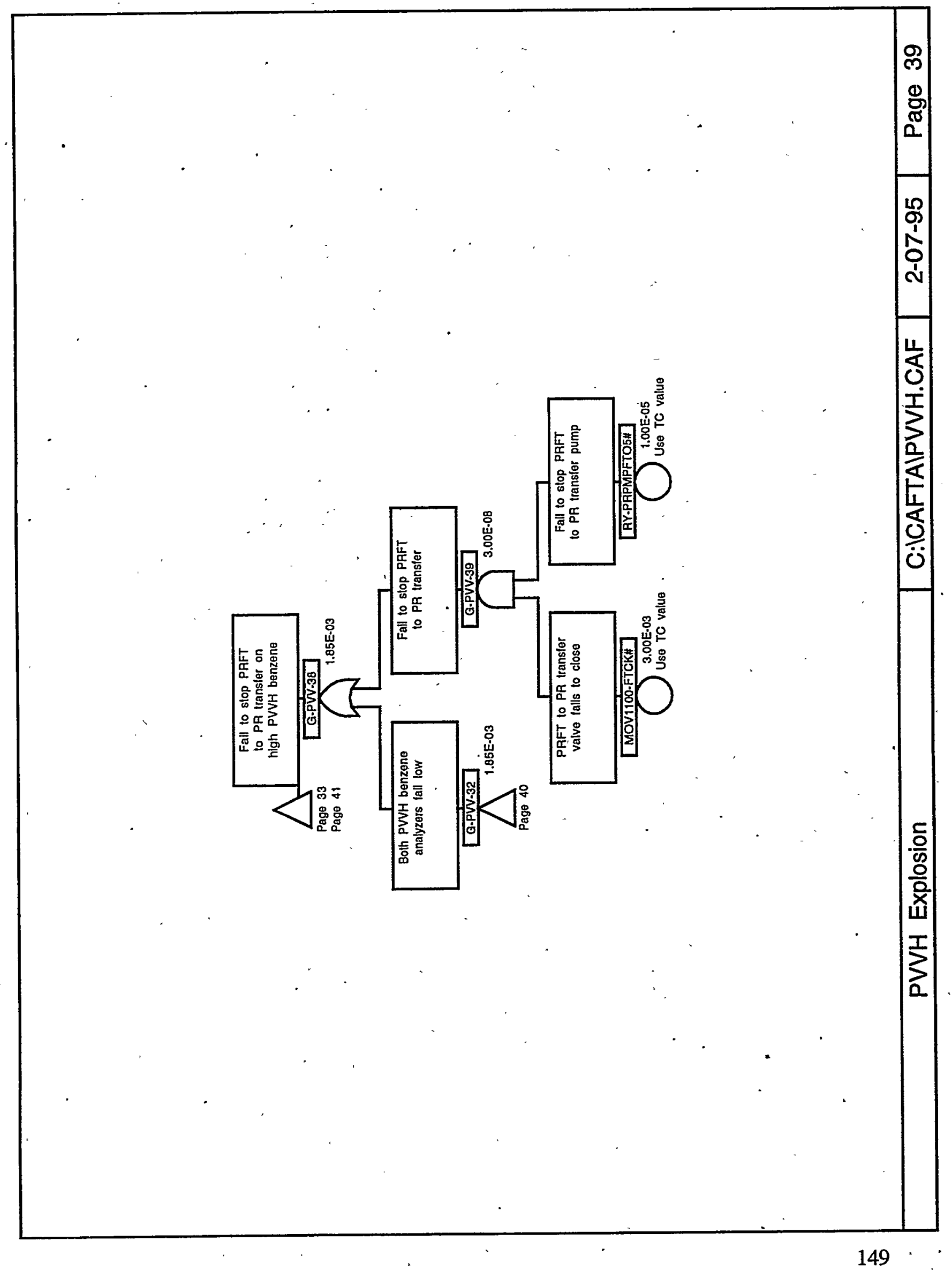




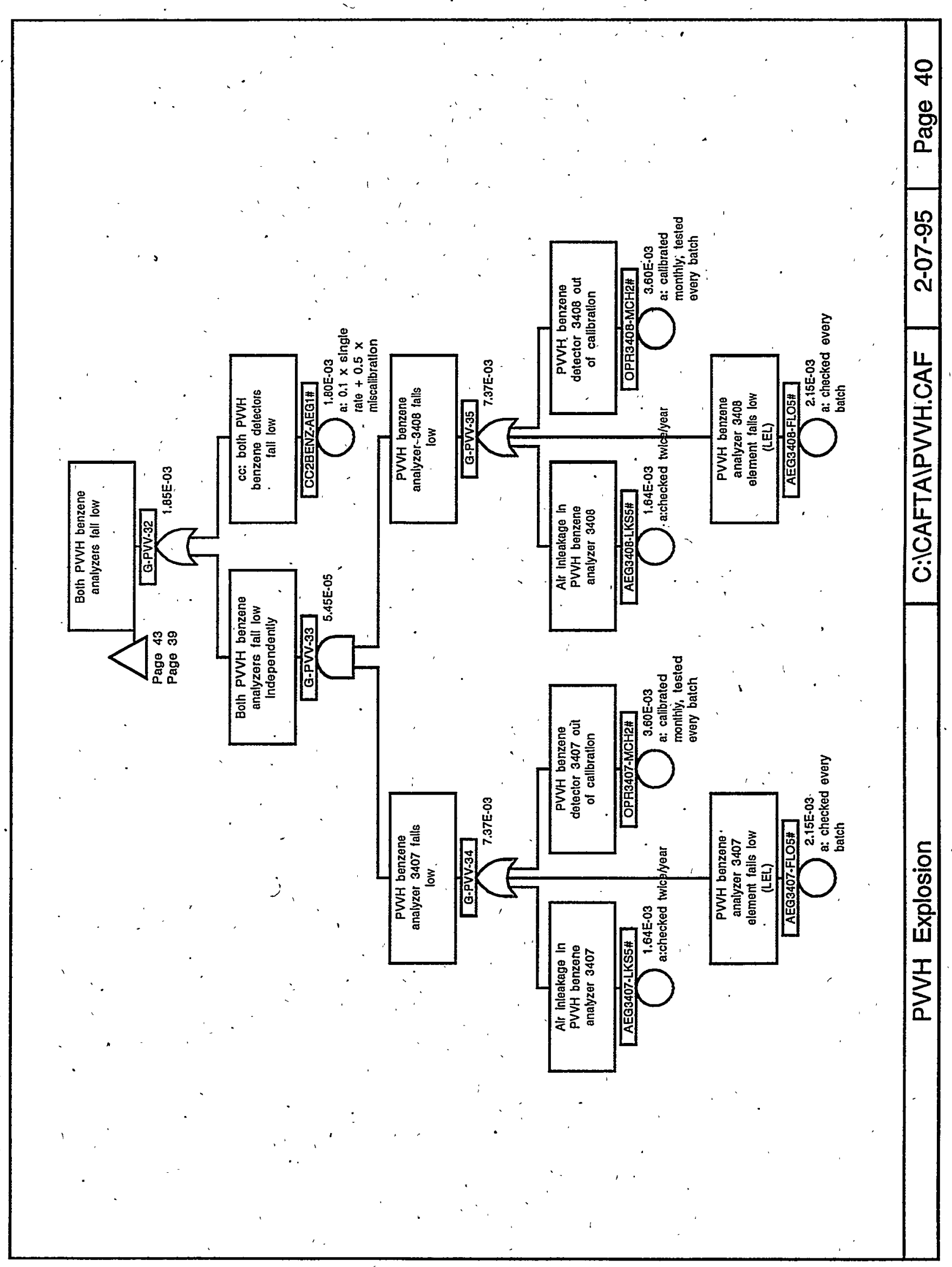




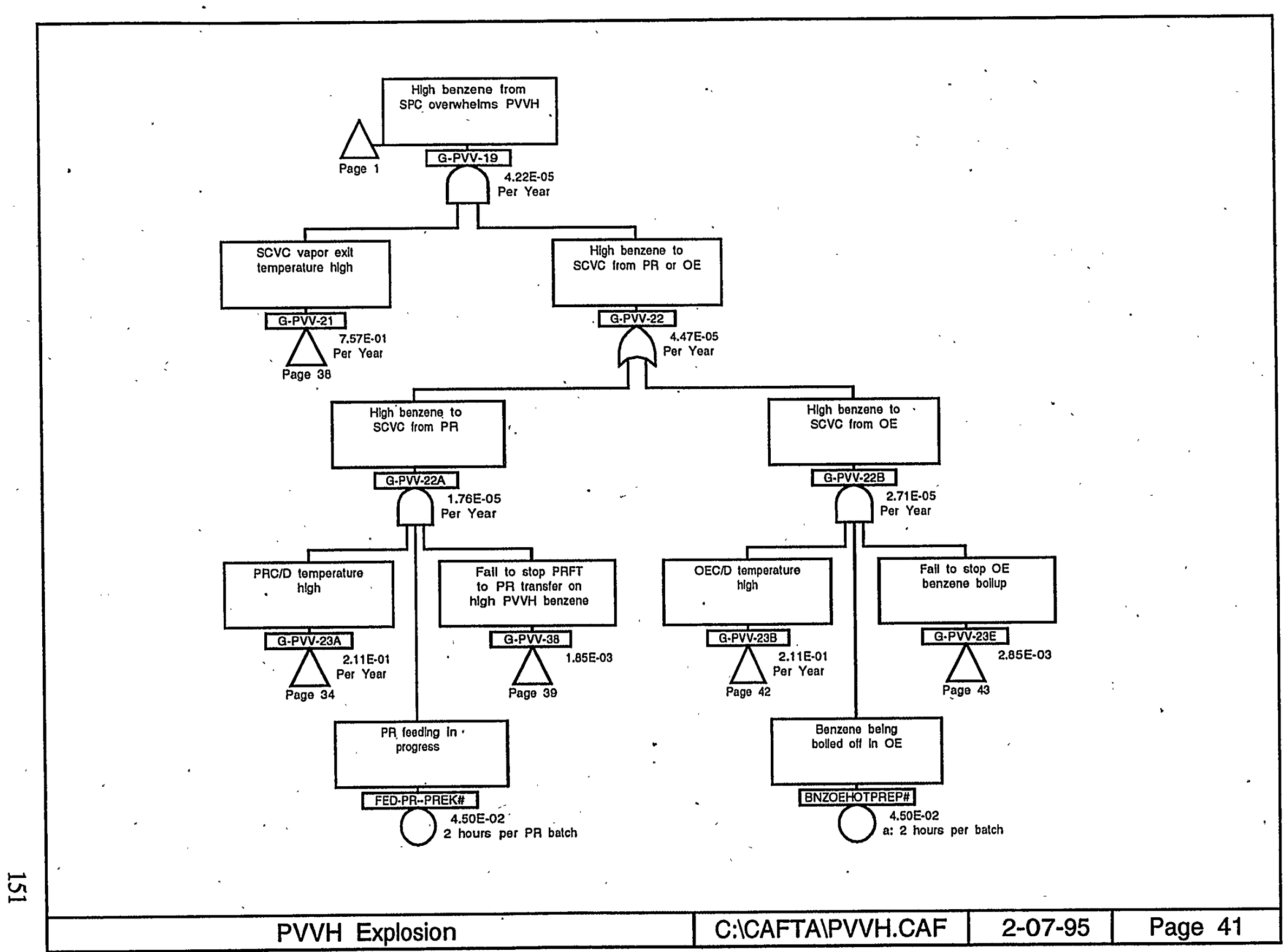


X-ESR-S-00001, REV. 0

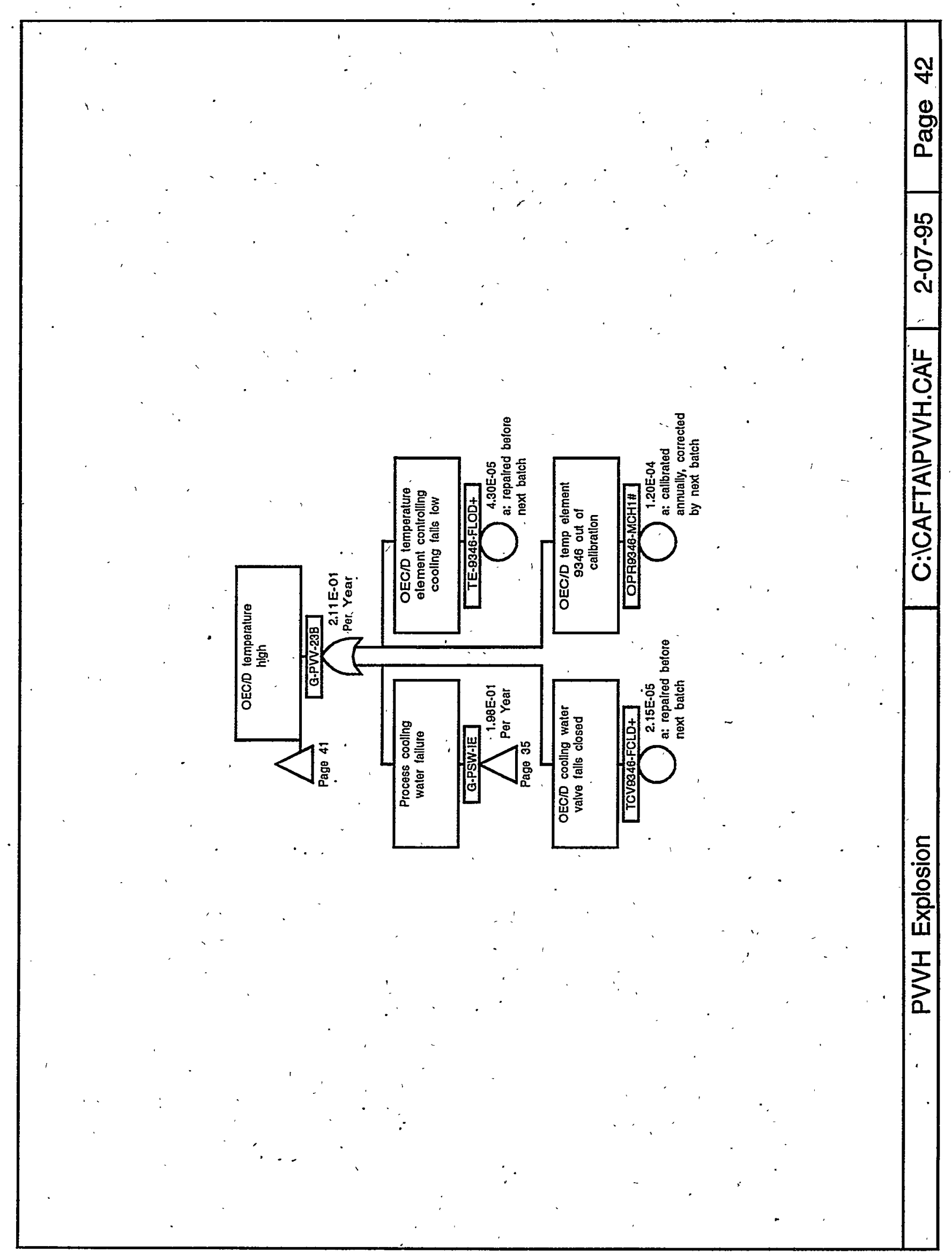




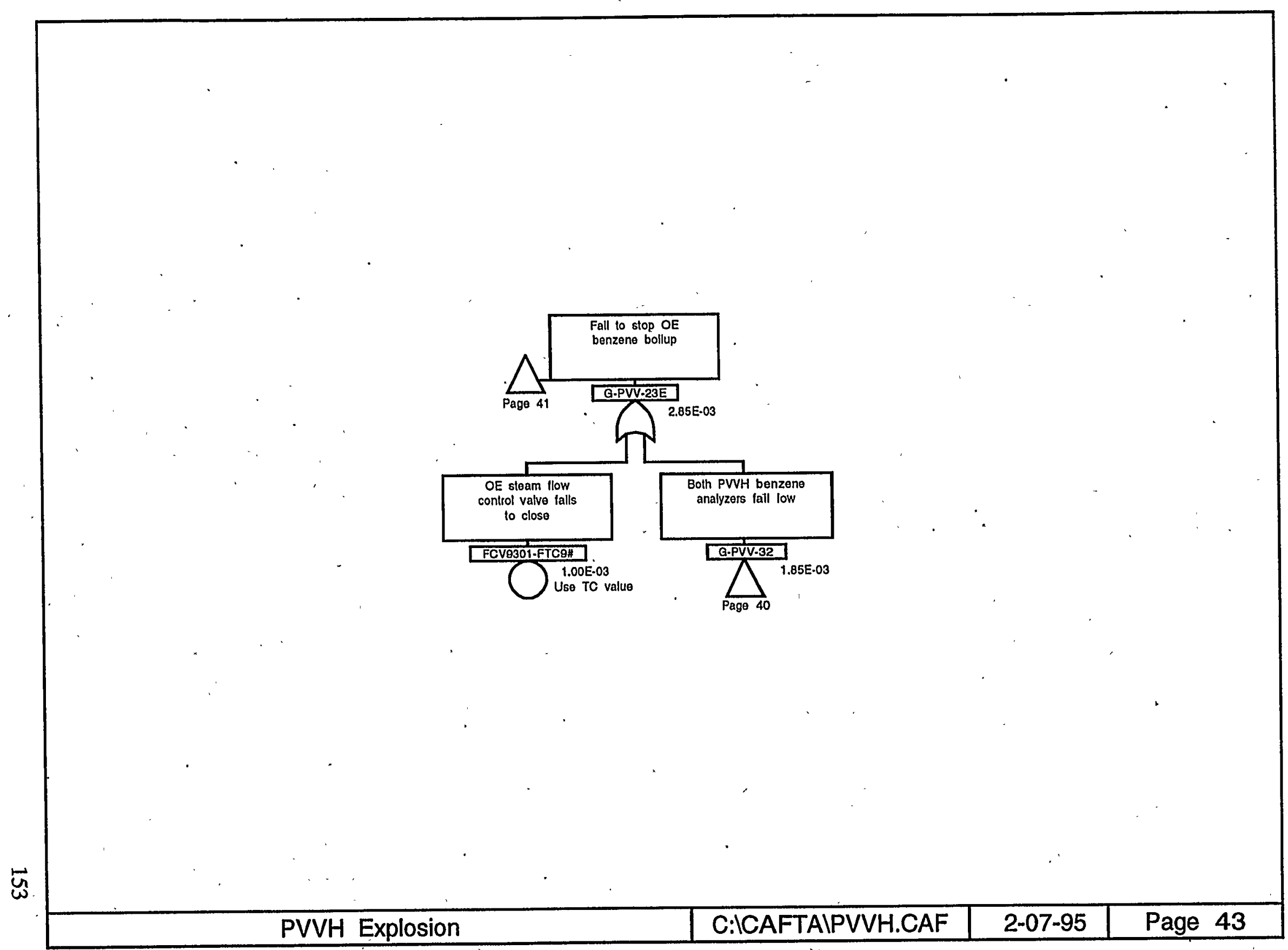


X-ESR-S-00001, REV. 0

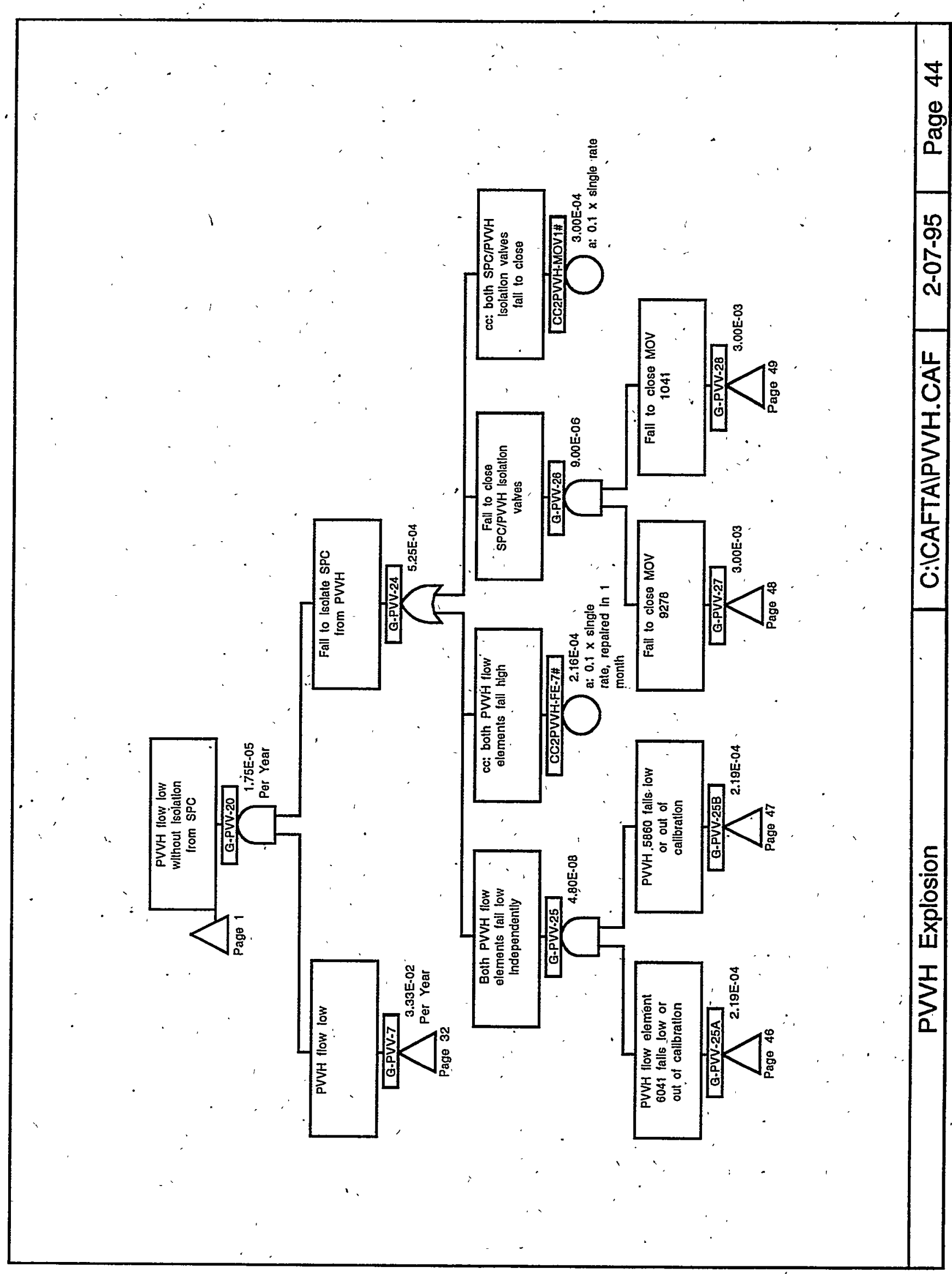




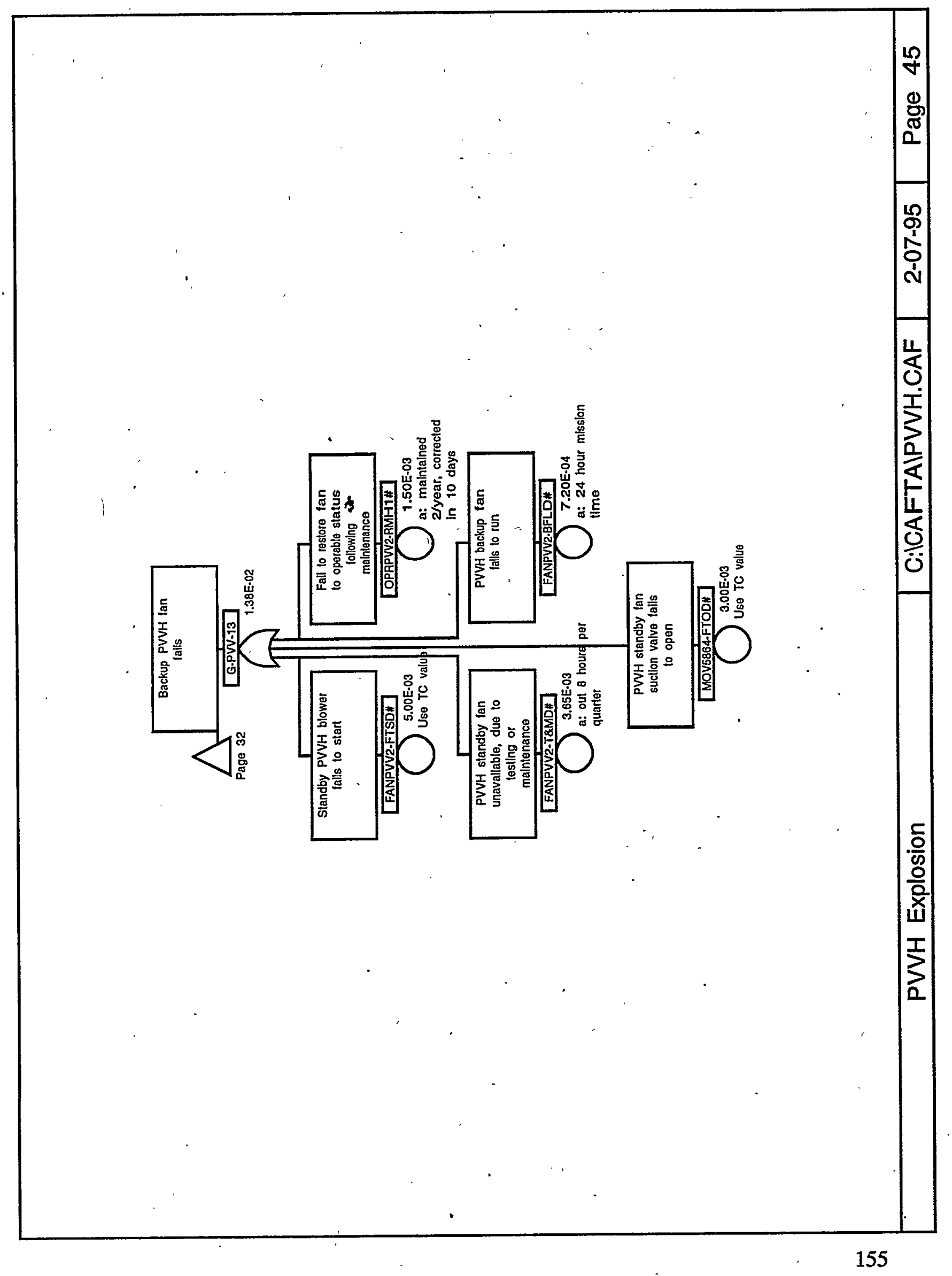


X-ESR-S-00001, REV. 0

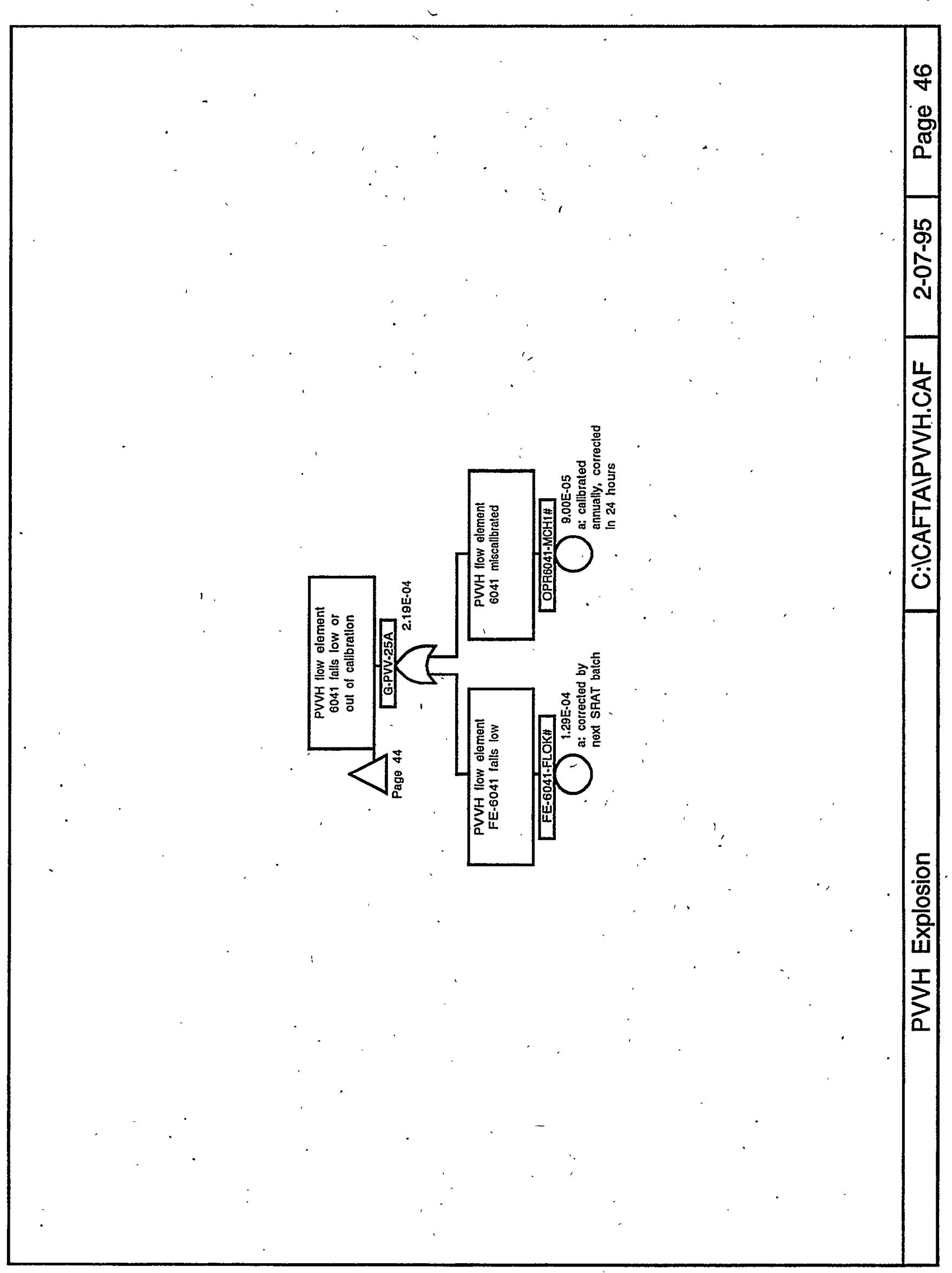




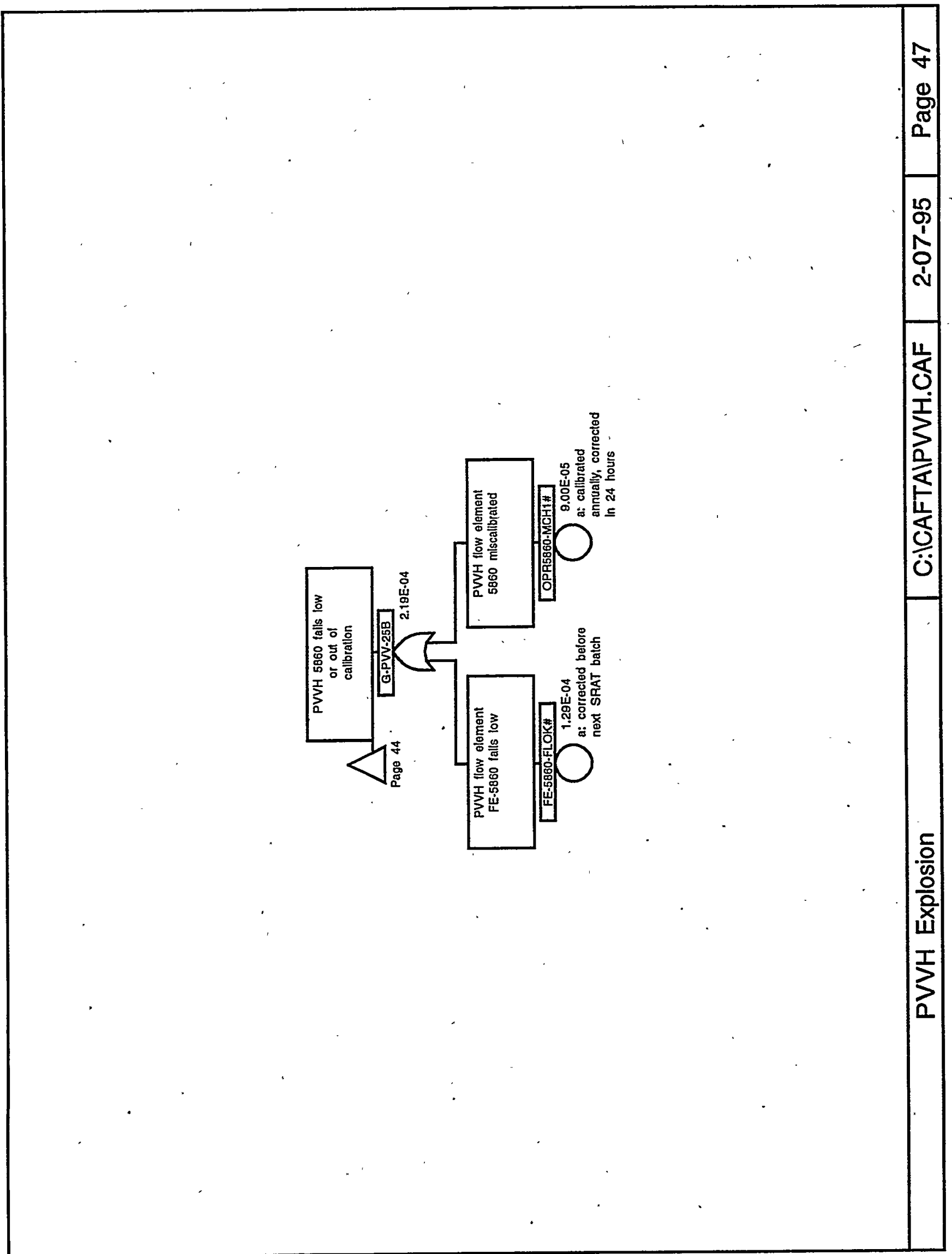


X-ESR-S-00001, REV. 0.

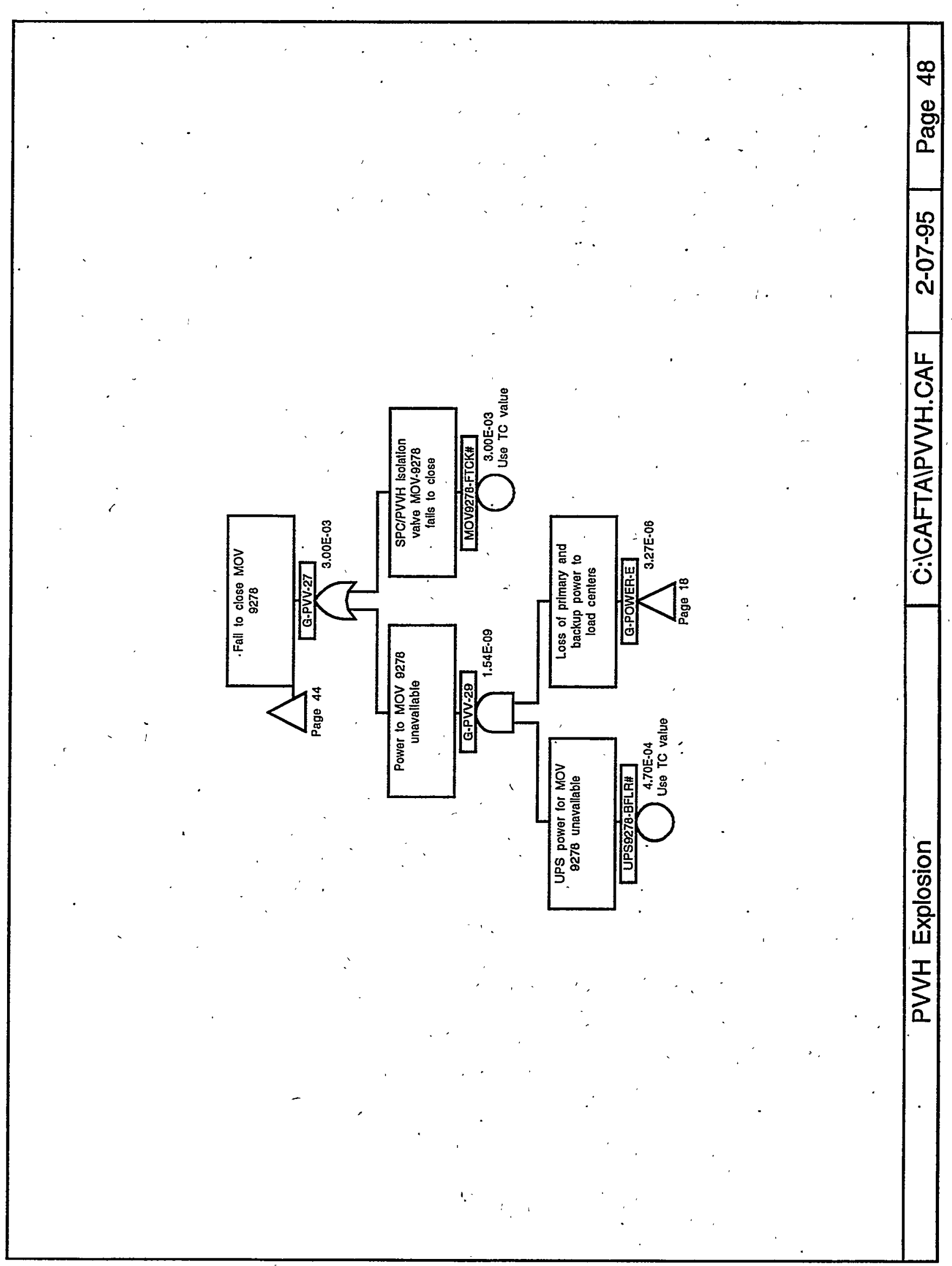




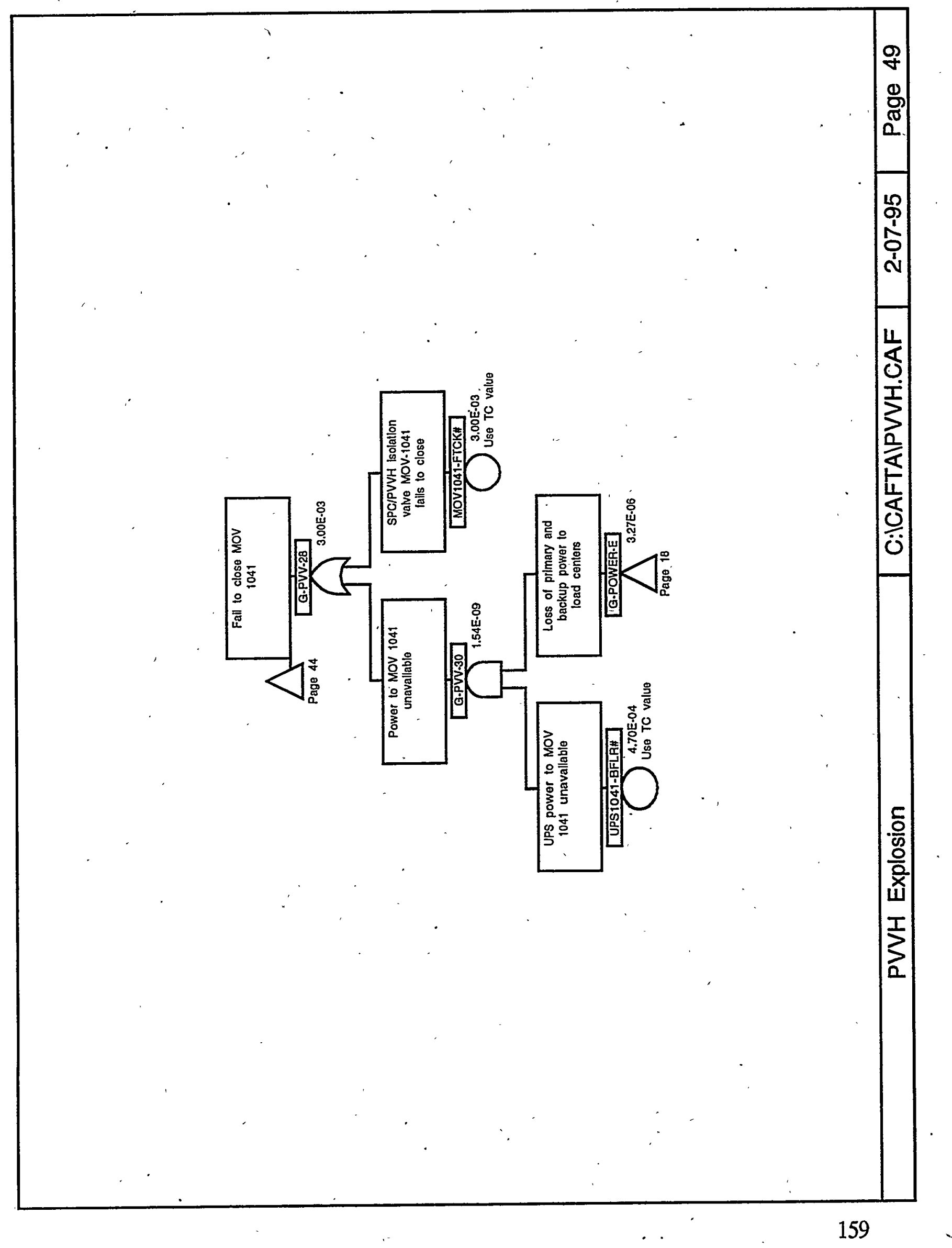


Gate/Event Name Page Zone AEG3407-FLO5\# - 40

AEG3 407-LKS5

AEG3 408-FLO5\#

AEG3408-LKS5\#

AEG8795-FLO5H

AEG8795-LKS5\#

AEG8796-FLO5\#

AEG8796-LKS5 \#

AEG8797-FLOD\#

AEG.8797-LKS4 \#

AEG8798-FLOD\#

AEG8798-LKS4 \#

AEGSRAT-LKS5 \#

$A X-8795-B F L 5 \#$

$A X-8797-B F L 4 \#$

BCHSRAT-DBL $5 \#$

BCHSRAT-PRE $5+$

BKRSRATSFOPD\#

BNZOEHOTPREP\#

CC2-SME-H2-1\#

CC2BENZ-AÉG1\#

CC2MAINTHCV1 \#

CC2 PVVH-FAN1+

CC2PVVH-FE-7\#

CC2PVVH-MOV1\#

CC2SRAT-LFL5 \#

CC2SRAT-SMP6\#

CC2STEAMHCV1+

CC3-SME-FSL 4 \#

CC3MAINTESL1\#

CC3 SRAT-FSLD\#

FANPVV1-BFLD+

FANPVV2 - BFLD\#

FANPVV2-FTSD\#

FANPVV2-T\&MD\#

FCV1027-FOP9+

FCV2 044-FTC4\#

FCV2056-ADJI+

33

14
Gate/Event Name Page Zone FCV2056-FOPI+ FCV3000-FCL5+ FCV3000-FTCG\# FCV3080-FTC4H FCV9301-FTC9\# FE-5860-FLOK\# FE-6041-FLOKH FED-PR- - PREK\# FSL8799-FTCD FSL8800-FTC4H FSL8801-FTC5\# FSL8802-FTCD \# FSL8803-FTC4\# FSL8804-FTC4\#

G-CPCBP

G-CPCBP-A

G-CPCPP

G-CPCPURGE

G-CPCPURGE

G-CTW-A-E

$G-C T W-A-I E$

G-CTW-A-IE

G-CTW-A-IE

G-CTW-B-E

G-CTW-B-IE

G-CTW-C-IE

G-CTW-IE

G-POWER-E

G-POWER-E

G-POWER-E

G-POWER-E

G-POWER-E

G-POWER-E

G-POWER-IE

G-POWER-IE

G-POWER-IE

G-POWER-IE

G-PRBT-1

11 zone

4

20

25

43

47

46

41

17

29

17

17

29

29

31

31

31

8

31

21

3

35

37.

21

37

3

3

16

18

21

30

48

49

3

3
32
Gate/Event Name Page Zone G-PRBT-1

G-PRBT -2

6

G-PRBT- 4

G-PRBT - 4 A

G-PRBT- $4 A$

G-PRBT- $4 B$

G-PRBT-5

G-PSW-E

G-PSW-E

G-PSW-E

G-PSW-IE

G-PSW-IE

G-PSW-IE

G-PVV-10

G-PVV-10

G-PVV-11

G-PVV-12

G-PVV-13

G-PVV-13

G-PVV-19

G-PVV -19

$G-P V V-20$

G-PVV-20

G-PVV-21

G-PVV-21

G-PVV-21

G-PVV-21B

G-PVV-22

G-PVV-22A

G-PVV-22B

$G-P V V-23$

G-PVV-23A

G-PVV-23A

G-PVV-23A

G-PVV-23B

G-PVV-23B

G-PVV-23C

G-PVV-23E
Gate/Event Name Page Zone G-PVV-23E

G-PVV-24

G-PVV-25

$G-P V V-25 A$

G-PVV-25A

$G-P V V-25 B$

G-PVV-25B

G-PVV-26

G-PVV-27

G-PVV-27.

G-PVV-28

G-PVV-28.

G-PVV-29

G-PVV-30

G-PVV-32

G-PVV-32

G-PVV-32

G-PVV-33

G-PVV-34

G-PVV-35

G-PVV-38

G-PVV-38

G-PVV-38

G-PVV-39

G-PVV- 4

G-PVV- 5

G-PVV-5A

G-PVV-5A

G-PVV-5B

G-PVV-5C

G-PVV-5C

G-PVV-5D

G-PVV-5E

G-PVV-6

G-PVV-7

G-PVV-7

G-PVV-7

G-PVV-8 


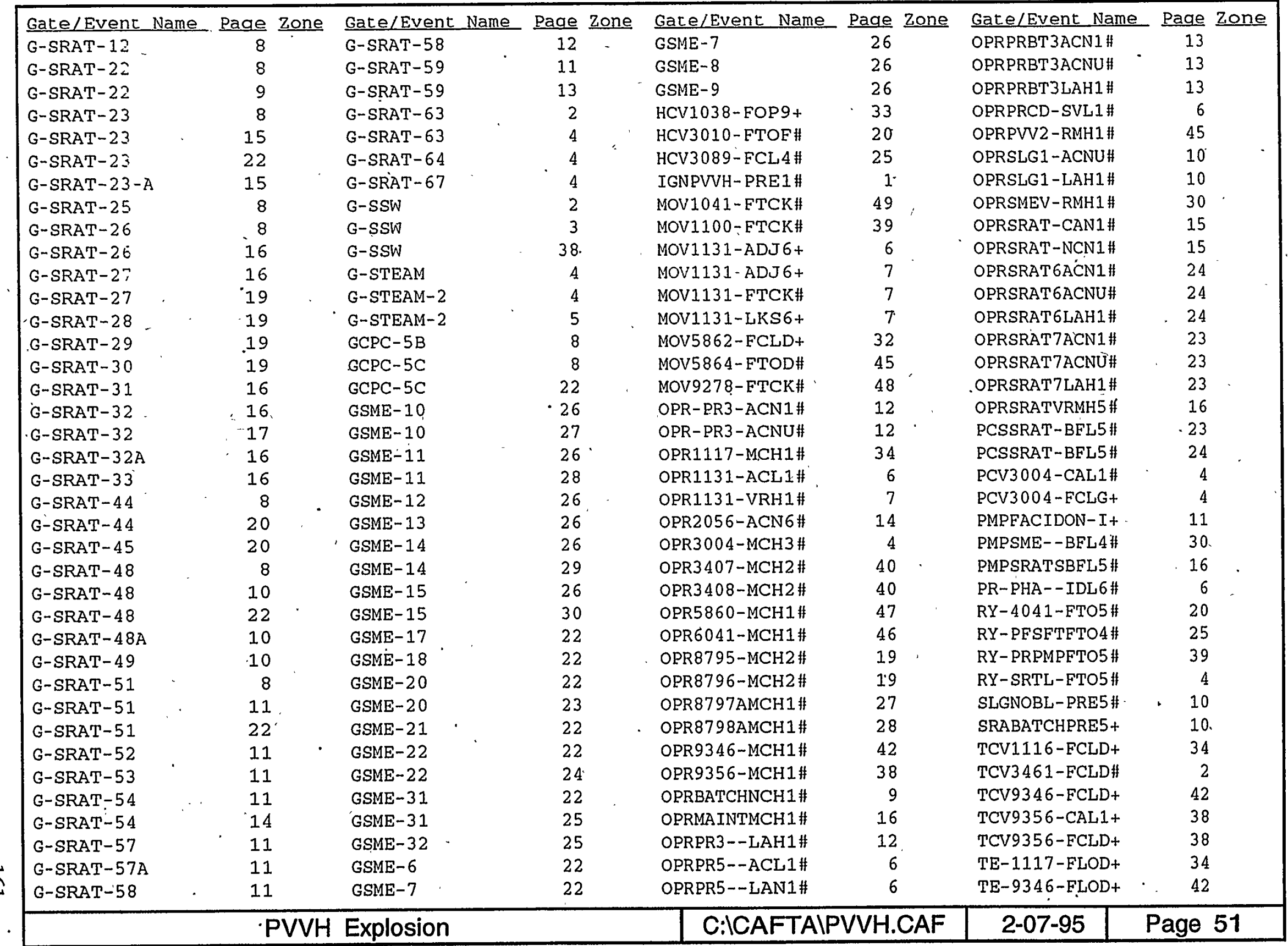




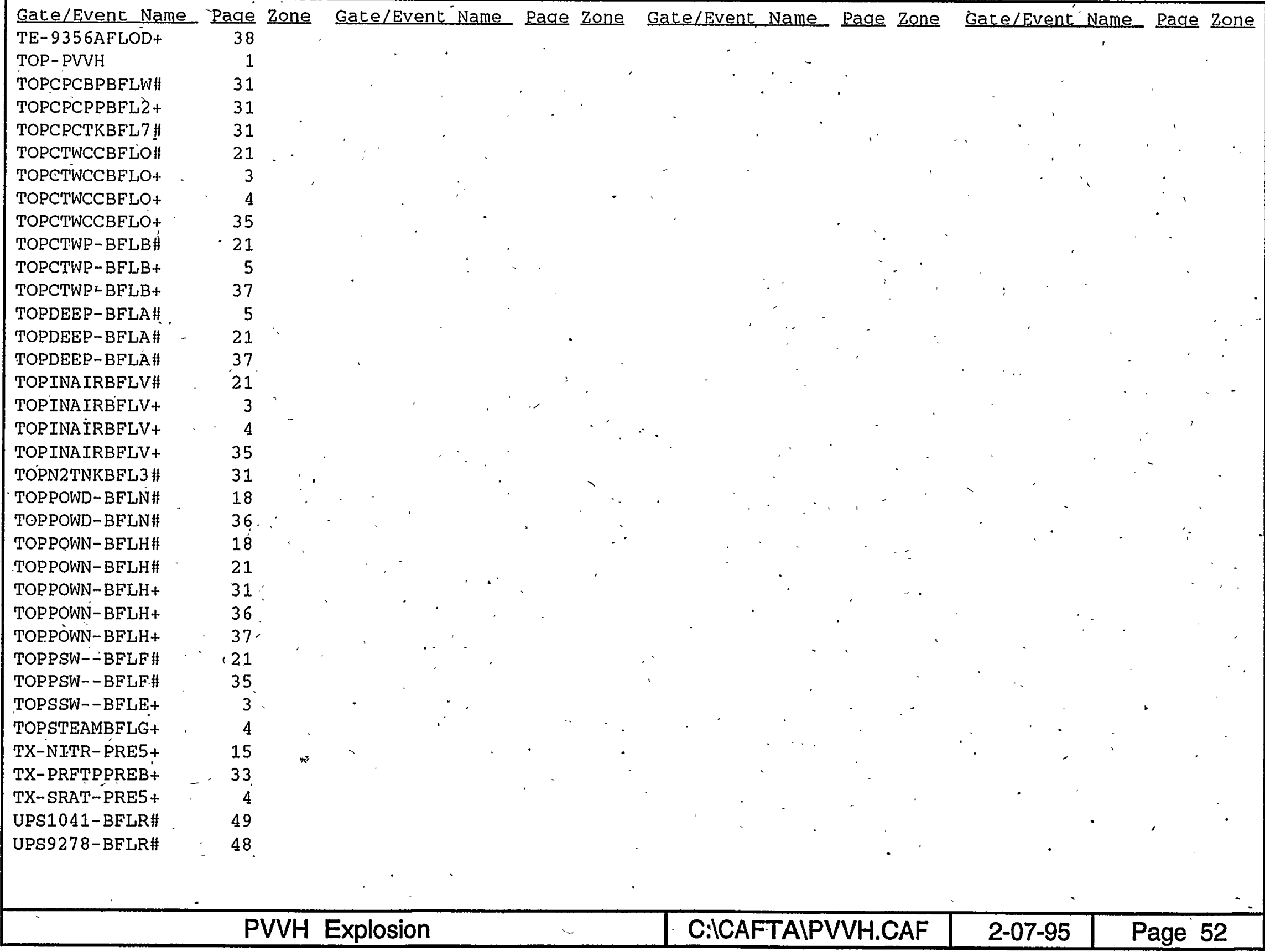


Cutsets for C:ICAFTAIPVVH.CSR 2/07/95 12:37 AM

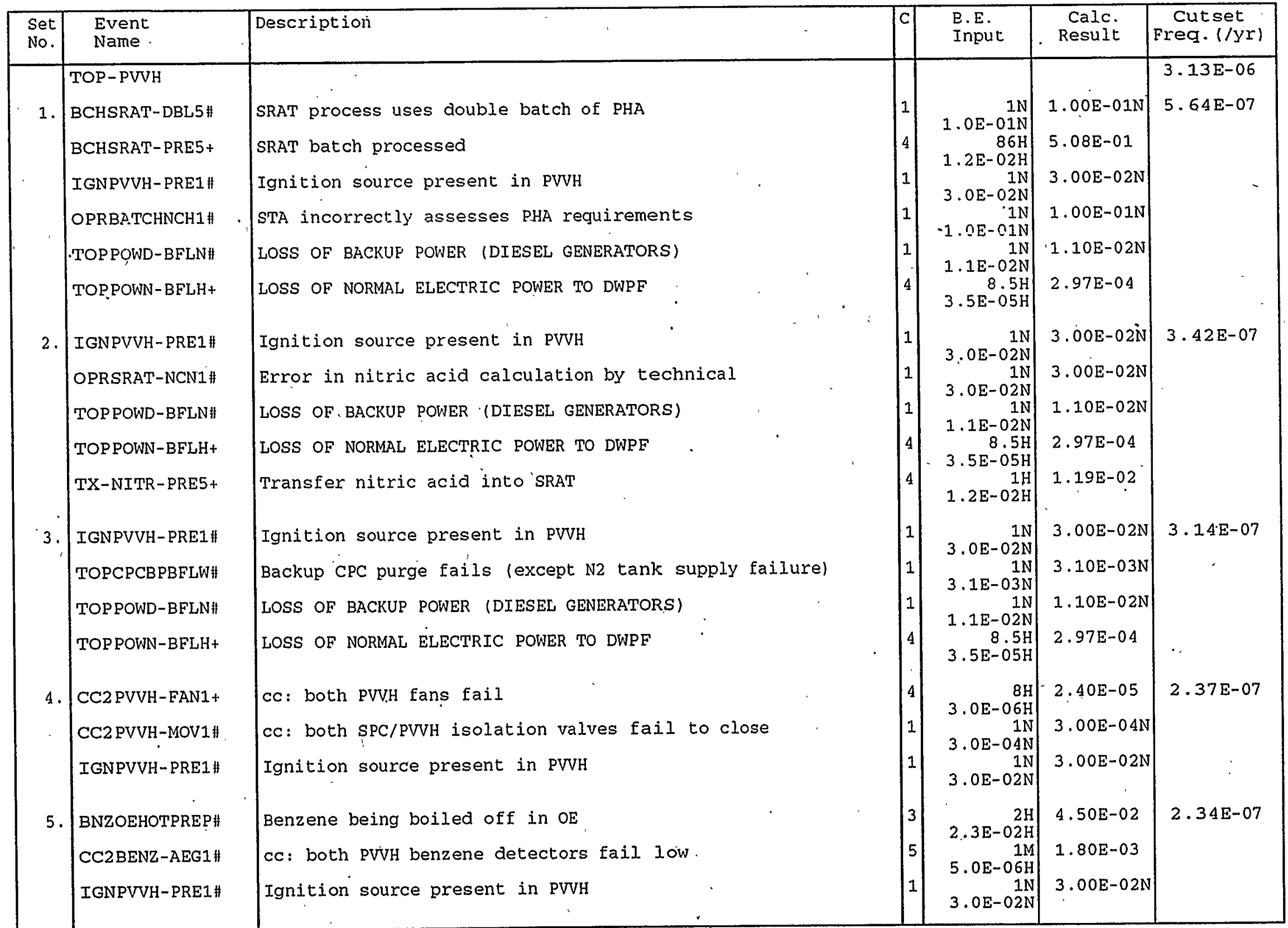


Cutsets for' C: ICAFTA \PVVH.CSR. 2/07/95 $12: 37$ AM (CONT.)

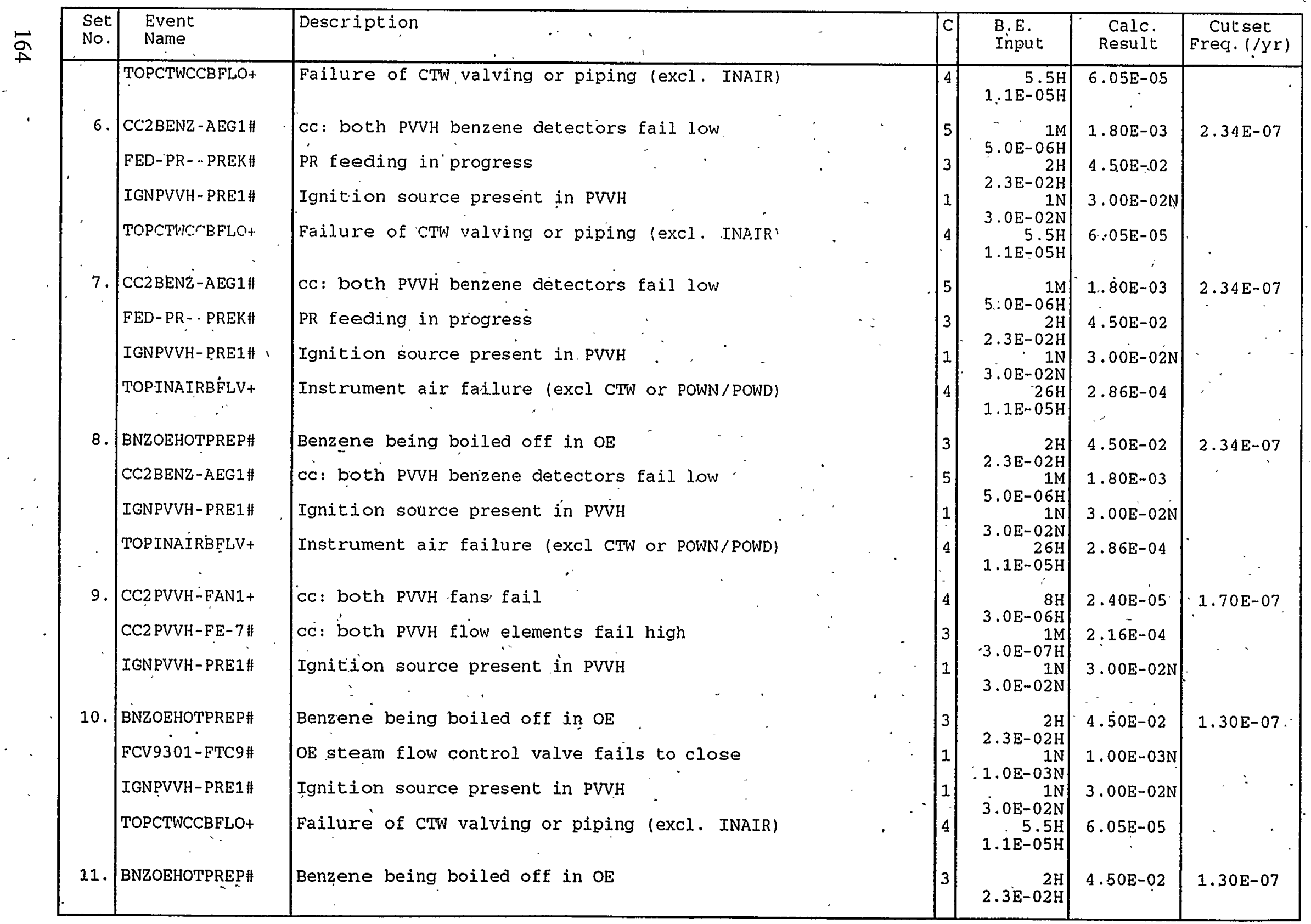


cutsets fol :: ICAFTA IPVVH.CSR

2/07/95 12:37 AM (CONT.)

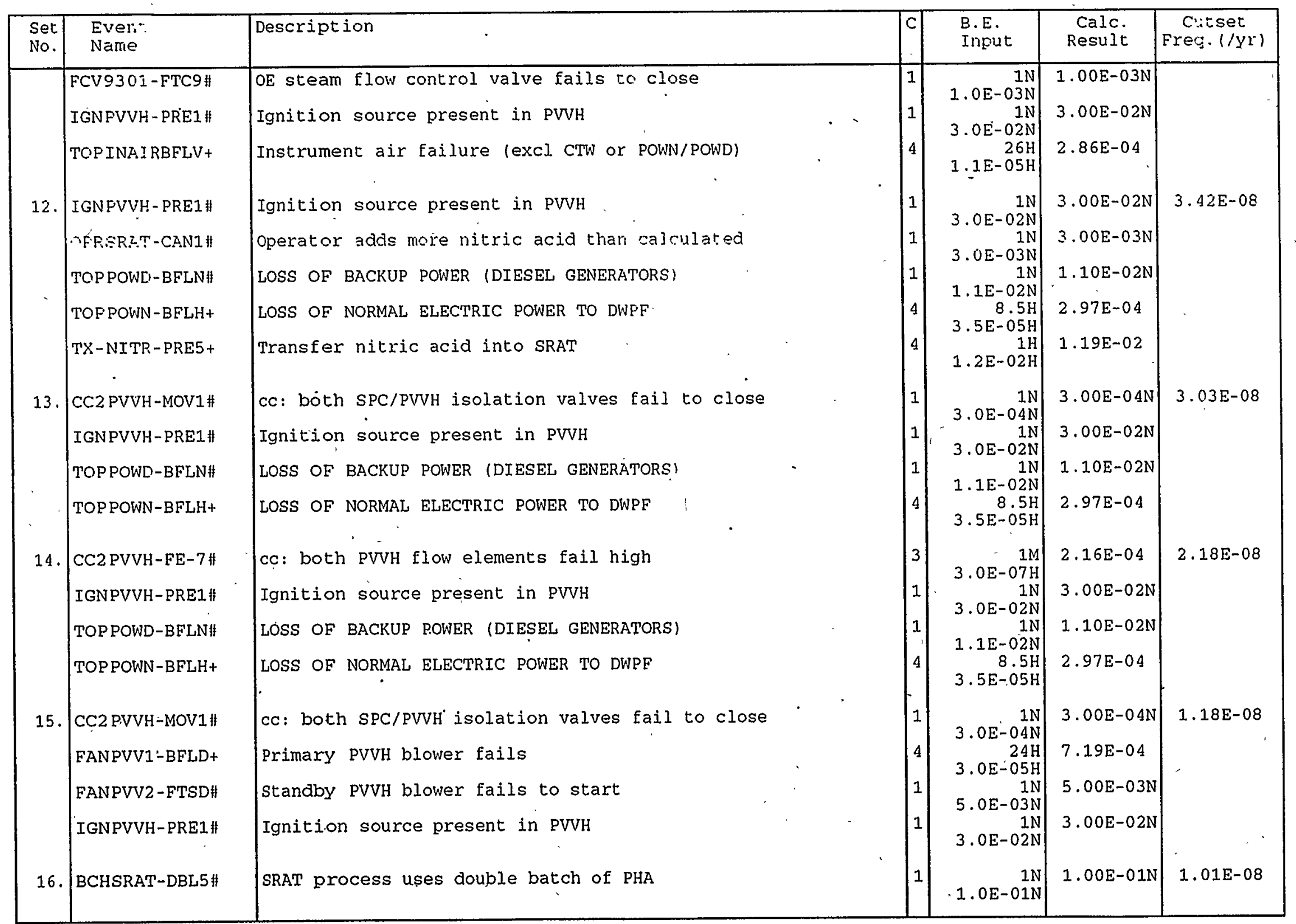




\section{X-ESR-S-00001, REV. 0}

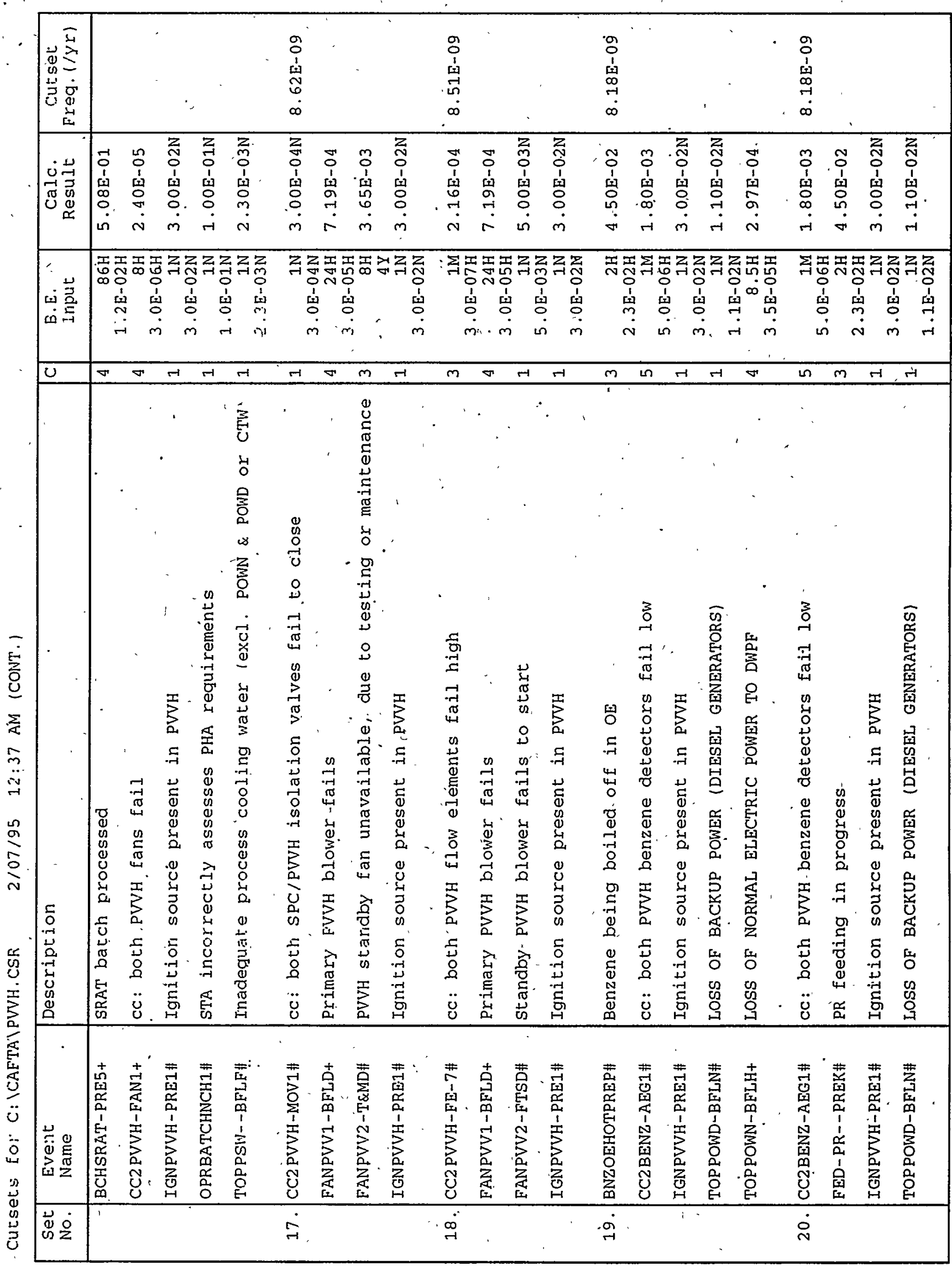


Cutsets for C: ICAFTA IFWH, CSK

$2 ; 07 / 95$ 12:37 AM (CONT.)

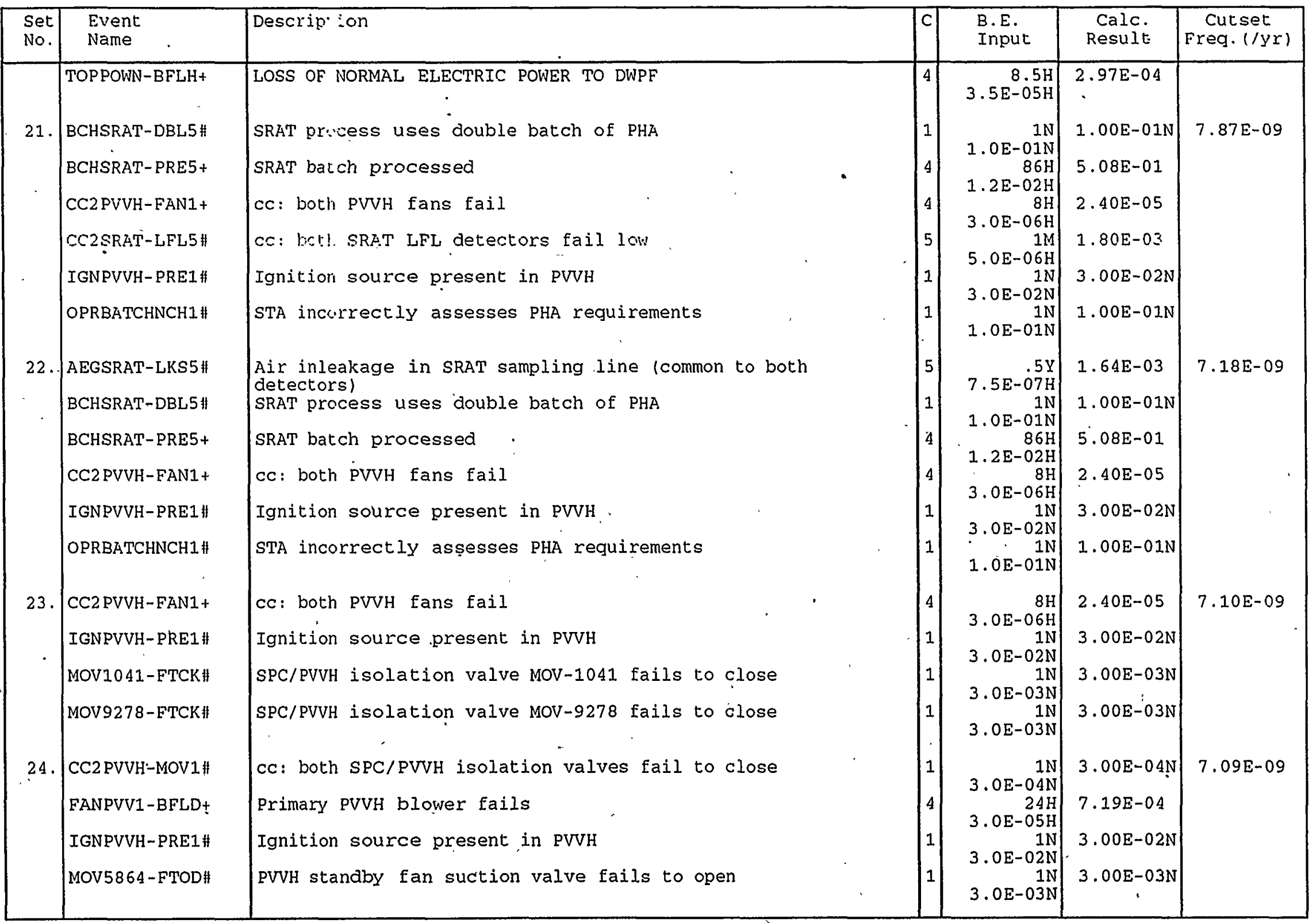


Basic Event Name CC2PVVH-FAN1+IC TOPCTWCCBFLO+IC TOPINAIRBFLV+-IC TOPPOWN-BFLH+-IC FANPVV1-BFLD+IC MOV5862-FCLD+-IC CC2PVVH-FE-7\# CC2PVVH-MOV1\# CC2BENZ-AEG1\# TOPCTWP-BFL B+-IC FCV9301-FTC9\# TOPSSW-BFLE+-IC TE-9356AFLOD+IC TCV9356-FCLD+-IC TOPPOWD-BFLN\# TOPCPCBPBFLW\# IGNPVVH-PRE1\# CC2STEAMHCV1+-IC TE-9346-FLOD+IC TCV9346-FCLD+IC CC2MAINTHCV1\# TE-1117-FLOD+IC TCV1116-FCLD+-IC TX-NITR-PRE5+-IC TOPCPCPPBFL 2+-IC BNZOEHOTPREP\# TOPPSW--BFLF\# OPRSRAT-CAN1\# OPRSRAT-NCN1\# FED-PR--PREK\# BCHSRAT-PRE5+-IC HCV3010-FTOF\# CC2SRAT-LFL5\# AX-8795-BFL5\# AEGSRAT-LKS5\# FCV3000-FTCG\# RY-4041-FTO5\# OPRBATCHNCH1\#

BCHSRAT-DBL5\# MOV5864-FTOD\# OPRPVV2-RMH1\# FANPVV2-T\&MD\# FANPVV2-FTSD\# FANPVV2-BFLD\# TCV9356-CAL1+IC

\section{Risk Achievement Worth} (page 1 of 2)

Description cc: both PVVH fan's fail Failure of CTW valving or piping (excl. INAIR) Instrument air failure (excl CTW or POWN/POWD) LOSS OF NORMAL ELECTRIC POWER TO DWPF Primary PVVH blower fails PVVH primary fan suction valve fails closed cc: both PVVH flow elements fail bigh cc: both SPC/PVVH isolation valves fail to close cc: both PVVH benzene detectors foil low CTW primary pumping failure (excl. POWN) $O E$ steam flow control valve fails to close Process chilled water failure SCVC vapor exit temperature element 9356A fails low SCVC chilled water supply valve fails closed LOSS OF BACKUP POWER (DIESEL GENERATORS) Backup CPC purge fails (except N2 tank supply failure) Ignition source present in PVVH cc: both PR steam supply valves fail open OEC/D temperature element controlling cooling fails low OEC/D cooling water valve fails closed cc: both PR steam valves miscalibrated PRCID temp element fails low PRCD cooling water valve fails closed Transfer nitric acid into SRAT CPC primary purge system fails (excl. normal power) Benzene being boiled off in $\mathrm{OE}$ Inadequate ooling water (excl. POWN \& POWD or CTW) Operator adds more nitric acid than calculated Error in nitric acid calculation by technical PR feeding in progress SRAT batch processed SRAT cooling water supply valve HCV3010 fails to open cc: both SRAT LFL detectors fail low SRAT \%LFL calculation switch failure Air inleakage in SRAT sampling line (both detectors)

SRAT steam supply valve fails to close Fail to open PRBT transfer pump switch (fail to stop pump) STA incorrectly assesses PHA requirements SRAT process uses double batch of PHA PVVH standby fan suction valve fails to open

Fail to restore fan to operable status following maintenance PVVH standby fan unavailable, due to $T \& M$ Standby PVVH blower fails to start PVVH backup fan fails to run SCVC chilled water supply valve 9356 calibrated

\begin{tabular}{ll} 
Prob/Freq & AchW \\
\hline $3.00 \mathrm{E}-06 / \mathrm{H}$ & $4.87 \mathrm{E}+04$ \\
$1.10 \mathrm{E}-05 / \mathrm{H}$ & $1.78 \mathrm{E}+04$ \\
$1.10 \mathrm{E}-05 / \mathrm{H}$ & $1.78 \mathrm{E}+04$ \\
$3.50 \mathrm{E}-05 / \mathrm{H}$ & $9.12 \mathrm{E}+03$ \\
$3.00 \mathrm{E}-05 / \mathrm{H}$ & $6.76 \mathrm{E}+02$ \\
$3.00 \mathrm{E}-07 / \mathrm{H}$ & $6.69 \mathrm{E}+02$ \\
$2.16 \mathrm{E}-04$ & $3.20 \mathrm{E}+02$ \\
$3.00 \mathrm{E}-04$ & $3.20 \mathrm{E}+02$ \\
$1.80 \mathrm{E}-03$ & $1.73 \mathrm{E}+02$ \\
$6.29 \mathrm{E}-06 / \mathrm{H}$ & $9.69 \mathrm{E}+01$ \\
$1.00 \mathrm{E}-03$ & $8.68 \mathrm{E}+01$ \\
$2.30 \mathrm{E}-05 / \mathrm{H}$ & $4.53 \mathrm{E}+01$ \\
$1.00 \mathrm{E}-06 / \mathrm{H}$ & $4.52 \mathrm{E}+01$ \\
$5.00 \mathrm{E}-07 / \mathrm{H}$ & $4.36 \mathrm{E}+01$ \\
$1.10 \mathrm{E}-02$ & $3.92 \mathrm{E}+01$ \\
$3.10 \mathrm{E}-03$ & $3.37 \mathrm{E}+01$ \\
$3.00 \mathrm{E}-02$ & $3.33 \mathrm{E}+01$ \\
$5.00 \mathrm{E}-08 / \mathrm{H}$ & $3.17 \mathrm{E}+01$ \\
$1.00 \mathrm{E}-06 / \mathrm{H}$ & $2.33 \mathrm{E}+01$ \\
$5.00 \mathrm{E}-07 / \mathrm{H}$ & $2.31 \mathrm{E}+01$ \\
$1.80 \mathrm{E}-05$ & $1.82 \mathrm{E}+01$ \\
$1.00 \mathrm{E}-06 / \mathrm{H}$ & $1.56 \mathrm{E}+01$ \\
$5.00 \mathrm{E}-07 / \mathrm{H}$ & $1.55 \mathrm{E}+01$ \\
$1.19 \mathrm{E}-02 / \mathrm{H}$ & $1.20 \mathrm{E}+01$ \\
$1.90 \mathrm{E}-05 / \mathrm{H}$ & $1.08 \mathrm{E}+01$ \\
$4.50 \mathrm{E}-02$ & $6.21 \mathrm{E}+00$ \\
$2.30 \mathrm{E}-03$ & $5.04 \mathrm{E}+00$ \\
$3.00 \mathrm{E}-03$ & $4.96 \mathrm{E}+00$ \\
$3.00 \mathrm{E}-02$ & $4.87 \mathrm{E}+00$ \\
$4.50 \mathrm{E}-02$ & $4.38 \mathrm{E}+00$ \\
$5.90 \mathrm{E}-03 / \mathrm{H}$ & $4.15 \mathrm{E}+00$ \\
$1.00 \mathrm{E}-03$ & $3.85 \mathrm{E}+00$ \\
$1.80 \mathrm{E}-03$ & $3.85 \mathrm{E}+00$ \\
$1.29 \mathrm{E}-03$ & $3.85 \mathrm{E}+00$ \\
$1.64 \mathrm{E}-03$ & $3.85 \mathrm{E}+00$ \\
$1.29 \mathrm{E}-04$ & $3.81 \mathrm{E}+00$ \\
$1.00 \mathrm{E}-05$ & $3.42 \mathrm{E}+00$ \\
$1.00 \mathrm{E}-01$ & $2.75 \mathrm{E}+00$ \\
$1.00 \mathrm{E}-01$ & $2.75 \mathrm{E}+00$ \\
$3.00 \mathrm{E}-03$ & $2.72 \mathrm{E}+00$ \\
$1.50 \mathrm{E}-03$ & $2.72 \mathrm{E}+00$ \\
$3.65 \mathrm{E}-03$ & $2.72 \mathrm{E}+00$ \\
$5.00 \mathrm{E}-03$ & $2.72 \mathrm{E}+00$ \\
$7.20 \mathrm{E}-04$ & $2.71 \mathrm{E}+00$ \\
$1.31 \mathrm{E}-03 / \mathrm{H}$ & $2.33 \mathrm{E}+00$ \\
&
\end{tabular}

Note: Events that make less than $1 \%$ difference to the importance factor are not included. Modified top event frequency $=$ (top event frequency) $\times$ (risk achievement worth). 
Explosion in the PVVH Top Event Frequency: 3.13E-06/YR

Basic EventNName
AEG3408-LKS5\#
AEG3407-LKS5\#
AEG3408-FLO5\#
AEG3407-FLO5\#
OPR3408-MCH2\#
OPR3407-MCH2\#
MOV9278-FTCK\#
MOV1041-FTCK\#
-TOPDEEP-BFLA\#
OPR9346-MCH1\#
OPR1117-MCH1\#
RY-PRPMPFTO5\#
MOV1131-LKS6+-IC
RY-SRTL-FTO5\#
UPS1041-BFLR\#
UPS9278-BFLR\#
OPRSRAT7ACNU\#
OPRSRAT7ACN1\#
PCSSRAT-BFL5\#
OPRSRATTLAH1\#
PMPSRATSBFL5\#
FE-5860-FLOK\#
FE-6041-FLOK\#
OPR6041-MCH1\#
OPR586-MCH1\#
OPR9356-MCH1\#
OPRSRATVRMH5\#
SRABATCHPRE5+-IC
OPR8796-MCH2\#
OPR8795-MCH2\#
AEG8796-FLO5\#
AEG8795-FLO5\#
AEG8796-LKS5\#
AEG8795-LKS5\#
TX-PRFTPPREB+-IC
AX-8797-BFLA\#

\section{Risk Achievement Worth}

Description (page 2 of 2)

Air inleakage in PVVH benzene analyzer 3408

Air inleakage in PVVH benzene andyzer 3407

PVVH benzene analyzer 3408 element fails low (LEL)

PVVH benzene analyzer 3407 element fails low (LEL)

PVVH benzene detector 3408 out of calibration

PVVH benzene detector 3407 out of calibration

SPC/PVVH isolation valve MOV-9278 fails to close

SPC/PVVH isolation valve MOV-1041 fails to close

CTW deep well pumping failure

OECDD temp element 9346 out of calibration

PRC/D temp element 1117 out of calibration

Fail to stop PRFT to PR transfer pump

PRCD drain valve leaks (benzene to PR)

Fail to open SRAT steam flow low interlock relay

UPS power to MOV 1041 unavailable

UPS power for MOV 9278 unavailable

Failure to sample SRAT product for nitric acid (pH)

Failure to follow procedure for SRAT nitric acid sampling

Fail to compensate: out of spec SRAT product data to PCCS

Error in SRAT product sample

SRAT sample pump fails

PVVH flow element FE-5860 fails low

PVVH flow element FE-6041 fails low

PVVH flow element 6041 miscalibrated

PVVH flow element 5860 miscalibrated

SCVC chilled water supply valve 9356 miscalibrated

Fail to open valve following maintenance

New batch of sludge for DWPF

SRAT offgas detector \#2 out of calibration

SRAT offgas detector \#1 out of calibration

SRAT offgas detector \#2 analyzer element fails low (LEL)

SRAT offgas detector \#1 analyzer element fails low (LEL)

Air inleakage in SRAT offgas detector \#2 sample line

Air inleakage in SRAT offgas detector \#1 sample line .

Transfer from PRFT to PR

SME \%LFL calculation and switch failure

\begin{tabular}{ll} 
Prob/Freg & AchW \\
\hline $1.64 \mathrm{E}-03$ & $2.27 \mathrm{E}+00$ \\
$1.64 \mathrm{E}-03$ & $2.27 \mathrm{E}+00$ \\
$2.15 \mathrm{E}-03$ & $2.27 \mathrm{E}+00$ \\
$2.15 \mathrm{E}-03$ & $2.27 \mathrm{E}+00$ \\
$3.60 \mathrm{E}-03$ & $2.27 \mathrm{E}+00$ \\
$3.60 \mathrm{E}-03$ & $2.27 \mathrm{E}+00$ \\
$3.00 \mathrm{E}-03$ & $1.97 \mathrm{E}+00$ \\
$3.00 \mathrm{E}-03$ & $1.97 \mathrm{E}+00$ \\
$5.40 \mathrm{E}-03$ & $1.73 \mathrm{E}+00$ \\
$1.20 \mathrm{E}-04$ & $1.68 \mathrm{E}+00$ \\
$1.20 \mathrm{E}-04$ & $1.50 \mathrm{E}+00$ \\
$1.00 \mathrm{E}-05$ & $1.26 \mathrm{E}+00$ \\
$1.00 \mathrm{E}-06 / \mathrm{H}$ & $1.25 \mathrm{E}+00$ \\
$1.00 \mathrm{E}-05$ & $1.18 \mathrm{E}+00$ \\
$4.70 \mathrm{E}-04$ & $1.11 \mathrm{E}+00$ \\
$4.70 \mathrm{E}-04$ & $1.11 \mathrm{E}+00$ \\
$5.00 \mathrm{E}-03$ & $1.08 \mathrm{E}+00$ \\
$5.00 \mathrm{E}-03$ & $1.08 \mathrm{E}+00$ \\
$1.00 \mathrm{E}-03$ & $1.07 \mathrm{E}+00$ \\
$1.00 \mathrm{E}-03$ & $1.07 \mathrm{E}+00$ \\
$2.40 \mathrm{E}-04$ & $1.07 \mathrm{E}+00$ \\
$1.29 \mathrm{E}-04$ & $1.07 \mathrm{E}+00$ \\
$1.29 \mathrm{E}-04$ & $1.07 \mathrm{E}+00$ \\
$9.00 \mathrm{E}-05$ & $1.06 \mathrm{E}+00$ \\
$9.00 \mathrm{E}-05$ & $1.06 \mathrm{E}+00$ \\
$3.00 \mathrm{E}-02$ & $1.06 \mathrm{E}+00$ \\
$7.00 \mathrm{E}-05$ & $1.04 \mathrm{E}+00$ \\
$5.68 \mathrm{E}-05 / \mathrm{H}$ & $1.03 \mathrm{E}+00$ \\
$3.60 \mathrm{E}-03$ & $1.02 \mathrm{E}+00$ \\
$3.60 \mathrm{E}-03$ & $1.02 \mathrm{E}+00$ \\
$2.15 \mathrm{E}-03$ & $1.02 \mathrm{E}+00$ \\
$2.15 \mathrm{E}-03$ & $1.02 \mathrm{E}+00$ \\
$1.64 \mathrm{E}-03$ & $1.01 \mathrm{E}+00$ \\
$1.64 \mathrm{E}-03$ & $1.01 \mathrm{E}+00$ \\
$2.20 \mathrm{E}-02 / \mathrm{H}$ & $1.01 \mathrm{E}+00$ \\
$6.34 \mathrm{E}-02$ & $1.01 \mathrm{E}+00$
\end{tabular}

Note: Events that make less than $1 \%$ difference to the importance factor are not included. Modified top event frequency = (top event frequency) $\mathrm{x}$ (risk achievement worth). 
Explosion in the PVVH Top Event Frequency: 3.13E-06/YR

\author{
Basic Event Name \\ *IGNPVVH-PRE1\# . \\ TOPPOWN-BFLH+-IC \\ TOPPOWD-BFLN\# \\ CC2BENZ-AEG1\# \\ BNZOEHOTPREP\# \\ TOPINAIRBFLV+-IC \\ TOPCTWCCBFLO+-IC \\ OPRBATCHNCH1\# \\ BCHSRAT-PRE5+-IC \\ BCHSRAT-DBL5\# \\ FED-PR-PREK\# \\ CC2PVVH-FAN1+-IC \\ TX-NITR-PRE5+IC \\ OPRSRAT-NCN1\# \\ TOPCPCBPBFLW\# \\ CC2PVVH-MOV1\# \\ FCV9301-FTC9\# \\ CC2PVVH-FE-7\# \\ FANPVV1-BFLD+-IC \\ OPRSRAT-CAN1\#
}

Risk Reduction Worth Description Ignition source present in PVVH LOSS OF NORMAL ELECTRIC POWER TO DWPF LOSS OF BACKUP POWER (DIESEL GENERATORS) cc: both.PVVH benzene detectors fail low. - Benzene being boiled off in $\mathrm{OE}$ Instrument air failure (excl CTW or POWN/POWD) Failure of CTW valving or piping (excl. INAIR) STA incorrectly assesses PHA requirements SRAT batch processed SRAT process uses double batch of PHA PR feeding in progress cc: both PVVH fans fail Transfer nitric acid into SRAT Error in nitric acid calculation by technical Backup CPC purge fails (except N2 tank supply failure) cc: both SPC/PVVH isolation valves fail to close $\mathrm{OE}$ steam flow control valve fails to close cc: both PVVH flow elements fail high Primary PVVH blower fails Operator adds more nitric acid than calculated

\begin{tabular}{ll} 
Prob/Frea & RedW \\
\hline $3.00 \mathrm{E}-02$ & $\frac{0.00 \mathrm{E}+00}{0.50 \mathrm{E}-05 / \mathrm{H}}$ \\
$1.75 \mathrm{E}+00$ \\
$1.10 \mathrm{E}-02$ & $1.74 \mathrm{E}+00$ \\
$1.80 \mathrm{E}-03$ & $1.45 \mathrm{E}+00$ \\
$4.50 \mathrm{E}-02$ & $1.32 \mathrm{E}+00$ \\
$1.10 \mathrm{E}-05 / \mathrm{H}$ & $1.24 \mathrm{E}+00$ \\
$1.10 \mathrm{E}-05 / \mathrm{H}$ & $1.24 \mathrm{E}+00$ \\
$1.00 \mathrm{E}-01$ & $1.24 \mathrm{E}+00$ \\
$5.90 \mathrm{E}-03 / \mathrm{H}$ & $1.24 \mathrm{E}+00$ \\
$1.00 \mathrm{E}-01$ & $1.24 \mathrm{E}+00$ \\
$4.50 \mathrm{E}-02$ & $1.19 \mathrm{E}+00$ \\
$3.00 \mathrm{E}-06 / \mathrm{H}$ & $1.18 \mathrm{E}+00$ \\
$1.19 \mathrm{E}-02 / \mathrm{H}$ & $1.15 \mathrm{E}+00$ \\
$3.00 \mathrm{E}-02$ & $1.14 \mathrm{E}+00$ \\
$3.10 \mathrm{E}-03$ & $1.11 \mathrm{E}+00$ \\
$3.00 \mathrm{E}-04$ & $1.11 \mathrm{E}+00$ \\
$1.00 \mathrm{E}-03$ & $1.09 \mathrm{E}+00$ \\
$2.16 \mathrm{E}-04$ & $1.07 \mathrm{E}+00$ \\
$3.00 \mathrm{E}-05 / \mathrm{H}$ & $1.02 \mathrm{E}+00$ \\
$3.00 \mathrm{E}-03$ & $1.01 \mathrm{E}+00$
\end{tabular}

* A Reduction Worth of $0.0 \mathrm{E}+00$ indicates event appears in every cutset could serve as a single event preventor for the accident.

Note: Events that make less than $1 \%$ difference to the importance factor are not included. Modified top event frequency = (top event frequency) / (risk reductionworth). 
X-ESR-S-00001, REV. 0

\section{SRAT Explosion}




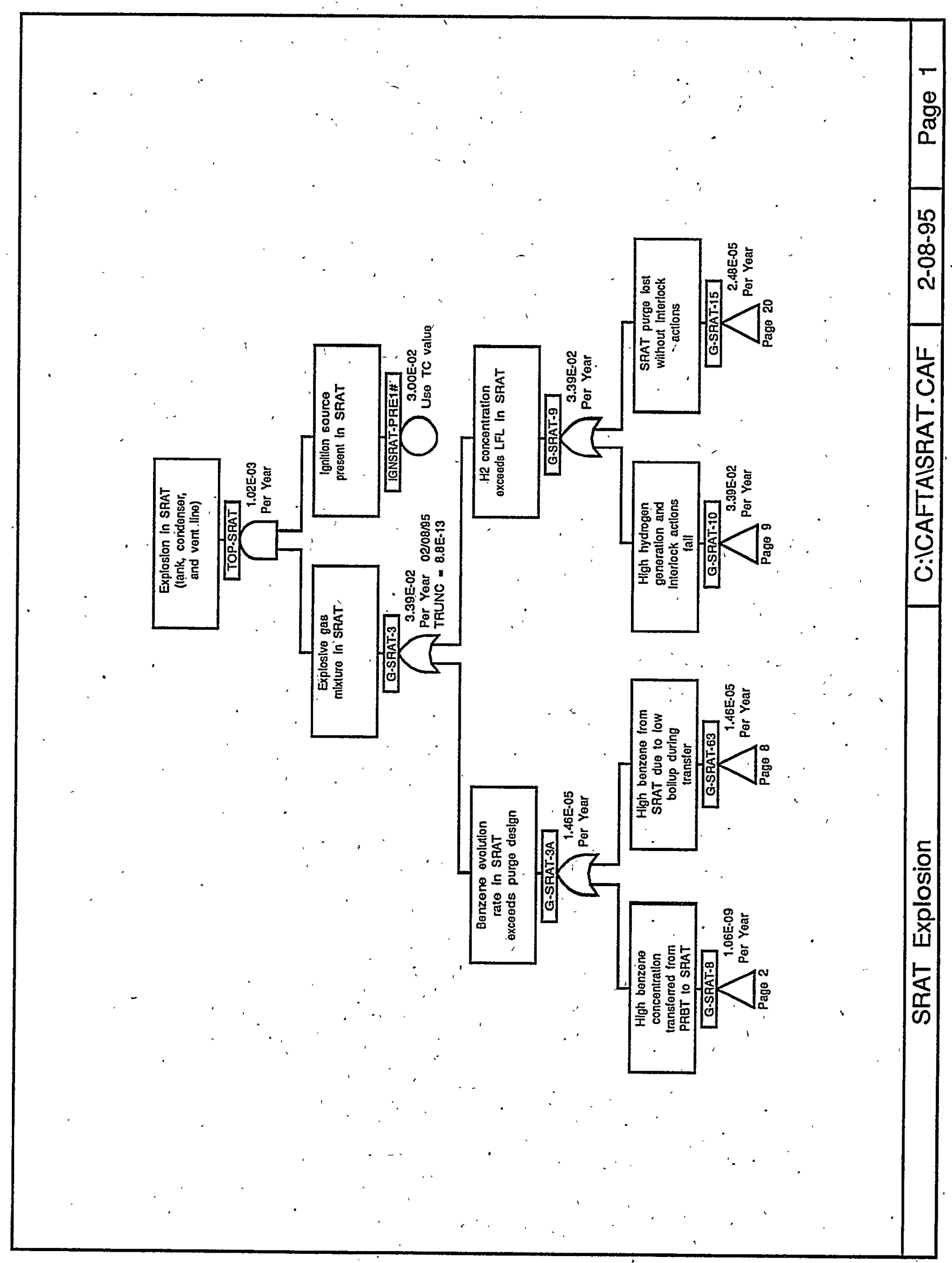




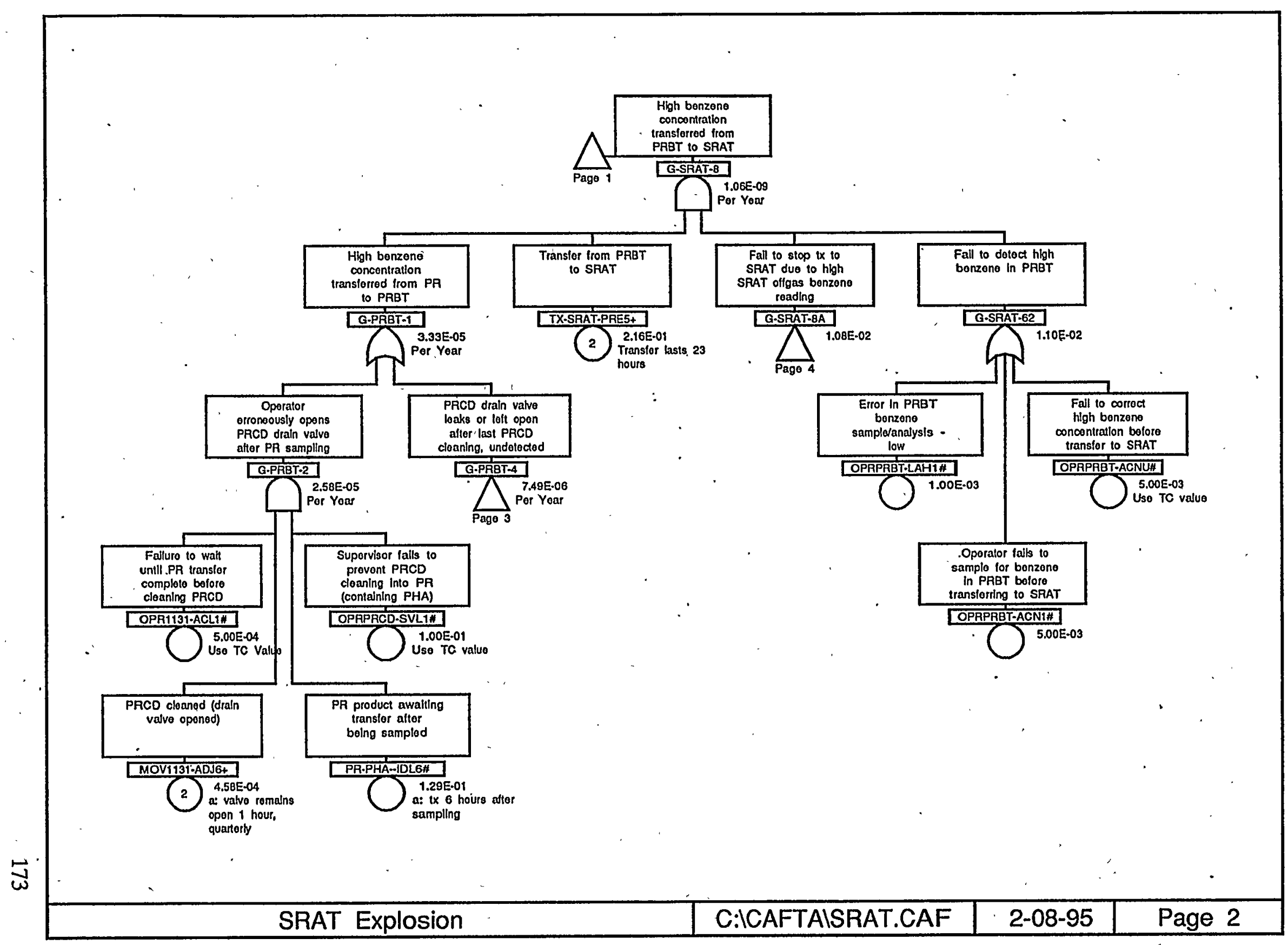




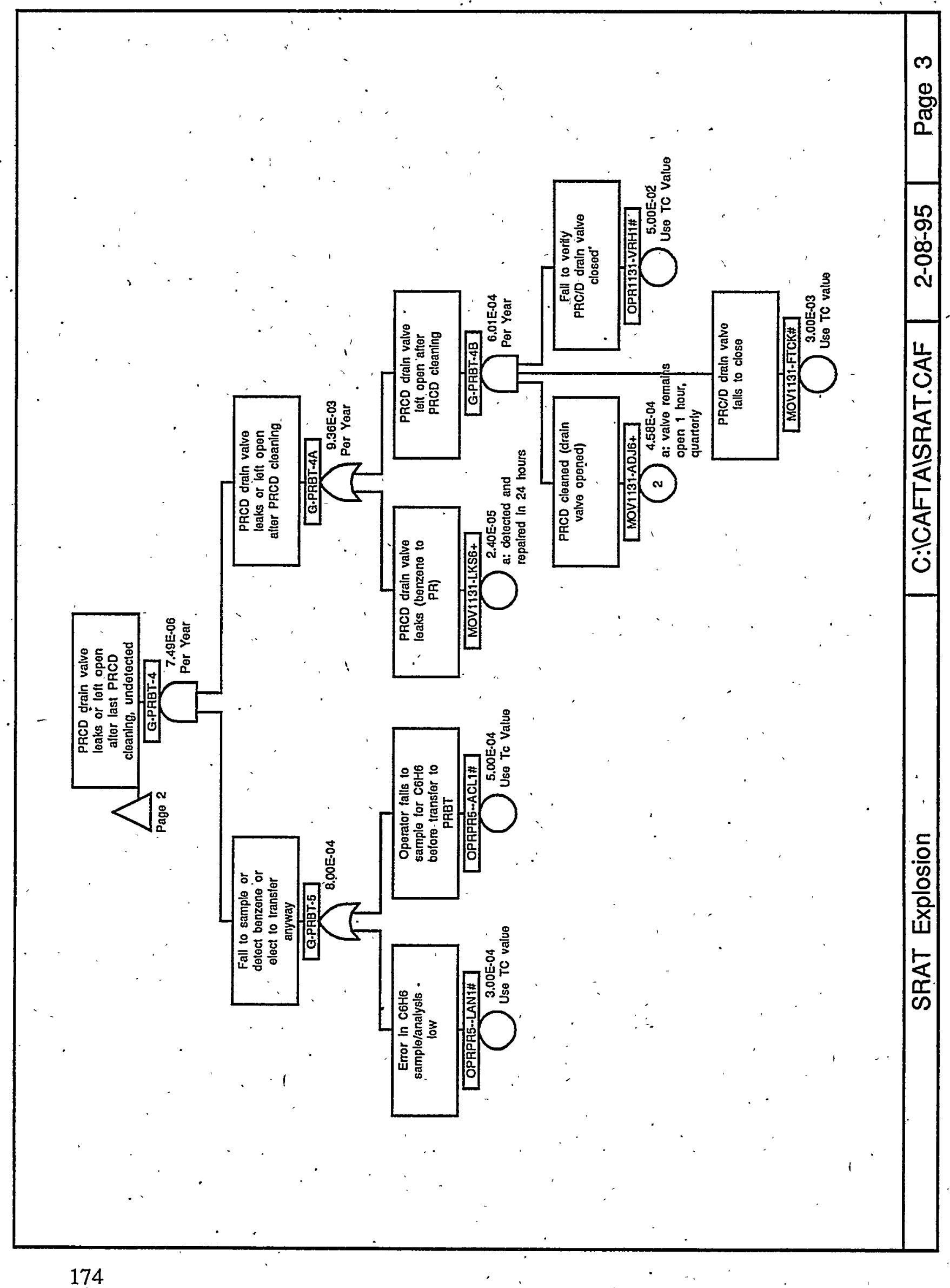




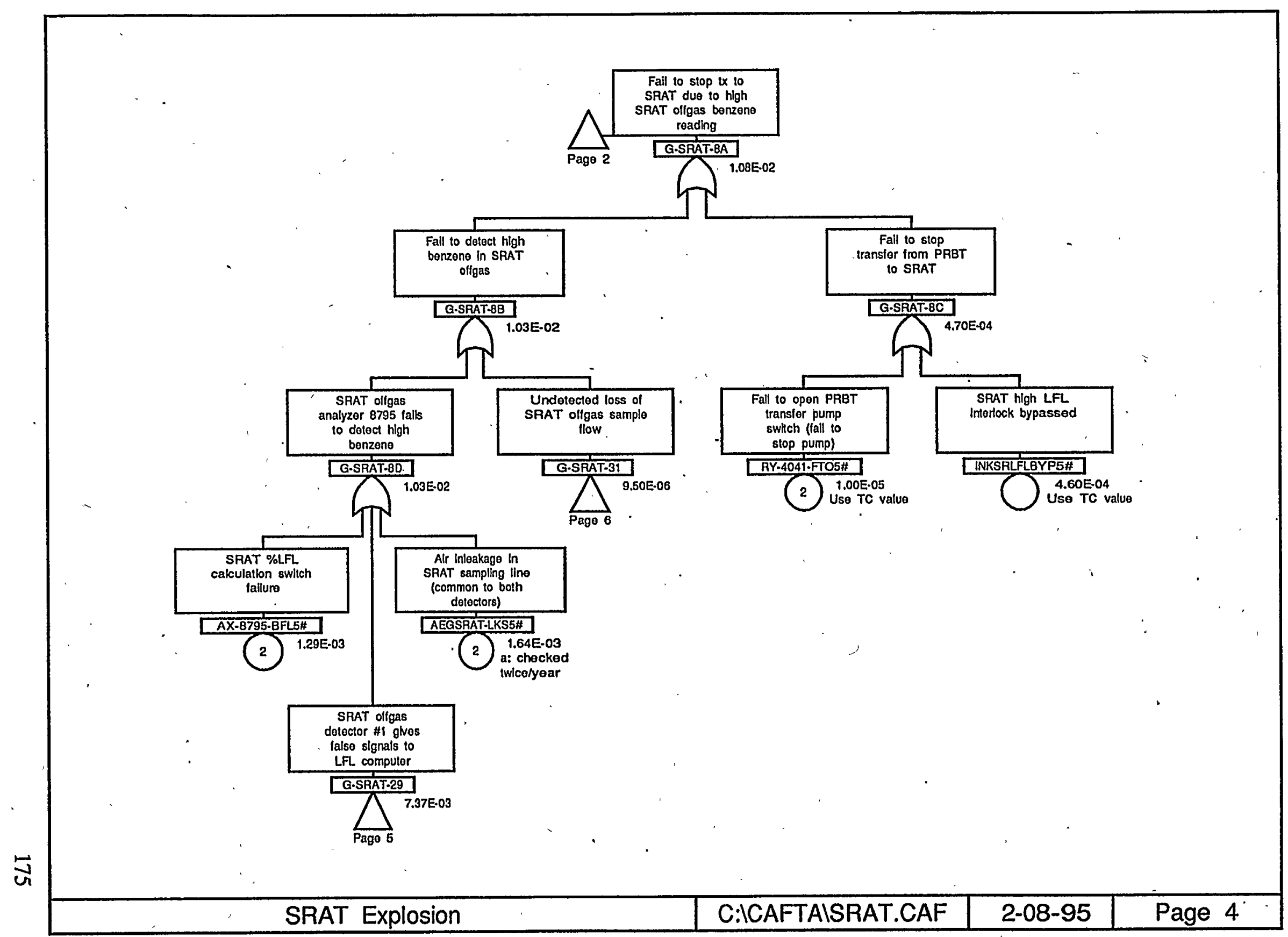


X-ESR-S-00001, REV. 0

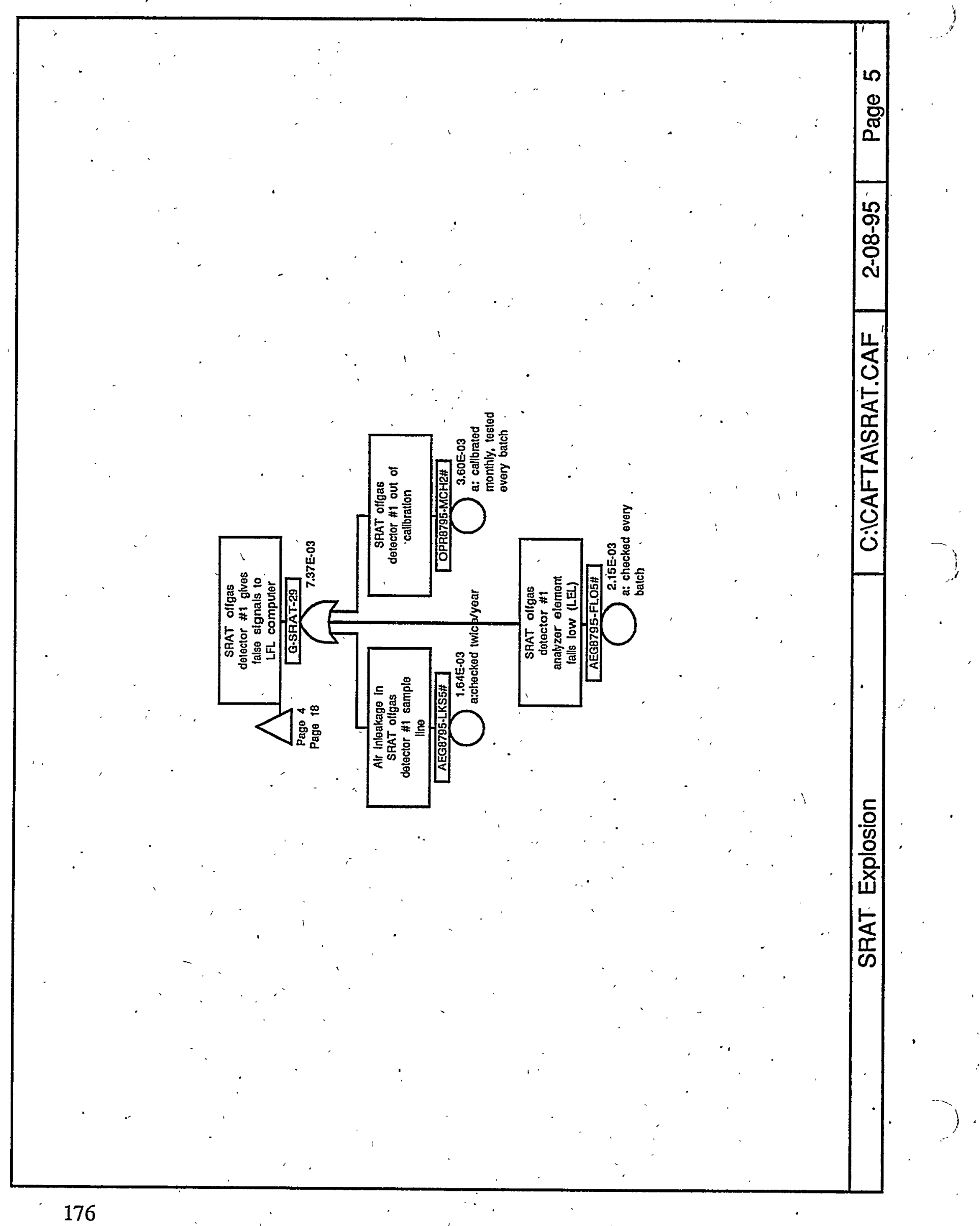




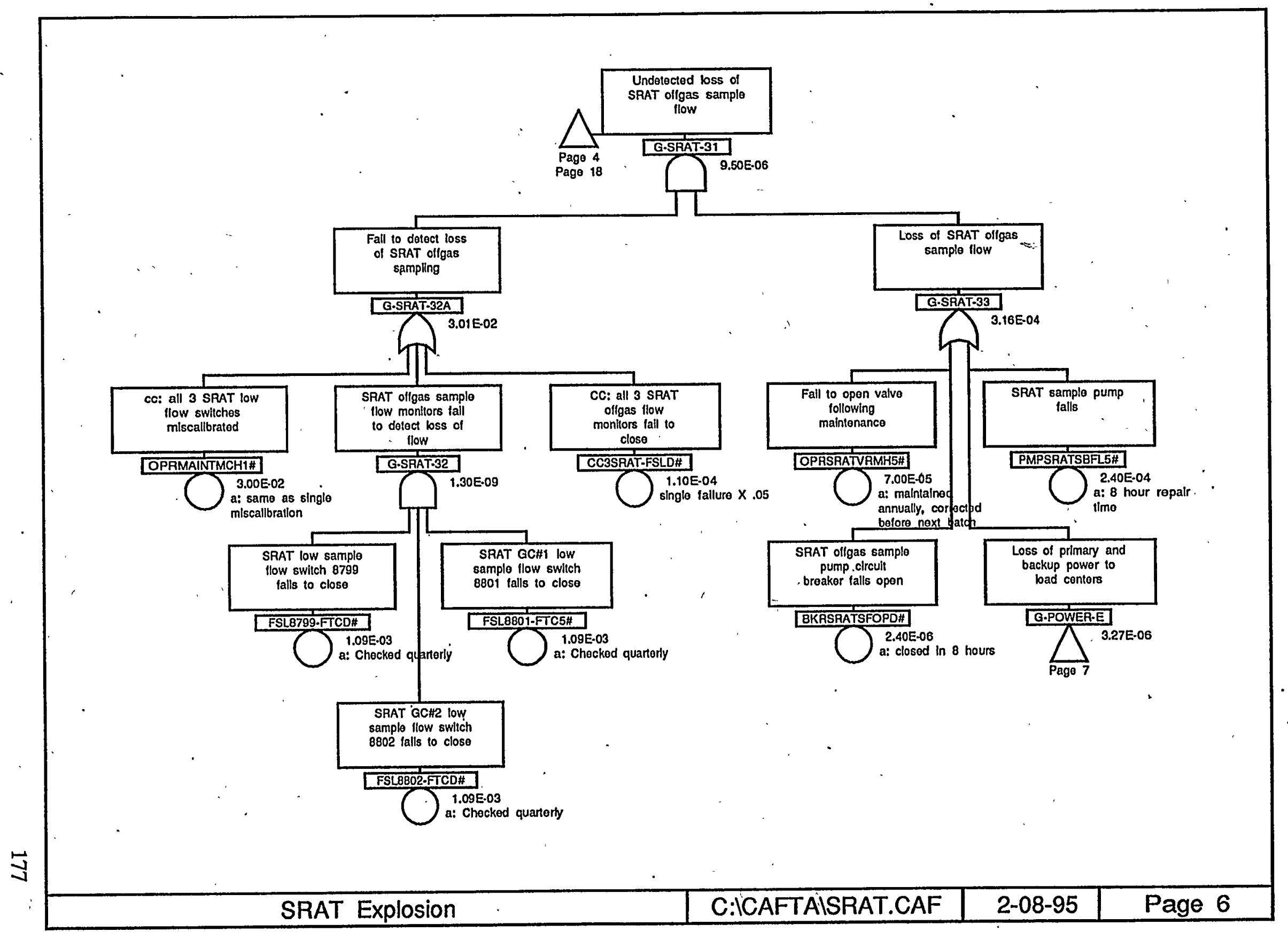


X-ESR-S-00001, REV. 0

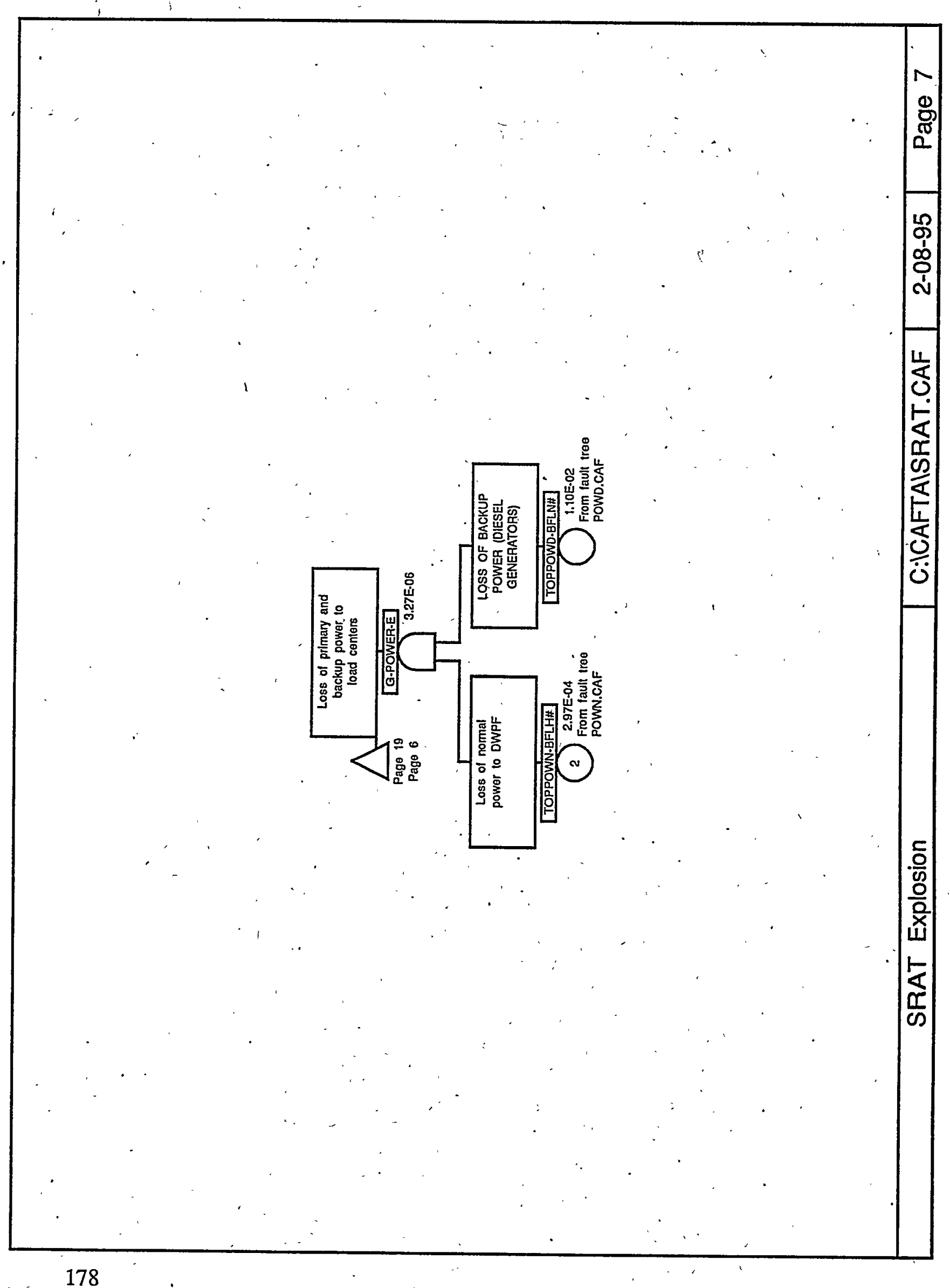




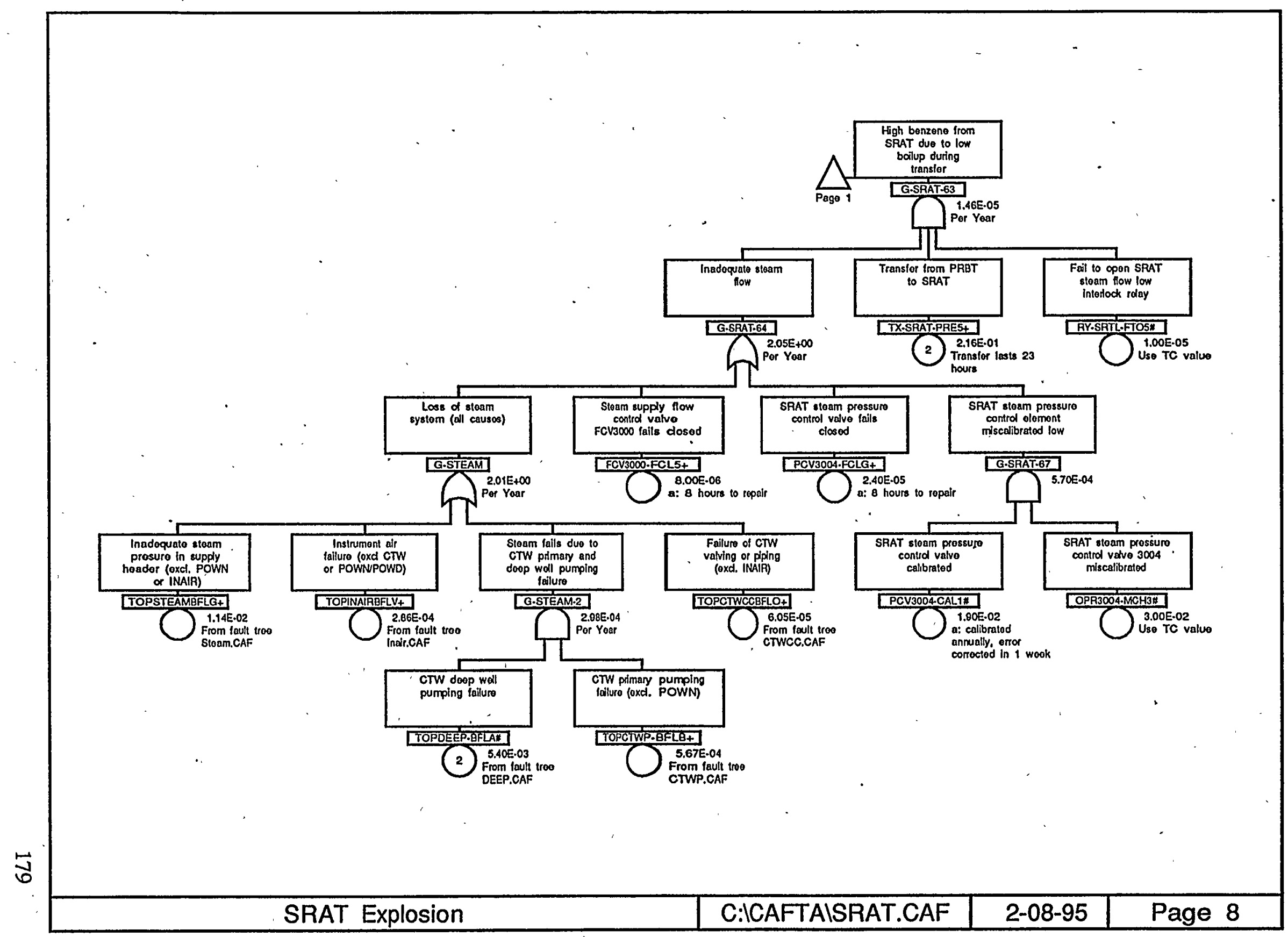




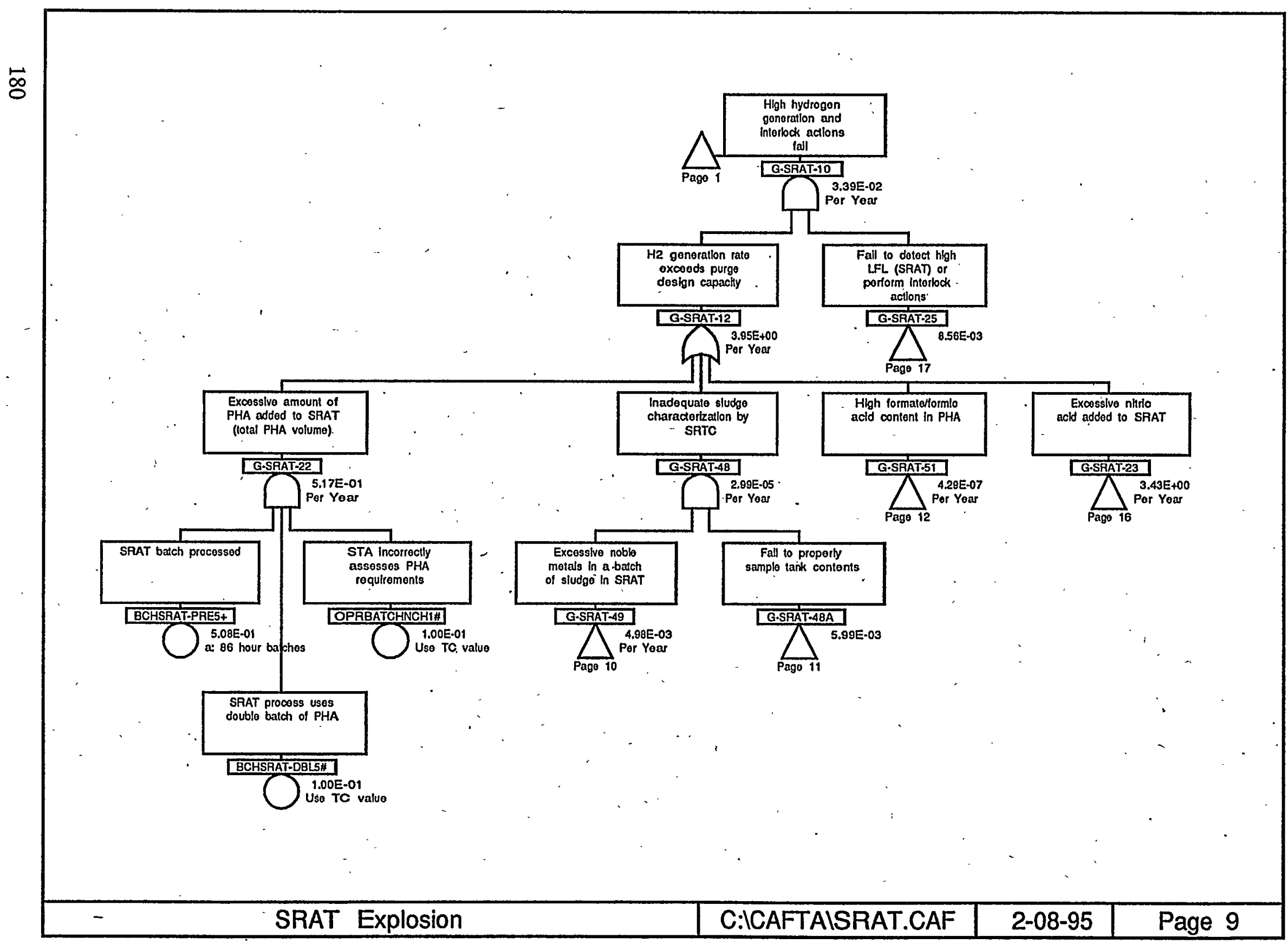




$$
g_{0}
$$


X-ESR-S-00001, REV. 0

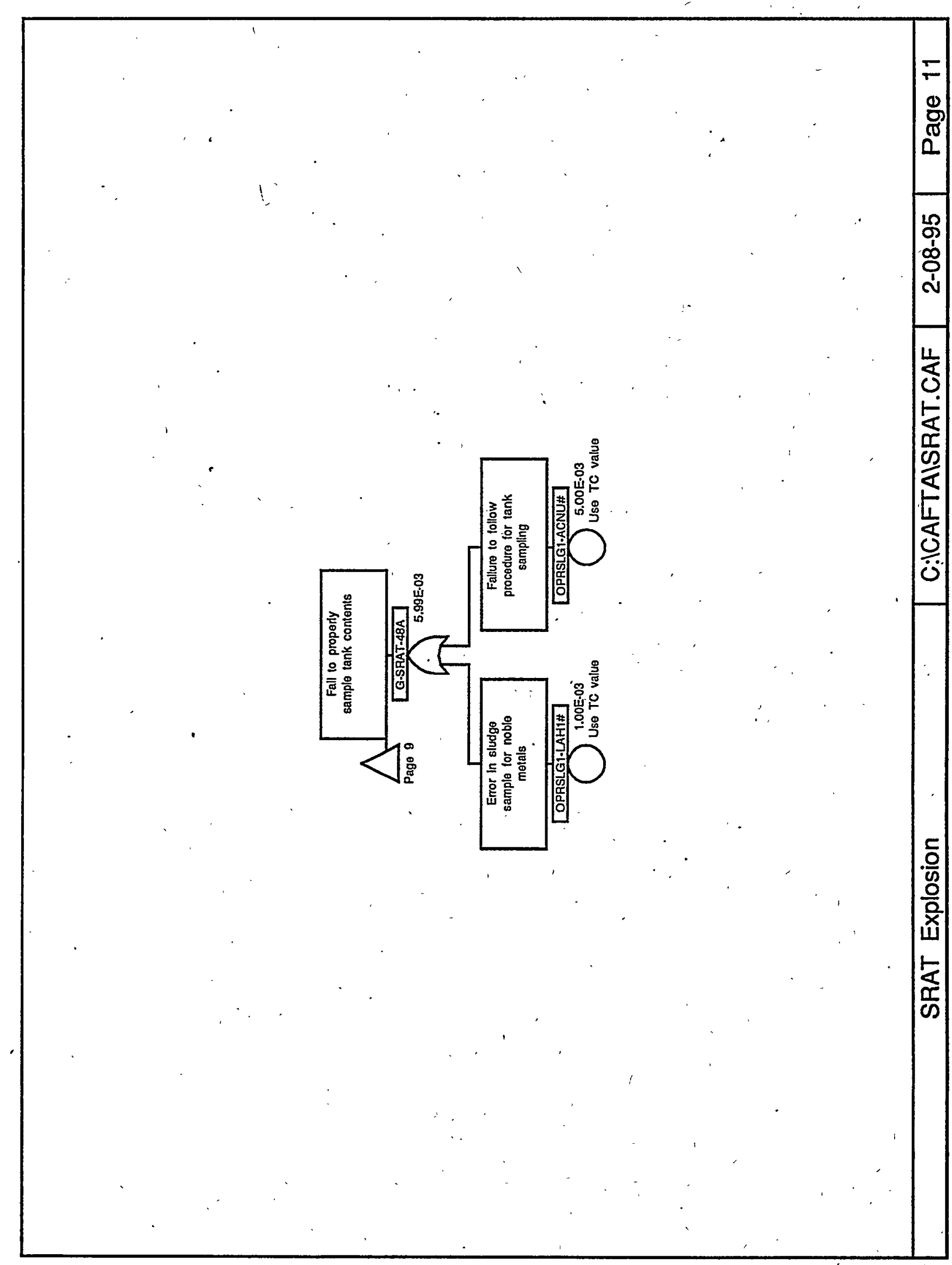




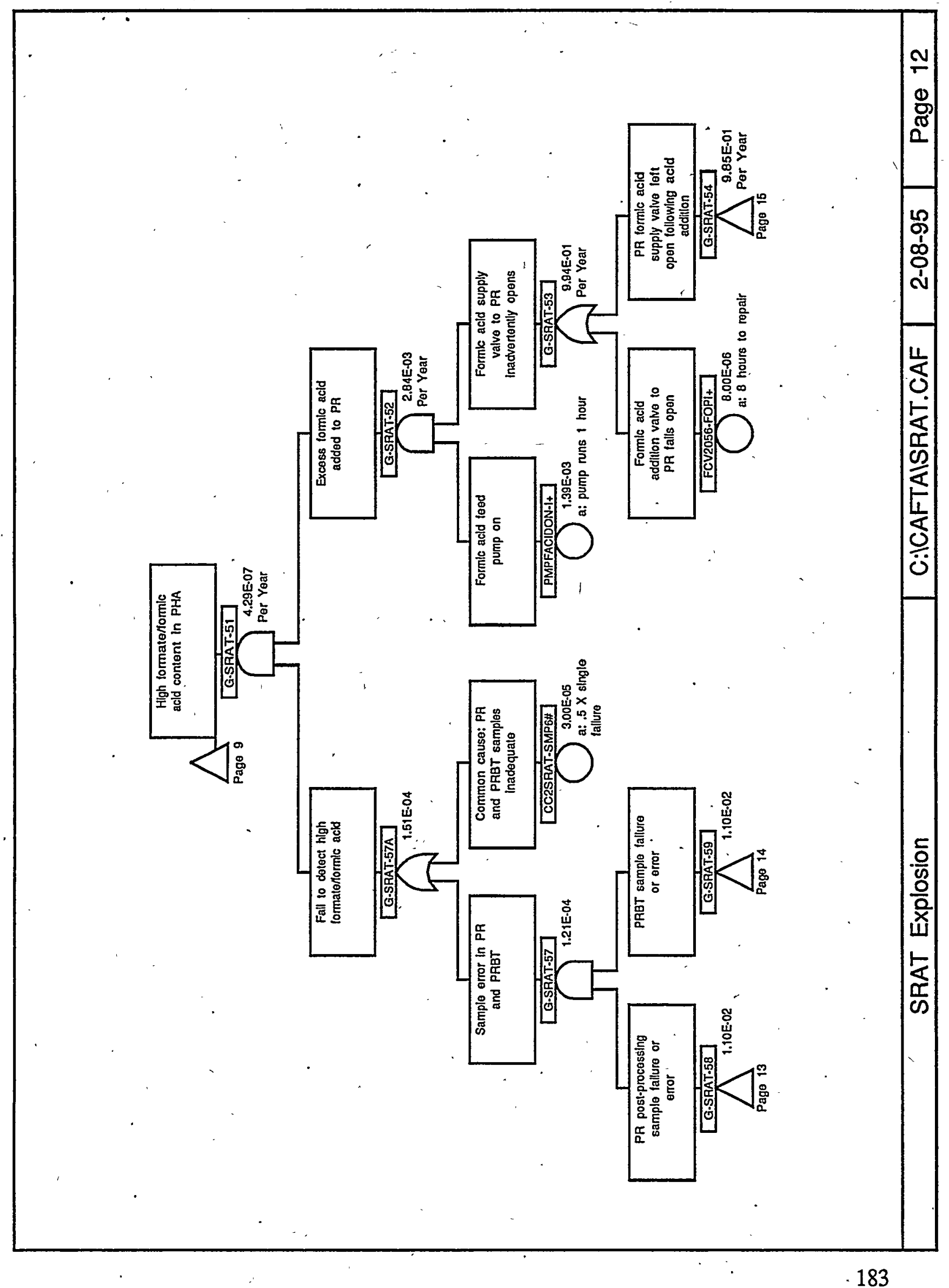


X-ESR-S-00001, REV. 0

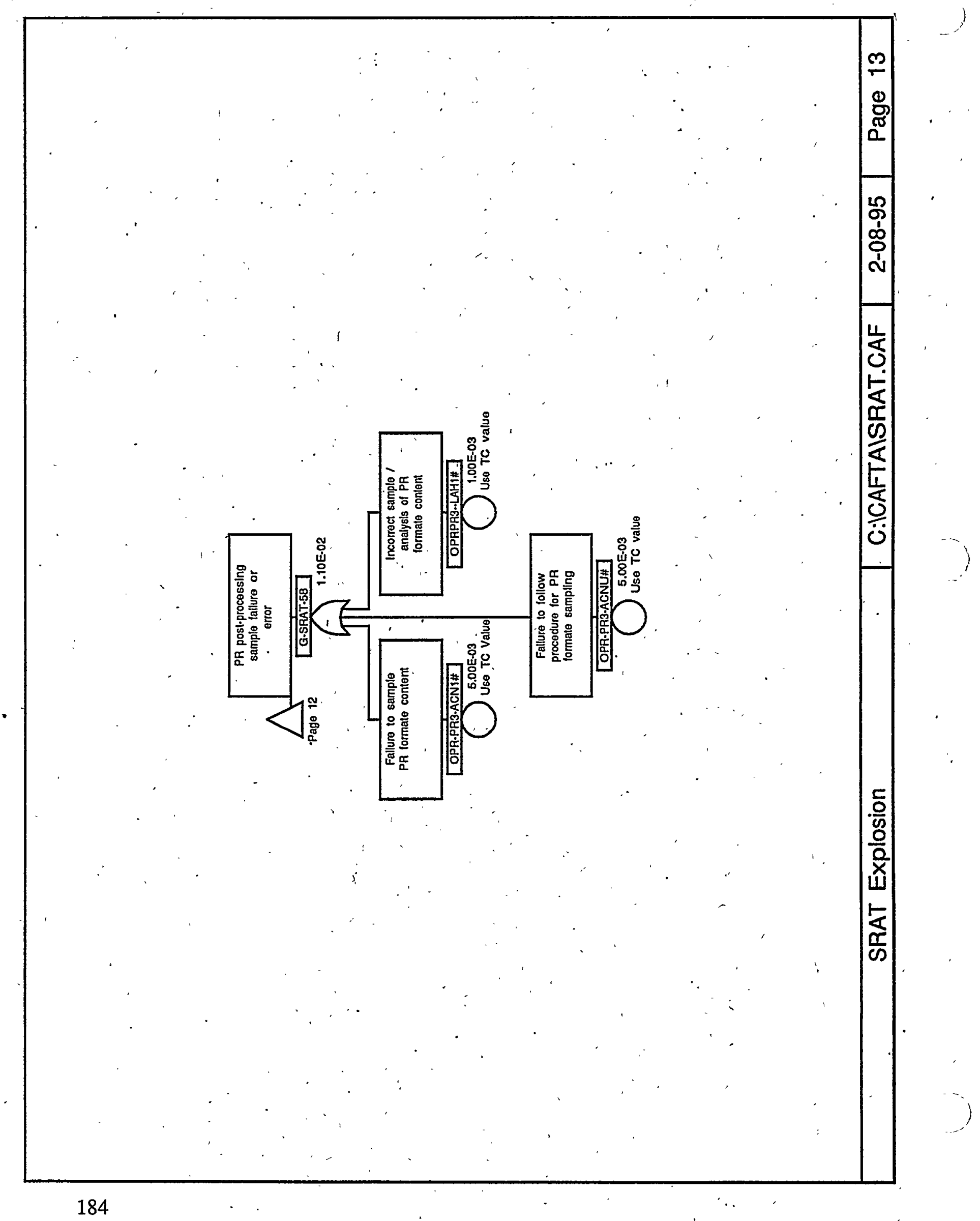


। X-ESR-S-00001, REV. 0

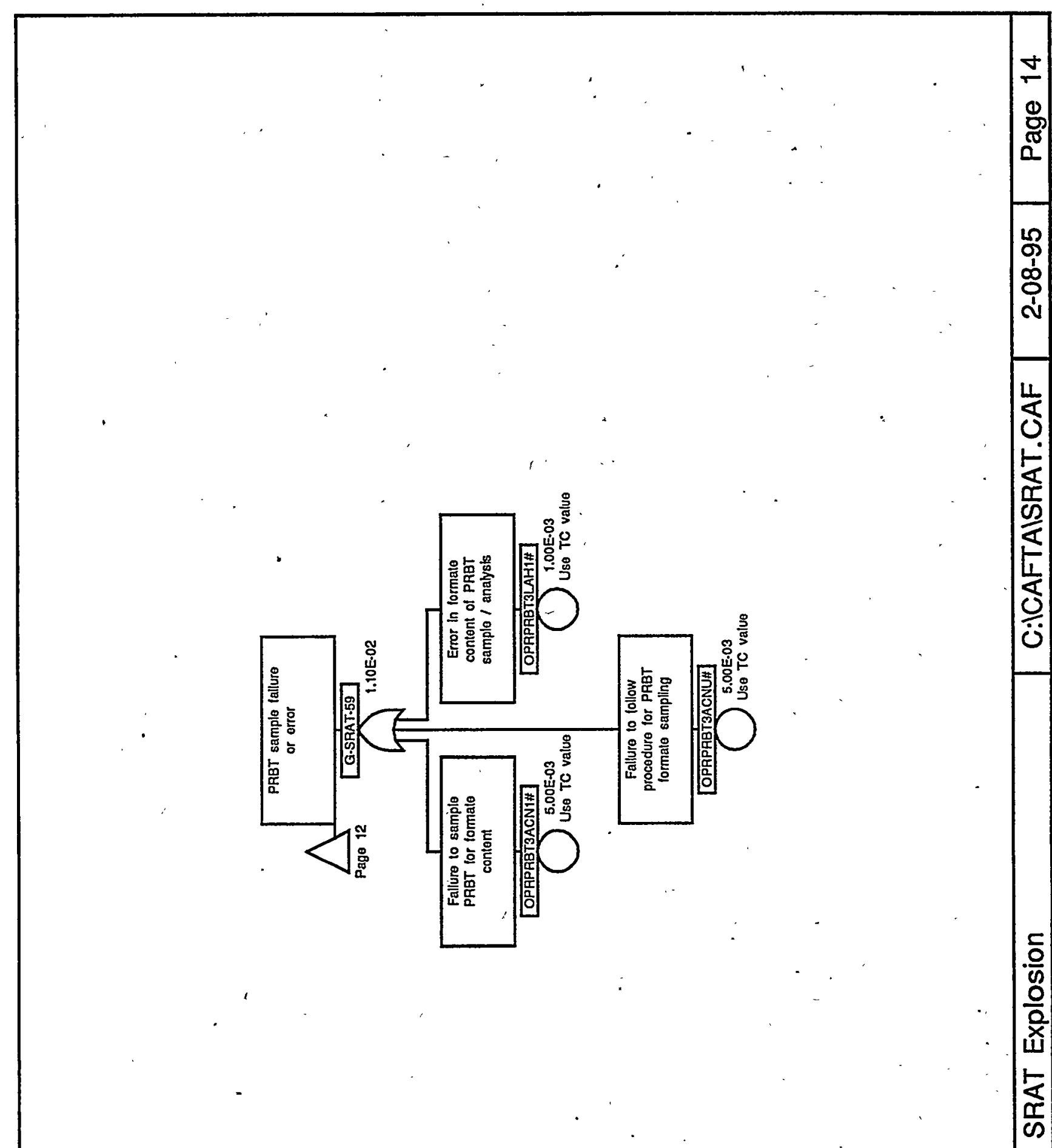


X-ESR-S-00001, REV. 0

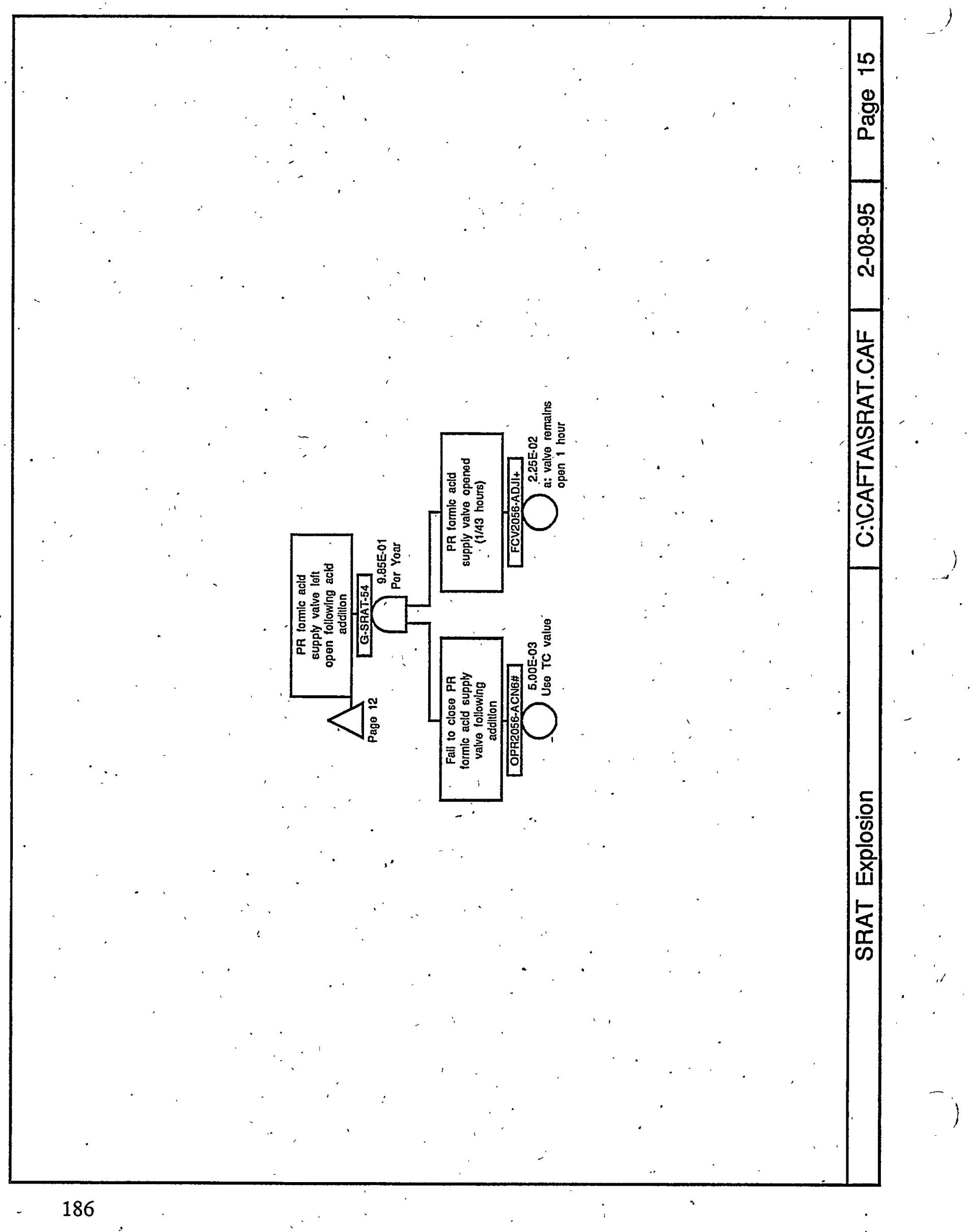




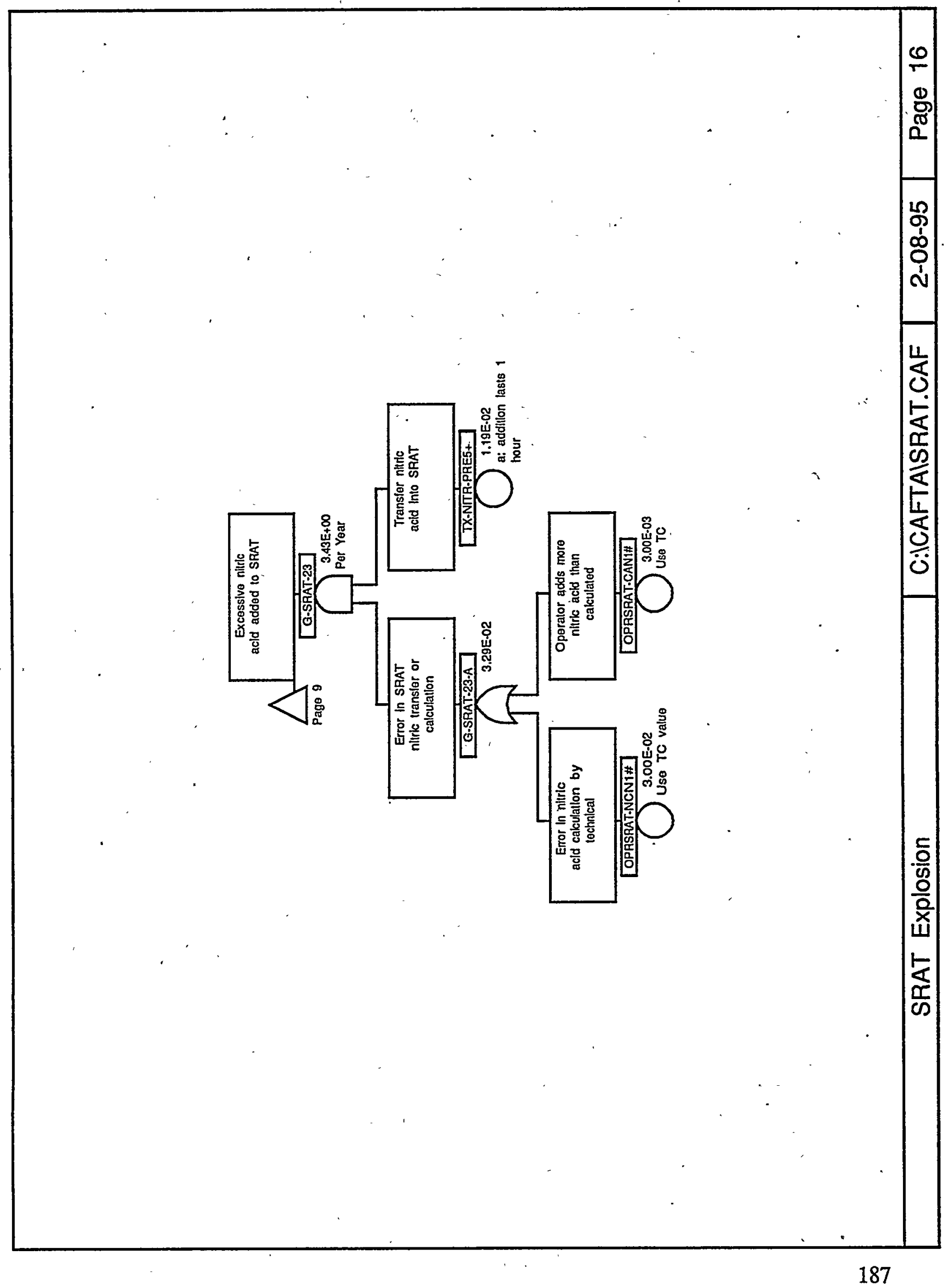


X-ESR-S-00001, REV. 0

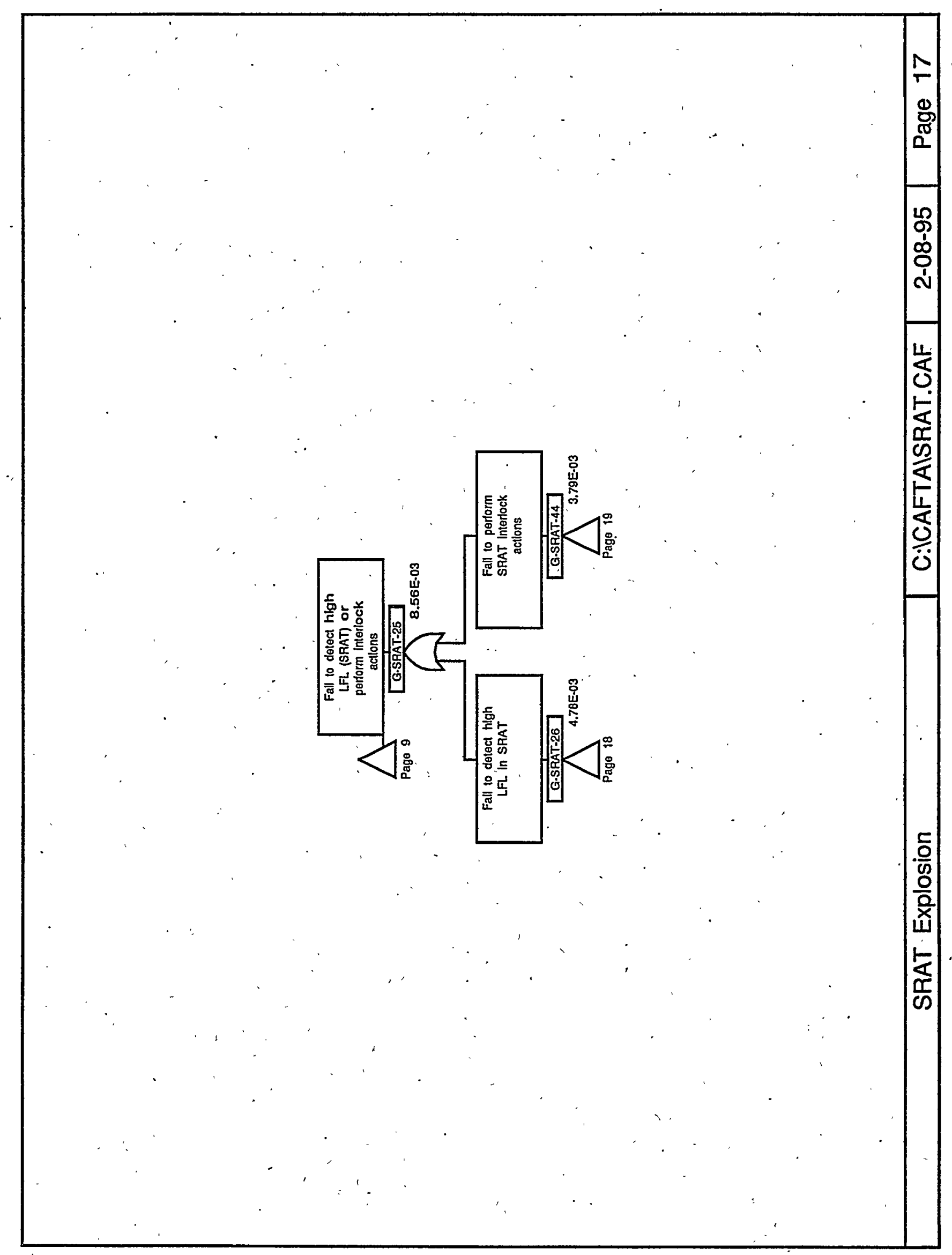




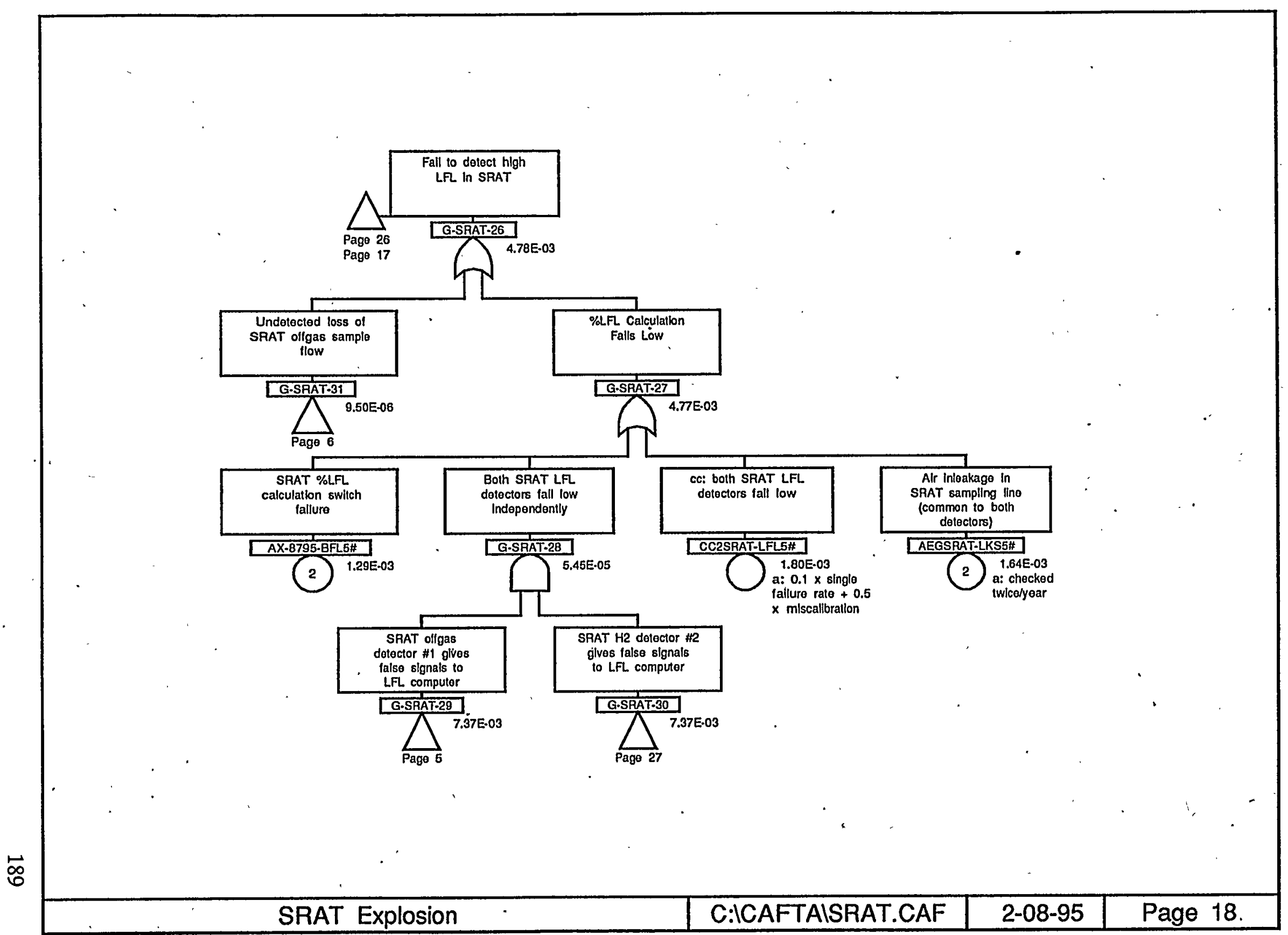


X-ESR-S-00001, REV. 0

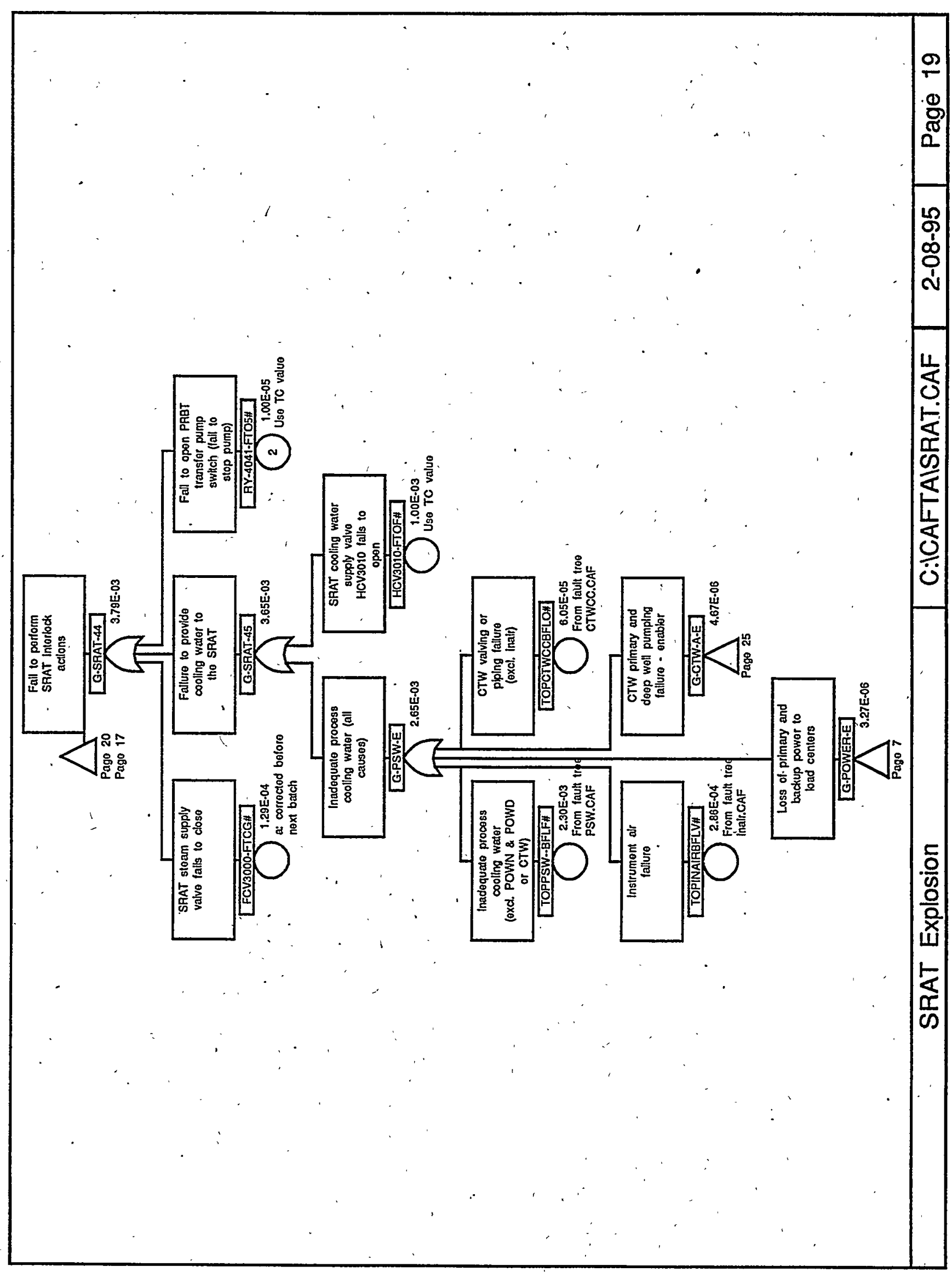




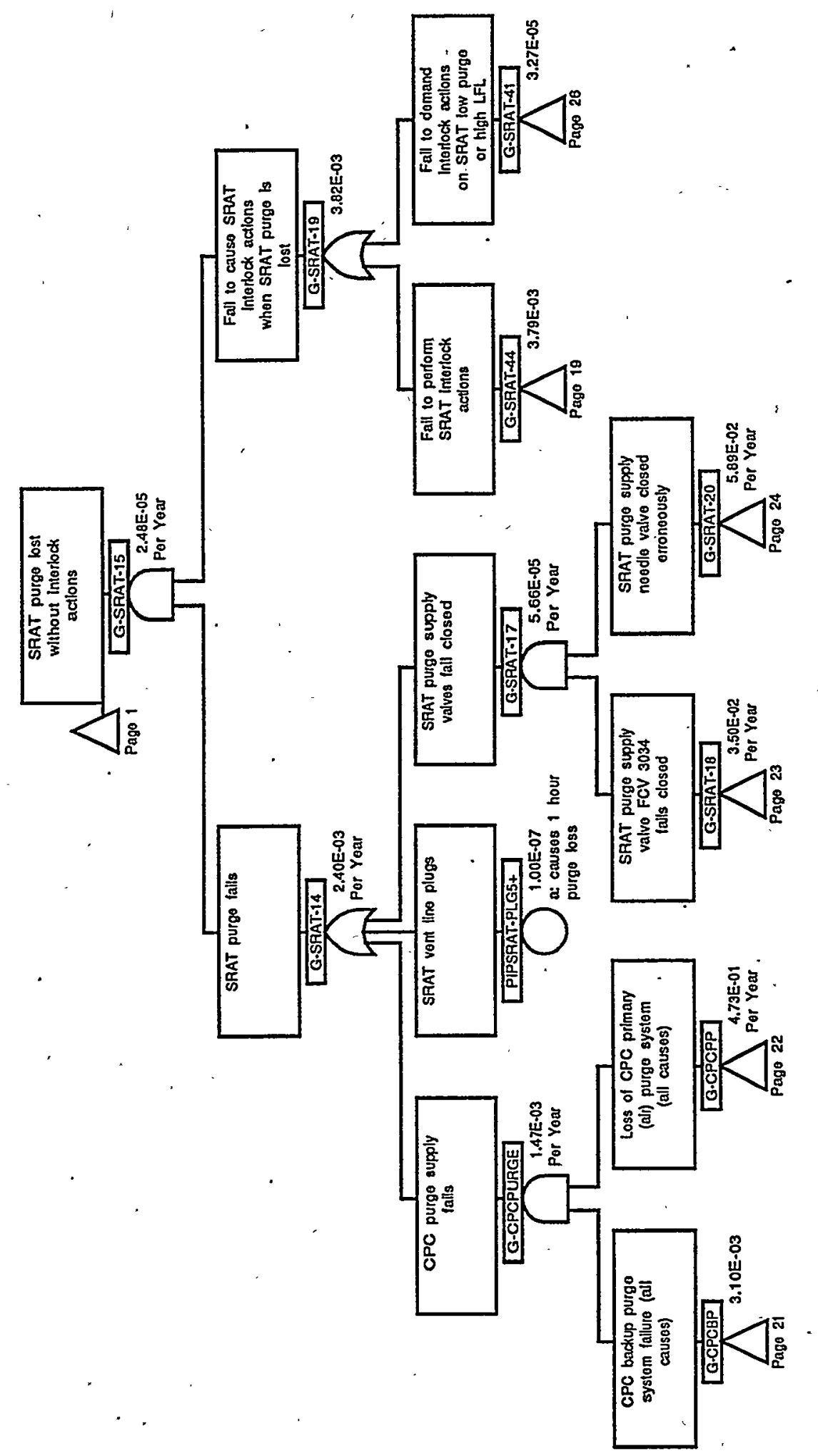


X-ESR-S-00001, REV. 0

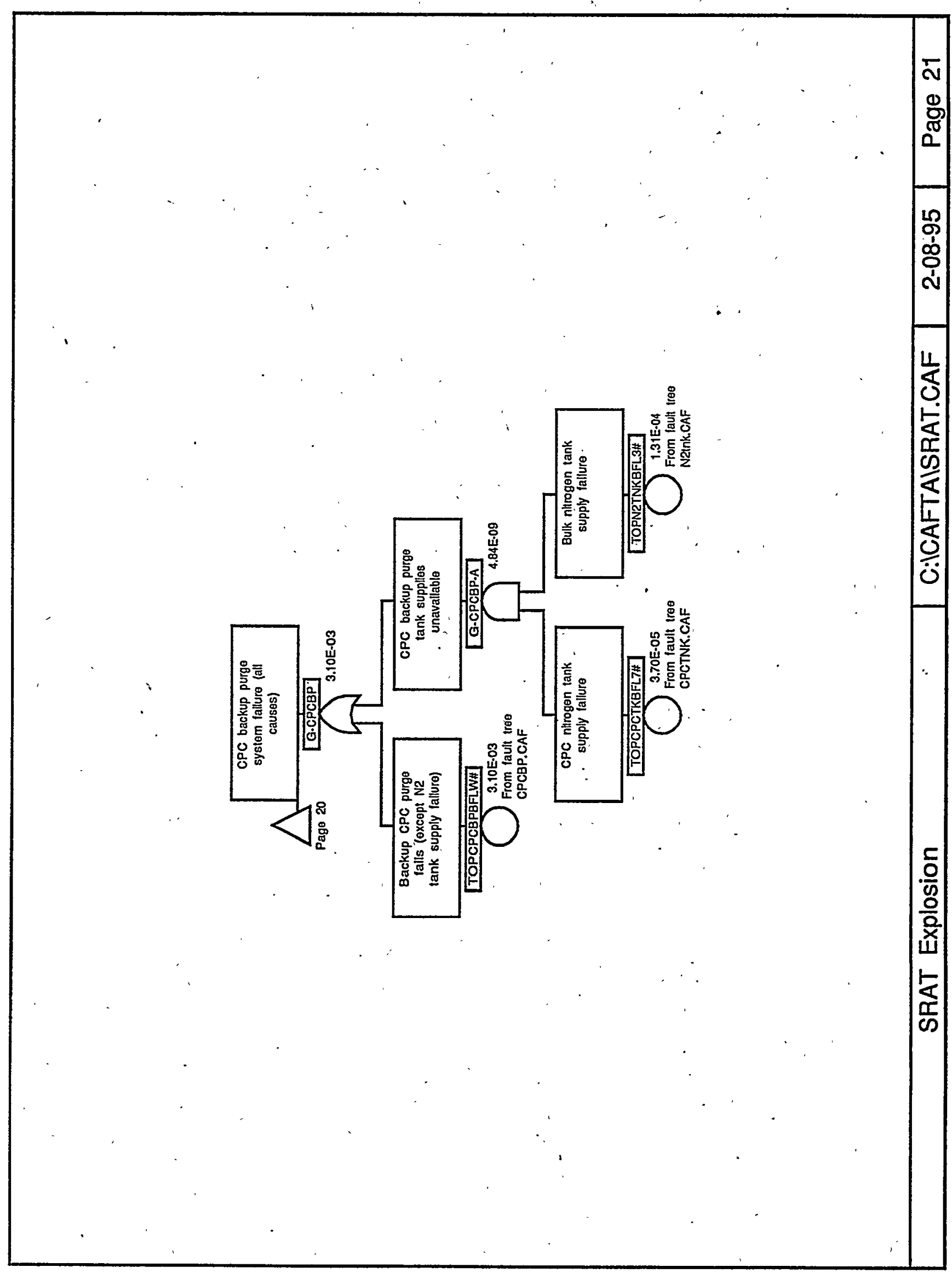




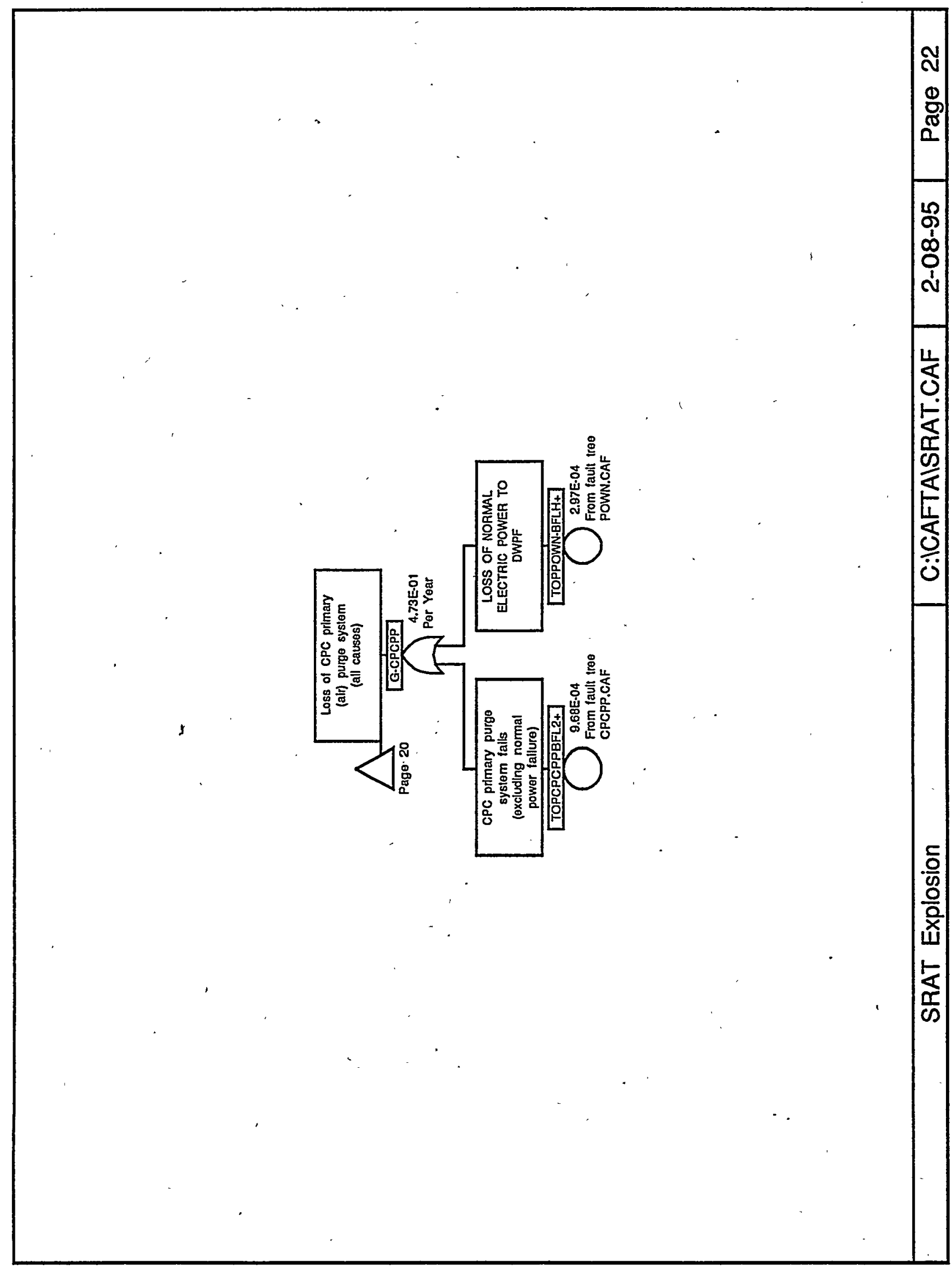


X-ESR-S-00001, REV. 0

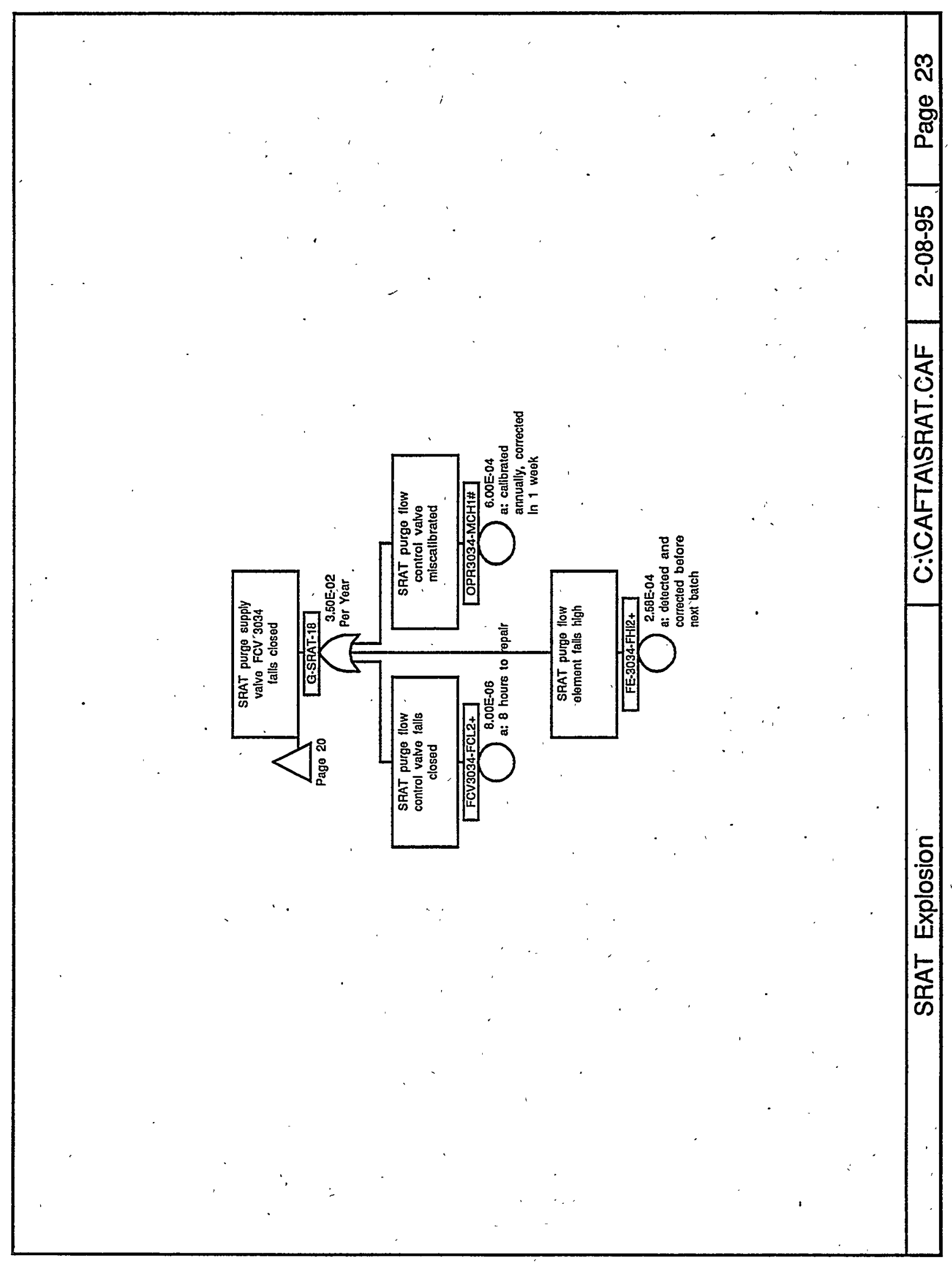




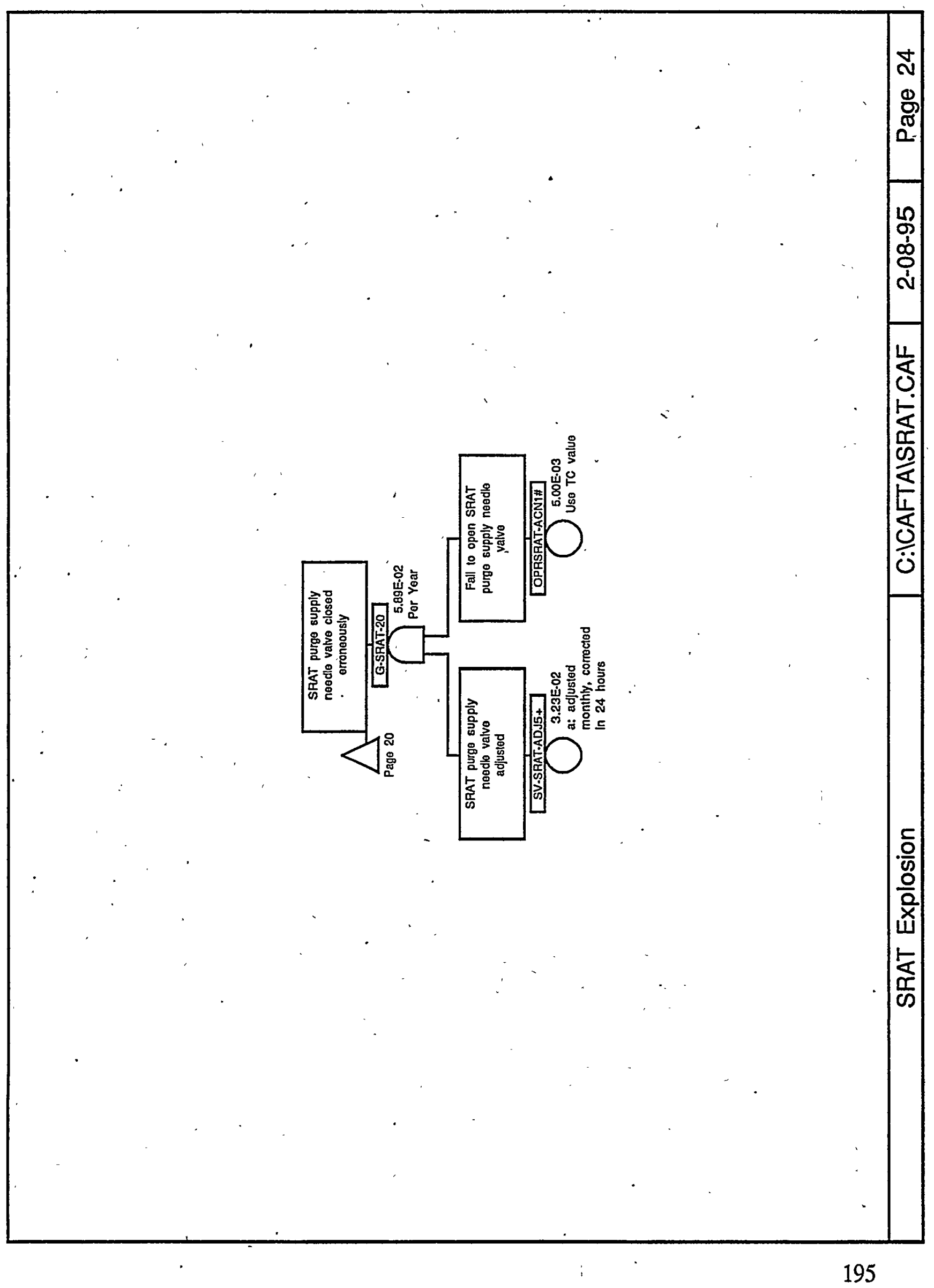


X-ESR-S-00001, REV. 0

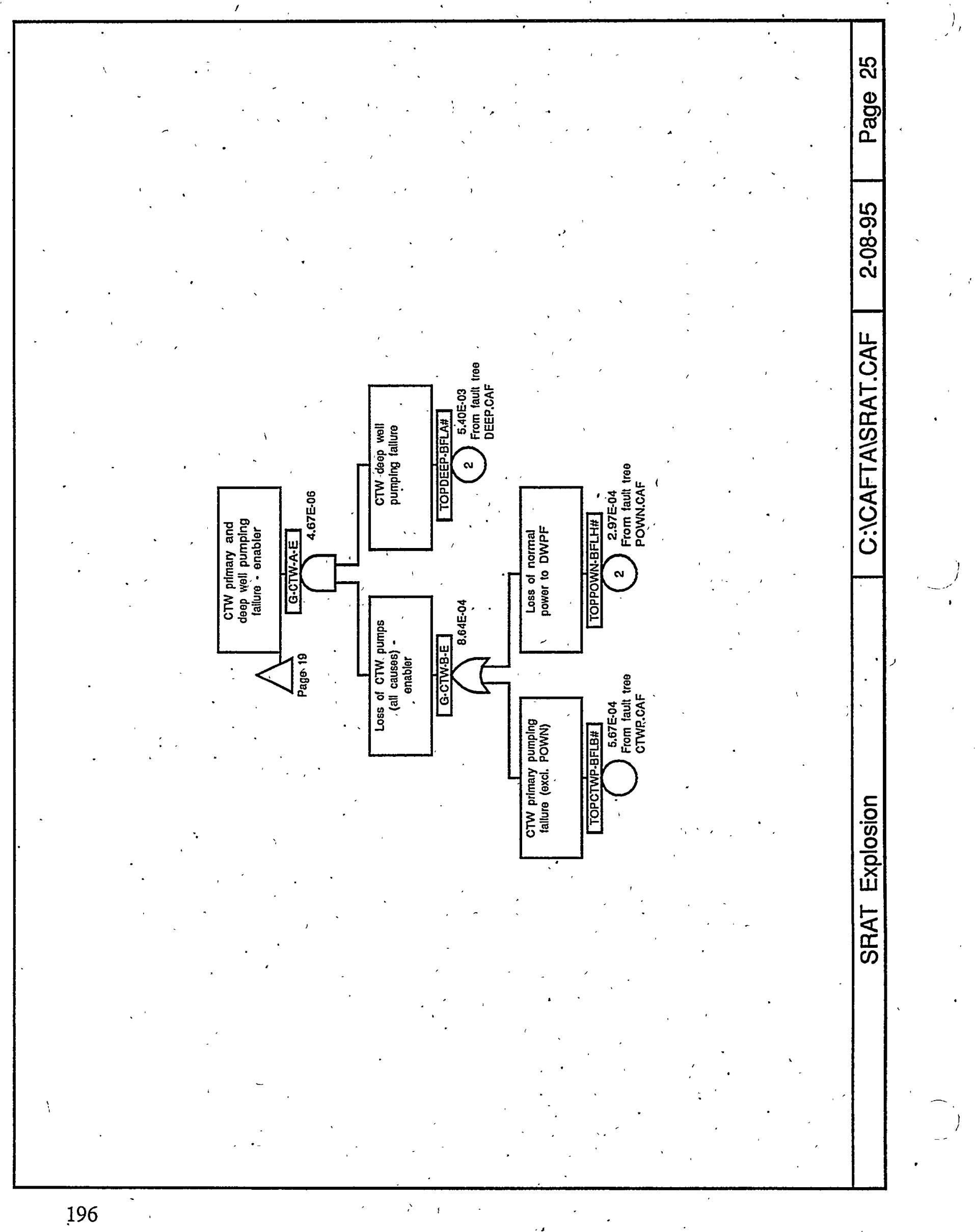




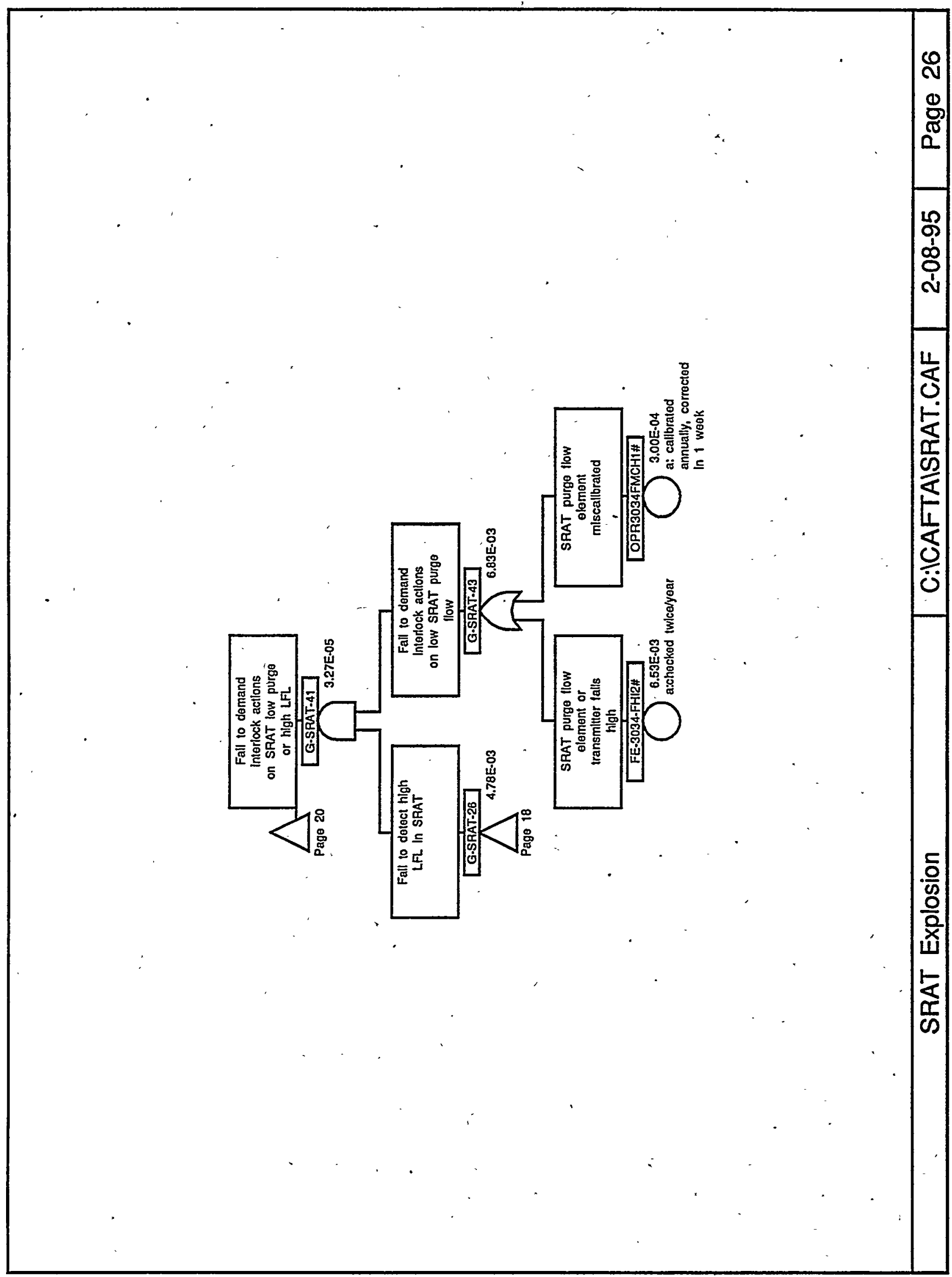


X-ESR-S-00001, REV. 0

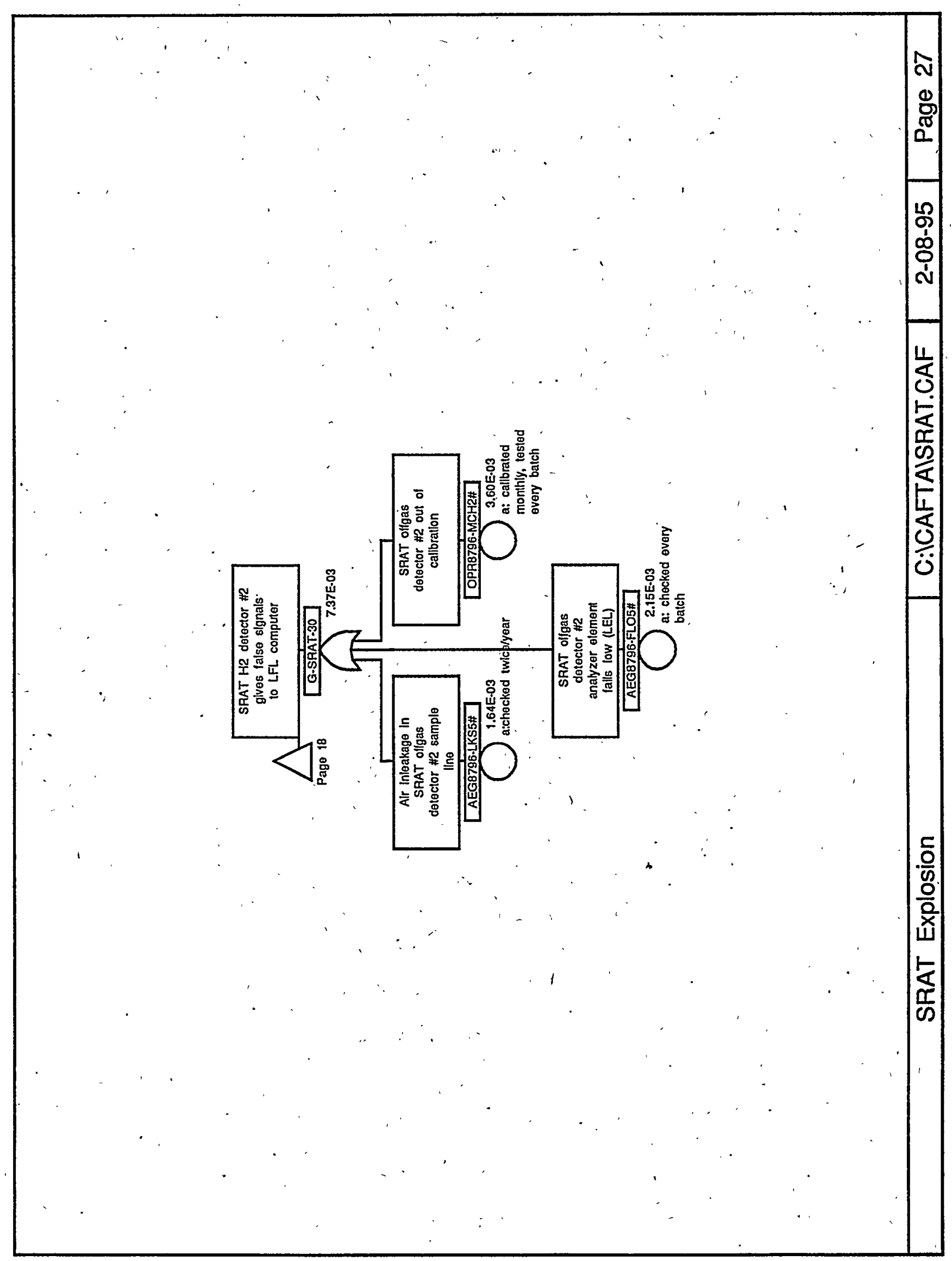




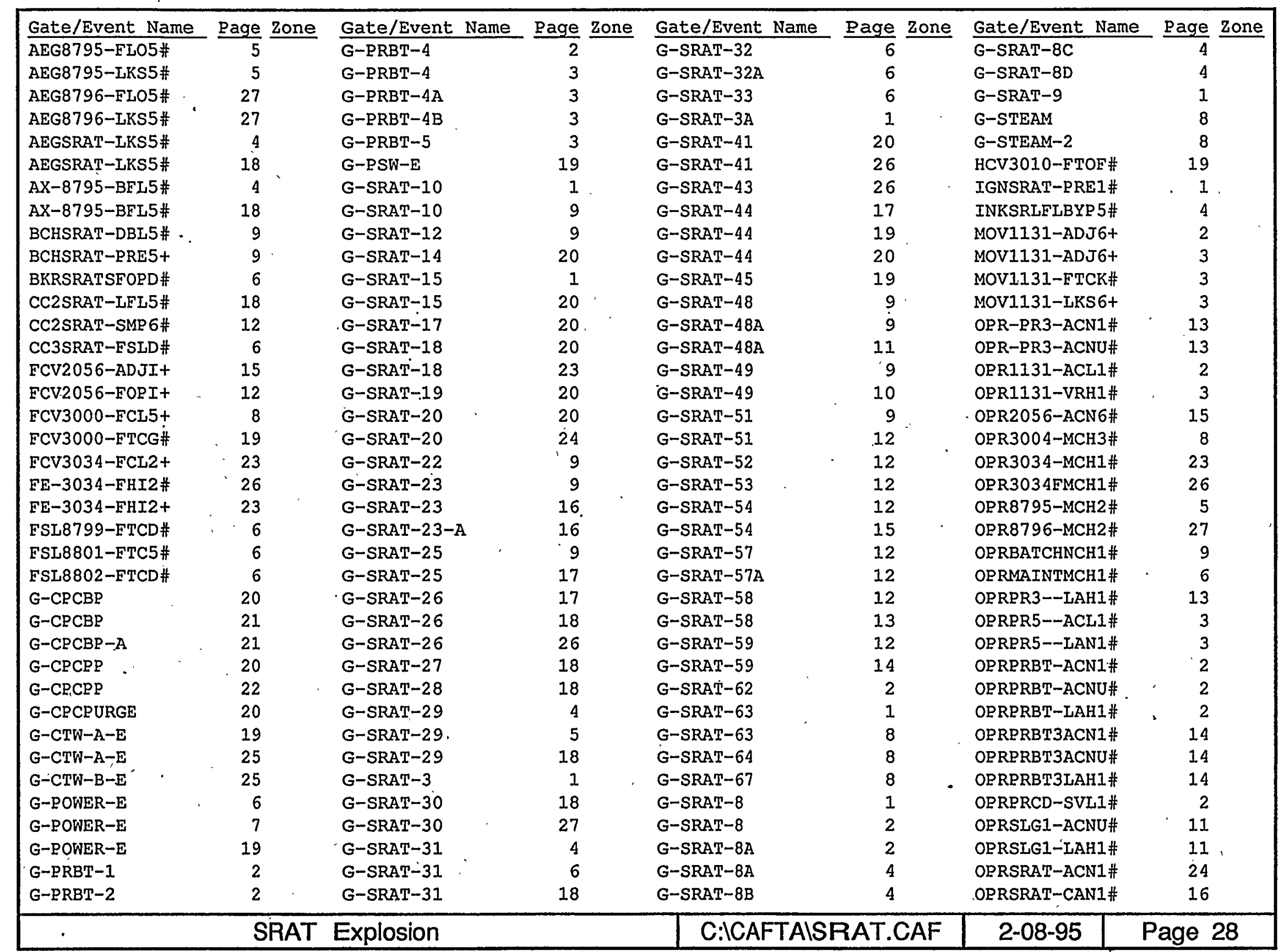




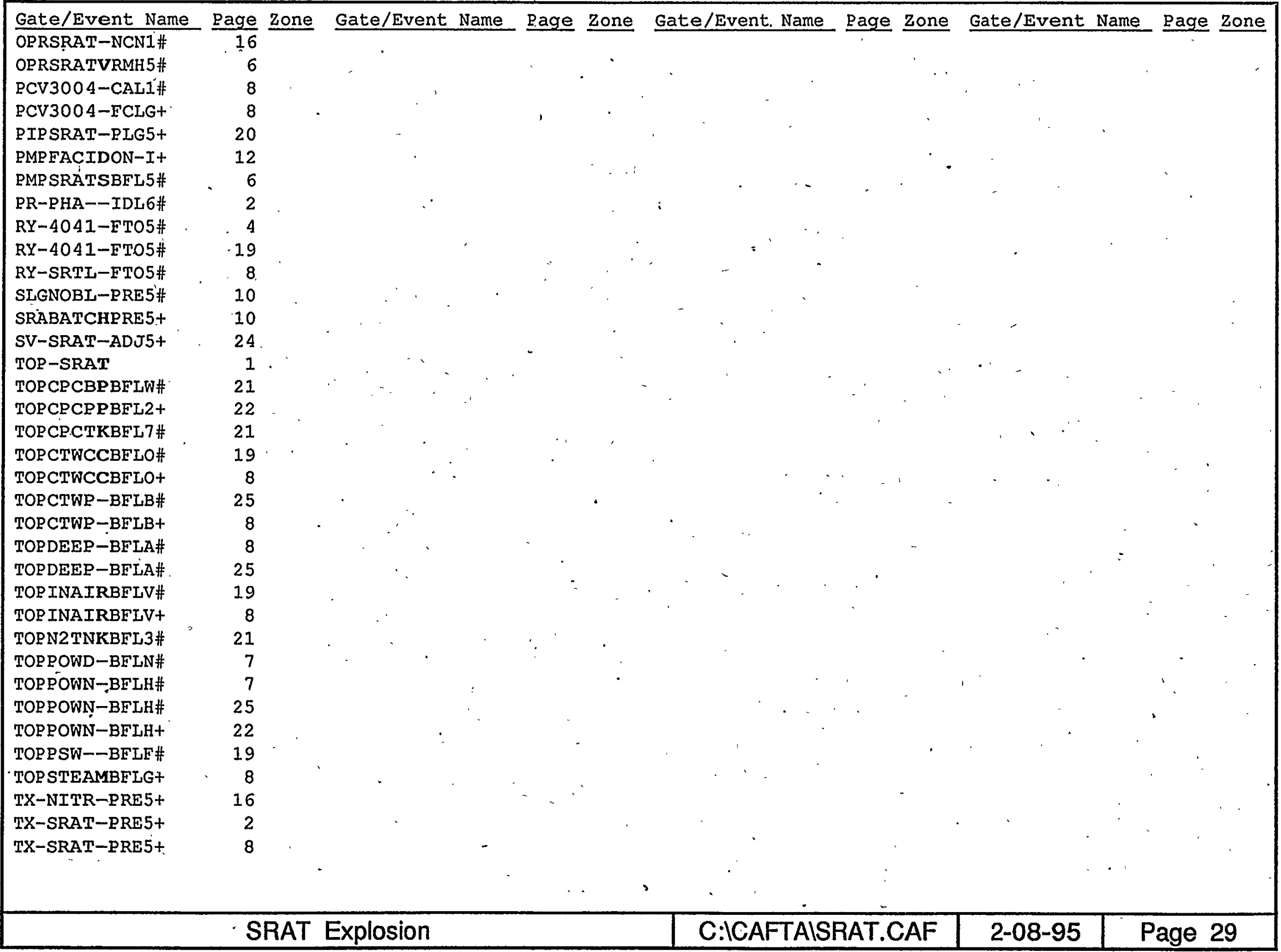




\begin{tabular}{|c|c|c|c|c|c|c|}
\hline $\begin{array}{l}\text { Set } \\
\text { No. }\end{array}$ & $\begin{array}{l}\text { Event } \\
\text { Name }\end{array}$ & Description & $\mathrm{c}$ & $\begin{array}{l}\text { B.E. } \\
\text { Input }\end{array}$ & $\begin{array}{l}\text { Calc. } \\
\text { Result }\end{array}$ & $\begin{array}{c}\text { Cutset } \\
\text { Ereq. }(/ y x)\end{array}$ \\
\hline 1. & $\begin{array}{l}\text { TOP-SRAT } \\
\text { IGNSRAT-PRE1\# } \\
\text { OPRSRAT-NCNI\# } \\
\text { TOPPSW--BELE\# } \\
\text { TX-NITR-PRE5+ }\end{array}$ & $\begin{array}{l}\text { Ignition source present in SRAT } \\
\text { Error in nitric acid calculation by technical } \\
\text { Inadequate process cooling water, (excl. POWN \& POWD or CTW) } \\
\text { Transfer nitric acid into SRAT }\end{array}$ & 1 & $\begin{array}{r}1 \mathrm{~N} \\
3.0 \mathrm{E}-02 \mathrm{~N} \\
1 \mathrm{~N} \\
3.0 \mathrm{E}-02 \mathrm{~N} \\
1 \mathrm{~N} \\
2.3 \mathrm{E}-03 \mathrm{~N} \\
1 \mathrm{H} \\
1.2 \mathrm{E}-02 \mathrm{H}\end{array}$ & $\begin{array}{l}3.00 \mathrm{E}-02 \mathrm{~N} \\
3.00 \mathrm{E}-02 \mathrm{~N} \\
2.30 \mathrm{E}-03 \mathrm{~N} \\
1.19 \mathrm{E}-02\end{array}$ & $\begin{array}{l}1.02 \mathrm{E}-03 \\
2.15 \mathrm{E}-04\end{array}$ \\
\hline 2. & $\begin{array}{l}\text { CC2SRAT-IEL5\# } \\
\text { IGNSRAT-ERE1\# } \\
\text { OPRSRAT-NCN1\# } \\
\text { TX-NITR-PRE5+ }\end{array}$ & $\begin{array}{l}\text { cc: both SRAT IFL detectors fail low } \\
\text { Ignition source present in SRAT } \\
\text { Error in nitric acid calculation by technical } \\
\text { Transfer nitric acid into SRAT }\end{array}$ & $\begin{array}{l}5 \\
1\end{array}$ & $\begin{array}{r}1 \mathrm{M} \\
5.0 \mathrm{E}-06 \mathrm{H} \\
1 \mathrm{~N} \\
3.0 \mathrm{E}-02 \mathrm{~N} \\
1 \mathrm{~N} \\
3.0 \mathrm{E}-02 \mathrm{~N} \\
1 \mathrm{H} \\
1.2 \mathrm{E}-02 \mathrm{H}\end{array}$ & $\begin{array}{c}1.80 \mathrm{E}-03 . \\
3.00 \mathrm{E}-02 \mathrm{~N} \\
3.00 \mathrm{E}-02 \mathrm{~N} \\
1.19 \mathrm{E}-02\end{array}$ & $1.68 \mathrm{E}-04$ \\
\hline 3. & $\begin{array}{l}\text { AEGSRAT-LKS5\# } \\
\text { IGNSRAT-PREI\# } \\
\text { ORRSRAT-NCN1\# } \\
\text { TX-NITR-PRE5+ }\end{array}$ & $\begin{array}{l}\text { Air inleakage in SRAT sampling line (common to both } \\
\text { detectors) } \\
\text { Ignition source present in SRAT } \\
\text { Error in nitric acid calculation by technical } \\
\text { Transfer nitric acid into SRAT }\end{array}$ & $\begin{array}{l}5 \\
1\end{array}$ & $\begin{array}{r}7.5 \mathrm{Y} \\
7.5 \mathrm{E}-07 \mathrm{H} \\
1 \mathrm{~N} \\
3.0 \mathrm{E}-02 \mathrm{~N} \\
1 \mathrm{~N} \\
3.0 \mathrm{E}-02 \mathrm{~N} \\
1 \mathrm{H} \\
1.2 \mathrm{E}-02 \mathrm{H}\end{array}$ & $\begin{array}{l}1.64 \mathrm{E}-03 \\
3.00 \mathrm{E}-02 \mathrm{~N} \\
3.00 \mathrm{E}-02 \mathrm{~N} \\
1.19 \mathrm{E}-02\end{array}$ & $1.53 \mathrm{E}-04$ \\
\hline 4. & $\begin{array}{l}\text { AX-8795-BEL5\# } \\
\text { IGNSRAT-PRE1\# } \\
\text { ORRSRAT-NCNI\# } \\
\text { TX-NITR-PRE5+ }\end{array}$ & $\begin{array}{l}\text { SRAT \&LEL calculation switch failure. } \\
\text { Ignition source present in SRAT } \\
\text { Error in nitric acid calculation by technical } \\
\text { Transfer nitric acid into SRAT }\end{array}$ & $\begin{array}{l}3 \\
1\end{array}$ & $\begin{array}{r}43 \mathrm{H} \\
3.0 \mathrm{E}-05 \mathrm{H} \\
1 \mathrm{~N} \\
3.0 \mathrm{E}-02 \mathrm{~N} \\
1 \mathrm{~N} \\
3.0 \mathrm{E}-02 \mathrm{~N} \\
1 \mathrm{H} \\
1.2 \mathrm{E}-02 \mathrm{H}\end{array}$ & $\begin{array}{l}1.29 \mathrm{E}-03 \\
3.00 \mathrm{E}-02 \mathrm{~N} \\
3.00 \mathrm{E}-02 \mathrm{~N} \\
1.19 \mathrm{E}-02\end{array}$ & $1.21 E-04$ \\
\hline 5. & $\begin{array}{l}\text { HCV3010--FTOF\# } \\
\text { IGNSRAT-PRE1\# } \\
\text { ORRSRAT-NCN1\# } \\
\text { TX-NITR-PRE5+ }\end{array}$ & $\begin{array}{l}\text { SRAT cooling water supply valve HCV3010 fails to open } \\
\text { Ignition source present in SRAT } \\
\text { Error in nitric acid calculation by technical } \\
\text { Transfer nitric acid into SRAT }\end{array}$ & $\begin{array}{l}1 \\
1\end{array}$ & $\begin{array}{r}1 \mathrm{~N} \\
1.0 \mathrm{E}-03 \mathrm{~N} \\
1 \mathrm{~N} \\
3.0 \mathrm{E}-02 \mathrm{~N} \\
1 \mathrm{~N} \\
3.0 \mathrm{E}-02 \mathrm{~N} \\
1 \mathrm{H} \\
1.2 \mathrm{E}-02 \mathrm{H}\end{array}$ & $\begin{array}{l}1.00 \mathrm{E}-03 \mathrm{~N} \\
3.00 \mathrm{E}-02 \mathrm{~N} \\
3.00 \mathrm{E}-02 \mathrm{~N} \\
1.19 \mathrm{E}-02\end{array}$ & $9.35 \mathrm{E}-05$ \\
\hline
\end{tabular}




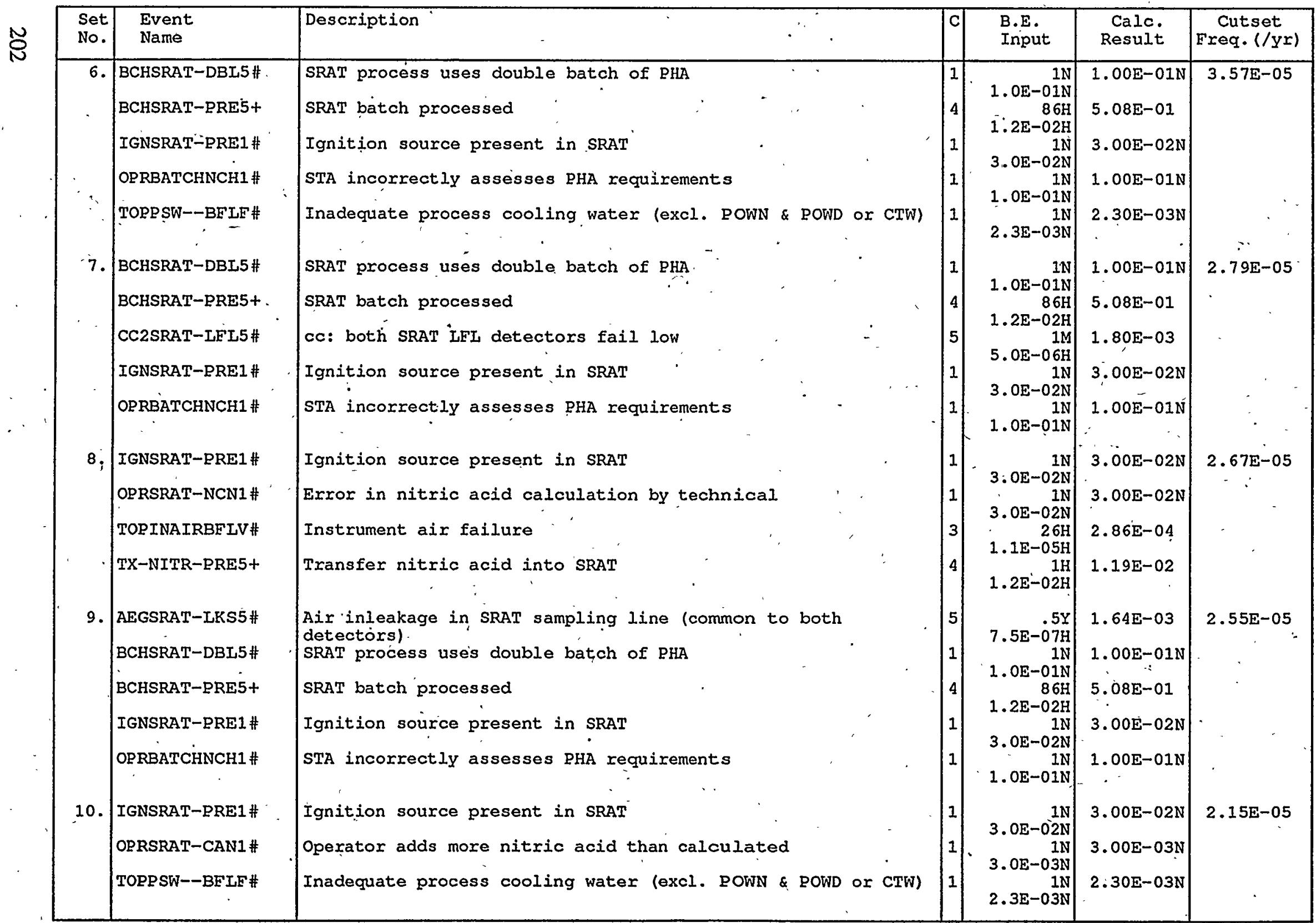


Cutsets for C: ICAFTAISRAT.CSR

2/08/95 10:36 AM (CONT.)

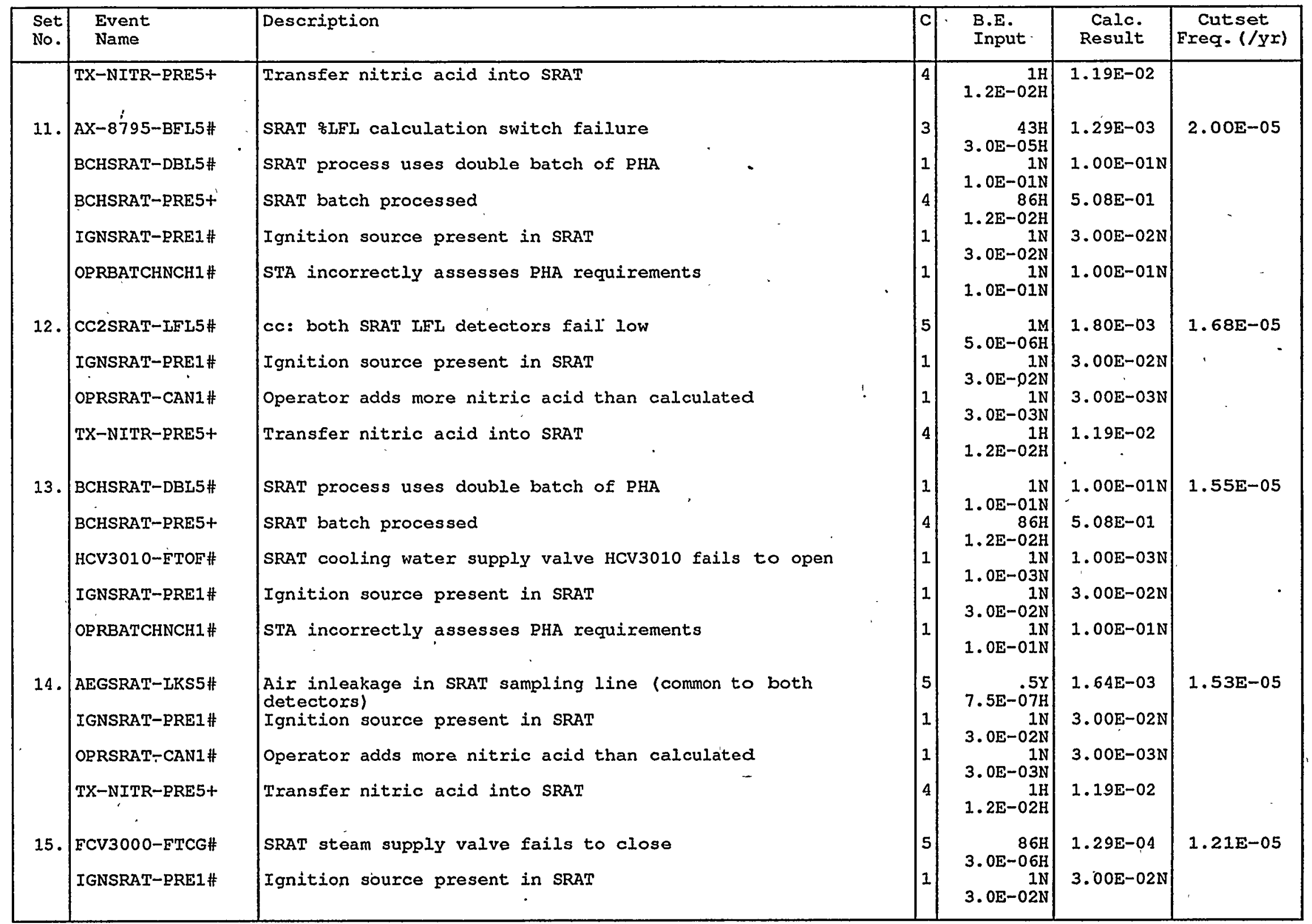


Cutsets for C: \CAFTA\SRAT.CSR 2/08/95 10:36 AM (CONT.)

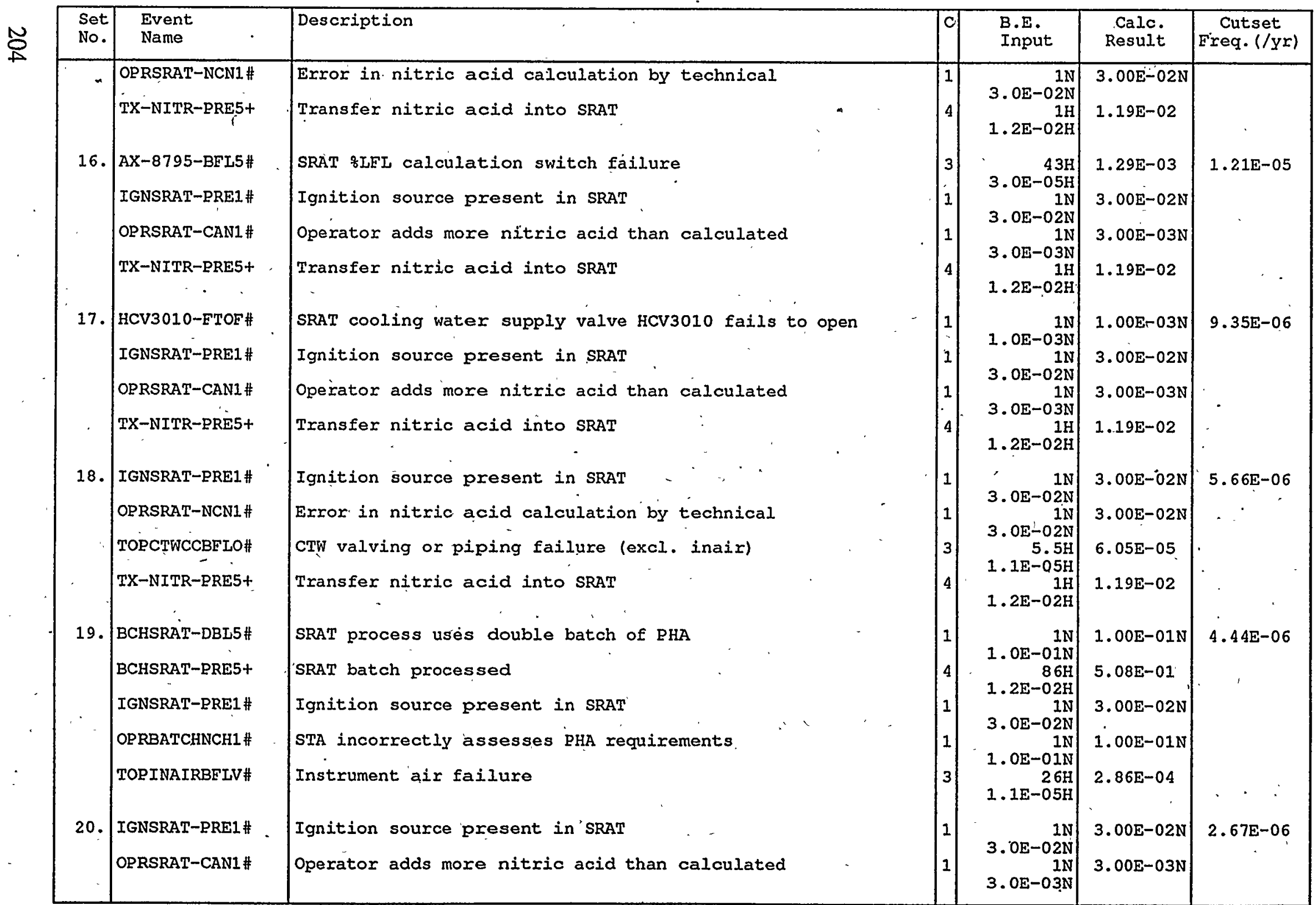




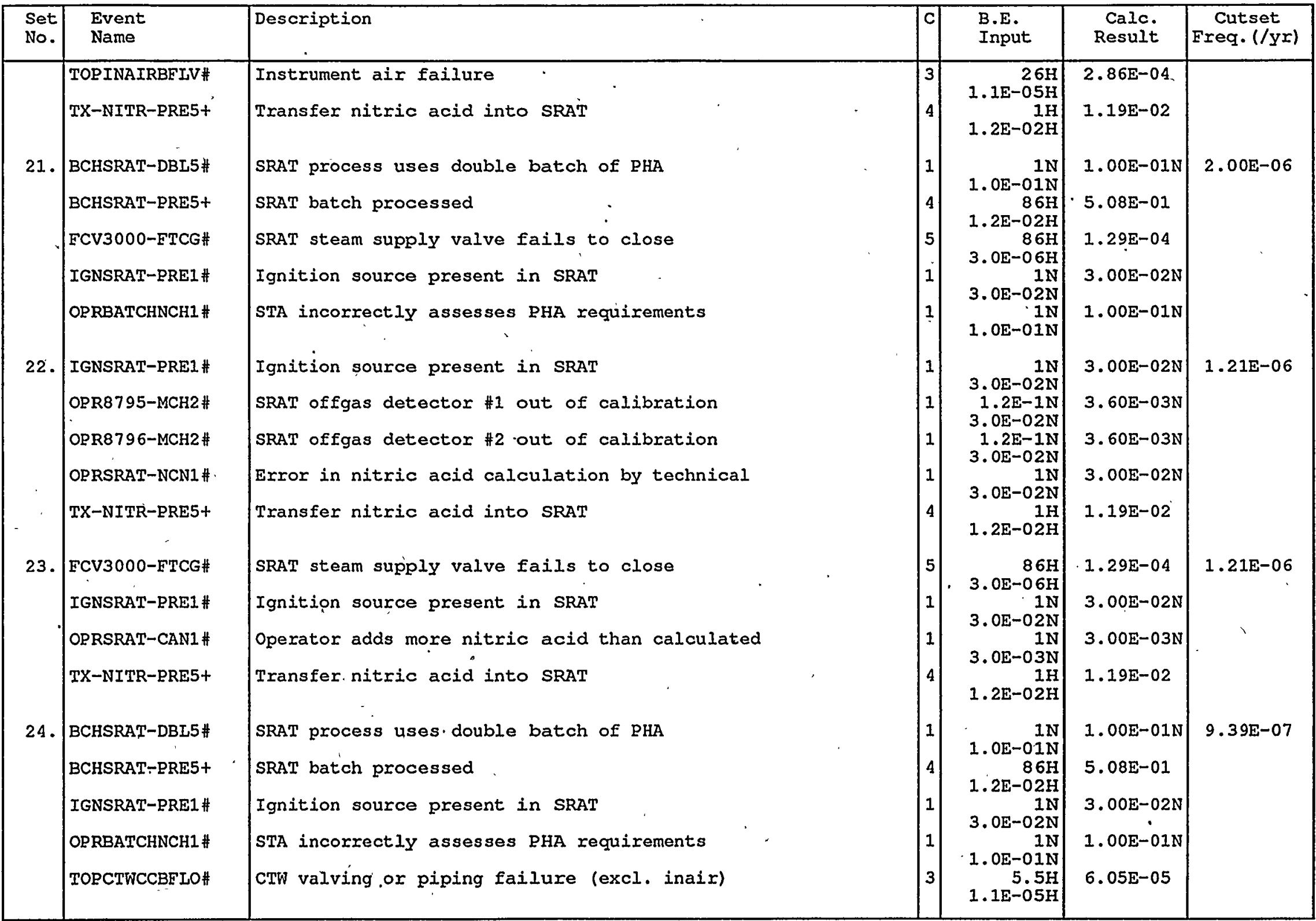


Explosion in SRAT

Top Event Frequency: 1.02E-03/YR

\begin{tabular}{|c|c|c|c|}
\hline Basic Event Name & Description & Prob/Frea & AchW \\
\hline PSRAT-PLG5+-I & SRAT vent line plugs & $1.00 \mathrm{E}-07 / \mathrm{H}$ & $9.90 \mathrm{E}+\mathrm{C}$ \\
\hline TOPCTWCCBFLO+IC & Failure of CTW valving or piping (excl. INAIR) & $1.10 \mathrm{E}-05 / \mathrm{H}$ & E+ \\
\hline TOPINAIRBFLV+-IC & Instrument air failure (excl CTW or POWN/POWD) & $1.10 \mathrm{E}-05 / \mathrm{H}$ & $1.18 \mathrm{E}+02$ \\
\hline RY-4041-FTO5\# & Fail to open PRBT transfer pump switch (fail to stop pump) & $1.00 \mathrm{E}-05$ & $1.17 \mathrm{E}+02$ \\
\hline FCV3000-FTCG\# & SRAT steam supply valve fails to close & $1.29 \mathrm{E}-04$ & 1.17E+02 \\
\hline HCV3010-FTOF\# & SRAT cooling water supply valve HCV3010 fails to open & $1.00 \mathrm{E}-03$ & $1.17 \mathrm{E}+02$ \\
\hline $\mathrm{AX}$ & AT \%LFL calculation switch failure & $1.29 \mathrm{E}-03$ & 1.17E+02 \\
\hline AEGSRAT-LKS5\# & Air inleakage in SRAT sampling line (both detectors) & $1.64 \mathrm{E}-03$ & 1.17E+02 \\
\hline TOPPSW-BFLF\# & Inadequate cooling water (excl. POWN \& POWD or CTW) & $2.30 \mathrm{E}-03$ & $1.17 \mathrm{E}+02$ \\
\hline CC2SRAT-LFL5\# & cc: both SRAT LFL detectors fail low & $1.80 \mathrm{E}-03$. & 1.17E+02 \\
\hline IR-PRE5+-I & Transfer nitric acid into SRAT & $1.19 \mathrm{E}-02 / \mathrm{H}$ & 7.33E+01 \\
\hline RY & Fail to open SRAT steam flow low interlock relay & $1.00 \mathrm{E}-05$ & 4.41E+01 \\
\hline IGNSRAT-PRE1\# & Ignition source present in SRAT & $3.00 \mathrm{E}-02$ & 3.33E+01 \\
\hline OPRSRAT-CAN1\# & Operator adds more nitric acid than calculated & $3.00 \mathrm{E}-03$ & 2.72E+01 \\
\hline RAT-NCN1\# & Error in nitric acid calculation by technical & $3.00 \mathrm{E}-02$ & $2.65 \mathrm{E}+01$ \\
\hline BCHSRAT-PRE5+-I & SRAT batch processed & $5.90 \mathrm{E}-03 / \mathrm{H}$ & 2.31E+01 \\
\hline TOPPOWN-BFLH+-IC & LOSS OF NÖRMAL ELECTRI & 05/H & $1.91 \mathrm{E}+01$ \\
\hline MH5\# & Ive following maintenance & $7.00 \mathrm{E}-05$ & $4.50 \mathrm{E}+00$ \\
\hline RATSBFLL\# & SRAT sample pump fails & $2.40 \mathrm{E}-04$ & $4.50 \mathrm{E}+00$ \\
\hline TSFOPD\# & SRAT offgas sample pump circuit breaker fails open & $2.40 \mathrm{E}-06$ & 30 \\
\hline L2+-I & CPC primary purge system fails & E-05/H & 00 \\
\hline BCF & SRAT $\mathrm{p}$ & 1.0 & +00 \\
\hline CH1\# & STA incorre & 1.00 & $2.18 \mathrm{E}+00$ \\
\hline S5\# & Air inleakage in SRAT offgas & $1.64 \mathrm{E}-03$ & $1.86 \mathrm{E}+00$ \\
\hline AF & nt fails low (LEL) & 2.15 & $1.86 \mathrm{E}+00$ \\
\hline KS5\# & 11 sample line & -03 & $1.86 \mathrm{E}+00$ \\
\hline AE & SRAT offgas detector \#1 anal & 03 & \\
\hline H2\# & etector \#2 & & \\
\hline 1CH2\# & SRAT 0 & $3.60 \mathrm{E}-03$ & $1.86 \mathrm{E}+00$ \\
\hline TOPCTWP-BFLB+IC & CTW primary pumping failure (excl. POWN) & $6.29 \mathrm{E}-06 / \mathrm{H}$ & $1.63 \mathrm{E}+00$ \\
\hline 000-FCL5+IC & Steam supply flow control valve FCV 3000 fails closed & $-06 / \mathrm{H}$ & +00 \\
\hline & SRAT steam & $-06 / \mathrm{H}$ & +00 \\
\hline MBFLG+-IC & excl. POWN or INAIR) & $2.08 \mathrm{E}-04 / \mathrm{H}$ & $1.58 \mathrm{E}+00$ \\
\hline FE-3034-FHI2+-IC & SRAT purge flow element fails high & $3.00 \mathrm{E}-06 / \mathrm{H}$ & $1.37 \mathrm{E}+00$ \\
\hline LW\# & Backup CPC purge fails (except N2 tank supply.failure) & $3.10 \mathrm{E}-03$ & $1.20 \mathrm{E}+00$ \\
\hline 4-FCL2+-IC & SRAT purge flow control valve fails closed & 1.00E-06/H & \\
\hline TCHPRE5+-I & New batch of sludge for DWPF & $-05 / \mathrm{H}$ & \\
\hline & CTW deep well pumping failure & $5.40 \mathrm{E}-03$ & $1.13 E+00$ \\
\hline D-BFLN\# & LOSS OF BACKUP POWER (DIESEL GENERATORS) & 1.10E-02 & $1.06 \mathrm{E}+00$ \\
\hline T-FSLD\# & CC: all 3 SRAT offgas flow monitors fail to close & 1.10E-04 & \\
\hline MCH1\# & Tlow flo & $3.00 \mathrm{E}-02$ & \\
\hline TX-SRAT-PRE5+-IC & Transfer from PRBT to SRAT & 9.41E-03/H & $1.03 \mathrm{E}+$ \\
\hline
\end{tabular}

Note: Events that make less than $1 \%$ difference to the importance factor are not included. Modified top event frequency $=$ (top event frequency) $\mathrm{x}$ (risk achievement worth). 
Explosion in SRAT

Top Event Frequency:.1.02E-03/YR

Basic Event Name
*IGNSRAT-PRE1\#
TX-NITR-PRE5+I
OPRSRAT-NCN1\#
TOPPSW-BFLF\#
CC2SRAT-LFL\#
AEGSRAT-LKS5\#
AX-8795-BFL5\#
OPRBATCHNCH1\#
BCHSRAT-PRE5+I
BCHSRAT-DBL5\#
HCV3010-FTOF\#
OPRSRAT-CAN1\#
TOPINAIRBFLV+IC
FCV3000-FTCG\#

\section{Risk Reduction Werth}

Description

Ignition source present in SRAT

Transfer nitric acid into SRAT

Error in nitric acid calculation by technical

Inadequate cooling water (excl. POWN \& POWD or CTW)

cc: both SRAT LFL detectors fail low

Air inleakage in SRAT sampling line (both detectors)

SRAT \%LFL calculation switch failure

STA incorrectly assesses PHA requirements

SRAT batch processed

SRAT process uses double batch of PHA

SRAT cooling water supply valve HCV3010 fails to open

Operator adds more nitric acid than calculated

Instrument air failure (excl CTW or POWN/POWD)

SRAT steam supply valve fails to close $=\frac{\text { Prob/Freg }}{3.00 \mathrm{E}-02} \cdot \frac{\text { RedW }}{0.00 \mathrm{E}+00}$

$1.19 \mathrm{E}-02 / \mathrm{H} \quad 7.57 \mathrm{E}+00$

$3.00 \mathrm{E}-02 \quad 4.74 \mathrm{E}+00$

$2.30 \mathrm{E}-03 \quad 1.37 \mathrm{E}+00$

$1.80 \mathrm{E}-03 \quad 1.26 \mathrm{E}+00$

$1.64 \mathrm{E}-03 \quad 1.24 \mathrm{E}+00$

$1.29 \mathrm{E}-03 \quad 1.18 \mathrm{E}+00$

$1.00 \mathrm{E}-01 \quad 1.15 \mathrm{E}+00$

$5.90 \mathrm{E}-03 / \mathrm{H} \quad 1.15 \mathrm{E}+00$

1.00E-01 1.15E+00

$1.00 \mathrm{E}-03 \quad 1.13 \mathrm{E}+00$

$3.00 \mathrm{E}-03, \quad 1.09 \mathrm{E}+00$

$1.10 \mathrm{E}-05 / \mathrm{H} \quad 1.03 \mathrm{E}+00$

$1.29 \mathrm{E}-04 \quad 1.02 \mathrm{E}+00$

* A Reduction Worth of $0.0 \mathrm{E}+00$ indicates event appears in every cutset could serve as a single event preventor for the accident.

Note: Events that make less than $1 \%$ difference to the importance factor are not included. Modified top event frequency = (top event fréquency) $/$ (risk reduction worth). 
X'E-ESR-S-00001, REV. 0

SME Explosion 


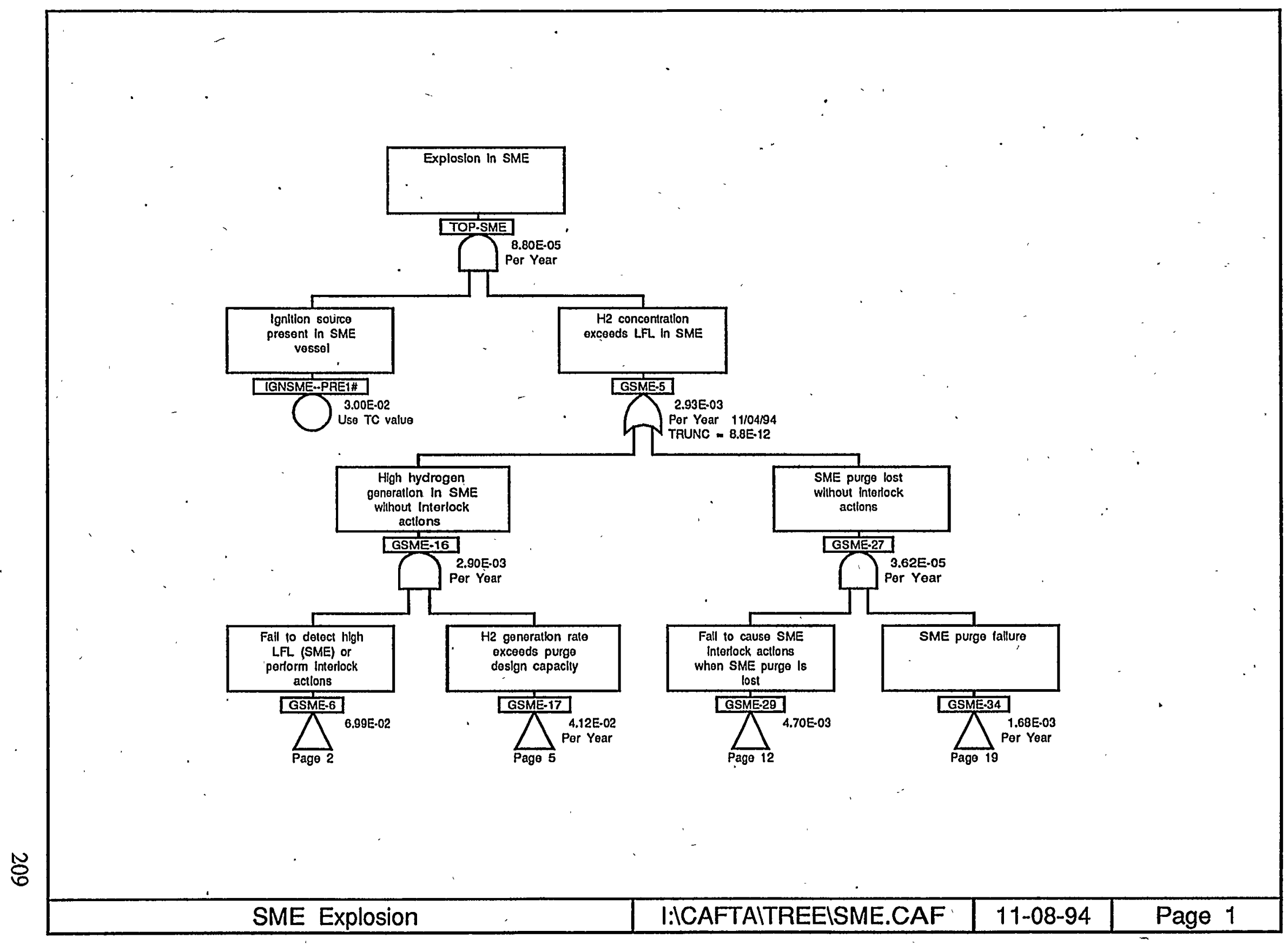




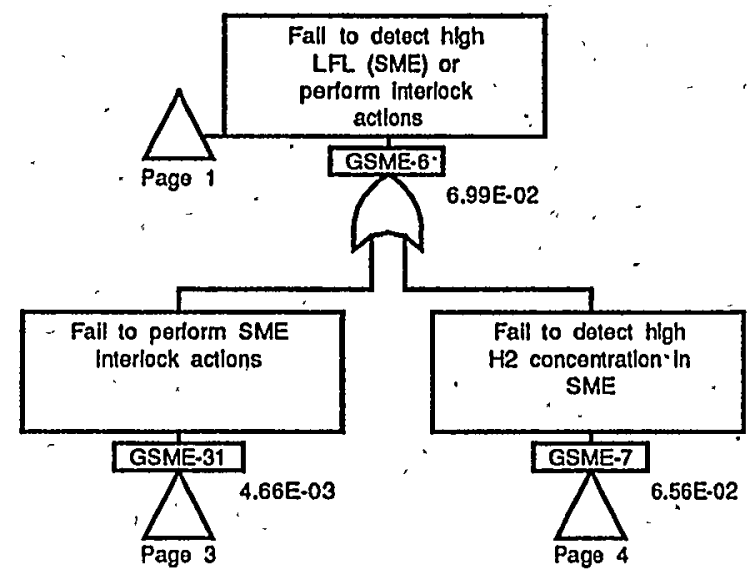




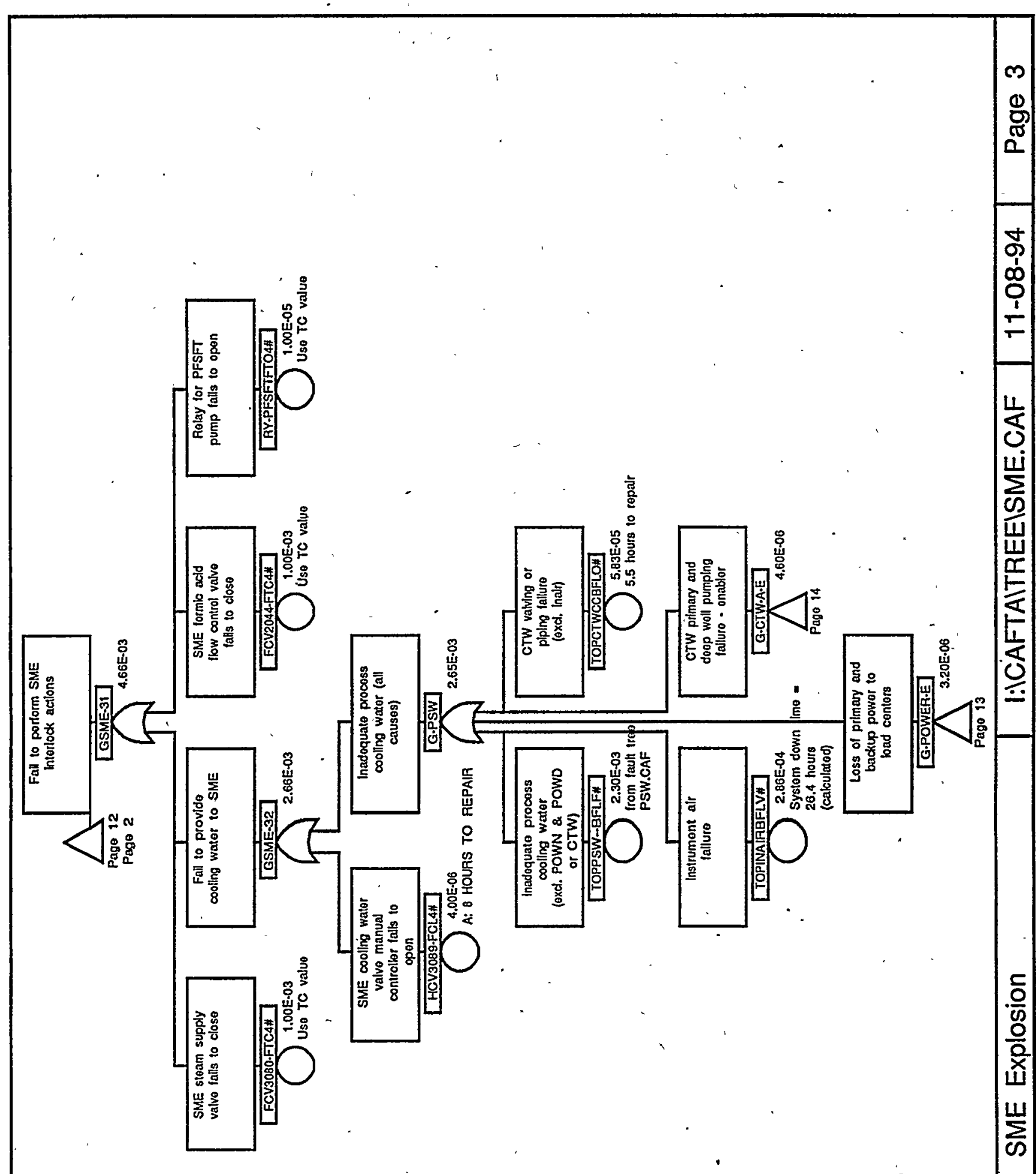


X-ESR-S-00001, REV. 0

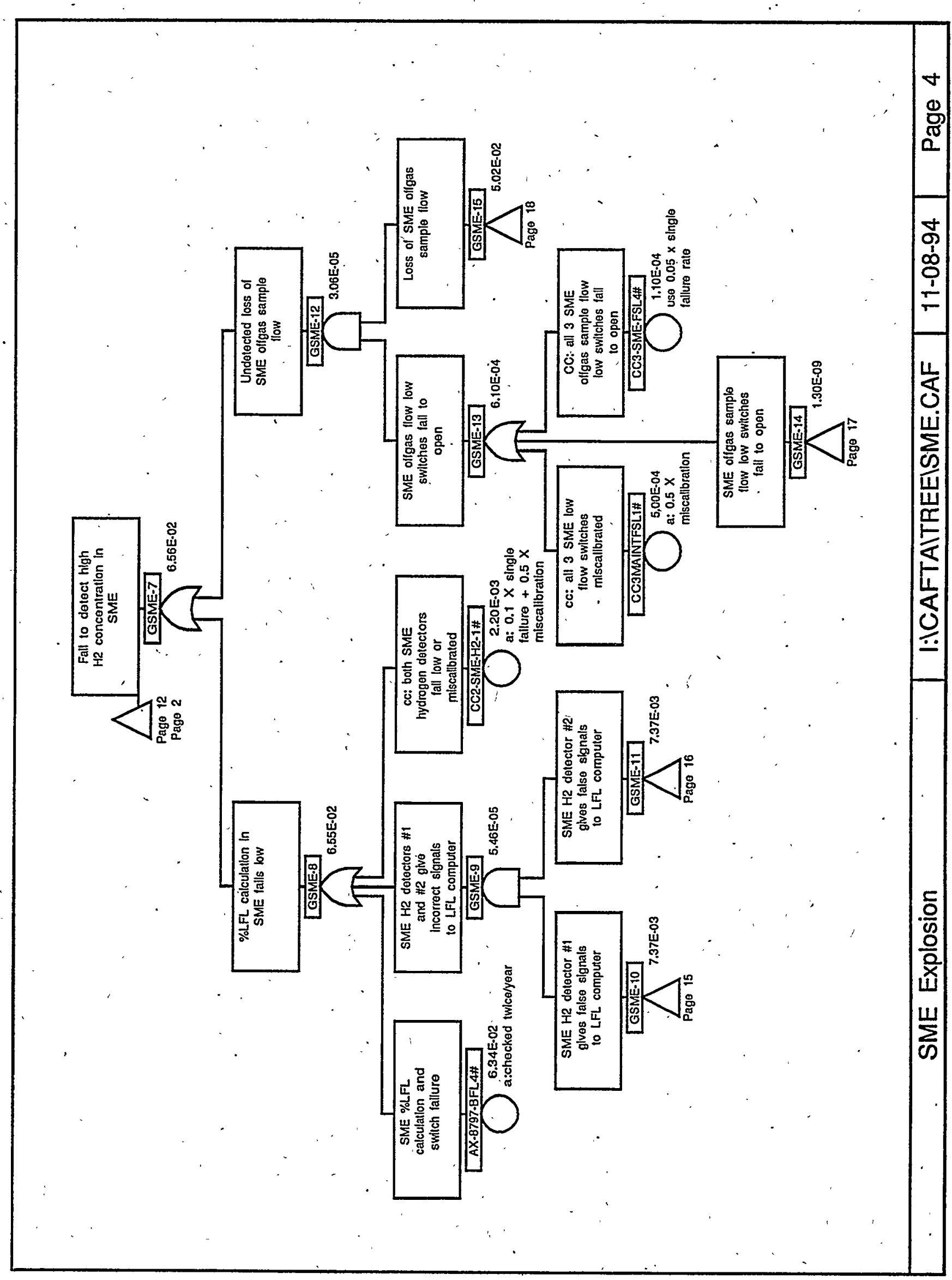


X-ESR-S-00001, REV. 0

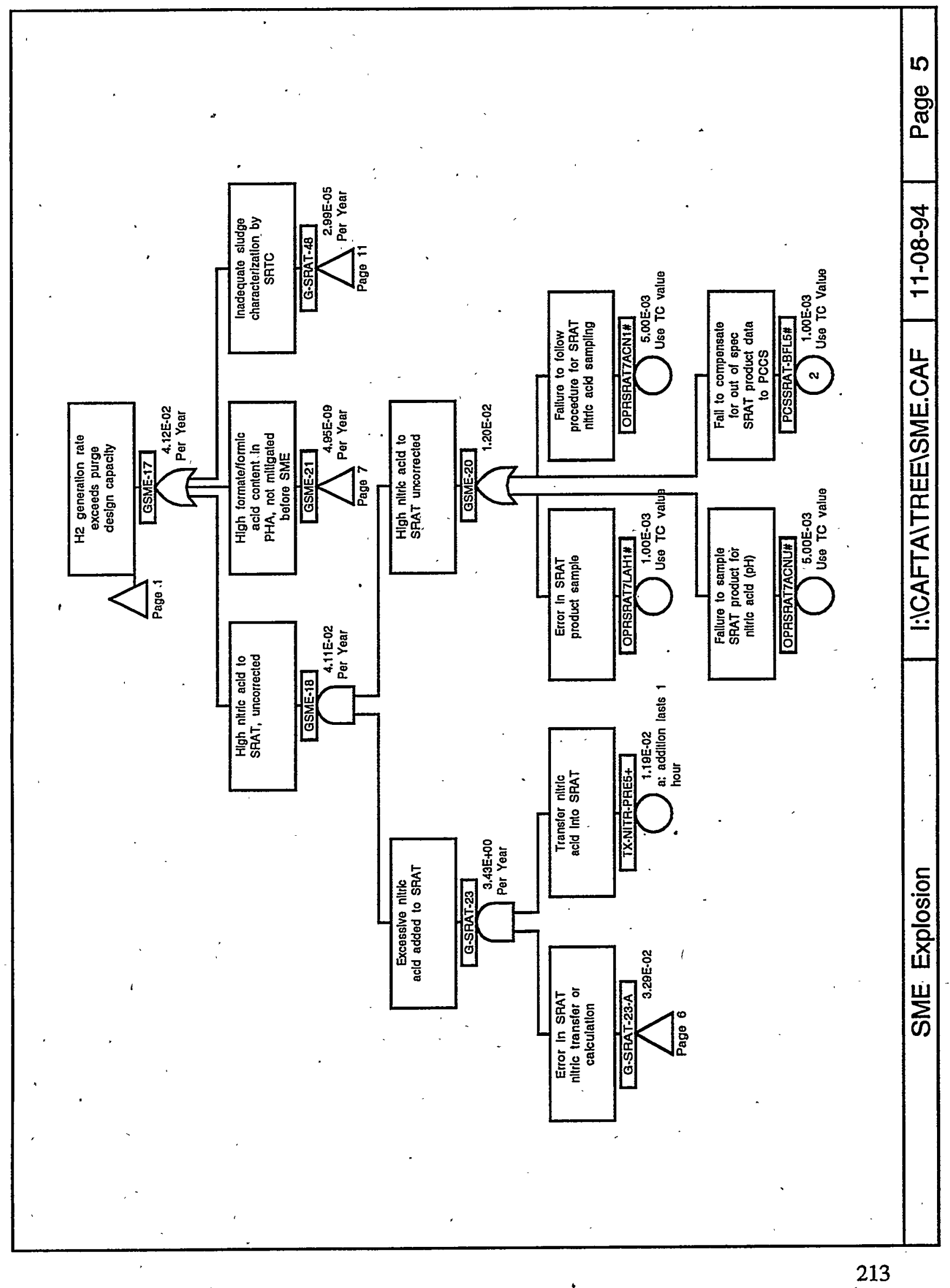


X-ESR-S-00001, REV. 0

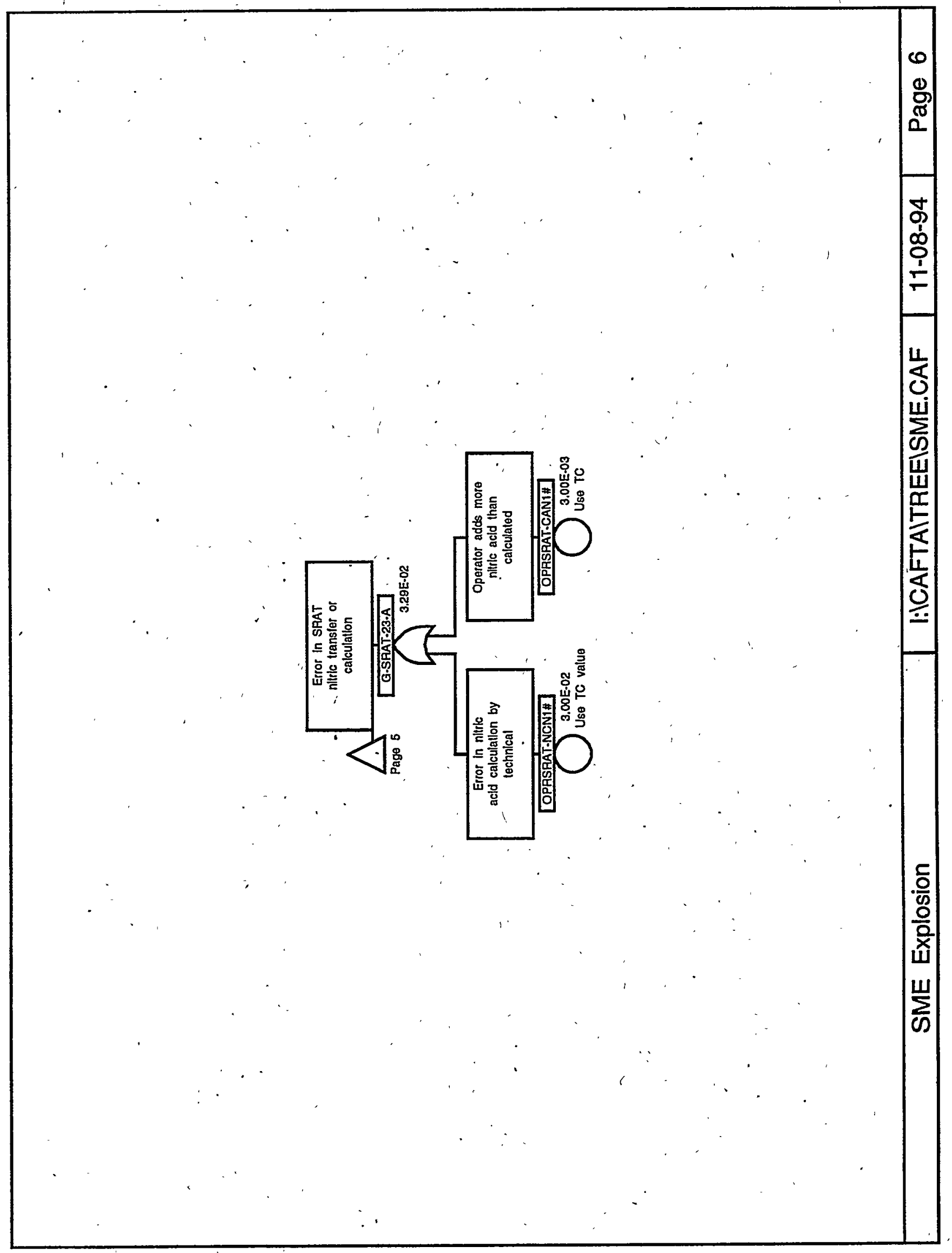




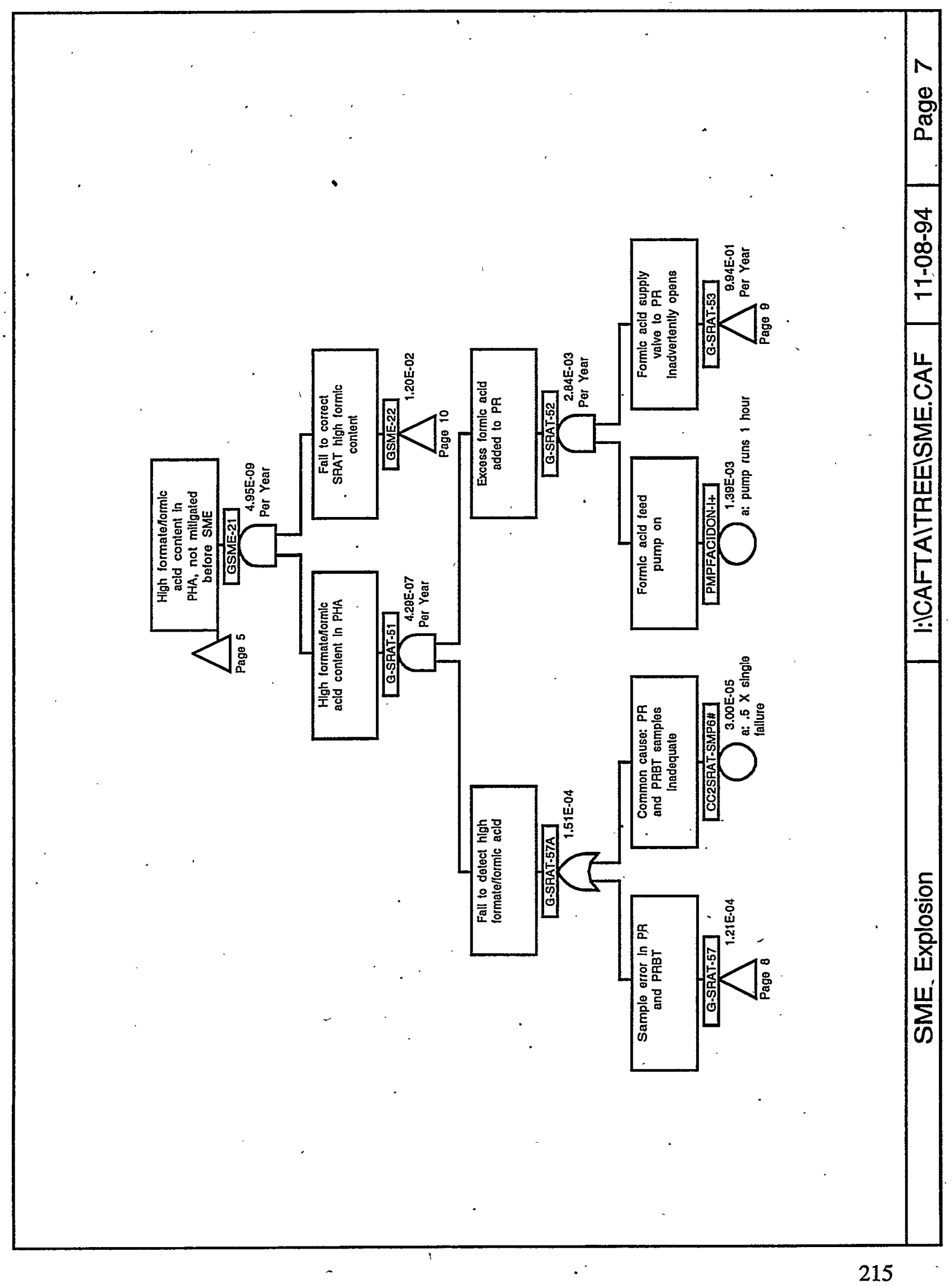


X-ESR-S-00001, REV. 0

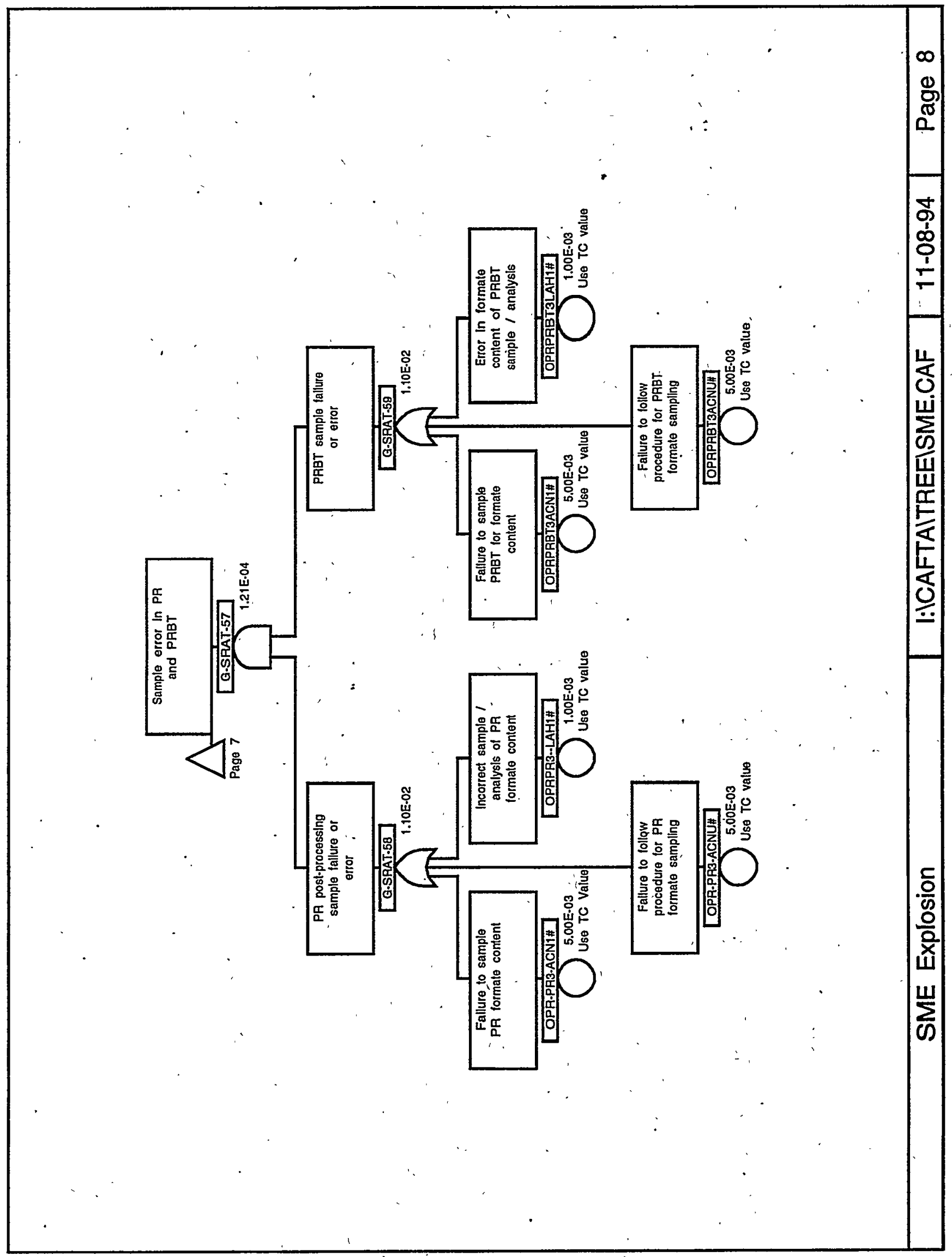




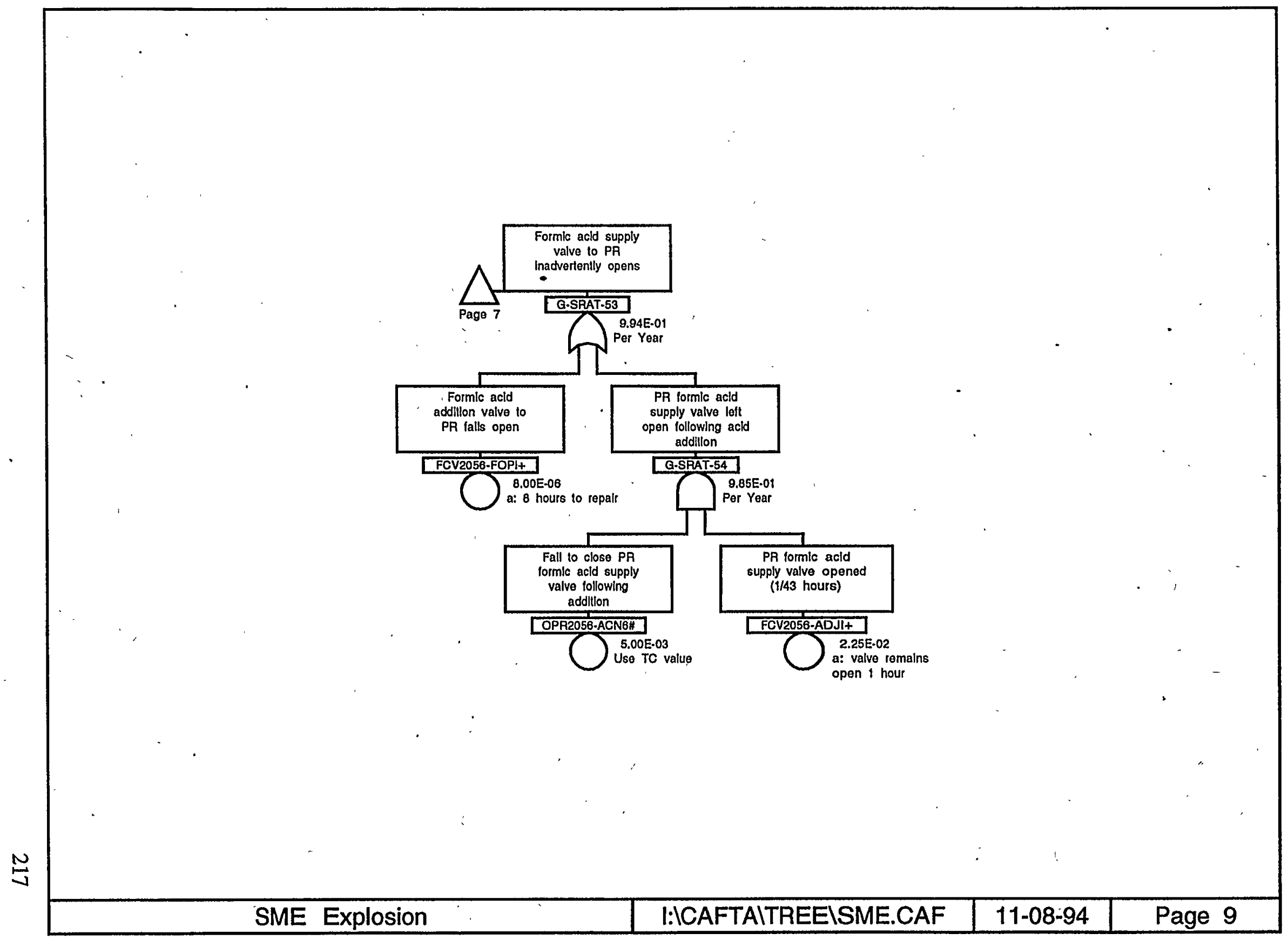


X-ESR-S-00001, REV. 0

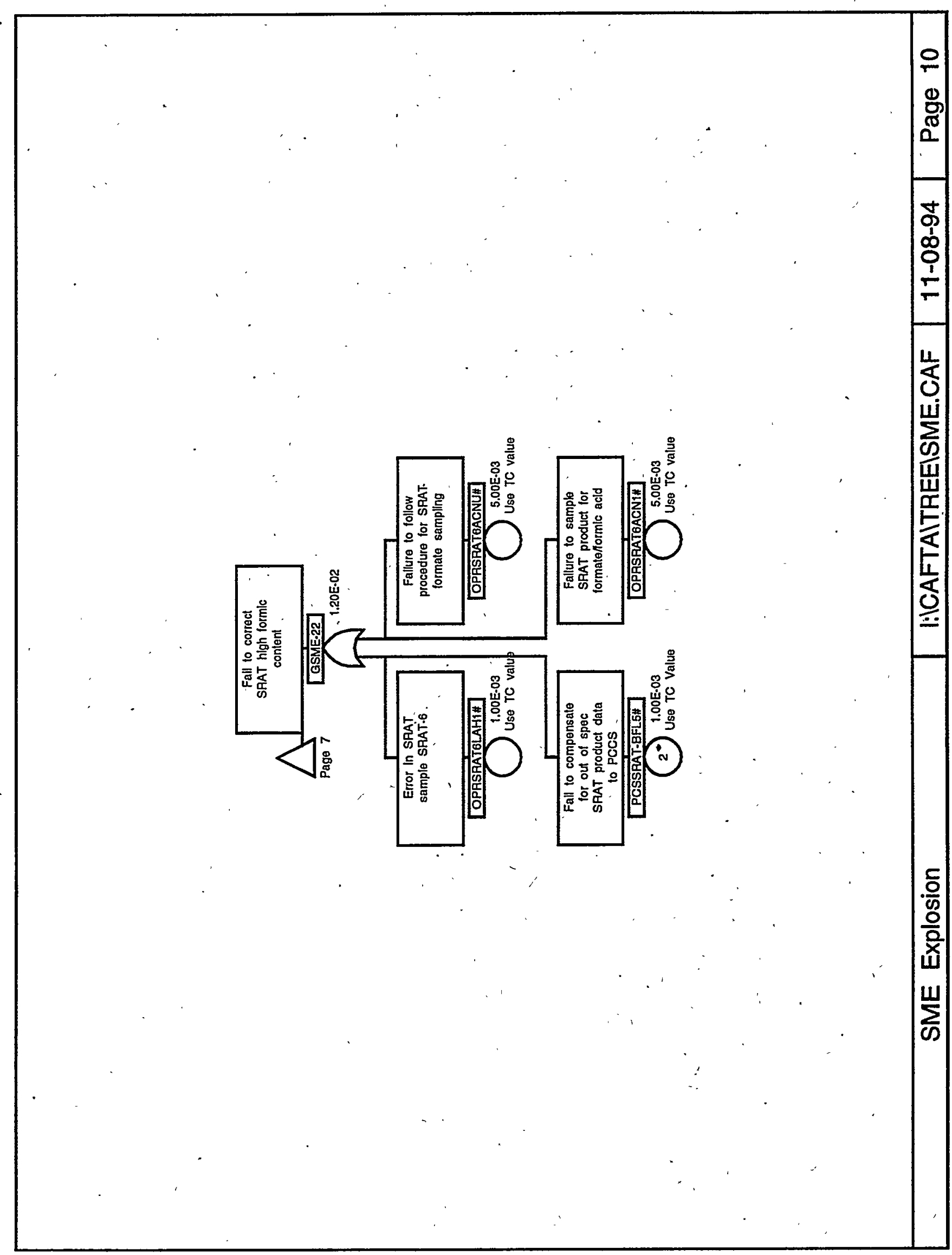




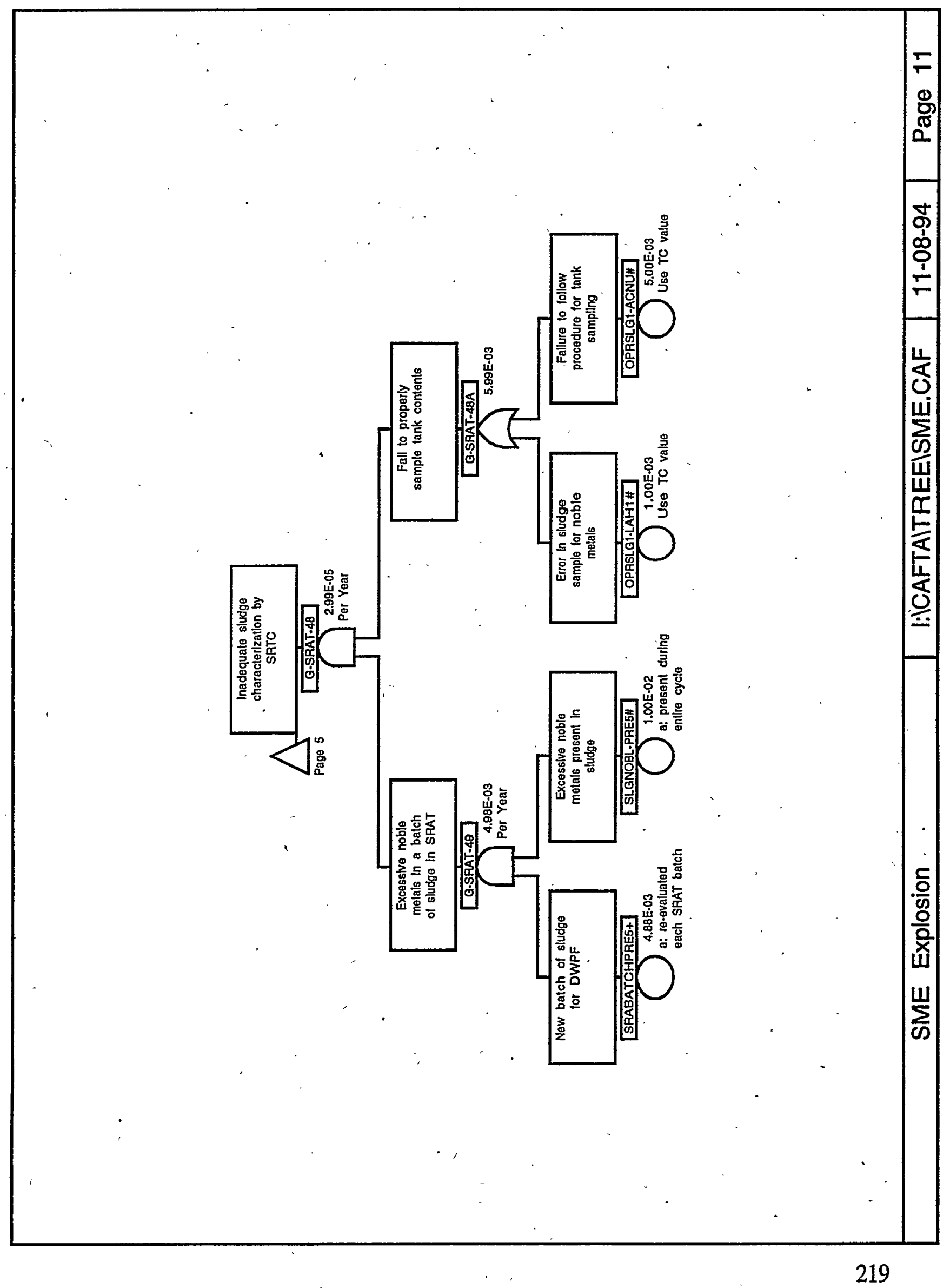


X-ESR-S-00001, REV. 0

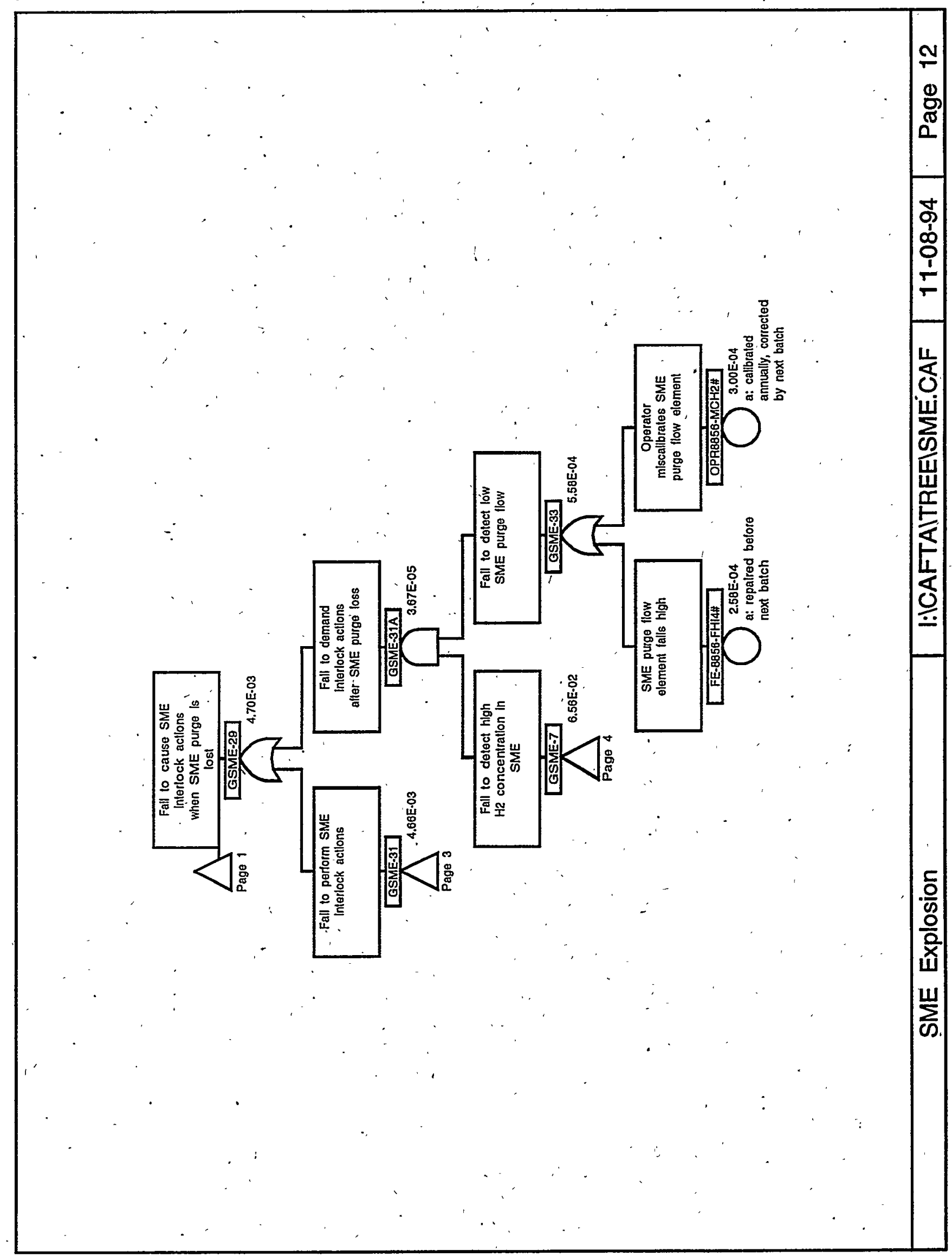



X-ESR-S-00001, REV. 0

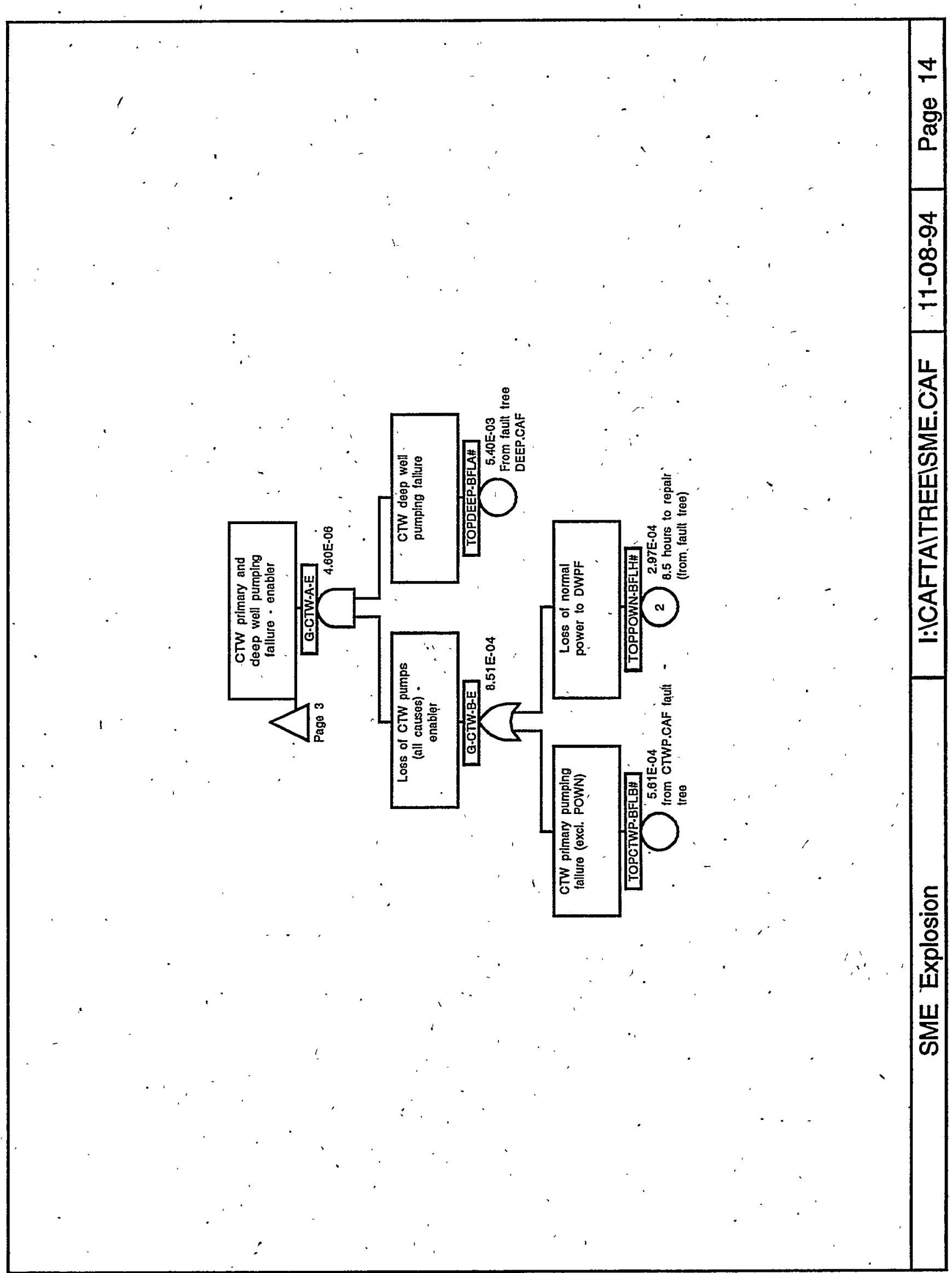




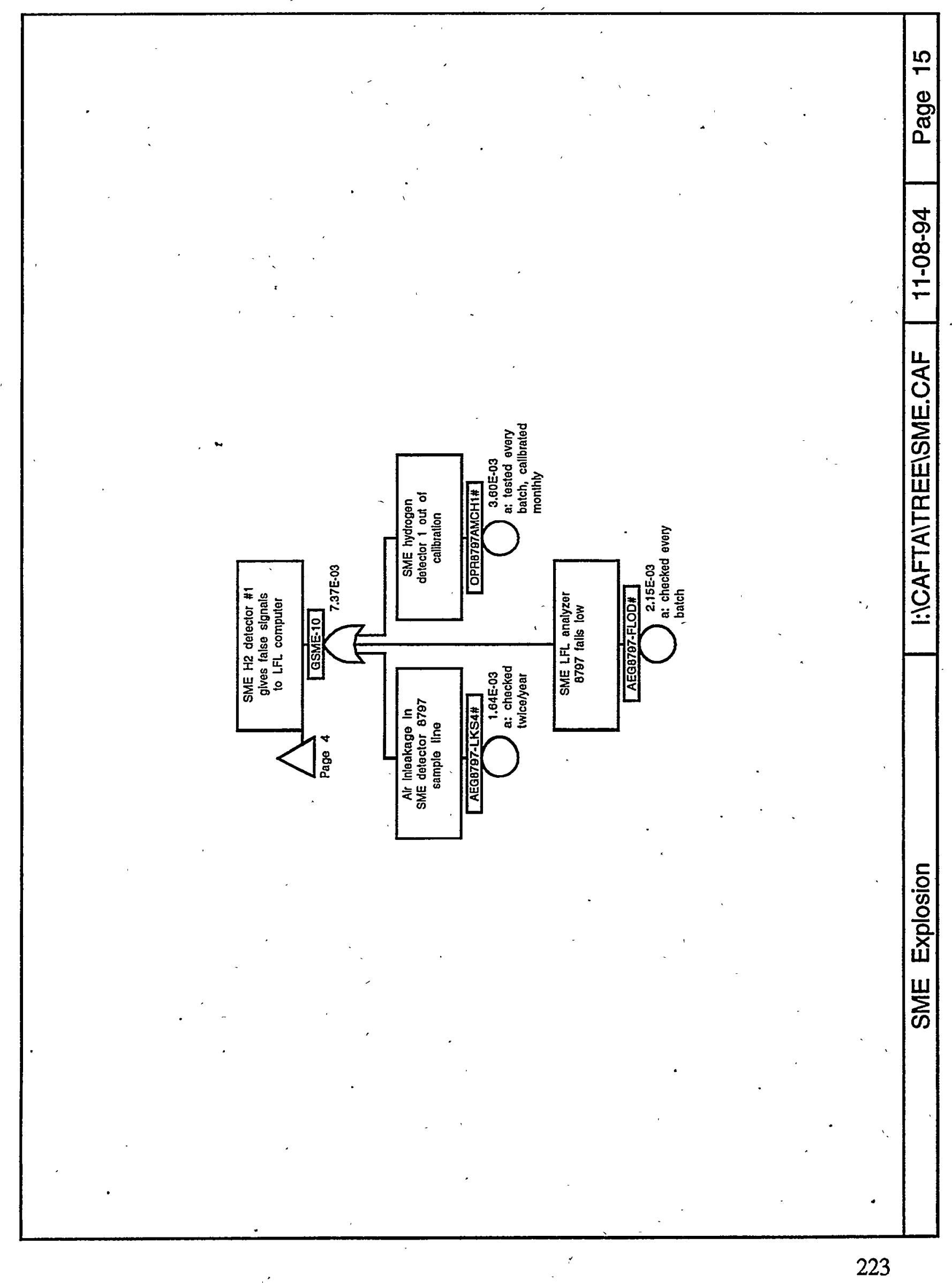


X-ESR-S-00001, REV. 0

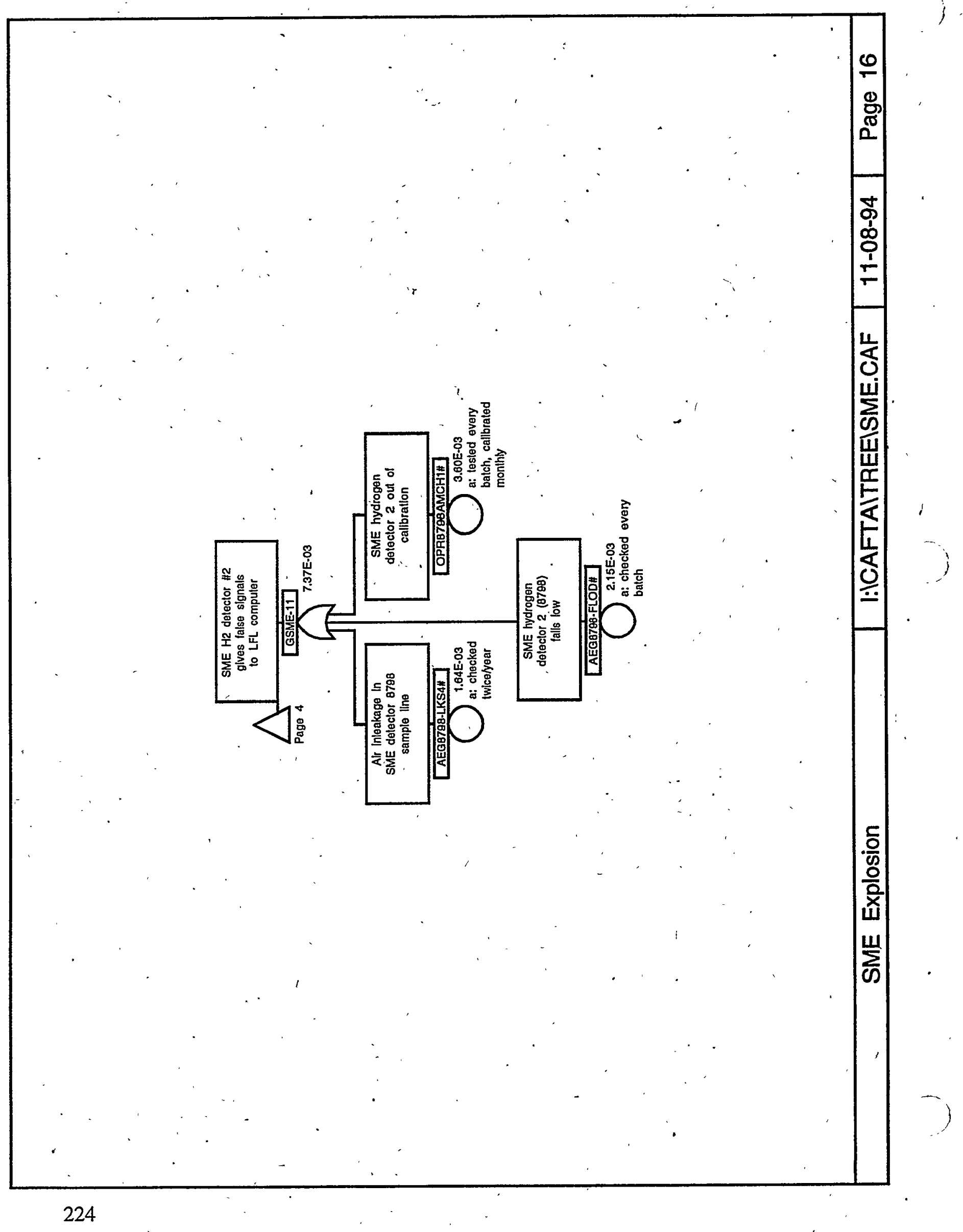




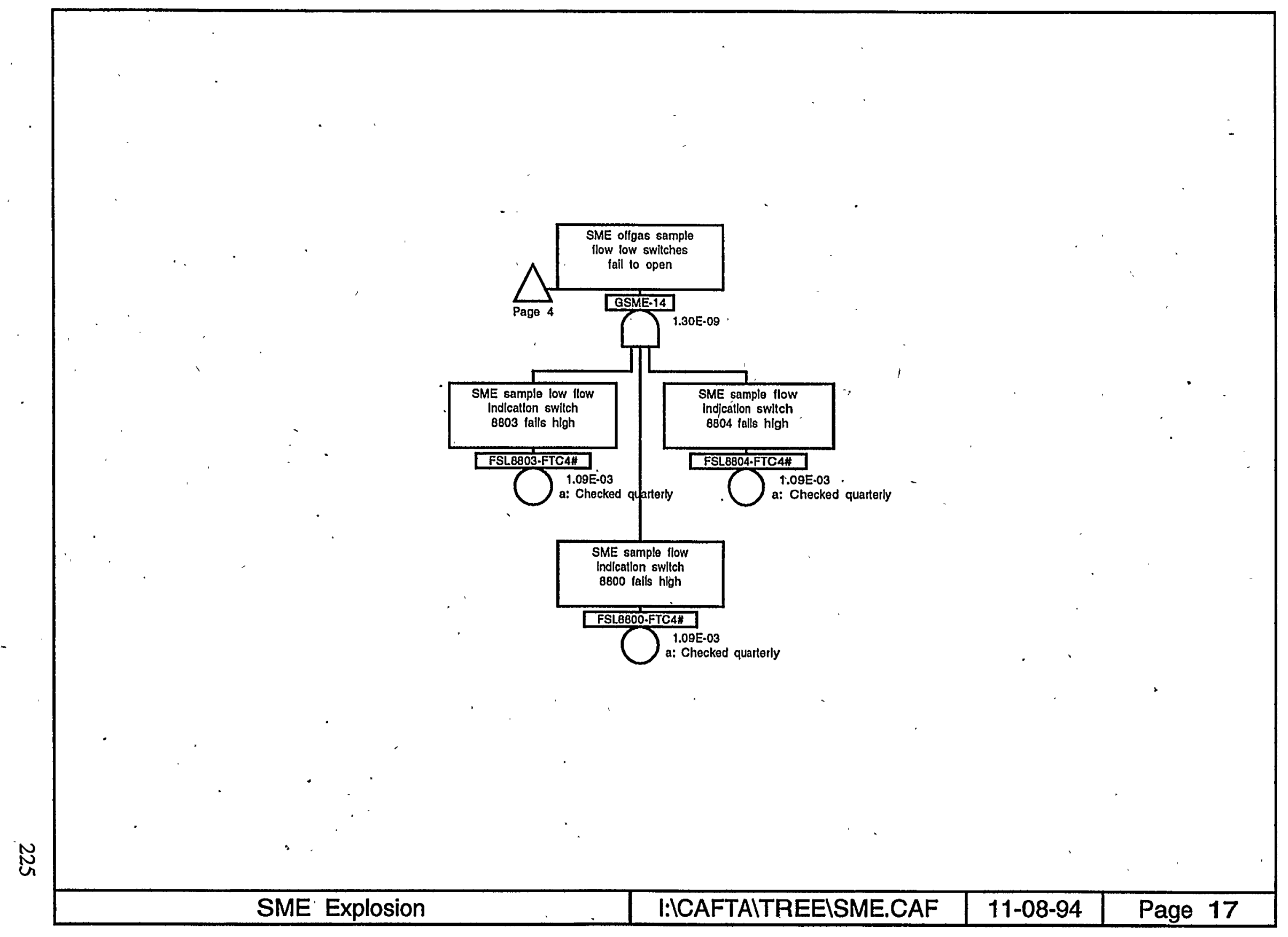




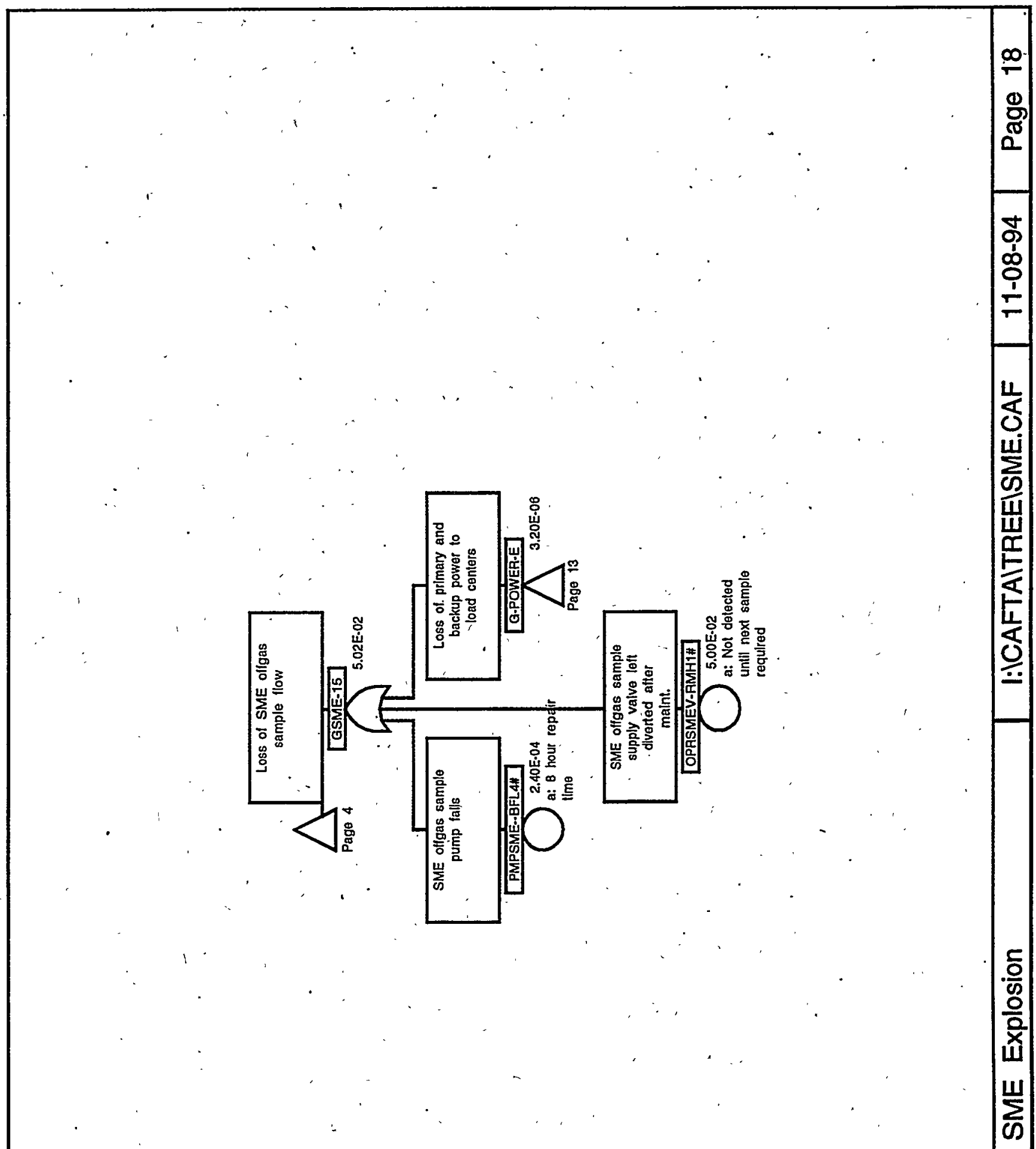




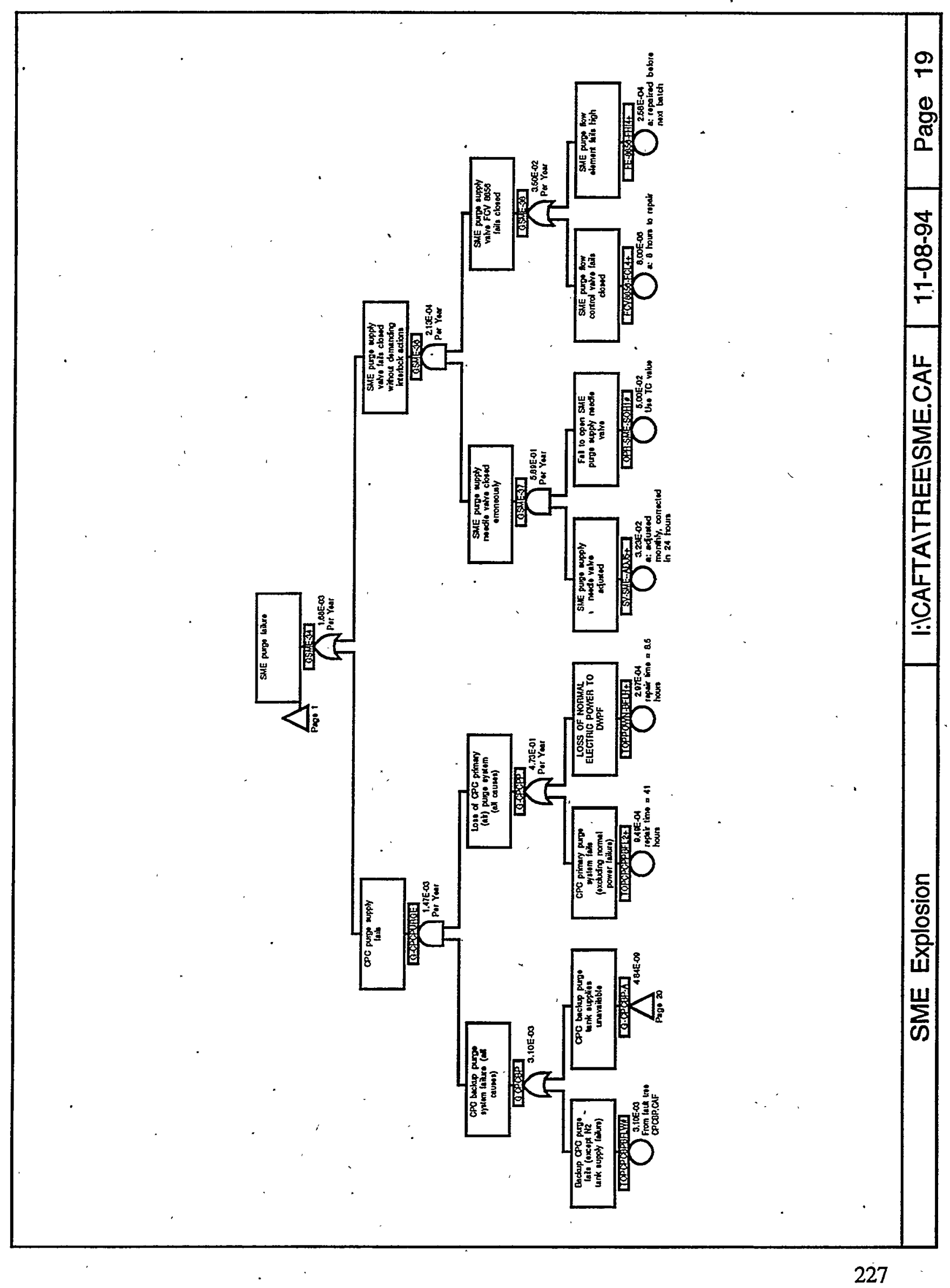


X-ESR-S-00001, REV. 0

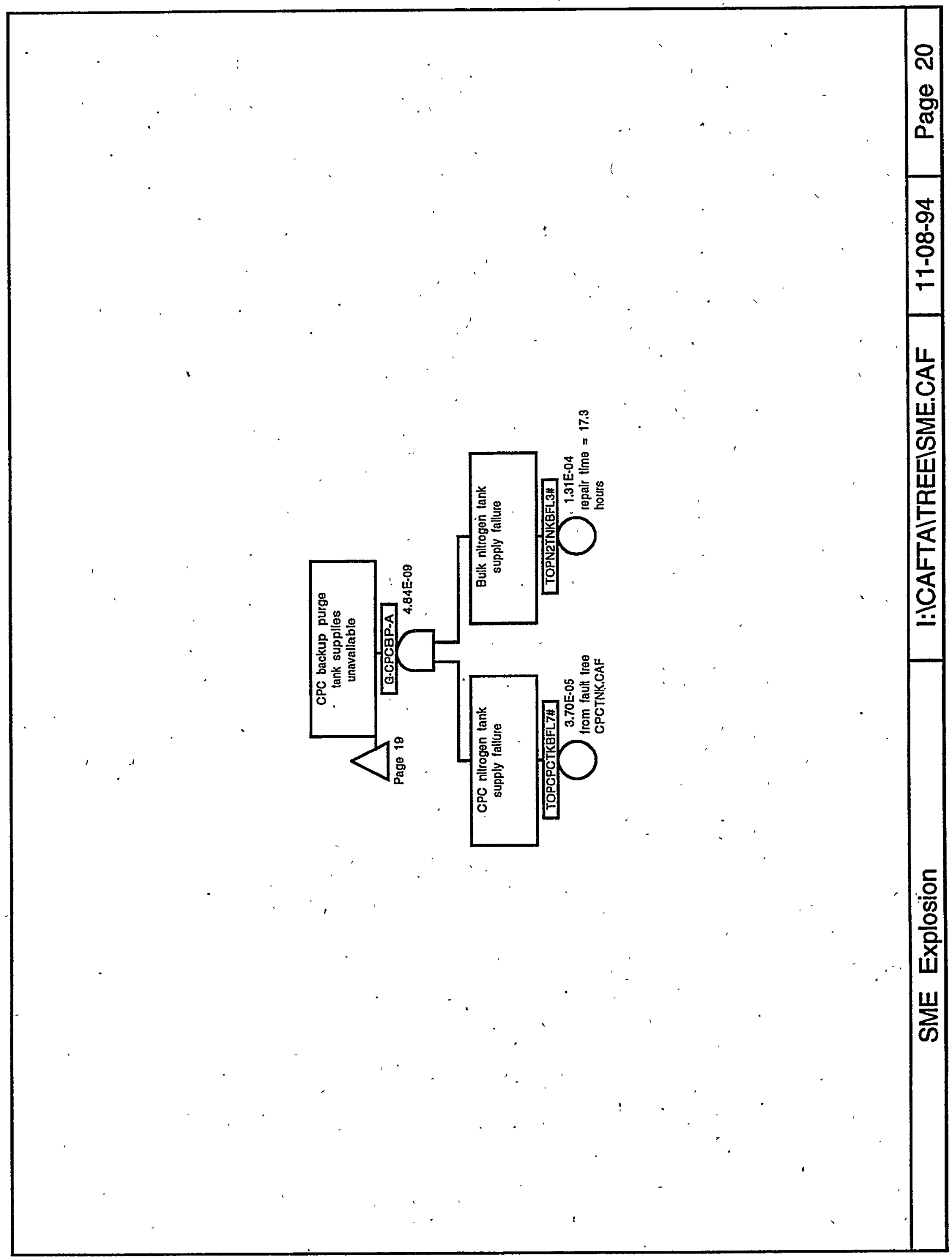




\begin{tabular}{|c|c|c|c|c|c|c|c|c|c|c|}
\hline Gate/Event Name & Page Zone & Gate/Event & Name & Page Zone & Gate/Event Name & Page & Zone & Gate/Event Name & Page & Zone \\
\hline AEG8797-FLOD\# & 15 & G-SRAT-51 & & 7 & GSME-34 & 1 & & PCSSRAT-BFL5\# & 10 & \\
\hline AEG8797-LKS4\# & 15 & G-SRAT-52 & & 7 & GSME- 34 & 19 & $=$ & PMPFACIDON-I+ & 7 & 7 \\
\hline AEG8798-ELOD\# & 16 & G-SRAT-53 & & 7 & GSME-36 & 19 & & PMPSME--BFL $4 \#$ & 18 & \\
\hline AEG8798-LKS4\# & 16 & G-SRAT-53 & & 9 & GSME-37 & 19 & & RY-PFSFTFTO $4 \#$ & 3 & 3 \\
\hline AX-8797-BEL4\# & 4 & G-SRAT-54 & & 9 & GSME -38 & 19 & & SLGNOBL-PRES\# & 11 & \\
\hline $\mathrm{CC} 2-\mathrm{SME}-\mathrm{H} 2-1 \#$ & 4 & G-SRAT-57 & & 7 & GSME-5 & 1 & & SRABATCHPRE5+ & 11 & \\
\hline CC2SRAT-SMP6\# & 7 & G-SRAT-57 & & 8 & GSME- 6 & 1 & & SV-SME--ADJ $5+$ & 19 & \\
\hline CC3-SME-FSL $4 \#$ & 4 & G-SRAT-57A & & 7 & GSME- 6 & 2 & . & TOP-SME & 1 & 1 \\
\hline CC3MAINTESL1\# & 4 & G-SRAT-58 & & 8 & GSME-7 & 2 & & TOPCPCBPBFLW\# & 19 & \\
\hline FCV2044-ETC4\# & 3 & G-SRAT-59 & & 8 & GSME-7 & 4 & & TOPCPCPPBFL2+ & 19 & \\
\hline ECV2056-ADJI+ & 9 & GSME- 10 & & 4 & GSME- 7 & 12 & & TOPCPCTKBFL7\# & 20 & \\
\hline FCV2056-FOPI+ & 9 & GSME-10 & & 15 & GSME- 8 & 4 & $\therefore$ & TOPCTWCCBFLO\# & 3 & 3 \\
\hline FCV3080-ETC4\# & 3 & GSME-11. & & 4 & GSME-9 & 4 & . & TOPCTWP-BFLB\# & 14 & \\
\hline FCV8856-FCL 4+ & 19 & GSME-11 & & 16 & HCV3089-FCL4\# & 3 & & TOPDEEP-BFLA\# & 14 & \\
\hline FE-8856-FHI4\# & 12 & GSME- 12 & & 4 & IGNSME--PRE1\# & 1 & & TOPINA IRBFLV\# & 3 & 3 \\
\hline FE-8856-FHI4+ & 19 & GSME- 13 & & 4 & OPR-PR3-ACN1\# & 8 & & TOPN2TNKBFL3\# & 20 & \\
\hline FSL8800-ETC4\# & 17 & GSME-14 & & 4 & OPR-PR3-ACNU\# & 8 & & TOPPOWD-BFLN\# & 13 & \\
\hline FSL8803-FTC4\# & 17 & GSME- 14 & & 17 & OPR-SME-SOH1\# & 19 & & TOPPOWN-BELH\# & 13 & \\
\hline FSL8804-FTC4\# & 17 & GSME-15 & & 4 & OPR2056-ACN6\# & 9 & & TOPPOWN-BFLH\# & 14 & \\
\hline G-CPCBP & 19 & GSME- 15 & & 18 & OPR8797AMCH1\# & 15 & & TOPPOWN-BFLH+ & 19 & \\
\hline G-CPCBP-A. & 19 & GSME- 16 & & 1 & OPR8798AMCH1\# & 16 & & TOPPSW--BFLE\# & 3 & 3 \\
\hline G-CPCBP-A & 20 & GSME-17 & & 1 & OPR8856-MCH2\# & 12 & & TX-NITR-PRE5+ & 5 & 5 \\
\hline G-CPCPP & 19 & GSME- 17 & & 5 & OPRPR3 --LAH1\# & 8 & . & & & \\
\hline G-CPCPURGE & 19 & GSME-18 & & 5 & OPRPRBT3ACN1\# & 8 & & & & - \\
\hline$G-C T W-A-E$ & 3 & GSME-20 & & 5 & OPRPRBT3ACNU\# & 8 & & & $\cdot$ & \\
\hline$G-C T W-A-E$ & 14 & GSME-21 & & 5 & OPRPRBT3LAH1\# & 8 & & & & \\
\hline$G-C T W-B-E$ & 14 & GSME-21 & & 7 & OPRSLG1-ACNU\# & 11 & & & & \\
\hline G-POWER-E & 3 & GSME-22 & , & 7 & OPRSLG1-LAH1\# & 11 & & & . & \\
\hline G-POWER-E & 13 & GSME-22 & & 10 & OPRSMEV-RMH1\#\# & 18 & & & & \\
\hline G-POWER-E & 18 & GSME-27 & & 1 & OPRSRAT-CAN1\# & 6 & & & • & \\
\hline G-PSW & 3 & GSME-29 & & 1 & OPRSRAT-NCN1\# & 6 & & - & & \\
\hline G-SRAT-23 & 5 & GSME-29 & & 12 & OPRSRAT6ACN1\# & 10 & & & & \\
\hline G-SRAT-23-A & 5 & GSME- 31 & & 2 & OPRSRAT6ACNU\# & 10 & & . & & \\
\hline G-SRAT-23-A & 6 & GSME-31 & & 3 & OPRSRAT6LAH1\# & 10 & & - & - & \\
\hline G-SRAT- 48 & 5 & GSME-31 & - & 12 & OPRSRAT7ACN1\# & 5 & & & & \\
\hline G-SRAT- 48 & 11 & GSME-31A & & 12 & OPRSRAT7ACNU\# & 5 & & & & \\
\hline G-SRAT-48A & 11 & GSME- 32 & & 3 & OPRSRAT7LAH 1\# & 5 & & & & \\
\hline G-SRAT- 49 & 11 & GSME-33 & & 12 & PCSSRAT-BEL5\# & 5 & . & & & \\
\hline \multicolumn{5}{|c|}{ SME Explosion } & I:ICAFTAITREEI & ME.C & AF & $11-08-94$ & Page & 21 \\
\hline
\end{tabular}




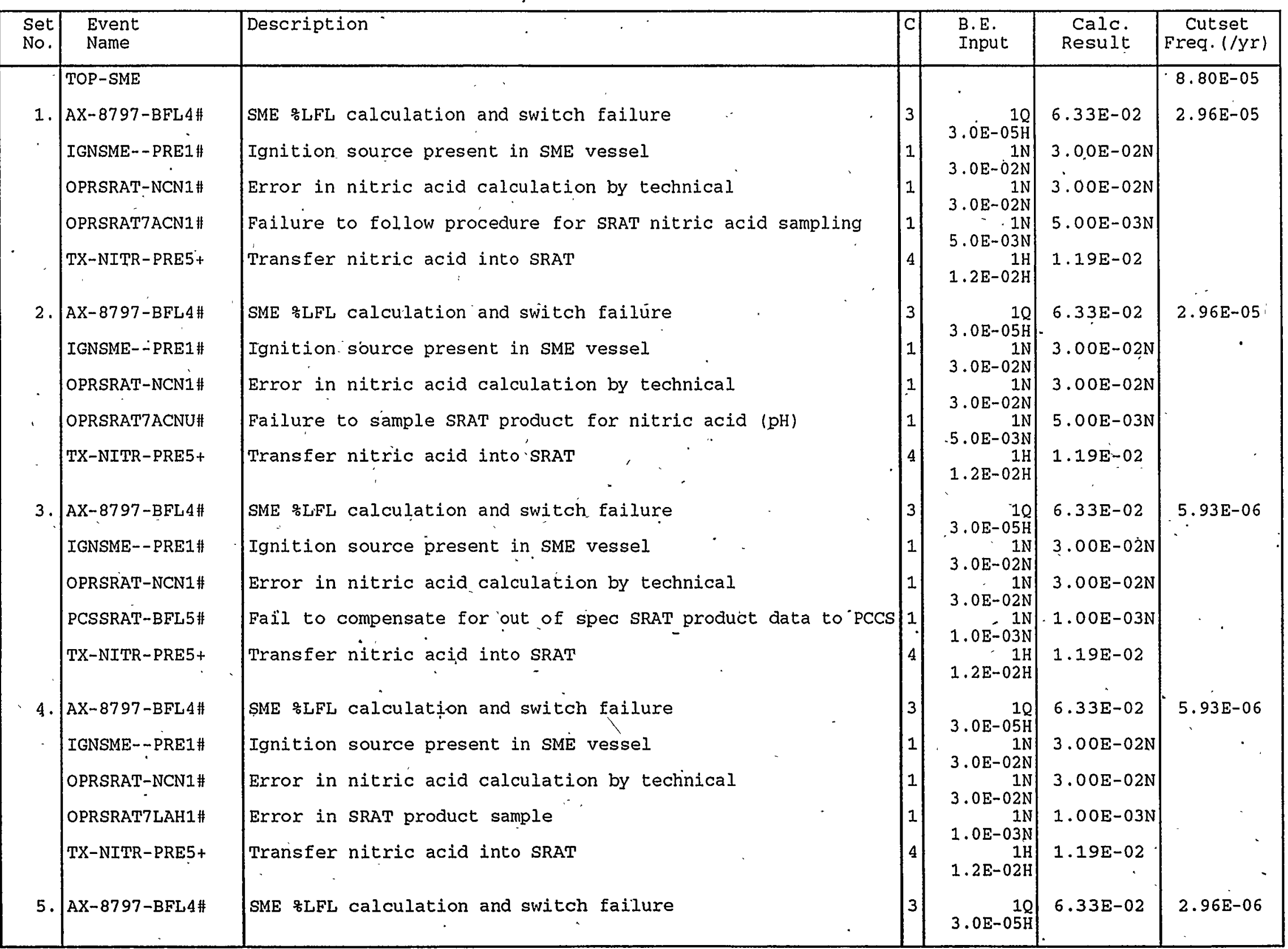




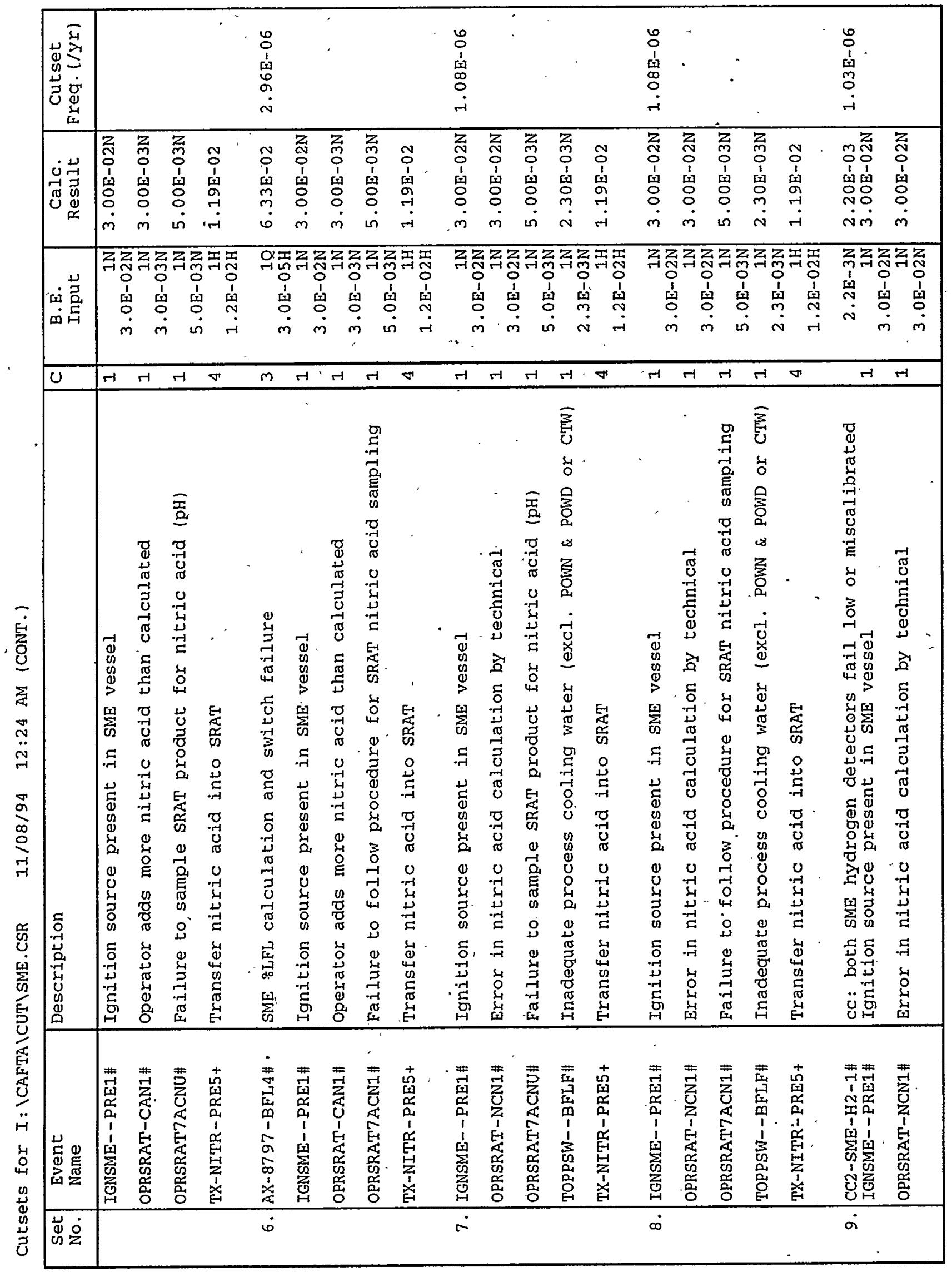




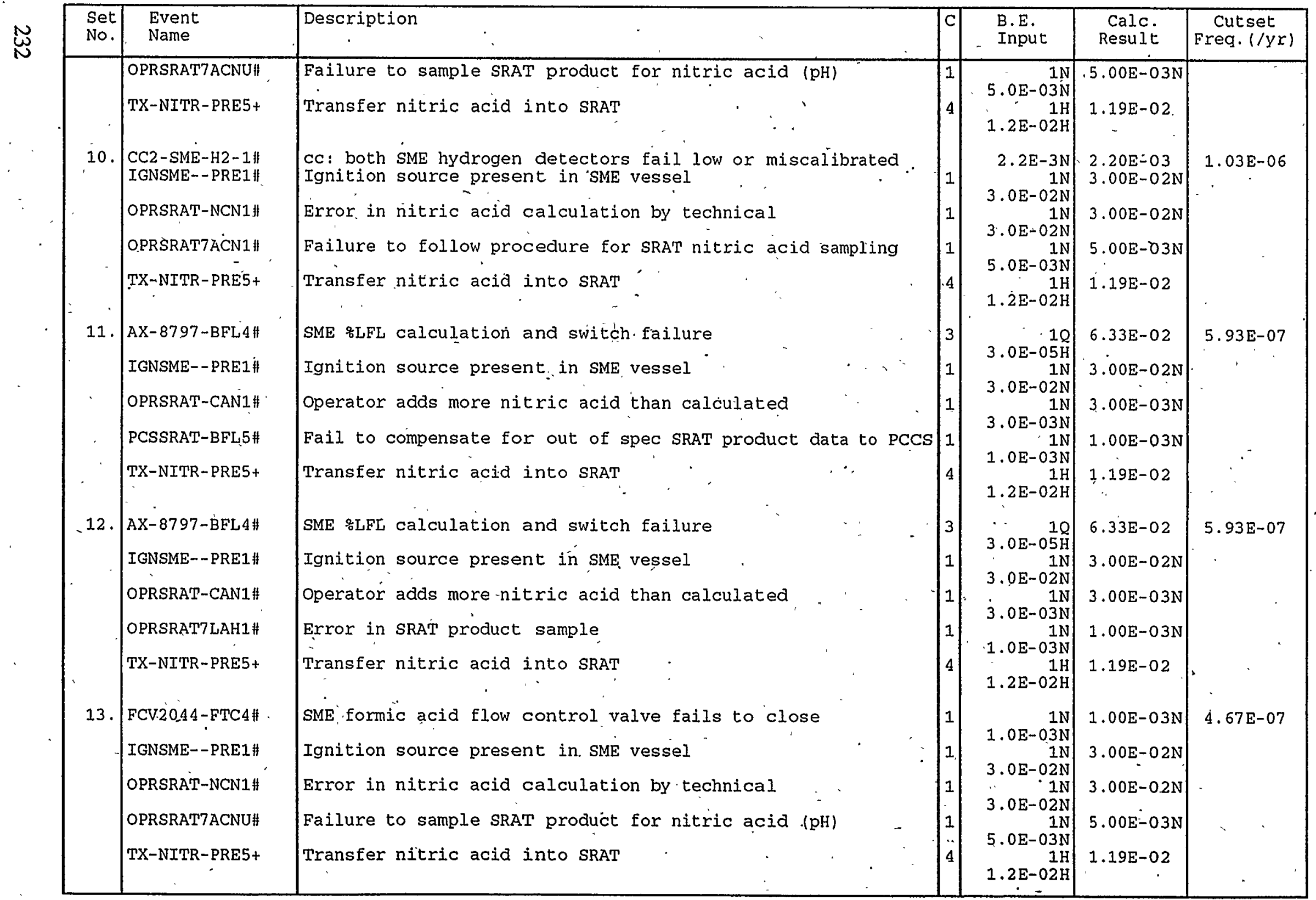


Cutsets for I: ICAFTAICUT\SME.CSR

11/08/94 12:24 AM (CONT.)

\begin{tabular}{|c|c|c|c|c|c|c|}
\hline $\begin{array}{l}\text { Set } \\
\text { No. }\end{array}$ & $\begin{array}{l}\text { Event } \\
\text { Name }\end{array}$ & Description & c| & $\begin{array}{l}\text { B.E. } \\
\text { Input }\end{array}$ & $\begin{array}{l}\text { Calc. } \\
\text { Result }\end{array}$ & $\begin{array}{c}\text { Cutset } \\
\text { Freq. (/yr) }\end{array}$ \\
\hline \multirow[t]{6}{*}{14.} & FCV $3080-$ FTC4\# & SME steam supply valve fails to close & 1 & & $1.00 \mathrm{E}-03 \mathrm{~N}$ & $4.67 \mathrm{E}-07$ \\
\hline & IGNSME- -PREI\# & Ignition source present in SME vessel & 1 & $\begin{array}{r}1.0 \mathrm{E}-03 \mathrm{~N} \\
1 \mathrm{~N}\end{array}$ & $3.00 \mathrm{E}-02 \mathrm{~N}$ & \\
\hline & OPRSRAT-NCN1\# & Error in nitric acid calculation by technical & 1 & $3.0 \mathrm{E}-02 \mathrm{~N}$ & $3.00 \mathrm{E}-02 \mathrm{~N}$ & $r$ \\
\hline & OPRSRAT7ACNU\# & Failure to sample SRAT product for nitric acid (DH) & 1 & $3.0 \mathrm{E}-02 \mathrm{~N}$ & $5.00 \mathrm{E}-03 \mathrm{~N}$ & \\
\hline & 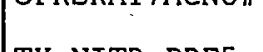 & 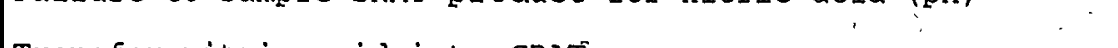 & & $5.0 \mathrm{E}-03 \mathrm{~N}$ & $3.00 \mathrm{E}-\mathrm{STN}$ & \\
\hline & TX-NITR-PRE5+ & Transfer nitric acid into SRAT & 4 & $1.2 \mathrm{E}-02 \mathrm{H}$ & $1.19 \mathrm{E} \div 02$ & \\
\hline \multirow[t]{5}{*}{15.} & FCV3080-FTC4\# & SME steam supply valve fails to close & 1 & $10,0-03 N$ & $1.00 \mathrm{E}-03 \mathrm{~N}$ & $4.67 \mathrm{E}-07$ \\
\hline & IGNSME- - PREI\# & Ignition source present in SME vessel $1^{\circ}$ & 1 & $\begin{aligned} 1.0 \mathrm{E}-03 \mathrm{~N} \\
1 \mathrm{~N}\end{aligned}$ & $3.00 \mathrm{E}-02 \mathrm{~N}$ & \\
\hline & OPRSRAT-NCN1\# & Error in nitric acid calculation by technical & 1 & $\begin{aligned} 3.0 \mathrm{E}-02 \mathrm{~N} \\
1 \mathrm{~N}\end{aligned}$ & $3.00 \mathrm{E}-02 \mathrm{~N}$ & \\
\hline & OPRSRAT7ACN1\# & Failure to follow procedure for SRAT nitric acid sampling & 1 & $\begin{aligned} 3.0 \mathrm{E}-02 \mathrm{~N} \\
1 \mathrm{~N} \\
5-0 \mathrm{E}-03 \mathrm{~N}\end{aligned}$ & $5.00 \mathrm{E}-03 \mathrm{~N}$ & \\
\hline & TX-NITR-PRE5+ & Transfer nitric acid into SRAT & 4 & $\begin{array}{r}3.0 \mathrm{E}-03 \mathrm{~N} \\
1 \mathrm{H} \\
1.2 \mathrm{E}-02 \mathrm{H}\end{array}$ & $1.19 \mathrm{E}-02$ & . \\
\hline \multirow[t]{5}{*}{16.} & FCV2044-FTC4\# & SME formic acid flow control valve fails to close & 1 & & $1.00 \mathrm{E}-03 \mathrm{~N}$ & $4.67 \mathrm{E}-07$ \\
\hline & IGNSME--PRE1\| & Ignition source present in SME vessel & 1 & $\begin{aligned} 1.0 \mathrm{E}-0.5 \mathrm{~N} \\
\mathrm{IN}\end{aligned}$ & $3.00 \mathrm{E}-02 \mathrm{~N}$ & \\
\hline & OPRSRAT-NCN1\# & Error in nitric acid calculation by technical. & 1 & $\begin{array}{r}3.0 \mathrm{E}-02 \mathrm{~N} \\
1 \mathrm{~N}\end{array}$ & $3.00 \mathrm{E}-02 \mathrm{~N}$ & \\
\hline & OPRSRAT7ACN1\# & Failure to follow procedure for SRAT nitric acid sampling & 1 & $3.0 \mathrm{E}-02 \mathrm{~N}$ & $5.0 .0 \mathrm{E}-0.03 \mathrm{~N}$ & \\
\hline & TX-NITR-PRE5+ & Transfer nitric acid into SRAT, & 4 & $\begin{array}{r}5.0 \mathrm{E}-03 \mathrm{~N} \\
1 \mathrm{H} \\
1.2 \mathrm{E}-02 \mathrm{H}\end{array}$ & $1.19 \mathrm{E}-02$ & \\
\hline \multirow[t]{5}{*}{17.} & AX-8797-BFL4\# & SME 8 LFL calculation and switch failure & 3 & $\begin{array}{r}10 \\
3.0 \mathrm{E}-05 \mathrm{H}\end{array}$ & $6.33 \mathrm{E}-02$ & $.3 .70 \mathrm{E}-07$ \\
\hline & FE-8856-FHI4+ & SME purge flow element fails high & 4 & $\begin{array}{r}86 \mathrm{H} \\
30 \mathrm{~F}-06 \mathrm{H}\end{array}$ & $2.58 \mathrm{E}-04$ & \\
\hline & IGNSME- -PRE1\# & Ignition source present in SME vessel & 1 & 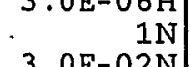 & $3.00 \mathrm{E}-02 \mathrm{~N}$ & \\
\hline & OPR-SME-SOH1\# & Fail to open SME purge supply needle valve & 1 & $\begin{array}{r}3.0 \mathrm{E}-0 . \mathrm{N} \\
1 \mathrm{~N} \\
5.0 \mathrm{E}-02 \mathrm{~N}\end{array}$ & $5.00 \mathrm{E}-02 \mathrm{~N}$ & \\
\hline & SV-SME--ADंJ5+ & SME purge supply needle valve adjusted & 4 & $\begin{array}{r}5.0 \mathrm{E}-02 \mathrm{~N} \\
24 \mathrm{H} \\
1.0 \mathrm{E}+00 \mathrm{M}\end{array}$ & $3.23 \mathrm{E}-02$ & \\
\hline 18. & |IGNSME- -PREI\# & Ignition source present in SME vessel & 1 & $3.0 \mathrm{E}-02 \mathrm{~N} N$ & $3.00 \mathrm{E}-02 \mathrm{~N}$ & $3.14 \mathrm{E}-07$ \\
\hline
\end{tabular}


Cutsèts for I: \CAFTA ICUTISME.CSR 11/08/94 $12: 24$ AM (CONT.)

\begin{tabular}{|c|c|c|c|c|c|c|}
\hline No. & & on & 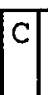 & & & \\
\hline 20 . & $\begin{array}{l}\text { IOPPOWN-BFLH+ } \\
\text { IGNSME--PRE1 } \\
\text { OPRSRAT-NCN1 } \\
\text { PCSSRAT-BFL5\# } \\
\text { TOPPSW--BFLF\# } \\
\text { TX-NITR-PRE5+ } \\
\text { IGNSME--PRE1\# } \\
\text { OPRSRAT-NCN1\# } \\
\text { OPRSRAT7LAH1\# } \\
\text { TOPPSW--BFLF\# } \\
\text { TX-NITR-PRE5+ } \\
\text { CC2-SME-H2-1\# } \\
\text { IGNSME--PRE1\# } \\
\text { OPRSRAT-NCN1\# } \\
\text { OPRSRAT7LAH1\# } \\
\text { TX-NITR-PRE5+ } \\
\text { CC2-SME-H2-1\# } \\
\text { IGNSME--PRE1\# } \\
\text { OPRSRAT-NCN1\# } \\
\text { PCSSRAT-BFL5\# } \\
\text { TX-NITR-PRE5+ }\end{array}$ & 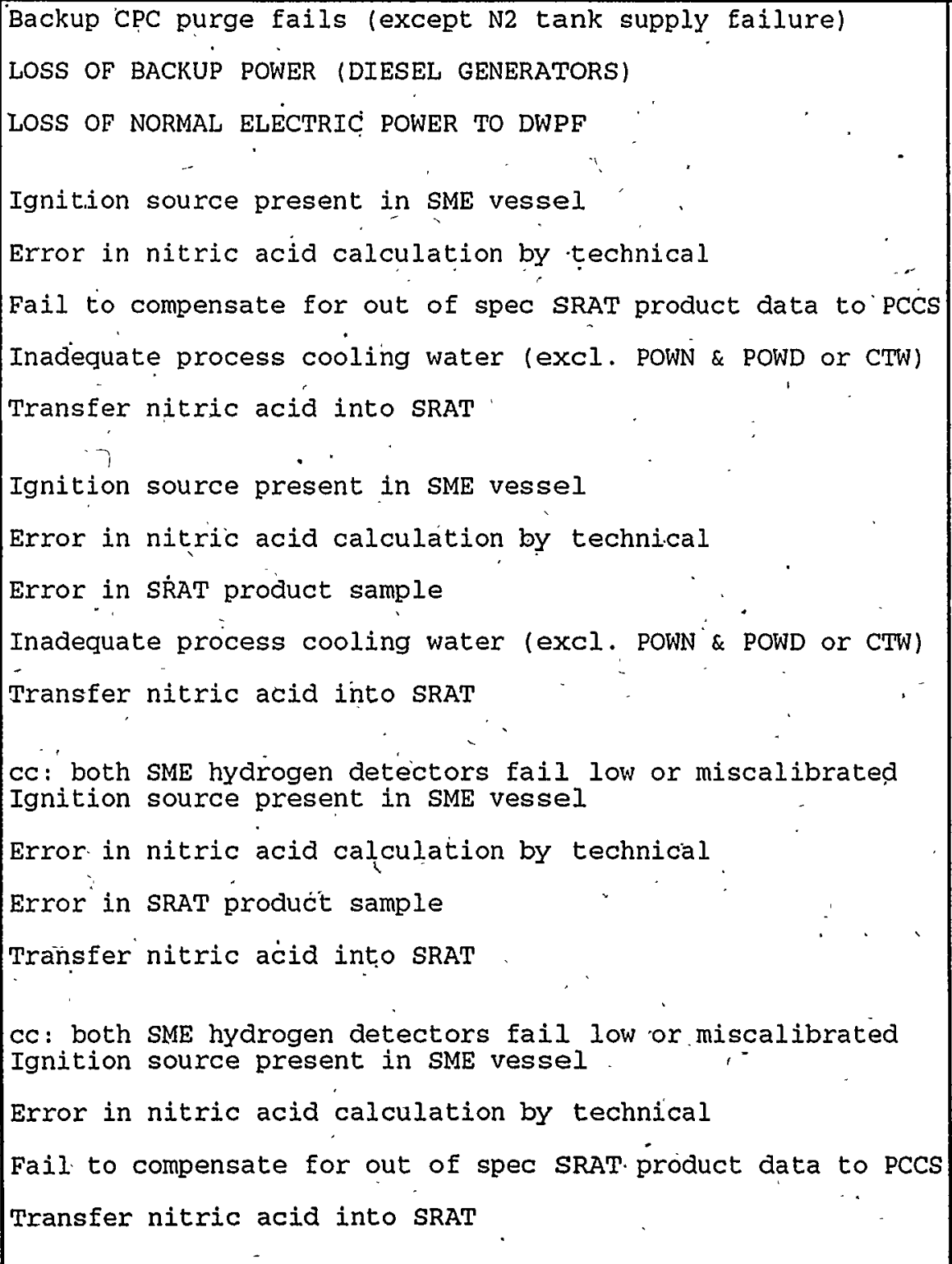 & 1 & $\begin{array}{r}1 \mathrm{~N} \\
3.0 \mathrm{E}-02 \mathrm{~N} \\
1 \mathrm{~N} \\
3.0 \mathrm{E}-02 \mathrm{~N} \\
1 \mathrm{~N} \\
1.0 \mathrm{E}-03 \mathrm{~N} \\
1 \mathrm{~N} \\
2.3 \mathrm{E}-03 \mathrm{~N} \\
1 \mathrm{H} \\
1.2 \mathrm{E}-02 \mathrm{H} \\
1 \mathrm{~N} \\
3.0 \mathrm{E}-02 \mathrm{~N} \\
1 \mathrm{~N} \\
3.0 \mathrm{E}-02 \mathrm{~N} \\
1 \mathrm{~N} \\
1.0 \mathrm{E}-03 \mathrm{~N} \\
1 \mathrm{~N} \\
2.3 \mathrm{E}-03 \mathrm{~N} \\
1 \mathrm{H} \\
-1.2 \mathrm{E}-02 \mathrm{H} \\
2.2 \mathrm{E}-3 \mathrm{~N} \\
1 \mathrm{~N} \\
3.0 \mathrm{E}-02 \mathrm{~N} \\
1 \mathrm{~N} \\
3.0 \mathrm{E}-02 \mathrm{~N} \\
1 \mathrm{~N} \\
1.0 \mathrm{E}-03 \mathrm{~N} \\
1 \mathrm{H} \\
1.2 \mathrm{E}-02 \mathrm{H} \\
2.2 \mathrm{E}-3 \mathrm{~N} \\
1 \mathrm{~N} \\
3.0 \mathrm{E}-02 \mathrm{~N} \\
1 \mathrm{~N} \\
3.0 \mathrm{E}-02 \mathrm{~N} \\
1 \mathrm{~N} \\
1.0 \mathrm{E}-03 \mathrm{~N} \\
1.2 \mathrm{E}-02 \mathrm{H}\end{array}$ & $\begin{array}{c}2.30 \mathrm{E}-03 \mathrm{~N} \\
1.19 \mathrm{E}-02 .\end{array}$ & $2.06 \mathrm{E}-07$ \\
\hline
\end{tabular}


cutsets for I: \CAFTA \CUT\SME.CSR

11/08/94. 12:24 AM (CONT.)

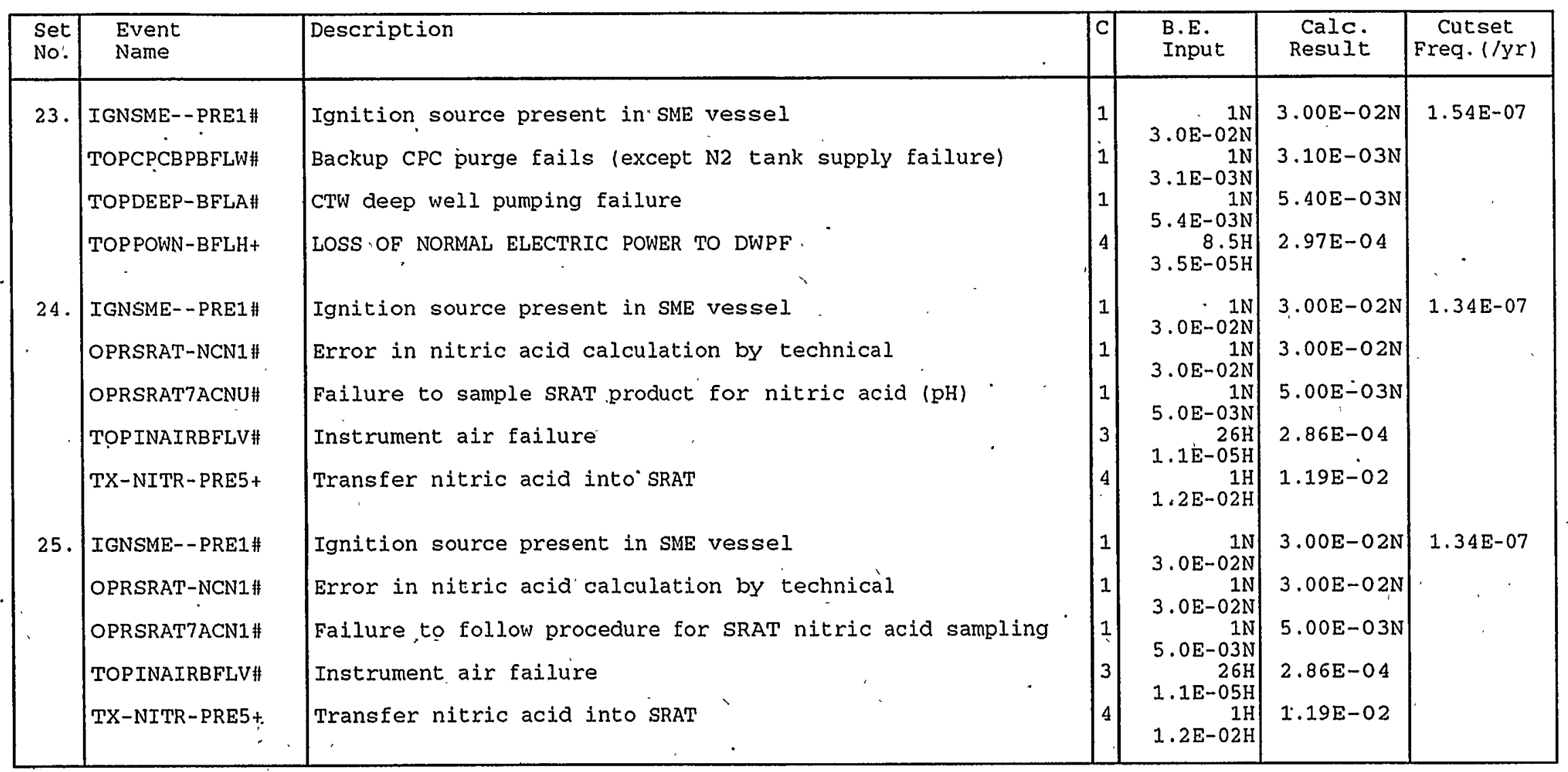


Explosion in SME

Basic Event Name

FE-8856-FHI4+-IC

TOPPOWN-BFLH+-IC

TX-NITR-PRE5+-I

OPRSRATTLAH1\#

PCSSRAT-BFL5\#

OPRSRAT7ACN1\#

OPRSRAT7ACNU\#

TOPCPCPPBFL2+I

IGNSME-PRE1\#

OPRSRAT-CAN1\#

OPRSRAT-NCN1\#

FCV8856-FCLA+-IC

HCV3089-FCLA\#

RY-PFSFTFTO4\#

TOPCTWCCBFLO\#

TOPINAIRBFLV\#

FCV2044-FTC4\# -

FCV3080-FTC4\#

TOPPSW--BFLF\#

CC2-SME-H2-1\#

AX-8797-BFLA\#

SRABATCHPRE5+I

SV-SME-ADJ5+IC

TOPCPCBPBFLW\#

CC3MAINTFSL1\#

CC3-SME-FSLA\#

TOPDEEP-BFLA\#

TOPPOWD-BFLN\#

OPRSLG1-LAH1\#

OPRSLG1-ACNU\#

AEG8797-LKS4\#

AEG8798-LKS4\#

AEG8798-FLOD\#

AEG8797-FLOD\#

OPR8798AMCH1\#

OPR8797AMCH1\#

OPR-SME-SOH1\#

TOPCTWP-BFLB\#

SLGNOBL-PRES\#

OPR8856-MCH2\#
Top Event Frequency: 8:75E-05/YR

\section{Risk Achievement Worth} Description

SME purge flow element fails high

LOSS OF NORMAL ELECTRIC POWER TO DWPF .

Transfer nitric acid into SRAT

Error in SRAT product sample

Fail to compensate out of spec SRAT product data to PCCS

Failure to follow procedure for SRAT nitric acid sampling

Failure to sample SRAT product for nitric acid $(\mathrm{pH})$

CPC primary purge system fails (excluding normal power)

Ignition source present in SME vessel

Operator adds more nitric acid than calculated

Error in nitric acid calculation by technical ,

SME purge flow control valve fails closed

SME cooling water valve manual controller fails to open

Relay for PFSFT pump fails to open

CTW valving or piping failure (excl. inair).

Instrument air failure

SME formic acid flow control valve fails to close

SME steam supply valve fails to close

Inadequate cooling water (excl. POWN \& POWD or CTW)

cc: both SME hydrogen detectors fail low or miscalibrated

SME \%LFL calculation and switch failure

New batch of sludge for DWPF

SME purge supply needle valve adjusted

Backup CPC purge fails (except N2 tank supply failure)

cc: all 3 SME low flow switches miscalibrated

CC: all 3 SME offgas sample flow low switches fail to open

CTW deep well pumping failure

LOSS OF BACKUP POWER (DIESEL GENERATORS)

Error in sludge sample for noble metals

Failure to follow procedure for tank sampling

Air inleakage in SME detector 8797 sample line

Air inleakage in SME detector 8798 sample line

SME hydrogen detector 2 (8798) fails low

SME LFL analyzer 8797 fails low

SME hydrogen detector 2 out of calibration

SME hydrogen detector 1 out of calibration

Fail to open SME purge supply needle valve

CTW primary pumping failure (excl. POWN)

Excessive noble metals present in sludge

Operator miscalibrates SME purge flow element
Prob/Freq

$3.00 \mathrm{E}-06 / \mathrm{H}$

$3.50 \mathrm{E}-05 / \mathrm{H}$

$1.19 \mathrm{E}-02 / \mathrm{H}$

$1.00 \mathrm{E}-03$

$1.00 \mathrm{E}-03$

5.00E-03

5.00E-03

$1.90 \mathrm{E}-05 / \mathrm{H}$

$3.00 \mathrm{E}-02$

$3.00 \mathrm{E}-03$

3.00E-02

$1.00 \mathrm{E}-06 / \mathrm{H}$

4.00E-06

$1.00 \mathrm{E}-05$

6.05E-05

2.86E-04

$1.00 \mathrm{E}-03$

$1.00 \mathrm{E}-03$

$2.30 \mathrm{E}-03$

$1.80 \mathrm{E}-03$

6.34E-02

$5.68 \mathrm{E}-05 / \mathrm{H}$

$1.34 \mathrm{E}-03 / \mathrm{H}$

$3.10 \mathrm{E}-03$

3.00E-04

$1.10 \mathrm{E}-04$

$5.40 \mathrm{E}-03$

$1.10 \mathrm{E}-02$

$1.00 \mathrm{E}-03$

$5.00 \mathrm{E}-03$

1.64E-03

1.64E-03

2.15E-03

2.15E-03

$3.60 \mathrm{E}-03$

$3.60 \mathrm{E}-03$

5.00E-02

5.67E-04

1.00E-02

3.00E-04 .
AchW

$3.54 \mathrm{E}+02$

$1.98 \mathrm{E}+02$

$8.33 \mathrm{E}+01$

8.32E+01

$8.32 \mathrm{E}+01$

$8.28 \mathrm{E}+01$

$8.28 \mathrm{E}+01$

$4.48 \mathrm{E}+01$

$3.33 \mathrm{E}+01$

$3.08 \mathrm{E}+01$

$3.00 \mathrm{E}+01$

2.47E+01

1.57E+01

$1.57 \mathrm{E}+01$

1.57E+01

1.57E+01

1.57E+01

$1.57 \mathrm{E}+01$

$1.57 \mathrm{E}+01$

1.52E+01

1.43E+01

1.36E+01

$3.74 \mathrm{E}+00$

$3.48 \mathrm{E}+00$

$1.71 \mathrm{E}+00$

$1.71 \mathrm{E}+00$

$1.34 \mathrm{E}+00$

$1.33 \mathrm{E}+00$

$1.12 \mathrm{E}+00$

$1.12 \mathrm{E}+00$

$1.10 \mathrm{E}+00$

$1.10 \mathrm{E}+00$

$1.10 \mathrm{E}+00$

$1.10 \mathrm{E}+00$

$1.10 \mathrm{E}+00$

$1.10 \mathrm{E}+00$

$1.09 \mathrm{E}+00$

$1.08 \mathrm{E}+00$

$1.07 \mathrm{E}+00$

$1.03 E+00$

Note: Events that make less than $1 \%$ difference to the importance factor are not included. Modified top event frequency $=$ (top event frequency) $\mathrm{x}$ (risk achievement worth). 
Explosion in SME Top Event Frequency: 8.75E-05/YR

Basic Event Name

*IGNSME--PRE1\#

TX-NITR-PRE5+I

AX-8797-BFLA\#

OPRSRAT-NCN1\#

OPRSRAT7ACN1\#

OPRSRAT7ACNU\#

OPRSRAT-CAN1\#

PCSSRAT-BFL5\#

OPRSRATTLAH1\#

TOPPSW-BFLF\#

CC2-SME-H2-1\#

FCV2044-FTC4\#

FCV3080-FTC4\#
Risk Reduction Worth

Description

Ignition source present in SME vessel

Transfer nitric acid into SRAT

SME \%LFL calculation and switch failure

Error in nitric acid calculation by technical

Failure to follow procedure for SRAT nitric acid sampling

Failure to sample SRAT product for nitric acid (pH)

Operator adds more nitric acid than calculated

Fail to compensate out of spec SRAT product data to PCCS

Error in SRAT product sample

Inadequate cooling water (excl. POWN \& POWD or CTW)

cc: both SME hydrogen detectors fail low or miscalibrated

SME formic acid flow control valve fails to close

SME steam supply valve fails to close
Prob/Freq RedW

$3.00 \mathrm{E}-02 \quad 0.00 \mathrm{E}+00$

$1.19 \mathrm{E}-02 / \mathrm{H} \quad 7.62 \mathrm{E}+01$

$6.34 \mathrm{E}-02 \quad 9.95 \mathrm{E}+00$

$3.00 \mathrm{E}-02 \quad 9.72 \mathrm{E}+00$

$5.00 \mathrm{E}-03 \quad 1.70 \mathrm{E}+00$

$5.00 \mathrm{E}-03, \quad 1.70 \mathrm{E}+00$

$3.00 \mathrm{E}-03 \quad 1.10 \mathrm{E}+00$

$1.00 \mathrm{E}-03 \quad 1.09 \mathrm{E}+00$

$1.00 \mathrm{E}-03 \quad 1.09 \mathrm{E}+00$

$2.30 \mathrm{E}-03 \quad 1.03 \mathrm{E}+00$

$1.80 \mathrm{E}-03 \quad 1.03 \mathrm{E}+00$

$1.00 \mathrm{E}-03 \quad 1.01 \mathrm{E}+00$

$1.00 \mathrm{E}-03$ $1.01 \mathrm{E}+00$

* A Reduction Worth of $0.0 \mathrm{E}+00$ indicates event appears in every cutset could serve as a single event preventor for the accident.

Note: Events that make less than $1 \%$ difference to the importance factor are not included. Modified top event frequency $=$ (top event frequency) / (risk reduction worth). 
X-ESR-S-00001, REV. 0

\section{PRBT Explosion}




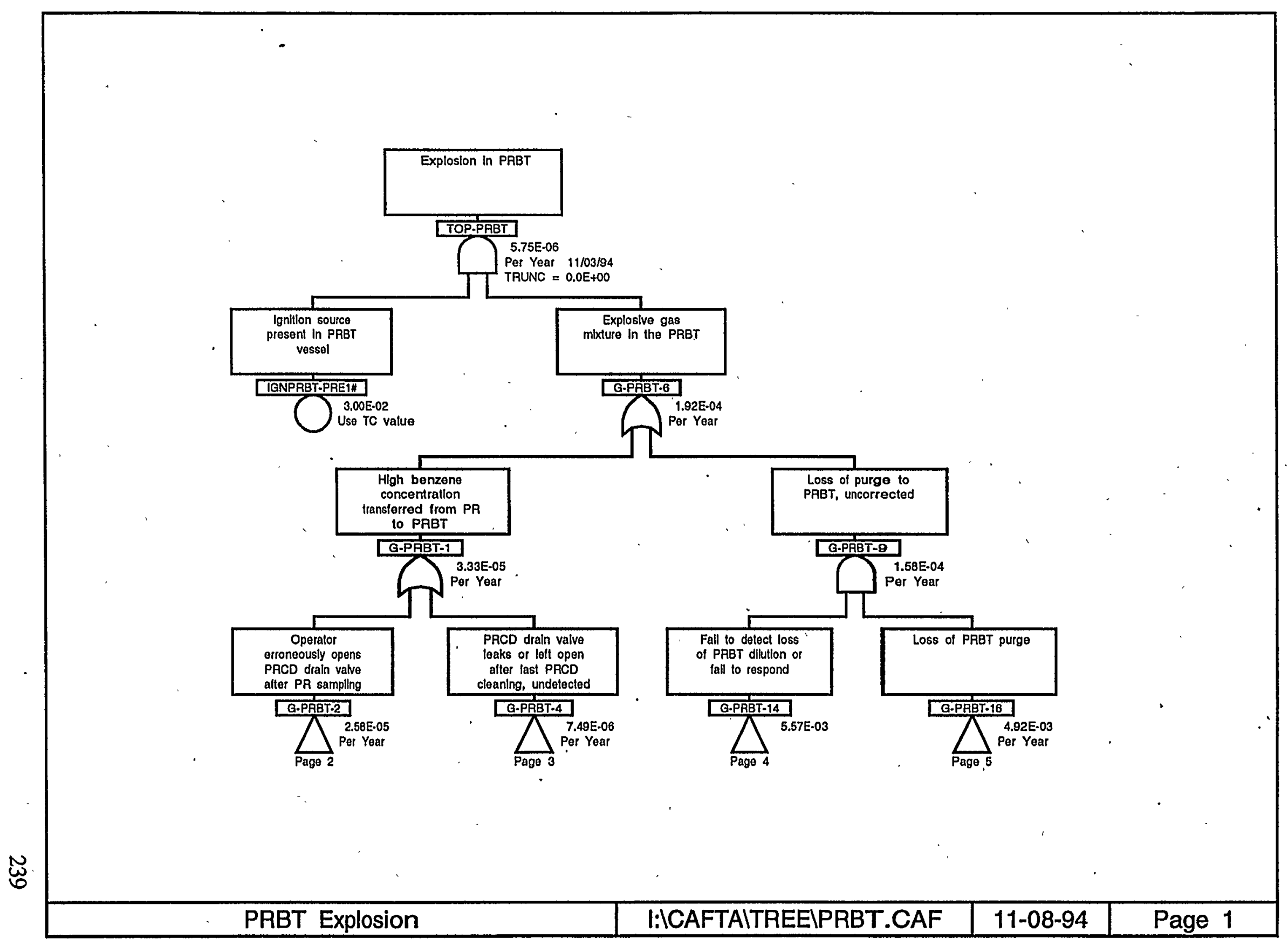


X-ESR-S-00001, REV. 0

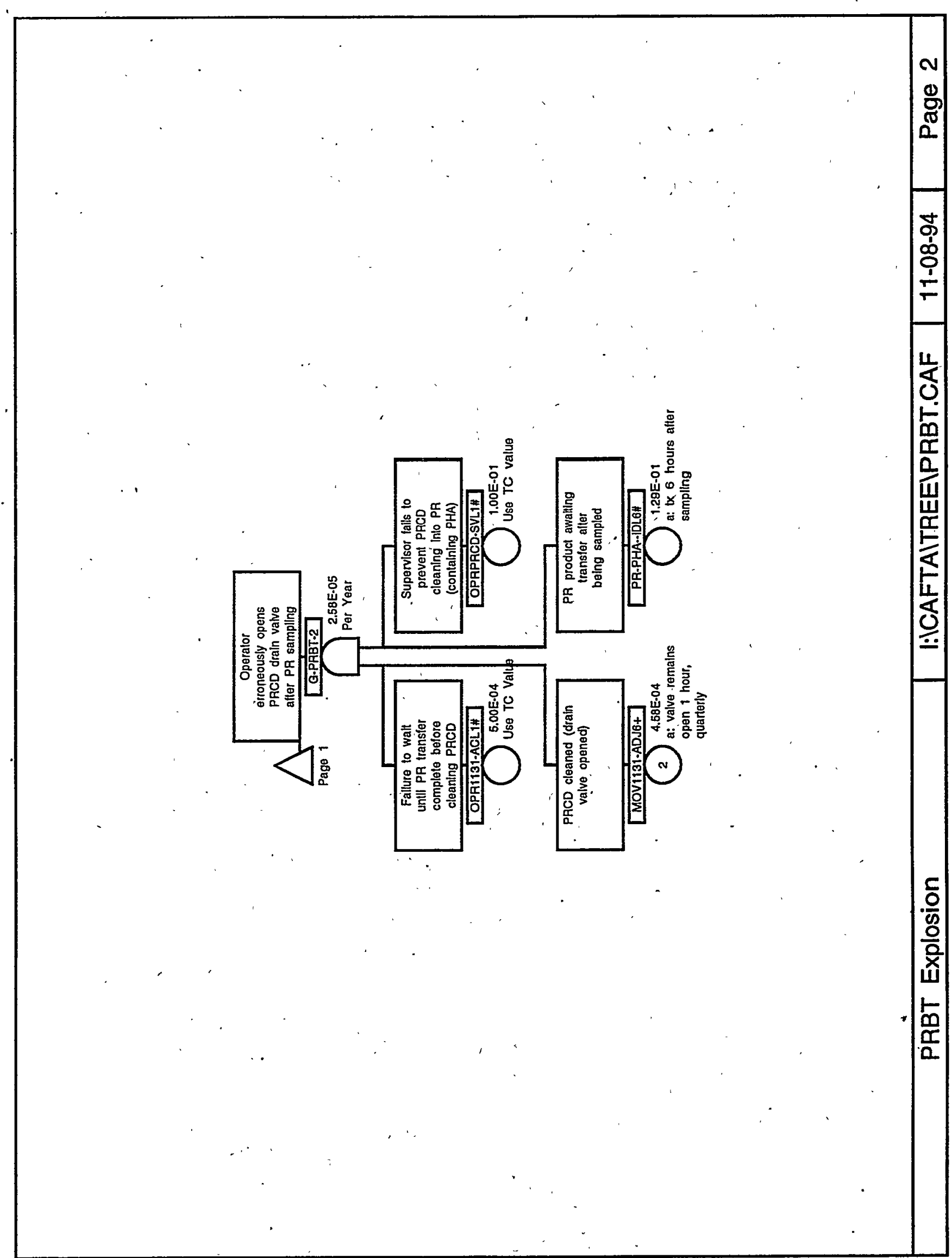




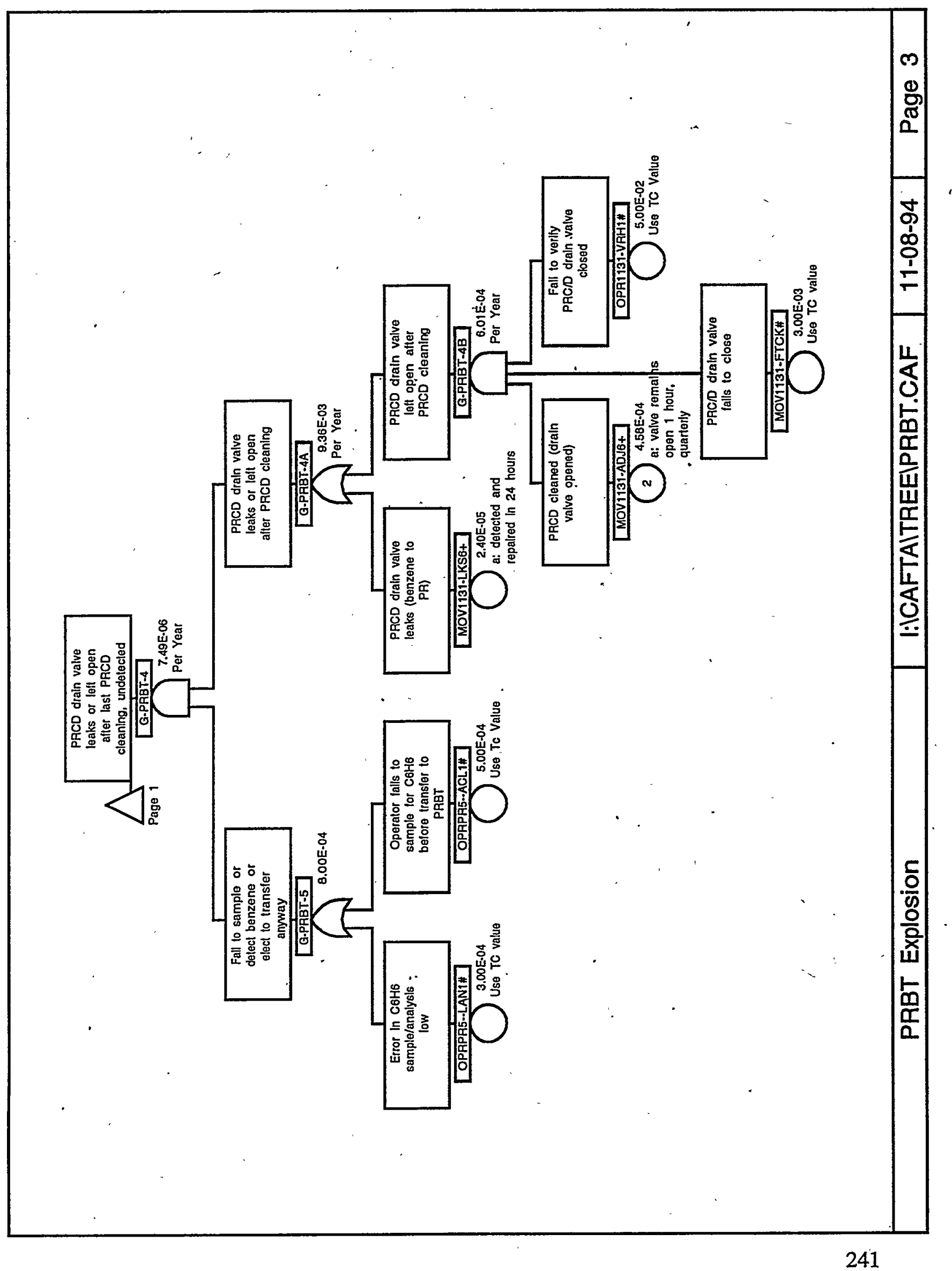




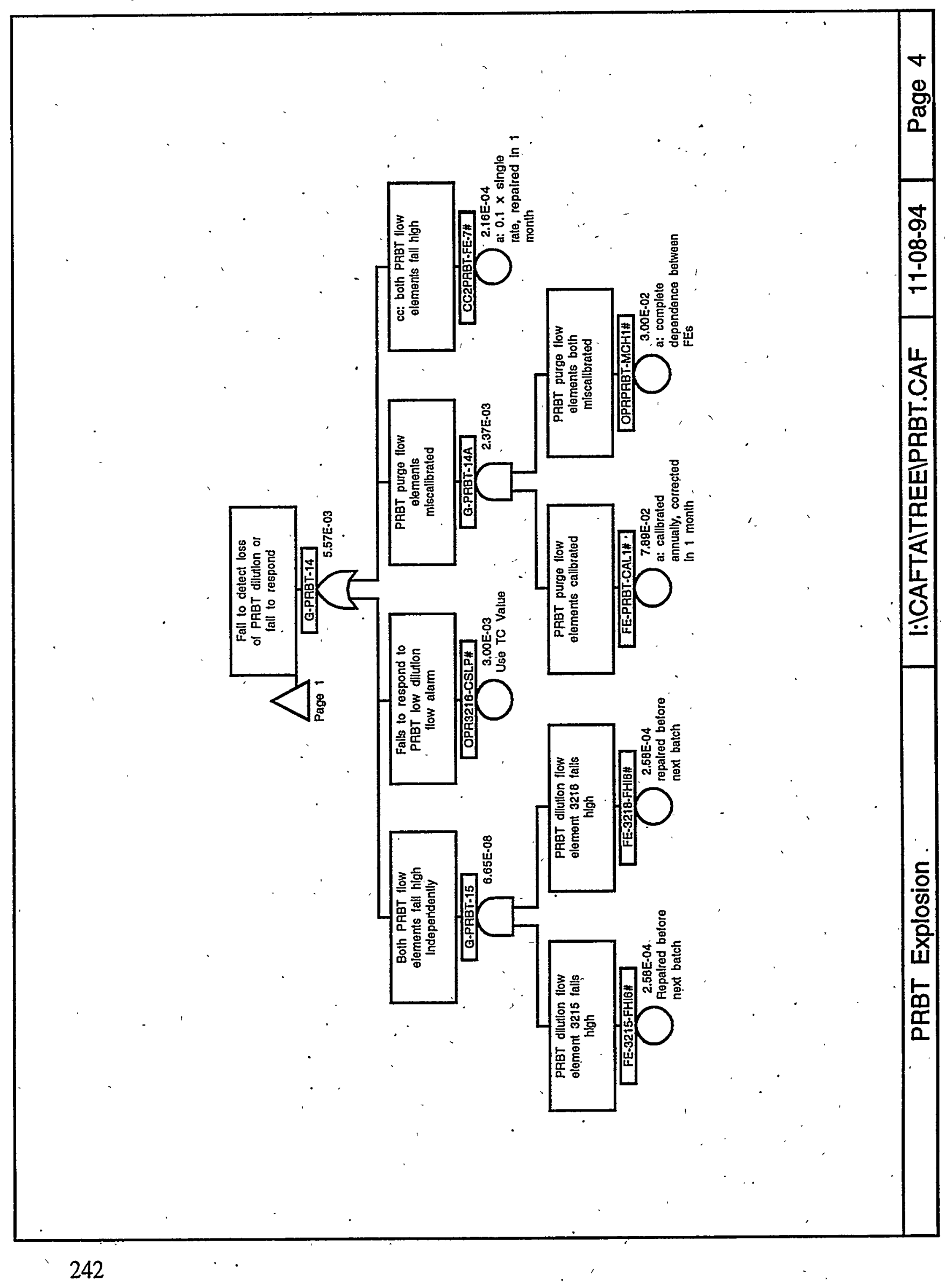







X-ESR-S-00001, REV. 0

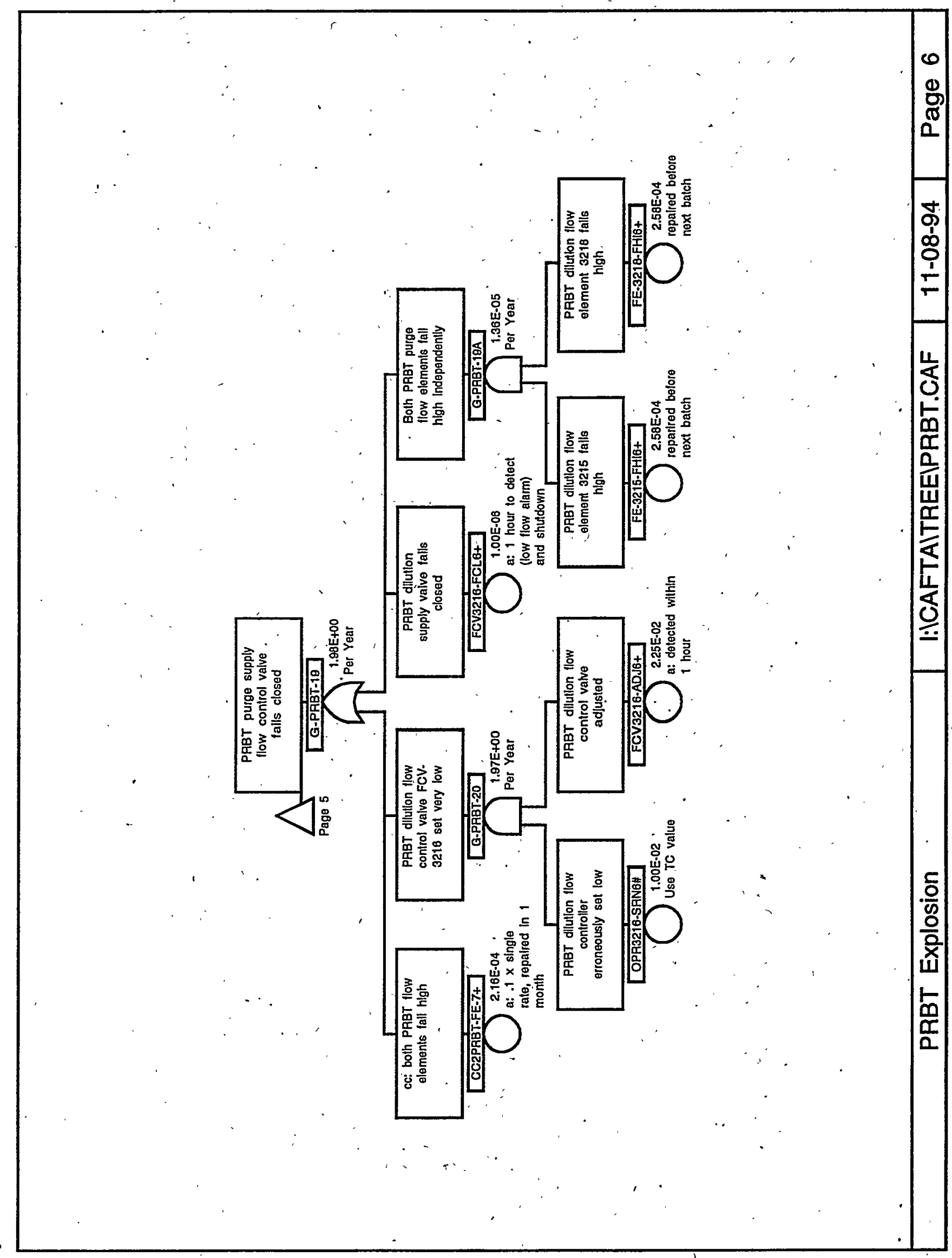




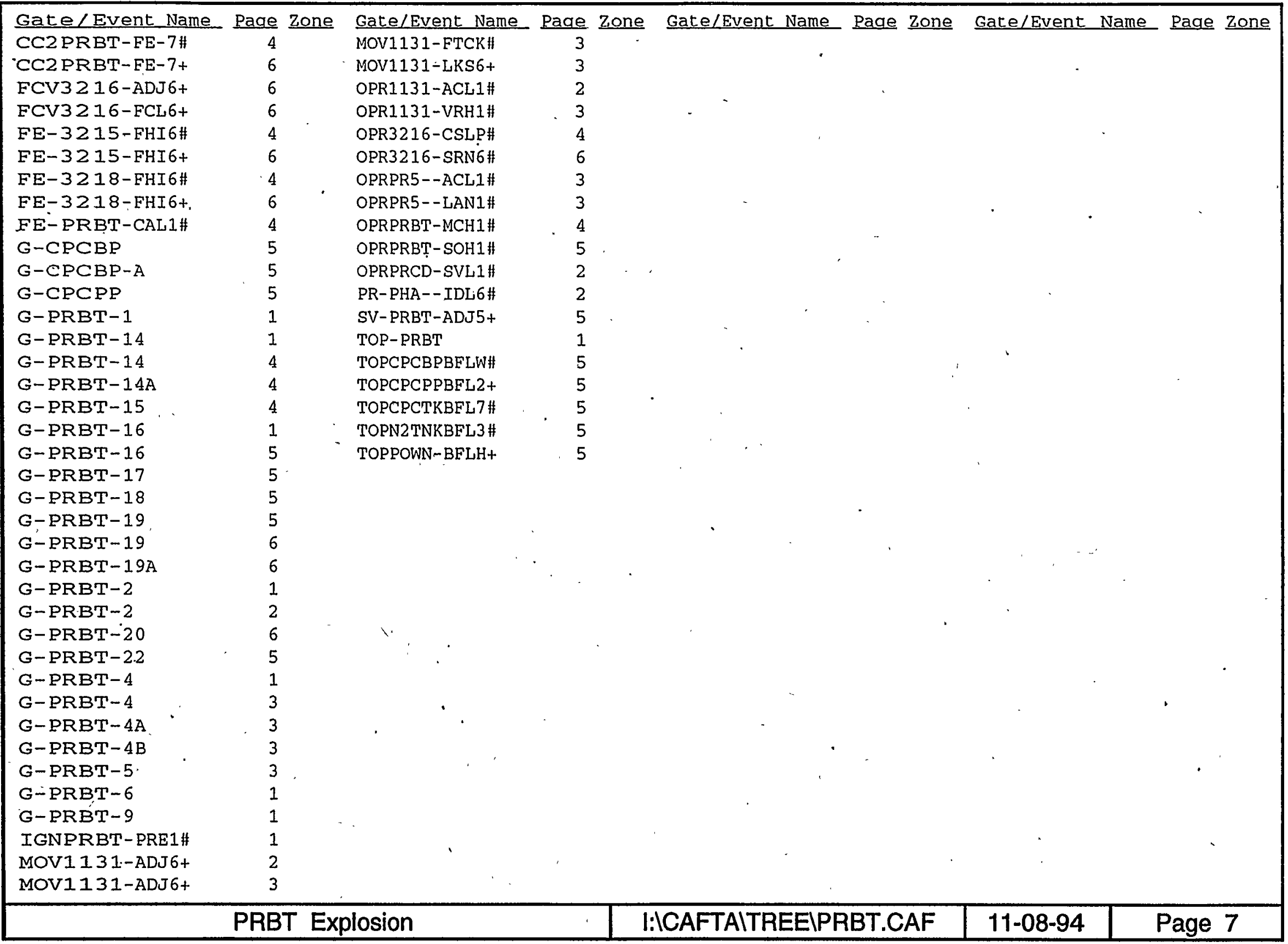




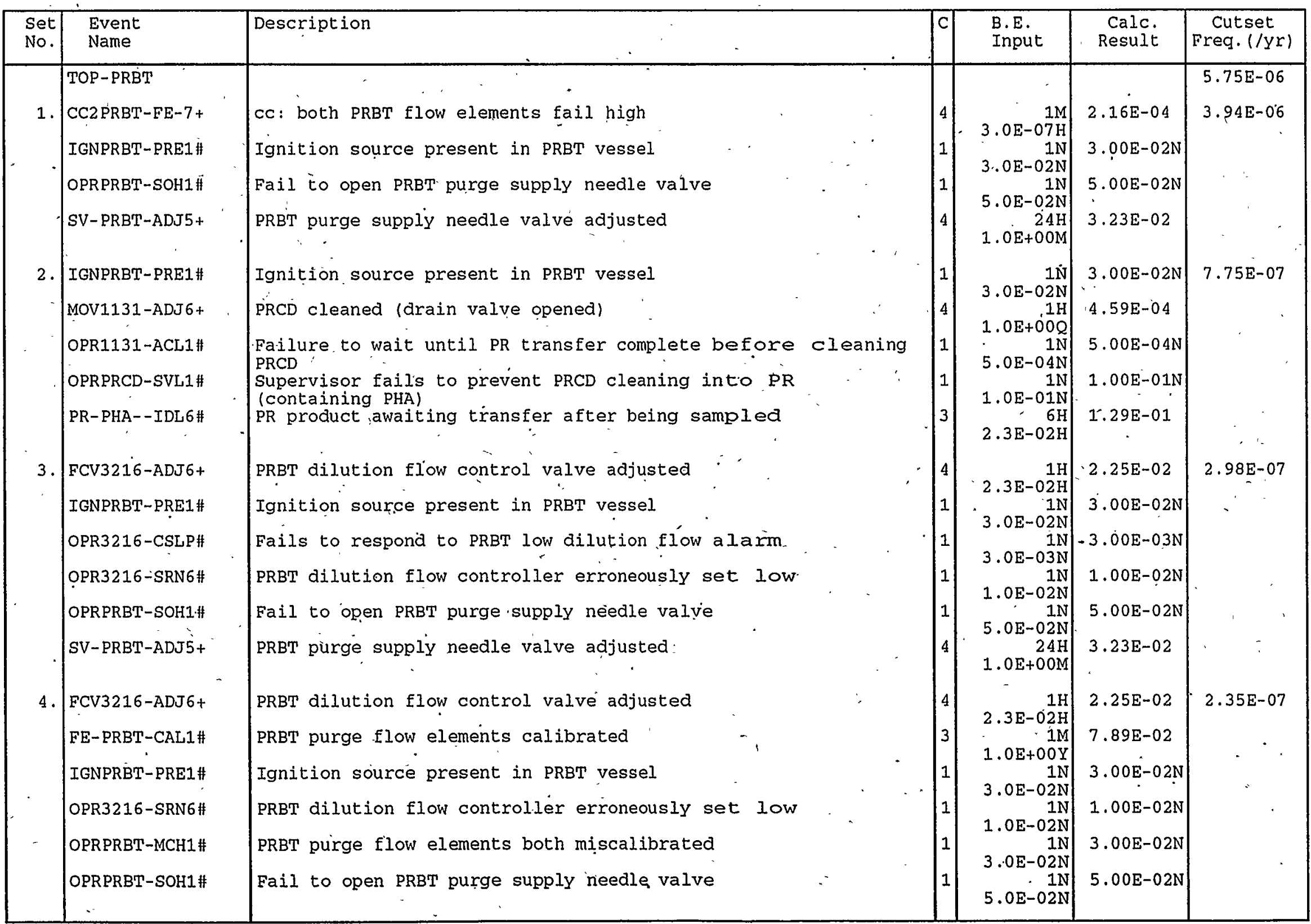


Cutsets for I: ICAFTA ICUT $\backslash P R B T . \dot{C} S R$

11/08/94 12:26 AM (CONT.)

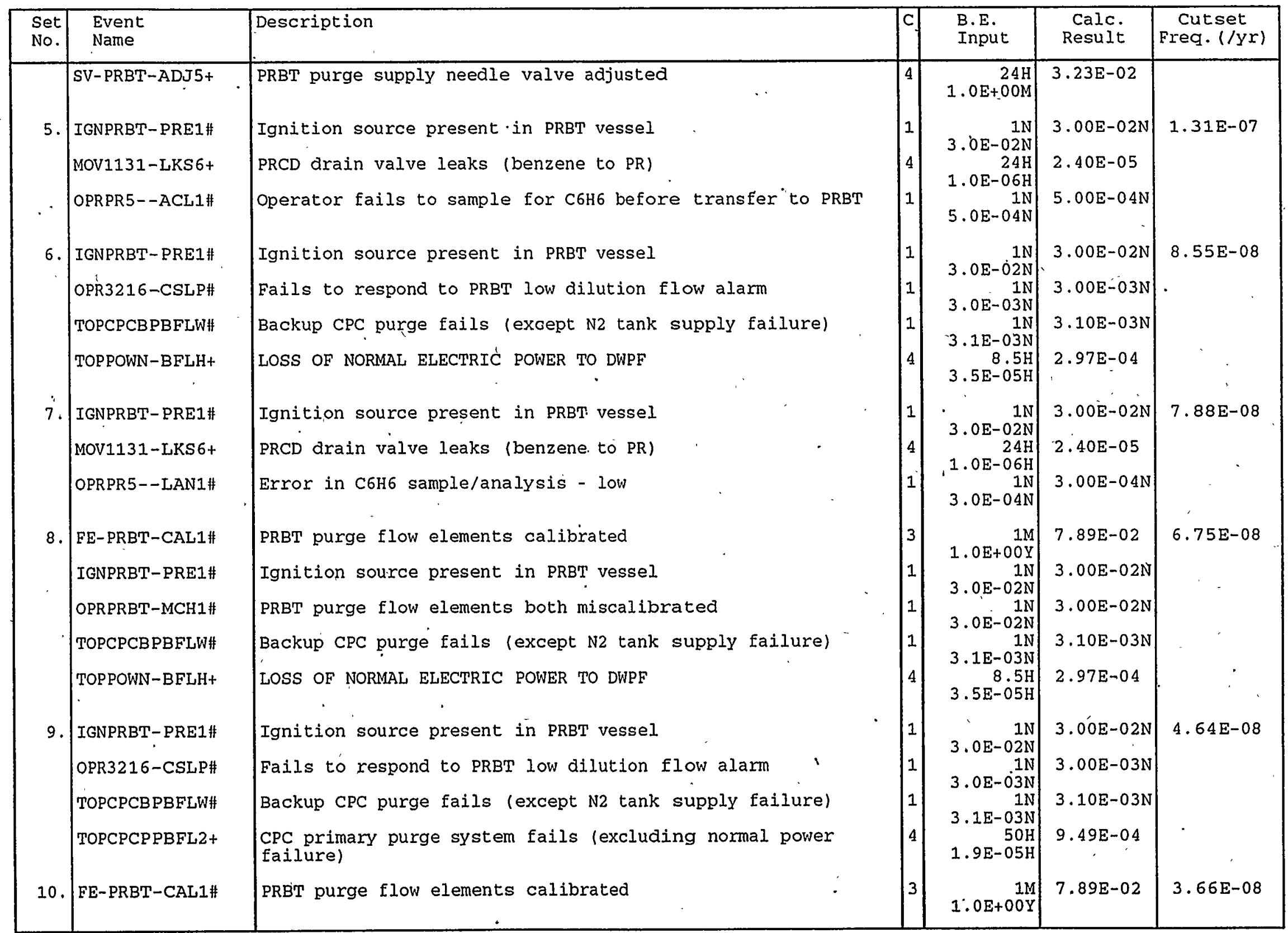




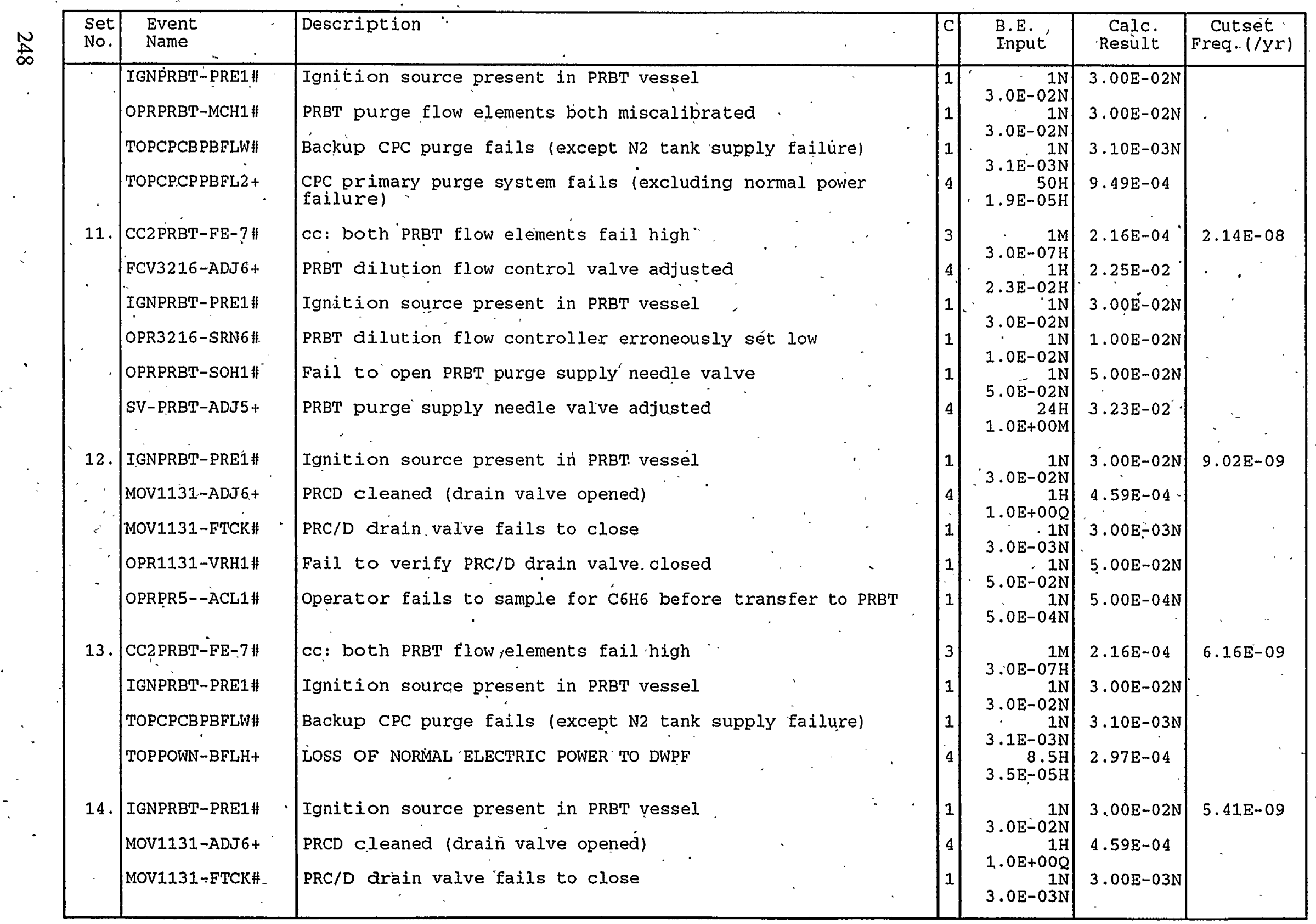


Cutsets for I: \CAFTA ICUT\PRBT.CSR. 11/08/94 12:26 AM (CONT.)

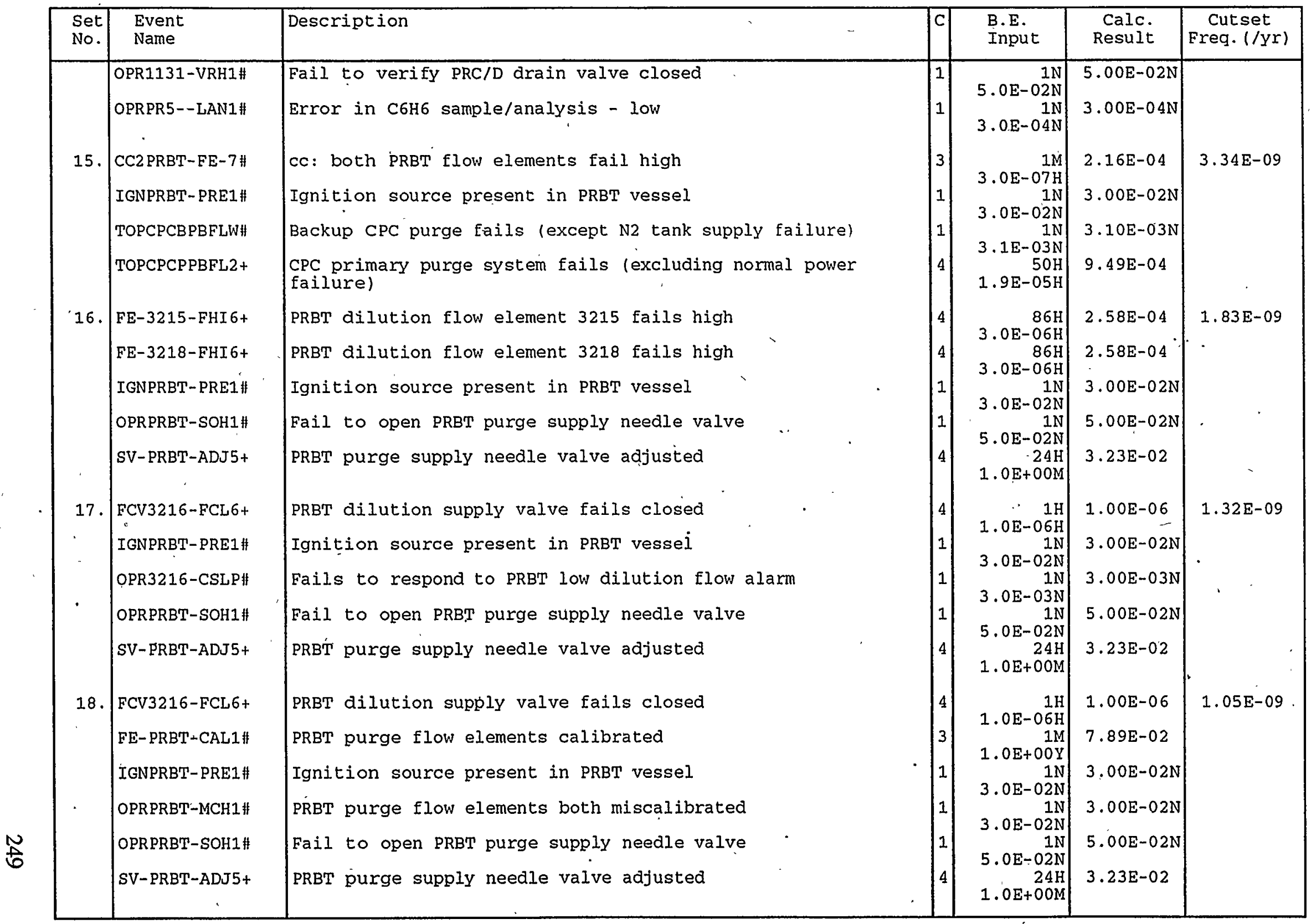


Cutsets for I: \CAFTA \CUT\PRBT.CSR. 11/08/94 12:26 AM (CONT.)

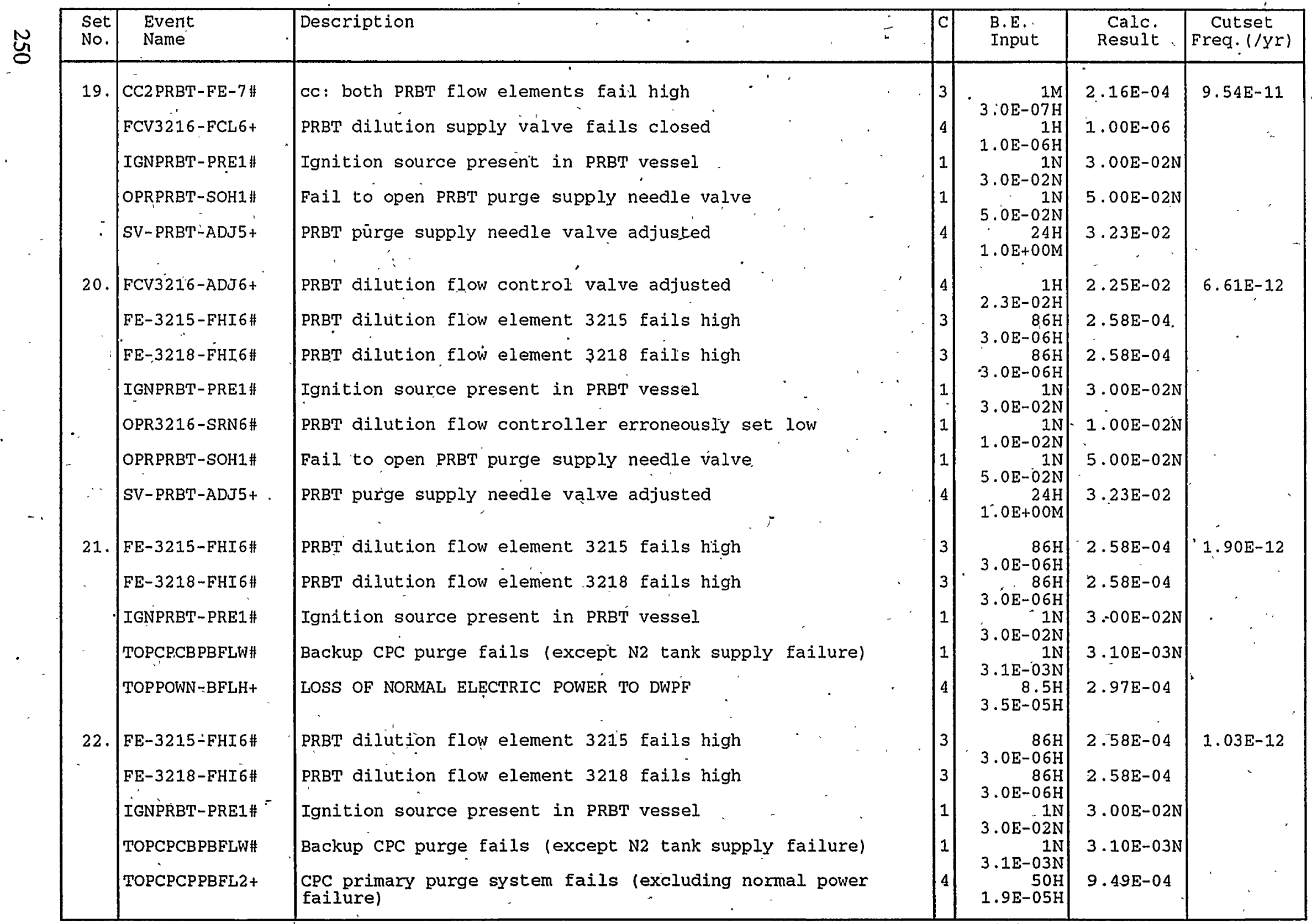


Cutsets for I : \CAFTA \CUT \PRBT.CSR

11/08/94 12:26 AM (CONT.)

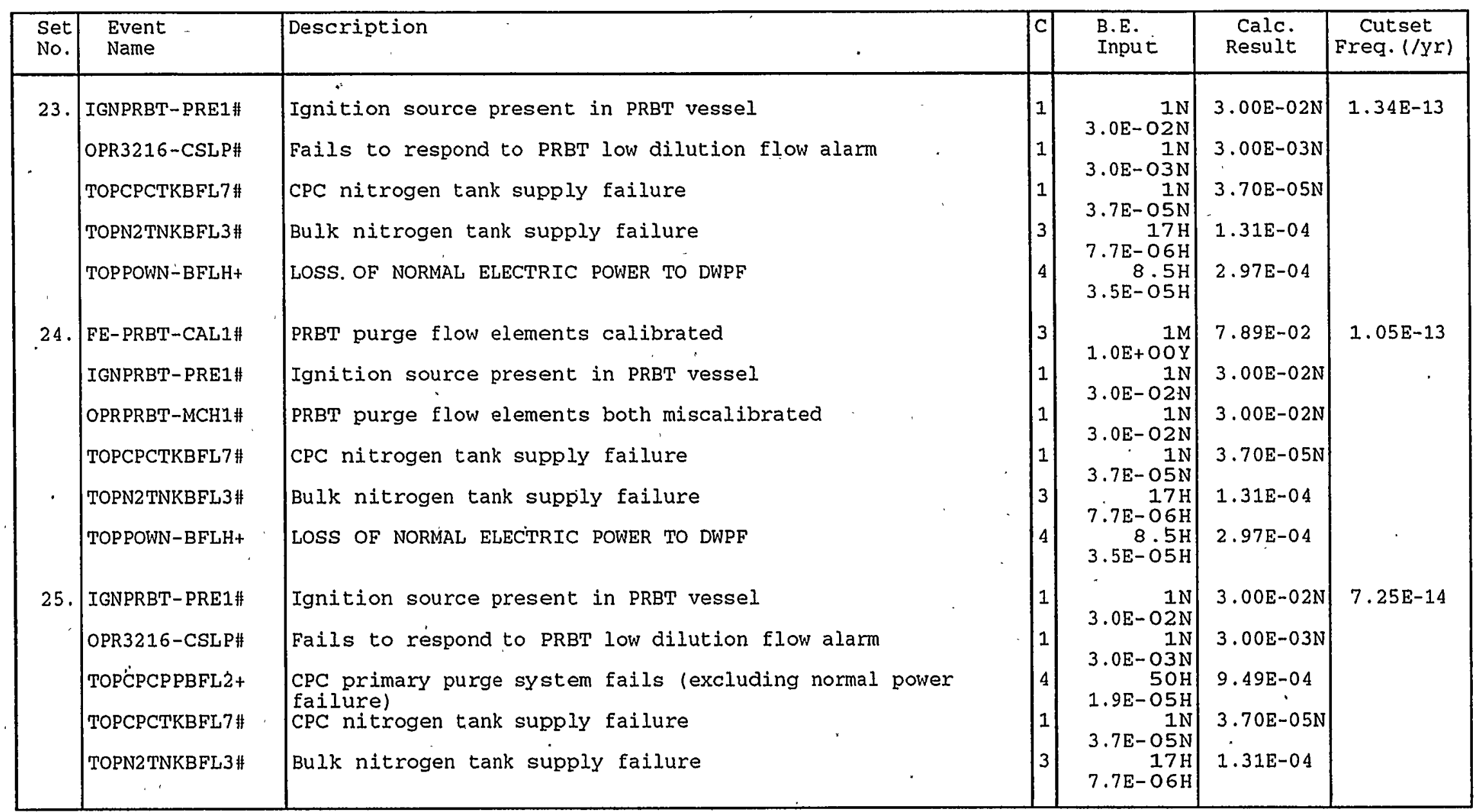


Explosion in PRBT

Basic Event Name

CC2PRBT-FE-7+IC MOV1131-LKS6+I TOPCPCPPBFL2+-I TOPPOWN-BFLH+-I SV-PRBT-ADJ5+IC FCV3216-FCL6+-IC MOV1131-ADJ6+I

OPR1131-ACL1\#

OPRPR5-LAN1\#

OPRPR5-ACL1\#

IGNPRBT-PREI\#

OPR3216-CSLP\#

FE-3215-FHI6+-IC

FE-3218-FH16+-IC

OPRPRBT-SOH1\#

TOPCPCBPBFLW\#

OPR3216-SRN6\#

FCV3216-ADJ6+-IC

OPRPRBT-MCH1\#

OPRPRCD-SVL1\#

PR-PHA-IDL6\#

MOV1131-FTCK\#

FE-PRBT-CAL1\#

OPR1131-VRH1\#
Top Event Frequency: 5.75E-06/YR

\section{Risk Achievement Worth} Description

cc: both PRBT flow elements fail high

$P R C D$ drain valve leaks (benzene to $P R$ )

Prob/Freq AchW

$3.00 \mathrm{E}-07 / \mathrm{H} \quad 7.69 \mathrm{E}+04$

$1.00 \mathrm{E}-06 / \mathrm{H} \quad 3.66 \mathrm{E}+04$

$1.90 \mathrm{E}-05 / \mathrm{H} \quad 7.92 \mathrm{E}+02$

$3.50 \mathrm{E}-05 / \mathrm{H} \quad 7.92 \mathrm{E}+02$

$1.34 \mathrm{E}-03 / \mathrm{H} \quad 5.01 \mathrm{E}+02$

$1.00 \mathrm{E}-06 / \mathrm{H} \quad 4.30 \mathrm{E}+02$

$4.58 \mathrm{E}-04 / \mathrm{H} \quad 3.01 \mathrm{E}+02$

$5.00 \mathrm{E}-04 \quad 2.71 \mathrm{E}+02$

$3.00 \mathrm{E}-04 \quad 4.99 \mathrm{E}+01$

$5.00 \mathrm{E}-04 \quad 4.98 \mathrm{E}+01$

$3.00 \mathrm{E}-02 \quad 3.33 \mathrm{E}+01$

$3.00 \mathrm{E}-03 \quad 2.59 \mathrm{E}+01$

$3.00 \mathrm{E}-06 / \mathrm{H} \cdot 2.10 \mathrm{E}+01$

$3.00 \mathrm{E}-06 / \mathrm{H} \quad 2.10 \mathrm{E}+01$

$5.00 \mathrm{E}-02 \quad 1.59 \mathrm{E}+01$

3.10E-03 1.47E+01

$1.00 \mathrm{E}-02 \quad 1.06 \mathrm{E}+01$

$2.25 \mathrm{E}-02 / \mathrm{H} \quad 5.19 \mathrm{E}+00$

$3.00 \mathrm{E}-02 \cdot \quad 2.91 \mathrm{E}+00$

1.00E-01 2.21E+00

$1.29 \mathrm{E}-01 \quad 1.91 \mathrm{E}+00$

$3.00 \mathrm{E}-03 \quad 1.84 \mathrm{E}+00$

$7.89 \mathrm{E}-02 \quad 1.69 \mathrm{E}+00$

$5.00 \mathrm{E}-02 \quad 1.05 \mathrm{E}+00$

Note: Events that make less than $1 \%$ difference to the importance factor are not included. Modified top event frequency $=$ (top event frequency) $\mathrm{x}$ (risk achievement worth). 


\section{Explosion in PRBT}

\begin{tabular}{l} 
Basic Event Name \\
\hline *IGNPRBT-PRE1\# \\
OPRPRBT-SOH1\# \\
SV-PRBT-ADJ5+-IC \\
CC2PRBT-FE-7+-IC \\
MOV1131-ADJ6+-I \\
OPR1131-ACL1\# \\
OPRPRCD-SVL1\# \\
PR-PHA--IDL6\# \\
FCV3216-ADJ6+-IC \\
OPR3216-SRN6\# \\
OPR3216-CSLP\# \\
OPRPRBT-MCH1\# \\
FE-PRBT-CAL1\# \\
TOPCPCBPBFLW\# \\
MOV1131-LKS6+-I \\
TOPPOWN-BFLH+-I \\
OPRPR5--ACL1\# \\
TOPCPCPPBFL2+-I \\
OPRPR5-LAN1\#
\end{tabular}

Top Event Frequency: 5.75E-06/YR

\section{Risk Reduction Worth}

\author{
Descrintion
}

Ignition source present in PRBT vessel

Fail to open PRBT purge supply needle valve

PRBT purge supply needle valve adjusted

cc: both PRBT flow elements fail high

PRCD cleaned (drain valve opened)

Failure to wait until PR transfer before cleaning PRCD

Supervisor fails to prevent PRCD cleaning into PR

PR product awaiting transfer after being sampled

PRBT dilution flow control valve adjusted

PRBT dilution flow controller erroneously set low

Fails to respond to PRBT low dilution flow alarm

PRBT purge flow elements both miscalibrated

PRBT purge flow elements calibrated

Backup CPC purge fails (except N2 tank supply failure)

PRCD drain valve leaks (benzene to $P R$ )

LOSS OF NORMAL ELECTRIC POWER TO DWPF

Operator fails to sample for $\mathrm{C} 6 \mathrm{H} 6$ before transfer to PRBT

CPC primary purge system fails (excluding normal power)

Error in $\mathrm{C} 6 \mathrm{H} 6$ sample/analysis - low

\begin{tabular}{ll} 
Prob/Freq & RedW \\
\hline $3.00 \mathrm{E}-02$ & $0.00 \mathrm{E}+00$ \\
$5.00 \mathrm{E}-02$ & $4.61 \mathrm{E}+00$ \\
$1.34 \mathrm{E}-03 / \mathrm{H}$ & $4.61 \mathrm{E}+00$ \\
$3.00 \mathrm{E}-07 / \mathrm{H}$ & $3.24 \mathrm{E}+00$ \\
$4.58 \mathrm{E}-04 / \mathrm{H}$ & $1.16 \mathrm{E}+00$ \\
$5.00 \mathrm{E}-04$ & $1.16 \mathrm{E}+00$ \\
$1.00 \mathrm{E}-01$ & $1.16 \mathrm{E}+00$ \\
$1.29 \mathrm{E}-01$ & $1.16 \mathrm{E}+00$ \\
$2.25 \mathrm{E}-02 / \mathrm{H}$ & $1.11 \mathrm{E}+00$ \\
$1.00 \mathrm{E}-02$ & $1.11 \mathrm{E}+00$ \\
$3.00 \mathrm{E}-03$ & $1.08 \mathrm{E}+00^{\prime}$ \\
$3.00 \mathrm{E}-02$ & $1.06 \mathrm{E}+00$ \\
$7.89 \mathrm{E}-02$ & $1.06 \mathrm{E}+00$ \\
$3.10 \mathrm{E}-03$ & $1.04 \mathrm{E}+00$ \\
$1.00 \mathrm{E}-06 / \mathrm{H}$ & $1.04 \mathrm{E}+00$ \\
$3.50 \mathrm{E}-05 / \mathrm{H}$ & $1.03 \mathrm{E}+00$ \\
$5.00 \mathrm{E}-04$ & $1.03 \mathrm{E}+00$ \\
$1.90 \mathrm{E}-05 / \mathrm{H}$ & $1.02 \mathrm{E}+00$ \\
$3.00 \mathrm{E}-04$ & $1.01 \mathrm{E}+00$
\end{tabular}

* A Reduction Worth of $0.0 \mathrm{E}+00$ indicates event appears in every cutset could serve as a single event preventor for the accident.

Note: Events that make less than $1 \%$ difference to the importance factor are not included. Modified top event frequency $=$ (top event frequency) $/$ (risk reduction worth). 
X-ESR-S-00001, REV. 0

\section{MFT Explosion}




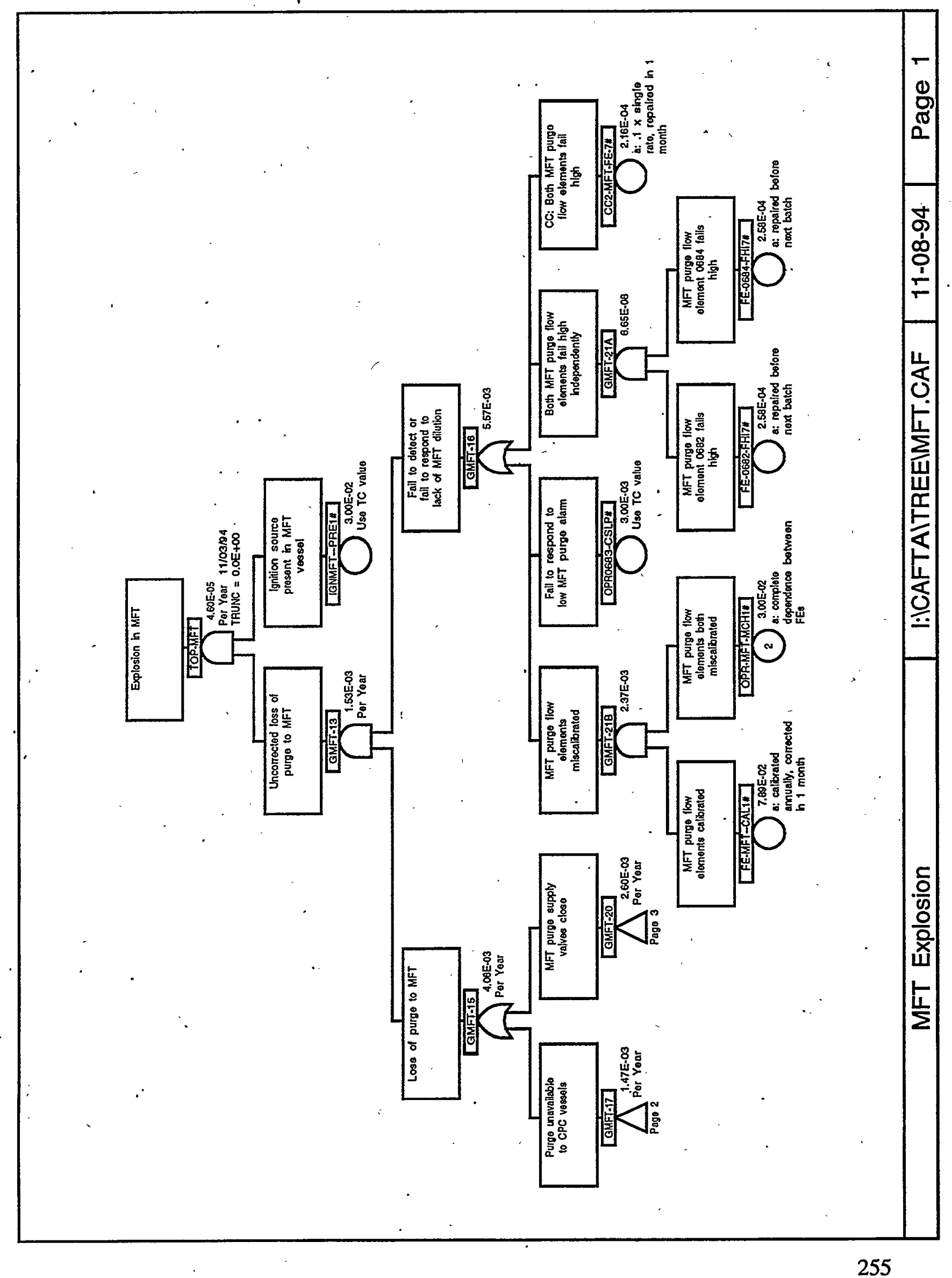


X-ESR-S-00001, REV. 0

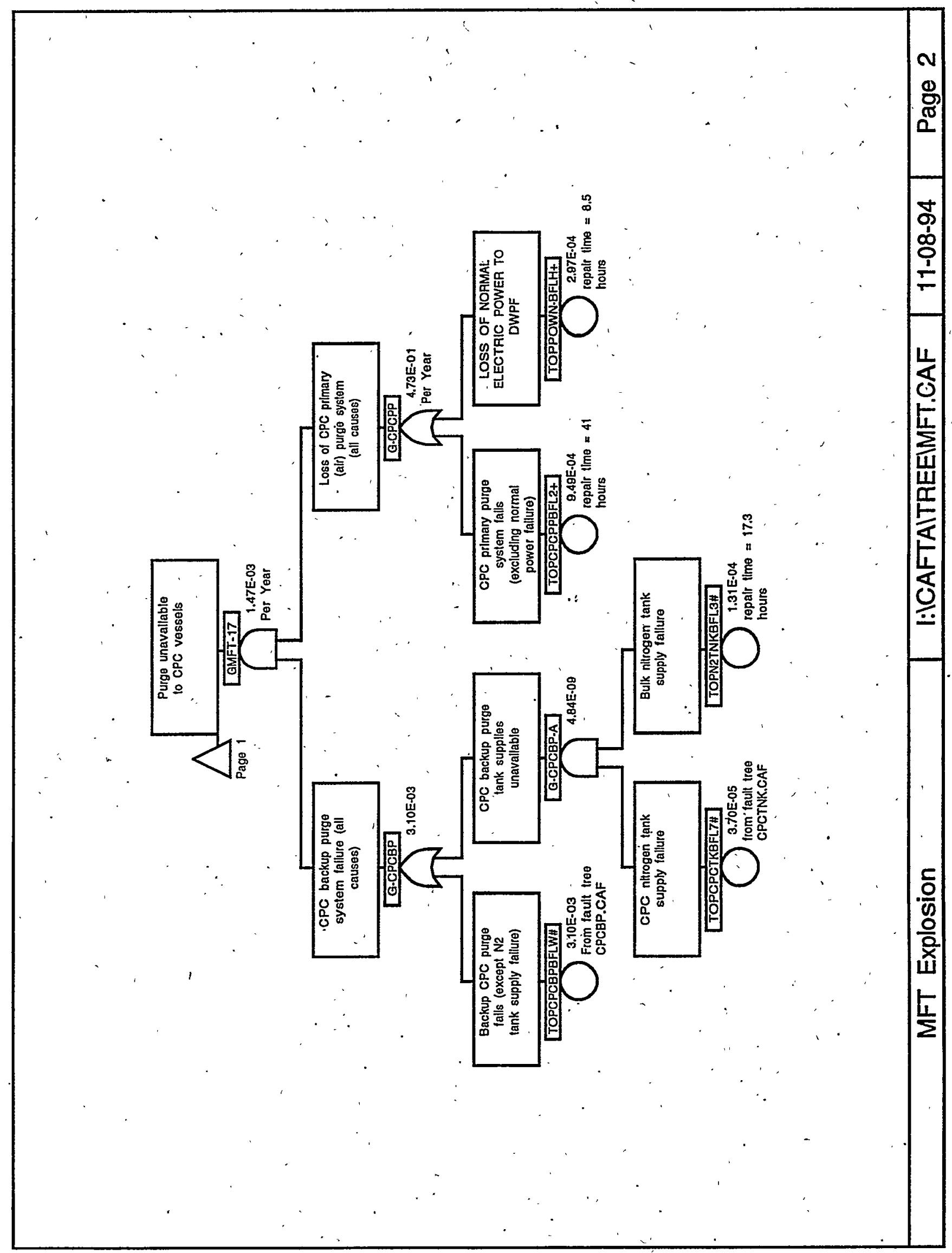




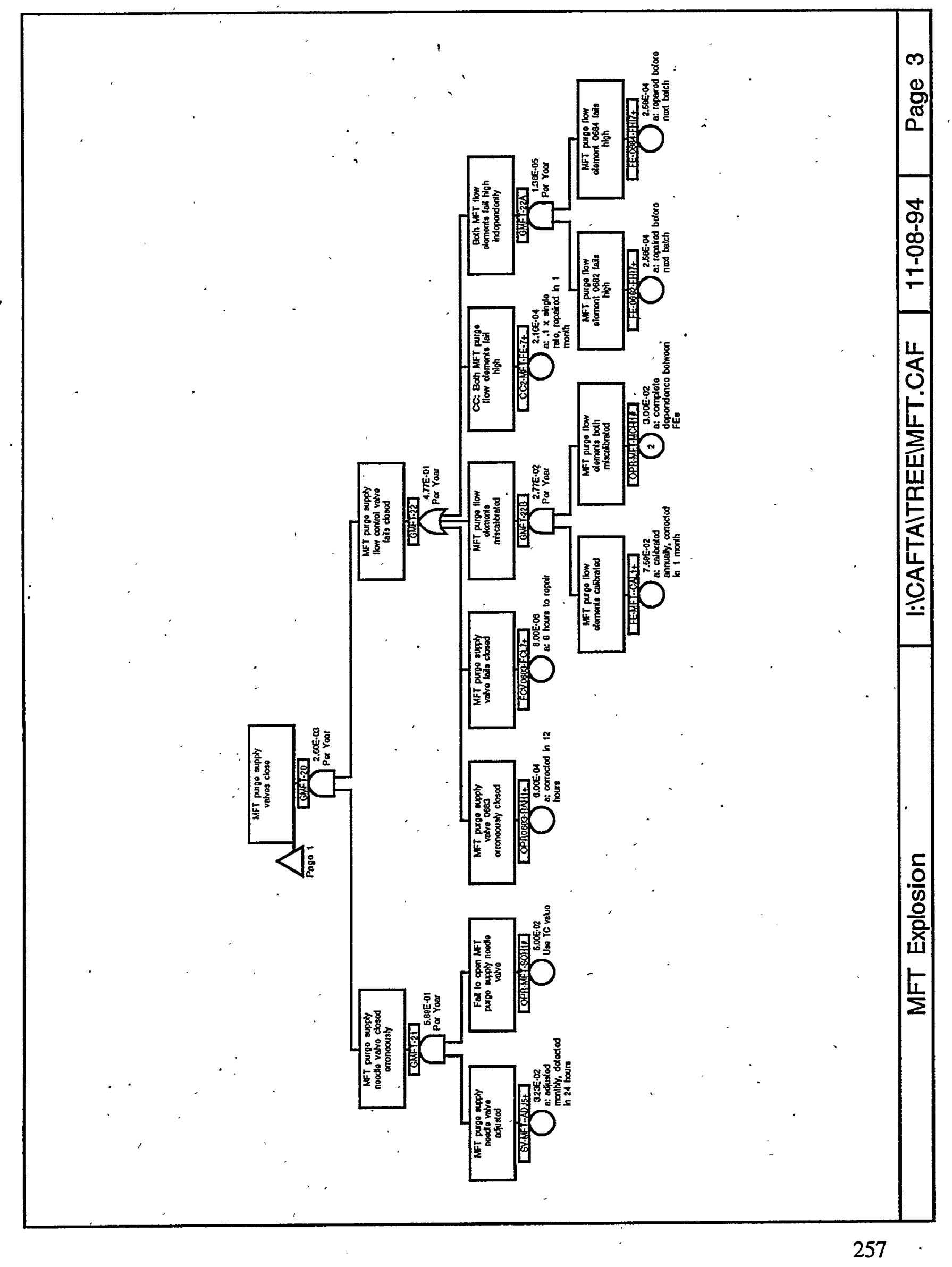




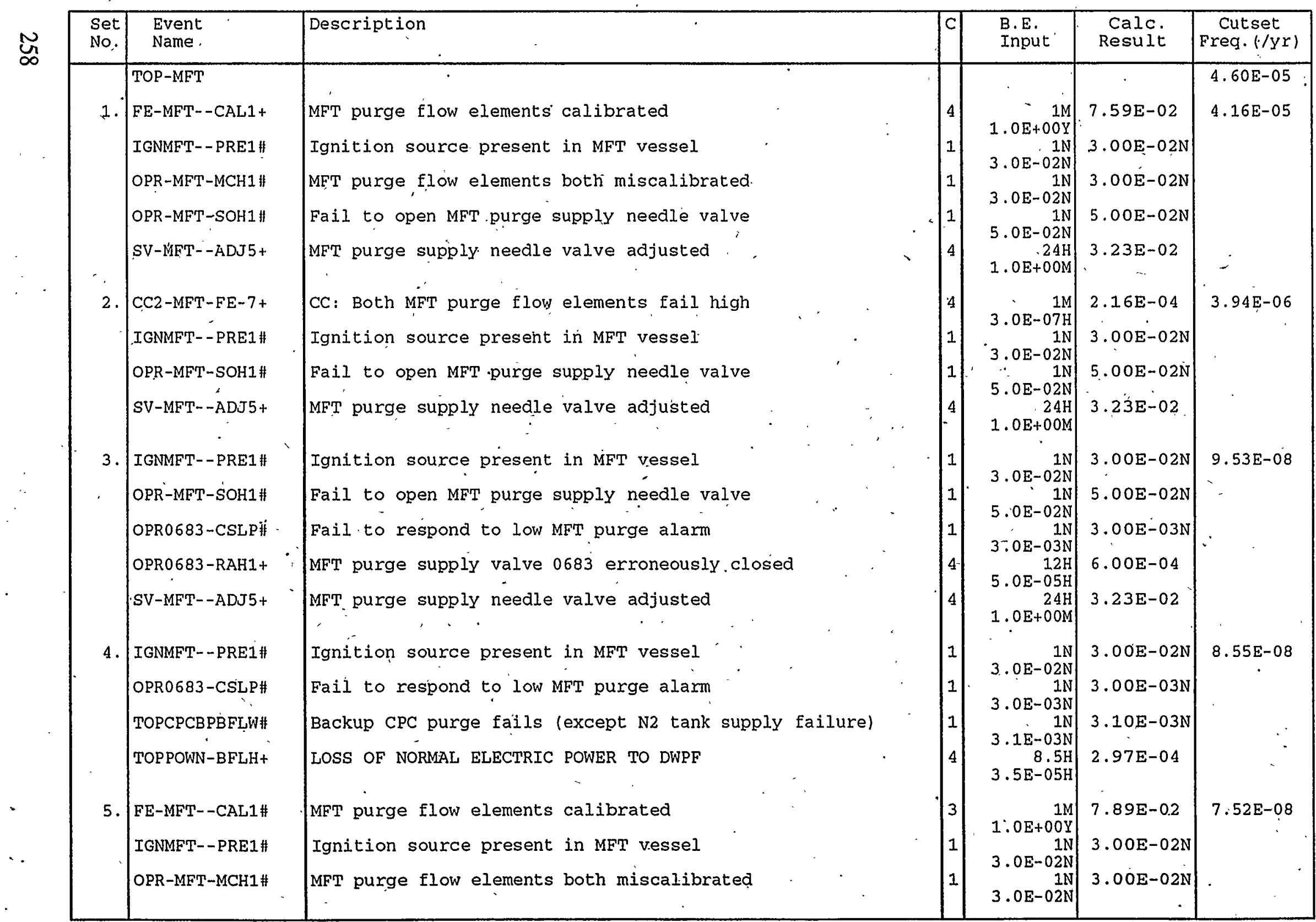


Cutsets for I: ICAFTA \CUT\MFT.CSR

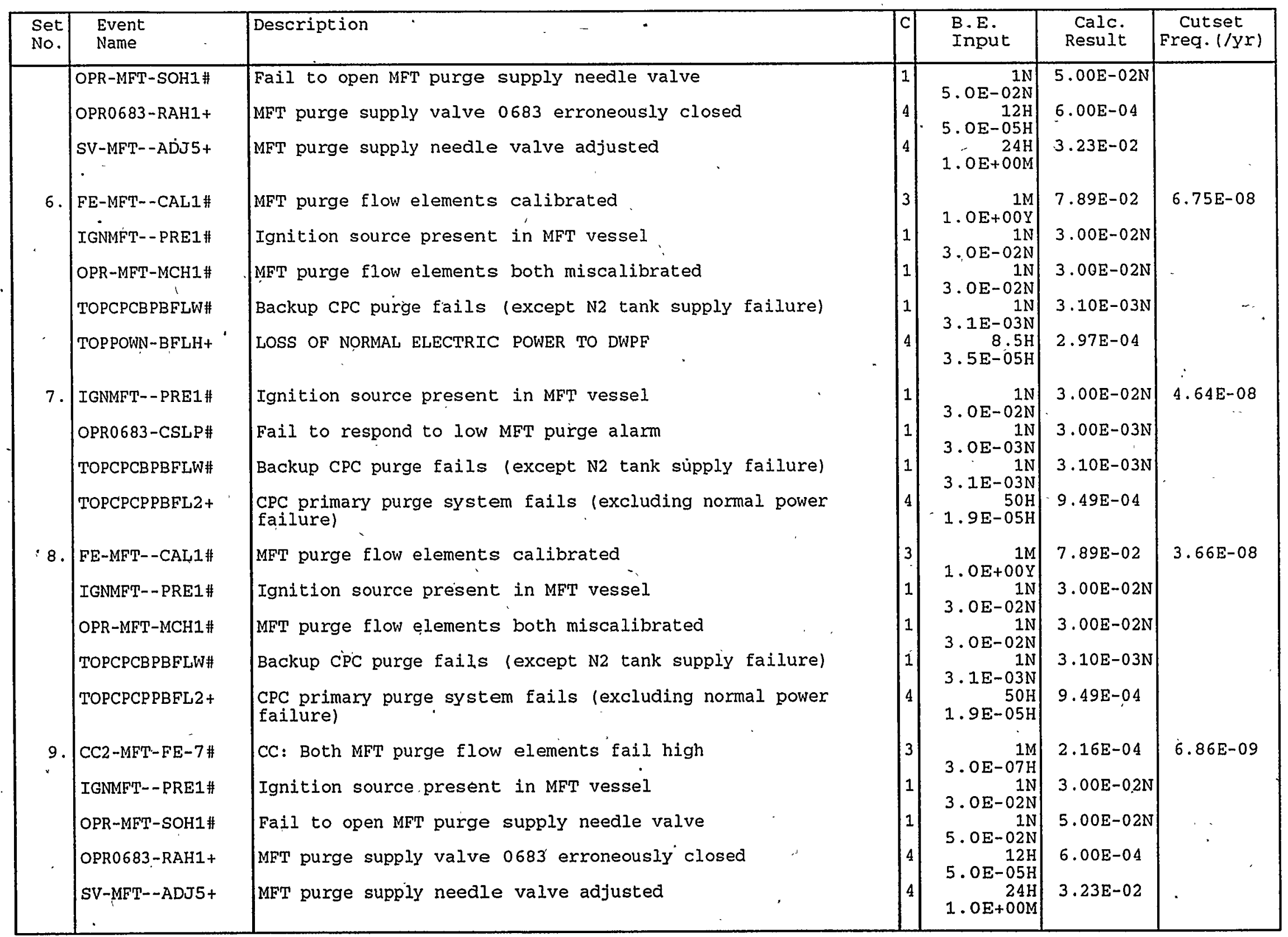




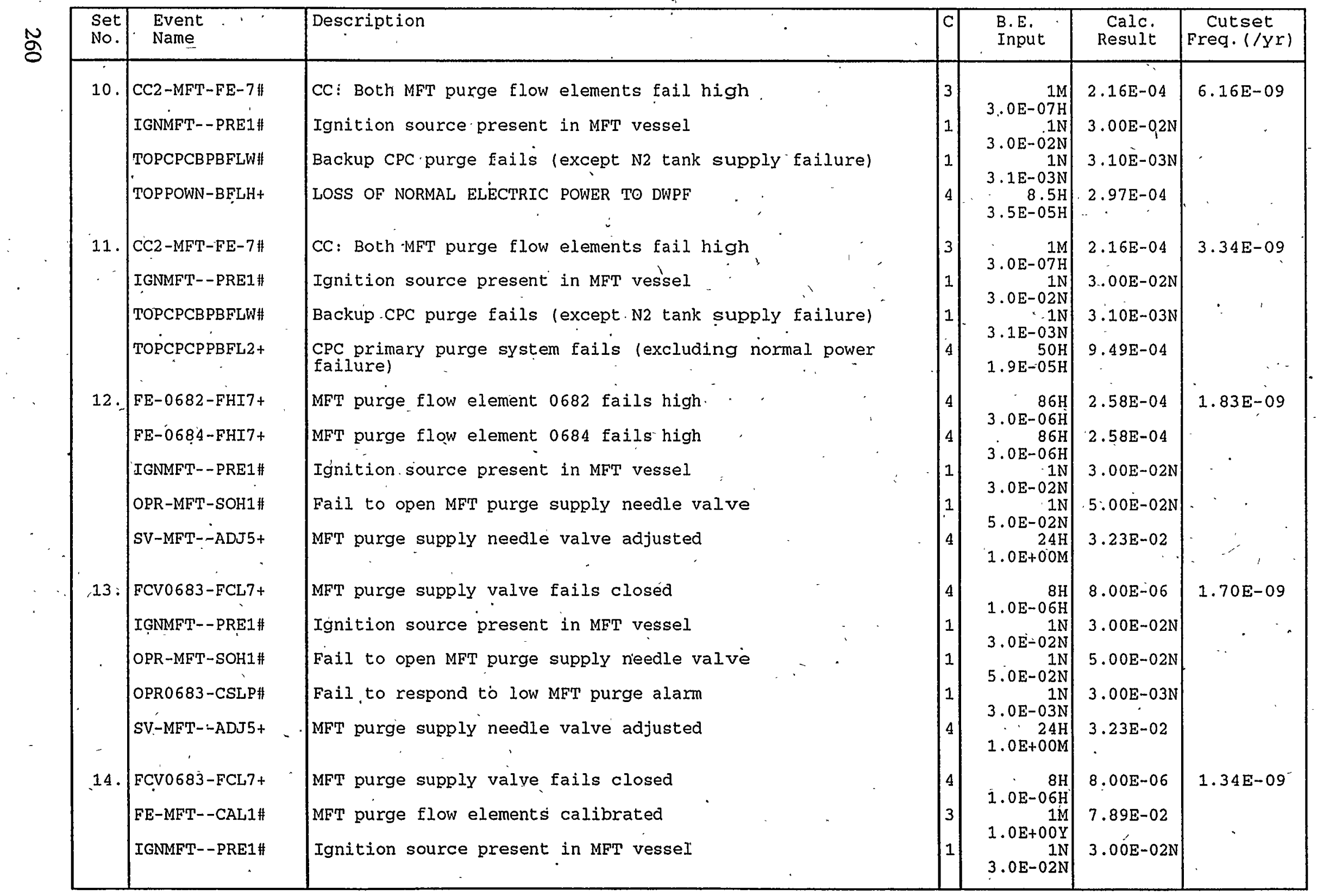


Cutsets for I: \CAFTA \CUT\MFT.CSR

11/08/94 12:27 AM (CONT.)

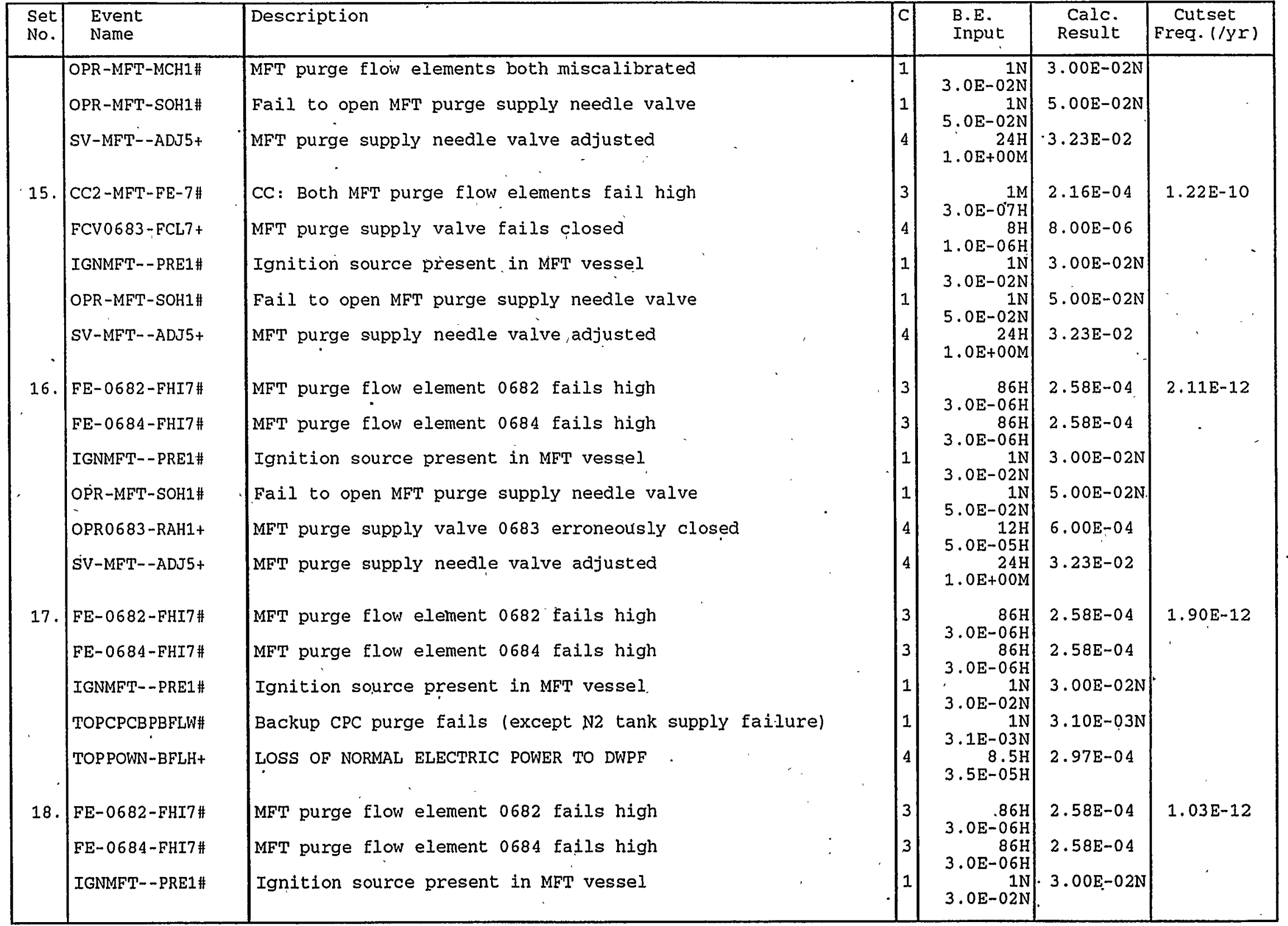


Cuisets . for I : \CAFTA \CUT\MFT.CSR

11/08/94 12:27 AM (CONT.)

\begin{tabular}{|c|c|c|c|c|c|c|c|}
\hline & $\begin{array}{l}\text { Set } \\
\text { No. }\end{array}$ & $\begin{array}{l}\text { Event } \\
\text { Name }\end{array}$ & Description & $\mathrm{C}$ & $\begin{array}{l}\text { B.E. } \\
\text { Input }\end{array}$ & $\begin{array}{l}\text { Calc: } \\
\text { Result }\end{array}$ & $\begin{array}{c}\text { cutset } \\
\text { Freq. }(/ \text { yr })\end{array}$ \\
\hline & 21. & $\begin{array}{l}\text { TOPCPCBPBFLW\# } \\
\text { TOPCPCPPBFL2+ } \\
\text { IGNMFT--PRE1\# } \\
\text { OPR0683-CSLP\# } \\
\text { TOPCPCTKBEL7\# } \\
\text { TOPN2TNKBFL3\# } \\
\text { TOPPOWN-BFLH+ } \\
\text { FE-MFT--CAL1\# } \\
\text { IGNMFT--PRE1\# } \\
\text { OPR-MFT-MCH1\# } \\
\text { TOPCPCTKBFL7\# } \\
\text { TOPN2TNKBFL3\# } \\
\text { TOPPOWN-BFLH+ } \\
\text { IGNMFT--PRE1\# } \\
\text { OPRO683-CSLP\# } \\
\text { TOPCPCPPBFL2+ } \\
\text { TOPCPCTKBFL7\# } \\
\text { TOPN2TNKBFL3\# } \\
\text { FOPCPCPPBFL2+ } \\
\text { IGNMFT--PRE1\# } \\
\text { OPR-MFT-MCH1\# } \\
\text { TOPI-CAL1\# }\end{array}$ & 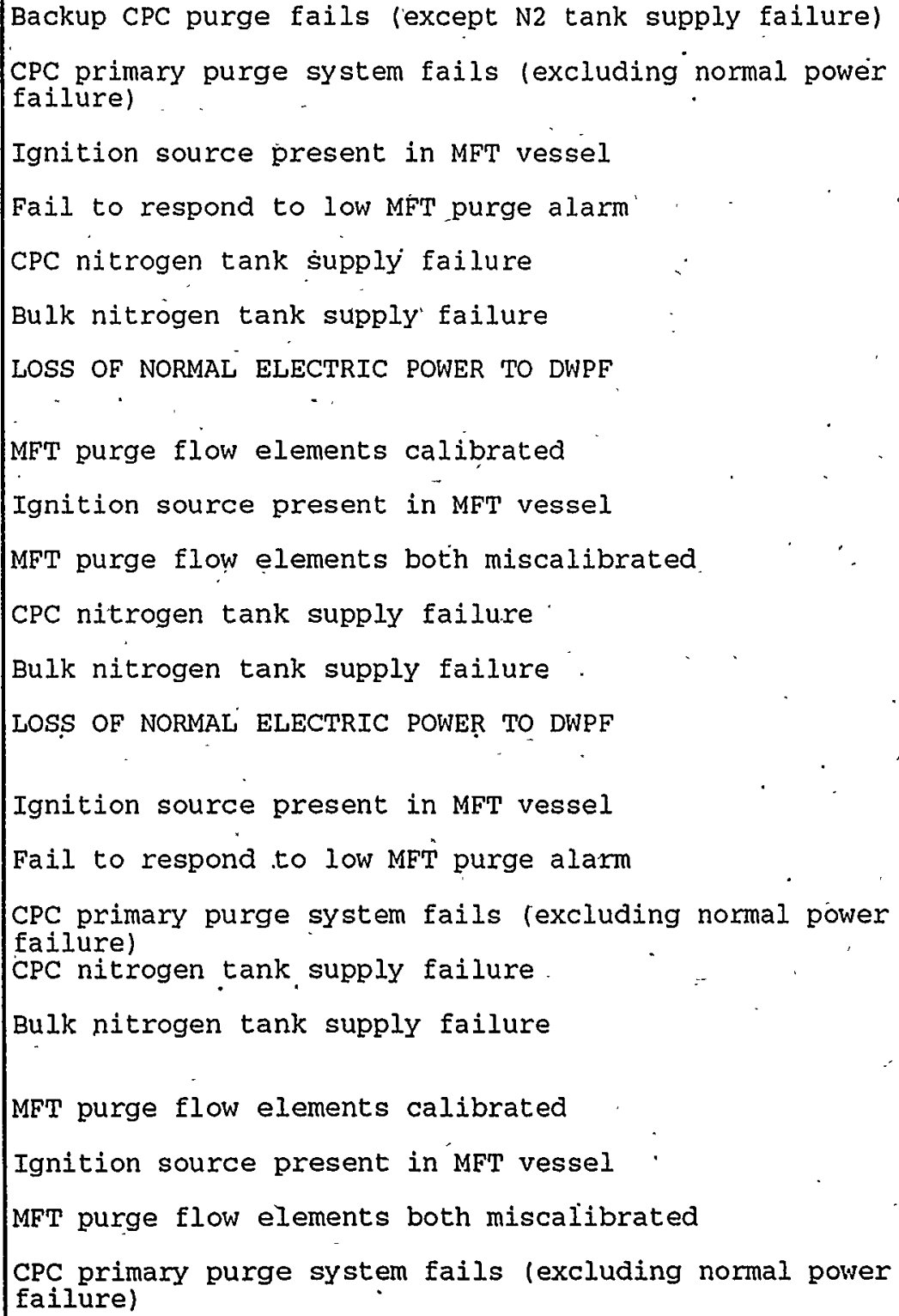 & 1 & $\begin{array}{r}1 \mathrm{~N} \\
3.1 \mathrm{E}-03 \mathrm{~N} \\
50 \mathrm{H} \\
1.9 \mathrm{E}-05 \mathrm{H} \\
1 \mathrm{~N} \\
3.0 \mathrm{E}-02 \mathrm{~N} \\
1 \mathrm{~N} \\
3.0 \mathrm{E}-03 \mathrm{~N} \\
1 \mathrm{~N} \\
3.7 \mathrm{E}-05 \mathrm{~N} \\
17 \mathrm{H} \\
7.7 \mathrm{E}-06 \mathrm{H} \\
8.5 \mathrm{H} \\
3.5 \mathrm{E}-05 \mathrm{H} \\
1.0 \mathrm{E}+00 \mathrm{M} \\
1 \mathrm{~N} \\
3.0 \mathrm{E}-02 \mathrm{~N} \\
1 \mathrm{~N} \\
3.0 \mathrm{E}-02 \mathrm{~N} \\
1 \mathrm{~N} \\
3.7 \mathrm{E}-05 \mathrm{~N} \\
17 \mathrm{H} \\
7.7 \mathrm{E}-06 \mathrm{H} \\
8.5 \mathrm{H} \\
3.5 \mathrm{E}-05 \mathrm{H} \\
3.0 \mathrm{E}-02 \mathrm{~N} \\
5.9 \mathrm{E}-05 \mathrm{H}\end{array}$ & $\begin{array}{l}3.10 \mathrm{E}-03 \mathrm{~N} \\
9.49 \mathrm{E}-04 \\
3.00 \mathrm{E}-02 \mathrm{~N} \\
3.00 \mathrm{E}-03 \mathrm{~N} \\
3.70 \mathrm{E}-05 \mathrm{~N} \\
1.31 \mathrm{E}-04 \\
2.97 \mathrm{E}-04 \\
7 \\
7.89 \mathrm{E}-02 \\
3.00 \mathrm{E}-02 \mathrm{~N} \\
3.00 \mathrm{E}-02 \mathrm{~N} \\
3.70 \mathrm{E}-05 \mathrm{~N} \\
1.31 \mathrm{E}-04 \\
2.97 \mathrm{E}-04 \\
3.00 \mathrm{E}-02 \mathrm{~N} \\
3.00 \mathrm{E}-03 \mathrm{~N} \\
9.49 \mathrm{E}-04 \\
3.70 \mathrm{E}-05 \mathrm{~N} \\
1.31 \mathrm{E}-04 \\
7.89 \mathrm{E}-02 \\
3.00 \mathrm{E}-02 \mathrm{~N} \\
3.00 \mathrm{E}-02 \mathrm{~N} \\
9.49 \mathrm{E}-04\end{array}$ & $\begin{array}{c}1.34 \mathrm{E}-13 \\
1.05 \mathrm{E}-13 \\
\vdots \\
7.72 \mathrm{E}-14 \\
7.25 \mathrm{E}-14\end{array}$ \\
\hline
\end{tabular}


Cutsets for I: \CAFTA ICUT\MFT.CSR

11/08/94 12:27 AM (CONT.)

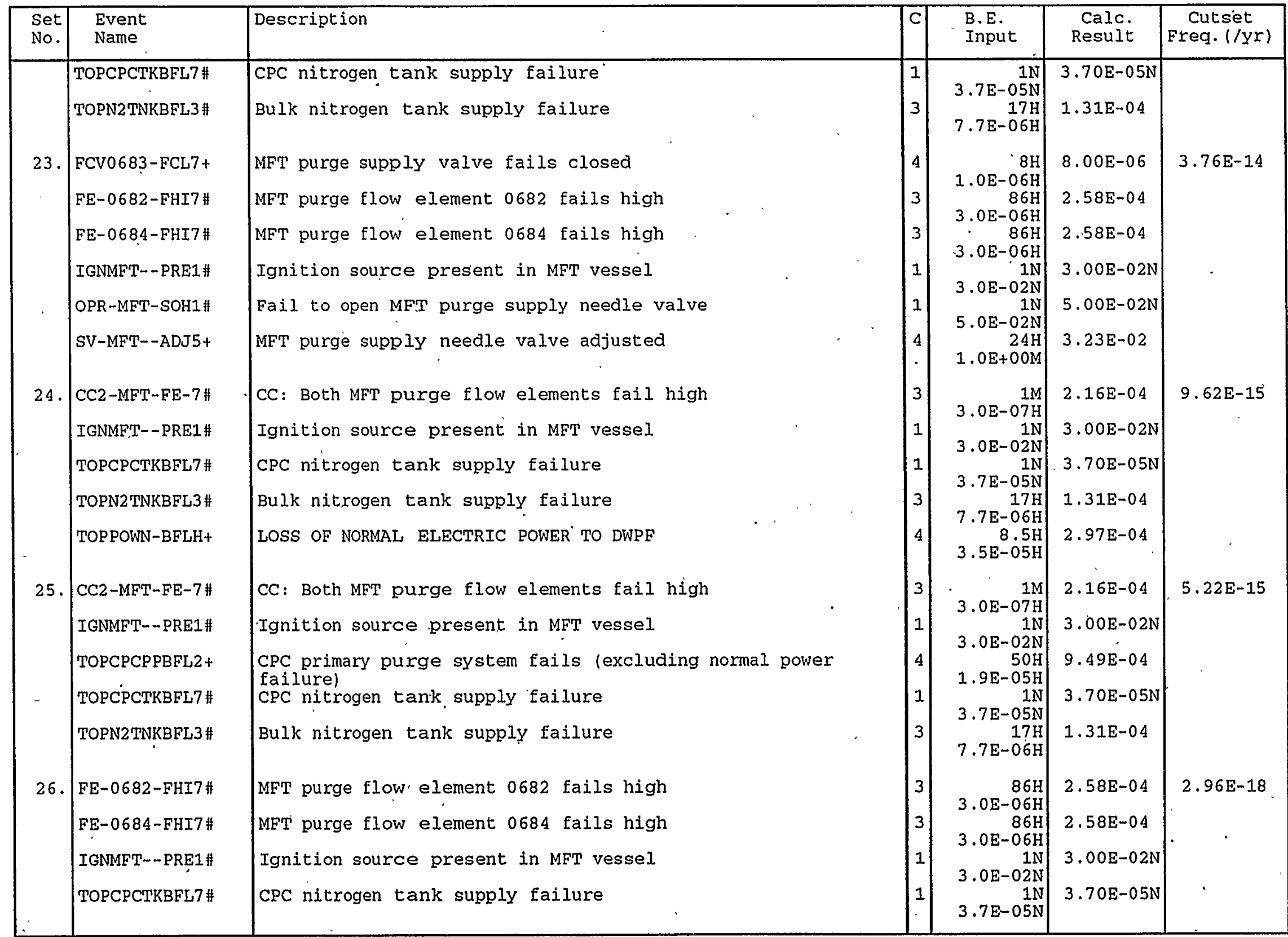


Cutsets for I: \CAFTA \CUT\MFT.CSR

11/08/94 12:27 AM (ĊONT.)

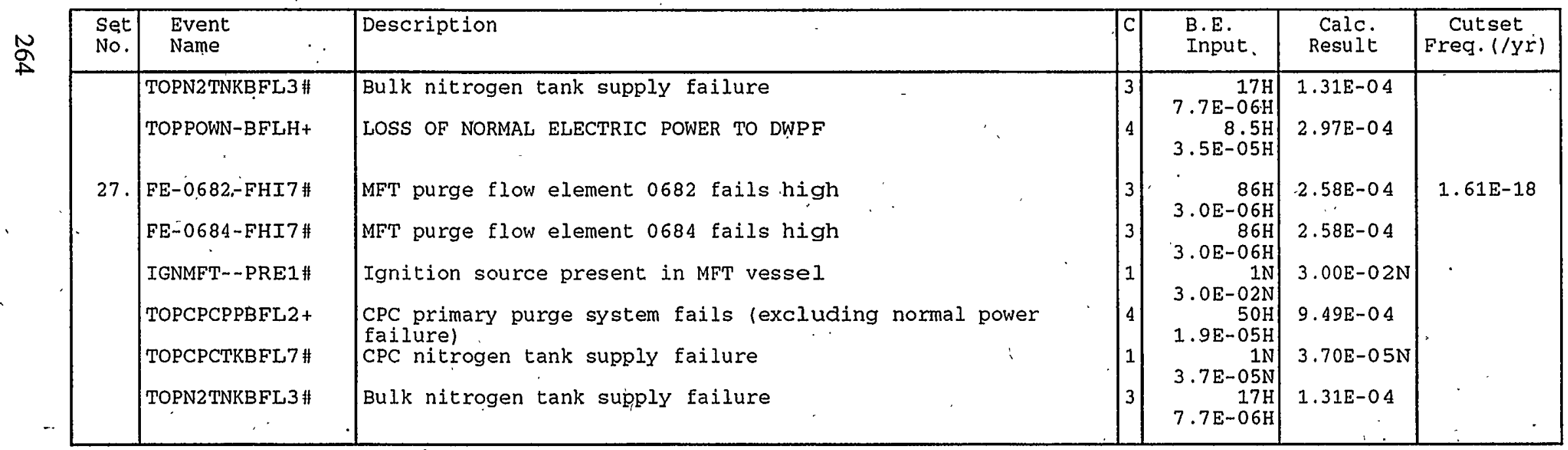


Explosion in MFT

Basic Event Name

CC2-MFT-FE-7+-IC

SV-MFT-ADJ5+-IC

FE-MFT--CAL1+IC

TOPCPCPPI3FL 2+-I

TOPPOWN-BFLH+-I

FCV0683-FCL7+-IC

OPR0683-RAH1+-IC

IGNMFT-PRE1\#

OPR-MFT-MCH1\#

OPR-MFT-SOH1\#

FE-0684-FHI7+-IC

FE-0682-FHI7+-IC

TOPCPCBPIBELW\#

OPR0683-C'SLP\#
Top Event Frequency: 4.60E-05/YR

\section{Risk Achievement Worth} Description

CC: Both MFT purge flow elements fail high

Prob/Freg AchW

3.00E-07/H $\quad 9.61 \mathrm{E}+03$

MFT purge supply needle valve adjusted $\quad 1.34 \mathrm{E}-03 / \mathrm{H} \quad 7.15 \mathrm{E}+02$

MFT purge flow elements calibrated $\quad-\quad 1.05 \mathrm{E}-04 / \mathrm{H} \quad 2.88 \mathrm{E}+02$

CPC primary purge system fails (excluding normal power)

LOSS OF NORMAL ELECTRIC POWER TO DWPF

MFT purge supply valve fails closed

MFT purge supply valve 0683 erroneously closed

Ignition source present in MFT vessel

MFT purge flow elements both miscalibrated

Fail to open MFT purge supply needle valve

MFT purge flow element 0684 fails high

MFT purge flow element 0682 fails high

Backup CPC purge fails (except N2 tank supply failure)

Fail to respond to low MFT purge alarm
$1.90 \mathrm{E}-05 / \mathrm{H} \quad 1.00 \mathrm{E}+02$

$3.50 \mathrm{E}-05 / \mathrm{H} \quad 1.00 \mathrm{E}+02$

$1.00 \mathrm{E}-06 / \mathrm{H} \quad 5.46 \mathrm{E}+01$

$4.99 \mathrm{E}-05 / \mathrm{H} \quad 5.46 \mathrm{E}+01$

$3.00 \mathrm{E}-02 \quad 3.33 \mathrm{E}+01$

$3.00 \mathrm{E}-02 \quad 3.04 \mathrm{E}+01$

$5.00 \mathrm{E}-02 \quad 1.99 \mathrm{E}+01$

$3.00 \mathrm{E}-06 / \mathrm{H} \quad 3.51 \mathrm{E}+00$

$3.00 \mathrm{E}-06 / \mathrm{H} \cdot 3.51 \mathrm{E}+00$

$3.10 \mathrm{E}-03 \quad 2.72 \mathrm{E}+00$

$3.00 \mathrm{E}-03 \quad 2.66 \mathrm{E}+00$

Modified top event frequency $=($ top event frequency $) /$ (risk reduction worth).

Basic Event Name

*IGNMFT-.PRE1\# OPR-MFT-SOH1\#

SV-MFT--ADI5+-IC

FE-MFT--CAL1+-IC

OPR-MFT-MCH1\#

CC2-MFT-IFE-7+IC

\section{Risk Reduction Worth}

Description

Ignition source present in MFT vessel

Fail to open MFT purge supply needle valve

MFT purge supply needle valve adjusted

MFT purge flow elements calibrated

MFT purge flow elements both miscalibrated

CC: Both MFT purge flow elements fail high

\begin{tabular}{ll} 
Prob/Freg & RedW \\
\hline $3.00 \mathrm{E}-02$ & $0.00 \mathrm{E}+00$ \\
$5.00 \mathrm{E}-02$ & $1.87 \mathrm{E}+02$ \\
$1.34 \mathrm{E}-03 / \mathrm{H}$ & $1.87 \mathrm{E}+02$ \\
$1.05 \mathrm{E}-04 / \mathrm{H}$ & $1.10 \mathrm{E}+01$ \\
$3.00 \mathrm{E}-02$ & $1.10 \mathrm{E}+01$ \\
$3.00 \mathrm{E}-07 / \mathrm{H}$ & $1.09 \mathrm{E}+00$
\end{tabular}

* A Reduction Worth of $0.0 \mathrm{E}+00$ indicates event appears in every cutset could serve as a single event preventor for the accident.

Note: Events that make less than $1 \%$ difference to the importance factor are not included. Modified top event frequency = (top event frequency) $/$ (risk reduction worth). 
X-ESR-S-00001, REV. 0

PR Explosion 


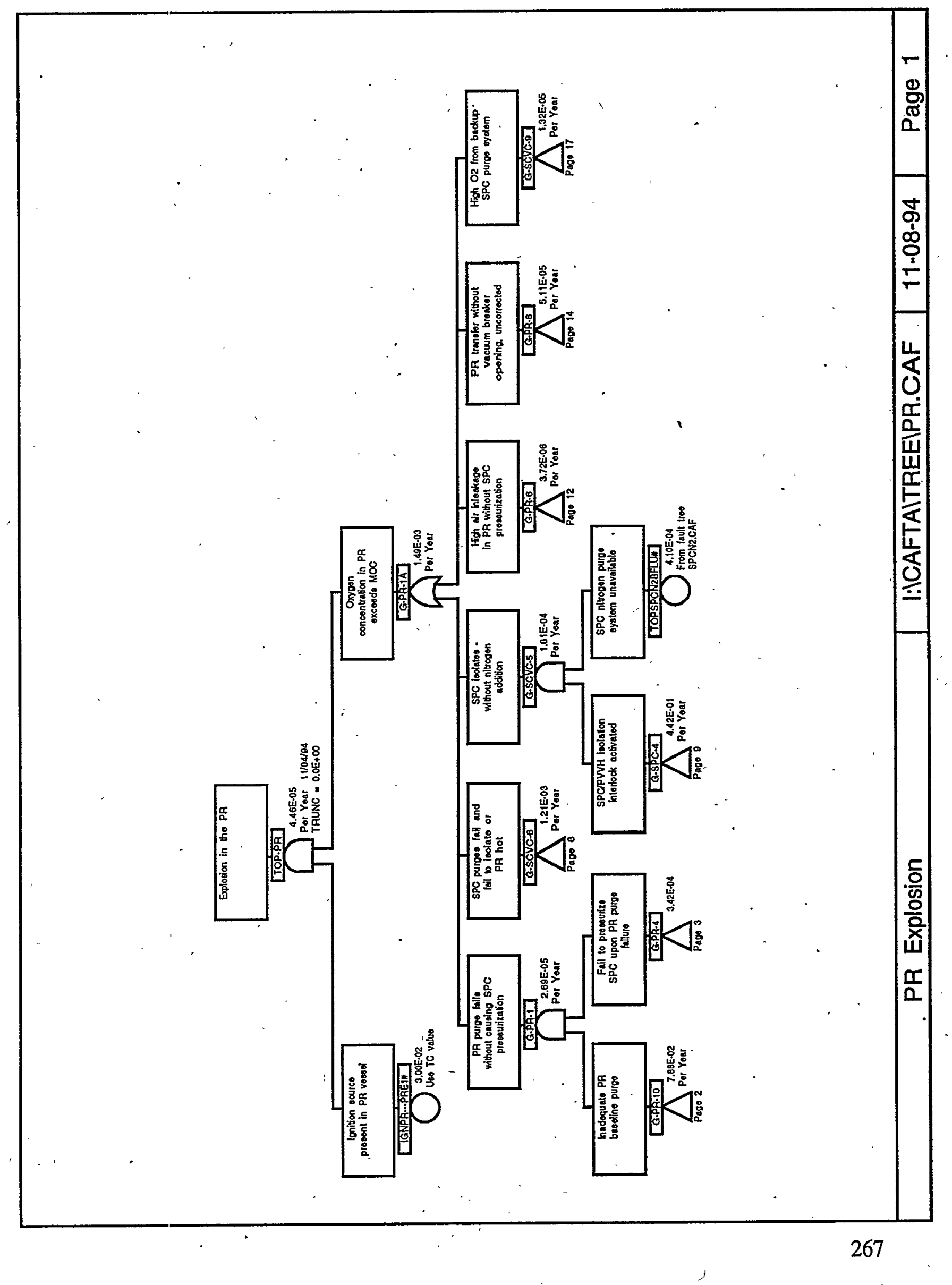




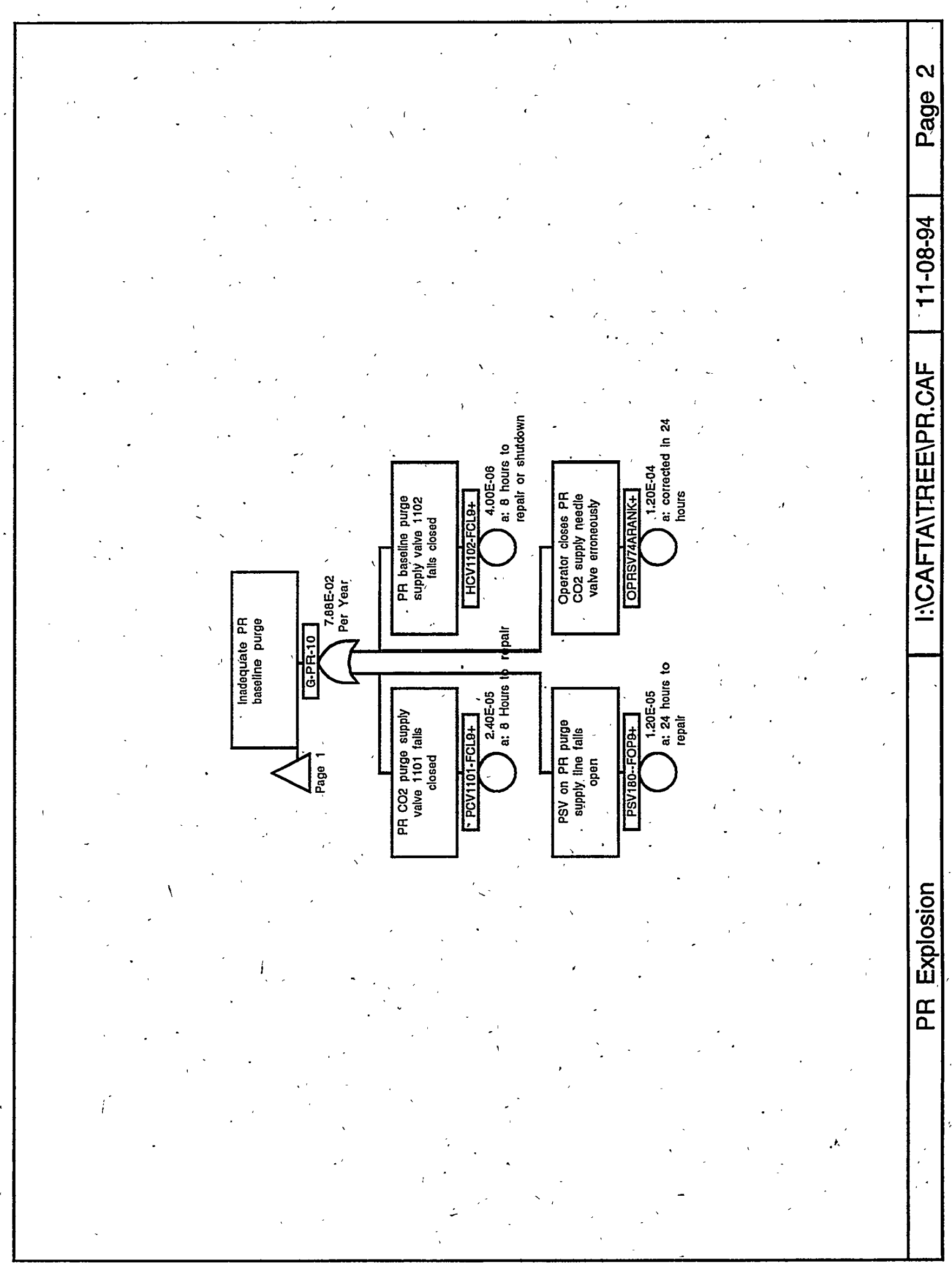




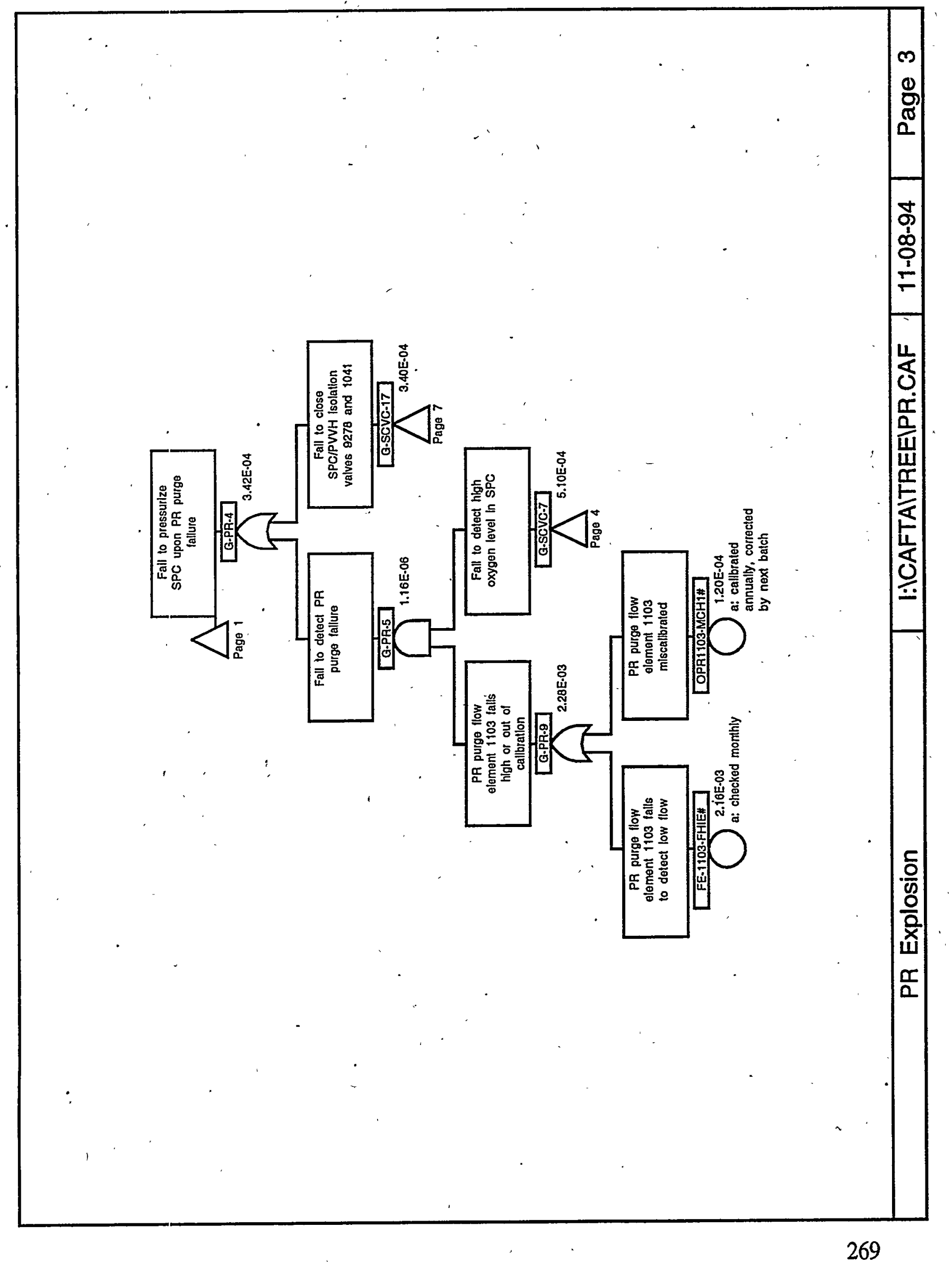




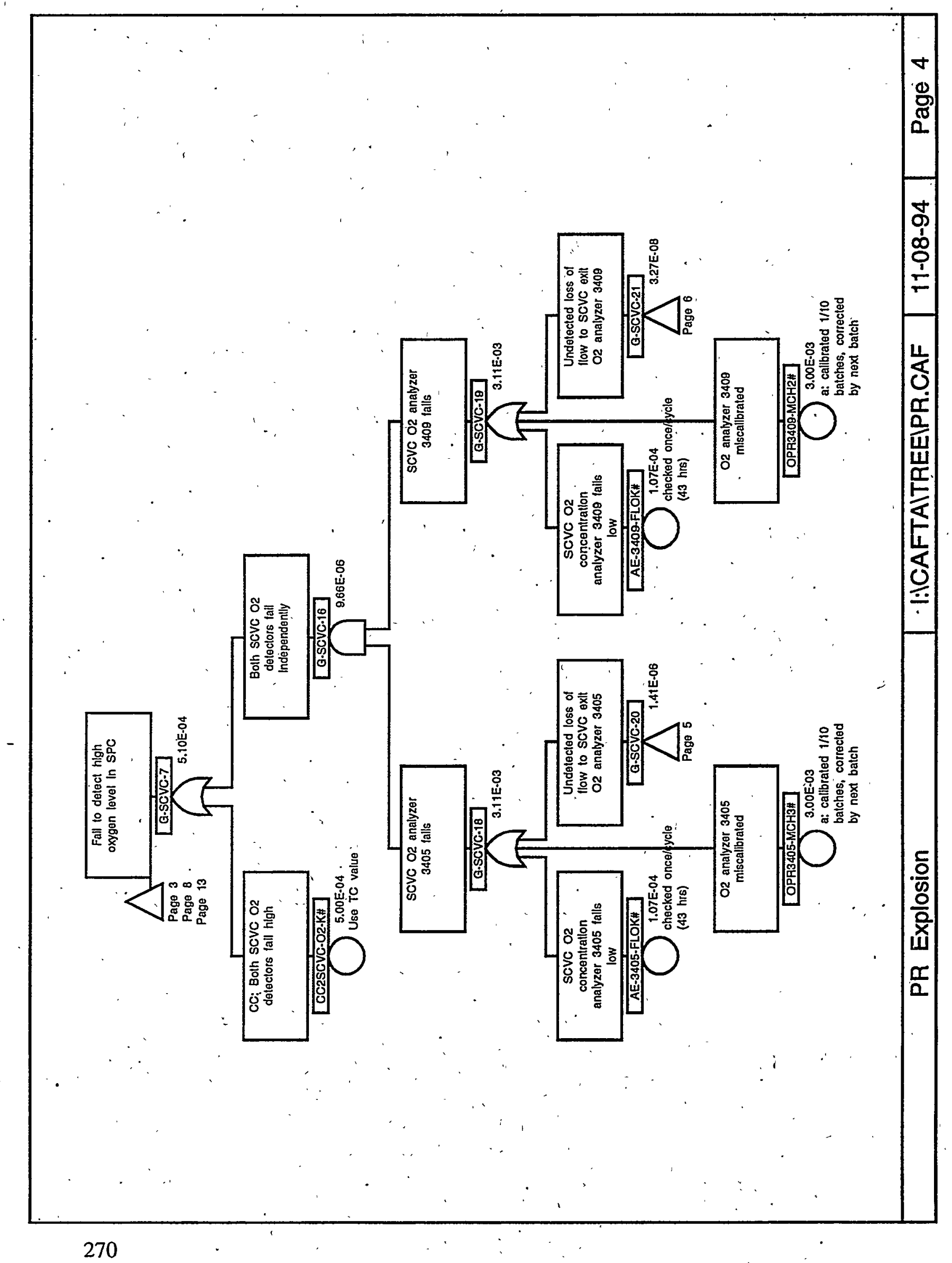




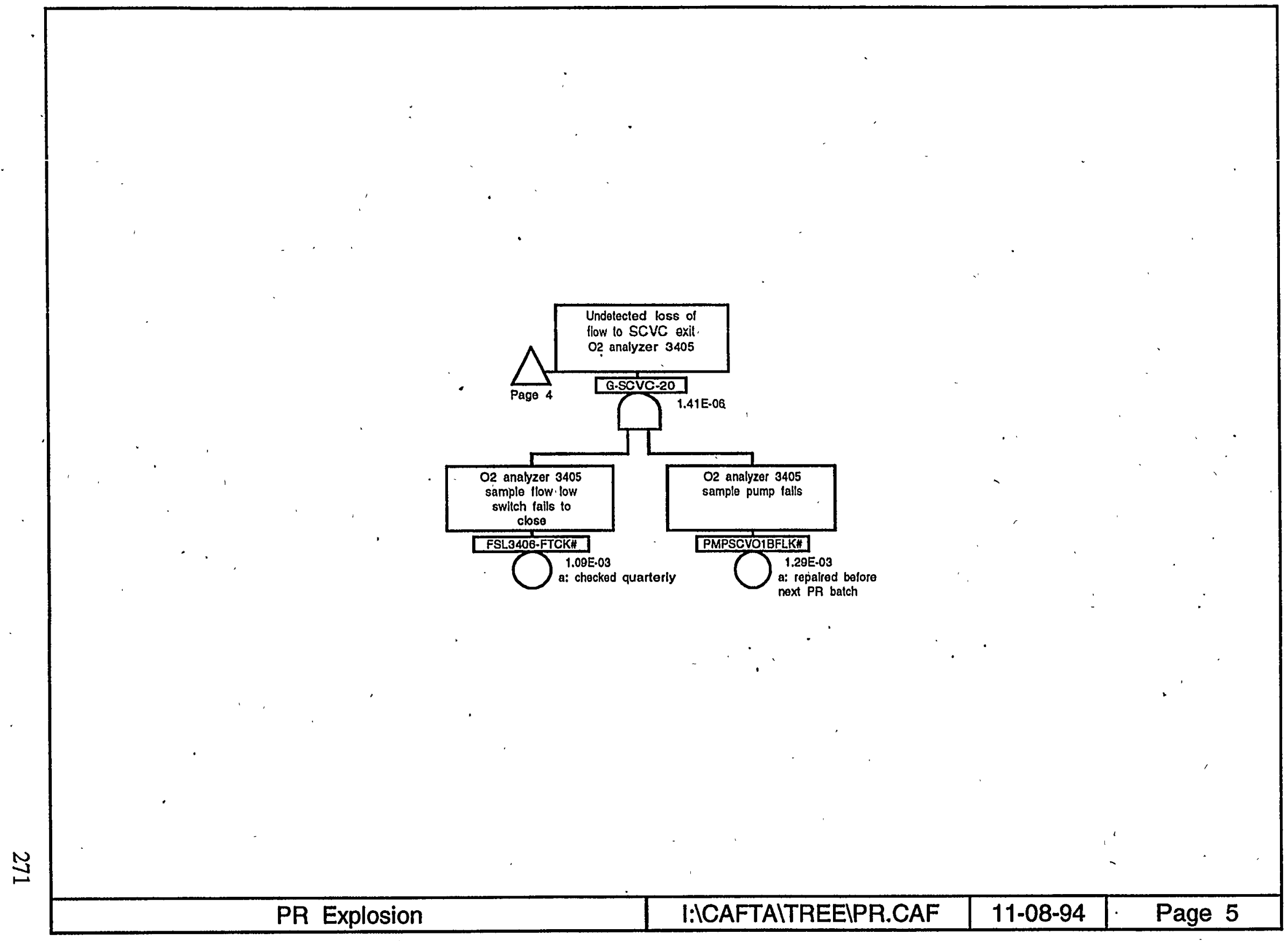


X-ESR-S-00001, REV. 0

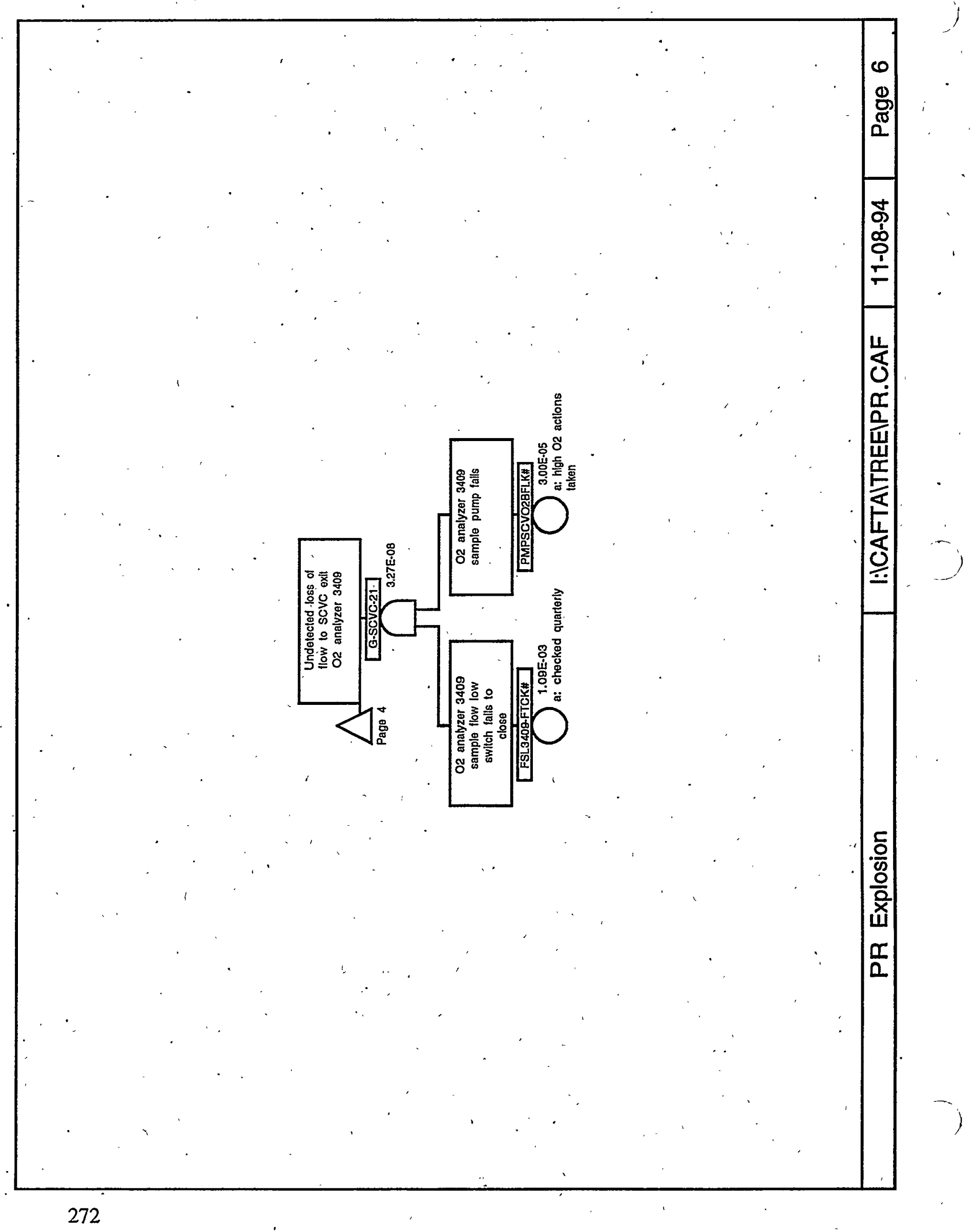




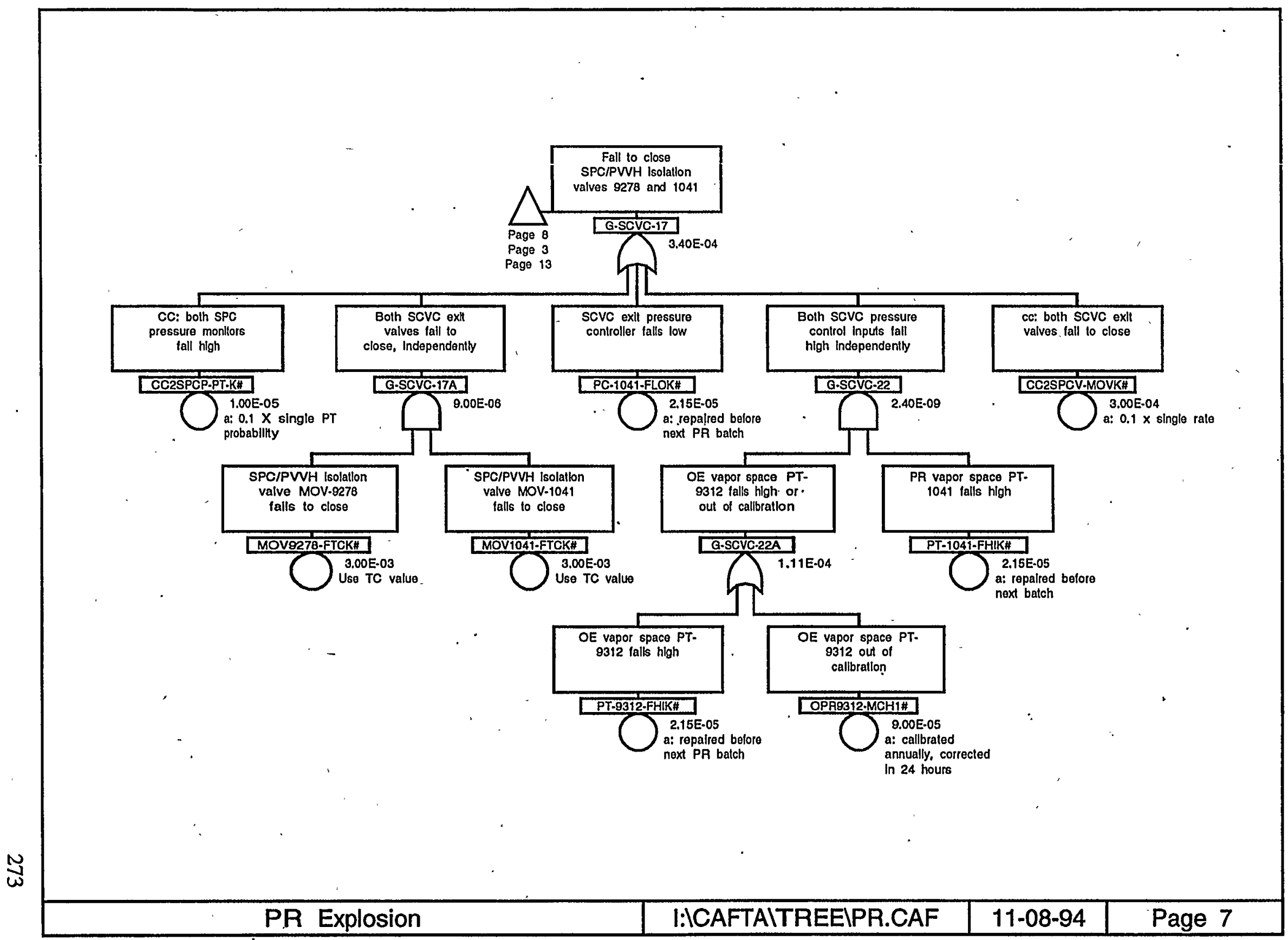




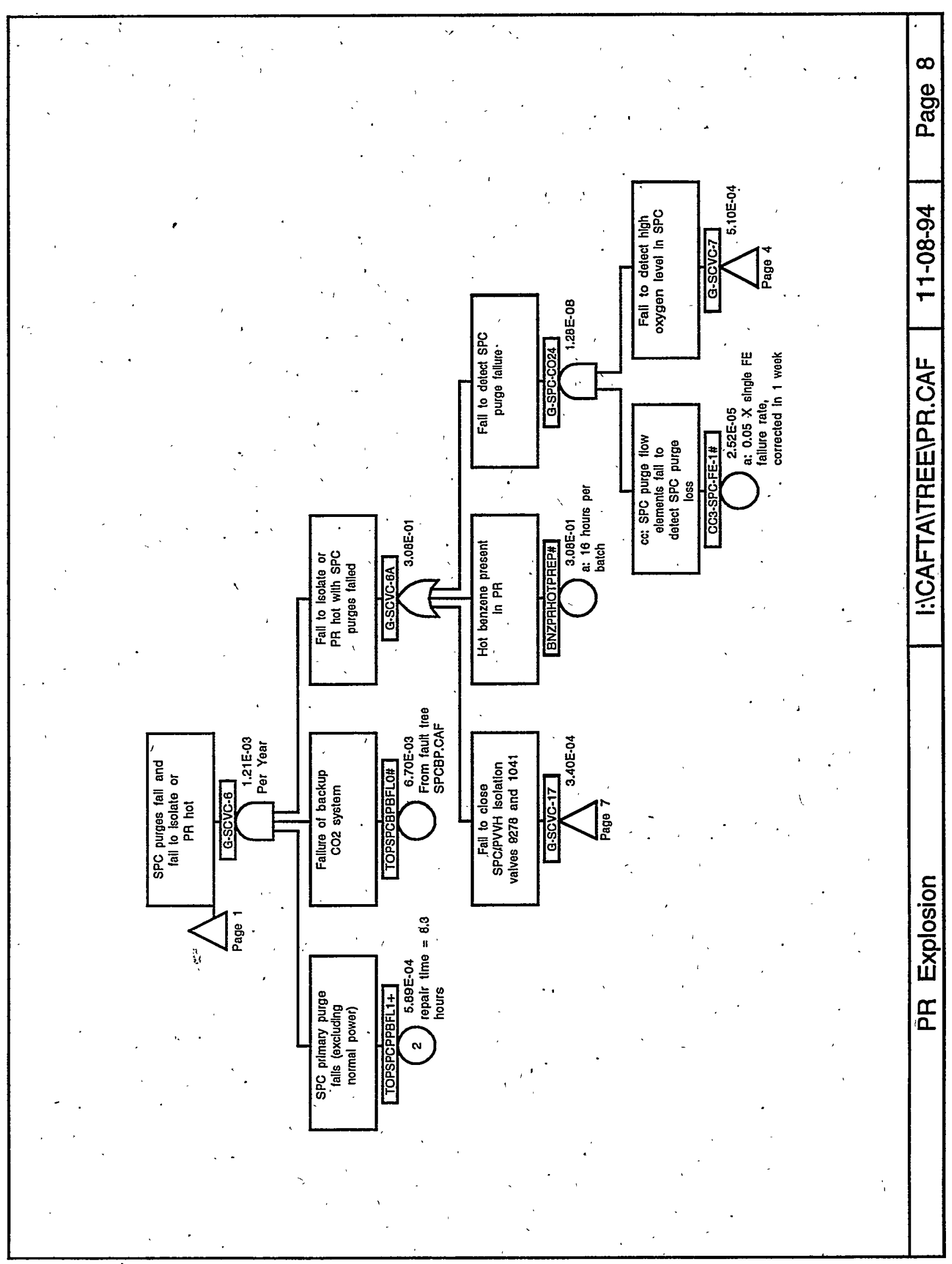




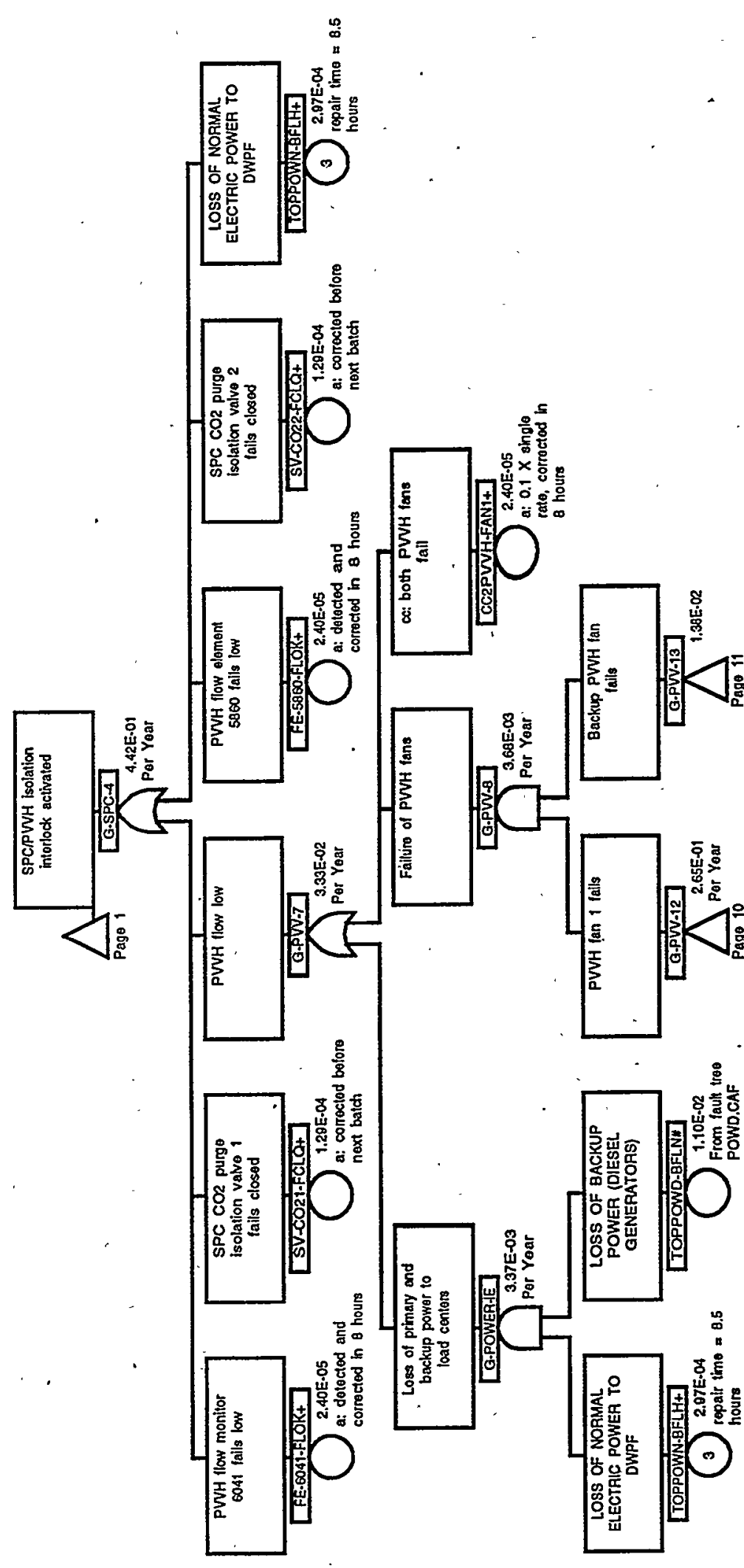


X-ESR-S-00001, REV. 0

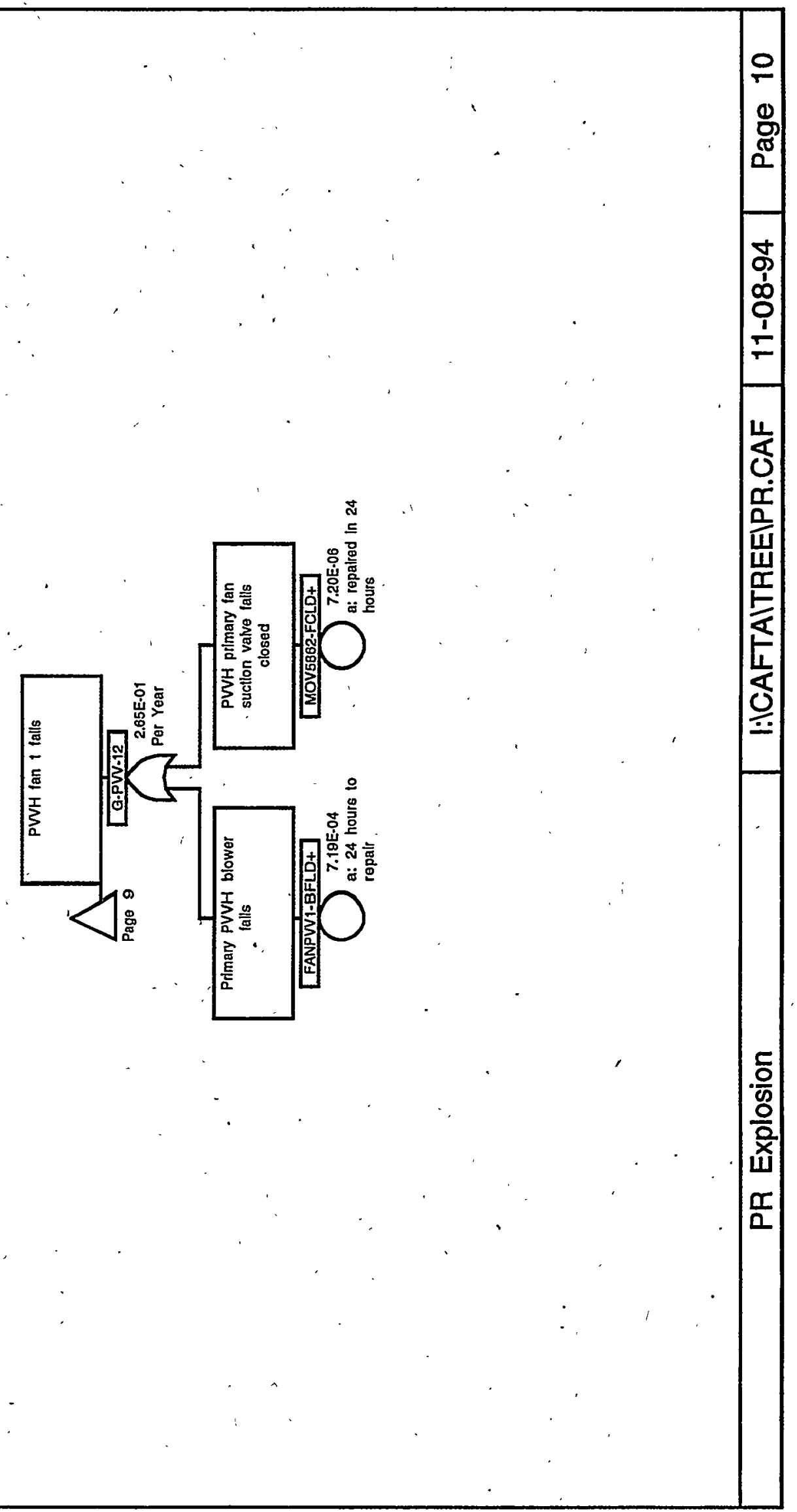




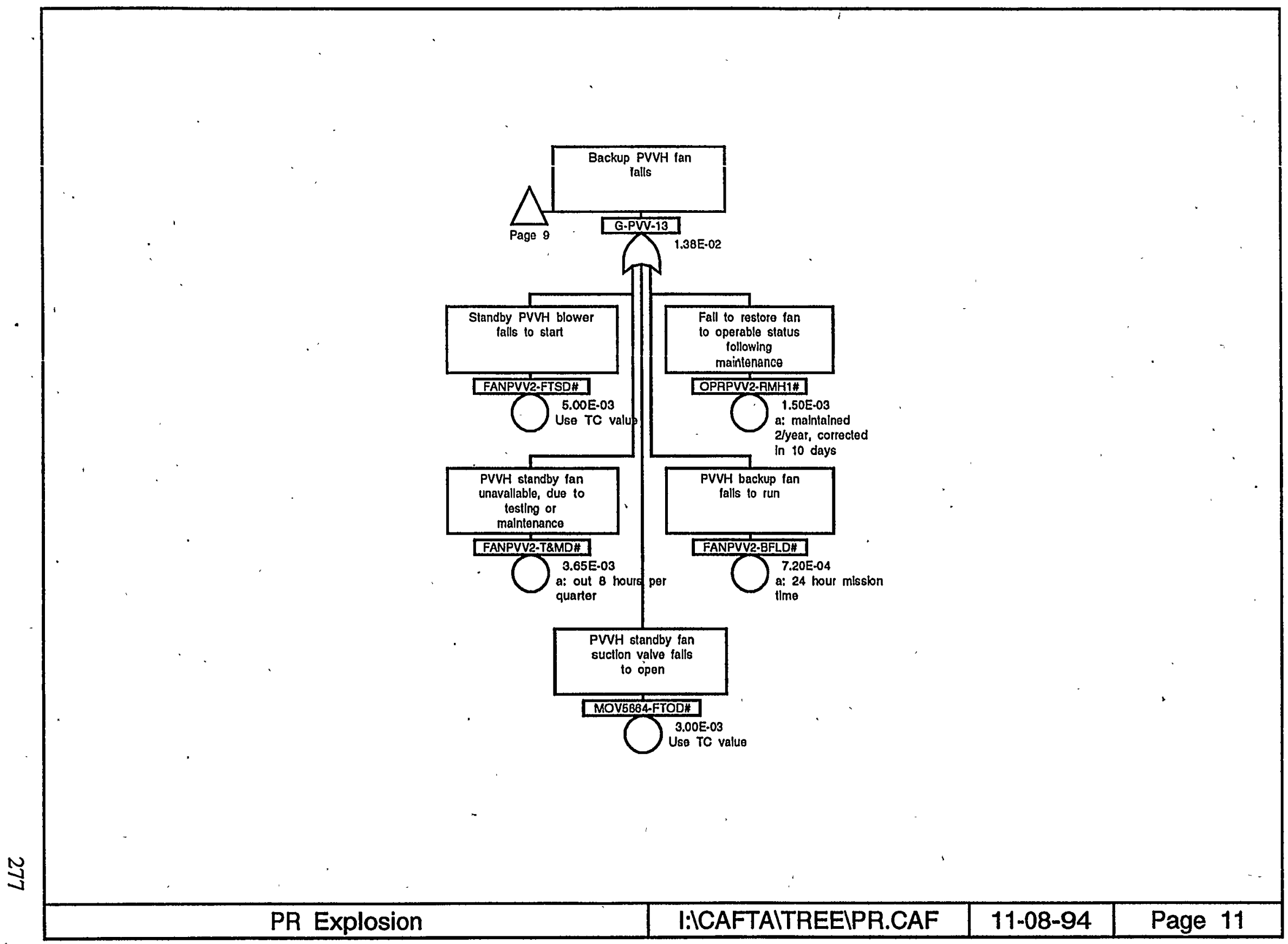


X-ESR-S-00001, REV. 0

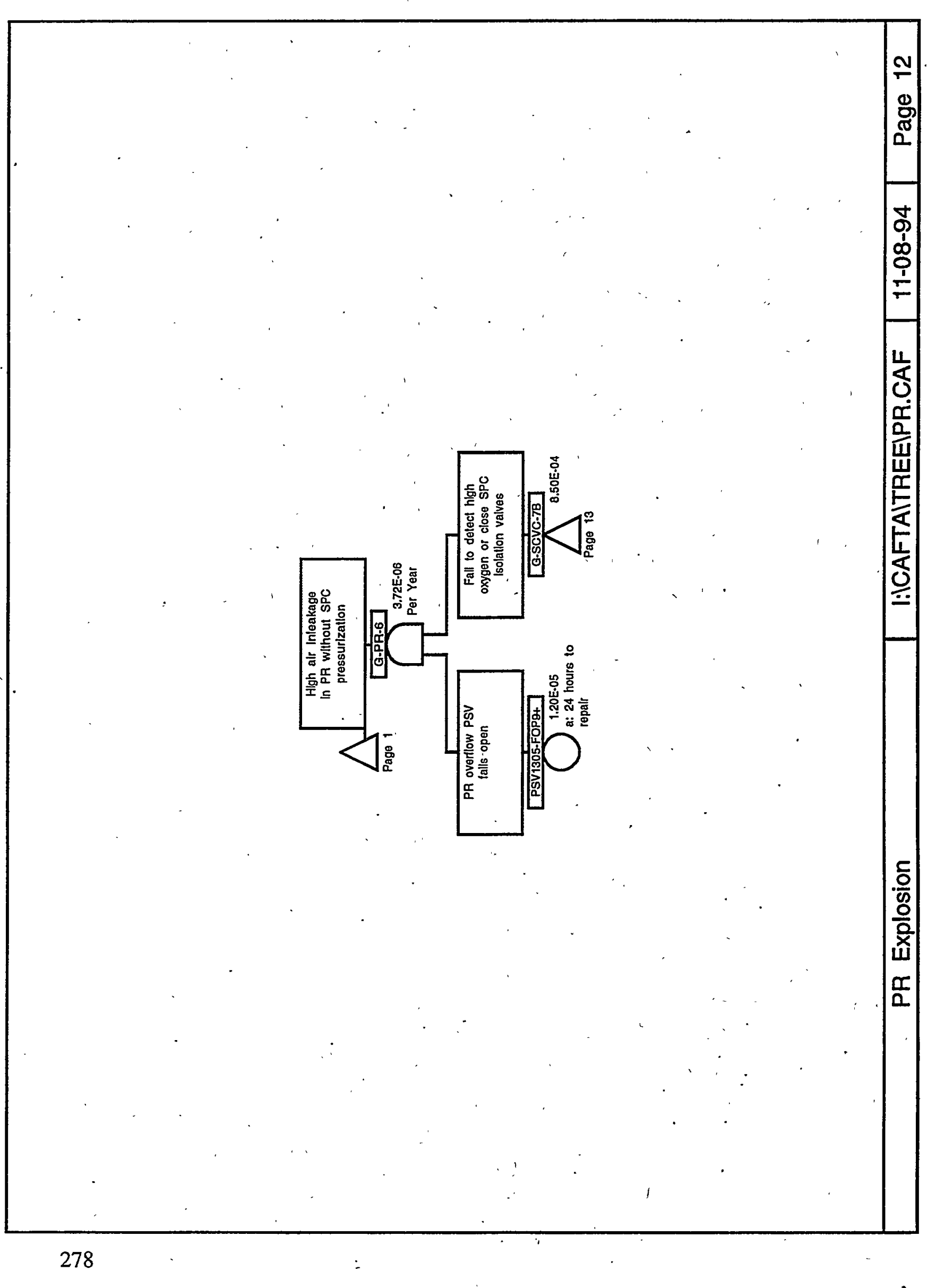




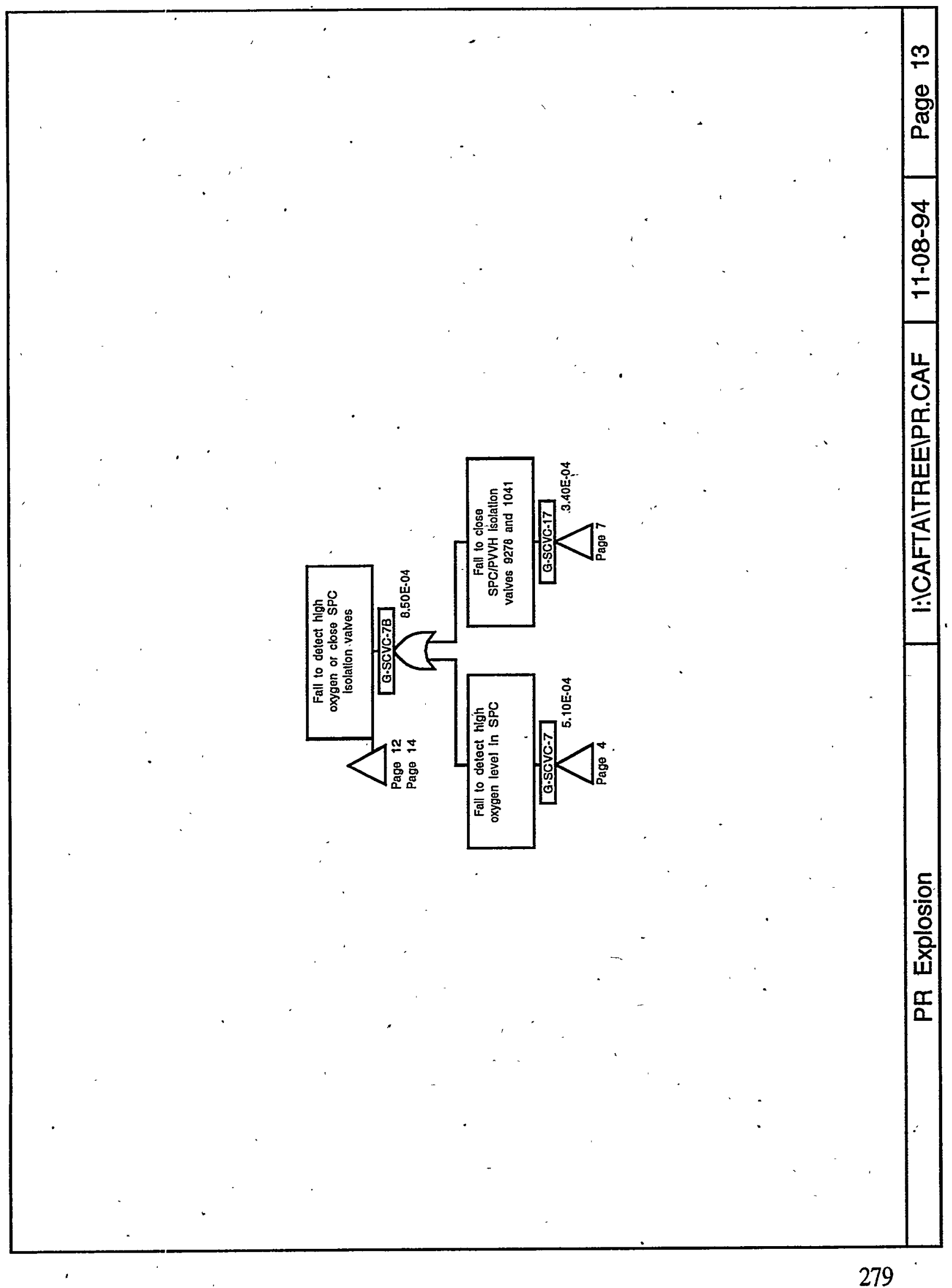




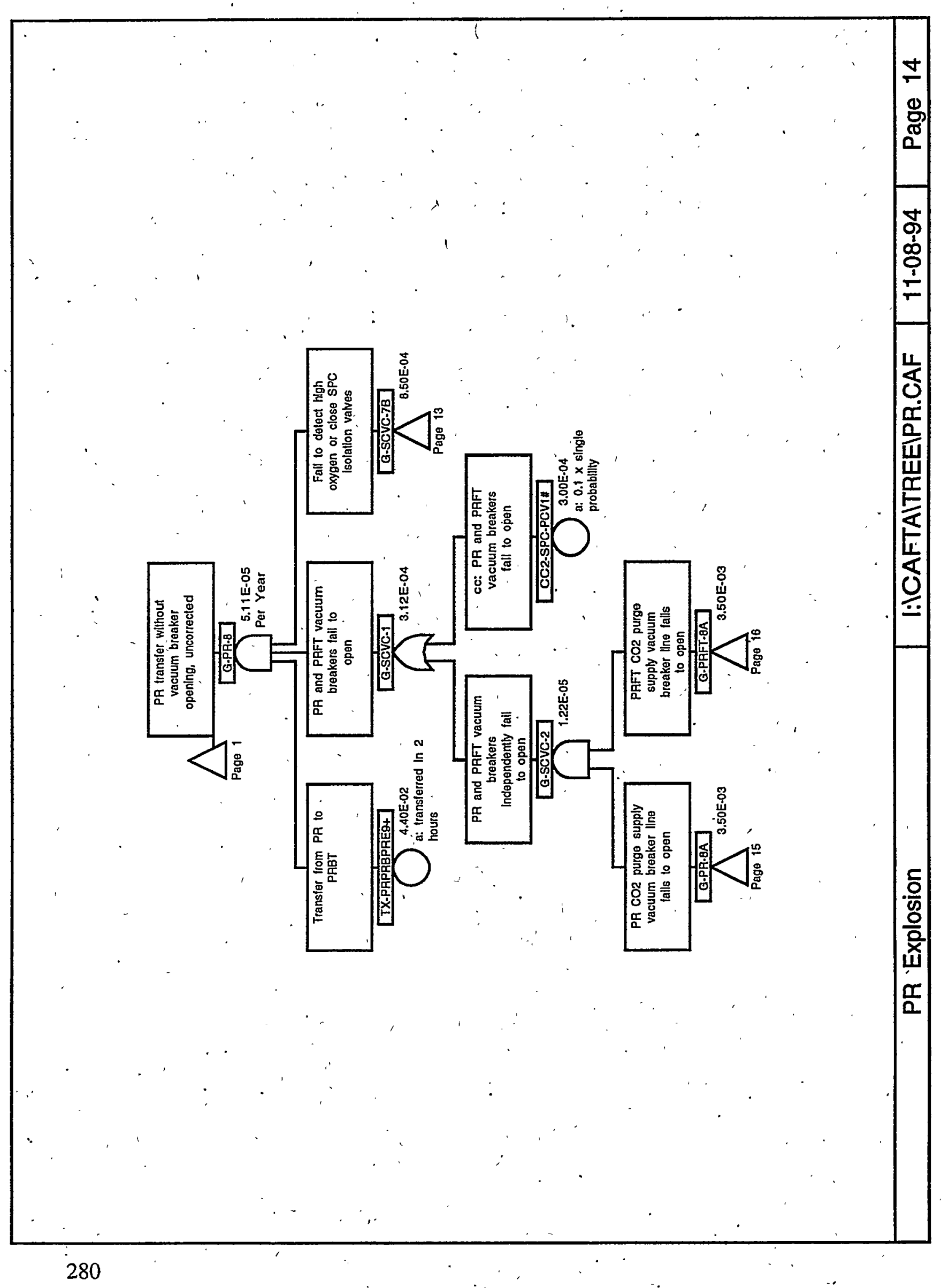




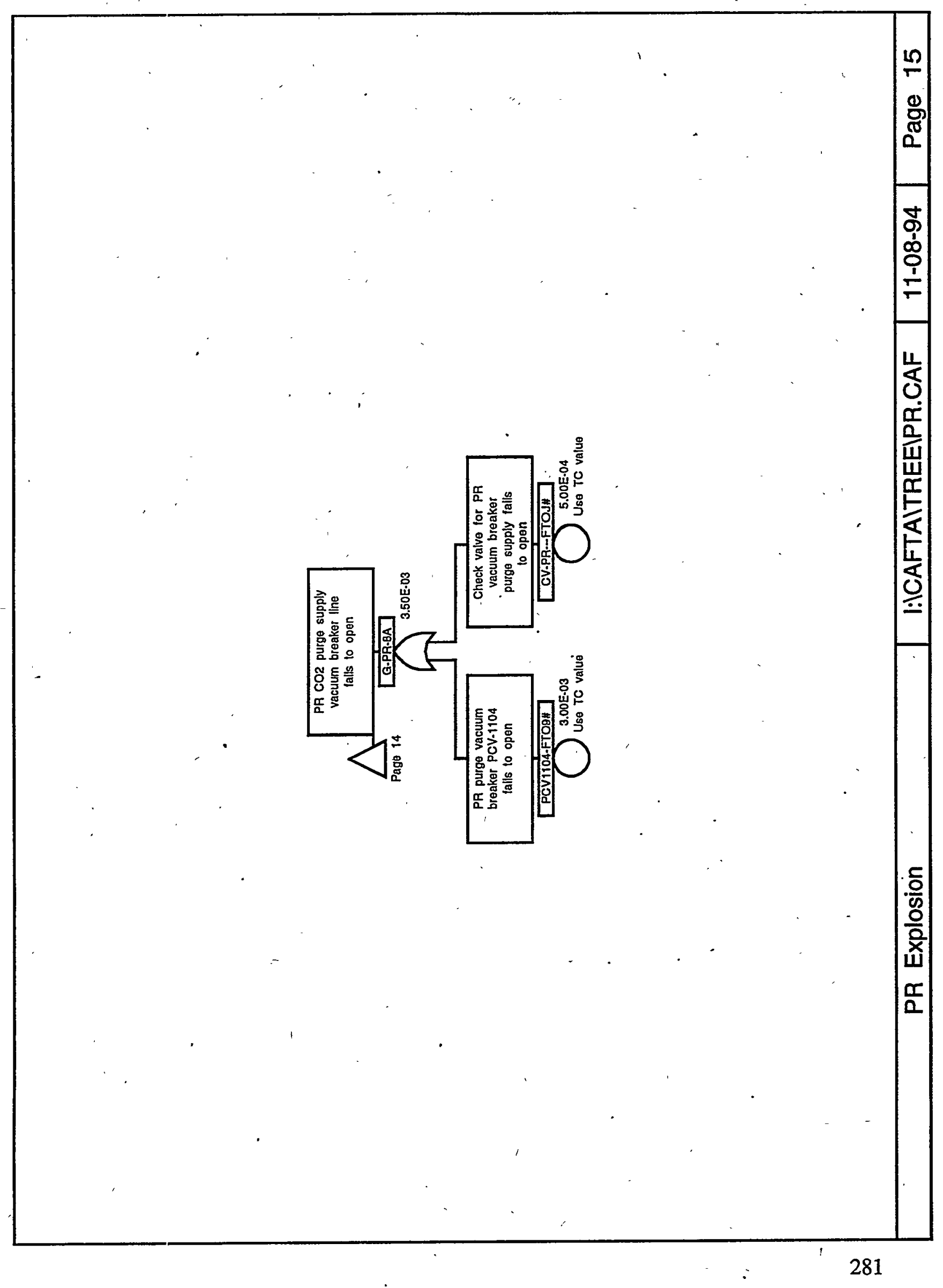




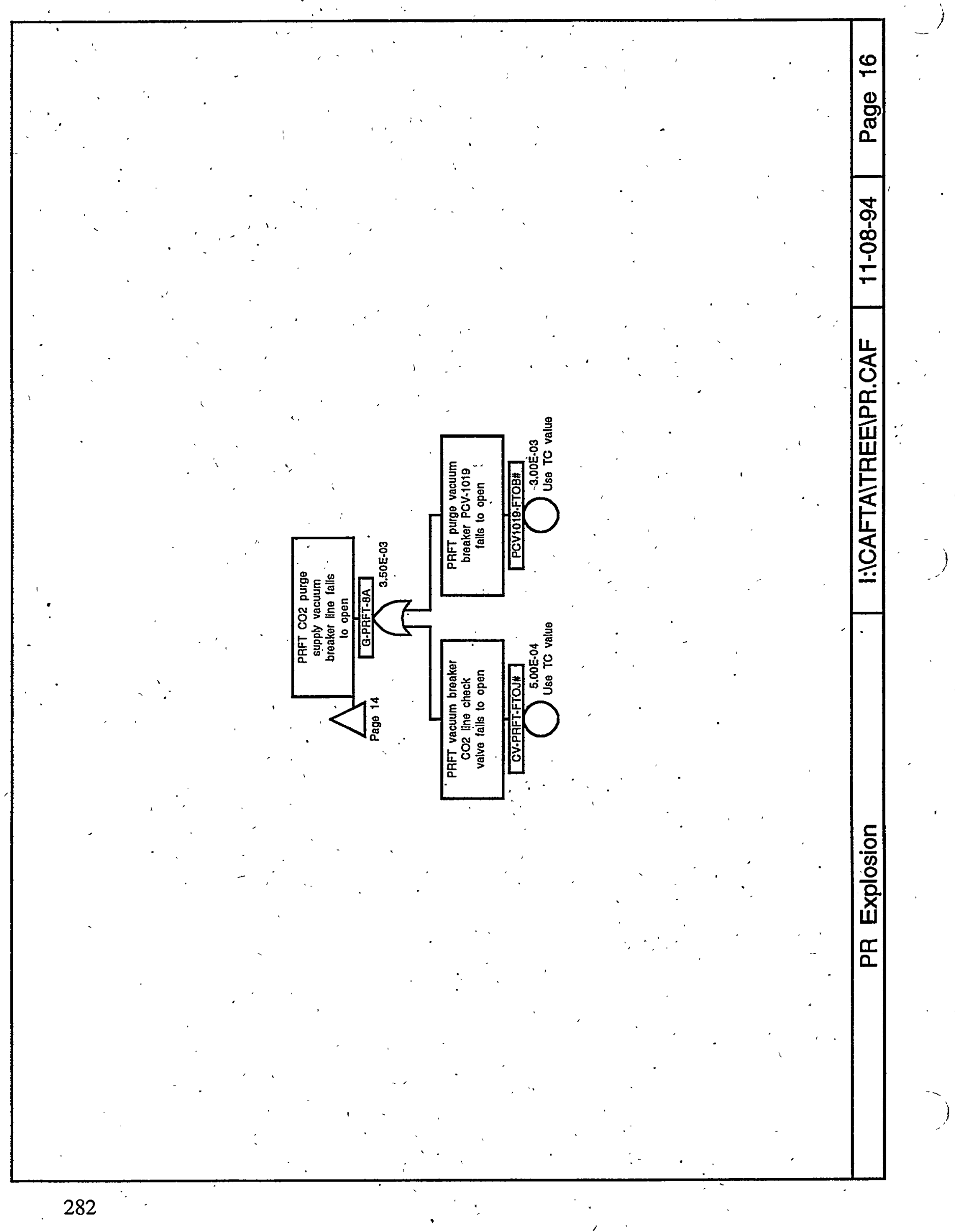




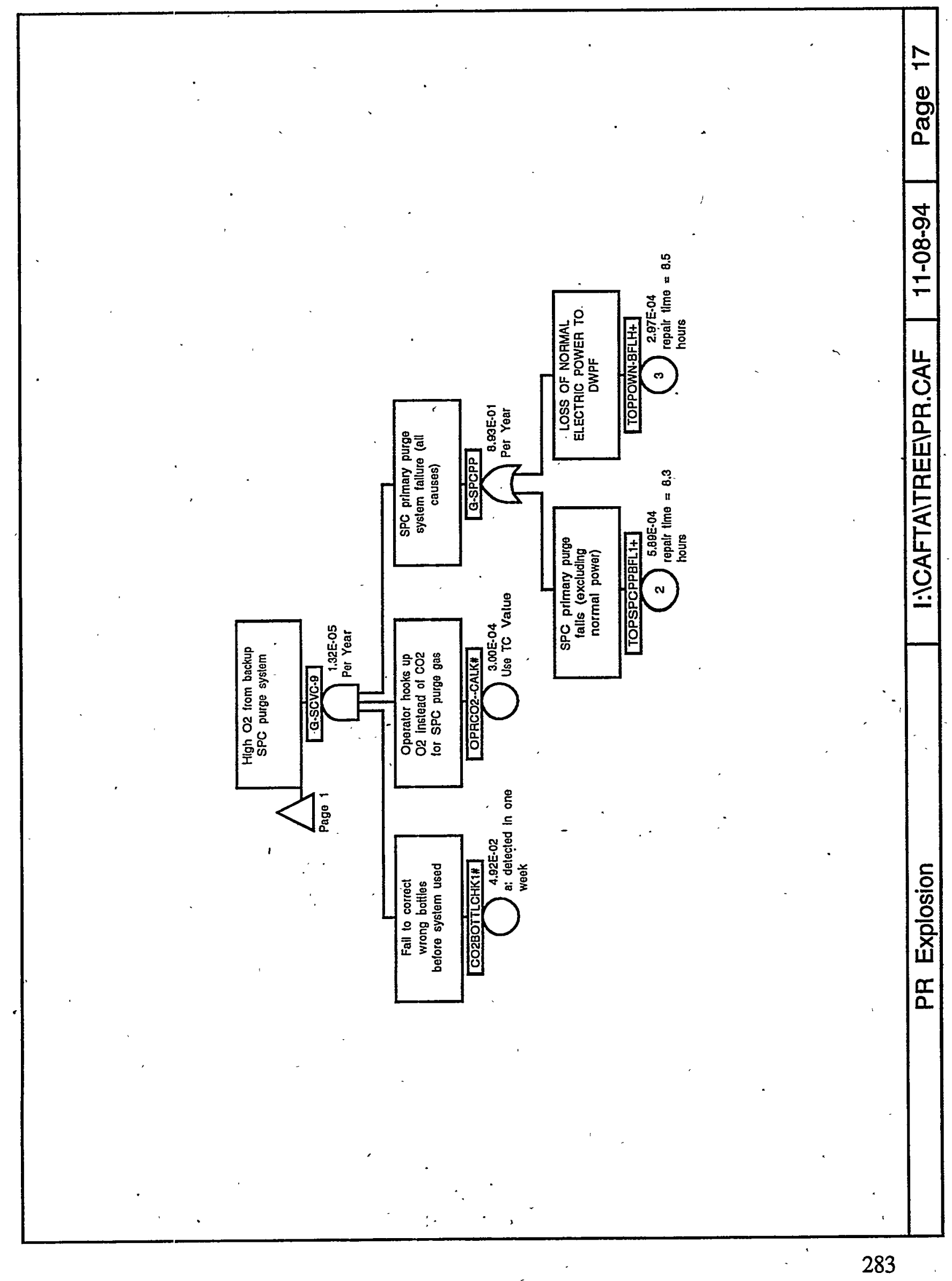


Gate/Event Name Page zone Gate/Event Name Page Zone.

AE-3405-ELOK\#

AE-3409-FLOK\#

BNZ PRHOTPREP\#

CC2-SPC-PCVI\#

CC2 PVVH-FAN1+

$\mathrm{CC} 2 \mathrm{SCVC}-\mathrm{O} 2-\mathrm{K} \#$

CC2 SPCP-PT-K\#

CC2 SPCV-MOVK\#

CC3-SPC- $\mathrm{FE}-1 \mathrm{H}$

CO2BOTTLCHK1\#

CV-PR-- -ETOJ\#

CV-PRFT-FTOJ\#

FANPVV1-BFLD+

FANPVV2-BFLD\#

FANPVV2-ETSD

FANPVV2 -T\&MD\#

FE-1103-FHIE\#

FE-5860-FLOK+

FE-6041-FLOK+

FSL3406-FTCK\#

FSL3409-FTCK\#

G-POWER-IE

G-PR- 1

G-PR-10

$G-P R-10$

$G-P R-1 A$

$G-P R=4$

$G-P R-4$

G-PR- 5

G-PR- 6

$G-P R-6$

G-PR- 8 .

$G-P R-8$

G-PR- $8 A$

$G-P R-8 A$

G-PR- 9

G-PRET- $8 A$

G-PRET- $8 A$

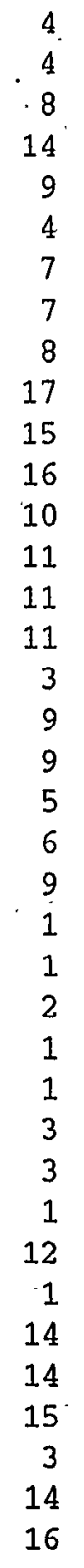

G-PVV-12

G-PVV-12

G-PVV-13

G-PVV- 13

G-PVV-7

G-PVV-8

G-SCVC-1

G-SCVC-16

G-SCVC-17

G- SCVC-17

G-SCVC-17

G-SCVC- 17

G-SCVC-17A

G-SCVC- 18

G-SCVC- 19

G-SCVC-2

G-SCVC-20

G-SCVC-20

G-SCVC-21

G-SCVC-21

G-SCVC-22

G-SCVC-22A

G-SCVC- 5

G-SCVC- 6

G-SCVC- 6

G-SCVC-6A

G-SCVC-7

G-SCVC-7

G-SCVC-7

G-SCVC- 7

G-SCVC-7B

G- SCVC-7B

G-SCVC-7B

G-SCVC-9

G-SCVC- 9

G-SPC- 4

G-SPC-4

G-SPC-CO24
Gate/Event Name Page Zone Gate/Event Name Page Zone G-SPCPP

HCV1102-FCL9+

IGNPR---PRE1\#

MOV1041-FTCK\#

MOV5862-FCLD+

MOV5864-FTOD\#

MOV9278-FTCK\#

OPR1103-MCH1\#

OPR3 405-MCH3\#

OPR3 409-MCH2 \#

OPR9312-MCH1\#

OPRCO2--CALK\#

OPRPVV2-RMH1\#

OPRSV7,4ARANK+

PC-1041-FLOK\#

PCV1019-ETOB\#

PCV1101-FCL9+

PCV1104-FTO9\#

PMPSCVO1BELK\#

PMPSCVO2BELK\#

PSV1305-FOP9+

PSV180--FOP9+

PT-1041-FHIK\#

PT-9312-FHIK\#

SV-CO21-FCLQ+

SV-CO22 -FCLQ+

TOP-PR

TOPPOWD-BFLN\#

TOPPOWN-BFLH+

TOPPOWN-BFLH+

TOPPOWN-BFLH+

TOPSPCBPBFLO\#

TOPSPCN2BFLU\#

TOPSPCPPBEL1+

TOPSPCPPBFL1+

TX-PRPRBPRE9+ 
Cutsets for I: \CAFTA \CUT\PR.CSR

$11 / 08 / 94 \quad 12: 29 \quad \mathrm{AM}$

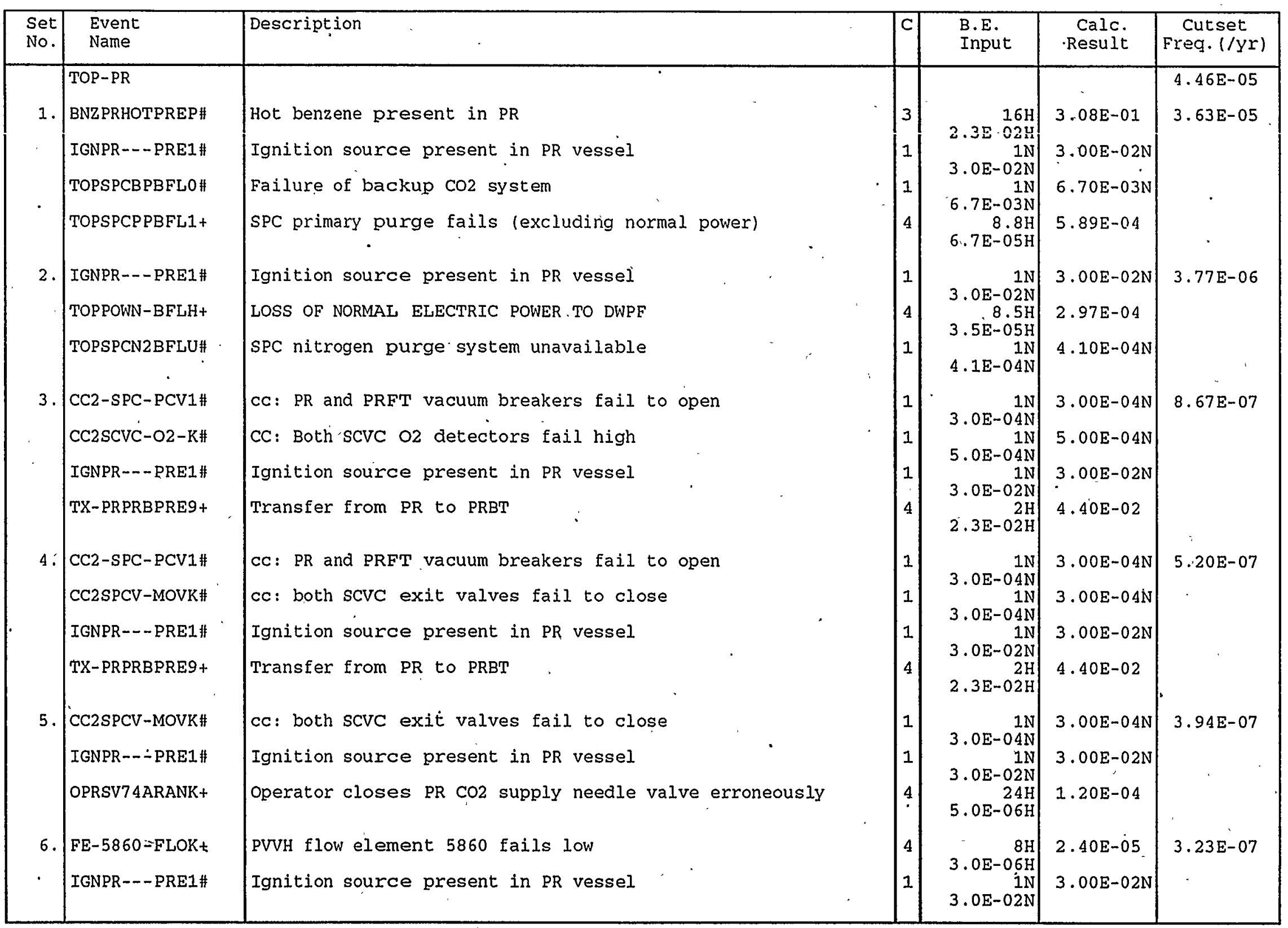


Cutsets for I: ICAFTA \CUT \PR.CSR

11/08/94 12:29 AM (CONT.)

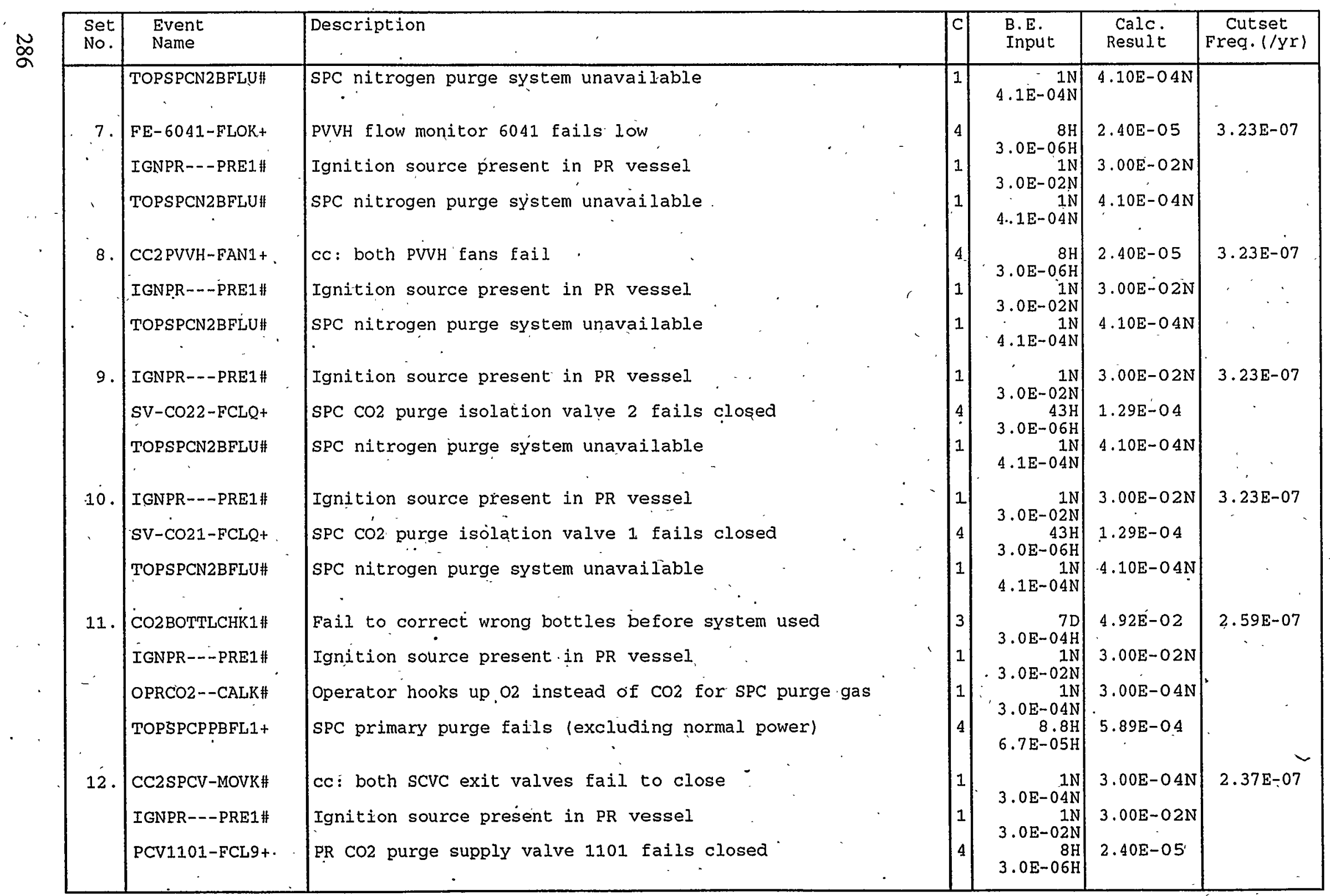


Cutsets for I: ICAFTA ICUT\PR.CSR

11/08/94 12:29 AM (CONT.)

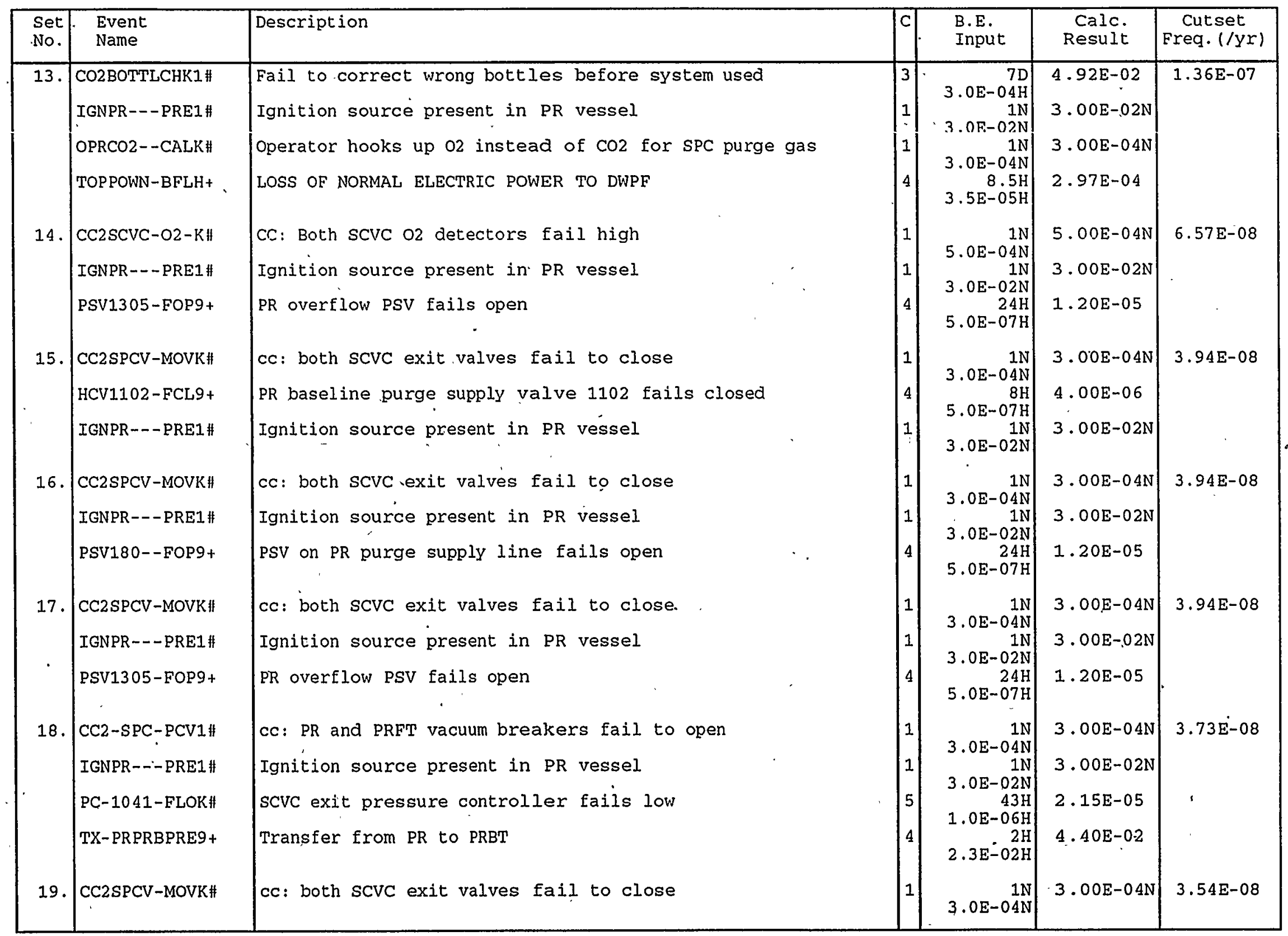


Cutsets for I: ICAFTA ICUT\PR.CSR

11/08/94 12:29 AM (CONT.)

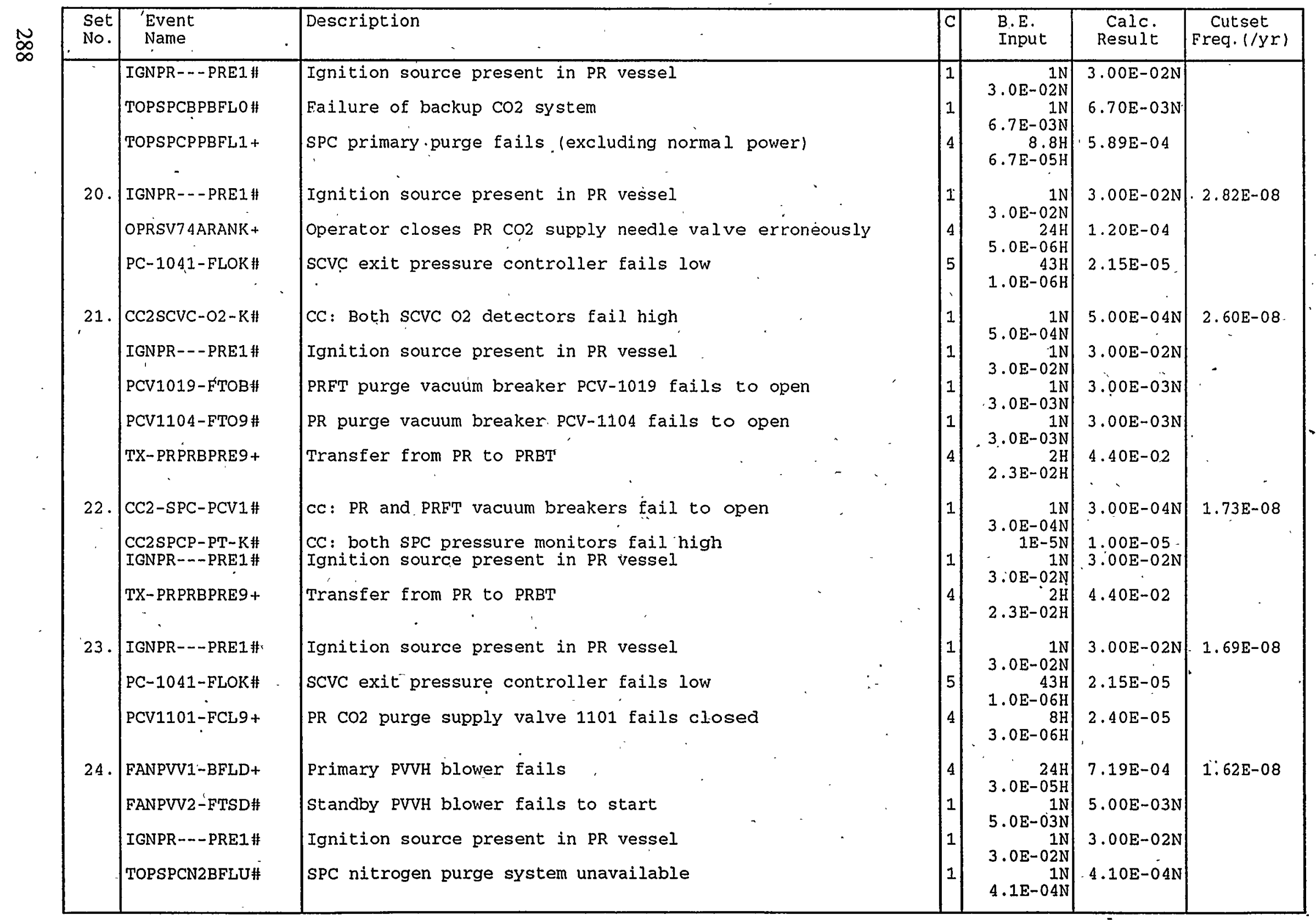


Cutsets for I: ICAFTA ICUT\PR. CSR

11/08/94 12:29 AM (CONT.)

\begin{tabular}{|c|c|c|c|c|c|c|}
\hline $\begin{array}{l}\text { Set } \\
\text { No. }\end{array}$ & $\begin{array}{l}\text { Event } \\
\text { Name }\end{array}$ & Description & $\mathrm{C}$ & $\begin{array}{l}\text { B.E. } \\
\text { Input }\end{array}$ & $\begin{array}{l}\text { Calc. } \\
\text { Result }\end{array}$ & $\begin{array}{c}\text { Cutset } \\
\text { Freq. (/yr) }\end{array}$ \\
\hline 25 . & $\begin{array}{l}\text { CC2-SPC-PCV1\# } \\
\text { IGNPR---PRE1\# } \\
\text { MOV1041-FTCK\# } \\
\text { MOV9278-FTCK\# } \\
\text { TX-PRPRBPRE9+ }\end{array}$ & $\begin{array}{l}\text { CC: PR and PRFT vacuum breakers fail to open } \\
\text { Ignition source present in PR vessel } \\
\text { SPC/PVVH isolation valve MOV-1041 fails to close } \\
\text { SPC/PVVH isolation valve MOV-9278 fails to close } \\
\text { Transfer from PR to PRBT }\end{array}$ & $\begin{array}{l}1 \\
1\end{array}$ & $\begin{array}{r}1 \mathrm{~N} \\
3.0 \mathrm{E}-04 \mathrm{~N} \\
1 \mathrm{~N} \\
3.0 \mathrm{E}-02 \mathrm{~N} \\
1 \mathrm{~N} \\
3.0 \mathrm{E}-03 \mathrm{~N} \\
1 \mathrm{~N} \\
3.0 \mathrm{E}-03 \mathrm{~N} \\
2 \mathrm{H} \\
2.3 \mathrm{E}-02 \mathrm{H}\end{array}$ & $\begin{array}{l}3.00 \mathrm{E}-04 \mathrm{~N} \\
3.00 \mathrm{E}-02 \mathrm{~N} \\
3.00 \mathrm{E}-03 \mathrm{~N} \\
3.00 \mathrm{E}-03 \mathrm{~N} \\
4.40 \mathrm{E}-02\end{array}$ & $1.56 \mathrm{E}-08$ \\
\hline
\end{tabular}


Explosion in the PR Top Event Frequency: 4.46E-05/YR

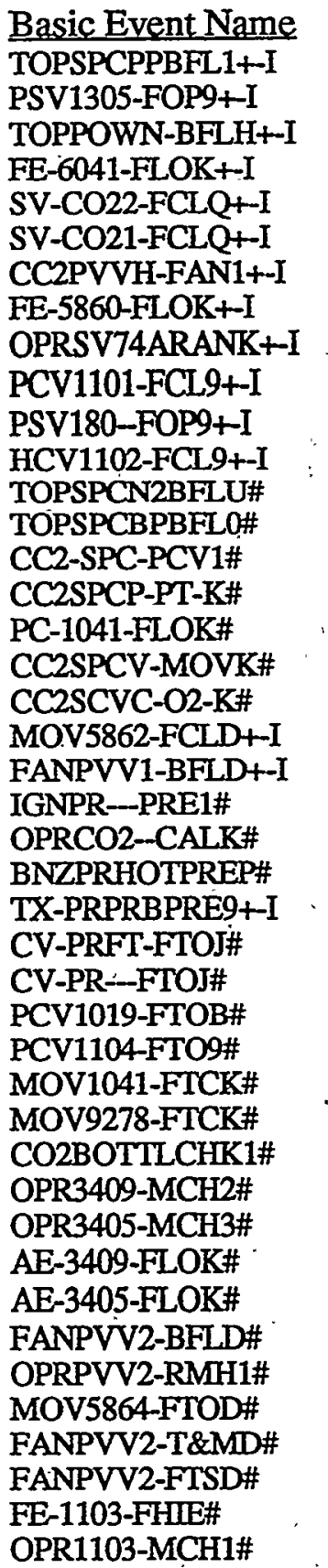

\section{Risk Achievement Worth Description}

SPC primary purge fails (excluding normal power)

PR overflow PSV fails open

LOSS OF NORMAL ELECTRIC POWER TO DWPF *

PVVH flow monitor 6041 fails low

SPC CO2 purge isolation valve 2 fails closed

SPC CO2 purge isolation valve 1 fails closed

cc: both PVVH fans fail

PVVH flow element 5860 fails low

Operator closes PR CO2 supply needle valve erroneously

PR CO2 purge supply valve 1101 fails closed

PSV on PR purge supply line fails open

PR baseline purge supply valve 1102 fails closed

SPC nitrogen purge system umavailable

Failure of backup $\mathrm{CO} 2$ system

cc: PR and PRFT vacuum breakers fail to open

CC: both SPC pressure monitors fail high

SCVC exit pressure controller fails low

cc: both SCVC exit valves fail to close

CC: Both SCVC O2 detectors fail high

PVVH primary fan suction valve fails closed

Primary PVVH blower fails

Ignition source present in $P R$ vessel

Operator hooks up $\mathrm{O} 2$ instead of $\mathrm{CO} 2$ for SPC purge gas

Hot benzene present in PR

Transfer from PR to PRBT

PRFT vacuum breaker $\mathrm{CO} 2$ line check valve fails to open

Check valve for $P R$ vacuim breaker purge fails to open

PRFT.purge vacuum breaker PCV-1019 fails to open

PR purge vacuum breaker PCV-1104 fails to open

SPC/PVVH isolation valve MOV-1041 fails to close

SPC/PVVH isolation valve MOV-9278 fails to close

Fail to correct wrong bottles before system used

O2 analyzer 3409 miscalibrated

$\mathrm{O} 2$ analyzer 3405 miscalibrated

SCVC 02 concentration analyzer 3409 fails low

SCVC 02 concentration analyzer 3405 fails low.

PVVH backup fan fails to run

Fail to restore fan to operable status following maintenance

PVVH standby fan suction valve fails to open

PVVH standby fan unavailable, due to T\&M

Standby PVVH blower fails to start

PR purge flow element 1103 fails to detect low flow

PR purge flow element 1103 miscalibrated
Prob/Freq AchW

6.69E-05/H $1.23 \mathrm{E}+04$

$5.00 \mathrm{E}-07 / \mathrm{H} \quad 5.00 \mathrm{E}+03$

$3.50 \mathrm{E}-05 / \mathrm{H} \quad 2.50 \mathrm{E}+03$

$3.00 \mathrm{E}-06 / \mathrm{H} \quad 2.42 \mathrm{E}+03$

3.00E-06/H 2.42E+03

$3.00 \mathrm{E}-06 / \mathrm{H} \quad 2.42 \mathrm{E}+03$

$3.00 \mathrm{E}-06 / \mathrm{H} \quad 2.42 \mathrm{E}+03$

$3.00 \mathrm{E}-06 / \mathrm{H} \quad 2.42 \mathrm{E}+03$

$5.00 \mathrm{E}-06 / \mathrm{H}, 2.00 \mathrm{E}+03$

$3.00 \mathrm{E}-06 / \mathrm{H} \quad 2.00 \mathrm{E}+03$

$5.00 \mathrm{E}-07 / \mathrm{H} \quad 2.00 \mathrm{E}+03$

$5.00 \mathrm{E}-07 / \mathrm{H} \quad 2.00 \mathrm{E}+03$

4.10E-04 2.98E+02

6.70E-03 1.22E+02

3.00E-04 1.11E+02

8.40E-06 1.00E+02

2.15E-05 1.00E +02

3.00E-04 1.00E+02

5.00E-04 4.45E+01

$3.00 \mathrm{E}-07 / \mathrm{H} \quad 3.45 \mathrm{E}+01$

$3.00 \mathrm{E}-05 / \mathrm{H} \quad 3.45 \mathrm{E}+01$

$3.00 \mathrm{E}-02 \quad 3.33 \mathrm{E}+01$

$3.00 \mathrm{E}-044^{\prime} \quad 3.05 \mathrm{E}+01$

3.08E-01 2.83E+00

$2.20 \mathrm{E}-02 / \mathrm{H} \quad 2.53 \mathrm{E}+00$

5.00E-04 1.38E+00

5.00E-04 1.38E+00

3.00E-03 1.38E+00

3.00E-03 1.38E+00

$3.00 \mathrm{E}-03-1.30 \mathrm{E}+00$

3.00E-03 $\quad 1.30 \mathrm{E}+00$

4.92E-02 1.17E+00

$3.00 \mathrm{E}-03 \quad 1.13 \mathrm{E}^{\prime}+00$

3.00E-03 1.13E+00

$1.07 \mathrm{E}-04 \quad 1.13 \mathrm{E}+00$

$1.07 \mathrm{E}-04 \quad 1.13 \mathrm{E}+00$

7.20E-04 1.07E+00

$1.50 \mathrm{E}-03 \quad 1.07 \mathrm{E}+00$

. 3.00E-03 1.07E+00

3.65E-03 1.07E+00

5.00E-03 1.07E+00

2.16E-03 1.03E+00

$1.20 \mathrm{E}-04 \quad 1.03 \mathrm{E}+00$

Note: Events that make less than $1 \%$ difference to the importance factor are not included. Modified top event frequency $=$ (top event frequency) $\mathrm{x}$ (risk achievement worth). 
Explosion in the PR Top Event Frequency: 4.46E-05/YR

Basic Event Name

*IGNPR---PRE1\#

TOPSPCPPIBFL1+-I

TOPSPCBPBFLO\#

BNZPRHOTPREP\#

TOPSPCN2BFLU\#

TOPPOWN-BFLH+-I

TX-PRPRBI?RE9+-I

CC2-SPC-PCV1\#

CC2SPCV-MOVK\#

CC2SCVC-O2-K\#

OPRSV74ARANK+-I
Risk Reduction Worth

\section{Descrintion}

Ignition source present in $P R$ vessel

SPC primary purge fails (excluding normal power)

Failure of backup $\mathrm{CO} 2$ system

Hot benzene present in PR

SPC nitrogen purge system unavailable

LOSS OF NORMAL ELECTRIC POWER TO DWPF

Transfer from PR to PRBT

cc: PR and PRFT vacuum breakers fail to open

cc: both SCVC exit valves fail to close

CC: Both SCVC O2 detectors fail high

Operator closes PR CO2 supply needle valve erroneously
Prob/Freg RedW

$3.00 \mathrm{E}-02 \quad 0.00 \mathrm{E}+00$

$6.69 \mathrm{E}-05 / \mathrm{H} \quad 5.56 \mathrm{E}+00$

$6.70 \mathrm{E}-03 \quad 5.39 \mathrm{E}+00$

$3.08 \mathrm{E}-01 \quad 5.36 \mathrm{E}+00$

$4.10 \mathrm{E}-04 \quad 1.14 \mathrm{E}+00$

3.50E-05/H $\quad 1.10 \mathrm{E}+00$

$2.20 \mathrm{E}-02 / \mathrm{H} \quad 1.04 \mathrm{E}+00$

$3.00 \mathrm{E}-04 \quad 1.03 \mathrm{E}+00$

3.00E-04 1.03E+00

$5.00 \mathrm{E}-04 \quad 1.02 \mathrm{E}+00$

$5.00 \mathrm{E}-06 / \mathrm{H} \quad 1.01 \mathrm{E}+00$

* A Reduction Worth of $0.0 \mathrm{E}+00$ indicates event appears in every cutset could serve as a single event preventor for the accident.

Note: Events that make less than $1 \%$ difference to the importance factor are not included. Modified top event frequency = (top event frequency) $/$ (risk reduction worth). 
X-ESR-S-00001, REV. 0

\section{OE Explosion}




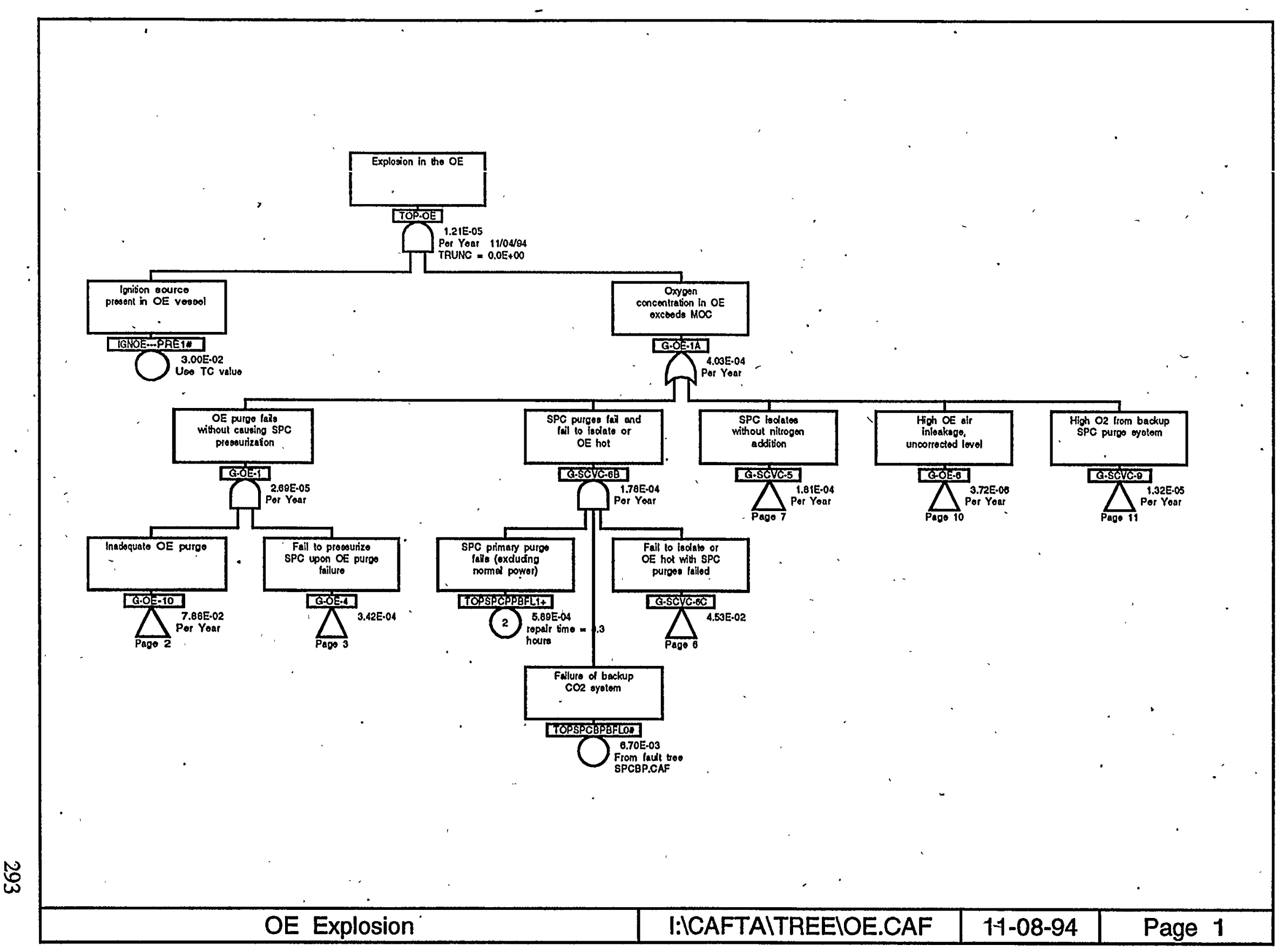


X-ESR-S-00001, REV. 0

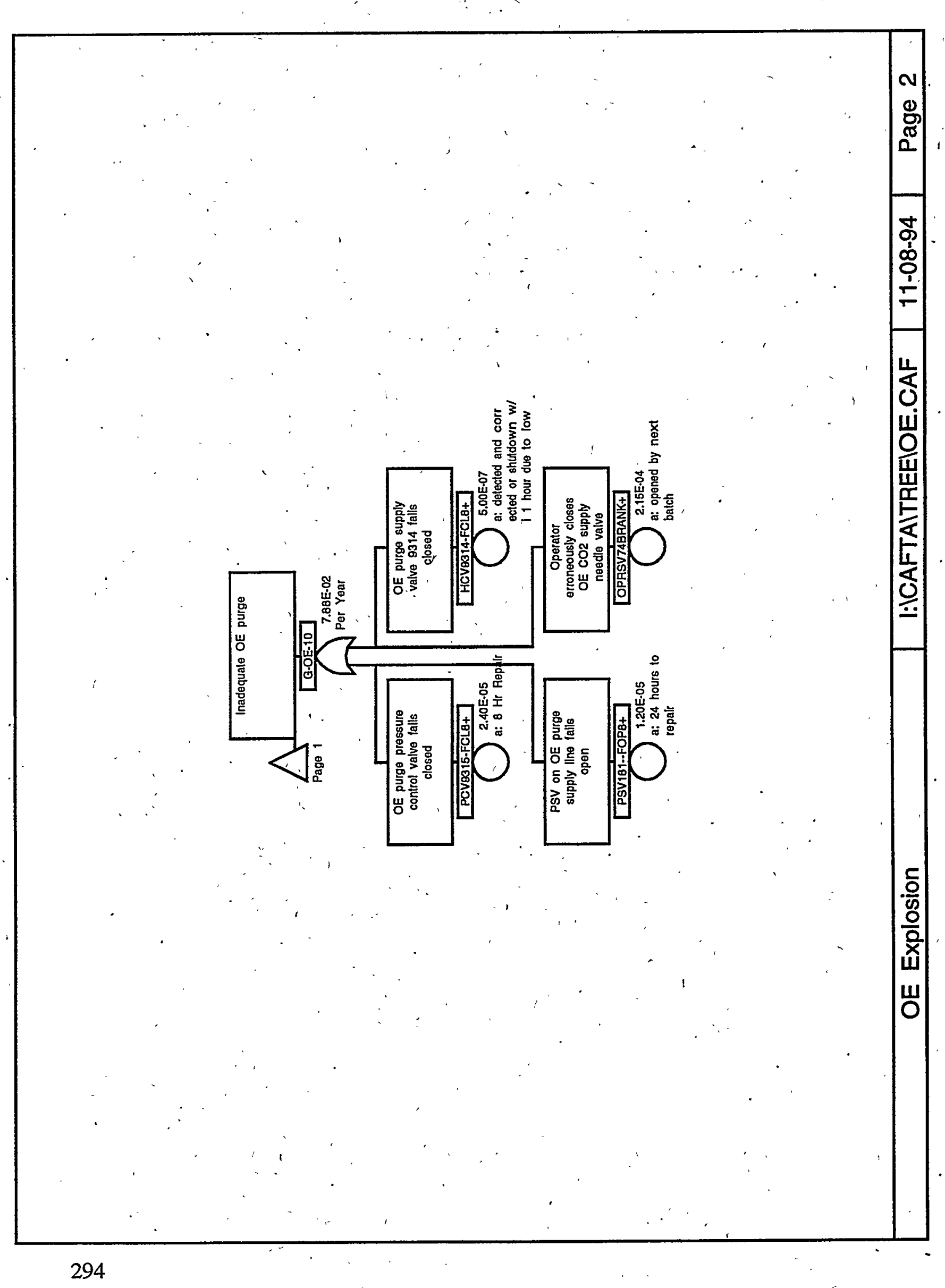




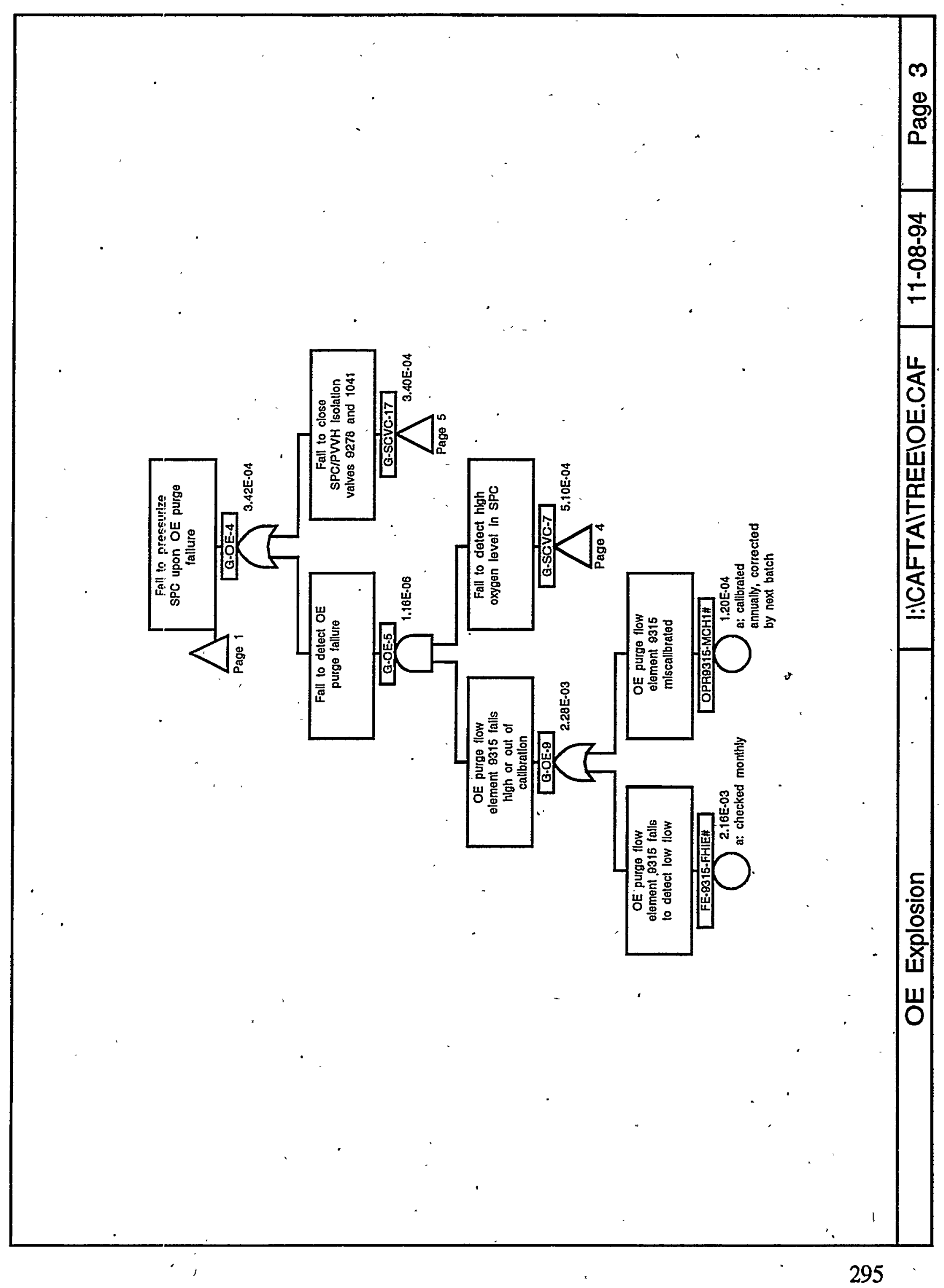




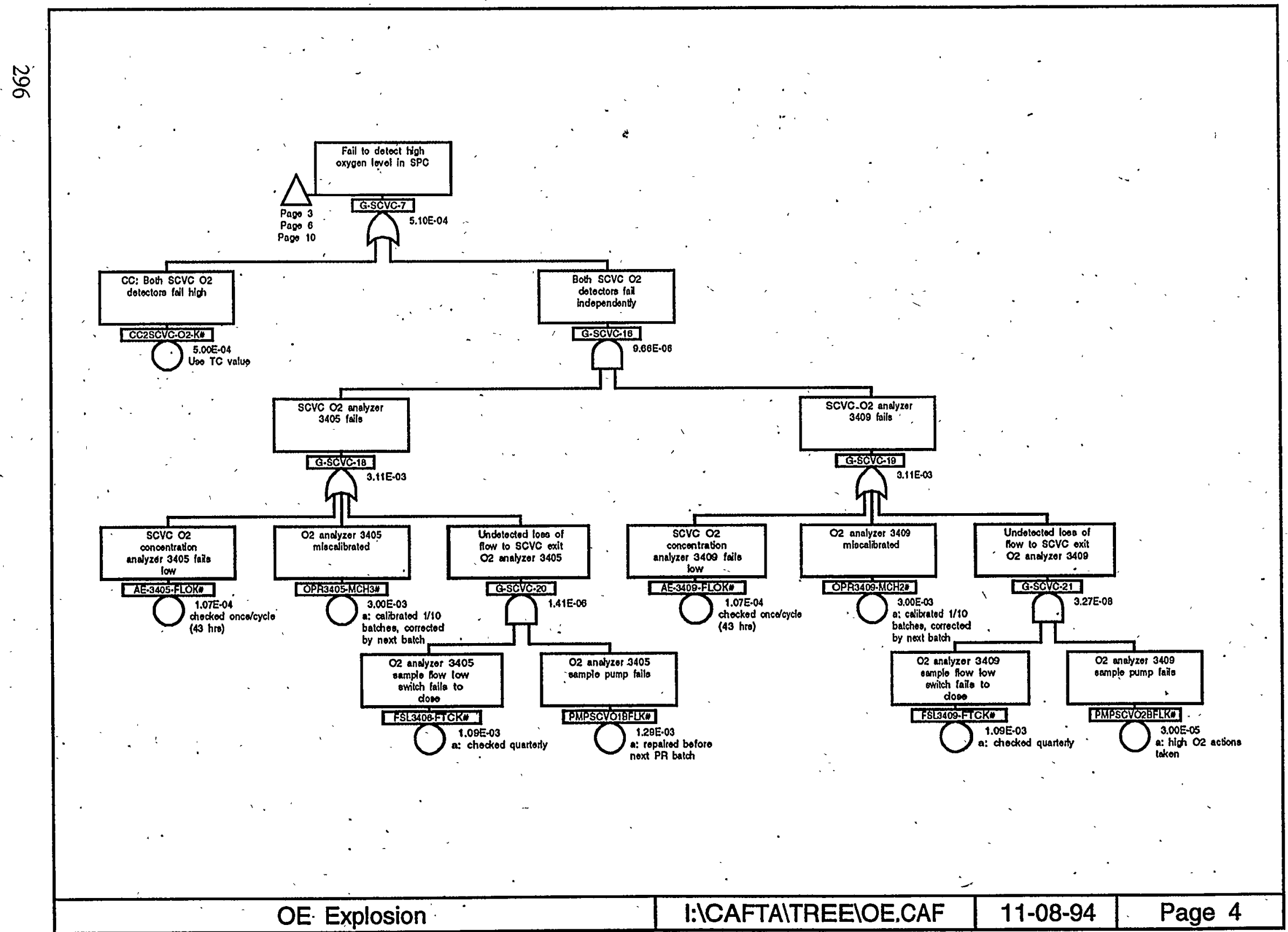


X-ESR-S-00001, REV. 0

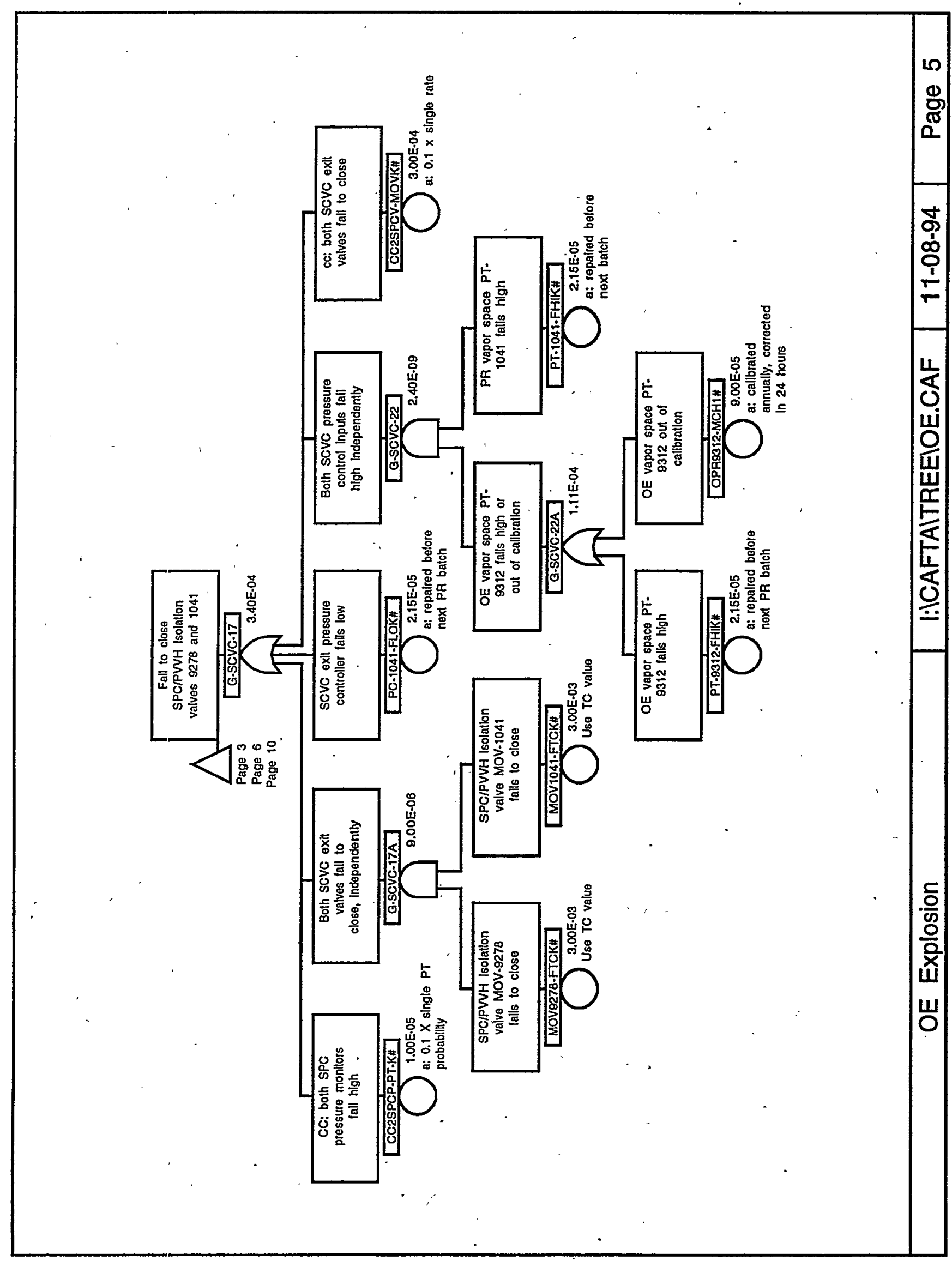




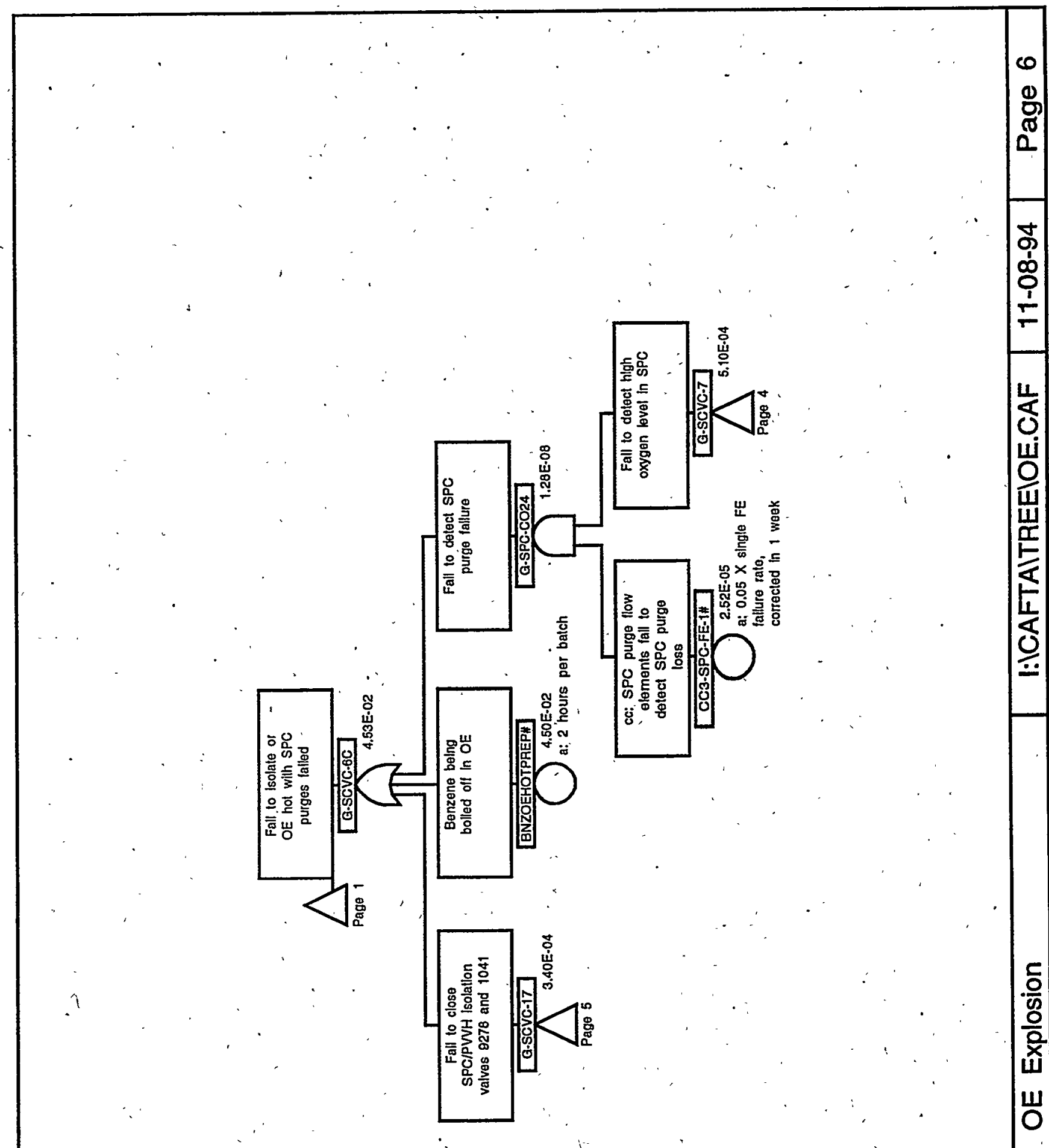




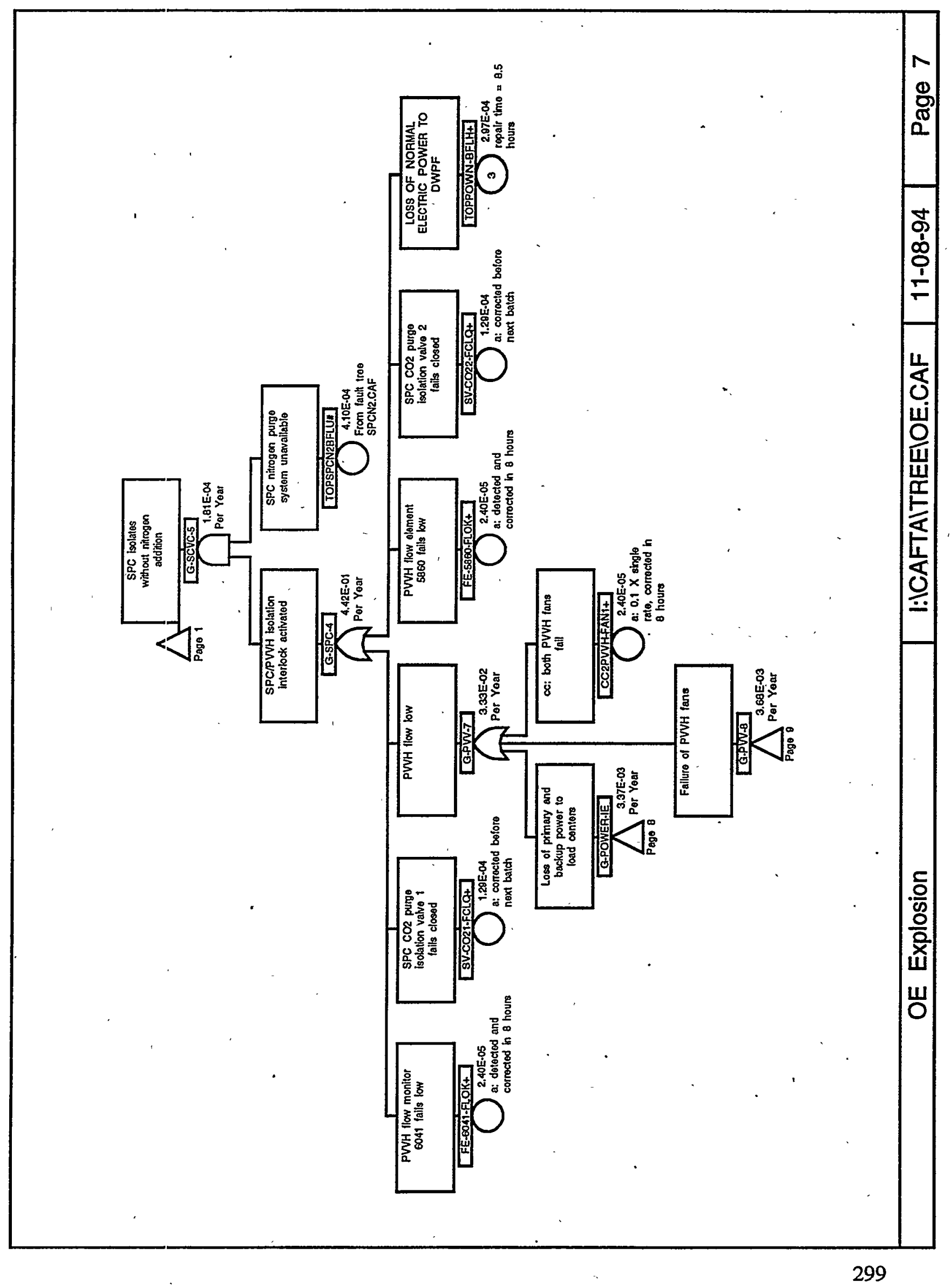


X-ESR-S-00001, REV. 0

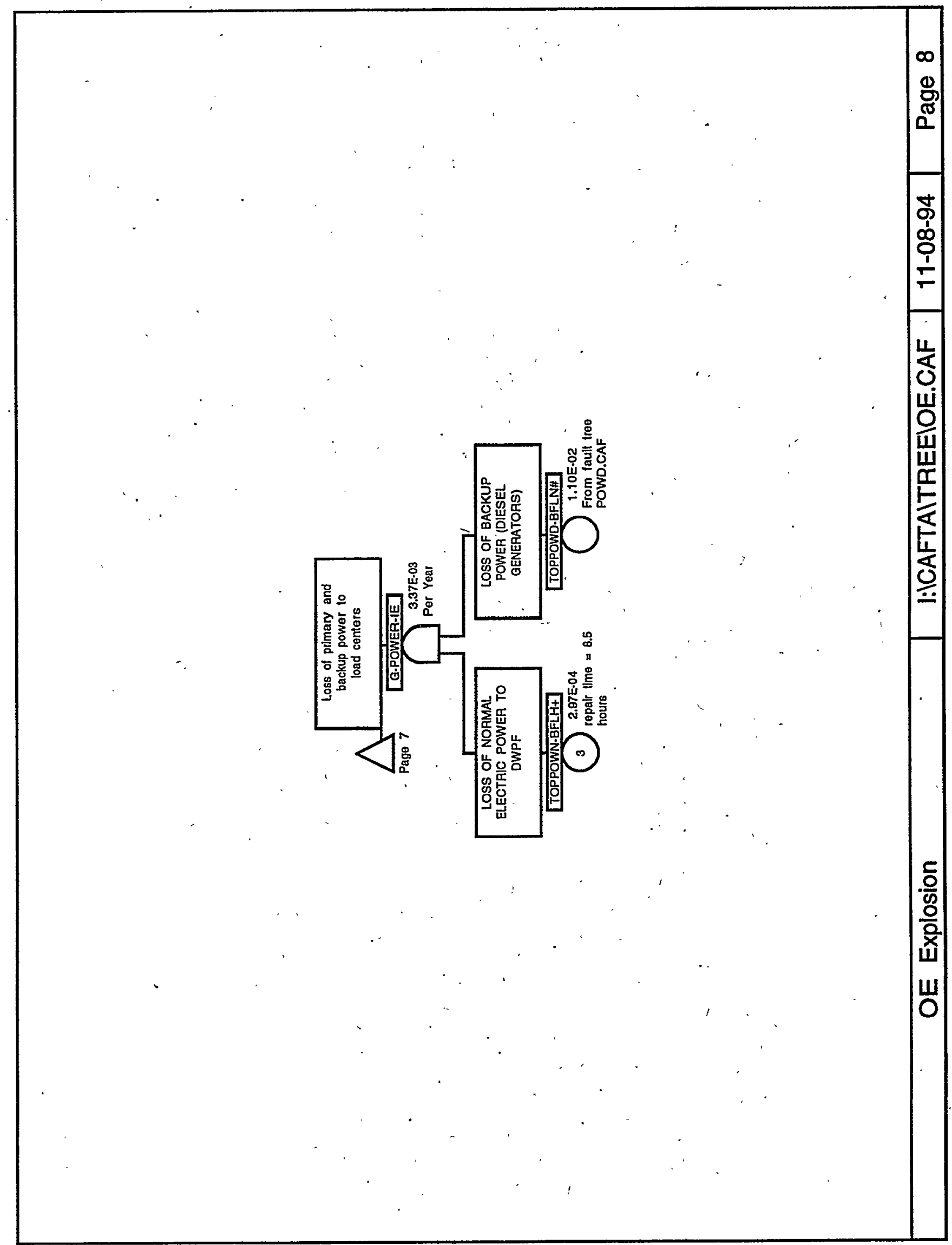




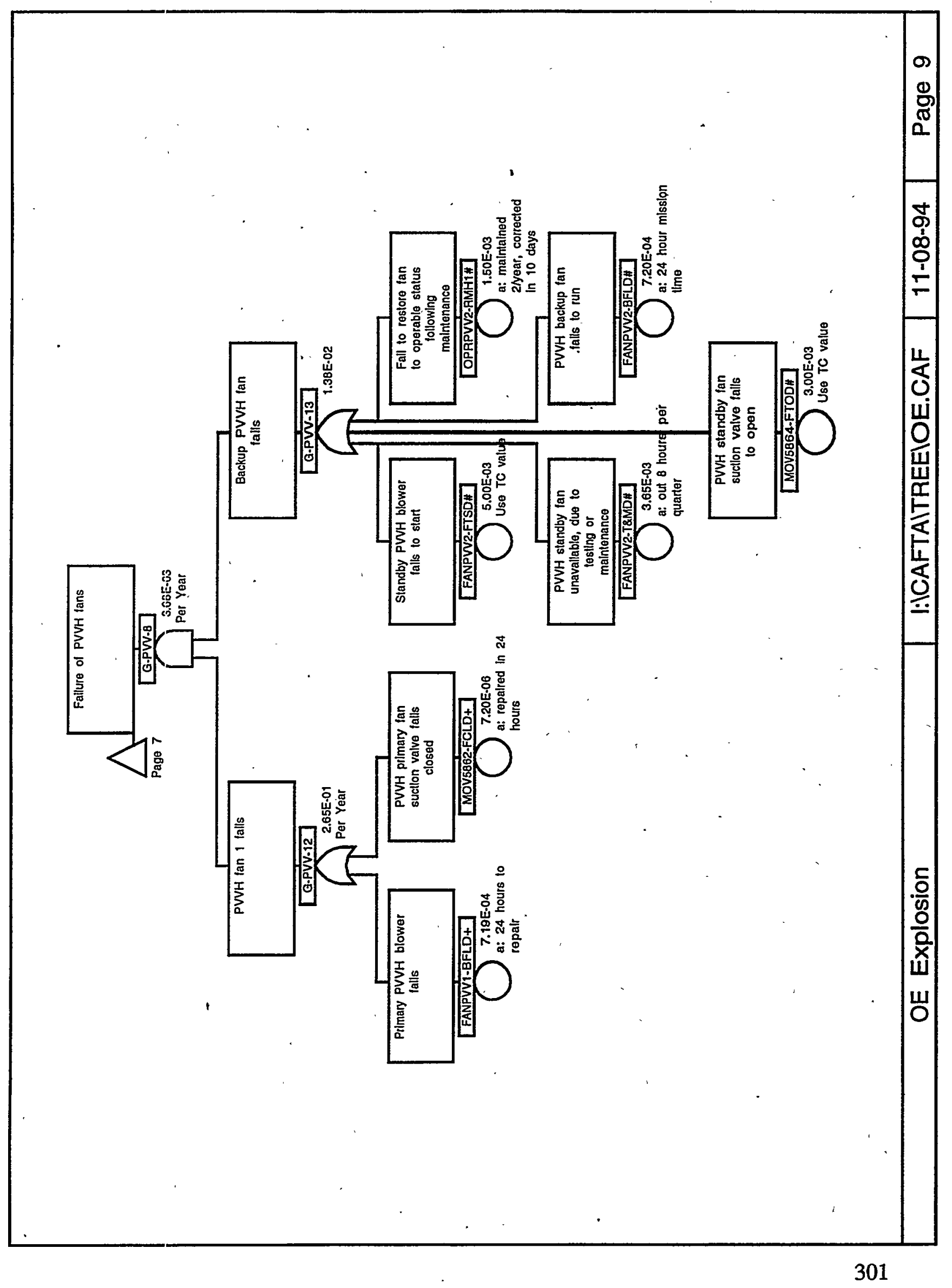


X-ESR-S-00001, REV. 0

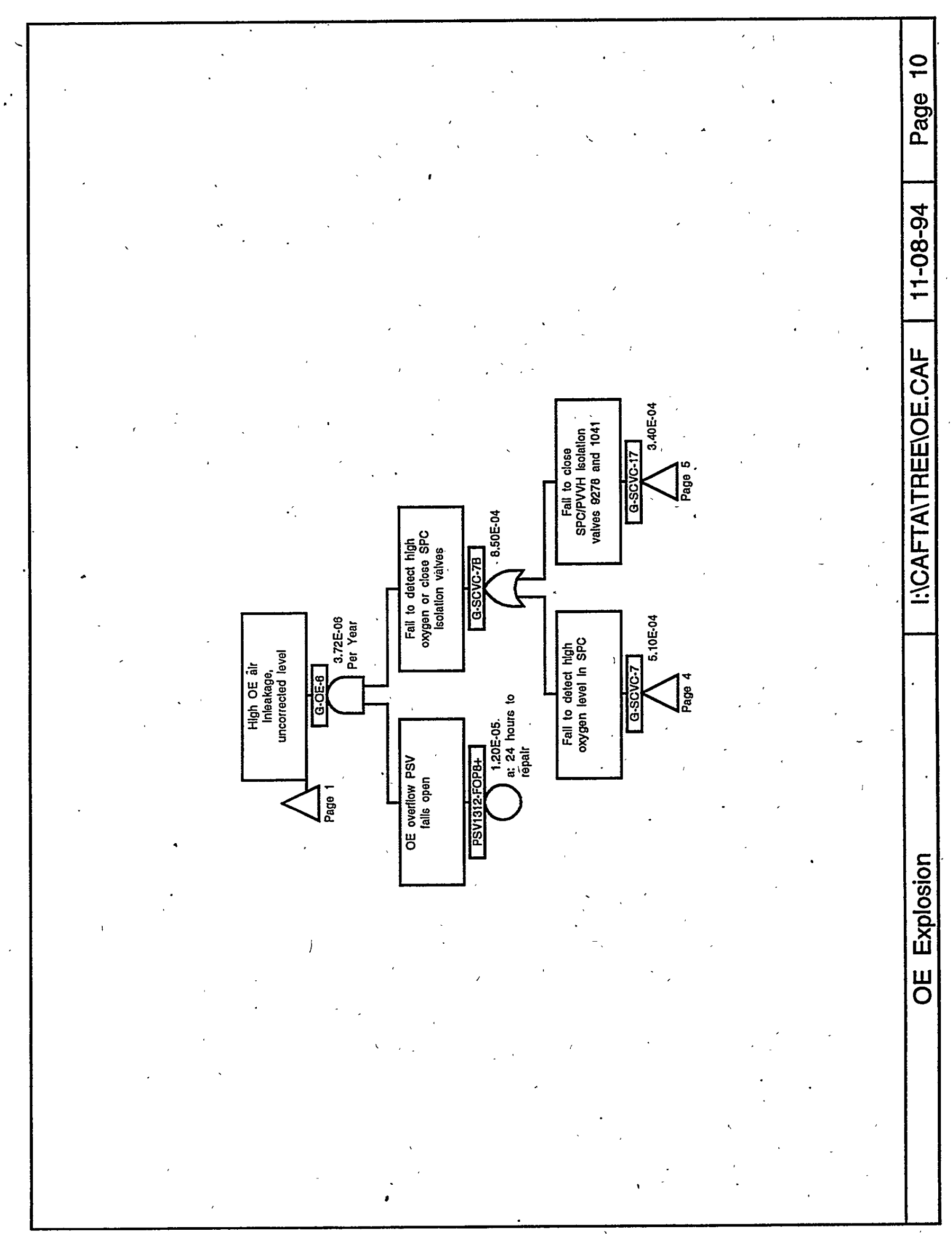




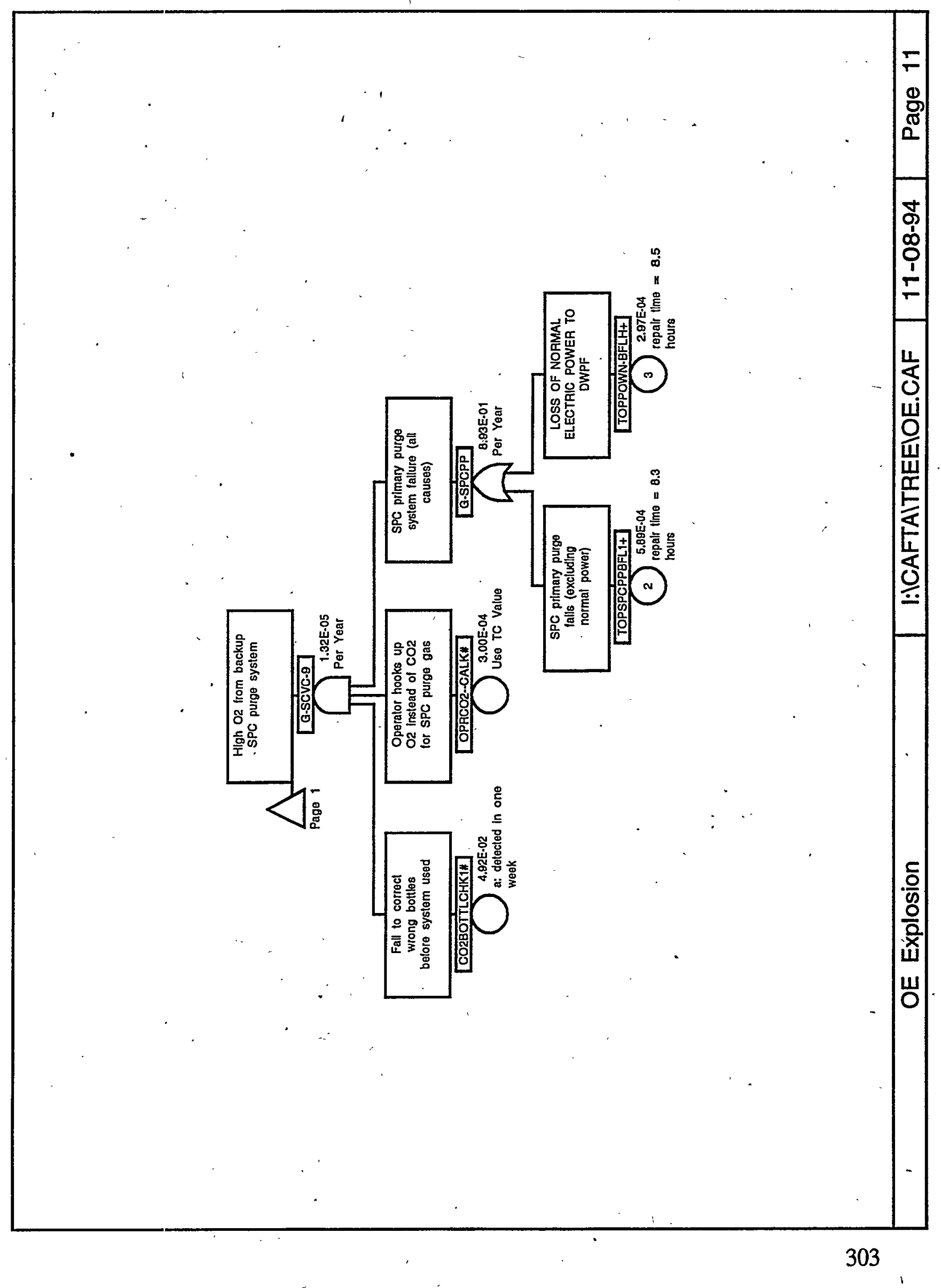




\section{X-ESR-S-00001, REV. 0}

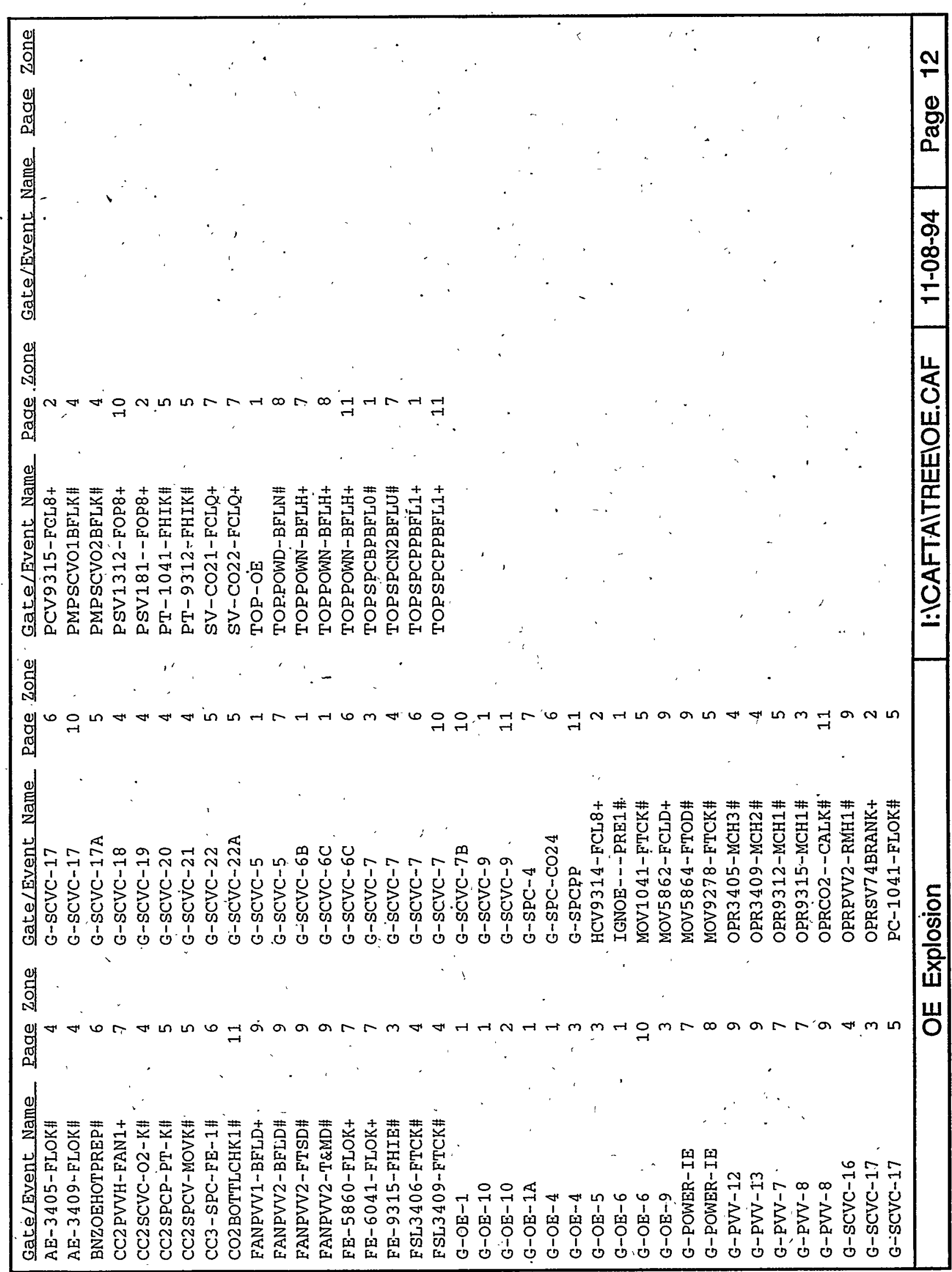


Cutsets for I: ICAFTAICUT\OE.CSR $\quad 11 / 08 / 94 \quad 12: 30$ AM

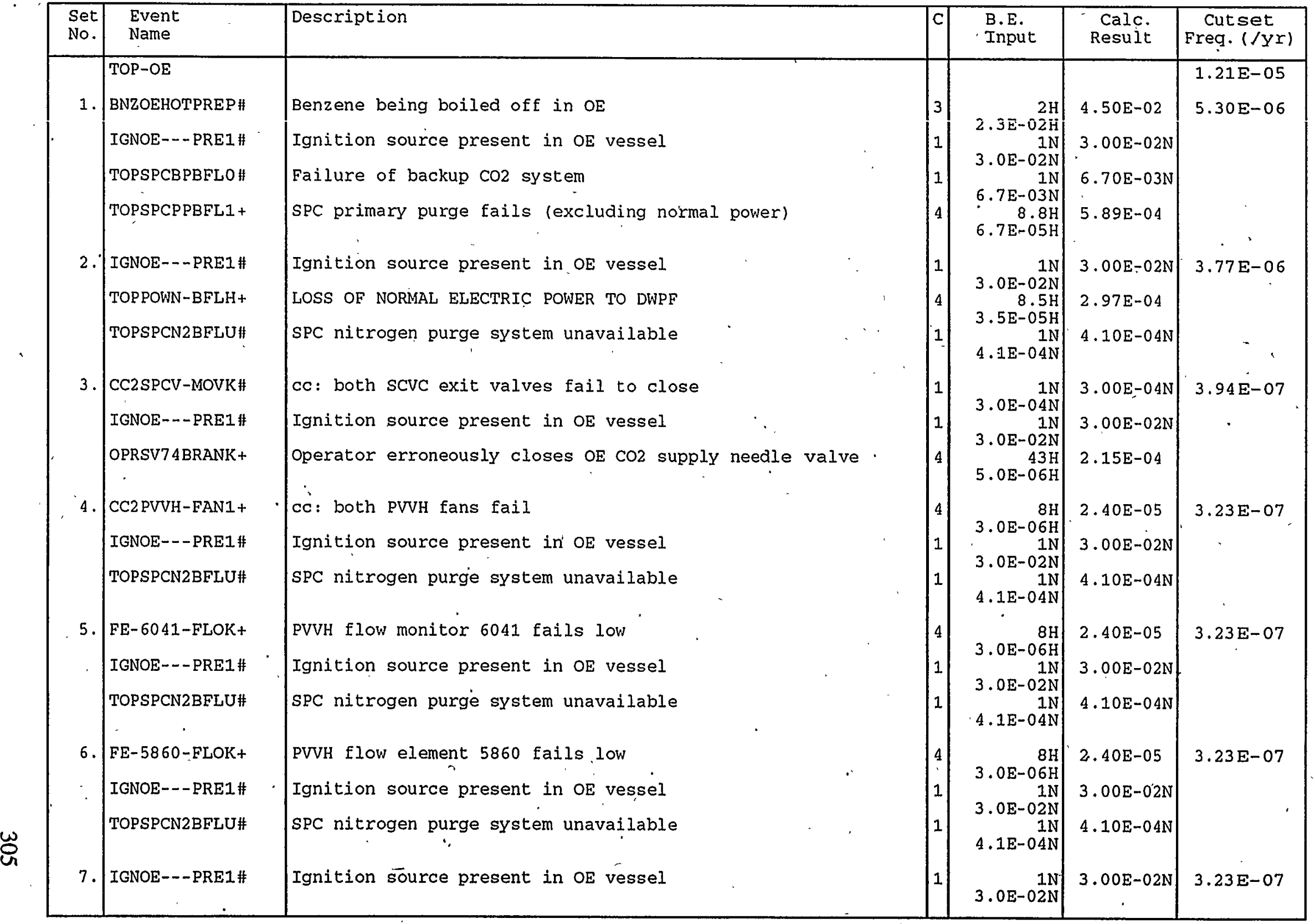


Cutsets for I: ICAFTA ICUTIOE.CSR - 11/08/94 12:30 AM (CONT.)

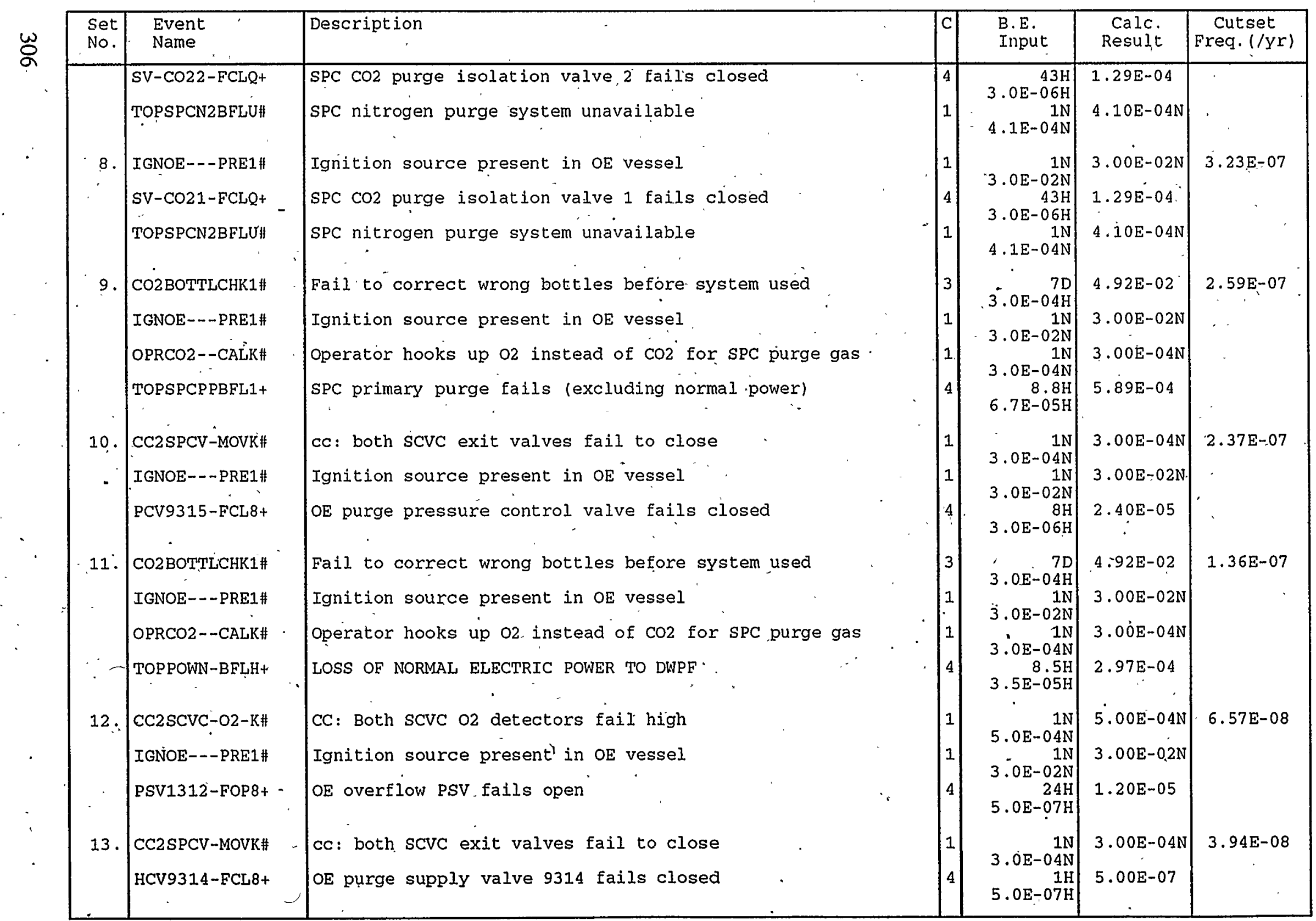


Cutsets for I: ICAFTAICUTIOE.CSR

11/08/94 12:30 AM (CONT.)

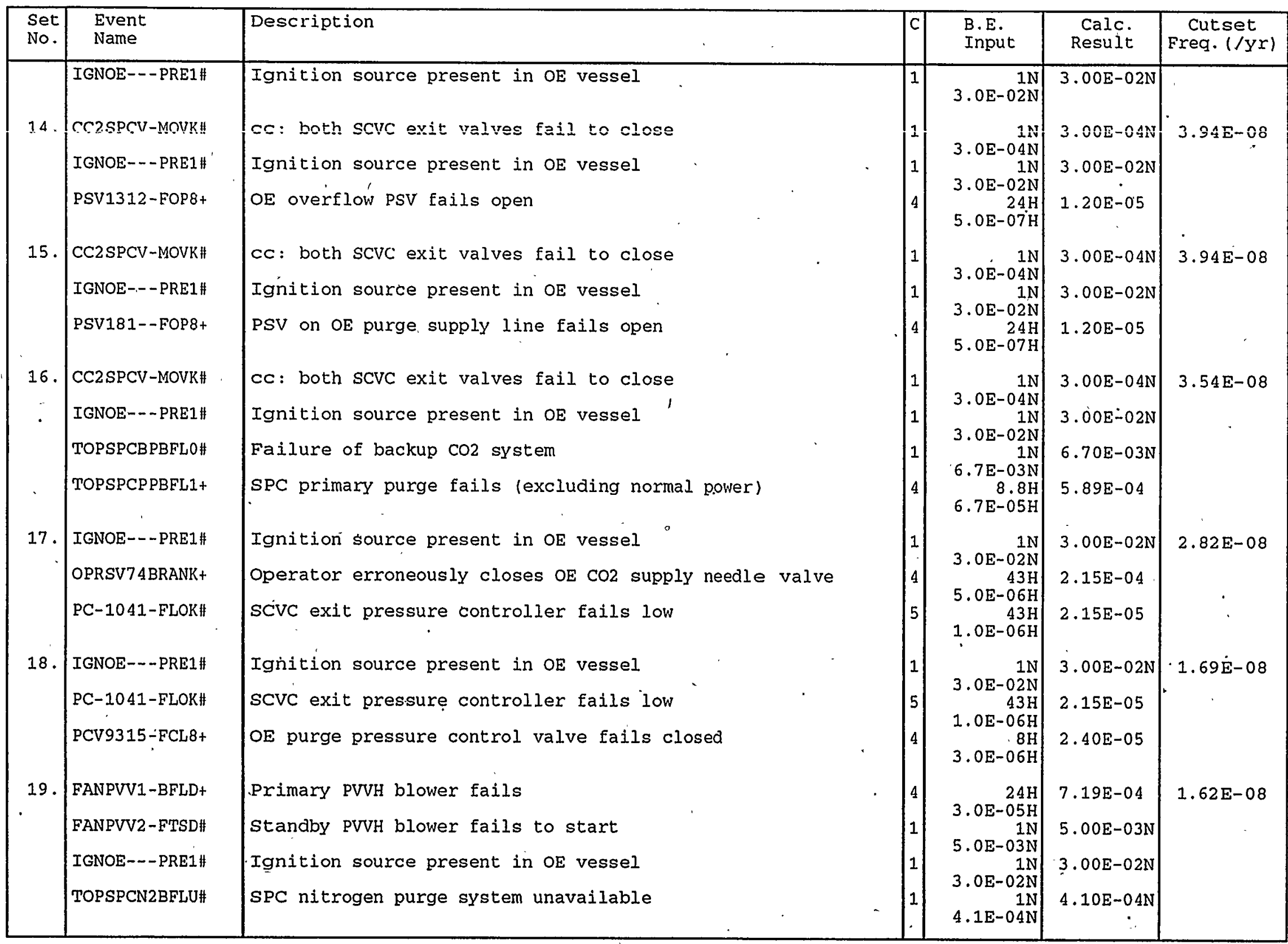




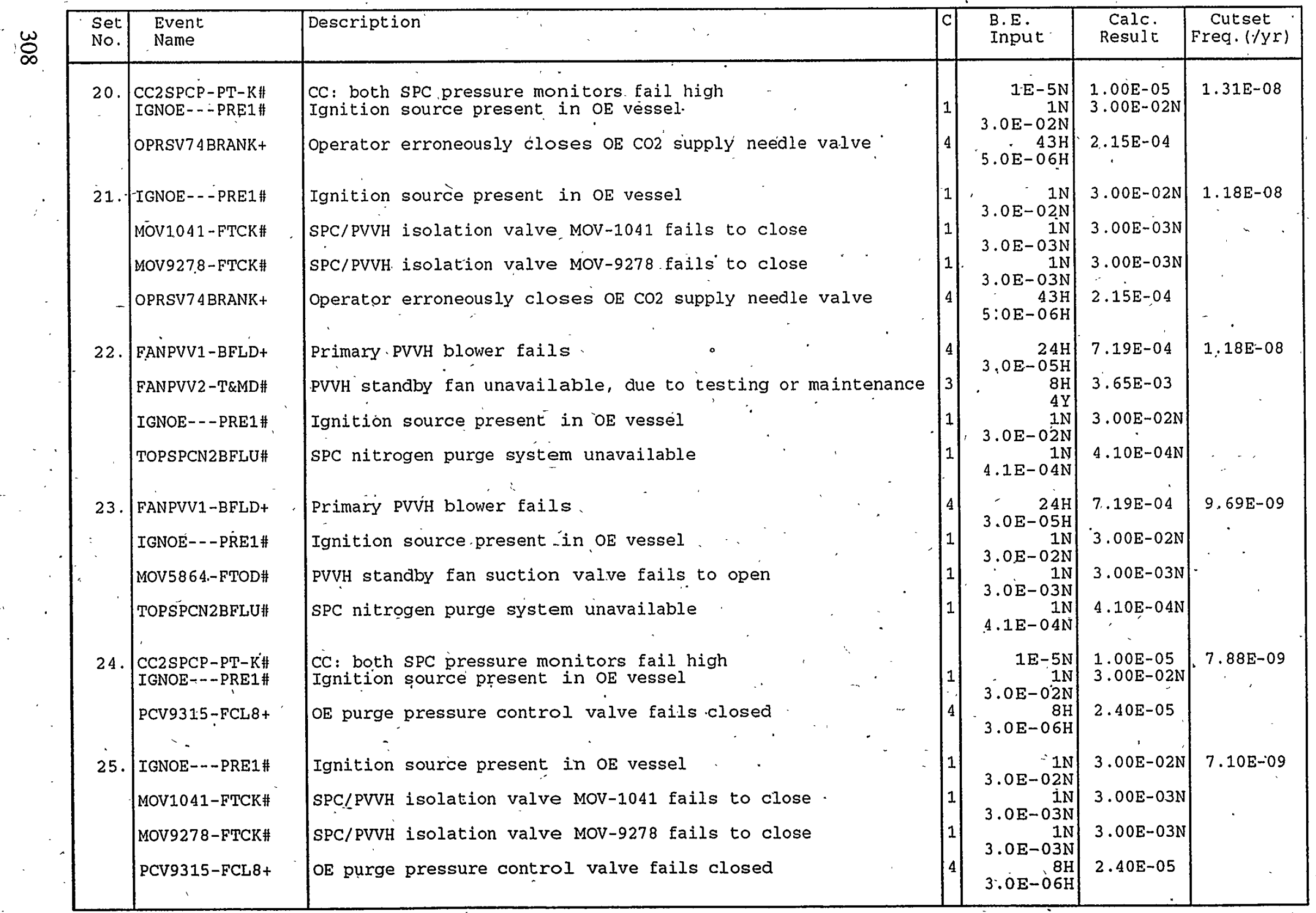


Explosion in the $\mathrm{OE}$

Basic EVent Name
PSV1312-FOP8+-I
TOPPOWN-BFLH+I
CC2PVVH-JAAN1+-I
FE-6041-FLOK+-I
FE-5860-FLOK+-I
SV-CO21-FILQ+-I
SV-CO22-FILQ+-I
OPRSV74BIRANK+I
PCV9315-FCL8+-I
PSV181-FCP8+-I
HCV9314-FCL8+-I
TOPSPCPPI3FL1+-I
TOPSPCN2]3FLU\#
CC2SPCP-PT-K\#
PC-1041-FLOK\#
CC2SPCV-MOVK\#
MOV5862-FCLD+-I
FANPVV1-13FLD+-I
OPRCO2--CALK\#
TOPSPCBPI3FL0\#
IGNOE--PFE1\#
CC2SCVC-O2-K\#
BNZOEHO1.PREP\#
MOV1041-FTCK\#
MOV9278-FTCK\#
CO2BOTTLCHK1\#
FANPVV2-HFLD\#
OPRPVV2-FMH1\#
MOV5864-FTOD\#
FANPVV2-I[\&MD\#
FANPVV2-FTSD\#
FE-9315-FHIE\#
OPR9315-MCH1\#
OPR3409-MCH2\#
OPR3405-MCH3\#
AE-3405-FLOK\#
AE-3409-FLOK\#
PT-1041-FHKK\#

Top Event Frequency: 1.21E-05/YR

\section{Risk Achievement Worth .} Description

OE overflow PSV fails open LOSS OF NORMAL ELECTRIC POWER TO DWPF cc: both PVVH fans fail PVVH flow monitor 6041 fails low PVVH flow element 5860 fails low SPC CO2 purge isolation valve 1 fails closed SPC CO2 purge isolation valve 2 fails closed Operator erroneously closes $\mathrm{OE} \mathrm{CO} 2$ supply needle valve $O E$ purge pressure control valve fails closed PSV on OE purge supply line fails open OE purge supply valve 9314 fails closed SPC primary purge fails (excluding normal power) SPC nitrogen purge system unavailable CC: both SPC pressure monitors fail high SCVC exit pressure controller fails low oc: both SCVC exit valves fail to close PVVH primary fan suction valve fails closed Primary PVVH blower fails Operator hooks up $\mathrm{O} 2$ instead of $\mathrm{CO} 2$ for SPC purge gas * Failure of backup $\mathrm{CO} 2$ system

Ignition source present in $\mathrm{OE}$ vessel CC: Both SCVC O2 detectors fail high Benzene being boiled off in $\mathrm{OE}$ SPC/PVVH isolation valve MOV-1041 fails to close SPC/PVVH isolation valve MOV-9278 fails to close Fail to correct wrong bottles before system used PVVH backup fan fails to run Fail to restore fan to operable status following maintenance PVVH standby fan suction valve fails to open PVVH standby fan unavailable, due to T\&M Standby PVVH blower fails to start OE purge flow element 9315 fails to detect low flow OE purge flow element 9315 miscalibrated O2 analyzer 3409 miscalibrated O2 analyzer 3405 miscalibrated SCVC O2 concentration analyzer 3405 fails low. SCVC O2 concentration analyzer 3409 fails low $P R$ vapor space $P T-1041$ fails high

\begin{tabular}{ll} 
Prob/Freq & AchW \\
\hline $5.00 \mathrm{E}-07 / \mathrm{H}$ & $1.85 \mathrm{E}+04$ \\
$3.50 \mathrm{E}-05 / \mathrm{H}$ & $9.24 \mathrm{E}+03$ \\
$3.00 \mathrm{E}-06 / \mathrm{H}$ & $8.92 \mathrm{E}+03$ \\
$3.00 \mathrm{E}-06 / \mathrm{H}$ & $8.92 \mathrm{E}+03$ \\
$3.00 \mathrm{E}-06 / \mathrm{H}$ & $8.92 \mathrm{E}+03$ \\
$3.00 \mathrm{E}-06 / \mathrm{H}$ & $8.92 \mathrm{E}+03$ \\
$3.00 \mathrm{E}-06 / \mathrm{H}$ & $8.92 \mathrm{E}+03$ \\
$5.00 \mathrm{E}-06 / \mathrm{H}$ & $7.40 \mathrm{E}+03$ \\
$3.00 \mathrm{E}-06 / \mathrm{H}$ & $7.40 \mathrm{E}+03$ \\
$5.00 \mathrm{E}-07 / \mathrm{H}$ & $7.40 \mathrm{E}+03$ \\
$5.00 \mathrm{E}-07 / \mathrm{H}$ & $7.40 \mathrm{E}+03$ \\
$6.69 \mathrm{E}-05 / \mathrm{H}$ & $6.92 \mathrm{E}+03$ \\
$4.10 \mathrm{E}-04$ & $1.10 \mathrm{E}+03$ \\
$8.40 \mathrm{E}-06$ & $2.17 \mathrm{E}+02$ \\
$2.15 \mathrm{E}-05$ & $2.17 \mathrm{E}+02$ \\
$3.00 \mathrm{E}-04$ & $2.17 \mathrm{E}+02$ \\
$3.00 \mathrm{E}-07 / \mathrm{H}$ & $1.25 \mathrm{E}+02$ \\
$3.00 \mathrm{E}-05 / \mathrm{H}$ & $1.25 \mathrm{E}+02$ \\
$3.00 \mathrm{E}-04$ & $1.10 \mathrm{E}+02$ \\
$6.70 \mathrm{E}-03$ & $6.65 \mathrm{E}+01$ \\
$3.00 \mathrm{E}-02$ & $3.33 \mathrm{E}+01$ \\
$5.00 \mathrm{E}-04$ & $1.23 \mathrm{E}+01$ \\
$4.50 \mathrm{E}-02$ & $1.03 \mathrm{E}+01$ \\
$3.00 \mathrm{E}-03$ & $1.65 \mathrm{E}+00$ \\
$3.00 \mathrm{E}-03$ & $1.65 \mathrm{E}+00$ \\
$4.92 \mathrm{E}-02$ & $1.63 \mathrm{E}+00$ \\
$7.20 \mathrm{E}-04$ & $1.27 \mathrm{E}+00$ \\
$1.50 \mathrm{E}-03$ & $1.27 \mathrm{E}+00$ \\
$3.00 \mathrm{E}-03$ & $1.27 \mathrm{E}+00$ \\
$3.65 \mathrm{E}-03$ & $1.27 \mathrm{E}+00$ \\
$5.00 \mathrm{E}-03$ & $1.27 \mathrm{E}+00$ \\
$2.16 \mathrm{E}-03$ & $1.10 \mathrm{E}+00$ \\
$1.20 \mathrm{E}-04$ & $1.09 \mathrm{E}+00$ \\
$3.00 \mathrm{E}-03$ & $1.03 \mathrm{E}+00$ \\
$3.00 \mathrm{E}-03$ & $1.03 \mathrm{E}+00$ \\
$1.07 \mathrm{E}-04$ & $1.03 \mathrm{E}+00$ \\
$1.07 \mathrm{E}-04$ & $1.03 \mathrm{E}+00$ \\
$2.15 \mathrm{E}-05$ & $1.02 \mathrm{E}+00$ \\
&
\end{tabular}

Note: Events that make less than $1 \%$ difference to the importance factor are not included. Modified top event frequency $=$ (top event frequency) $\mathrm{x}$ (risk achievement worth). 
Explosion in the OE Top Event Frequency: 1.21E-05/YR

Basic Event Name
*IGNOE-PRE1\#
TOPSPCPPBFL1+-I
TOPSPCN2BFLU\#
TOPSPCBPBFLO\#
BNZOEHOTPREP\#
TOPPOWN-BFLH+-I
CC2SPCV-MOVK\#
OPRSV74BRANK+-I
CO2BOTTLCHK1\#
OPRCO2-CALK\#
SV-CO22-FCLQ+-I
SV-CO21-FCLQ+I
FE-6041-FLOK+-I
FE-5860-FLOK+-I
CC2PVVH-FAN1+-I
PCV9315-FCL8+-I

Risk Reduction Worth

\section{Description}

Ignition source present in $O E$ vessel SPC primary purge fails (excluding normal power) SPC nitrogen purge system unavailable

Failure of backup $\mathrm{CO} 2$ system

Benzene being boiled off in $\mathrm{OE}$ LOSS OF NORMAL EIECTRIC POWER TO DWPF cc: both SCVC exit valves fail to close Operator erroneously closes $\mathrm{OE} \mathrm{CO} 2$ supply needle valve Fail to correct wrong bottles before system used Operator hooks up O2 instead of CO2 for SPC purge gas SPC $\mathrm{CO} 2$ purge isolation valve 2 fails closed SPC CO2 purge isolation valve 1 fails closed PVVH flow monitor 6041 fails low PVVH flow element 5860 fails low cc: both PVVH fans fail $\mathrm{OE}$ purge pressure control valve fails closed
Prob/Freg RedW

3.00E-02 0.00E+00

$6.69 \mathrm{E}-05 / \mathrm{H} \quad 1.86 \mathrm{E}+00$

4.10E-04

$1.82 \mathrm{E}+00$

$6.70 \mathrm{E}-03 \quad 1.79 \mathrm{E}+00$

$4.50 \mathrm{E}-02 \quad 1.78 \mathrm{E}+00$

$3.50 \mathrm{E}-05 / \mathrm{H} \quad 1.48 \mathrm{E}+00$

3.00E-04 1.07E+00

$5.00 \mathrm{E}-06 / \mathrm{H} \quad 1.04 \mathrm{E}+00$

4.92E-02 1.03E+00

$3.00 \mathrm{E}-04 \quad 1.03 \mathrm{E}+00$

$3.00 \mathrm{E}-06 / \mathrm{H} \quad 1.03 \mathrm{E}+00$

$3.00 \mathrm{E}-06 / \mathrm{H} \quad 1.03 \mathrm{E}+00$

$3.00 \mathrm{E}-06 / \mathrm{H} \quad 1.03 \mathrm{E}+00$

$3.00 \mathrm{E}-06 / \mathrm{H} \quad 1.03 \mathrm{E}+00$

$3.00 \mathrm{E}-06 / \mathrm{H} \quad 1.03 \mathrm{E}+00$

$3.00 \mathrm{E}-06 / \mathrm{H} \quad 1.02 \mathrm{E}+00$

* A Reduction Worth of $0.0 \mathrm{E}+00$ indicates event appears in every cutset could serve as a single event preventor for the accident.

Note: Events that make less than $1 \%$ difference to the importance factor are not included. Modified top event frequency = (top event frequency) / (risk: reduction worth). 


\section{OECT Explosion}


X-ESR-S-00001, REV. 0

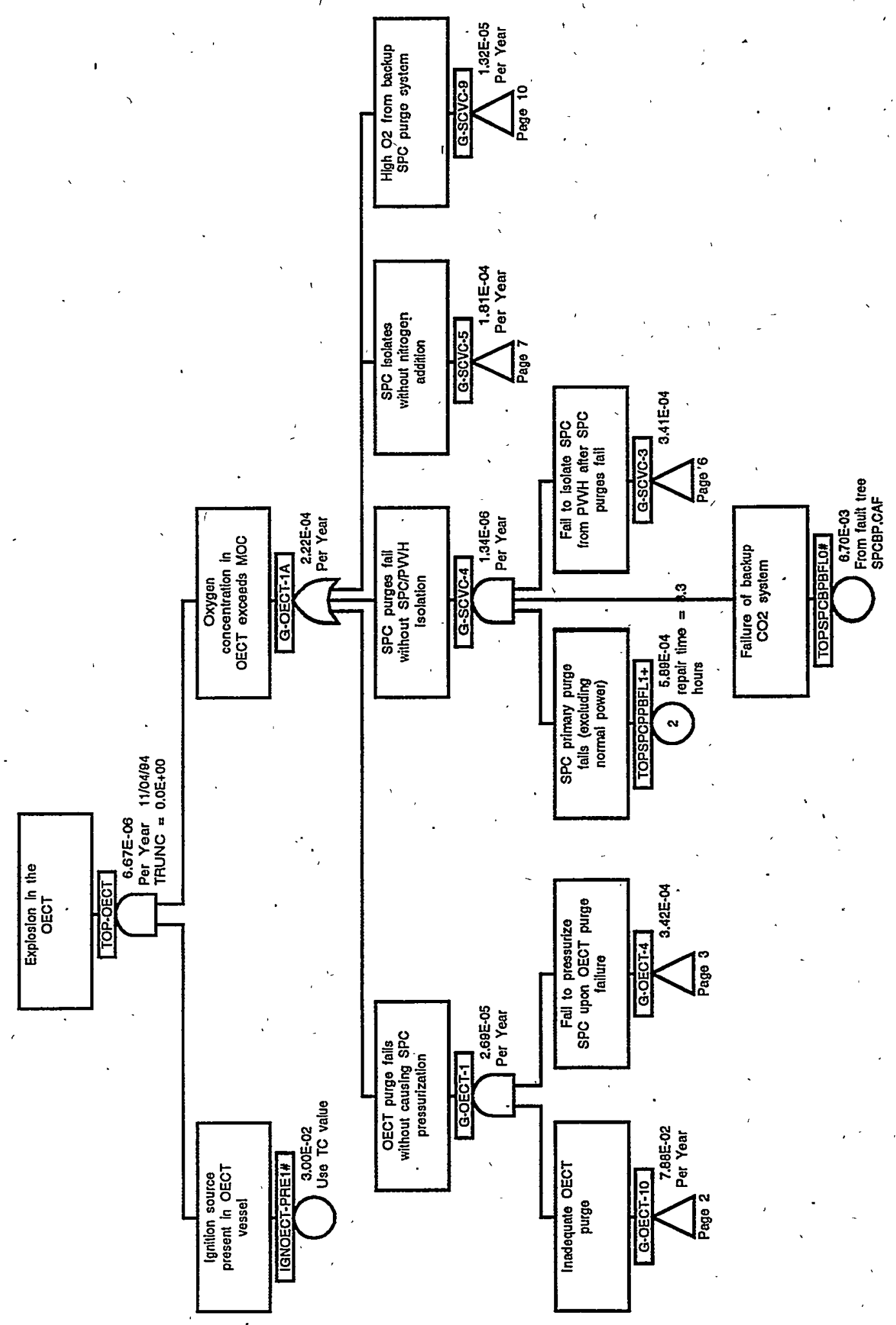

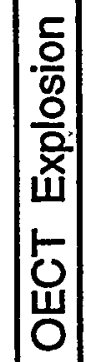




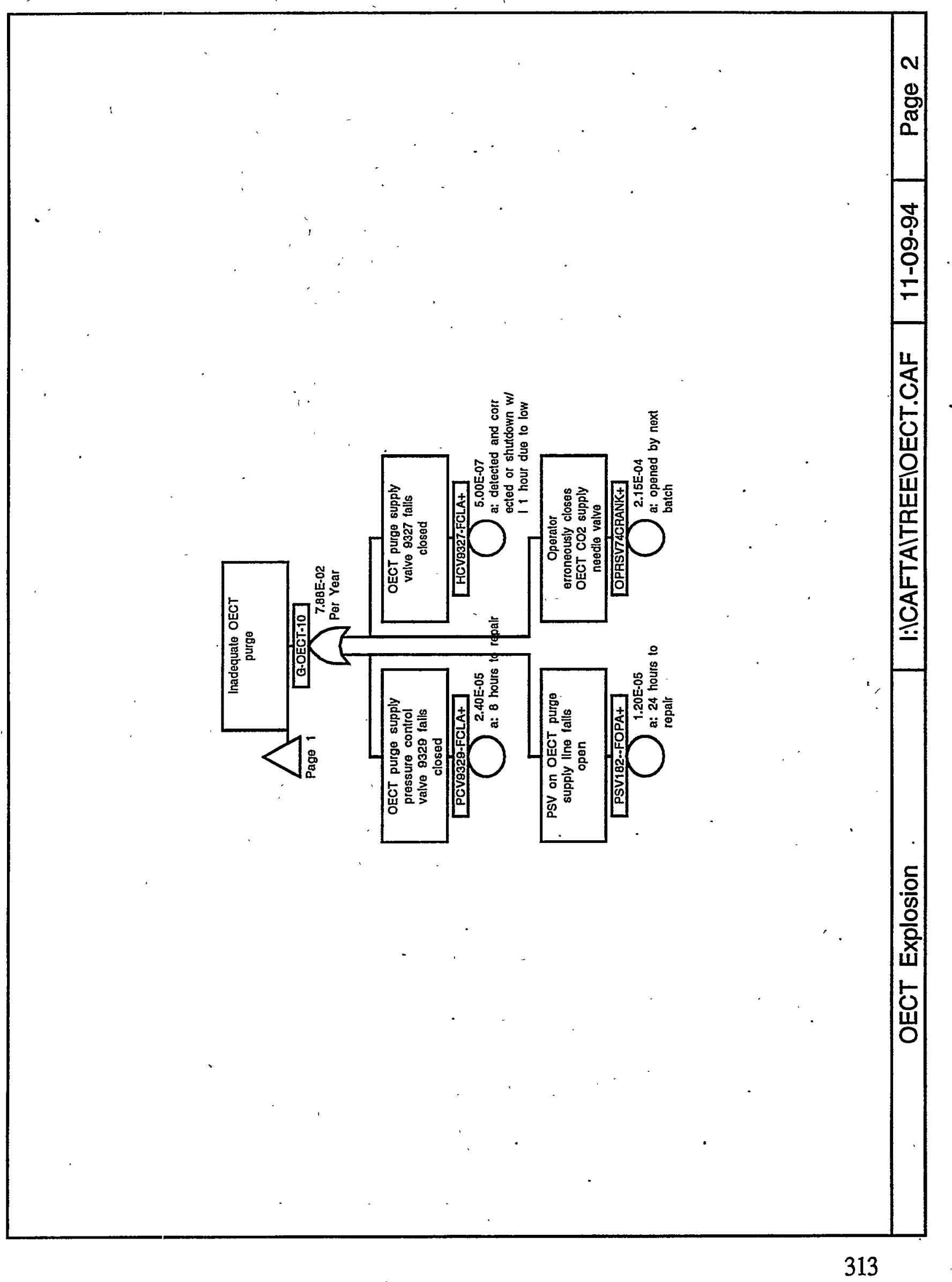


X-ESR-S-00001, REV. 0

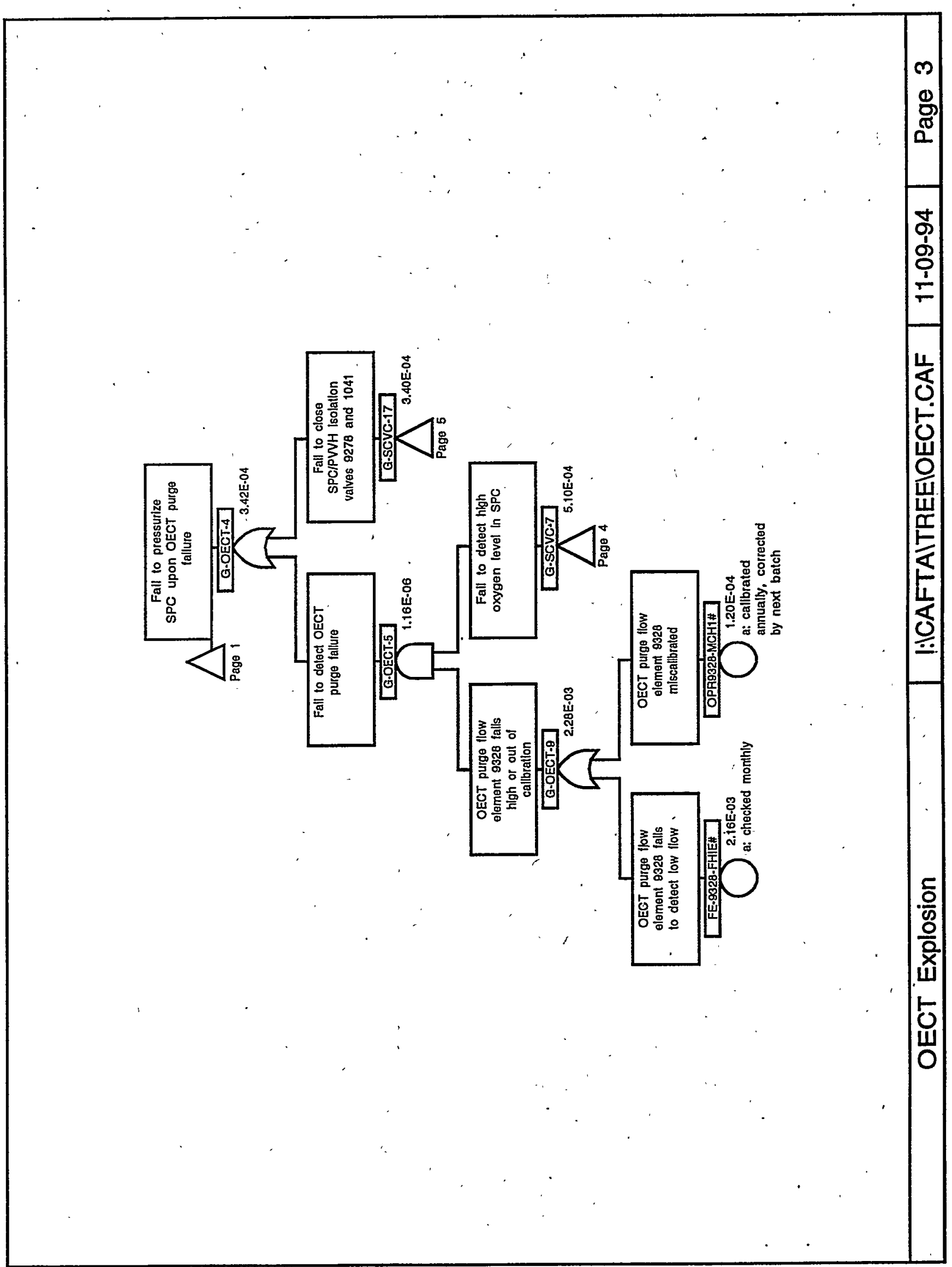




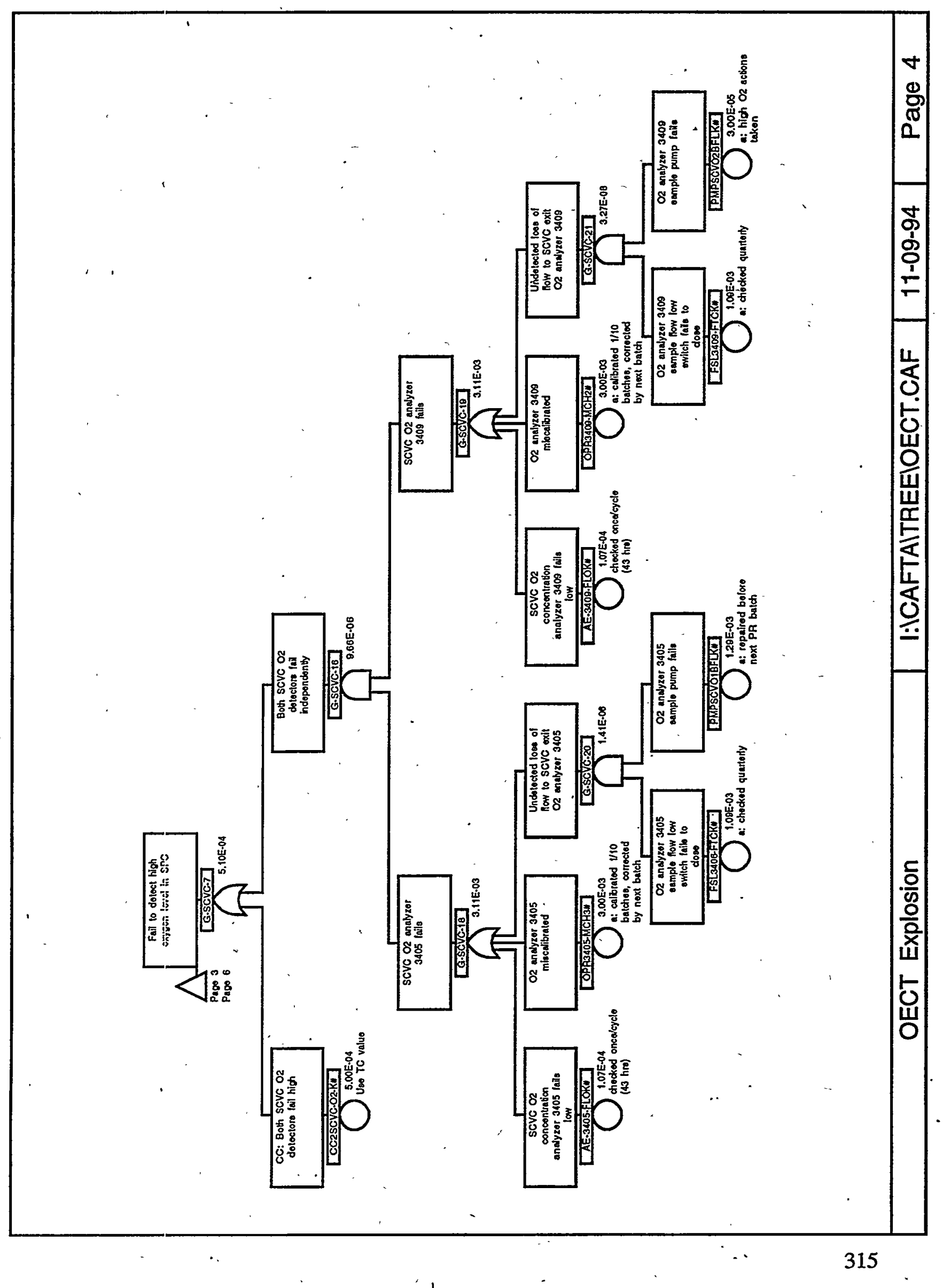




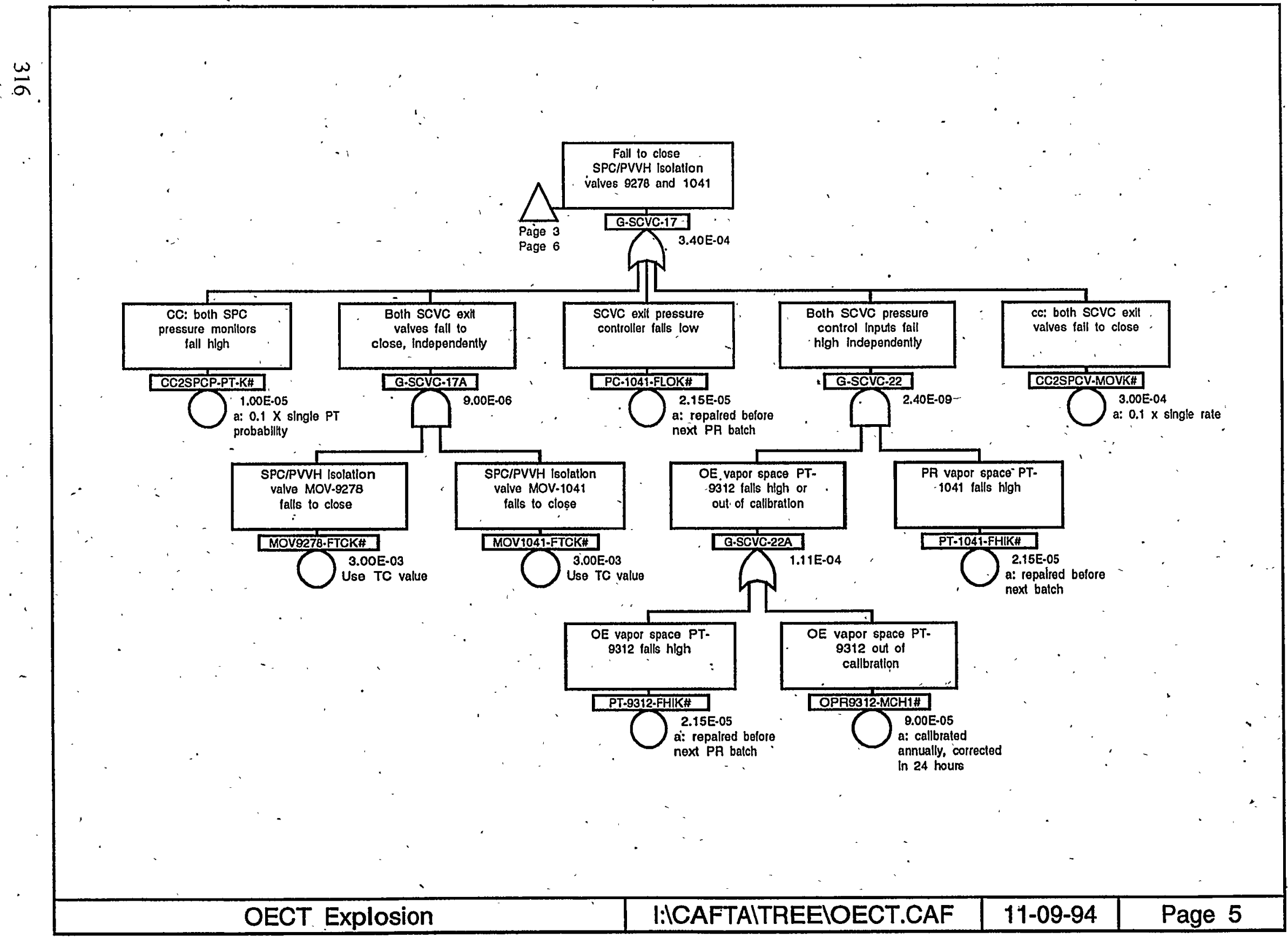




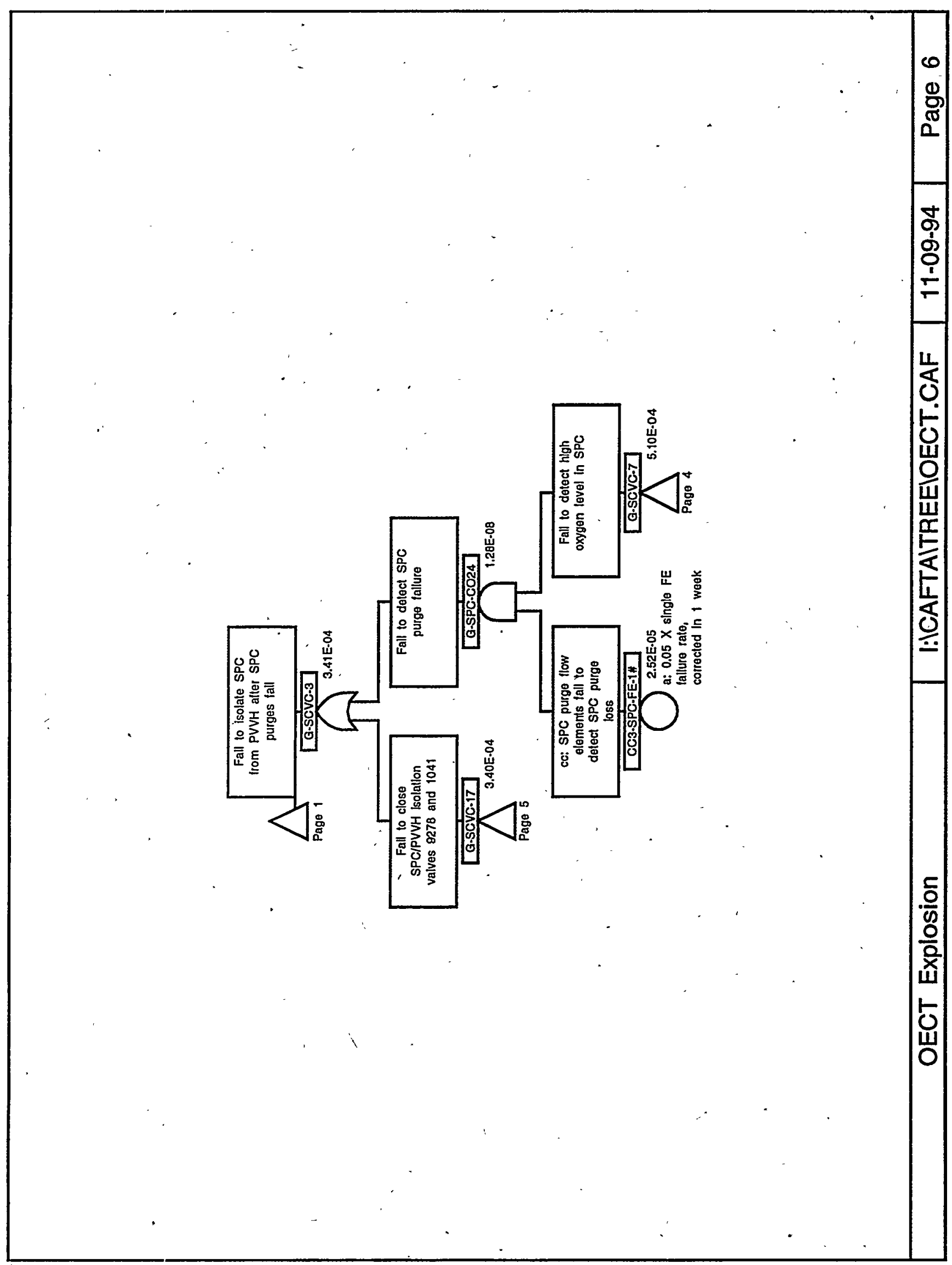


X-ESR-S-00001, REV. 0

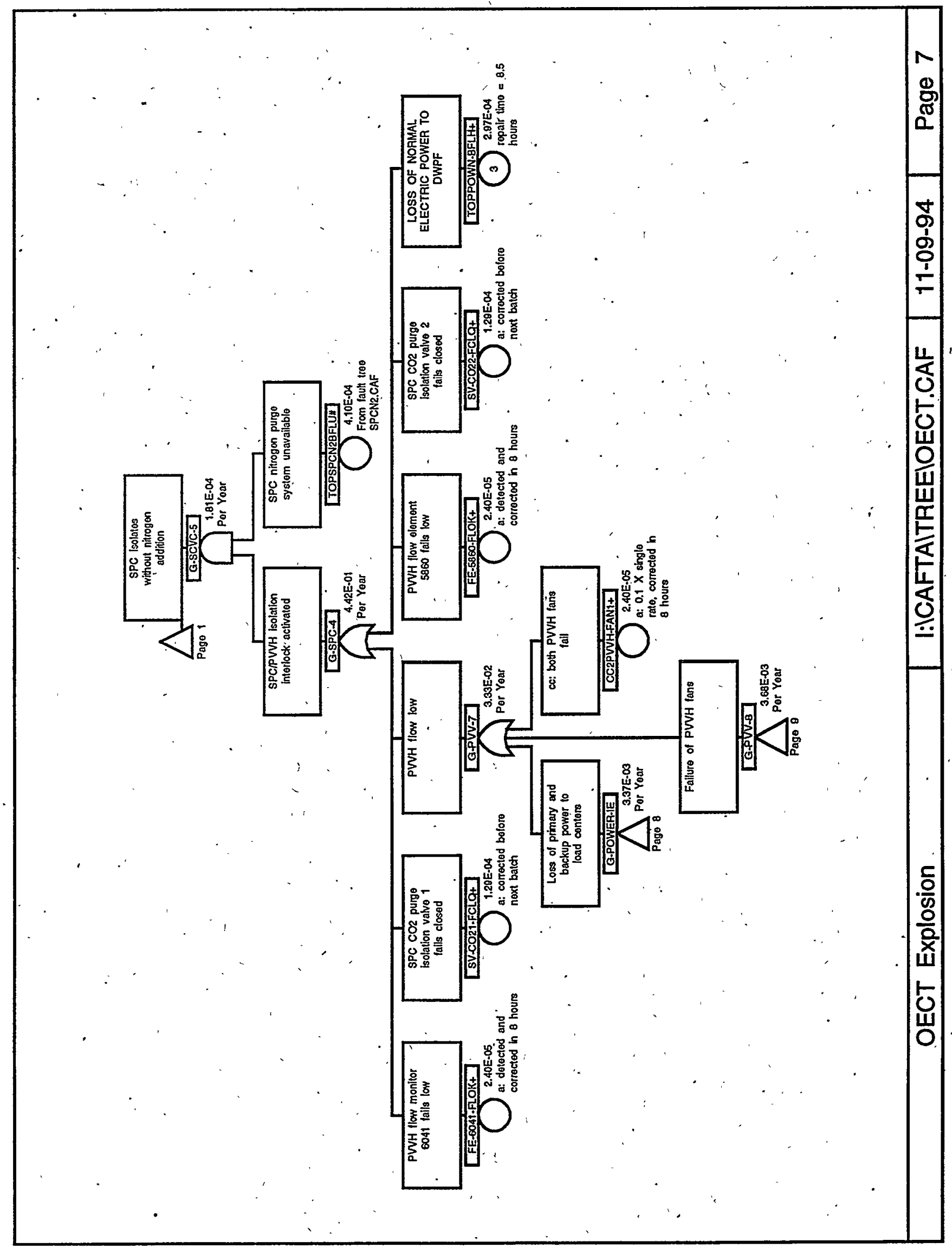




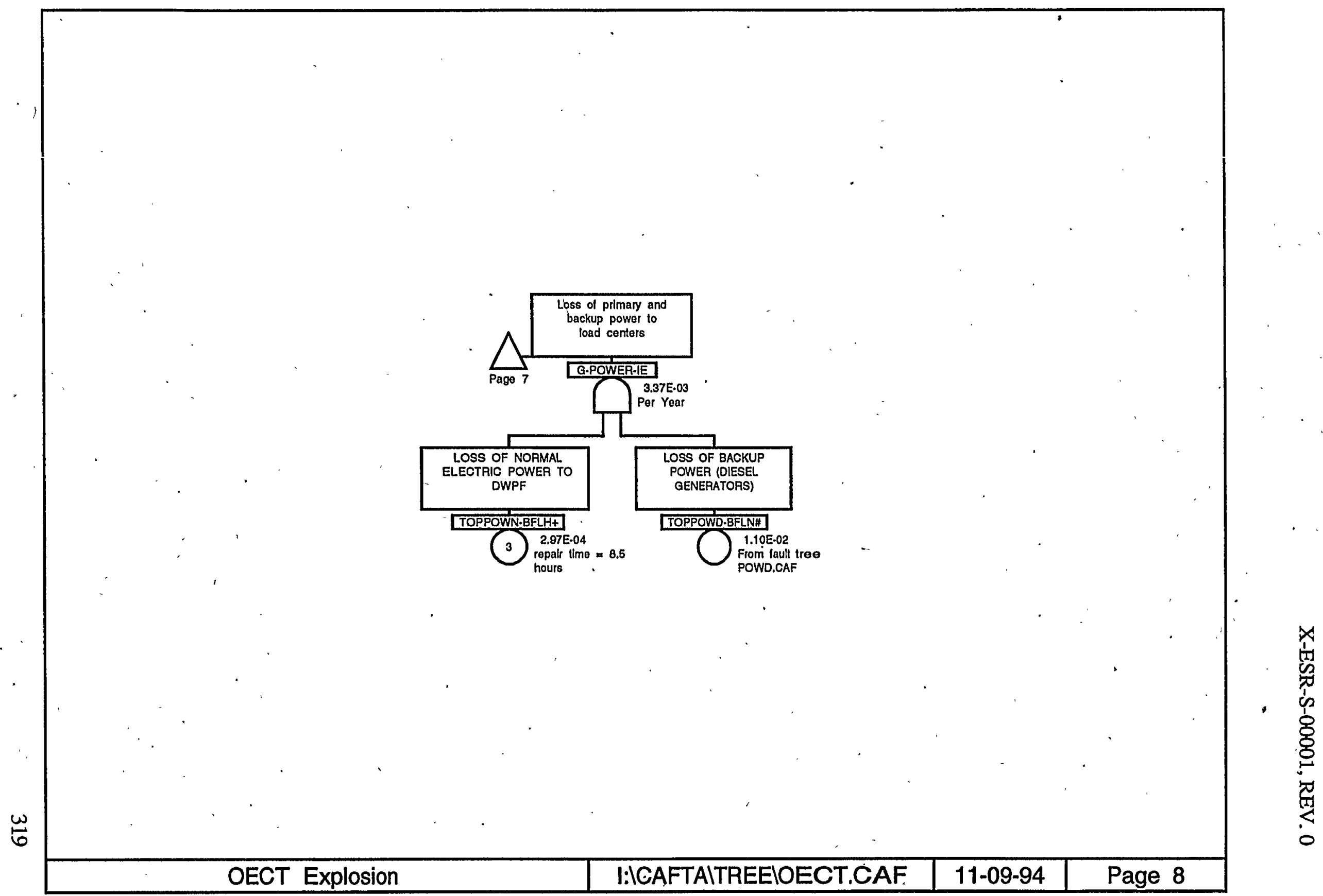


X-ESR-S-00001, REV. 0

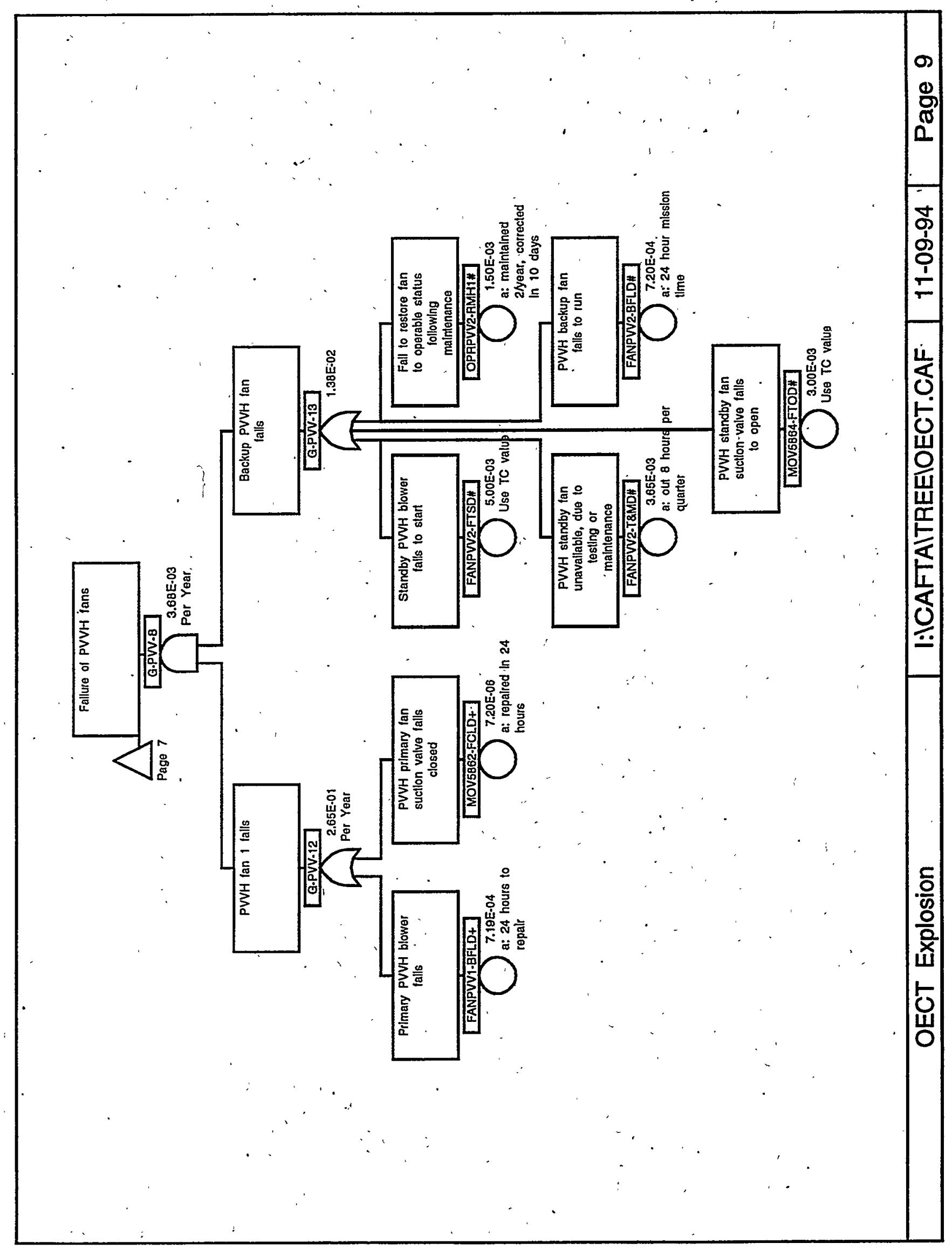




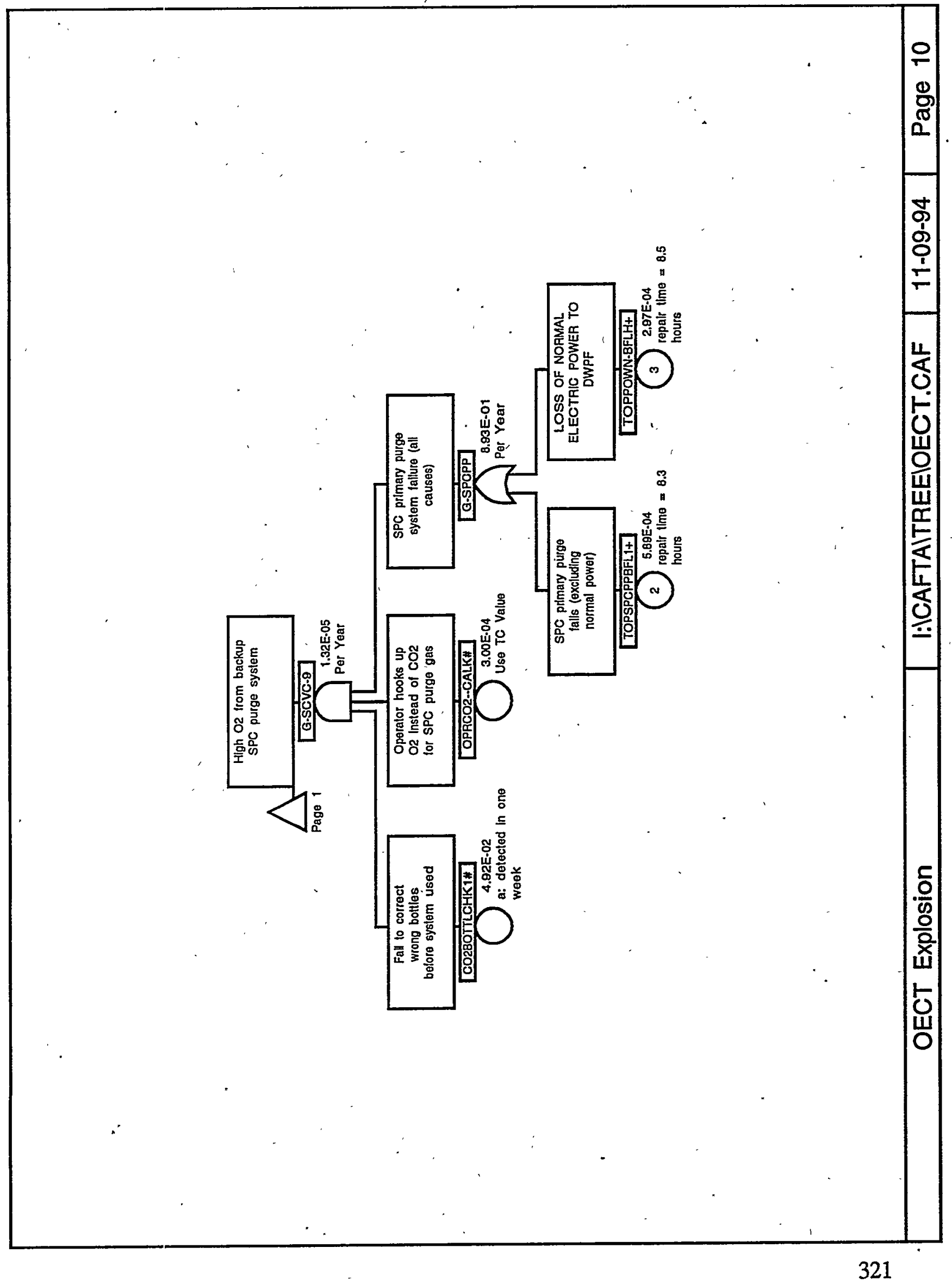




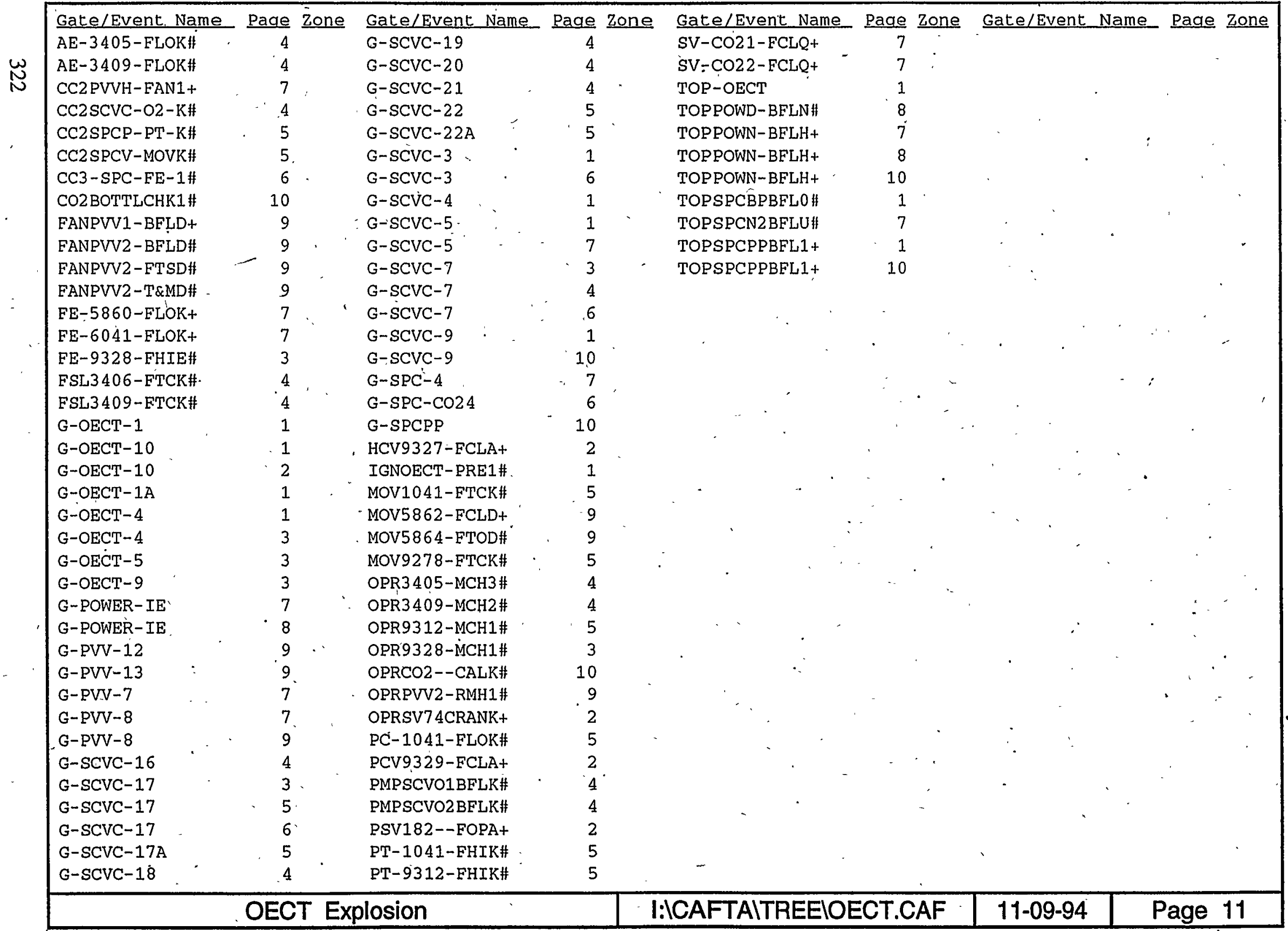




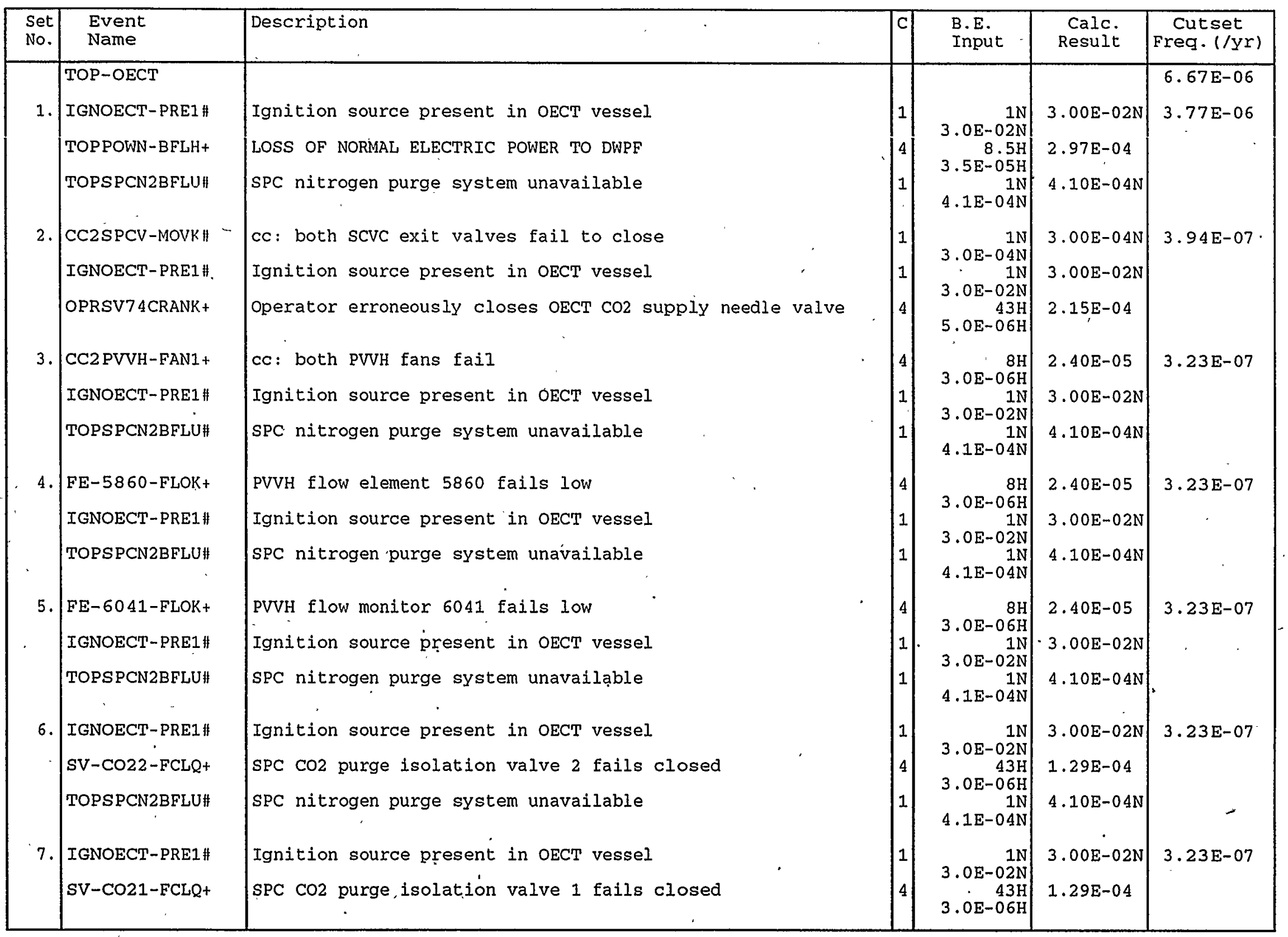




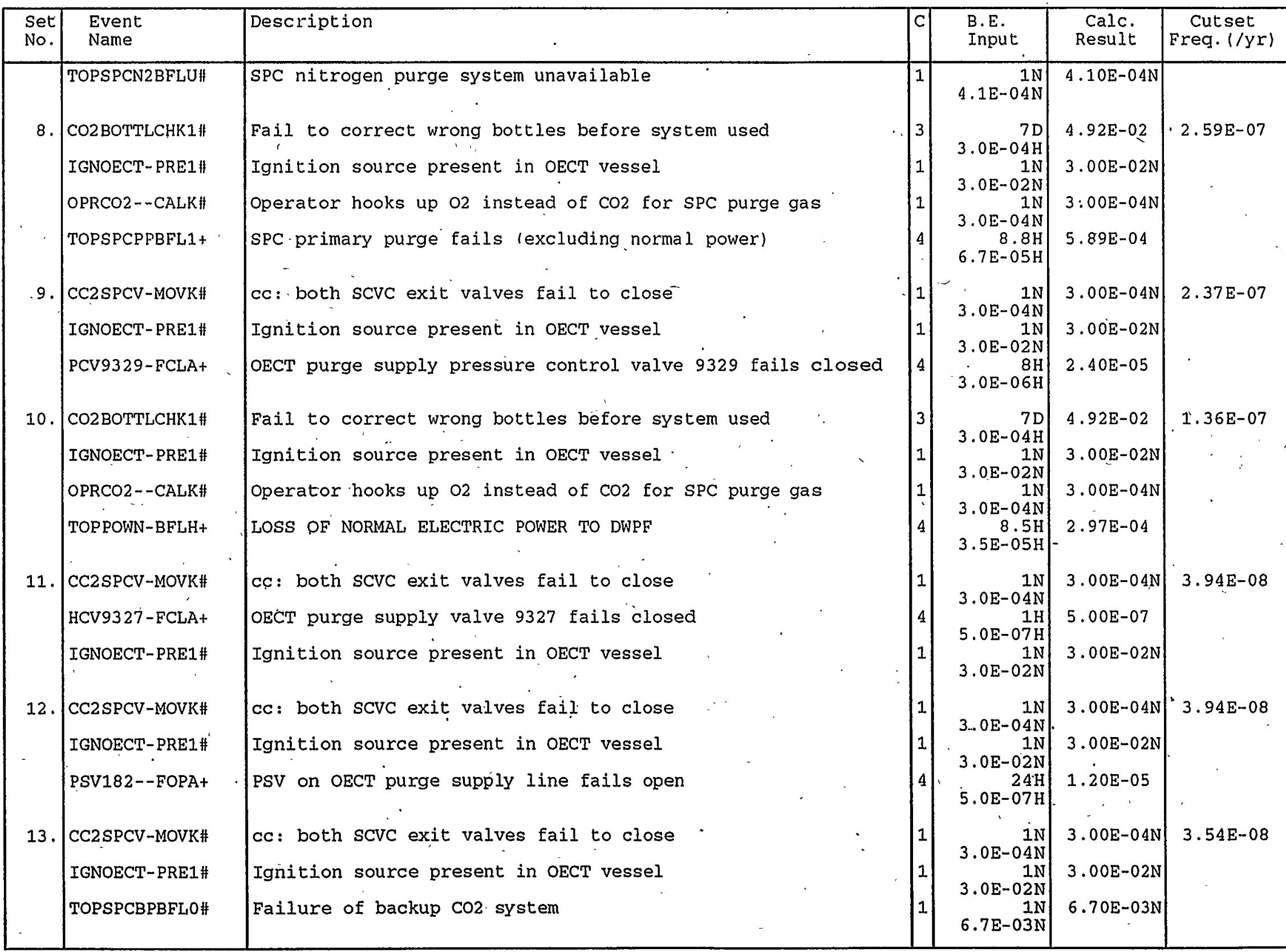


Cutsets fol I: ICAFTA ICUTं\OECT.CSR 11/09/94 10:25 AM (CONT.)

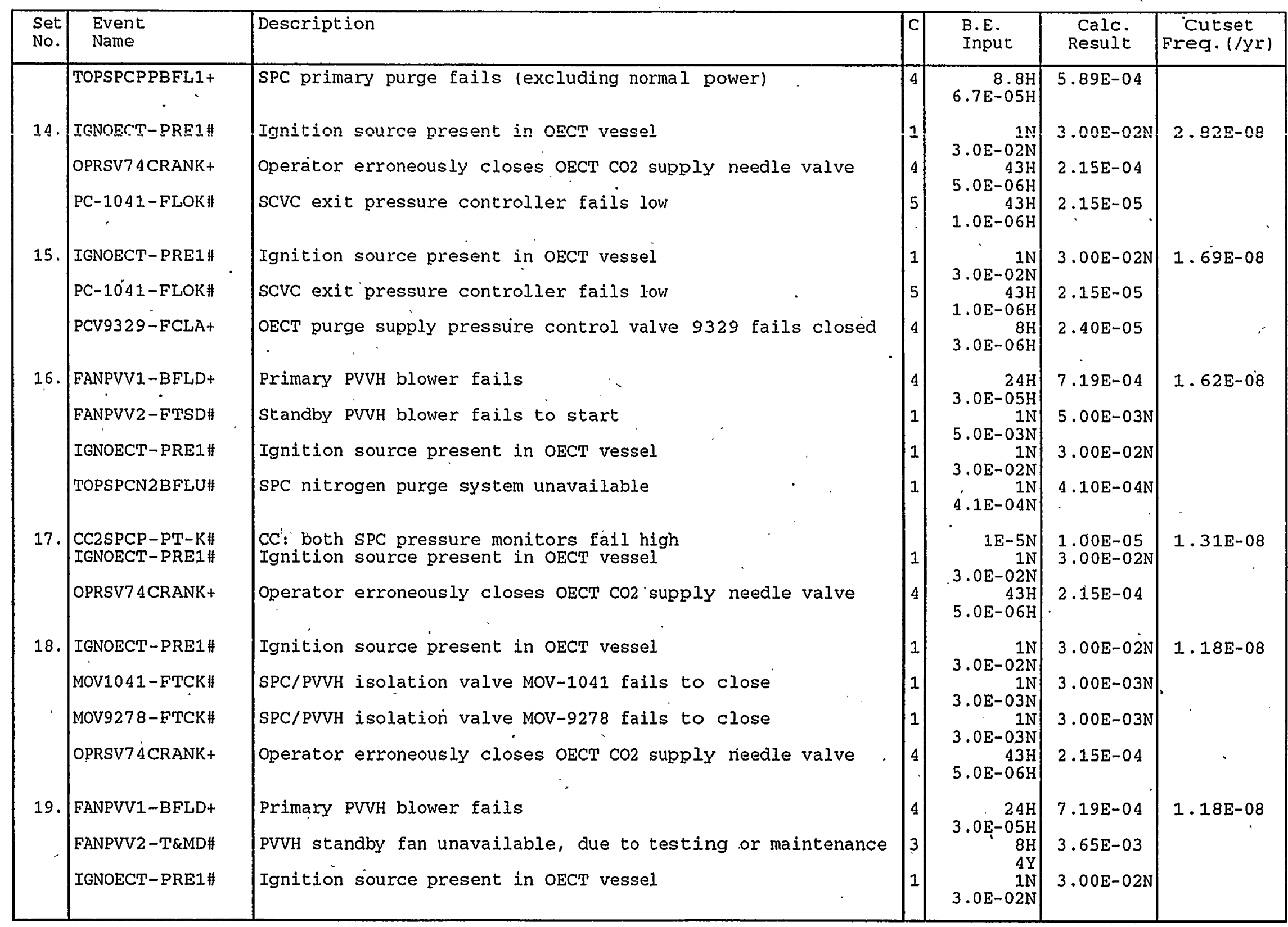




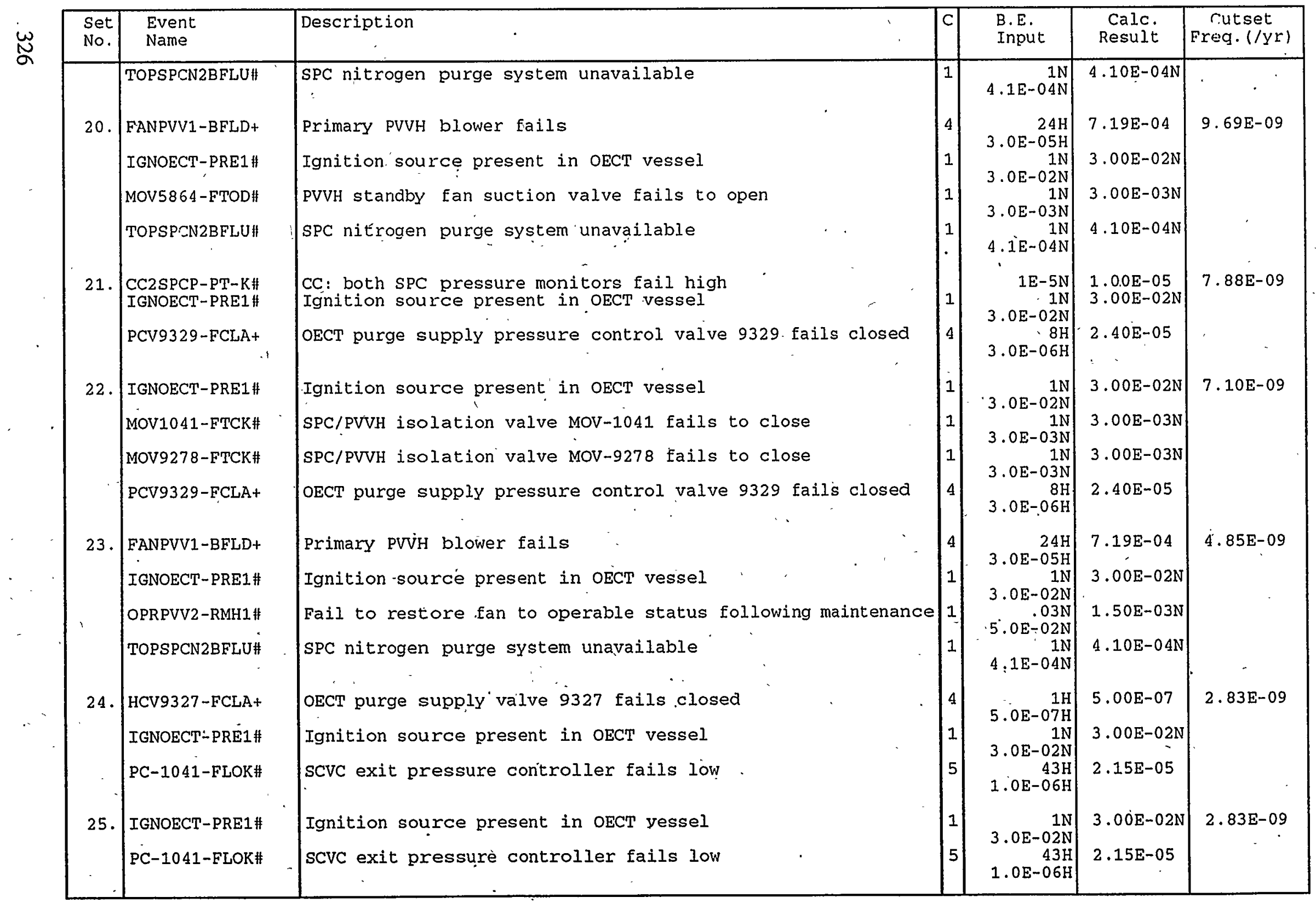


X-ESR-S-00001, REV. 0

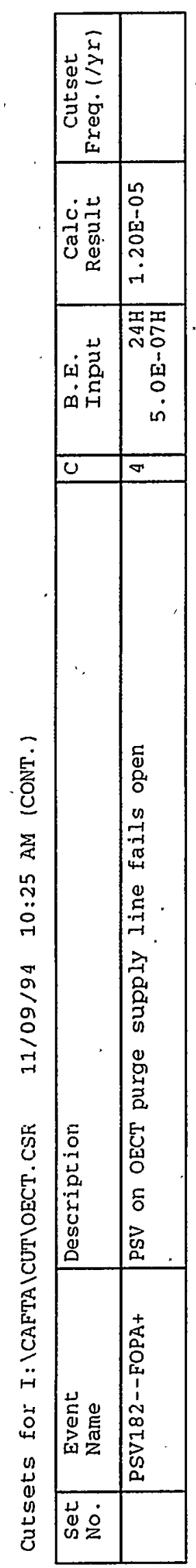


Basic Event Name TOPPOWN-BFLH+I FE-5860-FLOK+I FE-6041-FLOK+I CC2PVVH-FAN1+-I SV-CO22-FCLQ+-I SV-CO21-FCLQ+-I OPRSV74CRANK+-I PCV9329-FCLA+I PSV182-FOPA+I HCV9327-FCLA+I TOPSPCN2BFLU\# TOPSPCPPBFL1+I CC2SPCP-PT-K\# PC-1041-FLOK\# CC2SPCV-MOVK\# MOV5862-FCLD+I FANPVV1-BFLD+-I OPRCO2--CALK\# IGNOECT-PRE1\# CO2BOTTLCHK1\# MOV1041-FTCK\# MOV9278-FTCK\# TOPSPCBPBFLO\# CC2SCVC-O2-K\# FANPVV2-BFLD\# OPRPVV2-RMH1\# MOV5864-FTOD\# FANPVV2-T\&MD\# FANPVV2-FTSD\# FE-9328-FHIE\# OPR9328-MCH1\# PT-1041-FHIK\#

\section{Risk Achievement Worth} Description LOSS OFNORMAL ELECTRIC POWER TO DWPF PVVH flow element 5860 fails low PVVH flow monitor 6041 fails low cc: both PVVH fans fail SPC CO2 purge isolation valve 2 fails closed SPC CO2 purge isolation valve 1 fails closed Operator erroneously closes OECT CO2 supply needle valve OECT purge supply pressure control valve 9329 fails closed PSV on OECT purge supply line fails open OECT purge supply valve 9327 fails closed SPC nitrogen purge system unavailable SPC primary purge fails (excluding normal power) CC: both SPC pressure monitors fail high SCVC exit pressure controller fails low cc: both SCVC exit valves fail to close PVVH primary fan suction valve fails closed Primary PVVH blower fails Operator hooks up 02 instead of $\mathrm{CO} 2$ for $\mathrm{SPC}$ purge gas Ignition source present in OECT vessel Fail to correct wrong bottles before system used SPC/PVVH isolation valve MOV-1041 fails to close SPC/PVVH isolation valve MOV-9278 fails to close Failure of backup $\mathrm{CO} 2$ system CC: Both SCVC O2 detectors fail high PVVH backup fan fails to run Fail to restore.fan to operable status following maintenance PVVH standby fan suction valve fails to open PVVH standby fan unavailable, due to $T \& M$ Standby PVYH blower fails to start OECT purge flow element 9328 fails to detect low flow OECT purge flow element 9328 miscalibrated $P R$ vapor space $P T-1041$ fails high
Prob/Freq $3.50 \mathrm{E}-05 / \mathrm{H}$ $3.00 \mathrm{E}-06 / \mathrm{H}$ $3.00 \mathrm{E}-06 / \mathrm{H}$ $3.00 \mathrm{E}-06 / \mathrm{H}$ $3.00 \mathrm{E}-06 / \mathrm{H}$ $3.00 \mathrm{E}-06 / \mathrm{H}$

$5.00 \mathrm{E}-06 / \mathrm{H}$ $3.00 \mathrm{E}-06 / \mathrm{H}$ $5.00 \mathrm{E}-07 / \mathrm{H}$ $5.00 \mathrm{E}-07 / \mathrm{H}$ 4.10E-04 $6.69 \mathrm{E}-05 / \mathrm{H}$ 8.40E-06

2.15E-05

3.00E-04

3.00E- $07 / \mathrm{H}$ $3.00 \mathrm{E}-05 / \mathrm{H}$ 3.00E-04 3.00E-02

4.92E-02

3.00E-03

3.00E-03

$6.70 \mathrm{E}-03$

5.00E-04

7.20E-04

$1.50 \mathrm{E}-03$

3.00E-03

3.65E-03

$5.00 \mathrm{E}-03$

2.16E-03

$1.20 \mathrm{E}-04$

2.15E-05
AchW

$1.67 \mathrm{E}+04$

$1.62 \mathrm{E}+04$

$1.62 \mathrm{E}+04$

$1.62 \mathrm{E}+04$

$1.62 \mathrm{E}+04$

$1.62 \mathrm{E}+04$

$1.34 \mathrm{E}+04$

$1.34 \mathrm{E}+04$

$1.34 \mathrm{E}+04$

$1.34 \mathrm{E}+04$

$1.99 \mathrm{E}+03$

$6.71 \mathrm{E}+02$

$3.73 \mathrm{E}+02$

$3.73 \mathrm{E}+02$

$3.73 E+02$

$2.25 \mathrm{E}+02$

$2.25 \mathrm{E}+02$

$1.98 \mathrm{E}+02$

$3.33 \mathrm{E}+01$

$2.15 \mathrm{E}+00$

$2.11 \mathrm{E}+00$

$2.11 \mathrm{E}+00$

$1.89 \mathrm{E}+00$

$1.81 \mathrm{E}+00$

$1.49 \mathrm{E}+00$

$1.49 \mathrm{E}+00$

$1.49 \mathrm{E}+00$

$1.49 \mathrm{E}+00$

$1.49 \mathrm{E}+00$

$1.18 \mathrm{E}+00$

$1.17 \mathrm{E}+00$

$1.03 E+00$

Note: Events that make less than $1 \%$ difference to the importance factor are not included. Modified top event frequency $=$ (top event frequency) $\mathrm{x}$ (risk achievement worth). 
Explosion in the OECT Top Event Frequency: 6.67E-06/YR

Basic Event Name
*IGNOECT-PRE1\#
TOPSPCN2BFLU\#
TOPPOWN-BFLH+-I
CC2SPCV-JMOVK\#
OPRSV74CRANK+-I
OPRCO2-CALK\#
CO2BOTTI_CHK1\#
SV-CO21-FCLQ+I
SV-CO22-FCLQ+I
CC2PVVH-FAN1+-I
FE-6041-FL.OK+-I
FE-5860-FI.OK+-I
TOPSPCPP.BFL1+-I.
PCV9329-FCLA+I

\section{Risk Reduction Worth}

Description

Ignition source present in OECT vessel

SPC nitrogen purge system unavailable

LOSS OF NORMAL ELECTRIC POWER TO DWPF

cc: both SCVC exit valves fail to close

Operator erroneously closes OECT CO2 supply needle valve

Operator hooks up $\mathrm{O} 2$ instead of $\mathrm{CO} 2$ for SPC purge gas

Fail to correct wrong bottles before system used

SPC CO2 purge isolation valve 1 fails closed

SPC CO2 purge isolation valve 2 fails closed

cc: both PVVH fans fail

PVVH flow monitor 6041 fails low

PVVH flow element 5860 fails low

SPC primary purge fails (excluding normal power)

OECT purge supply pressure control valve 9329 fails closed

\begin{tabular}{ll} 
Prob/Freg & RedW \\
\hline $3.00 \mathrm{E}-02$ & $0.00 \mathrm{E}+00$ \\
$4.10 \mathrm{E}-04$ & $5.38 \mathrm{E}+00$ \\
$3.50 \mathrm{E}-05 / \mathrm{H}$ & $2.41 \mathrm{E}+00$ \\
$3.00 \mathrm{E}-04$ & $1.13 \mathrm{E}+00$ \\
$5.00 \mathrm{E}-06 / \mathrm{H}$ & $1.07 \mathrm{E}+00$ \\
$3.00 \mathrm{E}-04$ & $1.06 \mathrm{E}+00$ \\
$4.92 \mathrm{E}-02$ & $1.06 \mathrm{E}+00$ \\
$3.00 \mathrm{E}-06 / \mathrm{H}$ & $1.05 \mathrm{E}+00$ \\
$3.00 \mathrm{E}-06 / \mathrm{H}$ & $1.05 \mathrm{E}+00$ \\
$3.00 \mathrm{E}-06 / \mathrm{H}$ & $1.05 \mathrm{E}+00$ \\
$3.00 \mathrm{E}-06 / \mathrm{H}$ & $1.05 \mathrm{E}+00$ \\
$3.00 \mathrm{E}-06 / \mathrm{H}$ & $1.05 \mathrm{E}+00$ \\
$6.69 \mathrm{E}-05 / \mathrm{H}$ & $1.05 \mathrm{E}+00$ \\
$3.00 \mathrm{E}-06 / \mathrm{H}$ & $1.04 \mathrm{E}+00$
\end{tabular}

* A Reduction Worth of $0.0 \mathrm{E}+00$ indicates event appears in every cutset could serve as a single event preventor for the accident.

Note: Events that make less than $1 \%$ difference to the importance factor are not included. Modified top event frequency $=$ (top event frequency) $/$ (risk reduction worth). 
X-ESR-S-00001, REV. 0

\section{PRFT Explosion}




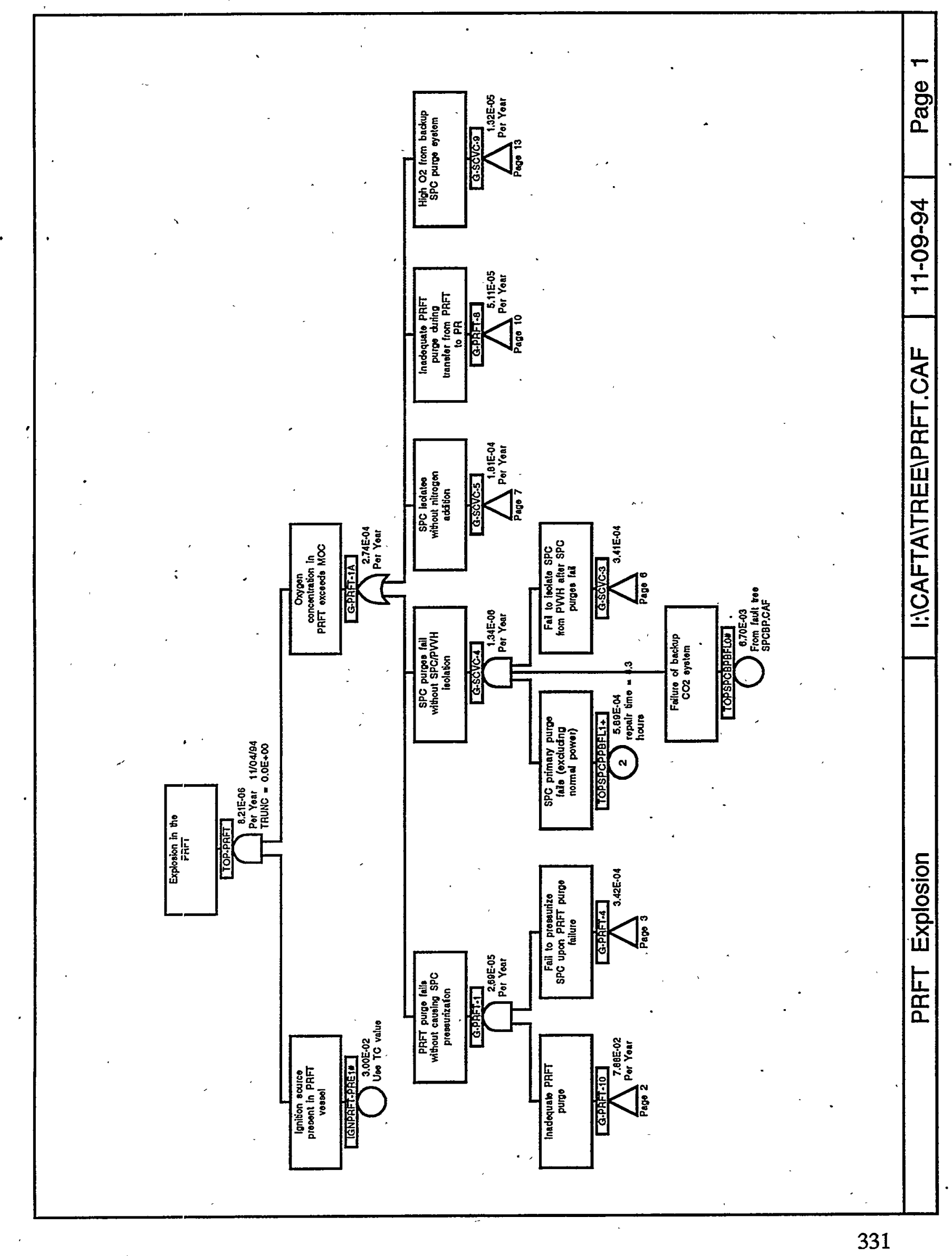




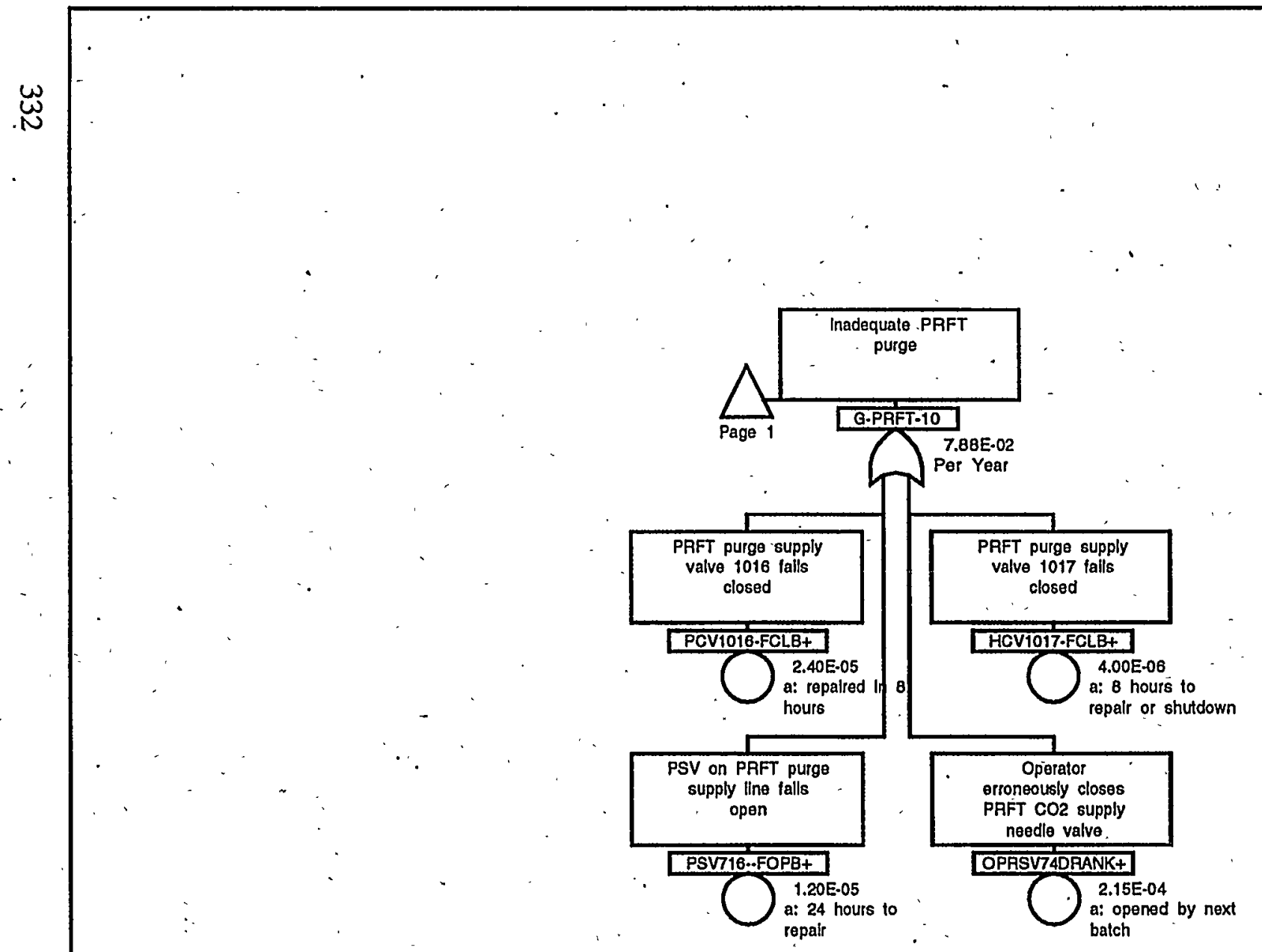




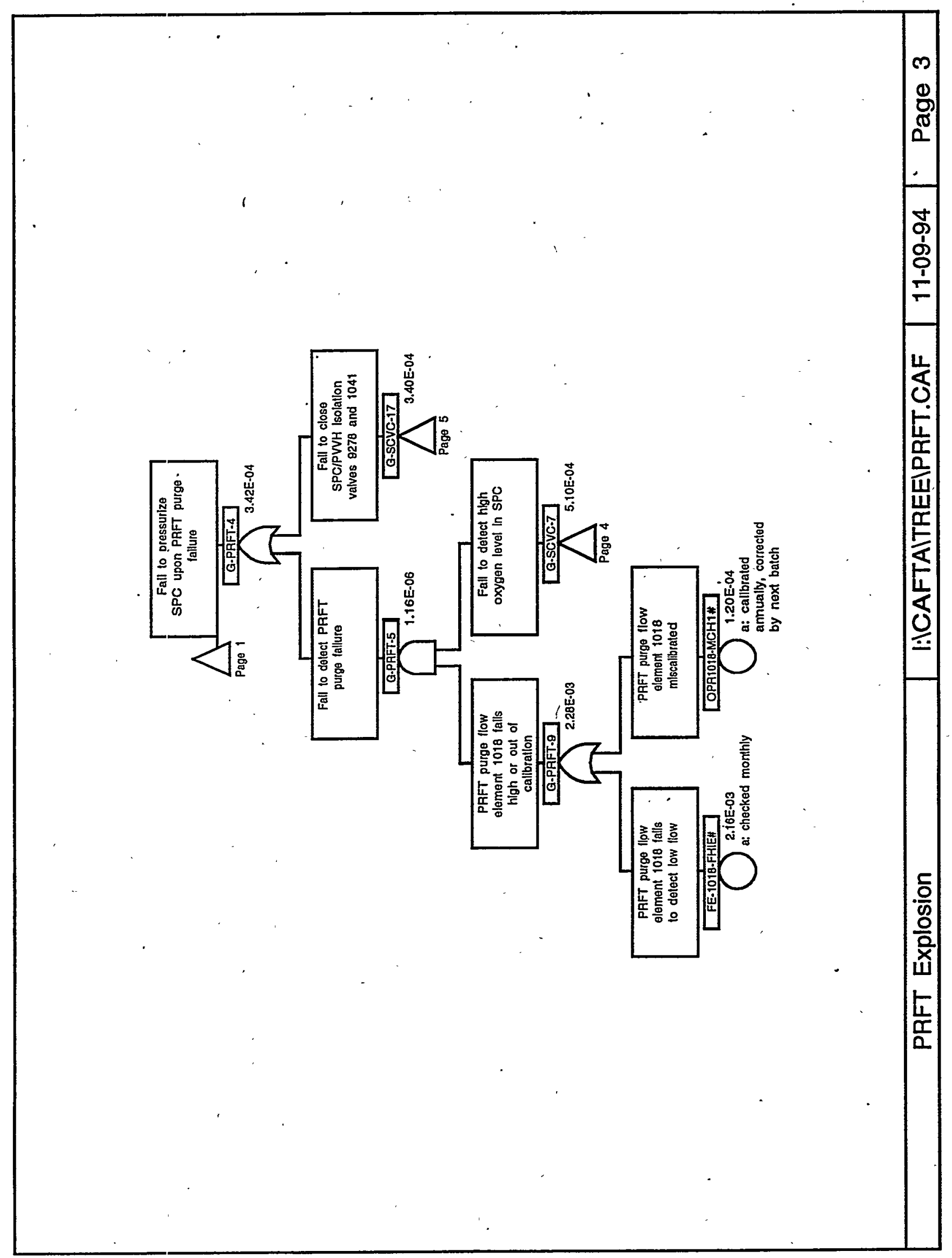



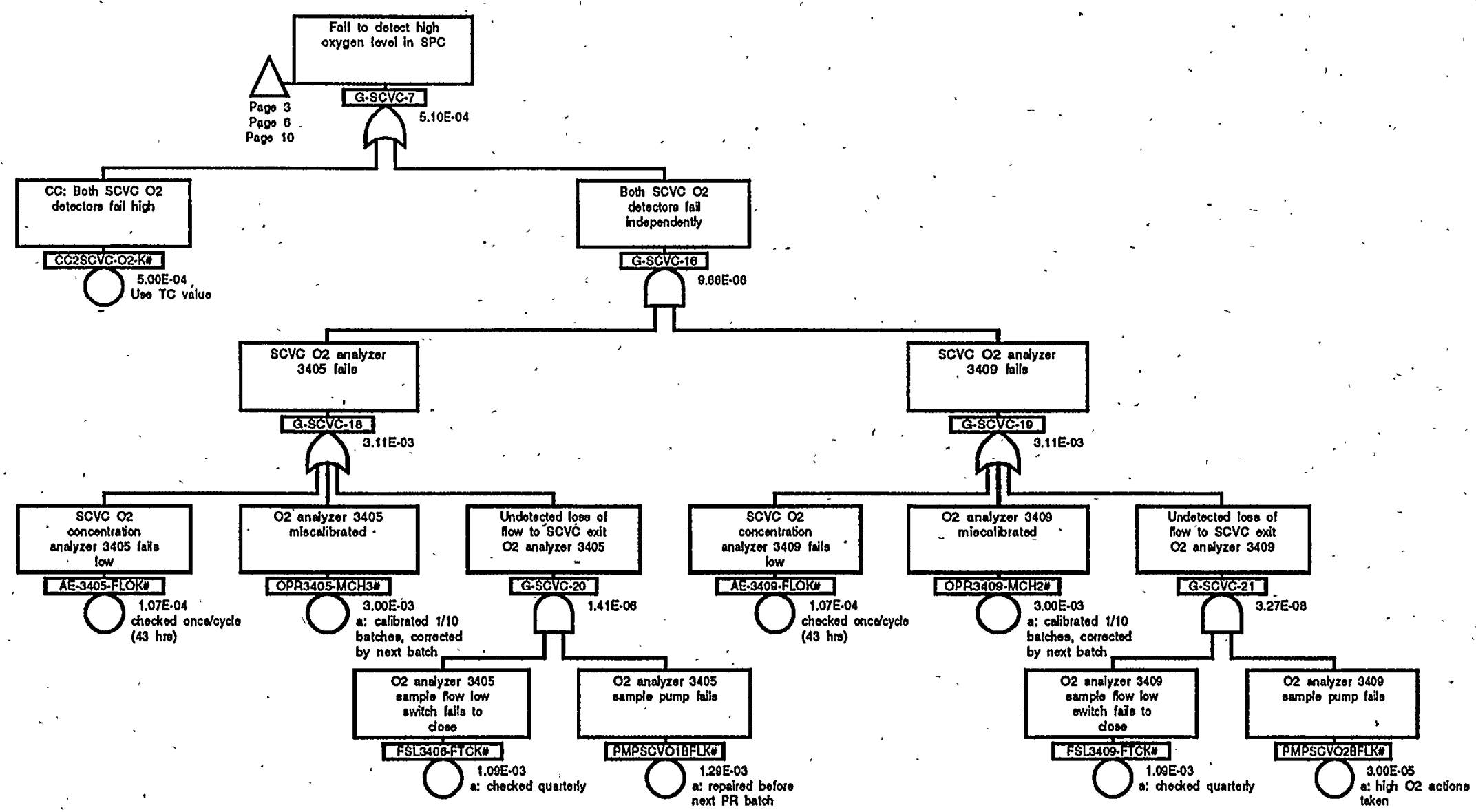


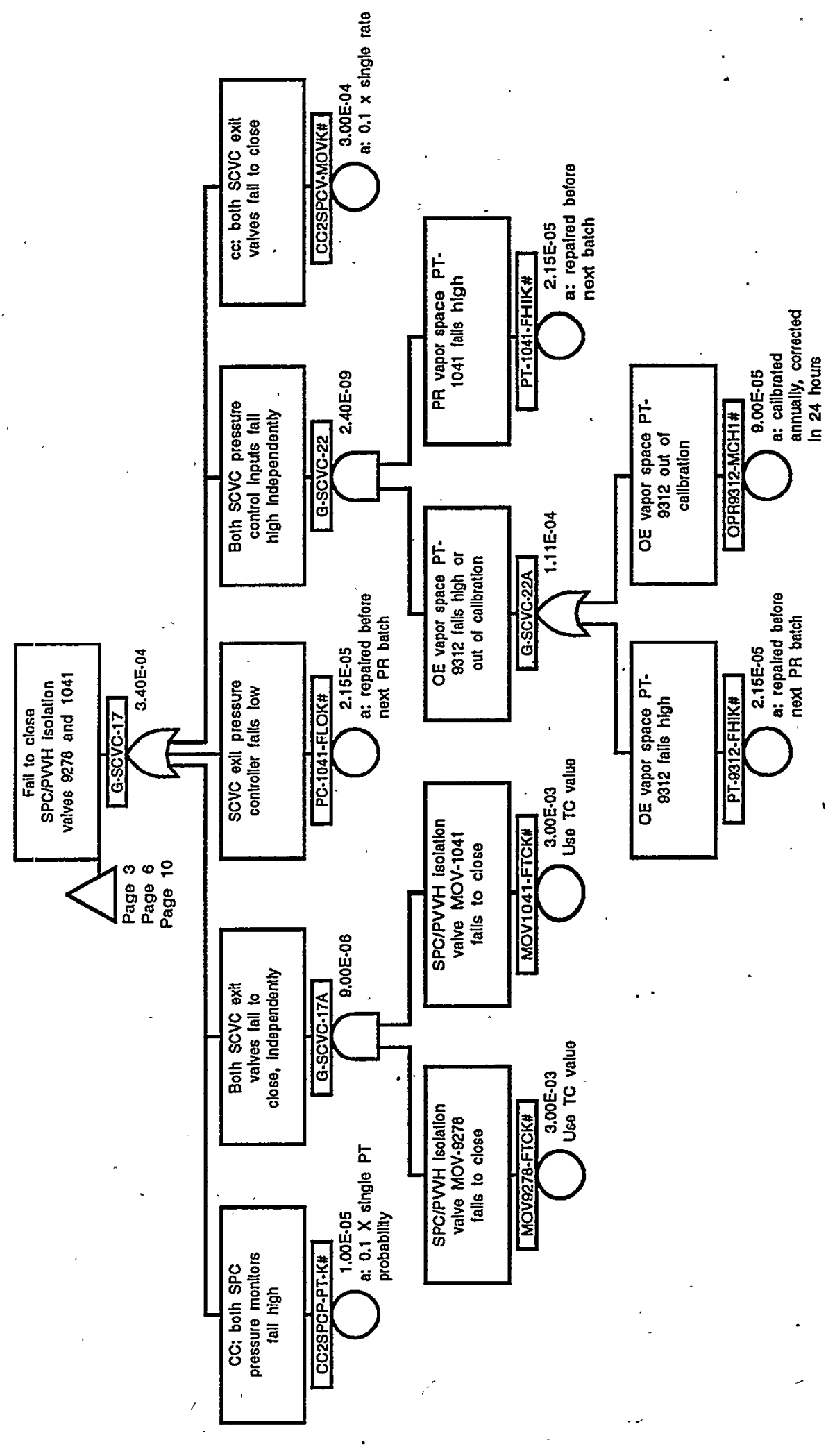


X-ESR-S-00001, REV. 0

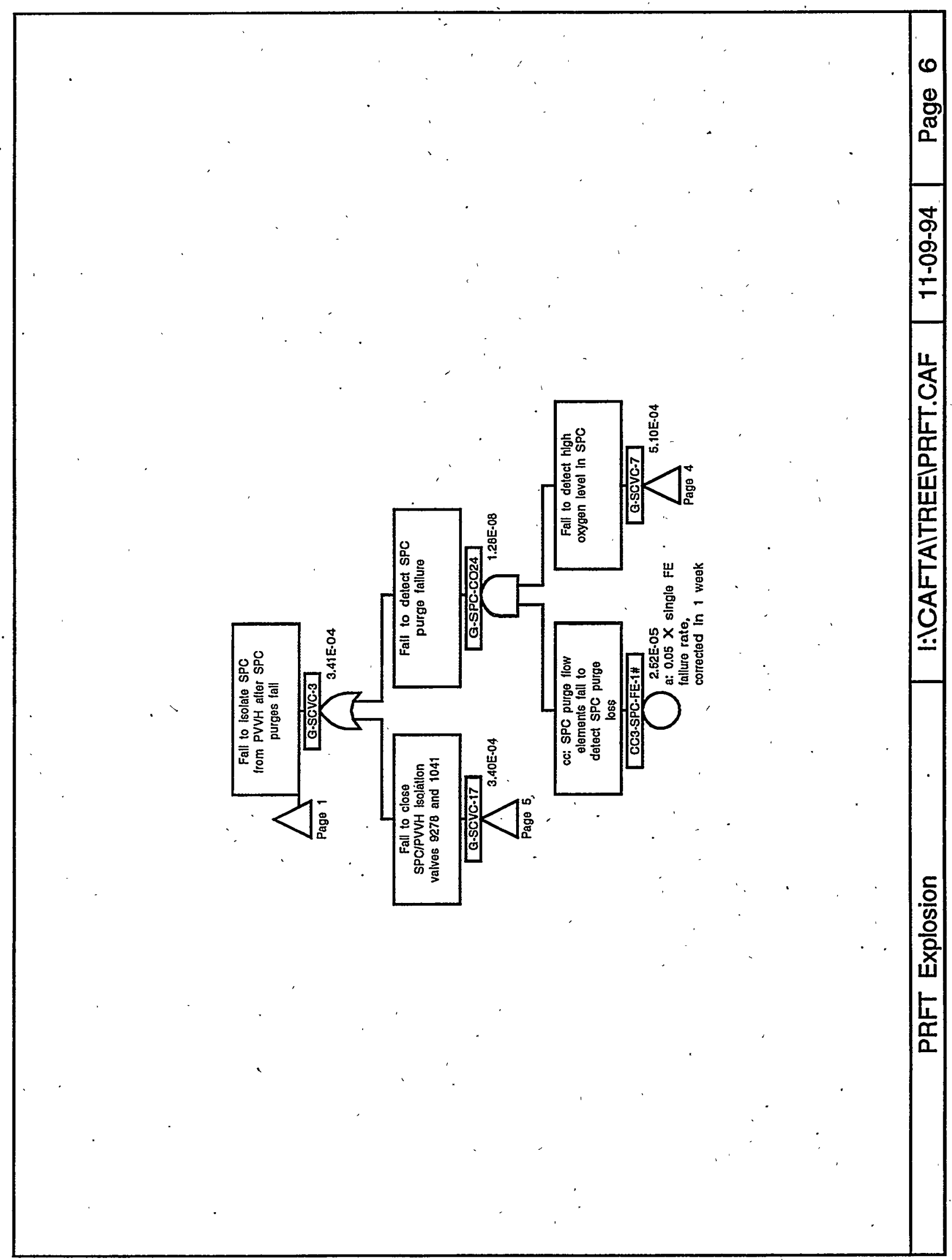




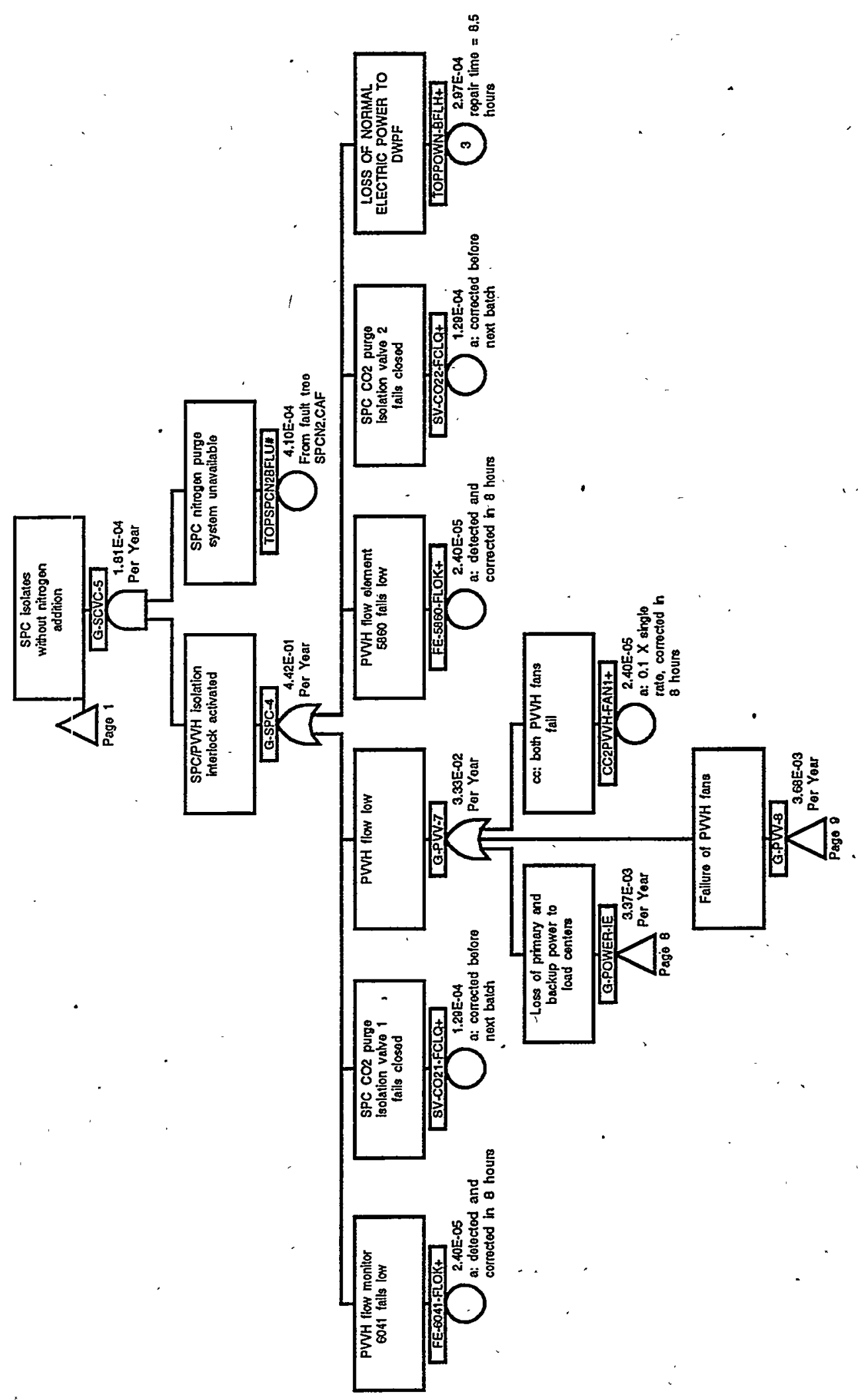




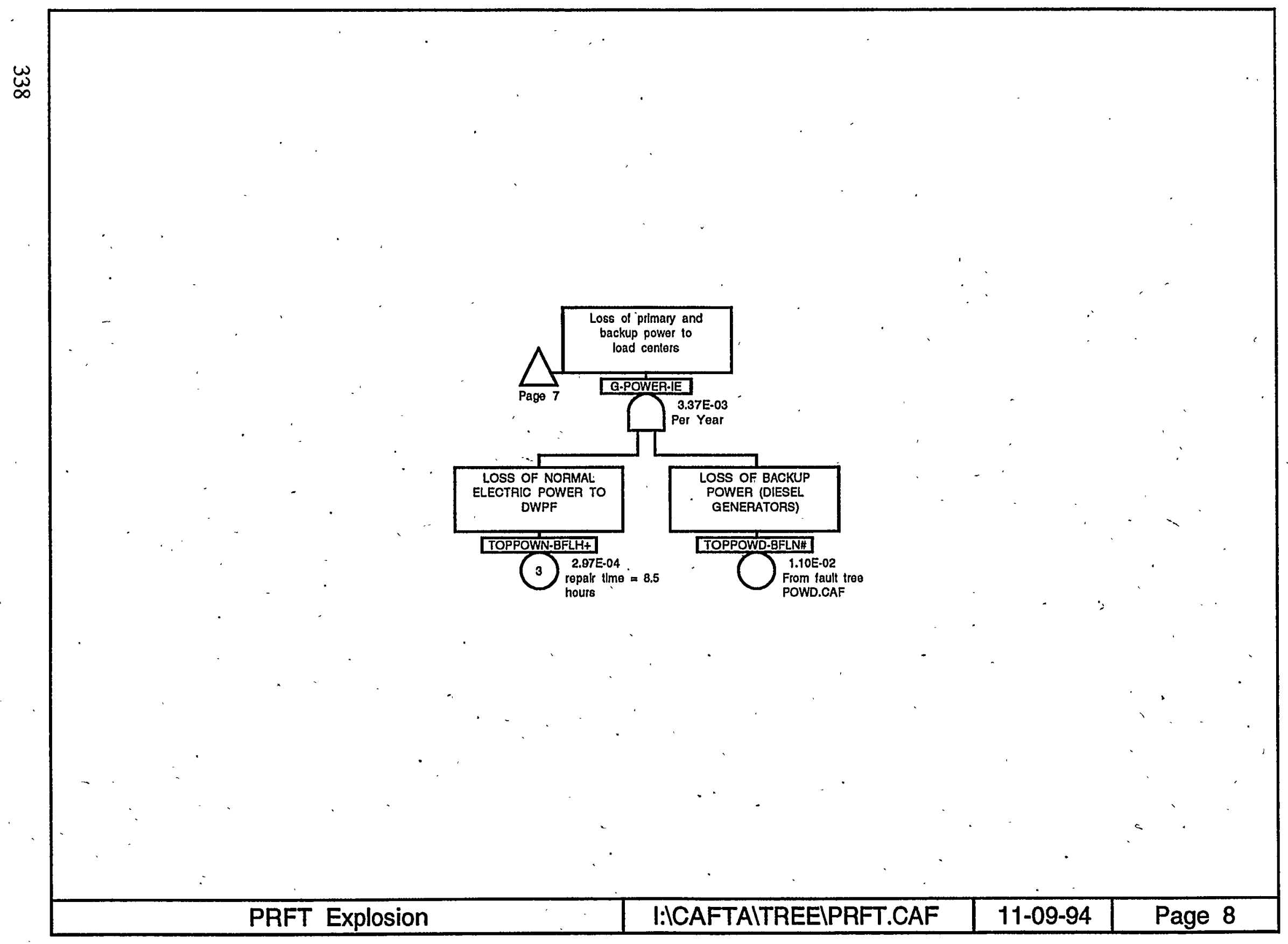




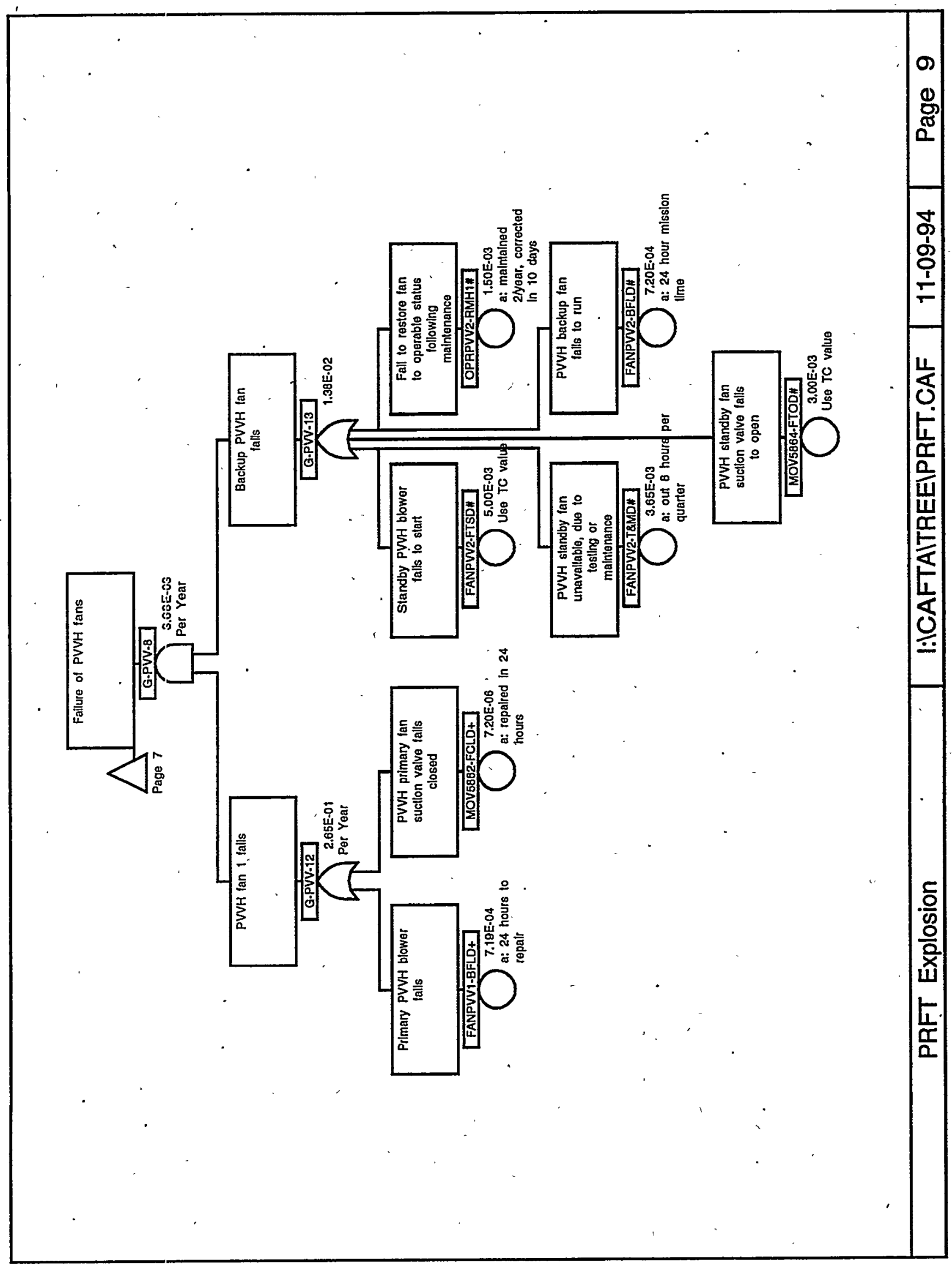


X-ESR-S-00001, REV. 0

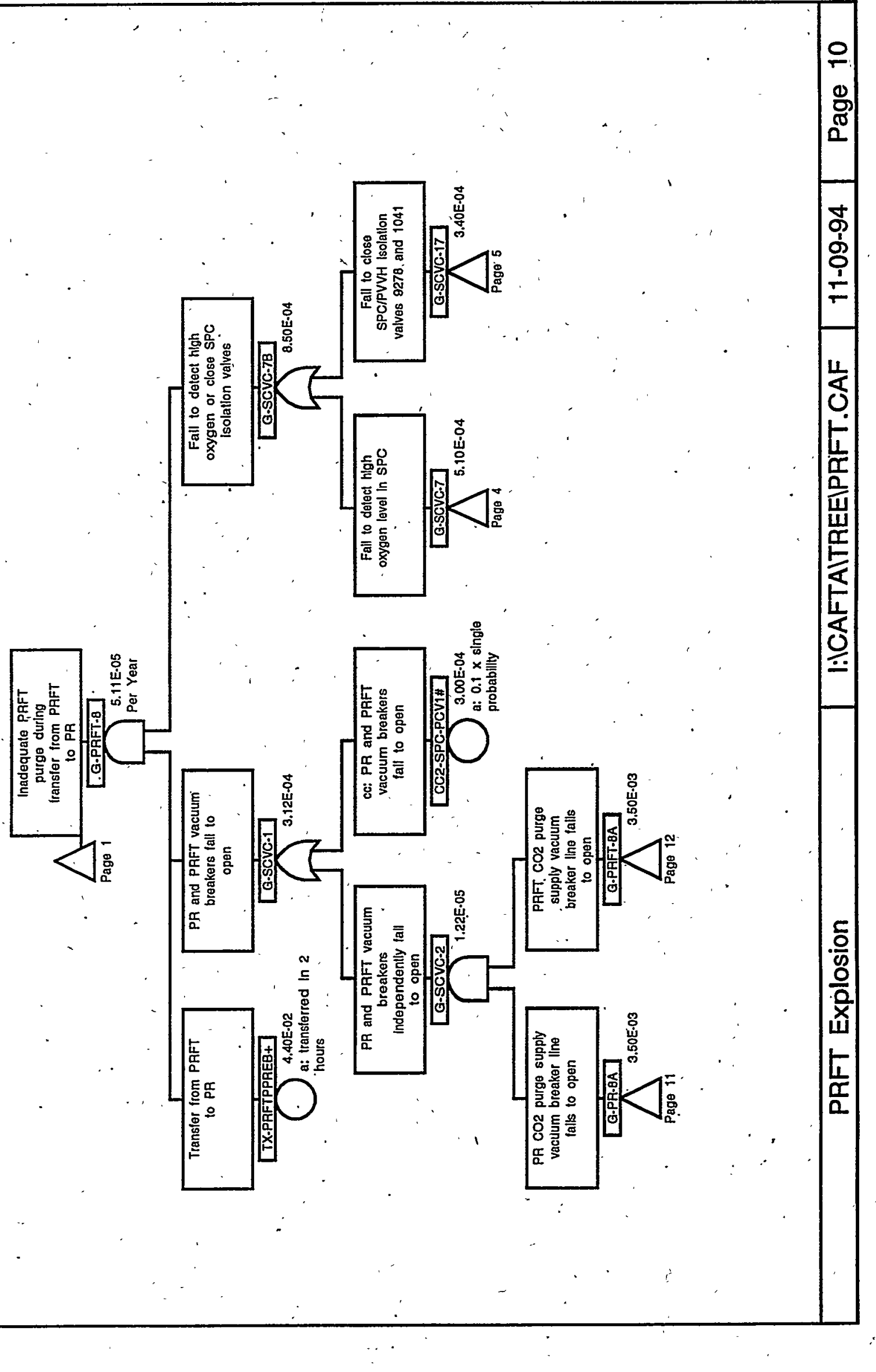




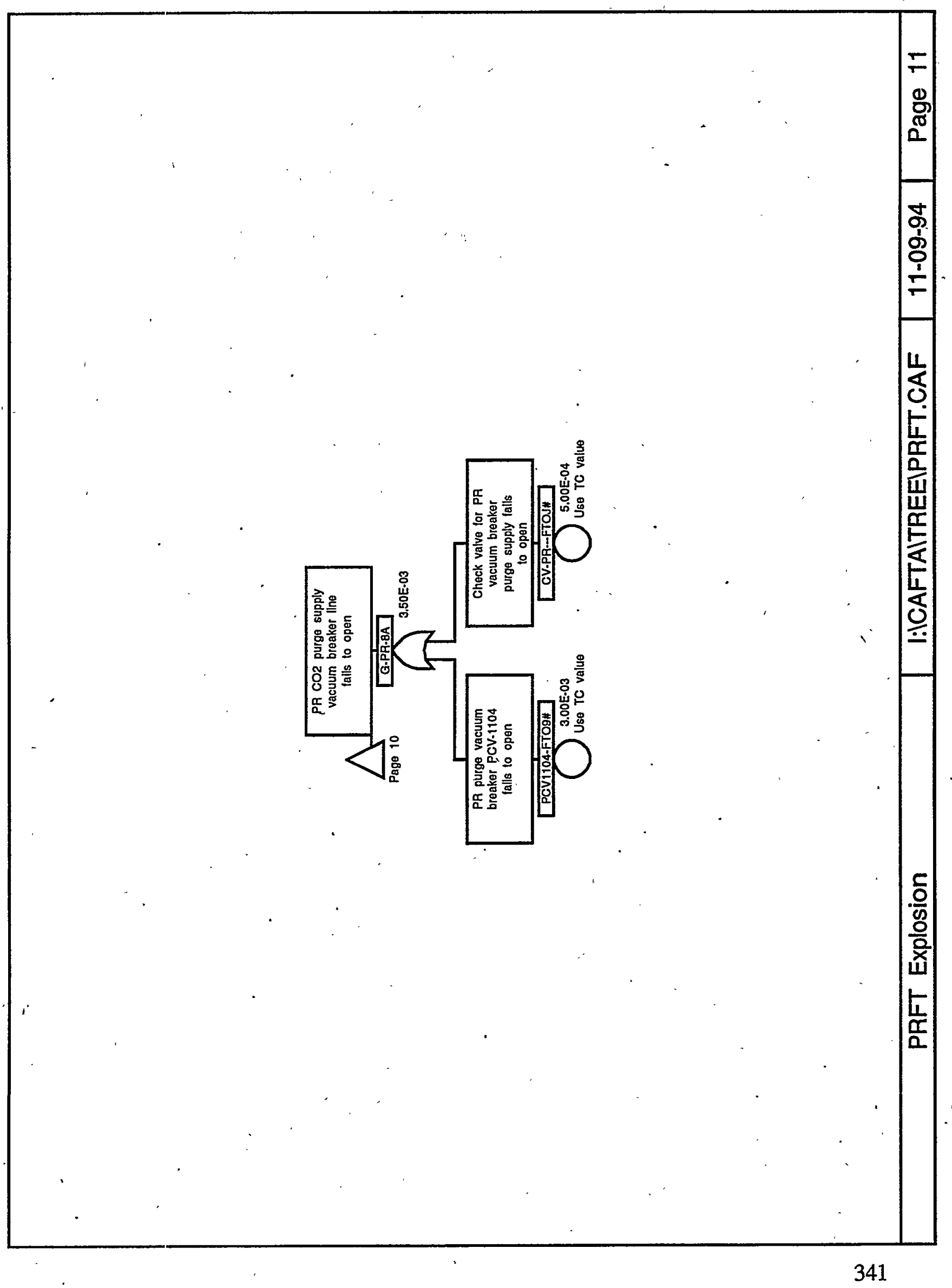


X-ESR-S-00001, REV. 0

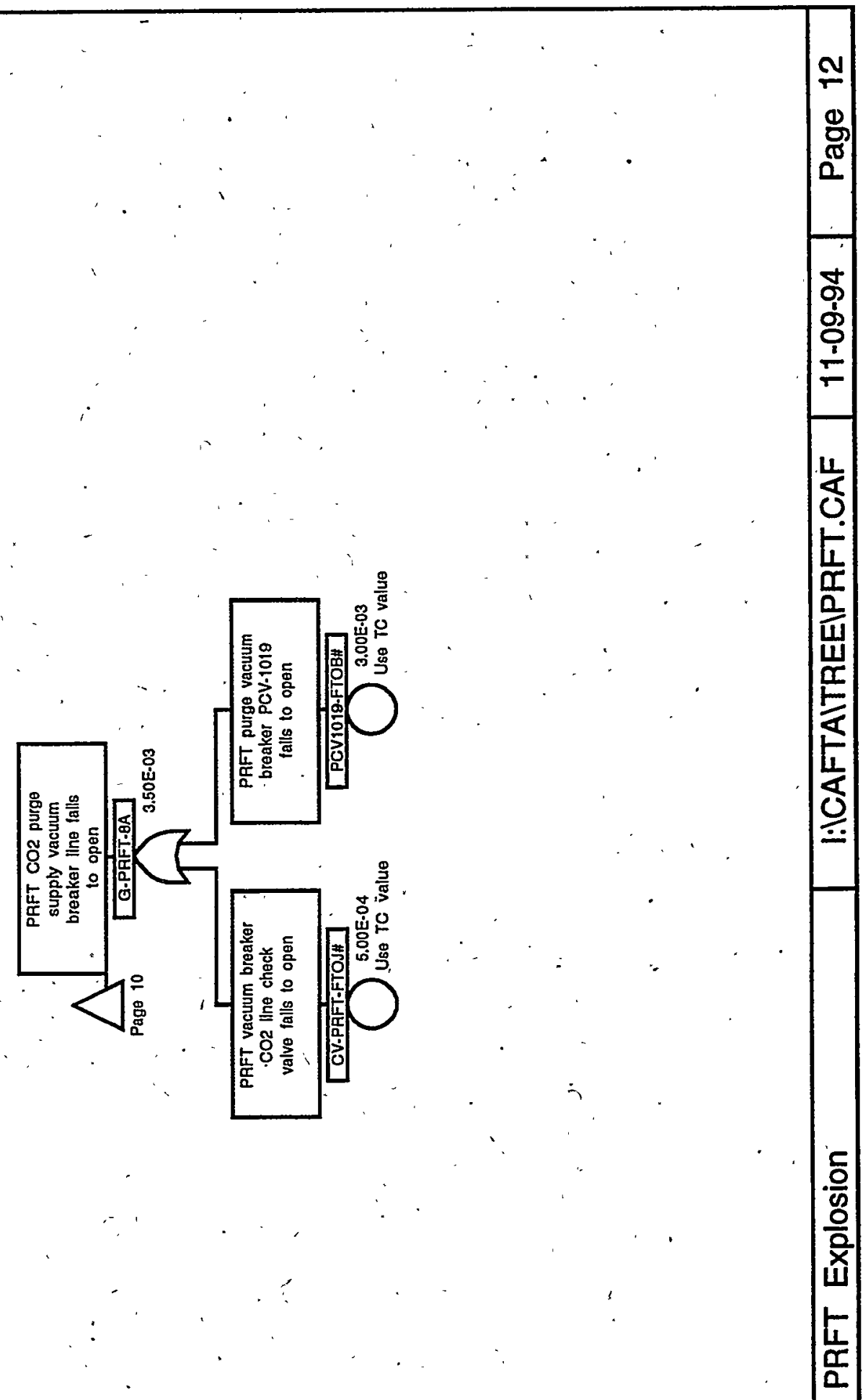




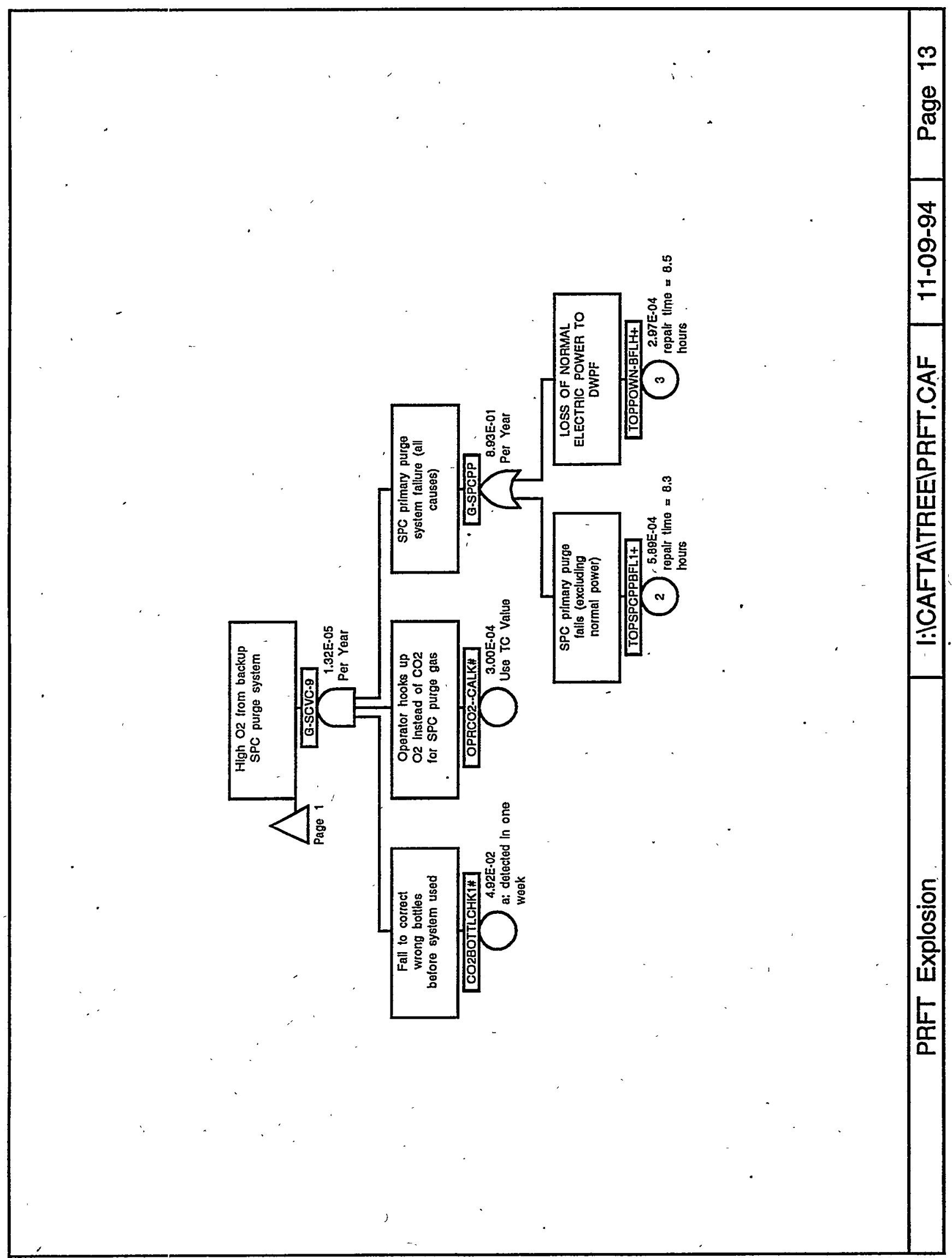




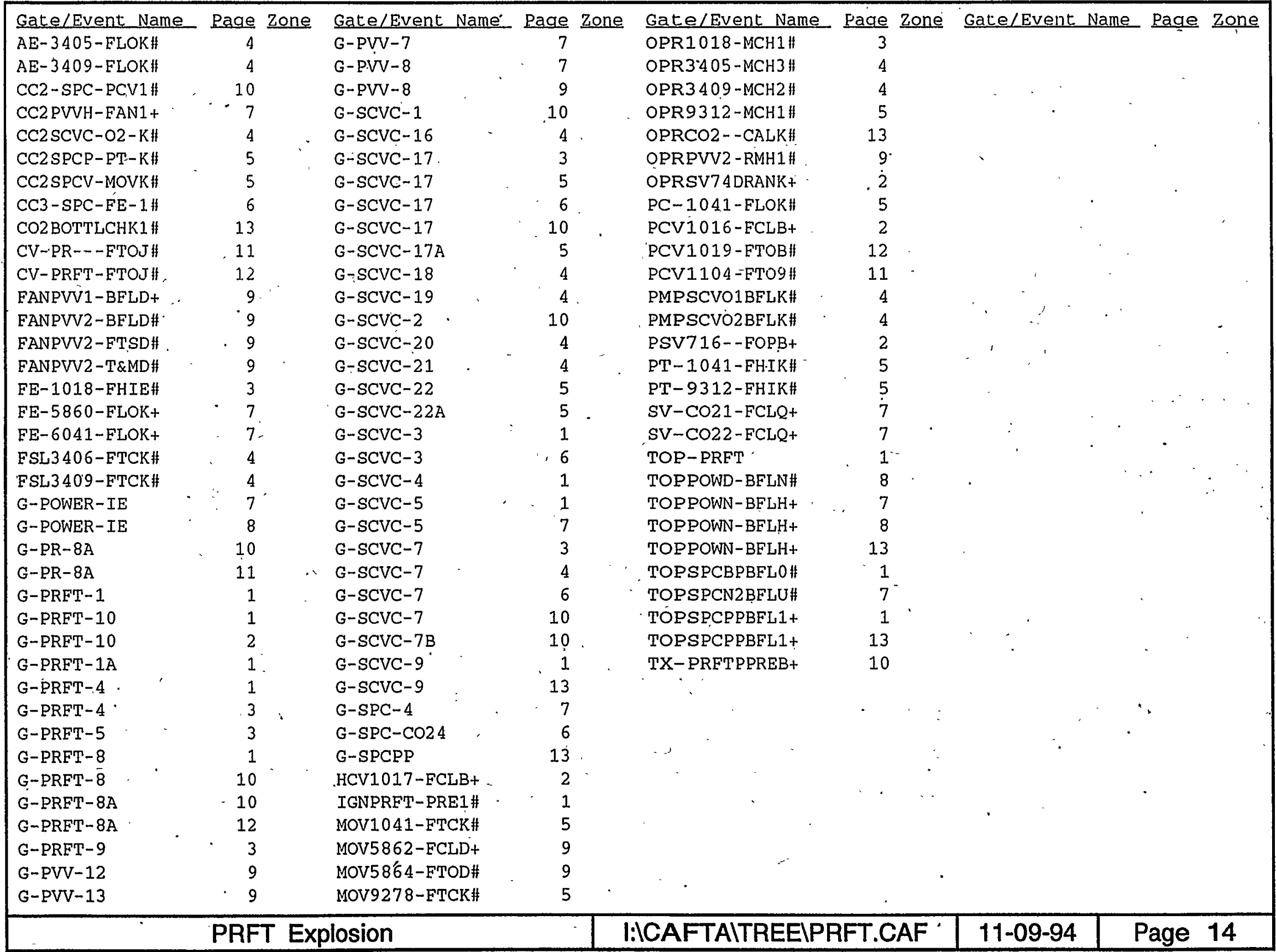


Cutsets for I : ICAFTAICUT\PRFT.CSR

$11 / 09 / 94 \quad 10: 26$ AM

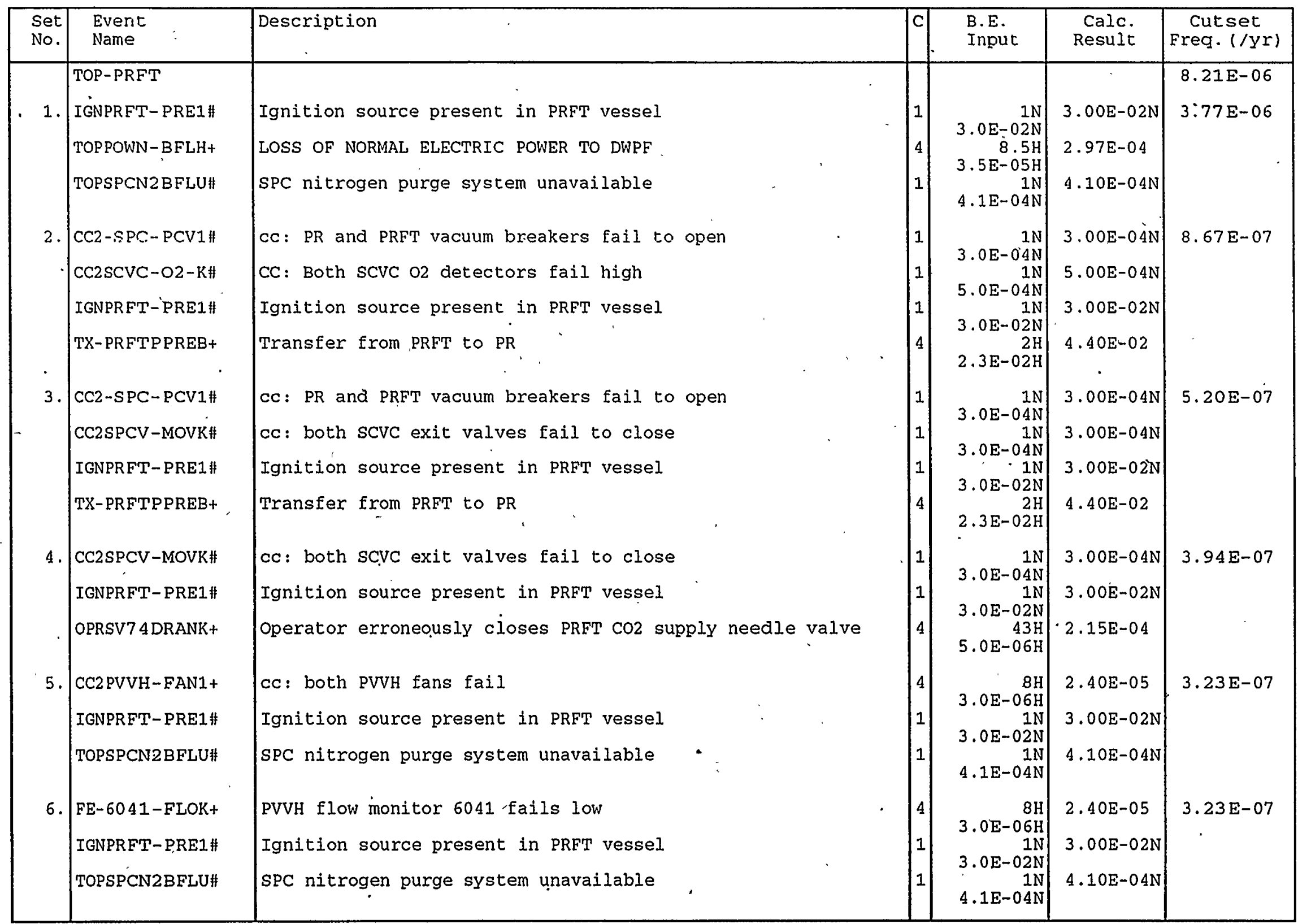




\section{X-ESR-S-00001, REV. 0}

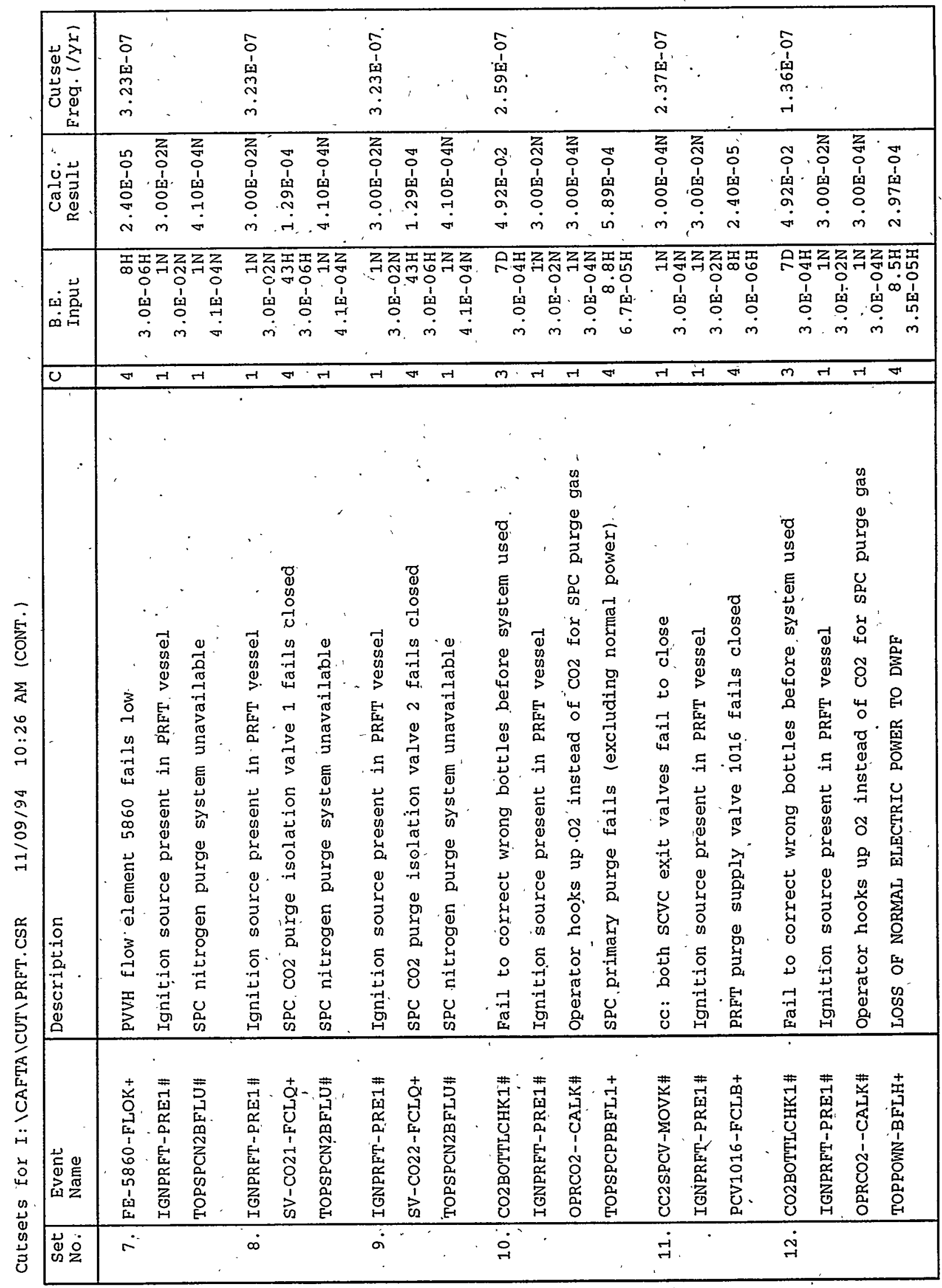


Cutsets for I: ICAFTAICUT $\backslash P R F T . C S R$

11/09/94 10:26 AM (CONT.)

\begin{tabular}{|c|c|c|c|c|c|c|}
\hline $\begin{array}{l}\text { Set } \\
\text { No. }\end{array}$ & $\begin{array}{l}\text { Event } \\
\text { Name }\end{array}$ & Description & C & $\begin{array}{l}\text { B.E. } \\
\text { Input }\end{array}$ & $\begin{array}{r}\text { Calc. } \\
\text { Result }\end{array}$ & $\begin{array}{c}\text { Cutset } \\
\text { Freg. }(/ Y Y)\end{array}$ \\
\hline 13. & $\begin{array}{l}\text { CC2SPCV-MOVK\# } \\
\text { HCV1017-FCLB+ } \\
\text { IGNPRFT-PRE1\# }\end{array}$ & $\begin{array}{l}\text { CC: both SCVC exit valves fail to close. } \\
\text { PRFT purge supply valve } 1017 \text { fails closed } \\
\text { Ignition source present in PRFT vessel }\end{array}$ & $\begin{array}{l}1 \\
4 \\
1\end{array}$ & $\begin{array}{r}1 \mathrm{~N} \\
3.0 \mathrm{E}-04 \mathrm{~N} \\
8 \mathrm{H} \\
5.0 \mathrm{E}-07 \mathrm{H} \\
1 \mathrm{~N} \\
3.0 \mathrm{E}-02 \mathrm{~N}\end{array}$ & $\begin{array}{l}.00 \mathrm{E}-04 \mathrm{~N} \\
4.00 \mathrm{E}-06 \\
3.00 \mathrm{E}-02 \mathrm{~N}\end{array}$ & $3.94 E-08$ \\
\hline 14. & $\begin{array}{l}\text { CC2SPCV-MOVK\# } \\
\text { IGNPRFT-PRE1\# } \\
\text { PSV716--FOPB+ }\end{array}$ & $\begin{array}{l}\text { cc: both SCVC exit valves fail to close } \\
\text { Ignition source present in PRFT vessel } \\
\text { PSV on PRFT purge supply line fails open - }\end{array}$ & $\begin{array}{l}1 \\
1 \\
4\end{array}$ & $\begin{array}{r}1 \mathrm{~N} \\
3.0 \mathrm{E}-04 \mathrm{~N} \\
1 \mathrm{~N} \\
3.0 \mathrm{E}-02 \mathrm{~N} \\
24 \mathrm{H} \\
5.0 \mathrm{E}-07 \mathrm{H}\end{array}$ & $\begin{array}{l}3.00 \mathrm{E}-04 \mathrm{~N} \\
3.00 \mathrm{E}-02 \mathrm{~N} \\
1.20 \mathrm{E}-05\end{array}$ & $3.94 E-08$ \\
\hline 15. & $\begin{array}{l}\text { CC2-SPC-PCV1\# } \\
\text { IGNPRFT-PRE1\# } \\
\text { PC-1041-FLOK\# } \\
\text { TX-PRFTPPREB+ }\end{array}$ & $\begin{array}{l}\text { CC: PR and PRFT vacuum breakers fail to open } \\
\text { Ignition source present in PRFT vessel } \\
\text { SCVC exit pressure controller fails low } \\
\text { Transfer from PRFT to PR }\end{array}$ & $\left|\begin{array}{l}1 \\
1 \\
5 \\
4\end{array}\right|$ & $\begin{array}{r}1 \mathrm{~N} \\
3.0 \mathrm{E}-04 \mathrm{~N} \\
1 \mathrm{~N} \\
3.0 \mathrm{E}-02 \mathrm{~N} \\
43 \mathrm{H} \\
1.0 \mathrm{E}-06 \mathrm{H} \\
2 \mathrm{H} \\
2.3 \mathrm{E}-02 \mathrm{H}\end{array}$ & $\begin{array}{l}3.00 \mathrm{E}-04 \mathrm{~N} \\
3.00 \mathrm{E}-02 \mathrm{~N} \\
2.15 \mathrm{E}-05 \\
4.40 \mathrm{E}-02\end{array}$ & $3.73 E-08$ \\
\hline 16. & $\begin{array}{l}\text { CC2SPCV-MOVK\# } \\
\text { IGNPRFT-PRE1\# } \\
\text { TOPSPCBPBFL0\# } \\
\text { TOPSPCPPBFL1+ }\end{array}$ & $\begin{array}{l}\text { CC: both SCVC exit valves fail to close } \\
\text { Ignition source present in PRFT vessel } \\
\text { Failure of backup CO2 system } \\
\text { SPC primary purge fails (excluding normal power) }\end{array}$ & $\begin{array}{l}1 \\
1 \\
1 \\
4\end{array} \mid$ & $\begin{array}{r}1 \mathrm{~N} \\
3.0 \mathrm{E}-04 \mathrm{~N} \\
1 \mathrm{~N} \\
3.0 \mathrm{E}-02 \mathrm{~N} \\
1 \mathrm{~N} \\
6.7 \mathrm{E}-03 \mathrm{~N} \\
8.8 \mathrm{H} \\
6.7 \mathrm{E}-05 \mathrm{H}\end{array}$ & $\begin{array}{l}3.00 \mathrm{E}-04 \mathrm{~N} \\
3.00 \mathrm{E}-02 \mathrm{~N} \\
6.70 \mathrm{E}-03 \mathrm{~N} \\
5.89 \mathrm{E}-04\end{array}$ & $3.54 \mathrm{E}-08$ \\
\hline 17. & $\begin{array}{l}\text { IGNPRFT-PRE1\# } \\
\text { OPRSV7 4DRANK+ } \\
\text { PC-1041-FLOK\# }\end{array}$ & $\begin{array}{l}\text { Ignition source present in PRFT vessel } \\
\text { Operator erroneously closes PRFT CO2 supply needle valve } \\
\text { SCVC exit pressure controller fails low }\end{array}$ & $\begin{array}{l}1 \\
4 \\
5\end{array} \mid$ & $\begin{array}{r}1 \mathrm{~N} \\
3.0 \mathrm{E}-02 \mathrm{~N} \\
43 \mathrm{H} \\
5.0 \mathrm{E}-06 \mathrm{H} \\
43 \mathrm{H} \\
1.0 \mathrm{E}-06 \mathrm{H}\end{array}$ & $\begin{array}{l}3.00 \mathrm{E}-02 \mathrm{~N} \\
2.15 \mathrm{E}-04 \\
2.15 \mathrm{E}-05\end{array}$ & $2.82 \mathrm{E}-08$ \\
\hline 18. & $\begin{array}{l}\text { CC2SCVC-O2-K\# } \\
\text { IGNPRFT-PRE1\# } \\
\text { PCV1019-FTOB\# } \\
\text { PCV1104-FTO9\# }\end{array}$ & $\begin{array}{l}\text { CC: Both SCVC O2 detectors fail high } \\
\text { Ignition source present in PRFT vessel } \\
\text { PRFT purge vacuum breaker PCV-1019 fails to open. } \\
\text { PR purge vacuum breaker PCV-1104 fails to open }\end{array}$ & $\left|\begin{array}{l}1 \\
1 \\
1 \\
1\end{array}\right|$ & $\begin{array}{r}1 \mathrm{~N} \\
5.0 \mathrm{E}-04 \mathrm{~N} \\
1 \mathrm{~N} \\
3.0 \mathrm{E}-02 \mathrm{~N} \\
1 \mathrm{~N} \\
3.0 \mathrm{E}-03 \mathrm{~N} \\
1 \mathrm{~N} \\
3.0 \mathrm{E}-03 \mathrm{~N}\end{array}$ & $\begin{array}{l}5.00 \mathrm{E}-04 \mathrm{~N} \\
3.00 \mathrm{E}-02 \mathrm{~N} \\
3.00 \mathrm{E}-03 \mathrm{~N} \\
3.00 \mathrm{E}-03 \mathrm{~N}\end{array}$ & $2.60 \mathrm{E}-08$ \\
\hline
\end{tabular}




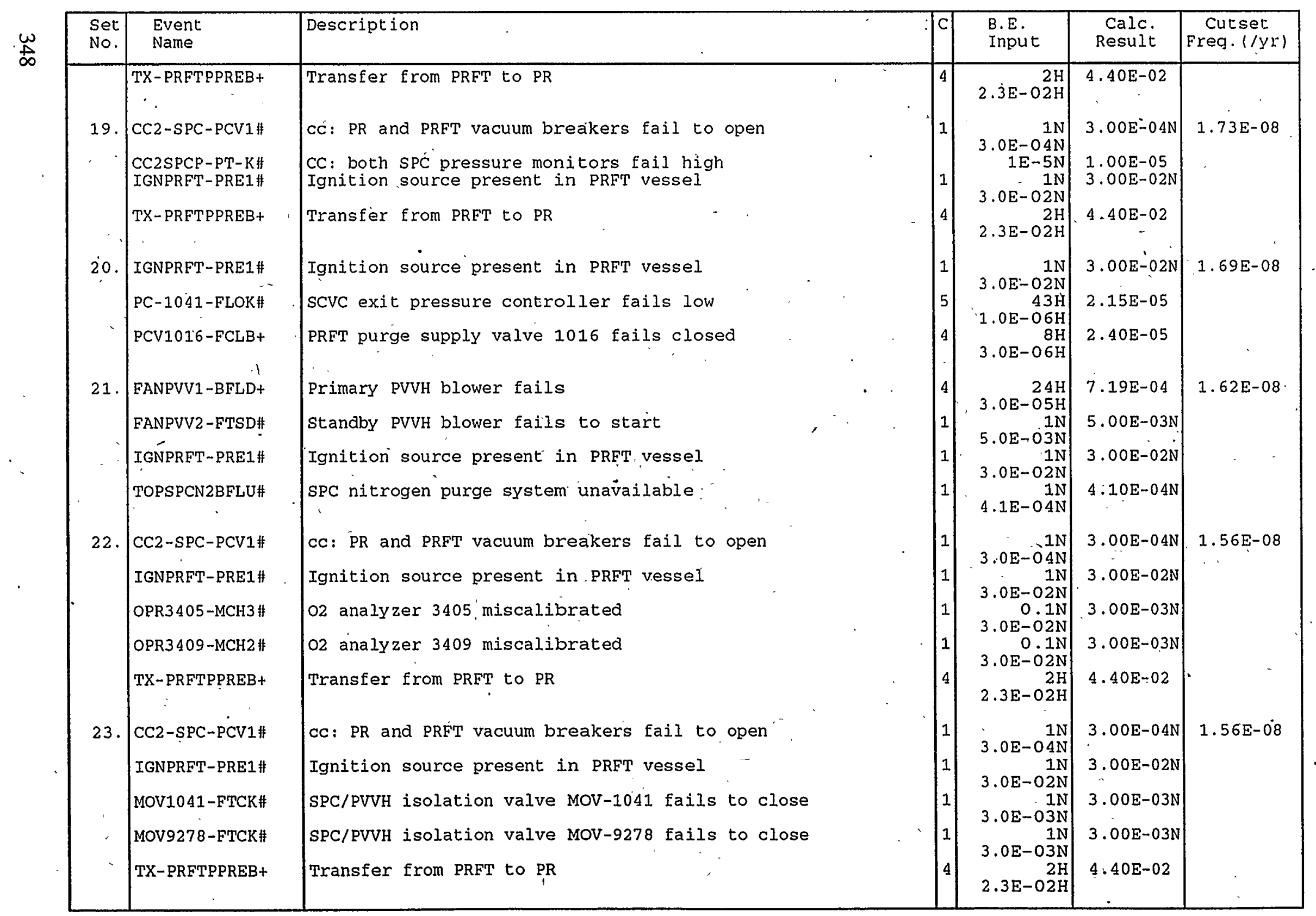


$10: 26$ AM (CONT.)

\begin{tabular}{|c|c|c|c|c|c|c|}
\hline $\begin{array}{l}\text { Set } \\
\text { No. }\end{array}$ & $\begin{array}{l}\text { Event } \\
\text { Name }\end{array}$ & Description & C & $\begin{array}{l}\text { B.E. } \\
\text { Input }\end{array}$ & $\begin{array}{l}\text { Calc. } \\
\text { Result }\end{array}$ & $\begin{array}{c}\text { Cutset } \\
\text { Freq. }(/ y r)\end{array}$ \\
\hline 24. & $\begin{array}{l}\text { CC2SPCV-MOVK\# } \\
\text { IGNPRFT-PRE1\# } \\
\text { PCV1019-FTOB\# } \\
\text { PCV1104-FTO9 } \\
\text { TX-PRFTPPREB+ }\end{array}$ & $\begin{array}{l}\text { cc: both scVC exit valves fail to close } \\
\text { Ignition source present in PRFT vessel } \\
\text { PRFT purge vacuum breaker PCV-1019 fails to open } \\
\text { PR purge vacuum breaker PCV-1104 fails to open } \\
\text { Transfer from PRFT to PR }\end{array}$ & $\begin{array}{l}1 \\
1 \\
1 \\
1 \\
4\end{array}$ & $\begin{array}{r}1 \mathrm{~N} \\
3.0 \mathrm{E}-04 \mathrm{~N} \\
1 \mathrm{~N} \\
3.0 \mathrm{E}-02 \mathrm{~N} \\
1 \mathrm{~N} \\
3.0 \mathrm{E}-03 \mathrm{~N} \\
1 \mathrm{~N} \\
3.0 \mathrm{E}-03 \mathrm{~N} \\
2 \mathrm{H} \\
2.3 \mathrm{E}-02 \mathrm{H}\end{array}$ & $\begin{array}{l}3.00 \mathrm{E}-04 \mathrm{~N} \\
3.00 \mathrm{E}-02 \mathrm{~N} \\
3.00 \mathrm{E}-03 \mathrm{~N} \\
3.00 \mathrm{E}-03 \mathrm{~N} \\
4.40 \mathrm{E}-02\end{array}$ & $1.56 \mathrm{E}-08$ \\
\hline 25. & $\begin{array}{l}\text { CC2 SPCP-PT-K\# } \\
\text { IGNPRFT-PRE1\# } \\
\text { OPRSV7 4DRANK+ }\end{array}$ & $\begin{array}{l}\text { CC: both SPC pressure monitors fail high } \\
\text { Ignition source present in PRFT vessel } \\
\text { Operator erroneously closes PRFT CO2 supply needle valve }\end{array}$ & $\begin{array}{l}i \\
4\end{array} \mid$ & $\begin{array}{r}1 \mathrm{E}-5 \mathrm{~N} \\
1 \mathrm{~N} \\
3.0 \mathrm{E}-02 \mathrm{~N} \\
43 \mathrm{H} \\
5.0 \mathrm{E}-06 \mathrm{H}\end{array}$ & $\begin{array}{l}1.00 \mathrm{E}-05 \\
3.00 \mathrm{E}-02 \mathrm{~N} \\
2.15 \mathrm{E}-04\end{array}$ & $1.31 E-08$ \\
\hline
\end{tabular}




Basic EVent Name
TOPPOWN-BFLH+-I
FE-5860-FLOK+I
FE-6041-FLOK+-I
CC2PVVH-FAN1+-I
SV-CO22-FCLQ+-I
SV-CO21-FCLQ+-I
OPRSV74DRANK+I
PCV1016-FCLB+-I
PSV716-FOPB+-I
HCV1017-FCLB+I
TOPSPCN2BFLU\#
CC2-SPC-PCV1\#
TOPSPCPPBFL1+I
CC2SPCP-PT-K\#
PC-1041-FLOK\#
CC2SPCV-MOVK\#
CC2SCVC-O2-K\#
MOV5862-FCLD+-I
FANPVV1-BFLD+I
OPRCO2-CALK\#
IGNPRFT-PRE1\#
TX-PRFTPPREB+I
CV-PRFT-FTÓI\#
CV-PR-FTOJ\#
PCV1104-FTO9\#
PCV1019-FTOB\#
MOV1041-FTCK\#
MOV9278-FTCK\#
CO2BOTTLCHK1\#
TOPSPCBPBFLO\#
OPR3409-MCH2\#
OPR3405-MCH3\#
AE-3409-FLOK\#
AE-3405-FLOK\#
FANPVV2-BFLD\#
OPRPVV2-RMH1\#
MOV5864-FTOD\#
FANPVV2-T\&MD\#
FANPVV2-FTSD\#
FE-1018-FHIE\#
OPR1018-MCH1\#
PT-1041-FHIK\#

\section{Risk Achievement Worth} Description LOSS OF NORMAL ELECTRIC POWER TO DWPF

PVVH flow element 5860 fails low

PVVH flow monitor 6041 fails low

cc: both PVVH fans fail

SPC $\mathrm{CO} 2$ purge isolation valve 2 fails closed

SPC CO2 purge isolation valve 1 fails closed

Operator erroneously closes PRFT CO2 supply needle valve

PRFT purge supply valve 1016 fails closed

PSV on PRFT purge supply line fails open

PRFT purge supply valve 1017 fails closed

SPC nitrogen purge system unavailable

cc: PR and PRFT vacuum breakers fail to open

SPC primary purge fails (excluding normal power)

CC: both SPC pressure monitors fail high

SCVC exit pressure controller fails low

cc: both SCVC exit valves fail to close

CC: Both SCVC O2 detectors fail high

PVVH primary fan suction valve fails closed

Primary PVVH blower fails

Operator hooks up $\mathrm{O} 2$ instead of $\mathrm{CO} 2$ for SPC purge gas

Ignition source present in PRFT vessel

Transfer from PRFT to PR

PRFT vacuum breaker $\mathrm{CO} 2$ line check valve fails to open

Check valve for $P R$ vacuum breaker purge fails to open

PR purge vacuum breaker PCV-1104 fails to open

PRFT purge vacuum breaker PCV-1019 fails to open

SPC/PVVH isolation valve MOV-1041 fails to close

SPC/PVVH isolation valve MOV-9278 fails to close

Fail to correct wrong bottles before system used

Failure of backup $\mathrm{CO} 2$ system

O2 analyzer 3409 miscalibrated

O2 analyzer 3405 miscalibrated

SCVC O2 concentration analyzer 3409 fails low

SCVC 02 concentration analyzer 3405 fails low

PVVH backup fan fails to run

Fail to restore fan to operable status following maintenance

PVVH standby fan suction valve fails to open

PVVH standby fan unavailable, due to T\&M

Standby PVVH blower fails to start

PRFT purge flow element 1018 fails to detect low flow

PRFT purge flow element 1018 miscalibrated

PR vapor space PT-1041 fails high
Prob/Freg

$3.50 \mathrm{E}-05 / \mathrm{H}$

$3.00 \mathrm{E}-06 / \mathrm{H}$

$3.00 \mathrm{E}-06 / \mathrm{H}$

$3.00 \mathrm{E}-06 / \mathrm{H}$

$3.00 \mathrm{E}-06 / \mathrm{H}$

$3.00 \mathrm{E}-06 / \mathrm{H}$

$5.00 \mathrm{E}-06 / \mathrm{H}$

$3.00 \mathrm{E}-06 / \mathrm{H}$

$5.00 \mathrm{E}-07 / \mathrm{H}$

$5.00 \mathrm{E}-07 / \mathrm{H}$

4.10E-04

3.00E-04

$6.69 \mathrm{E}-05 / \mathrm{H}$

8.40E-06

2.15E-05

3.00E-04

5.00E-04

$3.00 \mathrm{E}-07 / \mathrm{H}$

$3.00 \mathrm{E}-05 / \mathrm{H}$

3.00E-04

3.00E-02

$2.20 \mathrm{E}-02 / \mathrm{H}$

5.00E-04

$5.00 \mathrm{E}-04$

$3.00 \mathrm{E}-03$

$3.00 \mathrm{E}-03$

$3.00 \mathrm{E}-03$

$3.00 \mathrm{E}-03$

$4.92 \mathrm{E}-02$

$6.70 \mathrm{E}-03$

$3.00 \mathrm{E}-03$

3. $00 \mathrm{E}-03$

1.07E-04

1.07E-04

$7.20 \mathrm{E}-04$

$1.50 \mathrm{E}-03$

3.00E-03

3.65E-03

5.00E-03

$2.16 \mathrm{E}-03$

$1.20 \mathrm{E}-04$

2.15E-05
AchW

$1.36 \mathrm{E}+04$

$1.31 \mathrm{E}+04$

$1.31 \mathrm{E}+04$

$1.31 \mathrm{E}+04$

$1.31 \mathrm{E}+04$

$1.31 \mathrm{E}+04$

$1.09 \mathrm{E}+04$

$1.09 \mathrm{E}+04$

$1.09 \mathrm{E}+04$

$1.09 \mathrm{E}+04$

$1.62 \mathrm{E}+03$

$5.99 \mathrm{E}+02$

$5.46 \mathrm{E}+02$

$5.24 \mathrm{E}+02$

$5.24 \mathrm{E}+02$

$5.24 \mathrm{E}+02$

$2.22 \mathrm{E}+02$

$1.83 \mathrm{E}+02$

$1.83 \mathrm{E}+02$

$1.61 \mathrm{E}+02$

3.33E+01

$9.30 \mathrm{E}+00$

$3.09 \mathrm{E}+00$

$3.09 \mathrm{E}+00$

$3.09 \mathrm{E}+00$

$3.09 \mathrm{E}+00$

$2.56 \mathrm{E}+00$

$2.56 \mathrm{E}+00$

$1.93 \mathrm{E}+00$

$1.72 \mathrm{E}+00$

$1.68 \mathrm{E}+00$

$1.68 \mathrm{E}+00$

$1.68 \mathrm{E}+00$

$1.68 \mathrm{E}+00$

$1.40 \mathrm{E}+00$

$1.40 \mathrm{E}+00$

$1.40 \mathrm{E}+00$

$1.40 \mathrm{E}+00$

$1.40 \mathrm{E}+00$

$1.15 \mathrm{E}+00$

$1.15 \mathrm{E}+00$

$1.03 \mathrm{E}+00$

Note: Events that make less than $1 \%$ difference to the importance factor are not included. Modified top event frequency $=$ (top event frequency) $\mathrm{x}$ (risk achievement worth). 
Explosion in the PRFT Top Event Frequency: 8.20E-06/YR

Basic Event Name
*IGNPRFT-PRE1\#
TOPSPCN2BFLU\#
TOPPOWN-BFLH+-I
TX-PRFTPPREB+-I
CC2-SPC-PCV1\#
CC2SPCV-MOVK\#
CC2SCVC-O2-K\#
OPRSV74DRANK+-I
CO2BOTTLCHK1\#
OPRCO2-CALK\#
SV-CO22-FCLQ+-I
FE-5860-FLOK+-I
FE-6041-FLOK+-I
SV-CO21-FCLQ+-I
CC2PVVH-FAN1+I
TOPSPCPPBFL1+-I
PCV1016-FCLB+-I
PC-1041-FLOK\#

\section{- Risk Reduction Worth}

Description

Ignition source present in PRFT vessel

SPC nitrogen purge system unavailable

LOSS OF NORMAL ELECTRIC POWER TO DWPF *

Transfer from PRFT to PR

cc: PR and PRFT vacuum breakers fail to open

cc: both SCVC exit valves fail to close

CC: Both SCVC 02 detectors fail high

Operator erroneously closes PRFT CO2 supply needle valve

Fail to correct wrong bottles before system used

Operator hooks up $\mathrm{O} 2$ instead of $\mathrm{CO} 2$ for SPC purge gas

SPC CO2 purge isolation valve 2 fails closed

PVVH flow element 5860 fails low

PVVH flow monitor 6041 fails low

SPC CO2 purge isolation valve 1 fails closed

cc: both PVVH fans fail

SPC primary purge fails (excluding normal power)

PRFT purge supply valve 1016 fails closed

SCVC exit pressure controller fails low

\begin{tabular}{ll} 
Prob/Freq & RedW \\
\hline $3.00 \mathrm{E}-02$ & $0.00 \mathrm{E}+00$ \\
$4.10 \mathrm{E}-04$ & $2.96 \mathrm{E}+00$ \\
$3.50 \mathrm{E}-05 / \mathrm{H}$ & $1.91 \mathrm{E}+00$ \\
$2.20 \mathrm{E}-02 / \mathrm{H}$ & $1.23 \mathrm{E}+00$ \\
$3.00 \mathrm{E}-04$ & $1.22 \mathrm{E}+00$ \\
$3.00 \mathrm{E}-04$ & $1.19 \mathrm{E}+00$ \\
$5.00 \mathrm{E}-04$ & $1.12 \mathrm{E}+00$ \\
$5.00 \mathrm{E}-06 / \mathrm{H}$ & $1.06 \mathrm{E}+00$ \\
$4.92 \mathrm{E}-02$ & $1.05 \mathrm{E}+00$ \\
$3.00 \mathrm{E}-04$ & $1.05 \mathrm{E}+00$ \\
$3.00 \mathrm{E}-06 / \mathrm{H}$ & $1.04 \mathrm{E}+00$ \\
$3.00 \mathrm{E}-06 / \mathrm{H}$ & $1.04 \mathrm{E}+00$ \\
$3.00 \mathrm{E}-06 / \mathrm{H}$ & $1.04 \mathrm{E}+00$ \\
$3.00 \mathrm{E}-06 / \mathrm{H}$ & $1.04 \mathrm{E}+00$ \\
$3.00 \mathrm{E}-06 / \mathrm{H}$ & $1.04 \mathrm{E}+00$ \\
$6.69 \mathrm{E}-05 / \mathrm{H}$ & $1.04 \mathrm{E}+00$ \\
$3.00 \mathrm{E}-06 / \mathrm{H}$ & $1.03 \mathrm{E}+00$ \\
$2.15 \mathrm{E}-05$ & $1.01 \mathrm{E}+00$
\end{tabular}

* A Reduction Worth of $0.0 \mathrm{E}+00$ indicates event appears in every cutset could serve as a single event preventor for the accident.

Note: Events that make less than 1\% difference to the importance factor are not included. Modified top event frequency = (top event frequency) / (risk reduction worth). 
X-ESR-S-00001, REV. 0

Steam Explosion in the Melter 


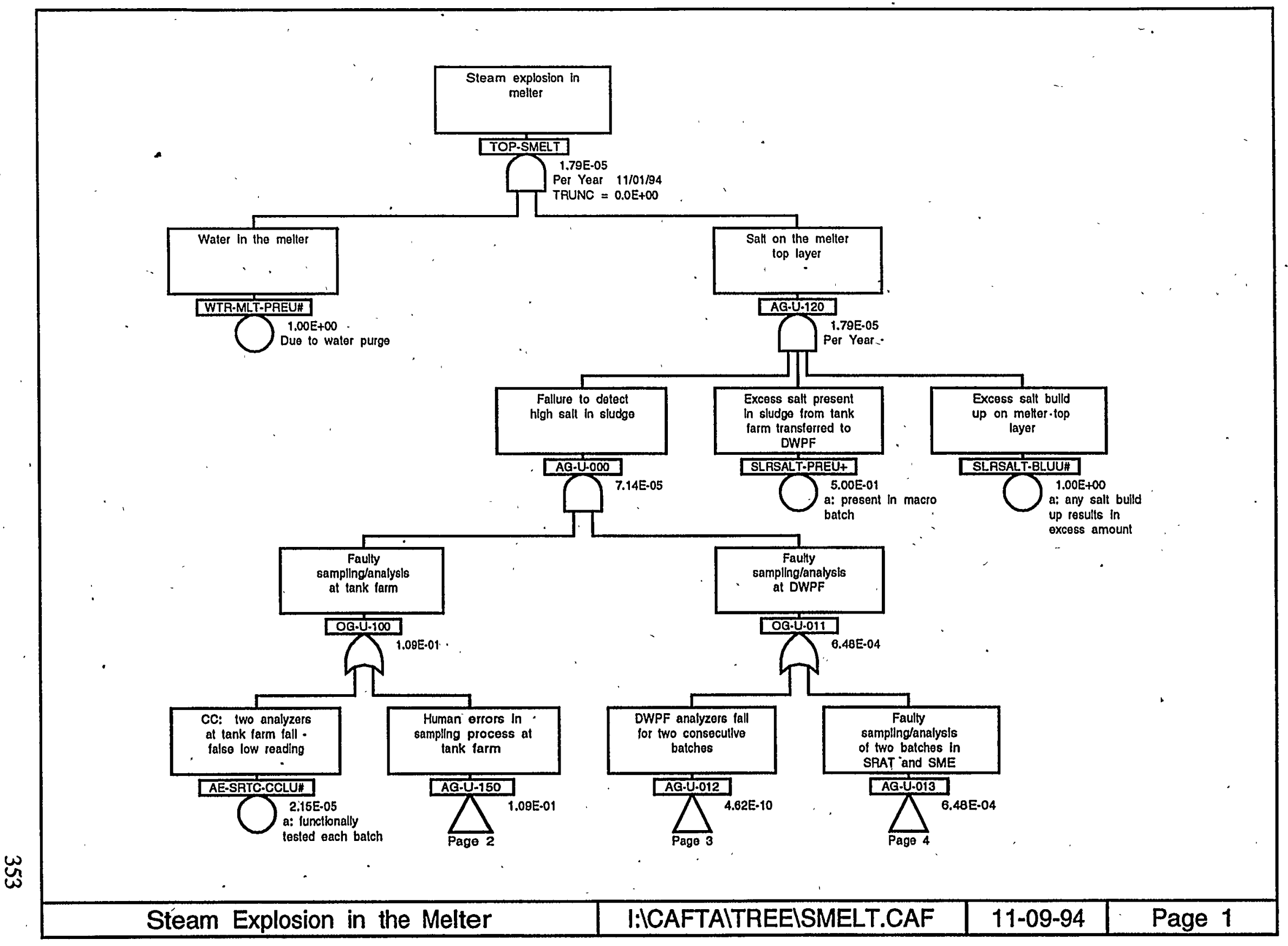




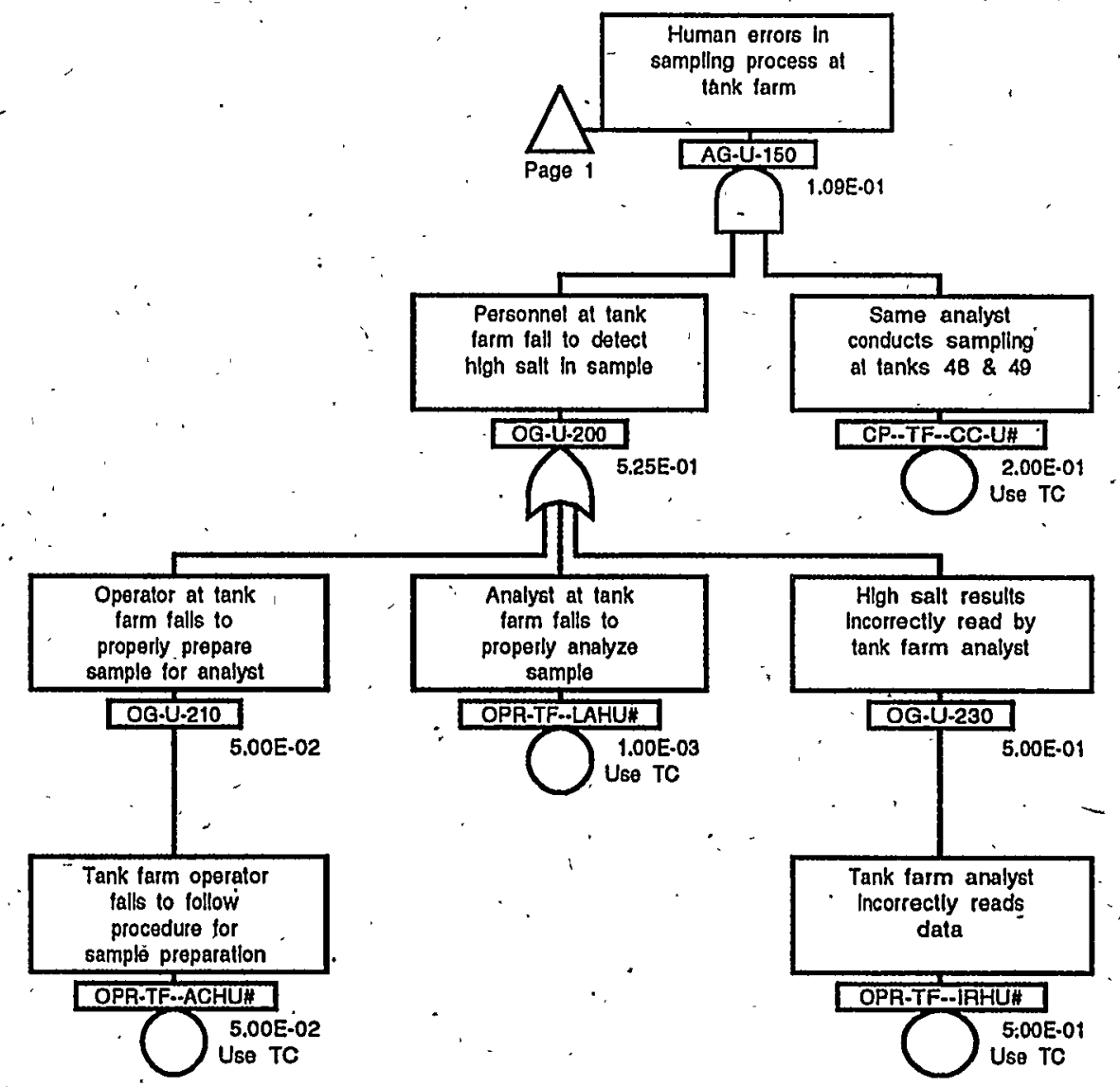




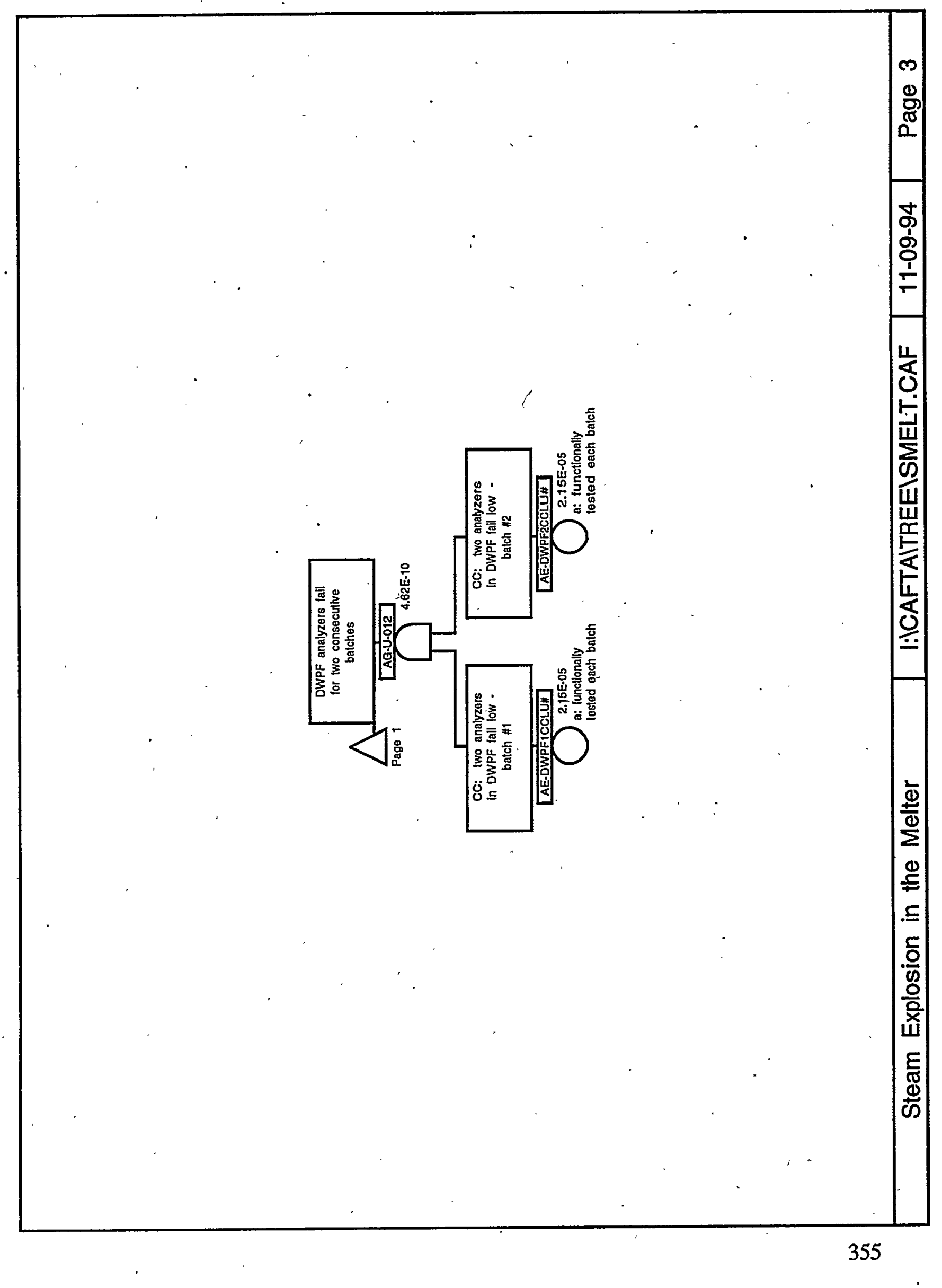




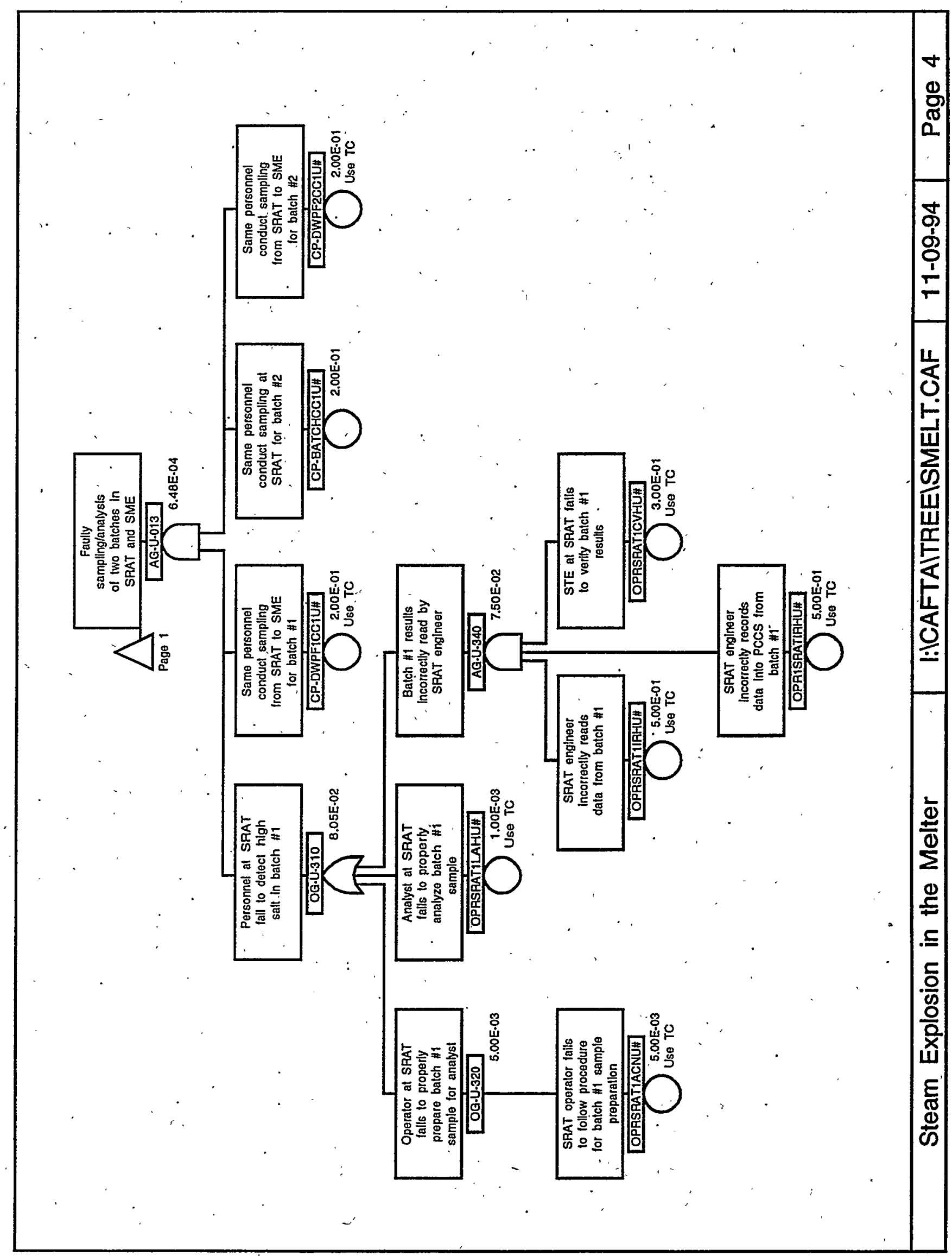




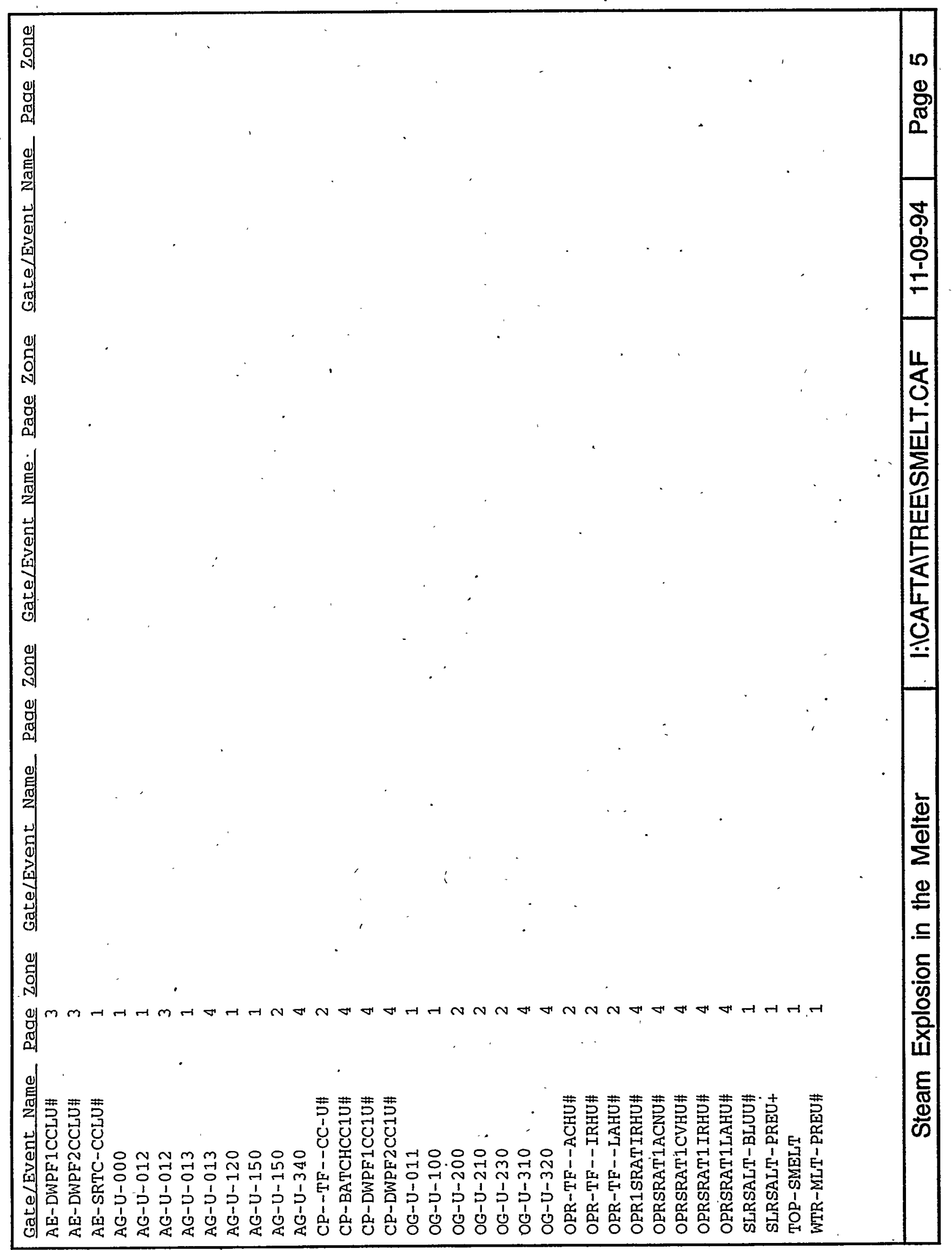




\begin{tabular}{|c|c|c|c|c|c|c|}
\hline $\begin{array}{l}\text { Set } \\
\text { No. }\end{array}$ & $\begin{array}{l}\text { Event } \\
\text { Name }\end{array}$ & Description & C & $\begin{array}{l}\text { B.E. } \\
\text { Input }\end{array}$ & $\begin{array}{l}\text { Calc. } \\
\text { Result }\end{array}$ & $\begin{array}{c}\text { Cutset } \\
\text { Freq. } / \text { /yr) }\end{array}$ \\
\hline \multirow{10}{*}{1.} & TOP-SMELT & & & & & $1.79 \mathrm{E}-05$ \\
\hline & CP- -TF- -CC-U\# & Same analyst conducts sampling at tanks $48 \& 49$ & 1 & & $2.00 \mathrm{E}-01 \mathrm{~N}$ & i. $50 \mathrm{E}-05$ \\
\hline & CP-BATCHCC1U\# & Same personnel conduct sampling at SRAT for batch \#2 & $11 \mid$ & $2.0 \mathrm{E}-01 \mathrm{~N}$ & $2.00 \mathrm{E}-01 \mathrm{~N}$ & \\
\hline & CP-DWPF1CC1U\# & Same personnel conduct sampling from SRAT to SME for batch & 1 & $2.0 \mathrm{E}-01 \mathrm{~N}$ & 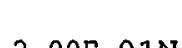 & - \\
\hline & 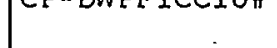 & 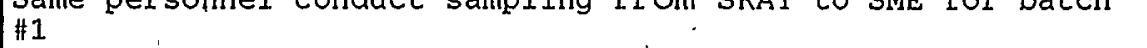 & $1+1$ & $2.0 \mathrm{E}-01 \mathrm{~N}$ & $2.00 \mathrm{E}-0,0 \underline{\mathrm{N}} \mathrm{N}$ & \\
\hline & CF- DWPF2CC1U\# & Same personnel conduct sampling from SRAT to SME for batch & 1 & $1 \mathrm{~N}$ & $2.00 \mathrm{E}-01 \mathrm{~N}$ & \\
\hline & OPR-TF- - IRHUH & Tank farm analyst incorrectly reads data. & 1 & $1 \mathrm{~N}$ & $5.00 \mathrm{E}-0.1 \mathrm{~N}$ & \\
\hline & OPRISRATIRHU\# & SRAT engineer incorrectly records data into PCCS from batch & 1 & $\begin{array}{r}01 N \\
1 N\end{array}$ & $5.00 \mathrm{E}-01 \mathrm{~N}$ & \\
\hline & JOPRSRAT1CVHU\# & $\begin{array}{l}\# 1 \\
\text { STE at SRAT fails to verify batch \#1 results. }\end{array}$ & $1 \mid$ & $5.0 \mathrm{E}-01 \mathrm{~N}$ & $300 \mathrm{E}-01 \mathrm{~N}$ & \\
\hline & ( & 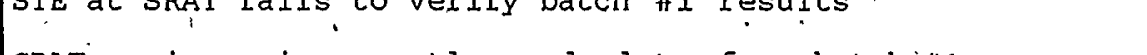 & 1 & $3.0 \mathrm{E}-01 \mathrm{~N}$ & $3.00 \mathrm{E}-\mathrm{U} 1 \mathrm{IV}$ & \\
\hline & OPRSRATIIRHU\# & SRAT engineer incorrectly reads data from batch \#1 & 1 & & $5.00 \mathrm{E}-01 \mathrm{~N}$ & \\
\hline & SLRSALT-BLUU\# & Excess salt build up on melter top layer & 1 & $1 \mathrm{n}$ & $1.00 \mathrm{E}+00 \mathrm{~N}$ & \\
\hline & SLRSALLT-PREU+ & Excess salt present in sludge from tank farm transferred to & 4 & $\begin{array}{r}1.0 \mathrm{E}+00 \mathrm{~N} \\
2 \mathrm{Y}\end{array}$ & $5.00 \mathrm{E}-01$ & \\
\hline & WTR-MLT-PREU\# & in the melter & 1 & $5.0 \mathrm{E}-01 \mathrm{Y}$ & & \\
\hline & 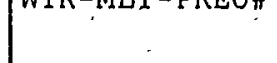 & Waler in line inester & & $1.0 \mathrm{E}+00 \mathrm{~N}$ & $1.00 \mathrm{E}+00 \mathrm{~N}$ & \\
\hline \multirow[t]{12}{*}{2 . } & CP- -TF--CC-U\# & Same analyst conducts sampling at tanks $48 \& 49$ & 1 & $2.0 \mathrm{E}-01 \mathrm{NN}$ & $2.00 \mathrm{E}-01 \mathrm{~N}$ & $1.50 \mathrm{E}-06$ \\
\hline & CP-BATCHCC1U\# & Same personnel conduct sampling at SRAT for batch \#2 & 1 & & $2.00 \mathrm{E}-01 \mathrm{~N}$ & \\
\hline & CP-DWPF1C̣C1U\# & ne personnel conduct sampling from SRAT to SME for batch. & 1 & 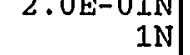 & $2.00 \mathrm{E}-01 \mathrm{~N}$ & \\
\hline & CP-DWPF2CC1U\# & $\begin{array}{l}\text { \#1 } \\
\text { Same personnel conduct sampling from-SRAT to SME for batch }\end{array}$ & 1 & $.0 \mathrm{E}-01 \mathrm{~N}$ & 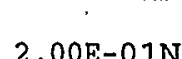 & - \\
\hline & & 1. & & $2.0 \mathrm{E}-01 \mathrm{~N}$ & & \\
\hline & OPR-TYF--ACHU\# & $\begin{array}{l}\text { Tank farm operator fails to follow procedure for sample } \\
\text { preparation }\end{array}$ & 1 & $5.0 \mathrm{E}-02 \mathrm{~N}$ & $5.00 \mathrm{E}-02 \mathrm{~N}$ & - \\
\hline & OPRISRATIRHU\# & $\begin{array}{l}\text { SRAT engineer incorrectly records data into PCCS from batch } \\
\# 1 \text {. }\end{array}$ & 1 & $5.0 \mathrm{E}-01 \mathrm{NN}$ & $5.00 \mathrm{E}-01 \mathrm{~N}$ & \\
\hline & OPRSRAT1CVHU\# & STE at SRAT fails to verify batch \#1 results & 1 & & $3.00 \mathrm{E}-01 \mathrm{~N}$ & \\
\hline & OPRSRATIIRHU\# & SRAT engineer incorrectly reads data from batch \#1 & 1 & & $5.00 \mathrm{E}-01 \mathrm{~N}$ & \\
\hline & SLRSALT-BLUU\# & Excess salt build up on melter top layer & 1 & & $1.00 \mathrm{E}+00 \mathrm{~N}$ & 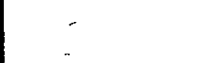 \\
\hline & SLRSALT-PREU+ & $\begin{array}{l}\text { Excess salt present in sludge from tank farm transferred to } \\
\text { DWPF }\end{array}$ & 4 & $\begin{array}{r}1.0 \mathrm{E}+\mathrm{ON} \\
5.0 \mathrm{E}-01 \mathrm{Y}\end{array}$ & $5.00 \mathrm{E}-01$ & \\
\hline & WTR-MLT-PREU\# & Water in the melter & 1 & $1.0 \mathrm{E}+00 \mathrm{~N}$ & $1.00 \mathrm{E}+0 \mathrm{ON}$ & \\
\hline
\end{tabular}


Cutsets for I : \CAFTA \CUT\SMELT.CSR

11/09/94 10:28 AM iCONT.)

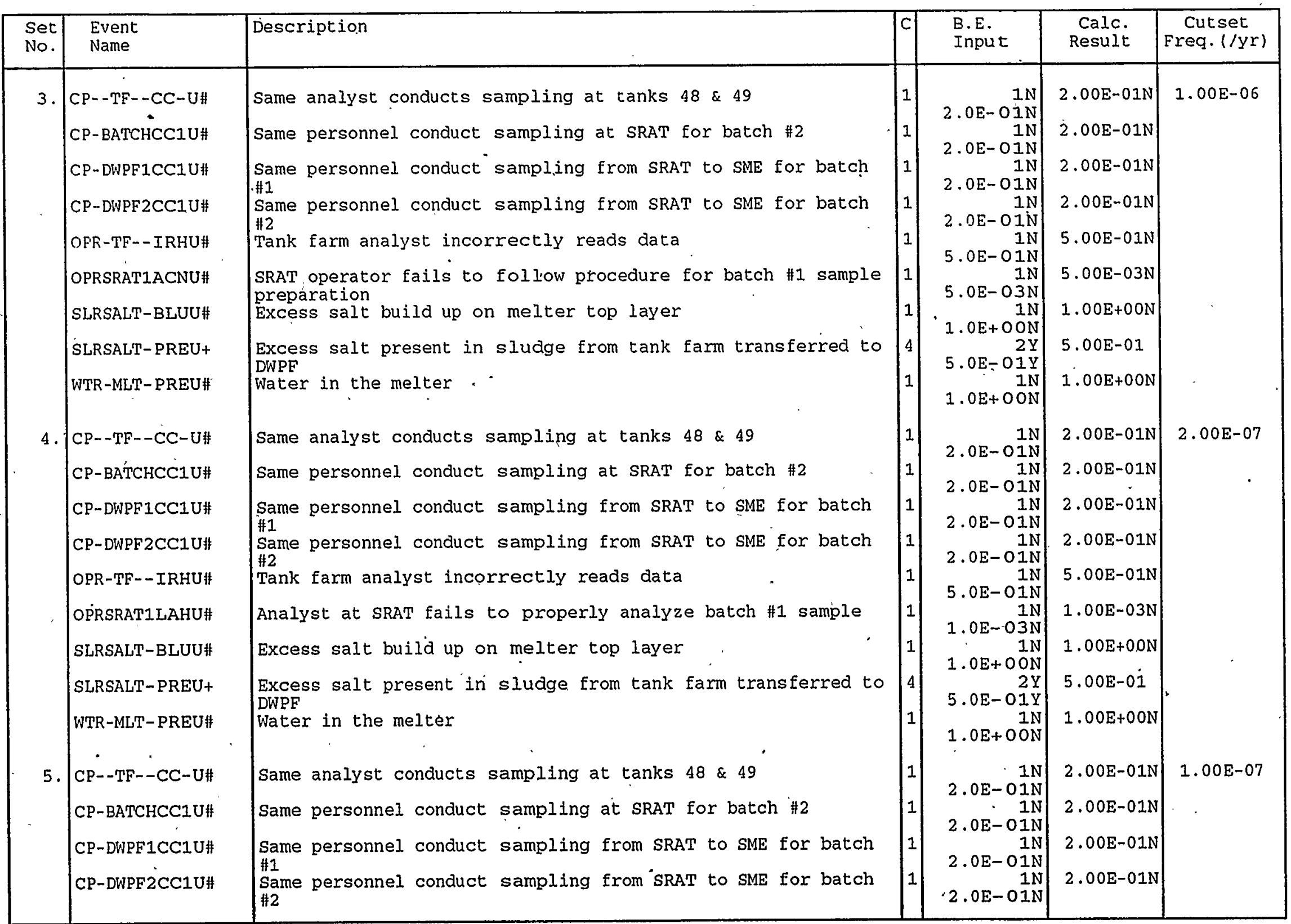




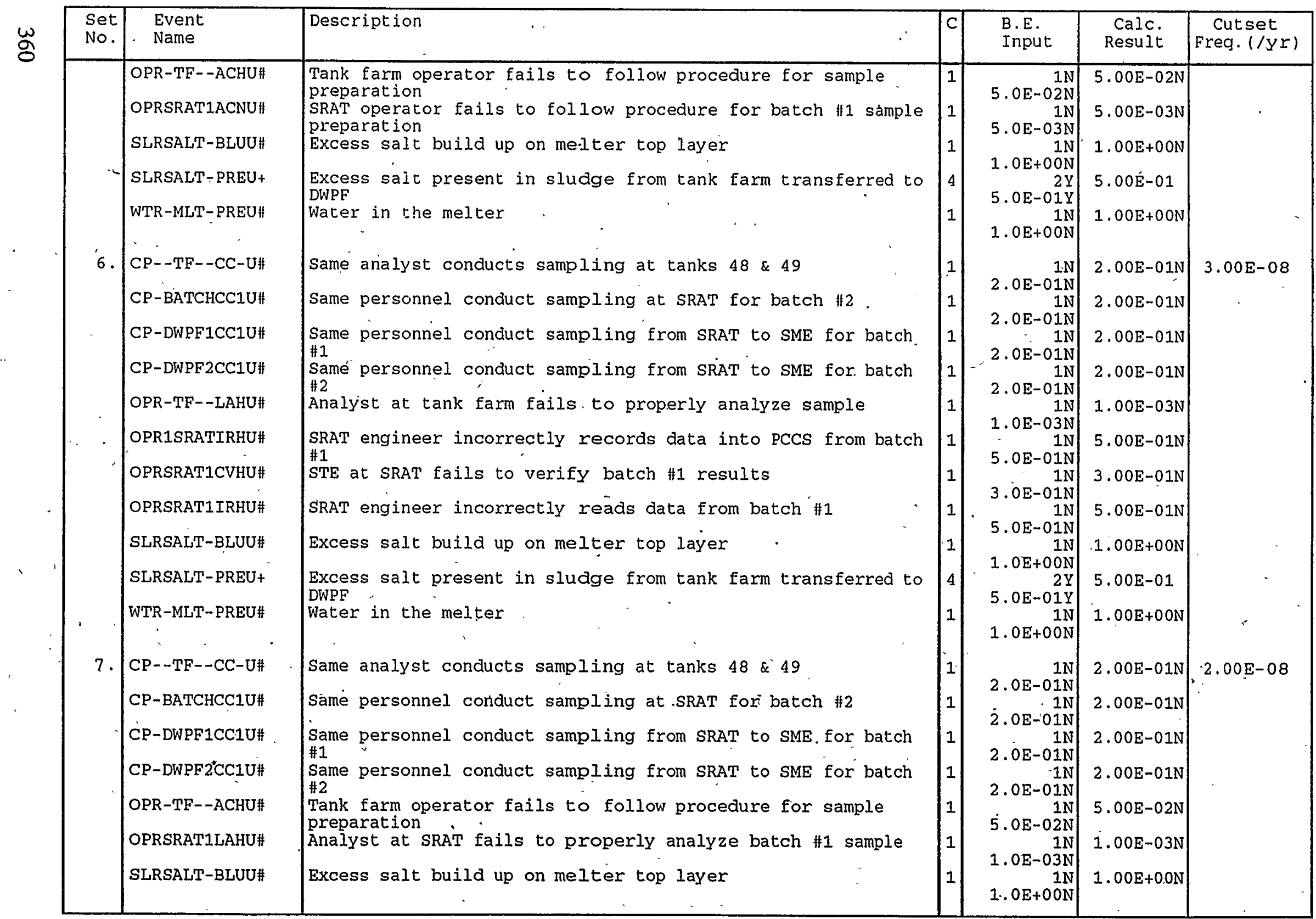




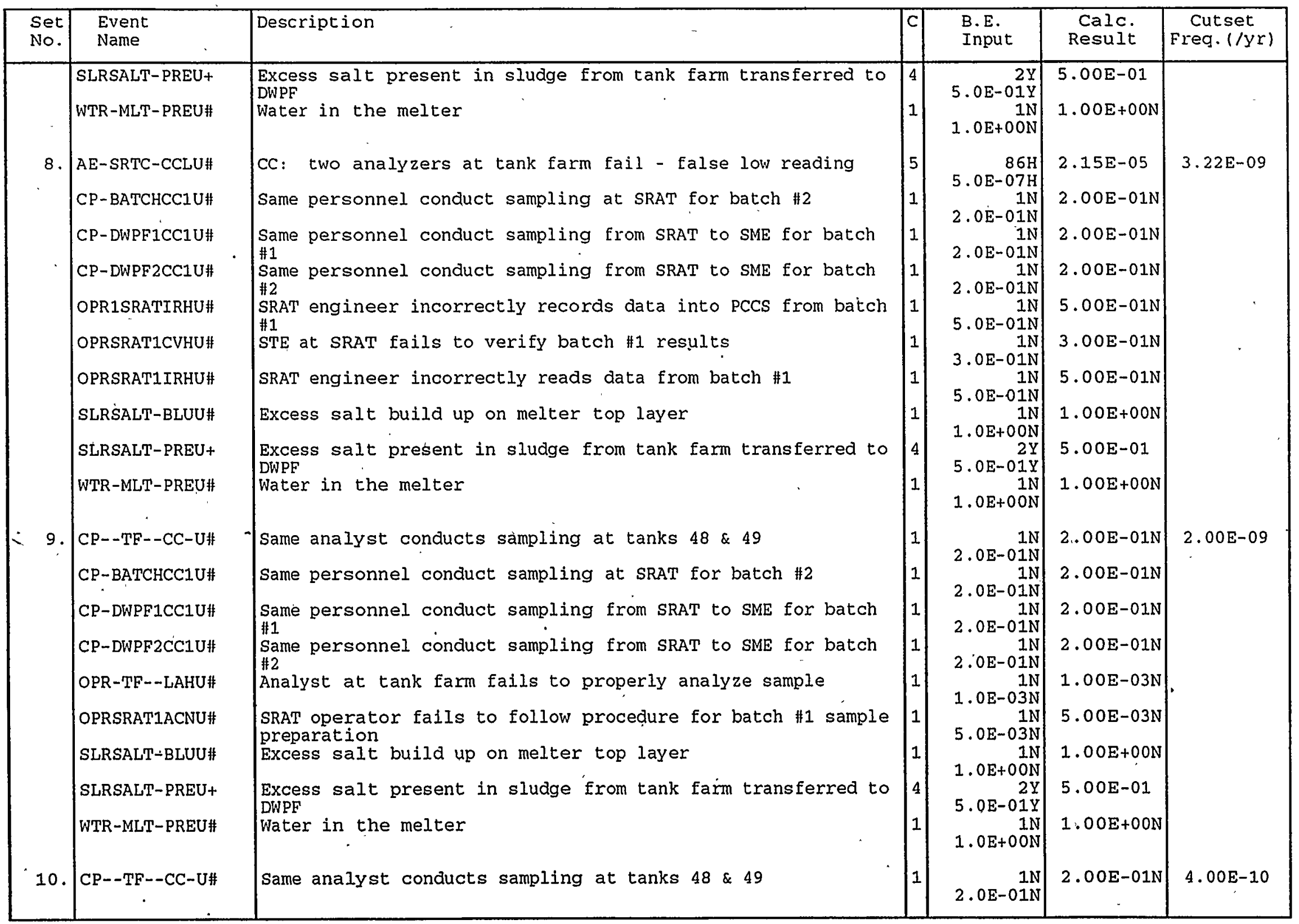




\begin{tabular}{|c|c|c|c|c|c|c|c|}
\hline & $\begin{array}{l}\text { Set } \\
\text { No. }\end{array}$ & Name & ription & $\mathrm{C}$ & $\begin{array}{l}\text { B.E. } \\
\text { Input }\end{array}$ & $\begin{array}{l}\text { Calc. } \\
\text { Resuli }\end{array}$ & $\begin{array}{l}\text { Cut } \\
\text { Freq. }\end{array}$ \\
\hline - & 11. & $\begin{array}{l}\text { CP-BATCHCC1U\# } \\
\text { CP-DWPF1CC1U\# } \\
\text { CP-DWPF2CC1U\# } \\
\text { OPR-TF--LAHU\#, } \\
\text { OPRSRAT1LAHU\# } \\
\text { SLRSALT-BLUU\# } \\
\text { SLRSALT-PREU+ } \\
\text { WTR-MLT-PREU\# } \\
\text { AE-SRTC-CCLU\# } \\
\text { CP-BATCHCC1U\# } \\
\text { CP-DWPF1CC1U\# } \\
\text { CP-DWPF2CC1U\# } \\
\text { OPRSRAT1ACNU\# } \\
\text { SLRSALT-BLUU\# } \\
\text { SLRSALT-PREU+ } \\
\text { WTR-MLT-PREU\# } \\
\text { AE-SRTC-CCLU\# } \\
\text { CP-BATCHCC1U\# } \\
\text { CP-DWPF1CC1U\# } \\
\text { CP-DWPF2CC1U\# } \\
\text { OPRSRAT1LAHU\# } \\
\text { SLRSALT-BLUU\# } \\
\text { SLRSALT-PREU+ }\end{array}$ & 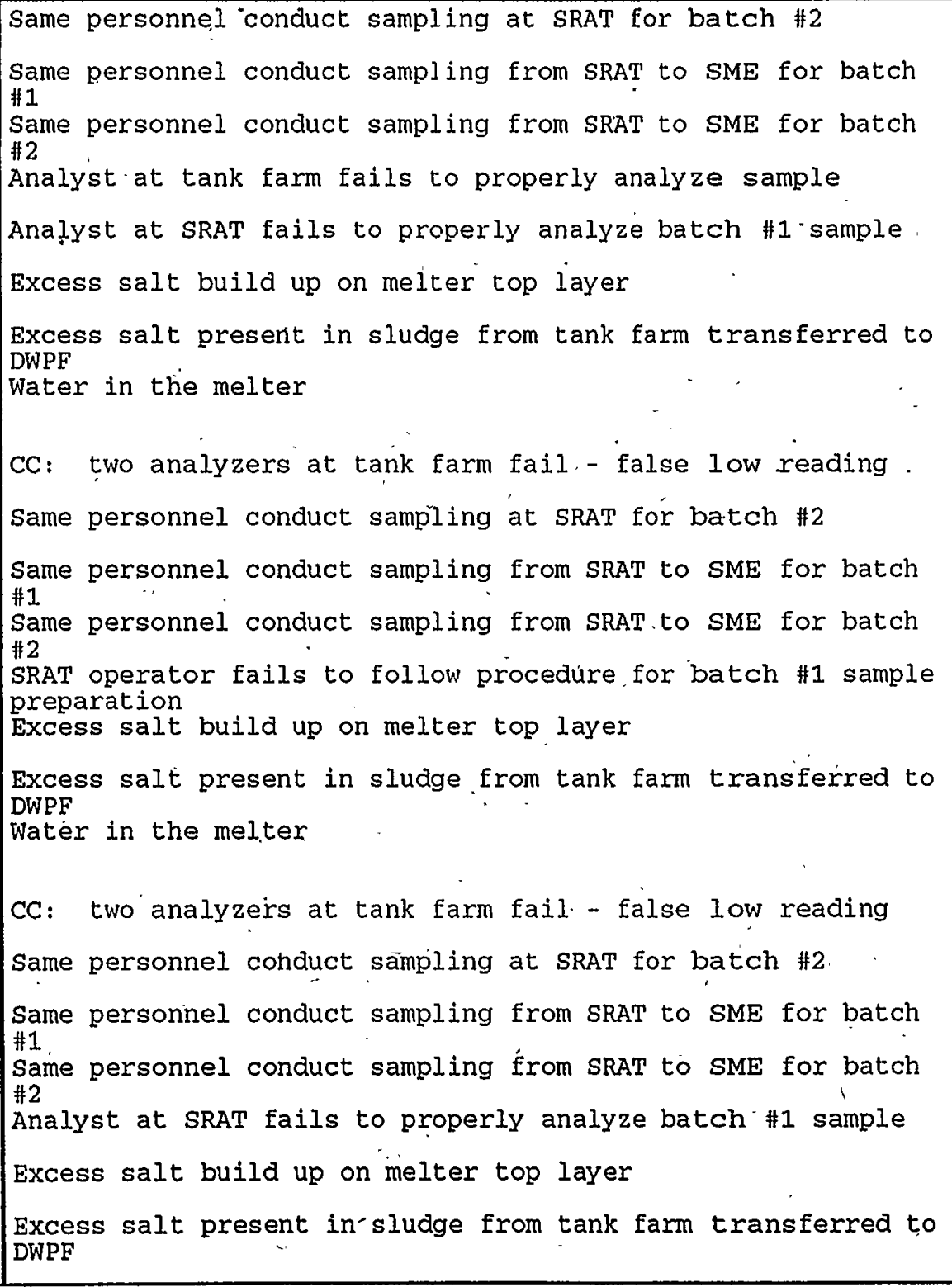 & $\begin{array}{l}1 \\
1 \\
1\end{array}$ & 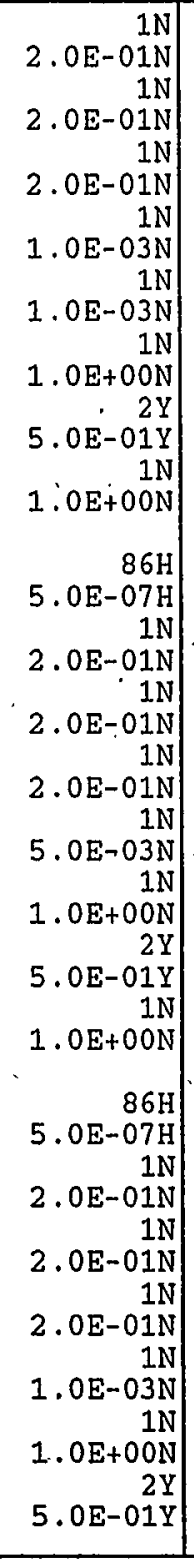 & $\begin{array}{l}2.00 \mathrm{E}-01 \mathrm{~N} \\
2.00 \mathrm{E}-01 \mathrm{~N} \\
1.00 \mathrm{E}-03 \mathrm{~N} \\
1.00 \mathrm{E}-03 \mathrm{~N} \\
1.00 \mathrm{E}+00 \mathrm{~N} \\
5.00 \mathrm{E}-01 \\
1.00 \mathrm{E}+00 \mathrm{~N} \\
2.15 \mathrm{E}-05 \\
2.00 \mathrm{E}-01 \mathrm{~N} \\
2.00 \mathrm{E}-01 \mathrm{~N} \\
2.00 \mathrm{E}-01 \mathrm{~N} \\
5.00 \mathrm{E}-03 \mathrm{~N} \\
1.00 \mathrm{E}+00 \mathrm{~N} \\
5.00 \mathrm{E}-01 \\
1.00 \mathrm{E}+00 \mathrm{~N} \\
2.15 \mathrm{E}-05 \\
2.00 \mathrm{E}-01 \mathrm{~N} \\
2.00 \mathrm{E}-01 \mathrm{~N} \\
2.00 \mathrm{E}-01 \mathrm{~N} \\
1.00 \mathrm{E}-03 \mathrm{~N} \\
1.00 \mathrm{E}+00 \mathrm{~N} \\
5.00 \mathrm{E}-01\end{array}$ & $2.15 \mathrm{E}-10$ \\
\hline
\end{tabular}




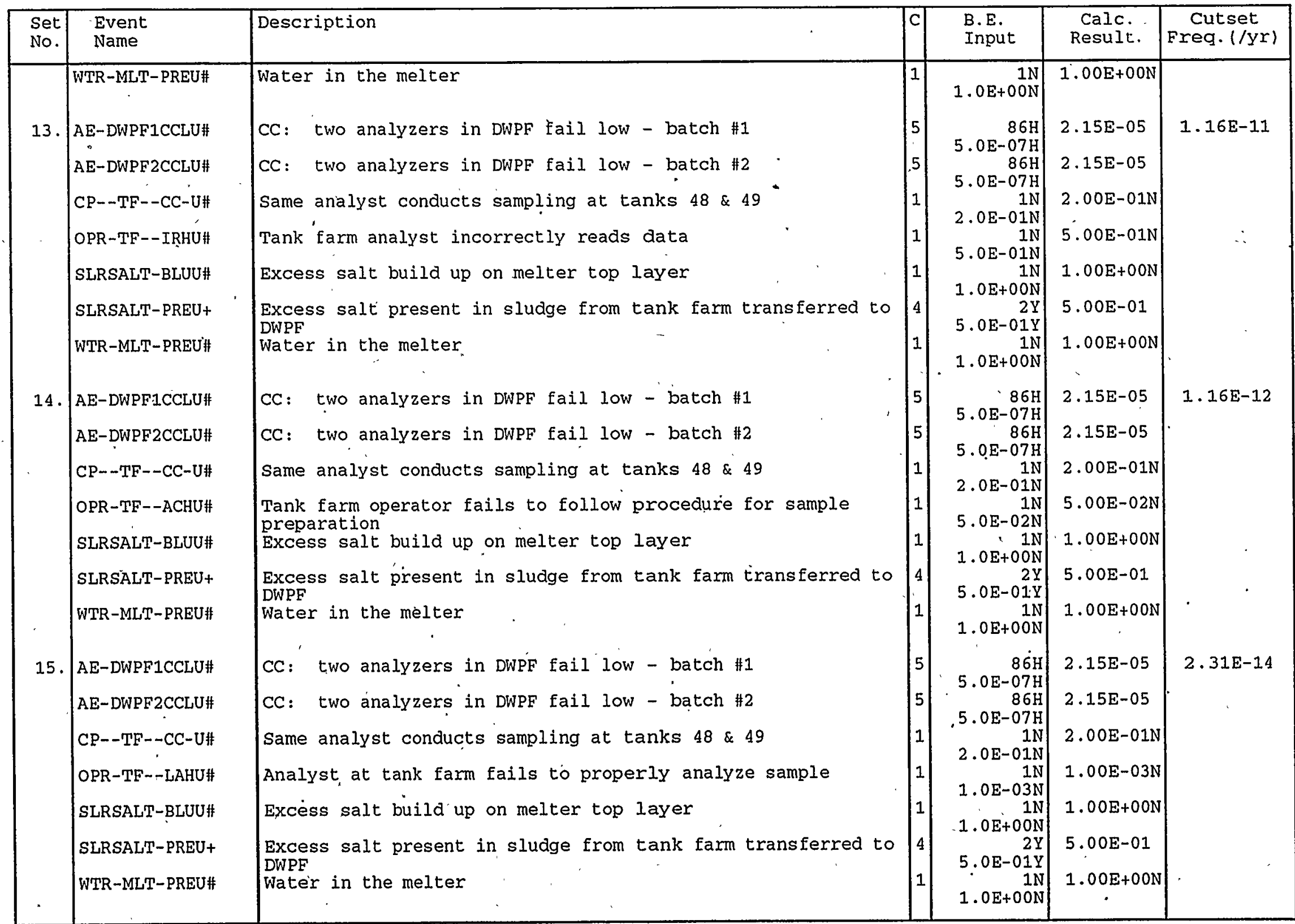


Cutsets for I: \CAFTA \CUT\SMELT.CSR

11/09/94 10:28. AM (CONT:)

\begin{tabular}{|c|c|c|c|c|c|c|}
\hline $\begin{array}{l}\text { Set } \\
\text { No. }\end{array}$ & $\begin{array}{l}\text { Event } \\
\text { Name }\end{array}$ & Description & C & $\begin{array}{l}\text { B.E. } \\
\text { Input }\end{array}$ & $\begin{array}{l}\text { Calc. } \\
\text { Result. }\end{array}$ & $\begin{array}{c}\text { Cutset } \\
\text { Freq. (/Yr) }\end{array}$ \\
\hline 16. & $\begin{array}{l}\text { AE-DWPF1CCLU\# } \\
\text { AE-DWPF2CCLU\# } \\
\text { AE-SRTC-CCLU\# } \\
\text { SLRSALT-BLUU\# } \\
\text { SLRSALT-PREU+ } \\
\text { WTR-MLT-PREU\# }\end{array}$ & $\begin{array}{l}\text { CC: two analyzers in DWPF fail low - batch \#1 } \\
\text { CC: two analyzers in DWPF fail low - batch \#2 } \\
\text { CC: two analyzers at tank farm fail - false low reading } \\
\text { Excess salt build up on melter top layer } \\
\text { Excess salt present in sludge from tank farm transferred to } \\
\text { DWPF } \\
\text { Water in the melter } \\
\text {. }\end{array}$ & $\begin{array}{l}5 \\
5 \\
1\end{array}$ & $\begin{array}{r}86 \mathrm{H} \\
5.0 \mathrm{E}-07 \mathrm{H} \\
86 \mathrm{H} \\
5.0 \mathrm{E}-07 \mathrm{H} \\
86 \mathrm{H} \\
5.0 \mathrm{E}-07 \mathrm{H} \\
1 \mathrm{~N} \\
1.0 \mathrm{E}+00 \mathrm{~N} \\
2 \mathrm{Y} \\
5.0 \mathrm{E}-01 \mathrm{Y} \\
1 \mathrm{~N} \\
1.0 \mathrm{E}+00 \mathrm{~N}\end{array}$ & $\begin{array}{l}2.15 \mathrm{E}-05 \\
2.15 \mathrm{E}-05 \\
2.15 \mathrm{E}-05 \\
1.00 \mathrm{E}+00 \mathrm{~N} \\
5.00 \mathrm{E}-01 \\
1.00 \mathrm{E}+00 \mathrm{~N}\end{array}$ & $\begin{array}{c}2.48 \mathrm{E}-15 \\
-\end{array}$ \\
\hline
\end{tabular}


Steam explosion in melter Top Event Frequency: 1.79E-05/YR

\author{
Basic Event Name \\ SLRSALT-PREUT+-I \\ OPRSRATILAHU\# \\ OPRSRATIACNU\# \\ AE-SRTC-CCLU\# \\ CP-BATCHCC1U\# \\ CP-DWPF1CC1U\# \\ CP-DWPF2CC1U\# \\ CP--TF--CC-U\# \\ OPRSRATICVHÜ\# \\ OPR-TF--LAHU\# \\ OPR-TF-ACHU\# \\ OPRSRATIIRHU\# \\ OPR1SRATTRHU\# \\ OPR-TF--IRHU\# \\ AE-DWPFICCLU\# \\ AE-DWPF2CCLU\#
}

Basic Event Name

*SLRSALT-BLUU\#

*SLRSALT-PREU+-I

*WTR-MLT-PREU\#

CP-DWPF1CC1U\#

CP-DWPF2CC1U\#

CP-BATCHCCIÜ\#

CP-TF--CC-U\#

OPRSRAT1CVHU\#

OPRSRATIIRHU\#

OPR1SRATIRHU\#

OPR-TF--IRHU\#

OPR-TF--ACHU\#

OPRSRAT1ACNU\#

OPRSRATILAHU\#

\section{Risk Achievement Worth}

Description

Excess salt in sludge from tank farm transferred to DWPF

Analyst at SRAT fails to properly analyze batch \#1 sample

Fail to follow procedure batch.\#1 sample prep.

CC: two analyzers at tank farm fail - false low reading

Same personnel conduct sampling at SRAT for batch \#2

Same personnel conduct sampling: SRAT to SME: batch \#1

Same personnel conduct sampling :SRAT to SME: batch \#2

Same analyst conducts sampling at tanks 48 \& 49

STE at SRAT fails to verify batch \#1 results

Analyst at tank farm fails to properly analyze sample

Tank farm :fails to follow procedure for sample preparation

SRAT engineer incorrectly reads data from batch \#1

SRAT engineer incorrectly records data into PCCS: batch \#1

Tank farm analyst incorrectly reads data

CC: two analyzers in DWPF fail low - batch \#1

CC: two analyzers in DWPF fail low - batch $\# 2$.
Prob/Freq AchW

2.85E-05/H $3.50 \mathrm{E}+04$

$1.00 \mathrm{E}-03 \quad 1.33 \mathrm{E}+01$

5.00E-03 1.33E+01

2.15E-05 1.01E+01

$2.00 \mathrm{E}-01 \quad 5.00 \mathrm{E}+00$

$2.00 \mathrm{E}-01 \quad 5.00 \mathrm{E}+00$

$2.00 \mathrm{E}-01 \quad 5.00 \mathrm{E}+00$

$2.00 \mathrm{E}: 01 \quad 5.00 \mathrm{E}+00$

3.00E-01 3.16E+00

$1.00 \mathrm{E}-03 \quad 2.81 \mathrm{E}+00$

$5.00 \mathrm{E}-02 \quad 2.72 \mathrm{E}+00$

$5.00 \mathrm{E}-01 \quad 1.93 \mathrm{E}+00$

5.00E-01 1.93E +00

$5.00 \mathrm{E}-01 \quad 1.91 \mathrm{E}+00$

$2.15 \mathrm{E}-05-1.03 \mathrm{E}+00$

2.15E-05 1.03E+00

\section{Risk Reduction Worth}

Description

Excess salt build up on melter top layer

Excess salt in sludge from tank farm transferred to DWPF

Water in the melter

Same personnel conduct sampling : SRAT to SME: batch \#1

Same personnel conduct sampling: SRAT to SME: batch \#2

Same personnel conduct sampling at SRAT for batch \#2

Same analyst conducts sampling at tanks 48 \& 49

STE at SRAT fails to verify batch \#1 results

SRAT engineer incorrectly reads data from batch \#1

SRAT engineer incorrectly records data into PCCS: batch \#1

Tank farm analyst incorrectly reads data

Tank farm operator fails to follow procedure: sample prep.

Fail to follow procedure for batch \#1 sample preparation

Analyst at SRAT fails to properly analyze batch \#1 sample

\begin{tabular}{|c|c|}
\hline Freq & $\mathbf{W}$ \\
\hline $1.00 \mathrm{E}+00$ & $0.00 \mathrm{E}+00$ \\
\hline $2.85 \mathrm{E}-05 / \mathrm{H}$ & $0.00 E+0$ \\
\hline $1.00 \mathrm{E}+00$ & $0.00 \mathrm{E}+0$ \\
\hline 2.00 & $1.53 \mathrm{E}$ \\
\hline & $53 E$ \\
\hline & $1.53 \mathrm{I}$ \\
\hline 2.00 & $5.13 \mathrm{E}$ \\
\hline $3.00 \mathrm{E}-$ & $.35 \mathrm{E}$ \\
\hline $5.00 \mathrm{E}-01$ & $5 E+0$ \\
\hline & \\
\hline & $F$ \\
\hline & \\
\hline & \\
\hline 1.0 & \\
\hline
\end{tabular}

* A Reduction Worth of $0.0 \mathrm{E}+00$ indicates event appears in every cutset could serve as a single event preventor for the accident.

Note: Events that make less than $1 \%$ difference to the importance factor are not included. 
X-ESR-S-00001, REV. 0

Melter Offgas Explosion 


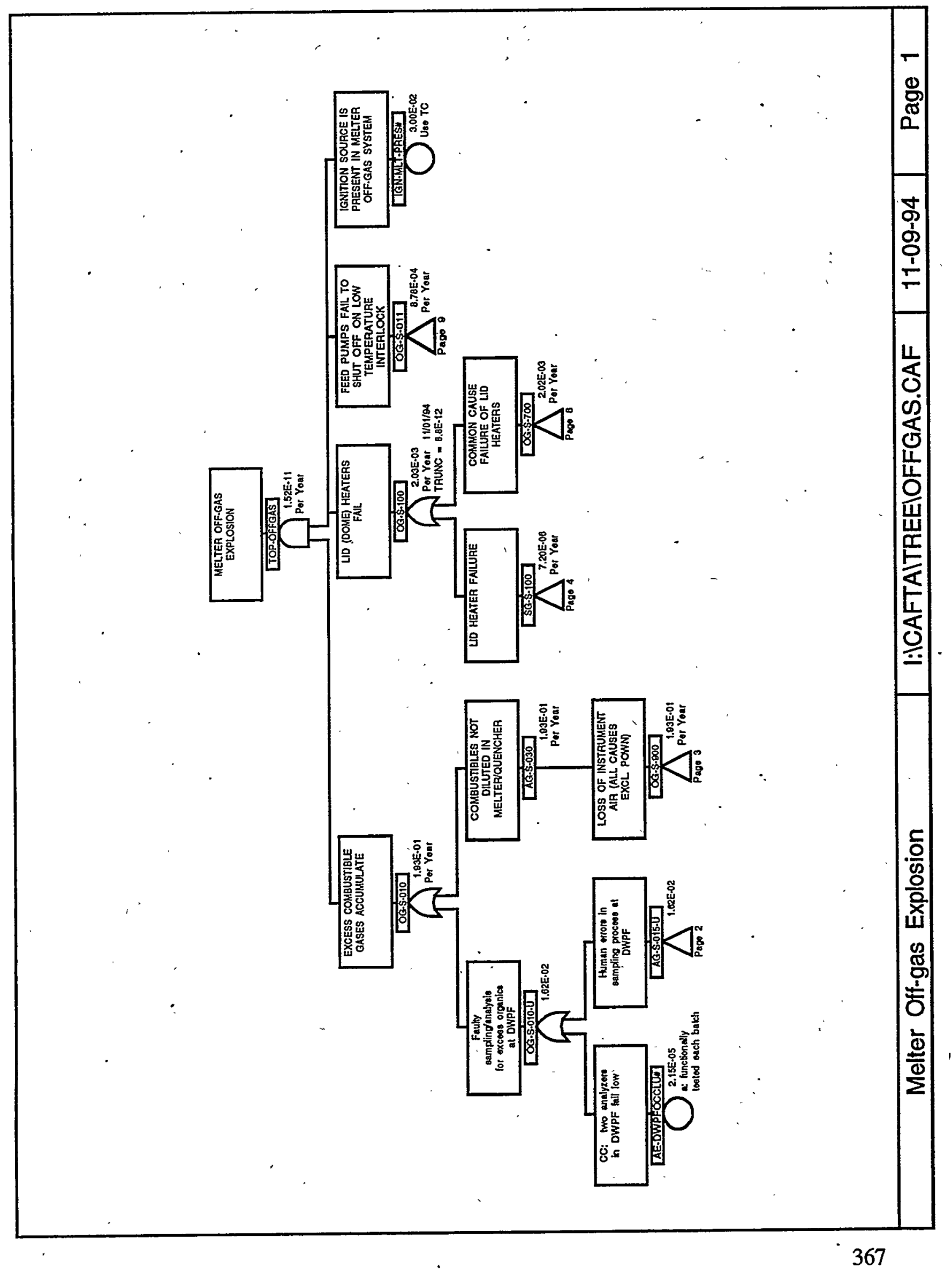




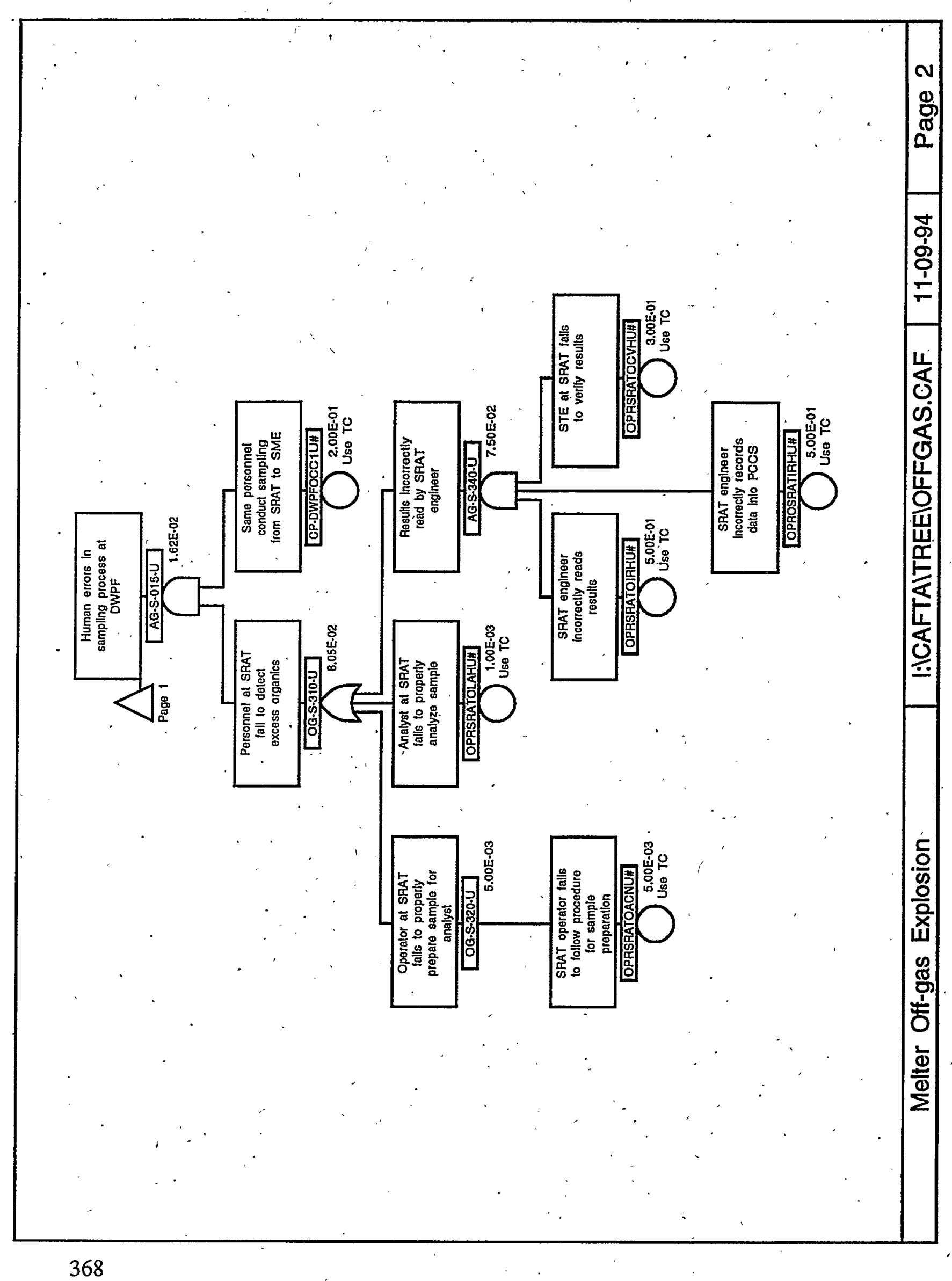




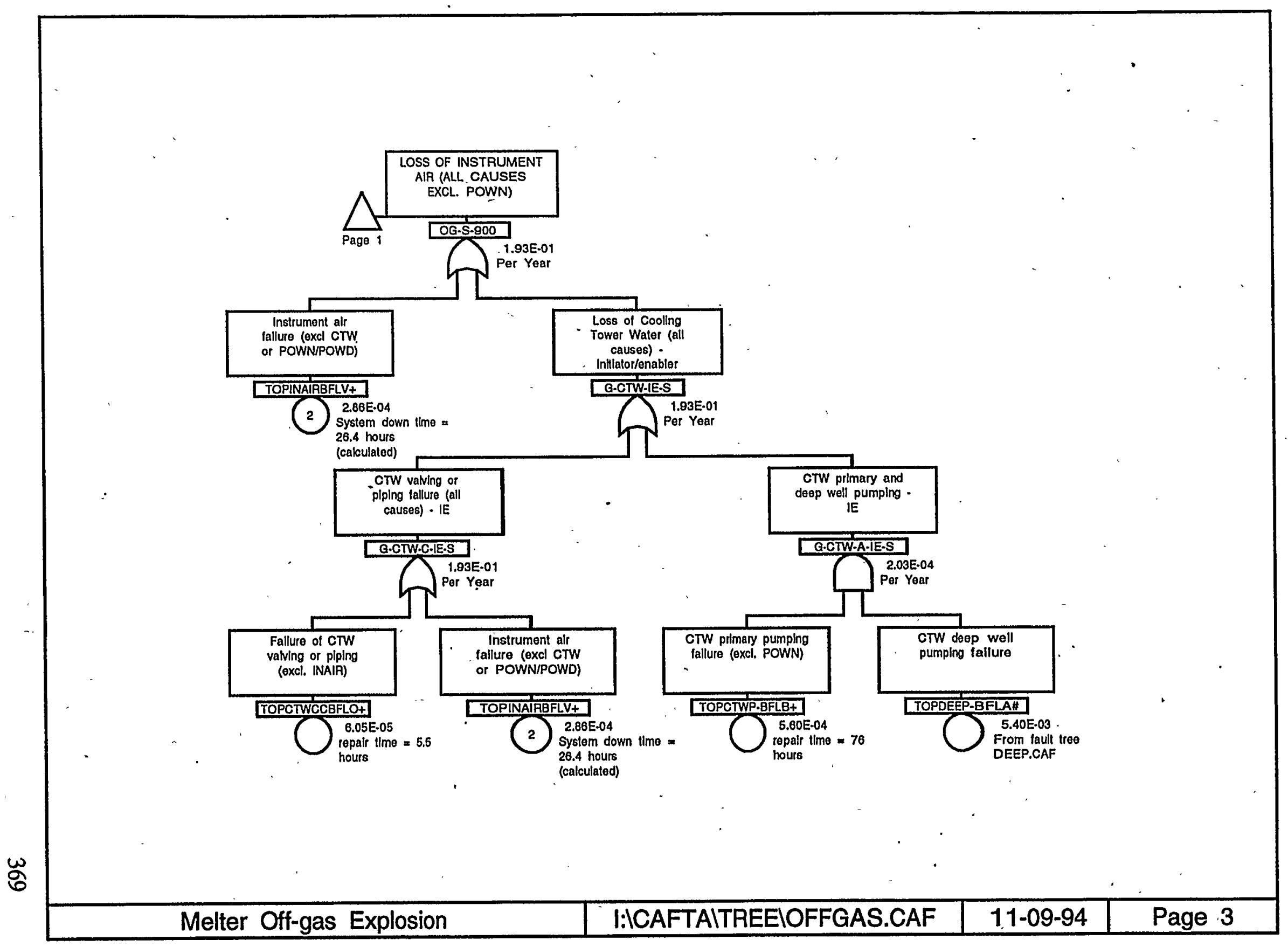




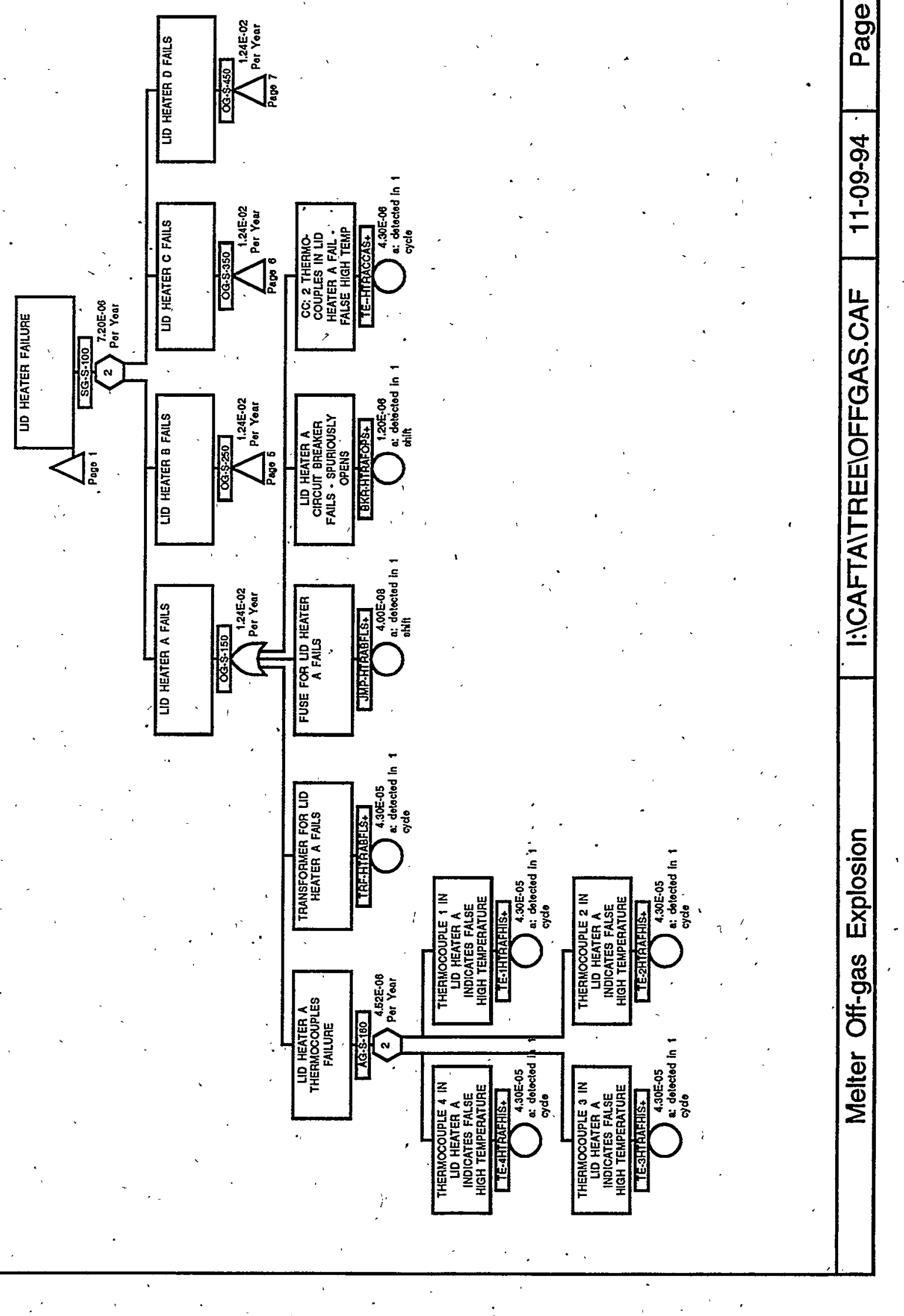




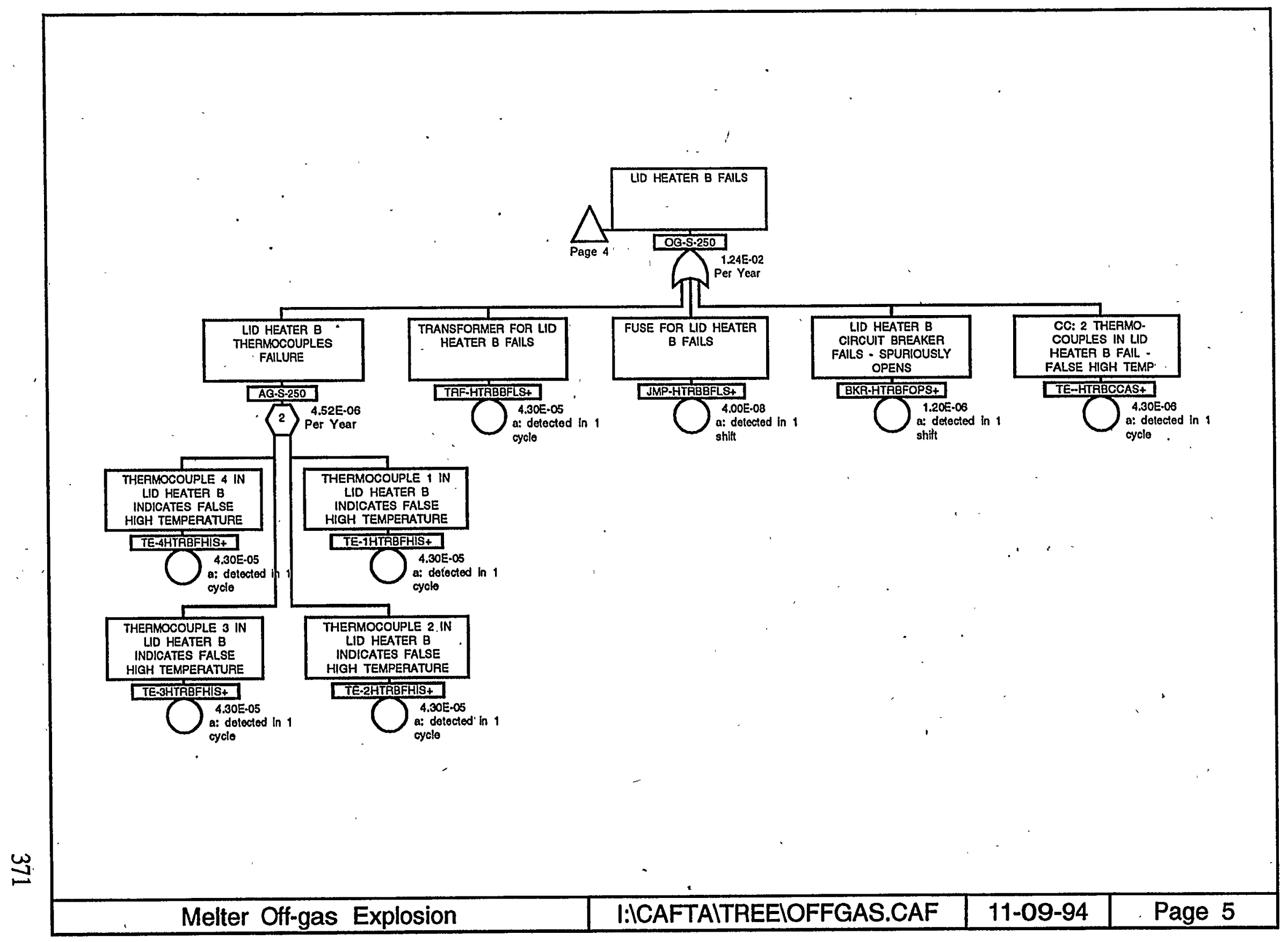


X-ESR-S-00001, REV. 0

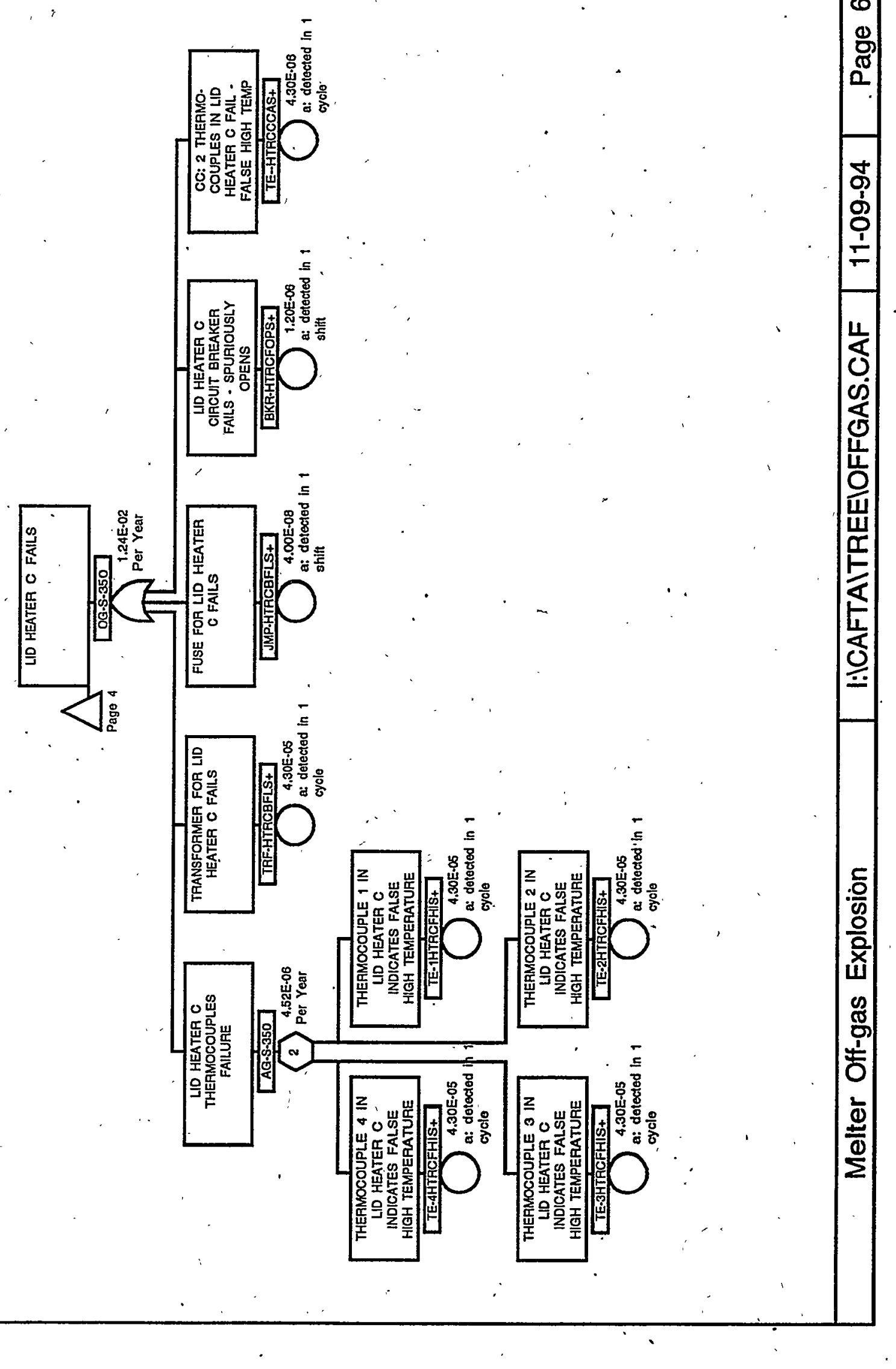




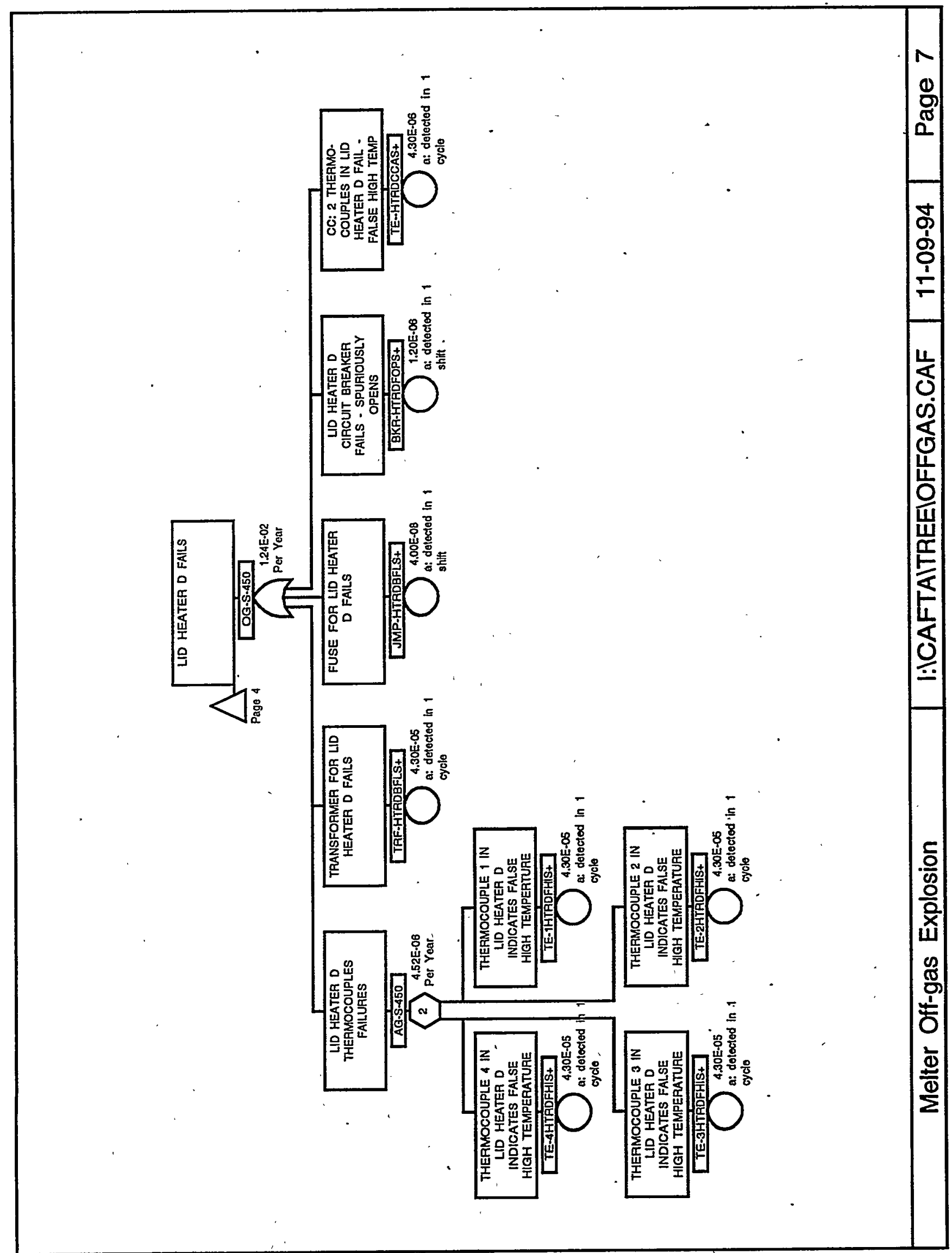


X-ESR-S-00001, REV. 0

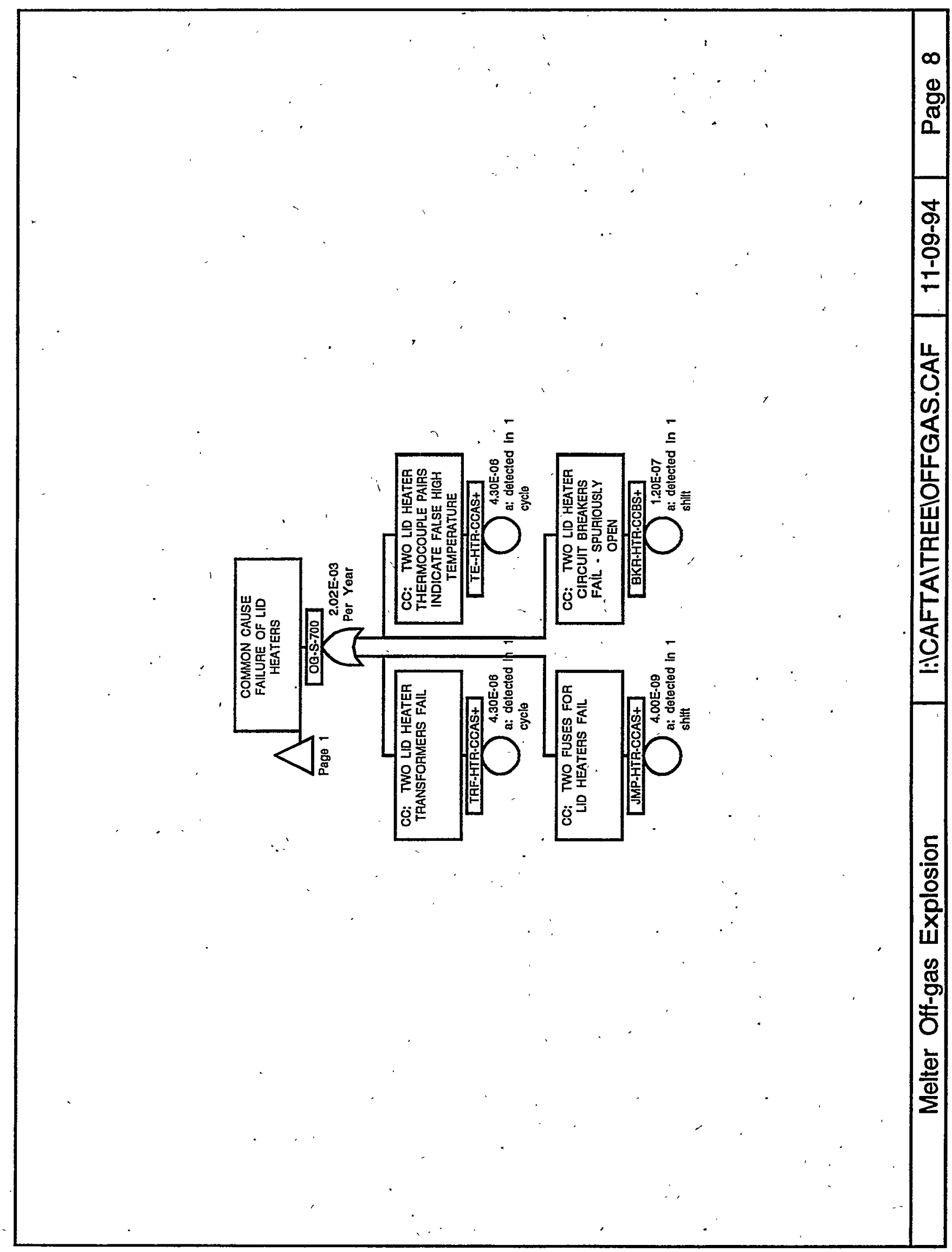




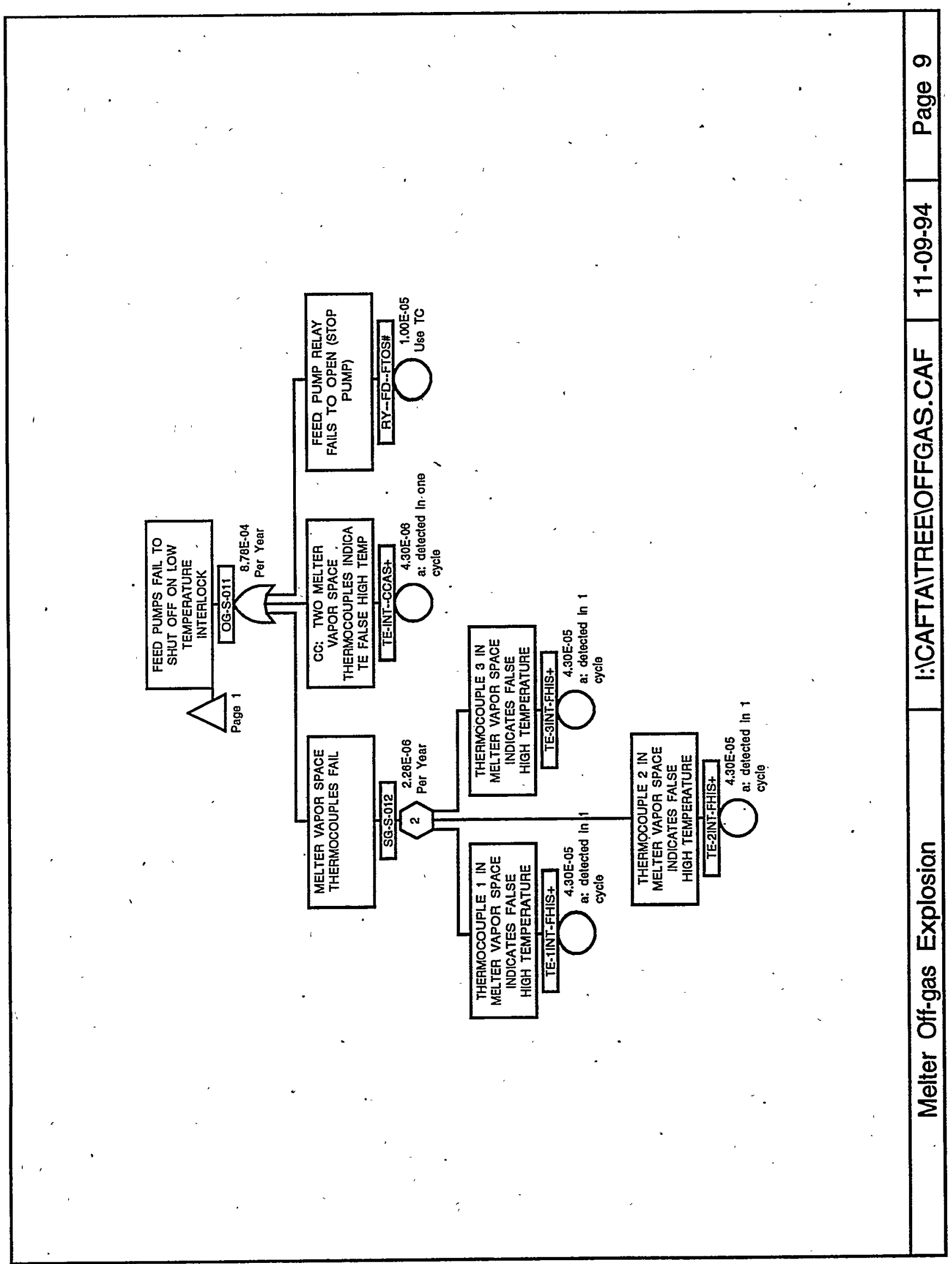




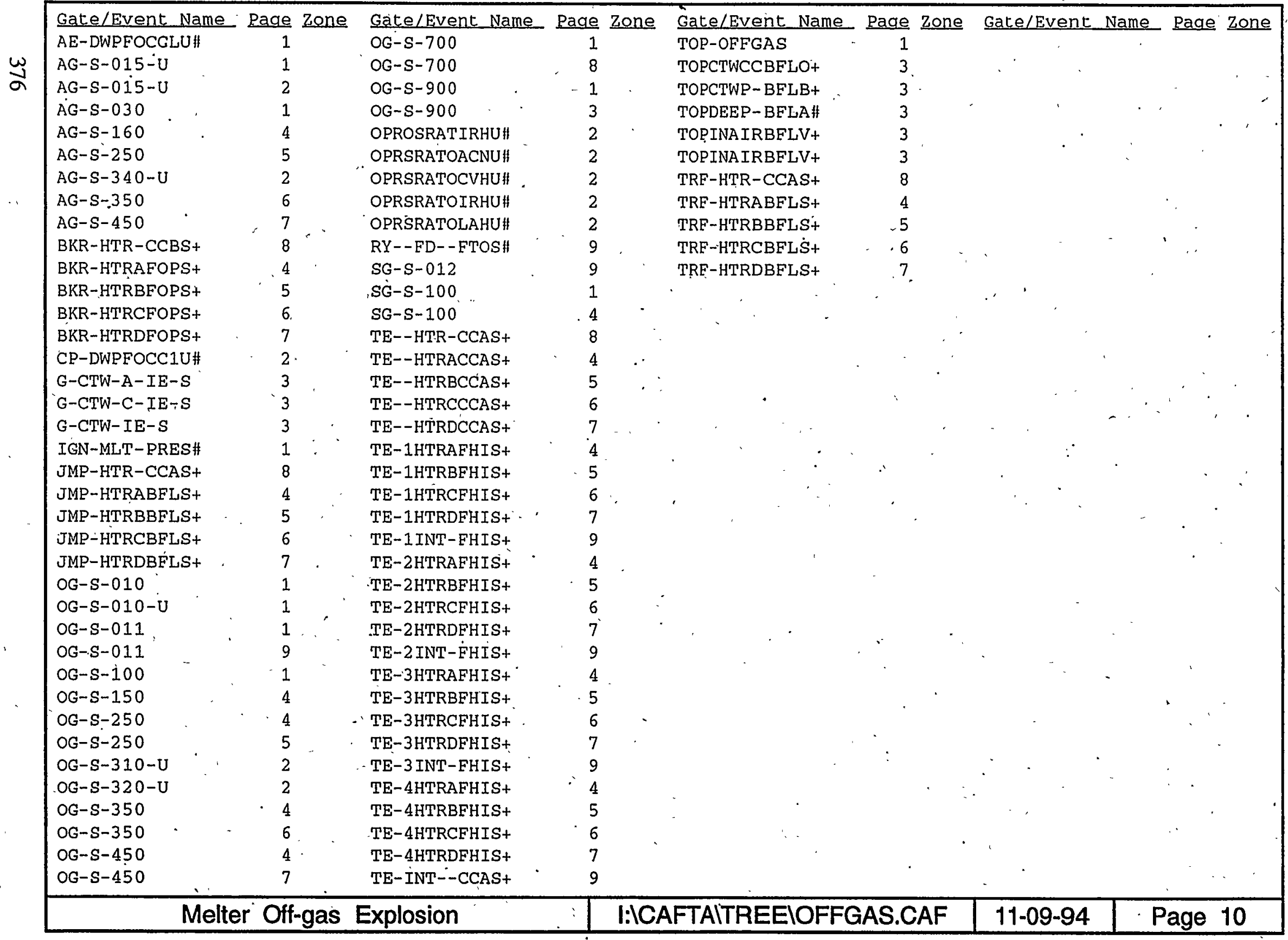




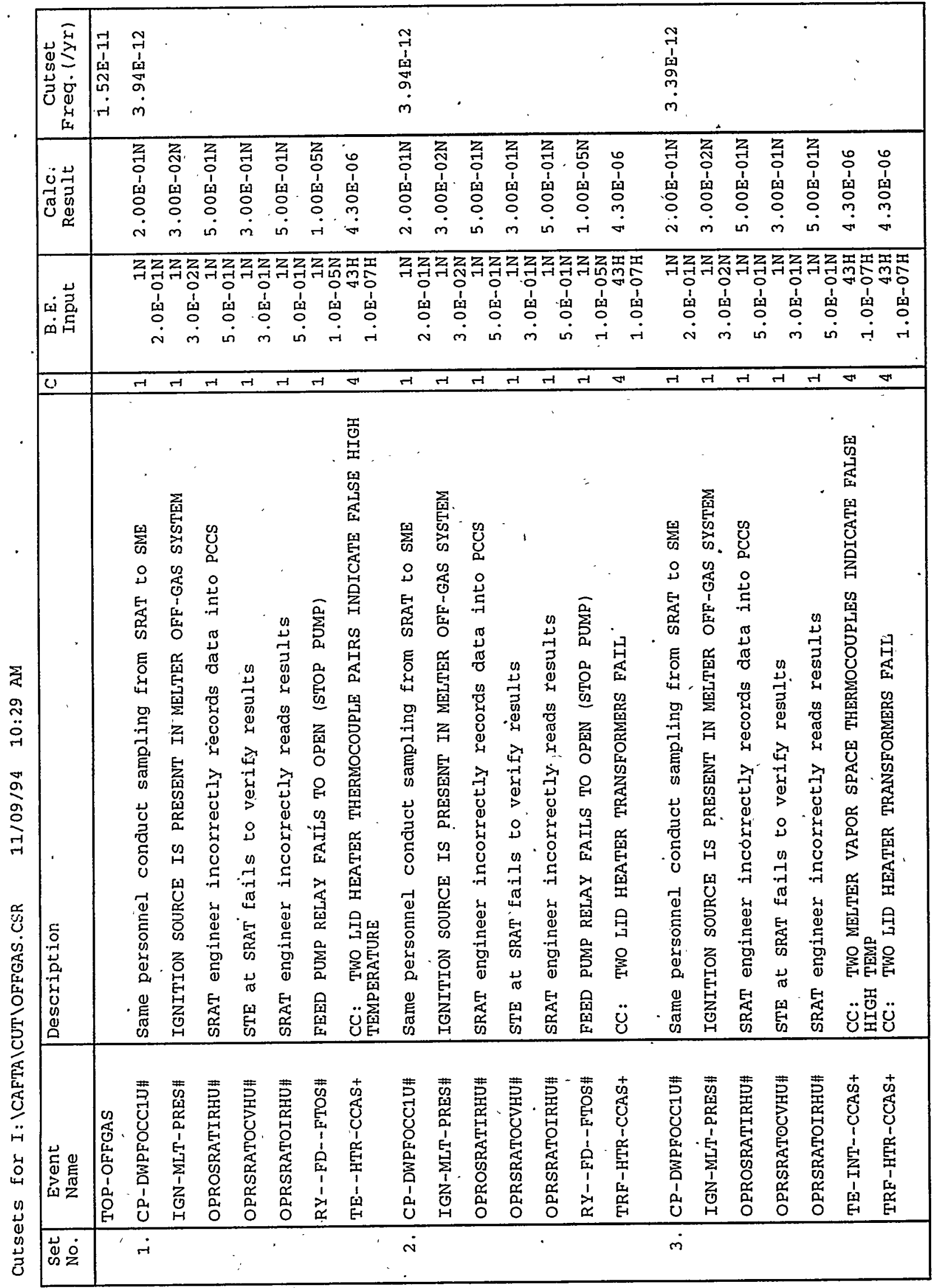


Cutsets for I: ICAFTA ICUT\OFFGAS.CSR

$11 / 09 / 94 \quad 10: 29 \cdot \mathrm{AM}$ (CONT.)

\begin{tabular}{|c|c|c|c|c|c|c|c|}
\hline & $\begin{array}{l}\text { Set } \\
\text { No. }\end{array}$ & $\begin{array}{l}\mathrm{Ev} \\
\mathrm{Na}\end{array}$ & Description & C & ut & it & $\begin{array}{l}\text { t } \\
y r)\end{array}$ \\
\hline & 4. & $\begin{array}{l}\text { CP-DWPFOCCIU\# } \\
\text { IGN-MLT-PRES\# } \\
\text { OPROSRATIRHU\# } \\
\text { OPRSRATOCVHU\# } \\
\text { OPRSRATOIRHU\# } \\
\text { TE- - HTR-CCÁS+ } \\
\text { TE-INT--CCAS+ } \\
\text { CP-DWPFOCCIU\# } \\
\text { IGN-MLT-PRES\# } \\
\text { OPRSRATOACNU\# } \\
\text { RY--FD--FTOS\# } \\
\text { TE--HTR-CCAS+ } \\
\text { CP-DWPFOCCIU\# } \\
\text { IGN-MLT-PRES\# } \\
\text { OPRSRATOACNU\# } \\
\text { RY- - FD--FTOS\# } \\
\text { TRE-HTR-CCAS+ }+\end{array}$ & 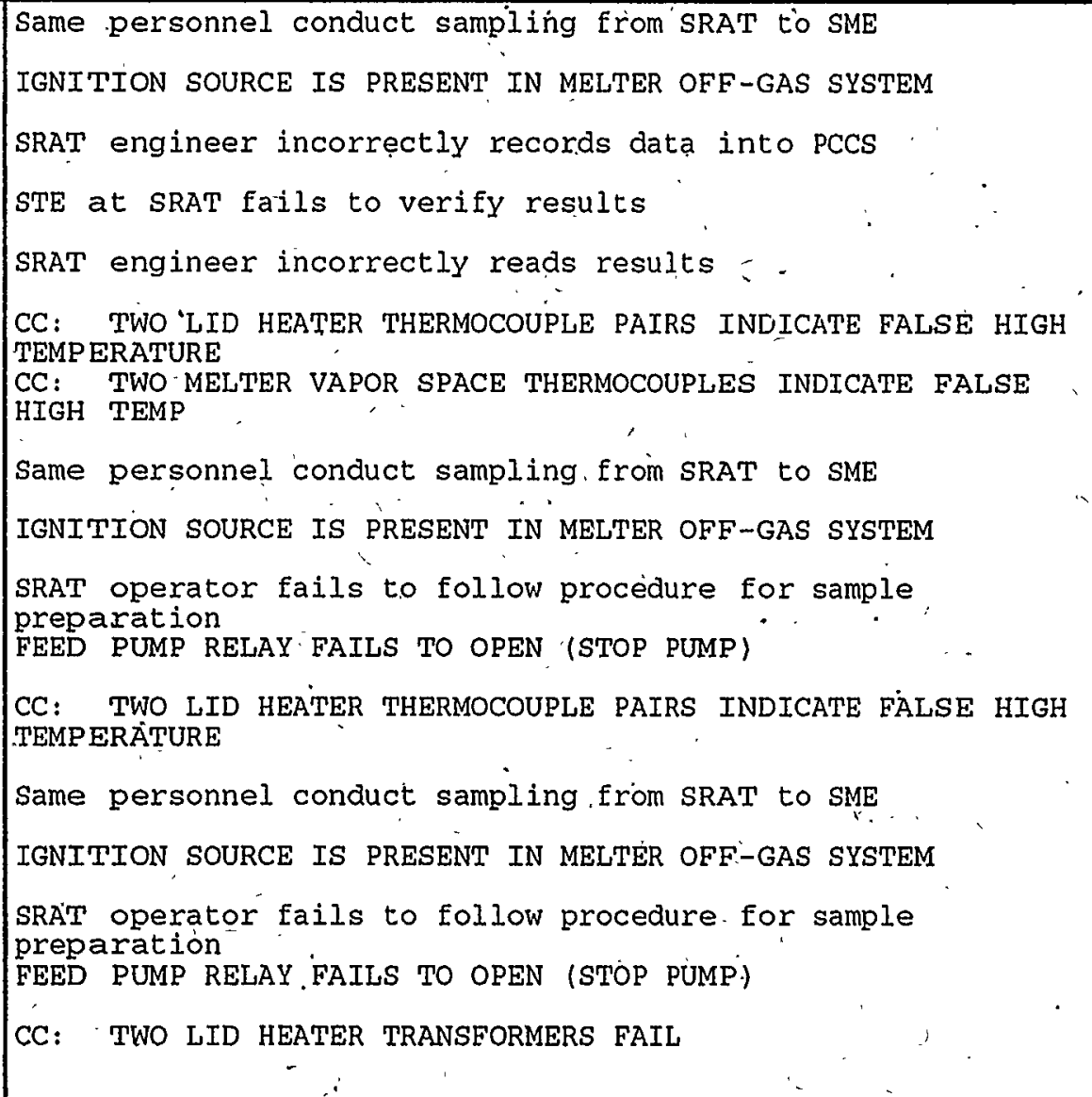 & 1 & $\begin{array}{r}1 \mathrm{~N} \\
2.0 \mathrm{E}-01 \mathrm{~N} \\
1 \mathrm{~N} \\
3.0 \mathrm{E}-02 \mathrm{~N} \\
1 \mathrm{~N} \\
5.0 \mathrm{E}-01 \mathrm{~N} \\
1 \mathrm{~N} \\
3.0 \mathrm{E}-01 \mathrm{~N} \\
1 \mathrm{~N} \\
5.0 \mathrm{E}-01 \mathrm{~N} \\
43 \mathrm{H} \\
1.0 \mathrm{E}-07 \mathrm{H} \\
43 \mathrm{H} \\
1.0 \mathrm{E}-07 \mathrm{H} \\
1 \mathrm{~N} \\
2.0 \mathrm{E}-01 \mathrm{~N} \\
1 \mathrm{~N} \\
3.0 \mathrm{E}-02 \mathrm{~N} \\
1 \mathrm{~N} \\
5.0 \mathrm{E}-03 \mathrm{~N} \\
1 \mathrm{~N} \\
1.0 \mathrm{E}-05 \mathrm{~N} \\
43 \mathrm{H} \\
1.0 \mathrm{E}-07 \mathrm{H} \\
1.0 \mathrm{E}-07 \mathrm{H} \\
1.0 \mathrm{E}-05 \mathrm{~N} \\
1 \mathrm{~N} \\
2.0 \mathrm{E}-01 \mathrm{~N} \\
1 \mathrm{~N} \\
3.0 \mathrm{E}-02 \mathrm{~N} \\
1 \mathrm{~N} \\
5.0 \mathrm{E}-03 \mathrm{~N} \\
1 \mathrm{~N} \\
1.06\end{array}$ & $\begin{array}{l}2.00 \mathrm{E}-01 \mathrm{~N} \\
3.00 \mathrm{E}-02 \mathrm{~N} \\
5.00 \mathrm{E}-01 \mathrm{~N} \\
3.00 \mathrm{E}-01 \mathrm{~N} \\
5.00 \mathrm{E}-01 \mathrm{~N} \\
4.30 \mathrm{E}-06 \\
4.30 \mathrm{E}-06 \\
2.00 \mathrm{E}-01 \mathrm{~N} \\
3.00 \mathrm{E}-02 \mathrm{~N} \\
5.00 \mathrm{E}-03 \mathrm{~N} \\
1.00 \mathrm{E}-05 \mathrm{~N} \\
4.30 \mathrm{E}-06 \\
2.00 \mathrm{E}-01 \mathrm{~N} \\
3.00 \mathrm{E}-02 \mathrm{~N} \\
5.00 \mathrm{E}-03 \mathrm{~N} \\
1.00 \mathrm{E}-05 \mathrm{~N} \\
4.30 \mathrm{E}-06\end{array}$ & $2.63 E-13$ \\
\hline
\end{tabular}


Basic Event Name TE-HTR-CCAS+IC TRF-HTR-CCAS+-IC TE-INT-CCAS+-IC BKR-HTR-CCBS+-IC JMP-HTR-CCAS+-IC RY-FD-FTOS\# TOPINAIRBFLV+-IC TOPCTWCCBFLO+IC IGN-MLT-PRES\# TOPCTWP-BFLB+-IC OPRSRATOACNU\# CP-DWPFOCCIU\# OPRSRATOCVHU\# OPROSRATTRHU\# OPRSRATOIRHU\#

\section{Risk Achievement Worth}

\section{Description}

CC: TWO LID HEATER TC PAIRS FAIL HGH

CC: TWO LID HEATER TRANSFORMERS FAII

CC: TWO MELTER VAPOR SPACE TCS FAII HIGH

CC: TWO LID HEATER CIRCUIT BRKRS FAIL OPEN

CC: TWO FUSES FOR LID HEATERS FAIL

FEED PUMP RELAY FAILS TO OPEN (STOP PUMP)

Instrument air failure (excl CTW o POWN/POWD)

Failure of CTW valving or piping (excl. INAIR)

IGNITTON SOURCE IS PRESENT IN MELTER OFF-GAS

CTW primary pumping failure (excl. POWN)

Fail to follow procedure for SRAT simple preperstion -

Same personnel conduct sampling from SRAT to SME

STE at SRAT fails to verify results

SRAT engineer incorrectly records data into PCCS .

SRAT engineer incorrectly reads results

\begin{tabular}{ll} 
Prob/Ereq & AchW \\
\hline $1.00 \mathrm{E}-07 / \mathrm{H}$ & $3.73 \mathrm{E}+06$ \\
$1.00 \mathrm{E}-07 / \mathrm{H}$ & $3.73 \mathrm{E}+06$ \\
$1.00 \mathrm{E}-07 / \mathrm{H}$ & $2.18 \mathrm{E}+06$ \\
$3.00 \mathrm{E}-08 / \mathrm{H}$ & $8.62 \mathrm{E}+04$ \\
$1.00 \mathrm{E}-09 / \mathrm{H}$ & $5.99 \mathrm{E}+04$ \\
$1.00 \mathrm{E}-05$ & $5.60 \mathrm{E}+04$ \\
$1.10 \mathrm{E}-05 / \mathrm{H}$ & $2.08 \mathrm{E}+03$ \\
$1.10 \mathrm{E}-05 / \mathrm{H}$ & $2.08 \mathrm{E}+03$ \\
$3.00 \mathrm{E}-02$ & $3.33 \mathrm{E}+01$ \\
$6.29 \mathrm{E}-06 / \mathrm{H}$ & $1.23 \mathrm{E}+01$ \\
$5.00 \mathrm{E}-03$ & $7.45 \mathrm{E}+00$ \\
$2.00 \mathrm{E}-01$ & $4.70 \mathrm{E}+00$ \\
$3.00 \mathrm{E}-01$ & $3.15 \mathrm{E}+00$ \\
$5.00 \mathrm{E}-01$ & $1.72 \mathrm{E}+00$ \\
$5.00 \mathrm{E}-01$ & $1.72 \mathrm{E}+00$
\end{tabular}

Modified top event frequency $=$ (top event frequency) $\mathrm{x}$ (risk achievement worth).

Basic Event Name *IGN-MLT-PRES\# OPRSRATOCVHU\# OPRSRATOIRHU\# CP-DWPFOCCIU\# OPROSRATIRHU\# RY--FD--FTOS\# TRF-HTR-CCAS+-IC TE-INT-CCAS+IC TE-HTR-CCAS+-IC

\section{Risk Reduction Worth}

Description IGNITION SOURCE IS PRESENT IN MELTER OFF-GAS STE at SRAT fails to verify results SRAT engineer incorrectly reads results Same personnel conduct sampling from SRAT to SME SRAT engineer incorrectly records data into PCCS FEED PUMP RELAY FAIIS TO OPEN (STOP PUMP). CC: TWOLID HEATER TRANSFORMERS FAIL CC: TWO MELTER VAPOR SPACE TTCS FAIL HIGH CC: TWO LID HEATER TC PAIRS FAIL HIGH

\begin{tabular}{ll} 
Prob/Freg & RedW \\
\hline $3.00 \mathrm{E}-02$ & $0.00 \mathrm{E}+00$ \\
$3.00 \mathrm{E}-01$ & $2.22 \mathrm{E}+01$ \\
$5.00 \mathrm{E}-01$ & $2.22 \mathrm{E}+01$ \\
$2.00 \mathrm{E}-01$ & $2.22 \mathrm{E}+01$ \\
$5.00 \mathrm{E}-01$ & $2.22 \mathrm{E}+01$ \\
$1.00 \mathrm{E}-05$ & $1.91 \mathrm{E}+00$ \\
$1.00 \mathrm{E}-07 / \mathrm{H}$ & $1.91 \mathrm{E}+00$ \\
$1.00 \mathrm{E}-07 / \mathrm{H}$ & $1.91 \mathrm{E}+00$ \\
$1.00 \mathrm{E}-07 / \mathrm{H}$ & $1.91 \mathrm{E}+00$
\end{tabular}

* A.Reduction Worth of $0.0 \mathrm{E}+00$ indicates event appears in every cutset could serve as a single event preventor for the accident.

Note: Events that make less than $1 \%$ difference to the importance factor are not included. Modified top event frequency $=($ top event frequency) $/$ (risk reduction worth). 
X-ESR-S-00001, REV. 0

\section{LPPP SPT Explosion}




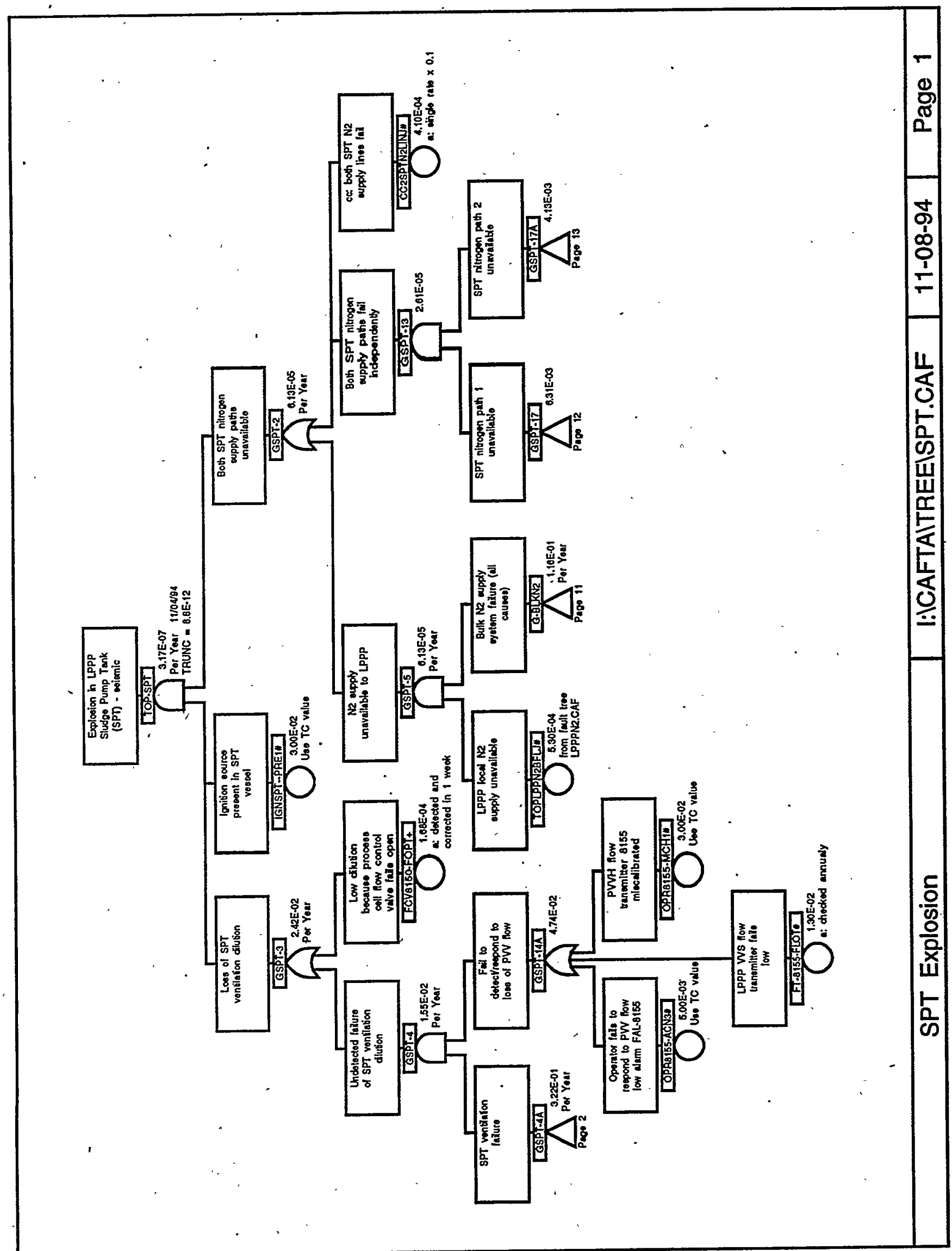


X-ESR-S-00001, REV. 0

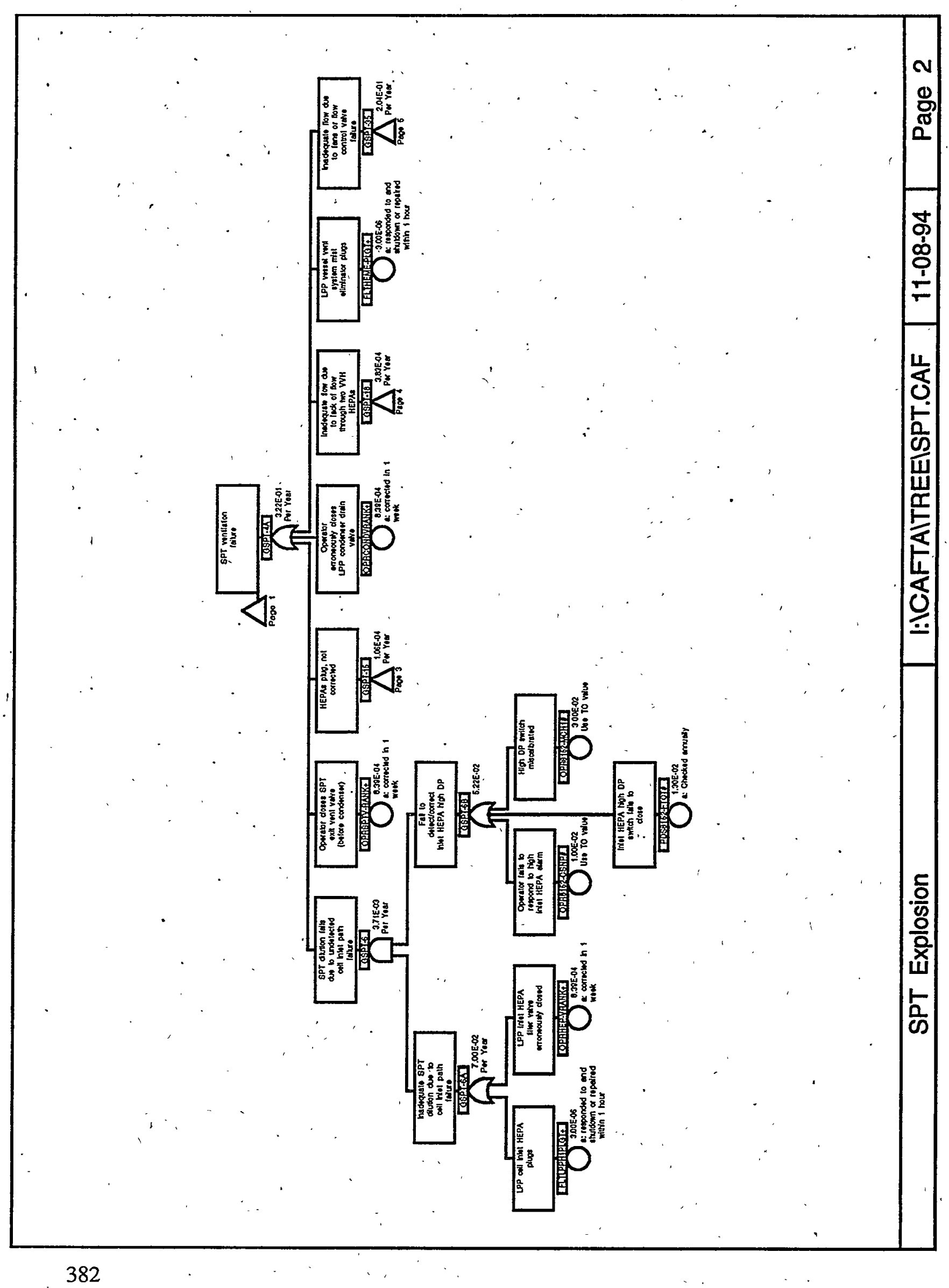




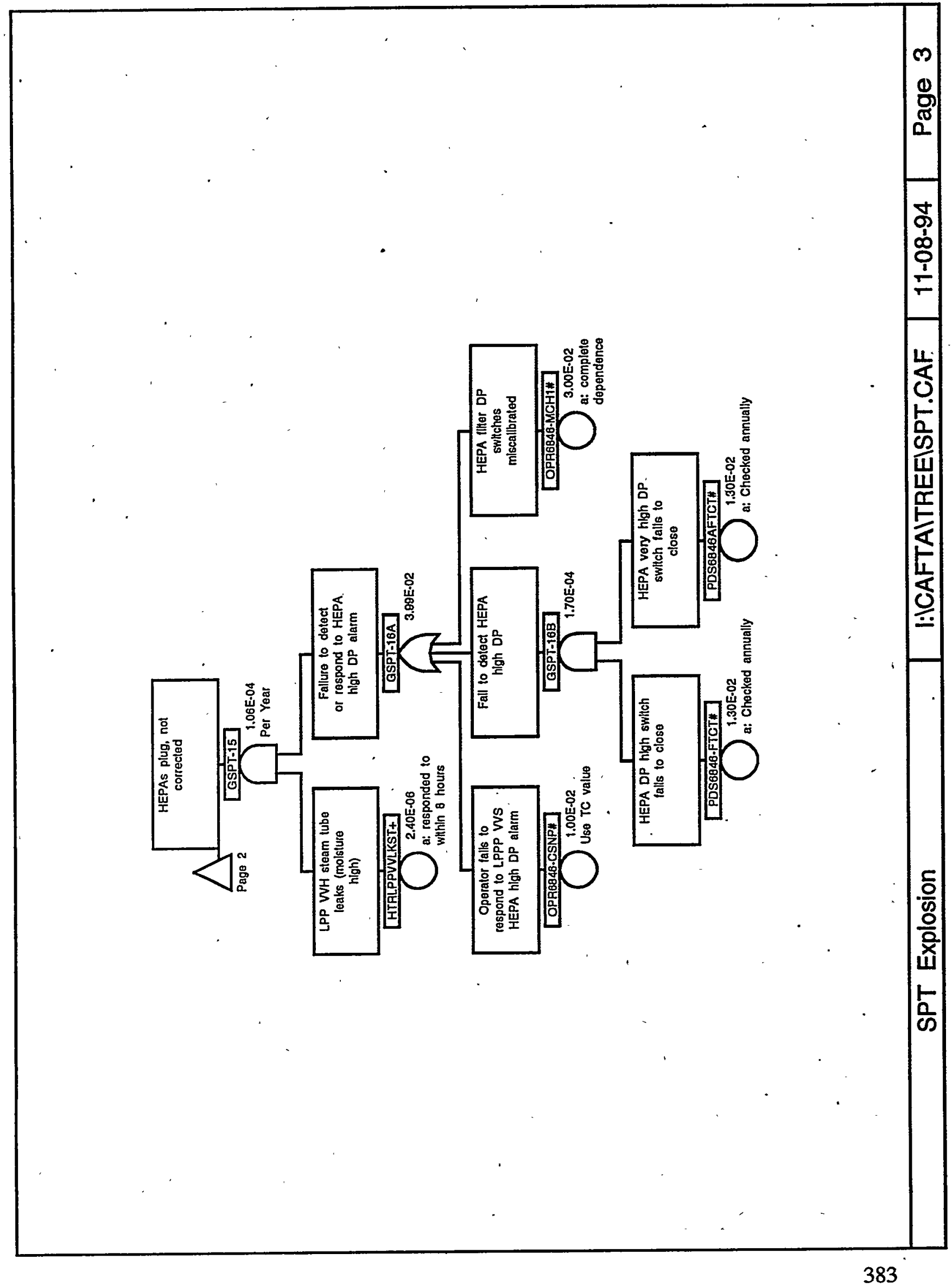




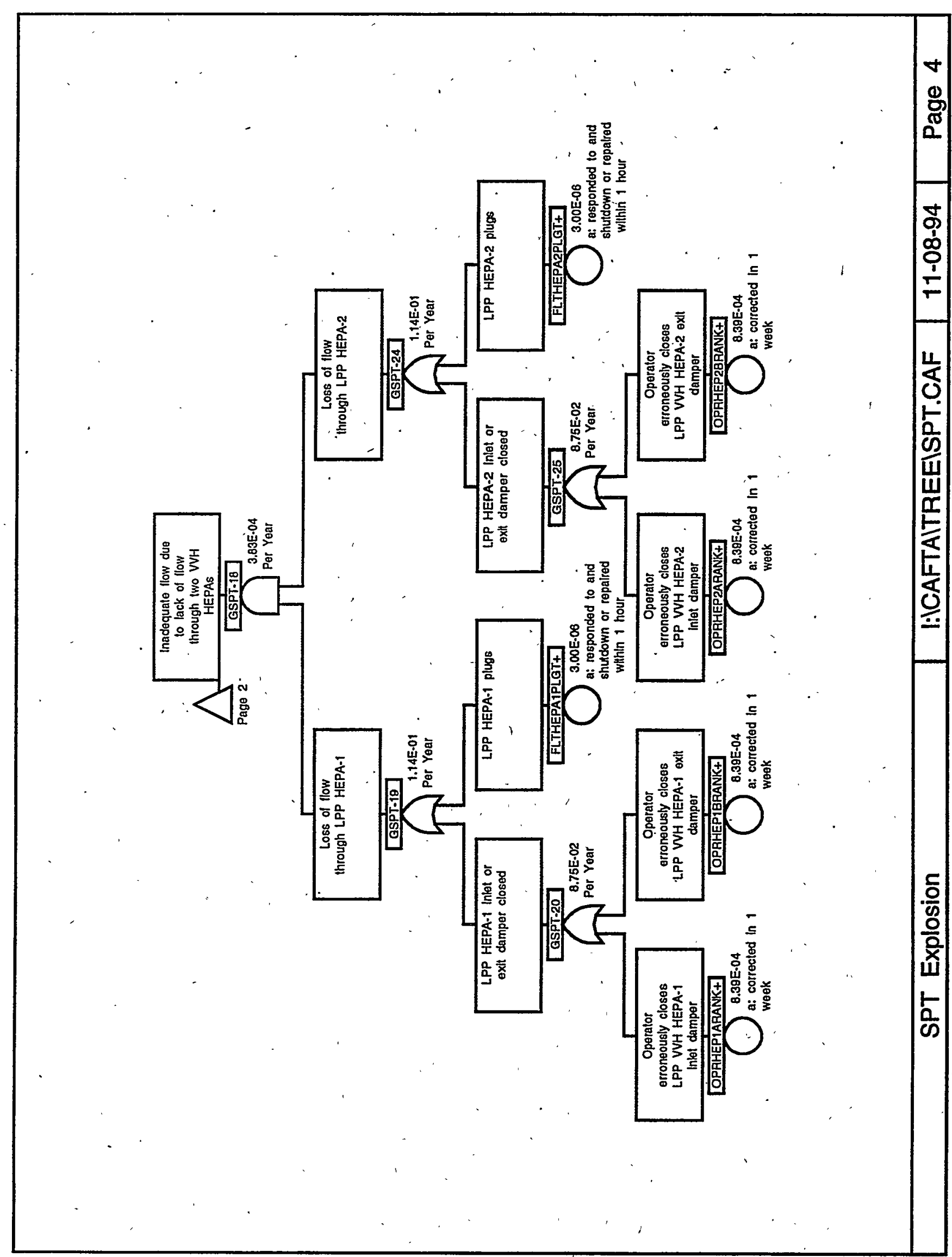




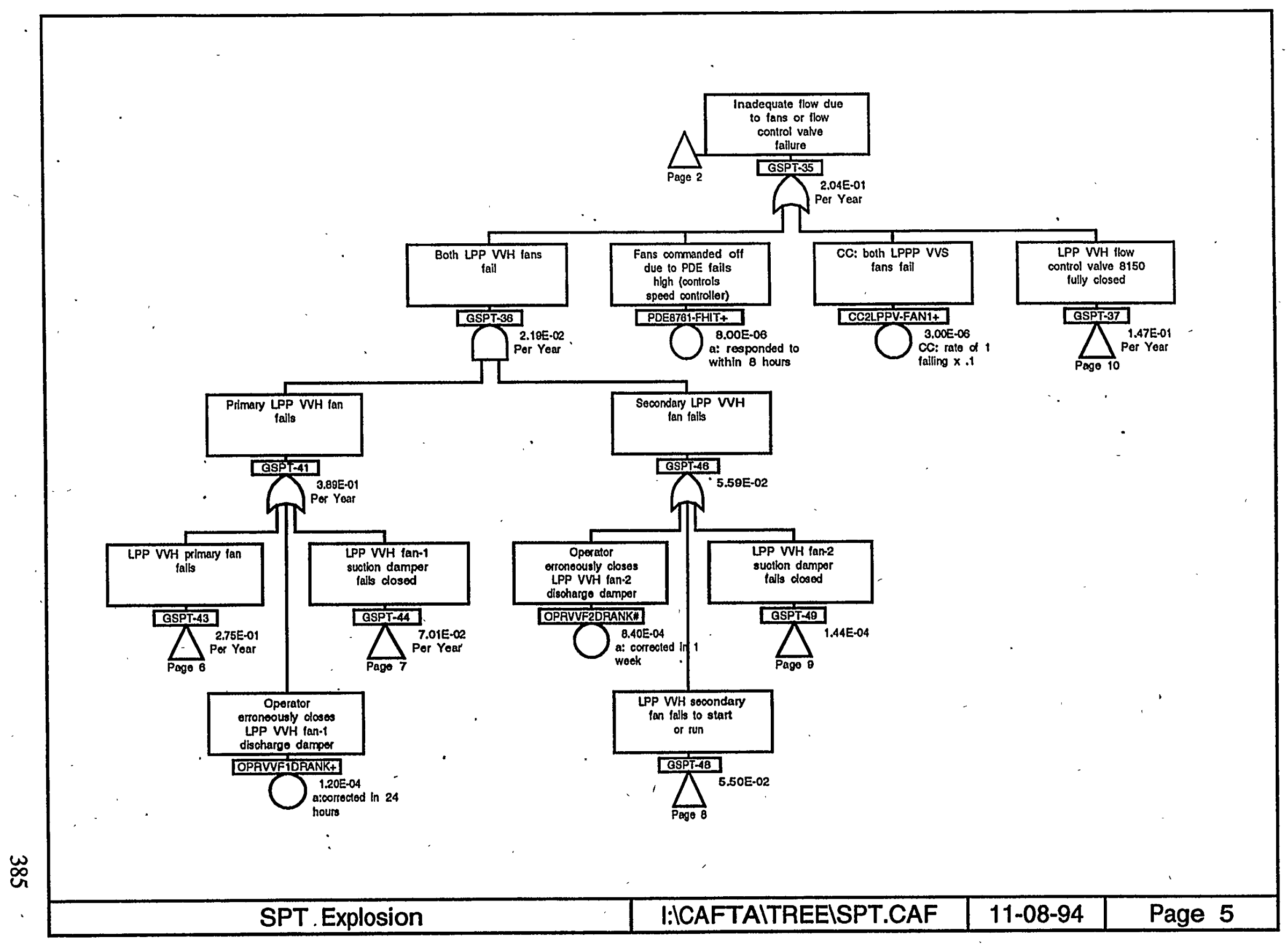


X-ESR-S-00001, REV. 0

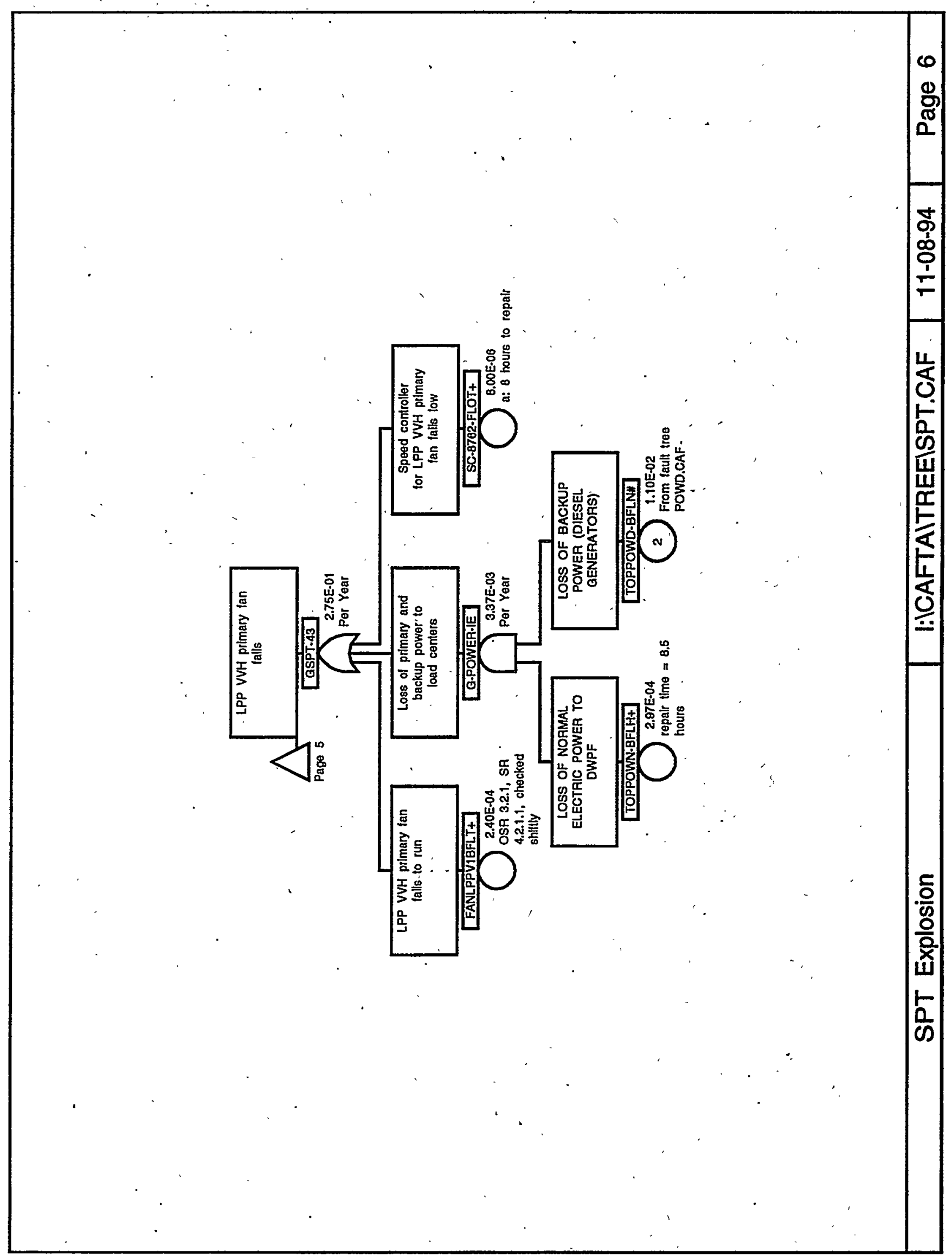




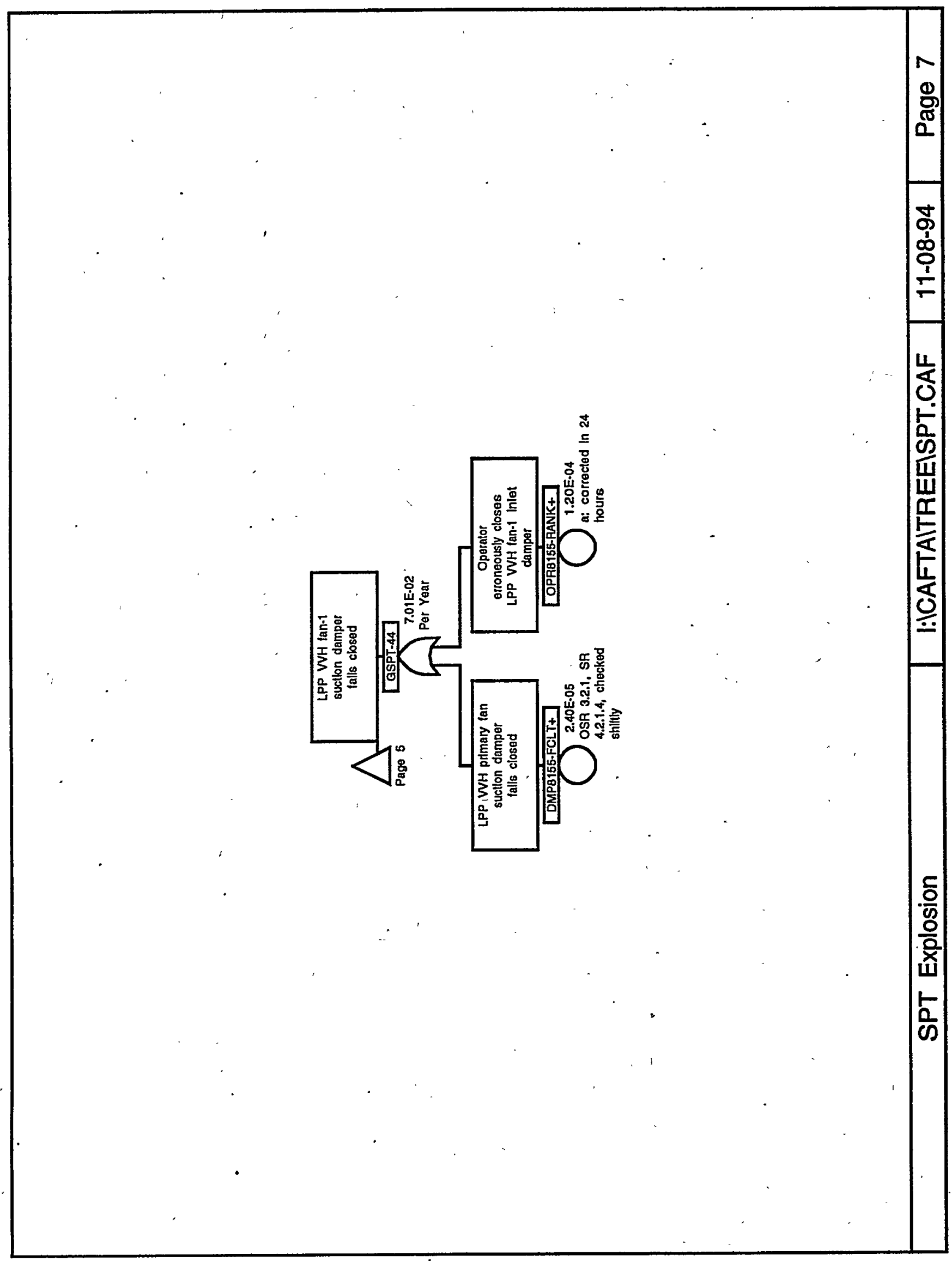




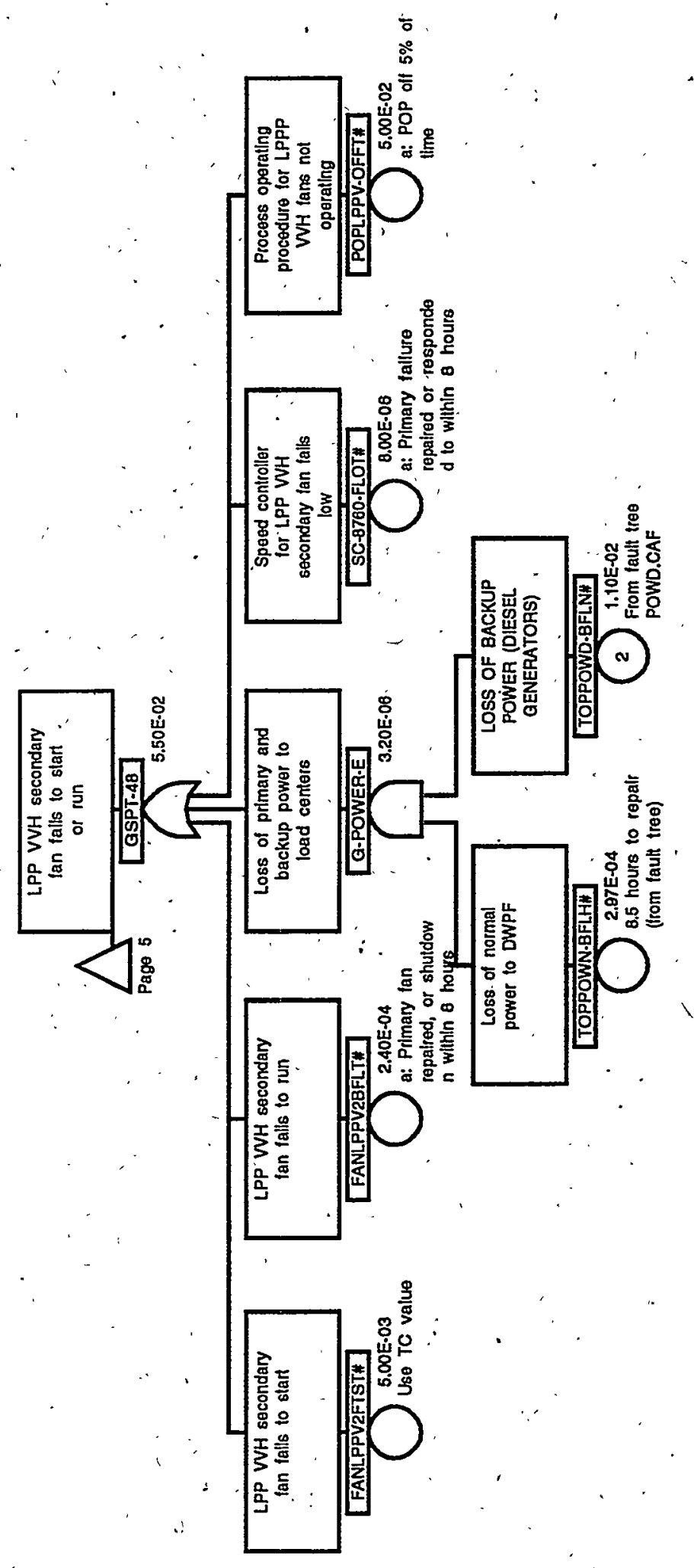




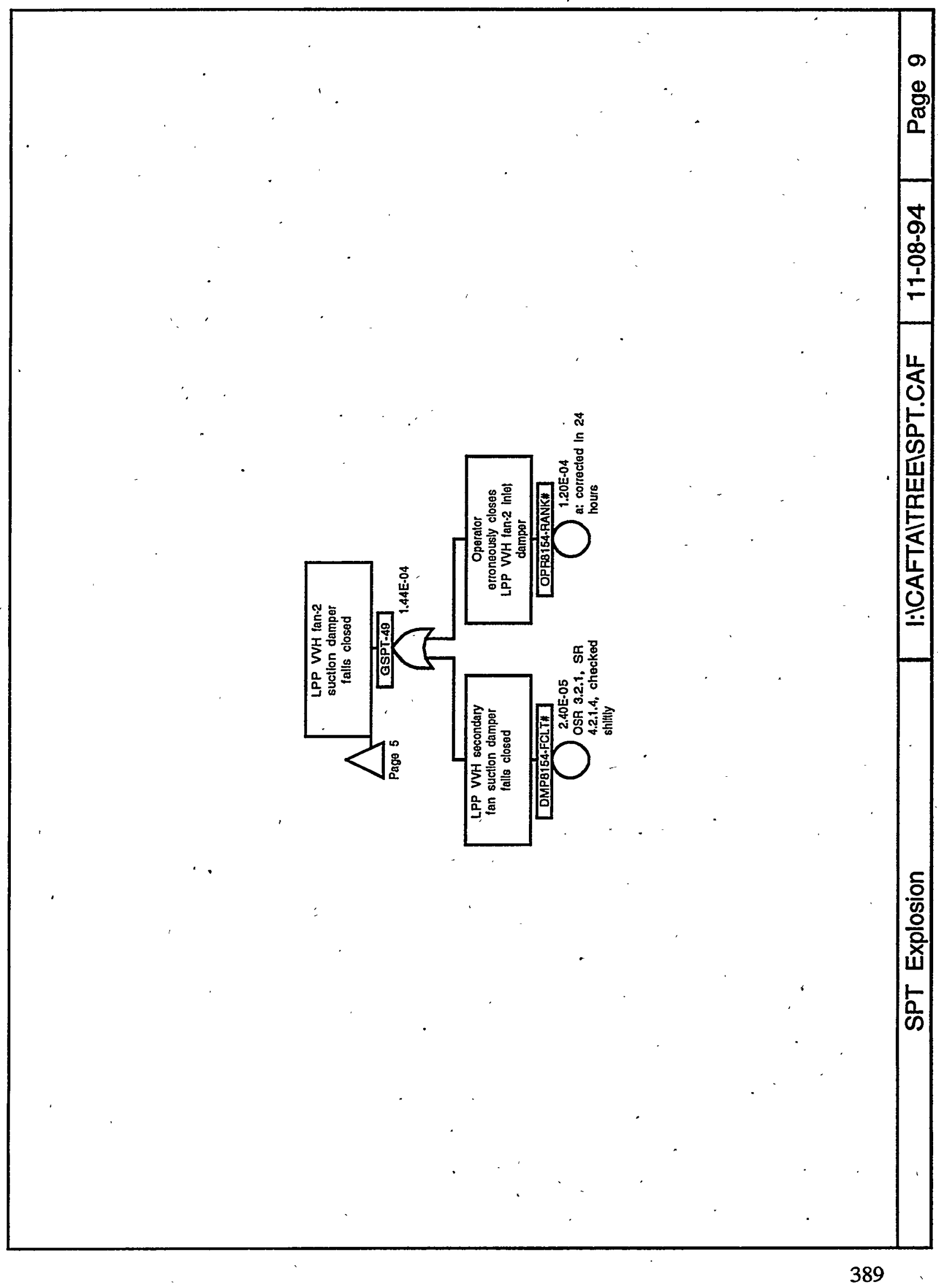




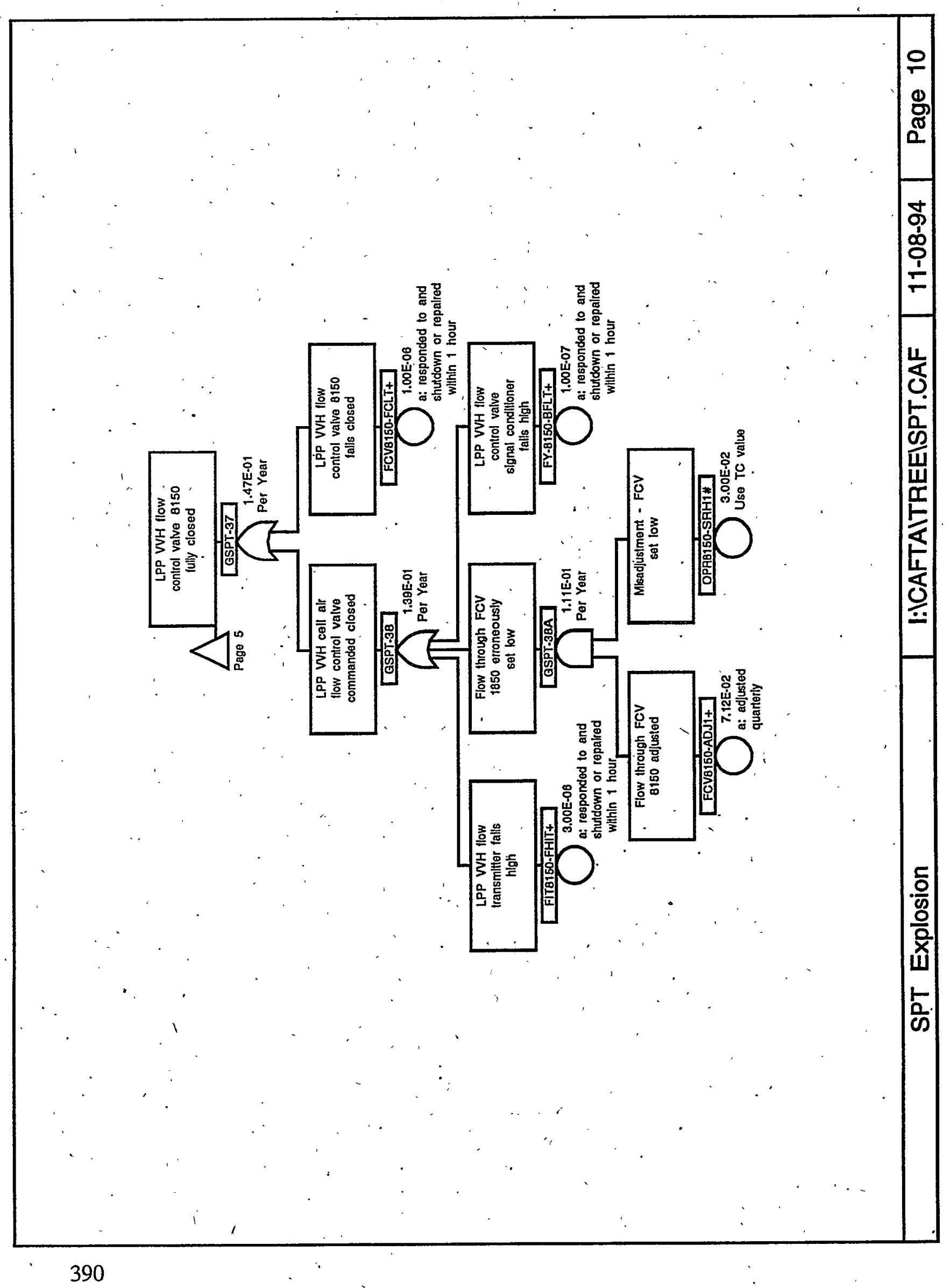




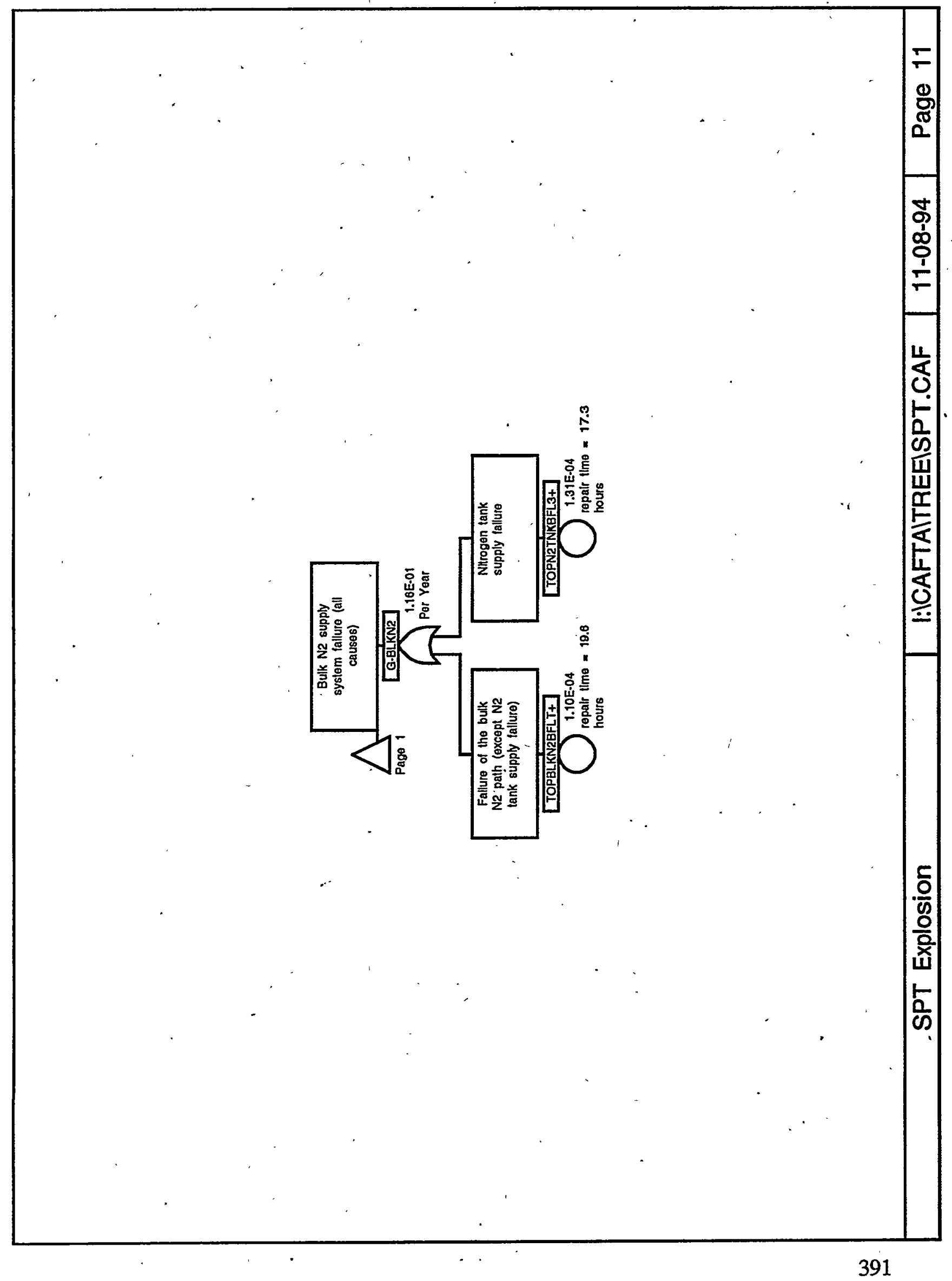


X-ESR-S-00001, REV. 0

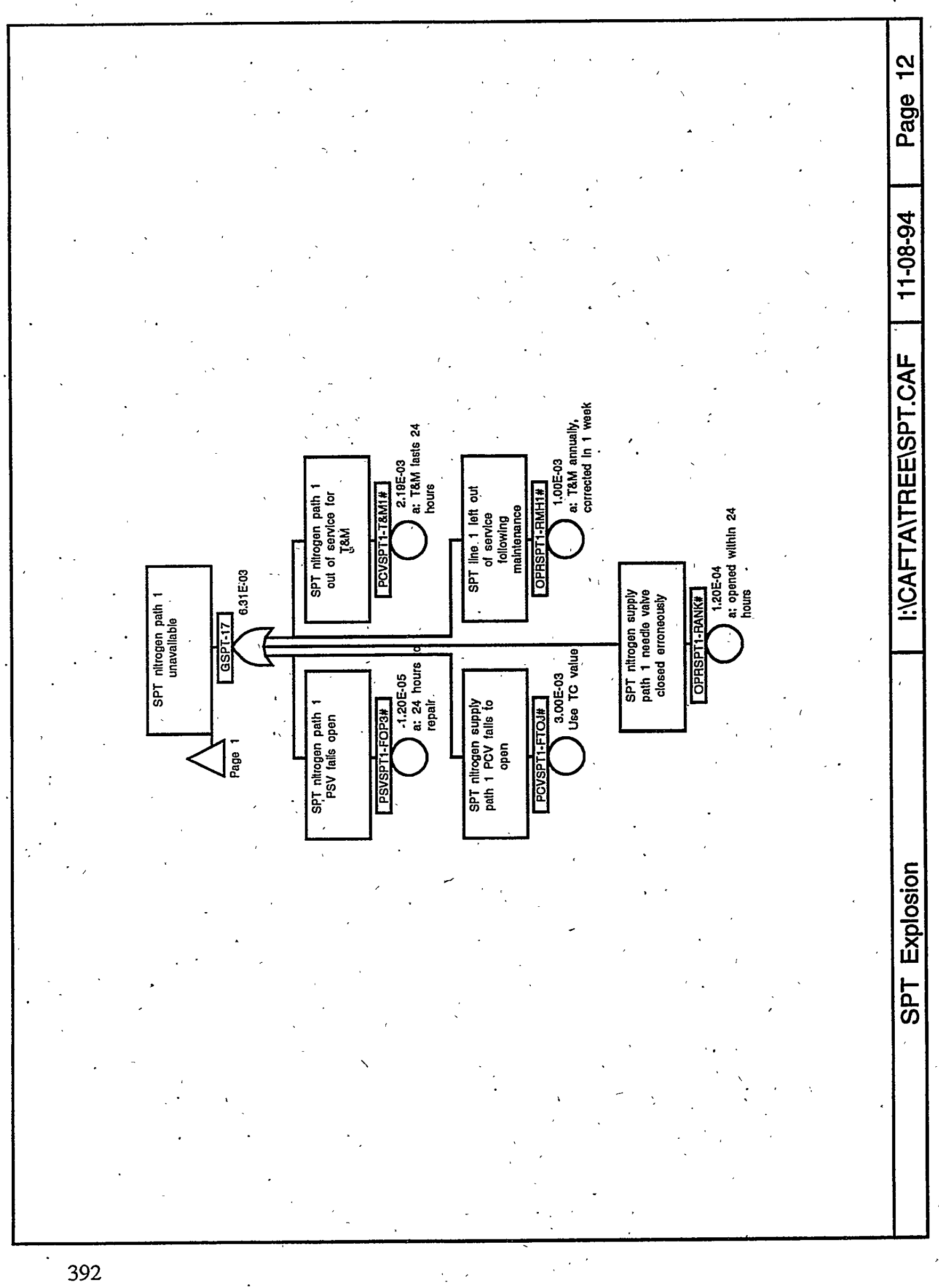




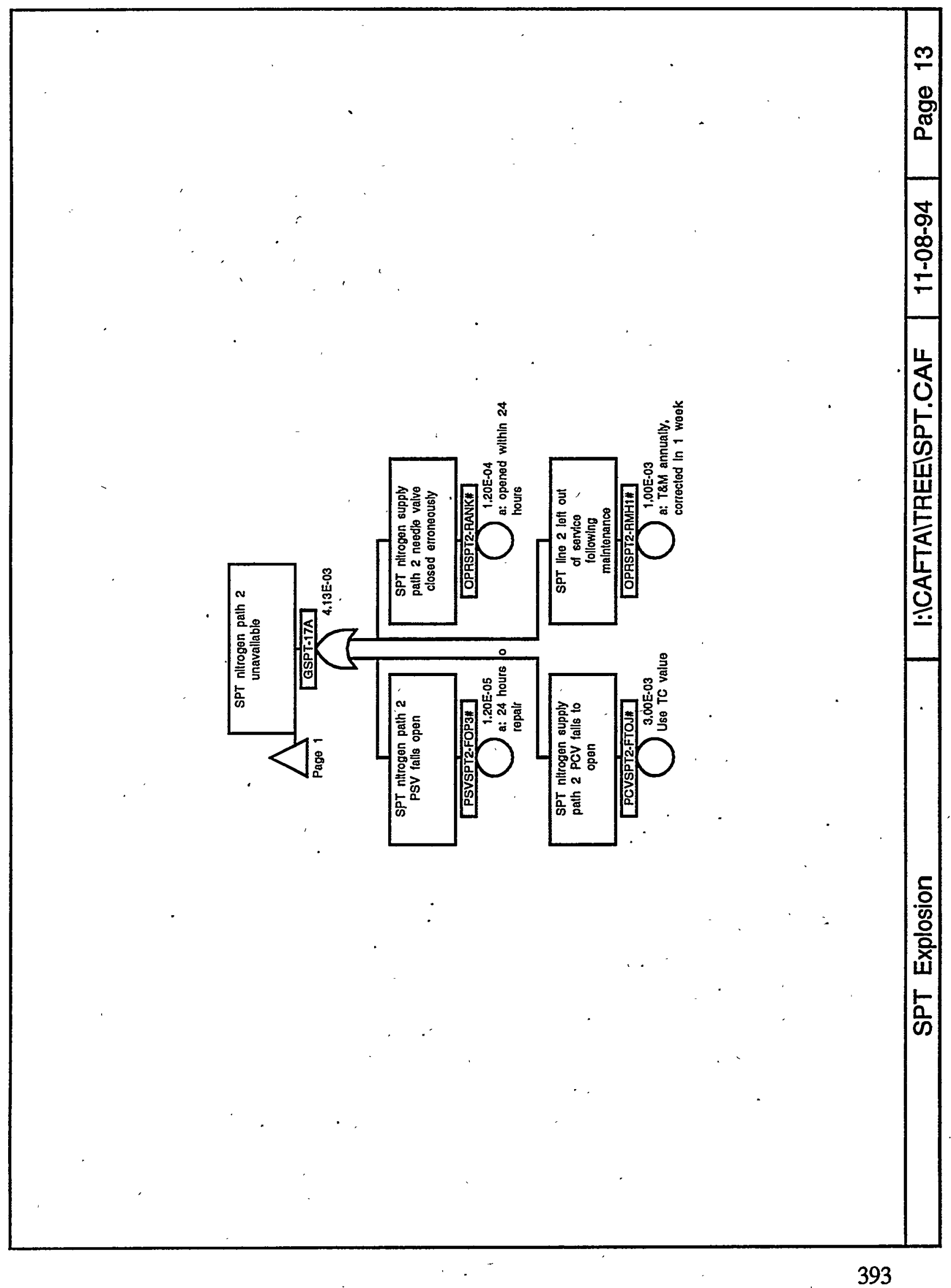




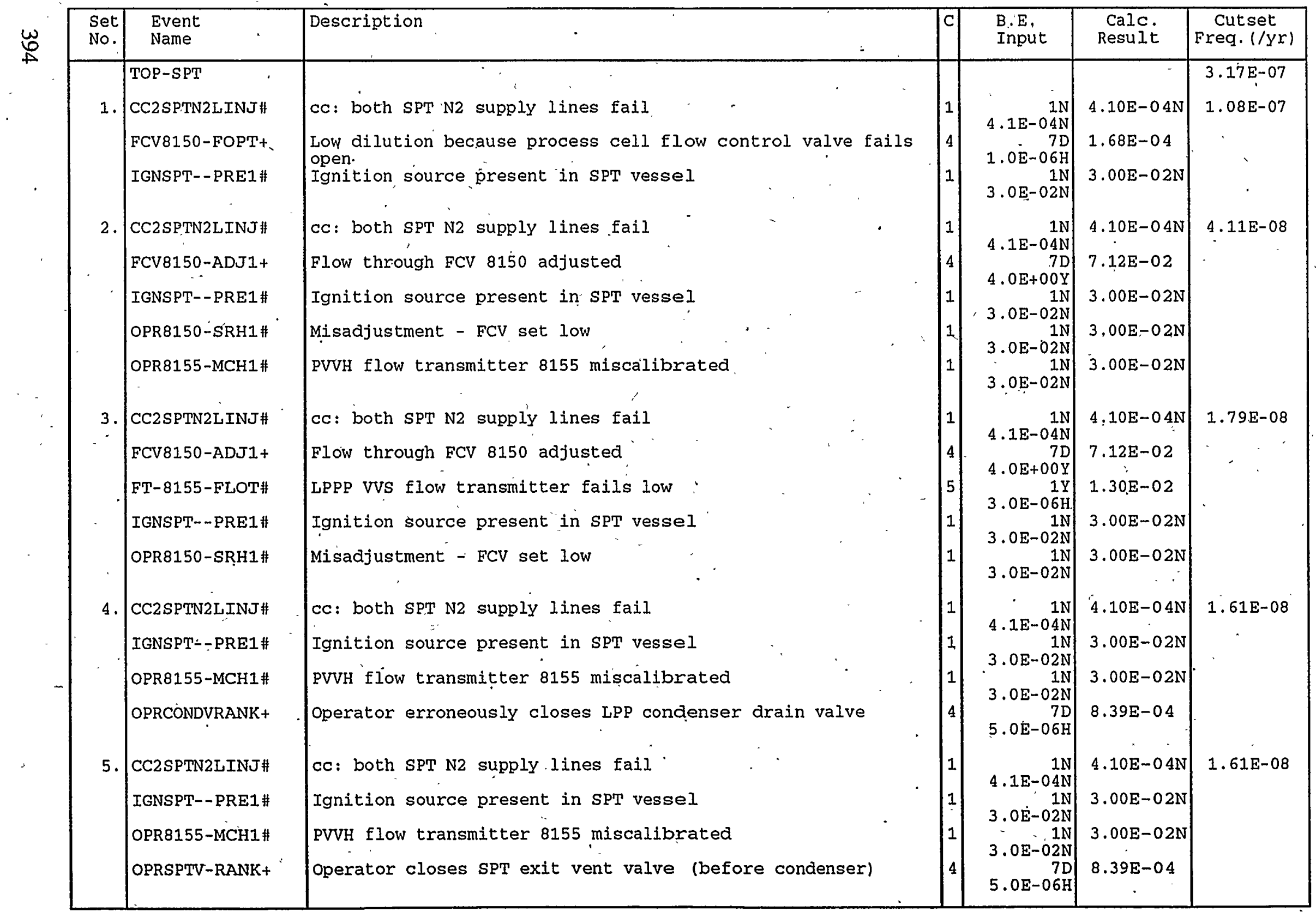




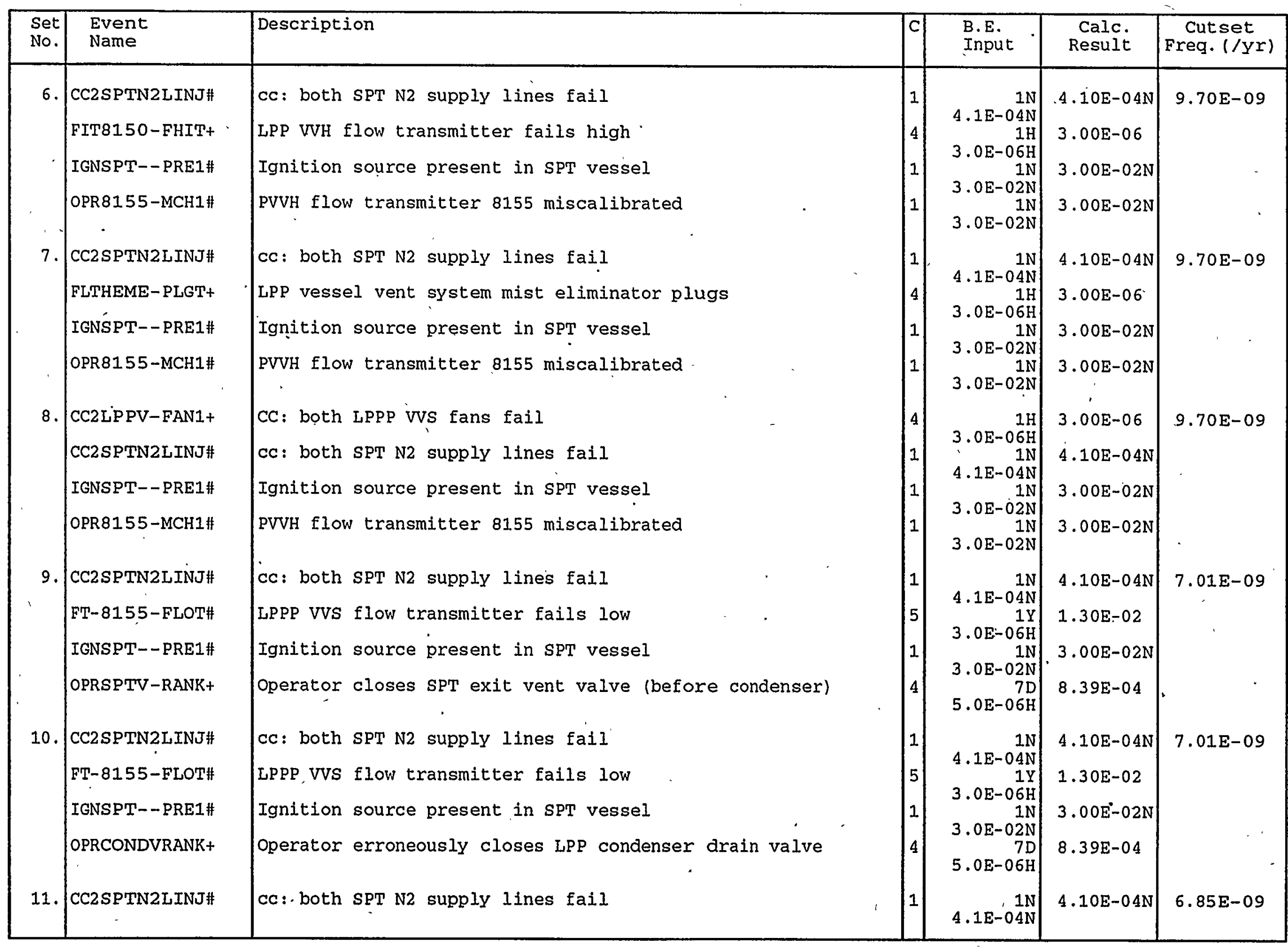


Cutsets for I: ICAFTA ICUT\SPT.CSR $11 / 08 / 94 \quad 12: 39$ AM (CONT.)

\begin{tabular}{|c|c|c|c|c|c|c|c|}
\hline $\begin{array}{l}\text { Set } \\
\text { No. }\end{array}$ & $\begin{array}{l}\text { Event } \\
\text { Name }\end{array}$ & ption & & C & $\begin{array}{l}\text { B.E. } \\
\text { Input }\end{array}$ & $\begin{array}{l}\text { Calc. } \\
\text { Result }\end{array}$ & $\begin{array}{c}\text { Cutset } \\
\text { Freq. }(/ \mathrm{yr})\end{array}$ \\
\hline 12. & $\begin{array}{l}\text { FCV8150-ADJ1+ } \\
\text { IGNSPT- - PRE1\# } \\
\text { OPR8150-SRH1\# } \\
\text { OPR8155-ACN3\# } \\
\text { CC2SPTN2LINJ\# } \\
\text { FANLPPV1BFLT+ } \\
\text { IGNSPT--PRE1\# } \\
\text { OPR8155-MCH1\# } \\
\text { POPLPPV-OFFT\# } \\
\text { CC2SPTN2LINJ\# } \\
\text { FLTHEME-PLGT+ } \\
\text { FT-8155-FLOT\# } \\
\text { IGNSPT--PRE1\# } \\
\text { CC2SPTN2LINJ\# - } \\
\text { FIT8150-FHIT+ } \\
\text { FT-8155-FLOT\# } \\
\text { IGNSPT--PRE1\# } \\
\text { CC2LPPV-FAN1+ } \\
\text { CC2SPTN2LINJ\# } \\
\text { FT-8155-FLOT\# } \\
\text { IGNSPT--PRE1\# } \\
\text { - }\end{array}$ & 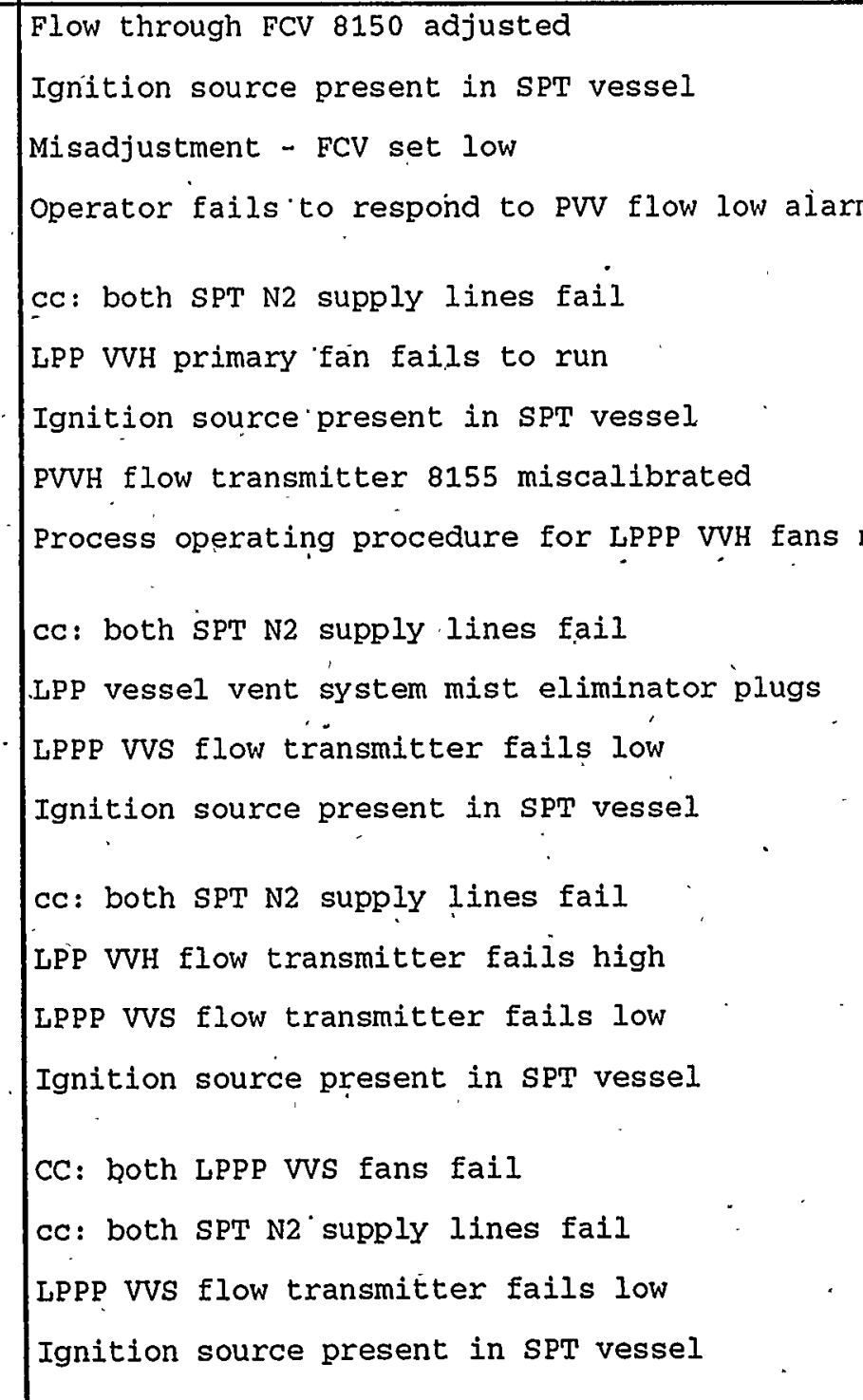 & rm FAL-8155 - & 1 & $\begin{array}{r}7.7 \mathrm{D} \\
4.0 \mathrm{E}+00 \mathrm{Y} \\
1 \mathrm{~N} \\
3.0 \mathrm{E}-02 \mathrm{~N} \\
1 \mathrm{~N} \\
3.0 \mathrm{E}-02 \mathrm{~N} \\
1 \mathrm{~N} \\
5.0 \mathrm{E}-03 \mathrm{~N} \\
1 \mathrm{~N} \\
4.1 \mathrm{E}-04 \mathrm{~N} \\
8 \mathrm{H} \\
3.0 \mathrm{E}-05 \mathrm{H} \\
1 \mathrm{~N} \\
3.0 \mathrm{E}-02 \mathrm{~N} \\
1 \mathrm{~N} \\
3.0 \mathrm{E}-02 \mathrm{~N} \\
1 \mathrm{~N} \\
5.0 \mathrm{E}-02 \mathrm{~N} \\
4.1 \mathrm{E}-04 \mathrm{~N} \\
1 \mathrm{H} \\
3.0 \mathrm{E}-06 \mathrm{H} \\
1 \mathrm{Y} \\
3.0 \mathrm{E}-06 \mathrm{H} \\
1 \mathrm{~N} \\
3.0 \mathrm{E}-02 \mathrm{~N} \\
3.1 \mathrm{E}-04 \mathrm{~N} \\
1 \mathrm{H} \\
3.0 \mathrm{E}-06 \mathrm{H} \\
1 \mathrm{Y} \\
3.0 \mathrm{E}-06 \mathrm{H} \\
1 \mathrm{~N} \\
3.0 \mathrm{E}-02 \mathrm{~N} \\
3.0 \mathrm{E}-06 \mathrm{H} \\
1 \mathrm{~N} \\
4.1 \mathrm{E}-04 \mathrm{~N} \\
1 \mathrm{Y} \\
3.0 \mathrm{E}-06 \mathrm{H} \\
1 \mathrm{~N} \\
3.0 \mathrm{E}-02 \mathrm{~N}\end{array}$ & $\begin{array}{l}7.12 \mathrm{E}-02 \\
3.00 \mathrm{E}-02 \mathrm{~N} \\
3.00 \mathrm{E}-02 \mathrm{~N} \\
5.00 \mathrm{E}-03 \mathrm{~N} \\
4.10 \mathrm{E}-04 \mathrm{~N} \\
2.40 \mathrm{E}-04 \\
3.00 \mathrm{E}-02 \mathrm{~N} \\
3.00 \mathrm{E}-02 \mathrm{~N} \\
5.00 \mathrm{E}-02 \mathrm{~N} \\
4.10 \mathrm{E}-04 \mathrm{~N} \\
3.00 \mathrm{E}-06 \\
1.30 \mathrm{E}-02 \\
3.00 \mathrm{E}-02 \mathrm{~N} \\
4.10 \mathrm{E}-04 \mathrm{~N} \\
3.00 \mathrm{E}-06 \\
1.30 \mathrm{E}-02 \\
3.00 \mathrm{E}-02 \mathrm{~N} \\
3.00 \mathrm{E}-06 \\
4.10 \mathrm{E}-04 \mathrm{~N} \\
1.30 \mathrm{E}-02 \\
3.00 \mathrm{E}-02 \mathrm{~N}\end{array}$ & $\begin{array}{r}4.85 \mathrm{E}-09 \\
4.21 \mathrm{E}-09\end{array}$ \\
\hline
\end{tabular}


Cutsets for I: ICAFTA ICUT\SPT.CSR

11/08/94 12:39 AM (CONT.)

\begin{tabular}{|c|c|c|c|c|c|c|}
\hline $\begin{array}{l}\text { Set } \\
\text { No. }\end{array}$ & $\begin{array}{l}\text { Event } \\
\text { Name }\end{array}$ & Description & C & $\begin{array}{l}\text { B.E. } \\
\text { Input }\end{array}$ & $\begin{array}{l}\text { Calc. } \\
\text { Result }\end{array}$ & $\begin{array}{c}\text { Cutset } \\
\text { Freq. }(/ y r)\end{array}$ \\
\hline 16. & $\begin{array}{l}\text { CC2 SPTN2LINJ\# } \\
\text { FCV8150-FCLT+ } \\
\text { IGNSPT--PRE1\# } \\
\text { OPR8155-MCH1\# }\end{array}$ & $\begin{array}{l}\text { CC: both SPT N2 supply lines fail } \\
\text { LPP VVH flow control valve } 8150 \text { fails closed } \\
\text { Ignition source present in SPT vessel } \\
\text { PVVH flow transmitter } 8155 \text { miscalibrated }\end{array}$ & 1 & $\begin{array}{r}1 \mathrm{~N} \\
4.1 \mathrm{E}-04 \mathrm{~N} \\
1 \mathrm{H} \\
1.0 \mathrm{E}-06 \mathrm{H} \\
1 \mathrm{~N} \\
3.0 \mathrm{E}-02 \mathrm{~N} \\
1 \mathrm{~N} \\
3.0 \mathrm{E}-02 \mathrm{~N}\end{array}$ & $\begin{array}{l}4.10 \mathrm{E}-04 \mathrm{~N} \\
1.00 \mathrm{E}-06 \\
3.00 \mathrm{E}-02 \mathrm{~N} \\
3.00 \mathrm{E}-02 \mathrm{~N}\end{array}$ & $3.23 \mathrm{E}-09$ \\
\hline 17. & $\begin{array}{l}\text { CC2 SPTN2LINJ\# } \\
\text { IGNSPT--PRE1\# } \\
\text { OPR8155-MCH1\# } \\
\text { PDE8761-FHIT+ }\end{array}$ & $\begin{array}{l}\text { Cc: both SPT N2 supply lines fail } \\
\text { Ignition source present in SPT vessel } \\
\text { PVVH flow transmitter } 8155 \text { miscalibrated } \\
\text { Fans commanded off due to PDE fails high (controls speed } \\
\text { controller) }\end{array}$ & 1 & $\begin{array}{r}1 \mathrm{~N} \\
4.1 \mathrm{E}-04 \mathrm{~N} \\
1 \mathrm{~N} \\
3.0 \mathrm{E}-02 \mathrm{~N} \\
1 \mathrm{~N} \\
3.0 \mathrm{E}-02 \mathrm{~N} \\
8 \mathrm{H} \\
1.0 \mathrm{E}-6 \mathrm{H}\end{array}$ & 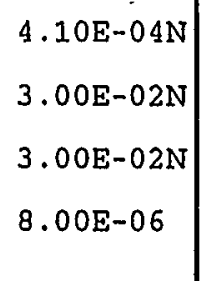 & $3.23 E-09$ \\
\hline 18. & $\begin{array}{l}\text { CC2 SPTN2LINU\# } \\
\text { IGNSPT--PRE1\# } \\
\text { OPRB155 -ACN3\# } \\
\text { OPRCONDVRANK+ }\end{array}$ & $\begin{array}{l}\text { cc: both SPT N2 supply Iines fail } \\
\text { Ignition source present in SPT vessel } \\
\text { Operator fails to respond to PVV flow low alarm FAL-8155 } \\
\text { operator erroneously closes LPP condenser drain valve }\end{array}$ & 1 & $\begin{array}{r}1 \mathrm{~N} \\
4.1 \mathrm{E}-04 \mathrm{~N} \\
1 \mathrm{~N} \\
3.0 \mathrm{E}-02 \mathrm{~N} \\
1 \mathrm{~N} \\
5.0 \mathrm{E}-03 \mathrm{~N} \\
7 \mathrm{D} \\
5.0 \mathrm{E}-06 \mathrm{H}\end{array}$ & $\begin{array}{l}4.10 \mathrm{E}-04 \mathrm{~N} \\
3.00 \mathrm{E}-02 \mathrm{~N} \\
5.00 \mathrm{E}-03 \mathrm{~N} \\
8.39 \mathrm{E}-04\end{array}$ & $\begin{array}{l}2.69 \mathrm{E}-09 \\
.\end{array}$ \\
\hline 19. & $\begin{array}{l}\text { CC2 SPTN2LINJ\# } \\
\text { IGNSPT--PRE1\# } \\
\text { OPR8155-ACN3\# } \\
\text { OPRSPTV-RANK+ }\end{array}$ & $\begin{array}{l}\text { cc: both SPT N2 supply lines fail } \\
\text { Ignition source present in SPT vessel. } \\
\text { Operator fails to respond to PVV flow low alarm FAL- } 8155 \\
\text { Operator closes SPT exit vent valve (before condenser) }\end{array}$ & 4 & $\begin{array}{r}1 \mathrm{~N} \\
4.1 \mathrm{E}-04 \mathrm{~N} \\
1 \mathrm{~N} \\
3.0 \mathrm{E}-02 \mathrm{~N} \\
1 \mathrm{~N} \\
5.0 \mathrm{E}-03 \mathrm{~N} \\
7 \mathrm{D} \\
5.0 \mathrm{E}-06 \mathrm{H}\end{array}$ & $\begin{array}{l}4.10 \mathrm{E}-04 \mathrm{~N} \\
3.00 \mathrm{E}-02 \mathrm{~N} \\
5.00 \mathrm{E}-03 \mathrm{~N} \\
8.39 \mathrm{E}-04\end{array}$ & $2.69 \mathrm{E}-09$ \\
\hline 20. & $\begin{array}{l}\text { FCV8150-FOPT+ } \\
\text { IGNSPT--PRE1\# } \\
\text { PCVSPT1-FTOJ\# } \\
\text { PCVSPT2-FTOJ\# }\end{array}$ & $\begin{array}{l}\text { Low dilution because process cell flow control valve fails } \\
\text { open } \\
\text { Ignition source present in SPT vessel } \\
\text { SPT nitrogen supply path } 1 \text { PCV fails to open } \\
\text { SPT nitrogen supply path } 2 \text { PCV fails to open }\end{array}$ & 4 & $\begin{array}{r}7 \mathrm{D} \\
1.0 \mathrm{E}-06 \mathrm{H} \\
1 \mathrm{~N} \\
3.0 \mathrm{E}-02 \mathrm{~N} \\
1 \mathrm{~N} \\
3.0 \mathrm{E}-03 \mathrm{~N} \\
1 \mathrm{~N} \\
3.0 \mathrm{E}-03 \mathrm{~N}\end{array}$ & $\begin{array}{l}1.68 \mathrm{E}-04 \\
3.00 \mathrm{E}-02 \mathrm{~N} \\
3.00 \mathrm{E}-03 \mathrm{~N} \\
3.00 \mathrm{E}-03 \mathrm{~N}\end{array}$ & $2.36 \mathrm{E}-09$ \\
\hline 21. & CC2SPTN2LINJ\# & cc: both SPT N2 supply lines fail & 1 & $\begin{array}{r}1 \mathrm{~N} \\
4.1 \mathrm{E}-04 \mathrm{~N}\end{array}$ & $4.10 \mathrm{E}-04 \mathrm{~N}$ & $2.10 \mathrm{E}-09$ \\
\hline
\end{tabular}




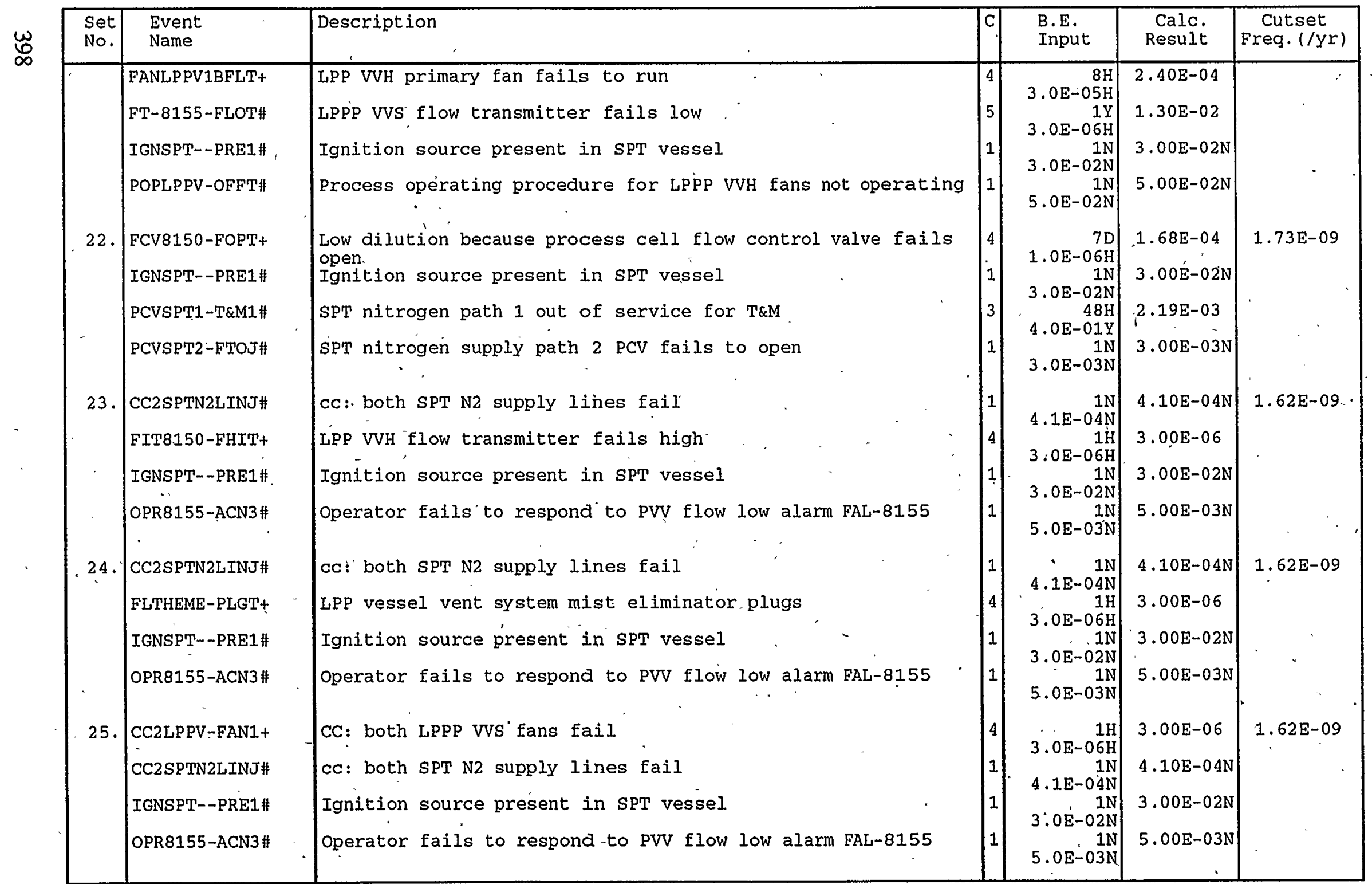




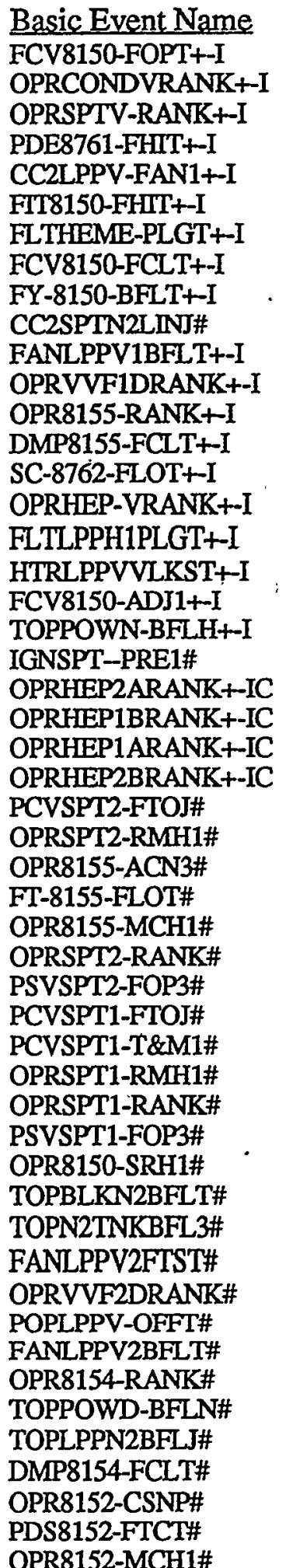

\section{Risk Achievement Worth Description}

Low dilution because process cell FCV fails open Operator erroneously closes LPP condenser drain valvé Operator closes SPT exit vent valve (before condenser) Fans commanded off due to PDE fails high CC: both LPPP VVS fans fail LPP VVH flow transmitter fails high LPP vessel vent system mist eliminator plugs LPP VVH flow control valve 8150 fails closed LPP VVH flow control valve signal conditioner fails high cc: both SPT N2 supply lines fail

LPP VVH primary fan fails to run Erroneaous closure of LPP VVH fan-1 discharge damper Operator erroneously closes LPP VVH fan-1 inlet damper LPP VVH primary fan suction damper fails closed Speed controller for LPP VVH primary fan fails low LPP inlet HEPA filter valve erroneously closed LPP cell inlet HEPA plugs LPP VVH steam tube leaks (moisture high)

Flow through FCV 8150 adjusted LOSS OF NORMAL ELECTRIC POWER TO DWPF Ignition source present in SPT vessel Operator closes LPP VVH HEPA-2 inlet damper Operator erroneously closes LPP VVH HEPA-1 exit damper Operator closes LPP VVH HEPA-1 inlet damper

Operator erroneously closes LPP VVH HEPA-2 exit damper SPT nitrogen supply path 2 PCV fails to open SPT line 2 left out of service following maintenance Operator fails to respond to PVV flow low alarm FAL-8155 LPPP VVS flow transmitter fails low PVVH flow transmitter 8155 miscalibrated SPT nitrogen supply path 2 needle valve closed erroneously SPT nitrogen path 2 PSV fails open SPT nitrogen supply path 1 PCV fails to open

SPT nitrogen path 1 out of service for T\&M SPT line 1 left out of service following maintenance SPT nitrogen supply path 1 needle valve closed erroneously SPT nitrogen path 1 PSV fails open Misadjustment - FCV set low Bulk N2 path unavailable Bulk nitrogen tank supply failure LPP VVH secondary fan fails to start Operator closes LPP VYH fan-2 discharge damper POP for LPPP VVH fans not operating

LPP VVH secondary fan fails to run Operator erroneously closes LPP VVH fan-2 inlet damper LOSS OF BACKUP POWER (DIESEL GENERATORS) LPPP local N2 supply unavailable LPP VVH secondary fan suction damper fails closed Operator fails to respond to high inlet HEPA alarm Inlet HEPA high DP switch fails to close High DP switch miscalibrated

\begin{tabular}{ll} 
Prob/Freq & AchW \\
\hline $1.00 \mathrm{E}-06 / \mathrm{H}$ & $3.61 \mathrm{E}+05$ \\
$4.99 \mathrm{E}-06 / \mathrm{H}$ & $1.73 \mathrm{E}+04$ \\
$4.99 \mathrm{E}-06 / \mathrm{H}$ & $1.73 \mathrm{E}+04$ \\
$1.00 \mathrm{E}-06 / \mathrm{H}$ & $1.73 \mathrm{E}+04$ \\
$3.00 \mathrm{E}-06 / \mathrm{H}$ & $1.73 \mathrm{E}+04$ \\
$3.00 \mathrm{E}-06 / \mathrm{H}$ & $1.73 \mathrm{E}+04$ \\
$3.00 \mathrm{E}-06 / \mathrm{H}$ & $1.73 \mathrm{E}+04$ \\
$1.00 \mathrm{E}-06 / \mathrm{H}$ & $1.71 \mathrm{E}+04$ \\
$1.00 \mathrm{E}-07 / \mathrm{H}$ & $1.63 \mathrm{E}+04$ \\
$4.10 \mathrm{E}-04$ & $2.30 \mathrm{E}+03$ \\
$3.00 \mathrm{E}-05 / \mathrm{H}$ & $9.72 \mathrm{E}+02$ \\
$5.00 \mathrm{E}-06 / \mathrm{H}$ & $9.70 \mathrm{E}+02$ \\
$5.00 \mathrm{E}-06 / \mathrm{H}$ & $9.70 \mathrm{E}+02$ \\
$3.00 \mathrm{E}-06 / \mathrm{H}$ & $9.61 \mathrm{E}+02$ \\
$1.00 \mathrm{E}-06 / \mathrm{H}$ & $9.44 \mathrm{E}+02$ \\
$4.99 \mathrm{E}-06 / \mathrm{H}$ & $9.19 \mathrm{E}+02$ \\
$3.00 \mathrm{E}-06 / \mathrm{H}$ & $8.64 \mathrm{E}+02$ \\
$3.00 \mathrm{E}-07 / \mathrm{H}$ & $6.52 \mathrm{E}+02$ \\
$4.24 \mathrm{E}-04 / \mathrm{H}$ & $5.20 \mathrm{E}+02$ \\
$3.50 \mathrm{E}-05 / \mathrm{H}$ & $1.91 \mathrm{E}+02$ \\
$3.00 \mathrm{E}-02$ & $3.34 \mathrm{E}+01$ \\
$4.99 \mathrm{E}-06 / \mathrm{H}$ & $2.98 \mathrm{E}+01$ \\
$4.99 \mathrm{E}-06 / \mathrm{H}$ & $2.98 \mathrm{E}+01$ \\
$4.99 \mathrm{E}-06 / \mathrm{H}$ & $2.98 \mathrm{E}+01$ \\
$4.99 \mathrm{E}-06 / \mathrm{H}$ & $2.98 \mathrm{E}+01$ \\
$3.00 \mathrm{E}-03$ & $1.53 \mathrm{E}+01$ \\
$1.00 \mathrm{E}-03$ & $1.51 \mathrm{E}+01$ \\
$5.00 \mathrm{E}-03$ & $1.43 \mathrm{E}+01$ \\
$1.30 \mathrm{E}-02$ & $1.42 \mathrm{E}+01$ \\
$3.00 \mathrm{E}-02$ & $1.40 \mathrm{E}+01$ \\
$1.20 \mathrm{E}-04$ & $1.26 \mathrm{E}+01$ \\
$1.20 \mathrm{E}-05$ & $1.20 \mathrm{E}+01$ \\
$3.00 \mathrm{E}-03$ & $1.04 \mathrm{E}+01$ \\
$2.19 \mathrm{E}-03$ & $1.03 \mathrm{E}+01$ \\
$1.00 \mathrm{E}-03$ & $1.03 \mathrm{E}+01$ \\
$1.20 \mathrm{E}-04$ & $8.58 \mathrm{E}+00$ \\
$1.20 \mathrm{E}-05$ & $8.16 \mathrm{E}+00$ \\
$3.00 \mathrm{E}-02$ & $8.13 \mathrm{E}+00$ \\
$1.10 \mathrm{E}-04$ & $2.22 \mathrm{E}+00$ \\
$1.31 \mathrm{E}-04$ & $2.22 \mathrm{E}+00$ \\
$5.00 \mathrm{E}-03$ & $1.75 \mathrm{E}+00$ \\
$8.40 \mathrm{E}-04$ & $1.73 \mathrm{E}+00$ \\
$5.00 \mathrm{E}-02$ & $1.72 \mathrm{E}+00$ \\
$2.40 \mathrm{E}-04$ & $1.68 \mathrm{E}+00$ \\
$1.20 \mathrm{E}-04$ & $1.66 \mathrm{E}+00$ \\
$1.10 \mathrm{E}-02$ & $1.60 \mathrm{E}+00$ \\
$5.30 \mathrm{E}-04$ & $1.55 \mathrm{E}+00$ \\
$2.40 \mathrm{E}-05$ & $1.41 \mathrm{E}+00$ \\
$1.13 \mathrm{E}+02$ & $1.13 \mathrm{E}+00$ \\
$3 \mathrm{~m}+00$ \\
\hline
\end{tabular}

Note: Events that make less than 1\% difference to the importance factor are not included. Modified top event frequency $=$ (top event frequency) $\mathrm{x}$ (risk achievement worth). 
Explosion in LPPP Sludge Pump Tank (SPT) . Top Event Frequency: 3.18E-07/YR

\begin{tabular}{|c|c|c|c|c|}
\hline Basic Event Name & Description & & Prob/Freg & RedW \\
\hline *IGNSPT-PRE1\# & Ignition source present in SPT vessel & & $3.00 \mathrm{E}-02$ & $0.00 \mathrm{E}+00$ \\
\hline CC2SPIN2LINJ\# & cc: both SPT N2 supply lines fail & & 4.10E-04 & $1.77 \mathrm{E}+01$ \\
\hline OPR8155-MCH1\# & PVVH flow transmitter 8155 miscalibrated & & $3.00 \mathrm{E}-02$ & $1.67 \mathrm{E}+00$ \\
\hline FCV8150-FOPT+I & Low dilution because process cell FCV fails open & & $1.00 \mathrm{E}-06 / \mathrm{H}$ & $1.56 \mathrm{E}+00$ \\
\hline FCV8150-ADJ1+-I & Flow through FCV 8150 adjusted & & $4.24 \mathrm{E}-04 / \mathrm{H}$ & $1.28 \mathrm{E}+00$ \\
\hline OPR8150-SRH1\# & Misadjustment - FCV set low & & $3,00 \mathrm{E}-02$ & $1.28 \mathrm{E}+00$ \\
\hline FT-8155-FLOT\# & LPPP VVS flow transmitter fails low & & $1.30 \mathrm{E}-02$ & $1.21 \mathrm{E}+00$ \\
\hline OPRSPTV-RANK+I & Operator closes SPT exit vent valve (before condenser & & $4.99 \mathrm{E}-06 / \mathrm{H}^{\circ}$ & $1.09 \mathrm{E}+00$ \\
\hline OPRCONDVRANK+-I & Operator erroneously closes LPP condenser drain valv & & $4.99 \mathrm{E}-06 / \mathrm{H}$ & $1.09 E+00$ \\
\hline OPR8155-ACN3\# & Operator fails to respond to PVV flow low alarm FAL & -8155 & $5.00 \mathrm{E}-03$ & $1.07 \mathrm{E}+00$ \\
\hline FLTHEME-PLGT+I & LPP vessel vent system mist eliminator plugs & . & $3.00 \mathrm{E}-06 / \mathrm{H}$ & $1.05 E+00$ \\
\hline FIT8150-FHIT+-I & LPP VVH flow transmitter fails high & & $3.00 \mathrm{E}-06 / \mathrm{H}$ & $1.05 E+00$ \\
\hline CC2LPPV-FAN1+I & CC: both LPPP VVS fans fail & & $3.00 \mathrm{E}-06 / \mathrm{H}$ & $1.05 E+00$ \\
\hline PCVSPT2-FTOJ\# & SPT nitrogen supply path 2 PCV fails to & & $3.00 \mathrm{E}-03$ & $1.05 E+00$ \\
\hline POPLPPV-OFFT\# & POP for LPPP VVH fans not operating & & -02 & $1.04 \mathrm{E}+00$ \\
\hline FANLPPV1BFLT+I & LPP VVH primary fan fails to run & & $3.00 \mathrm{E}-05 / \mathrm{H}$ & $1.03 E+00$ \\
\hline PCVSPT1-FTOJ\# & SPT nitrogen supply path 1 PCV fails to open & & $3.00 \mathrm{E}-03$ & $1.03 E+00$ \\
\hline PCVSPT1-T\&M1\# & SPT nitrogen path 1 out of service for $T \& M$ & & $2.19 \mathrm{E}-03$ & $1.02 \mathrm{E}+00$ \\
\hline PDE & Fans commanded off due to PDE fails high & & $1.00 \mathrm{E}-06 / \mathrm{H}$ & $1.02 \mathrm{E}+00$ \\
\hline FCV8150-FCLT+I & lve 8150 fails closed & & $.1 .00 \mathrm{E}-06 / \mathrm{H}$ & $1.02 \mathrm{E}+00$ \\
\hline OPRSPT2-RMH1\# & SPT line 2 left out of service following maintenance & & $1.00 \mathrm{E}-03$ & $1.01 \mathrm{E}+00$ \\
\hline
\end{tabular}

* $\dot{\mathrm{A}}$ Reduction Worth of $0.0 \mathrm{E}+00$ indicates event appears in every cutset could serve as a single event preventor for the accident.

Note: Events that make less than $1 \%$ difference to the importance factor are not included. Modified top event frequency $=$ (top event frequency) $/$ (risk reduction worth). 
X-ESR-S-00001, REV. 0

\section{LPPP PPT Explosion}




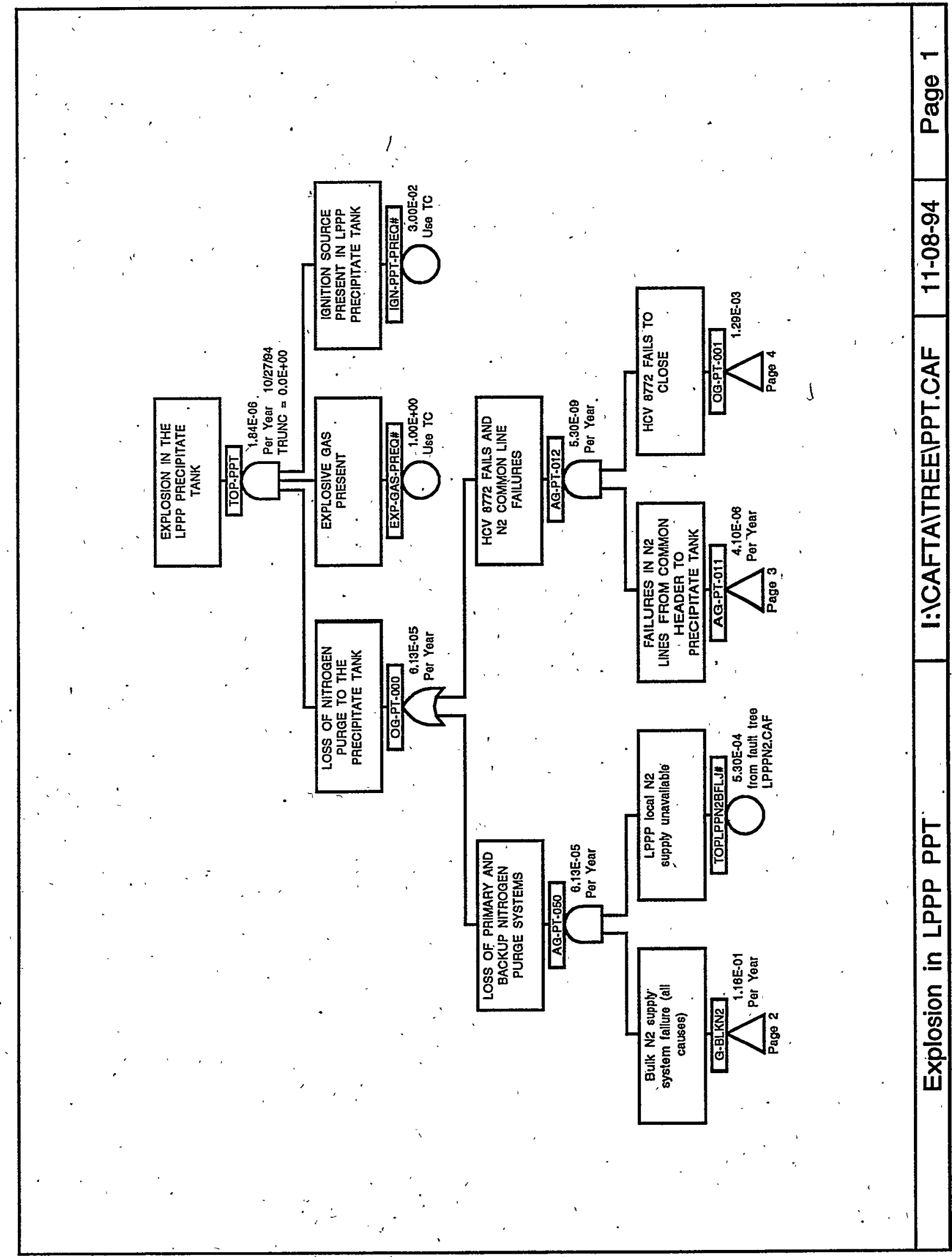




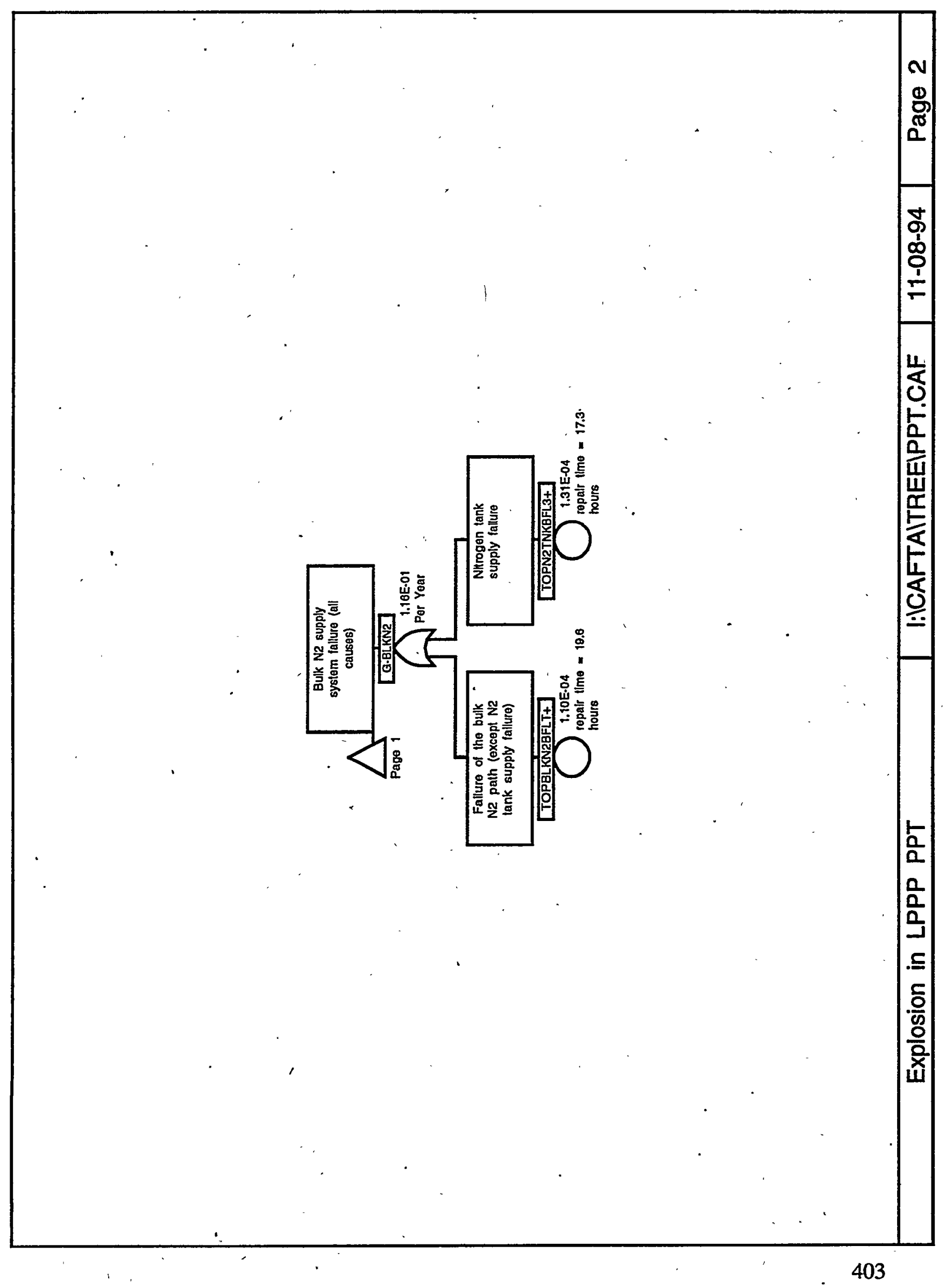


X-ESR-S-00001, REV. 0

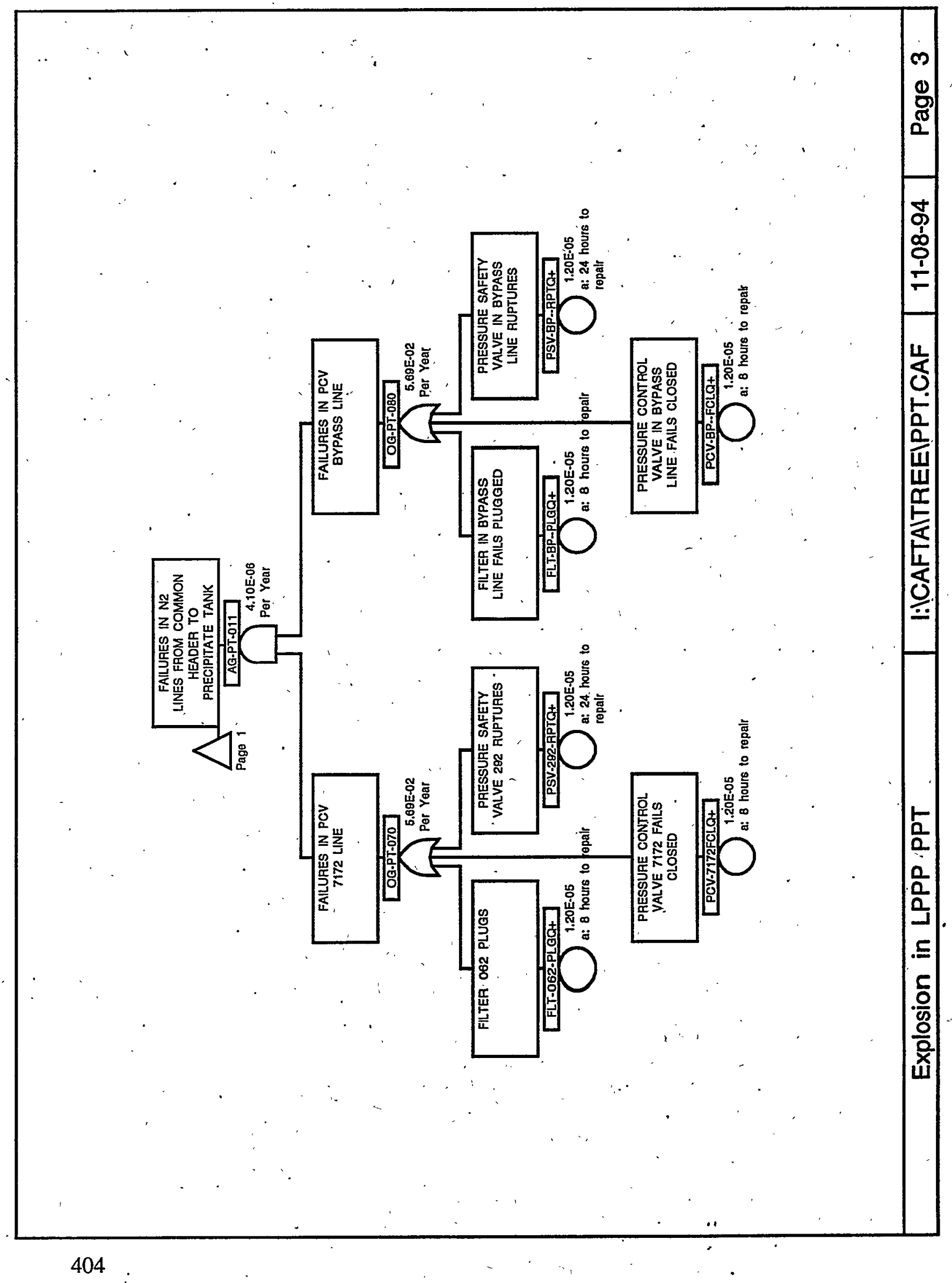




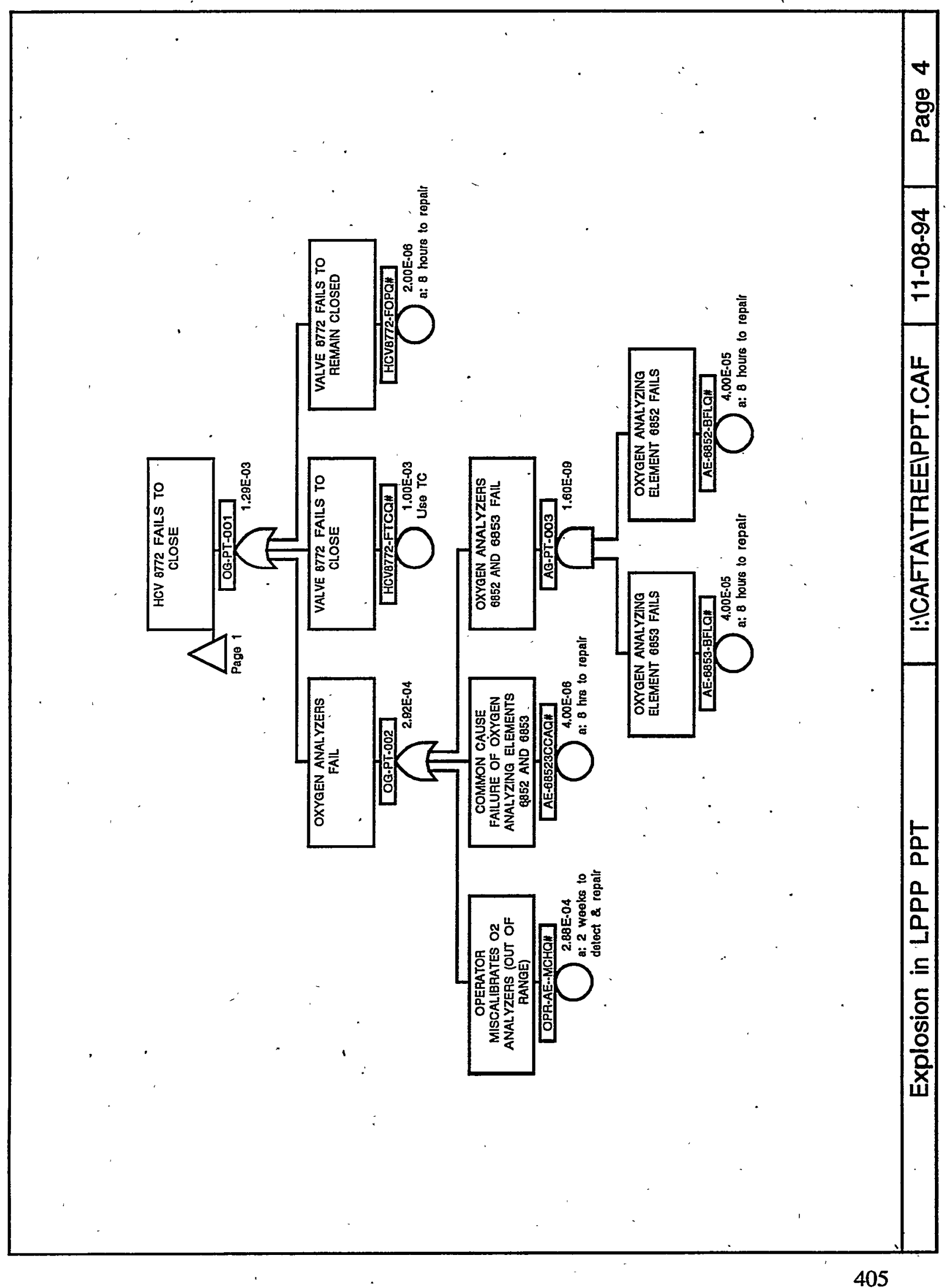




$$
\square
$$


Cutsets for I: \CAFTA \CUT\PPT:CSR

$11 / 08 / 94 \quad 12: 41 \mathrm{AM}$

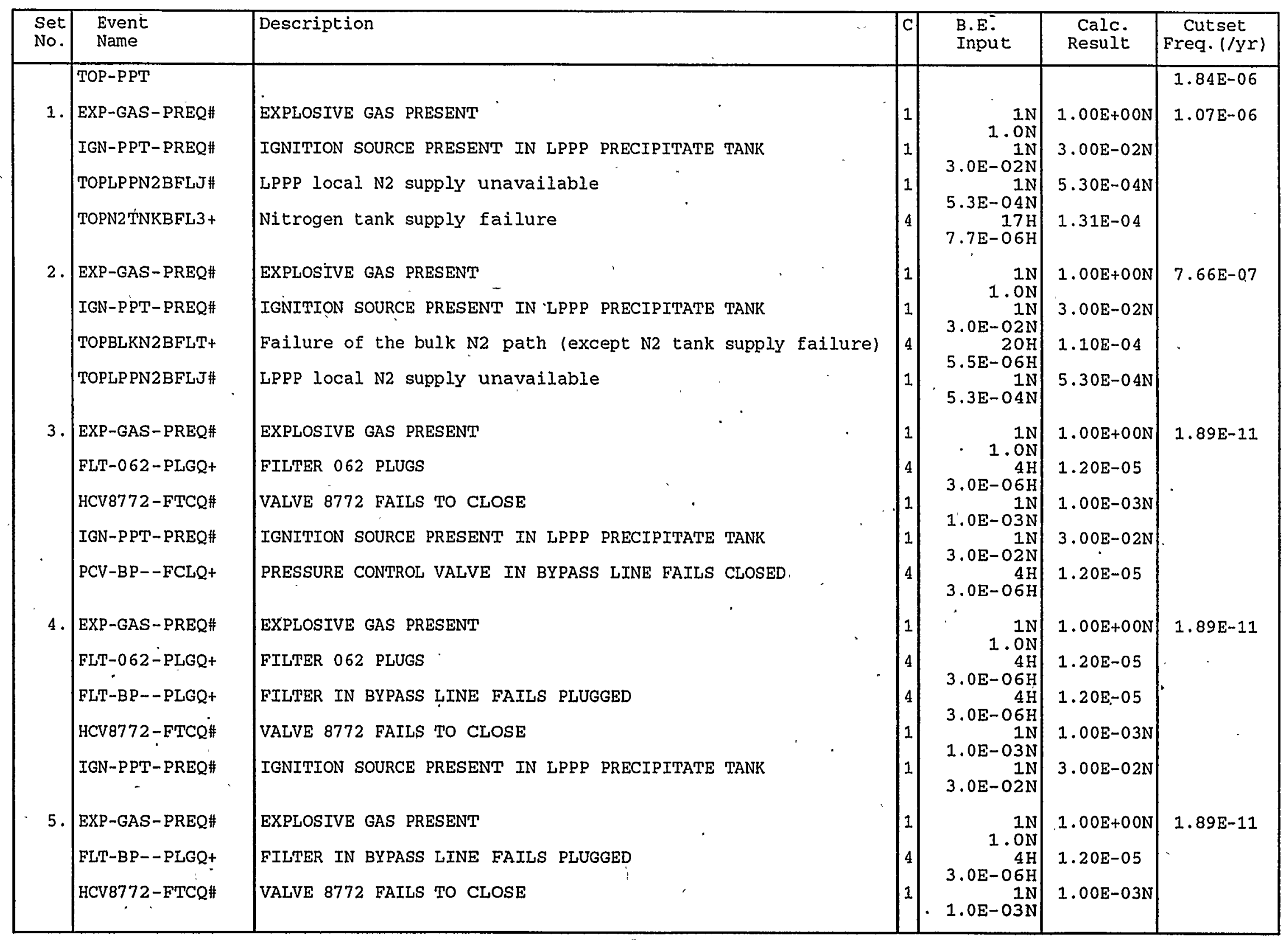




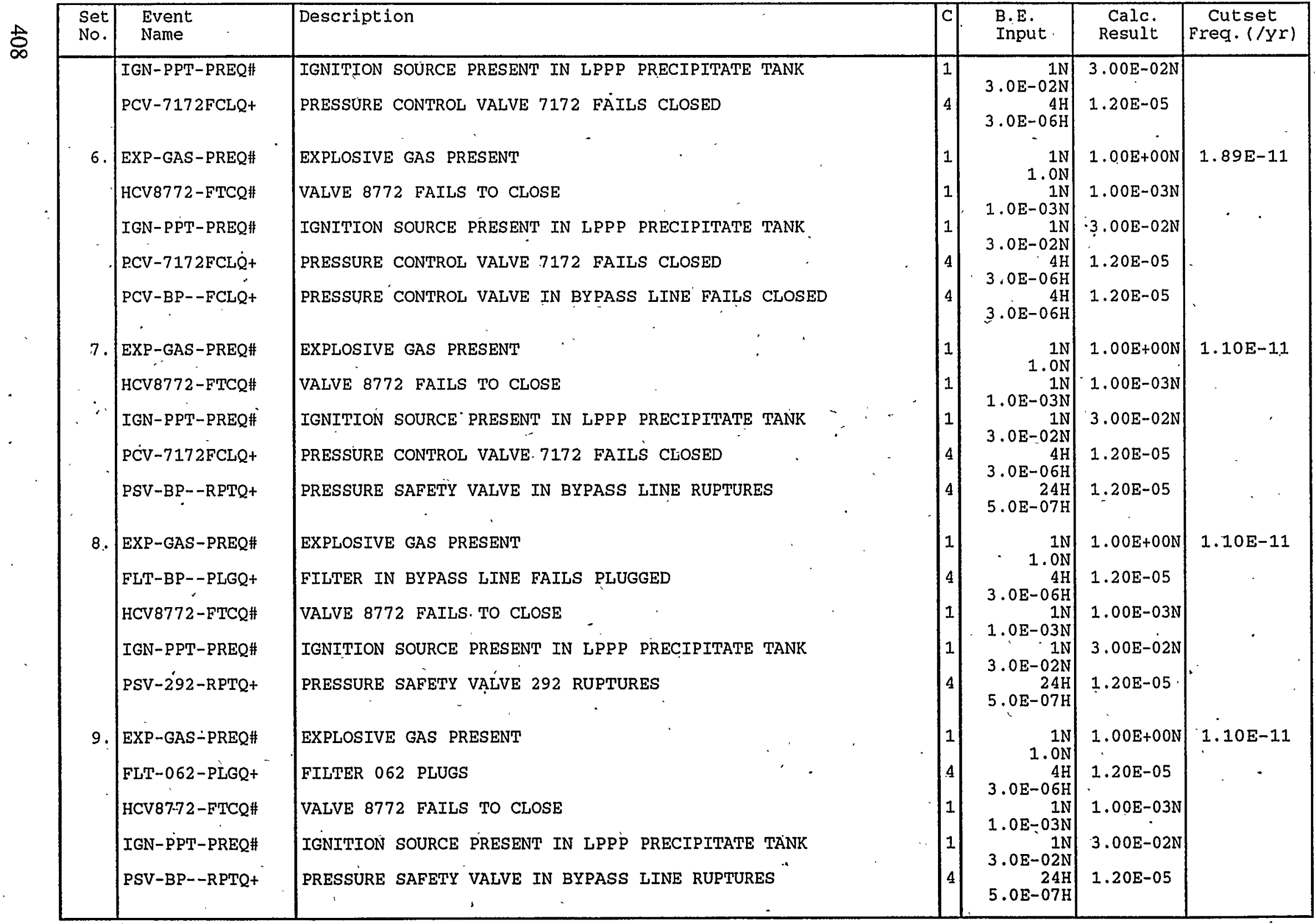


Cutsets for I: ICAFTA \CUT\PPT.CSR 11/08/94 12:41 AM (CONT.)

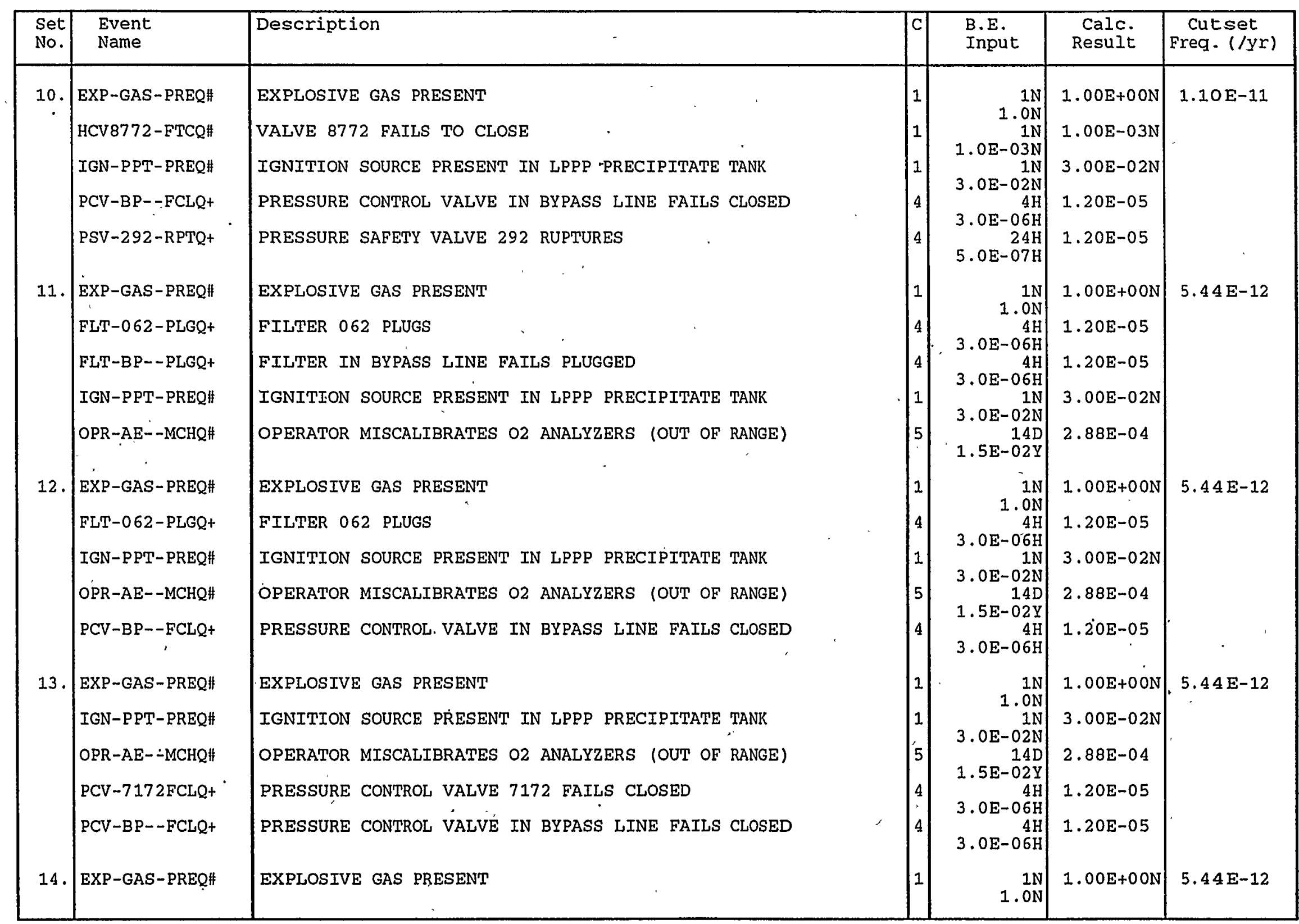




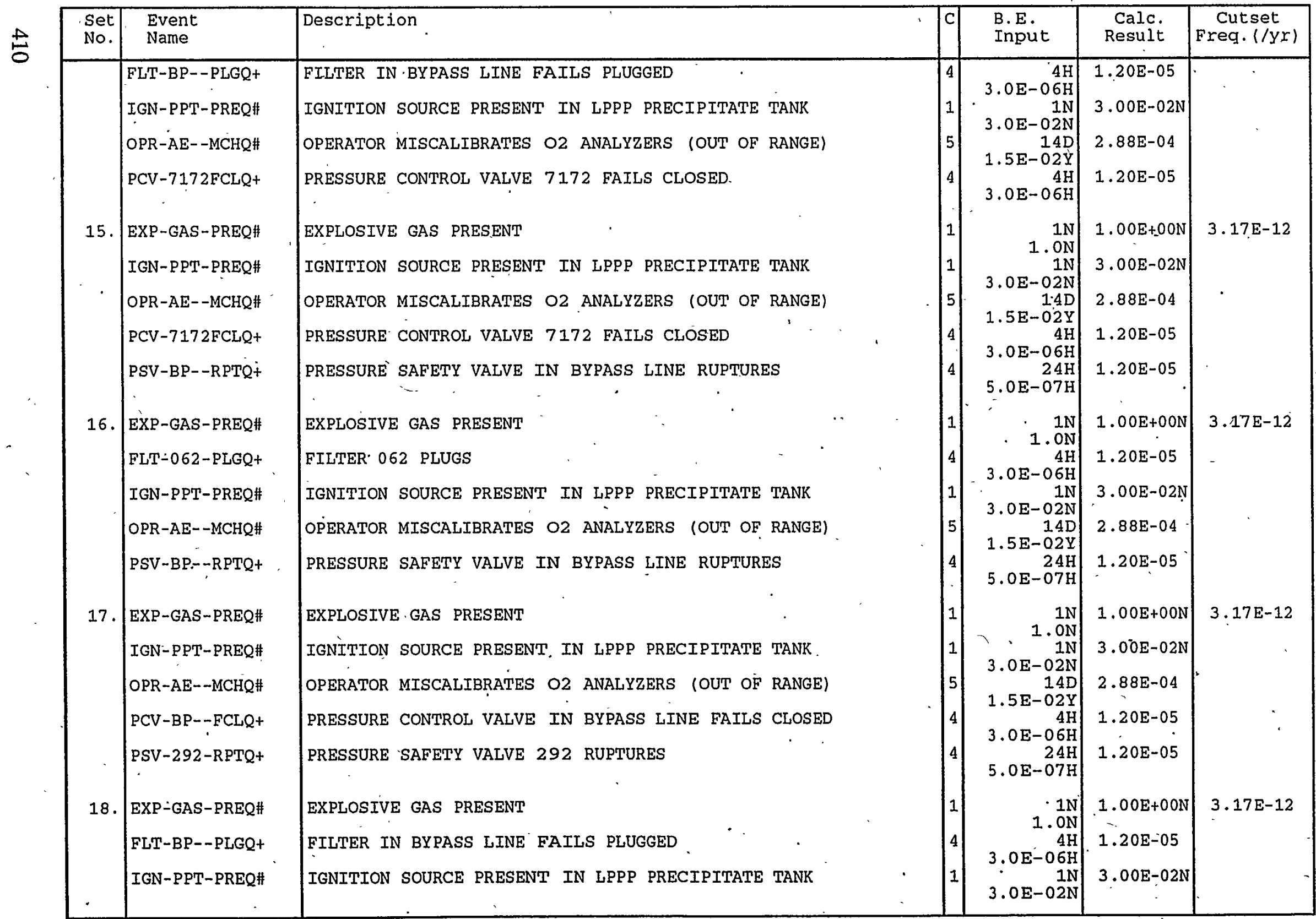


Cutsets for I: \CAFT'A \CUT\PPT'.CSR

11/08/94 12:41 AM (CONT.)

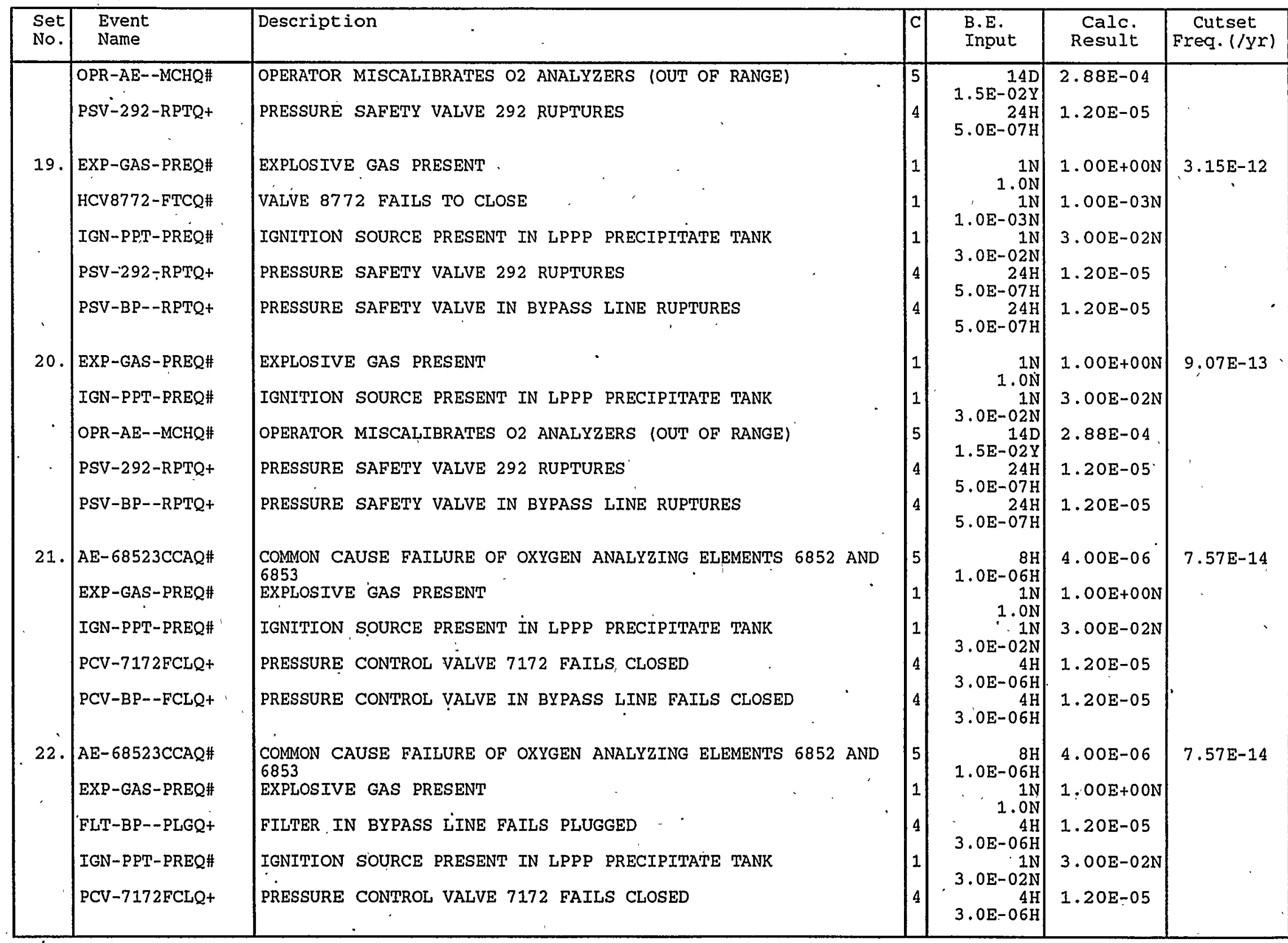




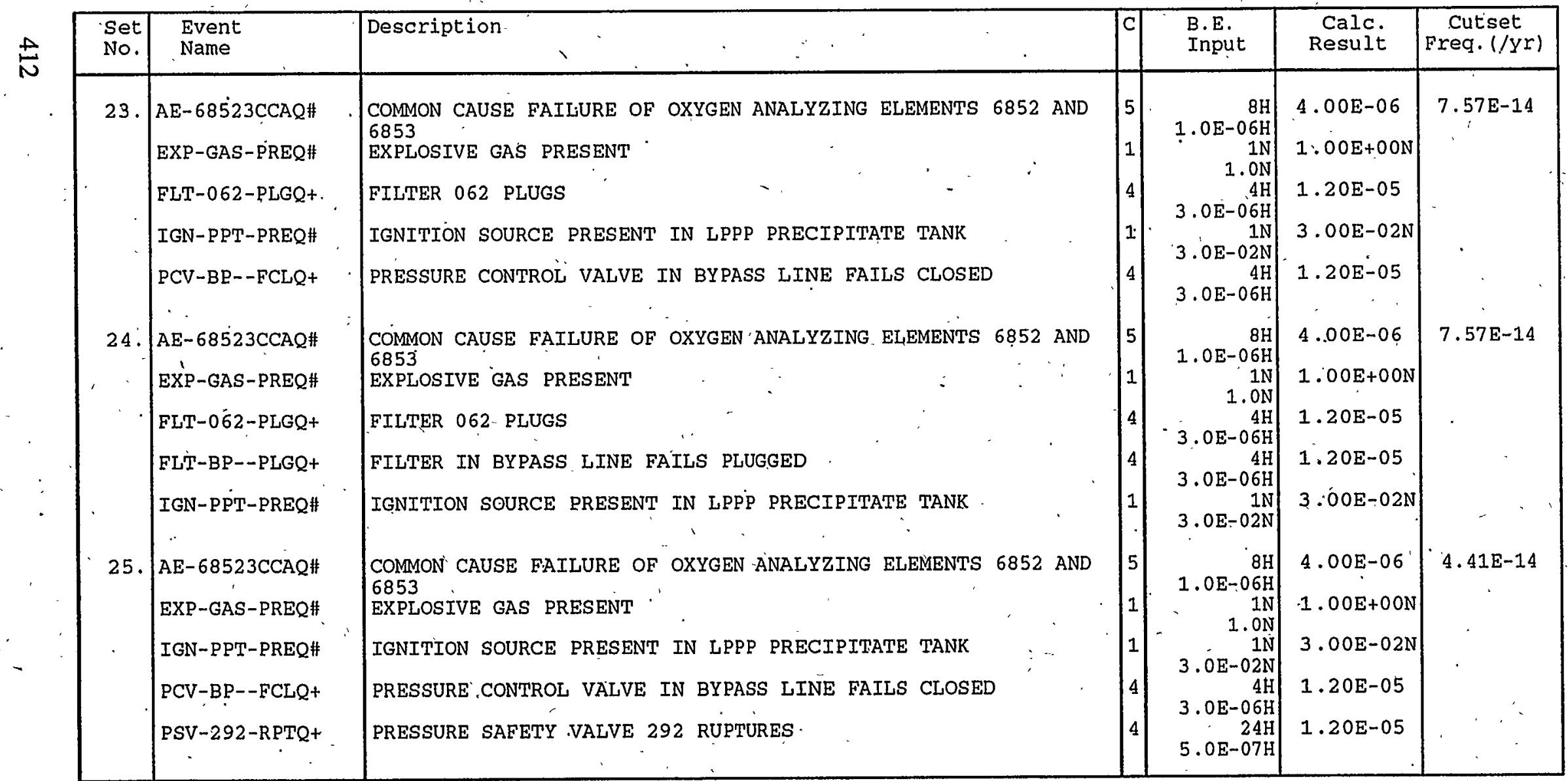




\section{EXPLOSION IN THE LPPP PRECIPITATE TANK}

Top Event Frequency: 1.84E-06/YR

\begin{abstract}
Basic Event Name TOPBLKN2BFLT+I TOPN2TNKBFL3+I TOPLPPN2BFLJ\# IGN-PPT-PREQ\# PSV-292-RPTQ+-IC PSV-BP--RPTQ+-IC PCV-7172FCLQ+IC FLT-BP-PLGQ+-IC FLT-062-PLGQ+-IC PCV-BP-FCLQ+-IC OPR-AE-MCHQ\# HCV8772-FTCQ\#
\end{abstract}

Risk Achievement Worth Description Failure of the bulk N2 path Nitrogen tank supply failure LPPP local N2 supply unavailable IGNITION SOURCE PRESENT IN PRECIPITATE TANK PRESSURE SAFETY VALVE 292 RUPTURES PSV IN BYPASS LINE RUPTURES PRESSURE CONTROL VALVE 7172 FAILS CLOSED FILTER IN BYPASS LINE FAIIS PLUGGED FILTER 062 PLUGS

PCV IN BYPASS LINE FAILS CLOSED OPERATOR MISCALIBRATES O2 ANALYZERS

VALVE 8772 FAIIS TO CLOSE

Modified top event frequency $=$

\begin{tabular}{ll} 
Prob/Freg & AchW \\
\hline $5.50 \mathrm{E}-06 / \mathrm{H}$ & $7.58 \mathrm{E}+04$ \\
$7.70 \mathrm{E}-06 / \mathrm{H}$ & $7.58 \mathrm{E}+04$ \\
$5.30 \mathrm{E}-04$ & $1.89 \mathrm{E}+03$ \\
$3.00 \mathrm{E}-02$ & $3.33 \mathrm{E}+01$ \\
$5.00 \mathrm{E}-07 / \mathrm{H}$ & $8.82 \mathrm{E}+00$ \\
$5.00 \mathrm{E}-07 / \mathrm{H}$ & $8.82 \mathrm{E}+00$ \\
$3.00 \mathrm{E}-06 / \mathrm{H}$ & $8.82 \mathrm{E}+00$ \\
$3.00 \mathrm{E}-06 / \mathrm{H}$ & $8.82 \mathrm{E}+00$ \\
$3.00 \mathrm{E}-06 / \mathrm{H}$ & $8.82 \mathrm{E}+00$ \\
$3.00 \mathrm{E}-06 / \mathrm{H}$ & $8.82 \mathrm{E}+00$ \\
$2.88 \mathrm{E}-04$ & $1.07 \mathrm{E}+00$ \\
$1.00 \mathrm{E}-03$ & $1.07 \mathrm{E}+00$
\end{tabular}

Basic Event Name *EXP-GAS-PREQ\# *IGN-PPT-PREQ\# TOPLPPN2BFL \#\# TOPN2TNKBFL3+I

$\therefore$ TOPBLKN2BFLT+I

\section{Risk Reduction Worth}

\section{Description} EXPLOSIVE GAS PRESENT IGNITION SOURCE PRESENT IN PRECIPITATE TANK LPPP local N2 supply unavailable Nitrogen tank supply failure Failure of the bulk 2 path

$\begin{array}{ll}\frac{\text { Prob/Freg }}{1.00 \mathrm{E}+00} & \frac{\text { RedW }}{0.00 \mathrm{E}+00} \\ 3.00 \mathrm{E}-02 & 0.00 \mathrm{E}+00 \\ 5.30 \mathrm{E}-04 & 1.13 \mathrm{E}+04 \\ 7.70 \mathrm{E}-06 / \mathrm{H} & 2.40 \mathrm{E}+00 \\ 5.50 \mathrm{E}-06 / \mathrm{H} & 1.71 \mathrm{E}+00\end{array}$

* A Reduction Worth of $0.0 \mathrm{E}+00$ indicates event appears in every cutset could serve as a single event preventor for the accident.

Note: Events that make less than $1 \%$ difference to the importance factor are not included. Modified top event frequency = (top event frequency) / (risk reduction worth). 
X-ESR-S-00001, REV. 0

\section{OWST Explosion}




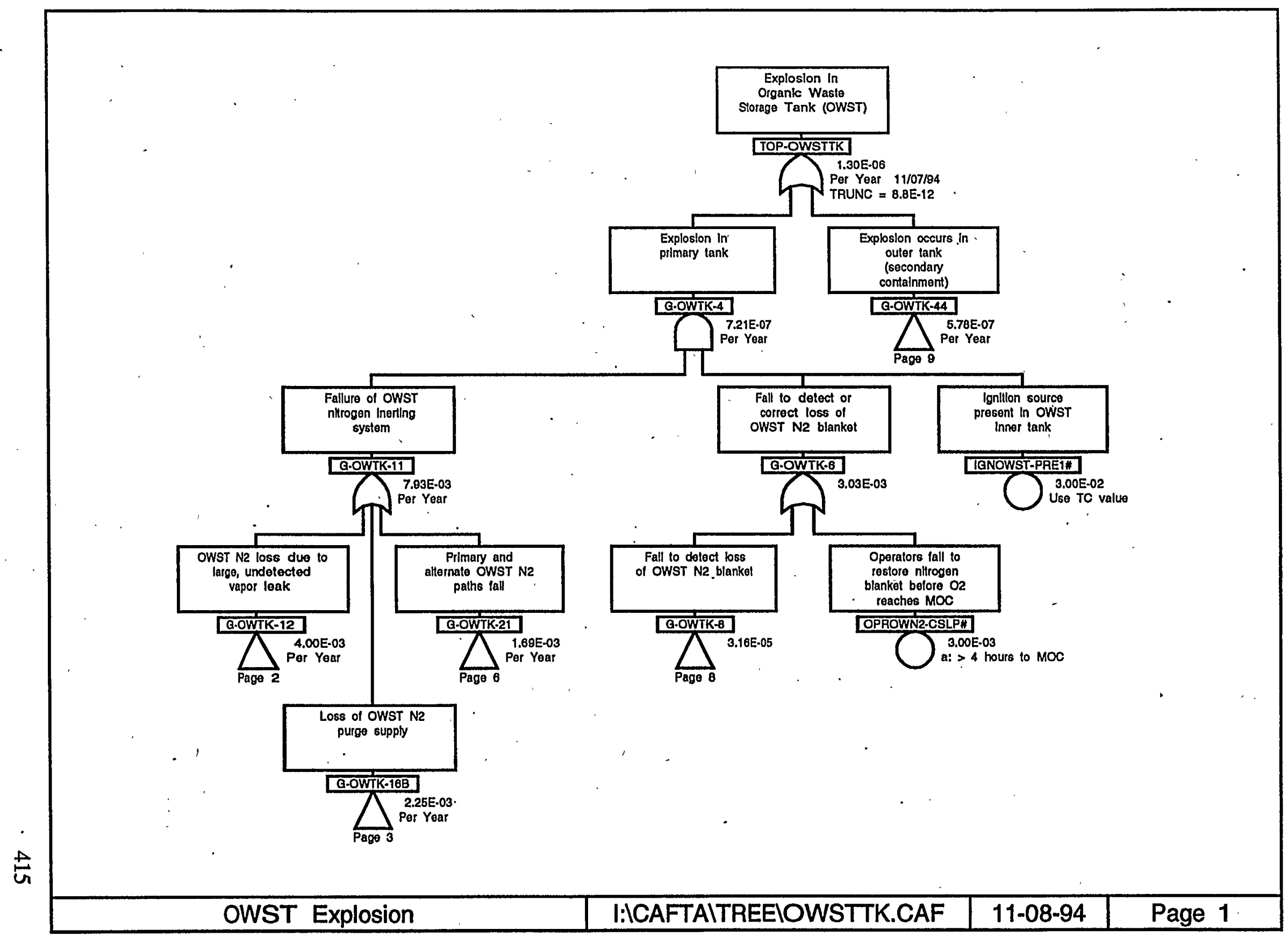




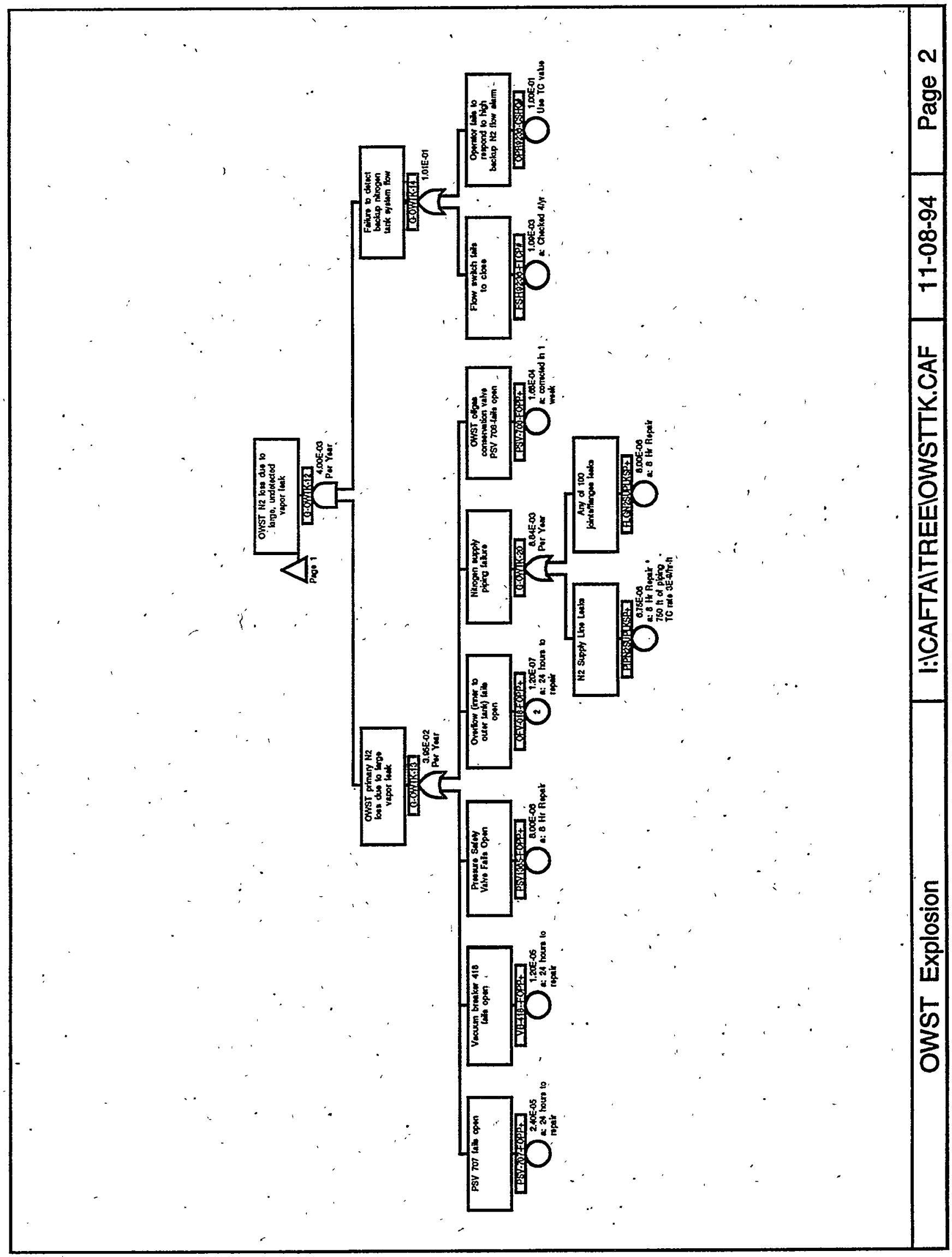




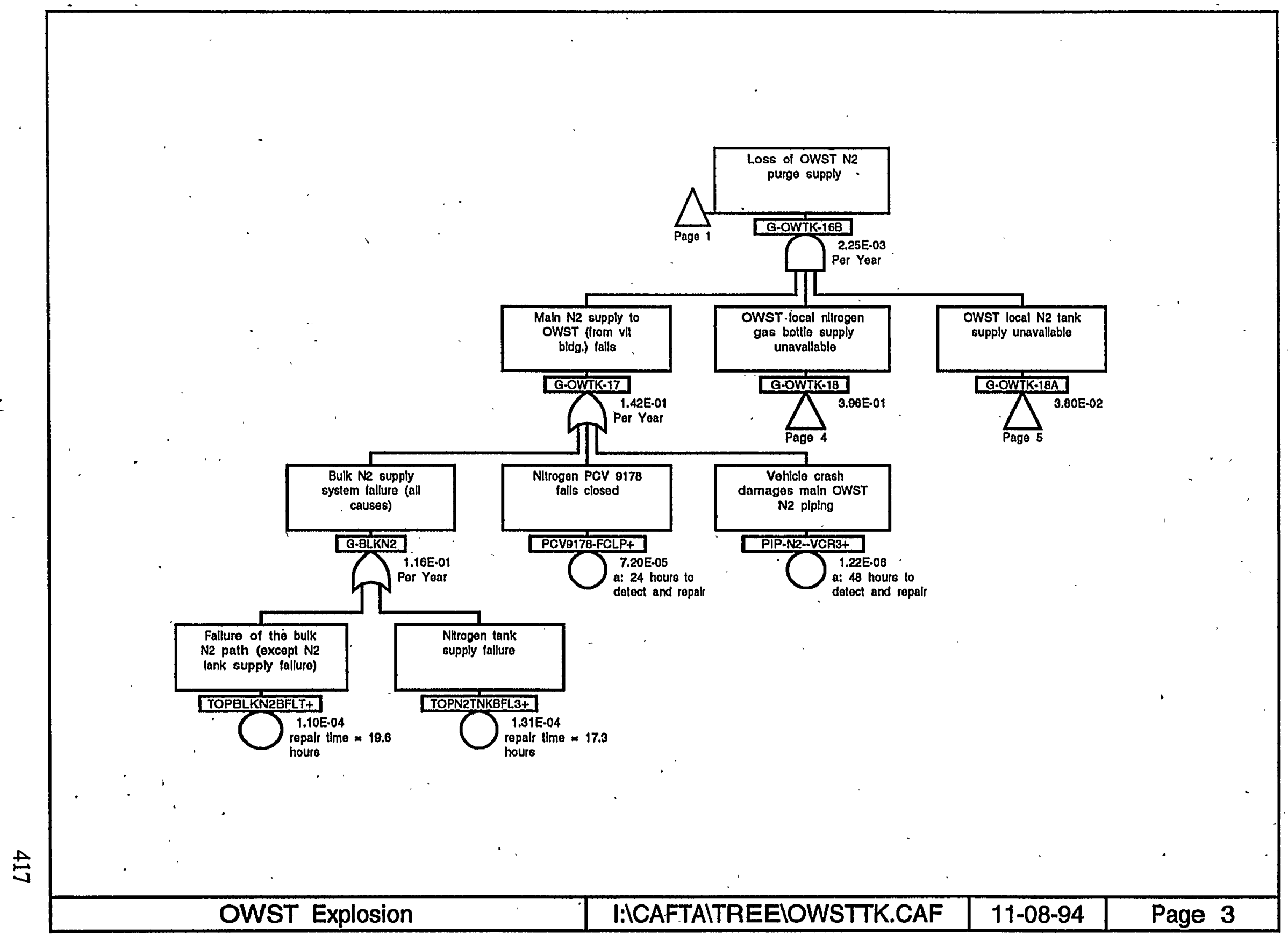




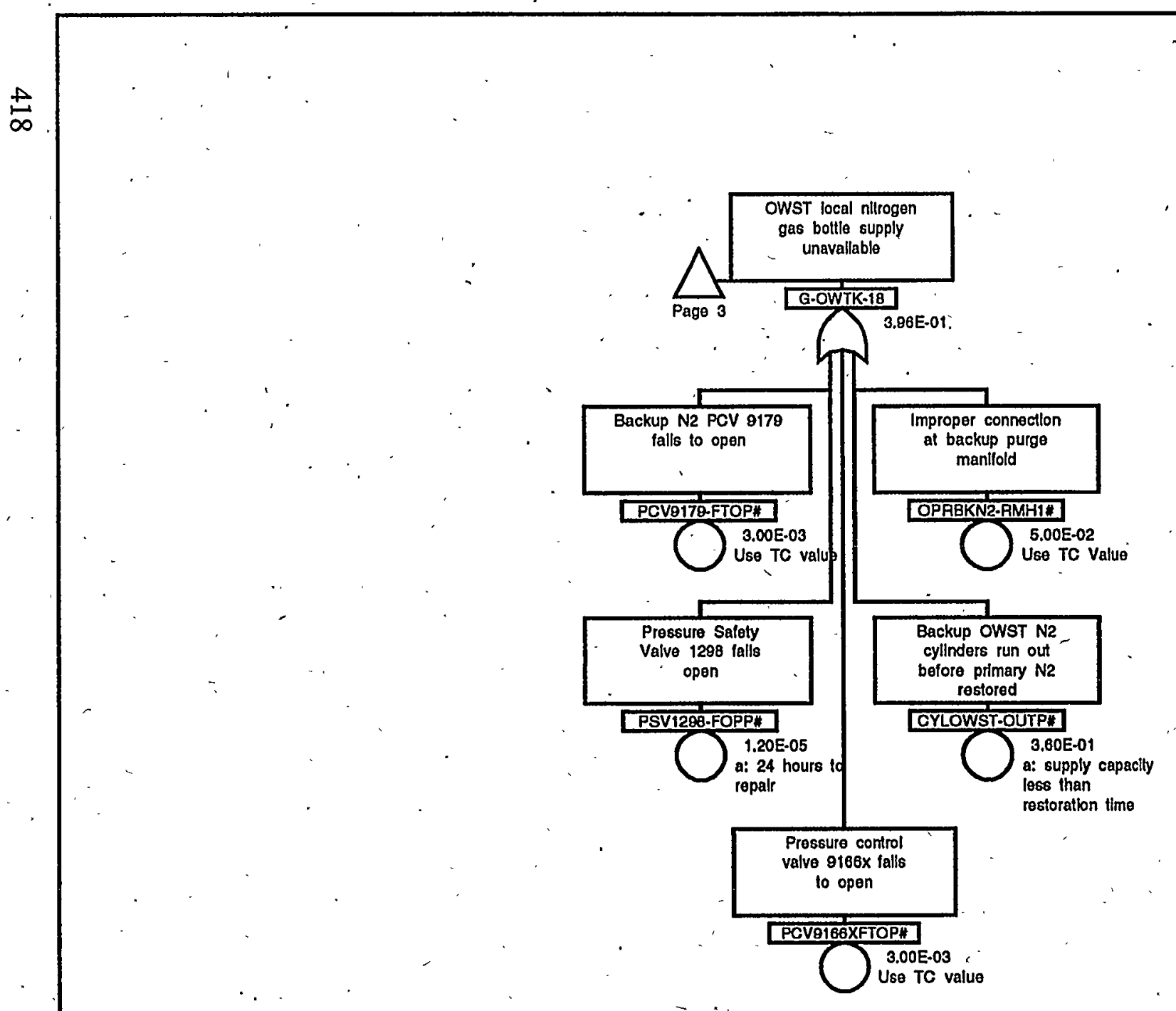




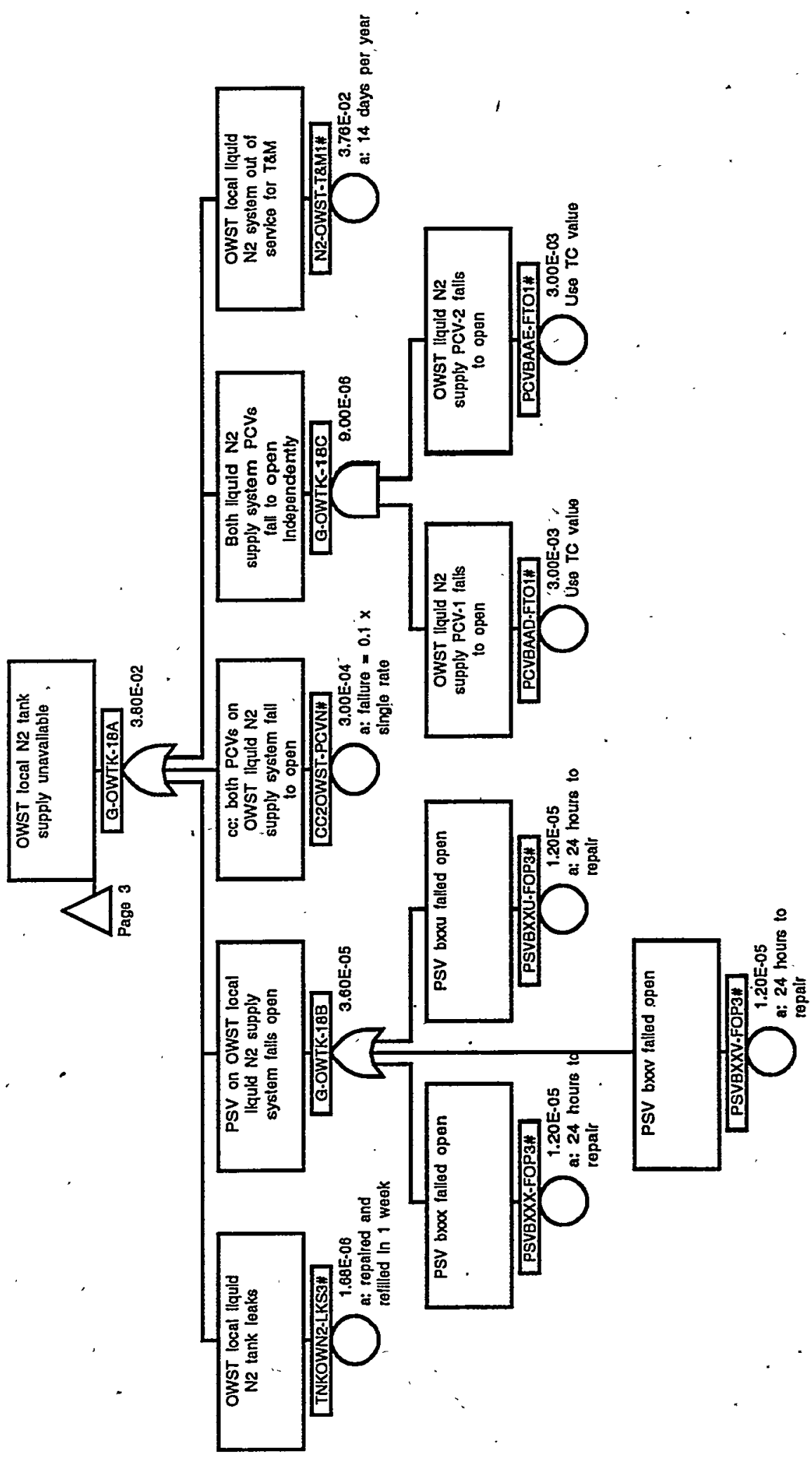


X-ESR-S-00001, REV. 0

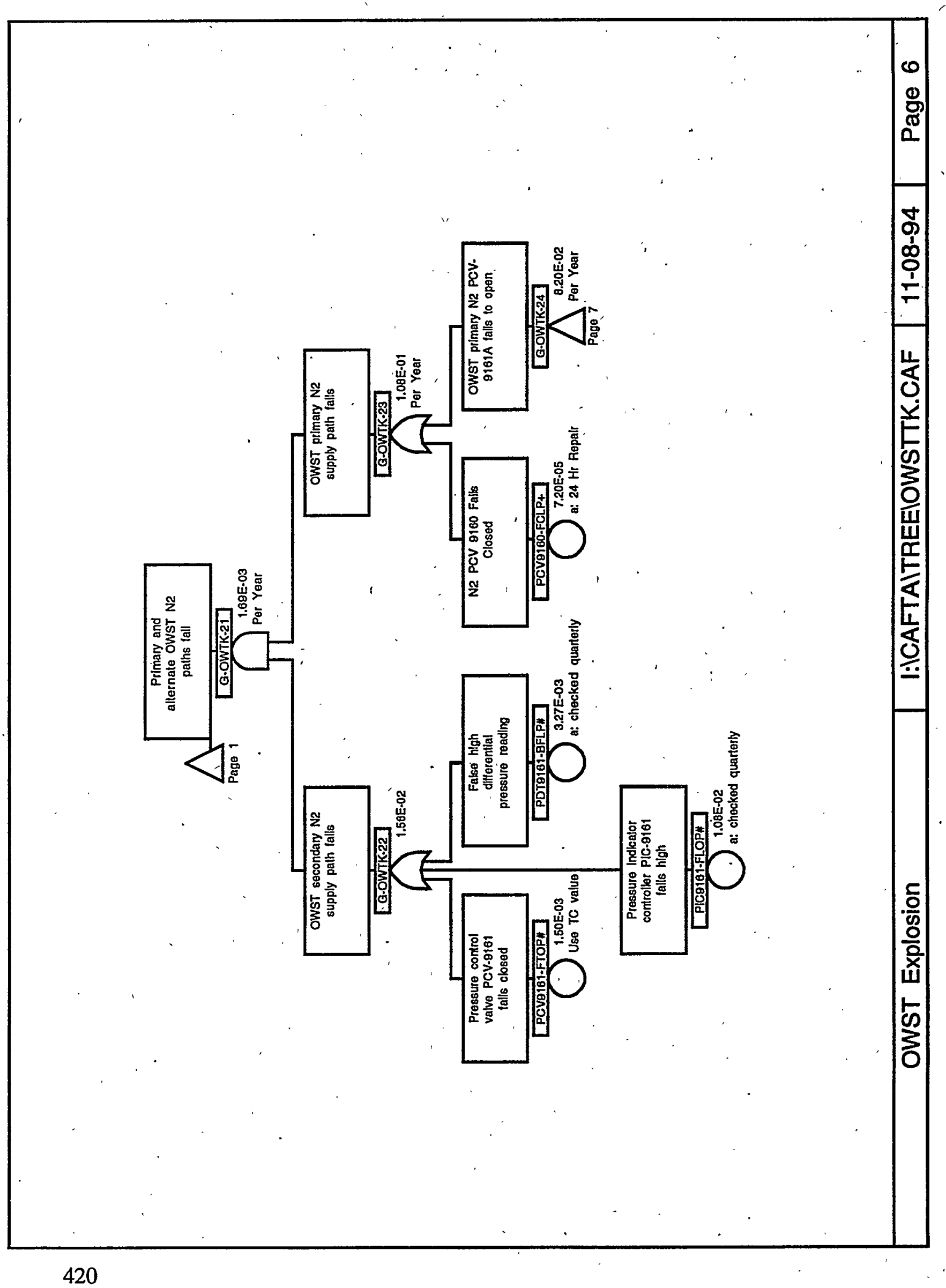




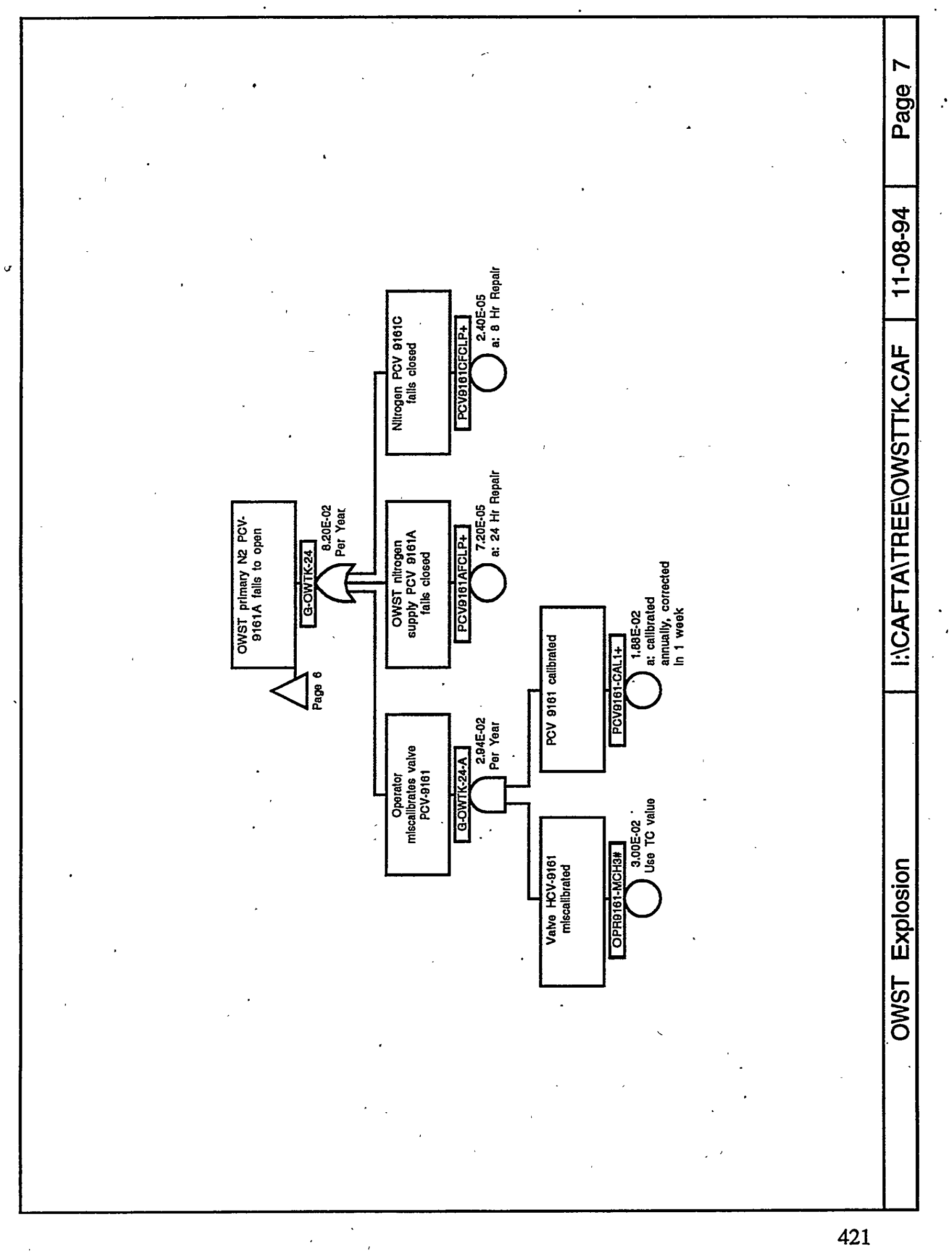


X-ESR-S-00001, REV. 0

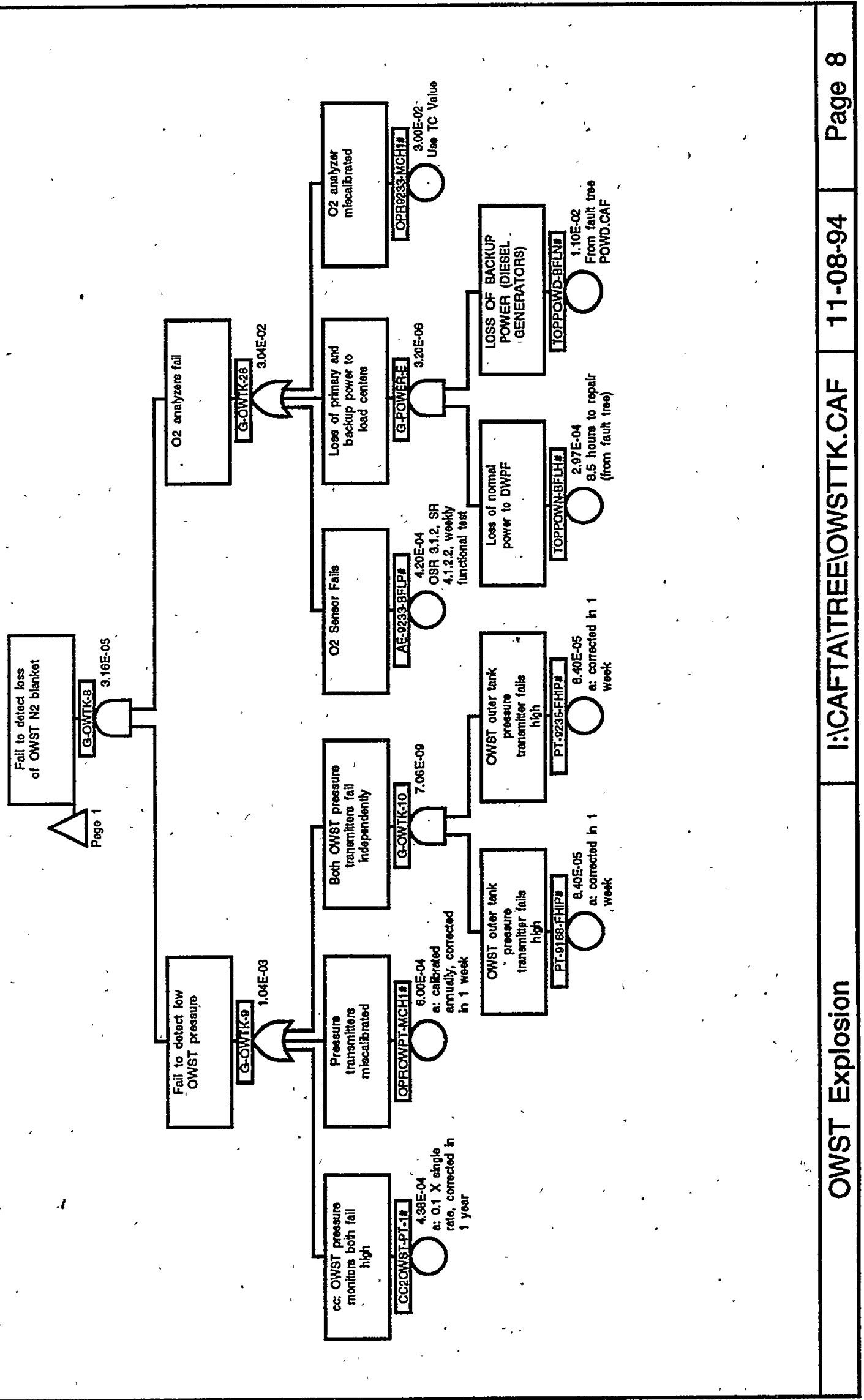




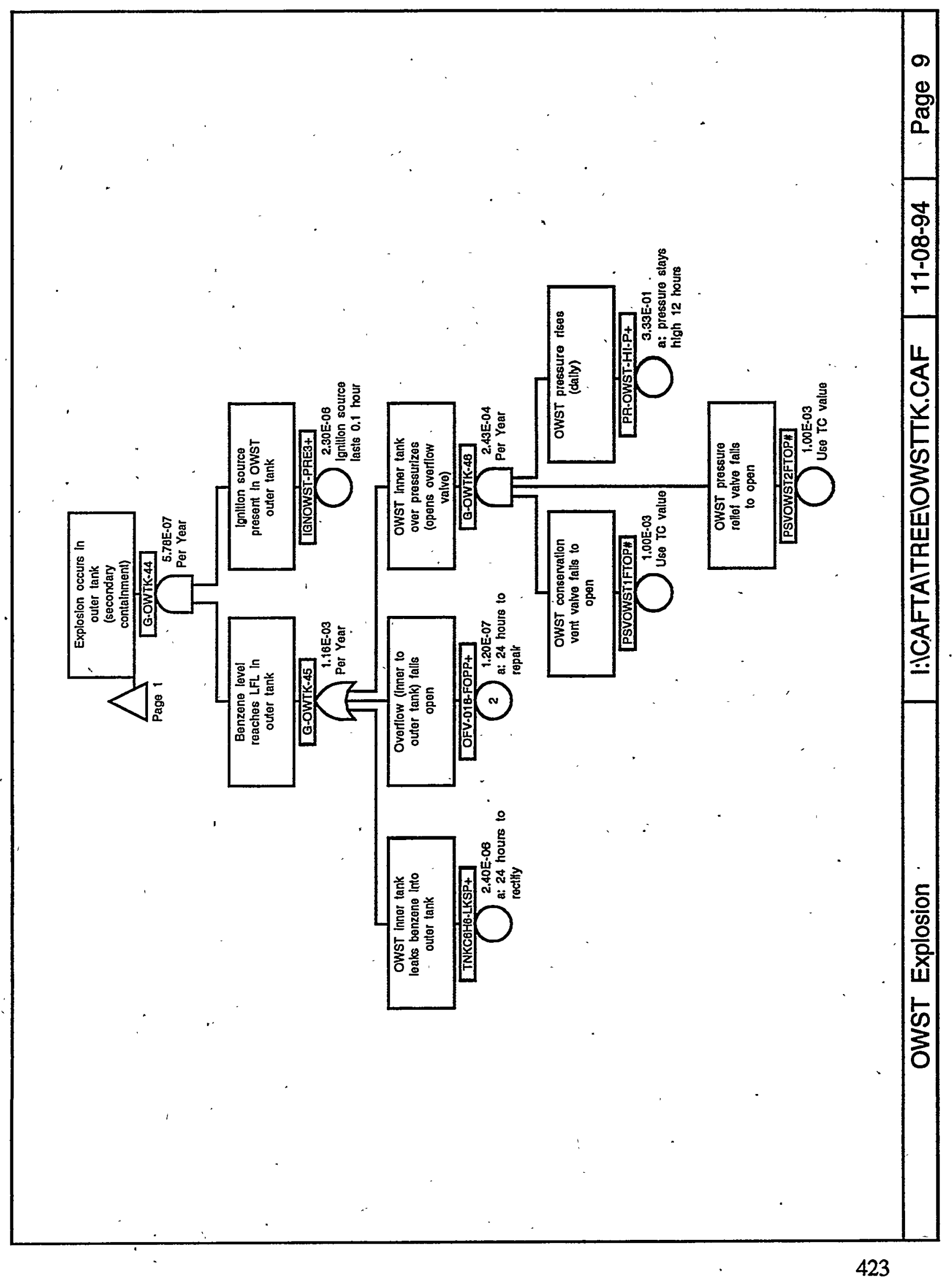


X-ESR-S-00001, REV. 0

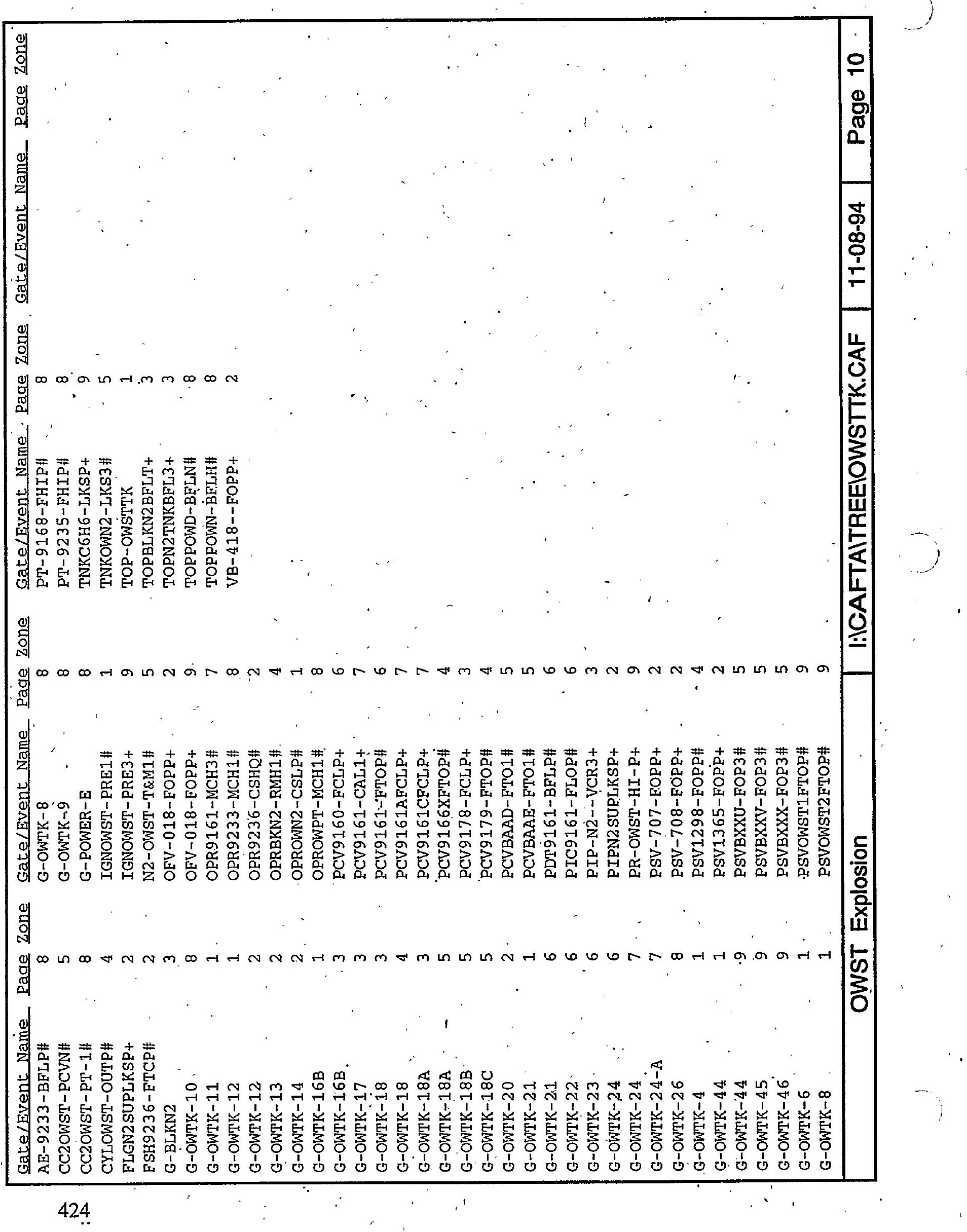




\begin{tabular}{|c|c|c|c|c|c|c|}
\hline $\begin{array}{l}\text { Set } \\
\text { No. }\end{array}$ & $\begin{array}{l}\text { Event } \\
\text { Name }\end{array}$ & Description & C & o. & $\begin{array}{l}\text { Calc. } \\
\text { Result }\end{array}$ & $\begin{array}{c}\text { Cutiset } \\
\text { Freq. }(/ Y Y)\end{array}$ \\
\hline 1. & $\begin{array}{l}\text { TOP-OWSTPK } \\
\text { IGNOWST-PRE3+ } \\
\text { TNKC6H6-LKSP+ }\end{array}$ & $\begin{array}{l}\text { Ignition source present in OWST outer tank } \\
\text { OWST inner tank leaks benzene into outer tank }\end{array}$ & 4 & $\begin{array}{r}2.3 \mathrm{E}-5 \mathrm{H} \\
24 \mathrm{H} \\
1.0 \mathrm{E}-07 \mathrm{H}\end{array}$ & $\begin{array}{l}2.30 \mathrm{E}-06 \\
2.40 \mathrm{E}-06\end{array}$ & $\begin{array}{l}1.30 \mathrm{E}-06 \\
4.86 \mathrm{E}-07\end{array}$ \\
\hline 2 . & $\begin{array}{l}\text { CYLOWST-OUTP\# } \\
\text { IGNOWST-PRE1\# } \\
\text { N2-OWST-T\&M1\# } \\
\text { OPROWN2-CSLP\# } \\
\text { TOPN2TNKBFL3+ }\end{array}$ & $\begin{array}{l}\text { Backup OWST N2 cylinders run out before primary N2 restored } \\
\text { Ignition source present in OWST inner tank } \\
\text { OWST local liquid N2 system out of service for T\&M } \\
\text { Operators fail to restore nitrogen blanket before } 02 \text { reaches } \\
\text { MOC } \\
\text { Nitrogen tank supply failure }\end{array}$ & $\begin{array}{l}3 \\
1 \\
4\end{array}$ & $\begin{array}{r}3.6 \mathrm{E}-01 \mathrm{~N} \\
1 \mathrm{~N} \\
3.0 \mathrm{E}-02 \mathrm{~N} \\
14 \mathrm{D} \\
1 \mathrm{Y} \\
1 \mathrm{~N} \\
3.0 \mathrm{E}-03 \mathrm{~N} \\
17 \mathrm{H} \\
7.7 \mathrm{E}-06 \mathrm{H}\end{array}$ & $\begin{array}{l}3.60 \mathrm{E}-01 \mathrm{~N} \\
3.00 \mathrm{E}-02 \mathrm{~N} \\
3.76 \mathrm{E}-02 \\
3.00 \mathrm{E}-03 \mathrm{~N} \\
1.31 \mathrm{E}-04\end{array}$ & . \\
\hline 3. & $\begin{array}{l}\text { FLGN2 SUPLKSP+ } \\
\text { IGNOWST-PRE1\# } \\
\text { OPR9236-CSHQ\# } \\
\text { OPROWN2-CSLP\# }\end{array}$ & $\begin{array}{l}\text { Any of } 100 \text { joints/flanges leaks } \\
\text { Ignition source present in OWST inner tank } \\
\text { operator fails to respond to high backup N2 flow alarm } \\
\text { Operators fail to restore nitrogen blanket before } 02 \text { reaches } \\
\text { MOC }\end{array}$ & 1 & $\begin{array}{r}8 \mathrm{H} \\
1.0 \mathrm{E}-06 \mathrm{H} \\
1 \mathrm{~N} \\
3.0 \mathrm{E}-02 \mathrm{~N} \\
1 \mathrm{~N} \\
1.0 \mathrm{E}-01 \mathrm{~N} \\
1 \mathrm{~N} \\
3.0 \mathrm{E}-03 \mathrm{~N}\end{array}$ & $\begin{array}{l}8.00 \mathrm{E}-06 \\
3.00 \mathrm{E}-02 \mathrm{~N} \\
1.00 \mathrm{E}-01 \mathrm{~N} \\
3.00 \mathrm{E}-03 \mathrm{~N}\end{array}$ & $7.88 \mathrm{E}-08$ \\
\hline 4. & $\begin{array}{l}\text { IGNOWST-PRE1\# } \\
\text { OPR9236-CSHQ\# } \\
\text { OPROWN2-CSLP\# } \\
\text { PSV1365-FOPP+ }\end{array}$ & $\begin{array}{l}\text { Ignition source present in OWST inner tank } \\
\text { Operator fails to respond to high backup N2 flow alarm } \\
\text { Operators fail to restore nitrogen blanket before } 02 \text { reaches } \\
\text { MOC } \\
\text { Pressure Safety Valve Fails Open }\end{array}$ & $\begin{array}{l}1 \\
1 \\
1\end{array}$ & $\begin{array}{r}1 \mathrm{~N} \\
3.0 \mathrm{E}-02 \mathrm{~N} \\
1 \mathrm{~N} \\
1.0 \mathrm{E}-01 \mathrm{~N} \\
1 \mathrm{~N} \\
3.0 \mathrm{E}-03 \mathrm{~N} \\
8 \mathrm{H} \\
1.0 \mathrm{E}-06 \mathrm{H}\end{array}$ & $\begin{array}{l}3.00 \mathrm{E}-02 \mathrm{~N} \\
1.00 \mathrm{E}-01 \mathrm{~N} \\
3.00 \mathrm{E}-03 \mathrm{~N} \\
8.00 \mathrm{E}-06\end{array}$ & $7.88 \mathrm{E}-08$ \\
\hline 5. & $\begin{array}{l}\text { IGNOWST-PRE1\# } \\
\text { OPR9236-CSHQ\# } \\
\text { OPROWN2-CSLP\# } \\
\text { PSV-707-FOPP+ }\end{array}$ & $\begin{array}{l}\text { Ignition source present in OWST inner tank } \\
\text { Operator fails to respond to high backup N2 flow alarm } \\
\text { Operators, fail to restore nitrogen blanket before } 02 \text { reaches } \\
\text { MOC } \\
\text { PSV } 707 \text { fails open }\end{array}$ & 1 & $\begin{array}{r}1 \mathrm{~N} \\
3.0 \mathrm{E}-02 \mathrm{~N} \\
1 \mathrm{~N} \\
1.0 \mathrm{E}-01 \mathrm{~N} \\
1 \mathrm{~N} \\
3.0 \mathrm{E}-03 \mathrm{~N} \\
.24 \mathrm{H} \\
1.0 \mathrm{E}-06 \mathrm{H}\end{array}$ & $\begin{array}{l}3.00 \mathrm{E}-02 \mathrm{~N} \\
1.00 \mathrm{E}-01 \mathrm{~N} \\
3.00 \mathrm{E}-03 \mathrm{~N} \\
2.40 \mathrm{E}-05\end{array}$ & $7.88 \mathrm{E}-08$ \\
\hline 6. & IGNOWST-PRE1\# & Ignition source present in OWST inner tank & 1 & $3.0 \mathrm{E}-02 \mathrm{~N}$ & $3.00 \mathrm{E}-02 \mathrm{~N}$ & $7.88 E-08$ \\
\hline
\end{tabular}


Cutsets for I: \CAFTA ICUT\OWSTTK.CSR

11/08/94 12:42 AM (CONT.)

\begin{tabular}{|c|c|c|c|c|c|c|c|}
\hline & $\begin{array}{l}\text { Set } \\
\text { No. }\end{array}$ & $\begin{array}{l}\text { Event } \\
\text { Name }\end{array}$ & Description & C & $\begin{array}{l}\text { B.E. } \\
\text { Input. }\end{array}$ & $\begin{array}{l}\text { Calc. } \\
\text { Result }\end{array}$ & $\begin{array}{l}\text { Cutset } \\
\text { Freq. }(/ y r)\end{array}$ \\
\hline . & 10. & $\begin{array}{l}\text { OPR9236-CSHQ\# } \\
\text { OPROWN2-CSLP\# } \\
\text { PSV-708-FOPP+ } \\
\text { IGNOWST-PRE3+ } \\
\text { PR-OWST-HI-P+ } \\
\text { PSVOWST1FTOP\# } \\
\text { PSVOWST2FTOP\# } \\
\text { CYLOWST-OUTP\# } \\
\text { IGNOWST-PRE1\# } \\
\text { N2-OWST-T\&M1\# } \\
\text { OPROWN2-CSLP\# } \\
\text { TOPBLKN2BFLT+ } \\
\text { IGNOWST-PRE1\# } \\
\text { OPR9236-CSHQ\# } \\
\text { OPROWN2-CSLP\# } \\
\text { VB-418--FOPP+ } \\
\text { CYLOWST-OUTP\# } \\
\text { IGNOWST-PRE1\# } \\
\text { N2-OWST-T\&M1\# } \\
\text { OPROWN2-CSLP\# } \\
\text { PCV9178-FCLP+ }\end{array}$ & 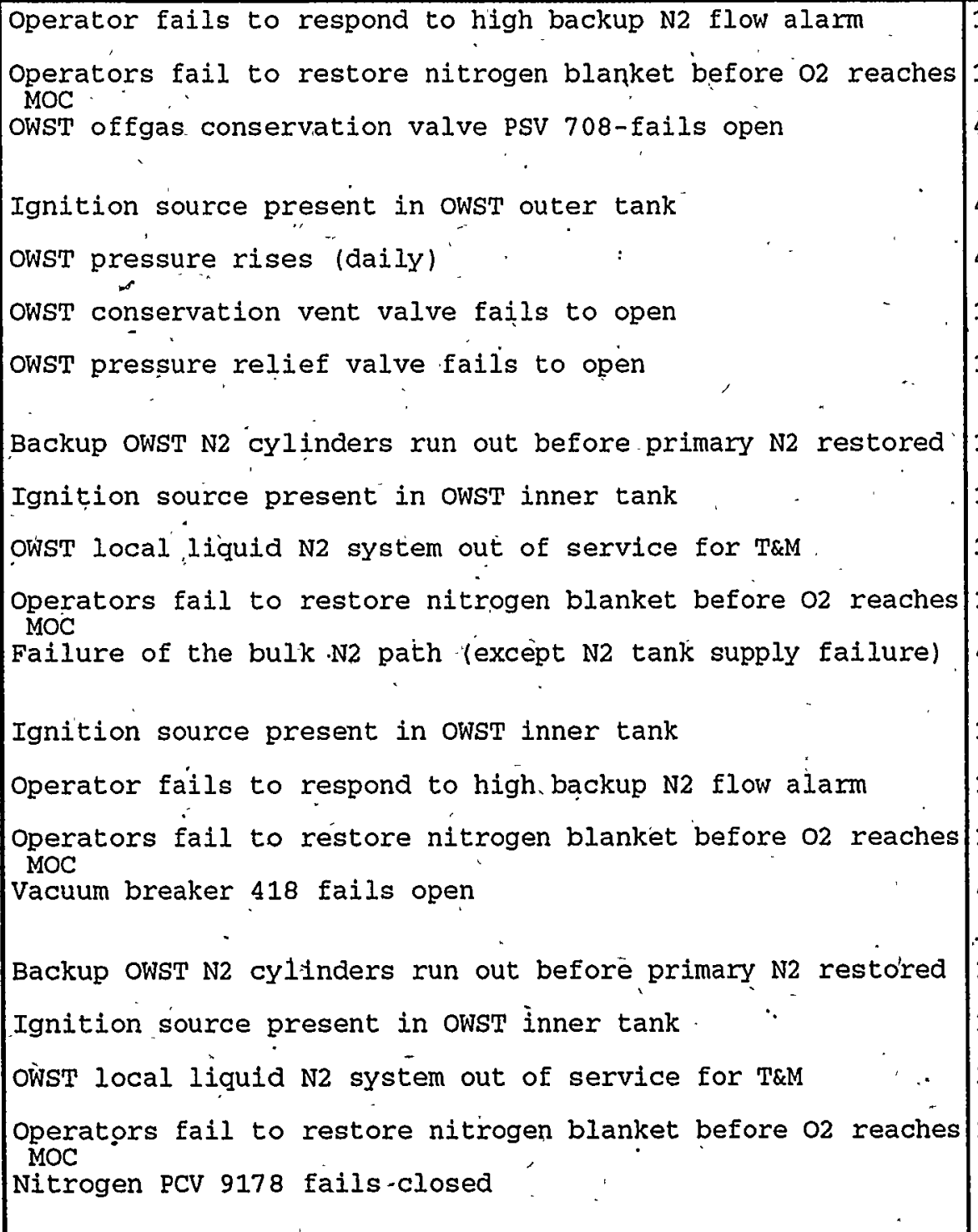 & 1 & 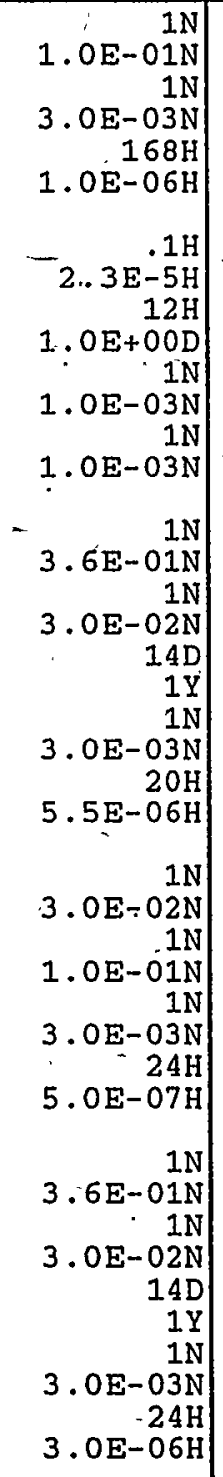 & $\begin{array}{l}3.33 \mathrm{E}-01 \\
1.00 \mathrm{E}-03 \mathrm{~N} \\
1.00 \mathrm{E}-03 \mathrm{~N} \\
3.60 \mathrm{E}-01 \mathrm{~N} \\
3.00 \mathrm{E}-02 \mathrm{~N} \\
3.76 \mathrm{E}-02 \\
3.00 \mathrm{E}-03 \mathrm{~N} \\
1.10 \mathrm{E}-0.4 \\
3.00 \mathrm{E}-02 \mathrm{~N} \\
1.00 \mathrm{E}-01 \mathrm{~N} \\
3.00 \mathrm{E}-03 \mathrm{~N} \\
1.20 \mathrm{E}-05 \\
3.60 \mathrm{E}-01 \mathrm{~N} \\
3.00 \mathrm{E}-02 \mathrm{~N} \\
3.76 \mathrm{E}-02 \\
3.00 \mathrm{E}-03 \mathrm{~N} \\
7.20 \mathrm{E}-05\end{array}$ & $\begin{array}{l}5.87 \mathrm{E}-08 \\
3.94 \mathrm{E}-08 \\
. \\
3.20 \mathrm{E}-08 \\
. \\
.\end{array}$ \\
\hline
\end{tabular}




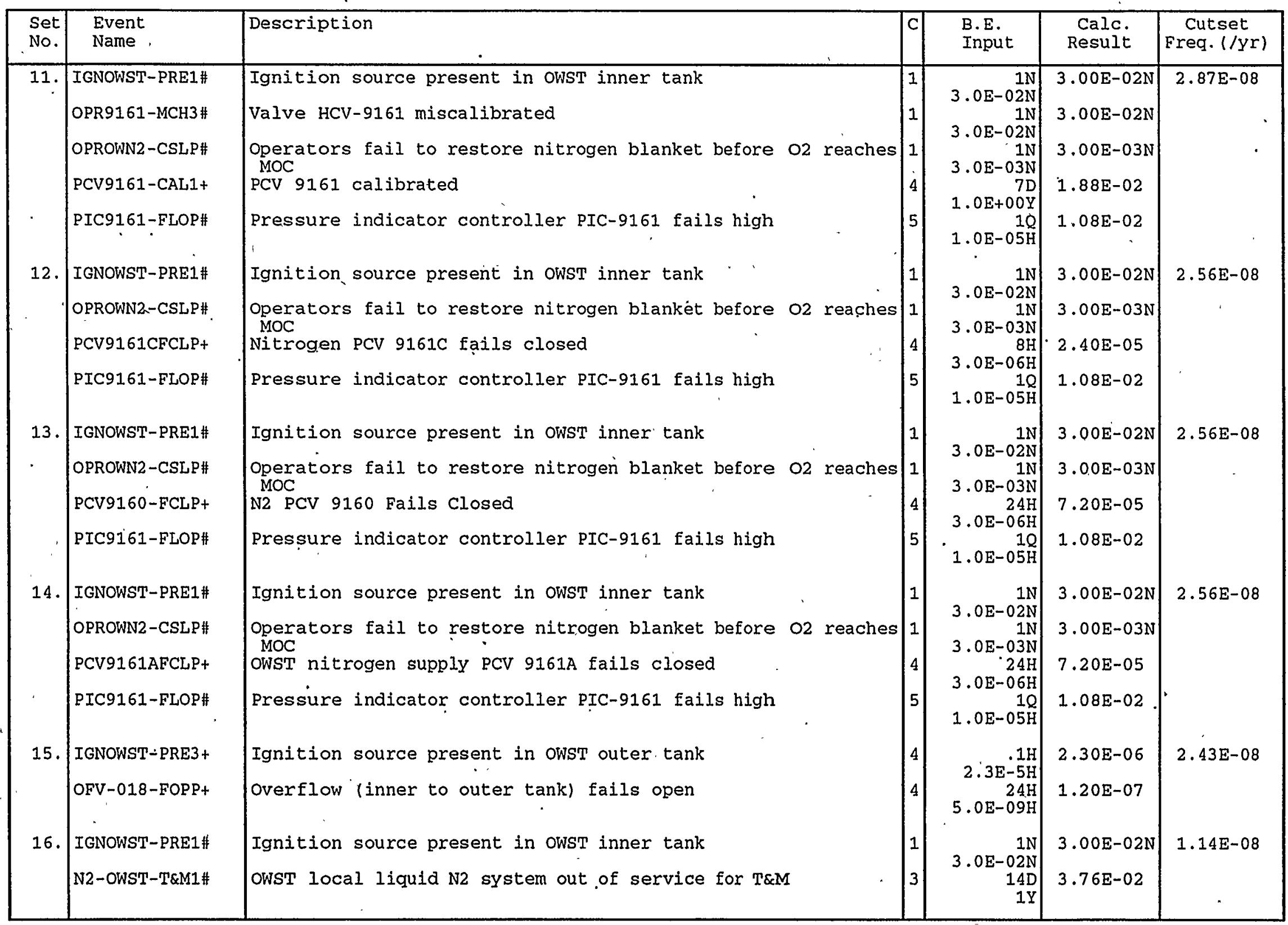




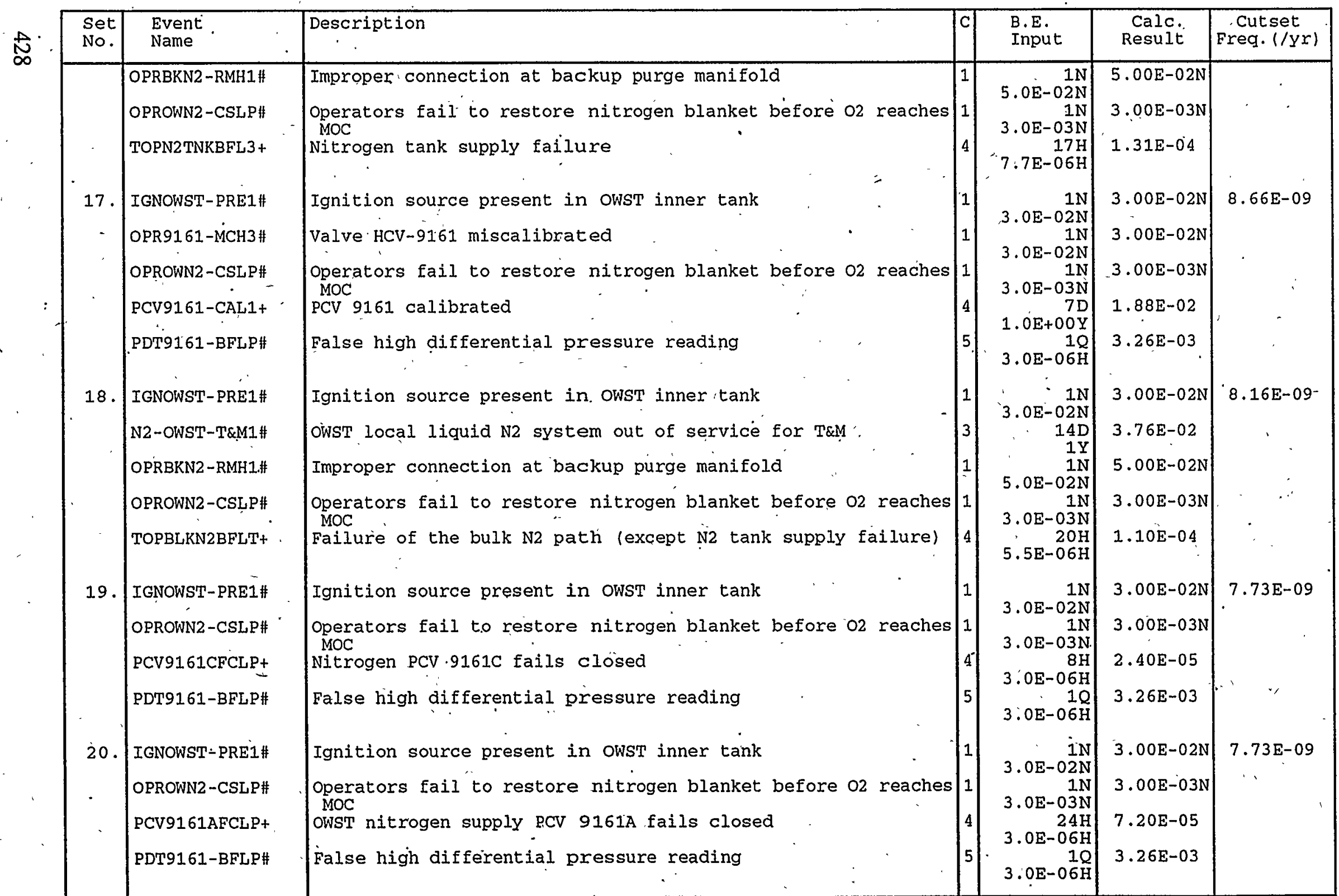


Cutsets for I: \CAFTA ICUT\OWSTTK. CSR

11/08/94 12:42 AM (CONT.)

\begin{tabular}{|c|c|c|c|c|c|c|}
\hline $\begin{array}{l}\text { Set } \\
\text { No. }\end{array}$ & Name & Description & C & $\begin{array}{l}\text { B.E. } \\
\text { Input }\end{array}$ & $\begin{array}{l}c . \\
l t\end{array}$ & $\begin{array}{c}\text { Cutset } \\
\text { Freg. }(/ \text { yr })\end{array}$ \\
\hline 21 & $\begin{array}{l}\text { IGNOWST-PRE1\# } \\
\text { OPROWN2-CSLP\# } \\
\text { PCV9160-FCLP+ } \\
\text { PDT9161-BFLP\# }\end{array}$ & $\begin{array}{l}\text { Ignition source present in OWST inner tank } \\
\text { Operators fail to restore nitrogen blanket before } 02 \text { reaches } \\
\text { MOC } \\
\text { N2 PCV } 9160 \text { Fails Closed } \\
\text { False high differential pressure reading }\end{array}$ & 5 & $\begin{array}{r}1 \mathrm{~N} \\
3.0 \mathrm{E}-02 \mathrm{~N} \\
1 \mathrm{~N} \\
3.0 \mathrm{E}-03 \mathrm{~N} \\
24 \mathrm{H} \\
3.0 \mathrm{E}-06 \mathrm{H} \\
10 \\
3.0 \mathrm{E}-06 \mathrm{H}\end{array}$ & $\begin{array}{l}3.00 \mathrm{E}-02 \mathrm{~N} \\
3.00 \mathrm{E}-03 \mathrm{~N} \\
7.20 \mathrm{E}-05 \\
3.26 \mathrm{E}-03\end{array}$ & \\
\hline 22 . & $\begin{array}{l}\text { IGNOWST-PRE1\# } \\
\text { N2-OWST-T\&M1\# } \\
\text { OPRBKN2-RMH1\# } \\
\text { OPROWN2-CSLP\# } \\
\text { PCV9178-FCLP+ }\end{array}$ & $\begin{array}{l}\text { Ignition source present in OWST inner tank } \\
\text { OWST local liquid N2 system out of service for T\&M. } \\
\text { Improper connection at backup purge manifold } \\
\text { Operators fail to restore nitrogen blanket before } 02 \text { reaches } \\
\text { MOC } \\
\text { Nitrogen PCV } 9178 \text { fails closed }\end{array}$ & 1 & $\begin{array}{r}1 \mathrm{~N} \\
3.0 \mathrm{E}-02 \mathrm{~N} \\
14 \mathrm{D} \\
1 \mathrm{Y} \\
1 \mathrm{~N} \\
5.0 \mathrm{E}-02 \mathrm{~N} \\
1 \mathrm{~N} \\
3.0 \mathrm{E}-03 \mathrm{~N} \\
24 \mathrm{H} \\
3.0 \mathrm{E}-06 \mathrm{H}\end{array}$ & $\begin{array}{l}3.00 \mathrm{E}-02 \mathrm{~N} \\
3.76 \mathrm{E}-02 \\
5.00 \mathrm{E}-02 \mathrm{~N} \\
3.00 \mathrm{E}-03 \mathrm{~N} \\
7.20 \mathrm{E}-05\end{array}$ & $4.45 E-09$ \\
\hline 23. & $\begin{array}{l}\text { IGNOWST-PRE1\# } \\
\text { OPR9161-MCH3\# } \\
\text { OPROWN2-CSLP\# } \\
\text { PCV9161-CAL1+ } \\
\text { PCV9161-FTOP\# }\end{array}$ & $\begin{array}{l}\text { Ignition source present in OWST inner tank } \\
\text { Valve HCV- } 9161 \text { miscalibrated } \\
\text { Operators fail to restore nitrogen blanket before } 02 \text { reaches } \\
\text { MOC } \\
\text { PCV } 9161 \text { calibrated } \\
\text { Pressure control valve PCV-9161 fails closed }\end{array}$ & 1 & $\begin{array}{r}1 \mathrm{~N} \\
3.0 \mathrm{E}-02 \mathrm{~N} \\
1 \mathrm{~N} \\
3.0 \mathrm{E}-02 \mathrm{~N} \\
1 \mathrm{~N} \\
3.0 \mathrm{E}-03 \mathrm{~N} \\
7 \mathrm{D} \\
1.0 \mathrm{E}+00 \mathrm{Y} \\
1 \mathrm{~N} \\
3.0 \mathrm{E}-03 \mathrm{~N}\end{array}$ & $\begin{array}{l}3.00 \mathrm{E}-02 \mathrm{~N} \\
3.00 \mathrm{E}-02 \mathrm{~N} \\
3.00 \mathrm{E}-03 \mathrm{~N} \\
1.88 \mathrm{E}-02 \\
1.50 \mathrm{E}-03 \mathrm{~N}\end{array}$ & $3.97 \mathrm{E}-09$ \\
\hline 24. & $\begin{array}{l}\text { IGNOWST-PRE1\# } \\
\text { OPROWN2-CSLP\# } \\
\text { PCV9161-FTOP\# } \\
\text { PCV9161CFCLP+ }\end{array}$ & $\begin{array}{l}\text { Ignition source present in OWST inner tank } \\
\text { Operators fail to restore nitrogen blanket before } 02 \text { reaches } \\
\text { MOC } \\
\text { Pressure control valve PCV-9161 fails closed } \\
\text { Nitrogen PCV } 9161 \mathrm{C} \text { fails closed }\end{array}$ & 1 & $\begin{array}{r}1 \mathrm{~N} \\
3.0 \mathrm{E}-02 \mathrm{~N} \\
1 \mathrm{~N} \\
3.0 \mathrm{E}-03 \mathrm{~N} \\
1 \mathrm{~N} \\
3.0 \mathrm{E}-03 \mathrm{~N} \\
8 \mathrm{H} \\
3.0 \mathrm{E}-06 \mathrm{H}\end{array}$ & $\begin{array}{l}3.00 \mathrm{E}-02 \mathrm{~N} \\
3.00 \mathrm{E}-03 \mathrm{~N} \\
1.50 \mathrm{E}-03 \mathrm{~N} \\
2.40 \mathrm{E}-05\end{array}$ & -09 \\
\hline 25. & $\begin{array}{l}\text { IGNOWST-PRE1\# } \\
\text { OPROWN2-CSLP\# } \\
\text { PCV9160-FCLP+ } \\
\text { PCV9161-FTOP\# }\end{array}$ & $\begin{array}{l}\text { Ignition source present in OWST inner tank } \\
\text { Operators fail to restore nitrogen blanket before } 02 \text { reaches } \\
\text { MOC } \\
\text { N2 PCV } 9160 \text { Fails Closed } \\
\text { Pressure control valve PCV-9161 fails closed }\end{array}$ & 1 & $\begin{array}{r}1 \mathrm{~N} \\
3.0 \mathrm{E}-02 \mathrm{~N} \\
1 \mathrm{~N} \\
3.0 \mathrm{E}-03 \mathrm{~N} \\
24 \mathrm{H} \\
3.0 \mathrm{E}-06 \mathrm{H} \\
1 \mathrm{~N} \\
3.0 \mathrm{E}-03 \mathrm{~N}\end{array}$ & $\begin{array}{l}3.00 \mathrm{E}-02 \mathrm{~N} \\
3.00 \mathrm{E}-03 \mathrm{~N} \\
7.20 \mathrm{E}-05 \\
1.50 \mathrm{E}-03 \mathrm{~N}\end{array}$ & $3.54 E-09$ \\
\hline
\end{tabular}


Explosion In Organic Waste Storage Tank (OWST)

Top Event Frequency: 1.30E-06/YR

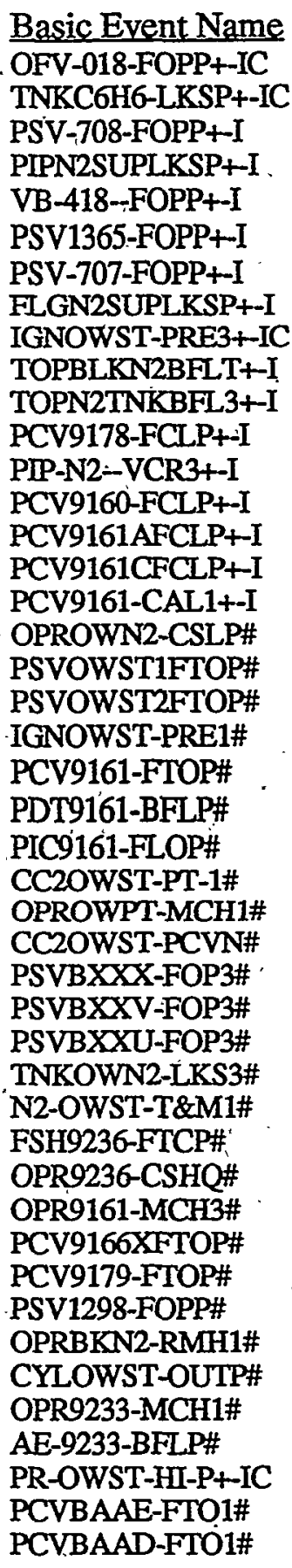

\section{Risk Achievement Worth}

\section{Description}

Overflow (inner to outer tank) fails open

OWST inner tank leaks benzene into outer tank

OWST offgas conservation valve PSV 708-fails open

N2 Supply Line Leaks

Vacuum breaker 418 fails open

Pressure Safety Valve Fails Open

PSV 707 fails open

Any of 100 joints/flanges leaks

Ignition source present in OWST outer tank

Failure of the builk N2 path

Nitrogen tank supply failure

Nitrogen PCV 9178 fails closed

Vehicle crash damages main OWST.N2 piping

N2 PCV 9160 Fails Closed

OWST nitrogen supply PCV 9161A fails closed

Nitrogen PCV 9161C fails closed

PCV 9161 calibrated

Fail to restore nitrogen blanket before $\mathrm{O} 2$ reaches $\mathrm{MOC}$

OWST conservation vent valve fails to open

OWST pressure relief valve fails to open

Ignition source present in OWST inner tank

Pressure control valve PCV-9161 fails closed

False high differential pressure reading

Pressure indicator controller PIC-9161 fails high

cc: OWST pressure monitors both fail high

Pressure transmitters miscalibrated

cc: both PCVs on OWST liquid N2 supply fail to open

PSV bxxx failed open

PSV bxxv failed open

PSV bxxu failed open

OWST local liquid N2 tank leaks

OWST local liquid N2 system out of service for T\&M

Flow switch fails to close

Operator fails to respond to high backup N2 flow alarm

Valve HCV.-9161 miscalibrated.

Pressure control valve $9166 \mathrm{x}$ fails to open

Backup N2 PCV 9179 fails to open

Pressure Safety Valve 1298 fails open

Improper connection at backup purge manifold

Backup OWST N2 cylinders run out before N2 restored

O2 analyzer miscalibrated

O2 Sensor Fails

OWST pressure rises (daily)

OWST liquid N2 supply PCV-2 fails to open

OWST liquid N2 supply PCV-1 fails to open

\begin{tabular}{cl} 
Prob/Freg & AchW \\
\hline $5.00 \mathrm{E}-09 / \mathrm{H}$ & $2.33 \mathrm{E}+05$ \\
$1.00 \mathrm{E}-07 / \mathrm{H}$ & $1.71 \mathrm{E}+05$ \\
$1.00 \mathrm{E}-06 / \mathrm{H}$ & $6.20 \mathrm{E}+04$ \\
$9.00 \mathrm{E}-09 / \mathrm{H}$ & $6.20 \mathrm{E}+04$ \\
$5.00 \mathrm{E}-07 / \mathrm{H}$ & $6.20 \mathrm{E}+04$ \\
$1.00 \mathrm{E}-06 / \mathrm{H}$ & $6.20 \mathrm{E}+04$ \\
$1.00 \mathrm{E}-06 / \mathrm{H}$ & $6.20 \mathrm{E}+04$ \\
$1.00 \mathrm{E}-06 / \mathrm{H}$ & $6.20 \mathrm{E}+04$ \\
$2.30 \mathrm{E}-05 / \mathrm{H}$ & $2.01 \mathrm{E}+04$ \\
$5.50 \mathrm{E}-06 / \mathrm{H}$ & $9.69 \mathrm{E}+03$ \\
$7.70 \mathrm{E}-06 / \mathrm{H}$ & $9.69 \mathrm{E}+03$ \\
$3.00 \mathrm{E}-06 / \mathrm{H}$ & $9.69 \mathrm{E}+03$ \\
$2.55 \mathrm{E}-08 / \mathrm{H}$ & $9.67 \mathrm{E}+03$ \\
$3.00 \mathrm{E}-06 / \mathrm{H}$ & $9.57 \mathrm{E}+03$ \\
$3.00 \mathrm{E}-06 / \mathrm{H}$ & $9.57 \mathrm{E}+03$ \\
$3.00 \mathrm{E}-06 / \mathrm{H}$ & $9.57 \mathrm{E}+03$ \\
$1.12 \mathrm{E}-04 / \mathrm{H}$ & $2.88 \mathrm{E}+02$ \\
$3.00 \mathrm{E}-03$ & $1.84 \mathrm{E}+02$ \\
$1.00 \mathrm{E}-03$ & $5.31 \mathrm{E}+01$ \\
$1.00 \mathrm{E}-03$ & $5.31 \mathrm{E}+01$ \\
$3.00 \mathrm{E}-02$ & $1.90 \mathrm{E}+01$ \\
$1.50 \mathrm{E}-03$ & $8.57 \mathrm{E}+00$ \\
$3.27 \mathrm{E}-03$ & $8.56 \mathrm{E}+00$ \\
$1.08 \mathrm{E}-02$ & $8.50 \mathrm{E}+00$ \\
$4.38 \mathrm{E}-04$ & $6.57 \mathrm{E}+00$ \\
$6.00 \mathrm{E}-04$ & $6.57 \mathrm{E}+00$ \\
$3.00 \mathrm{E}-04$ & $5.14 \mathrm{E}+00$ \\
$1.20 \mathrm{E}-05$ & $5.11 \mathrm{E}+00$ \\
$1.20 \mathrm{E}-05$ & $5.11 \mathrm{E}+00$ \\
$1.20 \mathrm{E}-05$ & $5.11 \mathrm{E}+00$ \\
$1.68 \mathrm{E}-06$ & $5.02 \mathrm{E}+00$ \\
$3.76 \mathrm{E}-02$ & $4.98 \mathrm{E}+00$ \\
$1.09 \mathrm{E}-03$ & $3.77 \mathrm{E}+00$ \\
$1.00 \mathrm{E}-01$ & $3.49 \mathrm{E}+00$ \\
$3.00 \mathrm{E}-02$ & $2.04 \mathrm{E}+00$ \\
$3.00 \mathrm{E}-03$ & $1.38 \mathrm{E}+00$ \\
$3.00 \mathrm{E}-03$ & $1.38 \mathrm{E}+00$ \\
$1.20 \mathrm{E}-05$ & $1.38 \mathrm{E}+00$ \\
$5.00 \mathrm{E}-02$ & $1.36 \mathrm{E}+00$ \\
$3.60 \mathrm{E}-01$ & $1.24 \mathrm{E}+00$ \\
$3.00 \mathrm{E}-02$ & $1.18 \mathrm{E}+00$ \\
$4.20 \mathrm{E}-04$ & $1.18 \mathrm{E}+00$ \\
$2.78 \mathrm{E}-02 / \mathrm{H}$ & $1.12 \mathrm{E}+00$ \\
$3.00 \mathrm{E}-03$ & $1.01 \mathrm{E}+00$ \\
$3.00 \mathrm{E}-03$ & $1.01 \mathrm{E}+00$ \\
&
\end{tabular}

Note: Events that make less than $1 \%$ difference to the importance factor are not included. Modified top event frequency $=$ (top event frequency) $\mathrm{x}$ (risk achievement worth). 
Explosion In Organic Waste Storage Tank (OWST)

Top Event Frequency: 1.30E-06/YR

\begin{tabular}{l} 
Basic EVent Name \\
\hline IGNOWST-PRE1\# \\
OPROWN2-CSLP\# \\
IGNOWST-PRE3+-IC \\
TNKC6H6-LKSP+IC \\
OPR9236-CSHQ\# \\
N2-OWST-T\&M1\# \\
CYLOWST-OUTP\# \\
PIC9161-FLOP\# \\
TOPN2TNKBFL3+-I \\
FLGN2SUPLKSP+I \\
PSV-708-FOPP+I \\
PSV-707-FOPP+I \\
PSV1365-FOPP+-I \\
TOPBLKN2BFLT+-I \\
PSVOWST1FTOP\# \\
PSVOWST2FTOP\# \\
PR-OWST-HI-P+-IC \\
OPR9161-MCH3\# \\
PCV9161-CAL1+I \\
VB-418-FOPP+I \\
PCV9178-FCLP+I \\
PCV9160-FCLP+I \\
PCV9161AFCLP+-I \\
PCV9161CFCLP+-I \\
PDT9161-BFLP\# \\
OFV-018-FOPP+-IC \\
OPRBKN2-RMH1\# \\
PCV9161-FTOP\#
\end{tabular}

\section{Risk Reduction Worth} Description

Ignition source present in OWST inner tank Fail to restore nitrogen blanket before $\mathrm{O} 2$ reaches $\mathrm{MOC}$ Ignition source present in OWST outer tank OWST inner tank leaks benzene into outer tank Operator fails to respond to high backup N2 flow alarm OWST local liquid N2 system out of service for T\&M Backup OWST N2 cylinders run out before $\mathrm{N} 2$ restored Pressure indicator controller PIC-9161 fails high Nitrogen tank supply failure Any of 100 joints/flanges leaks OWST offgas conservation valve PSV 708-fails open. PSV 707 fails open Pressure Safety Valve Fails Open

Failure of the bulk N2 path

OWST conservation vent valve fails to open OWST pressure relief valve fails to open

OWST pressure rises (daily)

Valve HCV-9161 miscalibrated

PCV 9161 calibrated

Vacuum breaker 418 fails open

Nitrogen PCV 9178 fails closed

N2 PCV 9160 Fails Closed .

OWST nitrogen supply PCV 9161A fails closed

Nitrogen PCV 9161C fails closed

False high differential pressure reading

Overflow (inner to outer tank) fails open

Improper connection at backup purge manifold

Pressure control valve PCV-9161 fails closed

\begin{tabular}{ll} 
Prob/Freg & RedW \\
\hline $3.00 \mathrm{E}-02$ & $2.25 \mathrm{E}+00$ \\
$3.00 \mathrm{E}-03$ & $2.22 \mathrm{E}+00$ \\
$2.30 \mathrm{E}-05 / \mathrm{H}$ & $1.80 \mathrm{E}+00$ \\
$1.00 \mathrm{E}-07 / \mathrm{H}$ & $1.60 \mathrm{E}+00$ \\
$1.00 \mathrm{E}-01$ & $1.38 \mathrm{E}+00$ \\
$3.76 \mathrm{E}-02$ & $1.18 \mathrm{E}+00$ \\
$3.60 \mathrm{E}-01$ & $1.16 \mathrm{E}+00$ \\
$1.08 \mathrm{E}-02$ & $1.09 \mathrm{E}+00$ \\
$7.70 \mathrm{E}-06 / \mathrm{H}$ & $1.08 \mathrm{E}+00$ \\
$1.00 \mathrm{E}-06 / \mathrm{H}$ & $1.07 \mathrm{E}+00$ \\
$1.00 \mathrm{E}-06 / \mathrm{H}$ & $1.07 \mathrm{E}+00$ \\
$1.00 \mathrm{E}-06 / \mathrm{H}$ & $1.07 \mathrm{E}+00$ \\
$1.00 \mathrm{E}-06 / \mathrm{H}$ & $1.07 \mathrm{E}+00$ \\
$5.50 \mathrm{E}-06 / \mathrm{H}$ & $1.06 \mathrm{E}+00$ \\
$1.00 \mathrm{E}-03$ & $1.05 \mathrm{E}+00$ \\
$1.00 \mathrm{E}-03$ & $1.05 \mathrm{E}+00$ \\
$2.78 \mathrm{E}-02 / \mathrm{H}$ & $1.05 \mathrm{E}+00$ \\
$3.00 \mathrm{E}-02$ & $1.03 \mathrm{E}+00$ \\
$1.12 \mathrm{E}-04 / \mathrm{H}$ & $1.03 \mathrm{E}+00$ \\
$5.00 \mathrm{E}-07 / \mathrm{H}$ & $1.03 \mathrm{E}+00$ \\
$3.00 \mathrm{E}-06 / \mathrm{H}$ & $1.03 \mathrm{E}+00$ \\
$3.00 \mathrm{E}-06 / \mathrm{H}$ & $1.03 \mathrm{E}+00$ \\
$3.00 \mathrm{E}-06 / \mathrm{H}$ & $1.03 \mathrm{E}+00$ \\
$3.00 \mathrm{E}-06 / \mathrm{H}$ & $1.03 \mathrm{E}+00$ \\
$3.27 \mathrm{E}-03$ & $1.03 \mathrm{E}+00$ \\
$5.00 \mathrm{E}-09 / \mathrm{H}$ & $1.02 \mathrm{E}+00$ \\
$5.00 \mathrm{E}-02$ & $1.02 \mathrm{E}+00$ \\
$1.50 \mathrm{E}-03$ & $1.01 \mathrm{E}+00$
\end{tabular}

Note: Events that make less than $1 \%$ difference to the importance factor are not included. Modified top event frequency = (top event frequency) $/$ (risk reduction worth). 
X-ESR-S-00001, REV. 0

\section{SPC Explosion}




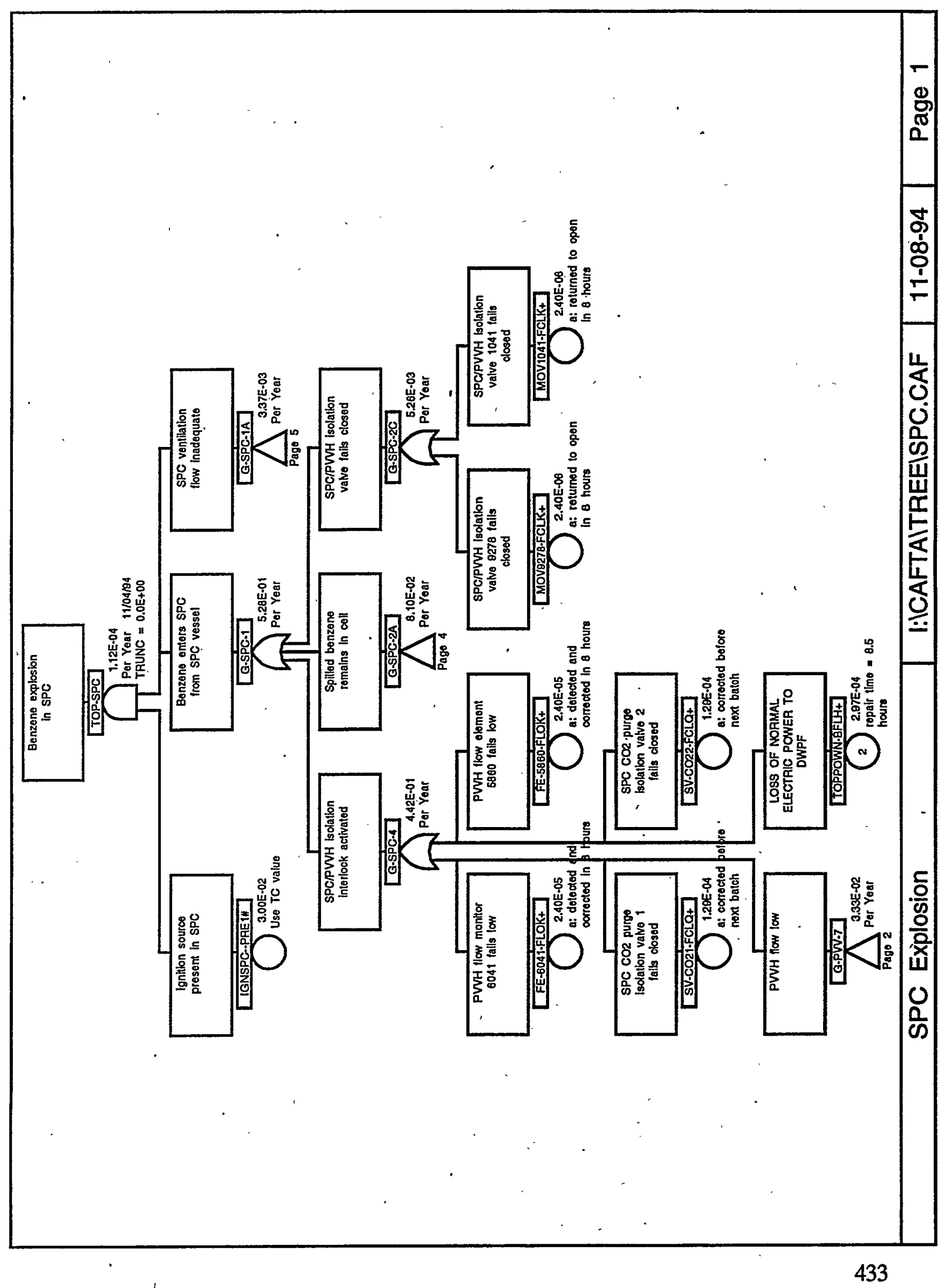


X-ESR-S-00001, REV. 0

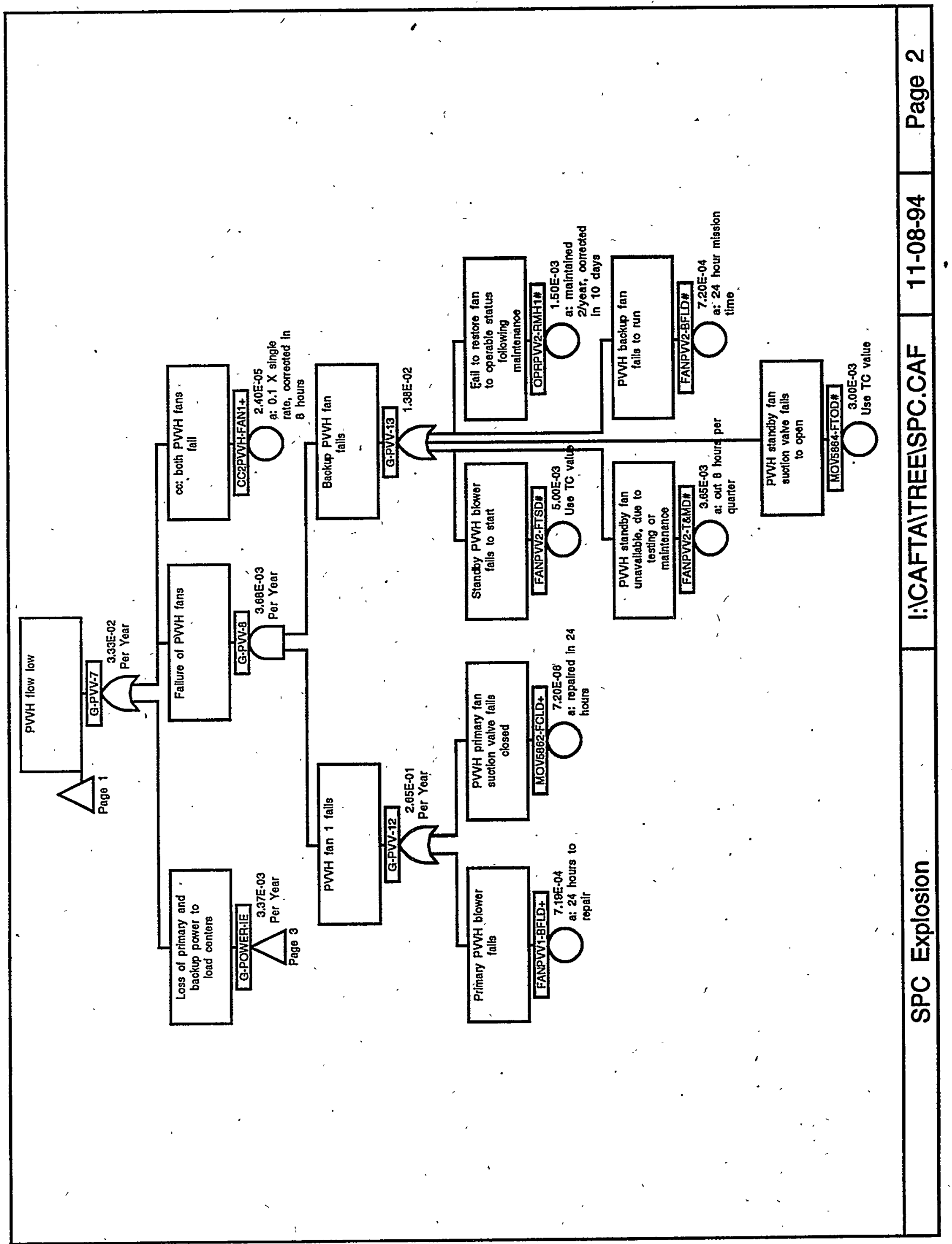




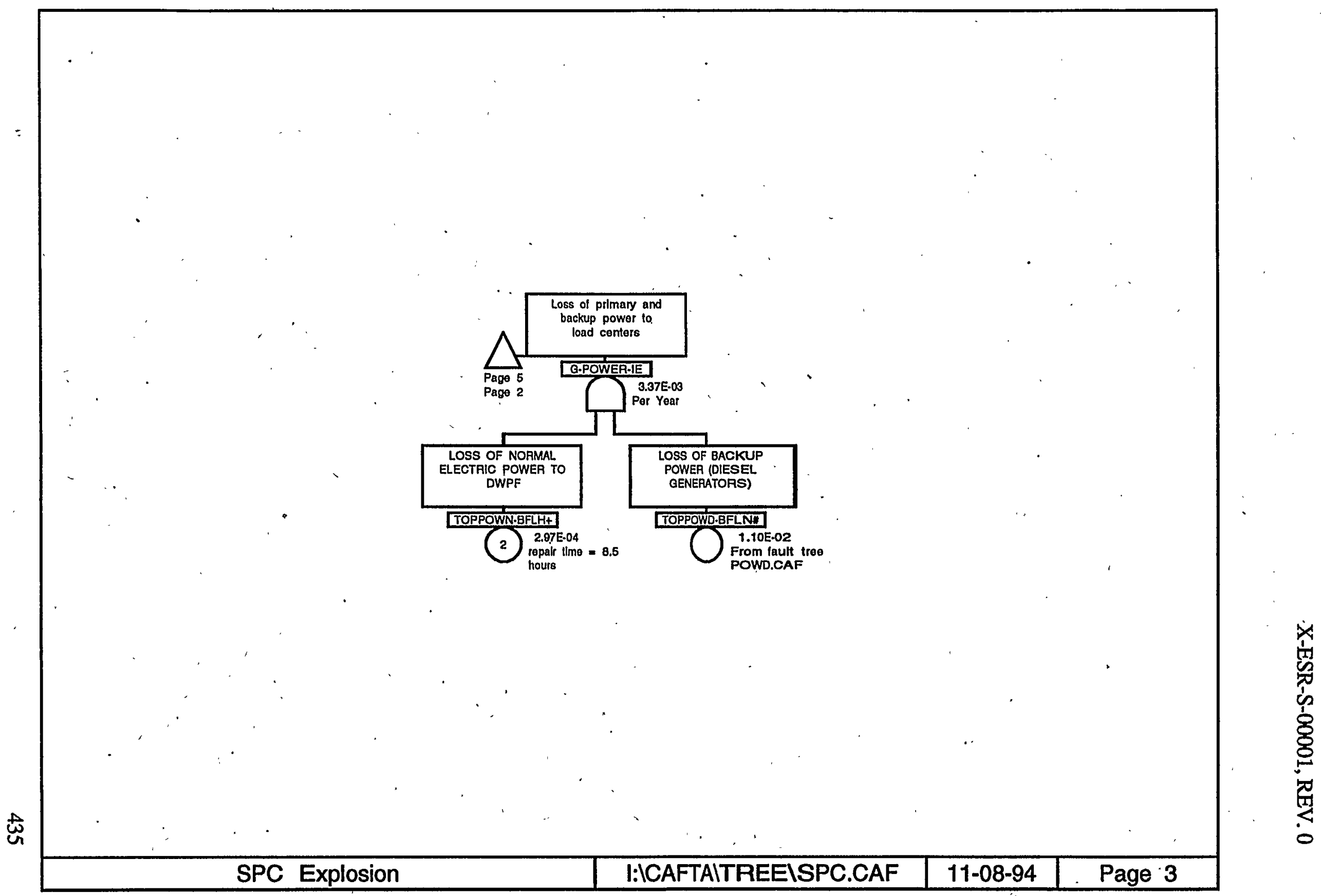


X-ESR-S-00001, REV. 0

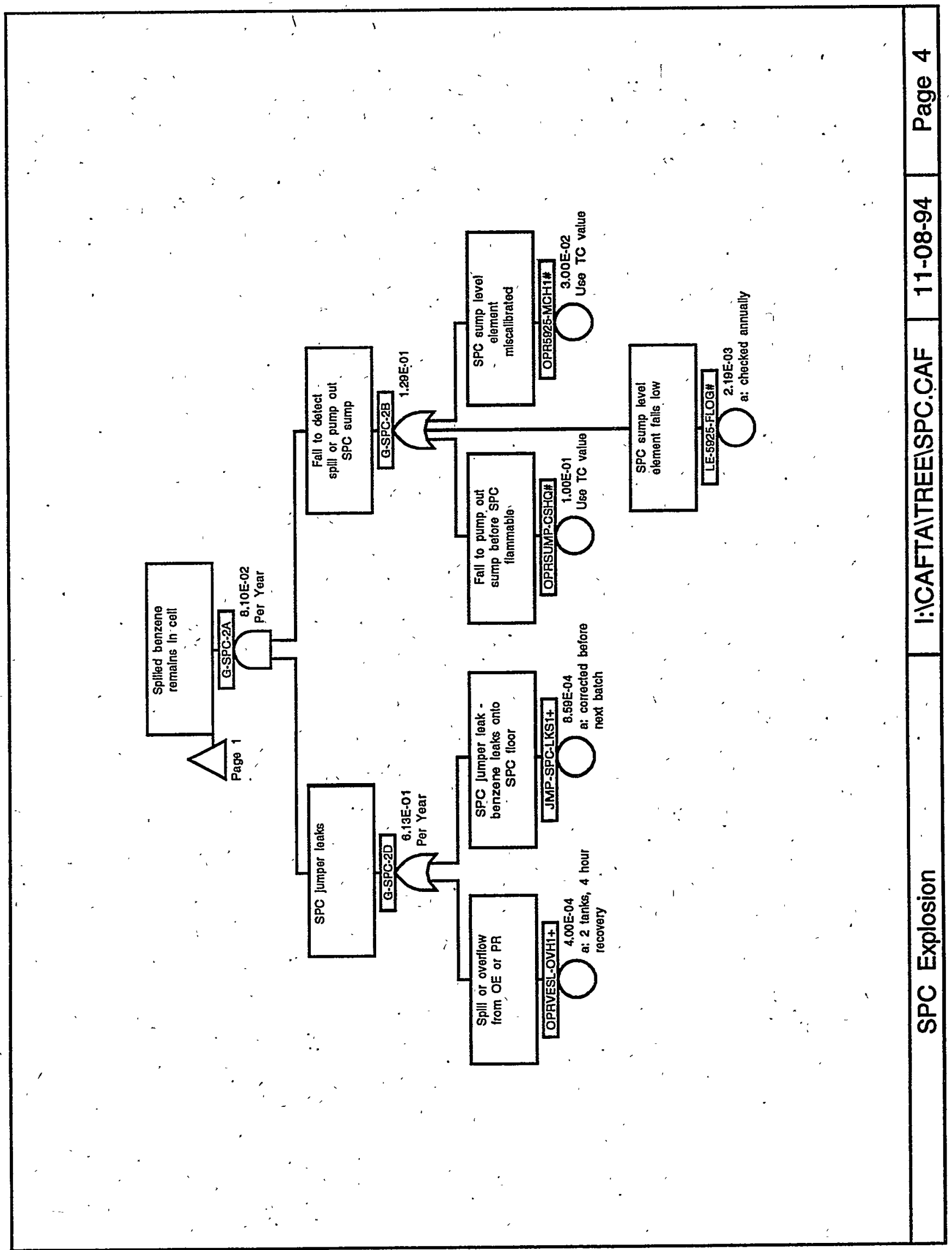




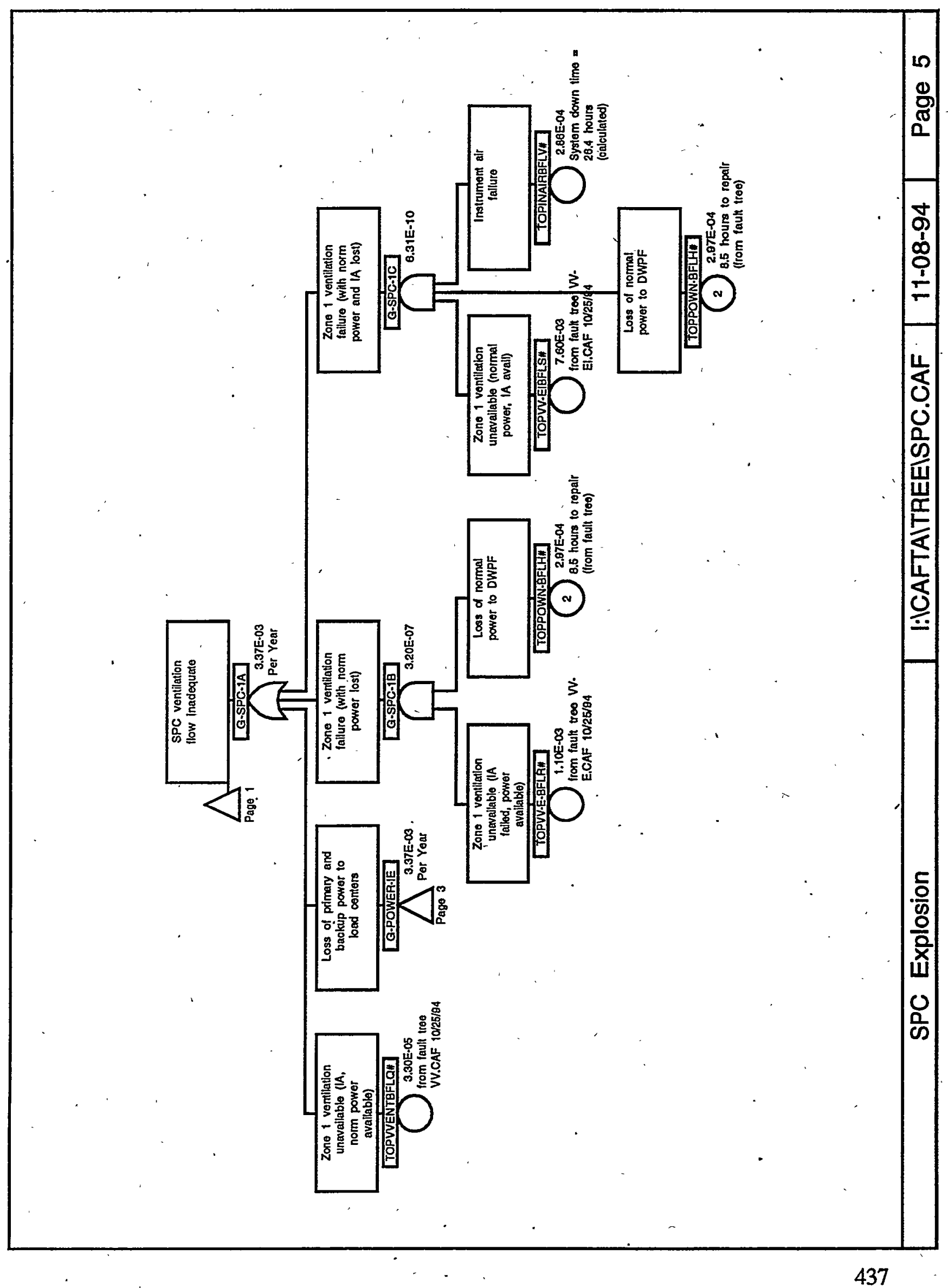




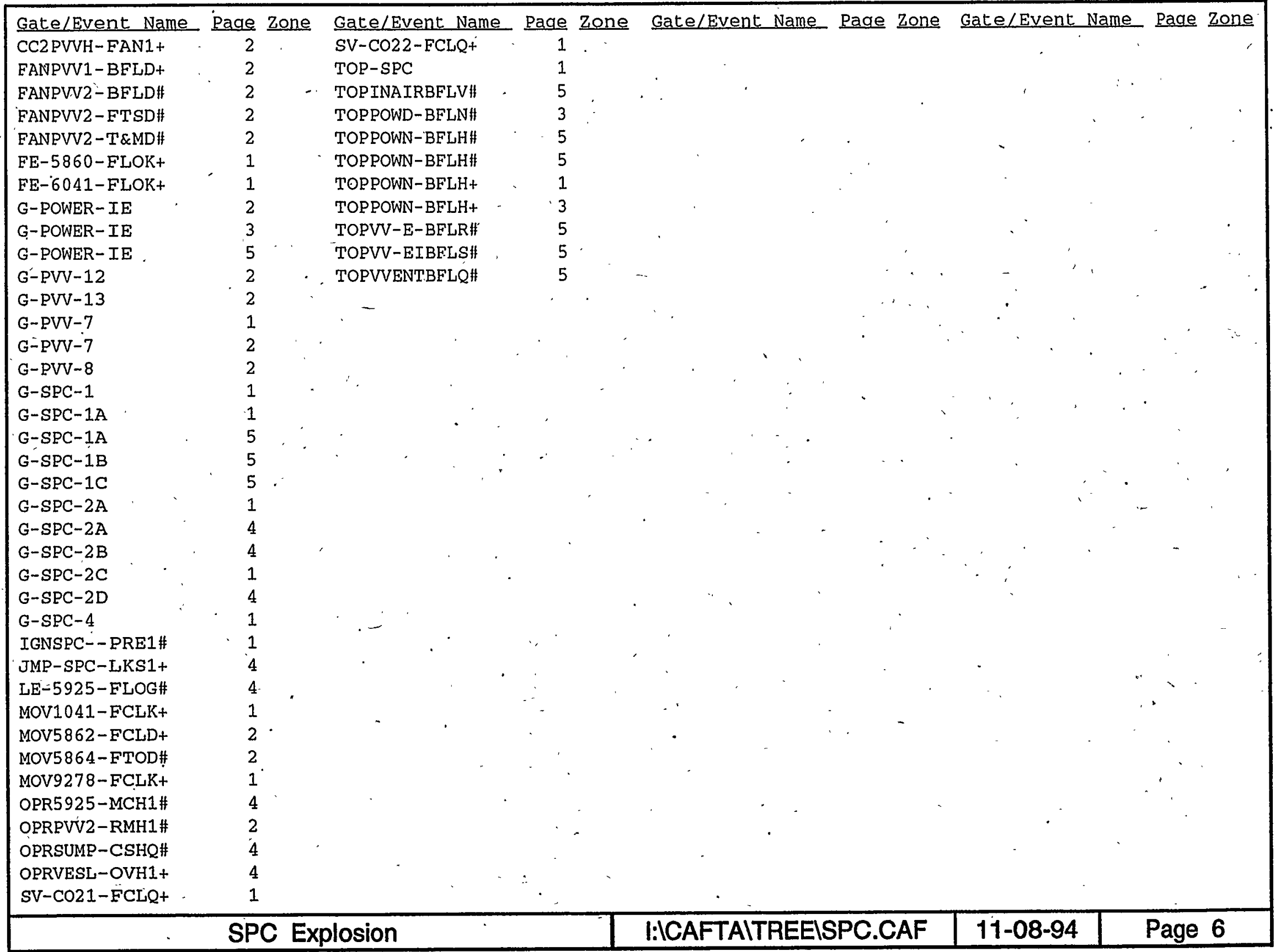


Cutsets for I: ICAFTA ICUT\SPC.CSR

$11 / 08 / 94 \quad 12: 43 \quad \mathrm{AM}$

\begin{tabular}{|c|c|c|c|c|c|c|}
\hline $\begin{array}{l}\text { Set } \\
\text { No. }\end{array}$ & $\begin{array}{l}\text { Event } \\
\text { Name. }\end{array}$ & Description & C & ut & $i$ & $\begin{array}{c}\text { Cutset } \\
\text { Freq. (/yr) }\end{array}$ \\
\hline 1. & $\begin{array}{l}\text { TOP-SPC } \\
\text { IGNSPC--PREI\# } \\
\text { TOPPOWD-BFLN\# } \\
\text { TOPPOWN-BFLH+ }\end{array}$ & $\begin{array}{l}\text { Ignition source present in SPC } \\
\text { LOSS OF BACKUP POWER (DIESEL GENERATORS) } \\
\text { LOSS. OF NORMAL ELECTRIC POWER TO DWPE }\end{array}$ & 1 & $\begin{array}{r}1 \mathrm{~N} \\
3,0 \mathrm{E}-02 \mathrm{~N} \\
1 \mathrm{~N} \\
1.1 \mathrm{E}-02 \mathrm{~N} \\
8.5 \mathrm{H} \\
3.5 \mathrm{E}-05 \mathrm{H}\end{array}$ & $\begin{array}{l}3.00 \mathrm{E}-02 \mathrm{~N} \\
1.10 \mathrm{E}-02 \mathrm{~N} \\
2.97 \mathrm{E}-04\end{array}$ & $\begin{array}{l}.12 \mathrm{E}-04 \\
.01 \mathrm{E}-04\end{array}$ \\
\hline 2 . & $\begin{array}{l}\text { IGNSPC--PRE1\# } \\
\text { TOPPOWN-BFLH+ } \\
\text { TOPVV-E-BFLR\# }\end{array}$ & $\begin{array}{l}\text { Ignition source present in SPC } \\
\text { LOSS OF NORMAL ELECTRIC POWER TO DWPF } \\
\text { Zone } 1 \text { ventilation unavailable (IA failed, power available) }\end{array}$ & $\begin{array}{l}4 \\
1\end{array}$ & $\begin{array}{r}1 \mathrm{~N} \\
3.0 \mathrm{E}-02 \mathrm{~N} \\
8.5 \mathrm{H} \\
3.5 \mathrm{E}-05 \mathrm{H} \\
1.1 \mathrm{~N}-03 \mathrm{~N}\end{array}$ & $\begin{array}{l}3.00 \mathrm{E}-02 \mathrm{~N} \\
2.97 \mathrm{E}-04 \\
1.10 \mathrm{E}-03 \mathrm{~N}\end{array}$ & $1.01 \mathrm{E}-05$ \\
\hline 3. & $\begin{array}{l}\text { IGNSPC- -PRE1\# } \\
\text { TOPPOWN-BFLH+ } \\
\text { TOPVVENTBFLQ\# }\end{array}$ & $\begin{array}{l}\text { Ignition source present in SPC } \\
\text { LOSS OF NORMAL ELECTRIC POWER TO DWPF } \\
\text { Zone } 1 \text { ventilation unavailable (IA, norm power available) }\end{array}$ & $\left|\begin{array}{l}1 \\
4 \\
1\end{array}\right|$ & $\begin{array}{r}1 \mathrm{~N} \\
3.0 \mathrm{E}-02 \mathrm{~N} \\
8.5 \mathrm{H} \\
3.5 \mathrm{E}-0.5 \mathrm{H} \\
3.3 \mathrm{E}-0.1 \mathrm{~N} \\
\mathrm{~N}\end{array}$ & $\begin{array}{l}3.00 \mathrm{E}-02 \mathrm{~N} \\
2.97 \mathrm{E}-04 \\
3.30 \mathrm{E}-05 \mathrm{~N}\end{array}$ & $3.03 \mathrm{E}-0.7$ \\
\hline 4. & $\begin{array}{l}\text { IGNSPC--PRE1\# } \\
\text { OPRSUMP-CSHQ\# } \\
\text { OPRVESL-OVH1+ } \\
\text { TOPVVENTBFLQ\# }\end{array}$ & $\begin{array}{l}\text { Ignition source present, in SPC } \\
\text { Fail to pump out sump before SPC flammable } \\
\text { Spill or overflow from OE or PR } \\
\text { Zone } 1 \text { ventilation unavailable (IA, norm power available) }\end{array}$ & $\begin{array}{l}1 \\
1 \\
4 \\
1\end{array}$ & $\begin{array}{r}1 \mathrm{~N} \\
3.0 \mathrm{E}-02 \mathrm{~N} \\
1 \mathrm{~N} \\
1.0 \mathrm{E}-01 \mathrm{~N} \\
8 \mathrm{H} \\
5.0 \mathrm{E}-05 \mathrm{H} \\
1 \mathrm{~N} \\
3.3 \mathrm{E}-05 \mathrm{~N}\end{array}$ & $\begin{array}{l}3.00 \mathrm{E}-02 \mathrm{~N} \\
1.00 \mathrm{E}-01 \mathrm{~N} \\
4.00 \mathrm{E}-04 \\
3.30 \mathrm{E}-05 \mathrm{~N}\end{array}$ & $4.33 \mathrm{E}-08$ \\
\hline 5. & $\begin{array}{l}\text { FE-6041-FLOK+ } \\
\text { IGNSPC--PRE1\# } \\
\text { TOPVVENTBFLQ\# }\end{array}$ & $\begin{array}{l}\text { PVVH flow monitor } 6041 \text { fails low } \\
\text { Ignition source present in SPC } \\
\text { Zone } 1 \text { ventilation unavailable (IA, norm power available) }\end{array}$ & $\begin{array}{l}4 \\
1 \\
1\end{array}$ & $\begin{array}{r}8 \mathrm{H} \\
3.0 \mathrm{E}-06 \mathrm{H} \\
1 \mathrm{~N} \\
3.0 \mathrm{E}-02 \mathrm{~N} \\
1 \mathrm{~N} \\
3.3 \mathrm{E}-05 \mathrm{~N}\end{array}$ & $\begin{array}{l}2.40 \mathrm{E}-05 \\
3.00 \mathrm{E}-02 \mathrm{~N} \\
3.30 \mathrm{E}-05 \mathrm{~N}\end{array}$ & $2.60 \mathrm{E}-08$ \\
\hline 6. & $\begin{array}{l}\text { CC2PVVH-FAN1+ } \\
\text { IGNSPC--PRE1\# } \\
\text { TOPVVENTBFLQ\# }\end{array}$ & $\begin{array}{l}\text { cc: both PVWH fans fail } \\
\text { Ignition source present in SPC } \\
\text { zone } 1 \text { ventilation unavailable (IA, norm power available) }\end{array}$ & $\begin{array}{l}4 \\
1 \\
1\end{array}$ & $\begin{array}{r}8 \mathrm{H} \\
3.0 \mathrm{E}-06 \mathrm{H} \\
1 \mathrm{~N} \\
3.0 \mathrm{E}-02 \mathrm{~N} \\
3.3 \mathrm{E}-05 \mathrm{~N}\end{array}$ & $\begin{array}{l}2.40 \mathrm{E}-05 \\
3.00 \mathrm{E}-02 \mathrm{~N} \\
3.30 \mathrm{E}-05 \mathrm{~N}\end{array}$ & $2.60 E-08$ \\
\hline 7. & FE-5860-FLOK+ & PVVH flow element 5860 fails low & 4 & $3.0 \mathrm{E}-06 \mathrm{HH}$ & $2.40 E-05$ & $2.60 \mathrm{E}-08$ \\
\hline
\end{tabular}


cutsets for I: \CAFTA \CUT\SPC.CSR . 11/08/94 12:43 AM (CONT.)

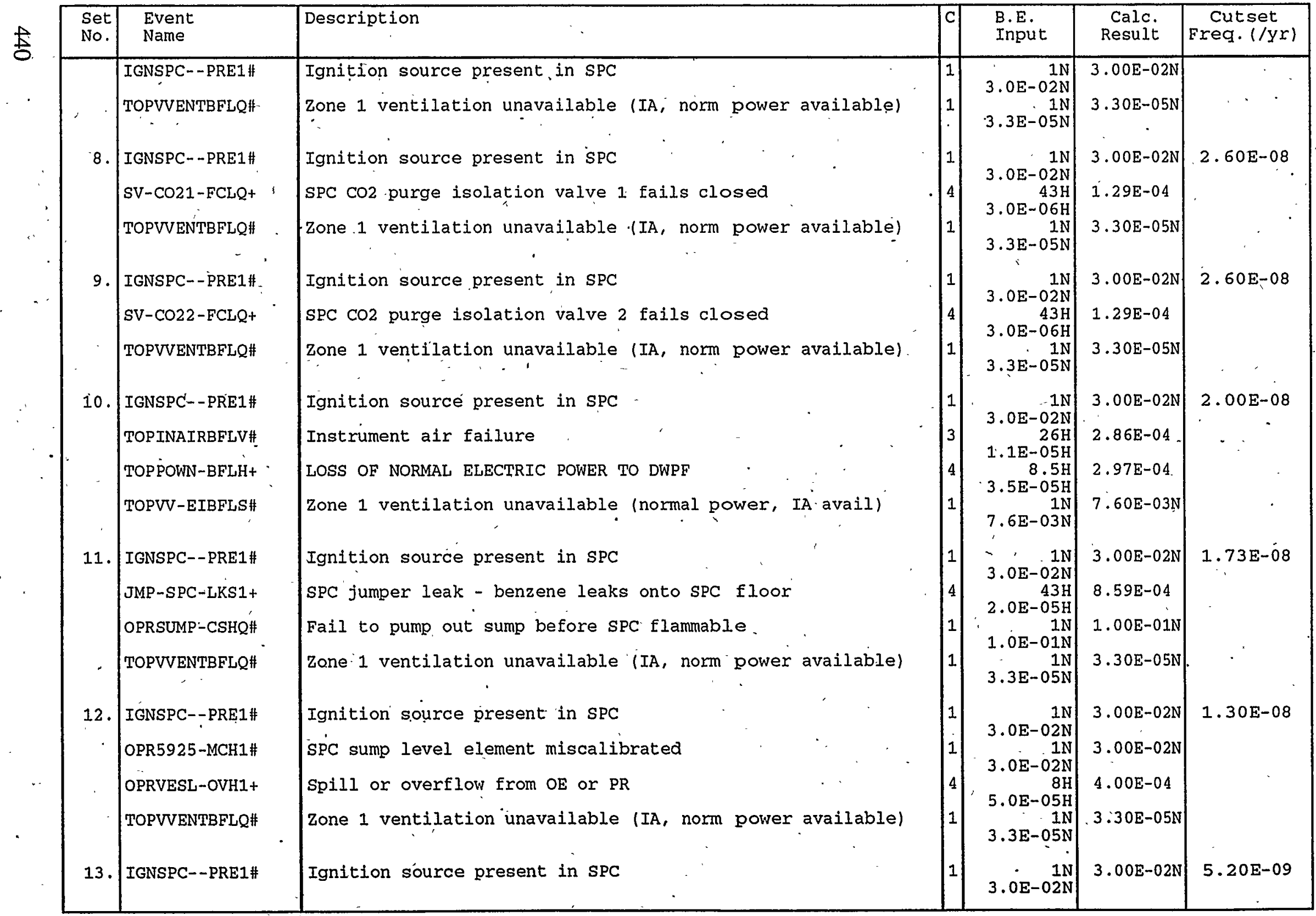


Cutsets for I: ICAFTA.ICUT\SPC.CSR

11/08/94 12:43 AM (CONT.)

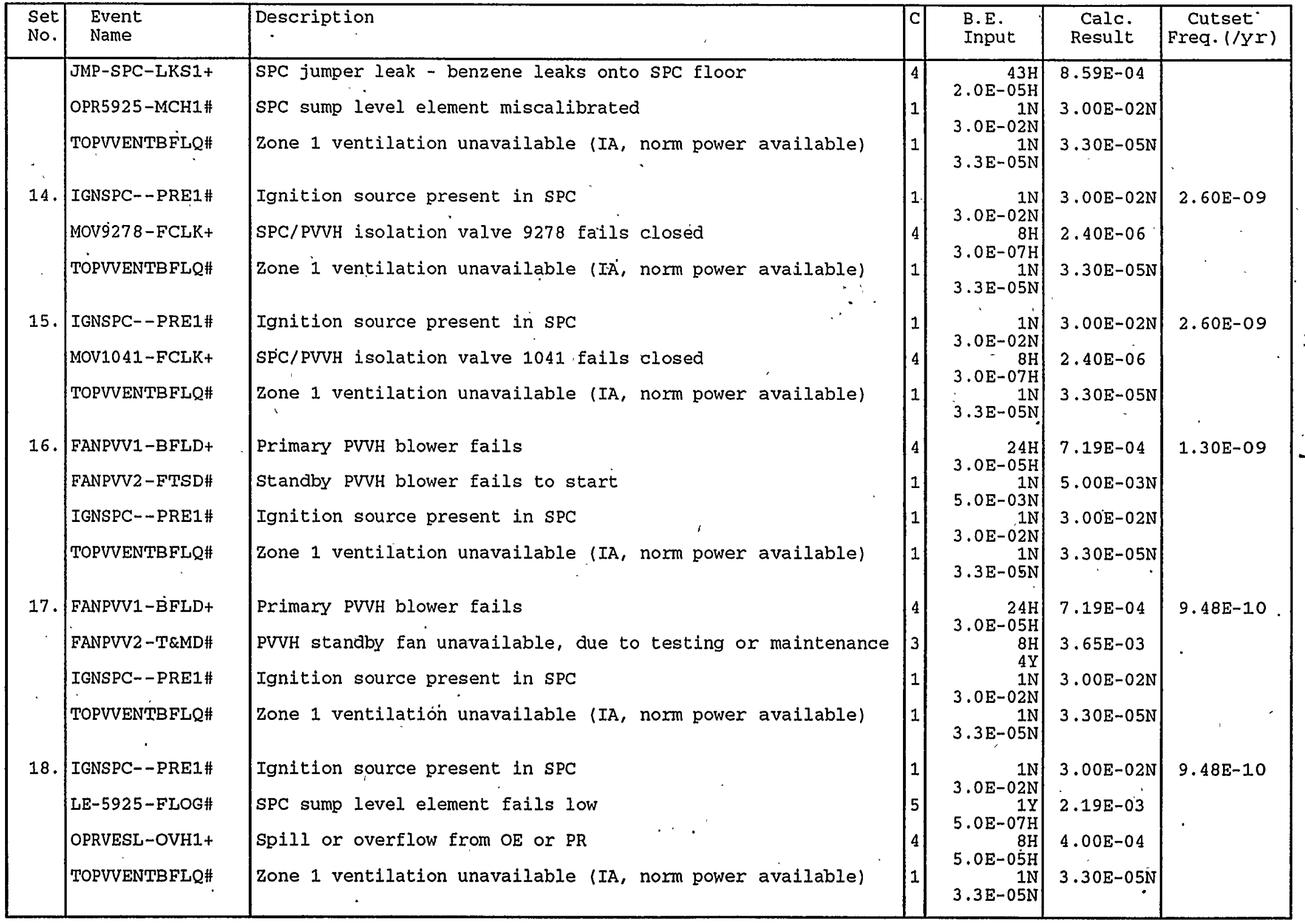


Cutsets for I: \CAFTA \CUT\SPC.CSR

11/08/94 12:43 AM (CONT.)

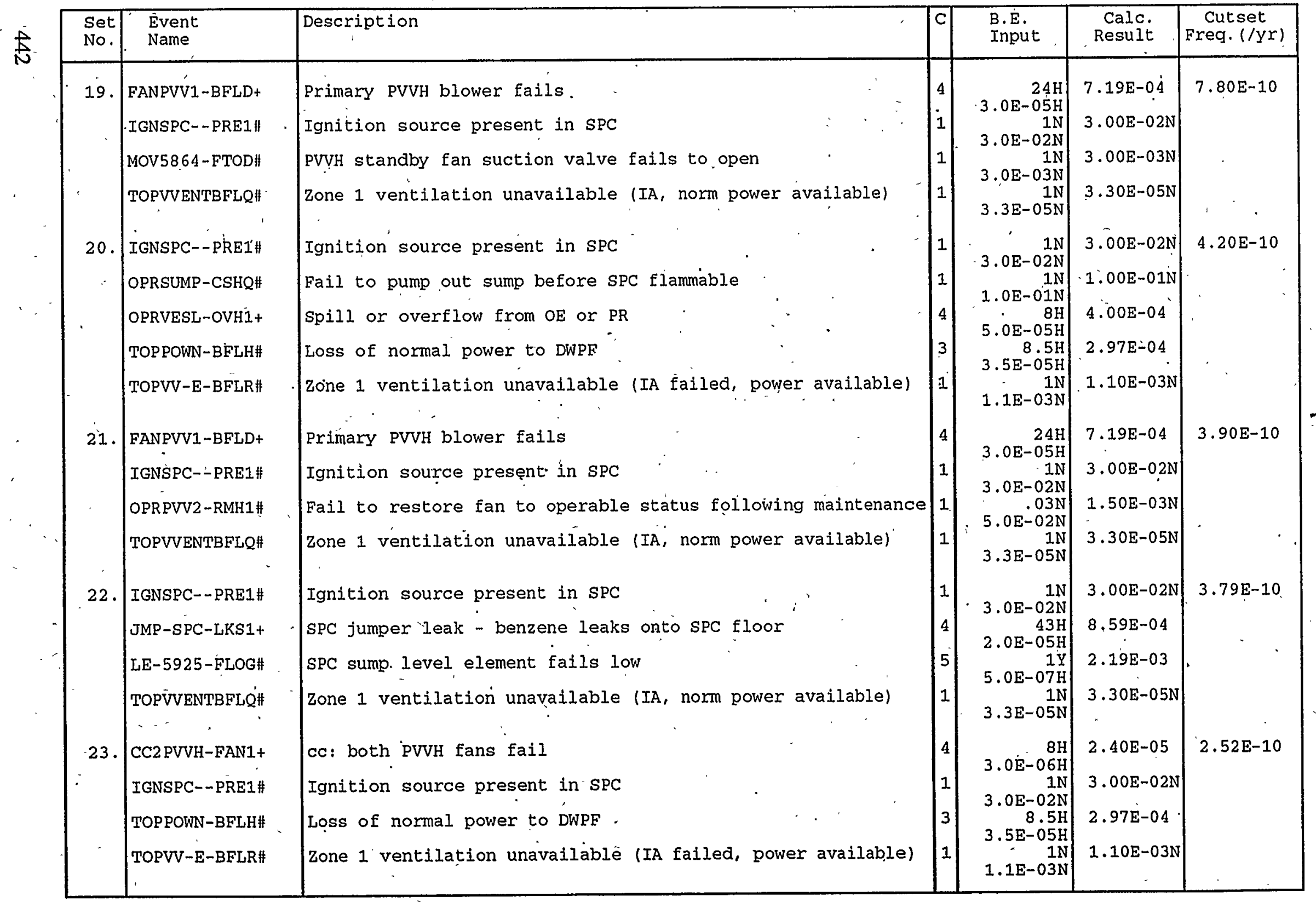


Cutsets for I: ICAFTA ICUT\SPC.CSR

11/08/94 12:43 AM (CONT.)

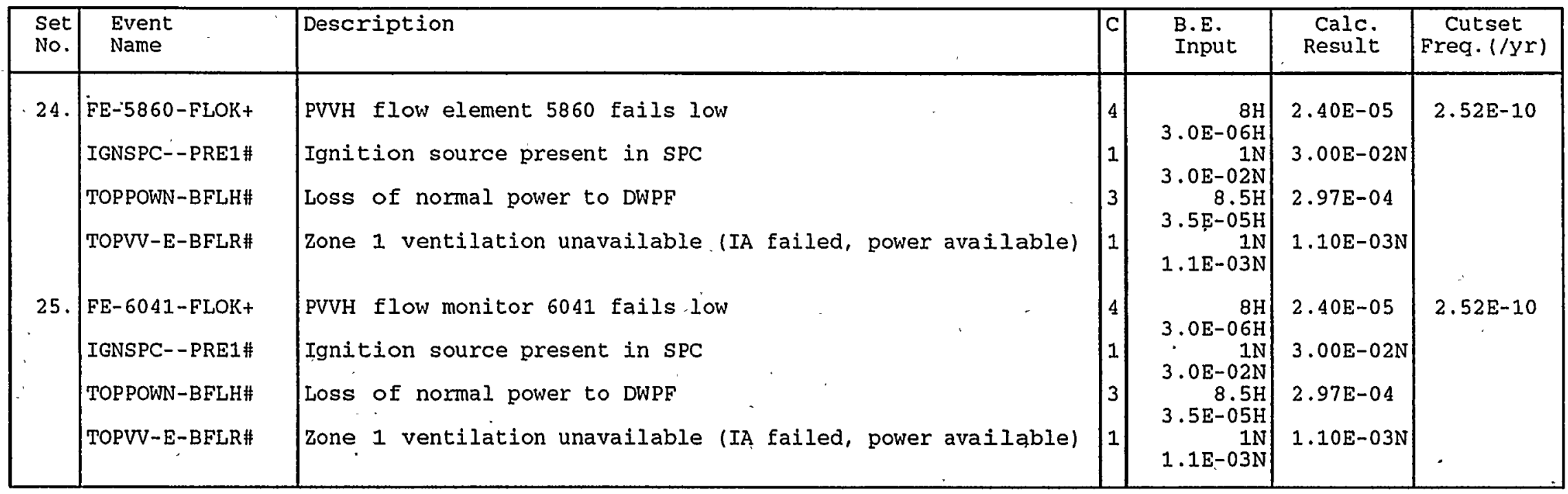


Benzene explosion in SPC Top Event Frequency: 1.12E-04/YR

\section{Risk Achievement Worth}

Basic Event Name

TOPPOWN-BFLH+-IC. TOPVVENTBFLQ\#

TOPVV-E-BFLR\#

TOPPOWD-BFLN\#

MOV1041-FCLK+I

MOV9278-FCLK+I

FE-5860-FLOK+I

SV-CO21-FCLQ+-I

SV-CO22-FCLQ+-I

FE-6041-FLOK+I

CC2PVVH-FAN1+-I

IGNSPC--PRE1\#

JMP-SPC-LKS1+-I

OPRVESL-OVH1+-I

MOV5862-FCLD+-I

FANPVV1-BFLD+-I

TOPINAIRBFLV\#

TOPVV-EIBFLS\#

\section{Description}

LOSS OF NORMAL ELECTRIC POWER TO DWPF

Zone 1 ventilation unavailable (IA, norm power ávailable)

Zone 1 ventilation unavailable (IA failed, power available)

LOSS OF BACKUP POWER (DIESEL GENERATORS)

SPC/PVVH isolation valve 1041 fails closed

SPC/PVVH isolation valve 9278 fails closed

PVVH flow element 5860 fails low

SPC CO2 purge isolation valve 1 fails closed

SPC CO2 purge isolation valve 2 fails closed

PVVH flow monitor 6041 fails low

cc: both PVVH fans fail

Ignition source present in SPC

SPC jumper leak - benzene leaks onto SPC floor

Spill or overflow from $O E$ or $P R$

PVVH primary fan suction valve' fails closed

Primary PVVH blower fails

Instrument air failure

Zone 1 ventilation unavailable (normal power, IA avail)

\begin{tabular}{ll} 
Prob/Freg & AchW \\
\hline $3.50 \mathrm{E}-05 / \mathrm{H}$ & $2.85 \mathrm{E}+04$ \\
$3.30 \mathrm{E}-05$ & $1.43 \mathrm{E}+02$ \\
$1.10 \mathrm{E}-03$ & $8.32 \mathrm{E}+01$ \\
$1.10 \mathrm{E}-02$ & $8.23 \mathrm{E}+01$ \\
$3.00 \mathrm{E}-07 / \mathrm{H}$ & $7.93 \mathrm{E}+01$ \\
$3.00 \mathrm{E}-07 / \mathrm{H}$ & $7.93 \mathrm{E}+01$ \\
$3.00 \mathrm{E}-06 / \mathrm{H}$ & $7.93 \mathrm{E}+01$ \\
$3.00 \mathrm{E}-06 / \mathrm{H}$ & $7.93 \mathrm{E}+01$ \\
$3.00 \mathrm{E}-06 / \mathrm{H}$ & $7.93 \mathrm{E}+01$ \\
$3.00 \mathrm{E}-06 / \mathrm{H}$ & $7.93 \mathrm{E}+01$ \\
$3.00 \mathrm{E}-06 / \mathrm{H}$ & $7.93 \mathrm{E}+01$ \\
$3.00 \mathrm{E}-02$ & $3.33 \mathrm{E}+01$ \\
$2.00 \mathrm{E}-05 / \mathrm{H}$ & $1.14 \mathrm{E}+01$ \\
$5.00 \mathrm{E}-05 / \mathrm{H}$ & $1.14 \mathrm{E}+01$ \\
$3.00 \mathrm{E}-07 / \mathrm{H}$ & $2.09 \mathrm{E}+00$ \\
$3.00 \mathrm{E}-05 / \mathrm{H}$ & $2.09 \mathrm{E}+00$ \\
$2.86 \mathrm{E}-04$ & $1.62 \mathrm{E}+00$ \\
$7.60 \mathrm{E}-03$ & $1.02 \mathrm{E}+00$
\end{tabular}

\section{Risk Reduction Worth}

Basic Event Name

*IGNSPC--PRE1\#

Description

Ignition source present in SPC

TOPPOWN-BFLH+-IC

TOPPOWD-BFLN\#

TOPVV-E-BFLR\#

Prob/Freg RedW

3.00E-02 0.00E+00

$3.50 \mathrm{E}-05 / \mathrm{H} \quad 5.10 \mathrm{E}+02$

$1.10 \mathrm{E}-02 \quad 1.05 \mathrm{E}+01$

$1.10 \mathrm{E}-03 \quad 1.10 \mathrm{E}+00$

* A Reduction Worth of $0.0 \mathrm{E}+00$ indicates event appears in every cutset could serve as a single event preventor for the accident.

Note: Events that make léss than $1 \%$ difference to the importance factor are not included. 


\section{Attachment 6}

\section{Support System Fault Tree Plots and Cut Sets}

Steam

SSW

PSW

CTWCC

DEEP

CTWP

BlkN2

N2tnk

CPCtnk

CPCBP

CPCPP

POWD

POWN

VV

VV-E

VV-EI

SPCPP

SPCBP

SPCN2

LPPPN2

InAir
Process steam failure

Process chilled water failure 446

Process cooling water failure

Cooling tower water (common cause) failure

Cooling tower water (deep well) failure

Cooling tower water (pumping) failure

Bulk nitrogen path failure..

Bulk nitrogen supply failure

CPC nitrogen tank supply unavailable

CPC backup purge path unavailable

CPC primary purge failure

Backup power unavailable

Normal power failure

Vitrification building ventilation failure

(Normal power and instrument air available)

Vitrification building ventilation failure

(Normal power failed, and instrument air available)

Vitrification building ventilation failure

(Normal power and instrument air failed).

SPC primary purge failure

SPC backup purge unavailable

SPC nitrogen blanket path unavailable

LPPP nitrogen supply path failure

Instrument air failure 
X-ESR-S-00001, Rev. 0

Process Steam Unavailable 


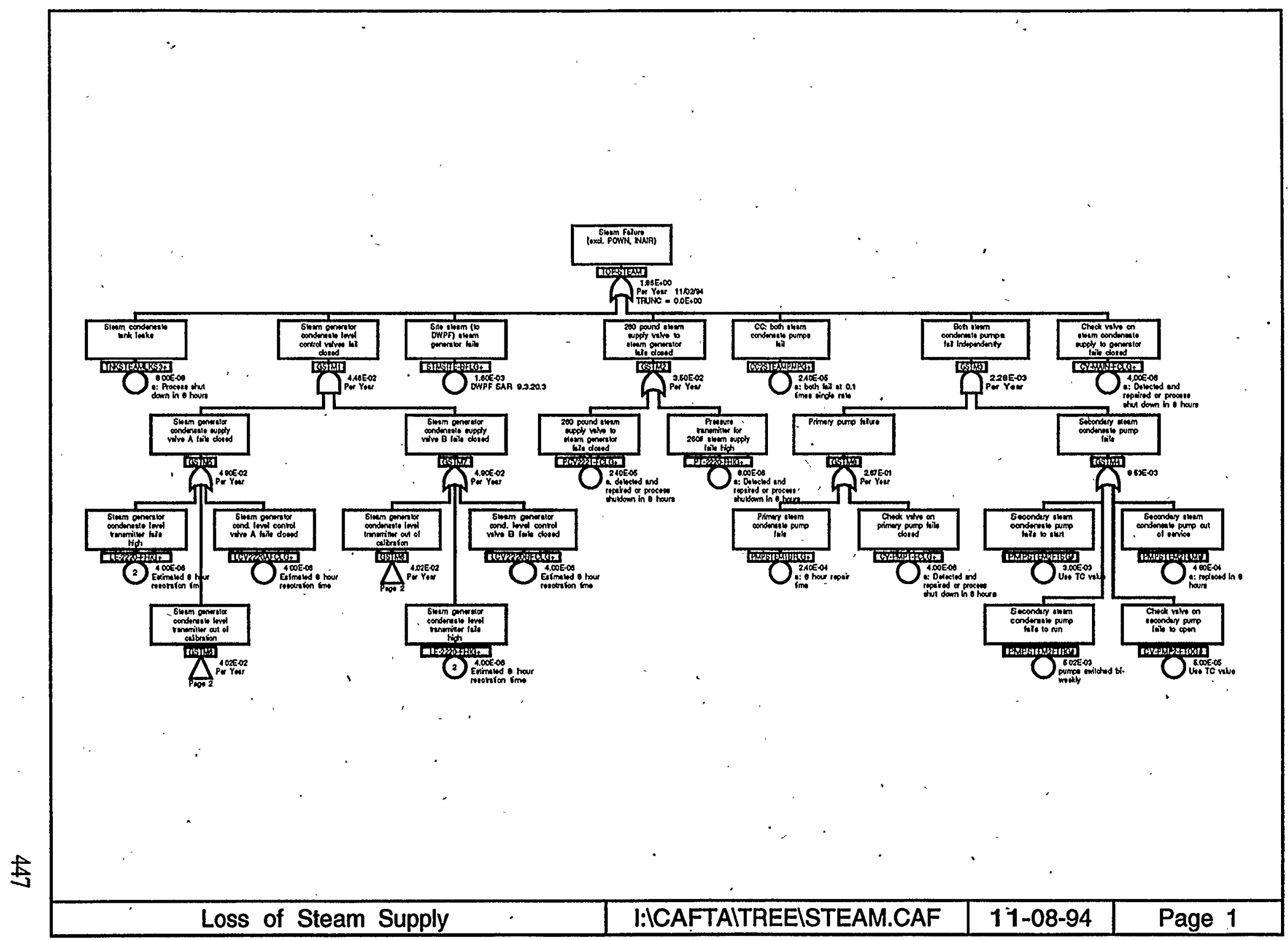


X-ESR-S-00001; Rev. 0

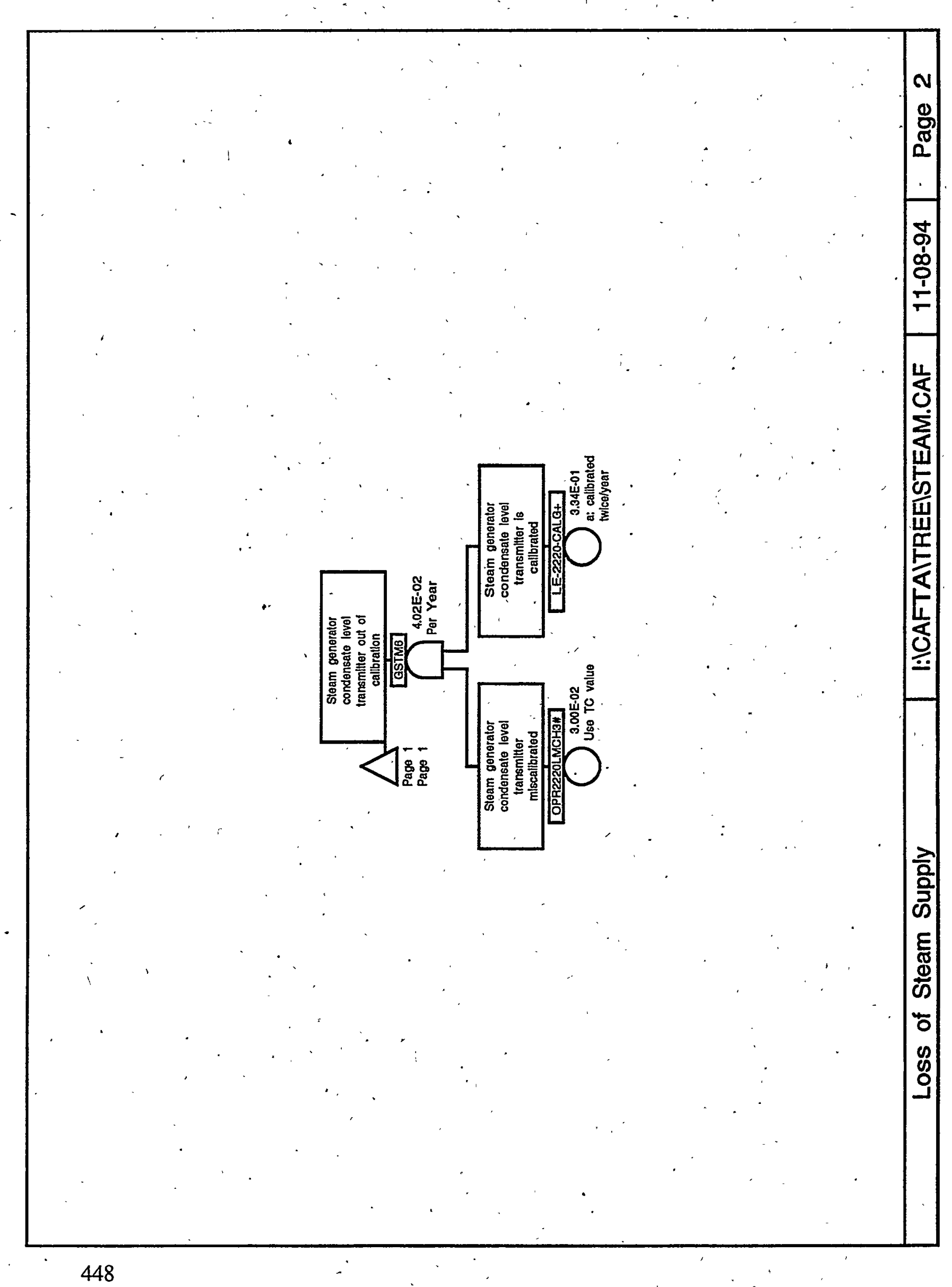


Cutsets for I: ICAFTA \CUT\STEAM.CSR

$11 / 08 / 94 \quad 11: 34$ AM

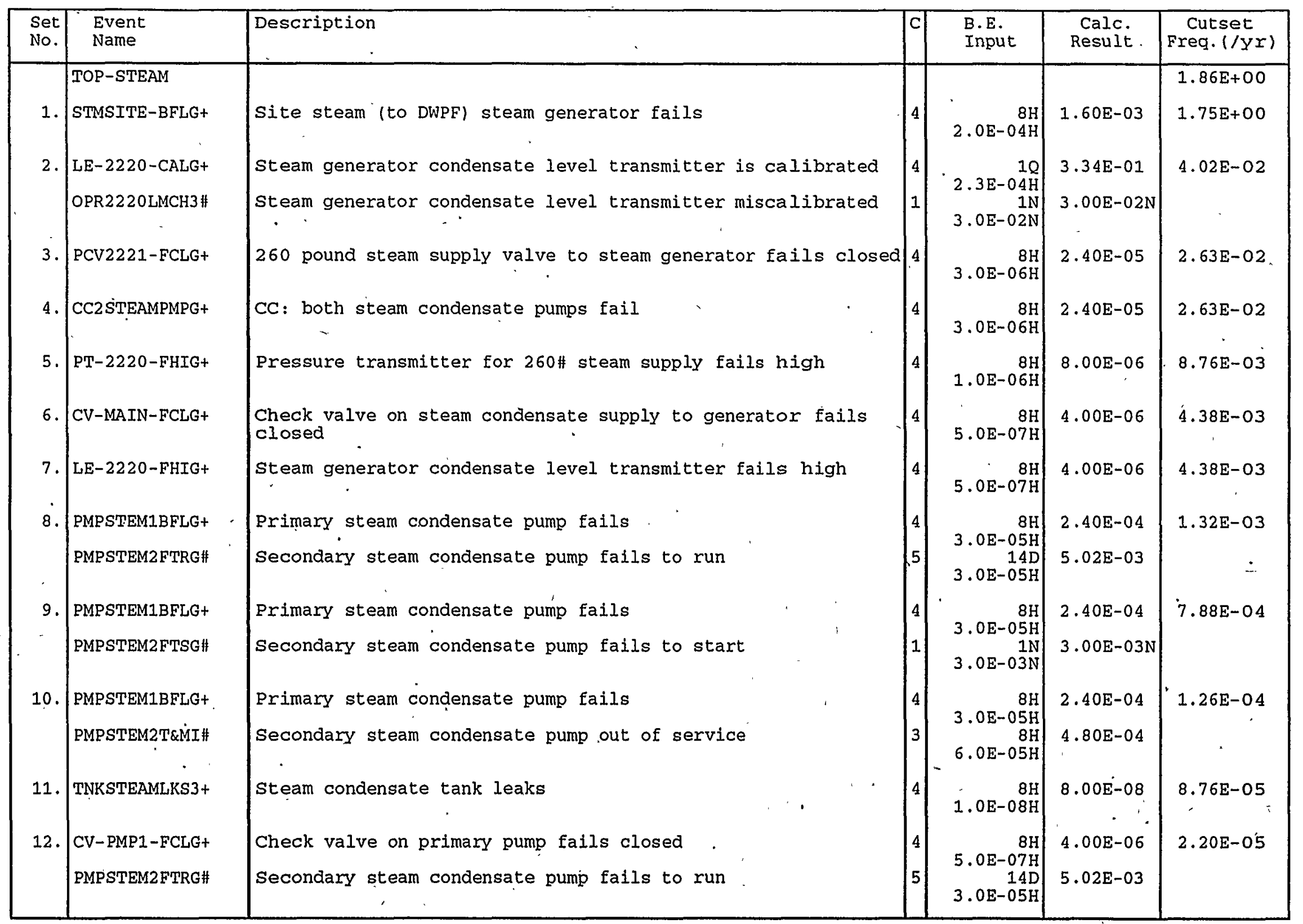




\begin{tabular}{|c|c|c|c|c|c|c|c|}
\hline & $\begin{array}{l}\text { Set } \\
\text { No. }\end{array}$ & $\begin{array}{l}\text { Event } \\
\text { Name }\end{array}$ & Description & C & $\begin{array}{l}\text { B.E. } \\
\text { Input }\end{array}$ & $\begin{array}{l}\text { Calc. } \\
\text { Result }\end{array}$ & $\begin{array}{c}\text { Cutset } \\
\text { Freq. }(/ y r)\end{array}$ \\
\hline & 17. & $\begin{array}{l}\text { CV-PMP1-FCLG+ } \\
\text { PMPSTEM2ETSG } \\
\text { CV-PMP2-FTOG\# } \\
\text { PMPSTEM1BFLG+ } \\
\text { CV-PMP1-FCLG+ } \\
\text { PMPSTEM2T\&MI\# } \\
\text { CV-PMP1-FCLG+ } \\
\text { CV-PMP2-FTOG\# } \\
\text { LCV2220AFCLG+ } \\
\text { LCV2220BFCLG+ }\end{array}$ & $\begin{array}{l}\text { Check valve on primary pump fails closed } \\
\text { Secondary steam condensate pump fails to start } \\
\text { Check valve on secondary pump fails to open } \\
\text { Primary steam condensate pump fails. } \\
\text { Check valve on primary pump fails closed } \\
\text { secondary steam condensate pump out of service } \\
\text { Check valve on primary pump fails closed } \\
\text { Check valve on secondary pump fails to open } \\
\text { steam generator cond. level control valve A fails closed } \\
\text { steam generator cond. level control valvé B fails closed }\end{array}$ & $\begin{array}{l}1 \\
4\end{array}$ & $\begin{array}{r}5.0 \mathrm{E}-07 \mathrm{H} \\
-1 \mathrm{~N} \\
3.0 \mathrm{E}-03 \mathrm{~N} \\
\\
1 \mathrm{~N} \\
5.0 \mathrm{E}-05 \mathrm{~N} \\
8 \mathrm{H} \\
3.0 \mathrm{E}-05 \mathrm{H} \\
\mathrm{r} \\
8 \mathrm{H} \\
5.0 \mathrm{E}-07 \mathrm{H} \\
8 \mathrm{H} \\
6.0 \mathrm{E}-05 \mathrm{H} \\
\\
5.0 \mathrm{E}-07 \mathrm{H} \\
1 \mathrm{~N} \\
5.0 \mathrm{E}-05 \mathrm{~N} \\
\\
5.0 \mathrm{E}-07 \mathrm{H} \\
-8 \mathrm{H} \\
5.0 \mathrm{E}-07 \mathrm{H}\end{array}$ & $\begin{array}{l}4.00 \mathrm{E}-06 \\
3.00 \mathrm{E}-03 \mathrm{~N} \\
5.00 \mathrm{E}-05 \mathrm{~N} \\
2.40 \mathrm{E}-04 \\
4.00 \mathrm{E}-06 \\
4.80 \mathrm{E}-04 \\
4.00 \mathrm{E}-06 \\
5.00 \mathrm{E}-05 \mathrm{~N} \\
4.00 \mathrm{E}-06 \\
4.00 \mathrm{E}-06\end{array}$ & $\begin{array}{c}2.19 \mathrm{E}-07 \\
3.50 \mathrm{E}-08\end{array}$ \\
\hline
\end{tabular}


Steam Failure (excl. POWN, INAIR) Top Event Frequency: $1.86 \mathrm{E}+00 / \mathrm{YR}$

Basic Event Name
CV-MAIN-FCLG+-I
PT-2220-FHIG+-I
TNKSTEAMLKS3+-I
LE-2220-FHGH+I
CC2STEAMPMPG+-I
STMSITE-BFLG+-I
PCV2221-FCLG+-I
LE-2220-CALG+I
CV-PMP1-FCLG+-I
PMPSTEM1BFLG+-I
OPR2220LMCH3\#
CV-PMP2-FTOG\#'
PMPSTEM2T\&MI
PMPSTEM2FTSG\#
PMPSTEM2FTRG\#
LCV2220BFCLG+-IC
LCV2220AFCLG+-IC
Modified top event f

Basic EVEnt Name
STMSITE-BFLG+-I
OPR2220LMCH3\#
LE-2220-CALG+-I
CC2STEAMPMPG+-I
PCV2221-FCLG+-I

\section{Risk Achievement Worth} Description

Check valve on steam condensate supply fails closed Pressure transmitter for 260\# steam supply fails high Steam condensate tank leaks

Steam generator condensate level transmitter fails high

CC: both steam condensate pumps fail

Site steam (to DWPF) steam generator fails

260 pound steam valve to steam generator fails closed

Steam generator condensate level transmitter is calibrated

Check valve on primary pump fails closed

Primary steam condensate pump fails

Steam generator condensate level transmitter miscalibrated

Check valve on secondary pump fails to open -

Secondary steam condensate pump out of service

Secondary steam condensate pump fails to start

Secondary steam condensate pump fails to run

Steam generator cond. level control valve $B$ fails closed

Steam generator cond. level control valve $A$ fails closed

\begin{tabular}{ll} 
Prob/Freg & AchW \\
\hline $5.00 \mathrm{E}-07 / \mathrm{H}$ & $4.71 \mathrm{E}+03$ \\
$1.00 \mathrm{E}-06 / \mathrm{H}$ & $4.71 \mathrm{E}+03$ \\
$1.00 \mathrm{E}-08 / \mathrm{H}$ & $4.71 \mathrm{E}+03$ \\
$5.00 \mathrm{E}-07 / \mathrm{H}$ & $4.71 \mathrm{E}+03$ \\
$3.00 \mathrm{E}-06 / \mathrm{H}$ & $4.71 \mathrm{E}+03$ \\
$2.00 \mathrm{E}-04 / \mathrm{H}$ & $4.71 \mathrm{E}+03$ \\
$3.00 \mathrm{E}-06 / \mathrm{H}$ & $4.71 \mathrm{E}+03$ \\
$1.53 \mathrm{E}-04 / \mathrm{H}$ & $1.42 \mathrm{E}+02$ \\
$5.00 \mathrm{E}-07 / \mathrm{H}$ & $4.12 \mathrm{E}+01$ \\
$3.00 \mathrm{E}-05 / \mathrm{H}$ & $4.12 \mathrm{E}+01$ \\
$3.00 \mathrm{E}-02$ & $1.70 \mathrm{E}+00$ \\
$5.00 \mathrm{E}-05$ & $1.14 \mathrm{E}+00$ \\
$4.80 \mathrm{E}-04$ & $1.14 \mathrm{E}+00$ \\
$3.00 \mathrm{E}-03$ & $1.14 \mathrm{E}+00$ \\
$5.02 \mathrm{E}-03$ & $1.14 \mathrm{E}+00$ \\
$5.00 \mathrm{E}-07 / \mathrm{H}$ & $1.02 \mathrm{E}+00$ \\
$5.00 \mathrm{E}-07 / \mathrm{H}$ & $1.02 \mathrm{E}+00$ \\
worth). & \\
&
\end{tabular}

Prob/Freg AchW

$1.00 \mathrm{E}-06 / \mathrm{H} \quad 4.71 \mathrm{E}+03$

$1.00 \mathrm{E}-08 / \mathrm{H} \quad 4.71 \mathrm{E}+03$

$3.00 \mathrm{E}-06 / \mathrm{H} \quad 471 \mathrm{E}+03$

$2.00 \mathrm{E}-04 / \mathrm{H} \quad 4.71 \mathrm{E}+03$

$3.00 \mathrm{E}-06 / \mathrm{H} \quad 4.71 \mathrm{E}+03$

$5.00 \mathrm{E}-07 / \mathrm{H}$ - $4.12 \mathrm{E}+01$

$3.00 \mathrm{E}-05 / \mathrm{H} \quad 4.12 \mathrm{E}+01$

3.00E-02 1.70E+00

5.00E-05 1.14E+00

$3.00 \mathrm{E}-03 \quad 1.14 \mathrm{E}+00$

5.02E-03 1.14E+00

$5.00 \mathrm{E}-07 / \mathrm{H} \quad 1.02 \mathrm{E}+00$

$5.00 \mathrm{E}-07 / \mathrm{H} \quad 1.02 \mathrm{E}+00$

Note: Events that make less than $1 \%$ difference to the importance factor are not included. Modified top event frequency $=$ (top event frequency) $/$ (risk reduction worth). 
X-ESR-S-00001, Rev. 0

Process Chilled Water System Failure 


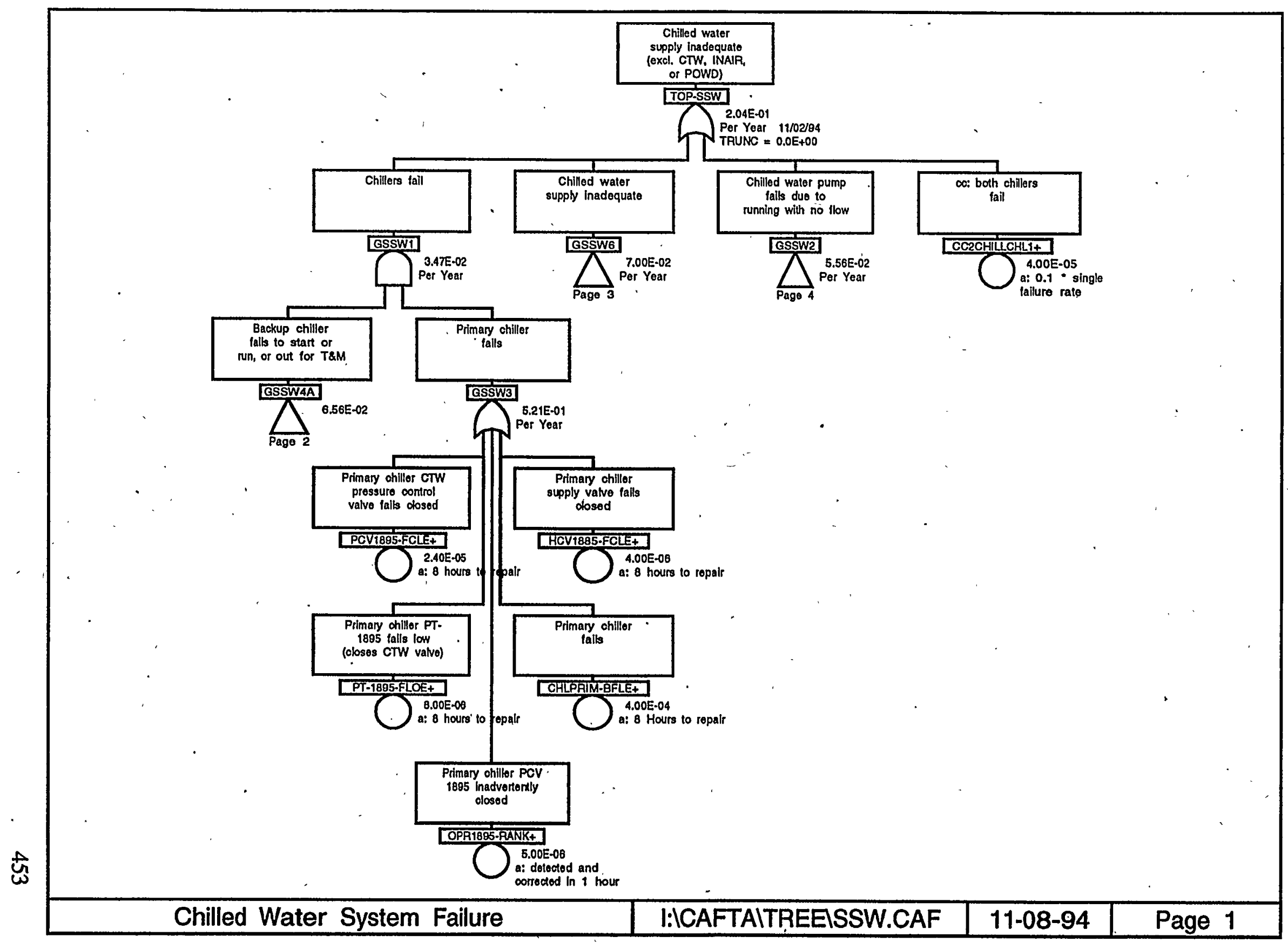


X-ESR-S-00001, Rev. 0

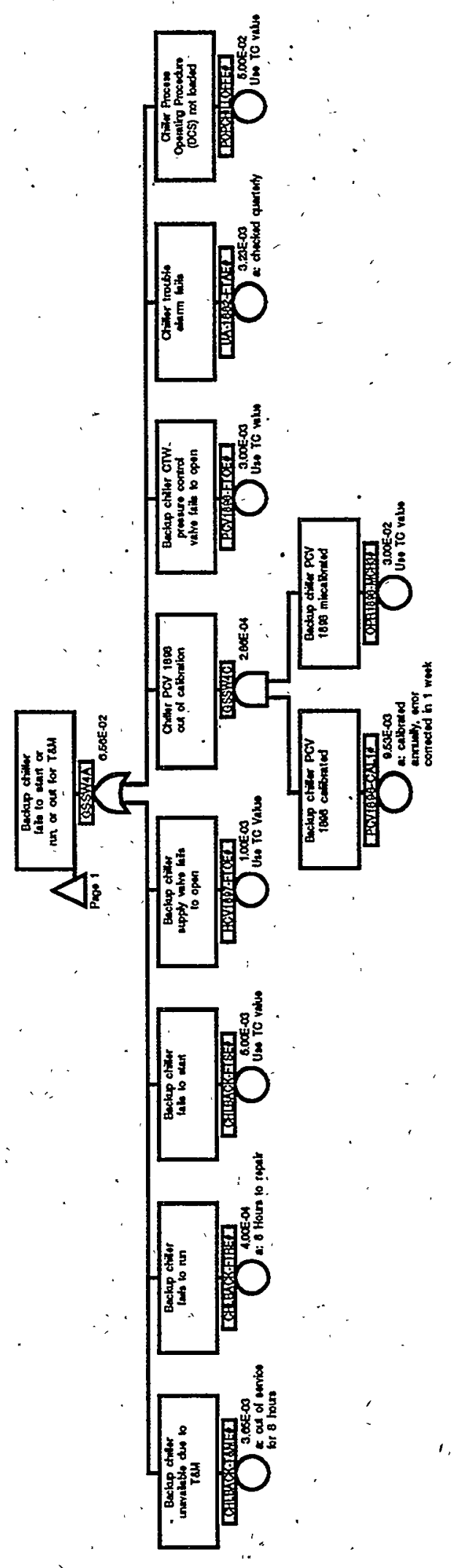




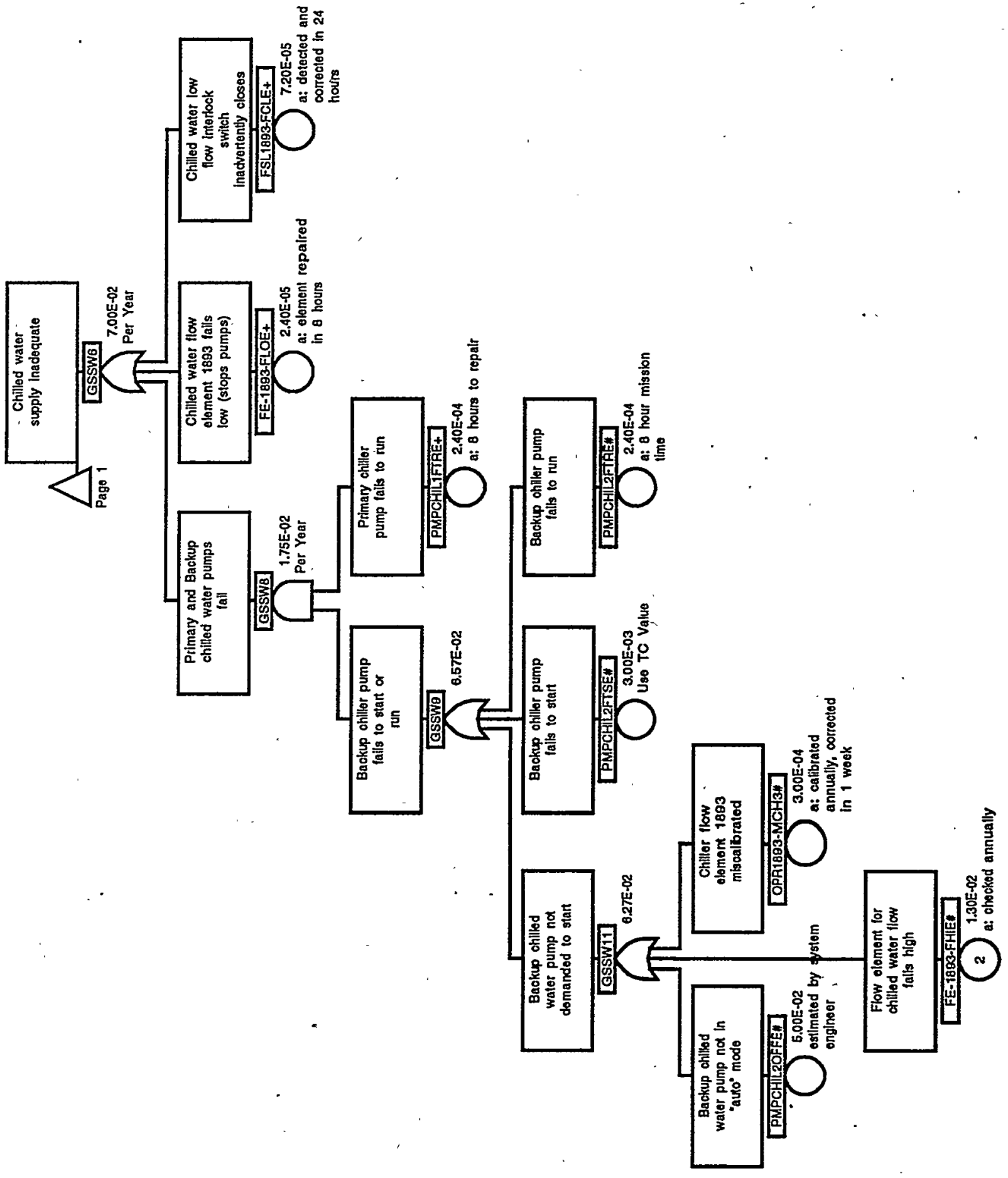




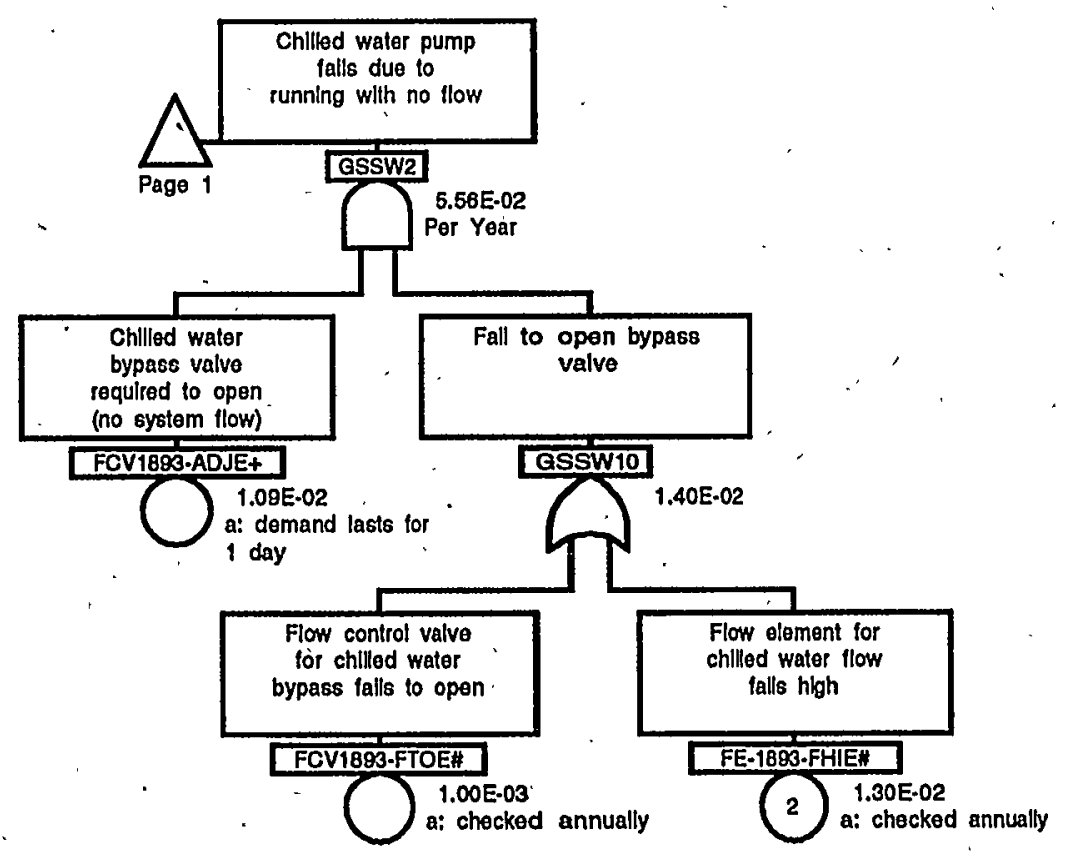


Cutsets for I: ICAFTA ICUT\SSW.CSR

$11 / 08 / 94 \quad 11: 51 \mathrm{AM}$

\begin{tabular}{|c|c|c|c|c|c|c|}
\hline $\begin{array}{l}\text { Set } \\
\text { No. }\end{array}$ & $\begin{array}{l}\text { Event } \\
\text { Name }\end{array}$ & Description & $\mathrm{C}$ & $\begin{array}{l}\text { B. E. } \\
\text { Input }\end{array}$ & $\begin{array}{l}\text { Calc. } \\
\text { Result }\end{array}$ & $\begin{array}{c}\text { Cutset } \\
\text { Freq. }(/ y r)\end{array}$ \\
\hline 1. & $\begin{array}{l}\text { TOP-SSW } \\
\text { FCV1893-ADJE+ } \\
\text { FE-1893-FHIE\# }\end{array}$ & $\begin{array}{l}\text { Chilled water bypass valve required to open (no system flow) } \\
\text { Flow element for chilled water flow fails high }\end{array}$ & 5 & $\begin{array}{r}1 \mathrm{D} \\
-1.0 \mathrm{Q} \\
1 \mathrm{Y} \\
3.0 \mathrm{E}-06 \mathrm{H}\end{array}$ & $\begin{array}{l}1.09 \mathrm{E}-02 \\
1.30 \mathrm{E}-02\end{array}$ & $\begin{array}{l}2.04 \mathrm{E}-01 \\
5.17 \mathrm{E}-02\end{array}$ \\
\hline 2 . & CC2CHILLCHL1+ & cc: both chillers fail & 4 & $5.0 \mathrm{E}-06 \mathrm{H}$ & $4.00 \mathrm{E}-05$ & $4.38 E-02$ \\
\hline 3. & FE-1893-FLOE+ & Chilled water flow element 1893 fails low (stops pumps) & 4 & $3.0 \mathrm{E}-06 \mathrm{H}$ & $2.40 E-05$ & $2.63 \mathrm{E}-02$ \\
\hline 4. & FSL1893-FCLE+ & Chilled water low flow interlock switch inadvertently closes & 4 & $3.0 \mathrm{E}-06 \mathrm{H}$ & $7.20 \mathrm{E}-05$ & $2.63 E-02$ \\
\hline 5. & $\begin{array}{l}\text { CHLPRIM-BFLE+ } \\
\text { POPCHILLOFFE\# }\end{array}$ & $\begin{array}{l}\text { Primary chiller fails } \\
\text { Chiller process operating procedure (DCS) not loaded }\end{array}$ & 4 & $\begin{array}{r}8 \mathrm{H} \\
5.0 \mathrm{E}-05 \mathrm{H} \\
1 \mathrm{~N} \\
5.0 \mathrm{E}-02 \mathrm{~N}\end{array}$ & $\begin{array}{l}4.00 \mathrm{E}-04 \\
5.00 \mathrm{E}-02 \mathrm{~N}\end{array}$ & $2.19 \mathrm{E}-02$ \\
\hline 6. & $\begin{array}{l}\text { PMPCHIL1FTRE+ } \\
\text { PMPCHIL2OFFE\# }\end{array}$ & $\begin{array}{l}\text { Primary chiller pump fails to run } \\
\text { Backup chilled water pump not in "auto" mode }\end{array}$ & 4 & $\begin{array}{r}8 \mathrm{H} \\
3.0 \mathrm{E}-05 \mathrm{H} \\
1 \mathrm{~N} \\
5.0 \mathrm{E}-02 \mathrm{~N}\end{array}$ & $\begin{array}{l}2.40 \mathrm{E}-04 \\
5.00 \mathrm{E}-02 \mathrm{~N}\end{array}$ & $1.31 E-02$ \\
\hline 7. & $\begin{array}{l}\text { FCV1893-ADJE+ } \\
\text { FCV1893-FTOE\# }\end{array}$ & $\begin{array}{l}\text { Chilled water bypass valve required to open (no system flow) } \\
\text { Flow control valve for chilled water bypass fails to open }\end{array}$ & 4 & $\begin{array}{r}1 \mathrm{D} \\
1.0 Q \\
1 \mathrm{~N} \\
1.0 \mathrm{E}-03 \mathrm{~N}\end{array}$ & $\begin{array}{l}1.09 \mathrm{E}-02 \\
1.00 \mathrm{E}-03 \mathrm{~N}\end{array}$ & $3.97 E-03$ \\
\hline 8. & $\begin{array}{l}\text { FE-1893-FHIE\# } \\
\text { PMPCHIL1FTRE+ }\end{array}$ & $\begin{array}{l}\text { Flow element for chilled water flow fails high } \\
\text { Primary chiller pump fails to run }\end{array}$ & 5 & $\begin{array}{r}1 \mathrm{Y} \\
3.0 \mathrm{E}-06 \mathrm{H} \\
8 \mathrm{H} \\
3.0 \mathrm{E}-05 \mathrm{H}\end{array}$ & $\begin{array}{l}1.30 \mathrm{E}-02 \\
2.40 \mathrm{E}-04\end{array}$ & $3.42 \mathrm{E}-03$ \\
\hline 9. & $\begin{array}{l}\text { OPR1895-RANK+ } \\
\text { POPCHILLOFFE\# }\end{array}$ & $\begin{array}{l}\text { Primary chiller PCV } 1895 \text { inadvertently closed } \\
\text { Chiller process operating procedure (DCS) not loaded }\end{array}$ & 4 & $\begin{array}{r}1 \mathrm{H} \\
5.0 \mathrm{E}-06 \mathrm{H} \\
1 \mathrm{~N} \\
5.0 \mathrm{E}-02 \mathrm{~N}\end{array}$ & $\begin{array}{l}5.00 \mathrm{E}-06 \\
5.00 \mathrm{E}-02 \mathrm{~N}\end{array}$ & $2.19 \mathrm{E}-03$ \\
\hline 10. & $\begin{array}{l}\text { CHLBACK-FTSE\# } \\
\text { CHLPRIM-BFLE+ }\end{array}$ & $\begin{array}{l}\text { Backup chiller fails to start } \\
\text { Primary chiller fails }\end{array}$ & 1 & $\begin{array}{r}1 \mathrm{~N} \\
5.0 \mathrm{E}-03 \mathrm{~N} \\
8 \mathrm{H} \\
5.0 \mathrm{E}-05 \mathrm{H}\end{array}$ & $\begin{array}{l}5.00 \mathrm{E}-03 \mathrm{~N} \\
4.00 \mathrm{E}-04\end{array}$ & $2.19 \mathrm{E}-03$ \\
\hline 11. & CHLBACK-T\&ME\# & Backup chiller unavailable due to T\&M & 3 & $\begin{array}{r}8 \mathrm{8H} \\
4.0 \mathrm{E}+00 \mathrm{Y}\end{array}$ & $3.65 \mathrm{E}-03$ & $1.60 \mathrm{E}-03$ \\
\hline
\end{tabular}


Cutsets for I: \CAFTA ICUT\SSW.CSR

11/08/94 11:51 AM (CONT.)

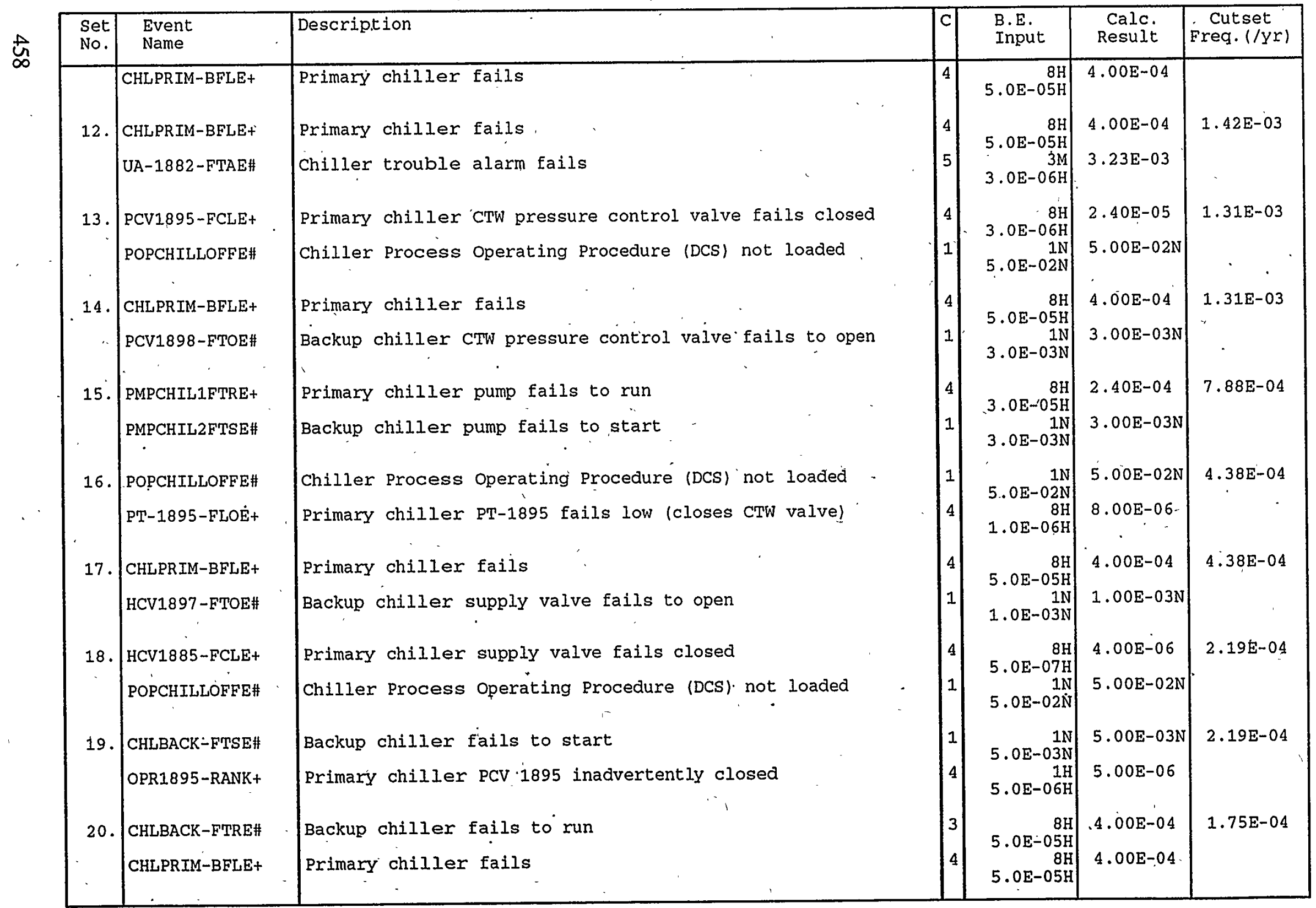


Cutsets for I: ICAFTAICUTISSW.CSR

11/08/94 11:51 AM (CONT.)

\begin{tabular}{|c|c|c|c|c|c|c|}
\hline $\begin{array}{l}\text { Set } \\
\text { No. }\end{array}$ & $\begin{array}{l}\text { Event } \\
\text { Name }\end{array}$ & Description & c & $\begin{array}{l}\text { B.E. } \\
\text { Input }\end{array}$ & $\begin{array}{l}\text { Calc. } \\
\text { Result }\end{array}$ & $\begin{array}{c}\text { Cutset } \\
\text { Freq. }(/ y r)\end{array}$ \\
\hline 21 & $\begin{array}{l}\text { CHLBACK-T\&ME\# } \\
\text { OPR1895-RANK+ }\end{array}$ & $\begin{array}{l}\text { Backup chiller unavailable due to T\&M } \\
\text { Primary chiller PCV } 1895 \text { inadvertently closed }\end{array}$ & $\begin{array}{l}3 \\
4\end{array}$ & $\begin{array}{r}8 \mathrm{H} \\
4.0 \mathrm{E}+00 \mathrm{Y} \\
1 \mathrm{H} \\
5.0 \mathrm{E}-06 \mathrm{H}\end{array}$ & $\begin{array}{l}3.65 \mathrm{E}-03 \\
5.00 \mathrm{E}-06\end{array}$ & 1. $60 \mathrm{E}-04$ \\
\hline 22 . & $\begin{array}{l}\text { OPR1895-RANK+ } \\
\text { UA-1882-FTAE\# }\end{array}$ & $\begin{array}{l}\text { Primary chiller PCV } 1895 \text { inadvertently closed } \\
\text { Chiller trouble alarm fails }\end{array}$ & 4 & $\begin{array}{r}1 \mathrm{H} \\
5.0 \mathrm{E}-06 \mathrm{H} \\
3 \mathrm{M} \\
3.0 \mathrm{E}-06 \mathrm{H}\end{array}$ & $\begin{array}{l}5.00 \mathrm{E}-06 \\
3.23 \mathrm{E}-03\end{array}$ & $1.42 \mathrm{E}-04$ \\
\hline 23. & $\begin{array}{l}\text { OPR1895-RANK+ } \\
\text { PCV1898-FTOE\# }\end{array}$ & $\begin{array}{l}\text { Primary chiller PCV } 1895 \text { inadvertently closed } \\
\text { Backup chiller CIW pressure control valve fails to open }\end{array}$ & $\begin{array}{l}4 \\
1\end{array}$ & $\begin{array}{r}1 \mathrm{H} \\
5.0 \mathrm{E}-06 \mathrm{H} \\
1 \mathrm{~N} \\
3.0 \mathrm{E}-03 \mathrm{~N}\end{array}$ & $\begin{array}{l}5.00 \mathrm{E}-06 \\
3.00 \mathrm{E}-03 \mathrm{~N}\end{array}$ & $1.31 E-04$ \\
\hline 24. & $\begin{array}{l}\text { CHLBACK-FTSE\# } \\
\text { PCV'1895-FCLE+ }\end{array}$ & $\begin{array}{l}\text { Backup chiller fails to start } \\
\text { Primary chiller CTW pressure control valve fails closed }\end{array}$ & 1 & $\begin{array}{r}1 \mathrm{~N} \\
5.0 \mathrm{E}-03 \mathrm{~N} \\
8 \mathrm{H} \\
3.0 \mathrm{E}-06 \mathrm{H}\end{array}$ & $\begin{array}{l}5.00 \mathrm{E}-03 \mathrm{~N} \\
2.40 \mathrm{E}-05\end{array}$ & $1.31 \mathrm{E}-04$ \\
\hline 25 . & $\begin{array}{l}\text { CHLPRIM-BFLE+ } \\
\text { OPR1898-MCH3\# } \\
\text { PCV1898-CAL1\# }\end{array}$ & $\begin{array}{l}\text { Primary chiller fails } \\
\text { Backup chiller PCV } 1898 \text { miscalibrated } \\
\text { Backup chiller PCV } 1898 \text { calibrated }\end{array}$ & $\mid \begin{array}{l}4 \\
1 \\
5\end{array}$ & $\begin{array}{r}8 \mathrm{H} \\
5.0 \mathrm{E}-05 \mathrm{H} \\
1 \mathrm{~N} \\
3.0 \mathrm{E}-02 \mathrm{~N} \\
7 \mathrm{D} \\
1.0 \mathrm{E}+00 \mathrm{Y}\end{array}$ & $\begin{array}{l}4.00 \mathrm{E}-04 \\
3.00 \mathrm{E}-02 \mathrm{~N} \\
9.53 \mathrm{E}-03\end{array}$ & 1. $25 \mathrm{E}-04$ \\
\hline
\end{tabular}


Chilled water supply inadequate (excl. CTW, INAIR, or POWD)

Top Event Frequency: 2.04E-01/YR'

\begin{tabular}{|c|c|c|}
\hline Basic Event Name & Description & Prob/Freg \\
\hline $\mathrm{CC} 2 \mathrm{CHILCHL1+- \textrm {I }}$ & cc: both chillers fail & $5.00 \mathrm{E}-06 / \mathrm{H}$ \\
\hline FE-1893-FLOE+I & Chilled water flow element 1893 fails low (stops pumps) & $06 / \mathrm{H}$ \\
\hline FSL1893-FCLE+I & Chilled water low flow interlock. switch inadvertently closes & $3.00 \mathrm{E}-06 / \mathrm{H}$ \\
\hline PMPCHIL1FTRE+-I & Primary chiller pump fails to run & $3.00 \mathrm{E}-05 / \mathrm{H}$ \\
\hline HCV1885-FCLE+-I & Primary chiller supply valve fails closed & $5.00 \mathrm{E}-07 / \mathrm{H}$ \\
\hline$-F L O E+I$ & Primary chiller PT-1895 fails low (closes CTW valve) & $-06 / \mathrm{H}$ \\
\hline .PCV1895-FCLE+-I & 'Primary chiller CTW pressure control valve fails closed & $-06 / \mathrm{H}$ \\
\hline OPR1895-RANK+I & Primary chiller PCV 1895 inadvertently closed & $5.00 \mathrm{E}-06 / \mathrm{H}$ \\
\hline CHLPRIM-BFLE+I & Primary chiller fails & $5.00 \mathrm{E}-05 / \mathrm{H}$ \\
\hline E+-I & Chilled w & $4 / \mathrm{H}$ \\
\hline FE- & chilled water flow fails high & \\
\hline FCT & Lve for chilled water bypass fails to open & \\
\hline $\mathrm{CH}$ & Backup chi & \\
\hline $\mathrm{HCr}$ & r supply valve fails to open. & 1.0 \\
\hline PC & CTW pressure control valve fails to open & \\
\hline UA & Chiller trouble alarm fails & \\
\hline CME\# & available due to $T \& M$ & \\
\hline CHLE & Báckup chil & 5.0 \\
\hline OFFE\# & s Operating Procedure (DCS) not loaded & \\
\hline RE\# & pump fails to run & -04 \\
\hline ICH3\# & ent 1893 miscalibrated & \\
\hline [SE\# & Backup chiller & 3.00 \\
\hline FE\# & not in "auto" mode & 5.001 \\
\hline & 'CV 1898 calibrated & 9.531 \\
\hline & Backup chiller PCV 1898 miscalibrated & \\
\hline
\end{tabular}

AchW

$4.29 \mathrm{E}+04$

$4.29 \mathrm{E}+04$

$4.29 \mathrm{E}+04$

$2.82 \mathrm{E}+03$

$2.82 \mathrm{E}+03$

$2.82 \mathrm{E}+03$

$2.82 \mathrm{E}+03$

$2.82 \mathrm{E}+03$

$2.82 \mathrm{E}+03$

$6.02 \mathrm{E}+02$

$2.14 \mathrm{E}+01$

$2.04 \mathrm{E}+01$

$3.55 \mathrm{E}+00$

$3.55 \mathrm{E}+00$

$3.54 \mathrm{E}+00$

$3.54 \mathrm{E}+00$

$3.54 \mathrm{E}+00$

$3.54 \mathrm{E}+00$

$3.42 \mathrm{E}+00$

$2.29 \mathrm{E}+00$

$2.29 \mathrm{E}+00$

$2.28 \mathrm{E}+00$

$2.22 \mathrm{E}+00$

$1.08 \mathrm{E}+00$

$1.02 \mathrm{E}+00$

Modified top event frequency $=$ (top event frequency) $\mathrm{x}$ (risk achievement worth).

\section{Basic Event Name FCV1893-ADJE+-I FE-1893-FHIE\# CC2CHILLCHII+I CHLPRIM-BFLE+-I FE-1893-FLOE+-I FSL1893-FCLE+-I POPCHILIOFFE\# PMPCHIL1FTRE+-I PMPCHII 2OFFE\# FCV1893-FTOE\# OPR1895-RANK+-I CHLBACK-FTSE\#}

\section{Risk Reduction Worth}

\section{Description}

Chilled water bypass valve required to open

Flow element for chilled water flow fails high

cc: both chillers fail

Primary chiller fails

Chilled water flow element 1893 fails low (stops pumps)

Chilled water low flow interlock switch inadvertently closes

Chiller Process Operating Procedure (DCS) not loaded

Primary chiller pump fails to run

Backup chilled water pump not in "auto" mode

Flow control valve for chilled water bypass fails to open

'Primary chiller PCV 1895 inadvértently closed

Backup.chiller fails to start

\begin{tabular}{|c|c|}
\hline reo & $\operatorname{edW}$ \\
\hline $4.53 \mathrm{E}-04 / \mathrm{H}$ & $1.37 \mathrm{E}_{t}$ \\
\hline $1.30 \mathrm{E}-0$ & 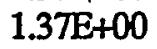 \\
\hline $5.00 \mathrm{E}-06 / \mathrm{H}$ & $1.27 \mathrm{E}$ \\
\hline $5.00 \mathrm{E}-05 / \mathrm{H}$ & $1.17 \mathrm{E}$ \\
\hline $3.00 \mathrm{E}-06 / \mathrm{H}$ & 1. \\
\hline $3.00 \mathrm{E}-06 / \mathrm{H}$ & $1.15 \mathrm{E}+$ \\
\hline $5.00 \mathrm{E}-02$ & $1.15 \mathrm{E}$ \\
\hline $3.00 \mathrm{E}-05 / \mathrm{H}$ & $1.09 \mathrm{E}$ \\
\hline $5.00 \mathrm{E}-$ & $1.07 \pi$ \\
\hline $1.00 \mathrm{E}-03$ & \\
\hline $5.00 \mathrm{E}-06 / \mathrm{H}$ & 1 \\
\hline $5.00 \mathrm{E}$ & \\
\hline
\end{tabular}

Note: Events that make less than $1 \%$ difference to the importance factor are not included. Modified top event frequency $=$ (top event frequency) $/$ (risk reduction worth). 
Process Cooling Water System Unavailable 


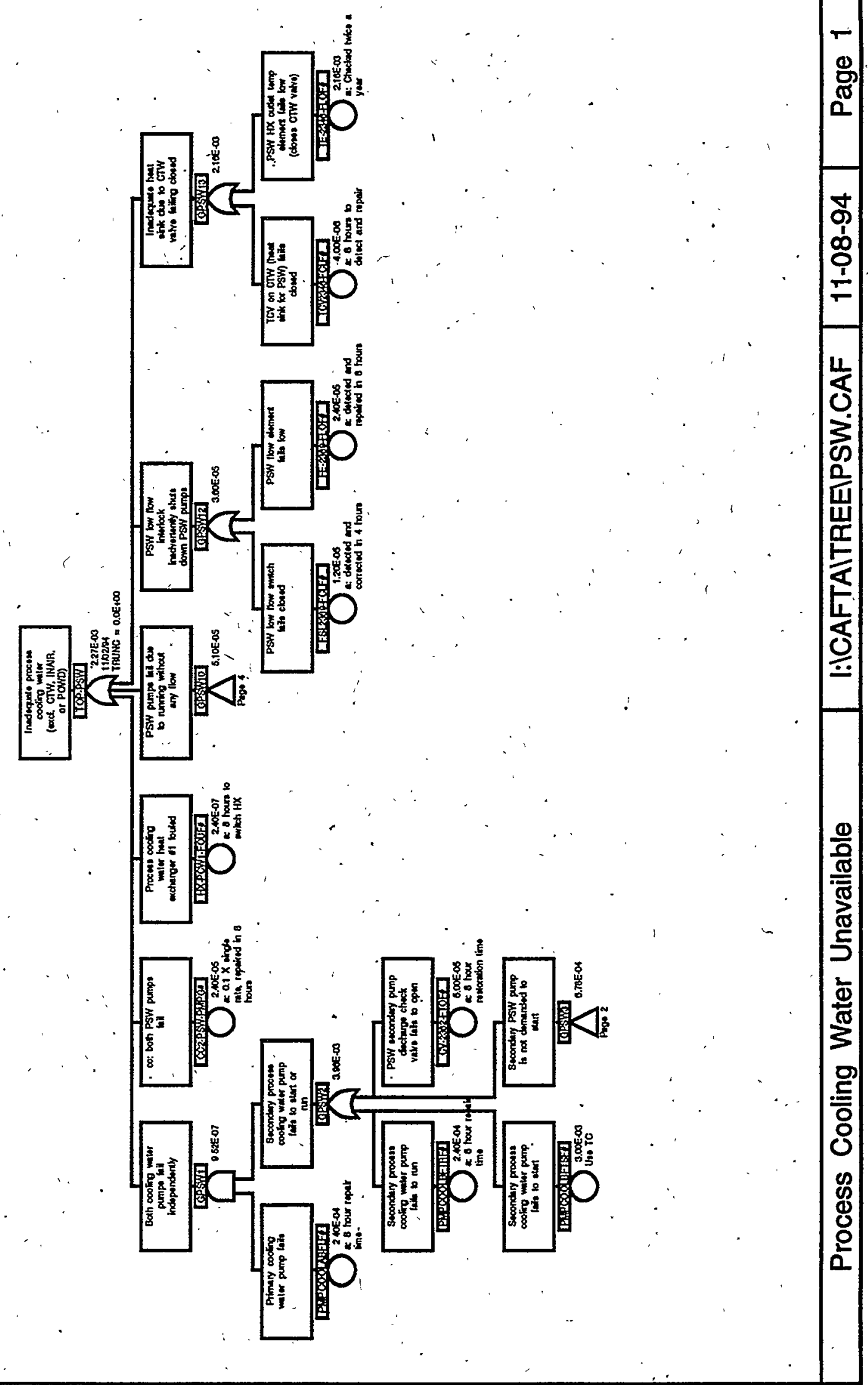




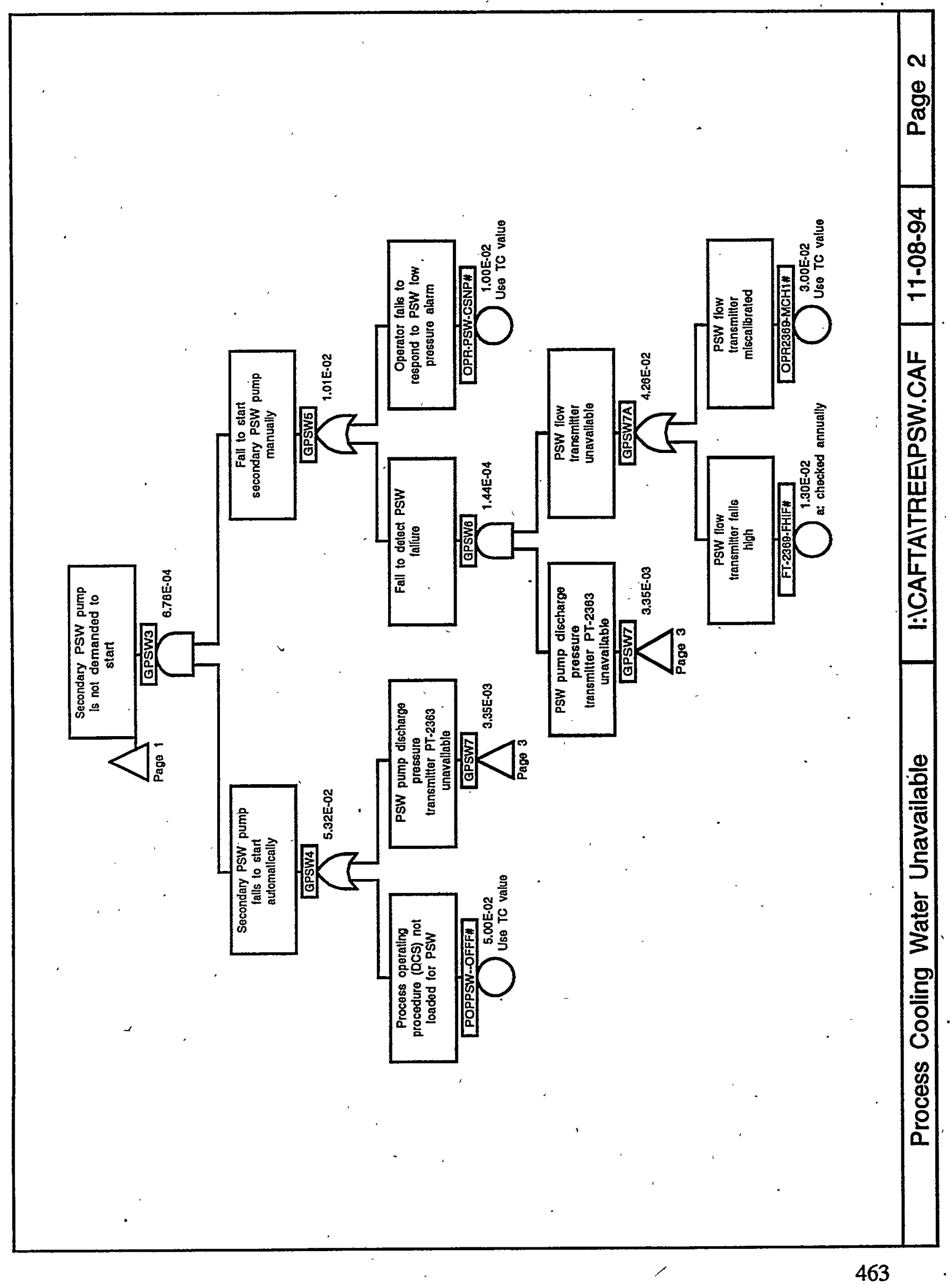




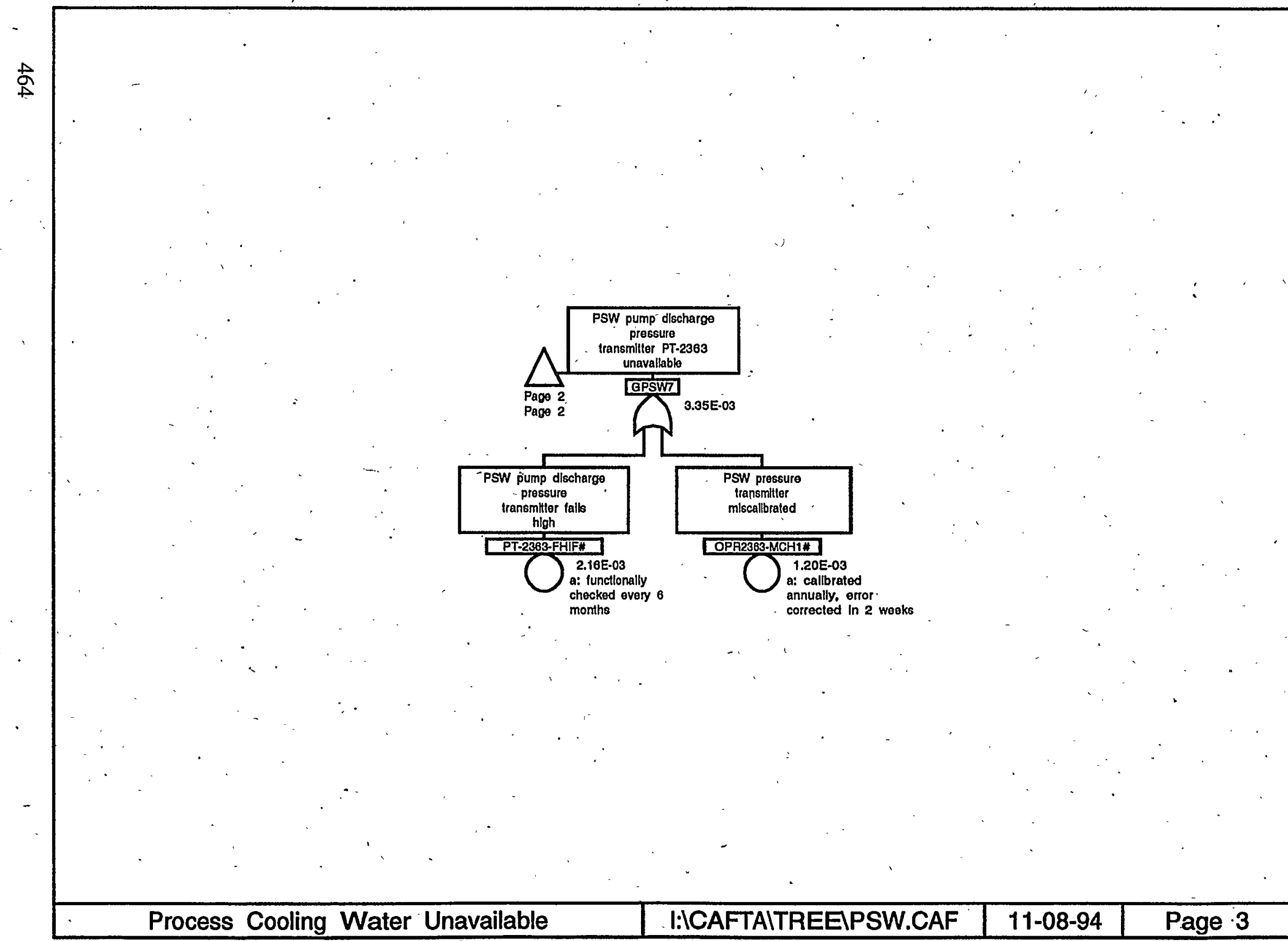


X-ESR-S-00001, Rev. 0

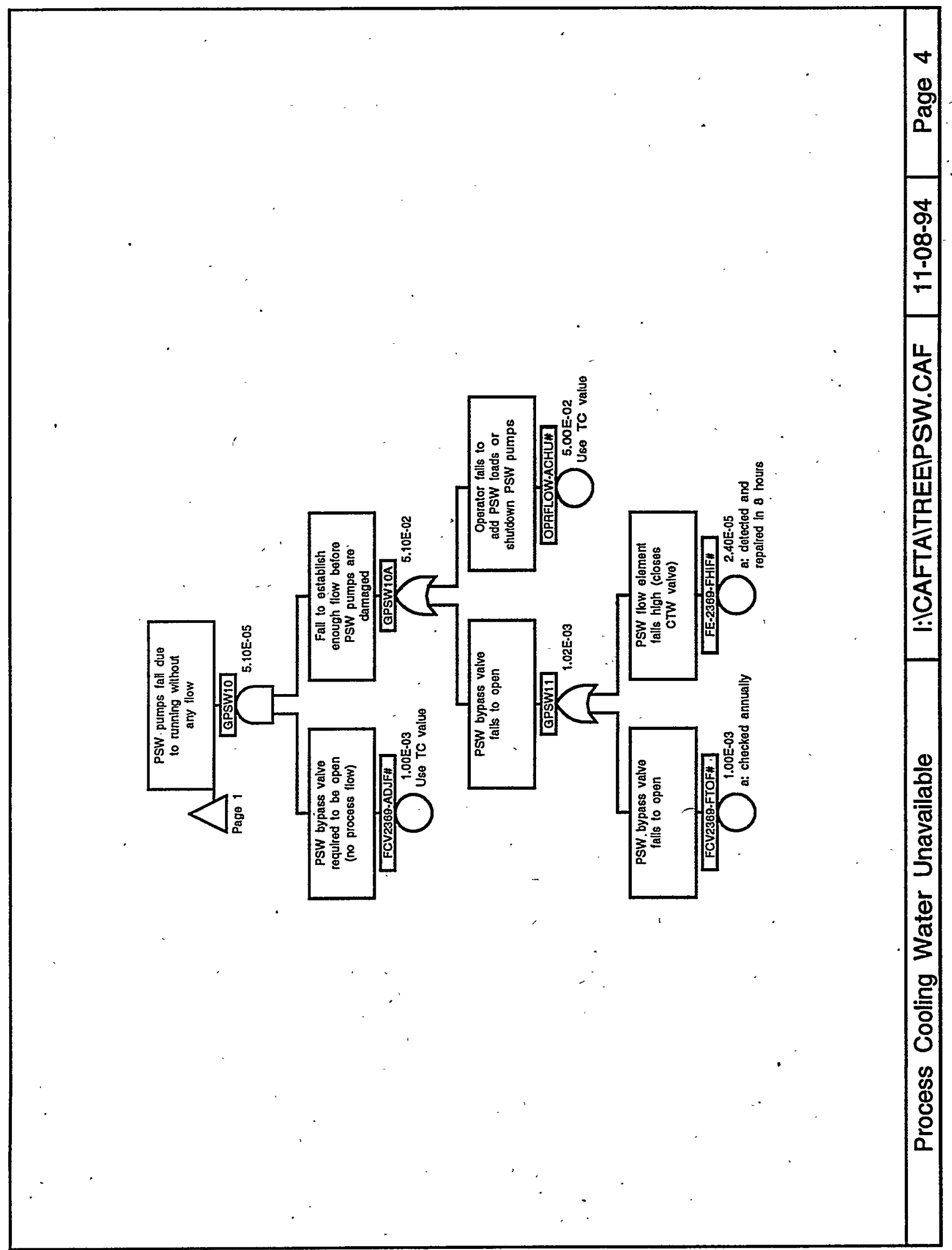




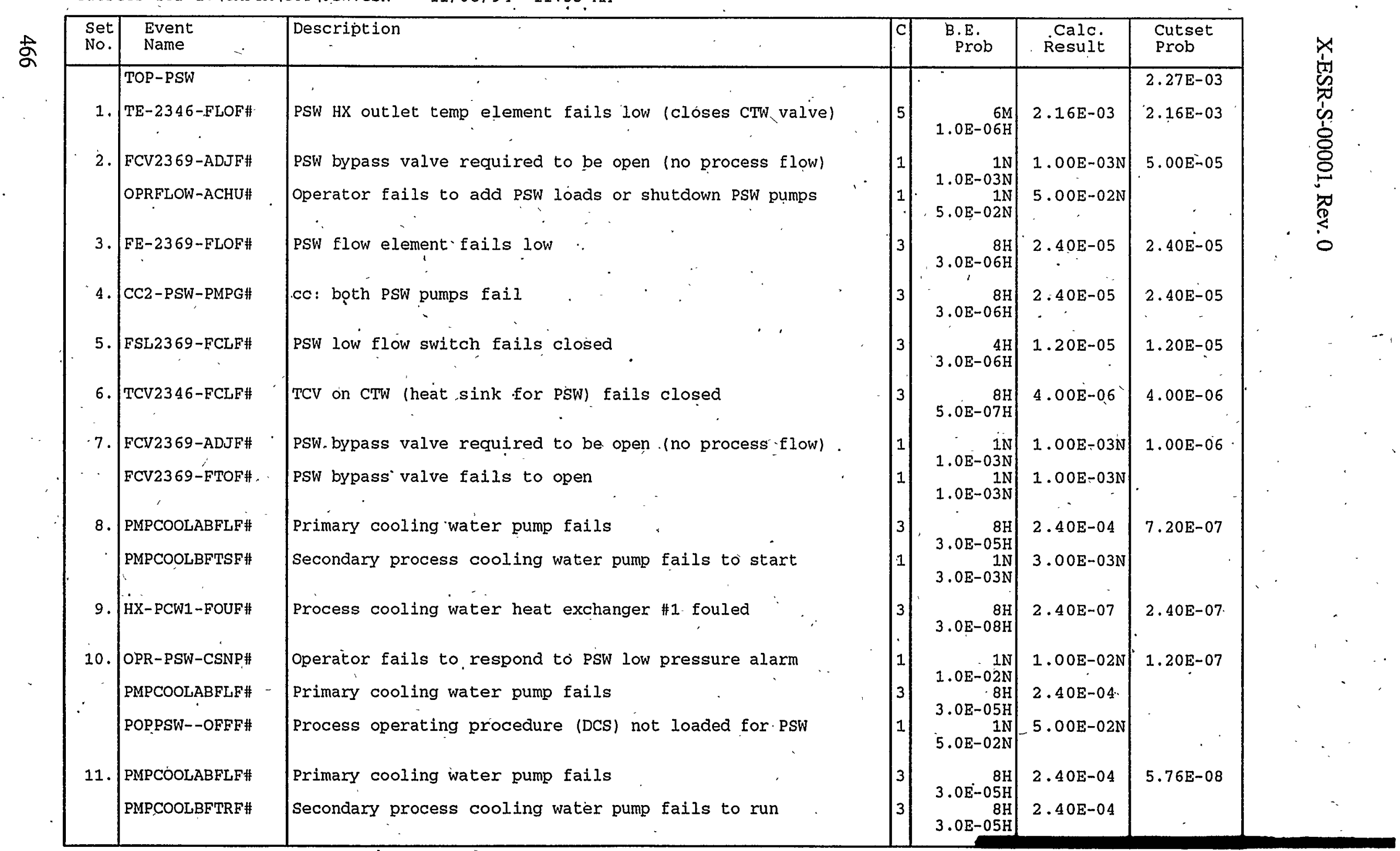


Cutsets for I: ICAFTA \CUT \PSW.CSR

11/08/94 11:53 AM (CONT.)

\begin{tabular}{|c|c|c|c|c|c|c|}
\hline $\begin{array}{l}\text { Set } \\
\text { No. }\end{array}$ & $\begin{array}{l}\text { Event } \\
\text { Name }\end{array}$ & Description & C & $\begin{array}{l}\text { B.E. } \\
\text { Prob }\end{array}$ & $\begin{array}{l}\text { Calc. } \\
\text { Result }\end{array}$ & $\begin{array}{l}\text { Cutset } \\
\text { Prob }\end{array}$ \\
\hline 12. & $\begin{array}{l}\text { FCV2369-ADJF\# } \\
\text { FE-2369-FHIF\# }\end{array}$ & $\begin{array}{l}\text { PSW bypass valve required to be open (no process flow) } \\
\text { PSW flow element fails high (closes CTW valve) }\end{array}$ & 1 & $\begin{array}{r}1 \mathrm{~N} \\
1.0 \mathrm{E}-03 \mathrm{~N} \\
8 \mathrm{H} \\
3.0 \mathrm{E}-06 \mathrm{H}\end{array}$ & $\begin{array}{l}1.00 \mathrm{E}-03 \mathrm{~N} \\
2.40 \mathrm{E}-05\end{array}$ & $2.40 E-08$ \\
\hline 13. & $\begin{array}{l}\text { OPR2369-MCH1\# } \\
\text { PMPCOOLABFLF\# } \\
\text { PT-2363-FHIF\# }\end{array}$ & $\begin{array}{l}\text { PSW flow transmitter miscalibrated } \\
\text { Primary cooling water pump fails } \\
\text { PSW pump discharge pressure transmitter fails high }\end{array}$ & $\begin{array}{l}1 \\
3 \\
5\end{array}$ & $\begin{array}{r}1 \mathrm{~N} \\
3.0 \mathrm{E}-02 \mathrm{~N} \\
8 \mathrm{H} \\
3.0 \mathrm{E}-05 \mathrm{H} \\
6 \mathrm{M} \\
1.0 \mathrm{E}-06 \mathrm{H}\end{array}$ & $\begin{array}{l}3.00 \mathrm{E}-02 \mathrm{~N} \\
2.40 \mathrm{E}-04 \\
2.16 \mathrm{E}-03\end{array}$ & 1. $55 \mathrm{E}-08$ \\
\hline 14. & $\begin{array}{l}\text { CV-2362-FTOF\# } \\
\text { PMPCOOLABFLF\# }\end{array}$ & $\begin{array}{l}\text { PSW secondary pump discharge check valve fails to open } \\
\text { Primary cooling water pump fails }\end{array}$ & $\begin{array}{l}1 \\
3\end{array}$ & $\begin{array}{r}1 \mathrm{~N} \\
5.0 \mathrm{E}-05 \mathrm{~N} \\
8 \mathrm{H} \\
3.0 \mathrm{E}-05 \mathrm{H}\end{array}$ & $\begin{array}{l}5.00 \mathrm{E}-05 \mathrm{~N} \\
2.40 \mathrm{E}-04\end{array}$ & $1.20 E-08$ \\
\hline 15. & $\begin{array}{l}\text { OPR2363-MCH1\# } \\
\text { OPR2369-MCH1\# } \\
\text { PMPCOOLABELF\# }\end{array}$ & $\begin{array}{l}\text { PSW pressure transmitter miscalibrated } \\
\text { PSW flow transmitter miscalibrated } \\
\text { Primary cooling water pump fails }\end{array}$ & $\begin{array}{l}1 \\
1 \\
3\end{array}$ & $\begin{array}{r}.04 \mathrm{~N} \\
3.0 \mathrm{E}-02 \mathrm{~N} \\
1 \mathrm{~N} \\
3.0 \mathrm{E}-02 \mathrm{~N} \\
8 \mathrm{H} \\
3.0 \mathrm{E}-05 \mathrm{H}\end{array}$ & $\begin{array}{l}1.20 \mathrm{E}-03 \mathrm{~N} \\
3.00 \mathrm{E}-02 \mathrm{~N} \\
2.40 \mathrm{E}-04\end{array}$ & $8.64 E-09$ \\
\hline 16. & $\begin{array}{l}\text { FT-2369-FHIF\# } \\
\text { PMPCOOLABFLF\# } \\
\text { PT-2363-FHIF\# }\end{array}$ & $\begin{array}{l}\text { PSW flow transmitter fails high } \\
\text { Primary cooling water pump fails } \\
\text { PSW pump discharge pressure transmitter fails high }\end{array}$ & 5 & $\begin{array}{r}1 \mathrm{Y} \\
3.0 \mathrm{E}-06 \mathrm{H} \\
8 \mathrm{H} \\
3,0 \mathrm{E}-05 \mathrm{H} \\
6 \mathrm{M} \\
1.0 \mathrm{E}-06 \mathrm{H}\end{array}$ & $\begin{array}{l}1.30 \mathrm{E}-02 \\
2.40 \mathrm{E}-04 \\
2.16 \mathrm{E}-03\end{array}$ & $6.74 \mathrm{E}-09$ \\
\hline 17. & $\begin{array}{l}\text { OPR-PSW-CSNP\# } \\
\text { PMPCOOLABFLF\# } \\
\text { PT-2363-FHIF\# }\end{array}$ & $\begin{array}{l}\text { Operator fails to respond to PSW low pressure alarm } \\
\text { Primary cooling water pump fails } \\
\text { PSW pump discharge pressure transmitter fails high }\end{array}$ & $\begin{array}{l}1 \\
3 \\
5\end{array} \mid$ & $\begin{array}{r}1 \mathrm{~N} \\
1.0 \mathrm{E}-02 \mathrm{~N} \\
8 \mathrm{H} \\
3.0 \mathrm{E}-05 \mathrm{H} \\
6 \mathrm{M} \\
1.0 \mathrm{E}-06 \mathrm{H}\end{array}$ & $\begin{array}{l}1.00 \mathrm{E}-02 \mathrm{~N} \\
2.40 \mathrm{E}-04 \\
2.16 \mathrm{E}-03\end{array}$ & $5.18 \mathrm{E}-09$ \\
\hline 18 . & $\begin{array}{l}\text { FT-2369-FHIF\# } \\
\text { OPR2363-MCH1\# } \\
\text { PMPCOOLABFLF\# }\end{array}$ & $\begin{array}{l}\text { PSW flow transmitter fails high } \\
\text { PSW pressure transmitter miscalibrated } \\
\text { Primary cooling water pump fails }\end{array}$ & $\begin{array}{l}5 \\
1 \\
3\end{array}$ & $\begin{array}{r}1 \mathrm{Y} \\
3.0 \mathrm{E}-06 \mathrm{H} \\
.04 \mathrm{~N} \\
3.0 \mathrm{E}-02 \mathrm{~N} \\
8 \mathrm{H} \\
3.0 \mathrm{E}-05 \mathrm{H}\end{array}$ & $\begin{array}{l}1.30 \mathrm{E}-02 \\
1.20 \mathrm{E}-03 \mathrm{~N} \\
2.40 \mathrm{E}-04\end{array}$ & $3.75 E-09$ \\
\hline 19. & OPR-PSW-CSNP\# & Operator fails to respond to PSW low pressure alarm & 1 & $1.0 \mathrm{E}-02 \mathrm{~N}$ & $1.00 \mathrm{E}-02 \mathrm{~N}$ & $2.88 E-09$ \\
\hline
\end{tabular}


Cutsets for I: \CAFTA \CUT\PSW.CSR

11/08/94 11:53 AM (CONT.)

\begin{tabular}{|c|c|c|c|c|c|c|c|}
\hline$\vec{a}$ & $\begin{array}{l}\text { Set } \\
\text { No. }\end{array}$ & $\begin{array}{l}\text { Event } \\
\text { Name }\end{array}$ & Description & $\mathrm{C}$ & $\begin{array}{l}\text { B.E. } \\
\text { Prob }\end{array}$ & $\begin{array}{l}\text { Calic. } \\
\text { Result }\end{array}$ & $\begin{array}{l}\text { Cutset } \\
\text { Prob }\end{array}$ \\
\hline & & $\begin{array}{l}\text { OPR2363-MCH1\# } \\
\text { PMPCOOLABFLE\# }\end{array}$ & $\begin{array}{l}\text { PSW pressure transmitter miscalibrated } \\
\text { Primary cooling water pump fails }\end{array}$ & $\begin{array}{l}1 \\
3\end{array}$ & $\begin{array}{r}.04 \mathrm{~N} \\
3.0 \mathrm{E}-02 \mathrm{~N} \\
8 \mathrm{H} \\
3.0 \mathrm{E}-05 \mathrm{H}\end{array}$ & $\begin{array}{l}1.20 \mathrm{E}-03 \mathrm{~N} \\
2.40 \mathrm{E}-04\end{array}$ & . \\
\hline
\end{tabular}


Inadequate process cooling water (excl. CTW, INAIR, or POWD)

Top Event Probability: 2.27E-03

Basic Event Name
FSL2369-FCLF\#
CC2-PSW-PMPG\#
HX-PCW1-FOUF\#
TCV2346-FCLF\#
TE-2346-FLOF\#
FE-2369-FLOF\#
FCV2369-ADJF\#
PMPCOOLABFLF\#
FE-2369-FHIF\#
FCV2369-FTOF\#
OPRFLOW-ACHU\#
CV-2362-FTOF\#
PMPCOOLBFTRF
PMPCOOLBFTSF\#

\section{Risk Achievement Worth}

\section{Description}

PSW low flow switch fails closed cc: both PSW pumps fail

Process cooling water heat exchanger \#1 fouled

TCV on CTW (heat sink for PSW) fails closed

PSW HX outlet temp element fails low (closes CTW valve)

PSW flow element fails low

PSW bypass valve required to be open (no process flow)

Primary cooling water pump fails

PSW flow element fails high (closes CTW valve)

PSW bypass valve fails to open

Operator fails to add PSW loads or shutdown PSW pumps

PSW secondary pump discharge check valve fails to open

Secondary process cooling water pump fails to run

Secondary process cooling water pump fails to start
Prob/Freq AchW

1.20E-05 4.40E+02

2.40E-05 4.40E+02

$2.40 \mathrm{E}-07 \quad 4.40 \mathrm{E}+02$

$4.00 \mathrm{E}-06 \quad 4.40 \mathrm{E}+02$

$2.1 \mathrm{E}-03 \quad 4.40 \mathrm{E}+02$

$2.40 \mathrm{E}-05 \quad 4.40 \mathrm{E}+02$

$1.00 \mathrm{E}-03 \quad 2.34 \mathrm{E}+01$

2.40E-04 2.74E+00

$2.40 \mathrm{E}-05 \quad 1.44 \mathrm{E}+00$

$1.00 \mathrm{E}-03 \quad 1.44 \mathrm{E}+00$

$5.00 \mathrm{E}-02 \quad 1.42 \mathrm{E}+00$

$5.00 \mathrm{E}-05 \quad 1.11 \mathrm{E}+00$

2.40E-04. 1.11E+00

3.00E-03 1.11E+00

Modified top event frequency $=$ (top event frequency) $\mathrm{x}$ (risk achievement worth).

\section{Basic Event Name TE-2346-FLOF\# FCV2369-ADJF\# OPRFLOW-ACHU\# CC2-PSW-PMPG\# FE-2369-FLOF\#}

\section{Risk Reduction Worth}

\section{Description}

PSW HX outlet temp element fails low (closes CTW valve) PSW bypass valve required to be open (no process flow) Operator fails to add PSW loads or shutdown PSW pumps cc: both PSW pumps fail PSW flow element fails low

\begin{tabular}{ll} 
Prob/Freq & RedW \\
\hline $2.16 \mathrm{E}-03$ & $1.96 \mathrm{E}+01$ \\
$1.00 \mathrm{E}-03$ & $1.02 \mathrm{E}+00$ \\
$5.00 \mathrm{E}-02$ & $1.02 \mathrm{E}+00$ \\
$2.40 \mathrm{E}-05$ & $1.01 \mathrm{E}+00$ \\
$2.40 \mathrm{E}-05$ & $1.01 \mathrm{E}+00$
\end{tabular}

Note: Events that make less than $1 \%$ difference to the importance factor are not included. Modified top event frequency = (top event frequency) $/$ (risk reduction worth). 
X-ESR-S-001001, Rev. 0

Cooling Tower Water System Unavailable - common equipment 


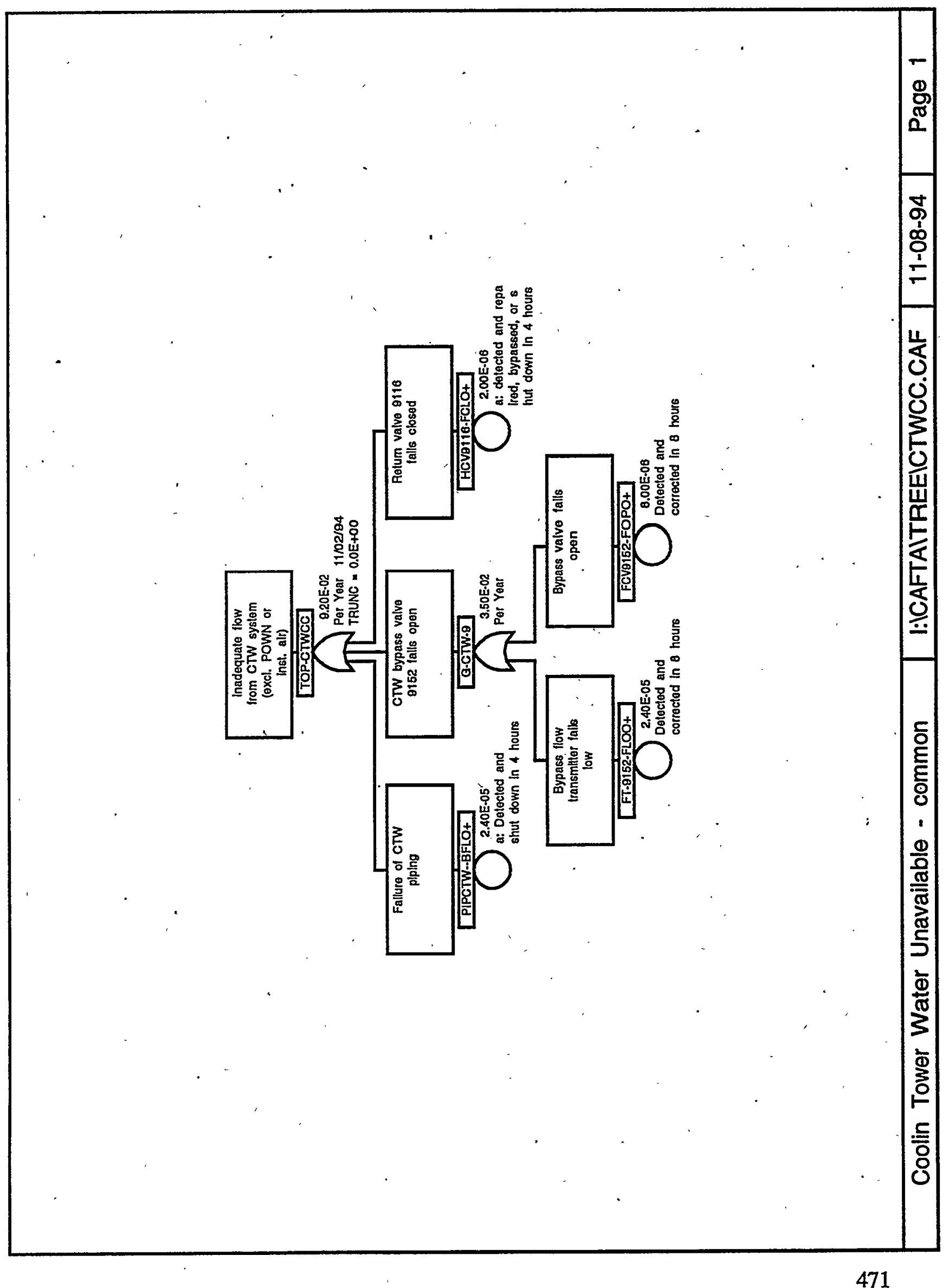


X-ESR-S-00001, Rev. 0

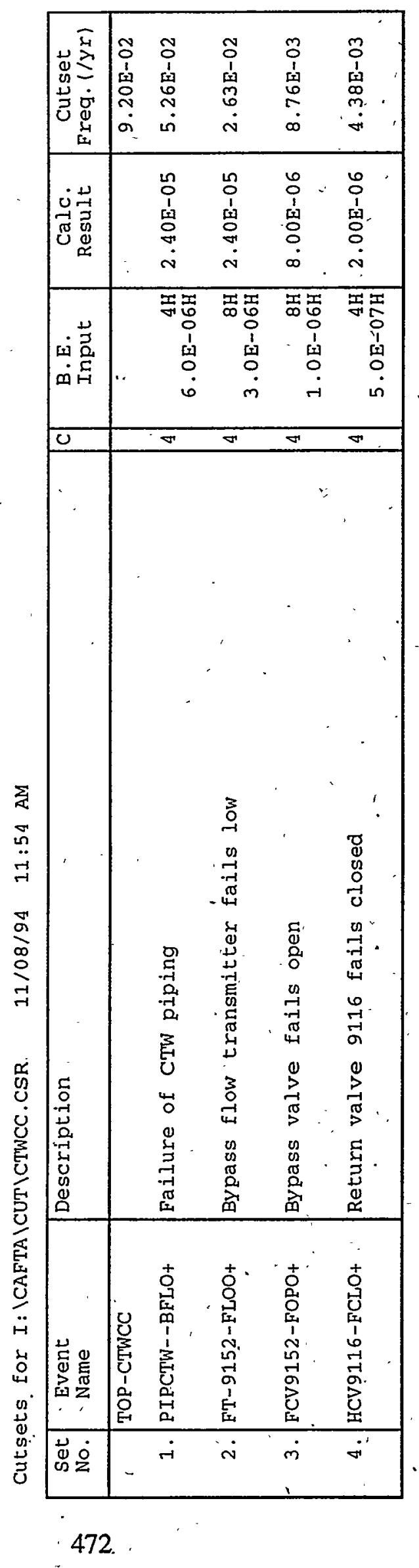


Inadequate flow from CTW system (excl. POWN or inst. air) Top Event Frequency: 9.20E-02/YR

\section{Risk Achievement Worth}

Basic Event Name

FCV9152-FOPO+-I HCV9116-FCLO+I PIPCTW-BFLO+-I FT-9152-FLOO+-I Bypass flow transmitter fails low

MT-9152-FLOO+-I $\quad$ Bypass flow transmitter fails low $\mathrm{x}$ (risk achievement worth).
Bypass valve fails open

Return valve 9116 fails closed

Failure of CTW piping
Prob/Freg AchW

$1.00 \mathrm{E}-06 / \mathrm{H} \quad 9.52 \mathrm{E}+04$

$5.00 \mathrm{E}-07 / \mathrm{H} \quad 9.52 \mathrm{E}+04$

$6.00 \mathrm{E}-06 / \mathrm{H} \quad 9.52 \mathrm{E}+04$

$3.00 \mathrm{E}-06 / \mathrm{H} \quad 9.52 \mathrm{E}+04$
Basic Event Name PIPCTW--BFLO+I FT-9152-FLOO+-I FCV9152-FOPO+-I HCV9116-FCLO+I

\section{Risk Reduction Worth}

\section{Description}

Failure of CTW piping

Bypass flow transmitter fails low

Bypass valve fails open

Return valve 9116 fails closed

\begin{tabular}{cc} 
Prob/Freg & RedW \\
\hline $6.00 \mathrm{E}-06 / \mathrm{H}$ & $\frac{2.33 \mathrm{E}+00}{3.00 \mathrm{E}-06 / \mathrm{H}}$ \\
$1.40 \mathrm{E}+00$ \\
$1.00 \mathrm{E}-06 / \mathrm{H}$ & $1.11 \mathrm{E}+00$ \\
$5.00 \mathrm{E}-07 / \mathrm{H}$ & $1.05 \mathrm{E}+00$
\end{tabular}
Modified top event frequency = (top event frequency) $/$ (risk reduction worth). 
X-ESR-S-00001, Rev. 0

\section{Deep Well System Unavailable}




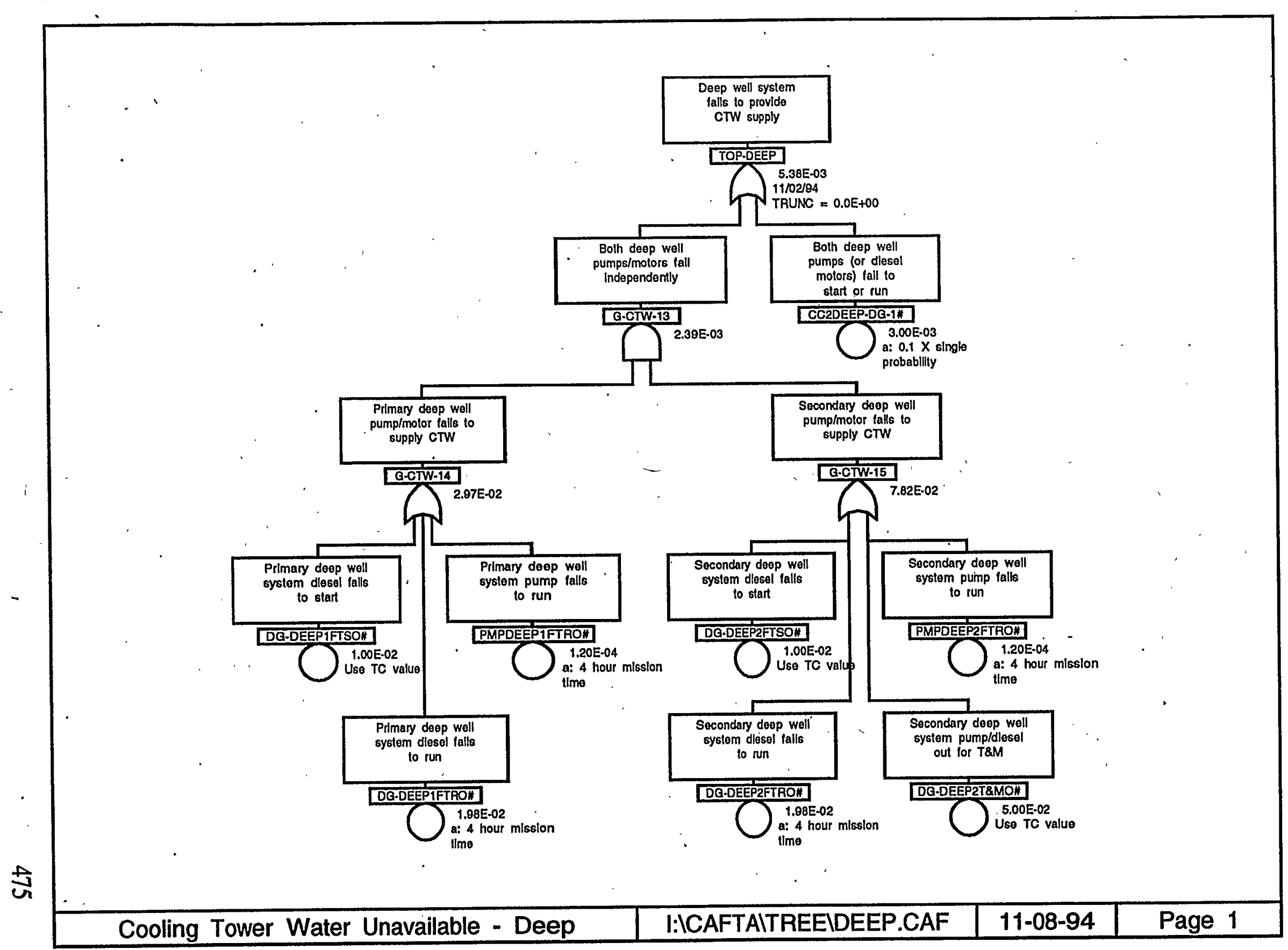


Cutsets for I: \CAFTA \CUT\DEEP.CSR. 11/08/94 11:55 AM

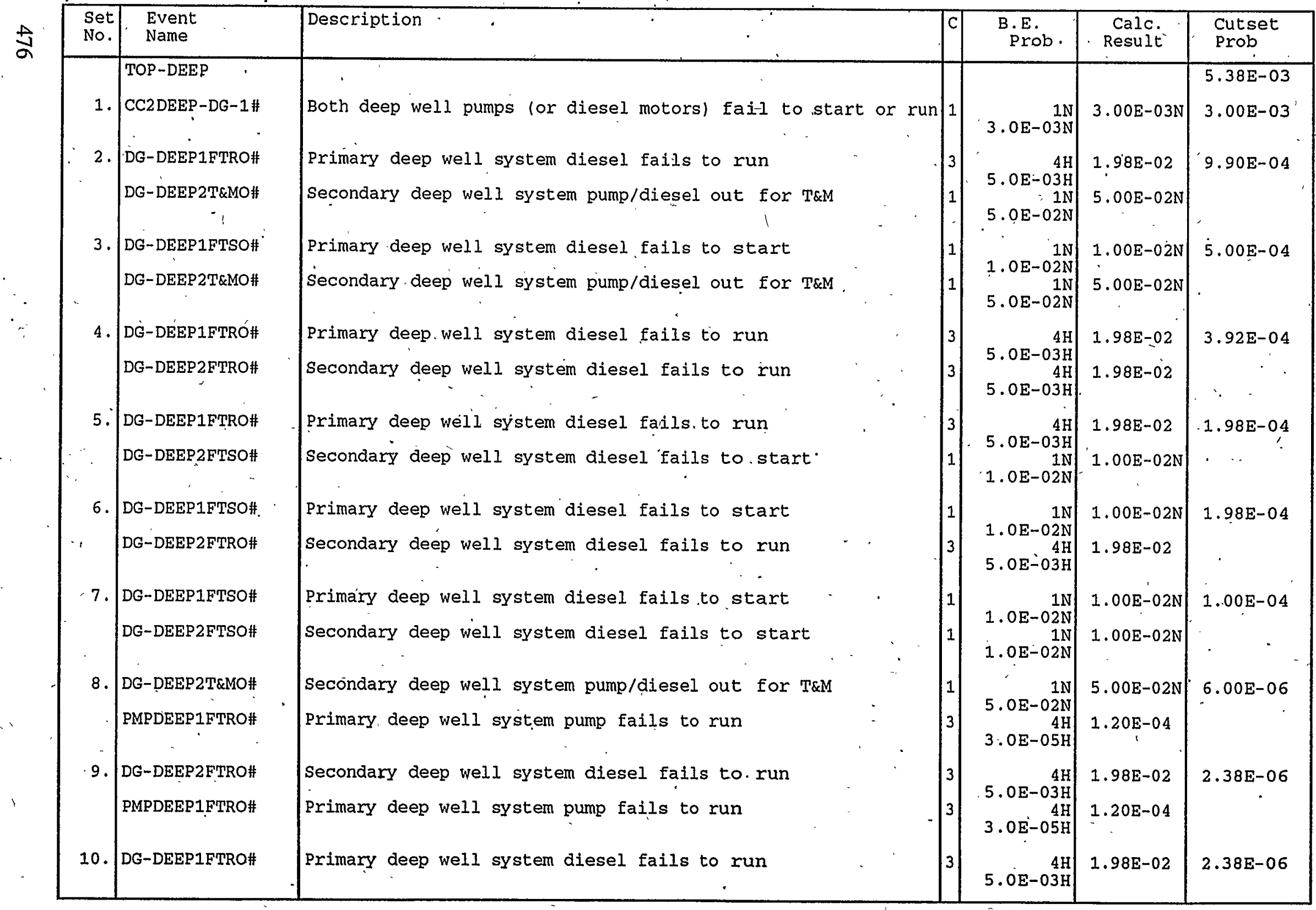


Cutsets for I: ICAFTA \CUT\DEEP.CSR

11/08/94 11:55 AM (CONT.)

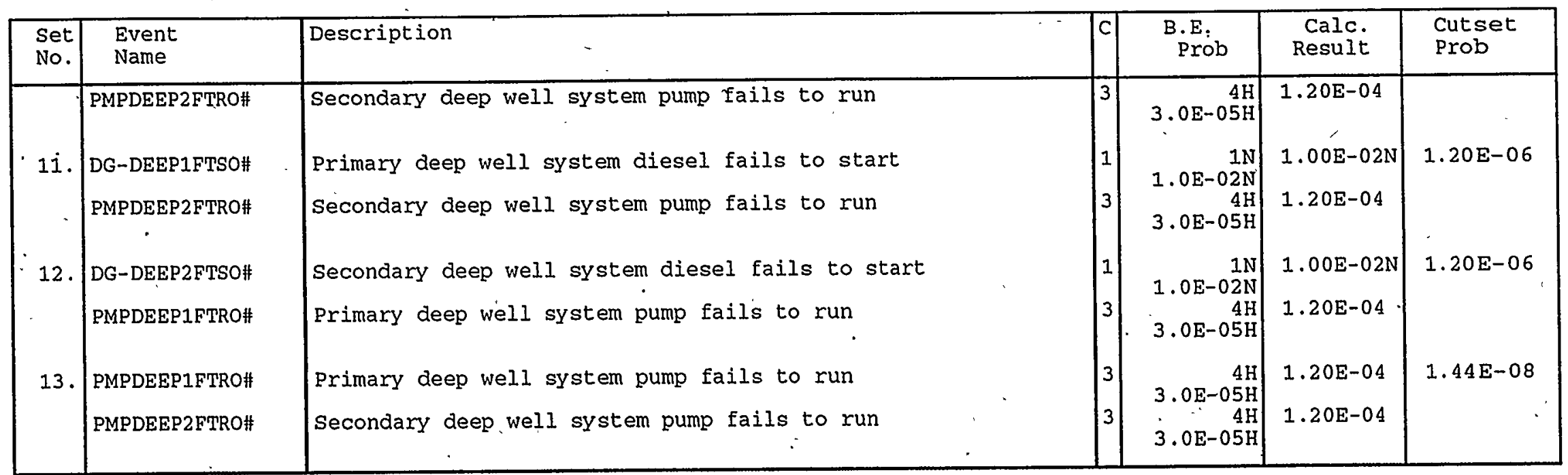


Deep well system fails to provide CTW supply Top Event-Probability: 5.38E-03

Basic Event Name

CC2DEEP-DG-1\# PMPDEEPIFTRO\# DG-DEEP1FTSO\# DG-DEEP1FTRO\# PMPDEEP2FTRO\# DG-DEEP2FTSO\# DG-DEEP2FTRO\# DG-DEEP2T\&MO\# Modified top event frequency $=$ (top event frequency) $x$ (risk achievement worth).

\section{Risk Achievement Worth}

\author{
Description.
}

Both deep well pumps (or-diesel motors) fail to start or run

Primary deep well system pump fails to run

Primary deep well system diesel fails to start

Primary deep well system diesel fails to run

Secondary deep well system pump fails to run

Prob/Freg

3.00E-03

$1.20 \mathrm{E}-04$

$1.00 \mathrm{E}-02$

$1.98 \mathrm{E}-02$

$1.20 \mathrm{E}-04$

$1.00 \mathrm{E}-02$

$1.98 \mathrm{E}-02$

Secondary deep well system diesel fails to run

$5.00 \mathrm{E}-02$

AchW

$1.86 \mathrm{E}+02$

$1.55 \mathrm{E}+01$

$1.53 \mathrm{E}+01$

$1.52 \mathrm{E}+01$

$6.49 \mathrm{E}+00$

$6.44 \mathrm{E}+00$

$6.39 \mathrm{E}+00$

$6.22 \mathrm{E}+00$
Basic Event Name

CC2DEEP-DG-1\#

DG-DEEP1FTRO\#

DG-DEEP2T\&MO\#

DG-DEEP1FTSO\#

DG-DEEP2FTRO\#

DG-DEEP2FTSO\#

\section{Risk Reduction. Worth}

\section{- Description}

Both deep well pumps (or diesel motors) fail to start or run

Primary deep well system diesel fails to run

Secondary deep well system pump/diesel out for T\&M

Primary deep well system diesel fails to start

Secondary deep well system diesel fails to run

Secondary deep well system diesel fails to start.
Prob/Freg

3.00E-03

$1.98 \mathrm{E}-02$

$5.00 \mathrm{E}-02$

$1.00 \mathrm{E}-02$

$1.98 \mathrm{E}-02$

$1.00 \mathrm{E}-02$
$\underline{R e d W}$

$2.25 \mathrm{E}+00$

$1.41 \mathrm{E}+00$

$1.38 \mathrm{E}+00$

$1.17 \mathrm{E}+00$

$1.12 \mathrm{E}+00$

$1.06 \mathrm{E}+00$

Note: Events that make less than $1 \%$ difference to the importance factor are not included.

Modified top event frequency = (top event frequency) / (risk-reduction worth). 
X-ESR-S-00001, Rev. 0

Cooling Tower Water System Ưnavailable - pumping

8 
X-ESR-S-00001, Rev. 0

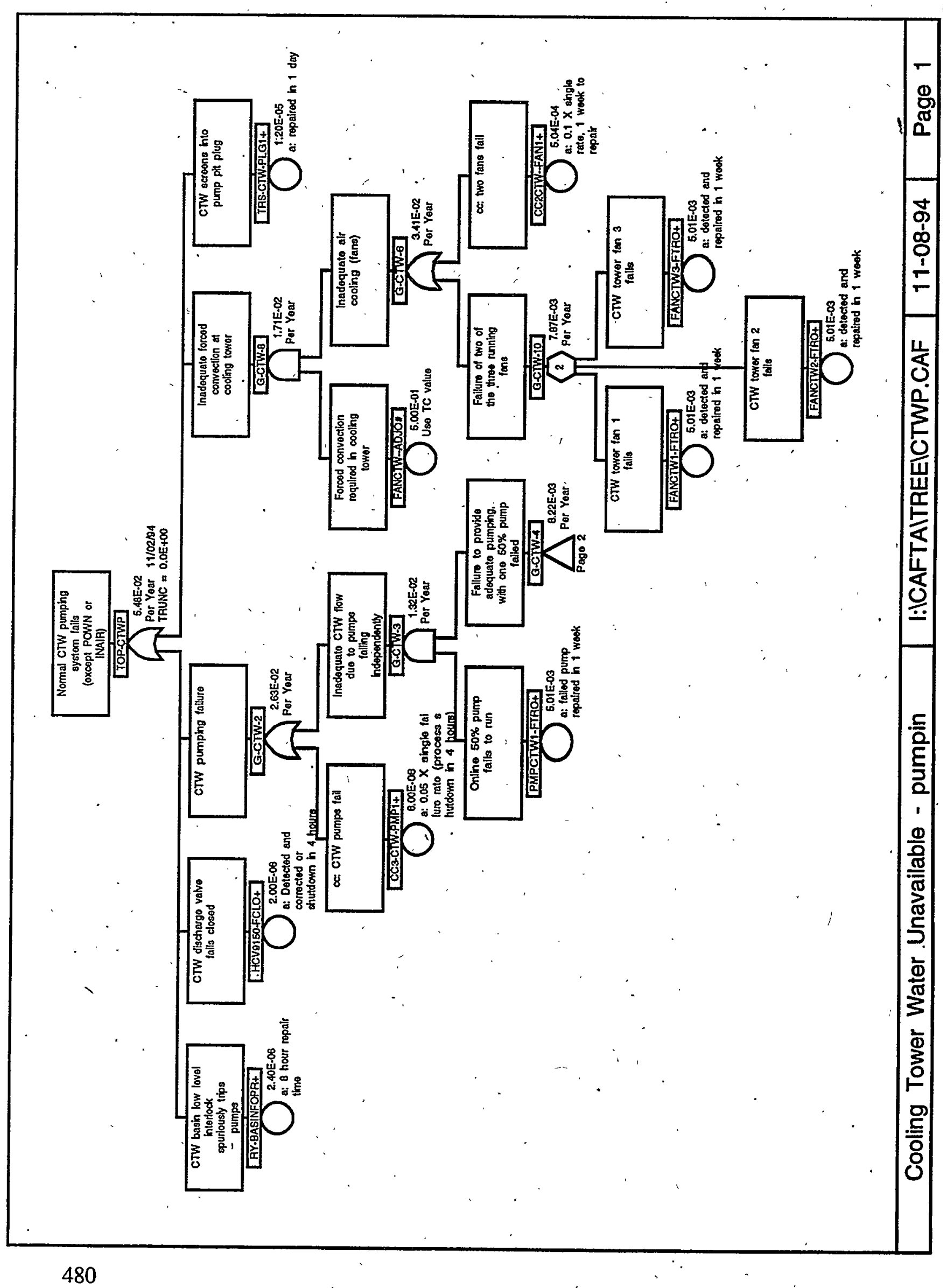




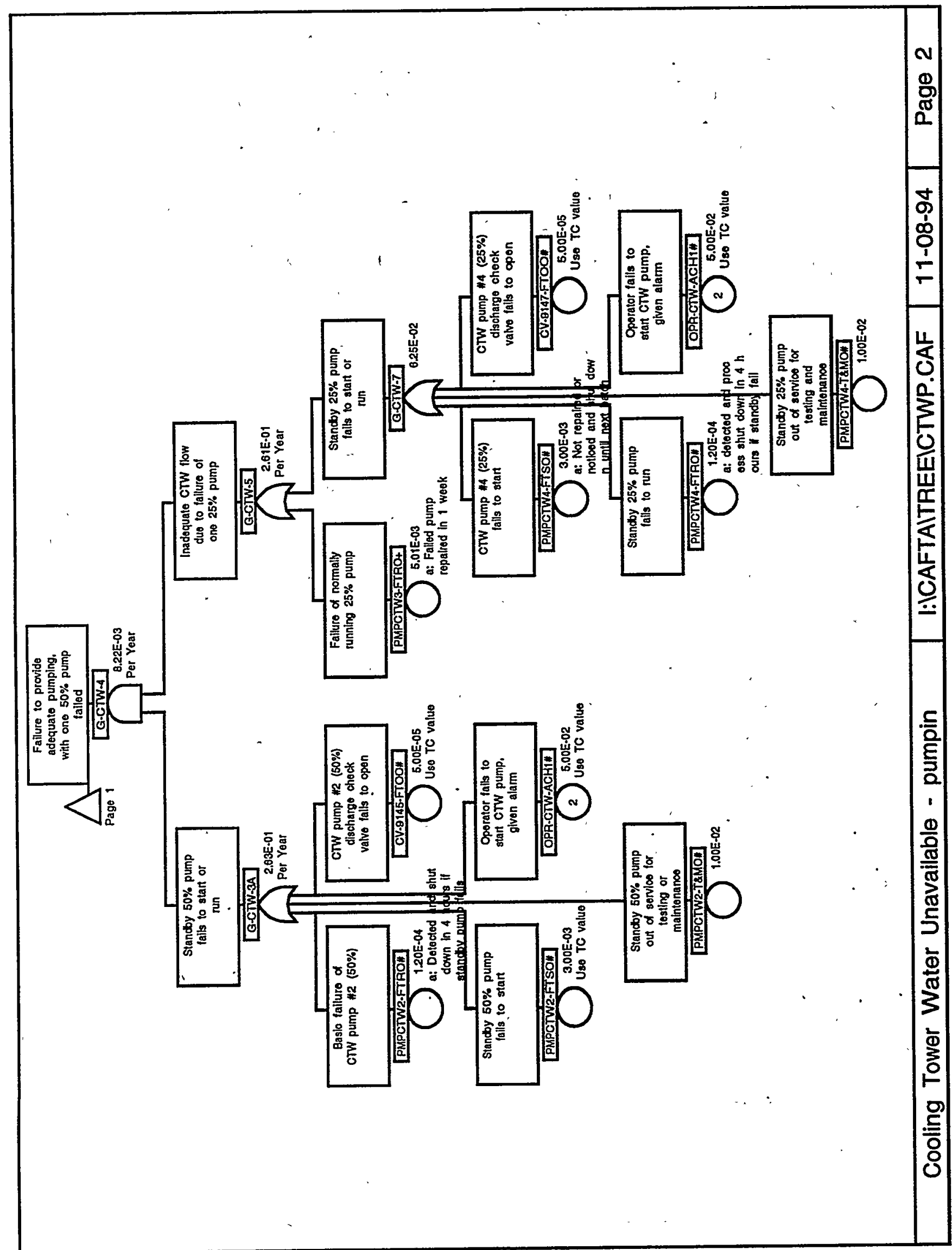




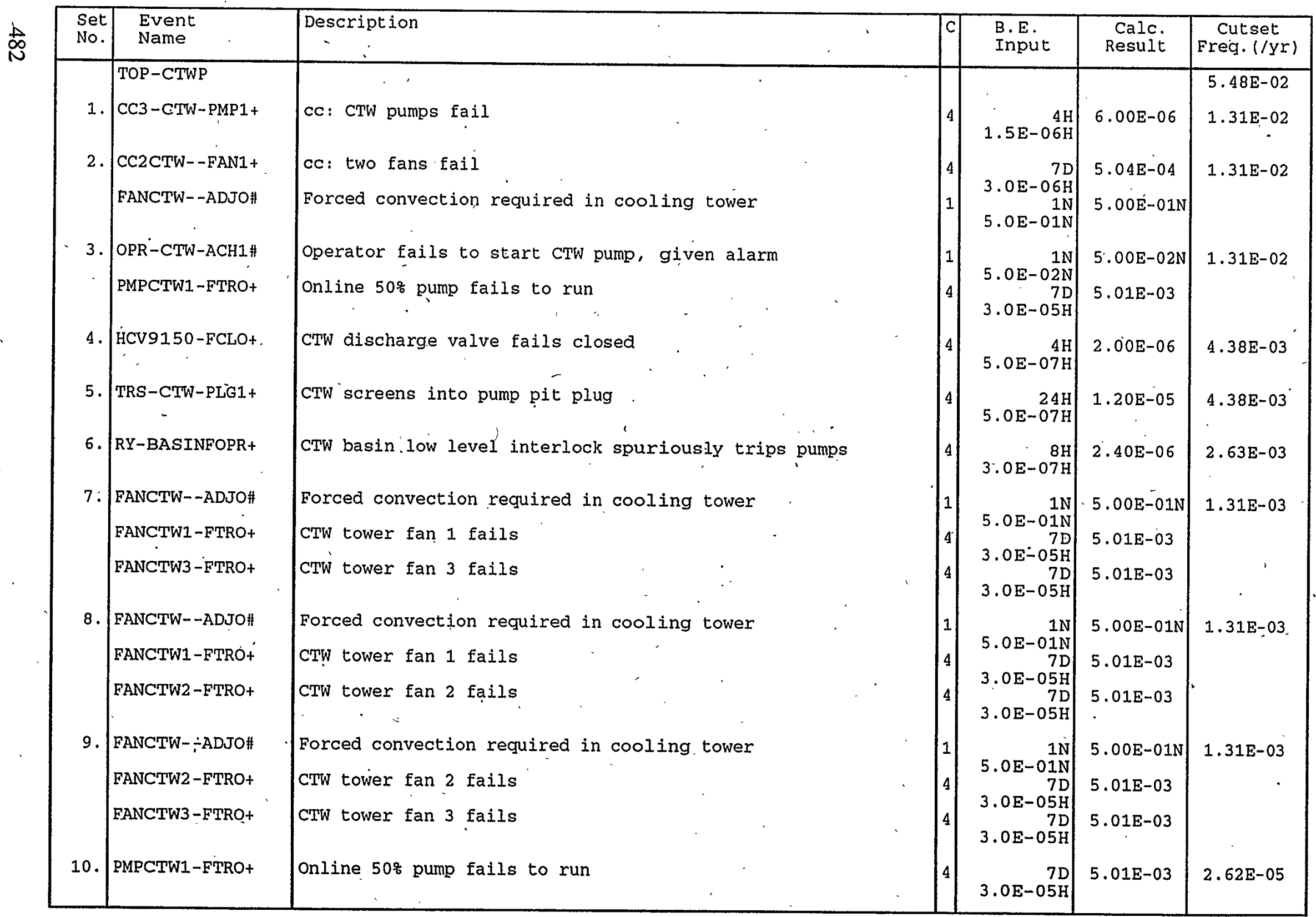




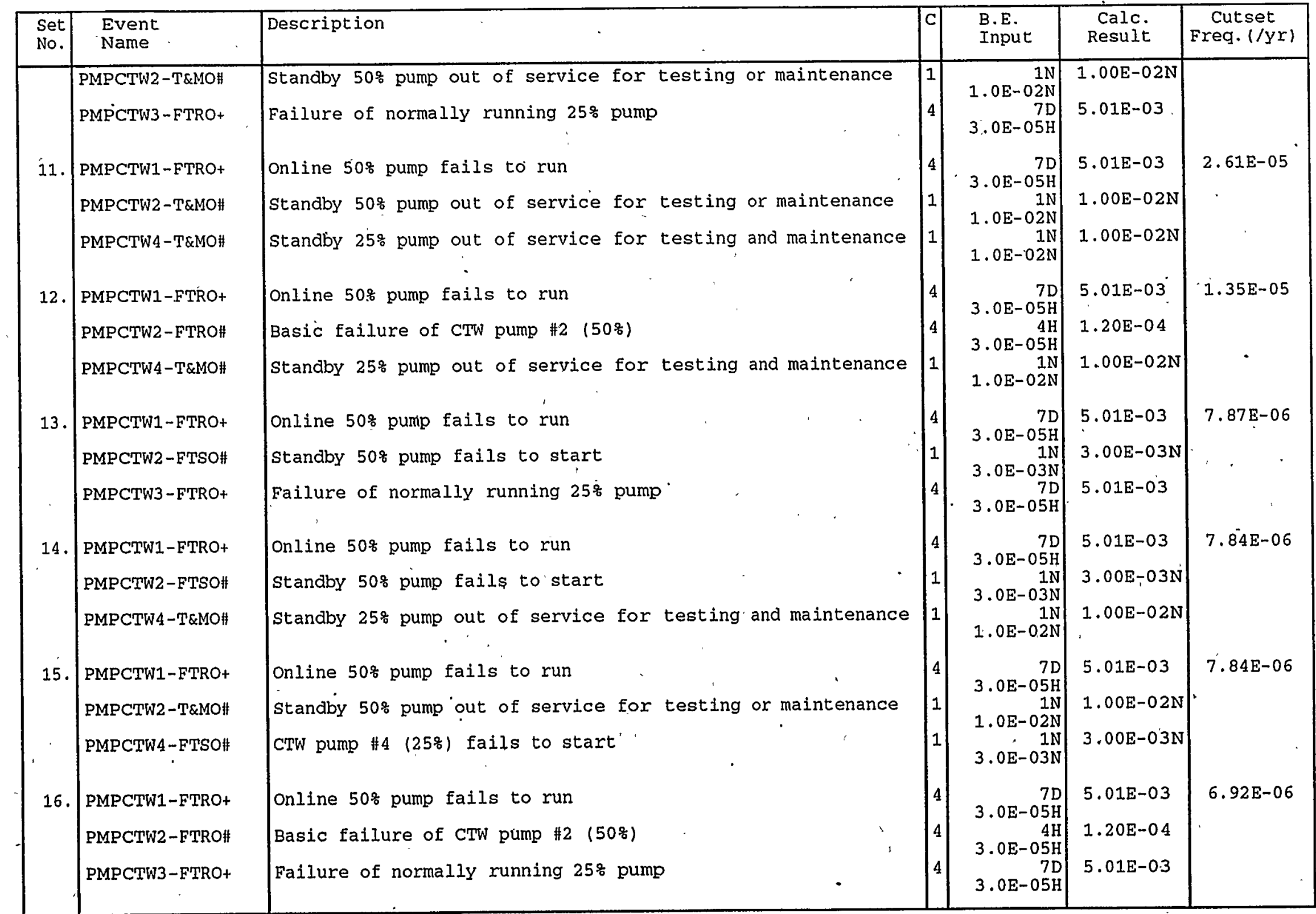


Cutsets for I.: \CAFTA \CUT\CTWP. CSR

11/08/94 11:56 AM (CONT.)

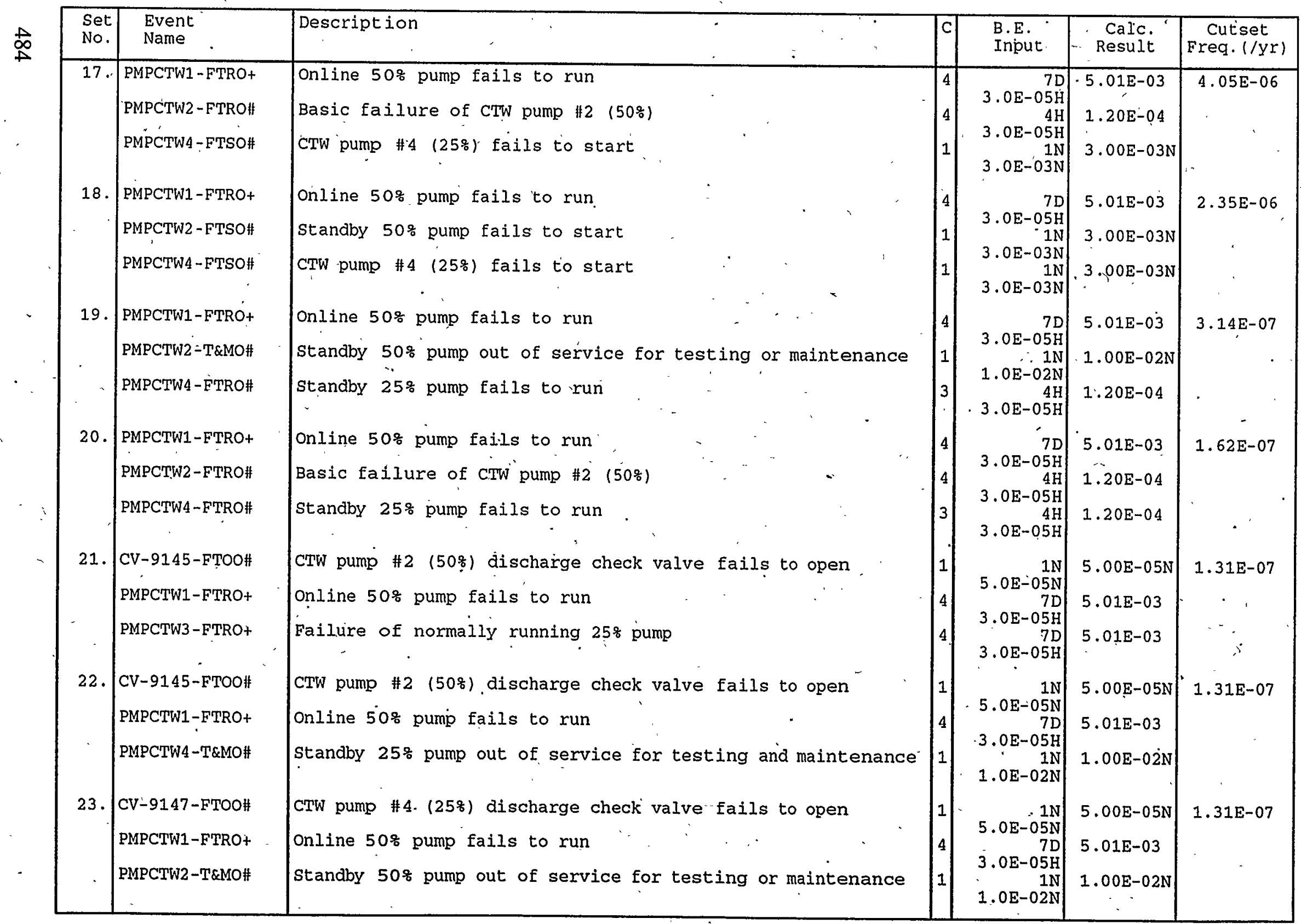


Cutsets for I: \CAFTA ICUT\CTWP.CSR

11/08/94 11:56 AM (CONT.)

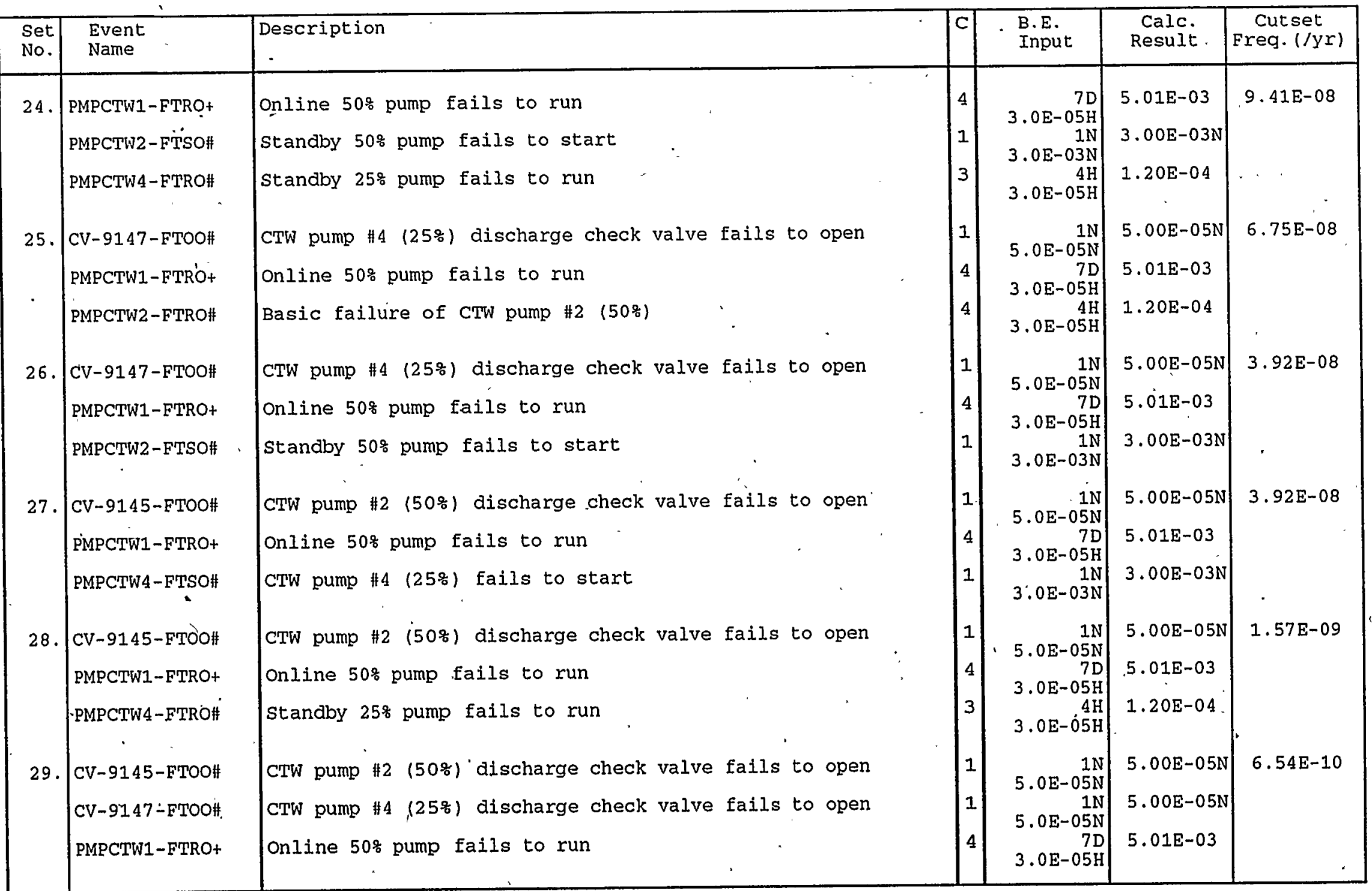


Normal CTW pumping system fails (except POWN or INAIR)

Top Event Frequency: 5.47E-02/YR

Basic Event Name HCV9150-FCLO+-I TRS-CTW-PLG1+-I RY-BASINFOPR+I CC3-CTW-PMP1+I CC2CTW-FAN1+I PMPCTW1-FTRO+-IC FANCTW2-FTRO+-IC FANCTW3-FTRO+-IC FANCTW1-FTRO+IC PMPCTW3-FTRO+-IC OPR-CTW-ACH1\# FANCTW-ADJO\# CV-9145-FTOO\# PMPCTW2-FTRO\# PMPCTW2-FTSO\# PMPCTW2-T\&MO\# CV-9147-FTOO\# PMPCTW4-FTRO\# PMPCTW4-FFSO\# PMPCTW4T\&MO\# Modified top event frequency = (top event frequency) $\mathrm{x}$ (risk achievement worth).

\section{Description}

CTW discharge valve fails closed

CTW screens into pump pit plug

CTW basin low level interlock spuriously trips pumps

cc: CTW pumpś fail

cc: two fans fail

Online 50\% pump fails to run

CTW tower fan 2 fails

CTW tower fan 3 fails

CTW tower fan 1 fails

Failure of normally running $25 \%$ pump

Operator fails to start CTW pump, given alarm

Forced convection required in cooling tower

CTW pump \#2 (50\%) discharge check valve fails to open

Basic failure of CT.W pump \#2 (50\%)

Standby $50 \%$ pump fails to start

Standby $50 \%$ pump out of service for testing or maintenance

CTW pump \#4 (25\%) discharge check valve fails to open

Standby $25 \%$ pump fails to run

CTW pump \#4 (25\%) fails to start

Standby $25 \%$ pump out of service for T\&M

\section{Risk Achievement Worth}

\begin{tabular}{cc} 
Prob/Freq & AchW \\
\hline $5.00 \mathrm{E}-07 / \mathrm{H}$ & $1.60 \mathrm{E}+05$ \\
$5.00 \mathrm{E}-07 / \mathrm{H}$ & $1.60 \mathrm{E}+05$ \\
$3.00 \mathrm{E}-07 / \mathrm{H}$ & $1.60 \mathrm{E}+05$ \\
$1.50 \mathrm{E}-06 / \mathrm{H}$ & $1.60 \mathrm{E}+05$ \\
$3.00 \mathrm{E}-06 / \mathrm{H}$ & $8.00 \mathrm{E}+04$ \\
$2.98 \mathrm{E}-05 / \mathrm{H}$ & $8.04 \mathrm{E}+03$ \\
$2.98 \mathrm{E}-05 / \mathrm{H}$ & $8.07 \mathrm{E}+02$ \\
$2.98 \mathrm{E}-05 / \mathrm{H}$ & $8.07 \mathrm{E}+02$ \\
$2.98 \mathrm{E}-05 / \mathrm{H}$ & $8.07 \mathrm{E}+02$ \\
$2.98 \mathrm{E}-05 / \mathrm{H}$ & $1.16 \mathrm{E}+01$ \\
$5.00 \mathrm{E}-02$ & $5.54 \mathrm{E}+00$ \\
$5.00 \mathrm{E}-01$ & $1.31 \mathrm{E}+00$ \\
$5.00 \mathrm{E}-05$ & $1.11 \mathrm{E}+00$ \\
$1.20 \mathrm{E}-04$ & $1.11 \mathrm{E}+00$ \\
$3.00 \mathrm{E}-03$ & $1.11 \mathrm{E}+00$ \\
$1.00 \mathrm{E}-02$ & $1.11 \mathrm{E}+00$ \\
$5.00 \mathrm{E}-05$ & $1.06 \mathrm{E}+00$ \\
$1.20 \mathrm{E}-04$ & $1.06 \mathrm{E}+00$ \\
$3.00 \mathrm{E}-03$ & $1.06 \mathrm{E}+00$ \\
$1.00 \mathrm{E}-02$ & $1.06 \mathrm{E}+00$
\end{tabular}

Basic Event Name

FANCTW-ADJO\# PMPCTW1-FTRO+-IC CC3-CTW-PMP1+-I CC2CTW-FAN1+-I OPR-CTW-ACH1\# TRS-CTW-PLG1+-I HCV9150-FCLO+I RY-BASINFOPR+-I FANCTW3-FTRO+-IC FANCTW2-FTRO+-IC FANCTW1-FTRO+-IC

\section{Risk Reduction Worth} Description

Forced convection required in cooling tower

Online 50\% pump fails to run

cc: CTW pumps fail

cc: two fans fail

Operator fails to start CTW pump, given alarm

CTW screens into pump pit plug

CTW discharge valve fails closed

CTW basin low level interlock spuriously trips pumps

CTW tower fan 3 fails

CTW tower fan 2 fails

CTW tower fan 1 fails \begin{tabular}{ll} 
Prob/Freq & RedW \\
\hline $5.00 \mathrm{E}-01$ & $1.45 \mathrm{E}+00$ \\
$2.98 \mathrm{E}-05 / \mathrm{H}$ & $1.32 \mathrm{E}+00$ \\
$1.50 \mathrm{E}-06 / \mathrm{H}$ & $1.32 \mathrm{E}+00$ \\
$3.00 \mathrm{E}-06 / \mathrm{H}$ & $1.32 \mathrm{E}+00$ \\
$5.00 \mathrm{E}-02$ & $1.31 \mathrm{E}+00$ \\
$5.00 \mathrm{E}-07 / \mathrm{H}$ & $1.09 \mathrm{E}+00$ \\
$5.00 \mathrm{E}-07 / \mathrm{H}$ & $1.09 \mathrm{E}+00$ \\
$3.00 \mathrm{E}-07 / \mathrm{H}$ & $1.05 \mathrm{E}+00$ \\
$2.98 \mathrm{E}-05 / \mathrm{H}$ & $1.05 \mathrm{E}+00$ \\
$2.98 \mathrm{E}-05 / \mathrm{H}$ & $1.05 \mathrm{E}+00$ \\
$2.98 \mathrm{E}-05 / \mathrm{H}$ & $1.05 \mathrm{E}+00$
\end{tabular}

Note: Events that make less than $1 \%$ difference to the importance factor are not included. Modified top event frequency = (top event frequency) / (risk reduction worth). 
X-ESR-S-00001, Rev. 0

Bulk Nitrogen Supply Path Failure 


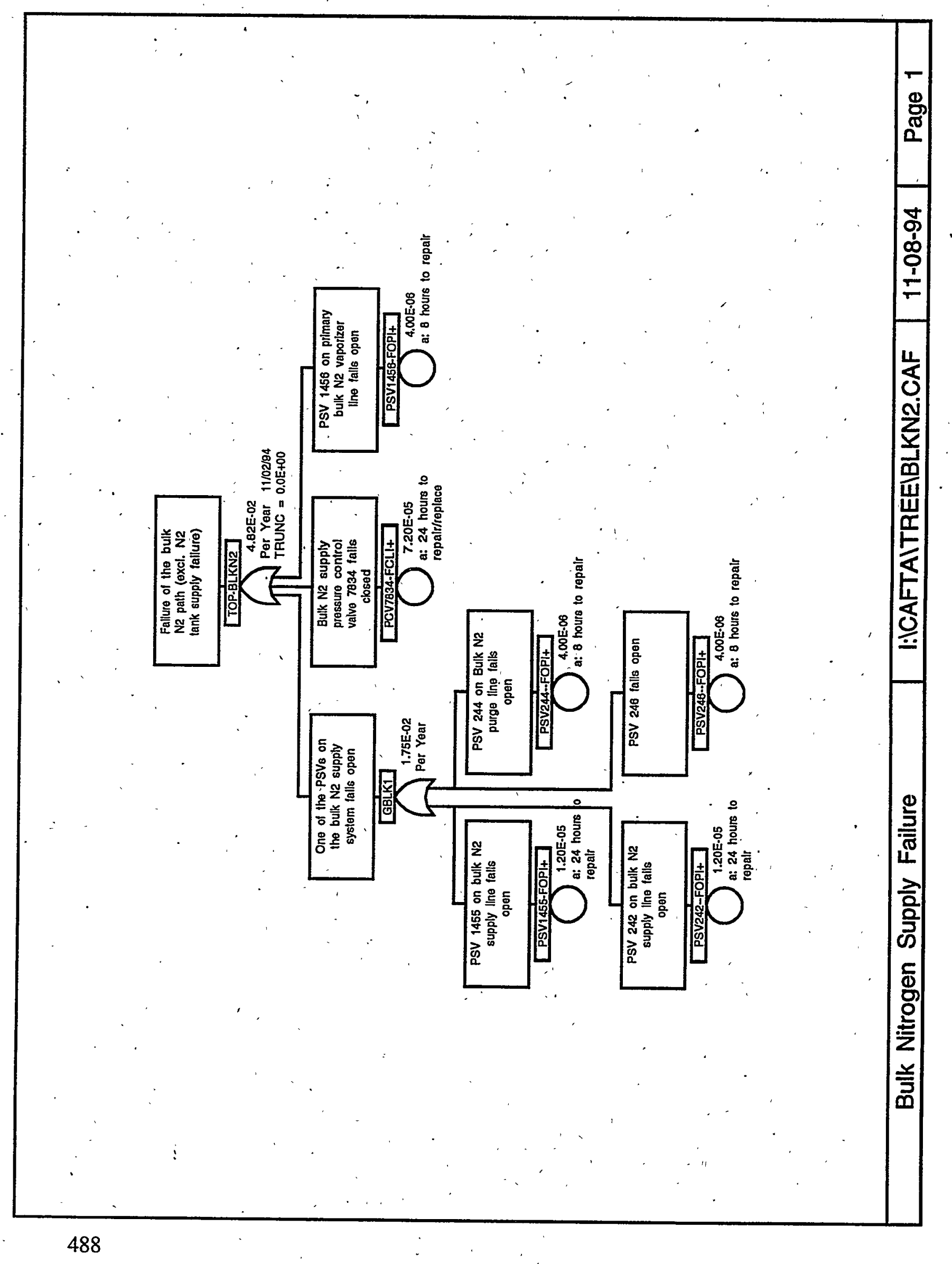


Cutsets for I: \CAFTA \CUT\BLKN2.CSR

11/08/94 11:57 AM

\begin{tabular}{|c|c|c|c|c|c|c|}
\hline $\begin{array}{l}\text { Set } \\
\text { No. }\end{array}$ & $\begin{array}{l}\text { Event } \\
\text { Name }\end{array}$ & Description & C & $\begin{array}{l}\text { B.E. } \\
\text { Input }\end{array}$ & $\begin{array}{l}\text { Calc. } \\
\text { Result }\end{array}$ & $\begin{array}{c}\text { Cutset } \\
\text { Freq. (/yr) }\end{array}$ \\
\hline & TOP-BLKN2 & & & & & $4.82 \mathrm{E}-02$ \\
\hline 1. & PCV7834-FCLIt & Bulk N2 supply pressure control valve 7834 fails closed & 4 & $\begin{array}{r}24 \mathrm{H} \\
3.0 \mathrm{E}-06 \mathrm{H}\end{array}$ & $7.20 \mathrm{E}-05$ & $2.63 \mathrm{E}-02$ \\
\hline 2. & PSV1456-FOPI+ & PSV 1456 on primary bulk N2 vaporizer line fails open & 4 & $5.0 \mathrm{E}-07 \mathrm{H}$ & $4.00 \mathrm{E}-06$ & $4.38 \mathrm{E}-03$ \\
\hline 3. & PSV246--FOPI+ & PSV 246. fails open & 4 & $5.0 \mathrm{E}-07 \mathrm{H}$ & $4.00 \mathrm{E}-06$ & $4.38 \mathrm{E}-03$ \\
\hline 4. & PSV244--FOPI+ & PSV 244 on Bulk N2 purge line fails open & 4 & $5.0 \mathrm{E}-07 \mathrm{H}$ & $4.00 \mathrm{E}-06$ & $4.38 \mathrm{E}-03$ \\
\hline 5 . & PSV242--FOPI+ & PSV 242 on bulk N2 supply line fails open & 4 & $\begin{array}{r}24 \mathrm{H} \\
5.0 \mathrm{E}-07 \mathrm{H}\end{array}$ & $1.20 \mathrm{E}-05$ & $4.38 \mathrm{E}-03$ \\
\hline 6. & PSV1455-FOPI+ & PSV 1455 on bulk N2 supply line fails open & 4 & $\begin{array}{r}24 \mathrm{H} \\
5.0 \mathrm{E}-07 \mathrm{H}\end{array}$ & $1.20 \mathrm{E}-05$ & $4.38 \mathrm{E}-03$ \\
\hline
\end{tabular}


Failure of the bulk N2 path (excl. N2 tank supply failure)

Top Event Frequency: 4.82E-02/YR

Basic Event Name

PSV244-FOPI+I

PSV242-FOPI+I

PSV1455-FOPI+I

PCV7834-FCLI+I

PSV1456-FOPI+I

PSV246-FOPI+-I.
Risk Achievement Worth

\section{Description}

PSV 244 on Bulk N2 purge line fails open

PSV 242 on bulk.N2 supply line fails open

PSV 1455 on bulk N2 supply line fails open

Bulk N2 supply pressure control valve 7834 fails closed

PSV 1456 on primary bulk N2 vaporizer line fails open

PSV 246 fails open
Prob/Freq

5.00E-07/H

$5.00 \mathrm{E}-07 / \mathrm{H}$

$5.00 \mathrm{E}-07 / \mathrm{H}$

$3.00 \mathrm{E}-06 / \mathrm{H}$

$5.00 \mathrm{E}-07 / \mathrm{H}$

$5.00 \mathrm{E}-07 / \mathrm{H}$

$\operatorname{Ach} W$

$1.82 \mathrm{E}+05$

$1.82 \mathrm{E}+05$

$1.82 \mathrm{E}+05$

$1.82 \mathrm{E}+05$

$1.82 \mathrm{E}+05$

Basic Event Name PCV7834-FCLI+I PSV242--FOPI+I PSV1455-FOPI+-I PSV244-FOPI+I PSV1456-FOPI+I PSV246-FOPI+I Modified top eve
$1.82 \mathrm{E}+05$

\section{Risk Reduction Worth}

Description

Bulk Ń2 supply pressure control valve 7834 fails closed

PSV 242 on bulk N2 supply line fails open

PSV 1455 on bulk N2 supply line fails open

PSV 244 on Bulk N2 purge line fails open

PSV 1456 on primary bulk $\mathrm{N} 2$ vaporizer line fails open

PSV 246 fails open \begin{tabular}{cc} 
Prob/Freg & RedW \\
\hline $3.00 \mathrm{E}-06 / \mathrm{H}$ & $2.20 \mathrm{E}+00$ \\
$5.00 \mathrm{E}-07 / \mathrm{H}$ & $1.10 \mathrm{E}+00$ \\
$5.00 \mathrm{E}-07 / \mathrm{H}$ & $1.10 \mathrm{E}+00$ \\
$5.00 \mathrm{E}-07 / \mathrm{H}$ & $1.10 \mathrm{E}+00$ \\
$5.00 \mathrm{E}-07 / \mathrm{H}$ & $1.10 \mathrm{E}+00$ \\
$5.00 \mathrm{E}-07 / \mathrm{H}$ & $1.10 \mathrm{E}+00$
\end{tabular}

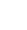


X-ESR-S-00001, Rev. 0

\section{Bulk Nitrogen Tank Supply Failure}




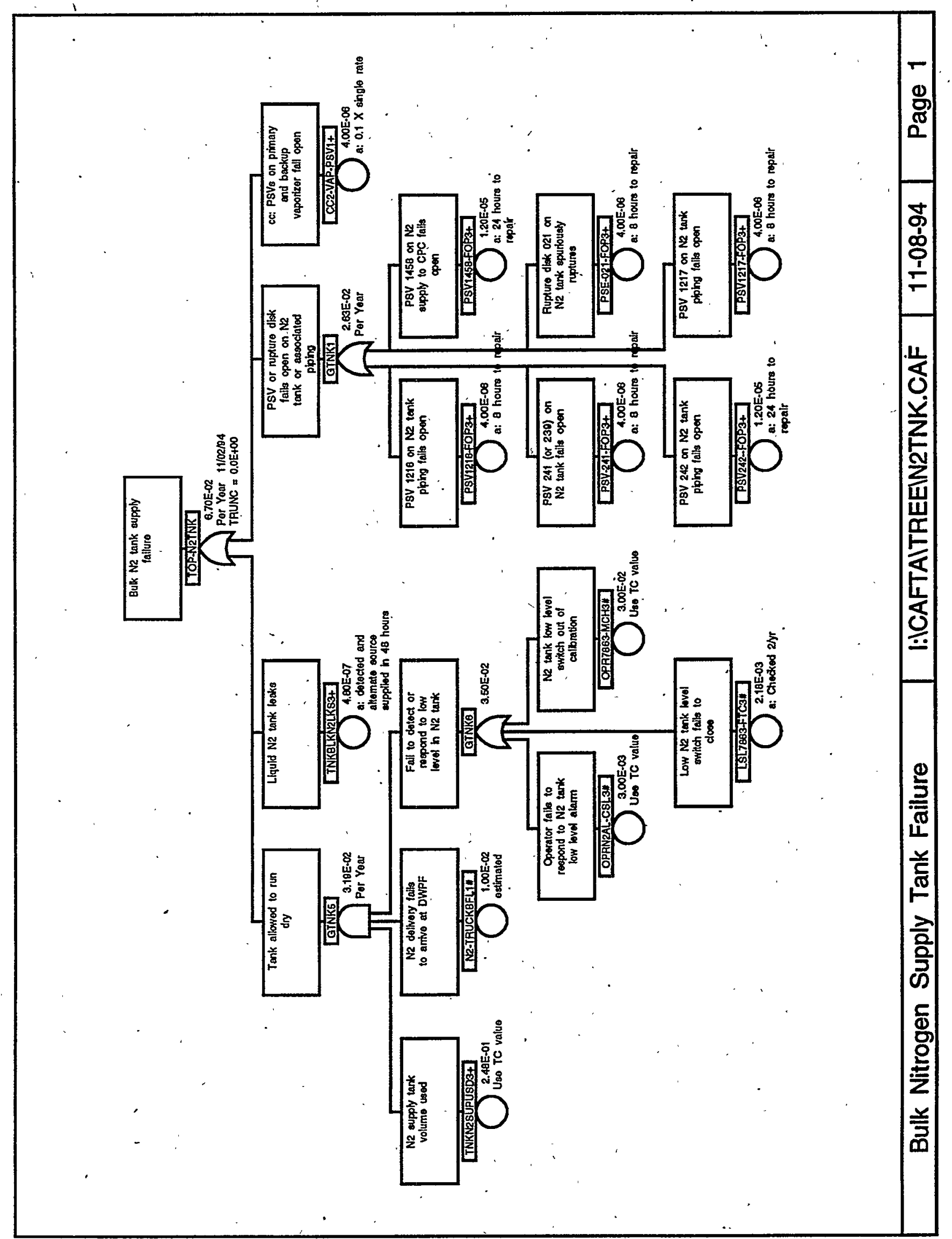


Cutsets for I: ICAFTA ICUTIN2TNK.CSR 11/08/94 11:59 AM

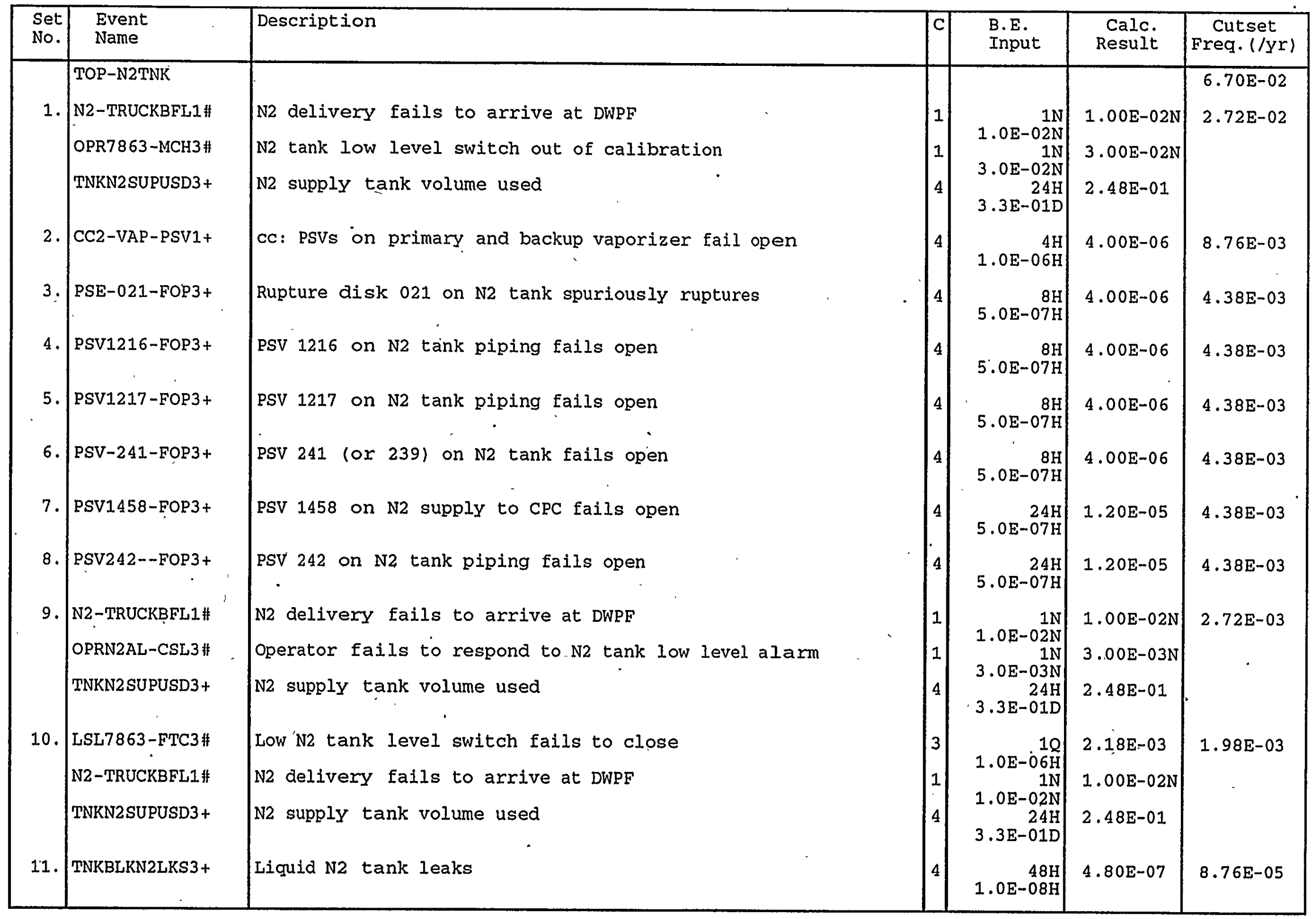


Bulk N2 tank supply failure Top Event Frequency: 6.70E-02/YR

Basic Event Name
PSV-241-FOP3+-I
PSV1217-FOP3+I
PSV1458-FOP3+I
TNKBLKN2LKS3+-I
PSV242-FOP3+I
PSV1216-FOP3+-I
CC2-VAP-PSV1+-1
PSE-021-FOP3+-I
N2-TRUCKBFL1\#
TNKN2SUPUSD3+-I
LSL7863-FTC3\#
OPRN2AL-CSL3\#
OPR7863-MCH3\#
Modified top event fred

Basic Event Name PSV1217-FOP3+I PSV1458-FOP3+I TNKBLKN2LKS3+-I PSV242-FOP3+-1 PSV1216-FOPH+I PSE-021-FOP3+-I N2-TRUCKBFL1\# LSL7863-FTC3\# OPR7863-MCH3\# Modified top event frequency = (top event frequency) $\mathrm{x}$ (risk achievement worth).

\section{Risk Achievement Worth}

\author{
Description
}

PSV 241 (or 239) on N2 tank fails open

PSV 1217 on N2 tank piping fails open PSV 1458 on N2 supply to CPC fails open

Liquid N2 tank leaks

PSV 242 on N2 tank piping fails open

PSV 1216 on N2 tank piping fails open

cc: PSVs on primary and backup vaporizer fail open

Rupture disk 021 on N2 tank spuriously ruptures

N2 delivery fails to arrive at DWPF

N2 supply tank volume used

Low N2 tank level switch fails to close

Operator fails to respond to $\mathrm{N} 2$ tank low level alarm

N2 tank low level switch out of calibration
Prob/Freg AchW

5.00E-07/H 1.31E+05

$5.00 \mathrm{E}-07 / \mathrm{H} \quad 1.31 \mathrm{E}+05$

$5.00 \mathrm{E}-07 / \mathrm{H} \quad 1.31 \mathrm{E}+05$

$1.00 \mathrm{E}-08 / \mathrm{H} \quad 1.31 \mathrm{E}+05$

5.00E-07/H 1.31E+05

$5.00 \mathrm{E}-07 / \mathrm{H}$ 1.31E+05

$1.00 \mathrm{E}-06 / \mathrm{H} \quad 1.31 \mathrm{E}+05$

$5.00 \mathrm{E}-07 / \mathrm{H} \quad 1.31 \mathrm{E}+05$

$1.00 \mathrm{E}-02$

$1.03 \mathrm{E}-02 / \mathrm{H}$

$2.18 \mathrm{E}-03$

$3.00 \mathrm{E}-03$

$3.00 \mathrm{E}-02$

\section{Risk Reduction Worth}

Basic Event Name

TNKN2SUPUSD3+-I

N2-TRUCKBFL1\#

OPR7863-MCH3\#

CC2-VAP-PSV1+-I

PSV-241-FOP3+I

PSV1458-FOP3+-I

PSV242--FOP3+-I

PSE-021-FOP3+I

PSV1216-FOP3+-I

PSV1217-FOP3+-I

OPRN2AL-CSL3\#

LSL7863-FTC3\#

\section{Description}

N2 supply tank volume used

N2 delivery fails to arrive at DWPF

N2 tank low level switch out of calibration

cc: PSVs on primary and backup vaporizer fail open

PSV 241 (or 239) on N2 tank fails open

PSV 1458 on N2 supply to CPC fails open

PSV 242 on N2 tank piping fails open

Rupture disk 021 on $\mathrm{N} 2$ tank spuriously ruptures

PSV 1216 on N2 tank piping fails open

PSV 1217 on N2 tank piping fails open

Operator fails to respond to $\mathrm{N} 2$ tank low level alarm

. Low.N2 tank level switch fails to close

\begin{tabular}{ll} 
Prob/Freg & RedW \\
\hline $1.03 \mathrm{E}-02 / \mathrm{H}$ & $1.91 \mathrm{E}+00$ \\
$1.00 \mathrm{E}-02$. & $1.91 \mathrm{E}+00$ \\
$3.00 \mathrm{E}-02$ & $1.68 \mathrm{E}+00$ \\
$1.00 \mathrm{E}-06 / \mathrm{H}$ & $1.15 \mathrm{E}+00$ \\
$5.00 \mathrm{E}-07 / \mathrm{H}$ & $1.07 \mathrm{E}+00$ \\
$5.00 \mathrm{E}-07 / \mathrm{H}$ & $1.07 \mathrm{E}+00$ \\
$5.00 \mathrm{E}-07 / \mathrm{H}$ & $1.07 \mathrm{E}+00$ \\
$5.00 \mathrm{E}-07 / \mathrm{H}$ & $1.07 \mathrm{E}+00$ \\
$5.00 \mathrm{E}-07 / \mathrm{H}$ & $1.07 \mathrm{E}+00$ \\
$5.00 \mathrm{E}-07 / \mathrm{H}$ & $1.07 \mathrm{E}+00$ \\
$3.00 \mathrm{E}-03$ & $1.04 \mathrm{E}+00$ \\
$2.18 \mathrm{E}-03$ & $1.03 \mathrm{E}+00$
\end{tabular}

Note: Events that make less than $1 \%$ difference to the importance factor are not included. Modified top event frequency = (top event frequency) / (risk reduction worth). 
X-ESR-S-00001, Rev. 0

\section{CPC Nitrogen Supply Tank Unavailable}




\section{X-ESR-S-00001, Rev. 0}

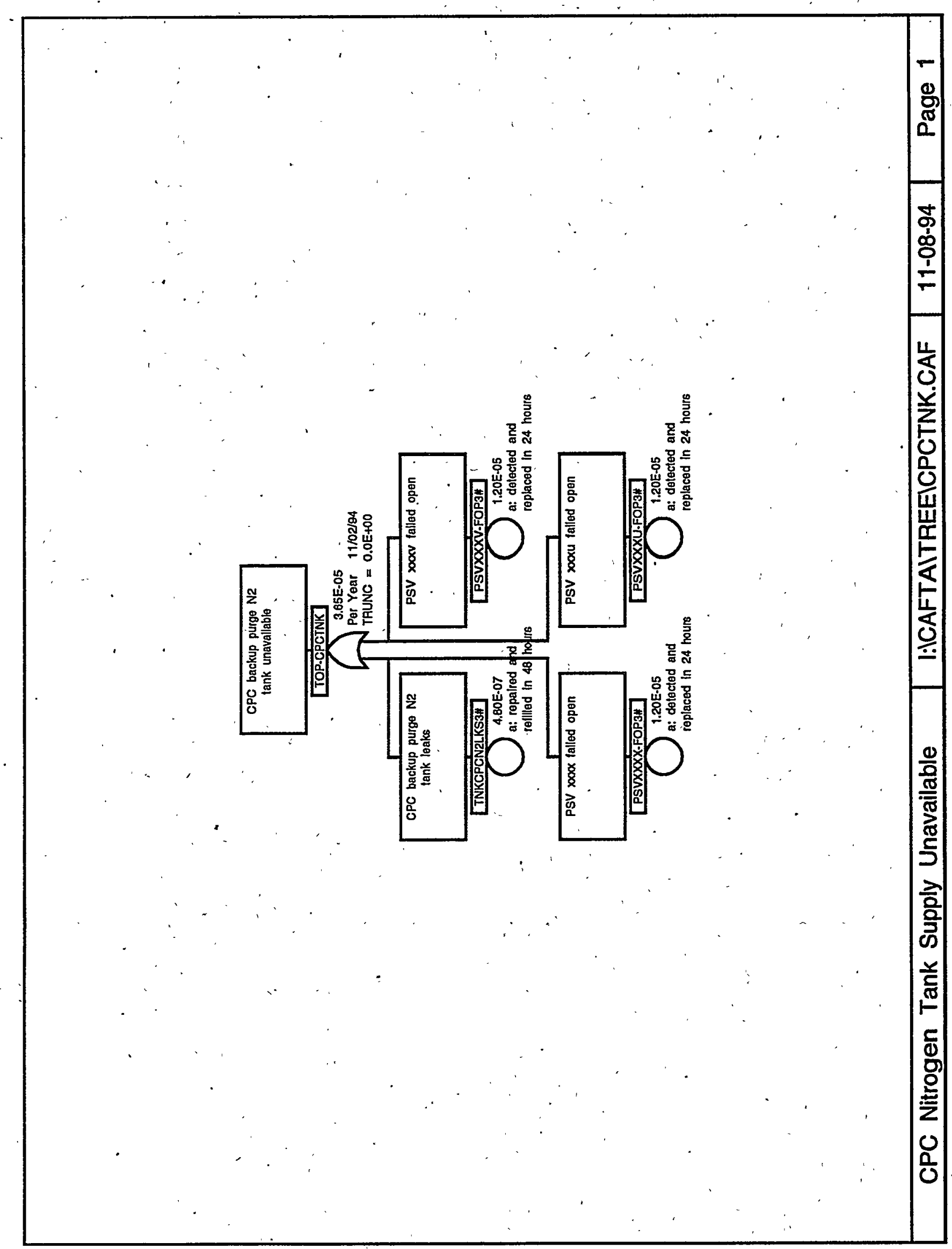


Cutsets for I: ICAFTA ICUT\CPCTNK.CSR

$11 / 08 / 94 \quad 12: 00 \mathrm{AM}$

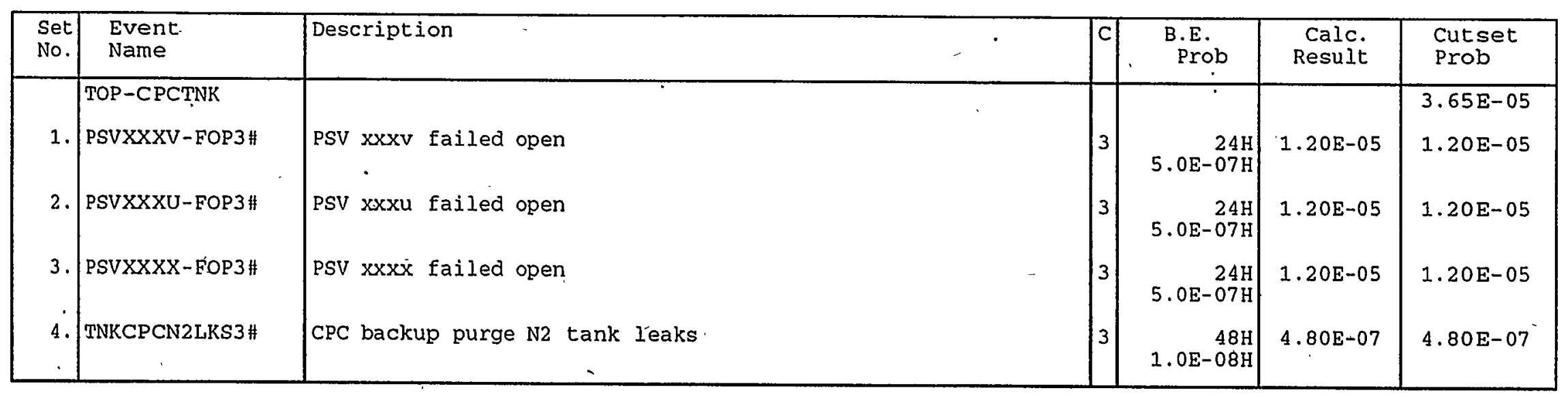


X-ESR-S-00001, Rev. 0

CPC backup purge N2 tank unavailable Top Event Probability: 3.65E-05

$\therefore \quad$ Risk Achievement Worth

Basic Event Name

PSVXXXX-FOP3\#

TNKCPCN2LKS3\#

PSVXXXV-FOP3\#

PSVXXXU-FOP3\#
Description

PSV $x x x x$ failed open

CPC backup purge N2 tank leaks

PSV xxxv failed open

PSV $x x x u$ failed open
Prob/Freg AchW

1.20E-05 2.74E+04

4.80E-07 2.74E+04

$1.20 \mathrm{E}-05 \quad 2.74 \mathrm{E}+04$

$1.20 \mathrm{E}-05, \quad 2.74 \mathrm{E}+04$

Modified top event frequency = (top event frequency) (risk achievement worth).

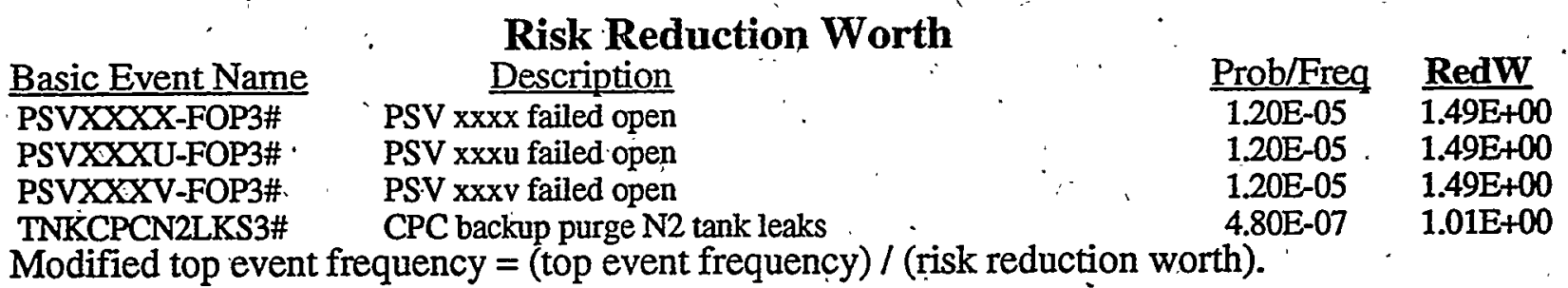


X-ESR-S-00001, Rev. 0

CPC Backup Purge Path Unavailable 
X-ESR-S-00001, Rev. 0

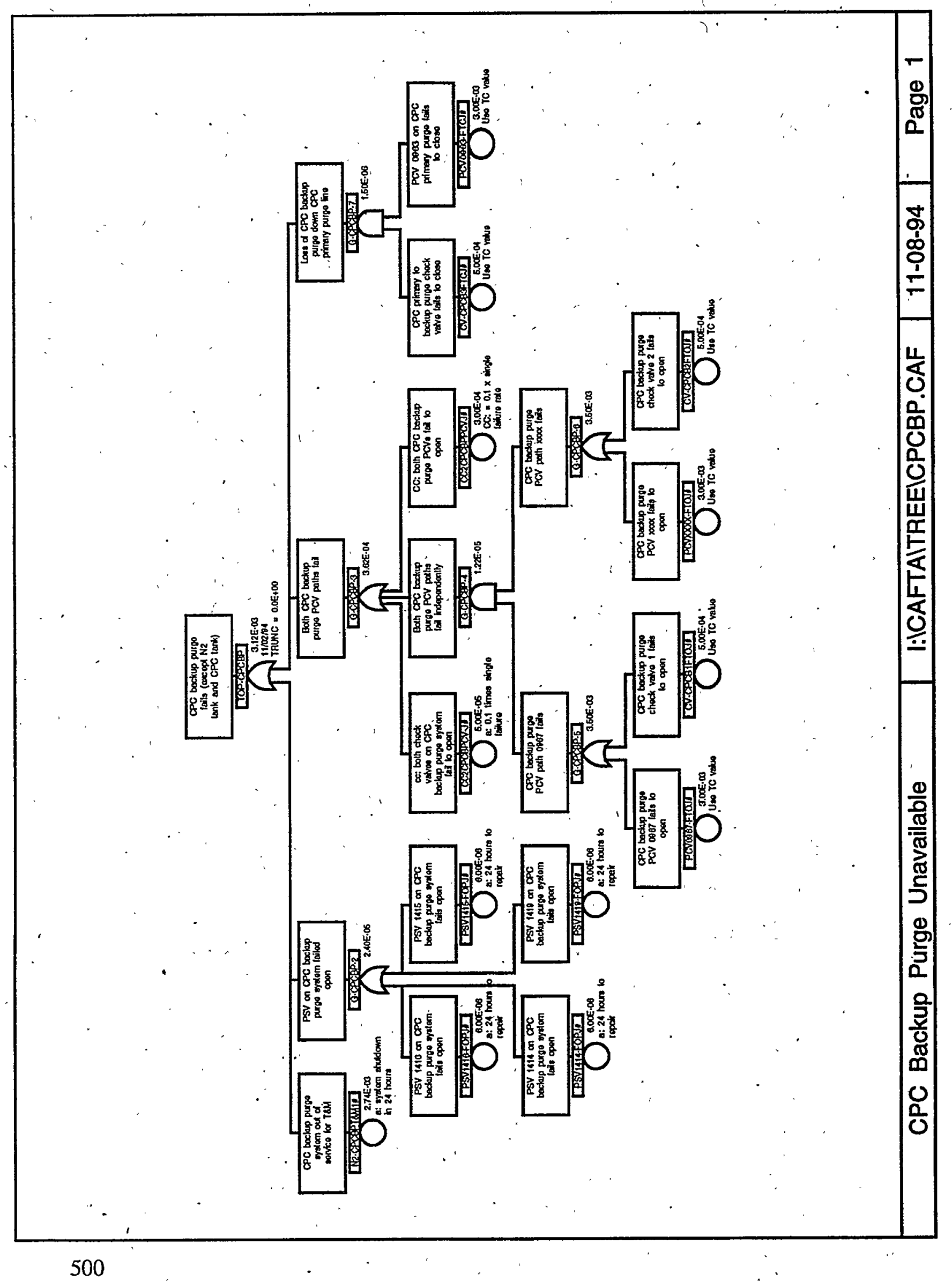


$12: 01 . \mathrm{AM}$

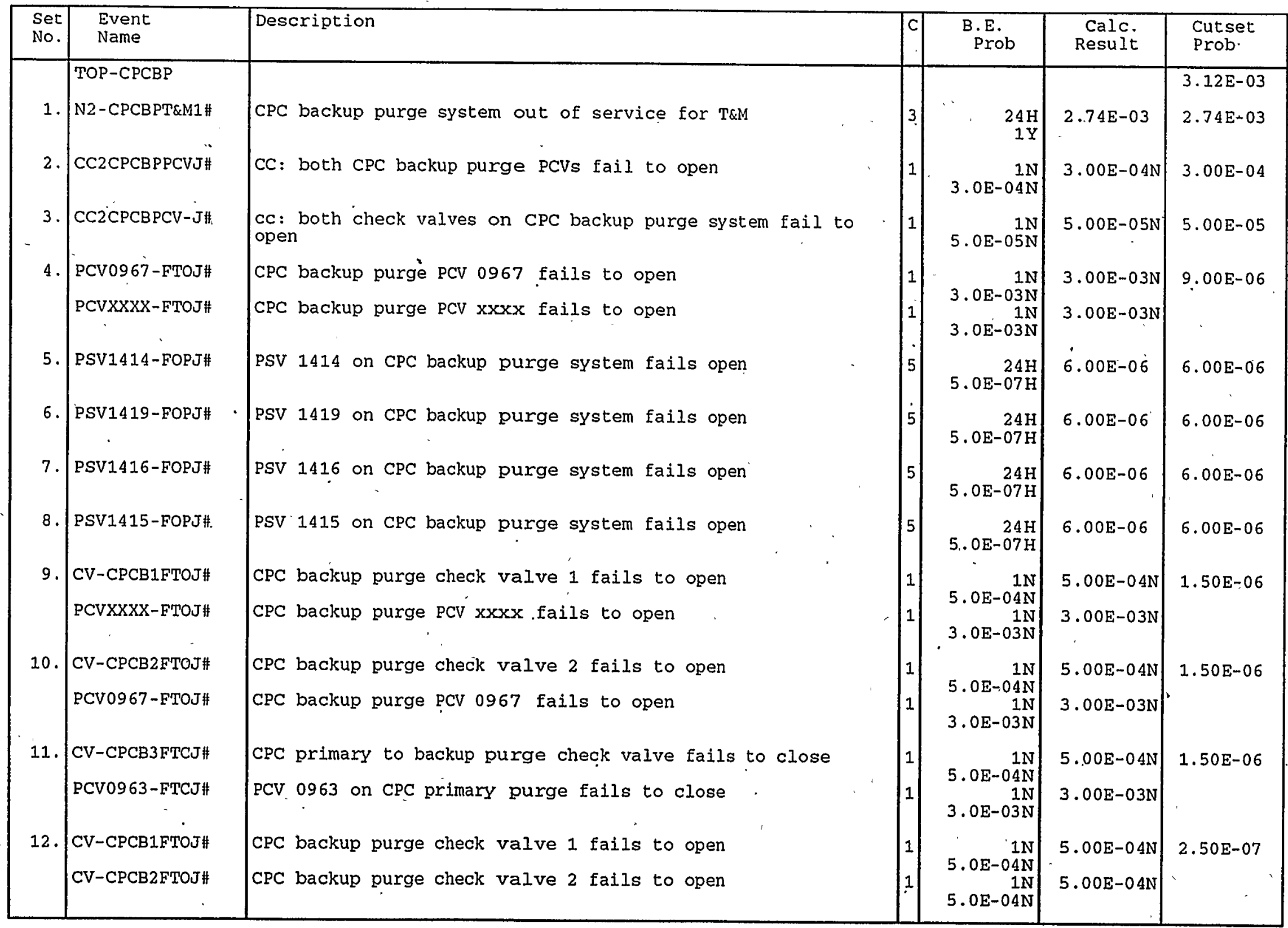


CPC backup purge fails (except $\mathrm{N} 2$ tank and $\mathrm{CPC}$ tank)

Top Event Probability: 3.12E-03

Basic Event Name
PSV1419-FOPJ\#
PSV1414-FOPJ\#
PSV1415-FOPJ\#
PSV1416-FOPJ\#
CC2CPCBPPCVJ
N2-CPCBPT\&M1\#
CC2CPCBPCV-J\#
CV-CPCB1FTOJ\#
CV-CPCB2FTOJ\#
PCV0967-FTOJ\#
PCVXXXX-FTOJ\#
CV-CPCB3FTCJ\#
PCV0963-FTCJ\#
MOdified top event

\section{- Risk Achievement Worth}

Description

PSV 1419 on CPC backup purge system fails open PSV 1414 on CPC backup purge system fails open PSV 1415 on CPC backup purge system fails open PSV 1416 on CPC backup purge system fails open CC: both CPC backup purge PCVs fail to open CPC backup purge system out of service for $T \& M$ cc: both check valves on CPC backup purge fail to open, CPC backup purge check valve 1 fails to open CPC backup purge check valve 2 fails to open CPC backup purge PCV 0967 fails to open CPC backup purge $P C V$ xxxx fails to open CPC primary to backup purge check valve fails to close PCV 0963 on CPC primary purge fails to close

\begin{tabular}{|c|c|c|}
\hline & Prob/Freq & AchW \\
\hline & $6.00 \mathrm{E}-06$ & $3.20 \mathrm{E}+02$ \\
\hline & 6.00E-06 & $\begin{array}{l}3.20 \mathrm{E}+02 \\
3.20 \mathrm{E}+02\end{array}$ \\
\hline & $6.00 \mathrm{E}-06$ & $3.20 E+02$ \\
\hline & $3.00 \mathrm{E}-04$ & $3.20 \mathrm{E}+02$ \\
\hline & $2.74 \mathrm{E}-03$ & $3.20 \mathrm{E}+02$ \\
\hline & 5.00E-05 & $3.20 \mathrm{E}+02$ \\
\hline & $5.00 \mathrm{E}-04$ & $2.12 E+00$ \\
\hline & $5.00 \mathrm{E}-04$ & $2.12 \mathrm{E}+00$ \\
\hline & $3.00 \mathrm{E}-03$ & $2.11 E+00$ \\
\hline & 3.00E-03 & $2.11 E+00$ \\
\hline & $5.00 \mathrm{E}-04$ & $1.96 \mathrm{E}+00$ \\
\hline & $3.00 \mathrm{E}-03$ & $1.16 E+00$ \\
\hline
\end{tabular}

Prob/Freq AchW

6.00E-06

$6.00 \mathrm{E}-06$

3.00E-04

$2.74 \mathrm{E}-03$

5.00E-05

$5.00 \mathrm{E}-04$

$5.00 \mathrm{E}-04$

$3.00 \mathrm{E}-03$

$5.00 \mathrm{E}-04$

worth).
$3.20 \mathrm{E}+02$

$320 \mathrm{E}+02$

$3.20 \mathrm{E}+02$

.

$3.20 \mathrm{E}+02$

$2.12 \mathrm{E}+00$

$2.12 \mathrm{E}+00$

$11 \mathrm{E}+00$

$1.96 \mathrm{E}+00$

$1.16 \mathrm{E}+00$

\section{Risk Reduction Worth}

\begin{tabular}{l} 
Basic Event Name \\
\hline N2-CPCBPT\&M1\# \\
CC2CPCBPPCVI\# \\
CC2CPCBPCV-J\#
\end{tabular}

\section{Description}

CPC backup purge system out of service for $T \& M$

CC: both CPC backup purge PCVs fail to open cc: both check valves on CPC backup purge fail to open

\begin{tabular}{cc} 
Prob/Freg & RedW \\
\hline $2.74 \mathrm{E}-03$ & $8.05 \mathrm{E}+00$ \\
$3.00 \mathrm{E}-04$ & $1.11 \mathrm{E}+00$ \\
$5.00 \mathrm{E}-05$ & $1.02 \mathrm{E}+00$
\end{tabular}

Note: Events that make-less than $1 \%$ difference to the importance factor are not included. Modified top event frequency $=$ (top event frequency) $/$ (risk reduction worth). 


\section{CPC Primary Purge Failure}




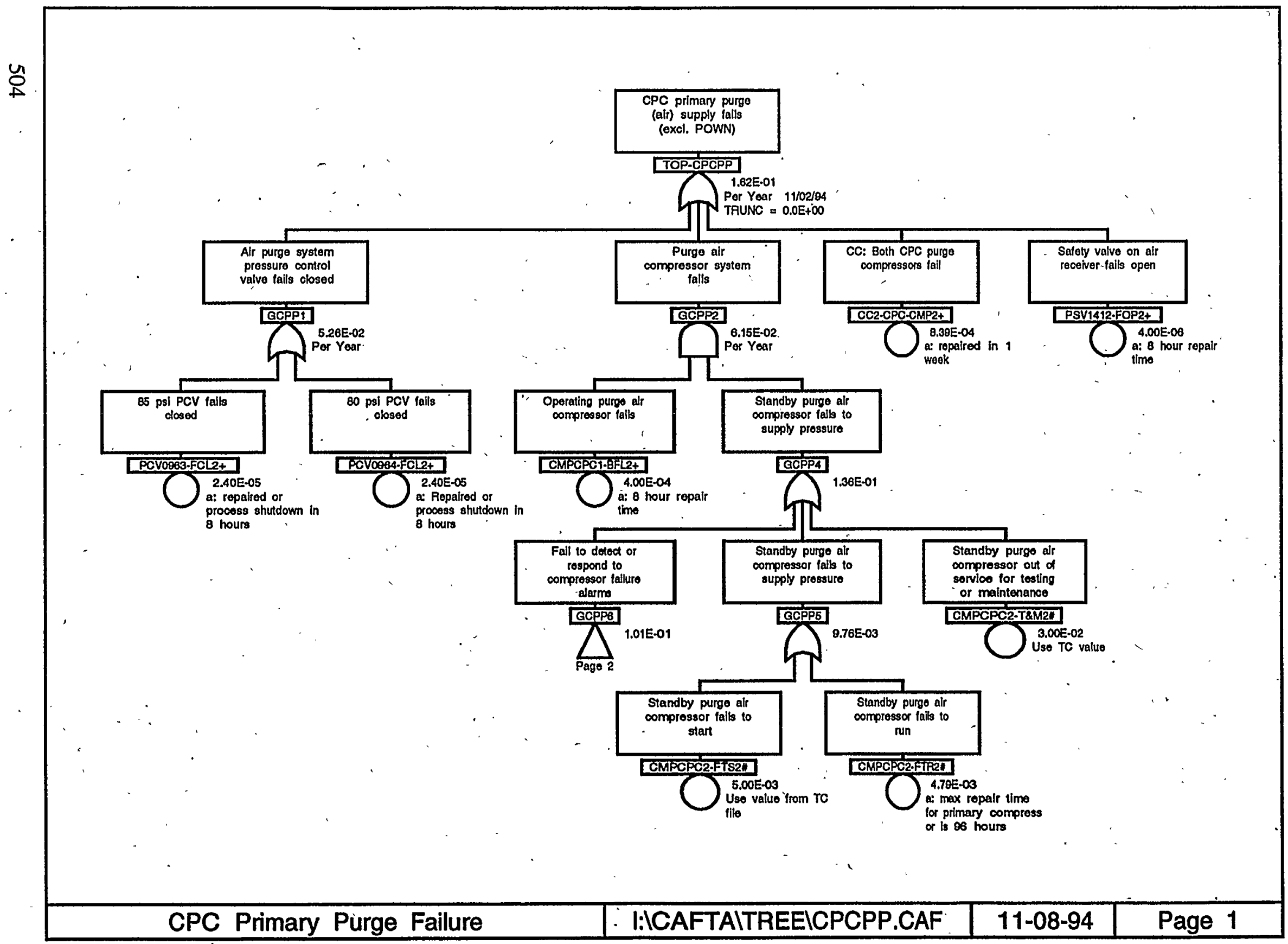




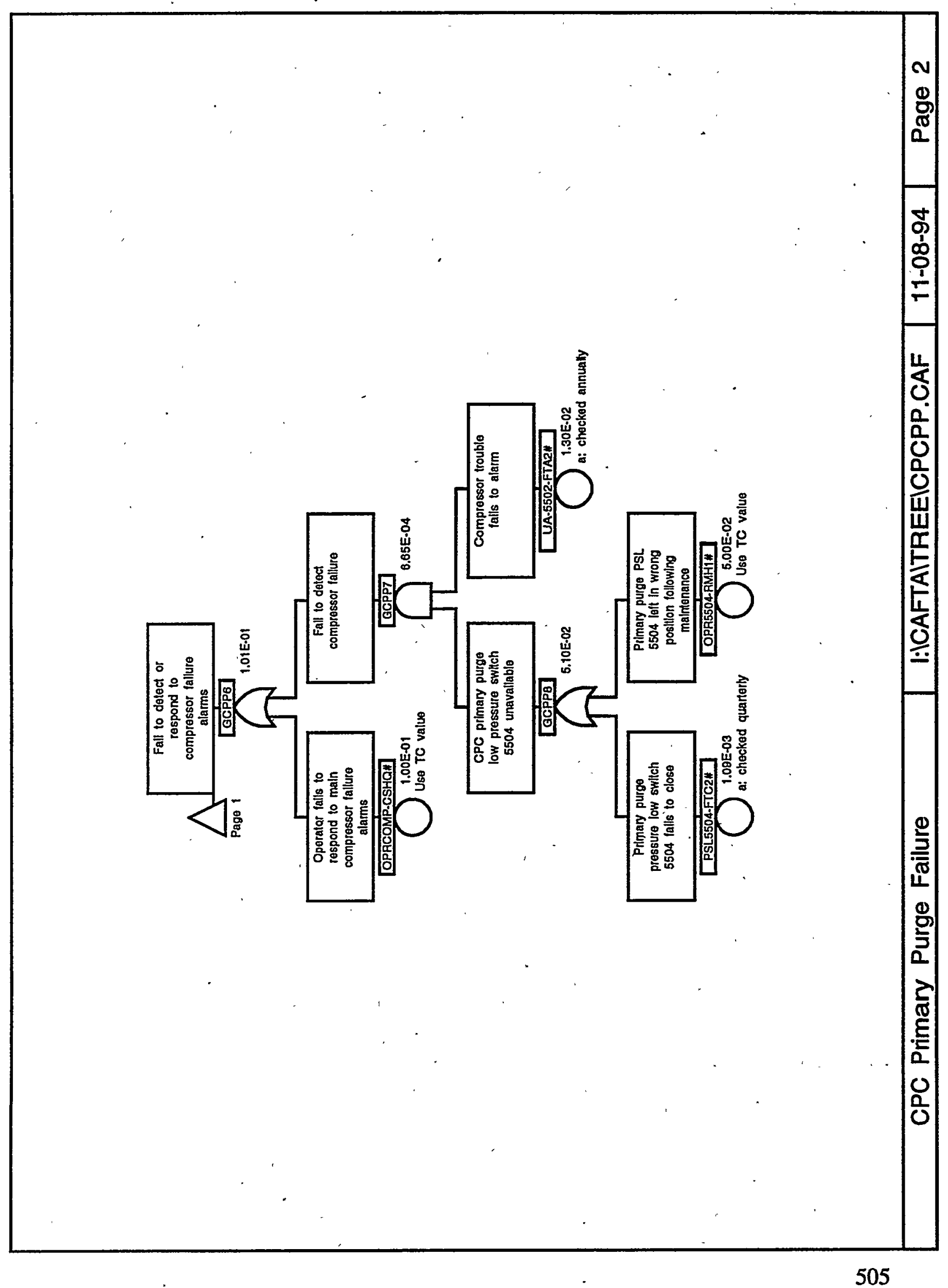




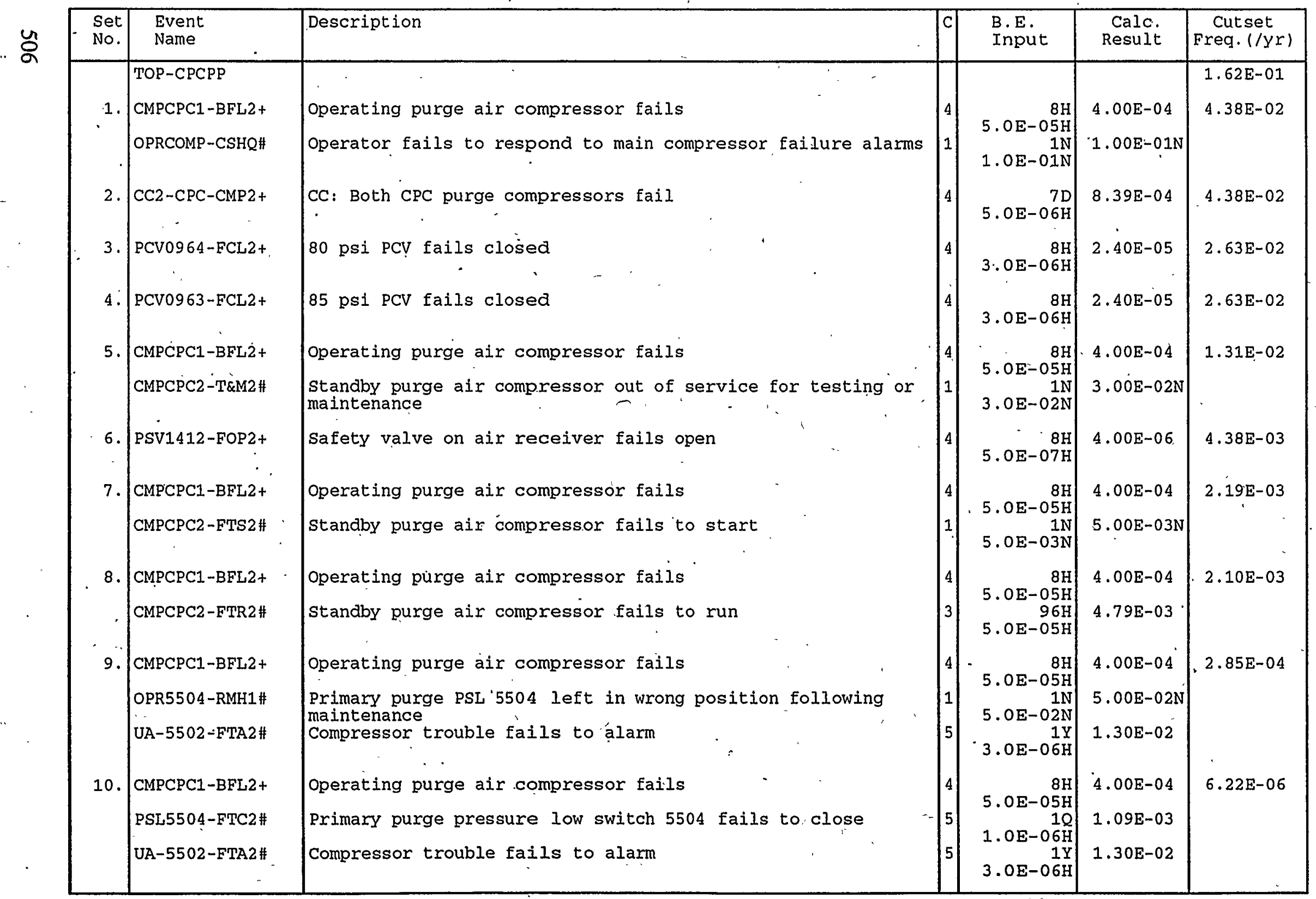


CPC primary purge (air) supply fails (excl. POWN)

Top Event Frequency: 1.62E-01/YR

Basic Event Name
PCV0964-FCL2+-I
PCV0963-FCL2+-I
PSV1412-FOP2+-I
CC2-CPC-CMP2+-I
CMPCPC1-BFL2+-I
CMPCPC2-FTR2\#
CMPCPC2-FTS2\#
CMPCPC2-T\&M2\#
OPRCOMP-CSHQ\#
UA-5502-FTA2\#
PSL5504-FTC2\#
OPR5504-RMH1\#
Modified top event fret

\section{Risk Achievement Worth}

\author{
Description
}

80 psi.PCV fails closed

85 psi PCV fails closed

Safety valve on air receiver fails open

CC: Both CPC purge compressors fail

Operating purge air compressor fails

Standby purge air compressor fails to run

Standby purge air compressor fails to start

Standby purge air compressor out of service for.T\&M

Operator fails to respond to main compressor failure alarms

Compressor trouble fails to alarm

Primary purge pressure low switch 5504 fails to close

Primary purge PSL 5504 left in wrong position after maint.

\begin{tabular}{|c|c|c|}
\hline & Prob/Freg & $\Delta \operatorname{chW}$ \\
\hline & $3.00 \mathrm{E}-06 / \mathrm{H}$ & $5.40 \mathrm{E}+$ \\
\hline & $3.00 \mathrm{E}-06 / \mathrm{H}$ & 5.4 \\
\hline & $5.00 \mathrm{E}$ & $5.40 \mathrm{E}$ \\
\hline & $4.99 \mathrm{I}$ & $5.40 \mathrm{I}$ \\
\hline & 5.0 & \\
\hline & 4.791 & 3.6 \\
\hline & $5.00 \mathrm{E}$ & $3.69 \mathrm{E}+0$ \\
\hline & 3.00E-0 & $3.62 \mathrm{E}+0$ \\
\hline & & \\
\hline & 1.3 & \\
\hline & 1.0 & \\
\hline & 5.00 & \\
\hline
\end{tabular}

Prob/Freg Achw

$3.00 \mathrm{E}-06 / \mathrm{H} \quad 5.40 \mathrm{E}+04$

$5.00 \mathrm{E}-07 / \mathrm{H} \quad 5.40 \mathrm{E}+04$

$4.99 \mathrm{E}-06 / \mathrm{H} \quad 5.40 \mathrm{E}+04$

$5.00 \mathrm{E}-05 / \mathrm{H} \quad 7.35 \mathrm{E}+03$

$4.79 \mathrm{E}-03$

$5.00 \mathrm{E}-03$

$1.00 \mathrm{E}-01$

$1.30 \mathrm{E}-02$

$5.00 \mathrm{E}-02$
Basic Event Name CMPCPC1-BFL2+-I OPRCOMP-CSHQ\# CC2-CPC-CMP2+-I PCV0963-FCL2+IPCV0964-FCL2+-I CMPCPC2-T\&M2\# PSV1412-FOP2+-I CMPCPC2-FTS2\# CMPCPC2-FTR2\#

\section{Risk Reduction Worth}

\section{Description}

Operating purge air compressor fails

Operator fails to respond to main compressor failure alarms

CC: Both CPC purge compressors fail

85 psi PCV fails closed

80 psi PCV fails closed

Standby purge air compressor out of service for $T \& M$

Safety valve on air receiver fails open

Standby purge air compressor fails to start

Standby purge air compressor fails to run

\begin{tabular}{ll} 
Prob/Freq & RedW \\
\hline $5.00 \mathrm{E}-05 / \mathrm{H}$ & $1.61 \mathrm{E}+00$ \\
$1.00 \mathrm{E}-01$ & $1.37 \mathrm{E}+00$ \\
$4.99 \mathrm{E}-06 / \mathrm{H}$ & $1.37 \mathrm{E}+00$ \\
$3.00 \mathrm{E}-06 / \mathrm{H}$ & $1.19 \mathrm{E}+00$ \\
$3.00 \mathrm{E}-06 / \mathrm{H}$ & $1.19 \mathrm{E}+00$ \\
$3.00 \mathrm{E}-02$ & $1.09 \mathrm{E}+00$ \\
$5.00 \mathrm{E}-07 / \mathrm{H}$ & $1.03 \mathrm{E}+00$ \\
$5.00 \mathrm{E}-03$ & $1.01 \mathrm{E}+0$ \\
$4.79 \mathrm{E}-03$ & $1.01 \mathrm{E}+00$
\end{tabular}

Note: Events that make less than $1 \%$ difference to the importance factor are not included. Modified top event frequency $=$ (top event frequency) $/$ (risk reduction worth). 
X-ESR-S-00001, REV: 0

Backup Power Unavailable

;

508 


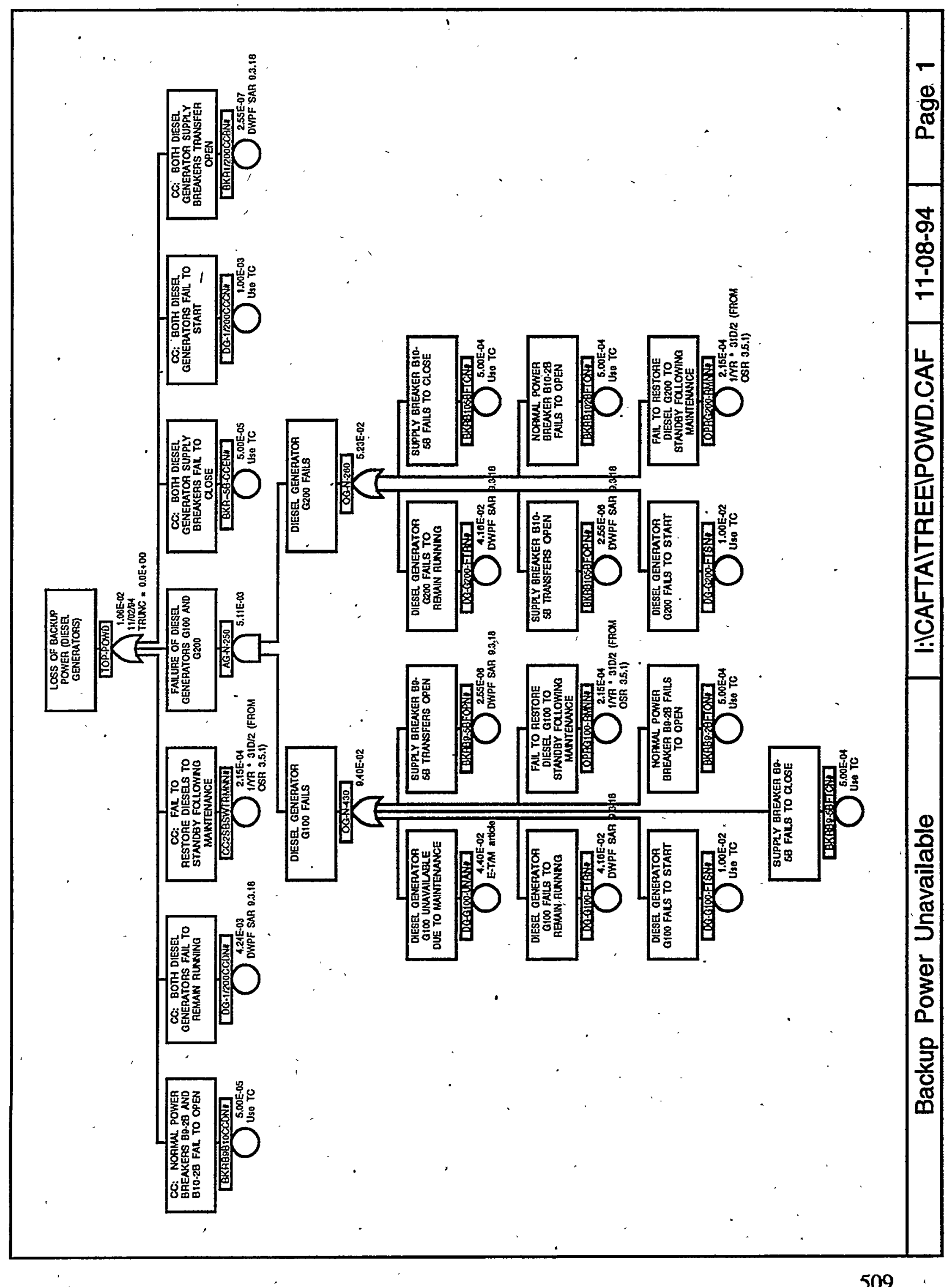


Cutsets for I: \CAFTA ICUT \POWD. CSR

11/08/94 12:05 AM (CONT.)

\begin{tabular}{|c|c|c|c|c|c|c|}
\hline $\begin{array}{l}\text { Set } \\
\text { No. }\end{array}$ & $\begin{array}{l}\text { Event } \\
\text { Name }\end{array}$ & Description & $\mathrm{C}$ & $\begin{array}{l}\text { B.E. } \\
\text { Prob }\end{array}$ & $\begin{array}{l}\text { Calc. } \\
\text { Result }\end{array}$ & $\begin{array}{l}\text { Cutset } \\
\text { Prob }\end{array}$ \\
\hline 12. & $\begin{array}{l}\text { BKRB105BFTCN\# } \\
\text { DG-G100-UNAN\# }\end{array}$ & $\begin{array}{l}\text { SUPPLY BREAKER B10-5B FAILS TO CLOSE } \\
\text { DIESEL GENERATOR G100 UNAVAILABLE DUE TO MAINTENANCE }\end{array}$ & 1 & $\begin{array}{r}1 \mathrm{~N} \\
5.0 \mathrm{E}-04 \mathrm{~N} \\
1 \mathrm{~N} \\
4.4 \mathrm{E}-02 \mathrm{~N}\end{array}$ & $\begin{array}{l}5.00 \mathrm{E}-04 \mathrm{~N} \\
4.40 \mathrm{E}-02 \mathrm{~N}\end{array}$ & $2.20 \mathrm{E}-05$ \\
\hline 13. & $\begin{array}{l}\text { BKRB102BFTON\# } \\
\text { DG-G100-UNAN\# }\end{array}$ & $\begin{array}{l}\text { NORMAL POWER BREAKER BI0-2B FAILS TO OPEN } \\
\text { DIESEL GENERATOR GIOO UNAVAILABLE DUE TO MAINTENANCE } \\
.\end{array}$ & $\begin{array}{l}1 \\
1\end{array}$ & $\begin{array}{r}1 \mathrm{~N} \\
5.0 \mathrm{E}-04 \mathrm{~N} \\
1 \mathrm{~N} \\
4.4 \mathrm{E}-02 \mathrm{~N}\end{array}$ & $\begin{array}{l}5.00 \mathrm{E}-04 \mathrm{~N} \\
4.40 \mathrm{E}-02 \mathrm{~N}\end{array}$ & $2.20 \mathrm{E}-05$ \\
\hline 14. & $\begin{array}{l}\text { BKRB9-5BFTCN\# } \\
\text { DG-G200-FTRN\# }\end{array}$ & $\begin{array}{l}\text { SUPPLY BREAKER B9-5B FAILS TO CLOSE } \\
\text { DIESEL GENERATOR G200 FAILS TO REMAIN RUNNING }\end{array}$ & $\begin{array}{l}1 \\
3\end{array}$ & $\begin{array}{r}1 \mathrm{~N} \\
5.0 \mathrm{E}-04 \mathrm{~N} \\
8.5 \mathrm{H} \\
5.0 \mathrm{E}-03 \mathrm{H}\end{array}$ & $\begin{array}{l}5.00 \mathrm{E}-04 \mathrm{~N} \\
4.16 \mathrm{E}-02\end{array}$ & $2.08 \mathrm{E}-05$ \\
\hline $15:$ & $\begin{array}{l}\text { BKRB102BFTON\# } \\
\text { DG-G100-FTRN\# }\end{array}$ & $\begin{array}{l}\text { NORMAL POWER BREAKER B10-2B FAILS TO OPEN } \\
\text { DIESEL GENERATOR G100 FAILS TO REMAIN RUNNING }\end{array}$ & $\begin{array}{l}1 \\
3\end{array}$ & $\begin{array}{r}1 \mathrm{~N} \\
5.0 \mathrm{E}-04 \mathrm{~N} \\
8.5 \mathrm{H} \\
5.0 \mathrm{E}-03 \mathrm{H}\end{array}$ & $\begin{array}{l}5.00 \mathrm{E}-04 \mathrm{~N} \\
4.16 \mathrm{E}-02\end{array}$ & $2.08 \mathrm{E}-05$ \\
\hline 16. & $\begin{array}{l}\text { BKRB9-2BETON\# } \\
\text { DG-G2 00-FTRN\# }\end{array}$ & $\begin{array}{l}\text { NORMAL POWER BREATER B9-2B FAILS TO OPEN. } \\
\text { DIESEL GENERATOR G200 FAILS TO REMAIN RUNNING }\end{array}$ & $\begin{array}{l}1 \\
3\end{array}$ & $\begin{array}{r}1 \mathrm{~N} \\
5.0 \mathrm{E}-04 \mathrm{~N} \\
8.5 \mathrm{H} \\
5.0 \mathrm{E}-03 \mathrm{H}\end{array}$ & $\begin{array}{l}5.00 \mathrm{E}-04 \mathrm{~N} \\
4.16 \mathrm{E}-02\end{array}$ & $2.08 \mathrm{E}-05$ \\
\hline 17. & $\begin{array}{l}\text { BKRB105BFTCN\# } \\
\text { DG-G100-FTRN\# }\end{array}$ & $\begin{array}{l}\text { SUPPLY BREAKER B10-5B FAILS TO CLOSE } \\
\text { DIESEL GENERATOR G100 FAILS TO REMAIN RUNNING }\end{array}$ & $\begin{array}{l}1 \\
3\end{array}$ & $\begin{array}{r}1 \mathrm{~N} \\
5.0 \mathrm{E}-04 \mathrm{~N} \\
8.5 \mathrm{H} \\
5.0 \mathrm{E}-03 \mathrm{H}\end{array}$ & $\begin{array}{l}5.00 \mathrm{E}-04 \mathrm{~N} \\
4.16 \mathrm{E}-02\end{array}$ & $2.08 \mathrm{E}-05$ \\
\hline 18. & $\begin{array}{l}\text { DG-G100-UNAN\# } \\
\text { OPRG200-RMNN\# }\end{array}$ & $\begin{array}{l}\text { DIESEL GENERATOR G100 UNAVAILABLE DUE TO MAINTENANCE } \\
\text { FAIL TO RESTORE DIESEL G200 TO STANDBY FOLLOWING MAINTENANCE }\end{array}$ & $\begin{array}{l}1 \\
1\end{array}$ & $\begin{array}{r}1 \mathrm{~N} \\
4.4 \mathrm{E}-02 \mathrm{~N} \\
4.3 \mathrm{E}-02 \mathrm{~N} \\
5.0 \mathrm{E}-03 \mathrm{~N}\end{array}$ & $\begin{array}{l}4.40 \mathrm{E}-02 \mathrm{~N} \\
2.15 \mathrm{E}-04 \mathrm{~N}\end{array}$ & $9.46 \mathrm{E}-06$ \\
\hline 19. & $\begin{array}{l}\text { DG-G100-FTRN\# } \\
\text { OPRG200-RMNN\# }\end{array}$ & $\begin{array}{l}\text { DIESEL GENERATOR G100 FAILS TO REMAIN RUNNING } \\
\text { FAIL TO RESTORE DIESEL G200 TO STANDBY FOLLOWING MAINTENANCE }\end{array}$ & 3 & $\begin{array}{r}8.5 \mathrm{H} \\
5.0 \mathrm{E}-03 \mathrm{H} \\
4.3 \mathrm{E}-02 \mathrm{~N} \\
5.0 \mathrm{E}-03 \mathrm{~N}\end{array}$ & $\begin{array}{l}4.16 \mathrm{E}-02 \\
2.15 \mathrm{E}-04 \mathrm{~N}\end{array}$ & $8.95 E-06$ \\
\hline 20. & $\begin{array}{l}\text { DG-G200-FTRN\# } \\
\text { OPRG100-RMNN\# }\end{array}$ & $\begin{array}{l}\text { DIESEL GENERATOR G200 FAILS TO REMAIN RUNNING } \\
\text { FAIL TO RESTORE DIESEL G100 TO STANDBY FOLLOWING MAINTENANCE }\end{array}$ & 3 & $\begin{array}{r}8.5 \mathrm{H} \\
5.0 \mathrm{E}-03 \mathrm{H} \\
4.3 \mathrm{E}-02 \mathrm{~N} \\
5.0 \mathrm{E}-03 \mathrm{~N}\end{array}$ & $\begin{array}{l}4.16 \mathrm{E}-02 \\
2.15 \mathrm{E}-04 \mathrm{~N}\end{array}$ & $8.95 \mathrm{E}-06$ \\
\hline $21:$ & BKRB9-2BFTON\# & NORMAL POWER BREAKER B9-2B FAILS TO OPEN & 1 & $\begin{array}{r}1 \mathrm{~N} \\
5.0 \mathrm{E}-04 \mathrm{~N}\end{array}$ & $5.00 \mathrm{E}-04 \mathrm{~N}$ & $5.00 \mathrm{E}-06$ \\
\hline
\end{tabular}


Cutsets for I: \CAFTA \CUT\POWD.CSR

11/08/94 12:05 AM (CONT.)

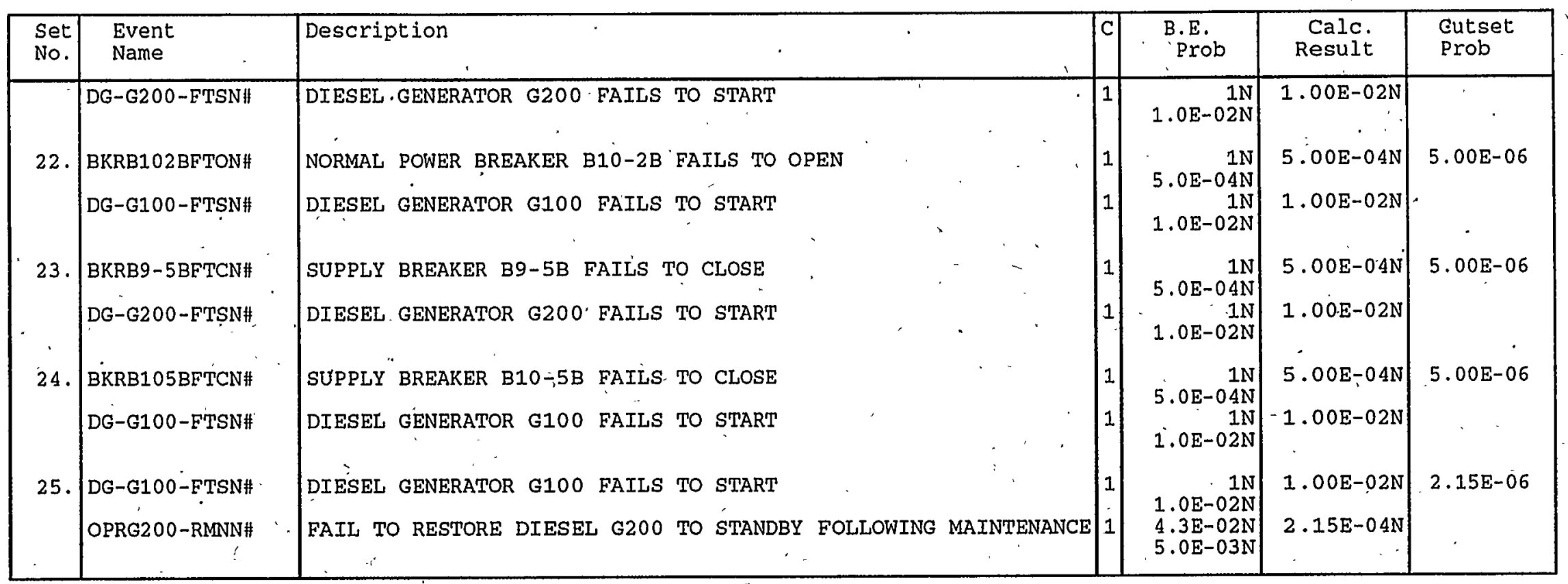


LOSS OF BACKUP POWER (DIESEL GENERATORS)

Top Event Probability: 1.06E-02

\begin{tabular}{|c|c|c|c|}
\hline Basic Event Name & $\begin{array}{l}\text { Risk Achievement Worth } \\
\text { Description }\end{array}$ & Prob/Freg & $\underline{A c h W}$ \\
\hline BKR-5B-CCEN\# & CC: BOTH DIESEL SUPPLY BRKRS FAIL TO CLOSE & $5.00 \mathrm{E}-05$ & $\overrightarrow{9.41 \mathrm{E}+01}$ \\
\hline CC2SBSWTRMNN\# • & CC: FAIL TO RESTORE DIESELS TO STANDBY & 2.15E-04 & $9.41 \mathrm{E}+01$ \\
\hline BKR1/200CCBN\# & CC: BOTH DIESEL SUPPLY BRKRS TRANSFER OPEN & $2.55 \mathrm{E}-07$ & $9.41 E+01$ \\
\hline BKRB9B10CCDN\# & CC: NORM BRKRS B9-2B AND B10-2B FAIL TO OPEN & $5.00 \mathrm{E}-05$ & $9.41 \mathrm{E}+01$ \\
\hline DG-1/200CCCN\# & CC: BOTH DIESEL GENERATORS FAIL TO START & $1.00 \mathrm{E}-03$ & $9.41 \mathrm{E}+01$ \\
\hline DG-1/200CCDN\# & CC: BOTH DIESELS FAIL TO REMAIN RUNNING & $4.24 \mathrm{E}-03$ & $9.41 E+01$ \\
\hline BKRB105BFOPN\# & SUPPLY BREAKER B10-5B TRANSFERS OPEN & $2.55 \mathrm{E}-06$ & $9.76 \mathrm{E}+00$ \\
\hline OPRG200-RMNN\# & FAIL TO RESTORE DIESEL G200 TO STANDBY & 2.15E-04 & $9.75 \mathrm{E}+00$ \\
\hline BKRB105BFTCN\# & SUPPLY BREAKER B10-5B FAILS TO CLOSE & 5.00E-04 & $9.75 \mathrm{E}+00$ \\
\hline BKRB102BFTON\# & NORMAL POWER BREAKER B10-2B FAILS TO OPEN & $5.00 \mathrm{E}-04$ & $9.75 E+00$ \\
\hline DG-G200-FTSN\# & DIESEL GENERATOR G200 FAILS TO START & $1.00 \mathrm{E}-02$ & $9.67 \mathrm{E}+00$ \\
\hline DG-G200-FTRN\# & DIESEL G200 FAIIS TO REMAIN RUNNING & $4.16 \mathrm{E}-02$ & $9.41 E+00$ \\
\hline BKRB9-5BFOPN\# & SUPPLY BREAKER B9.5B TRANSFERS OPEN & 2.55E-06 & $5.87 \mathrm{E}+00$ \\
\hline OPRG100-RMIN\# & FAIL TO RESTORE DIESEL G100 TO STANDBY & 2.15E-04 & $5.87 \mathrm{E}+00$ \\
\hline BKRB9-5BFTCN\# & SUPPLY BREAKER B9-5B FAILS TO CLOSE & 5.00E-04 & $5.87 \mathrm{E}+00$ \\
\hline BKRB9-2BFTON\# & NORMAL POWER BREAKER B9-2B FAIIS TO OPEN & $5.00 \mathrm{E}-04$ & $5.87 E+00$ \\
\hline DG-G100-FTSN\# & DIESEL GENERATOR G100 FAILS TO START & $1.00 \mathrm{E}-02$ & $5.83 E+00$ \\
\hline DG-G100-FTRN\# & DIESEL G100 FAILS TO REMAIN RUNNING & 4.16E-02 & $5.68 \mathrm{E}+00$ \\
\hline 100-UNAN\# & DIESEL G100 UNAVAILABLE DUE TO T\&M & 4.40E-02 & $5.67 \mathrm{E}+00$ \\
\hline
\end{tabular}

Modified top event frequency $=$ (top event frequency) $\mathrm{x}$ (risk achievement worth).

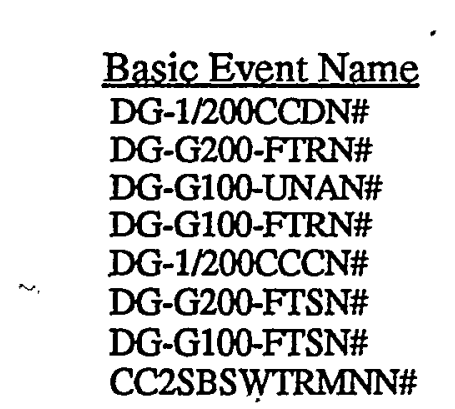

Basic Event Name

-1/200CCDN\#

DG-G100-FTRN\#

DG-1/200CCCN\#

CC2SBSWTRMNN\#

\section{Risk Reduction Worth}

CC: BOTH DIESELS FAIL TO REMAIN RUNNING
DIESEL G200 FAILS TO REMAIN RUNNING
DIESEL G100 UNAVAILABLE DUE TO T\&M
DIESEL G100 FAILS TO REMAIN RUNNING
CC: BOTH DIESEL GENERATORS FAIL TO START
DIESEL GENERATOR G200 FAILS TO START
DIESEL GENERATOR G100 FAILS TO START
CC: FAIL TO RESTORE DIESELS TO STANDBY

\begin{tabular}{|c|c|}
\hline $\mathrm{ob} /$ Frec & $\mathbf{W}$ \\
\hline $4.24 \mathrm{E}-03$ & $1.66 \mathrm{E}+00$ \\
\hline $4.16 \mathrm{E}-02$ & $1.60 \mathrm{E}+00$ \\
\hline 4.40 & $1.28 \mathrm{E}+$ \\
\hline 4.16 & $1.26 \mathrm{E}$ \\
\hline 1.00 & $1.10 \mathrm{E}$ \\
\hline $1.00 \mathrm{E}-02$ & $1.10 \mathrm{E}$ \\
\hline $1.00 \mathrm{E}-02$ & 1.0 \\
\hline & \\
\hline
\end{tabular}

Note: Events that make less than $1 \%$ difference to the importance factor are not included. Modified top event frequency = (top event frequency) / ('risk reduction worth). 
X-ESR-S-00001, REV. 0

\section{Normal Power Failure}

i1 


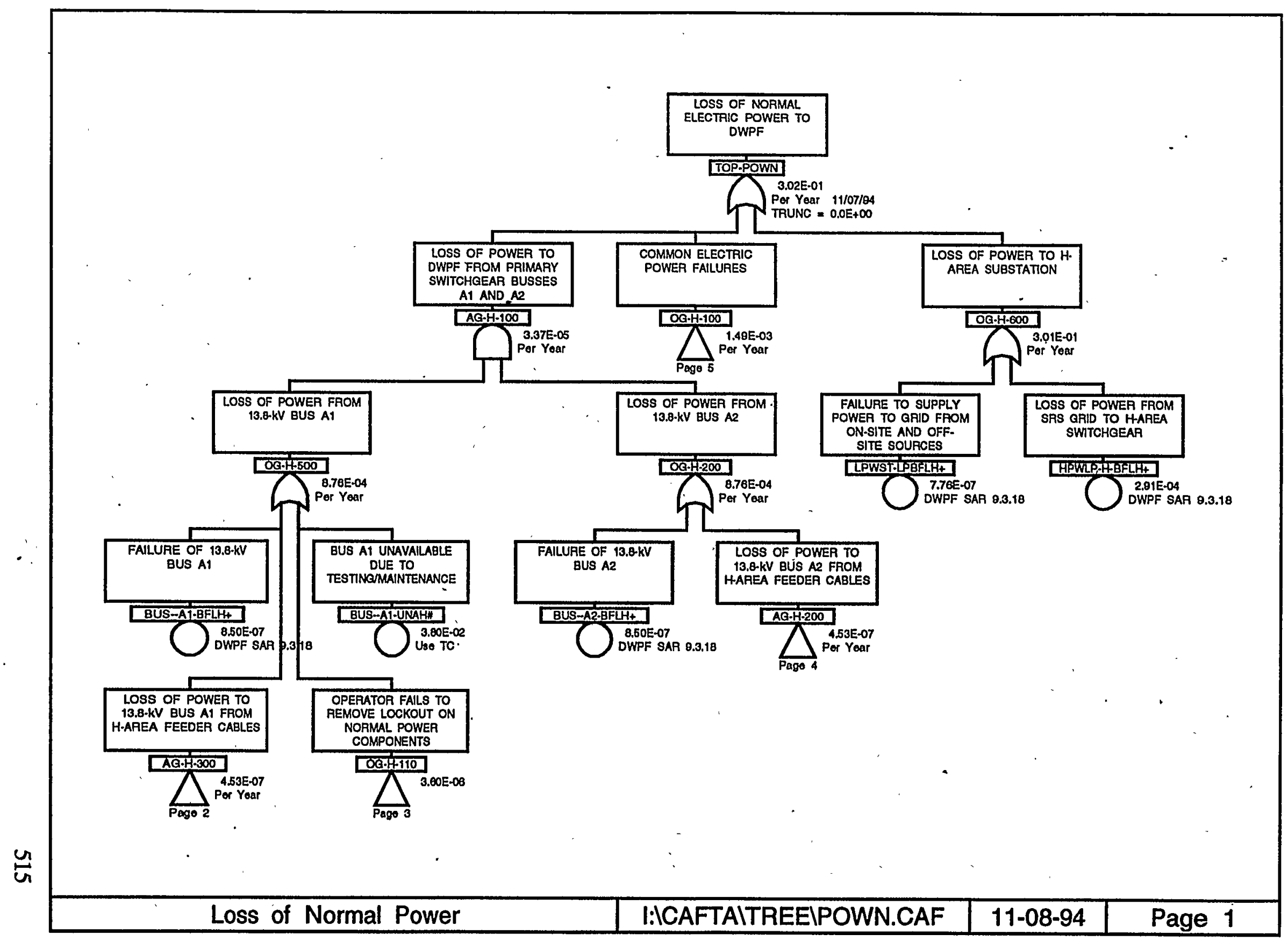


X-ESR-S-00001, REV. 0

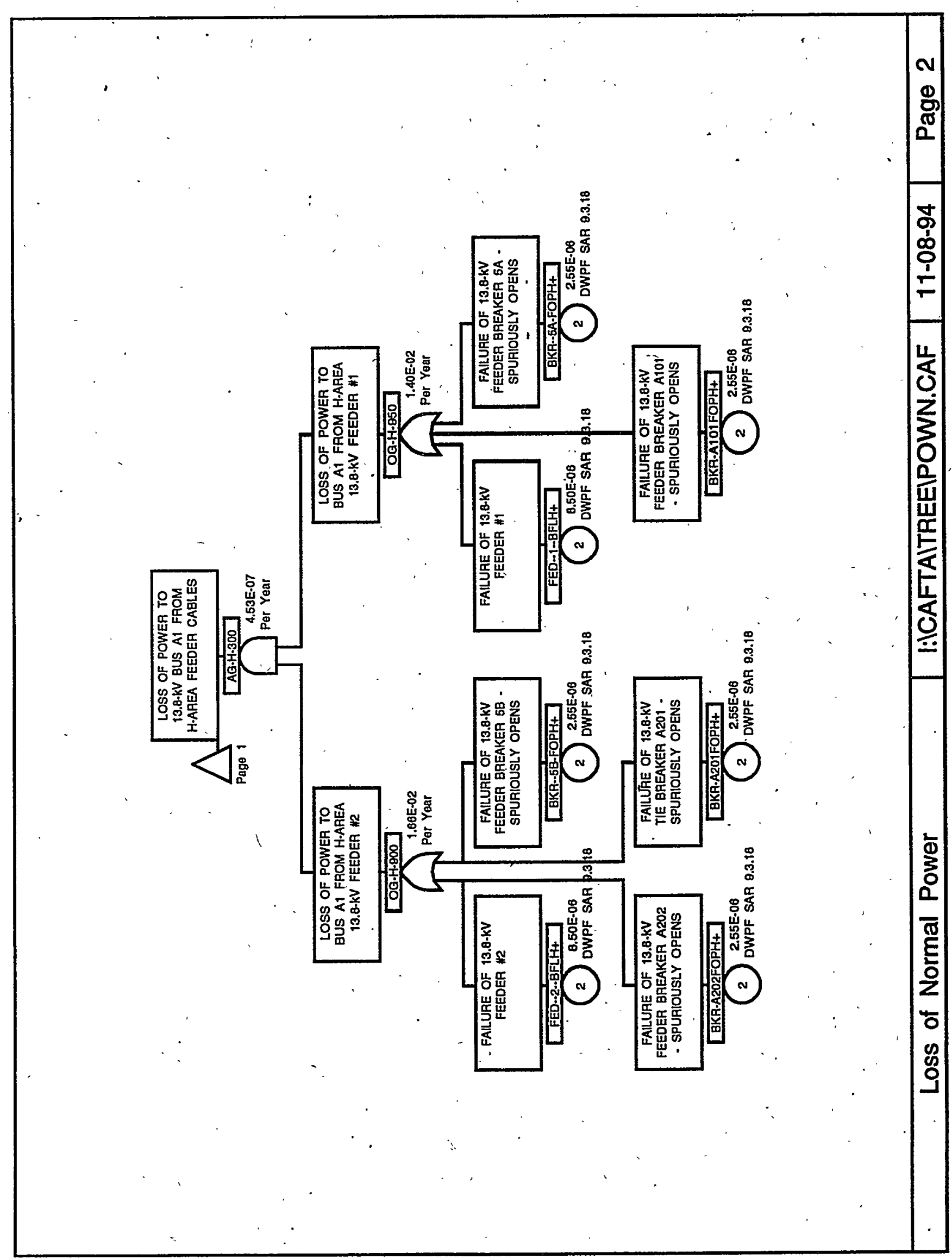




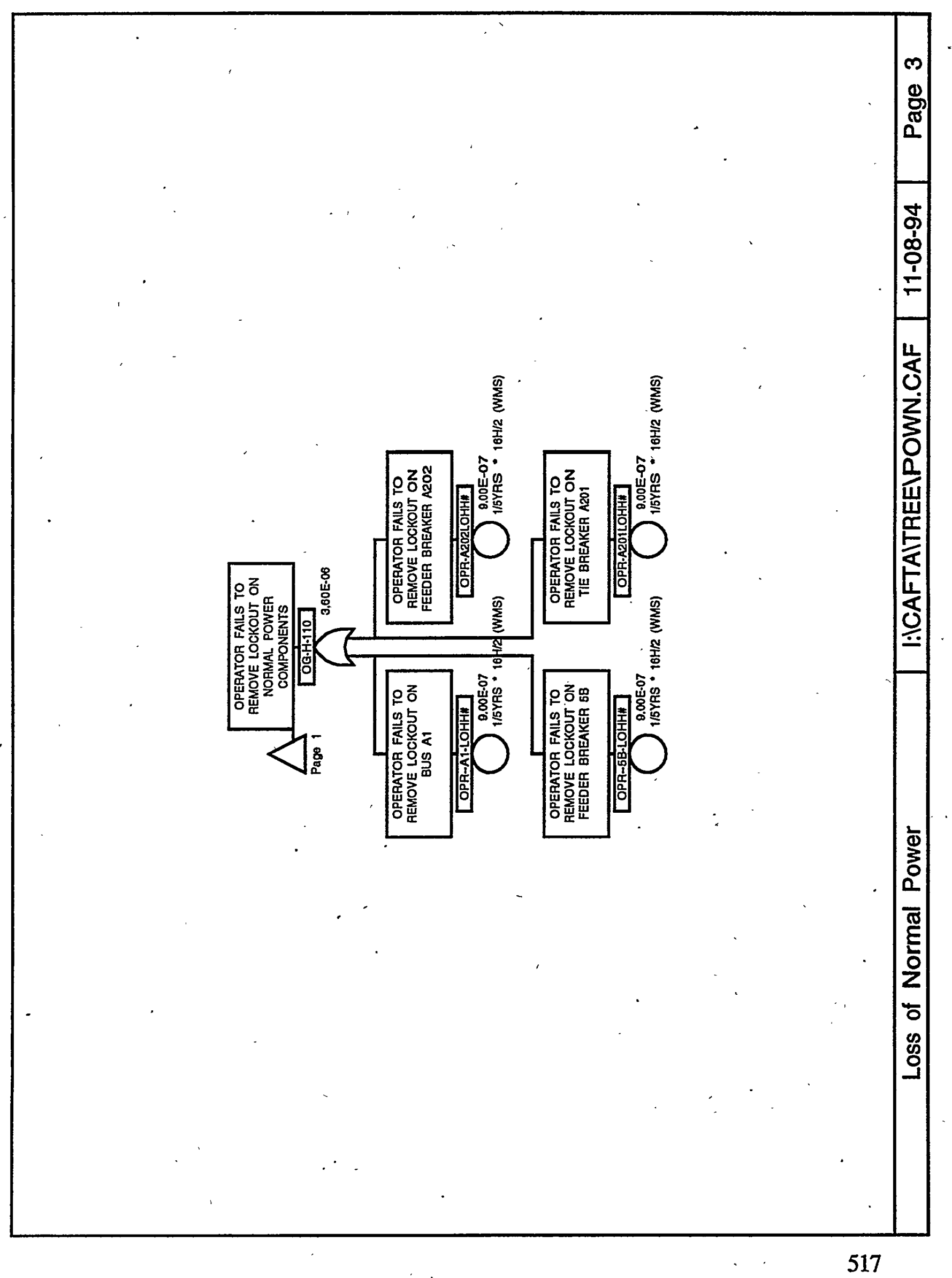


X-ESR-S-00001, REV. 0

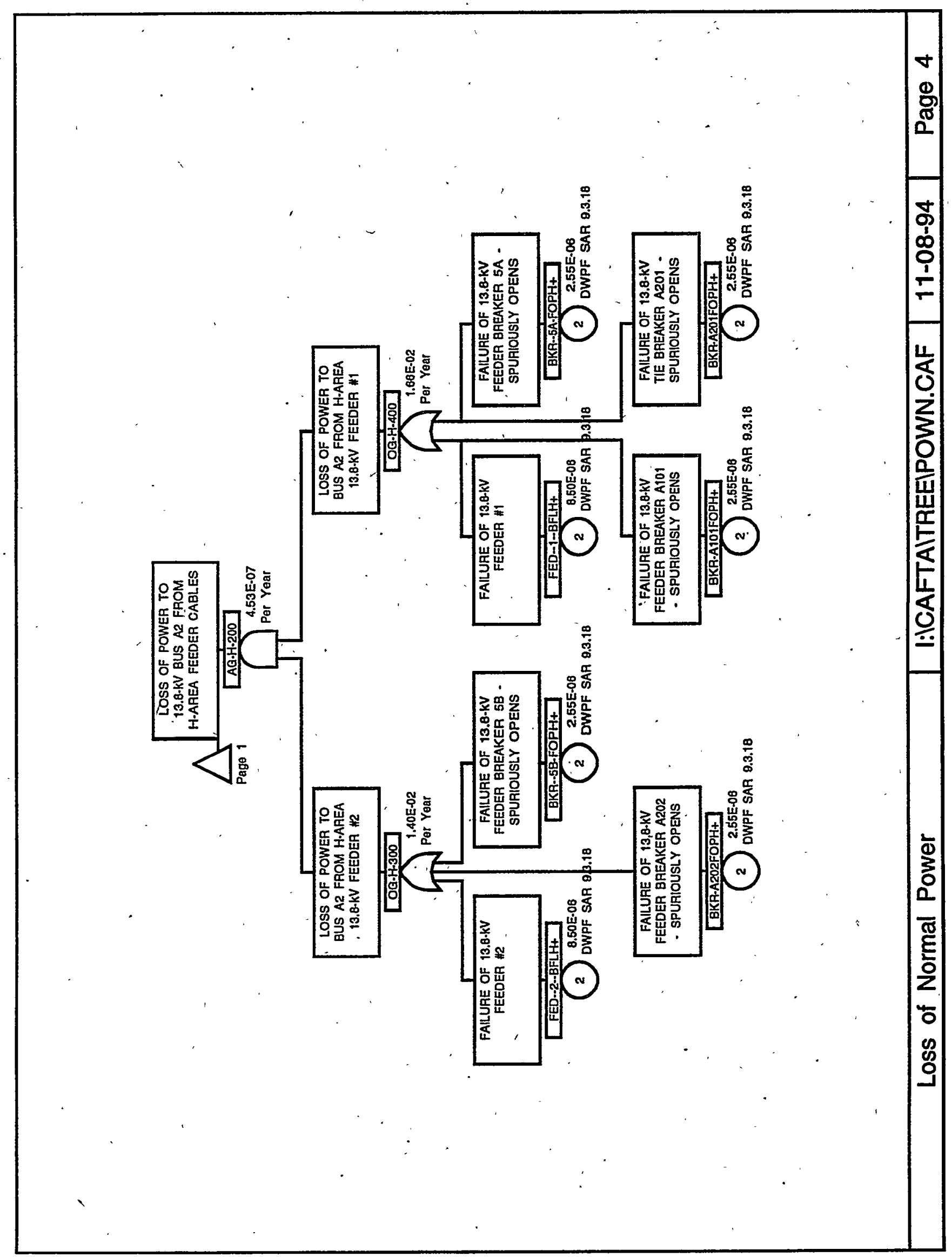




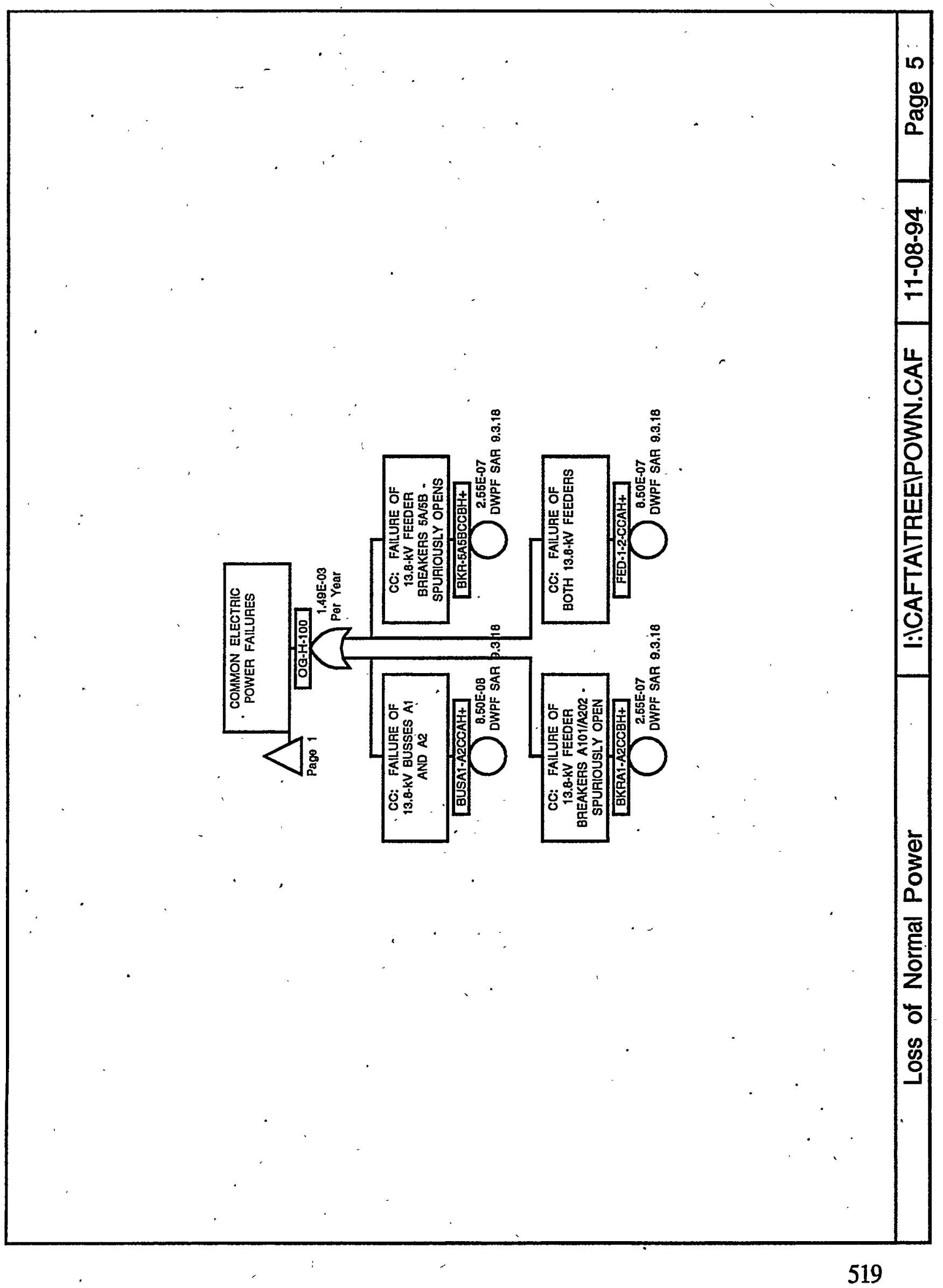




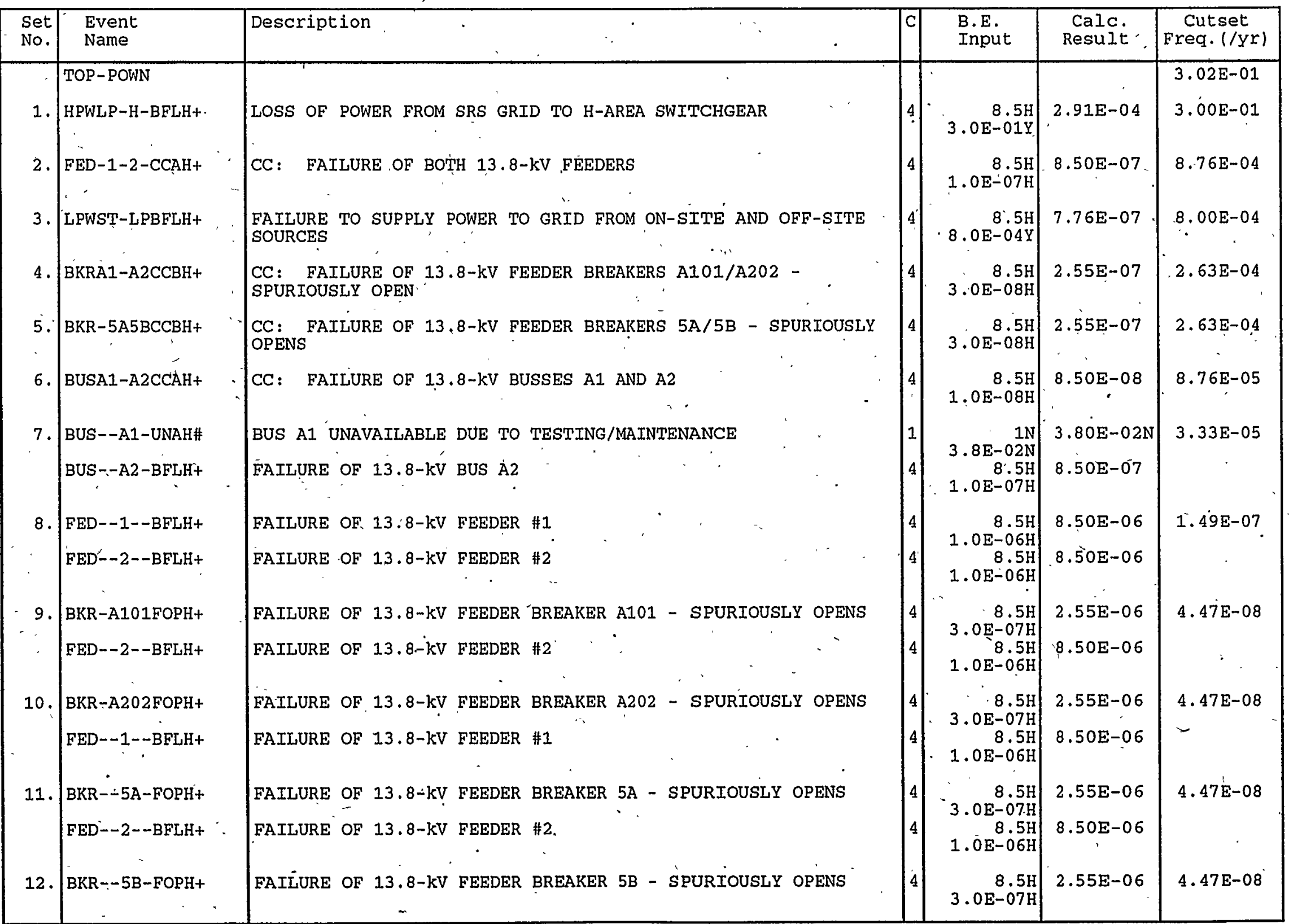


Cutsets for I: \CAFTA \CUT\POWN. CSR

11/08/94 12:06 AM (CONT.)

\begin{tabular}{|c|c|c|c|c|c|c|}
\hline $\begin{array}{l}\text { Set } \\
\text { No. }\end{array}$ & $\begin{array}{l}\text { Event } \\
\text { Name }\end{array}$ & Description & $\mathrm{C}$ & $\begin{array}{l}\text { B.E. } \\
\text { Input }\end{array}$ & $\begin{array}{l}\text { Calc. } \\
\text { Result }\end{array}$ & $\begin{array}{c}\text { Cutset } \\
\text { Freq. }(/ y r)\end{array}$ \\
\hline & FED--1--BFLH+ & FAILURE OF 13.8-kV FEEDER \#1 & 4 & $\begin{array}{r}8.5 \mathrm{H} \\
1.0 \mathrm{E}-06 \mathrm{H}\end{array}$ & $8.50 E-06$ & \\
\hline 13. & $\begin{array}{l}\text { BKR--5A-FOPH+ } \\
\text { BKR-A202FOPH+ }\end{array}$ & $\begin{array}{l}\text { FAILURE OF } 13.8-\mathrm{kV} \text { FEEDER BREAKER 5A - SPURIOUSLY OPENS } \\
\text { FAILURE OF } 13.8-\mathrm{kV} \text { FEEDER BREAKER A202 - SPURIOUSLY OPENS }\end{array}$ & 4 & $\begin{array}{r}8.5 \mathrm{H} \\
3.0 \mathrm{E}-07 \mathrm{H} \\
8.5 \mathrm{H} \\
3.0 \mathrm{E}-07 \mathrm{H}\end{array}$ & $\begin{array}{l}2.55 \mathrm{E}-06 \\
2.55 \mathrm{E}-06\end{array}$ & $1.34 \mathrm{E}-08$ \\
\hline 14. & $\begin{array}{l}\text { BKR--5A-FOPH+ } \\
\text { BKR--5B-FOPH+ }\end{array}$ & $\begin{array}{l}\text { FAILURE OF } 13.8-\mathrm{kV} \text { FEEDER BREAKER } 5 \mathrm{~A} \text { - SPURIOUSLY OPENS } \\
\text { FAILURE OF } 13.8-\mathrm{kV} \text { FEEDER BREAKER } 5 \mathrm{~B} \text { - SPURIOUSLY OPENS }\end{array}$ & 4 & $\begin{array}{r}.8 .5 \mathrm{H} \\
3.0 \mathrm{E}-07 \mathrm{H} \\
8.5 \mathrm{H} \\
3.0 \mathrm{E}-07 \mathrm{H}\end{array}$ & $\begin{array}{l}2.55 E-06 \\
2.55 E-06\end{array}$ & $1.34 \mathrm{E}-08$ \\
\hline 15 . & $\begin{array}{l}\text { BKR-A101FOPH+ } \\
\text { BKR-A202FOPH+ }\end{array}$ & $\begin{array}{l}\text { FAILURE OF } 13.8-\mathrm{kV} \text { FEEDER BREAKER A101 - SPURIOUSLY OPENS } \\
\text { FAILURE OF. } 13.8-\mathrm{kV} \text { FEEDER BREAKER A202 - SPURIOUSLY OPENS }\end{array}$ & 4 & $\begin{array}{r}8.5 \mathrm{H} \\
3.0 \mathrm{E}-07 \mathrm{H} \\
8.5 \mathrm{H} \\
3.0 \mathrm{E}-07 \mathrm{H}\end{array}$ & $\begin{array}{l}2.55 E-06 \\
2.55 E-06\end{array}$ & $1.34 E-08$ \\
\hline 16. & $\begin{array}{l}\text { BKR--5B-FOPH+ } \\
\text { BKR-A101FOPH+ }\end{array}$ & $\begin{array}{l}\text { FAILURE OF } 13.8-\mathrm{kV} \text { FEEDER BREAKER } 5 \mathrm{~B} \text { - SPURIOUSLY OPENS } \\
\text { FAILURE OF } 13.8-\mathrm{kV} \text { FEEDER BREAKER A101 - SPURIOUSLY OPENS }\end{array}$ & 4 & $\begin{array}{r}8.5 \mathrm{H} \\
3.0 \mathrm{E}-07 \mathrm{H} \\
8.5 \mathrm{H} \\
3.0 \mathrm{E}-07 \mathrm{H}\end{array}$ & $\begin{array}{l}2.55 E-06 \\
2.55 E-06\end{array}$ & $1.34 \mathrm{E}-08$ \\
\hline 17. & $\begin{array}{l}\text { BKR-A201FOPH+ } \\
\text { BUS--A1-UNAH\# } \\
\text { FED--2--BFLH+ }\end{array}$ & $\begin{array}{l}\text { FAILURE OF } 13.8-\mathrm{kV} \text { TIE BREAKER A201 - SPURIOUSLY OPENS } \\
\text { BUS A1 UNAVAILABLE DUE TO TESTING/MAINTENANCE } \\
\text { FAILURE OF } 13.8-\mathrm{kV} \text { FEEDER \#2. }\end{array}$ & 4 & $\begin{array}{r}8.5 \mathrm{H} \\
3.0 \mathrm{E}-07 \mathrm{H} \\
1 \mathrm{~N} \\
3.8 \mathrm{E}-02 \mathrm{~N} \\
8.5 \mathrm{H} \\
1.0 \mathrm{E}-06 \mathrm{H}\end{array}$ & $\begin{array}{l}2.55 \mathrm{E}-06 \\
3.80 \mathrm{E}-02 \mathrm{~N} \\
8.50 \mathrm{E}-06\end{array}$ & $1.70 \mathrm{E}-09$ \\
\hline 18. & $\begin{array}{l}\text { BUS--A1-BFLH+ } \\
\text { BUS--A2-BFLH+ }\end{array}$ & $\begin{array}{l}\text { FAILURE OF } 13.8-\mathrm{kV} \text { BUS } \mathrm{A} 1 \\
\text { FAILURE OF } 13.8-\mathrm{kV} \cdot \text { BUS A2 }\end{array}$ & 4 & $\begin{array}{r}8.5 \mathrm{H} \\
1.0 \mathrm{E}-07 \mathrm{H} \\
8.5 \mathrm{H} \\
1.0 \mathrm{E}-07 \mathrm{H}\end{array}$ & $\begin{array}{l}8.50 \mathrm{E}-07 \\
8.50 \mathrm{E}-07\end{array}$ & 1. $49 \mathrm{E}-09$ \\
\hline 19. & $\begin{array}{l}\text { BUS- }-\mathrm{A} 2-\mathrm{BFLH}+ \\
\text { OPR--5B-LOHH\# }\end{array}$ & $\begin{array}{l}\text { FAILURE OF } 13.8-\mathrm{kV} \text { BUS A2 } \\
\text { OPERATOR FAILS TO REMOVE LOCKOUT ON FEEDER BREAKER } 5 \mathrm{~B}\end{array}$ & 4 & $\begin{array}{r}8.5 \mathrm{H} \\
1.0 \mathrm{E}-07 \mathrm{H} \\
1.8 \mathrm{E}-04 \mathrm{~N} \\
5.0 \mathrm{E}-03 \mathrm{~N}\end{array}$ & $\begin{array}{l}8.50 \mathrm{E}-07 \\
9.00 \mathrm{E}-07 \mathrm{~N}\end{array}$ & $7.88 E-10$ \\
\hline 20. & $\begin{array}{l}\text { BUS--A2-BFLH+ } \\
\text { OPR--A1-LOHH\# }\end{array}$ & $\begin{array}{l}\text { FAILURE OF } 13.8-\mathrm{kV} \text { BUS A2 } \\
\text { OPERATTOR FAILS TO REMOVE LOCKOUT ON BUS A1 }\end{array}$ & 4 & $\begin{array}{r}1.0 \mathrm{E}-07 \mathrm{H} \\
1.8 \mathrm{E}-04 \mathrm{~N} \\
5.0 \mathrm{E}-03 \mathrm{~N}\end{array}$ & $\begin{array}{l}8.50 \mathrm{E}-07 \\
9.00 \mathrm{E}-07 \mathrm{~N}\end{array}$ & $7.88 \mathrm{E}-10$ \\
\hline 21. & BUS--A2-BFLH+ & FAILURE OF $13.8-\mathrm{kV}$ BUS A2 & 4 & $1.0 \mathrm{E}-07 \mathrm{H}$ & $8.50 \mathrm{E}-07$ & $7.88 \mathrm{E}-10$ \\
\hline
\end{tabular}


Cutsets for I: \CAFTA \CUT\ṔOWN,CSR

11/08/94 12:06 AM (CONT.)

\begin{tabular}{|c|c|c|c|c|c|c|}
\hline $\begin{array}{l}\text { Set } \\
\text { No. }\end{array}$ & $\begin{array}{l}\text { Event } \\
\text { Name }\end{array}$ & Description & C & $\begin{array}{l}\text { B.E: } \\
\text { Input }\end{array}$ & $\begin{array}{l}\text { Calc. } \\
\text { Result }\end{array}$ & $\begin{array}{c}\text { Cutset } \\
\text { Freq. }(/ y r)\end{array}$ \\
\hline & OPR-A201LOHH\# & OPERATOR FAILS TO REMOVE LOCKOUT ON TIE BREAKER A201 & 1 & $\begin{array}{l}1.8 \mathrm{E}-04 \mathrm{~N} \\
5.0 \mathrm{E}-03 \mathrm{~N}\end{array}$ & $9.00 \mathrm{E}-07 \mathrm{~N}$ & \\
\hline \multirow[t]{2}{*}{22.} & BUS--A2-BFLH+ & FAILURE OF $13.8-\mathrm{kV}$ BUS A2 & 4 & $1 \begin{array}{r}8.5 \mathrm{H} \\
1.0 \mathrm{E}-07 \mathrm{H}\end{array}$ & $8.50 \mathrm{E}-0.7$ & $7.88 \mathrm{E}-10$ \\
\hline & OPR-A202LOHH\# & OPERATOR FAILS TO REMOVE LOCKOUT ON FEEDER BREAKER A2O2 & 1 & $\begin{array}{r}1.8 \mathrm{E}-04 \mathrm{~N} \\
5.0 \mathrm{E}-03 \mathrm{~N}\end{array}$ & $9.00 \mathrm{E}-07 \mathrm{~N}$ & \\
\hline \multirow[t]{3}{*}{23.} & BKR-A201FOPH+ & FAILURE OF 13.8-kV TIE BREAKER A201 - SPUPIOUSLY OPENS & 4 & $3.0 \mathrm{E}-07 \mathrm{H}$ & $2.55 \mathrm{E}-06$ & $5.09 \mathrm{E}-10$ \\
\hline & $\mathrm{BKR}-\mathrm{A} 202 \mathrm{FOPH}+$ & FAILURE OF 13.8-KV FEEDER BREAKER A202 - SPURIOUSLY ORENS & 4 & $3.0 \mathrm{E}-07 \mathrm{H}$ & $2.55 E-06$ & \\
\hline & BUS--A1-UNAH\# & BUS A1 UNAVAILABLE DUE TO TESTING/MAINTENANCE & 1 & $3.8 \mathrm{E}-02 \mathrm{~N}$ & $3.80 \mathrm{E}-02 \mathrm{~N}$ & - \\
\hline \multirow[t]{3}{*}{$24 \cdot$} & BKR--5B-FOPH+ & $\begin{array}{l}\text { FAILURE OF 13.8-kV FEEDER BREAKER 5B - SPURIOUSLY OPENS } \\
: \text {. }\end{array}$ & 4 & $\begin{array}{r}8.5 \mathrm{H} \\
3.0 \mathrm{E}-07 \mathrm{H}\end{array}$ & $2.55+\mathrm{E}-06$ & $5.09 \mathrm{E}-10$ \\
\hline & $\mathrm{BKR}-\mathrm{A} 201 \mathrm{FOPH}+$ & FAILURE OF . 13:8-kV TIE BREAKER A201 - SPURIOUSLY OPENS & 4 & $\begin{array}{r}8.5 \mathrm{H} \\
3.0 \mathrm{E}-07 \mathrm{H}\end{array}$ & $2.55 \mathrm{E}-06$ & \\
\hline & BUS--A1-UNAH\# & BUS A1 UNAVAILABLE DUE TO TESTING/MAINTENANCE & 1 & $3.8 \mathrm{E}-02 \mathrm{~N}$ & $3.80 \mathrm{E}-02 \mathrm{~N}$ & \\
\hline \multirow[t]{3}{*}{25 . } & BKR-A201FOPH+ & FAILURE OF 13.8-kV TIE BREAKER A201 - SPURIOUSLY OPENS & 4 & $\begin{array}{r}8.5 \mathrm{H} \\
3.0 \mathrm{E}-07 \mathrm{H}\end{array}$ & $2.55 \mathrm{E}-06$ & $5.70 \mathrm{E}-14$ \\
\hline & BUS--A2-BFLH+ & FAILURE OF. $13.8-\mathrm{kV}$ BUS A2 & 4 & $\begin{array}{r}8.5 \mathrm{H} \\
1.0 \mathrm{E}-07 \mathrm{H}\end{array}$ & $8.50 \mathrm{E}-07$ & \\
\hline & $\begin{array}{c}\text { FED--1--BFLH+ } \\
\therefore\end{array}$ & FAILURE OF $13.8-\mathrm{kV}$ FEEDER \#1 & 4 & $1.0 \mathrm{E}-06 \mathrm{H}$ & $8.50 \mathrm{E}-06$ & . \\
\hline
\end{tabular}


LOSS OF NORMAL ELECTRIC POWER TO DWPF

Top Event Frequency: 3.02E-01/YR

Risk Achievement Worth

Basic Event Name
BKRA1-A2CCBH+I
BKR-5A5BCCBH+-I
BUSA1-A2CCAH+-I
HPWLP-H-BFLH+-I
FED-1-2-CCAH+-I
LPWST-LPBFLH+-I
BUS-A2-BFLH+-IC
BKR-A202FOPH+-IC
BKR--5B-FOPH+-IC
FED--2--BFLH+-IC
BKR-A101FOPH+-IC
BKR--5A-FOPH+-IC
FED--1--BFLH+-IC
BUS-A1-BFLH+-IC
BKR-A201FOPH+-IC

Description

CC: FEEDER BRKRS A101/A202 SPURIOUSLY OPEN

CC: FEEDER BREAKERS 5A/5B SPURIOUSLY OPEN

CC: FAILURE OF 13.8-kV BUSSES A1 AND A2

LOSS OF POWER FROM SRS GRID TO H-AREA

CC: FAILURE OF BOTH 13.8-kV FEEDERS

FAIIURE OF ON-SITE AND OFF-SITE SOURCES

FAIIURE OF 13.8-kV BUS A2

FEEDER BREAKER A202 - SPURIOUSLY OPENS 13.8-kV FEEDER BREAKER 5B - SPURIOUSLY OPENS

FAIIURE OF 13.8-kV FEEDER \#2

FEEDER BREAKER A101 SPURIOUSLY OPENS

13.8-kV FEEDER BREAKER 5A SPURIOUSLY OPENS

FAILURE OF 13.8-kV FEEDER \#1 .

FAIIURE OF 13.8-kV BUS A1

TE BREAKER A201 - SPURIOUSLY OPENS

\begin{tabular}{cc} 
Prob/Freg & AchW \\
\hline $3.00 \mathrm{E}-08 / \mathrm{H}$ & $2.90 \mathrm{E}+04$ \\
$3.00 \mathrm{E}-08 / \mathrm{H}$ & $2.90 \mathrm{E}+04$ \\
$1.00 \mathrm{E}-08 / \mathrm{H}$ & $2.90 \mathrm{E}+04$ \\
$3.42 \mathrm{E}-05 / \mathrm{H}$ & $2.90 \mathrm{E}+04$ \\
$1.00 \mathrm{E}-07 / \mathrm{H}$ & $2.90 \mathrm{E}+04$ \\
$9.13 \mathrm{E}-08 / \mathrm{H}$ & $2.90 \mathrm{E}+04$ \\
$1.00 \mathrm{E}-07 / \mathrm{H}$ & $1.10 \mathrm{E}+03$ \\
$3.00 \mathrm{E}-07 / \mathrm{H}$ & $1.44 \mathrm{E}+00$ \\
$3.00 \mathrm{E}-07 / \mathrm{H}$ & $1.44 \mathrm{E}+00$ \\
$1.00 \mathrm{E}-06 / \mathrm{H}$ & $1.44 \mathrm{E}+00$ \\
$3.00 \mathrm{E}-07 / \mathrm{H}$ & $1.44 \mathrm{E}+00$ \\
$3.00 \mathrm{E}-07 / \mathrm{H}$ & $1.44 \mathrm{E}+00$ \\
$1.00 \mathrm{E}-06 / \mathrm{H}$ & $1.44 \mathrm{E}+00$ \\
$1.00 \mathrm{E}-07 / \mathrm{H}$ & $1.03 \mathrm{E}+00$ \\
$3.00 \mathrm{E}-07 / \mathrm{H}$ & $1.02 \mathrm{E}+00$
\end{tabular}

Modified top event frequency $=$ (top event frequency) $\mathrm{x}$ (risk achievement worth).
Basic Event Name HPWLP-H-BFLH+-I

\section{Risk Reduction Worth}

Description LOSS OF POWER FROM SRS GRT TO H-AREA $\frac{\text { Prob/Freq }}{3.42 \mathrm{E}-05 / \mathrm{H}}$

RedW

$1.30 \mathrm{E}+02$

Note: Events that make less than $1 \%$ difference to the importance factor are not included. Modified top event frequency $=($ top event frequency) $/$ (risk reduction worth). 
X-ESR-S-00001, REV. 0

Zone 1 Ventilation Unavailable

(with normal power and instrument air available) 
X-ESR-S-00001, REV. 0

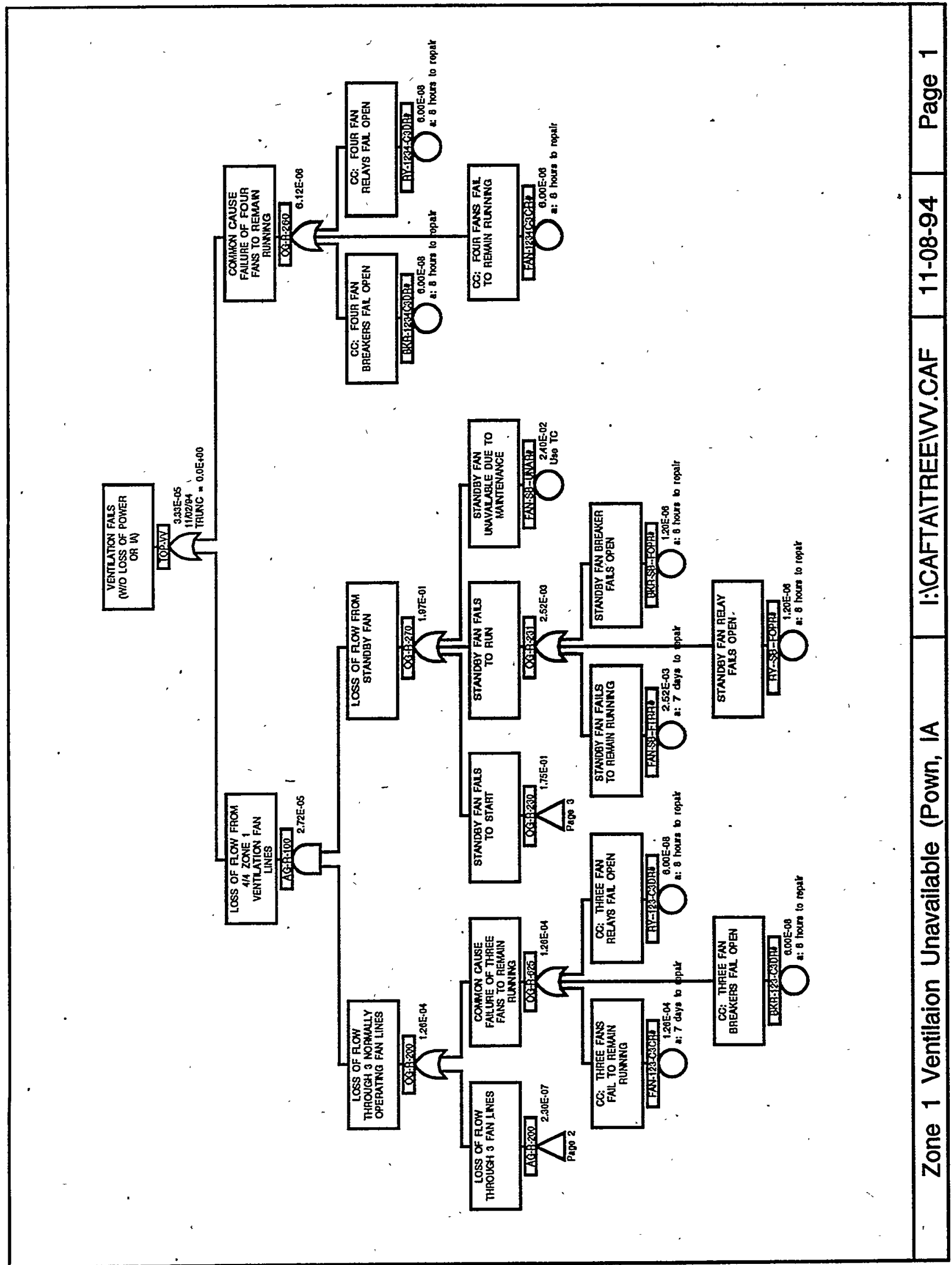


X-ESR-S-00001, REV. 0

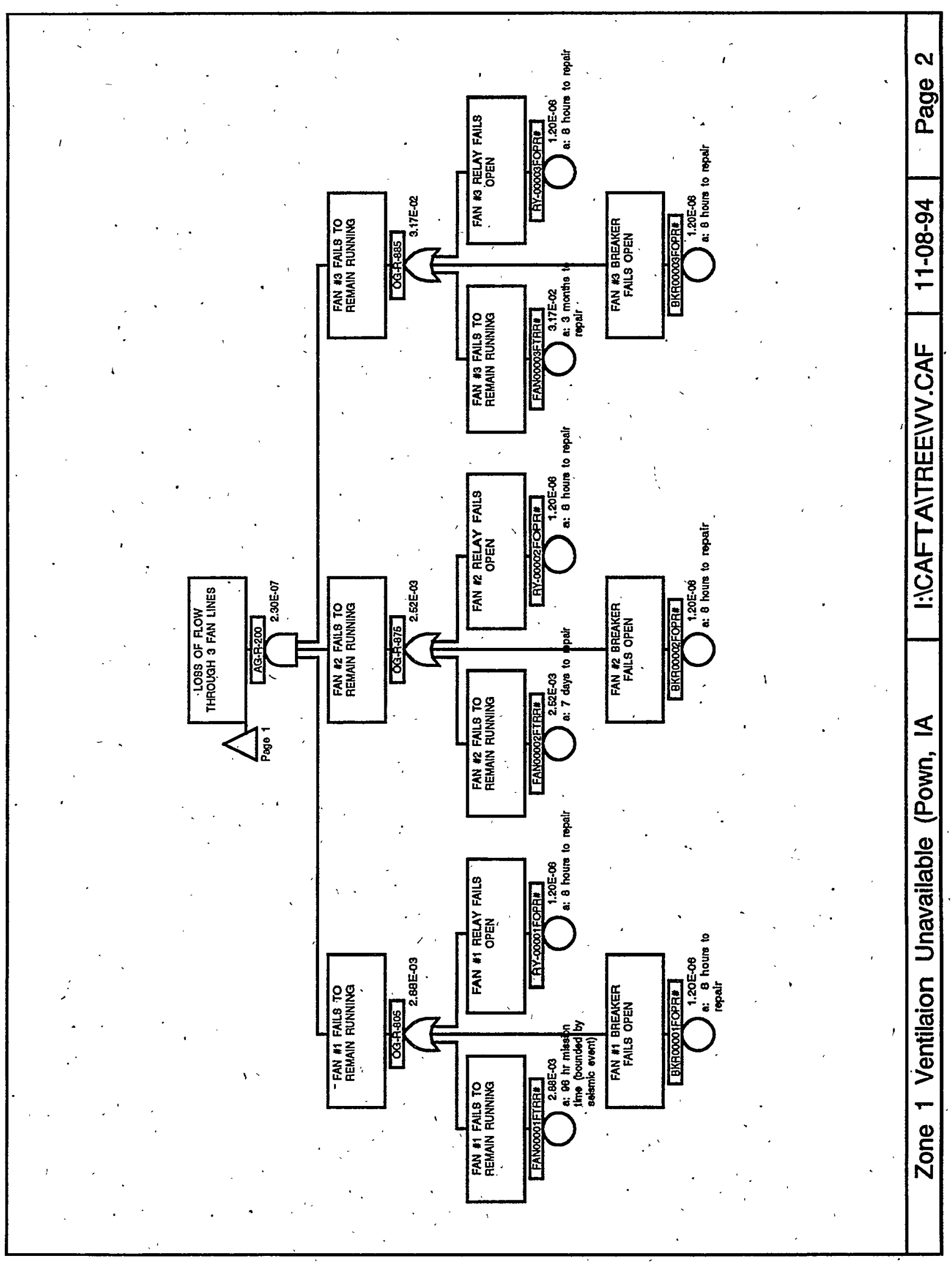




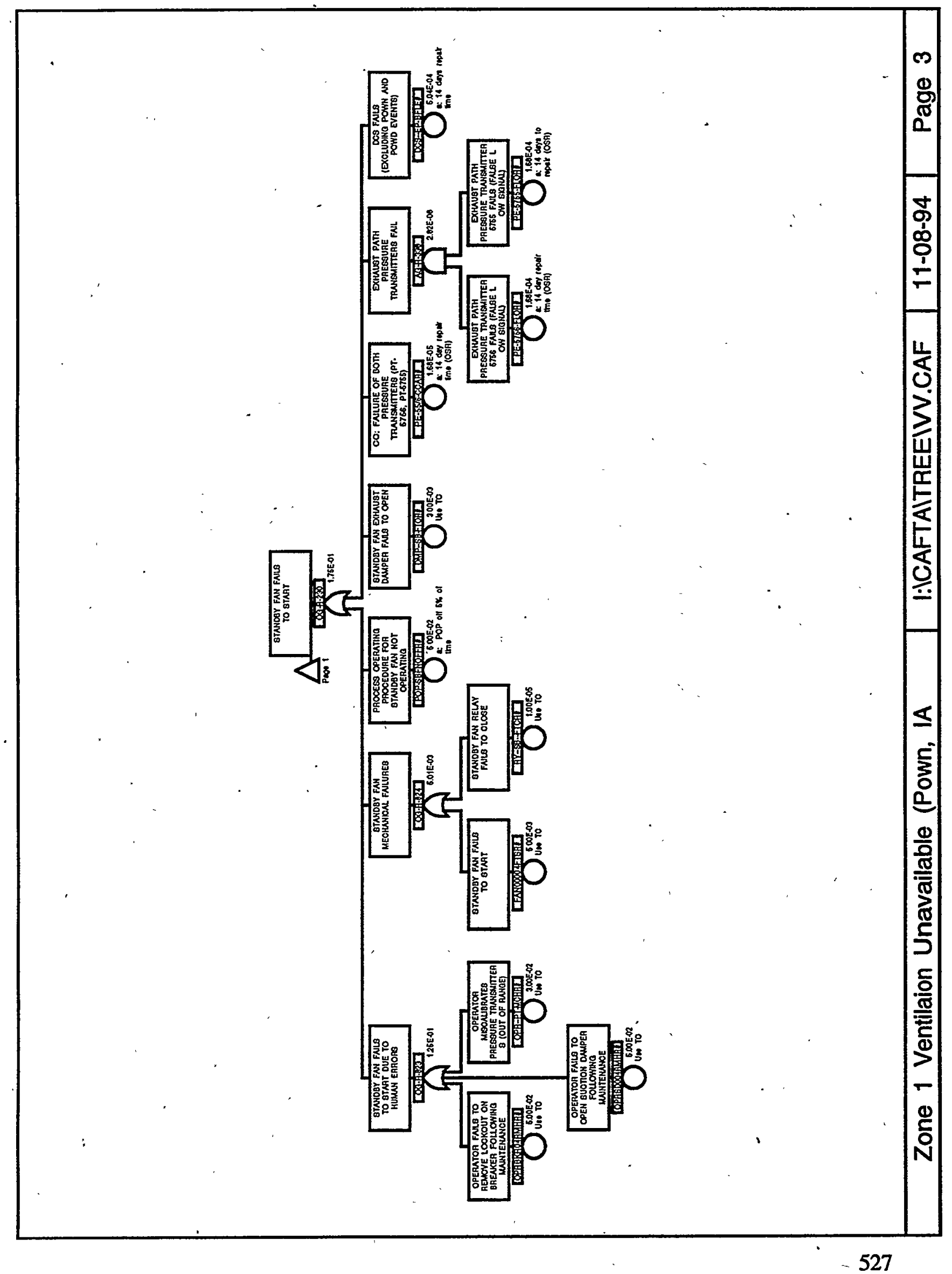


Cutsets for I: \CAFTA \CUT\VV.CSR

$11 / 08 / 94 \quad 12: 08 \quad \mathrm{AM}$

\begin{tabular}{|c|c|c|c|c|c|c|}
\hline $\begin{array}{l}\text { Set } \\
\text { No. }\end{array}$ & $\begin{array}{l}\text { Event } \\
\text { Name }\end{array}$ & Description & C & $\begin{array}{l}\text { B.E. } \\
\text { Prob }\end{array}$ & $\begin{array}{l}\text { Calc. } \\
\text { Result }\end{array}$ & $\begin{array}{l}\text { Cutset } \\
\text { Prob }\end{array}$ \\
\hline & TOP-VV & & & & & $3.33 \mathrm{E}-05$ \\
\hline \multirow[t]{2}{*}{1.} & FAN-123-C3CR\# & CC: THREE FANS FAIL TO REMAIN RUNNING & 5 & & $1.26 \mathrm{E}-04^{\circ}$ & $6.30 \mathrm{E}-06$ \\
\hline & OPRSD00 4RMHR\# & OPERATOR FAILS TO OPEN SUCTION DAMPER FOLLOWING MAINTENANCE & 1 & $5.0 \mathrm{E}-02 \mathrm{~N}$ & $5.00 \mathrm{E}-02 \mathrm{~N}$ & \\
\hline \multirow[t]{2}{*}{2.} & FAN-123-C3CR\# & CC: THREE FANS FAIL TO REMAIN RUNNING & 5 & & i. $26 \mathrm{E}-04$ & $6.30 \mathrm{E}-06$ \\
\hline & OPRBKR0 4RMHR\# & $\begin{array}{l}\text { OPERATOR FAILS TO REMOVE LOCKOUT ON BREAKER FOLLOWING - } \\
\text { MAINTENANCE }\end{array}$ & 1 & $\begin{array}{r}1.5 \mathrm{E}-06 \mathrm{H} \\
1 \mathrm{~N} \\
5.0 \mathrm{E}-02 \mathrm{~N}\end{array}$ & $5.00 \mathrm{E}-02 \mathrm{~N}$ & \\
\hline \multirow[t]{2}{*}{3 . } & FAN-123-C3CR\# & CC: THREE FANS FAIL TO REMAIN RUNNING & 5 & $7 \mathrm{D}$ & $1.26 \mathrm{E}-04$ & $6.30 \mathrm{E}-06$ \\
\hline & POP-SBFNOFER\# & PROCESS OPERATING PROCEDURE FOR STANDBY FAN NOT OPERATING & 1 & $\begin{array}{r}1 \mathrm{~N} \\
5.0 \mathrm{E}-02 \mathrm{~N}\end{array}$ & $5.00 \mathrm{E}-02 \mathrm{~N}$ & \\
\hline 4. & FAN-1234C3CR\# & CC: FOUR FANS FAIL TO REMAIN RUNNING & 5 & $1.5 \mathrm{E}-06 \mathrm{H}$ & $6.00 \mathrm{E}-06$ & $6.00 \mathrm{E}-06$ \\
\hline \multirow[t]{2}{*}{5 . } & FAN-123-C3CR\# & CC: THREE FANS FAIL TO REMAIN RUNNING & 5 & $\begin{array}{r}7 \mathrm{D} \\
1.5 \mathrm{E}-06 \mathrm{H}\end{array}$ & $1.26 \mathrm{E}-04$ & $3.78 \mathrm{E}-06$ \\
\hline & OPR--PT-MCHR\# & OPERATOR MISCALIBRATES PRESSURE TRANSMITTERS (OUT OF RANGE) & 1 & $3.0 \mathrm{E}-02 \mathrm{~N}$ & $3.00 \mathrm{E}-02 \mathrm{~N}$ & \\
\hline \multirow[t]{2}{*}{6.} & & CÉ: THREE FANS FAIL TO REMAIN RUNNING & 5 & $1.5 \mathrm{E}-06 \mathrm{H}$ & $1.26 \mathrm{E}-04$ & $3.02 \mathrm{E}-06$ \\
\hline & $\begin{array}{c}\text { FAN-SB--UNAR\# } \\
\cdots\end{array}$ & STANDBY FAN UNAVAILABLE DUE TO MAINTENANCE & 1 & $2.4 \mathrm{E}-02 \mathrm{~N}$ & $2.40 \mathrm{E}-02 \mathrm{~N}$ & \\
\hline \multirow[t]{2}{*}{7.} & FAN-123-C3CR\# & CC: THREE FANS FAIL TO REMAIN RUNNING & 5 & $\begin{array}{r}7 \mathrm{D} \\
1.5 \mathrm{E}-06 \mathrm{H}\end{array}$ & $1.26 \mathrm{E}-04$ & $6.30 \mathrm{E}-07$ \\
\hline & FANO0004FTSR\# & STANDBY FAN FAILS. TO START & 1 & $5.0 \mathrm{E}-03 \mathrm{~N}$ & $5.00 \mathrm{E}-03 \mathrm{~N}$ & - \\
\hline \multirow[t]{2}{*}{8 . } & DMP--SB-FTOR\# & STANDBY FAN EXHAUSTT DAMPER FAILS TO OPEN & 1 & $\begin{array}{r}1 \mathrm{~N} \\
3.0 \mathrm{E}-03 \mathrm{~N}\end{array}$ & $3.00 \mathrm{E}-03 \mathrm{~N}$ & $3.78 \mathrm{E}-07$ \\
\hline & FAN-123-C3CR\# & CC: THREE FANS FAIL TO REMAIN RUNNING & 5 & $\begin{array}{r}7 \mathrm{D} \\
1.5 \mathrm{E}-06 \mathrm{H}\end{array}$ & $1.26 \mathrm{E}-04$ & \\
\hline \multirow[t]{2}{*}{9.} & FAN-123-C3CR\# & CC: THREE FANS FAIL TO REMAIN RUNNING & 5 & $\begin{array}{r}7 \mathrm{D} \\
1.5 \mathrm{E}-06 \mathrm{H}\end{array}$ & $1.26 \mathrm{E}-0.4$ & $3.17 \mathrm{E}-07$ \\
\hline & FAN-SB--FTRR\# & STANDBY FAN FAILS TO REMAIN RUNNING & 5 & $3.0 \mathrm{E}-05 \mathrm{H}$ & $2.52 \mathrm{E}-03$ & \\
\hline 10. & DCS--EP-BFLE\# & DCS FAILS (EXCLUDING POWN AND POWD EVENTSS) & 5 & $3.0 \mathrm{E}-06 \mathrm{H}$ & $5.04 \mathrm{E}-04$ & $6.35 \mathrm{E}-08$ \\
\hline
\end{tabular}


Cutsets for I: ICAFTA ICUTIVV.CSR 11/08/94 12:08 AM .(CONT.)

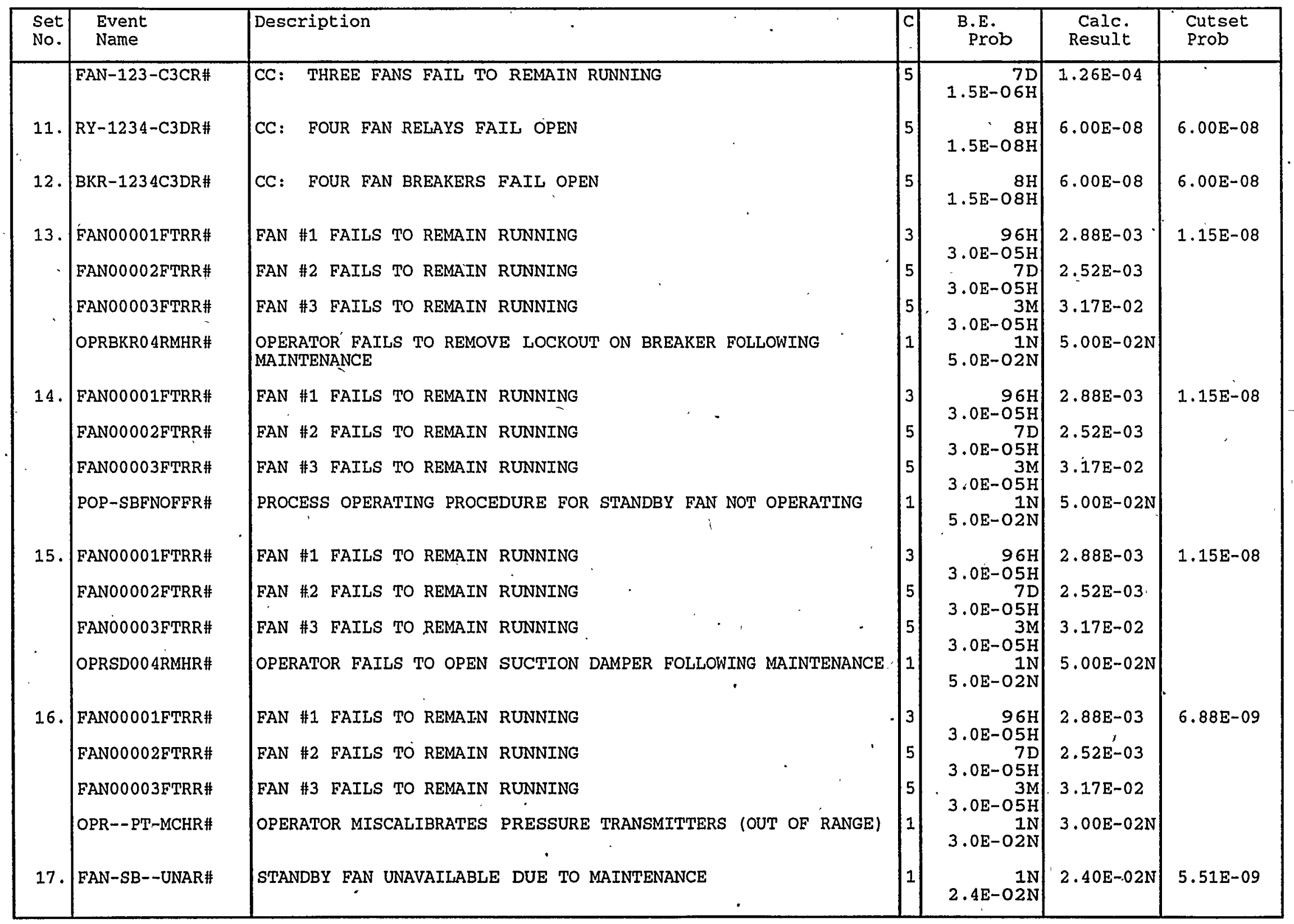


Cutsets for I: \CAFTA \CUT\VV.CSR 11/08/94 12:08 AM (CONT.)

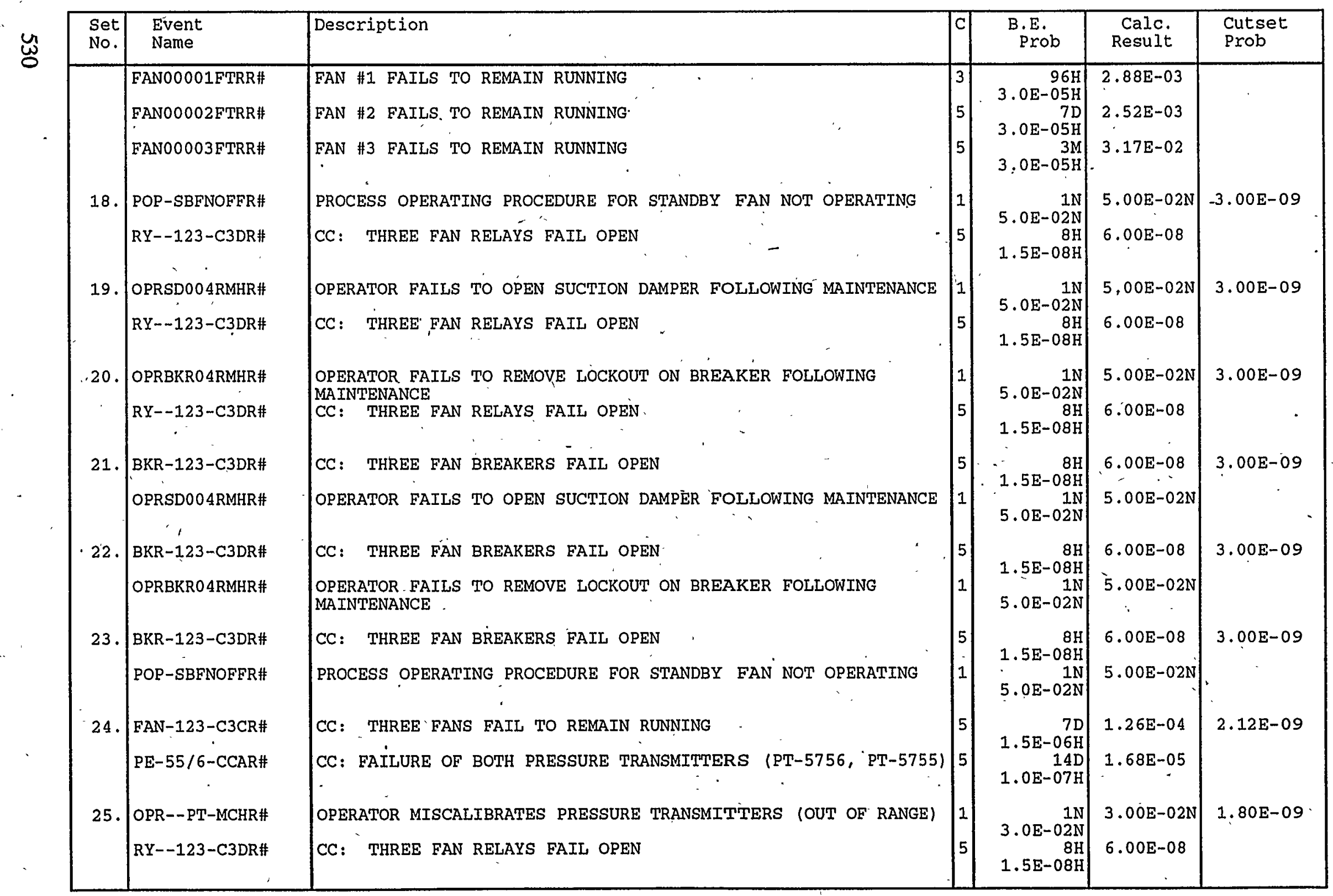


VENTILATION FAILS (W/O LOSS OF POWER OR IA)

Top Event Probability: 3.33E-05

\begin{tabular}{|c|c|c|c|}
\hline Basic Event Name & Description & Prob/Freg & AchW \\
\hline AN-1234C3CR\# & CC: FOUR FANS FAII TO REI & $6.00 \mathrm{E}-06$ & $3.00 E+04$ \\
\hline 234C3DR\# & CC: FOUR FAN BREAKERS FAIl OPEN & $6.00 \mathrm{E}-08$ & E+04 \\
\hline RY-1234-C3DR\# & CC: FOUR FAN RELAYS FAIL OPEN & 6.0 & E+04 \\
\hline BKR-123-C3DR\# & CC: THREE FAN BREAKERS FAIL OPEN & $6.00 \mathrm{E}-08$ & $5.93 E+03$ \\
\hline 23-C3DR\# & CC: THREE FAN RELAYS FAII OPEN & $6.00 \mathrm{E}-08$ & $93 E+03$ \\
\hline FAN-123-C3CR\# & CC: THREE FANS FAIl TO REMAIN RUNNING & $1.26 \mathrm{E}-04$ & $5.93 E+03$ \\
\hline BKR-SB-FOPR\# & STANDBY FAN BREAKER FAIIS OPEN & $1.20 \mathrm{E}-06$ & $480 \mathrm{E}+$ \\
\hline RY-SB-FOPR\# & STANDBY FAN RELAY FAI & $1.20 \mathrm{E}-06$ & \\
\hline B-FTCR\# & STANDBY FAN RELAY FAILS TO CLOSE & $1.00 \mathrm{E}-05$ & $4.80 \mathrm{E}+00$ \\
\hline 6-CCAR\# & CC: FAILURE OF BOTH PRESSURE TRANSMITTERS & $1.68 \mathrm{E}-05$ & $4.80 \mathrm{E}+00$ \\
\hline EP-BFLE\# & DCS FAILS (EXCLUDING POWN AND POWD) & E-04 & +00 \\
\hline FAN-SB--FTRR\# & STANDBY FAN FAIlS TO REMAIN RUNNING & -03 & 4. \\
\hline OR\# & STANDBY FAN EXHAUST DAMPER FAILS TO OPEN & -03 & $+\infty 0$ \\
\hline SR\# & STANDBY FAN FAILS TO START & & 4.78 \\
\hline AR\# & STANDBY FAN UNAVAILABLE DUE TO T\&M & $\mathrm{E}-02$ & $4.70 \mathrm{E}+00$ \\
\hline T-MCHR\# & PRESSURE TR & E-02 & +00 \\
\hline IHR\# & LOWING T\&M & $5.00 \mathrm{E}-02$ & $4.61 \mathrm{E}+00$ \\
\hline IHR\# & FAII TO OPEN SUCTION DAMPER AFTER T\&M & $5.00 \mathrm{E}-02$ & $4.61 \mathrm{E}+00$ \\
\hline FR\# & POP FOR STANDBY FAN NOT OPERATING & $5.00 \mathrm{E}-02$ & $4.61 \mathrm{E}+00$ \\
\hline PR\# & FAN \#2 BREAKER FAILS OPEN & $1.20 \mathrm{E}-06$ & $1.59 \mathrm{E}+00$ \\
\hline R\# & FAN \#2 RELAY FAILS OPEN & $1.20 \mathrm{E}-06$ & $1.59 \mathrm{E}+00$ \\
\hline R\# & REMAIN RUNNING & $2.52 \mathrm{E}-03$ & $+\infty 0$ \\
\hline R\# & REAKF & $1.20 \mathrm{E}-06$ & $1.52 E+00$ \\
\hline PR\# & FAN\#1 RELAY & $1.20 \mathrm{E}-06$ & $1.52 \mathrm{E}+00$ \\
\hline GTRR\# & FAN\#1 FAILS TO REMAIN RUNNING & $2.88 \mathrm{E}-03$ & $E+00$ \\
\hline DPR\# & RELAY FAILS OPEN & & \\
\hline & FAILS OPEN & & \\
\hline & EMAIN RUNNING & 3.17E-02 & \\
\hline
\end{tabular}

Risk Achievement Worth

\author{
Descriotion
}

CC: FOUR FANS FAII TO REMAIN RUNNING

CC: FOUR FAN BREAKERS FAIL OPEN

CC: FOUR FAN RELAYS FAIl OPEN

STANDBY FAN BREAKER FAIIS OPEN

STANDBY FAN RELAY FAIIS OPEN

CC: FAILURE OF BOTH PRESSURE TRANSMTTTERS

DCS FAILS (EXCLUDING POWN AND POWD)

STANDBY FAN FAILS TO START

FAIl TO REMOVE LOCKOUT FOLLOWING T\&M

FAN \#2 BREAKER FAIIS OPEN

FAN \#2 RELAY FAILS OPEN

FAN \#2 FAILS TO REMAIN RUNNING

FAN \#1 FAILS TO REMAIN RUNNING

FAN \#3 BREAKER FAIIS OPEN

FAN \#3 FAILS TO REMAIN RUNNING

Modified top event frequency $=$ (top event frequency) $\mathrm{x}$ (risk achievement worth).

Prob/Freg RedW

$1.26 \mathrm{E}-04 \quad 5.37 \mathrm{E}+00$

$5.00 \mathrm{E}-02 \quad 1.23 \mathrm{E}+00$

$5.00 \mathrm{E}-02 \cdot 1.23 \mathrm{E}+00$

$5.00 \mathrm{E}-02 \quad 1.23 \mathrm{E}+00$

$6.00 \mathrm{E}-06 \quad 1.22 \mathrm{E}+00$

3.00E-02 1.13E +00

$2.40 \mathrm{E}-02 \quad 1.10 \mathrm{E}+00$

$5.00 \mathrm{E}-03 \quad 1.02 \mathrm{E}+00$

3.00E-03 1.01E+00

Note: Events that make less than $1 \%$ difference to the importance factor are not included. Modified top event frequency = (top event frequency) / (risk reduction worth). 
X-ESR-S-00001, REV. 0

\section{Zone 1 Ventilation Unavailable}

(with instrument air available, normal power lost) 


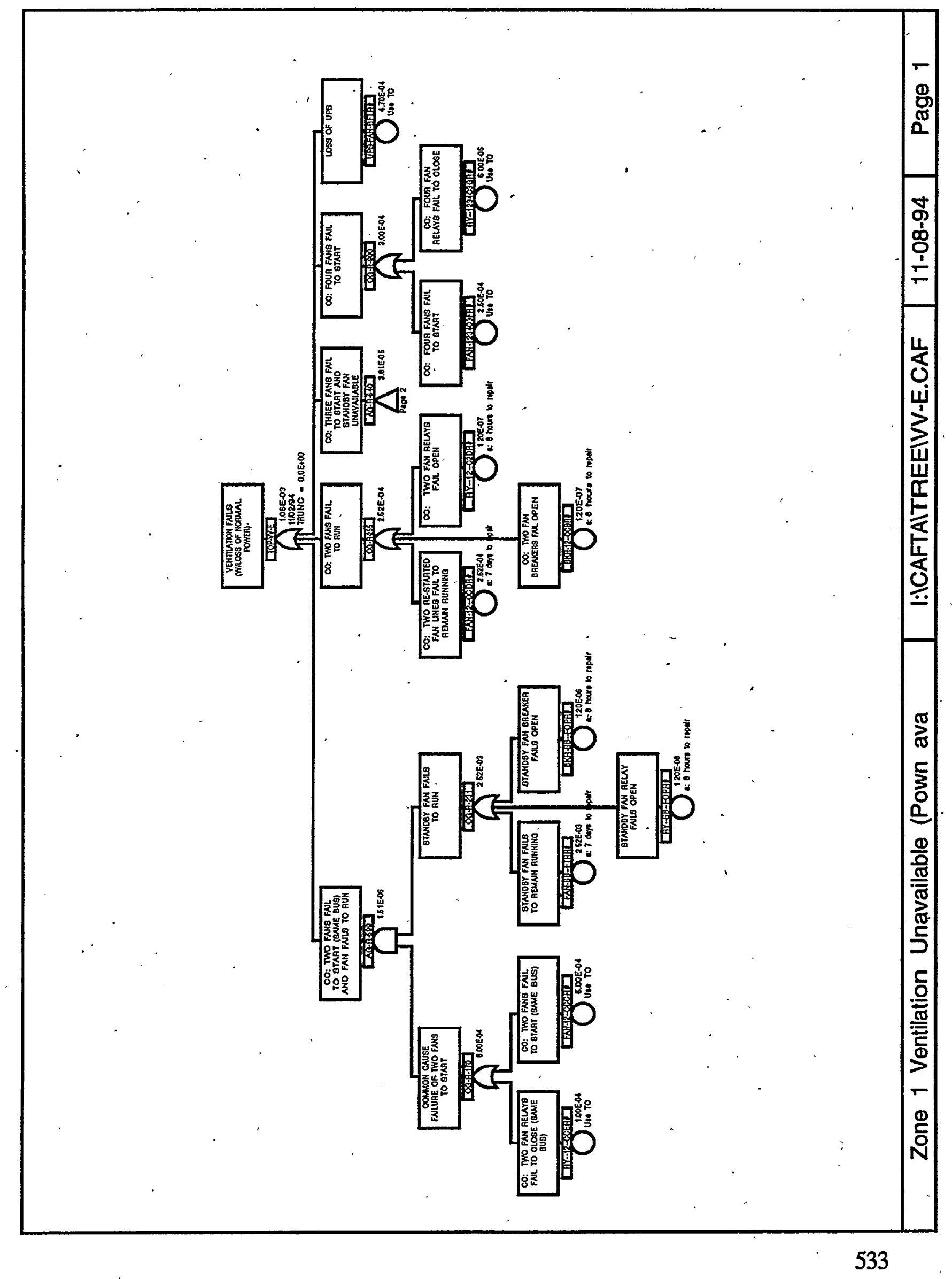




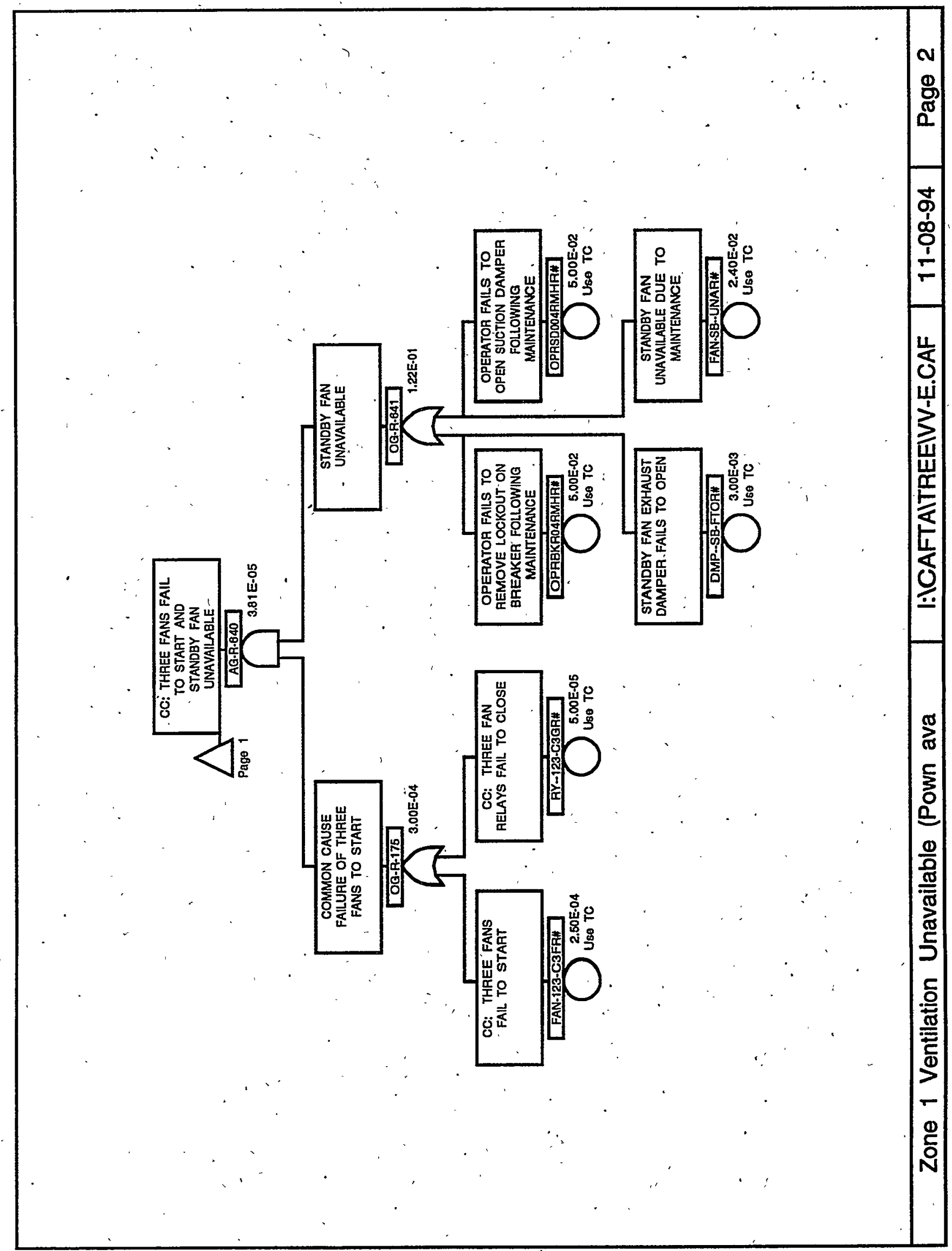


Cutsets for I: ICAFTA ICUTIVV-E. CSR

$11 / 08 / 94 \quad 12: 10$ AM

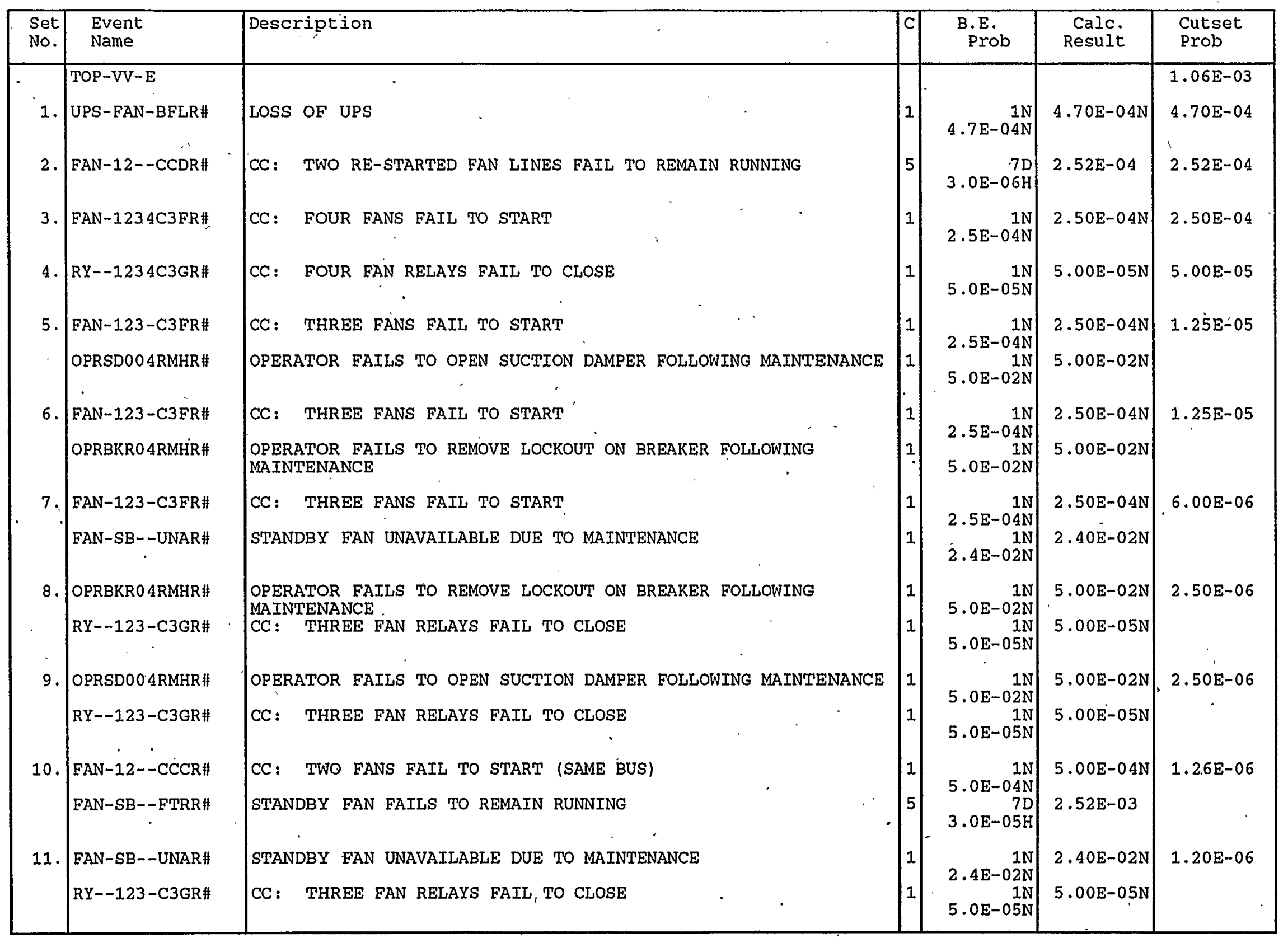




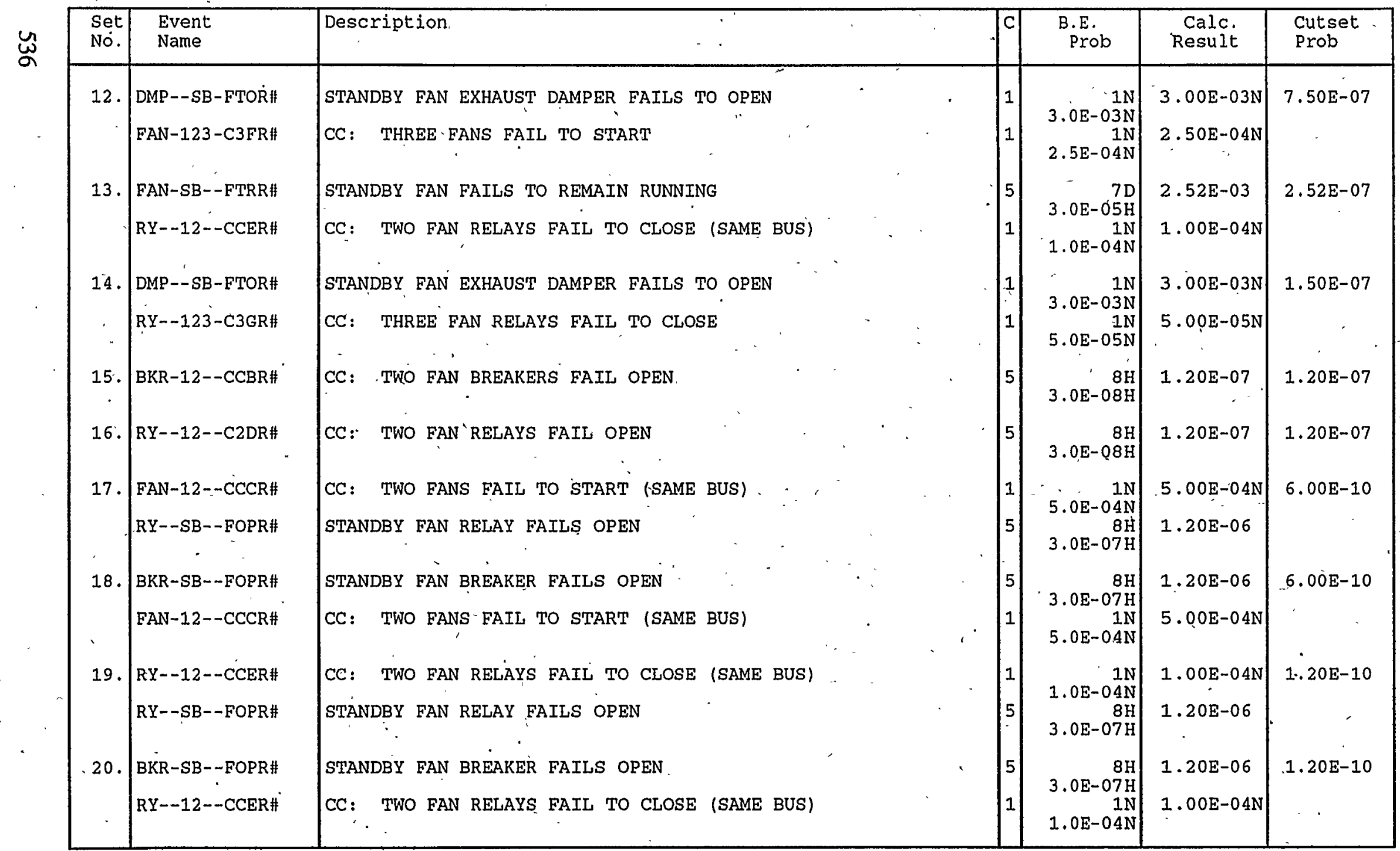


VENTILATION FAILS (W/LOSS OF NORMAL POWER)

Top Event Probability: 1.06E-03

\begin{tabular}{|c|c|c|c|}
\hline & Description & Prob/Frea & $\operatorname{AchW}$ \\
\hline RY--1234C3GR\# & FOUR FAN RELAYS FAIL TO CLOSE & $5.00 \mathrm{E}-05$ & $9.42 \mathrm{E}+02$ \\
\hline RY--12-C2DR\# & CC: TWO FAN RELAYS FAII OPEN & $1.20 \mathrm{E}-07$ & $9.42 \mathrm{E}+02$ \\
\hline BKR-12-CCBR\# & CC: TWO FAN BREAKERS FAIL OPEN & $1.20 \mathrm{E}-07$ & $9.42 \mathrm{E}+02$ \\
\hline UPS-FAN-BFLR\# & LOSS OF UPS & 4.70E-04 & $9.42 \mathrm{E}+02$ \\
\hline FAN-12--CCDR\# & CC: TWO RE-STARTED FAN LINES FAIL TO RUN & 2.52E-04 & $9.42 \mathrm{E}+02$ \\
\hline FAN-1234C3FR\# & CC: FOUR FANS FAII TO START & $2.50 \mathrm{E}-04$ & $9.42 \mathrm{E}+02$ \\
\hline C3GR\# & CC: THREE FAN RELAYS FAIL TO CLOSE & $5.00 \mathrm{E}-05$ & $1.16 \mathrm{E}+02$ \\
\hline 3FR\# & CC: THREE FANS FAIL TO START & $2.50 \mathrm{E}-04$ & $1.16 \mathrm{E}+02$ \\
\hline RY-12-CCER\# & CC: TWO FAN RELAYS FAIL TO CLOSE (SAME BUS) & 1.00E-04 & $3.37 \mathrm{E}+00$ \\
\hline FAN-12-CCCR\# & CC: TWO FANS FAIL TO START (SAME BUS) & $5.00 \mathrm{E}-04$ & $3.37 \mathrm{E}+00$ \\
\hline PRR\# & REAKER FAILS OPEN & $1.20 \mathrm{E}-06$ & $1.56 E+00$ \\
\hline PR\# & RELAY FAILS OPEN & $1.20 \mathrm{E}-06$ & $1.56 \mathrm{E}+00$ \\
\hline RR\# & STANDBY FAN FAILS TO REMAIN RUNNING & $2.52 \mathrm{E}-03$ & \\
\hline OR\# & STANDBY FAN EXHAUST DAMPER FAILS TO OPEN & $3.00 \mathrm{E}-03$ & $1.28 E+00$ \\
\hline & NAVAILABLE DUE TO T\&M & $2.40 \mathrm{E}-02$ & $1.28 \mathrm{E}+00$ \\
\hline RBKR04RMHR\# & FAII TO REMOVE LOCKOUT FOLLOWING T\&M & 5.00E-02 & $1.27 \mathrm{E}+00$ \\
\hline & DAMPER AFTER T\&M & $5.00 \mathrm{E}-02$ & $1.27 E+00$ \\
\hline
\end{tabular}

Basic Event Name UPS-FAN-BFLR\# FAN-12-CCDR\# FAN-1234C3FR\# RY--1234C3GR\# FAN-123-C3FR\# OPRBKR04RMHR\# OPRSD004RMHR\#
Risk Reduction Worth LOSS OF UPS

CC: TWO RE-STARTED FAN LINES FAIL TO RUN

CC: FOUR FANS FAIL TO START

CC: FOUR FAN RELAYS FAIL TO CLOSE

CC: THREE FANS FAIL TO START

FAII TO REMOVE LOCKOUT FOLLOWING T\&M FAIL TO OPEN SUCTION DAMPER AFTERT\&M

\begin{tabular}{cc} 
Prob/Erea & RedW \\
\hline $4.70 \mathrm{E}-04$ & $1.79 \mathrm{E}+00$ \\
$2.52 \mathrm{E}-04$ & $1.31 \mathrm{E}+00$ \\
$2.50 \mathrm{E}-04$ & $1.31 \mathrm{E}+00$ \\
$5.00 \mathrm{E}-05$ & $1.05 \mathrm{E}+00$ \\
$2.50 \mathrm{E}-04$ & $1.03 \mathrm{E}+00$ \\
$5.00 \mathrm{E}-02$ & $1.01 \mathrm{E}+00$ \\
$5.00 \mathrm{E}-02$ & $1.01 \mathrm{E}+00$
\end{tabular}

Note: Events that make less than $1 \%$ difference to the importance factor are not included. Modified top event frequency $=$ (top event frequency) $/$ (risk reduction worth). 
X-ESR-S-00001, REV. 0

Zone 1 Ventilation Unavailable

(with normal power and instrument air lost) 


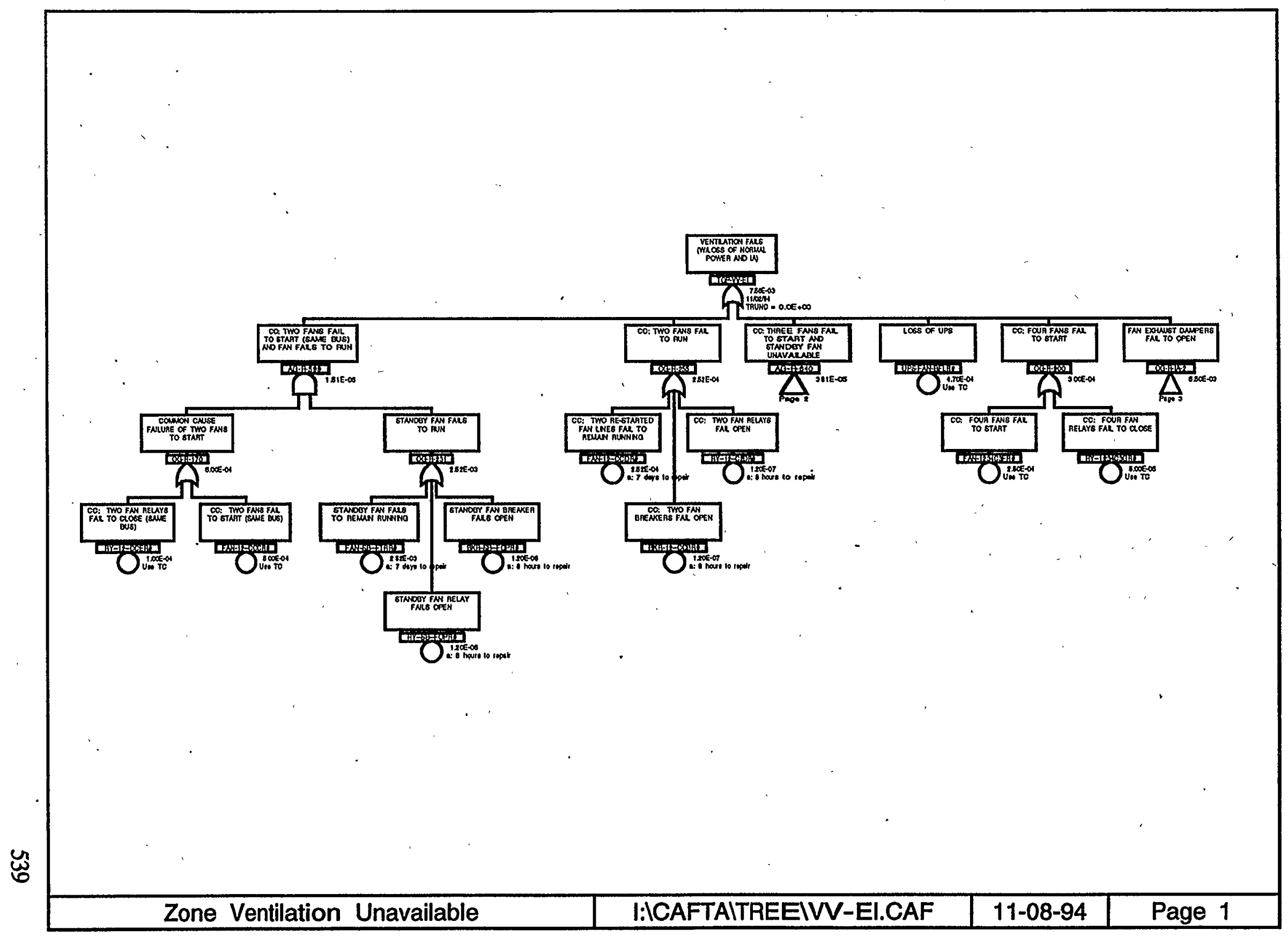




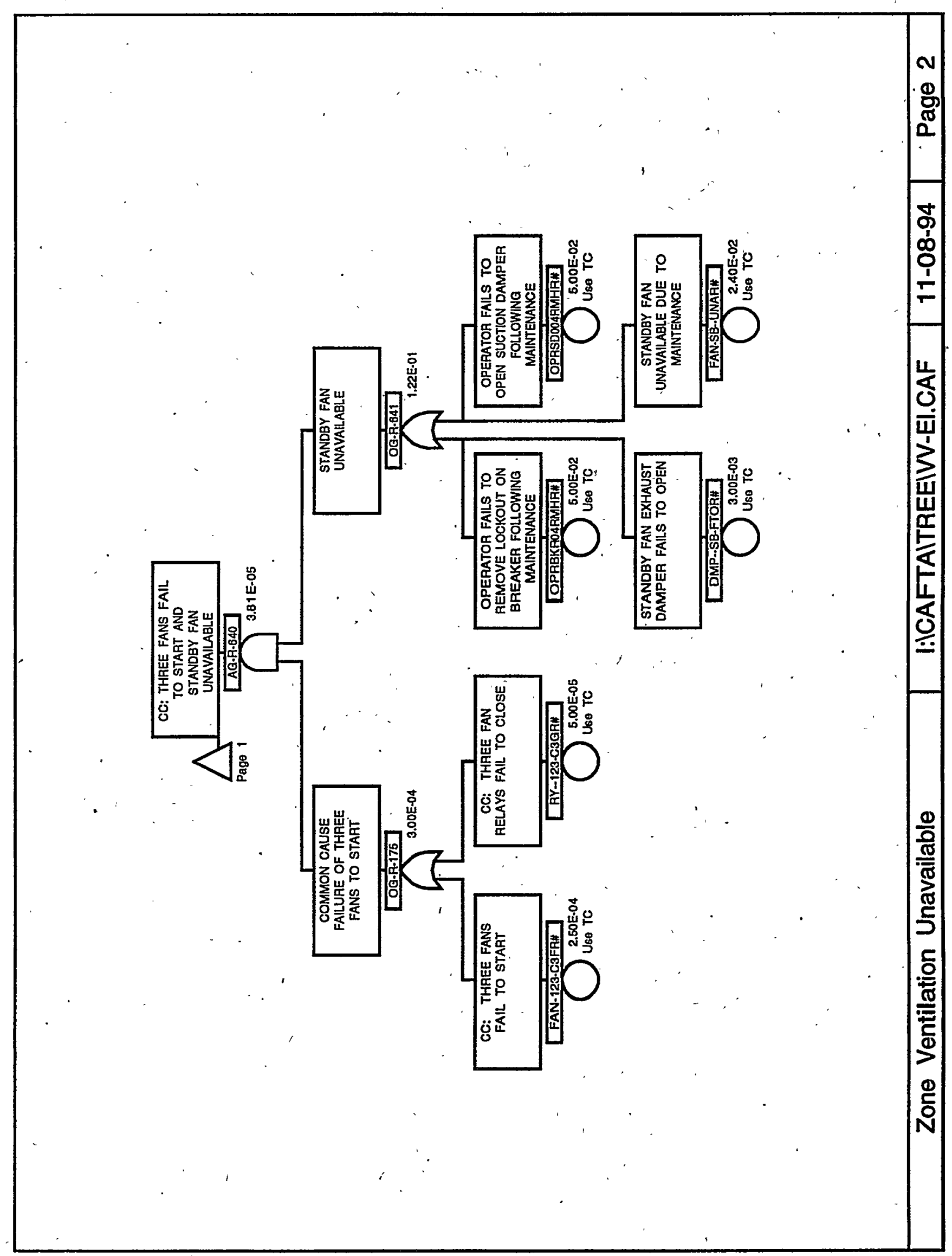




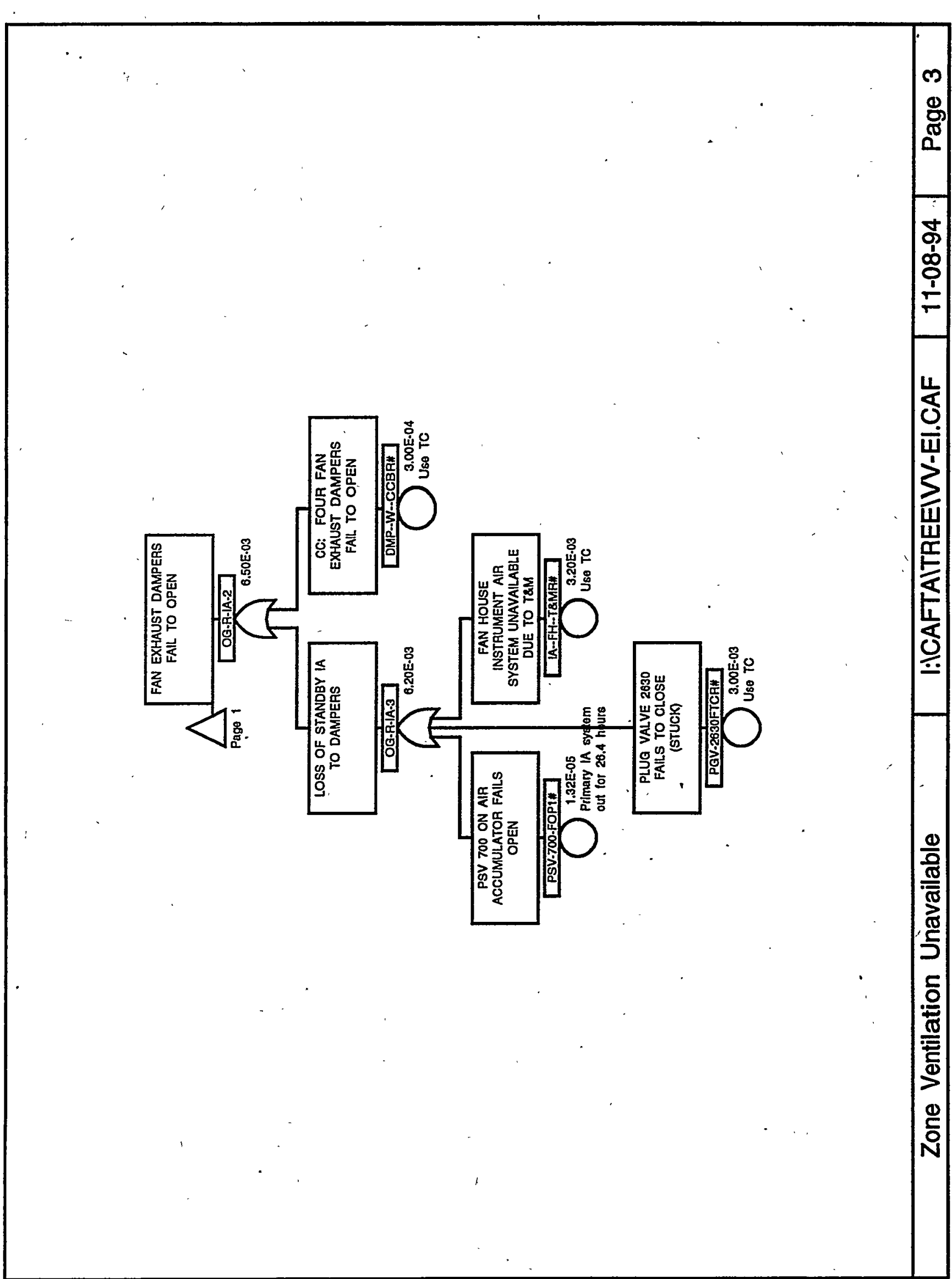




\begin{tabular}{|c|c|c|c|c|c|c|}
\hline $\begin{array}{l}\text { Set } \\
\text { No. }\end{array}$ & $\begin{array}{l}\text { Event } \\
\text { Name }\end{array}$ & Description & c) & $\begin{array}{l}\text { B.E. } \\
\text { Prob }\end{array}$ & $\begin{array}{l}\text { Calc. } \\
\text { Result }\end{array}$ & $\begin{array}{l}\text { Cutset } \\
\text { Prob }\end{array}$ \\
\hline & TOP-VV-EI & & & & & $7.56 \mathrm{E}-03$ \\
\hline 1. & IA- -FH- -T\&MR\# & FAN HOUSE INSTRUMENT. AIR SYSTEM UNAVAILABLE DUE TO T\&M & 1 & $3.2 \mathrm{E}-03 \mathrm{~N}$ & $3.20 \mathrm{E}-03 \mathrm{~N}$ & $3.20 \mathrm{E}-03$ \\
\hline $2:$ & PGV-2630FTCR\# & PLUG VALVE 2630 FAILS TO CLOSE (STUCK) & 1 & $3.0 \mathrm{E}-03 \mathrm{~N}$ & $3.00 \mathrm{E}-03 \mathrm{~N}$ & $3.00 \mathrm{E}-03$ \\
\hline 3. & ÚPS-FAN-BFLR\# & LOSS OF UPS & 1 & $4.7 \mathrm{E}-04 \mathrm{~N}$ & $4.70 \mathrm{E}-04 \mathrm{~N}$ & $4.70 \mathrm{E}-04$ \\
\hline 4. & DMP--W--CCBR\# & CC: FOUR FAN EXHAUST DAMPERS FAIL TO OPEN & 1 & $3.0 \mathrm{E}-04 \mathrm{~N}$ & $3.00 \mathrm{E}-0 \ddot{\mathrm{N}} \mathrm{N}$ & $3.00 \mathrm{E}-04$ \\
\hline 5. & FAN-12--CCDR\# & CC: TWO RE-STARTED FAN LINES FAIL TO REMAIN RUNNING & 5 & $3.0 \mathrm{E}-06 \mathrm{D}$ & $2.52 \mathrm{E}-04$ & $2.52 \mathrm{E}-04$ \\
\hline 6. & FAN-1234C3FR\# & CC: FOUR FANS FAIL TO START, & 1 & $2.5 \mathrm{E}-04 \mathrm{~N}$ & $2.50 \mathrm{E}-04 \mathrm{~N}$ & $2.50 \mathrm{E}-04$ \\
\hline 7. & RY--1234C3GR\# & CC: FOUR FAN RELAYS FAIL TO CLOSE & 1 & $5.0 \mathrm{E}-05 \mathrm{~N}$ & $5.00 \mathrm{E}-05 \mathrm{~N}$ & $5.00 \mathrm{E}-05$ \\
\hline 8. & PSV-700-FOP1\# & PSV 700 ON AIR ACCUMULATOR FAILS OPEN & 3 & $\begin{array}{r}26.4 \mathrm{H} \\
5.0 \mathrm{E}-07 \mathrm{H}\end{array}$ & $1.32 \mathrm{E}-05$ & $1.32 \mathrm{E}-05$ \\
\hline \multirow[t]{2}{*}{9.} & FAN-123-C3FR\# & CC: THREE FANS FAIL TO START & 1 & $2.5 E-04 N$ & $2.50 \mathrm{E}-04 \mathrm{~N}$ & $1.25 \mathrm{E}-05$ \\
\hline & OPRBKR04RMHR\# & $\begin{array}{l}\text { OPERATOR FAILS TO REMOVE LOCKOUT ON BREAKER FOLLOWING - } \\
\text { MAINTENANCE }\end{array}$ & 1 & $5.0 \mathrm{E}-02 \mathrm{~N}$ & $5.00 \mathrm{E}-02 \mathrm{~N}$ & . \\
\hline \multirow[t]{2}{*}{10.} & FAN-123-C3FR\# & CC: THREE FANS FAIL TO START & 1 & $2.5 \mathrm{E}-04 \mathrm{~N}$ & $2.50 \mathrm{E}-04 \mathrm{~N}$ & $1.25 \mathrm{E}-05$ \\
\hline & OPRSDO04RMHR\# & $\begin{array}{c}\text { OPERATOR FAILS TO OPEN SUCTION DAMPER FOLLOWING MAINTENANCE } \\
\ddots\end{array}$ & 1 & $5.0 \mathrm{E}-02 \mathrm{~N}$ & $5.00 \mathrm{E}-02 \mathrm{~N}$ & \\
\hline \multirow[t]{2}{*}{11.} & FAN-123-C3FR\# & CC: THREE FANS FAIL TO START - & 1 & $2.5 \mathrm{E}-04 \mathrm{~N}$ & $2.50 \mathrm{E}-04 \mathrm{~N}$ & $6.00 \mathrm{E}-06$ \\
\hline & FAN-SB--UNAR\# & STANDBY FAN UNAVAILABLE DUE TO MAINTENANCE & 1 & $2.4 \mathrm{E}-02 \mathrm{~N}$ & $2.40 \mathrm{E}-02 \mathrm{~N}$ & . \\
\hline \multirow[t]{2}{*}{12 . } & OPRSD004RMHR\# & OPERATOR FAILS TO OPEN SUCTION DAMPER FÖLLOWING MAINTENANCE & 1 & $5.0 \mathrm{E}-02 \mathrm{~N}$ & $5.00 \mathrm{E}-02 \mathrm{~N}$ & $2.50 \mathrm{E}-06$ \\
\hline & RY--123-C3GR\# & CC: THREE FAN RELAYS FAIL TO CLOSE & 1 & $5.0 \mathrm{E}-05 \mathrm{~N}$ & $5.00 \mathrm{E}-05 \mathrm{~N}$ & \\
\hline 13. & OPRBKR04RMHR\# & $\begin{array}{l}\text { OPERATOR FAILS TO REMOVE LOCKOUT ON BREAKER FOLLOWING } \\
\text { MAINTENANCE }\end{array}$ & 1 & $5.0 \mathrm{E}-02 \mathrm{~N}$ & $5.00 \mathrm{E}-02 \mathrm{~N}$ & $2.50 \mathrm{E}-06$ \\
\hline
\end{tabular}


cutsets for I: ICAFTA ICUTIVV-EI.CSR $11 / 08 / 94$ 12:11 AM (CONT.)

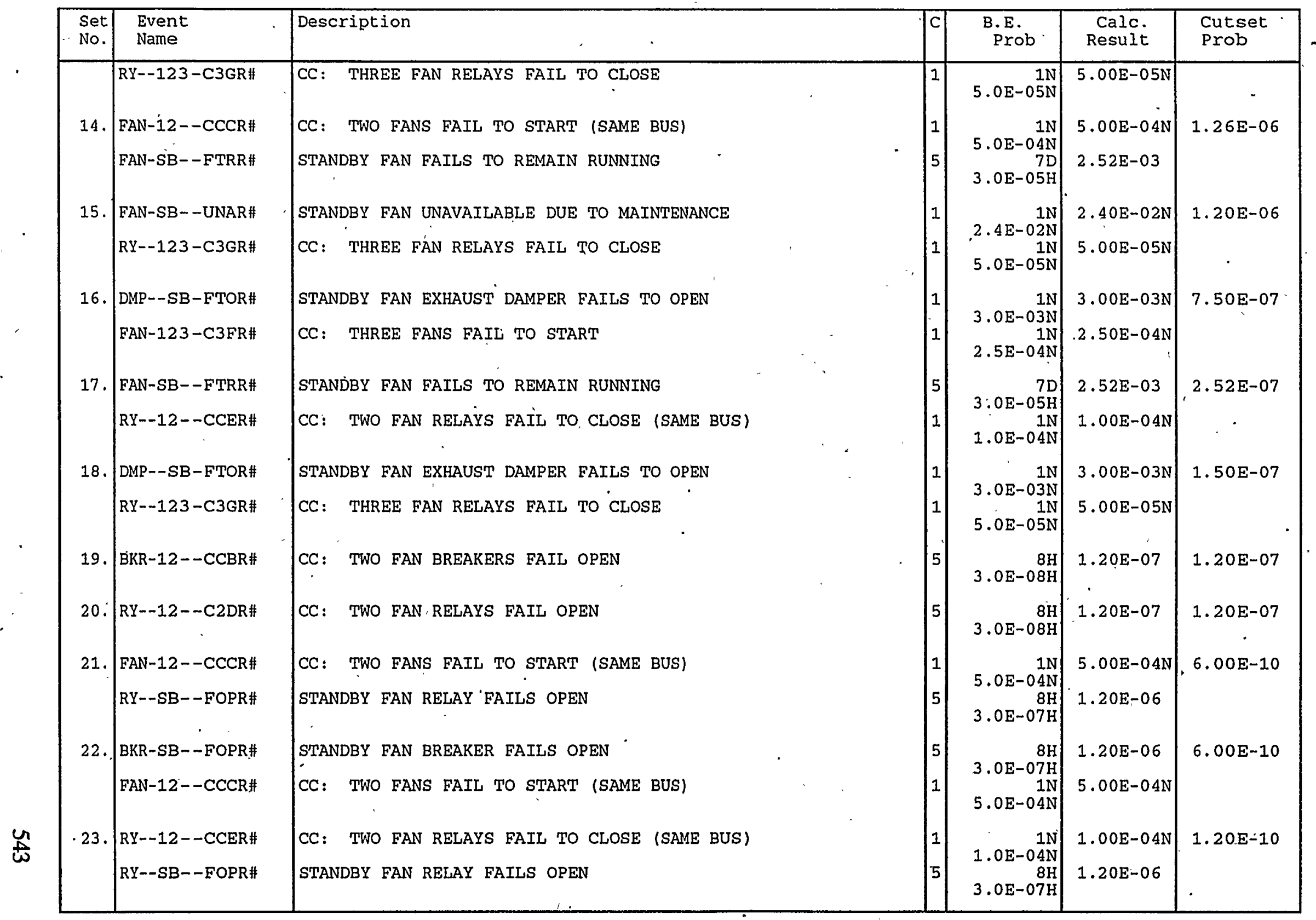


X-ESR-S-00001, REV. 0

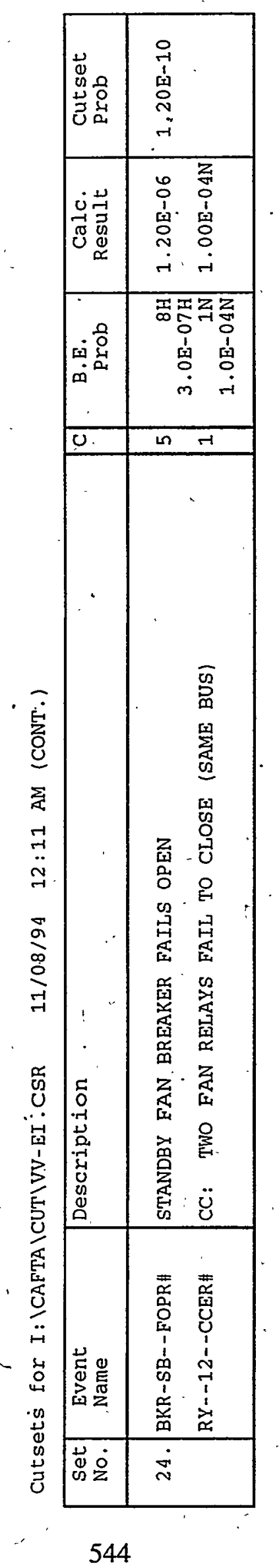


VENTILATION FAILS (W/LOSS OF NORMAL POWER AND IA)

Top Event Probability: 7.56E-03

\begin{tabular}{|c|c|c|c|}
\hline \multicolumn{4}{|c|}{ Risk Achievement Worth } \\
\hline Basic Évent Name & Description & Prob/Freg & AchW \\
\hline RY--1234C3GR\# & CC: FOUR FAN RELAYS FAIL TO CLOSE & $5.00 \mathrm{E}-05$ & $1.32 \mathrm{E}+02$ \\
\hline FAN-1234C3FR\# & CC: FOUR FANS FAIL TO START & $2.50 \mathrm{E}-04$ & $1.32 \mathrm{E}+$ \\
\hline PSV-700-FOP1\# & PSV 700 ON AIR ACCUMULATOR FAILS OPEN & $1.32 E-05$ & $1.32 E+02$ \\
\hline BKR-12--CCBR\# & CC: TWO FAN BREAKERS FAIL OPEN & $1.20 \mathrm{E}-07$ & $1.32 E+02$ \\
\hline RY-12-C2DR\# & CC: TWO FAN RELAYS FAIL OPEN & $1.20 \mathrm{E}-07$ & $1.32 E+02$ \\
\hline PGV-2630FTCR\# & PLUG VALVE 2630 FAIIS TO CLOSE (STUCK) & $3.00 \mathrm{E}-03$ & $1.32 E+02$ \\
\hline IA--FH-T\&MR\# & FAN HOUSE AIR SYSTEM UNAVAILABLE: T\&M & $3.20 \mathrm{E}-03$ & $1.32 E+02$ \\
\hline UPS-FAN-BFLR\# & LOSS OF UPS & 4.70E-04 & $1.32 \mathrm{E}+02$ \\
\hline FAN-12-CCDR\# & CC: TWO RE-STARTED FAN LINES FAIL TO RUN & $2.52 \mathrm{E}-04$ & $1.32 E+02$ \\
\hline DMP--W--CCBR\# & CC: FOUR FAN EXHAUST DAMPERS FAIL TO OPEN & $3.00 \mathrm{E}-04$ & $1.32 \mathrm{E}+02$ \\
\hline 123-C3GR\# & CC: THREE FAN RELAYS FAIL TO CLOSE & $5.00 \mathrm{E}-05$ & $1.70 \mathrm{E}+01$ \\
\hline FAN-123-C3FR\# & CC: THREE FANS FAIL TO START & $2.50 \mathrm{E}-04$ & $1.70 \mathrm{E}+01$ \\
\hline RY-12-CCER\# & CC: TWO FAN RELAYS FAIL TO CLOSE (SAME BUS) & $1.00 \mathrm{E}-04$ & $1.33 \mathrm{E}+00$ \\
\hline FAN-12-CCCR\# & CC: TWOFANS FAIL TO START (SAME BUS) & $5.00 \mathrm{E}-04$ & $1.33 E+00$ \\
\hline BKR-SB-FOPR\# & STANDBY FAN BREAKER FAILS OPEN & $1.20 \mathrm{E}-06$ & $1.08 \mathrm{E}+00$ \\
\hline RY-SB-FOPR\# & STANDBY FAN RELAY FAILS OPEN & $1.20 \mathrm{E}-06$ & $1.08 \mathrm{E}+00$ \\
\hline FAN-SB--FTRR\# & STANDBY FAN FAILS TO REMAIN RUNNING & $2.52 \mathrm{E}-03$ & $1.08 \mathrm{E}+00$ \\
\hline DMP-_SB-FTOR\# & STANDBY FAN EXHAUST DAMPER FAILS TO OPEN & $3.00 \mathrm{E}-03$ & $1.04 \mathrm{E}+00$ \\
\hline FAN-SB--UNAR\# & STANDBY FAN UNAVAILABLE DUE TO T\&M & $2.40 \mathrm{E}-02$ & $1.04 \mathrm{E}+00$ \\
\hline OPRBKR04RMHR\# & FAIL TO REMOVE LOCKOUT FOLLOWING T\&M & $5.00 \mathrm{E}-02$ & $1.04 E+00$ \\
\hline IHR\# & FAIL TO OPEN SUCTION DAMPER AFTER T\&M & $5.00 \mathrm{E}-02$ & $1.04 \mathrm{E}+00$ \\
\hline
\end{tabular}

Risk Reduction Worth

Basic Event Name

IA-FH-T\&MR\# PGV-2630FTCR\# UPS-FAN-BFLR\# DMP-.W--CCBR\# FAN-12-CCDR\# FAN-1234C3FR\#
Description FAN HOUSE AIR UNAVAILABLE DUE TO T\&M PLUG VALVE 2630 FAIIS TO CLOSE (STUCK) LOSS OF UPS CC: FOUR FAN EXHAUST DAMPERS FAIL TO OPEN CC: TWO RE-STARTED FAN LINES FAIl TO RUN CC: FOUR FANS FAIL TO START

\begin{tabular}{cc} 
Prob/Freg & RedW \\
\hline $3.20 \mathrm{E}-03$ & $1.73 \mathrm{E}+00$ \\
$3.00 \mathrm{E}-03$ & $1.65 \mathrm{E}+00$ \\
$4.70 \mathrm{E}-04$ & $1.07 \mathrm{E}+00$ \\
$3.00 \mathrm{E}-04$ & $1.04 \mathrm{E}+00$ \\
$2.52 \mathrm{E}-04$ & $1.03 \mathrm{E}+00$ \\
$2.50 \mathrm{E}-04$ & $1.03 \mathrm{E}+00$
\end{tabular}

Note: Events that make less than $1 \%$ difference to the importance factor are not included. Modified top event frequency = (top event frequency) / (risk reduction worth). 
X-ESR-S-00001, REV. 0

SPC Primary Purge Failure 


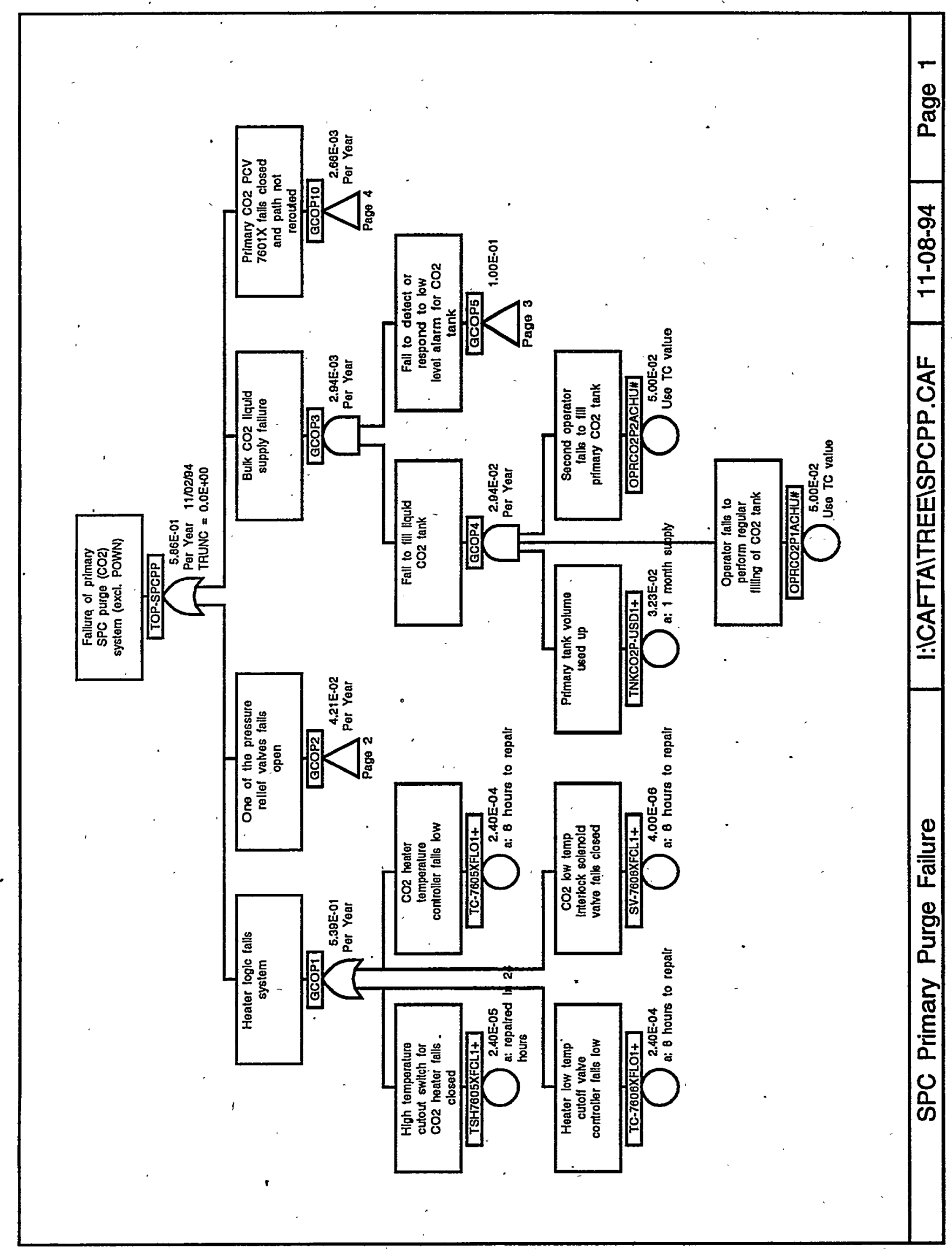


X-ESR-S-00001, REV. 0
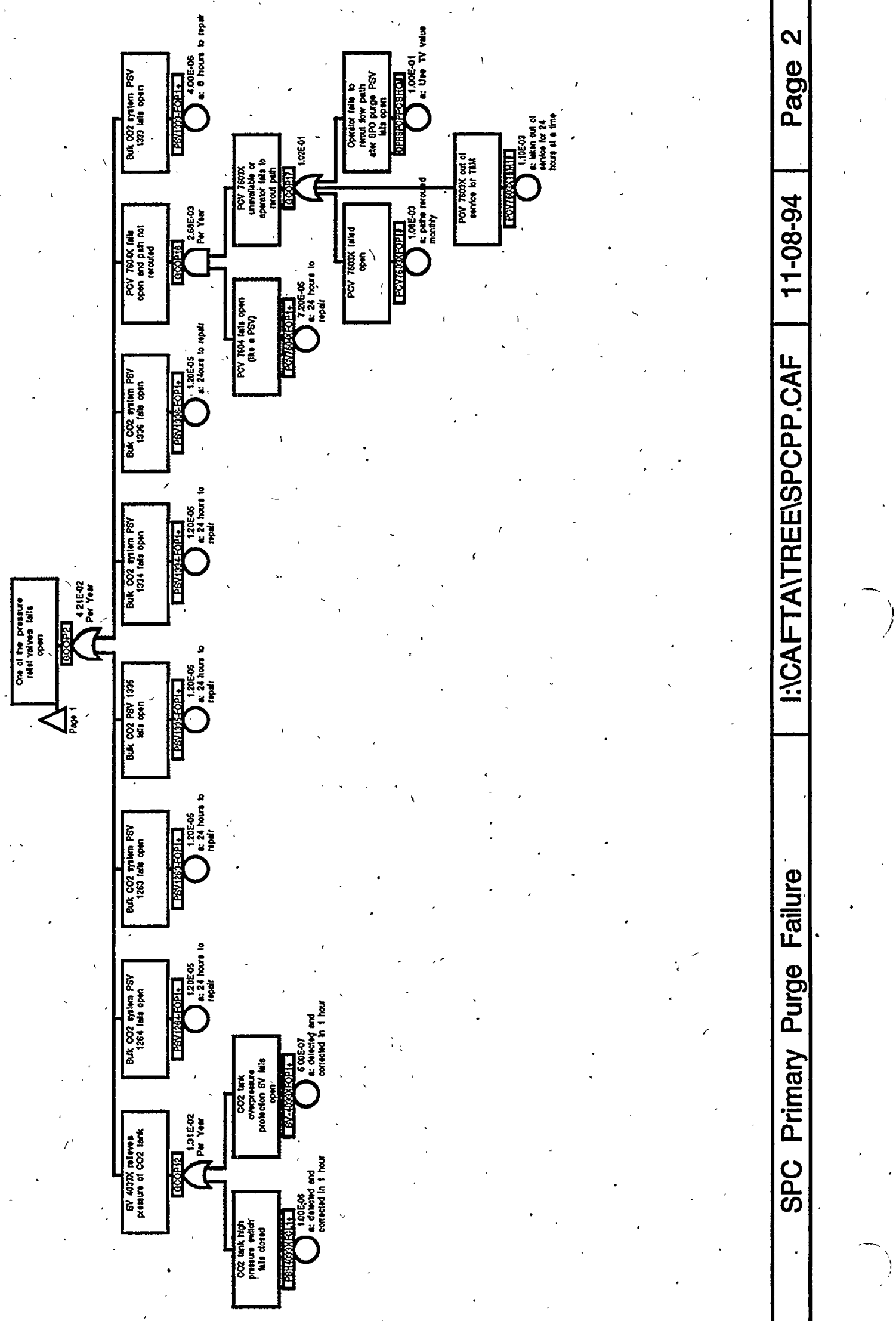

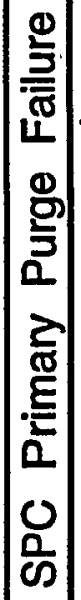




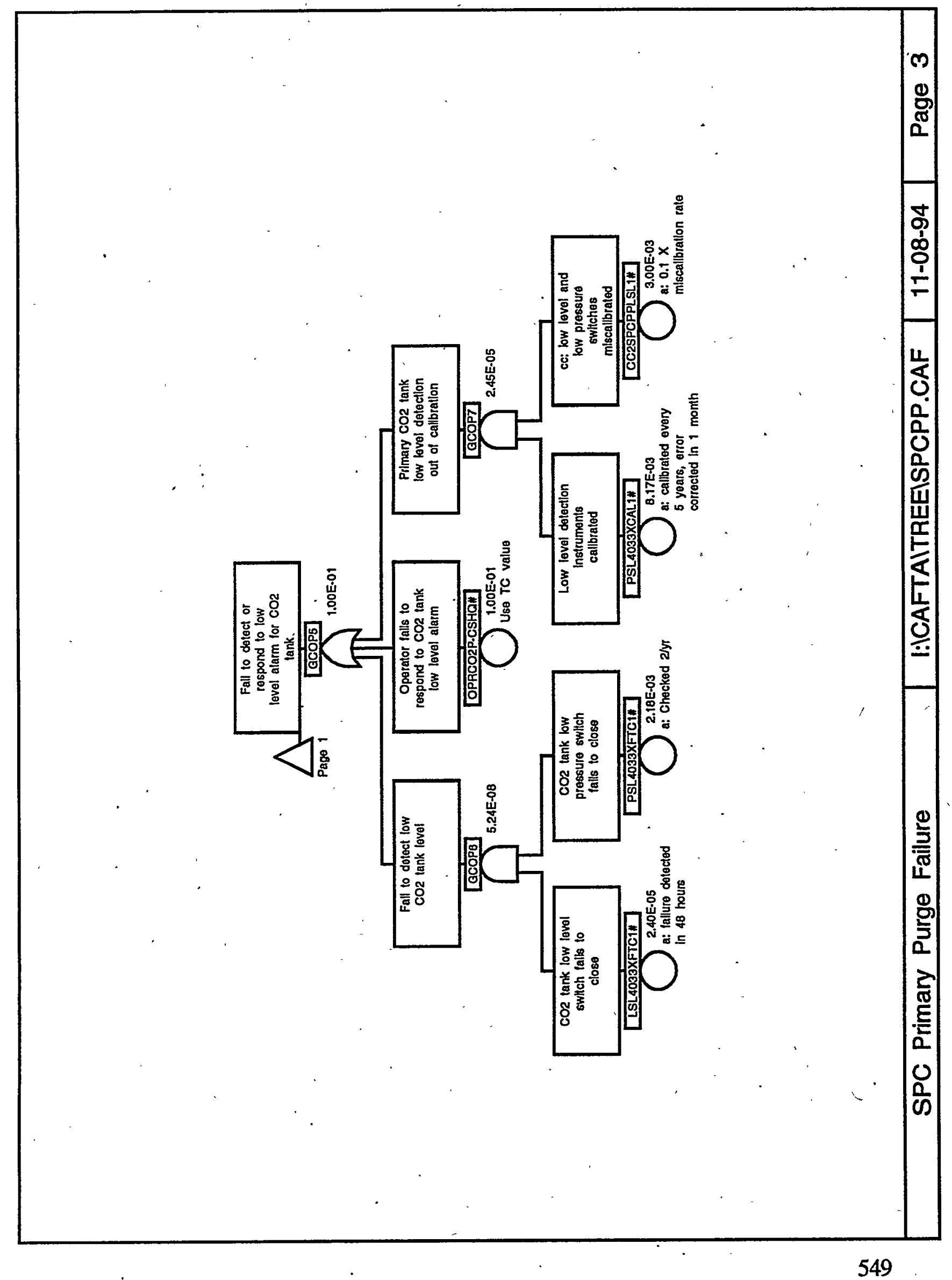


X-ESR-S-00001, REV, 0

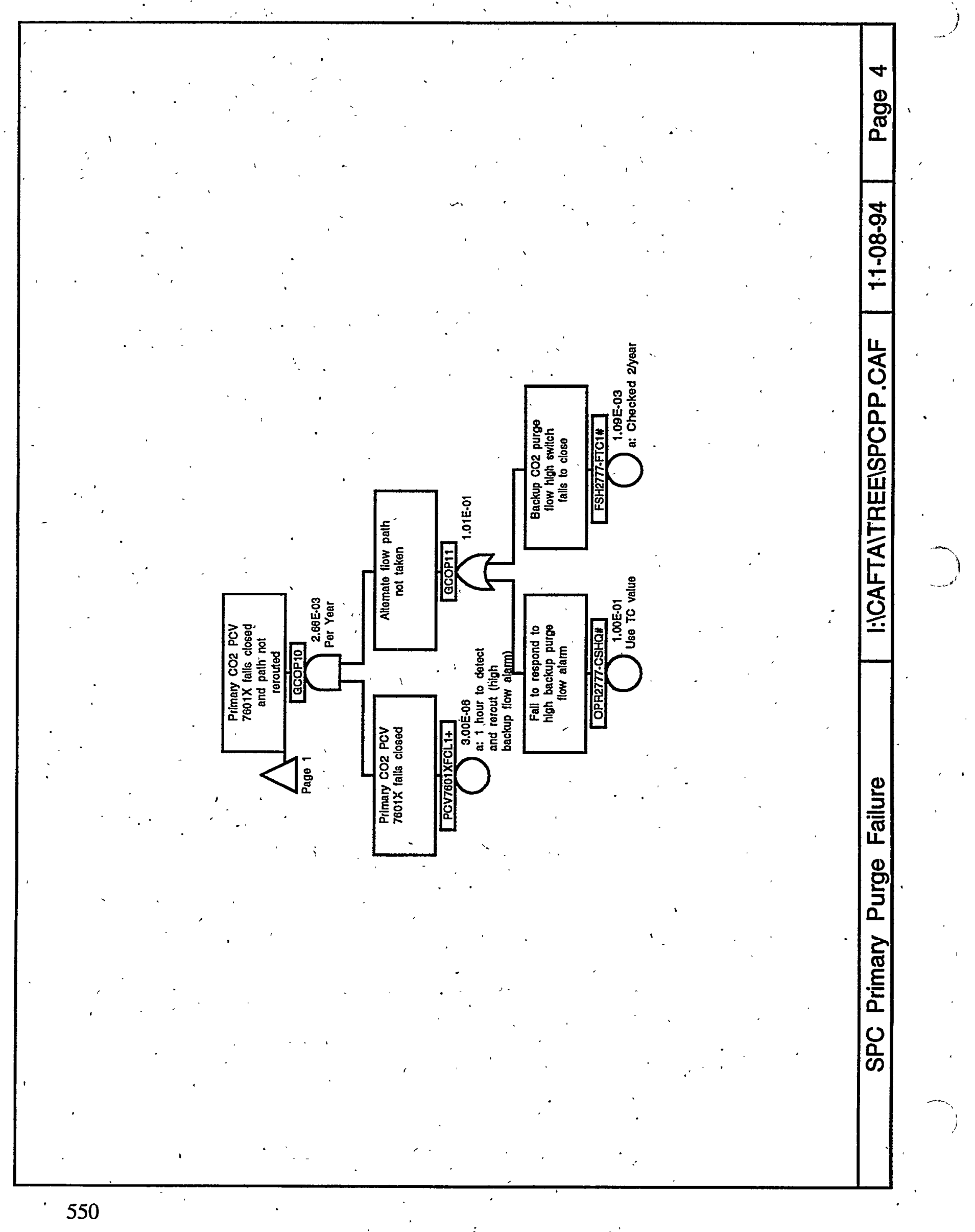


Cutsets for I: ICAFTA \CUT\SPCPP.CSR

$11 / 08 / 94 \quad 12: 12$ AM

\begin{tabular}{|c|c|c|c|c|c|c|}
\hline \begin{tabular}{l|} 
Set \\
No.
\end{tabular} & $\begin{array}{l}\text { Event } \\
\text { Name }\end{array}$ & Description & C) & $\begin{array}{l}\text { B. E. } \\
\text { Input }\end{array}$ & $\begin{array}{l}\text { Calc. } \\
\text { Result }\end{array}$ & $\begin{array}{l}\text { Cutset } \\
\text { Freg. }(/ Y x)\end{array}$ \\
\hline & TOP-SPCPP & & & & & $5.86 \mathrm{E}-01$ \\
\hline 1. & TC-7606XFLO1+ & Heater low temp cutoff valve controller fails low & 4 & $3.0 \mathrm{E}-05 \mathrm{H}$ & $2.40 E-04$ & $2.63 \mathrm{E}-01$ \\
\hline 2. & TC-7605XFLO1+ & CO2 heater temperature controller fails low & 4 & $3.0 \mathrm{E}-05 \mathrm{H}$ & $2.40 E-04$ & $2.63 \mathrm{E}-01$ \\
\hline 3 . & PSH4033XFCL1+ & $\mathrm{CO} 2$ tank high pressure switch fails closed. & 4 & $1.0 \mathrm{E}-06 \mathrm{H}$ & $1.00 \mathrm{E}-06$ & $8.76 \mathrm{E}-03$ \\
\hline 4. & TSH7 605XFCL1+ & High temperature cutout switch for $\mathrm{CO} 2$ heater fails, closed & 4 & $\begin{array}{r}24 \mathrm{H} \\
1.0 \mathrm{E}-06 \mathrm{H}\end{array}$ & $2.40 E-05$ & $8.76 \mathrm{E}-03$ \\
\hline 5. & SV-4033XFOP1+ & CO2 tank overpressure protection SV fails open & 4 & $5.0 \mathrm{E}-07 \mathrm{H}$ & $5.00 \mathrm{E}-07$ & $4.38 E-03$ \\
\hline 6. & SV-7606XFCL1+ & CO2 low temp interlock solenoid valve fails closed & 4 & $5.0 \mathrm{E}-07 \mathrm{H}$ & $4.00 \mathrm{E}-06$ & $4.38 E-03$ \\
\hline 7. & PSV1333-FOP1+ & Bulk CO2 system PSV 1333 fails open & 4 & $5.0 \mathrm{E}-07 \mathrm{H}$ & $4.00 \mathrm{E}-06$ & $4.38 \mathrm{E}-03$ \\
\hline 8. & PSV1334-FOP1+ & Bulk CO2 system PSV 1334 fails open & 4 & $5.0 \mathrm{E}-07 \mathrm{H}$ & $1.20 \mathrm{E}-05$ & $4.38 \mathrm{E}-03$ \\
\hline 9. & PSV1336-FOP1+ & Bulk CO2 system PSV 1336 fails open & 4 & $5.0 \mathrm{E}-07 \mathrm{H}$ & $1.20 \mathrm{E}-05$ & $4.38 E-03$ \\
\hline 10. & PSV1264-FOP1+ & Bulk CO2 system PSV 1264 fails open & 4 & $5.0 \mathrm{E}-07 \mathrm{H}$ & $1.20 \mathrm{E}-05$ & $4.38 \mathrm{E}-03$ \\
\hline 11 & PSV1263-FOP1+ & Bulk CO2 system PSV 1263 fails open & 4 & $\begin{array}{r}24 \mathrm{H} \\
5.0 \mathrm{E}-07 \mathrm{H}\end{array}$ & $1.20 \mathrm{E}-05$ & $4.38 \mathrm{E}-03$ \\
\hline 12. & PSV1335-FOP1+ & Bulk CO2 PSV 1335 , fails open & 4 & $5.0 \mathrm{E}-07 \mathrm{H}$ & $1.20 \mathrm{E}-05$ & $4.38 \mathrm{E}-03$ \\
\hline 13. & $\begin{array}{l}\text { OPRCO2P-CSHQ\# } \\
\text { OPRCO2P1ACHU\# } \\
\text { OPRCO2 P2ACHU\# } \\
\text { TNKCO2P-USD1+ }\end{array}$ & $\begin{array}{l}\text { Operator fails to respond to } \mathrm{CO} 2 \text { tank low level alarm } \\
\text { Operator fails to perform regular filling of } \mathrm{CO} 2 \text { tank } \\
\text { second operator fails to fill primary } \mathrm{CO} 2 \text { tank } \\
\text { Primary tank volume used up }\end{array}$ & $\left|\begin{array}{l}1 \\
1 \\
4\end{array}\right|$ & $\begin{array}{r}1 \mathrm{~N} \\
1.0 \mathrm{E}-01 \mathrm{~N} \\
1 \mathrm{~N} \\
5.0 \mathrm{E}-02 \mathrm{~N} \\
1 \mathrm{~N} \\
5.0 \mathrm{E}-02 \mathrm{~N} \\
24 \mathrm{H} \\
1 \mathrm{M}\end{array}$ & $\begin{array}{l}1.00 \mathrm{E}-01 \mathrm{~N} \\
5.00 \mathrm{E}-02 \mathrm{~N} \\
5.00 \mathrm{E}-02 \mathrm{~N} \\
3.23 \mathrm{E}-02\end{array}$ & $2.94 \mathrm{E}-03$ \\
\hline
\end{tabular}




\begin{tabular}{|c|c|c|c|c|c|c|c|}
\hline $\begin{array}{l}\text { Set } \\
\text { No. }\end{array}$ & $\begin{array}{l}\text { Event } \\
\text { Name }\end{array}$ & Description & & $\mathrm{C}$ & $\begin{array}{l}\text { B.E. } \\
\text { Input }\end{array}$ & $\begin{array}{r}\text { Calc. } \\
\text { Result }\end{array}$ & $\begin{array}{c}\text { Cutset } \\
\text { Freq. }(/ y r)\end{array}$ \\
\hline 14. & $\begin{array}{l}\text { OPR2777-CSHQ\# } \\
\text { PCV7601XFCL1+ }\end{array}$ & $\begin{array}{l}\text { Fail to respond to high backup purge flow alarm } \\
\text { Primary }{ }^{-} \mathrm{CO} \text { PCV } 7601 \mathrm{x} \text { fails closed }\end{array}$ & & $|4|$ & $\begin{array}{r}1 \mathrm{~N} \\
1.0 \mathrm{E}-01 \mathrm{~N} \\
1 \mathrm{H} \\
3.0 \mathrm{E}-06 \mathrm{H}\end{array}$ & $\begin{array}{l}1.00 \mathrm{E}-01 \mathrm{~N} \\
3.00 \mathrm{E}-06\end{array}$ & $2.63 \mathrm{E}-03$ \\
\hline 15 . & $\begin{array}{l}\text { OPRSPCPPCSSHQ\# } \\
\text { PCV7604XFOP1+ }\end{array}$ & $\begin{array}{l}\text { Operator fails to rerout flow path after SPC purge PSV } \\
\text { open } \\
\text { PCV } 7604 \text { fails open (like a PSV) } \\
\text {. }\end{array}$ & fails & $\begin{array}{l}1 \\
4\end{array}$ & $\begin{array}{r}1 \mathrm{~N} \\
1.0 \mathrm{E}-0.1 \mathrm{~N} \\
24 \mathrm{H} \\
3.0 \mathrm{E}-06 \mathrm{H}\end{array}$ & $\begin{array}{l}1.00 \mathrm{E}-01 \mathrm{~N} \\
7.20 \mathrm{E}-05\end{array}$ & $2.63 \mathrm{E}-03$ \\
\hline 16. & $\begin{array}{l}\text { PCV7 603XT\&M1 \# } \\
\text { PCV7.604XFOP1+ }\end{array}$ & $\begin{array}{l}\text { PCV } 7603 X \text { out of service fór T\&M } \\
\text { PCV } 7604 \text { fails open (like a PSV) }\end{array}$ & & 4 & $\begin{array}{r}24 \mathrm{H} \\
4.0 \mathrm{E}-01 \mathrm{Y} \\
24 \mathrm{H} \\
3.0 \mathrm{E}-06 \mathrm{H}\end{array}$ & $\begin{array}{l}1.10 \mathrm{E}-03 \\
7.20 \mathrm{E}-05\end{array}$ & $2.88 \mathrm{E}-05$ \\
\hline 17. & $\begin{array}{l}\text { FSH2777-FTC1\# } \\
\text { PCV7601XFCL1+ }\end{array}$ & $\begin{array}{l}\text { Backup CO2 purge flow high switch fails to close } \\
\text { Primary CO2 PCV } 7601 X \text { fails closed }\end{array}$ & & 5 & $\begin{array}{r}10 \\
1.0 \mathrm{E}-06 \mathrm{H} \\
1 \mathrm{H} \\
3.0 \mathrm{E}-06 \mathrm{H}\end{array}$ & $\begin{array}{l}1.09 \mathrm{E}-03 \\
3.00 \mathrm{E}-06\end{array}$ & $2.87 \mathrm{E}-05$ \\
\hline 18. & $\begin{array}{l}\text { PCV7603XFOP1\# } \\
\text { PCV7604XFOP1+ }\end{array}$ & $\begin{array}{l}\text { PCV } 7603 x \text { failed open } \\
\text { PCV } 7604 \text { fails open (iike a PSV) }\end{array}$ & & 5 & $\begin{array}{r}1 \mathrm{M} \\
3.0 \mathrm{E}-06 \mathrm{H} \\
24 \mathrm{H} \\
3.0 \mathrm{E}-06 \mathrm{H}\end{array}$ & $\begin{array}{l}1.08 \mathrm{E}-03 \\
7.20 \mathrm{E}-05\end{array}$ & $2.84 \mathrm{E}-05$ \\
\hline 19. & $\begin{array}{l}\text { CC2SPCP PLSL1\# } \\
\text { OPRCO2P1ACHÜ\# } \\
\text { OPRCO2P2ACHU\# } \\
\text { PSL4033XCAL1\# } \\
\text { TNKCO2P-USD1+ }\end{array}$ & $\begin{array}{l}\text { cc: low level and low pressure switches miscalibrated } \\
\text { Operator fails to perform regular fllling of } \mathrm{CO} 2 \text { tank } \\
\text { second operator fails to fill primary } \mathrm{CO} 2 \text { tank } \\
\text { Low level detection instruments calibrated } \\
\text { Primary tank volume used up }\end{array}$ & & 4 & $\begin{array}{r}1 \mathrm{~N} \\
3.0 \mathrm{E}-03 \mathrm{~N} \\
1 \mathrm{~N} \\
5.0 \mathrm{E}-02 \mathrm{~N} \\
1 \mathrm{~N} \\
5.0 \mathrm{E}-02 \mathrm{~N} \\
1 \mathrm{M} \\
2.0 \mathrm{E}-01 \mathrm{Y} \\
24 \mathrm{H} \\
1 \mathrm{M}\end{array}$ & $\begin{array}{l}3.00 \mathrm{E}-03 \mathrm{~N} \\
5.00 \mathrm{E}-02 \mathrm{~N} \\
5.00 \mathrm{E}-02 \mathrm{~N} \\
8.17 \mathrm{E}-03 \\
3.23 \mathrm{E}-02\end{array}$ & $7.22 \mathrm{E}-07$ \\
\hline 20. & $\begin{array}{l}\text { LSL4033XFTC1\# } \\
\text { OPRCO2P1ACHU\# } \\
\text { OPRCO2P2ACHU\# } \\
\text { PSL4033XFTC1\# } \\
\text { TNKCO2P-USD1+ }\end{array}$ & $\begin{array}{l}\mathrm{CO} 2 \text { tank low level switch fails to close } \\
\text { Operator fails to perform regular filling of } \mathrm{CO} 2 \text { tank } \\
\text { Second operator fails to fill primary } \mathrm{CO} 2 \text { tank } \\
\mathrm{CO} 2 \text { tank low pressure switch fails to close } \\
\text { Primary tank volume used up. }\end{array}$ & & 4 & $\begin{array}{r}2 \mathrm{D} \\
1.0 \mathrm{E}-06 \mathrm{H} \\
1 \mathrm{~N} \\
5.0 \mathrm{E}-02 \mathrm{~N} \\
1 \mathrm{~N} \\
5.0 \mathrm{E}-02 \mathrm{~N} \\
1 \mathrm{Q} \\
1.0 \mathrm{E}-06 \mathrm{H} \\
24 \mathrm{H} \\
1 \mathrm{M}\end{array}$ & $\begin{array}{l}2.40 \mathrm{E}-05 \\
5.00 \mathrm{E}-02 \mathrm{~N} \\
5.00 \mathrm{E}-02 \mathrm{~N} \\
2.18 \mathrm{E}-03 \\
3.23 \mathrm{E}-02\end{array}$ & $1.54 \mathrm{E}-09$ \\
\hline
\end{tabular}


Failure of primary SPC purge (CO2) system (excl. POWN)

Top Event Frequency: 5.86E-01/YR

Risk Achievement Worth

Basic Event Name
PSV1336-FOP1+-I
PSV1334-FOP1+-I
PSV1333-FOP1+-I
PSV1335-FOP1+-I
PSV1263-FOP1+-I
PSV1264-FOP1+-I
PSH4033XFCL1+-I
TC-7605XFLO1+-I
TC-7606XFLO1+-I
SV-7606XFCL1+-I
SV-4033XFOP1+-I
TSH7605XFCL1+-I
PCV7604XFOP1+-I
PCV7601XFCL1+-I
TNKCO2P-USD1+-I
OPRCO2P1ACHU\#
OPRCO2P2ACHU\#
OPRCO2P-CSHQ\#
PCV7603XFOP1\#
FSH2777-FTC1\#
PCV7603XT\&M1\#
OPR2777-CSHQ\#
OPRSPCPPCSHO\#

Description

Bulk CO2 system PSV 1336 fails open

Bulk CO2 system PSV 1334 fails open

Bulk CO2 system PSV 1333 fails open

Bulk CO2 PSV 1335 fails open

Bulk CO2 system PSV 1263 fails open

Bulk CO2 system PSV 1264 fails open

$\mathrm{CO} 2$ tank high pressure switch fails closed

$\mathrm{CO} 2$ heater temperature controller fails low

Heater low temp cutoff valve controller fails low

$\mathrm{CO} 2$ low temp interlock solenoid valve fails closed

$\mathrm{CO} 2$ tank overpressure protection SV fails open

High temperature cutout switch for $\mathrm{CO} 2$ heater fails closed

PCV 7604 fails open (like a PSV)

Primary CO2 PCV 7601X fails closed

Primary tank volume used up

Operator fails to perform regular filling of $\mathrm{CO} 2$ tank

Second operator fails to fill primary $\mathrm{CO} 2$ tank

Operator fails to respond to $\mathrm{CO} 2$ tank low level alarm

PCV 7603X failed open

Backup $\mathrm{CO} 2$ purge flow high switch fails to close

PCV 7603X out of service for $T \& M$

Fail to respond to high backup purge flow alarm

Fail to rerout flow path after SPC purge PSV fails open \begin{tabular}{ll} 
Prob/Freg & AchW \\
\hline $5.00 \mathrm{E}-07 / \mathrm{H}$ & $1.49 \mathrm{E}+04$ \\
$5.00 \mathrm{E}-07 / \mathrm{H}$ & $1.49 \mathrm{E}+04$ \\
$5.00 \mathrm{E}-07 / \mathrm{H}$ & $1.49 \mathrm{E}+04$ \\
$5.00 \mathrm{E}-07 / \mathrm{H}$ & $1.49 \mathrm{E}+04$ \\
$5.00 \mathrm{E}-07 / \mathrm{H}$ & $1.49 \mathrm{E}+04$ \\
$5.00 \mathrm{E}-07 / \mathrm{H}$ & $1.49 \mathrm{E}+04$ \\
$1.00 \mathrm{E}-06 / \mathrm{H}$ & $1.49 \mathrm{E}+04$ \\
$3.00 \mathrm{E}-05 / \mathrm{H}$ & $1.49 \mathrm{E}+04$ \\
$3.00 \mathrm{E}-05 / \mathrm{H}$ & $1.49 \mathrm{E}+04$ \\
$5.00 \mathrm{E}-07 / \mathrm{H}$ & $1.49 \mathrm{E}+04$ \\
$5.00 \mathrm{E}-07 / \mathrm{H}$ & $1.49 \mathrm{E}+04$ \\
$1.00 \mathrm{E}-06 / \mathrm{H}$ & $1.49 \mathrm{E}+04$ \\
$3.00 \mathrm{E}-06 / \mathrm{H}$ & $1.52 \mathrm{E}+03$ \\
$3.00 \mathrm{E}-06 / \mathrm{H}$ & $1.51 \mathrm{E}+03$ \\
$1.34 \mathrm{E}-03 / \mathrm{H}$ & $4.73 \mathrm{E}+00$ \\
$5.00 \mathrm{E}-02$ & $1.10 \mathrm{E}+00$ \\
$5.00 \mathrm{E}-02$ & $1.10 \mathrm{E}+00$ \\
$1.00 \mathrm{E}-01$ & $1.05 \mathrm{E}+00$ \\
$1.08 \mathrm{E}-03$ & $1.04 \mathrm{E}+00$ \\
$1.09 \mathrm{E}-03$ & $1.04 \mathrm{E}+00$ \\
$1.10 \mathrm{E}-03$ & $1.04 \mathrm{E}+00$ \\
$1.00 \mathrm{E}-01$ & $1.04 \mathrm{E}+00$ \\
$1.00 \mathrm{E}-01$ & $1.04 \mathrm{E}+00$ \\
&
\end{tabular}

Modified top event frequency $=$ (top event frequency) $\mathrm{x}$ (risk achievement worth).
Basic Event Name TC-7605XFLO1+-I TC-7606XFLO1+-I TSH7605XFCL1+I PSH4033XFCL1+-I

\section{Risk Reduction Worth}

\section{Description}

$\mathrm{CO} 2$ heater temperature controller fails low

Heater low temp cutoff valve controller fails low

High temperature cutout switch for $\mathrm{CO} 2$ heater fails closed

- $\mathrm{CO} 2$ tank high pressure switch fails closed

\section{Prob/Freg RedW \\ $3.00 \mathrm{E}-05 / \mathrm{H} \quad 1.81 \mathrm{E}+00$ \\ $3.00 \mathrm{E}-05 / \mathrm{H} \quad 1.81 \mathrm{E}+00$ \\ $1.00 \mathrm{E}-06 / \mathrm{H} \quad 1.02 \mathrm{E}+00$ \\ $1.00 \mathrm{E}-06 / \mathrm{H} \quad 1.02 \mathrm{E}+00$}

Note: Events that make less than $1 \%$ difference to the importance factor are not included. Modified top event frequency = (top event frequency) $/$ (risk reduction worth). 
X-ESR-S-00001, REV. 0

SPC Backup Purge Unavailable 


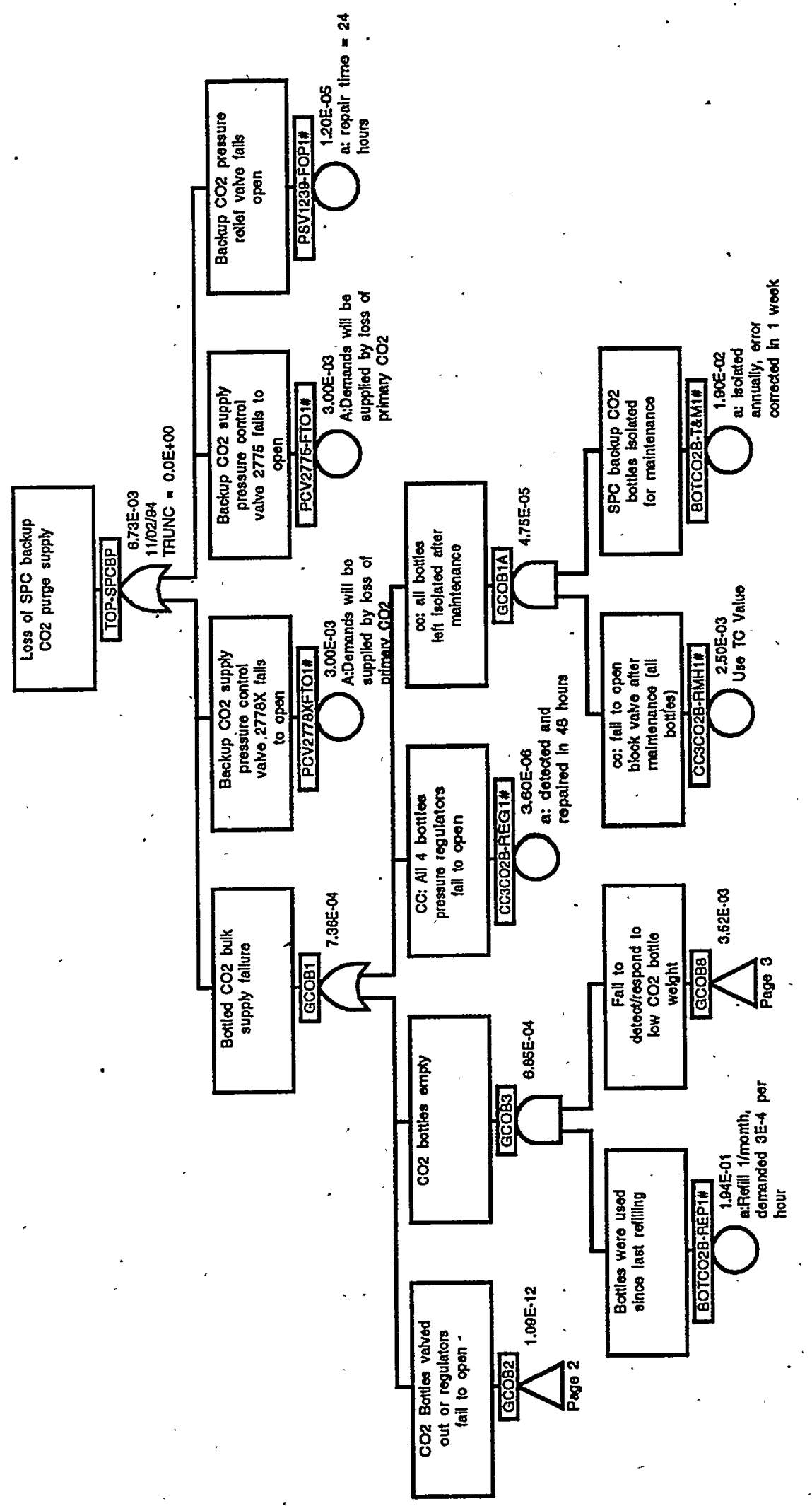




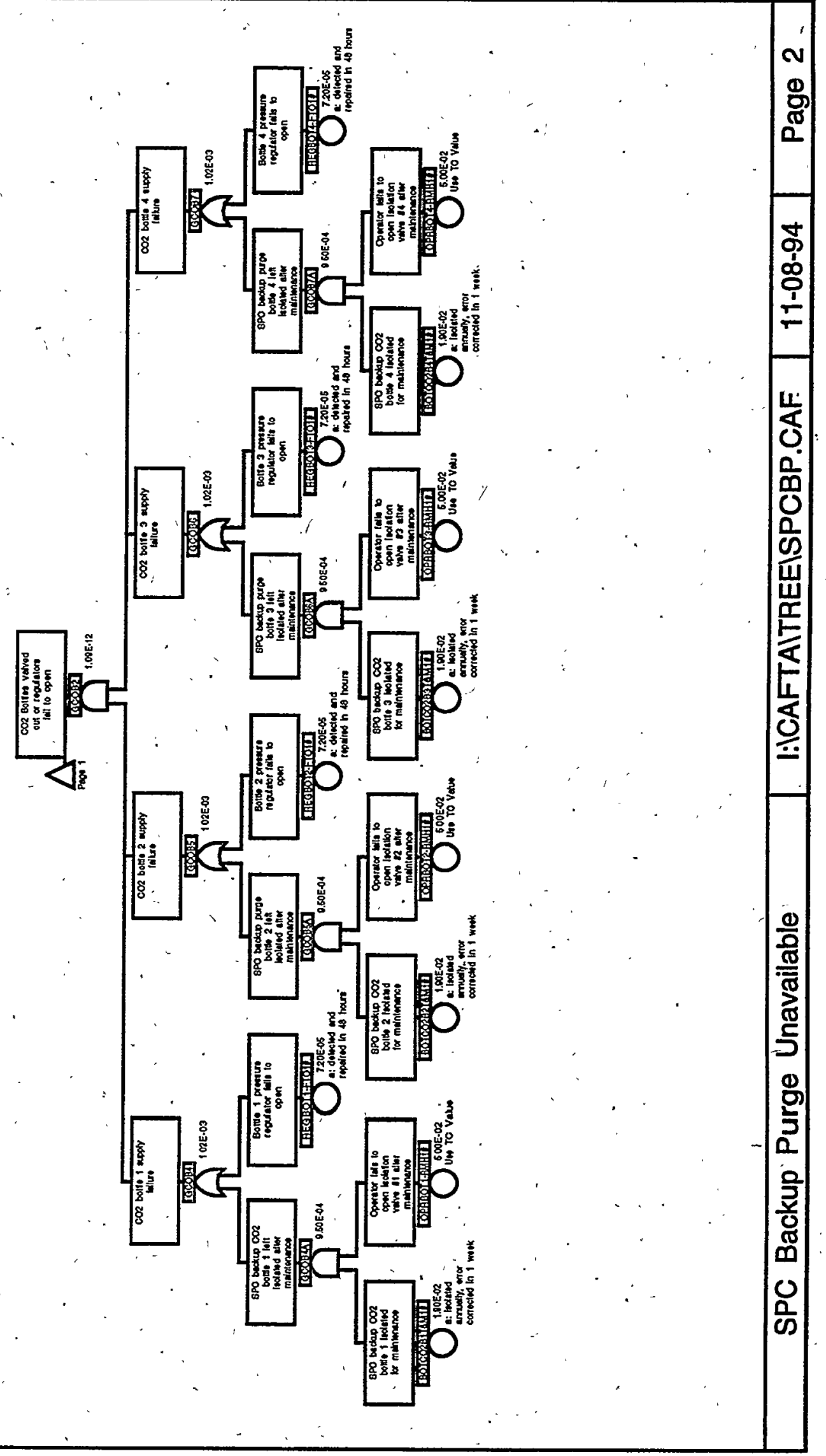




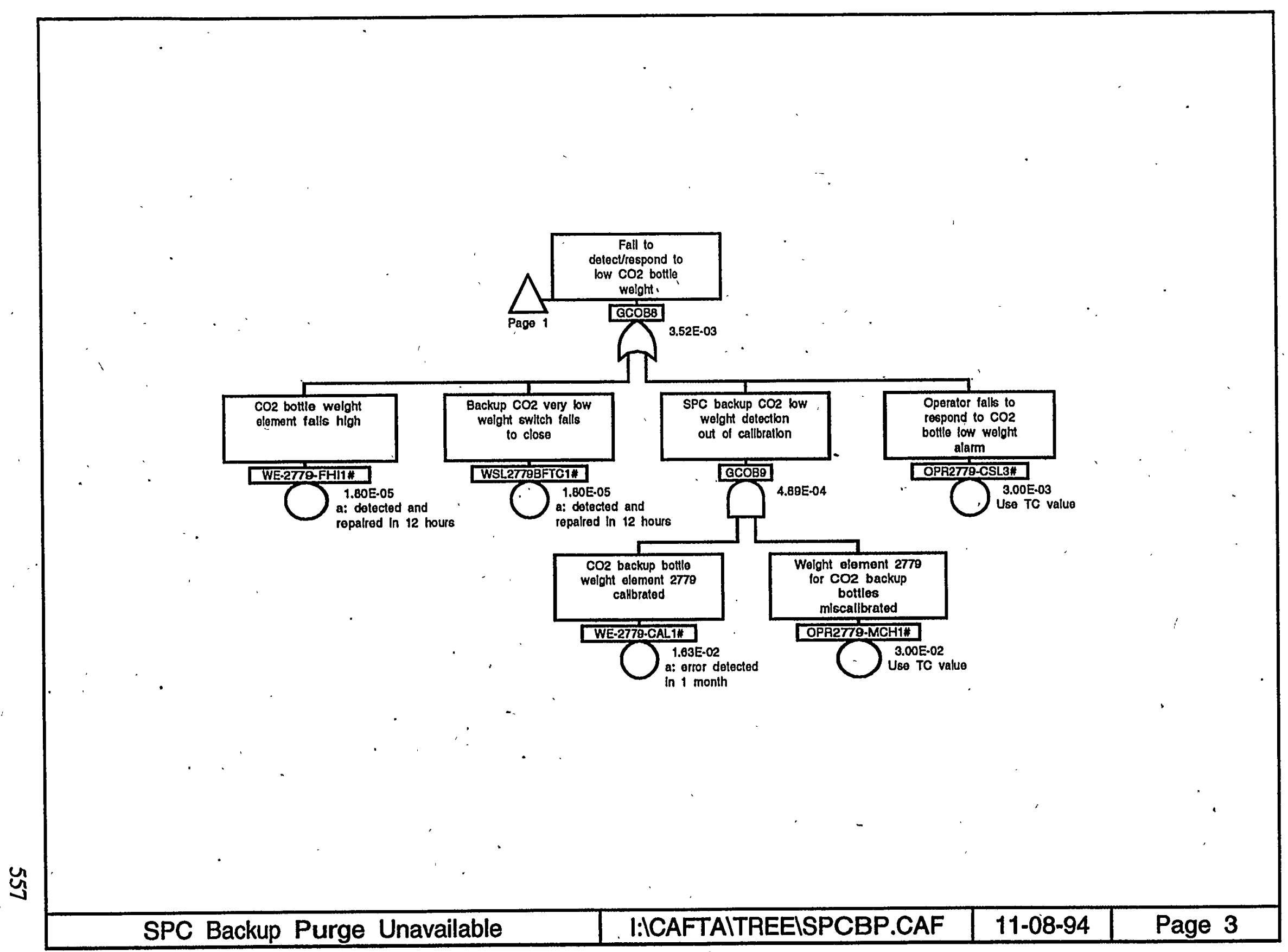




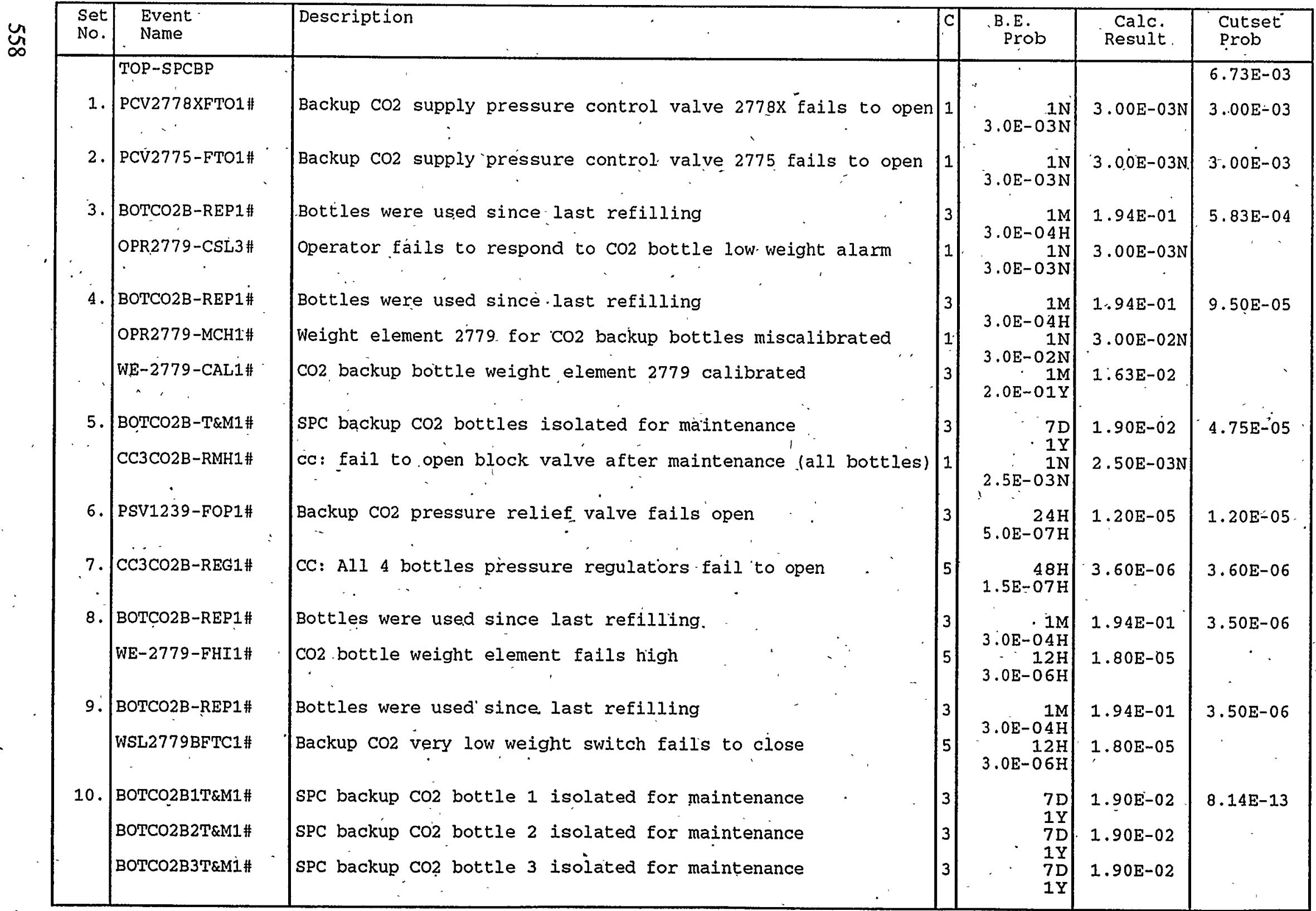




\begin{tabular}{|c|c|c|c|c|c|c|}
\hline $\begin{array}{l}\text { Set } \\
\text { No. }\end{array}$ & $\begin{array}{l}\text { Event } \\
\text { Name }\end{array}$ & Description & $c$ & $\begin{array}{c}\text { B.E. } \\
\text { Prob }\end{array}$ & $\begin{array}{l}\text { Calc. } \\
\text { Result }\end{array}$ & $\begin{array}{l}\text { Cutset } \\
\text { Prob }\end{array}$ \\
\hline 11. & $\begin{array}{l}\text { BOTCO2B4T\&M1\# } \\
\text { OPRBOT1-RMH1\# } \\
\text { OPRBOT2-RMH1\# } \\
\text { OPRBOT3-RMH1\# } \\
\text { OPRBOT4-RMH1\# } \\
\text { BOTCO2B1T\&M1\# } \\
\text { BOTCO2B3T\&M1\# } \\
\text { BOTCO2B4T\&M1\# } \\
\text { OPRBOT1-RMH1\# } \\
\text { OPRBOT3-RMH1\# } \\
\text { OPRBOT4-RMH1\# } \\
\text { REGBOT2-FTO1\# } \\
\text { BOTCO2B1T\&M1\# } \\
\text { BOTCO2B2T\&M1\# } \\
\text { BOTCO2B4T\&M1\# } \\
\text { OPRBOT1-RMH1\# } \\
\text { OPRBOT2-RMH1\# } \\
\text { OPRBOT4-RMH1\# } \\
\text { REGBOT3-FTO1\# } \\
\text { BOTCO2B2T\&M1\# } \\
\text { BOTCO2B3T\&M1\# } \\
\text { BOTCO2B4T\&M1\# }\end{array}$ & 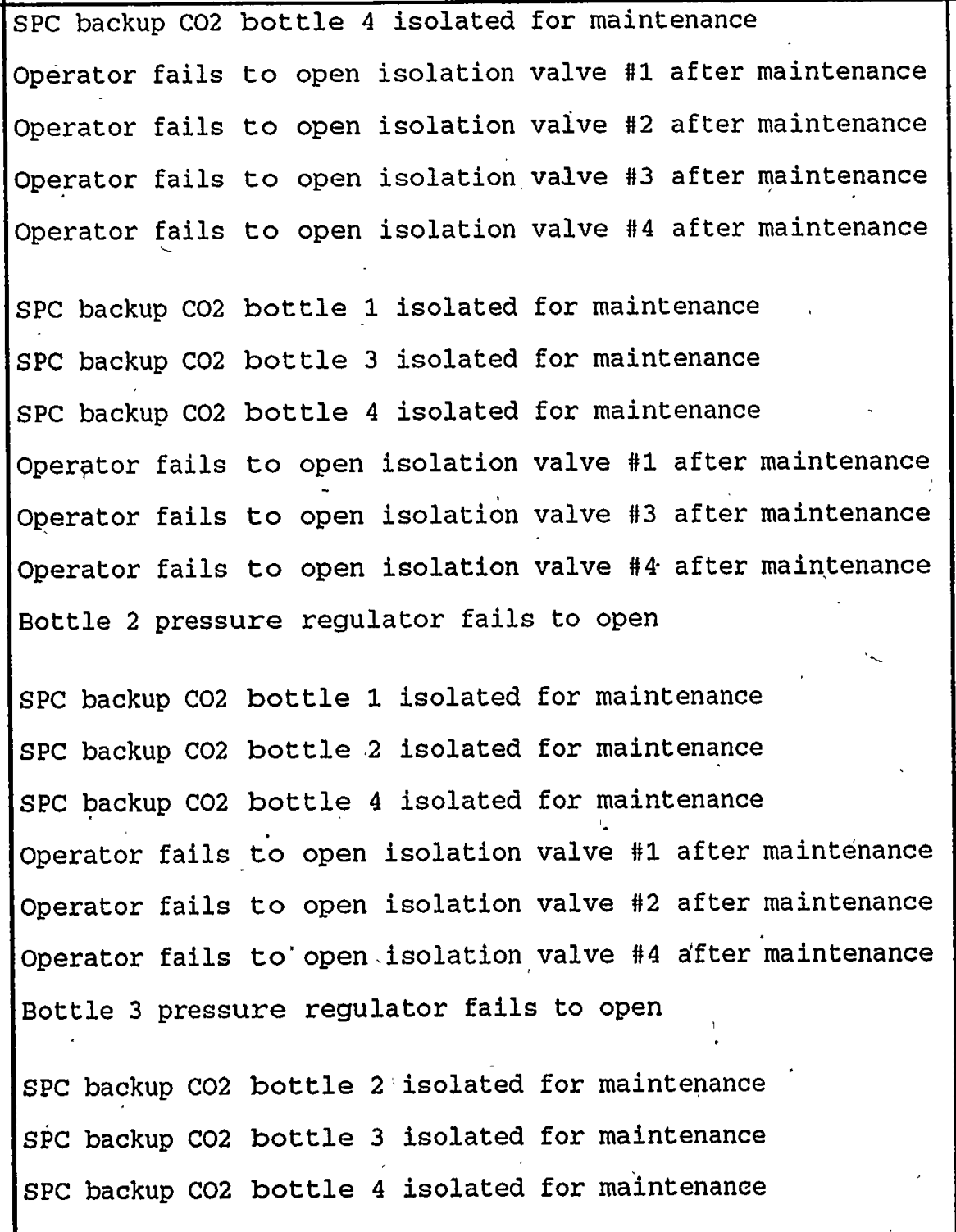 & $\begin{array}{l}1 \\
1 \\
5\end{array}$ & $\begin{array}{r}7 \mathrm{D} \\
1 \mathrm{Y} \\
1 \mathrm{~N} \\
5.0 \mathrm{E}-02 \mathrm{~N} \\
1 \mathrm{~N} \\
5.0 \mathrm{E}-02 \mathrm{~N} \\
1 \mathrm{~N} \\
5.0 \mathrm{E}-02 \mathrm{~N} \\
1 \mathrm{~N} \\
5.0 \mathrm{E}-02 \mathrm{~N} \\
7 \mathrm{D} \\
1 \mathrm{Y} \\
7 \mathrm{D} \\
1 \mathrm{Y} \\
7 \mathrm{D} \\
1 \mathrm{Y} \\
1 \mathrm{~N} \\
5.0 \mathrm{E}-02 \mathrm{~N} \\
1 \mathrm{~N} \\
5.0 \mathrm{E}-02 \mathrm{~N} \\
1 \mathrm{~N} \\
5.0 \mathrm{E}-02 \mathrm{~N} \\
48 \mathrm{H} \\
3.0 \mathrm{E}-06 \mathrm{H} \\
7 \mathrm{D} \\
1 \mathrm{Y} \\
7.0 \mathrm{D}-02 \mathrm{~N} \\
1 \mathrm{~N} \\
5.0 \mathrm{E}-02 \mathrm{~N} \\
1 \mathrm{~N} \\
5.0 \mathrm{E}-02 \mathrm{~N} \\
48 \mathrm{H} \\
3.0 \mathrm{E}-06 \mathrm{H}\end{array}$ & $\begin{array}{l}5.00 \mathrm{E}-02 \mathrm{~N} \\
5.00 \mathrm{E}-02 \mathrm{~N} \\
5.00 \mathrm{E}-02 \mathrm{~N} \\
1.90 \mathrm{E}-02 \\
1.90 \mathrm{E}-02 \\
1.90 \mathrm{E}-02 \\
5.00 \mathrm{E}-02 \mathrm{~N} \\
5.00 \mathrm{E}-02 \mathrm{~N} \\
5.00 \mathrm{E}-02 \mathrm{~N} \\
7.20 \mathrm{E}-05 \\
1.90 \mathrm{E}-02 \\
1.90 \mathrm{E}-02 \\
1.90 \mathrm{E}-02 \\
5.00 \mathrm{E}-02 \mathrm{~N} \\
5.00 \mathrm{E}-02 \mathrm{~N} \\
5.00 \mathrm{E}-02 \mathrm{~N} \\
7.20 \mathrm{E}-05 \\
1.90 \mathrm{E}-02 \\
1.90 \mathrm{E}-02 \\
1.90 \mathrm{E}-02\end{array}$ & $6.17 E-14$ \\
\hline
\end{tabular}




\begin{tabular}{|c|c|c|c|c|c|c|c|}
\hline & $\begin{array}{l}\text { Set } \\
\text { No. }\end{array}$ & $\begin{array}{l}\text { Event } \\
\text { Name }\end{array}$ & ion & & $b$ & te & \\
\hline 1 & 14. & $\begin{array}{c}\text { OPRBOT2-RMH1\# } \\
\text { OPRBOT3-RMH1\# } \\
\text { OPRBOT4-RMH1\# } \\
\text { REGBOT1-FTO1\# } \\
\text { BOTCO2B1T\&M1 \# } \\
\text { BOTCO2B2T\&M1\# } \\
\text { BOTCO2B3T\&M1\# } \\
\text { OPRBOT1-RMH1\# } \\
\text { OPRBOT2-RMH1\# } \\
\text { OPRBOT3-RMH1\# } \\
\text { REGBOT4-FTO1\# } \\
\text { BOTCO2B2T\&M1\# } \\
\text { BOTCO2B4T\&M1\# } \\
\text { OPRBOT2-RMH1\# } \\
\text { OPRBOT4-RMH1\# } \\
\text { REGBOT1-FTO1\# } \\
\text { REGBOT3-FTO1\# } \\
\text { BOTCO2B1T\&M1\# } \\
\text { BOTCO2B3T\&M1\# } \\
\text { OPRBOT1-RMH1\# } \\
\text { OPRBOT3-RMH1\# } \\
\text { REGBOT2-FTO1\# }\end{array}$ & 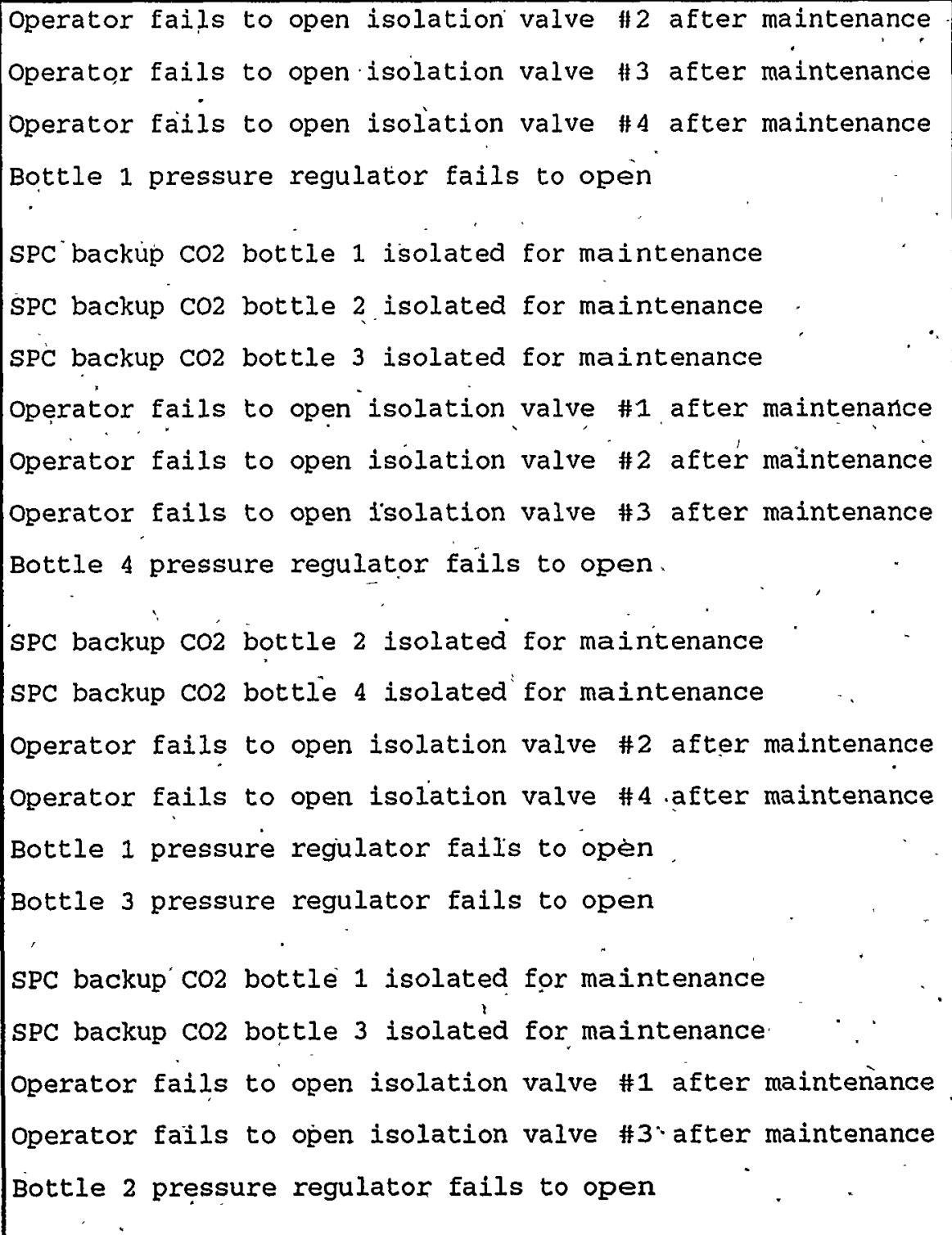 & 13 & $\begin{array}{r}1 \mathrm{~N} \\
5.0 \mathrm{E}-02 \mathrm{~N} \\
1 \mathrm{~N} \\
5.0 \mathrm{E}-02 \mathrm{~N} \\
1 \mathrm{~N} \\
5.0 \mathrm{E}-02 \mathrm{~N} \\
48 \mathrm{H} \\
3.0 \mathrm{E}-06 \mathrm{H} \\
7 \mathrm{D} \\
1 \mathrm{Y} \\
7 \mathrm{D} \\
1 \mathrm{Y} \\
7 \mathrm{D} \\
.1 \mathrm{Y} \\
1 \mathrm{~N} \\
5.0 \mathrm{E}-02 \mathrm{~N} \\
1 \mathrm{~N} \\
5.0 \mathrm{E}-02 \mathrm{~N} \\
1 \mathrm{~N} \\
5.0 \mathrm{E}-02 \mathrm{~N} \\
48 \mathrm{H} \\
3.0 \mathrm{E}-06 \mathrm{H} \\
\\
7 \mathrm{D} \\
1 \mathrm{Y} \\
7 \mathrm{D} \\
1 \mathrm{Y} \\
1.0 \mathrm{~N}-06 \mathrm{H} \\
48 \mathrm{H} \\
5.0 \mathrm{E}-02 \mathrm{~N} \\
1 \mathrm{~N} \\
5.0 \mathrm{E}-02 \mathrm{~N} \\
48 \mathrm{H} \\
3.06 \mathrm{H}\end{array}$ & $\begin{array}{l}5.00 \mathrm{E}-02 \mathrm{~N} \\
5.00 \mathrm{E}-02 \mathrm{~N} \\
5.00 \mathrm{E}-02 \mathrm{~N} \\
7.20 \mathrm{E}-05 \\
1.90 \mathrm{E}-02 \\
1.90 \mathrm{E}-02 \\
1.90 \mathrm{E}-02 \\
5.00 \mathrm{E}-02 \mathrm{~N} \\
5.00 \mathrm{E}-02 \mathrm{~N} \\
5.00 \mathrm{E}-02 \mathrm{~N} \\
7.20 \mathrm{E}-05 \\
1.90 \mathrm{E}-02 \\
1.90 \mathrm{E}-02 \\
5.00 \mathrm{E}-02 \mathrm{~N} \\
5.00 \mathrm{E}-02 \mathrm{~N} \\
7.20 \mathrm{E}-05 \\
7.20 \mathrm{E}-05 \\
1.90 \mathrm{E}-02 \\
1.90 \mathrm{E}-02 \\
5.00 \mathrm{E}-02 \mathrm{~N} \\
5.00 \mathrm{E}-02 \mathrm{~N} \\
7.20 \mathrm{E}-05\end{array}$ & $\begin{array}{l}. \\
\cdots \\
.\end{array}$ \\
\hline
\end{tabular}


Cutsets for I: \CAFTA ICUT\SPCBP.CSR

11/08/94 12:13 AM (CONT.)

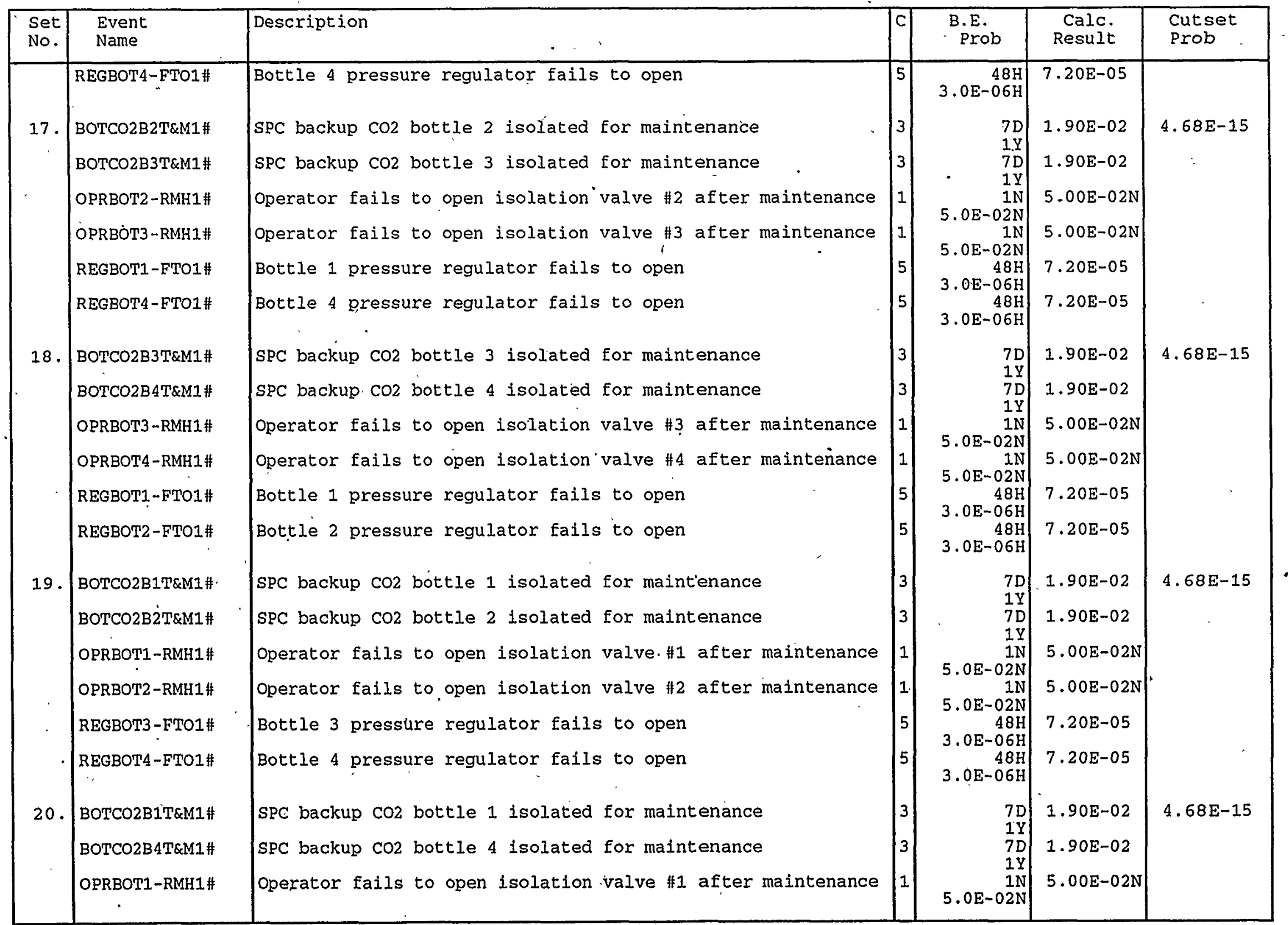




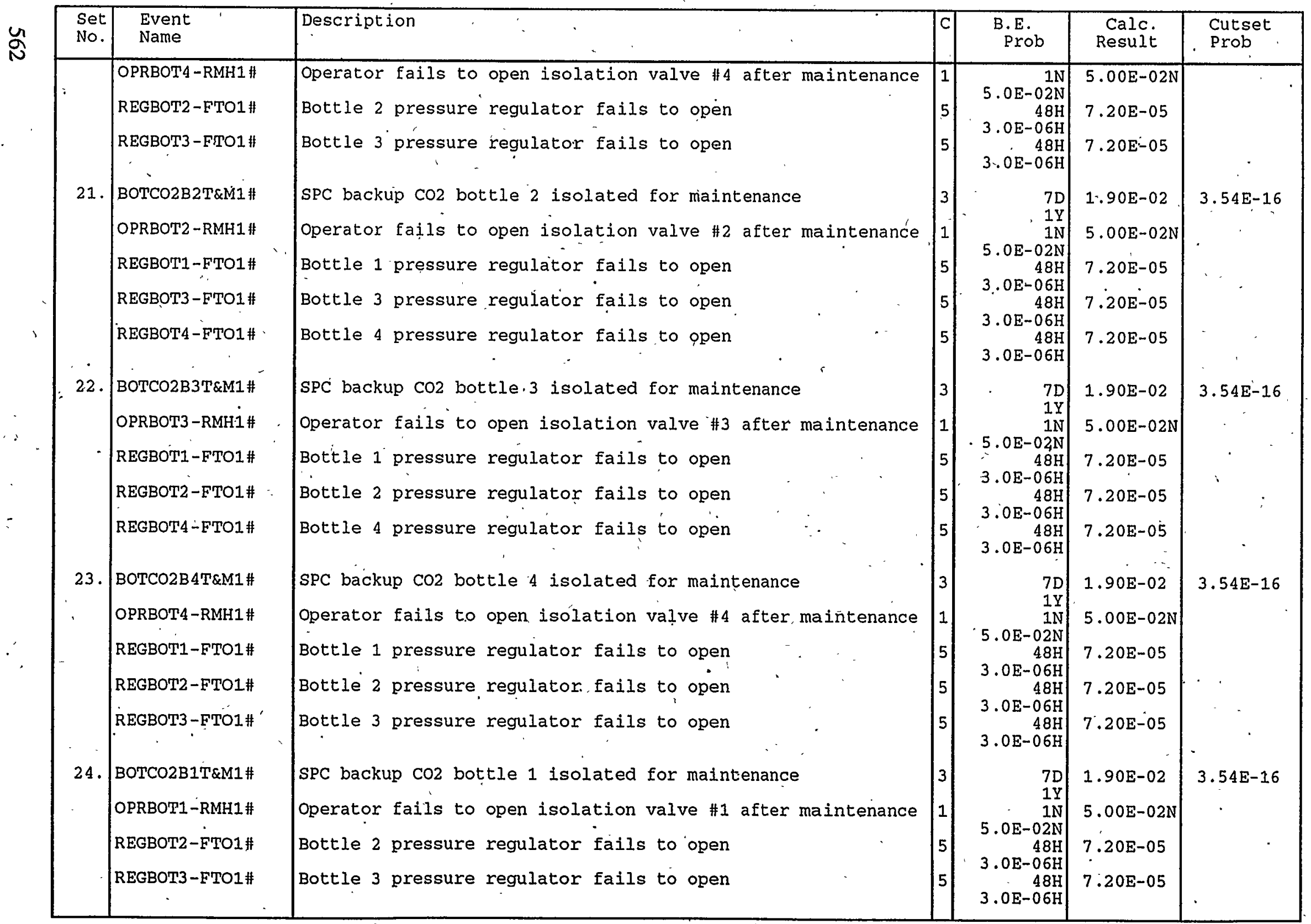


Cutsets for I: \CAFTA ICUT\SPCBP.CSR

11/08/94 12:13 AM (CONT.)

\begin{tabular}{|c|c|c|c|c|c|c|c|}
\hline $\begin{array}{l}\text { Set } \\
\text { No. }\end{array}$ & $\begin{array}{l}\text { Event } \\
\text { Name }\end{array}$ & Description & & C & $\begin{array}{l}\text { B.E. } \\
\text { Prob }\end{array}$ & $\begin{array}{l}\text { Calc. } \\
\text { Result }\end{array}$ & $\begin{array}{l}\text { Cutset } \\
\text { Prob }\end{array}$ \\
\hline 25 & $\begin{array}{l}\text { REGBOT4-FTO1\# } \\
\text { REGBOT1-FTO1\# } \\
\text { REGBOT2-FTO1\# } \\
\text { REGBOT3-FTO1\# } \\
\text { REGBOT4-FTO1\# }\end{array}$ & $\begin{array}{l}\text { Bottle } 4 \text { pressure regulator fails to open } \\
\text { Bottle } 1 \text { pressure regulator fails to open } \\
\text { Bottle } 2 \text { pressure regulator fails to open } \\
\text { Bottle } 3 \text { pressure regulator fails to open } \\
\text { Bottle } 4 \text { pressure regulator fails to open }\end{array}$ & , & $\left|\begin{array}{l}5 \\
5 \\
5 \\
5\end{array}\right|$ & $\begin{array}{r}48 \mathrm{H} \\
3.0 \mathrm{E}-06 \mathrm{H} \\
4 \mathrm{r} \\
4.0 \mathrm{H}-06 \mathrm{H} \\
48 \mathrm{H} \\
3.0 \mathrm{E}-06 \mathrm{H} \\
48 \mathrm{H} \\
3.0 \mathrm{E}-06 \mathrm{H} \\
48 \mathrm{H} \\
3.0 \mathrm{E}-06 \mathrm{H}\end{array}$ & $\begin{array}{l}7.20 \mathrm{E}-05 \\
7.20 \mathrm{E}-05 \\
7.20 \mathrm{E}-05 \\
7.20 \mathrm{E}-05 \\
7.20 \mathrm{E}-05\end{array}$ & $2.69 \mathrm{E}-17$ \\
\hline
\end{tabular}


Loss of SPC backup CO2 purge supply Top Event Probability: 6.22E-03

Basic Event Name PSV1239-FOP1\# CC3CO2B-REG1\# PCV2778XFTO1\# PCV2775-FTO1\# WSL2779BFTC1\# WE-2779-FHI1\# OPR2779-CSL3\# CC3CO2B-RMH1\# BOTCO2B-REP1\# BOTCO2B-T\&M1\# WE-2779-CAL1\# OPR2779-MCH1\#

Modified top event frequency = (top event frequency) $\times$ (risk achievement worth).
Bottles were used since last refilling

SPC backup $\mathrm{CO} 2$ bottles isolated for maintenance

CO2 backup bottle weight element 2779 calibrated
Backup CO2 pressure relief valve fails open
CC: All 4 bottles pressure regulators fail to open

Backup $\mathrm{CO} 2$ pressure control valve 2775 fails to open

$\mathrm{CO} 2$ bottle weight element fails high

Operator fails to respond to $\mathrm{CO} 2$ bottle low weight alarm
Prob/Freg AchW

1.20E-05 1.61E+02

$3.60 \mathrm{E}-06 \quad 1.61 \mathrm{E}+02$

3.00E-03 1.61E+02

3.00E-03 1.61E+02

$1.80 \mathrm{E}-05 \quad 8.53 \mathrm{E}+00$

$1.80 \mathrm{E}-05 \quad 8.53 \mathrm{E}+00$

3.00E-03 8.50E+00

$2.50 \mathrm{E}-03 \quad 4.03 \mathrm{E}+00$

4.71E-02 , 1.54E+00

$1.90 \mathrm{E}-02 \quad 1.39 \mathrm{E}+00$

$1.63 \mathrm{E}-02 \quad 1.22 \mathrm{E}+00$

$1.12 \mathrm{E}+00$

$.00 \mathrm{E}-02$

\section{Basic Event Name \\ PCV2775-FTO1\# PCV2778XFTO1\# BOTCO2B-REP1\# OPR2779-CSL3\#}

\section{Risk Reduction Worth}

Description

Backup $\mathrm{CO} 2$ pressure control valve 2775 fails to open Backup CO2 pressure control valve 2778X fails to open Bottles were used since last refilling Operator fails to respond to $\mathrm{CO} 2$ bottle low weight alarm

\begin{tabular}{ll} 
Prob/Freg & RedW \\
\hline $3.00 \mathrm{E}-03$ & $1.93 \mathrm{E}+00$ \\
$3.00 \mathrm{E}-03$ & $1.93 \mathrm{E}+00$ \\
$4.71 \mathrm{E}-02$ & $1.03 \mathrm{E}+00$ \\
$3.00 \mathrm{E}-03$ & $1.02 \mathrm{E}+00$
\end{tabular}

Note: Events that make less than $1 \%$ difference to the importance factor are not included. Modified top event frequency = (top event frequency) / (risk reduction worth). 
X-ESR-S-00001, REV. 0

SPC Nitrogen Purge Unavailable 
X-ESR-S-00001, REV. 0
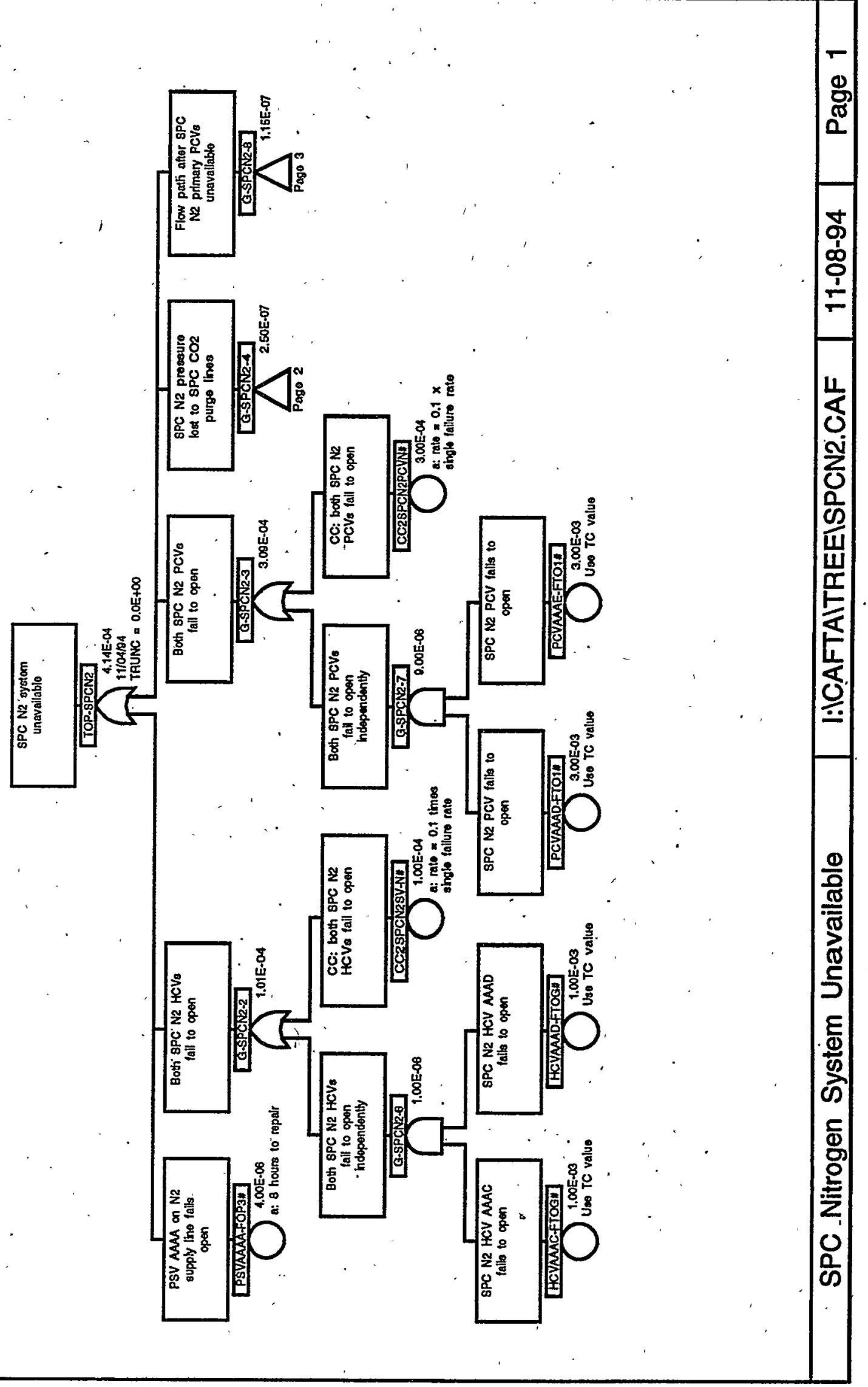


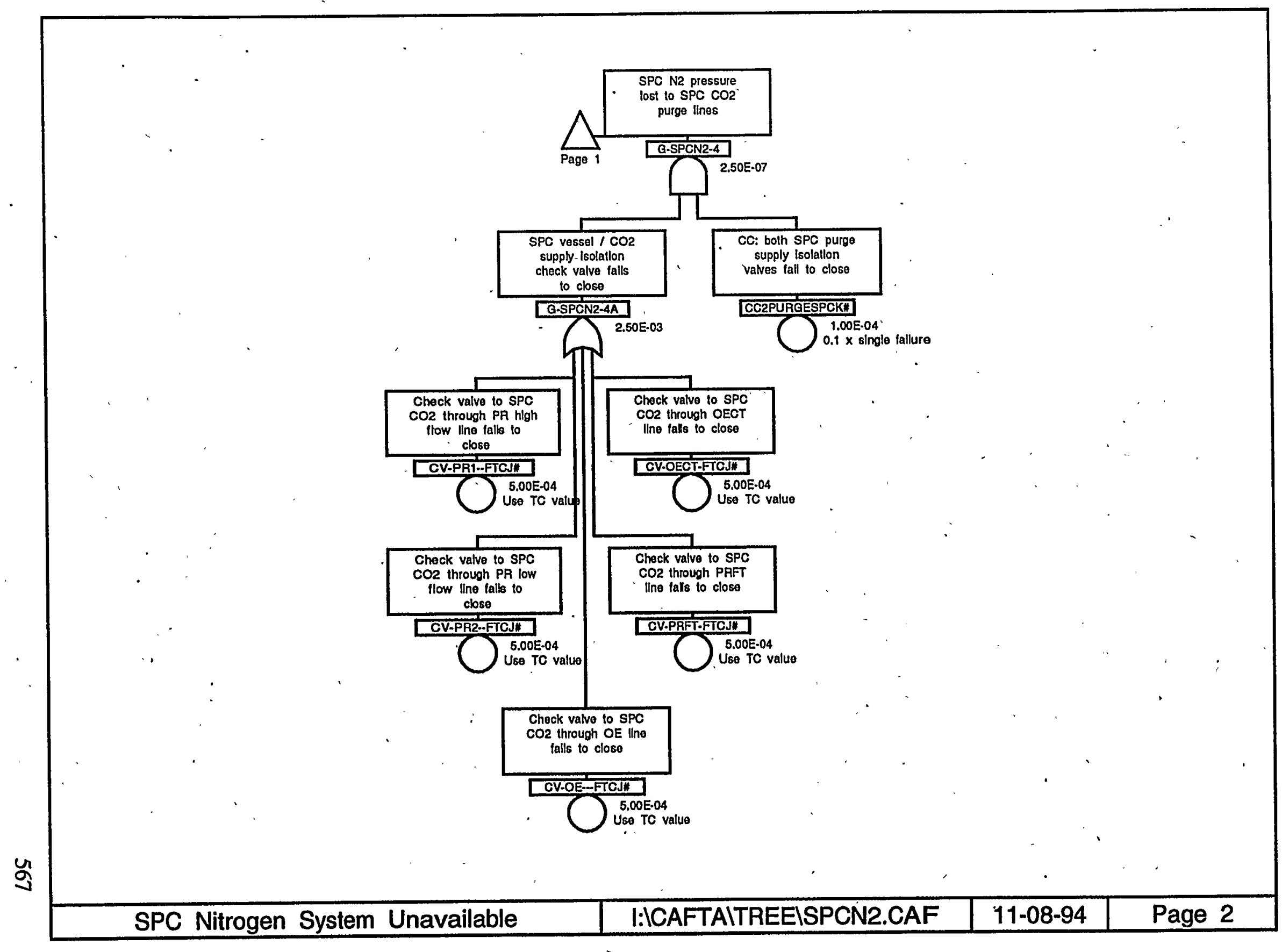




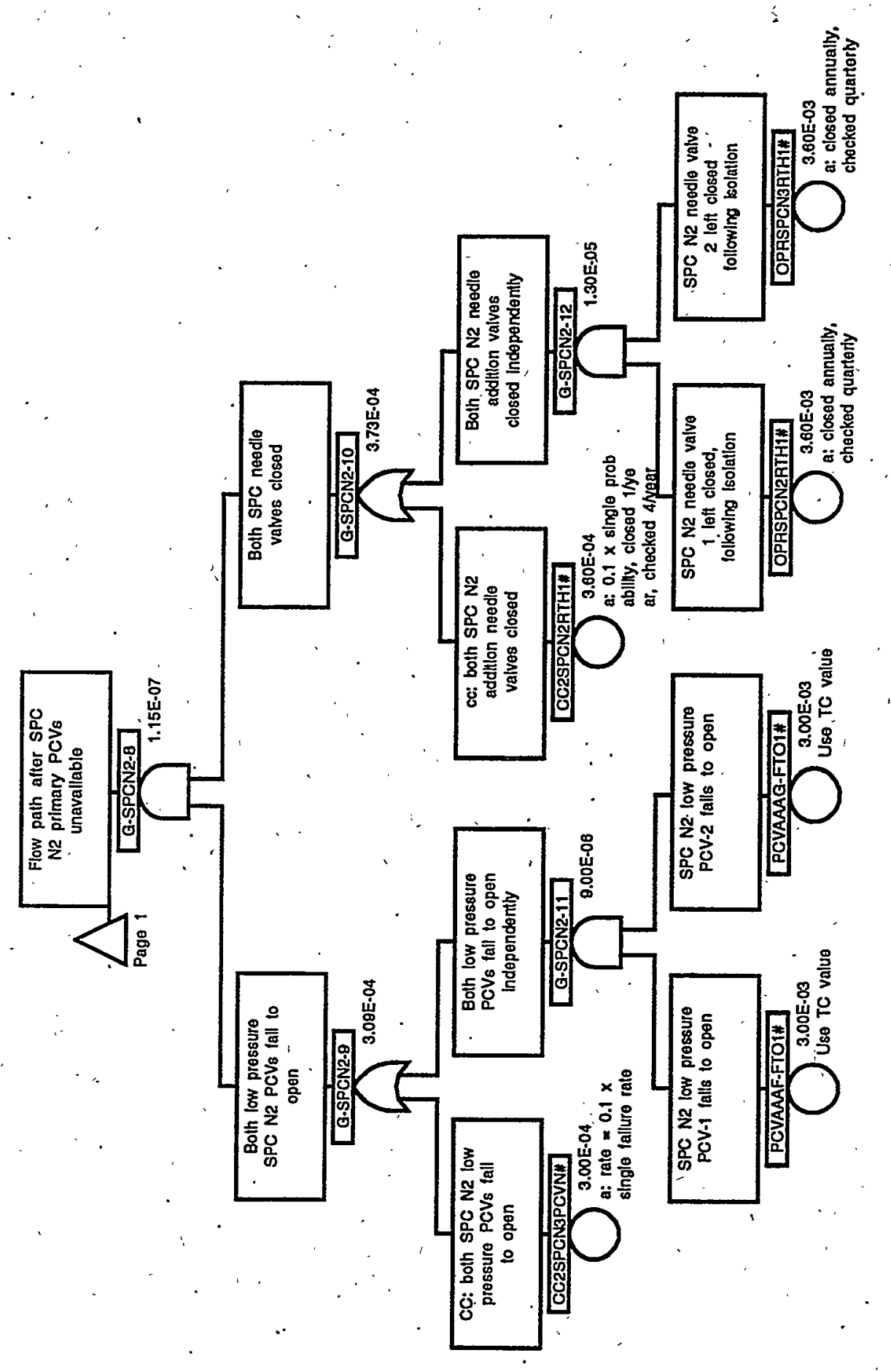


Cutsets for I : ICAFTA ICUT\SPCN2, CSR'

11/08/94 $12: 15$ AM

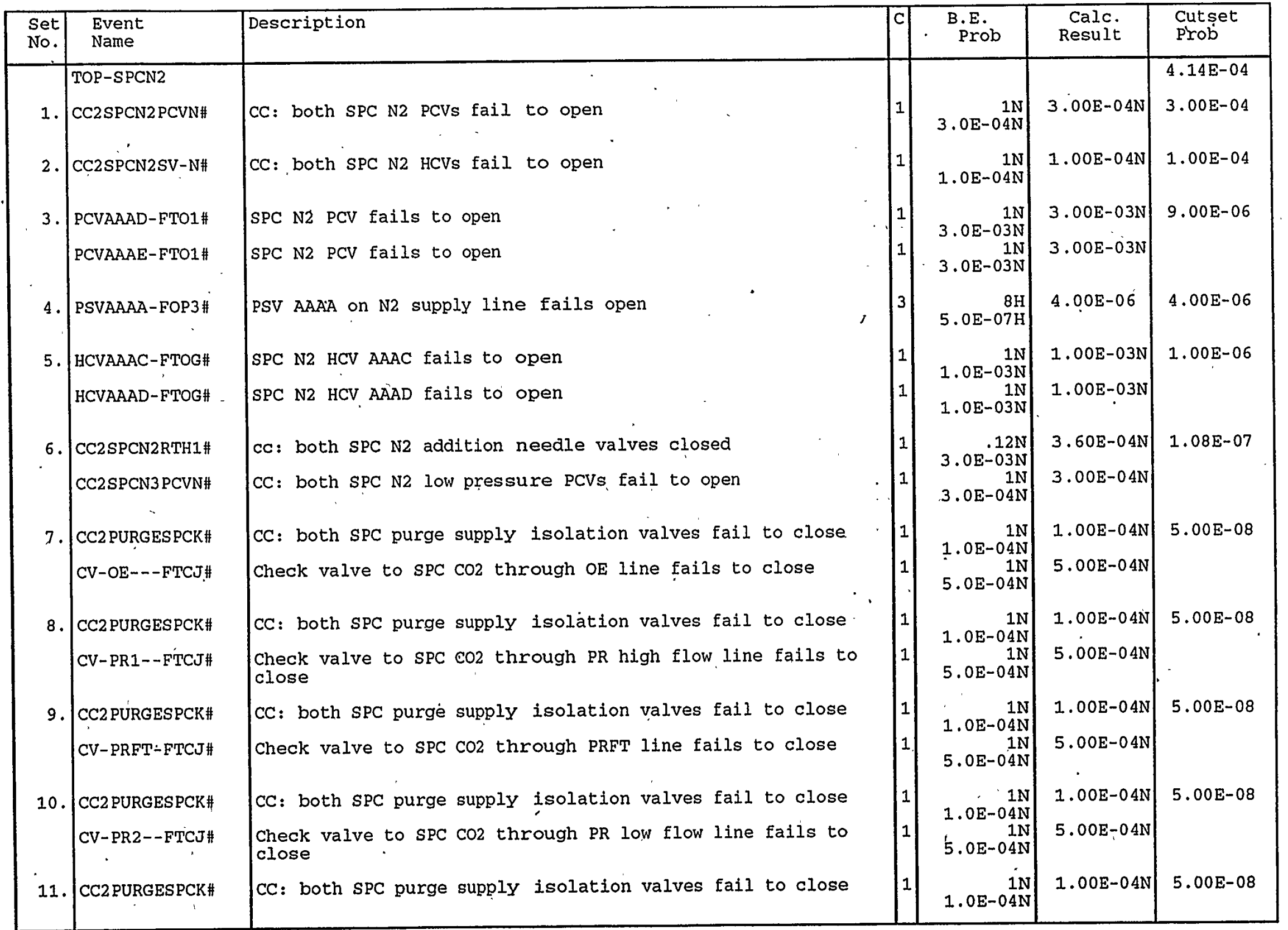


Cutsets for I: \CAFTA \CUT\SPCN2.CSR

11/08/94 12:15 AM (CONT.).

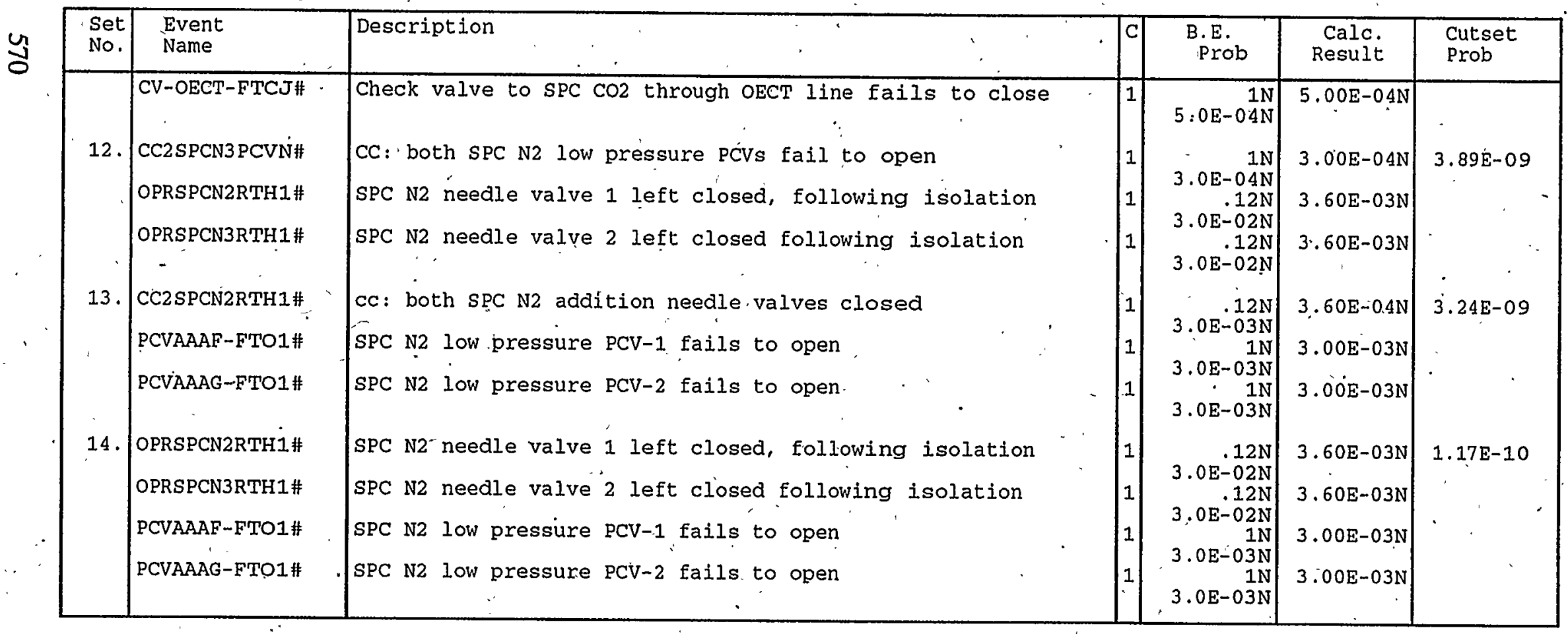


SPC N2 system unavailable Top Event Probability: 4.14E-04

Basic Event Name

PSVAAAA-FOP3\# CC2SPCN2SV-N\# CC2SPCN2PCVN\# PCVAAAE-FTO1\# PCVAAAD-FTO1\# CC2PURGESPCK\#

. HCVAAAC-FTOG\# HCVAAAD-FTOG\# CC2SPCN3PCVN\# CC2SPCN2RTH1\# CV-PR2--FTCJ\# CV-OECT-FTCJ\# CV-PRFT-FTCJ\# CV-OE--FTCJ\# CV-PR1-FTCJ\# MV-PR1--FT TH

\section{Risk Achievement Worth}

Description

PSV AAAA on N2 supply line fails open

CC: both SPC N2 HCVs fail to open

CC: both SPC N2 PCVs fail to open

SPC N2 PCV fails to open

SPC N2 PCV fails to open

CC: both SPC purge supply isolation valves fail to close

SPCN2 HCV AAAC fails to open

SPC N2 HCV AAAD fails to open

CC: both SPC N2 low pressure PCVs fail to open

cc: both SPC N2 addition needle valves closed

Check valve to SPC CO2 through PR fails to close

Check valve to SPC CO2 through OECT line fails to close

Check valve to SPC CO2 through PRFT line fails to close

Check valve to SPC CO2 through $\mathrm{OE}$ line fails to close

Check valve to SPC $\mathrm{CO} 2$ through $\mathrm{PR}$ high flow fails to close

\begin{tabular}{cc} 
Prob/Freq & AchW \\
\hline $4.00 \mathrm{E}-06$ & $2.41 \mathrm{E}+03$ \\
$1.00 \mathrm{E}-04$ & $2.41 \mathrm{E}+03$ \\
$3.00 \mathrm{E}-04$ & $2.41 \mathrm{E}+03$ \\
$3.00 \mathrm{E}-03$ & $8.22 \mathrm{E}+00$ \\
$3.00 \mathrm{E}-03$ & $8.22 \mathrm{E}+00$ \\
$1.00 \mathrm{E}-04$ & $7.02 \mathrm{E}+00$ \\
$1.00 \mathrm{E}-03$ & $3.41 \mathrm{E}+00$ \\
$1.00 \mathrm{E}-03$ & $3.41 \mathrm{E}+00$ \\
$3.00 \mathrm{E}-04$ & $1.90 \mathrm{E}+00$ \\
$3.60 \mathrm{E}-04$ & $1.75 \mathrm{E}+00$ \\
$5.00 \mathrm{E}-04$ & $1.24 \mathrm{E}+00$ \\
$5.00 \mathrm{E}-04$ & $1.24 \mathrm{E}+00$ \\
$5.00 \mathrm{E}-04$ & $1.24 \mathrm{E}+00$ \\
$5.00 \mathrm{E}-04$ & $1.24 \mathrm{E}+00$ \\
$5.00 \mathrm{E}-04$ & $1.24 \mathrm{E}+00$
\end{tabular}

,

Basic Event Name

. CC2SPCN2PCVN\#

CC2SPCN2SV-N\#

PCVAAAE-FTO1\#

PCVAAAD-FTO1\#

\section{Risk Reduction Worth}

Description

CC: both SPC N2 PCVs fail to open

CC: both SPC N2 HCVs fail to open

SPCN2 PCV fails to open

SPC N2 PCV fails to open

$\begin{array}{ll}\frac{\text { Prob/Freg }}{3.00 E-04} & \frac{\text { RedW }}{3.62 \mathrm{E}+00} \\ 1.00 \mathrm{E}-04 & 1.32 \mathrm{E}+00 \\ 3.00 \mathrm{E}-03 & 1.02 \mathrm{E}+00 \\ 3.00 \mathrm{E}-03 & 1.02 \mathrm{E}+00\end{array}$

Note: Events that make less than $1 \%$ difference to the importance factor are not included. Modified top event frequency $=$ (top event frequency) / (risk reduction worth). 
X-ESR-S-00001, REV. 0

\section{LPPP Nitrogen Purge Unnavailáble}




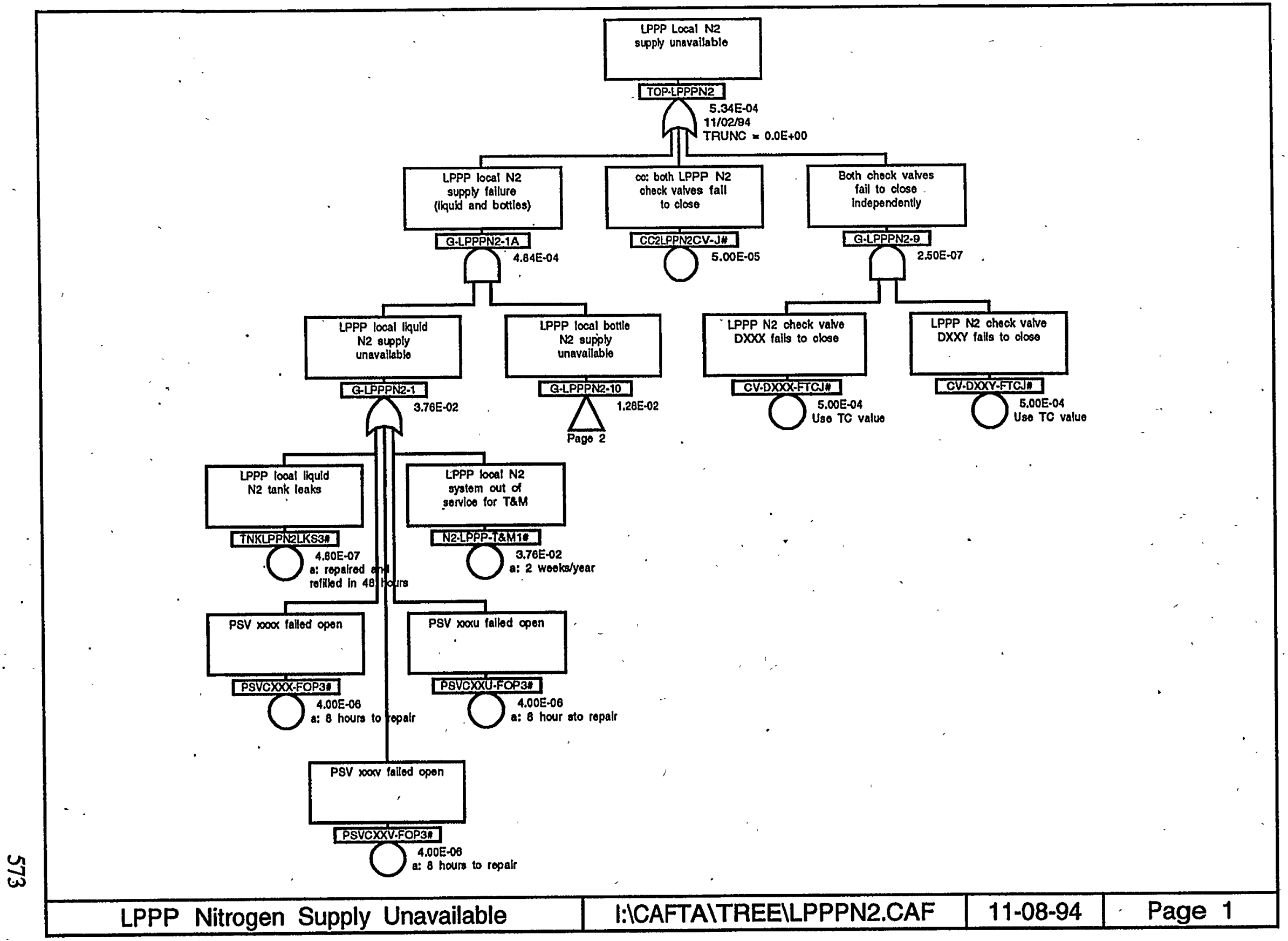


X-ESR-S-00001, REV. 0

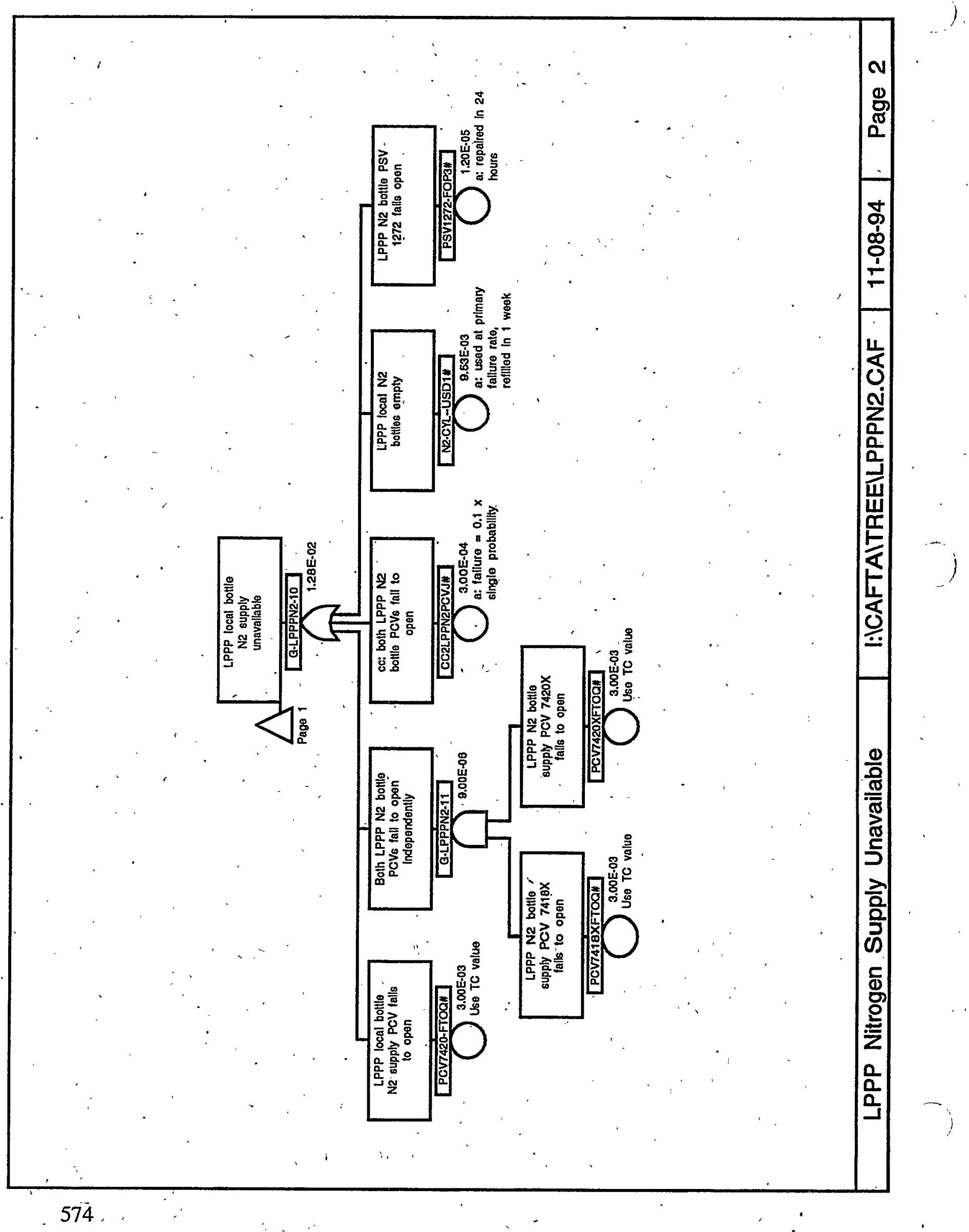




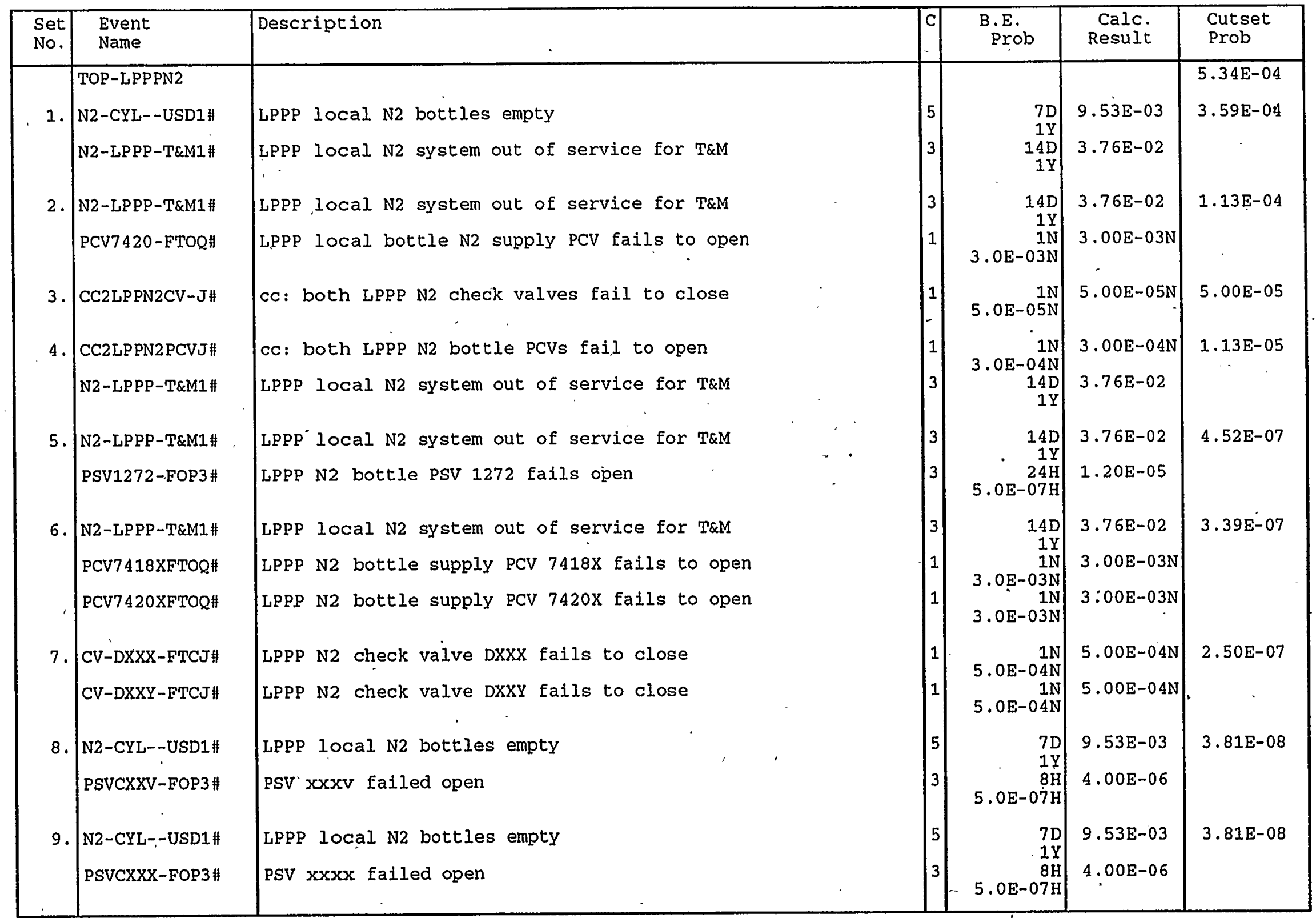


Cutsets for I: \CAFTA \CUT $\backslash L P P P N 2 . C S R$

11/08/94 12:16 AM (CONT.)

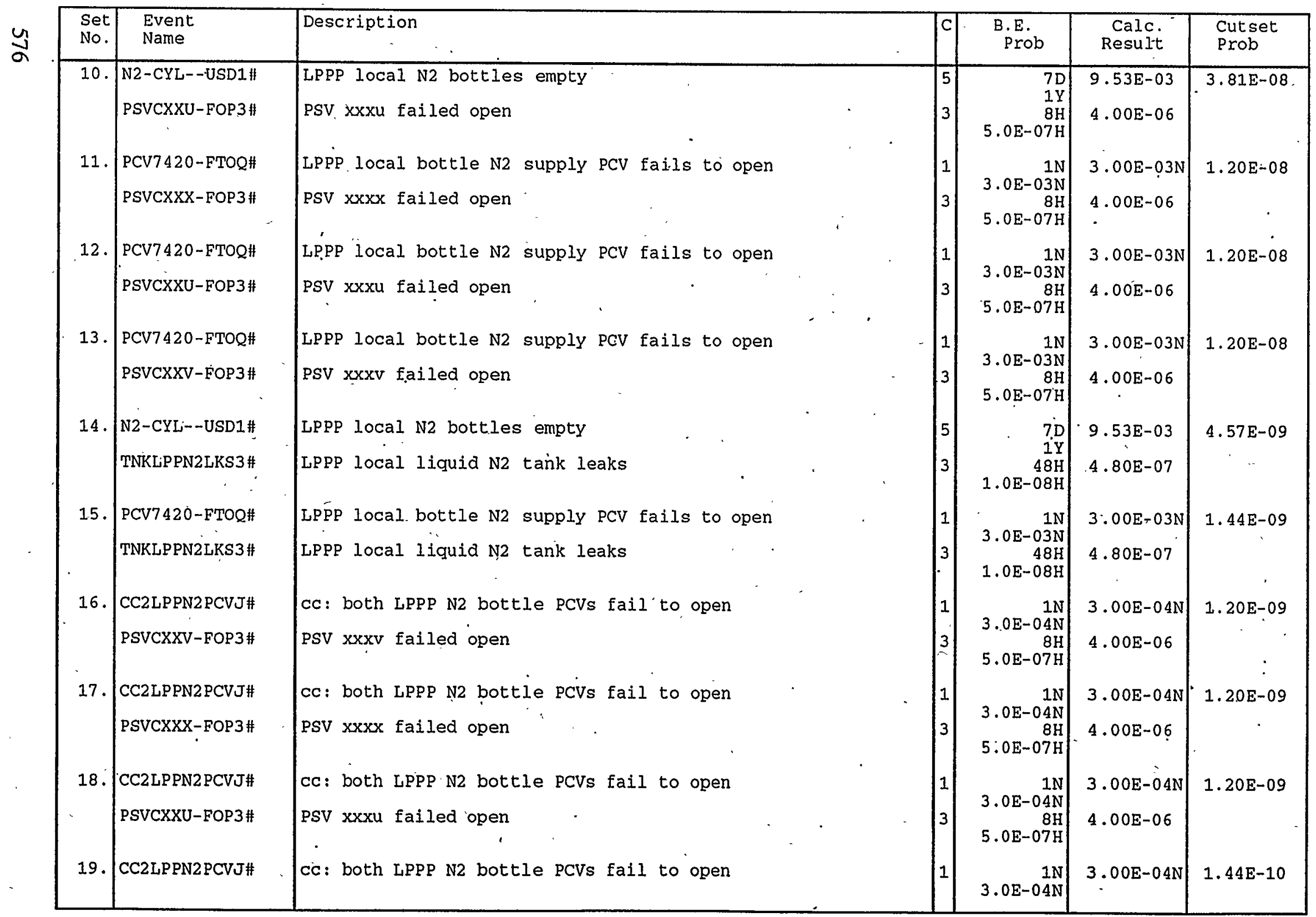


Cutsets for I: ICAFTA ICUT\LPPPN2.CSR $11 / 08 / 94 \quad 12: 16$ AM (CONT.)

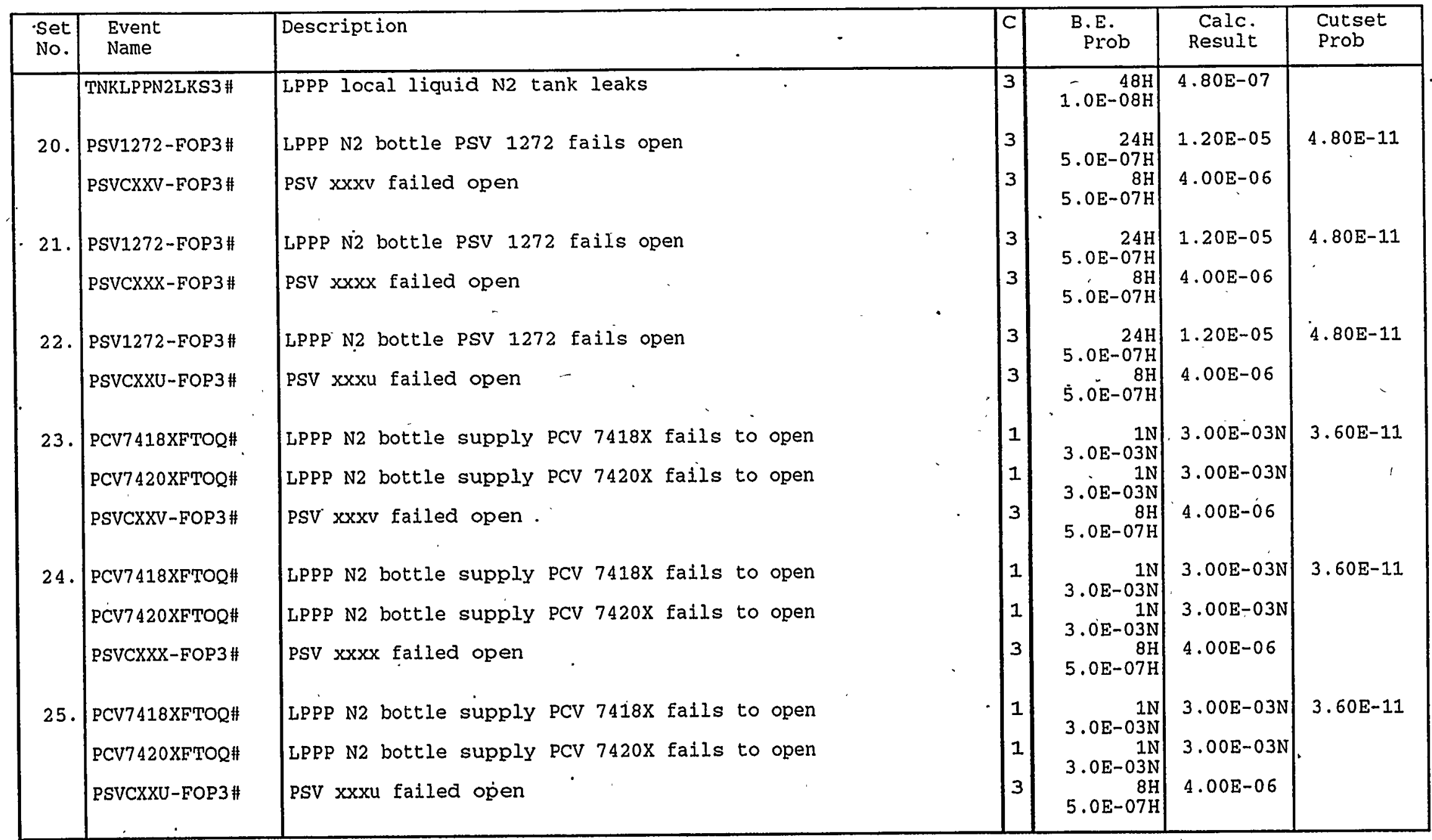


X-ESR-S-00001, REV. 0

LPPP Local N2 supply unavailable $\quad$ Top Event Probability: 5.34E-04

Basic Event Name

CC2LPPN2CV-J\#

PSV1272-FOP3\#

CC2LPPN2PCVJ\#

PCV7420-FTOQ\#

N2-CYL-USD1\#

TNKLPPN2LKS3\#

PSVCXXX-FOP3\#

PSVCXXV-FOP3\#

PSVCXXU-FOP3\#

N2-LPPP-T\&M1\#

CV-DXXX-FTCJ\#

CV-DXXY-FTCJ\#

PCV7418XFTOQ\#

PCV7420XFTOQ\#

Modified top event

\section{Risk Achievement Worth}

\section{Description}

cc: both LPPP N2 check valves fail to close

LPPP N2 bottle PSV 1272 fails open

cc: both LPPP N2 bottle PCVs fail to open

LPPP local bottle N2 supply PCV fails to open

LPPP local N2 bottles empty

LPPP local liquid N2 tank leaks

PSV $x x x x$ failed open

PSV $x x x v$ failed open

PSV xxxu failed open

LPPP local N2 system out of service for T\&M

LPPP N2 check valve DXXX fails to close

LPPP N2 check valve DXXY fails to close

LPPP N2 bottle supply PCV 7418X fails to open

LPPP N2 bottle supply PCV 7420X fails to open

en

\begin{tabular}{cc} 
Prob/Freg & AchW \\
\hline 5.00E-05 & $1.87 \mathrm{E}+03$ \\
$1.20 \mathrm{E}-05$ & $7.15 \mathrm{E}+01$ \\
$3.00 \mathrm{E}-04$ & $7.15 \mathrm{E}+01$ \\
$3.00 \mathrm{E}-03$ & $7.13 \mathrm{E}+01$ \\
$9.53 \mathrm{E}-03$ & $7.08 \mathrm{E}+01$ \\
$4.80 \mathrm{E}-07$ & $2.50 \mathrm{E}+01$ \\
$4.00 \mathrm{E}-06$ & $2.50 \mathrm{E}+01$ \\
$4.00 \mathrm{E}-06$ & $2.50 \mathrm{E}+01$ \\
$4.00 \mathrm{E}-06$ & $2.50 \mathrm{E}+01$ \\
$3.76 \mathrm{E}-02$ & $2.41 \mathrm{E}+01$ \\
$5.00 \mathrm{E}-04$ & $1.94 \mathrm{E}+00$ \\
$5.00 \mathrm{E}-04$ & $1.94 \mathrm{E}+00$ \\
$3.00 \mathrm{E}-03$ & $1.21 \mathrm{E}+00$ \\
$3.00 \mathrm{E}-03$ & $1.21 \mathrm{E}+00$
\end{tabular}

Prob/Freq AchW

$1.20 \mathrm{E}-05 \quad 7.15 \mathrm{E}+01$

$3.00 \mathrm{E}-04 \quad 7.15 \mathrm{E}+01$

3.00E-03 7.13E+01

$4.00 \mathrm{E}-06 \quad 250 \mathrm{E}+01$

4.00E-06 2.50E+01

5.00E-04 1.94E+00

5.00E-04 1.94E+00

$3.00 \mathrm{E}-03 \quad 1.21 \mathrm{E}+00$

\section{Risk Reduction Worth}

\section{Description}

LPPP local N2 system out of service for T\&M

LPPP local N2 bottles empty

LPPP local bottle N2 supply PCV fails to open

cc: both LPPP N2 check valves fail to close

cc: both LPPP N2 bottle PCVs fail to open

\begin{tabular}{cc} 
Prob/Freg & RedW \\
\hline $3.76 \mathrm{E}-02$ & $1.06 \mathrm{E}+01$ \\
$9.53 \mathrm{E}-03$ & $3.05 \mathrm{E}+00$ \\
$3.00 \mathrm{E}-03$ & $1.27 \mathrm{E}+00$ \\
$5.00 \mathrm{E}-05$ & $1.10 \mathrm{E}+00$ \\
$3.00 \mathrm{E}-04$ & $1.02 \mathrm{E}+00$
\end{tabular}

Note: Events that make less than $1 \%$ difference to the importance factor are not included. Modified top event frequency $=$ (top event frequency) $/$ (risk reduction worth). 


\section{Instrument Air Failure}




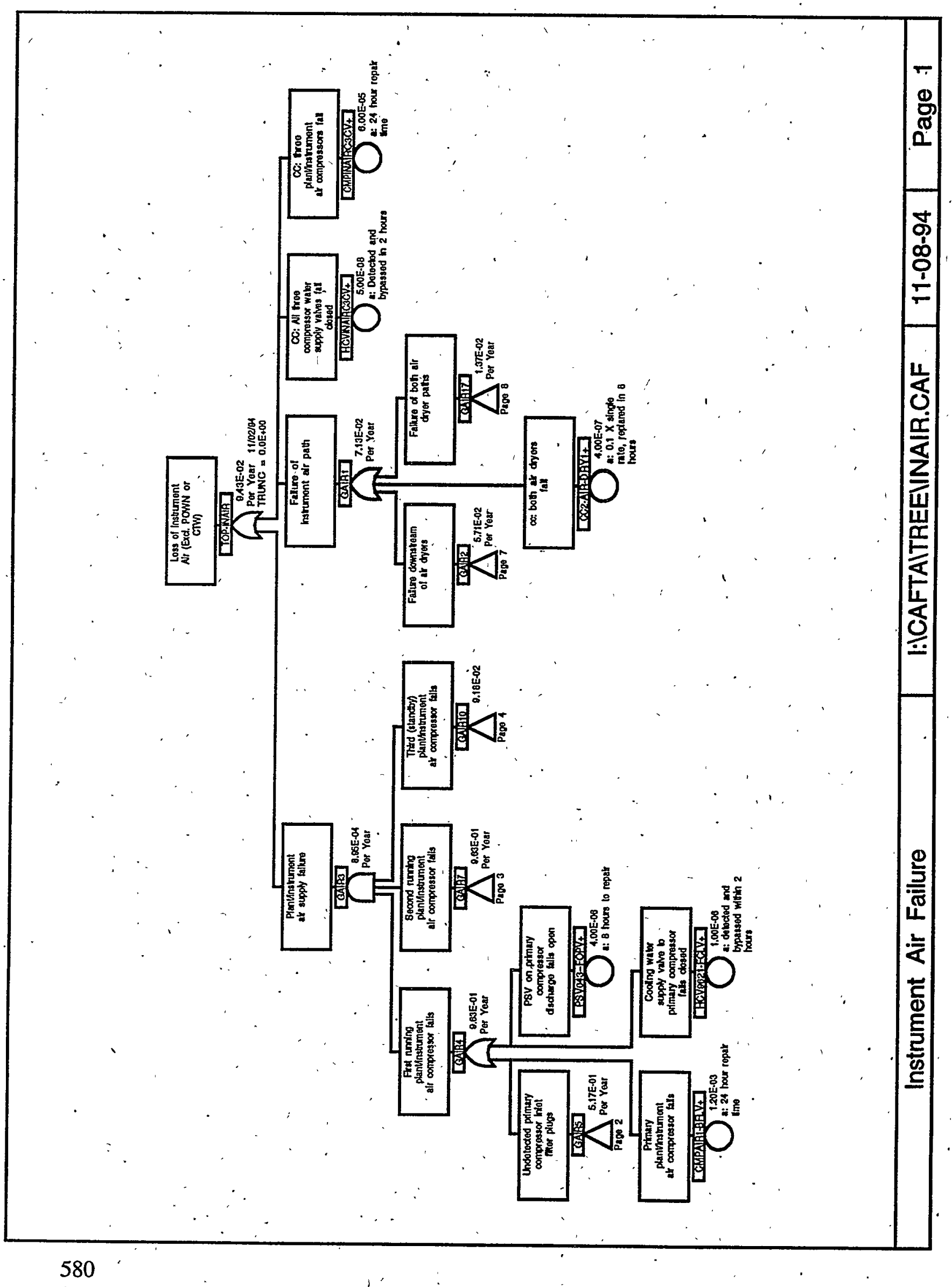




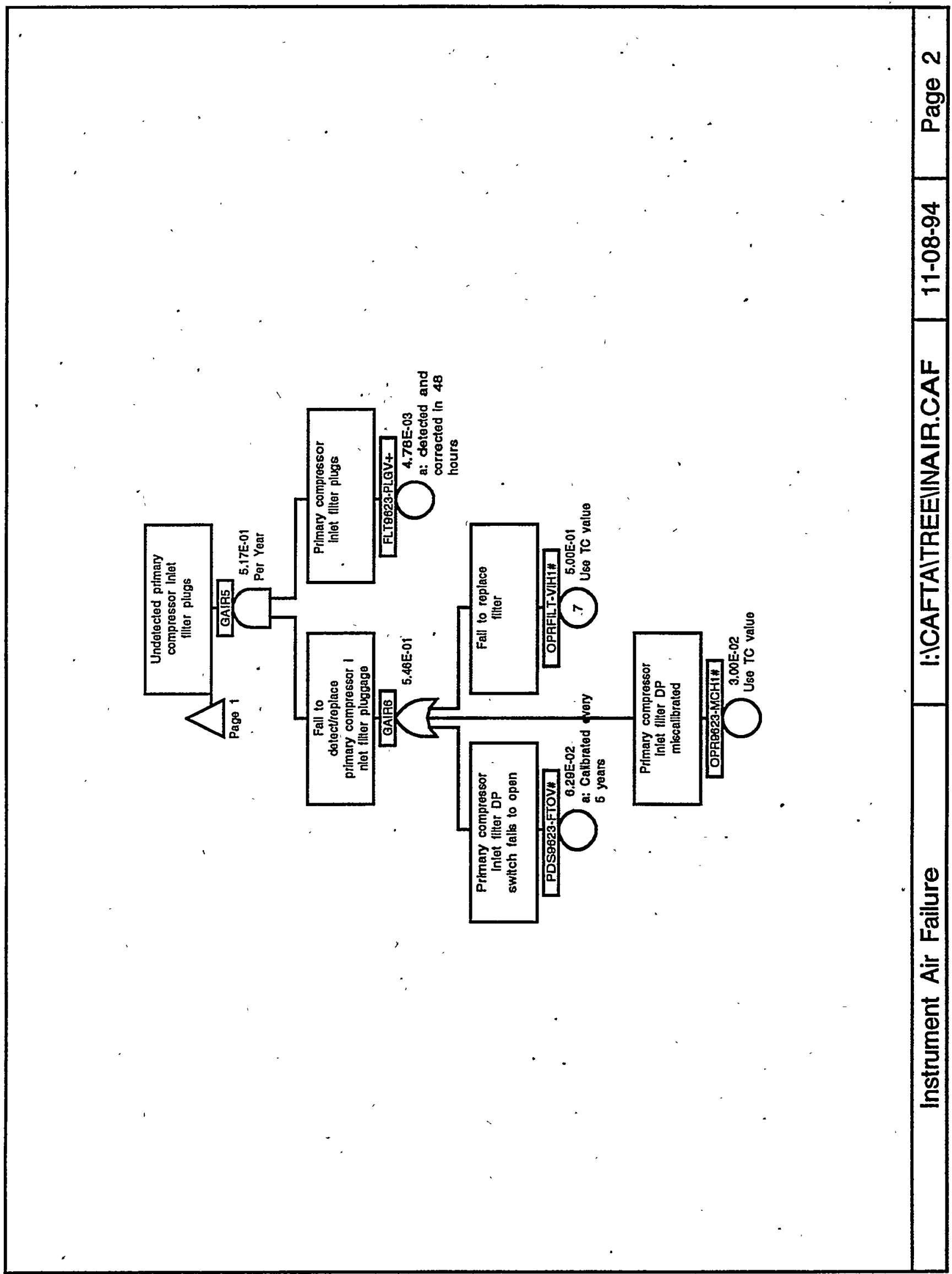




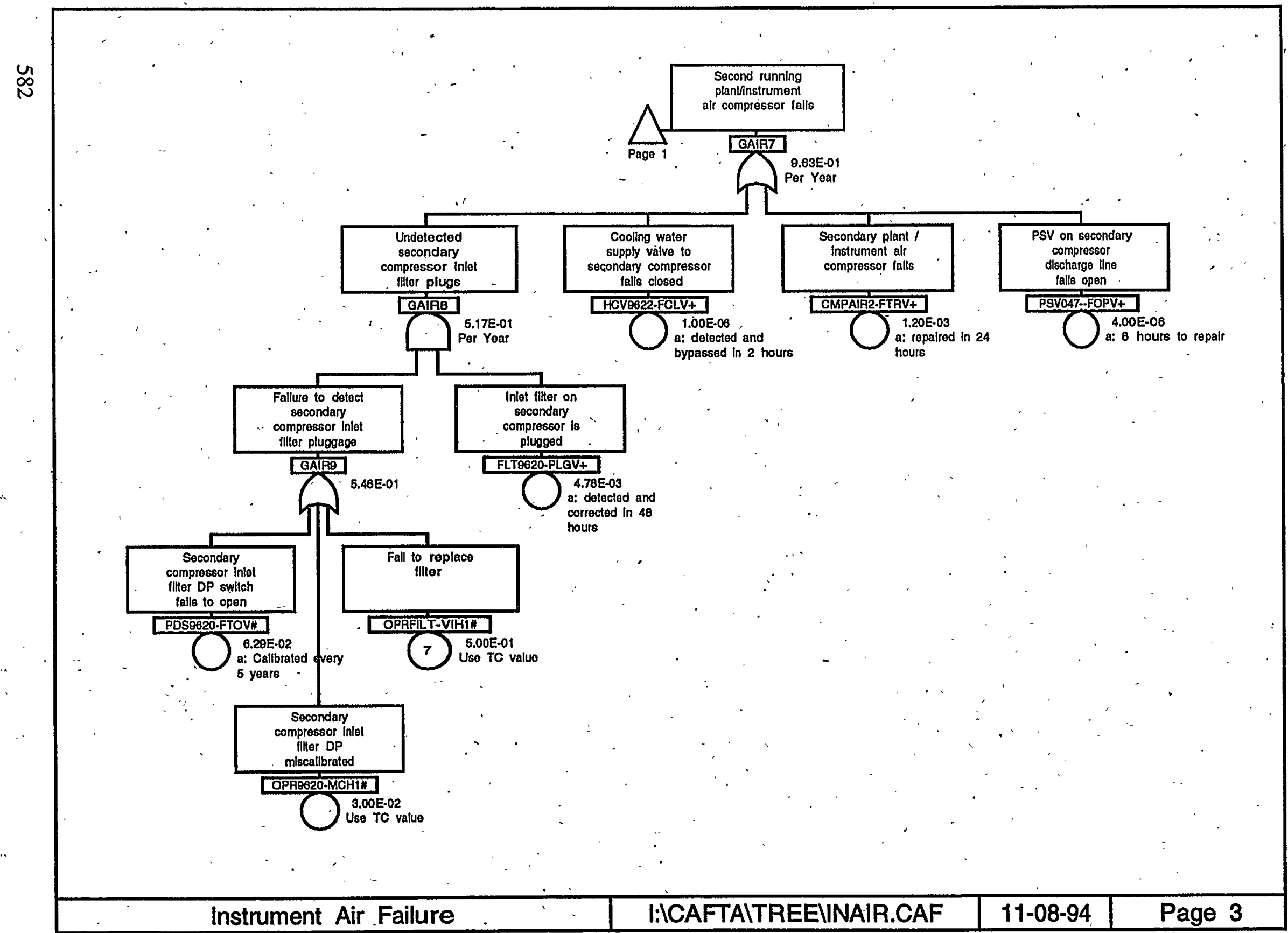




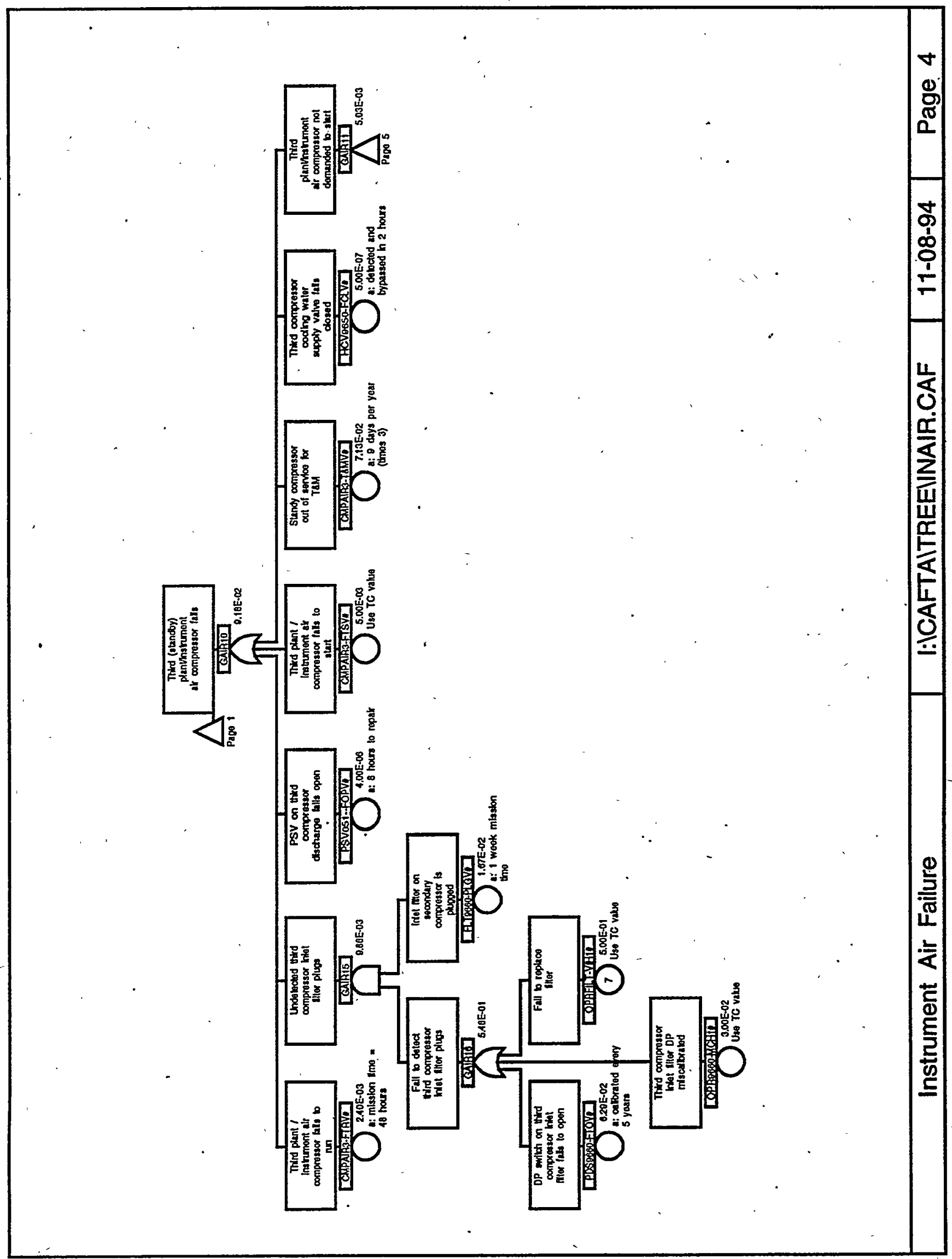




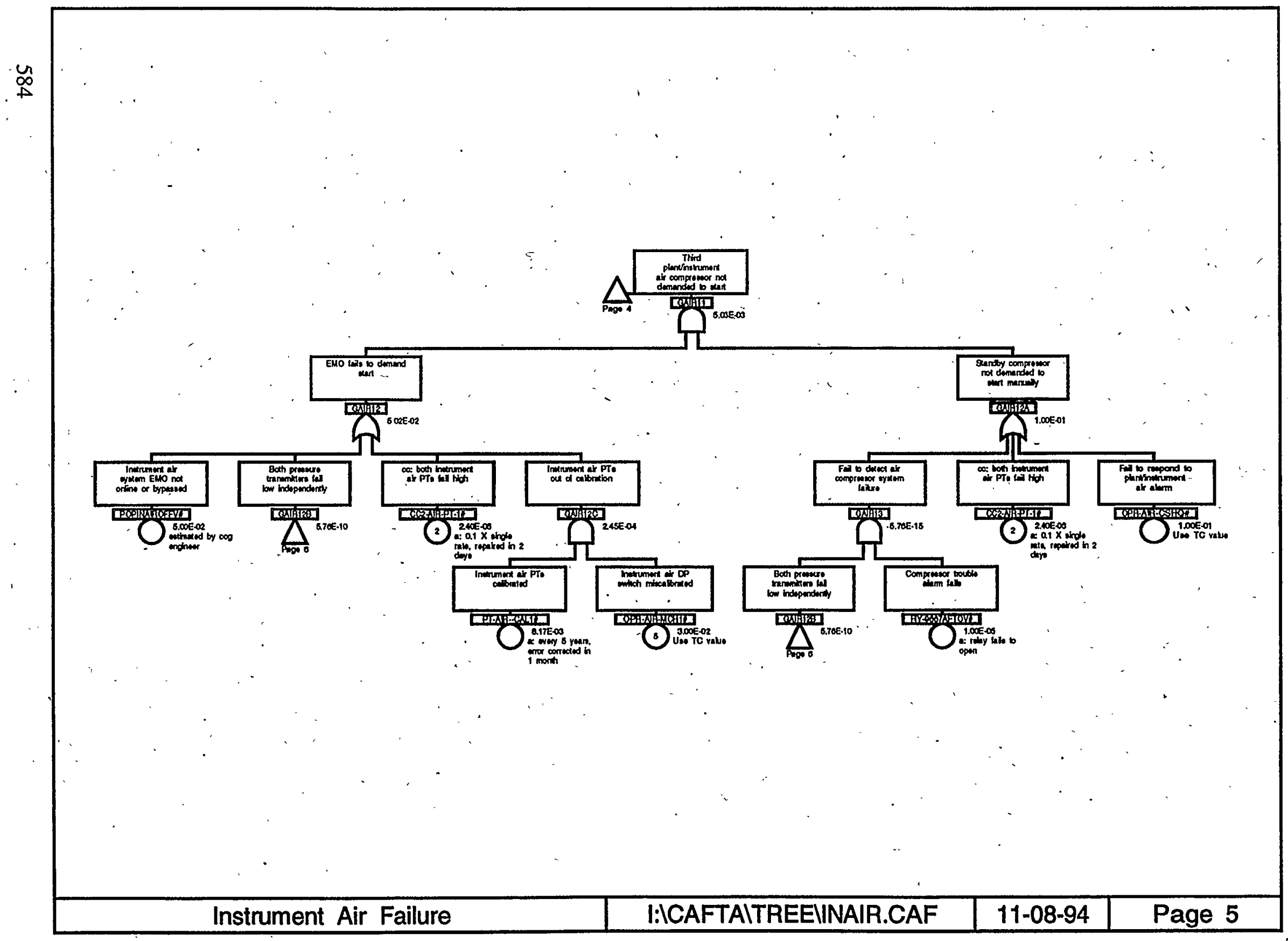


X-ESR-S-00001, REV. 0

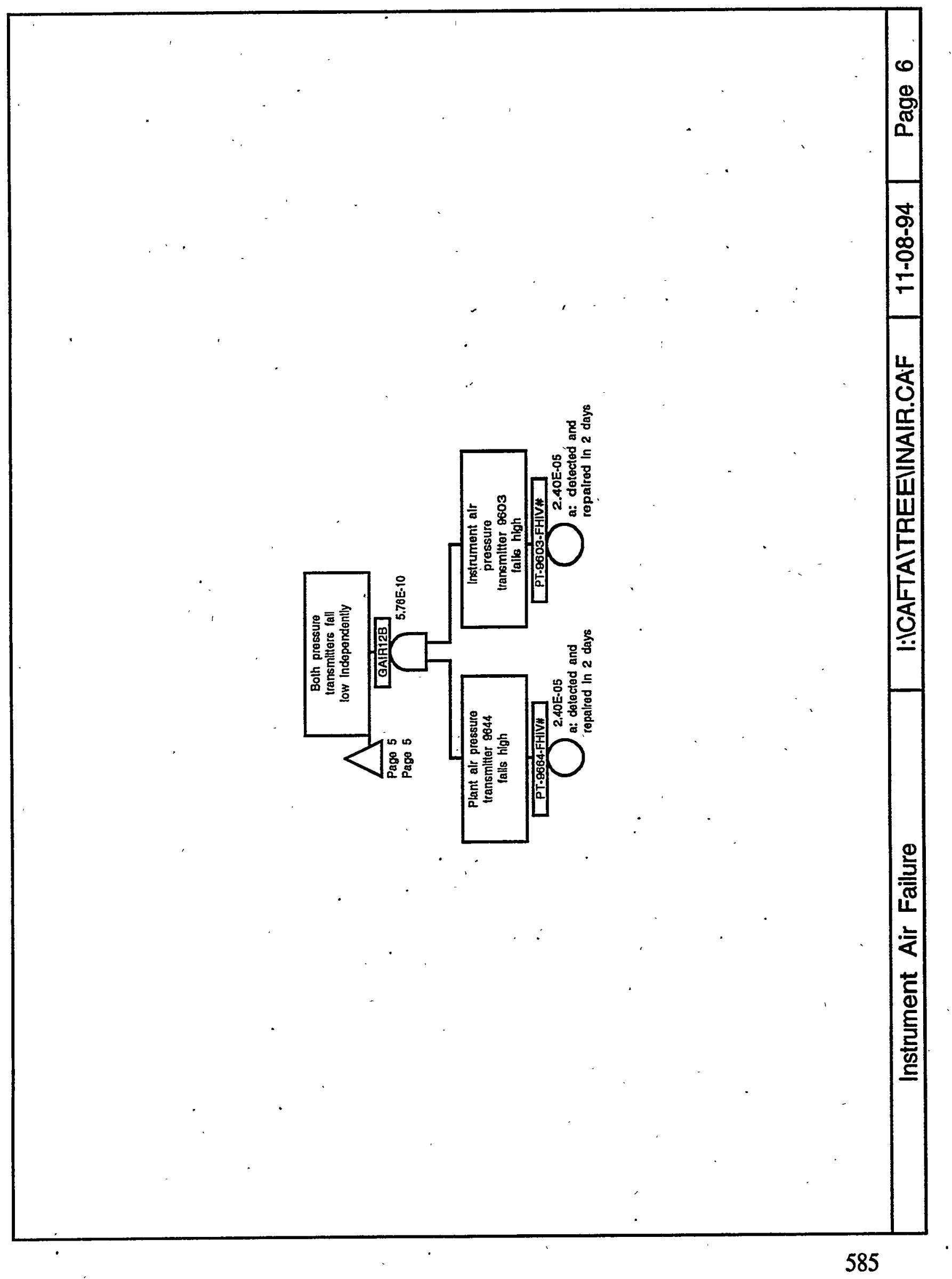


X-ESR-S-00001, REV. 0

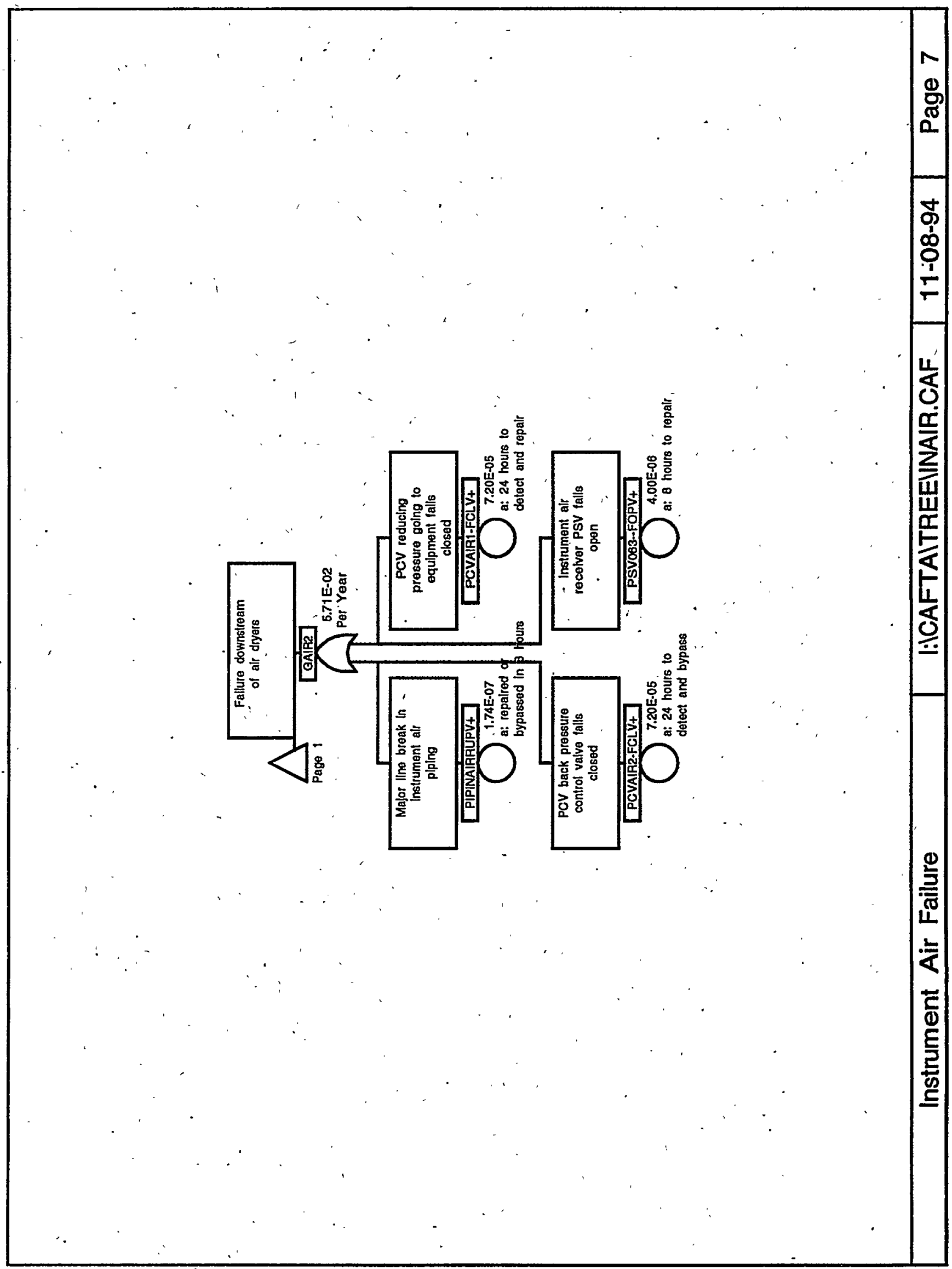




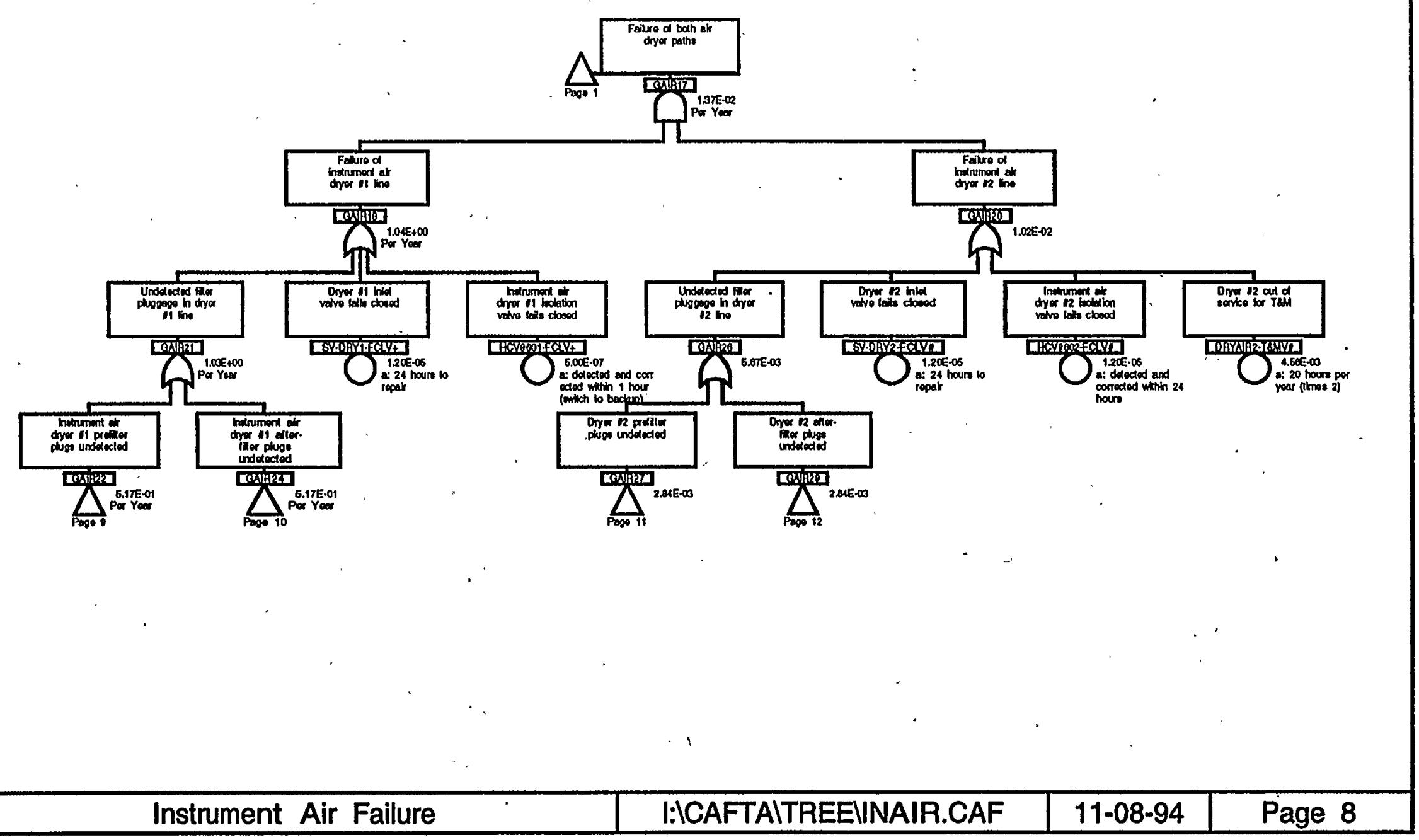




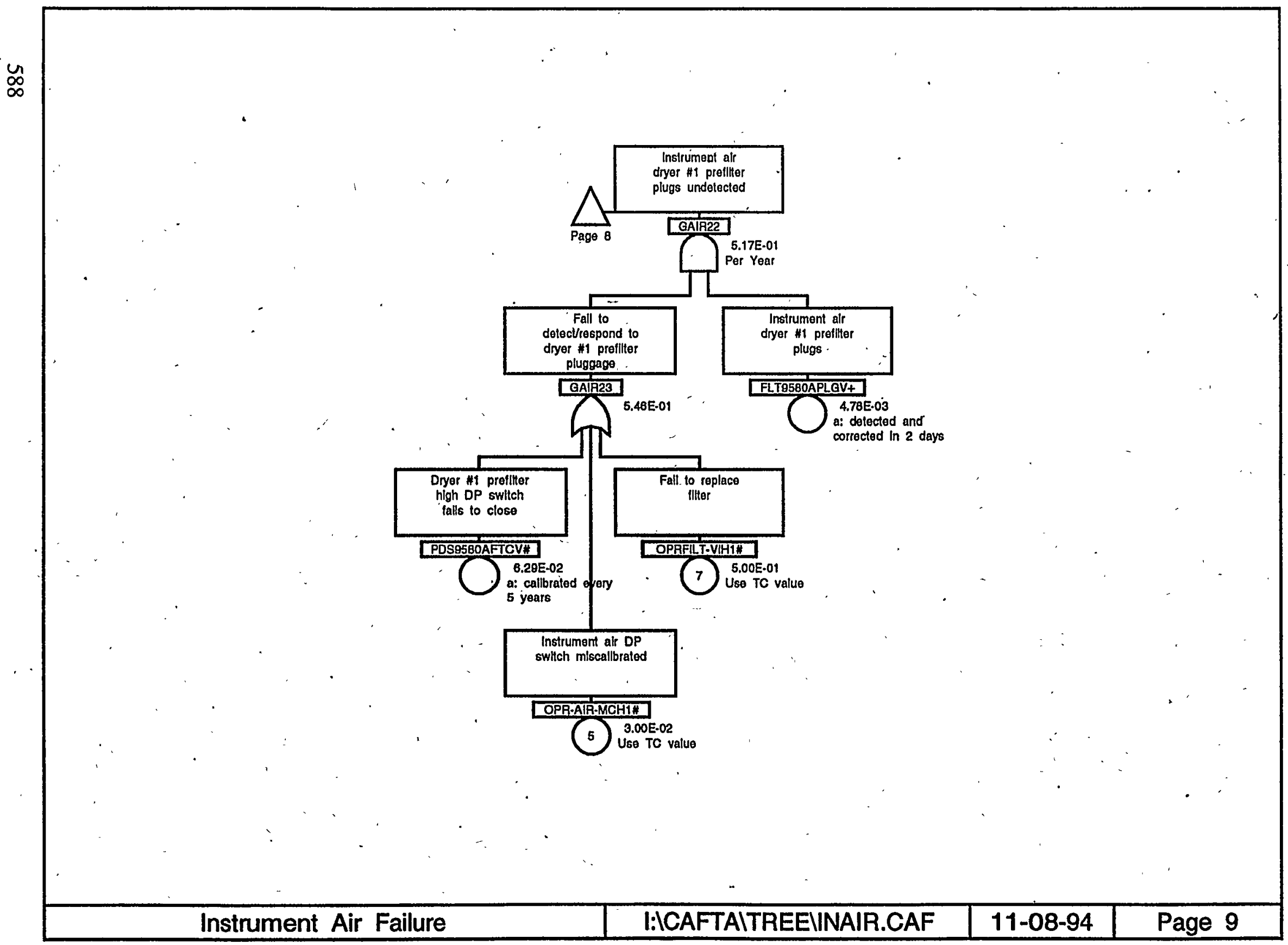




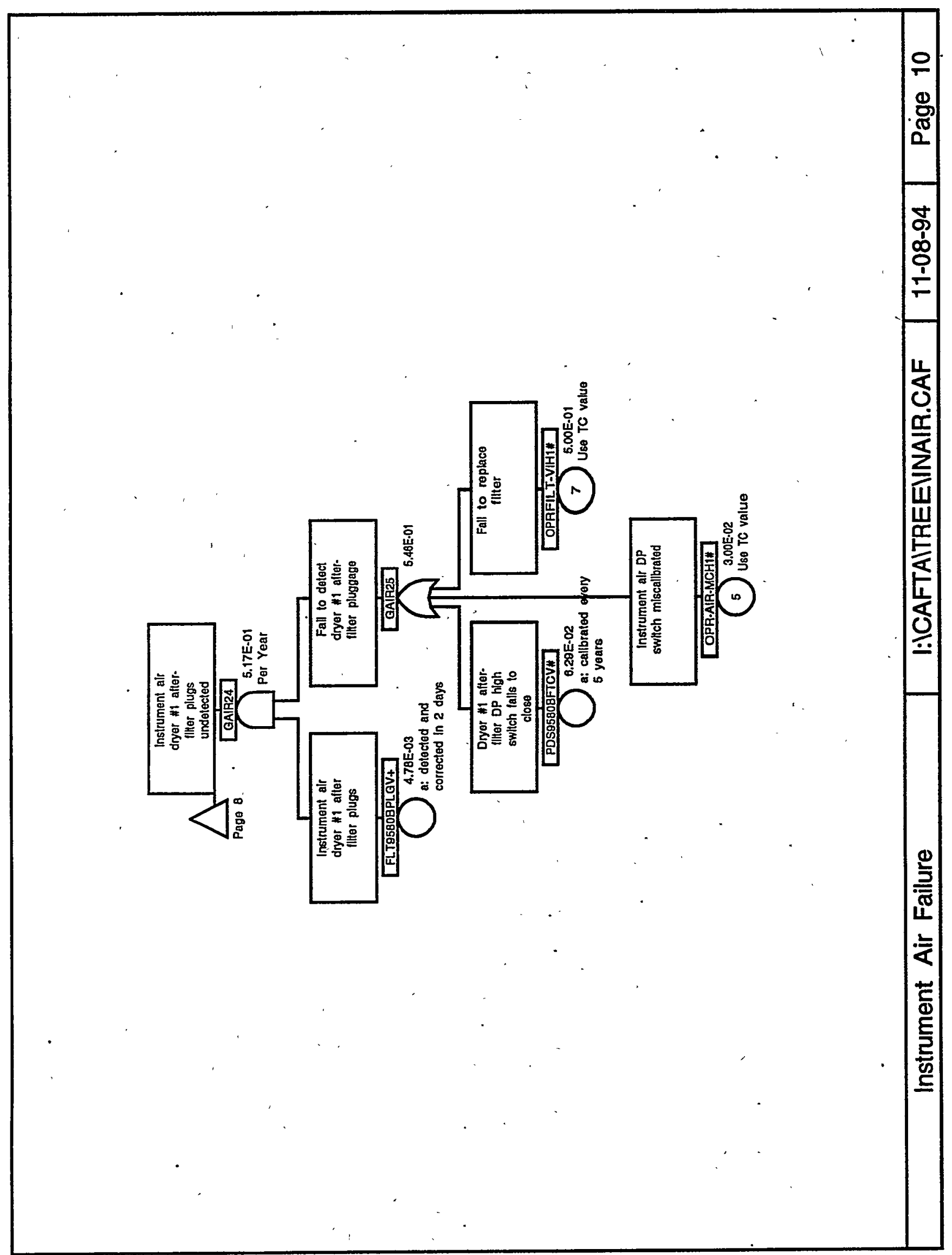




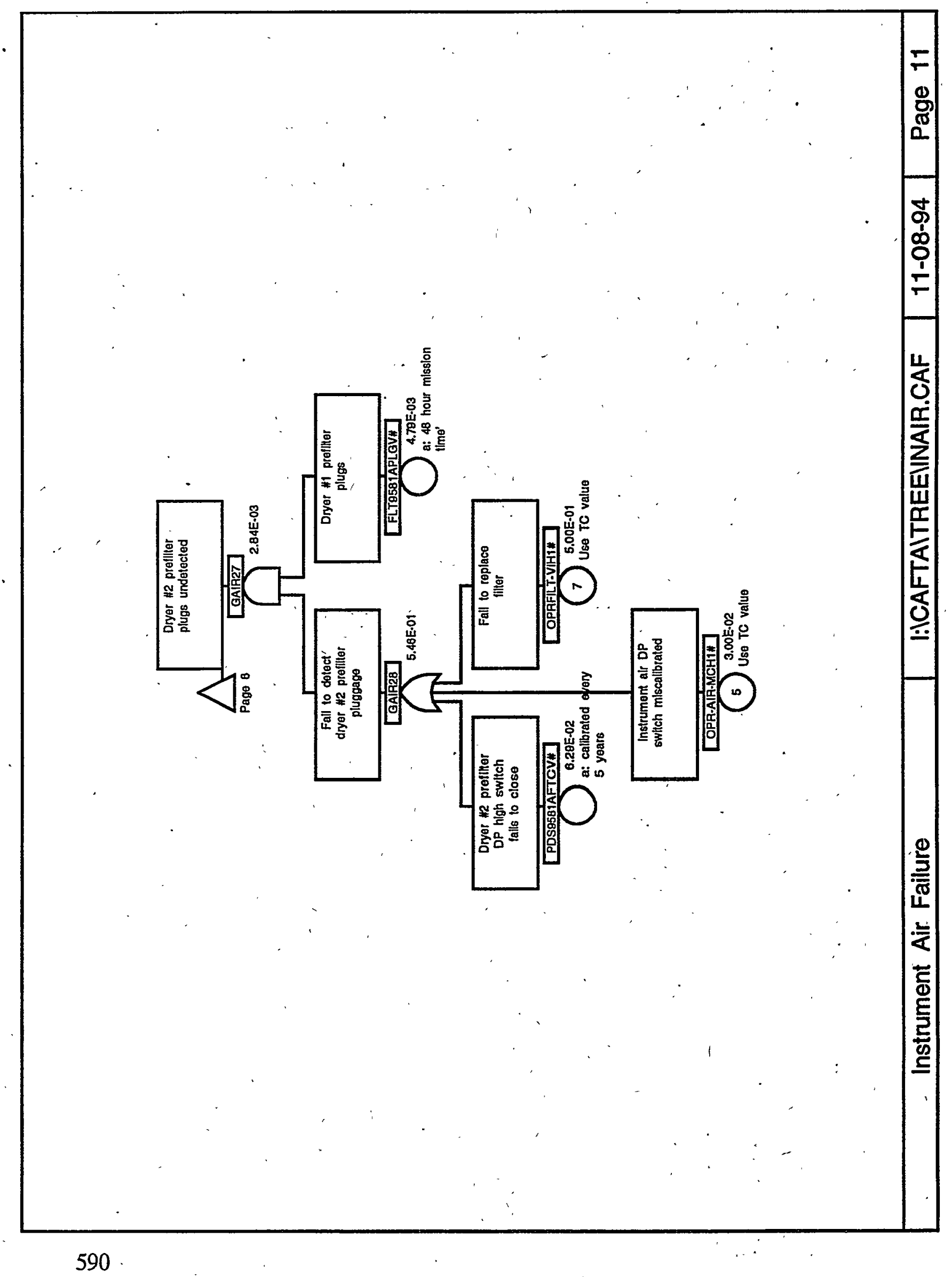




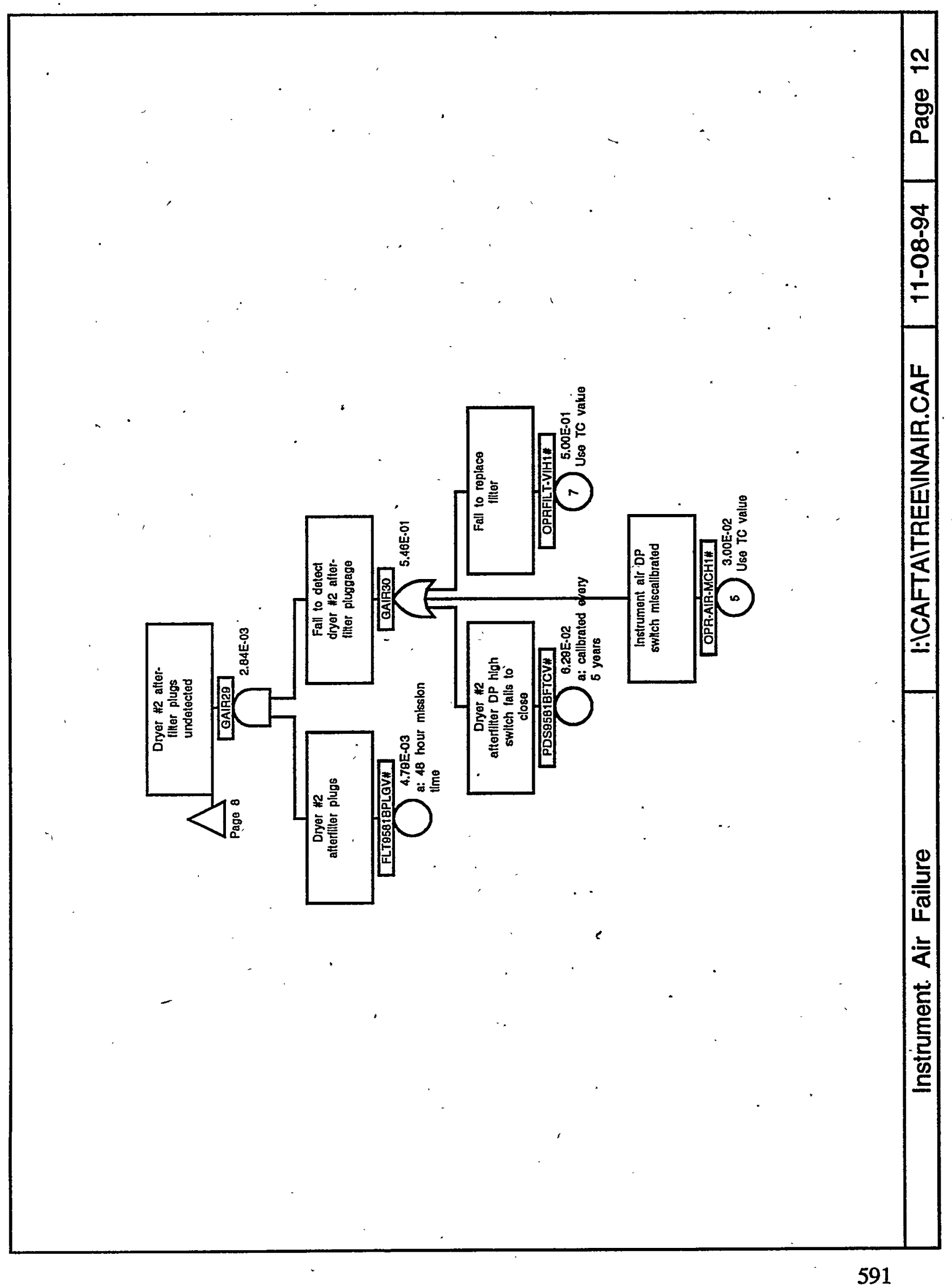


Cutsets for I: ICAFTA ICUT\INAIR.CSR

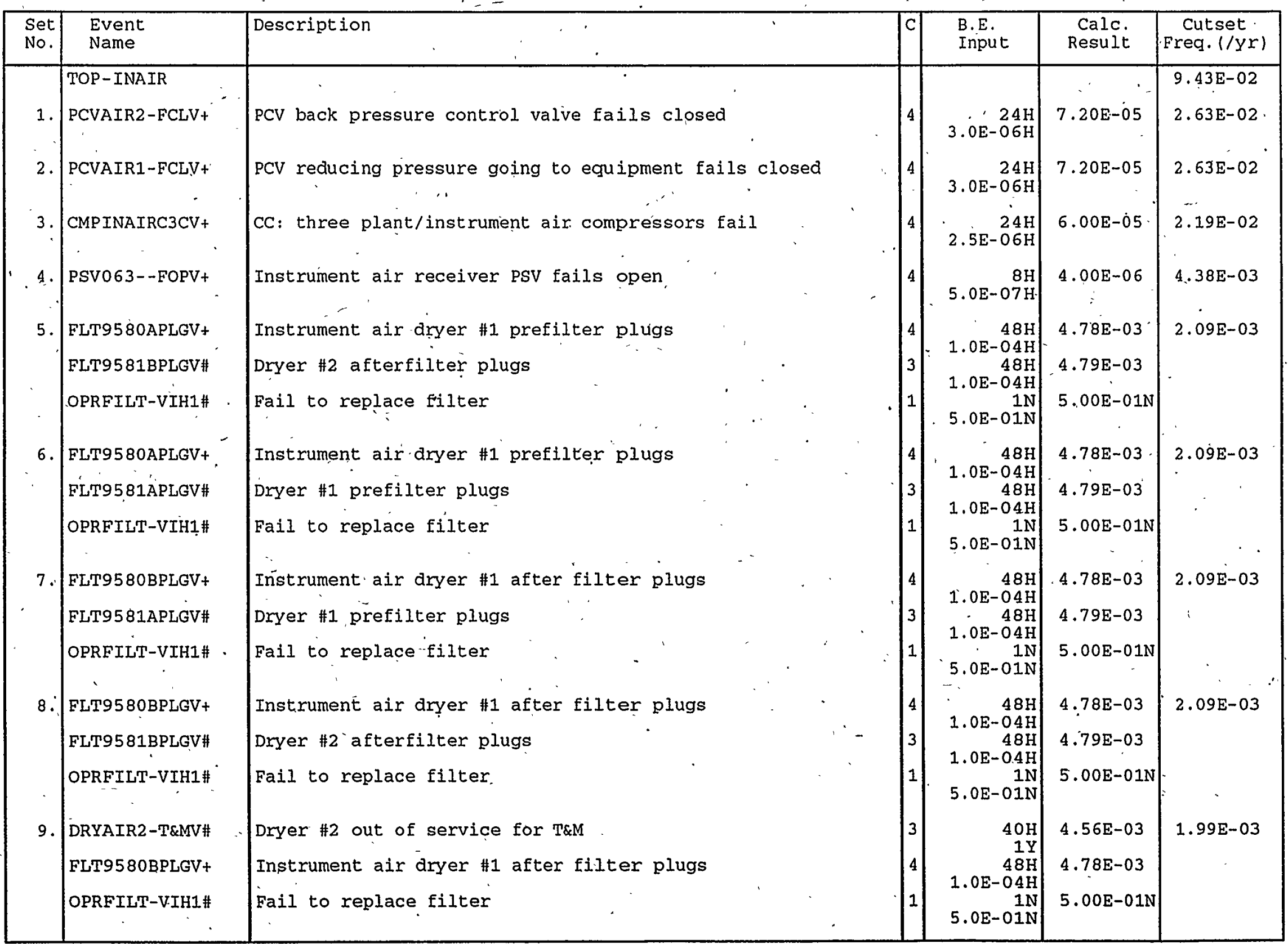


Cutsets for I: ICAFTA ICUT\INAIR.CSR $11 / 08 / 94 \quad 12: 17$ AM (CONT.)

\begin{tabular}{|c|c|c|c|c|c|c|}
\hline $\begin{array}{l}\text { Set } \\
\text { No. }\end{array}$ & $\begin{array}{l}\text { Event } \\
\text { Name }\end{array}$ & Description & c & $\begin{array}{l}\text { B.E. } \\
\text { Input }\end{array}$ & $\begin{array}{l}\text { Calc. } \\
\text { Result }\end{array}$ & $\begin{array}{c}\text { Cutset } \\
\text { Freq. (/yr) }\end{array}$ \\
\hline 10. & $\begin{array}{l}\text { DRYAIR2-T\&MV\# } \\
\text { FLT9580APLGV+ } \\
\text { OPRFILT-VIH1\# }\end{array}$ & $\begin{array}{l}\text { Dryer } \# 2 \text { out of service for T\&M. } \\
\text { Instrument air dryer \#1 prefilter plugs } \\
\text { Fail to replace filter }\end{array}$ & 4 & $\begin{array}{r}40 \mathrm{H} \\
1 \mathrm{Y} \\
48 \mathrm{H} \\
1.0 \mathrm{E}-04 \mathrm{H} \\
1 \mathrm{~N} \\
5.0 \mathrm{E}-01 \mathrm{~N}\end{array}$ & $\begin{array}{l}4.56 \mathrm{E}-03 \\
4.78 \mathrm{E}-03 \\
5.00 \mathrm{E}-01 \mathrm{~N}\end{array}$ & $1.99 \mathrm{E}-03$ \\
\hline 11. & CC2 -AIR-DRY $1+$ & cc: 'both air dryers fail' & 4 & $5.0 \mathrm{E}-08 \mathrm{H}$ & $4.00 \mathrm{E}-07$ & $4.38 \mathrm{E}-04$ \\
\hline 12. & $\begin{array}{l}\text { CMPAIR3-T\&MV\# } \\
\text { FLT9620-PLGV+ } \\
\text { FLT9623-PLGV+ } \\
\text { OPRFILT-VIH1\# }\end{array}$ & $\begin{array}{l}\text { Standy compressor out of service for T\&M } \\
\text { Inlet filter on secondary compressor is plugged } \\
\text { Primary compressor inlet filter plugs } \\
\text { Fail to replace filter }\end{array}$ & $\begin{array}{l}4 \\
4 \\
1\end{array}$ & $\begin{array}{r}27 \mathrm{D} \\
1 \mathrm{Y} \\
48 \mathrm{H} \\
1.0 \mathrm{E}-04 \mathrm{H} \\
48 \mathrm{H} \\
1.0 \mathrm{E}-04 \mathrm{H} \\
1 \mathrm{~N} \\
5.0 \mathrm{E}-01 \mathrm{~N}\end{array}$ & $\begin{array}{l}7.13 \mathrm{E}-02 \\
4.78 \mathrm{E}-03 \\
4.78 \mathrm{E}-03 \\
5.00 \mathrm{E}-01 \mathrm{~N}\end{array}$ & $2.97 \mathrm{E}-04$ \\
\hline 13. & $\begin{array}{l}\text { DRYAIR2-T\&MV\# } \\
\text { FLT9580BPLGV+ } \\
\text { PDS9580BFTCV\# }\end{array}$ & $\begin{array}{l}\text { Dryer \#2 out of service for T\&M } \\
\text { Instrument air dryer \#1 after filter plugs } \\
\text { Dryer } \# 1 \text { after-filter DP high switch fails to close }\end{array}$ & 3 & $\begin{array}{r}40 \mathrm{H} \\
1 \mathrm{Y} \\
48 \mathrm{H} \\
1.0 \mathrm{E}-04 \mathrm{H} \\
5 \mathrm{Y} \\
3.0 \mathrm{E}-06 \mathrm{H}\end{array}$ & $\begin{array}{l}4.56 \mathrm{E}-03 \\
4.78 \mathrm{E}-03 \\
6.29 \mathrm{E}-02\end{array}$ & $2.50 E-04$ \\
\hline 14. & $\begin{array}{l}\text { DRYAIR2-T\&MV\# } \\
\text { FLT9580APLGV+ } \\
\text { PDS9580AFTCV\# }\end{array}$ & $\begin{array}{l}\text { Dryer \#2 out of service for T\&M } \\
\text { Instrument air dryer \#1 prefilter plugs } \\
\text { Dryer \#1 prefilter high. DP switch fails to close }\end{array}$ & 4 & $\begin{array}{r}40 \mathrm{H} \\
1 \mathrm{Y} \\
48 \mathrm{H} \\
1.0 \mathrm{E}-04 \mathrm{H} \\
5 \mathrm{Y} \\
3.0 \mathrm{E}-06 \mathrm{H}\end{array}$ & $\begin{array}{l}4.56 \mathrm{E}-03 \\
4.78 \mathrm{E}-03 \\
6.29 \mathrm{E}-02\end{array}$ & $2.50 \mathrm{E}-04$ \\
\hline 15 & HCVINAIRC $3 \mathrm{CV}+$ & CC: All three compressor water supply valves fail closed & 4 & $2.5 \mathrm{E}-08 \mathrm{H}$ & $5.00 \mathrm{E}-08$ & $2.19 E-04$ \\
\hline 16 & PIPINAIRRUPV+ & Major line break in instrument air piping & 4 & $2.18 \mathrm{E}-08 \mathrm{H}$ & $1.74 \mathrm{E}-07$ & $1.91 \mathrm{E}-04$ \\
\hline 17. & $\begin{array}{l}\text { FLT9580BPLG̣V+ } \\
\text { FLT9581APLGV\# } \\
\text { OPR-AIR-MCH1\# }\end{array}$ & $\begin{array}{l}\text { Instrument air dryer } \# 1 \text { after filter plugs } \\
\text { Dryer } \# 1 \text { prefilter plugs } \\
\text { Instrument air DP switch miscalibrated }\end{array}$ & $\begin{array}{l}4 \\
3 \\
1\end{array}$ & $\begin{array}{r}48 \mathrm{H} \\
1.0 \mathrm{E}-04 \mathrm{H} \\
48 \mathrm{H} \\
1.0 \mathrm{E}-04 \mathrm{H} \\
1 \mathrm{~N} \\
3.0 \mathrm{E}-02 \mathrm{~N}\end{array}$ & $\begin{array}{l}4.78 \mathrm{E}-03 \\
4.79 \mathrm{E}-03 \\
3.00 \mathrm{E}-02 \mathrm{~N}\end{array}$ & $1.25 \mathrm{E}-04$ \\
\hline
\end{tabular}


Cutsets for I: \CAFTA \CUT\INAIR.CSR

$11 / 08 / 94$

$12: 17$ AM (CONT.)

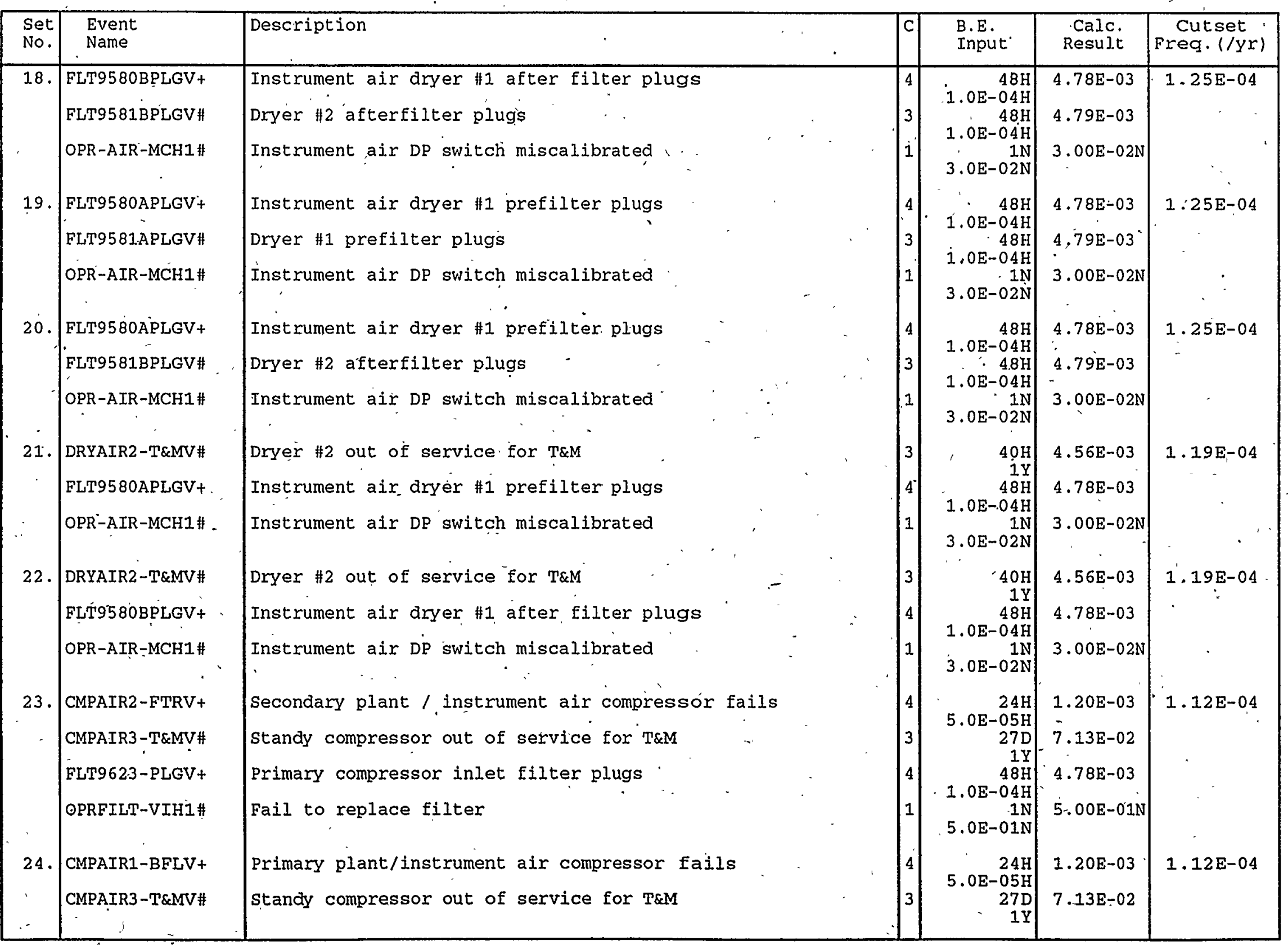


Cutsets for I: ICAFTA ICUT\INAIR.CSR

$12: 17$ AM (CONT.)

\begin{tabular}{|c|c|c|c|c|c|c|}
\hline $\begin{array}{l}\text { Set } \\
\text { No. }\end{array}$ & $\begin{array}{l}\text { Event } \\
\text { Name }\end{array}$ & Description & c & $\begin{array}{l}\text { B.E. } \\
\text { Input }\end{array}$ & $\begin{array}{l}\text { Calc. } \\
\text { Result }\end{array}$ & $\begin{array}{c}\text { Cutset } \\
\text { Freq. }(/ \mathrm{yr})\end{array}$ \\
\hline 25 & $\begin{array}{l}\text { FLT9620-PLGV+ } \\
\text { OPRFILT-VIH1\#" } \\
\text { CMPAIR1-BFLV+ } \\
\text { CMPAIR2-FTRV+ } \\
\text { CMPAIR3-T\&MV\# }\end{array}$ & $\begin{array}{l}\text { Inlet filter on secondary compressor is plugged } \\
\text { Fail to replace filter } \\
\text { Primary plant/instrument air compressor fails } \\
\text { Secondary plant / instrument air compressor fails } \\
\text { Standy compressor out of service for T\&M }\end{array}$ & $\begin{array}{l}4 \\
4 \\
3\end{array}$ & $\begin{array}{r}48 \mathrm{H} \\
1.0 \mathrm{E}-04 \mathrm{H} \\
1 \mathrm{~N} \\
5.0 \mathrm{E}-01 \mathrm{~N} \\
\\
24 \mathrm{H} \\
5.0 \mathrm{E}-05 \mathrm{H} \\
24 \mathrm{H} \\
5.0 \mathrm{E}-05 \mathrm{H} \\
27 \mathrm{D} \\
1 \mathrm{Y}\end{array}$ & $\begin{array}{l}4.78 \mathrm{E}-03 \\
5.00 \mathrm{E}-01 \mathrm{~N} \\
1.20 \mathrm{E}-03 \\
1.20 \mathrm{E}-03 \\
7.13 \mathrm{E}-02\end{array}$ & $\begin{array}{c}7.48 \mathrm{E}-05 \\
\sim\end{array}$ \\
\hline
\end{tabular}




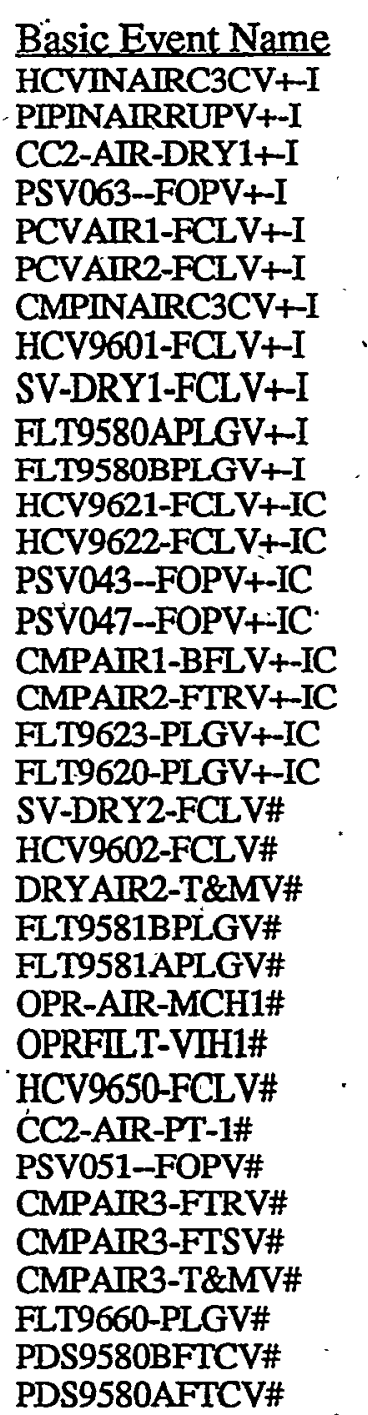

\section{Risk Achievement Worth}

Description

CC: All three compressor water supply valves fail closed

Major line break in instrument air piping

cc: both air dryers fail

Instrument air receiver PSV fails open

PCV reducing pressure going to equipment fails closed.

PCV back pressure control valve fails closed

CC: three plant/instrument air compressors fail

Instrument air dryer \#1 isolation valve fails closed

Dryer \#1 inlet valve fails closed

Instrument air dryer \#1 prefilter plugs

Instrument air dryer \#1 after filter plugs

Cooling water valve to primary compressor fails closed

Cooling water valve to secondary compressor fails closed

PSV on primary compressor discharge fails open

PSV on secondary compressor discharge line fails open

Primary plant/instrument air compressor fails

Secondary plant / instrument air compressor fails

Primary compressor inlet filter plugs

Inlet filter on secondary compressor is plugged

Dryer \#2 inlet valve fails closed

Instrument air dryer \#2 isolation valve fails closed

Dryer \#2 out of service for T\&M

Dryer \#2 afterfilter plugs

Dryer \#1 prefilter plugs

Instrument air DP switch miscalibrated

Fail to replace filter

Third compressor cooling water supply valve fails closed

cc: both instrument air PTs fail high

PSV on third compressor discharge fails open

Third plant / instrument air compressor fails to run

Third plant / instrument air compressor fails to start

Standy compressor out of service for T\&M

Inlet filter on secondary compressor is plugged

Dryer \#1 after-filter DP high switch fails to close

Dryer \#1 prefilter high DP switch fails to close
Prob/Freq

$2.50 \mathrm{E}-08 / \mathrm{H}$

$2.18 \mathrm{E}-08 / \mathrm{H}$

$5.00 \mathrm{E}-08 / \mathrm{H}$

$5.00 \mathrm{E}-07 / \mathrm{H}$

3.00E-06/H

$3.00 \mathrm{E}-06 / \mathrm{H}$

$2.50 \mathrm{E}-06 / \mathrm{H}$

$5.00 \mathrm{E}-07 / \mathrm{H}$

$5.00 \mathrm{E}-07 / \mathrm{H}$

9.95E-05/H

9.95E-05/H

$5.00 \mathrm{E}-07 / \mathrm{H}$

$5.00 \mathrm{E}-07 / \mathrm{H}$

$5.00 \mathrm{E}-07 / \mathrm{H}$

$5.00 \mathrm{E}-07 / \mathrm{H}$

$4.99 \mathrm{E}-05 / \mathrm{H}$

$4.99 \mathrm{E}-05 / \mathrm{H}$

9.95E-05/H

9.95E-05/H

$1.20 \mathrm{E}-05$

$1.20 \mathrm{E}-05$

$4.56 \mathrm{E}-03$

4.79E-03

4.79E-03

3.00E-02

5.00E-01

5.00E-07

2.40E-06

4.00E-06

$2.40 \mathrm{E}-03$

5.00E-03

7.13E-02

1.67E-02

6.29E-02

$6.29 \mathrm{E}-02$
AchW

9.29E+04

$9.29 \mathrm{E}+04$

$9.29 \mathrm{E}+04$

9.29E+04

$9.29 \mathrm{E}+04$

$9.29 \mathrm{E}+04$

$9.29 \mathrm{E}+04$

$9.50 \mathrm{E}+02$

$9.50 \mathrm{E}+02$

$7.26 \mathrm{E}+02$

$7.26 \mathrm{E}+02$

$3.82 \mathrm{E}+01$

$3.82 \mathrm{E}+01$

$3.82 E+01$

3.82E +01

$3.82 \mathrm{E}+01$

$3.82 \mathrm{E}+01$

$3.08 \mathrm{E}+01$

$3.08 \mathrm{E}+01$

$1.21 \mathrm{E}+01$

1.21E+01

$1.20 \mathrm{E}+01$

1.09E+01

$1.09 \mathrm{E}+01$

$1.25 \mathrm{E}+00$

$1.14 \mathrm{E}+00$

1.10E+00

$1.10 \mathrm{E}+00$

1.10E+00

$1.10 \mathrm{E}+00$

$1.10 \mathrm{E}+00$

$1.09 \mathrm{E}+00$

$1.08 \mathrm{E}+00$

$1.04 \mathrm{E}+00$

$1.04 \mathrm{E}+00$

Modified top event frequency = (top event frequency) $\mathrm{x}$ (rișk achievement worth).

\begin{tabular}{l} 
Basic Event Name \\
\hline PCVAIR1-FCLV+-I \\
PCVAIR2-FCLV+-I \\
CMPINAIRC3CV+I \\
OPRFILT-VIH1\# \\
FLT9580BPLGV+-I \\
FLT9580APLGV+-I \\
DRYAIR2-T\&MV\# \\
FLT9581APLGV\# \\
FLT9581BPLGV\# \\
PSV063-FOPV+-I
\end{tabular}

\section{Risk Reduction Worth}

\section{Description}

PCV reducing pressure going to equipment fails closed

PCV back pressure control valve fails closed

CC: three plant/instrument air compressors fail

Fail to replace filter

Instrument air dryer \#1 after filter plugs

Instrument air dryer \#1 prefilter plugs

Dryer \#2 out of service for T\&M

Dryer \#1 prefilter plugs

Dryer \#2 afterfilter plugs

Instrument air receiver PSV fails open
Prob/Freg

3.00E-06/H

$3.00 \mathrm{E}-06 / \mathrm{H}$

$2.50 \mathrm{E}-06 / \mathrm{H}$

$5.00 \mathrm{E}-01$.

9.95E-05/H

9.95E-05/H

$4.56 \mathrm{E}-03$

$4.79 \mathrm{E}-03$

4.79E-03

$5.00 \mathrm{E}-07 / \mathrm{H}$
RedW

$1.39 \mathrm{E}+00$

$1.39 \mathrm{E}+00$

$1.30 \mathrm{E}+00$

$1.16 \mathrm{E}+00$

$1.08 \mathrm{E}+00$

$1.08 \mathrm{E}+00$

$1.05 \mathrm{E}+00$

$1.05 \mathrm{E}+00$

$1.05 \mathrm{E}+00$

$1.05 \mathrm{E}+00$

Note: Events that make less than $1 \%$ difference to the importance factor are not included. Modified top event frequency $=($ top event frequency) $/$ (risk reduction worth). 


\section{Attachment 7 Seismic Initiator Accident Fault Trees}

PVVH Explosion

SRAT Explosion

SME Explosion

PRBT Explosion

MFT Explosion

PR Explosion

OE Explosion

OECT Explosion

PRFT Explosion

Melter Offgas Explosion

SPT Explosion

PPT Explosion

OWST Explosion

SPC Explosion
598

601

605

608

611

614

616

618

620

622

624

627

635

642 
X-ESR-S-00001, REV. 0

\section{PVVH Explosion - Seismic}




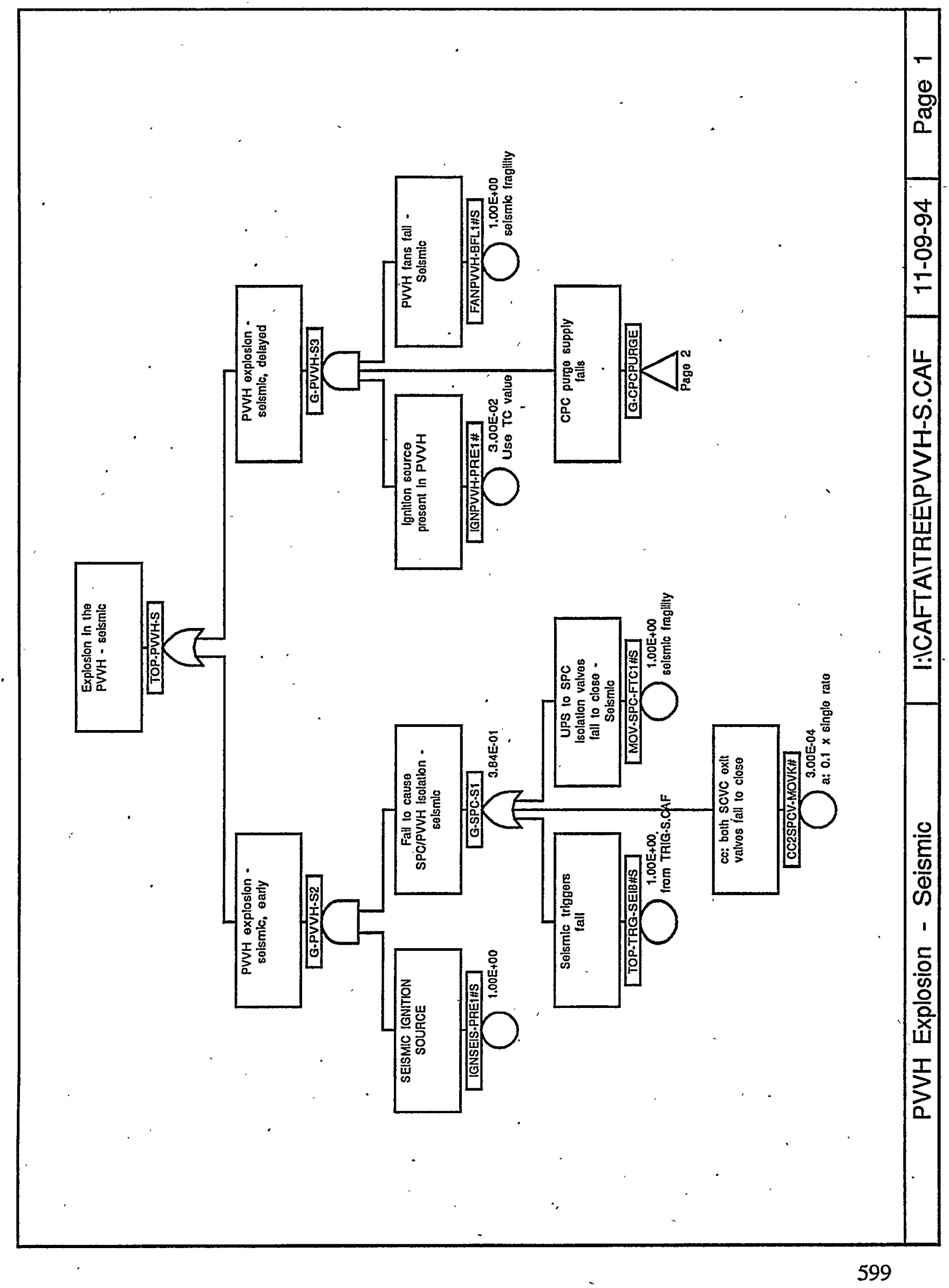




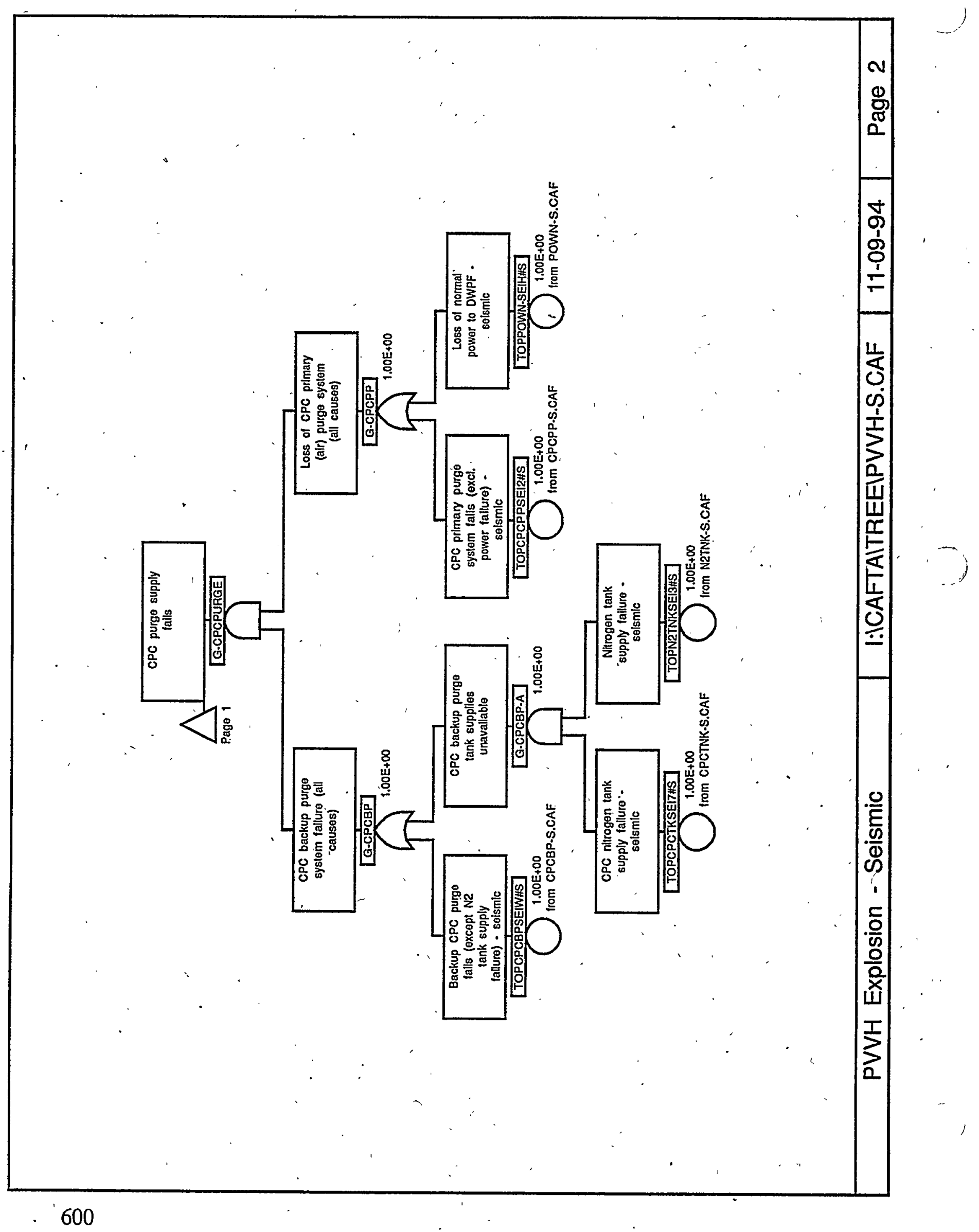


X-ESR-S-00001, REV. 0

\section{SRAT Explosion - Seismic}


X-ESR-S-00001, REV. 0

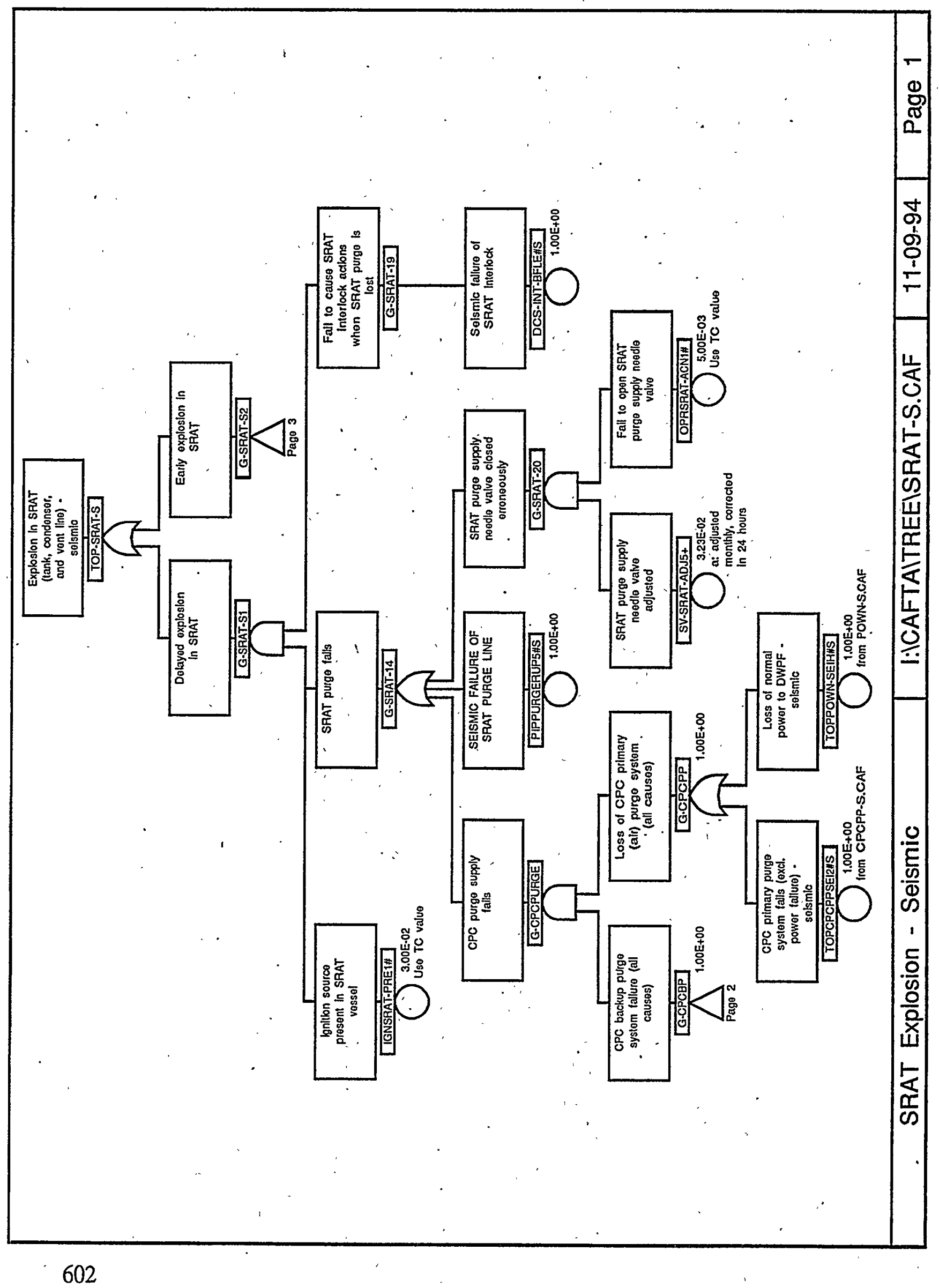




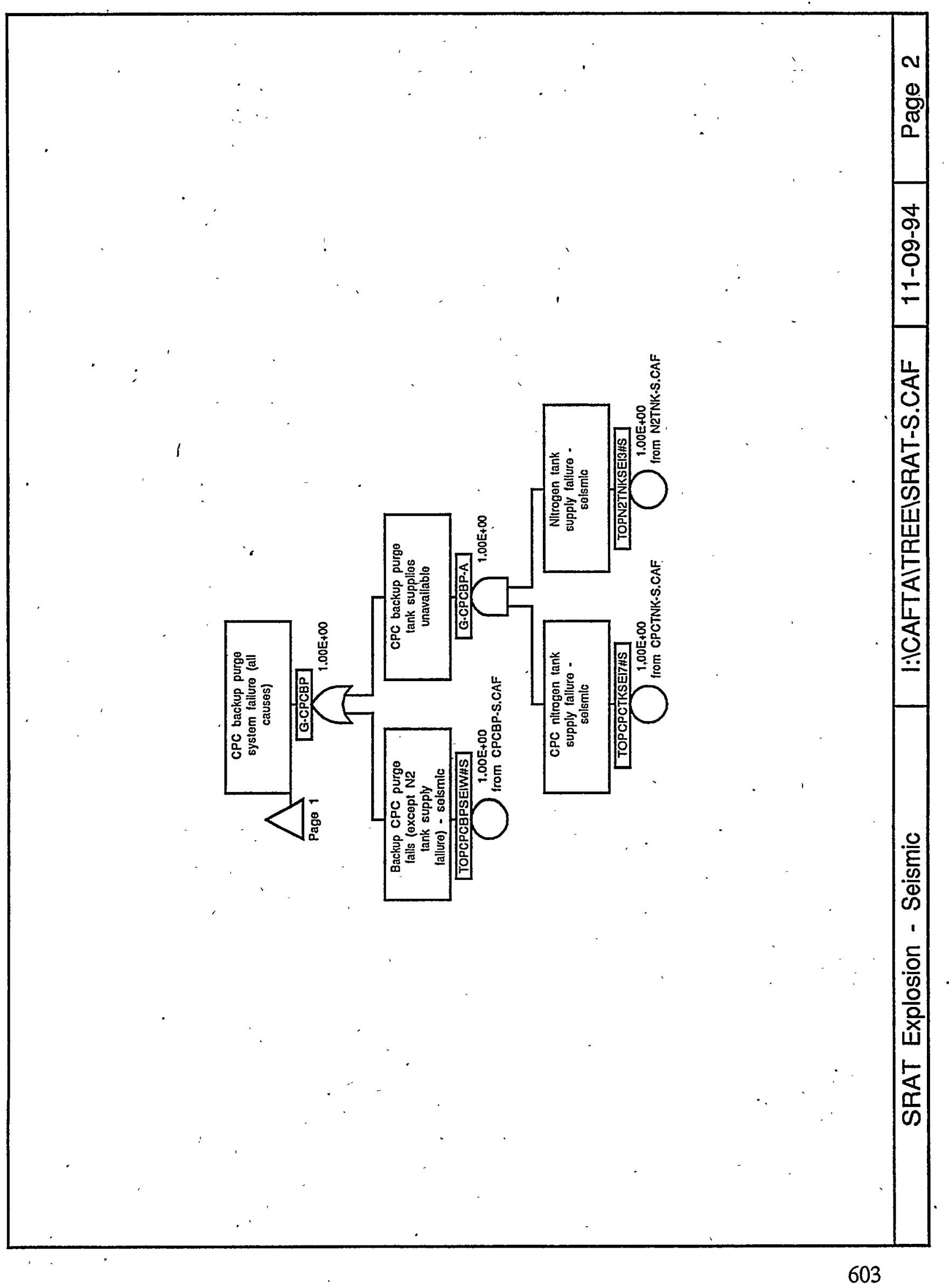


X-ESR-S-00001, REV. 0

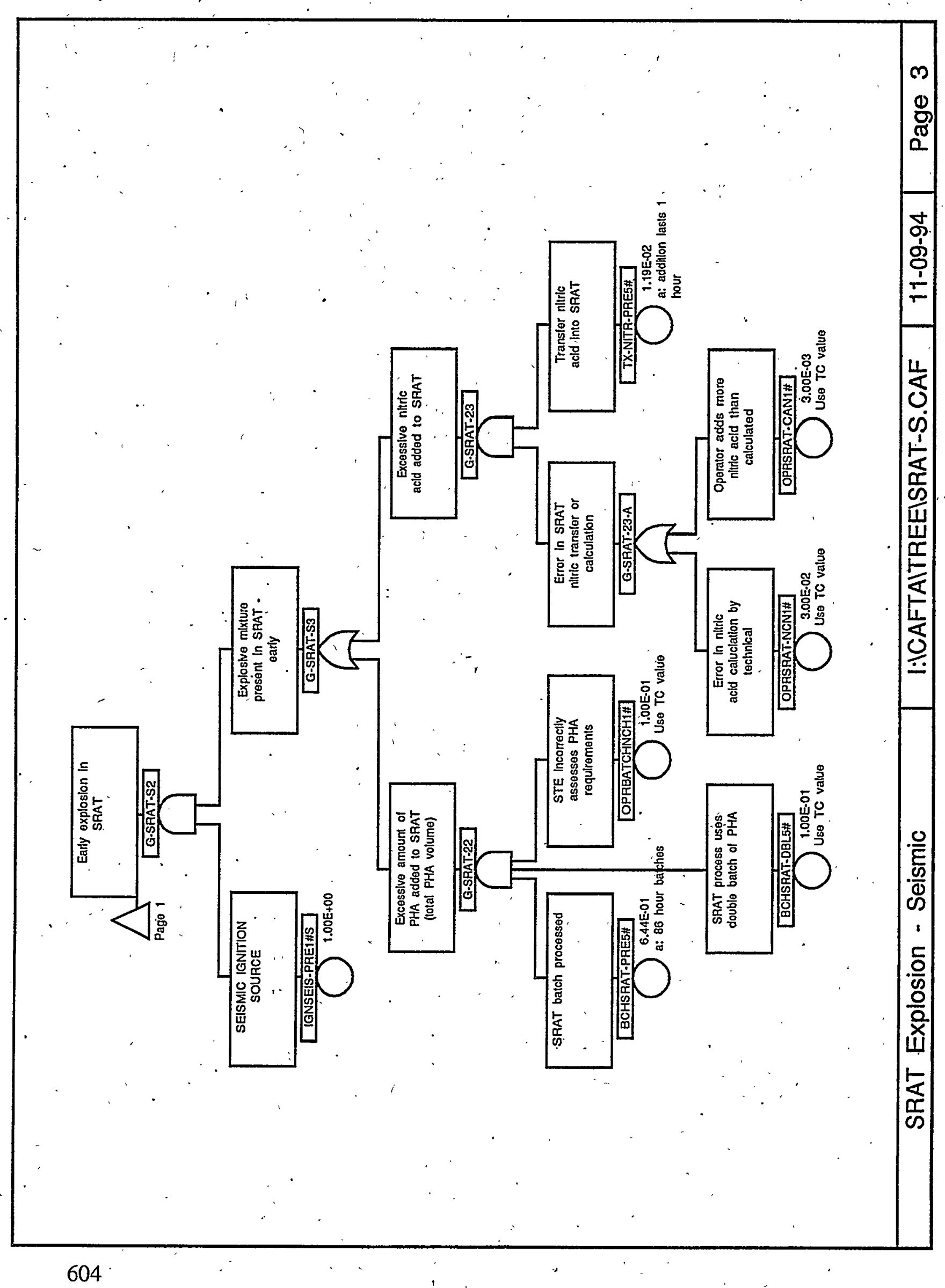




\section{SME Explosion:- Seismic}




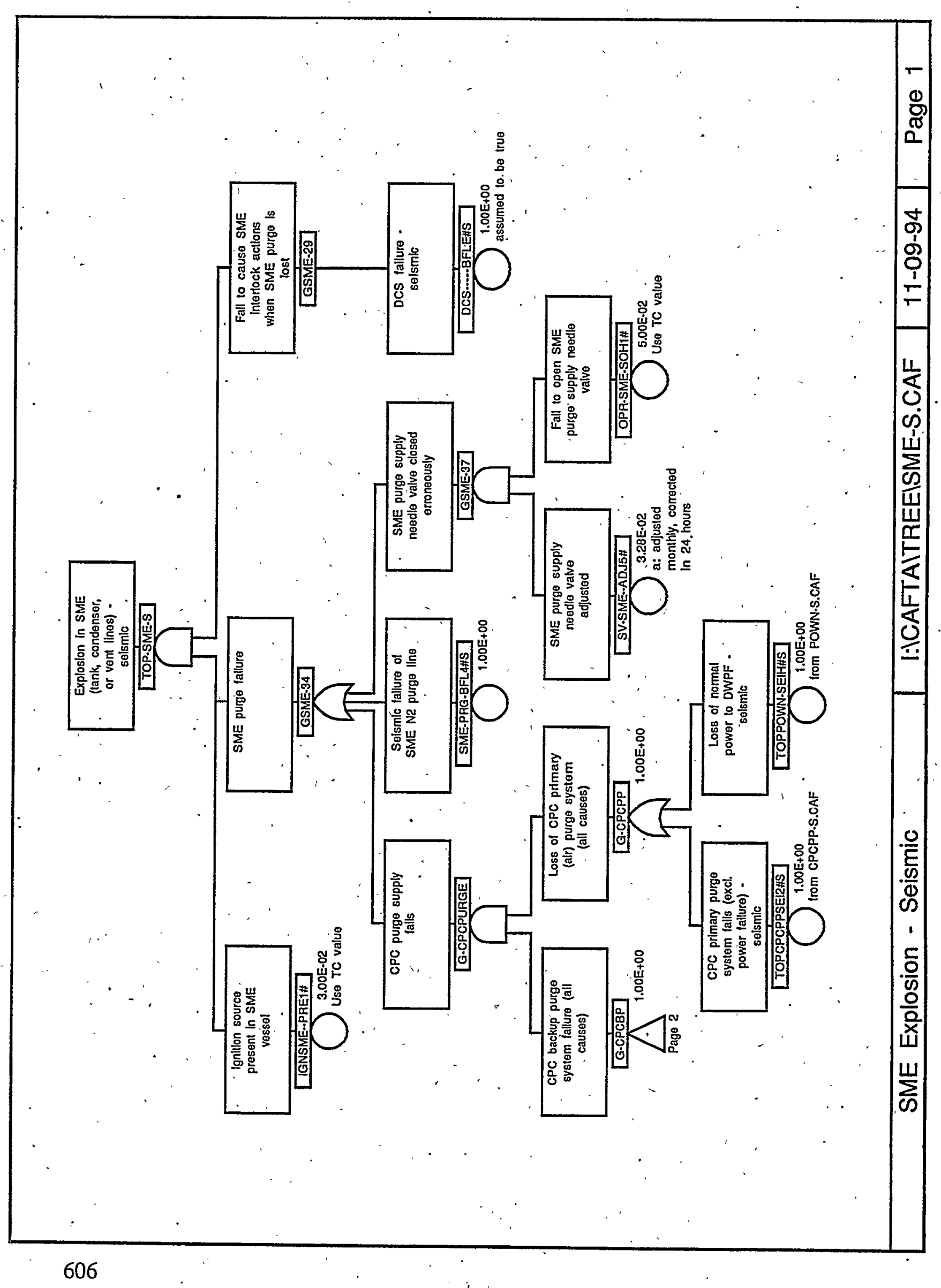




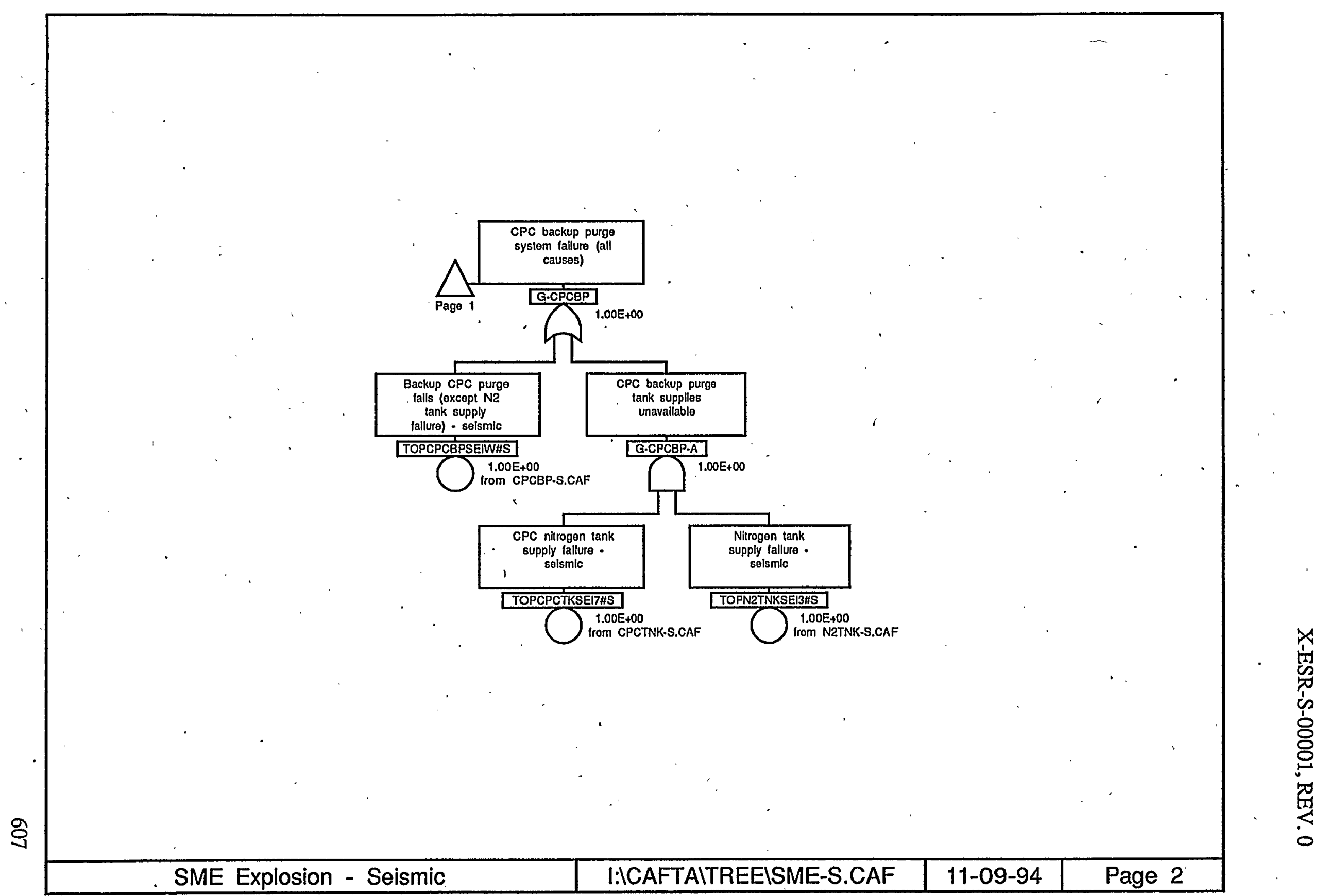


X-ESR-S-00001, REV. 0

\section{PRBT Explosion - Seismic}




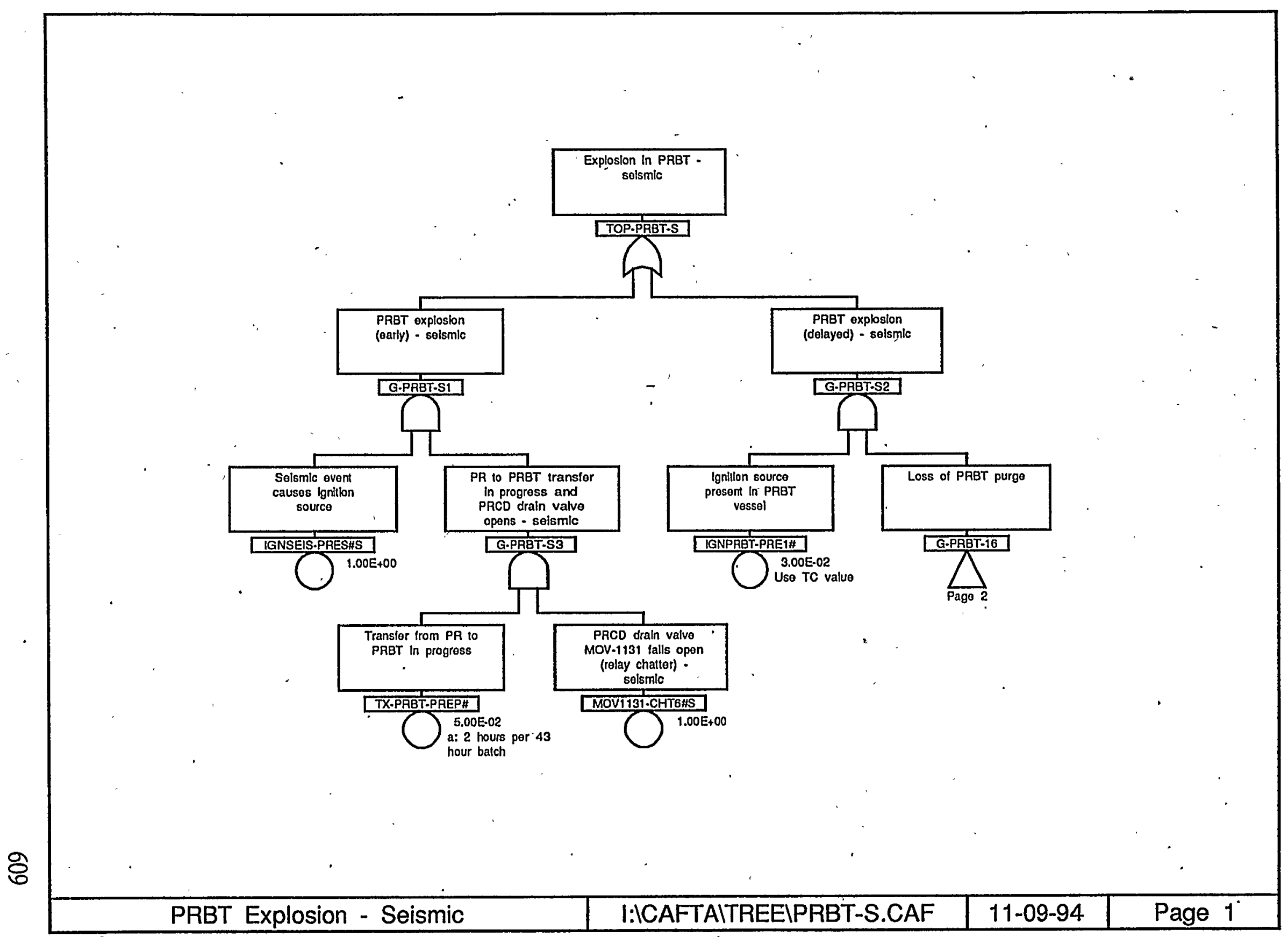




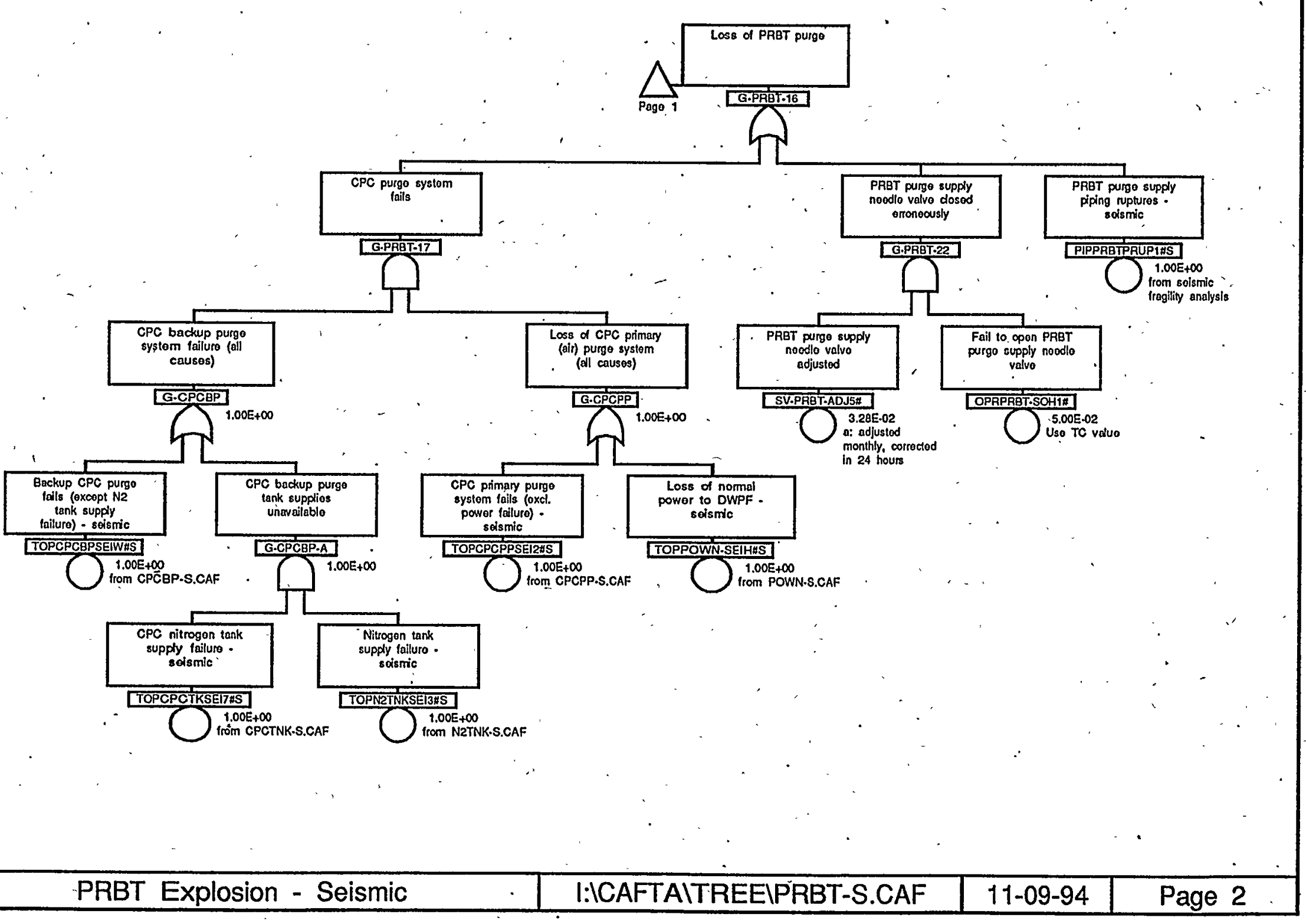


MFT Explosion - Seismic 
X-ESR-S-00001, REV. 0

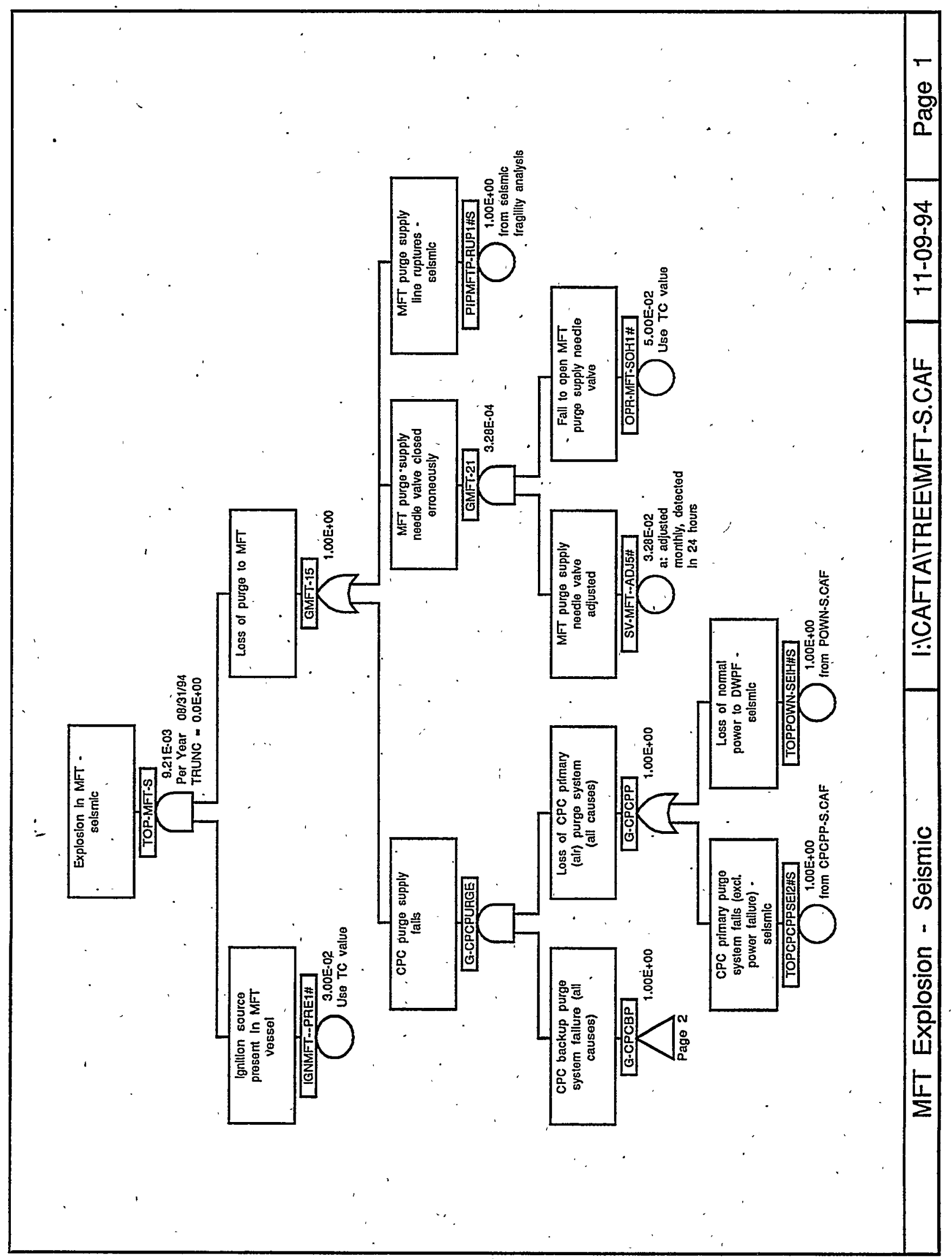




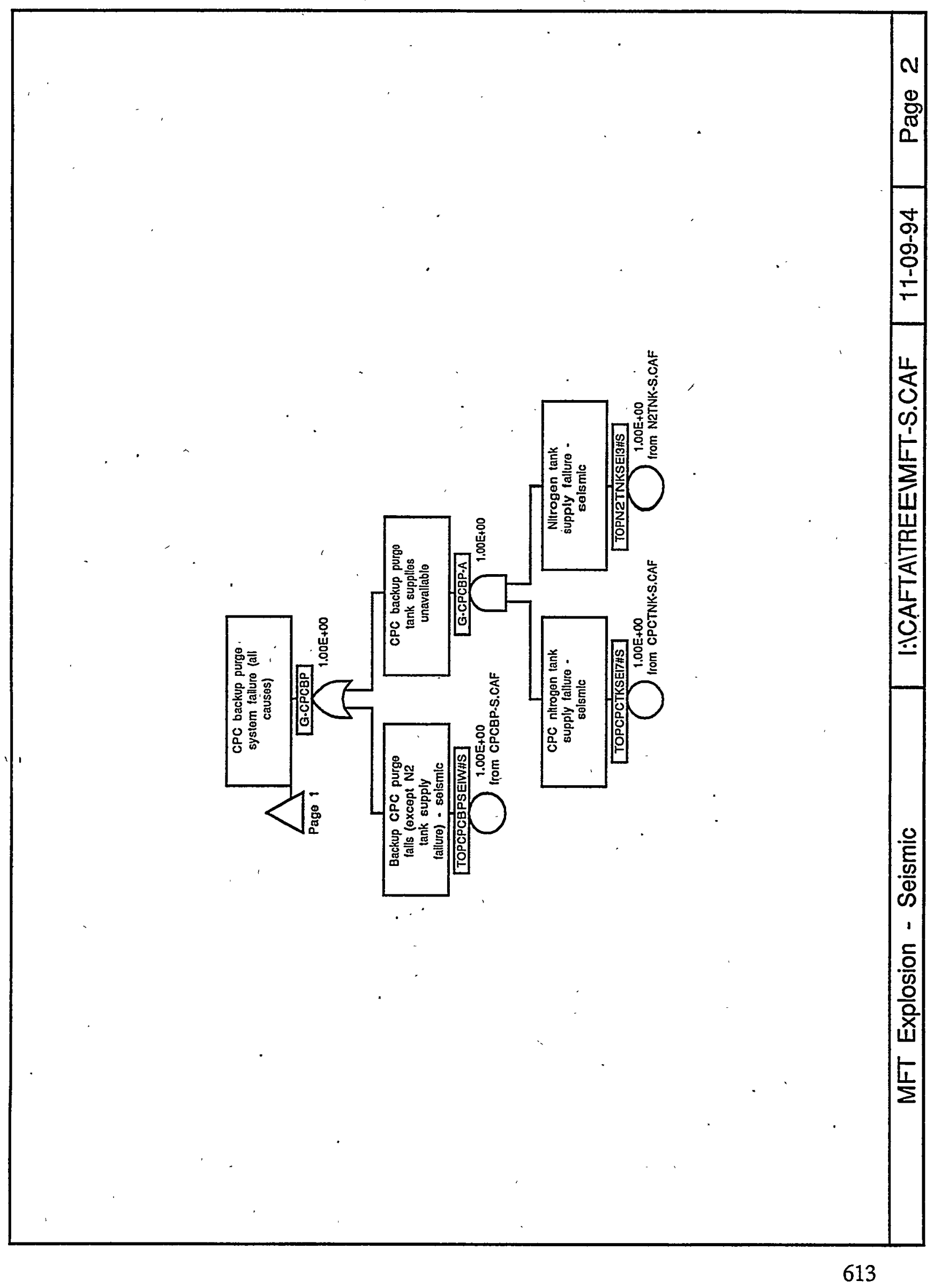


X-ESR-S-00001, REV. 0

\section{PR Explosion - Seismic}




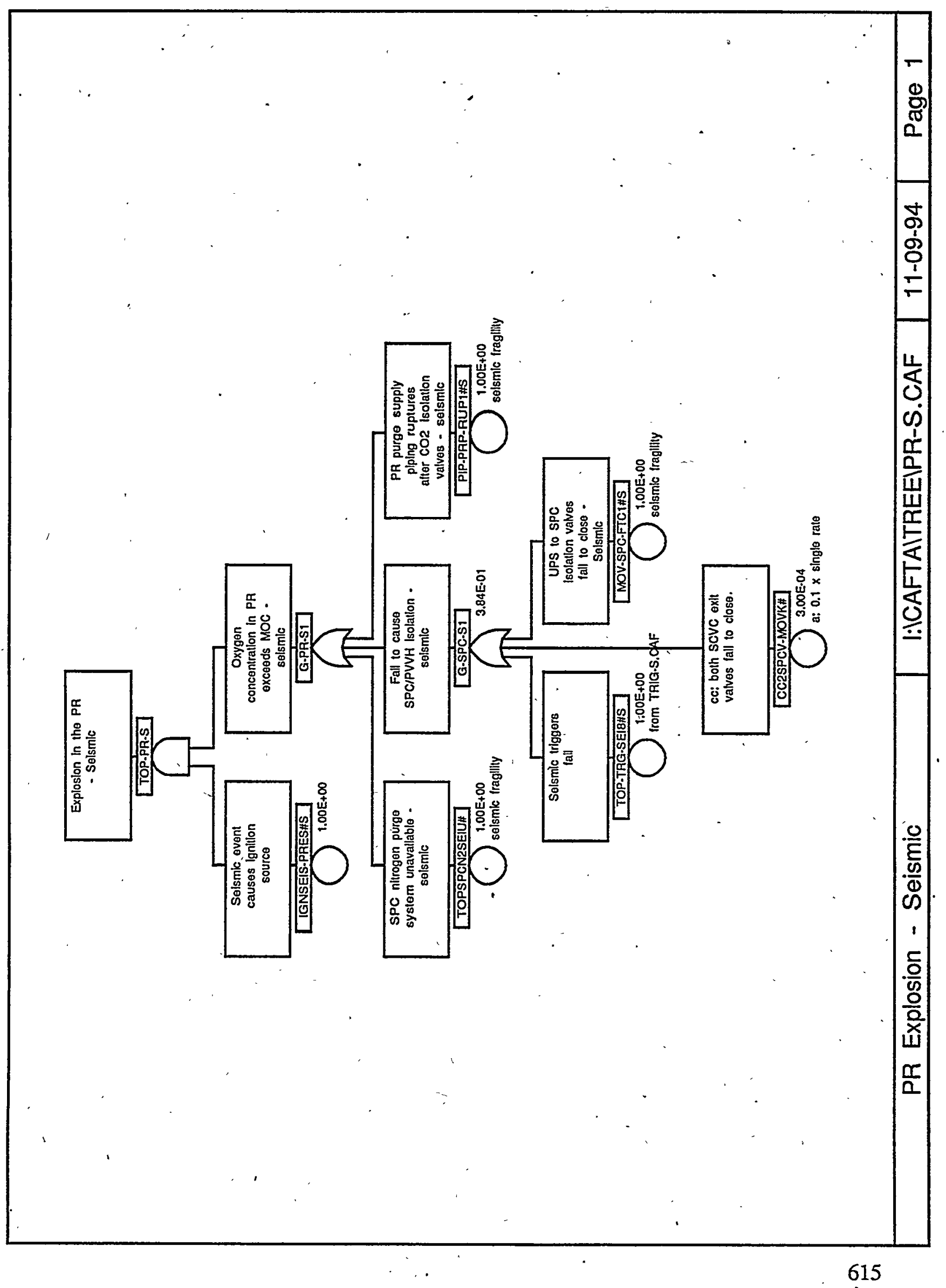


X-ESR-S-00001, REV. 0

\section{OE Explosion - Seismic}




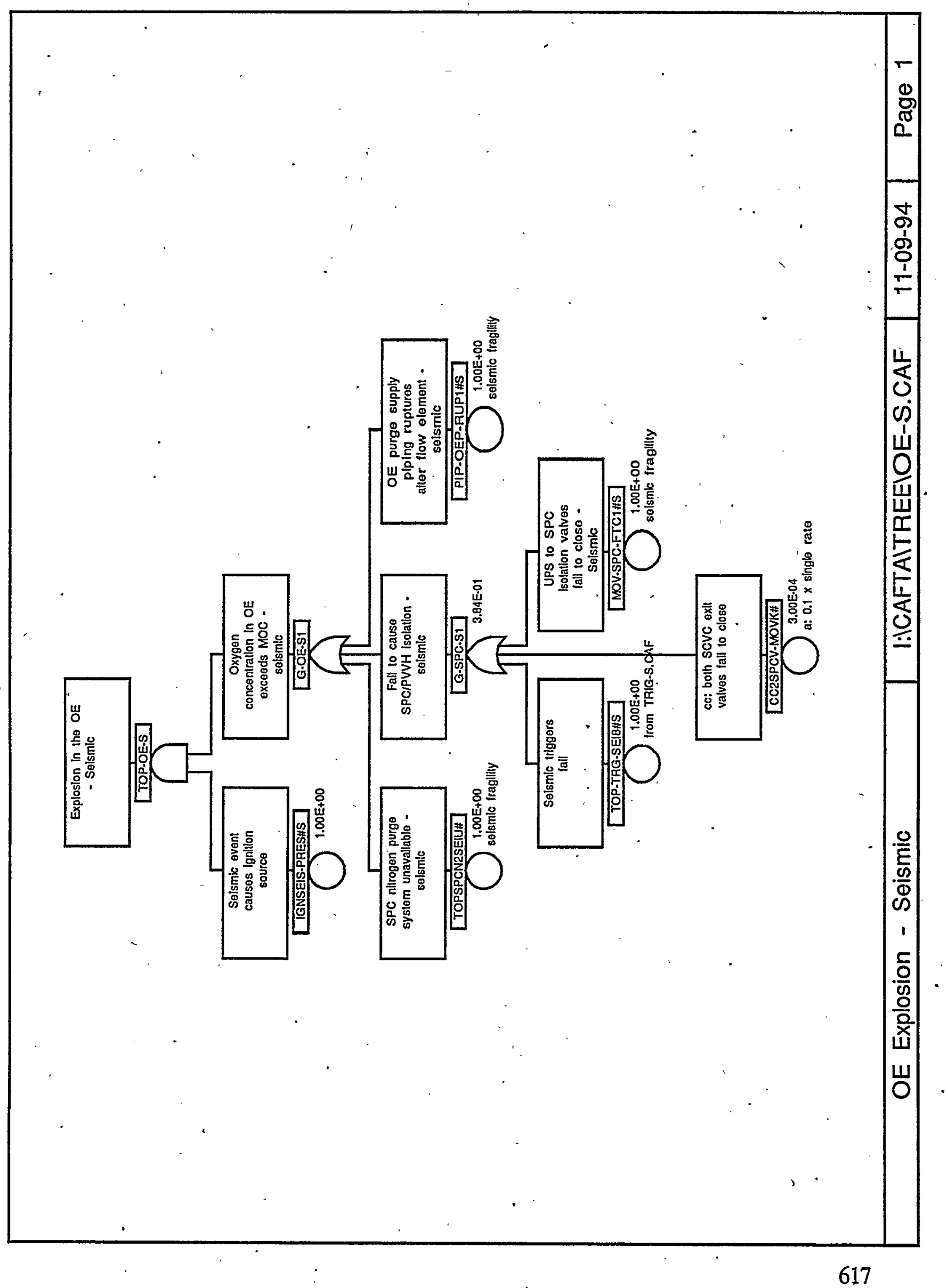


X-ESR-S-00001, REV. 0

\section{OECT Explosion - Seismic}




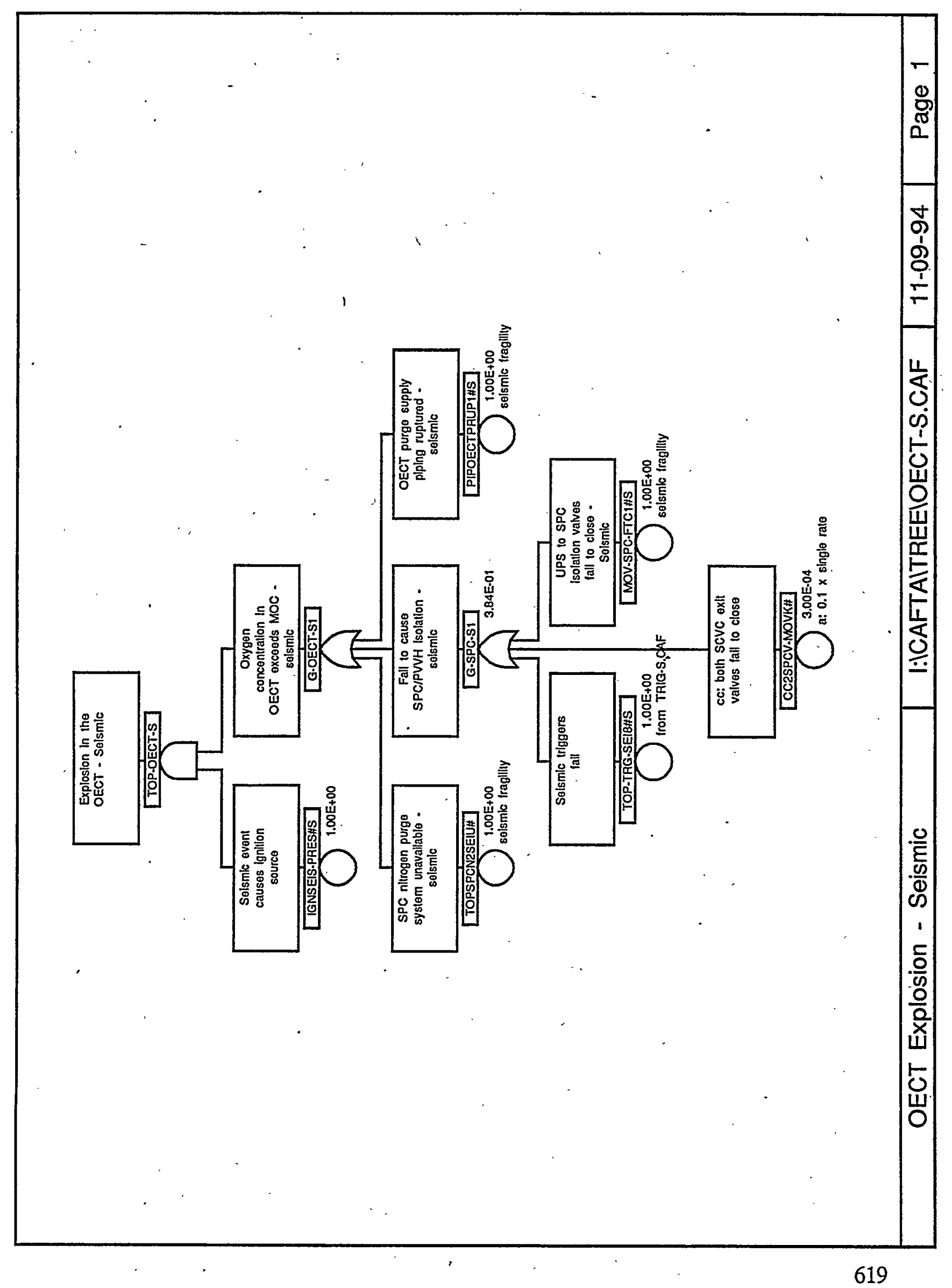


X-ESR-S-00001, REV. 0

\section{PRFT Explosion - Seismic}

1 


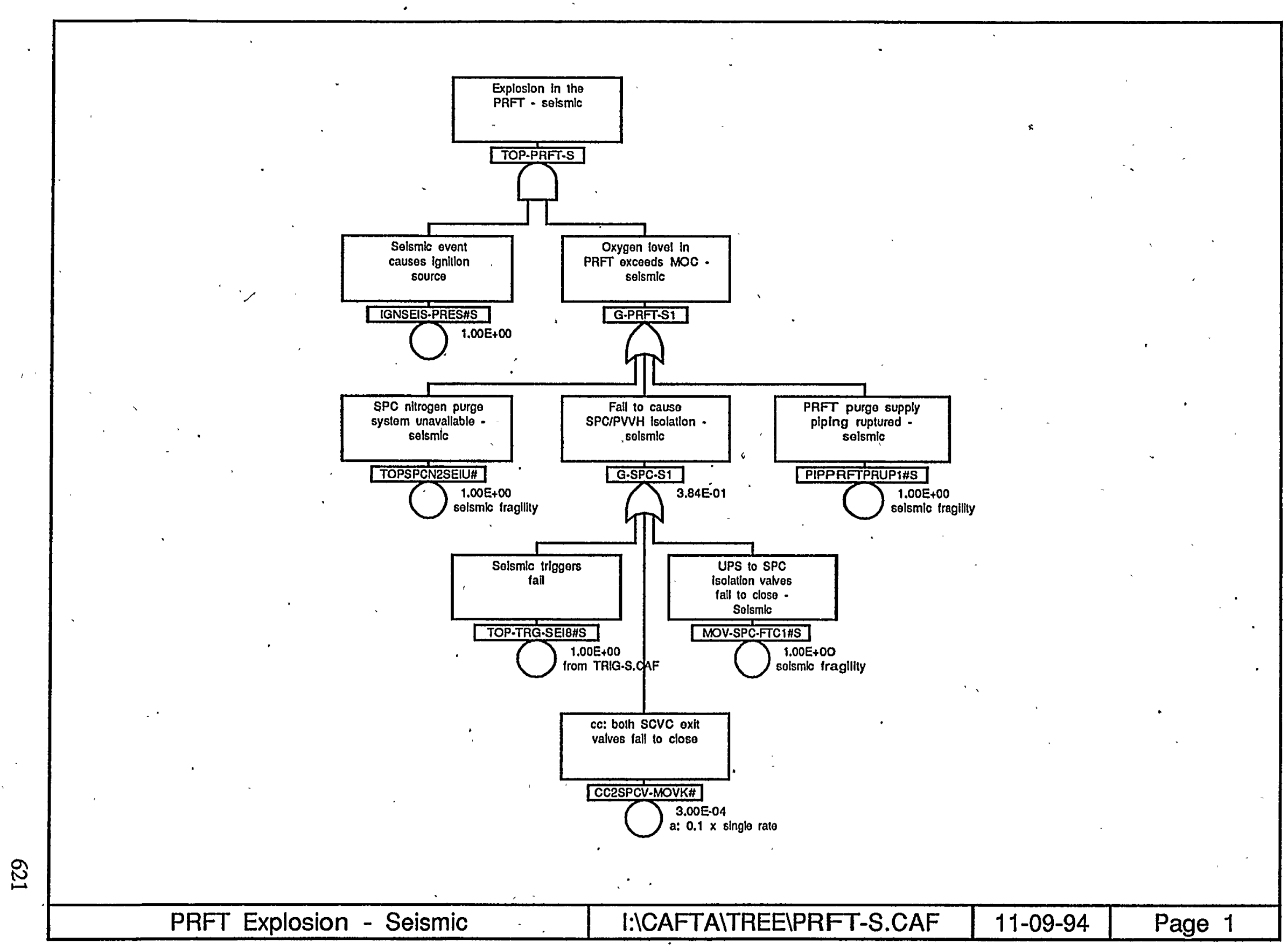


X-ESR-S-00001, REV. 0

\section{Melter Offgas Explosion}




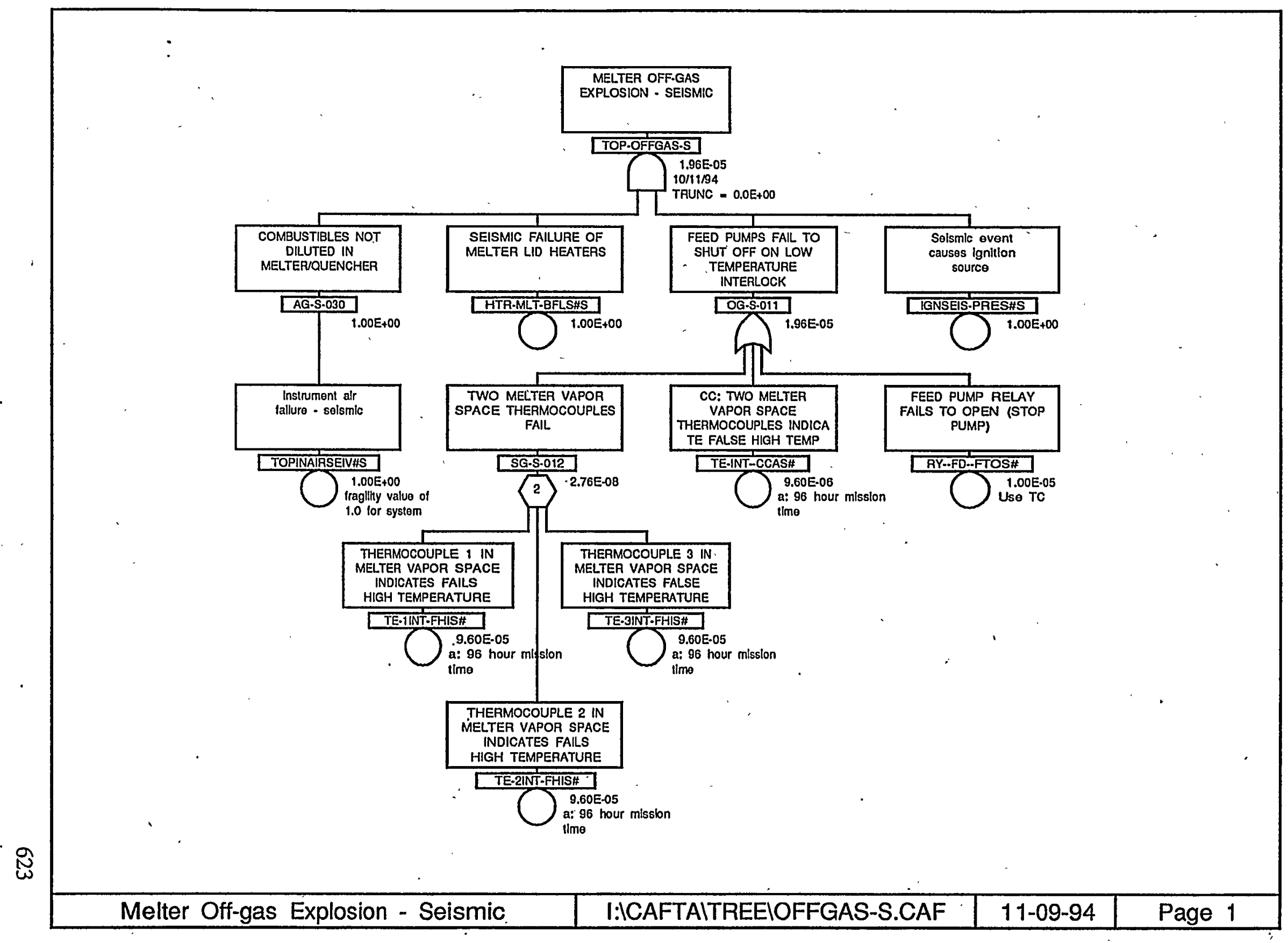


X-ESR-S-00001, REV. 0

LPPP Sludge Pump Tank Explosion - Seismic 


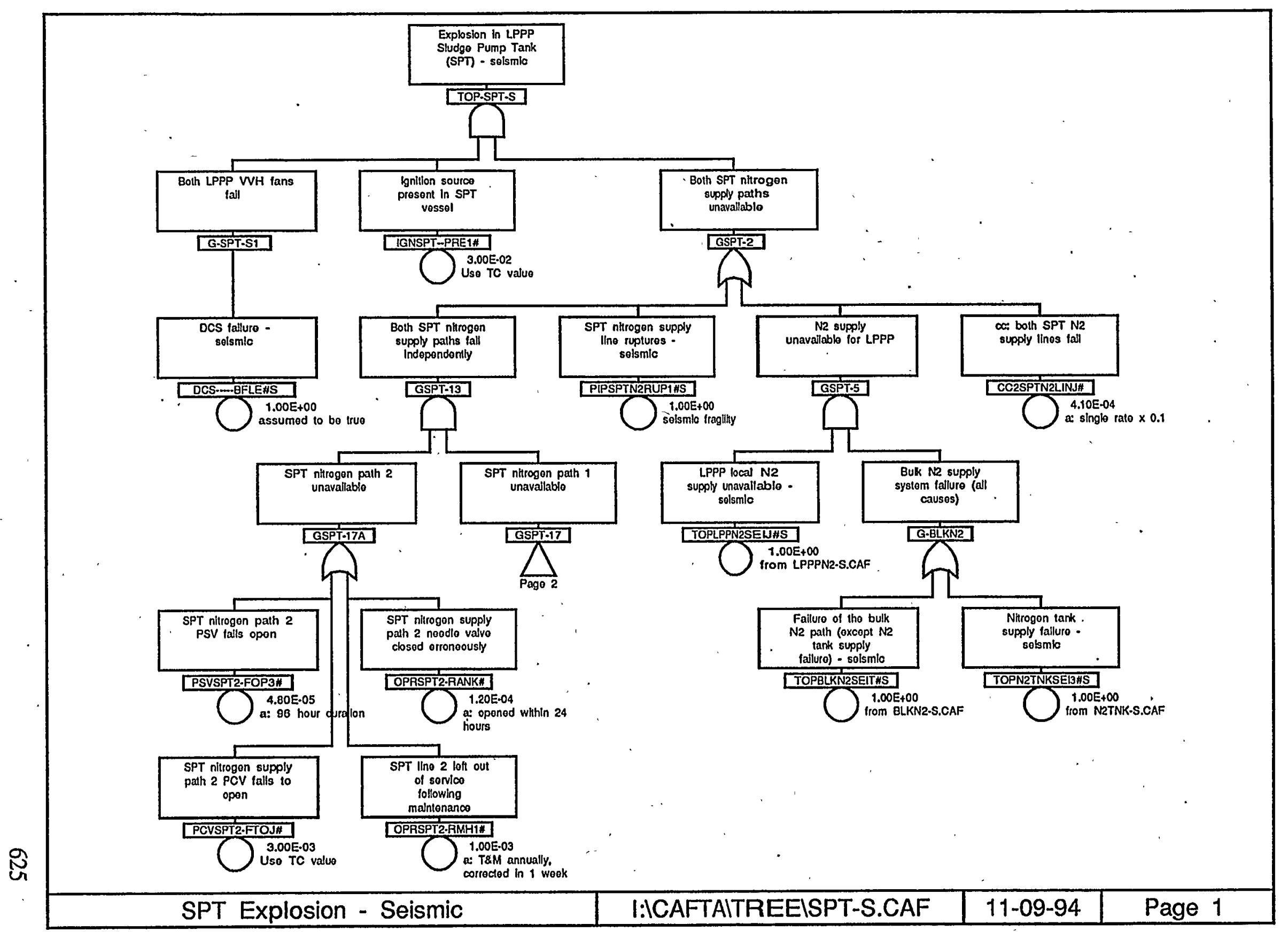




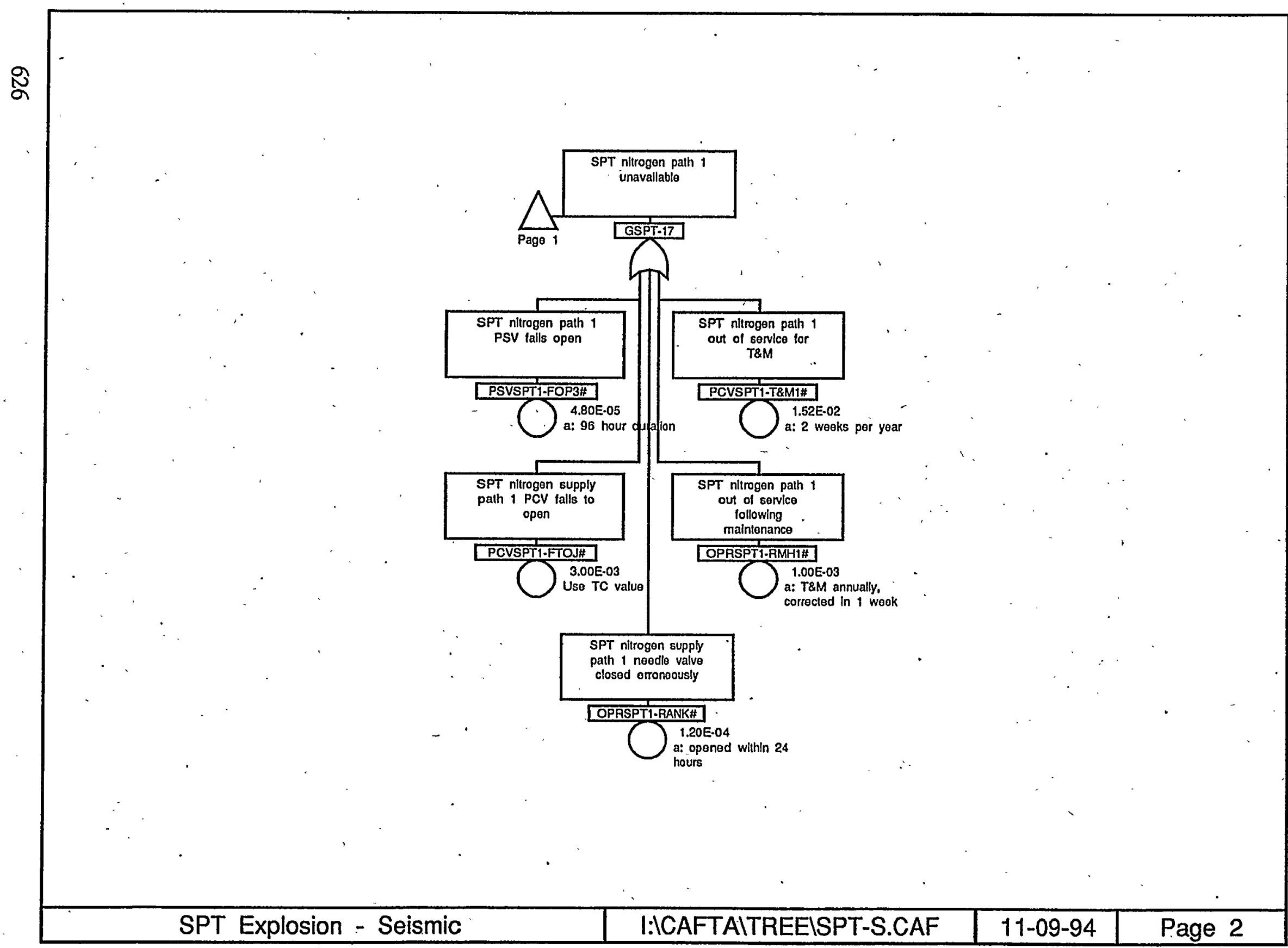




\section{LPPP PPT Explosion - Seismic}




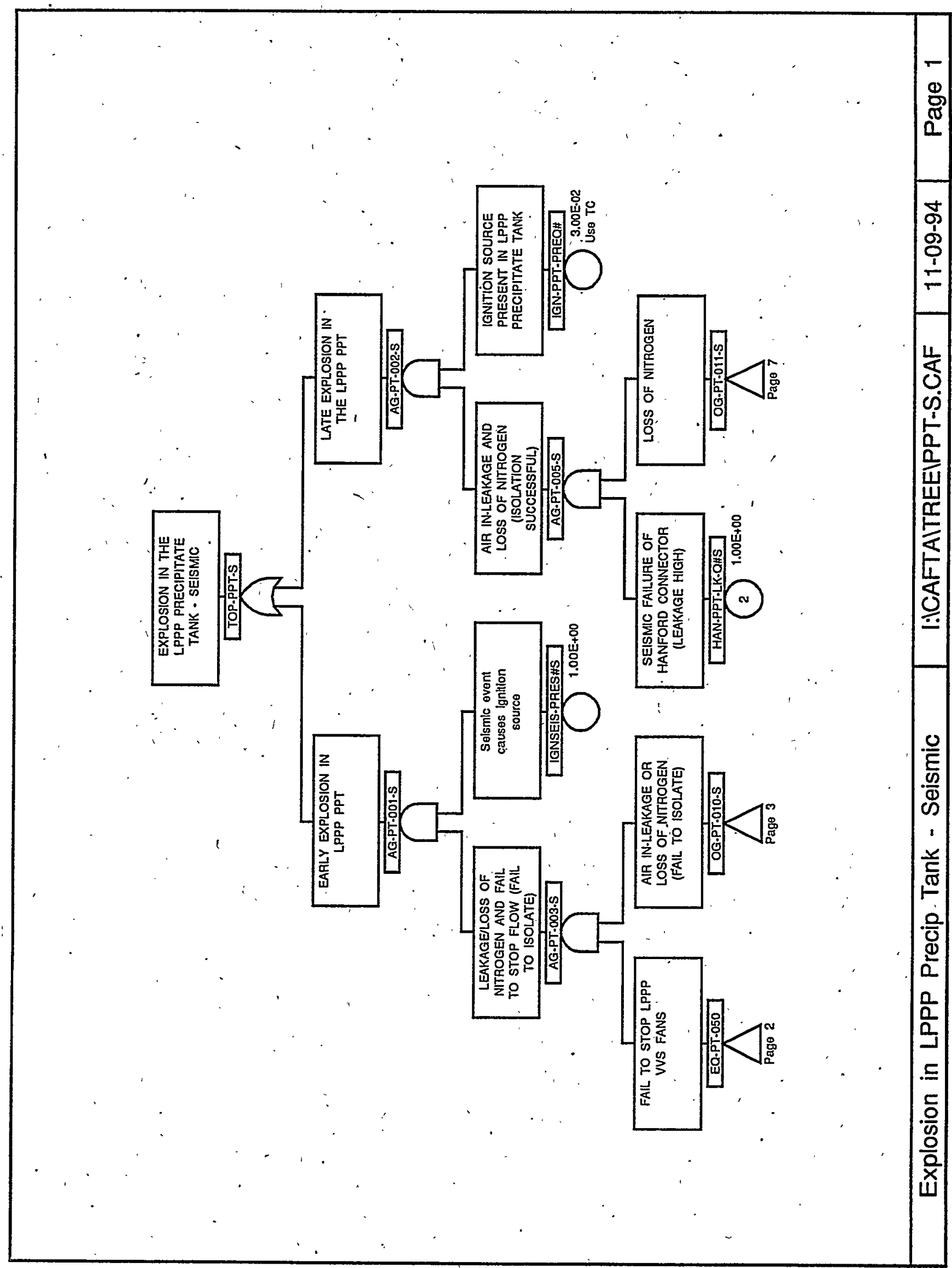




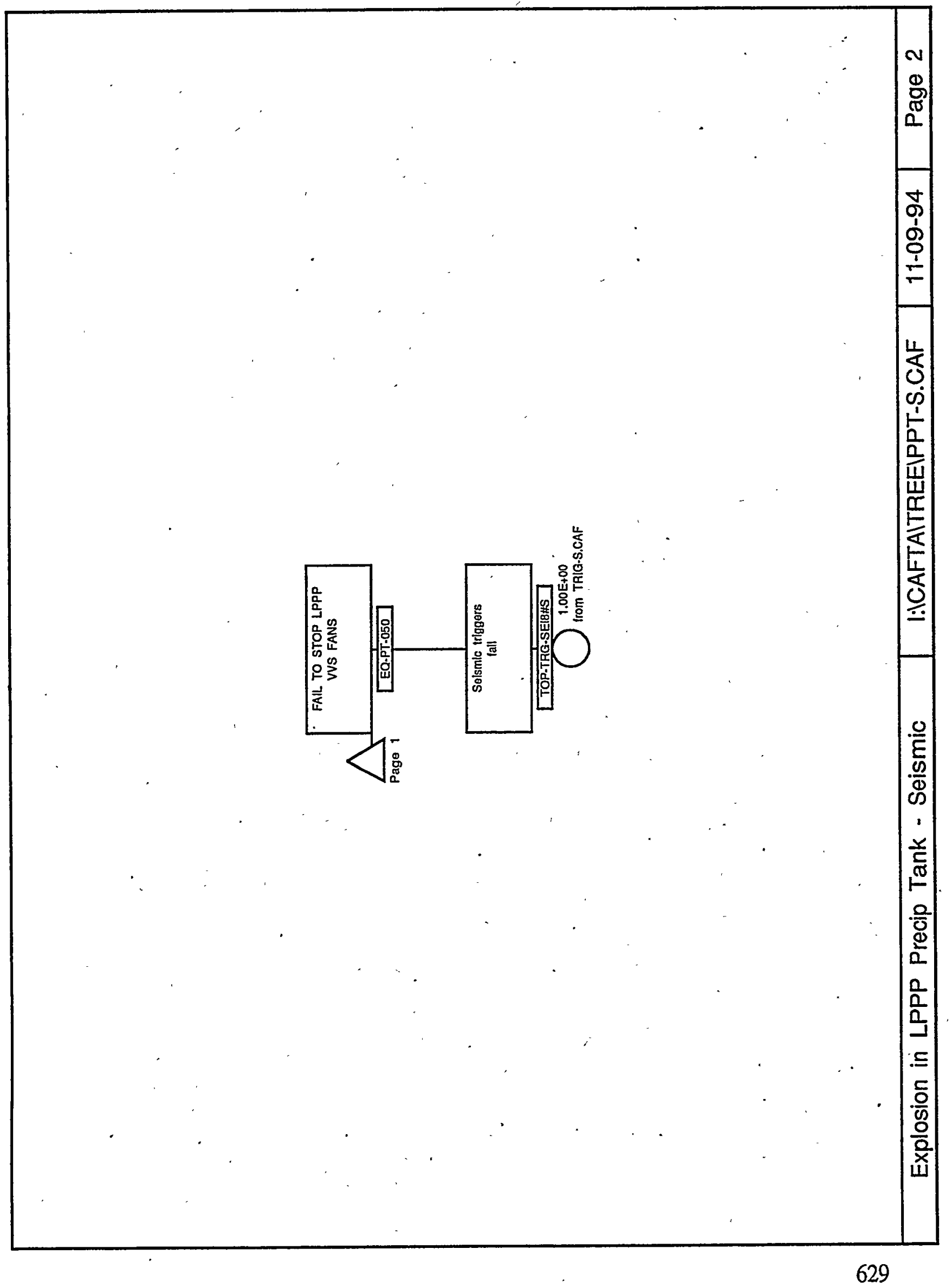


X-ESR-S-00001, REV. 0

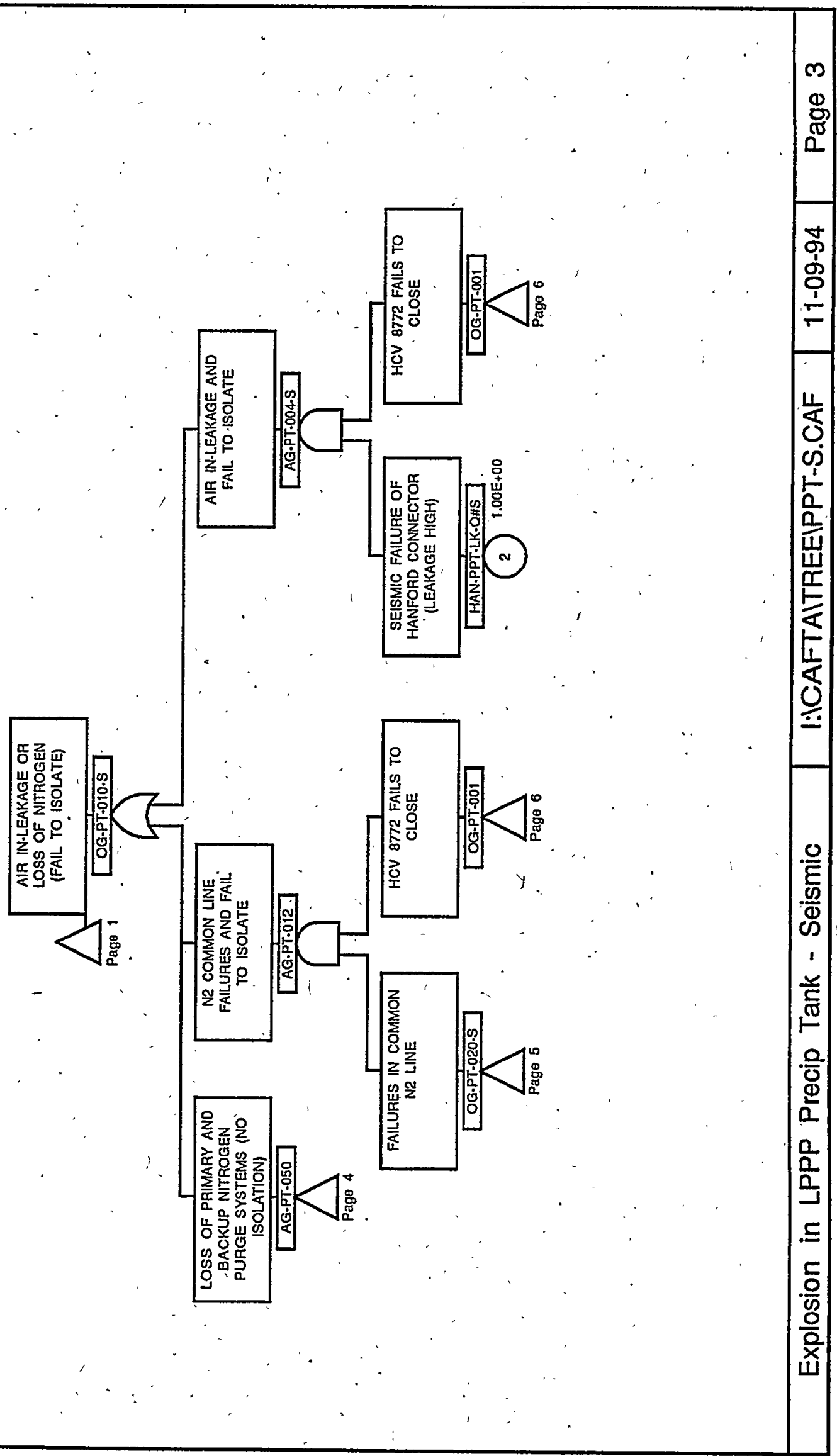




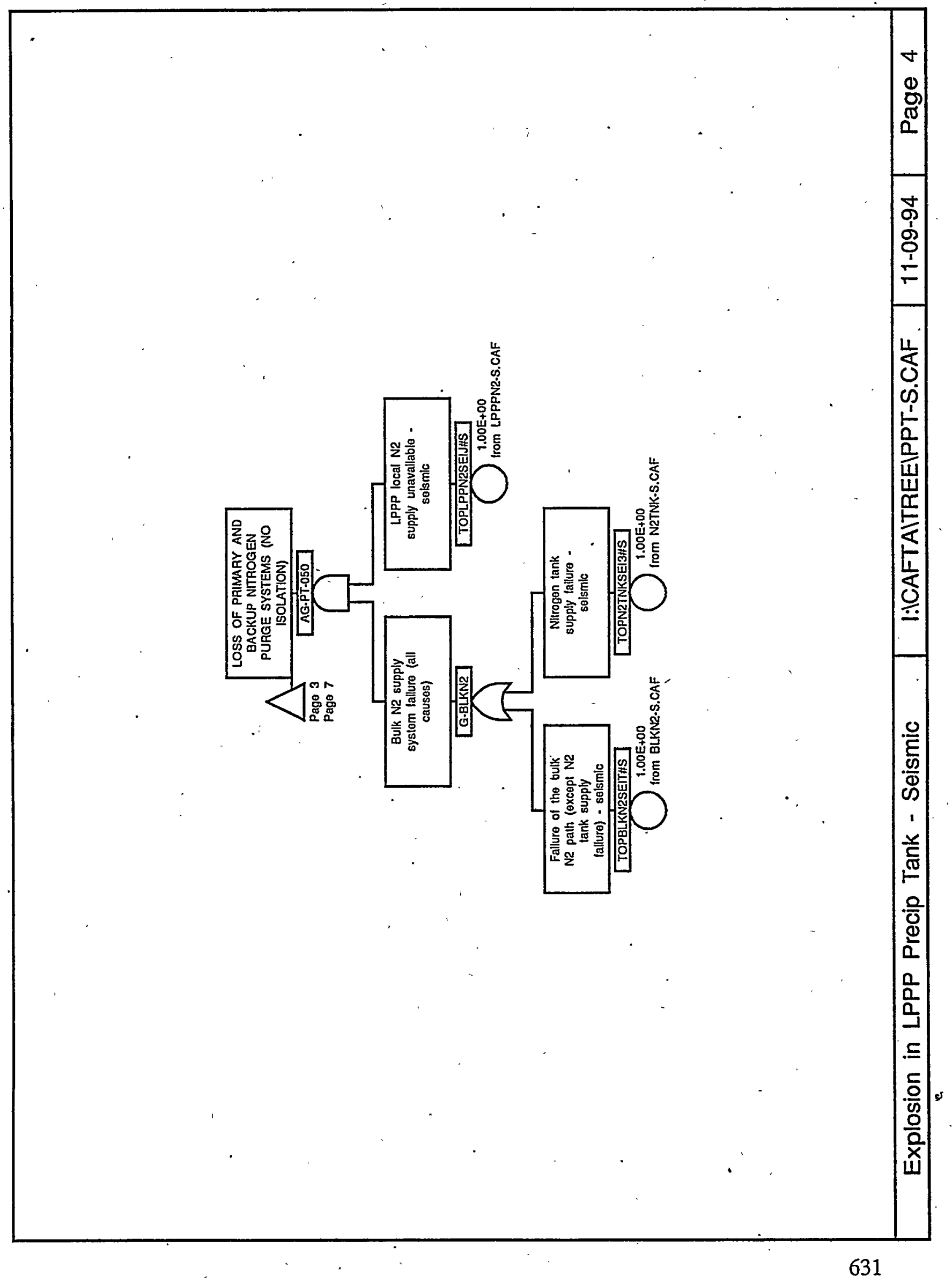




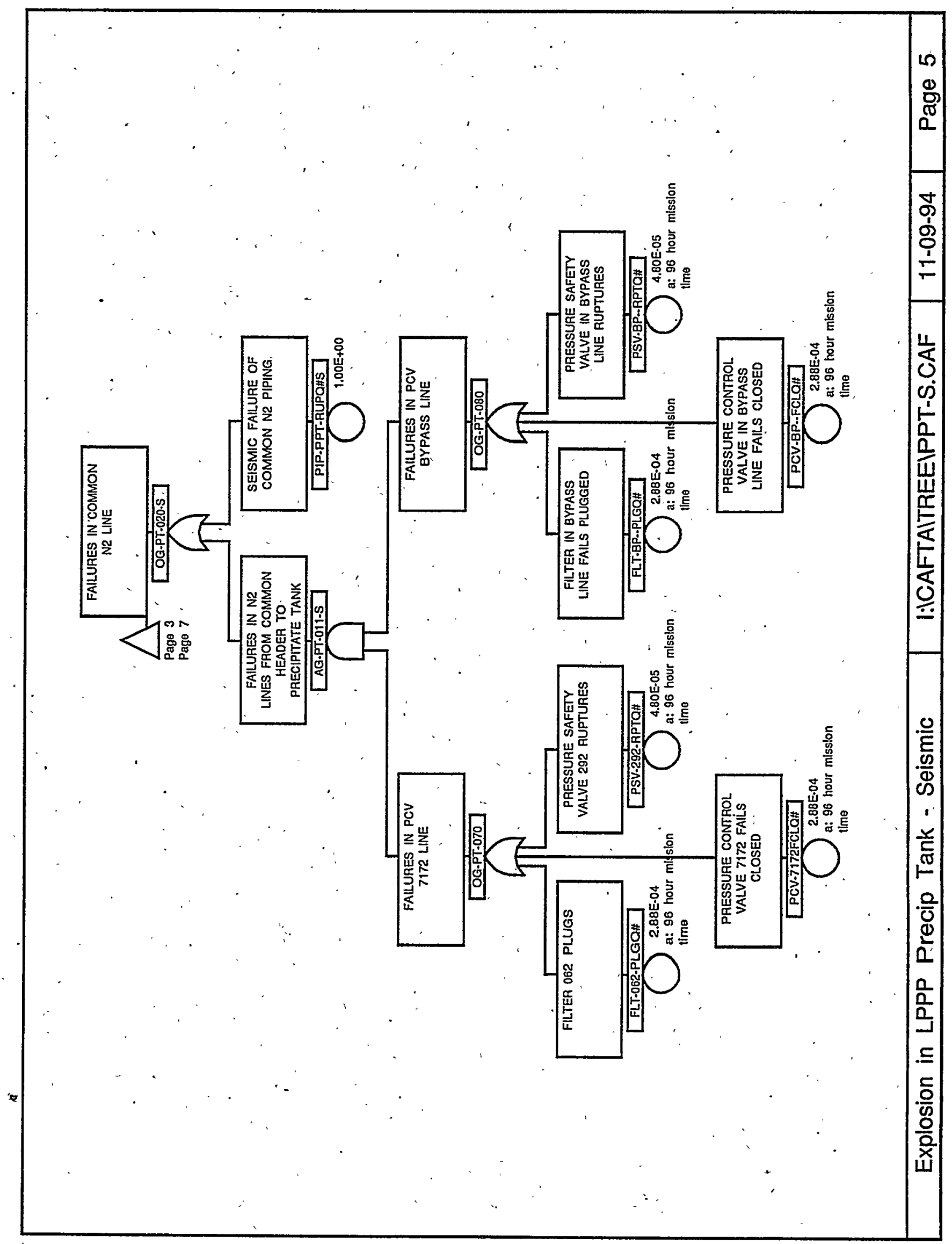


<smiles></smiles> 
X-ESR-S-00001, REV. 0

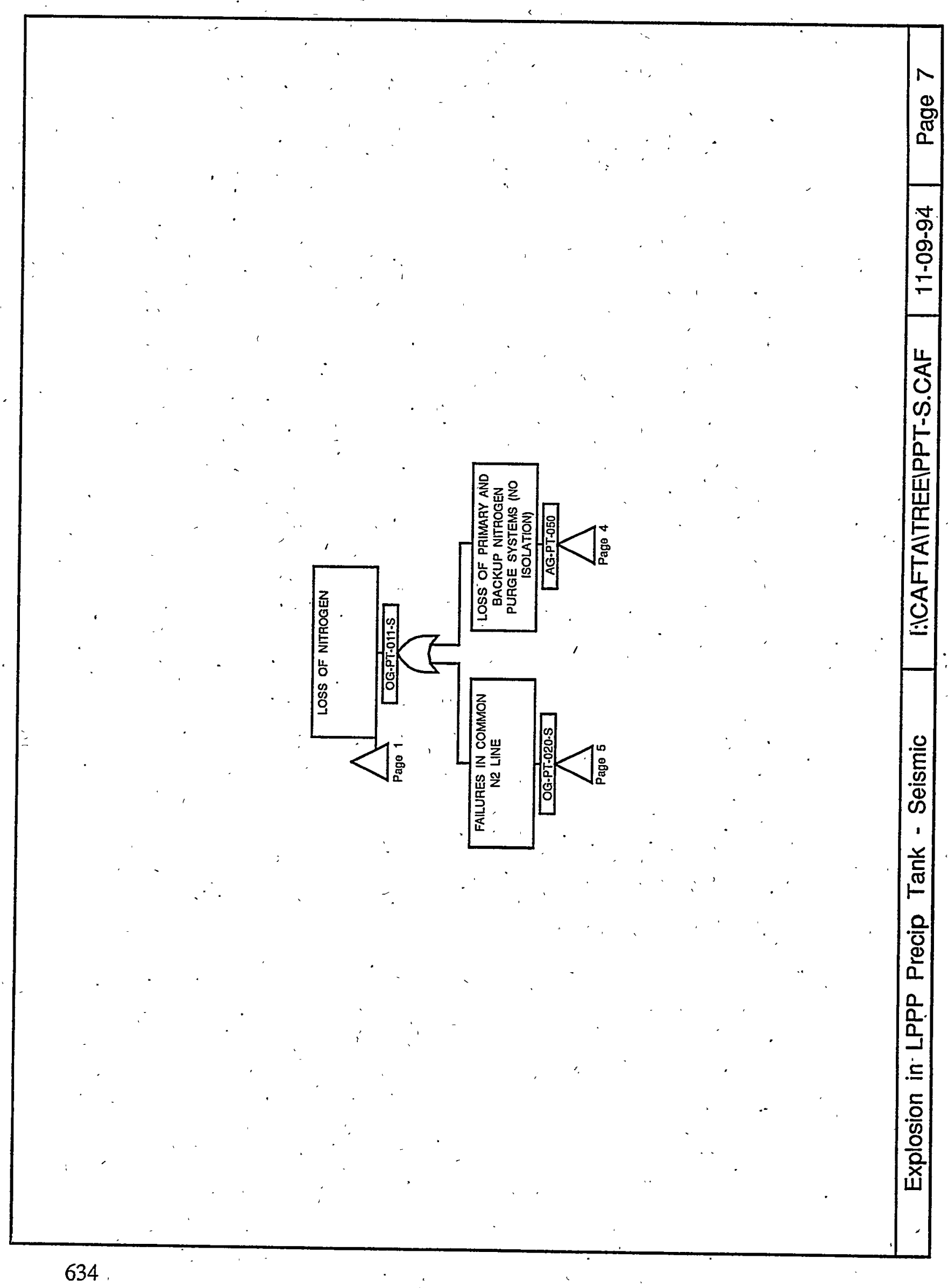




\section{OWST Explosion - Seismic}




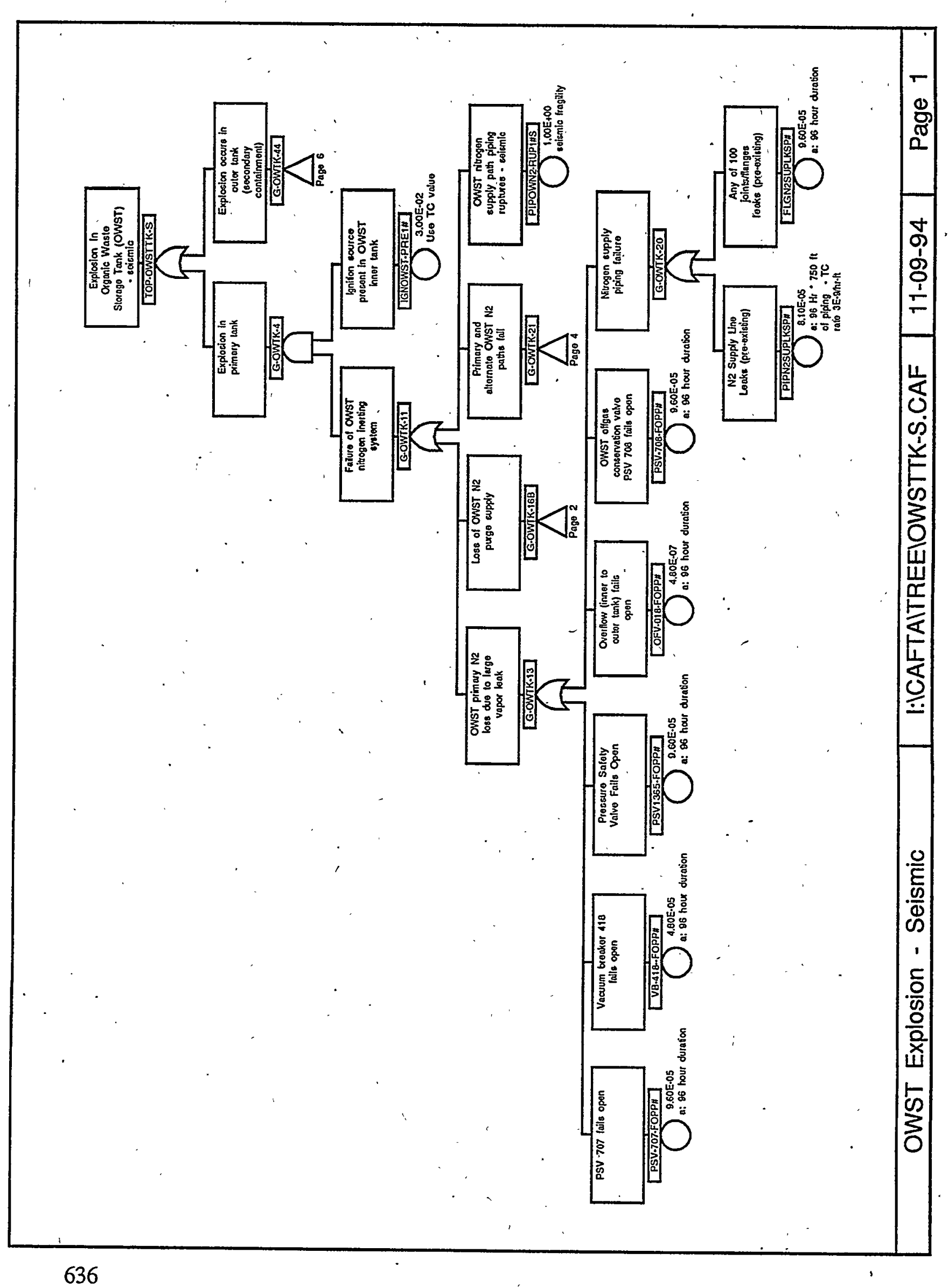


X-ESR-S-00001, REV. 0

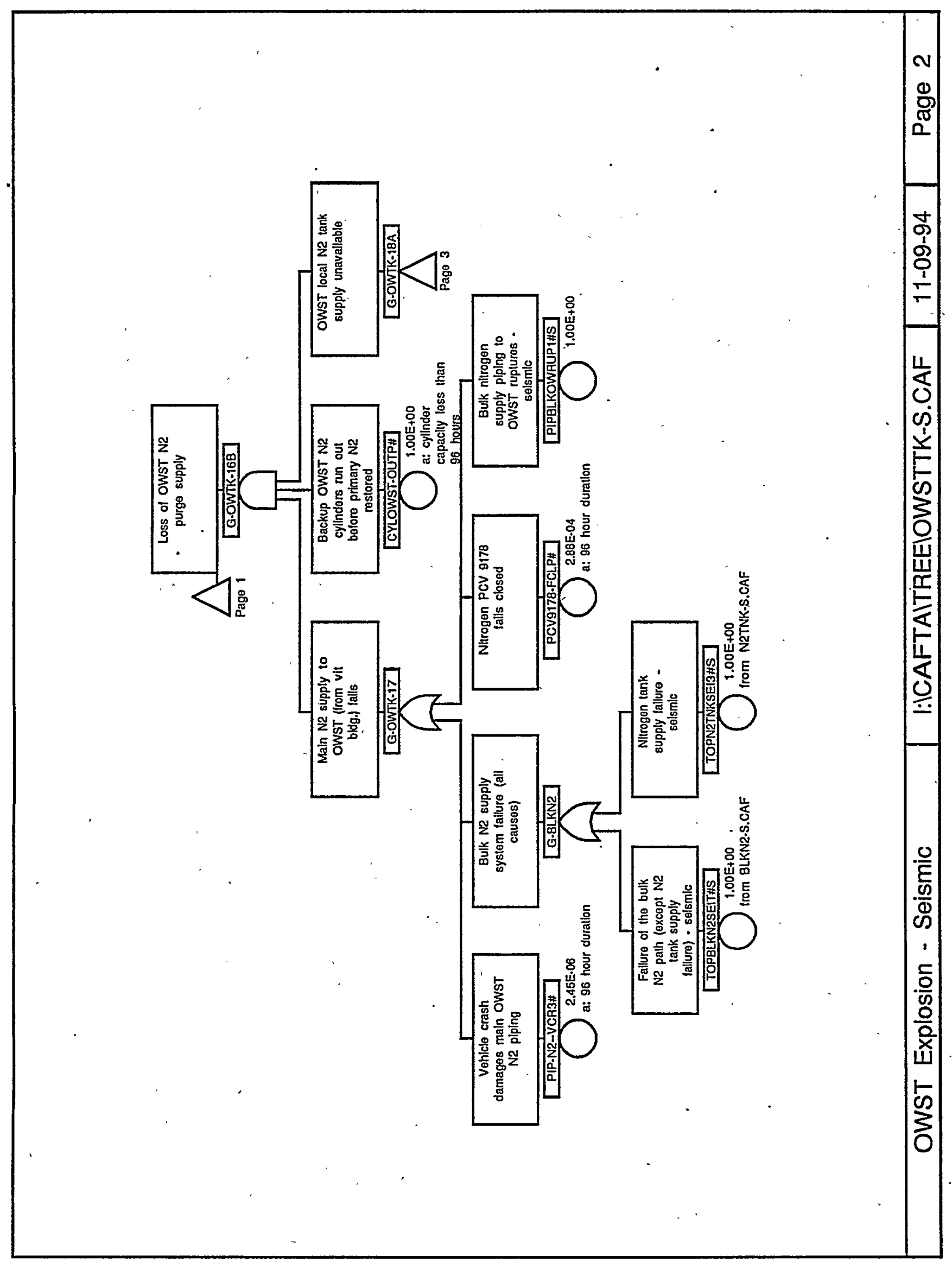




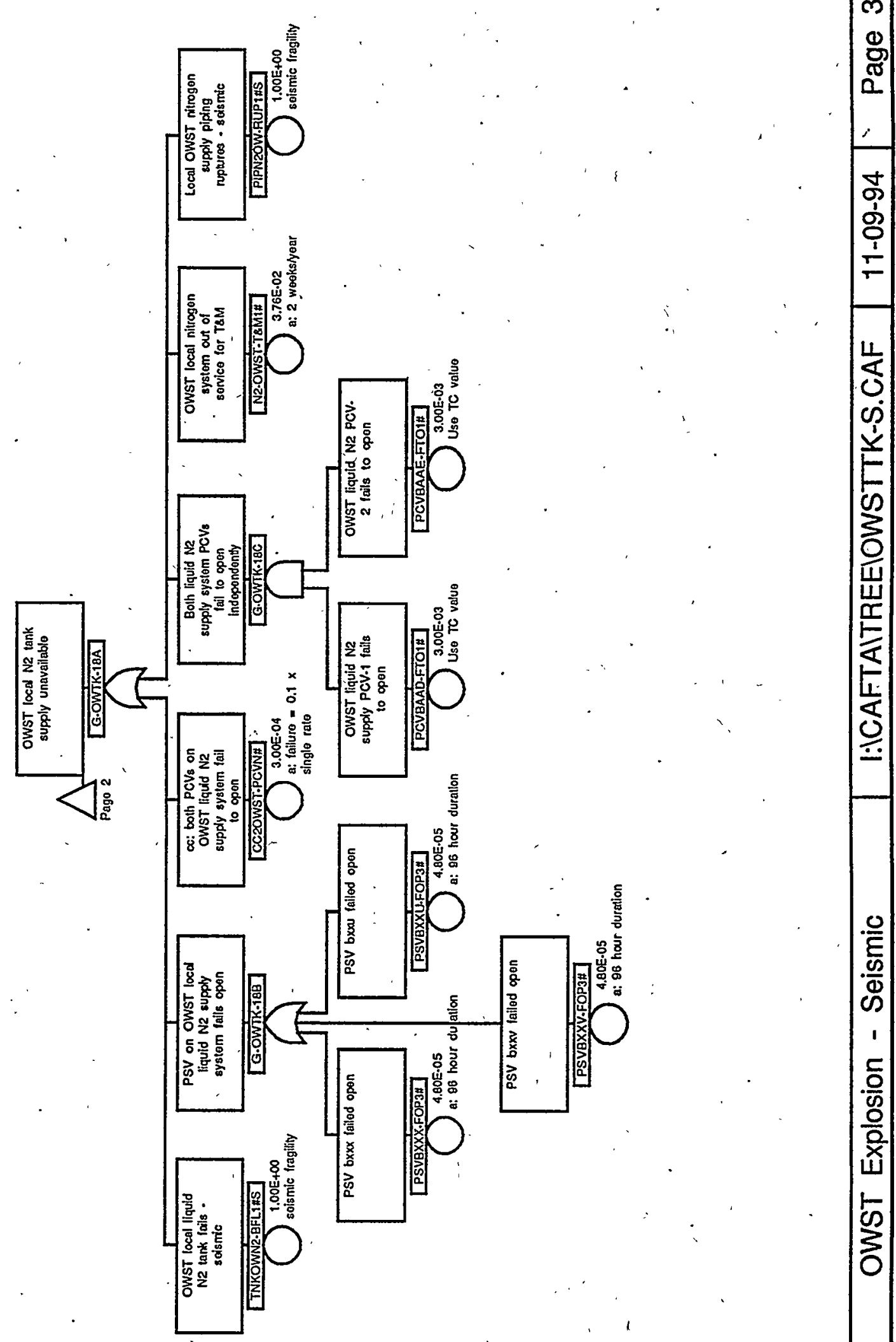




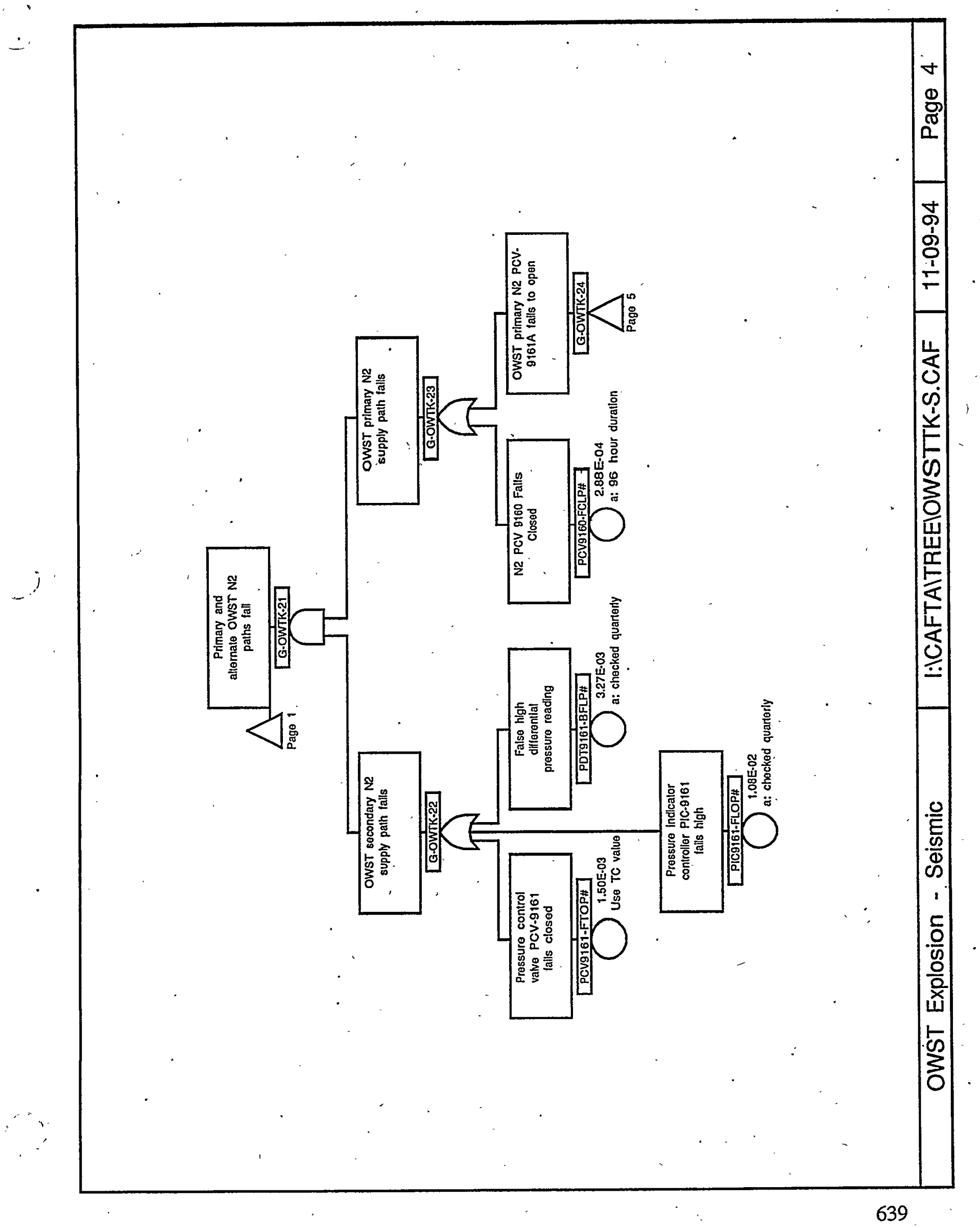


X-ESR-S-00001, REV. 0

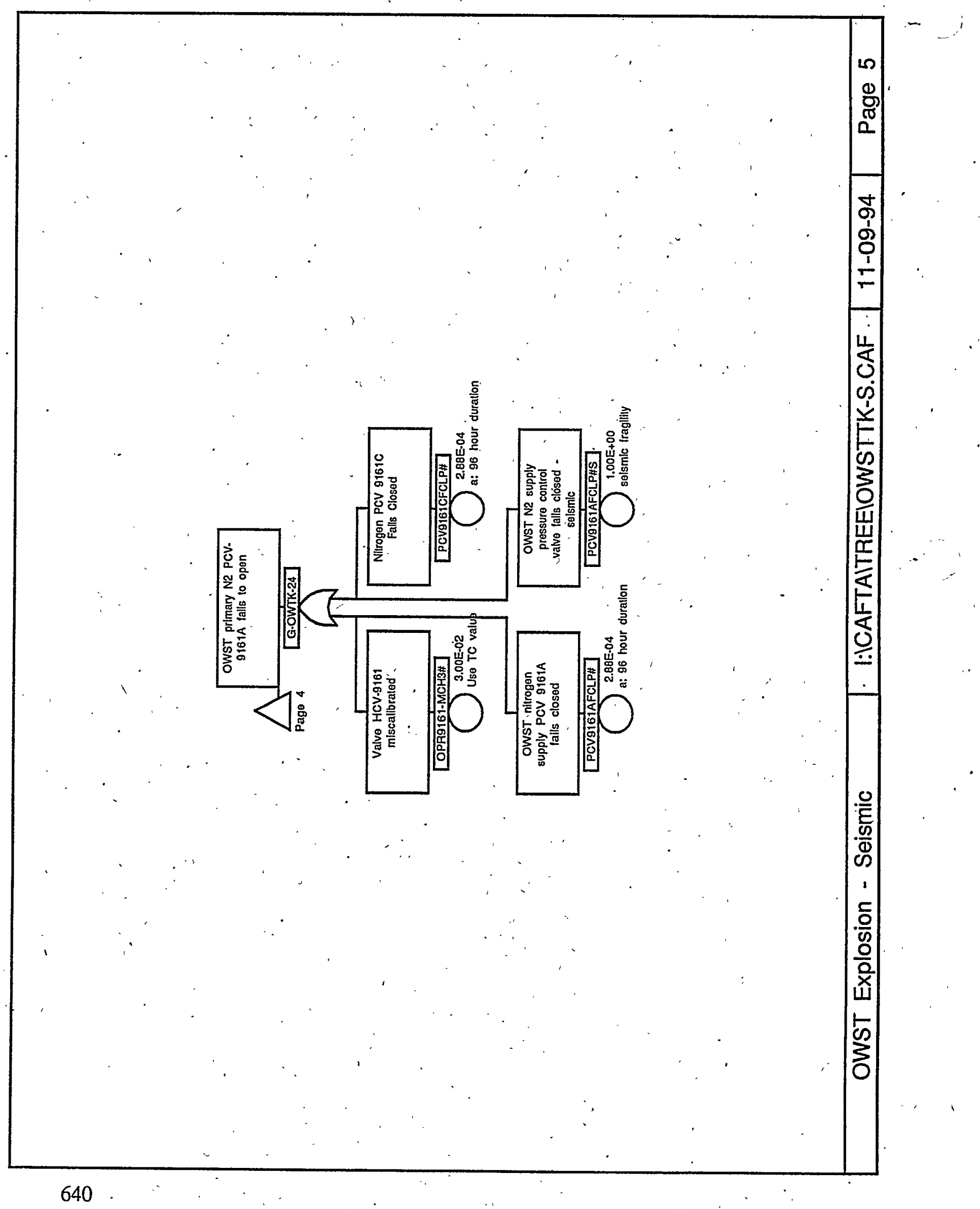




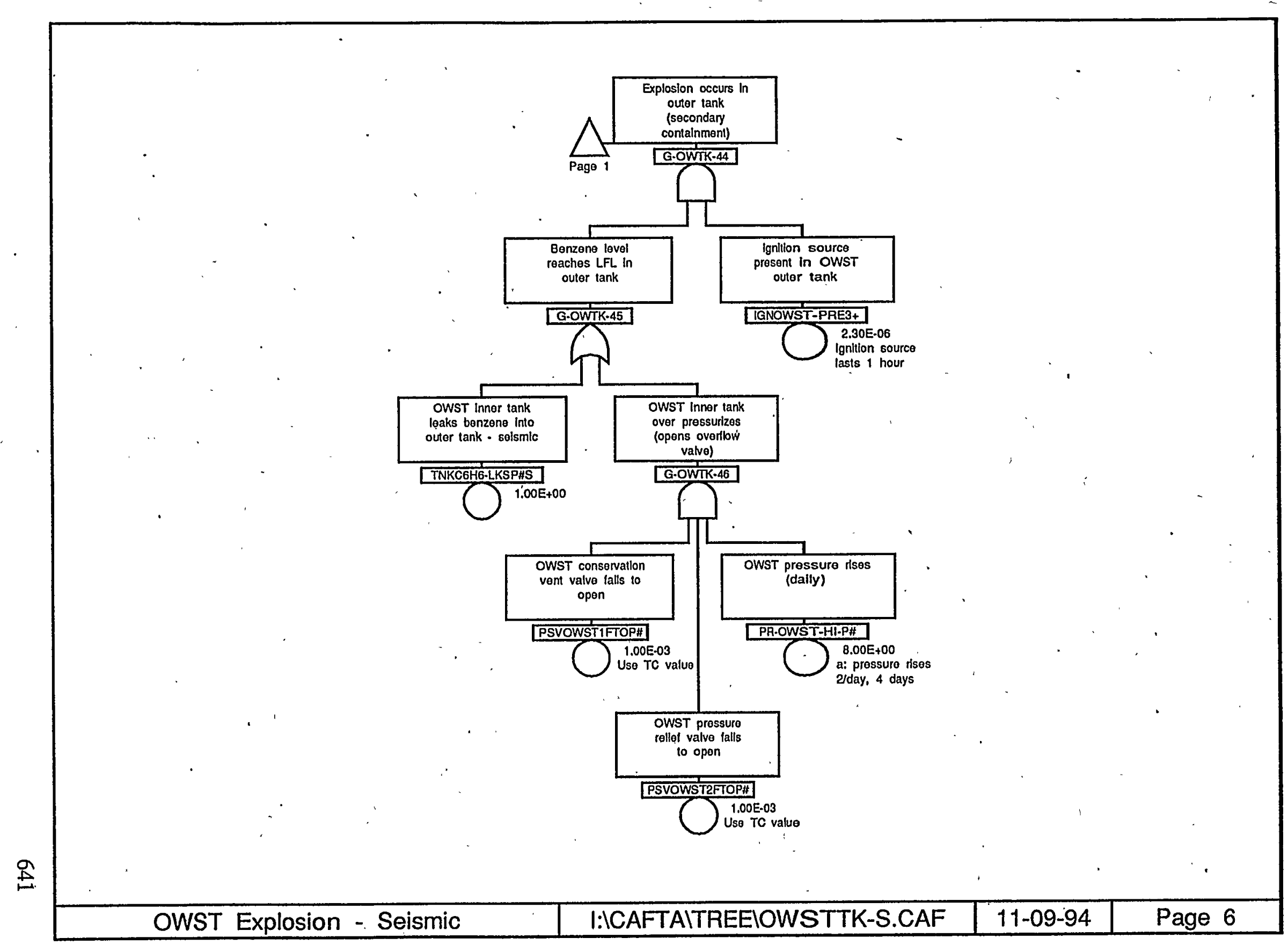


X-ESR-S-00001, REV. 0

SPC Explósion - Seismic

tom 


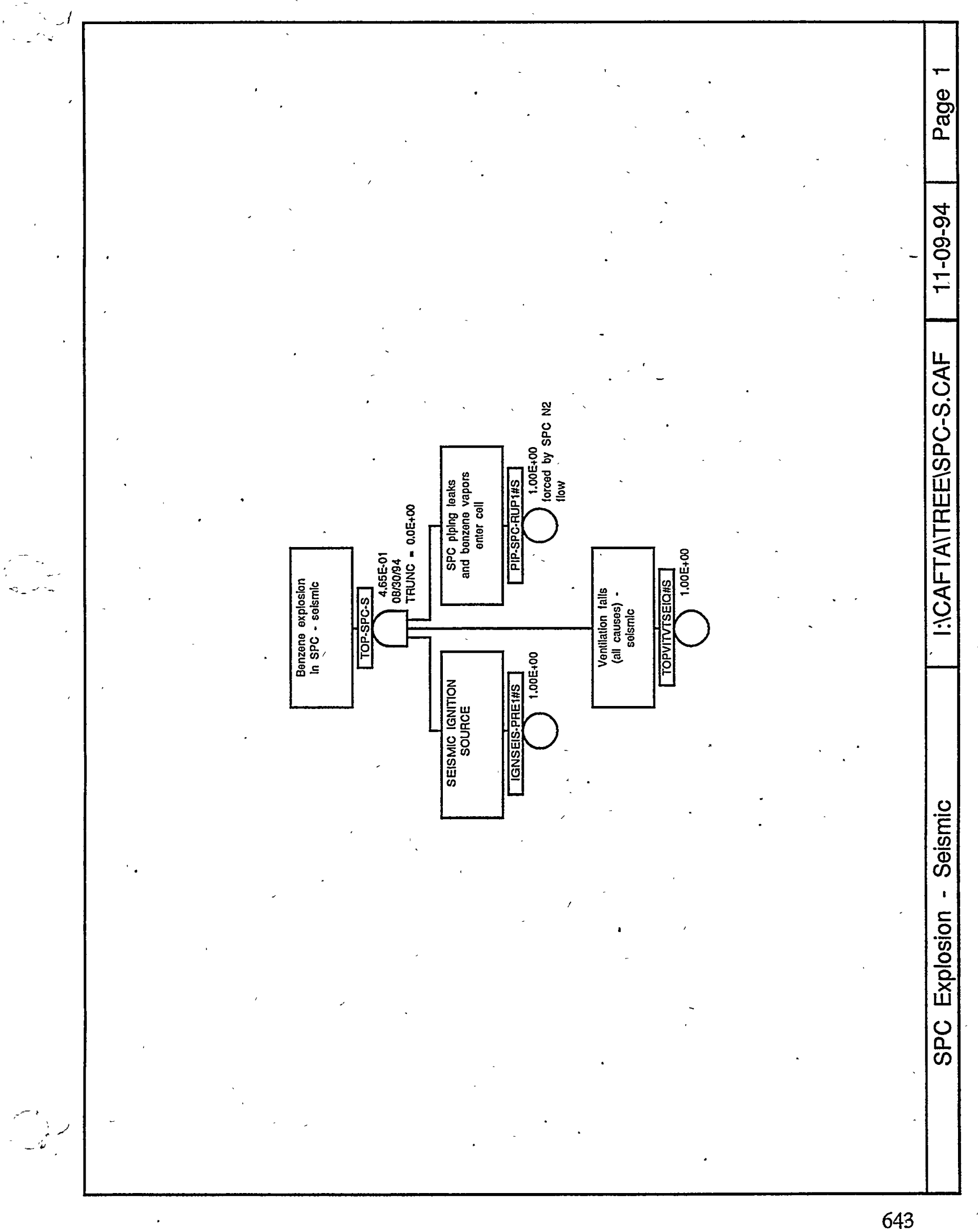




\section{Attachment 8}

\section{DWPF Basic Event Importance Factors}

The top event frequency (or probability) for each fault tree is a function of the failure frequency and failure probabilities of the various basic events in the tree. While some of these basic events contribute significantly to the top event frequency, others have less impact on that value. The relative significance of a basic event may be assessed using an importance analysis, a systematic reevaluation of the top event frequency based on independent change of individual events. Results of an importance analysis are typically expressed through presentation of importance measures, numeric rankings that represent each component's impact on the top event. A variety of importance measures may be calculated to express different perspectives of component significance. For purposes of the DWPF analysis, the Risk Reduction'Worth and Risk Achievement Worth measures were chosen to provide information most relevant to facility maintenance and improvement goals.

The Risk Reduction Worth measure is defined as the decrease in risk if the basic event is assumed to be optimized (assumed to never fail). Mathmatically, it is the ratio of the original, nominal top event value $\left(\mathrm{P}_{\text {top }}\right)$ to the reduced top event value resulting from optimization of the basic event being evaluated $\left(\mathrm{P}_{0}\right)$.

$$
\mathrm{RedW}=\mathrm{P}_{\text {top }} / \mathrm{P}_{\mathbf{0}}
$$

In the case in which the basic event is an initiating event, the failure is expressed as a frequency of occurrence per hour $(/ \mathrm{H})$. Optimization of such an event is represented by a failure frequency of 0 occurrences per hour. In the case in which the basic event is an enabling event, the failure is expressed as a probability of occurrence and optimization is represented as a probability of 0.0. Consideration of the Risk Reduction Worth measure offers insight into the level of potential risk reduction available through improvement of individual components. Events that display a large Risk Reduction Worth value are most significant in contributing to the top event value. Those events with a Risk Reduction Worth value near 1.0 contribute less significantly, on an individual basis, to the top event value.

In the unique situation in which a single event must occur in each of the failure combinations (cutśets) that lead to top event occurrence, such as the presence of the ignition source in explosion fault trees, a very high risk reduction worth value will occur indicating that optimization, elimination of that event, would result in elimination of top event occurrence $\left(P_{0} \rightarrow 0 \Rightarrow \operatorname{RedW} \rightarrow \infty\right)$. For this special case; the Risk Reduction Worth is defined, by convention, to be $1 / \mathrm{P}_{\text {top }}$.

The Risk Achievement Worth measure is defined as the increase in risk if the basic event is considered to assume the least optimum configuration. It is expressed as the ratio of the increased top event value that results from increased failure potential of the selected basic event $\left(\mathrm{Pl}^{\prime}\right)$ to the original, nominal top event value (Ptop).

$$
\text { AchW }=\mathrm{P}_{1} / \mathrm{P}_{\text {top }} \text {. }
$$


As previously stated, initiating basic events are expressed as a frequency of occurrence $(/ \mathrm{H})$ and reliability reduction of such events is represented by assignment of a high frequency, $1.0 / \mathrm{H}$. Enabling basic events are expressed as probabilities of failure and reliability reduction is represented as certainty of failure, an assigned value of 1.0. Consideration of the Risk Achievement Worth measure provides insight into the significance of assuring performance of individual components. Basic events that display a large value of Risk Achievement Worth contribute most significantly to the top event value. A Risk Achievement Worth value near 1.0 indicates that failure of that component has little impact on the top event.

Two importance ranking tables are included for each DWPF fault tree. The first presents a list of the fault tree basic events in order of descending Risk Achievement Worth value, the second is ordered according to descending Risk Reduction Worth value. In each, the basic event is described and the associated frequency for initiator events or probability for enabling events is included. Due to the method used for fault tree development, it is common for a given event to contribute both in an initiating capacity and in an enabling capacity. For ease of interpretation, these contributions have been combined. Components that function as both initiators and as enablers are identified in the importance ranking tables by a "-C" at the end of the basic event name and the importance measures associated with these components include both aspects of performance.

It should be noted that the importance measure of each event is an independent, noncumulative representation. It is not appropriate to consider the rankings in a summary manner as representation of the combined effect of multiple basic event value modifications. The impact of multiple event changes can only be determined through full reevaluation of the top event value. 
$X$ ESR $=S=00001$, REV. 0

$\therefore \quad 3$

(This page is left blank intentionally.) 